$$
\text { DOE AL-050509-AdLDI }
$$

BADIOLOGIC CHALACIEIZATION OF IHE MESTCN FAT, ITAB,

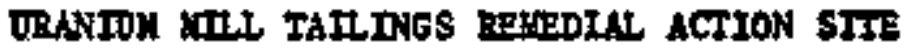

\author{
John R. Ludien \\ Beadir Field Engisenting Corperetion \\ Grand Jopetion Operations
}

Janoury 1985

EUSTRIBUTION OF THIS DOCLMENT IS UNLMMTID O

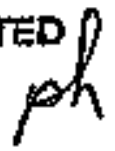

Propaced for

T.S. Depurtwent of Enezcy

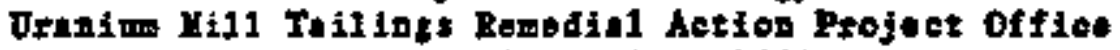

Albsqueque Operatians Offieo

Dader Contraez No. DE-ACO7-766ro1664

U.S. Depertaent of Energy

Grand Janetion Projects office

Gend $I$ unetton, cal tretso
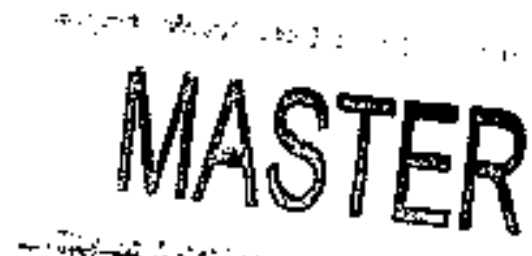

$::$ 


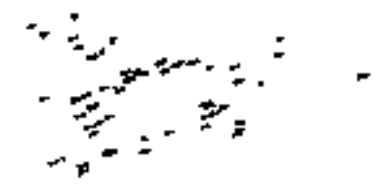

\section{RECENED \\ AU6 07 1995 \\ OSTI}

AODENOUM DI

RADIOLOGIC CHARACTERIZATION OF THE MEXICAN HAT, UTAH, URANIUM MILL TAILIHGS REHEDTAL ACTION SITE

(Note: The data on microfiche and the Plate 1 referenced in this document are avatrable tn the DOE UHTRA Project office, Albuquerque, New Hextco.) 


\section{DISCLADMER}

This repart was prepared as an account of work spansored by an agency of the Iniled States Goverament. Neither the United Stotes Govetament nor any egetacy thereof, nor any of their employees, makes any warranty, express or imptied, or assumes any legal liability or responsibility fat the accuracy, completeness, of usefiblness of any information, apparatus, product, or procest disclosed, or represents that its use woukd no infinge privateiy owned rights. Reference herein to any speciffe conmercial product, process, or service by trade name, trademerk, mantufacturer, ar otherwise does not necessarily constitute or inapty jis endocsoment, reconmendation, or favoring by the United States Gorernment or any agency thereof. The views and opinions of authots expressed berein do not necessarity state or reflect those of the United States Gorerament or any agency thereof. 


\section{DISCLAMMER}

Portions of this document may be illegible in electronic image products. Inages are produced from the best avallable original document. 


\section{cowitaits}

1.

ITISODJCEON

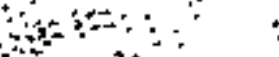
in

2,1 Loction .......................... 1

2.2 Gootoste and Egdzolotic Setting .............. 1

2.3 Sito History .......................

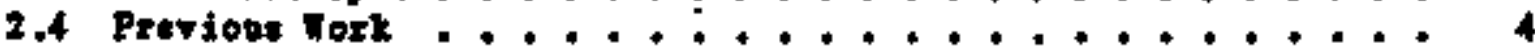

2.5 site condttiots ....................... 4

9.0 PROCEDURES ............................. s

3.1 Aree Dositations $\ldots \ldots \ldots$

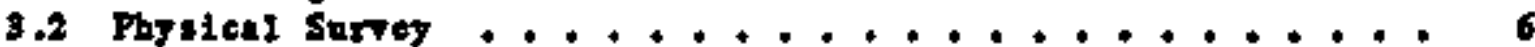

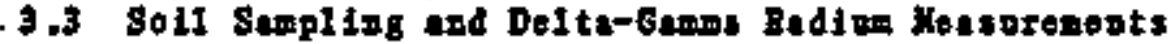

at Gridpointa. . . . . . . . . ........... 6

3.3.1 Soll Sampling..................... 7

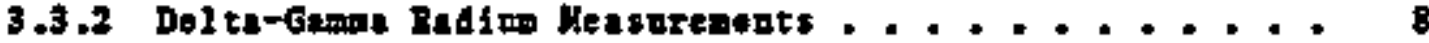

3.4 Arroyo Delte-Gamen Keasmeants. . . . . . . . . . . . 9

3.5 Borebole Sappling, Drillint, end Losglas . . . . . . . . . 10

3.5 .1 sumplins ...................... 10

$3.5,2$ De111128 ......................... 10

3.5 .3 Geophysical Logsiog .................... 10

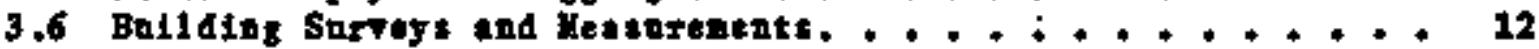

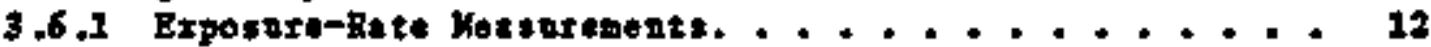

3.6 .2 AIphr Heaspements. .................. 13

3.7 Beckgroved Mearirgenerts. ................... 13

4.0 RESULTS ................................ 24

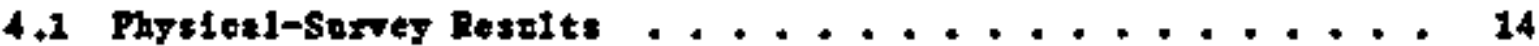

4.2 Soll Sempligg Posults ................... I5

4.3 Del ta-Gamie Hessoreacats .................. 18

4.3.1 Soll-Moluture Cotzetion Fattars ........... 18

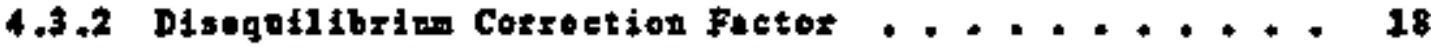

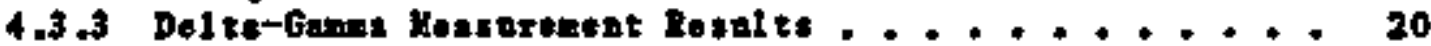

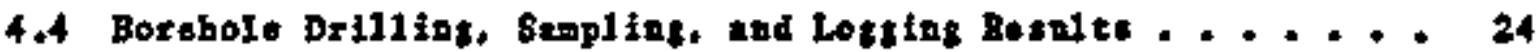

4.4.2 Drilligg and Stoplint Besults .............. 24

4.4.2 Goophyaloal Borebole-Logkits Resalts ......... 25

4.4 .2 .1 Borthole Losging with the

Treok-Monnted syetem ........... 25

4.4.2,2 Borthole Lotsing tith the BAschi

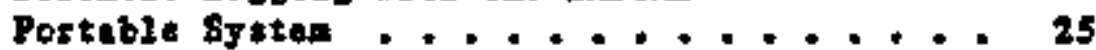

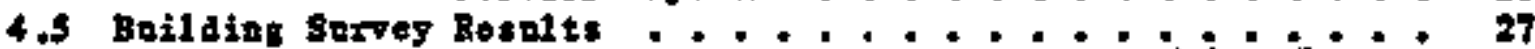

4.6 Beckgroard Cherecterization Results . . . . . . . . . 27

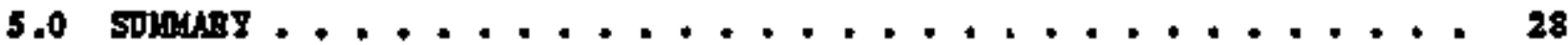

6.0 ACONOTLEDEGTHS ......................... 29 


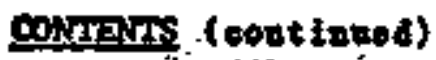

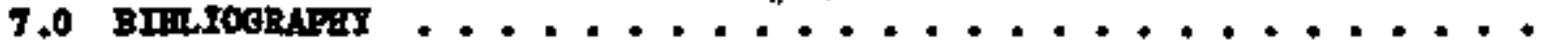

Pare

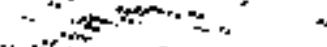

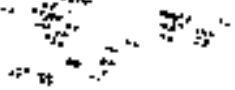

30

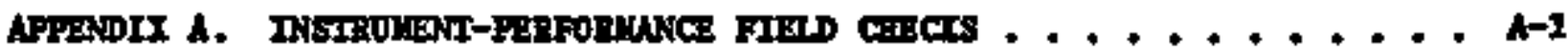

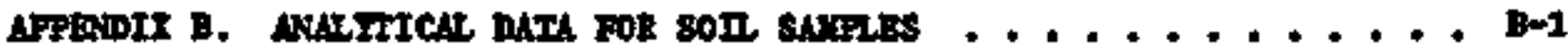

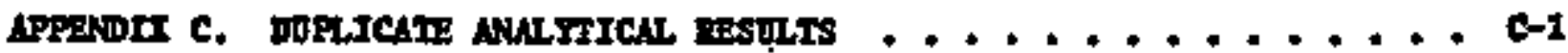

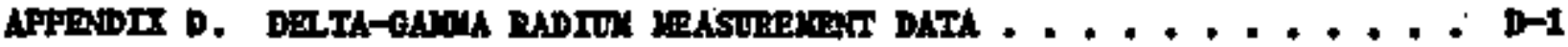

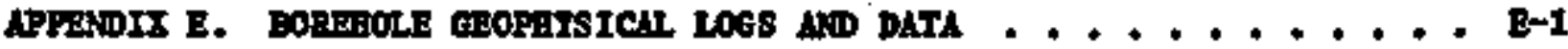

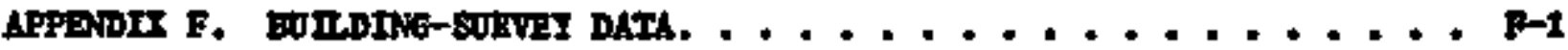

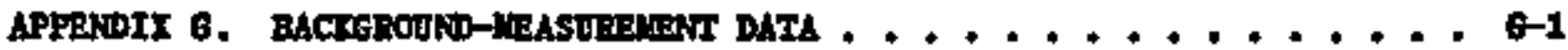

\section{EITSERTTONS}

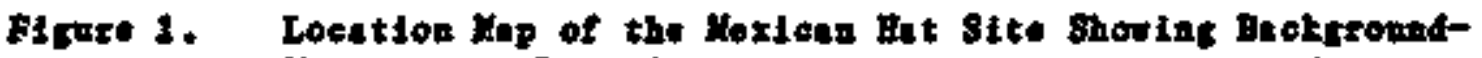

Measerenert Loentions .................... 2

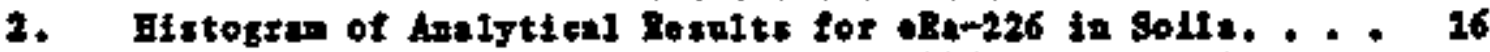

3. Locations of Soll Seples Eeplas eBt-226 Concentratlons

Greteter Than $\mathbf{p C H} / \mathrm{s}$ at Dopths of 0 to 6 Inches...... 17

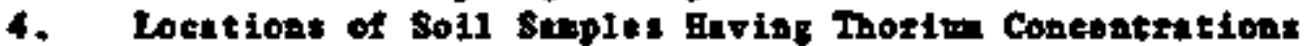

Gronter Than 15 pro it Deptrs of 0 to 6 Inehes . . . . . 19

5. Locktiors of Detta-Ganes Hessorenents Greater Thap

5 pCl(eRt-226)/s . . . . . . . . . . . . . . 22

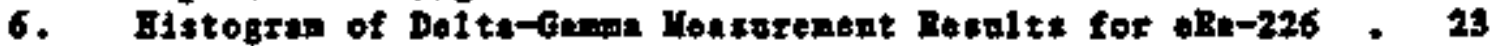

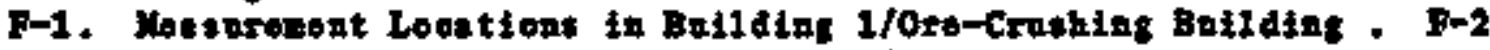

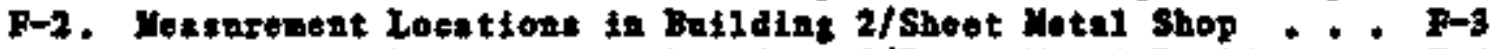

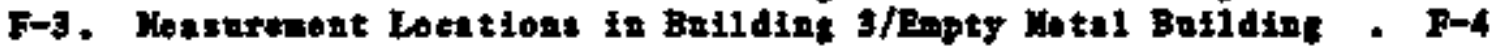

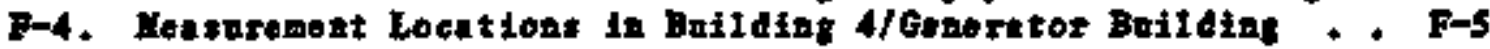

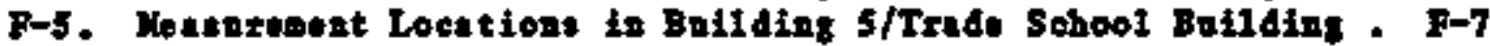

F-6. Meaturenest Locationt in Boilding 6/Brlet Brildins * . . F-8

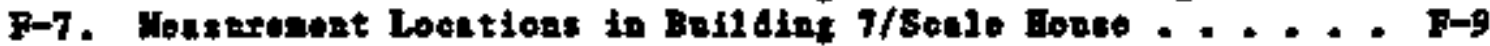

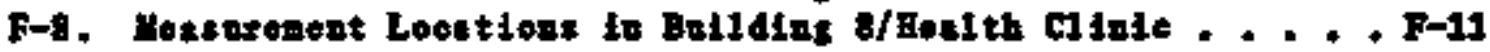

\section{TARL娶}

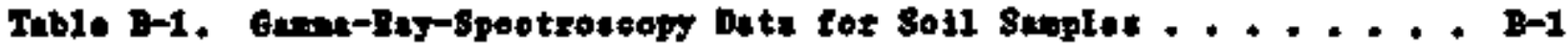

B-2. Lots-On-Dryiat Besult* . . . . . . . . . . . . . B-8

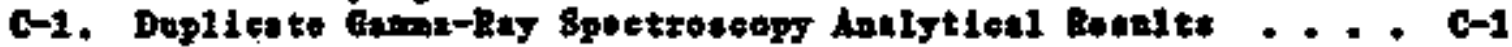

C-2. Soll \$neple/Deplitete Stoplo Compirfion .......... C-2

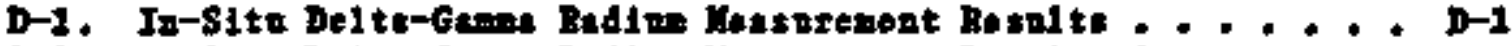

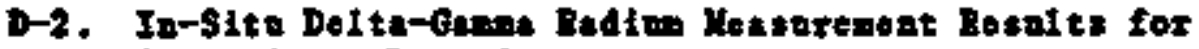

Approxinate Loektors .................. p-4

E-1. Borehole Date frod spectral systen . . . . . . . . . E-2

E-2. Borohole Deti frot total-Count 8jotin ........... E-3 


\section{ThBLS (oont inged)}

$\therefore$

Pase

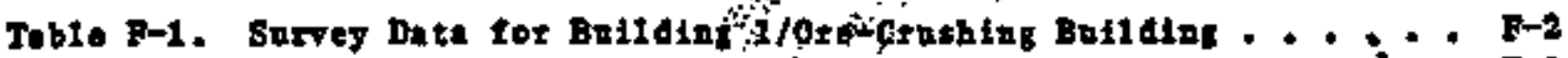

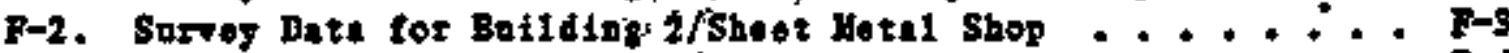

7-7. Survey Date for Beilding 3/2epty Hetel Building . . . . . F-4

F-4. Survoy Date for Building 4/Geserstor Boilding . . . . . . p-s

7-5. Survey Dets for Belldins s/Tredo sehool Bullding . . . . F-6

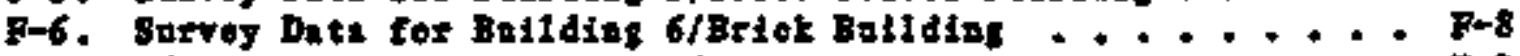

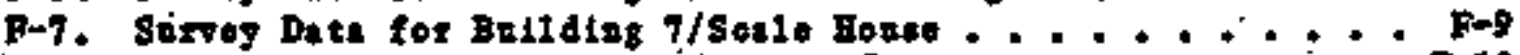

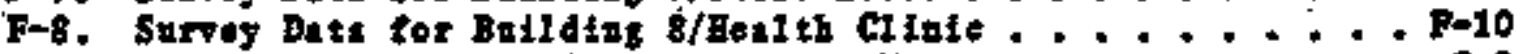

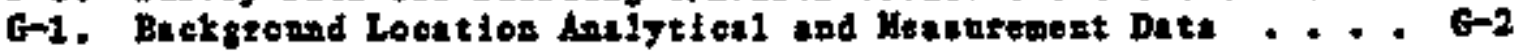

\section{PNATES}

(II pocket, bect iasta eoper)

Plate 1. Bese Ho for the Hexioas Hat, Uteb, Sito 


\subsection{INTODNCITON}

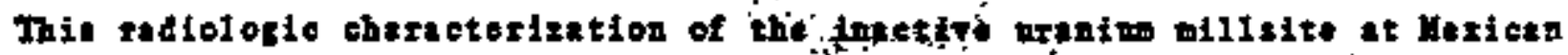

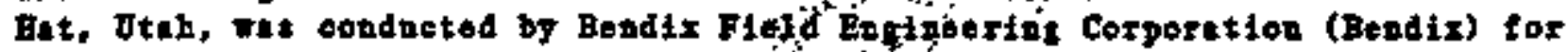
the U.S. Depkrtment of Energy (DOE), Gijad J wetion Projects Office, in

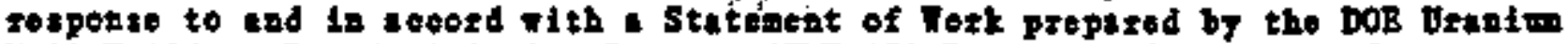

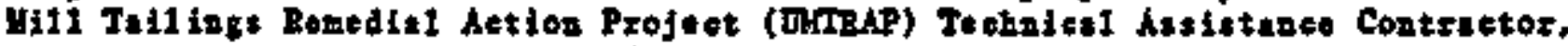
Jaeobs Englneering 6toop. Ine. (JEC). The objeotive of thit project vat to

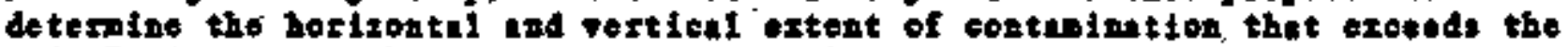

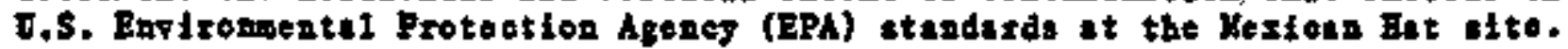
The deta presented th this sepozt aze zequited for oberzeterizetion of the

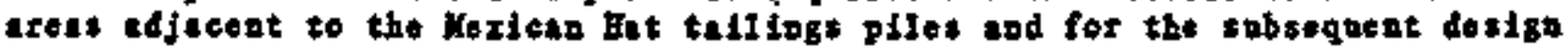

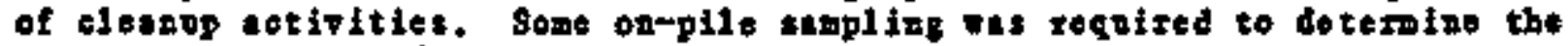

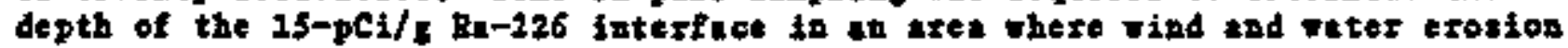
his takes plice.

An orlertation risit to the site was condected on 4-8 Juse 1984, In copjuction wth Jacobs Engineting Grotp, to determine tho epproximate extent of contar inetion thet orceds EPA standnris surtouding the piles, to establesh surver control points and bastines for later estrblishent of grid lints, to astes: drilling tequirements, and to gathor backgrond radiologic and geochemical date

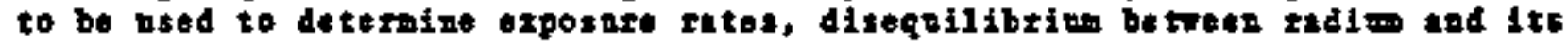

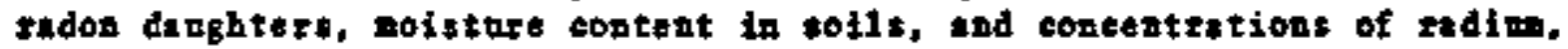
thorstm, and potastitim in sol1t. The faformation geinod froth this risit was used in determining the seope of rork required for the radiologic ste theract terization.

Fieldwerl at Werican Hat wa confocted on 10-24 september 1984. Radionetzic nesspesents, soil sample, and bullding sorvey vere eompleted on 20 septenber and drilling was completed on 24 Septesber 1984.

\subsection{BACKGROOND}

\subsection{LOCATION}

The Intctive nranitu villsite is loeeted on the Navajo Nation (Indion Resegration) eppeoximetely 1.5 miles couthest of the town of Hexiend Bat. Jtah (soe Fignte 1). The gitil housing arte, now the commenty of Filchite, 1s located

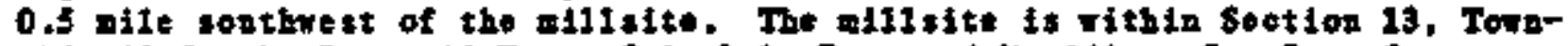
ohip 42 Soutb, Raste 18 Enst, Salt Lake Bane and Heridian, San Jun Coutg, Dtak.

\subsection{GEOLOGIC AND EYDROLOGIC SEITHNA}

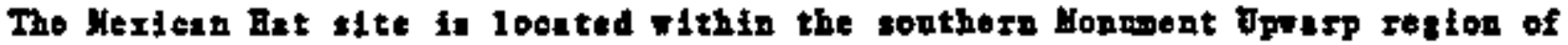
the Colorado Pletead. This portion of the acrtb-soth elongted uptift cortalat soperal spall ptanging antielines. Tho millate is in the Herloan Eat syodine which is a skerp cornfold between the gaplee fotioline to the oast and

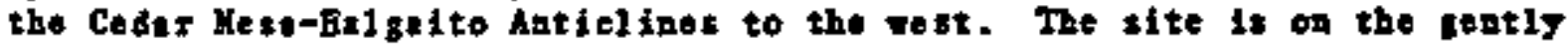
estsodipping (a to 6 dogreos) wont 1 imb of the syacline. 


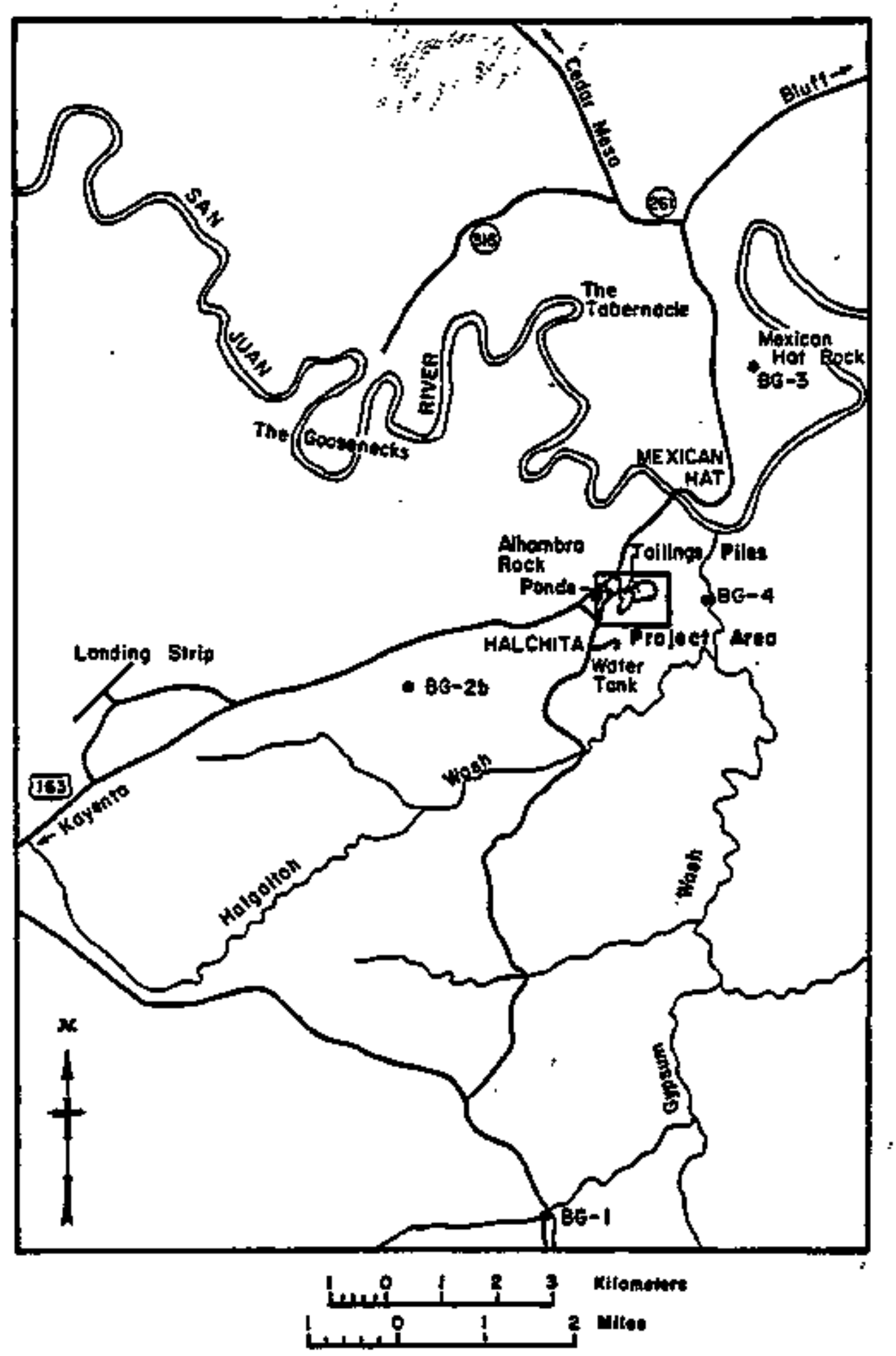

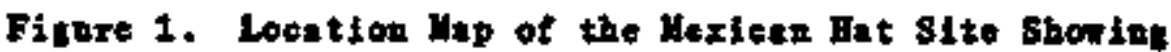
Beckground-Wlosureneat Locations 


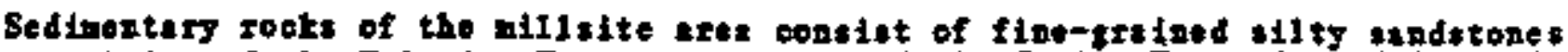
and shilet of the Ealgatto Jongue. a-medber of the Cotler Formetion of Penzsylranien age. The te beds ate a distinetiye, sed to chocolate-brong in color ad

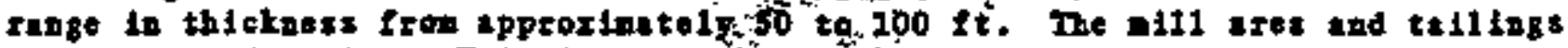
plles are nadezlain by Faltalto redbeds," To' the east of the tallings piles in the bottops of the asjor trroyes drifing the site cre resistant 1 inestone bed, frobubly pert of the Bico Formetion (or lactes of the Cutler Formation). Thich nodorlie rocks of the Balgatto Tongue.

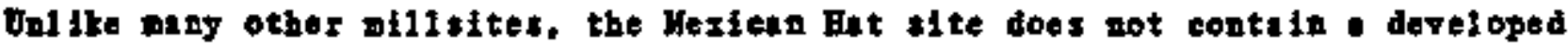

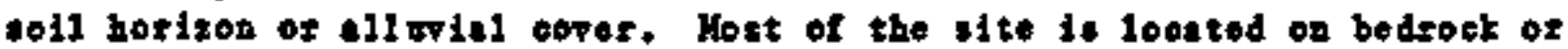
Testhered bodrock. Acomalations of neconsolidated auterial greater then $2 \mathrm{ft}$ thick are meinly rostrictid to man-made mixtmzas of bedrock. tallings, ore, tod debris. Dre to tho oolor contest between the Belgalto redbeds sod opptewinited meterials (often white to tray). it 1s beteraliy not diffiont to idestify contumintiod antorial*,

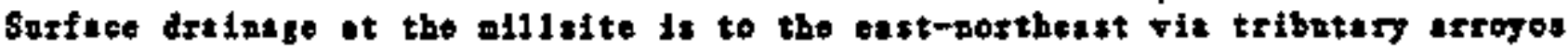
Thieb drafp isto Gypan Tath loss than oxe aile from where it enpties into the Sen Joun River. These errogos we osally dy, with weter flor zestrteted to Infrequent periods of hear reinfell.

Dte to the fine-trained nature of the Enltaito sendstones and sholes erd to the

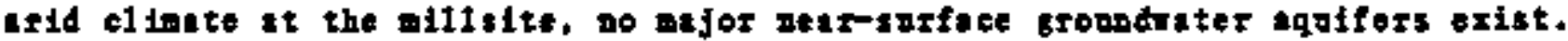

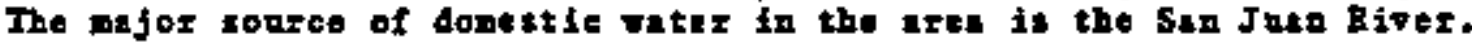

\section{2,3 STHE HTSTOR:}

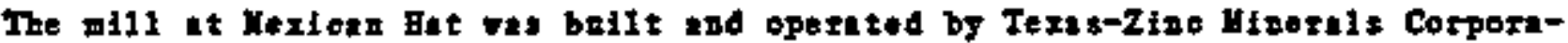
tion from 1957 to 1963 . The ofll was aegtifod by the Atlie Corporation in 1963 ad wan oporated by them netil operetionr cented fa 1965. A solforic actd plent continued to opertse at the ilte gnttl 1970. After the will elosore, control of the cite Ievertad to the Nayejo Netion. The ste res osed for a shert pefiod at a trede ebool (eerpegtry, velding, aleteleal, oto,) opersted by the Dtab Ttades Comistios. Preseotly tbo ouly costineonsty oconpied pos-

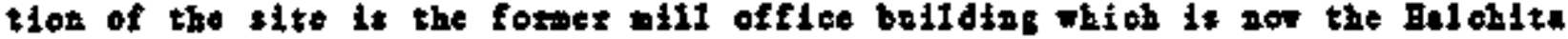
Besl th Center.

Wrah of the oxe proessed at tho aiIl cawe frod the Fite Caryon alstriet of

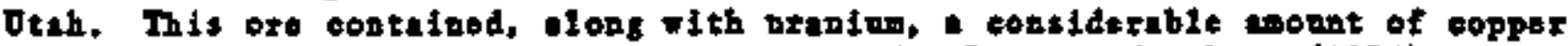
solfidec frow which copptr wis liso rocovertd. Bzors and others (1974)

deseribed the proess of recovery as a exoth-tiotation process followed by acid 164hing. Tho letched flotation concentrates were flltered to recover a find

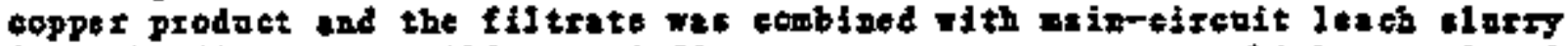

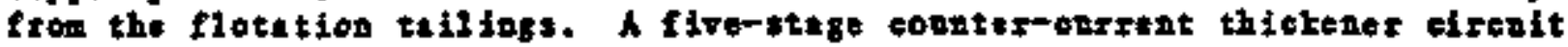

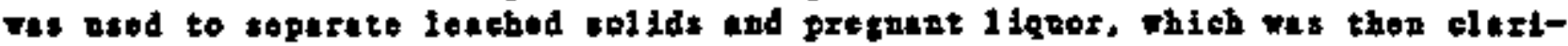
ilod before solveat extrection. The loeded organic ootront was strippted with

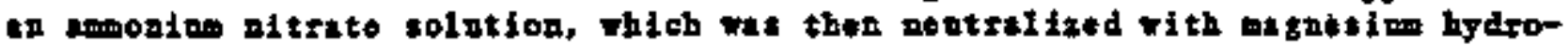
ride to procipltete the elosi orante prodect.

The tefling sere distribstod to two plles (referred to st the ppper and lorer pilea) by a cyclone separator which bojlt of the das with coarse, sind and 


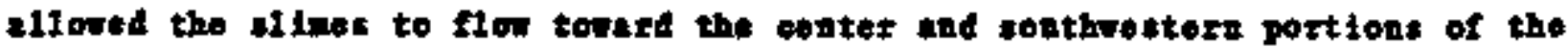
piles.

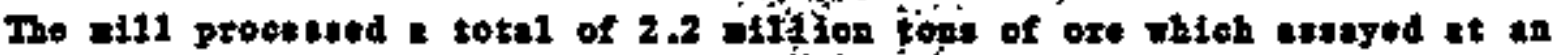

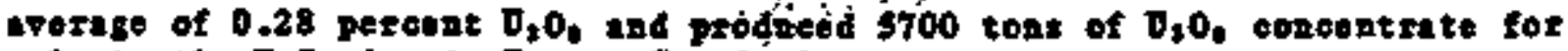
ato to the U.S. Atonic Enerty Comisision.

\subsection{PREVIOES TORK}

Priot to the wart of floldwork for thit thefweteritetion project, i thoroogh

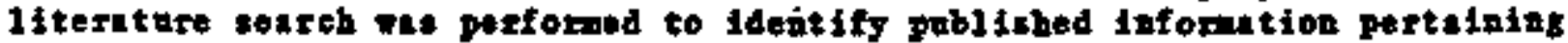
to remodilt action at the Nexion Bat alte. The resolt: of thit weareh are prosented in the bibllograply at the ond of thit roport (80etion 7,0$)$.

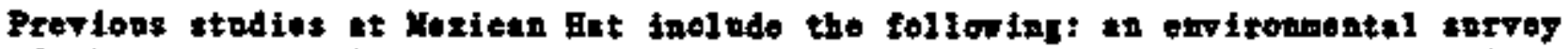

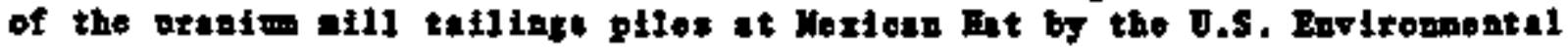

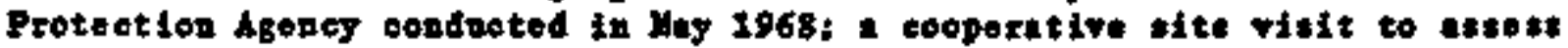

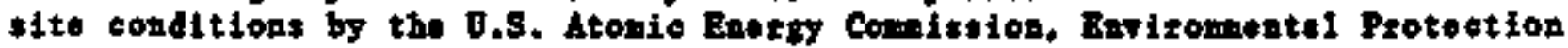

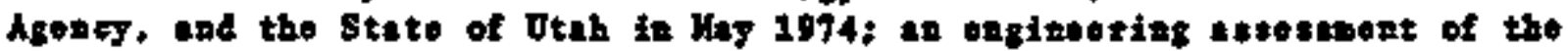

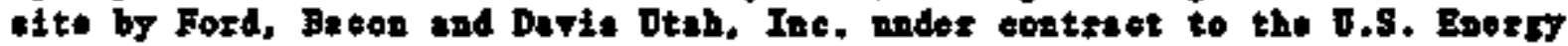

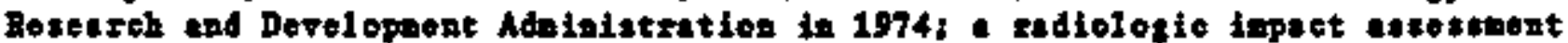

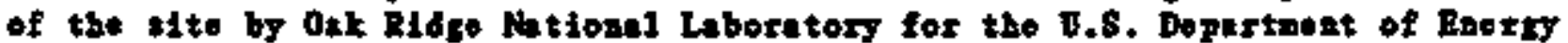

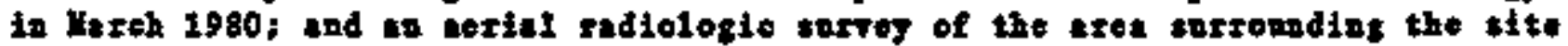

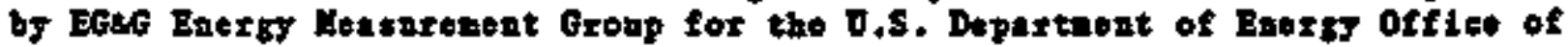
Opertionel sefoty In Suptember 1980.

\subsection{SITE CONDITIONS}

The reatipe of oight alli budldings exist wt the stec. Of the of phe brildiagt

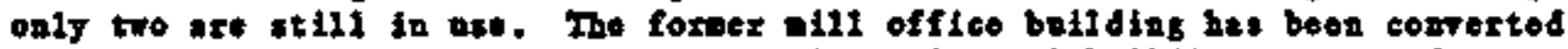
to bent th center and is in good condition. A total boilding onee goed al

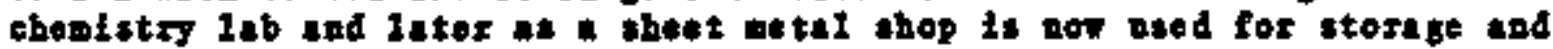

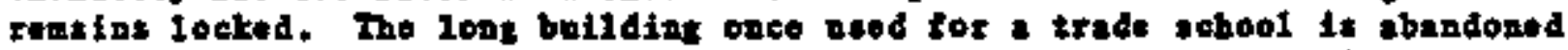

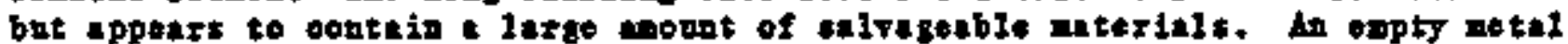
ttorage shod, in good condtion, aleo appears to be salrageable, Other buildings including the senlo house, ort-crnthing boilding. and generstor beilding are ither oontentated of do not land thentelves to strage.

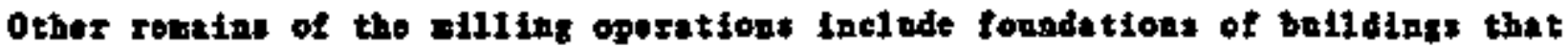

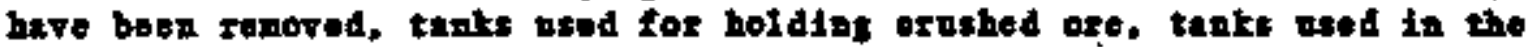

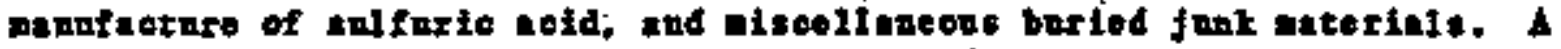
sewage poud, water libp, and olectrical traneformer are also present and in we st the itte.

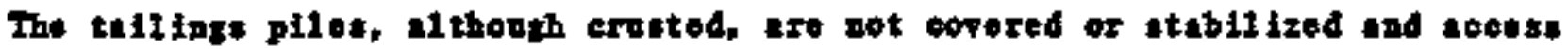

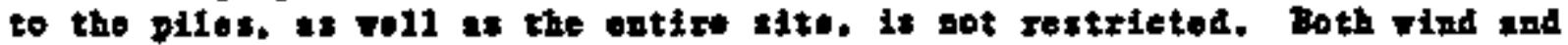

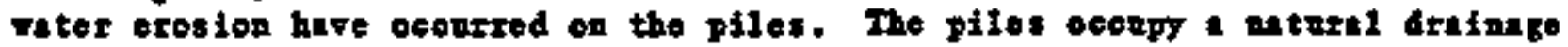
iref and al thotgh beras and dikes were boilt to reduee the amount of tallings being vashod of blow off of the piles. erroges dowatruan fron the pils contein teiliagt tor coagiderable distance. The provalling ind direction end drainige diroction aro both to the northost. Therefore ath aree surrond-

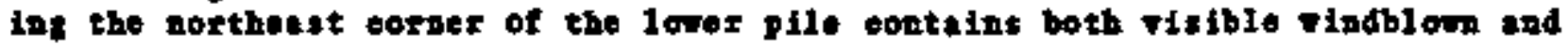


water-tensported talifrgo contabetion. Ortward frow the pilo to the cast

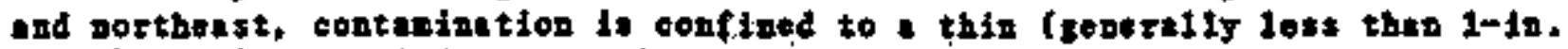

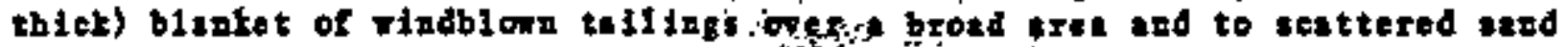

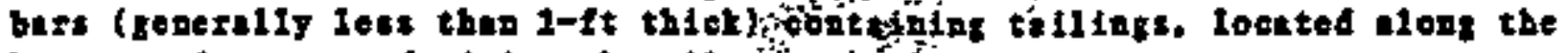

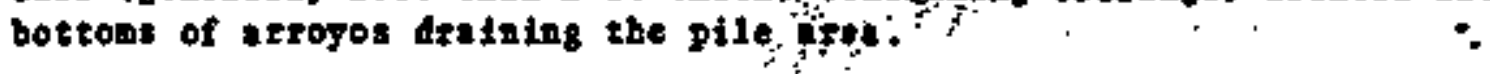

Dee to the rarisble topotzaphy at the stte (rolligg Hills, werron ridges, and

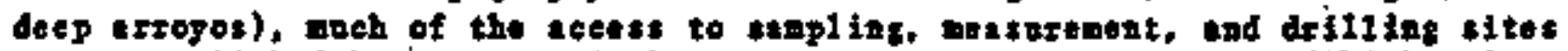

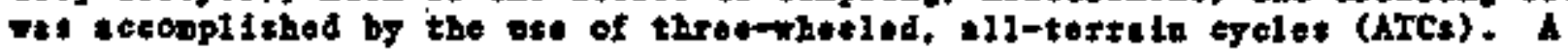

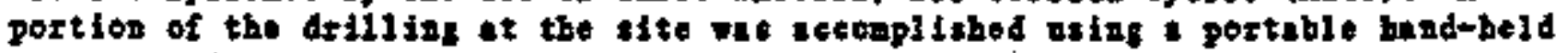
sta-porerad anger.

\subsection{PROCEDURES}

Tho measprewent techniques, Instrumenteflon, end procodures asto for the Kexicen Git rxdiologle charecterizetion wezo besed primaxily on protocols dereloped by the DOE Bivision of Renediel Aetion Projects (DRAP) Te ehafent Measnzements Center (THC) end on fleld-Implementetion oxperlonce geined during radiologic cheracterlzations of other DMTBAP t1tes (A11en, Showatter, and others, 1983; Allen, Steele, and others, 1983; Aller and Stzong, 19B4; Harntzky end othezt, 1983; Shog and othert. 1984; Shay and 8nth, 1984: and Goodrsikte ind watker. 1984).

A11 tetivitios te or in suppert of the project vere perforwed in cowpliance

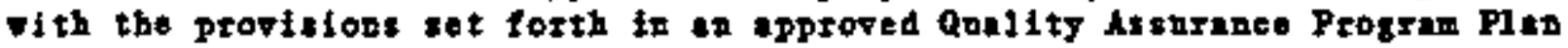

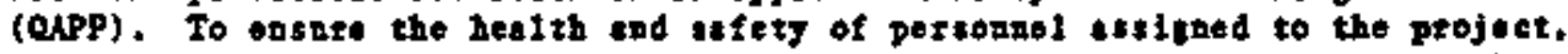

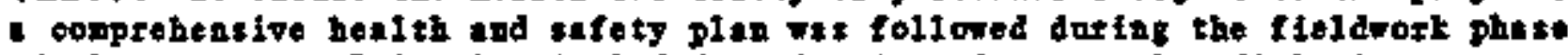
of the projet. This plan ineloded wonitortng of exterist tadiologte exporte. potent lil for futernal tadiologio exposte (1.0.. inhalat on of airborno rodionctivo dutt), peztonel conterintion, elte coese, nid equipnont oontuinination.

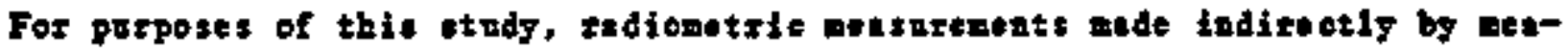
corement of redintion from sotrous othex than the decty of the adelide of intercst are reported s" "uquivalent" concentrations, denoted by an "ot prefix.

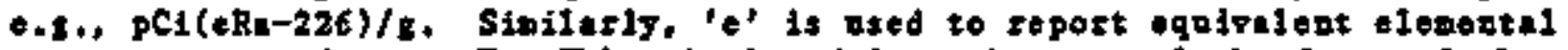

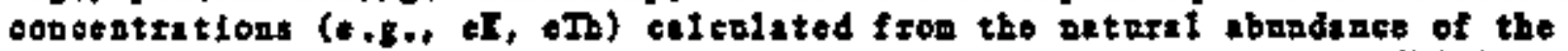

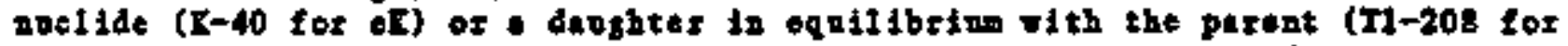
oTh). Bquivalept copcentration is thit concentration of anclide/element of

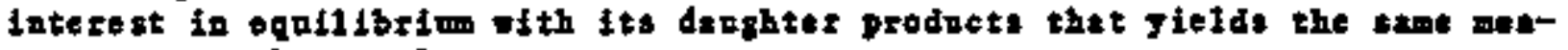
snetwent is the onwple.

AlI Instrment: bsed for radiologle cherecterization were celibrated at the bOE allibrition facliftiet in Grand Jenetion, Colorado, both before and after the

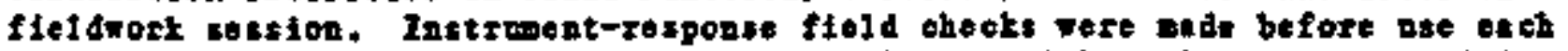
day, and the resilts were monticred for compl lance with quelity-eontzol 1 ivits established on the stntistieal besis of prevlous response ohecks. The wethods ased for inctrment-resporte field the tha e deseribod in Appendix A. 


\subsection{AREA DESTGKATOAS}

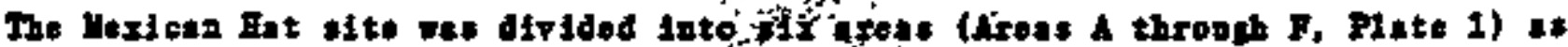
deseribed to the stetenent of Work; ditnefted on the basts of differing cor-

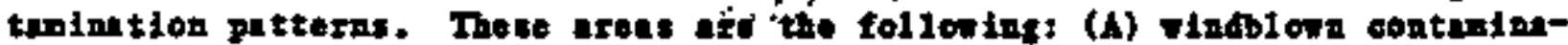
tion north, northeset, and east of the thilings plies sod arzoyos contufnins:

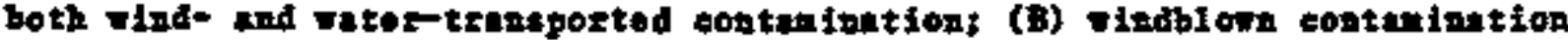

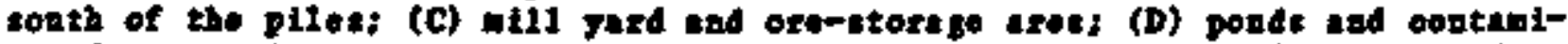
nated arrose ta the northetet portion of tho alke; snd (E) and (F) vind and

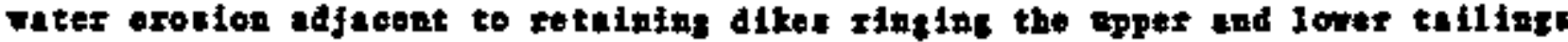

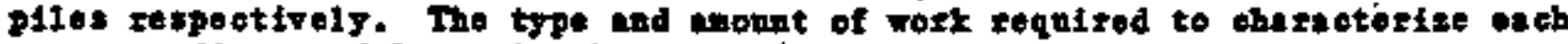
iros aro dictused later in thit repost.

\subsection{EXPSICAL SURVY}

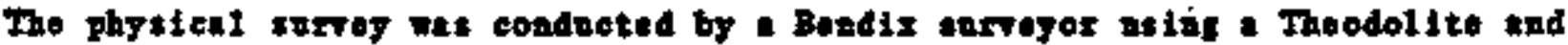

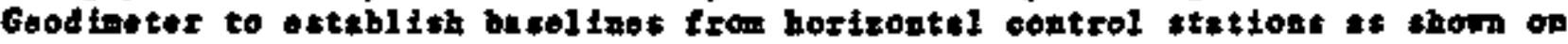

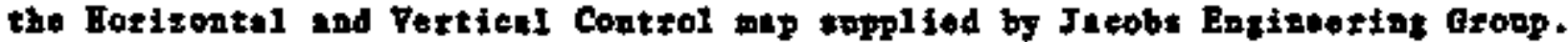
The aillsite at Hoxfens But differed from proviote oftes in thet the property

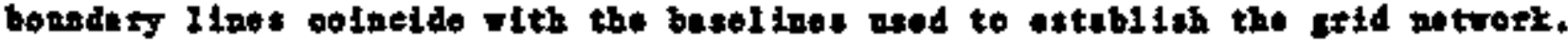

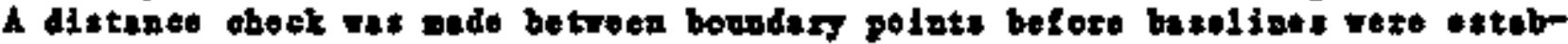
Itshed.

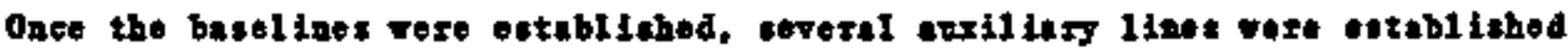
vith the lnstrubats ot ripht onglos to the bugelites to aid in sxidding the

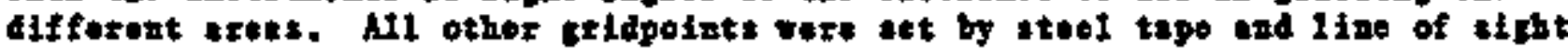

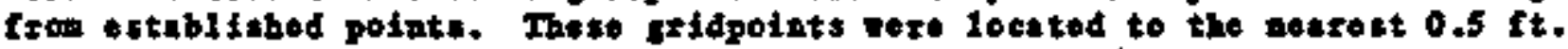

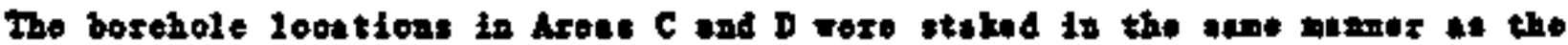

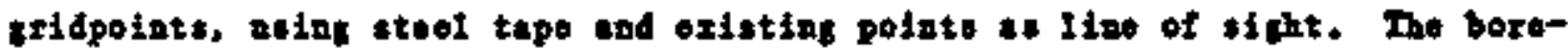

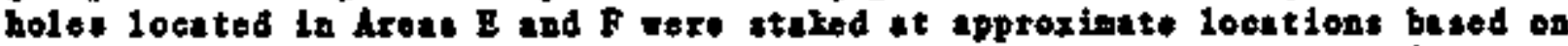
the Tork tatement saple-desigh mep provided by Jacobe Esgirestat Group.

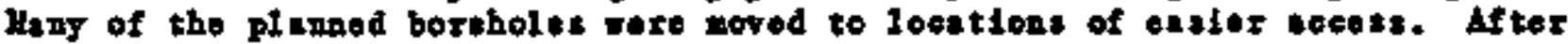

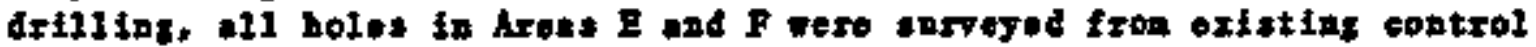
stetlons. Rlertions of etl boreboles vere tsablished by differential leralIng betroad control stations.

Iany of these holes rere noved and restaked by the Berdix site Hanger and the

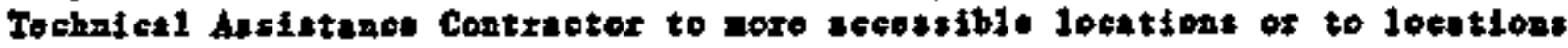

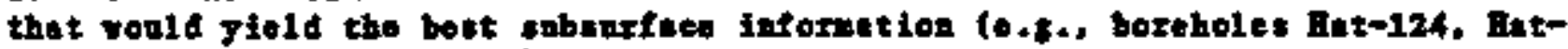
225, and Bat-158 in teet A).

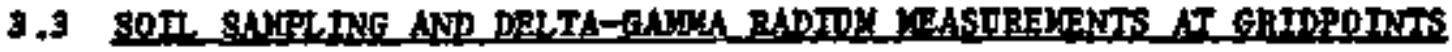

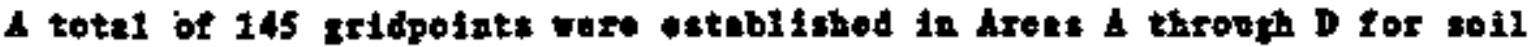

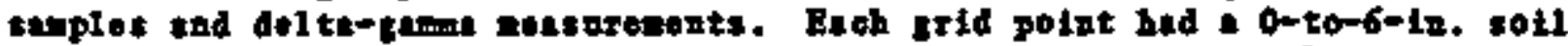

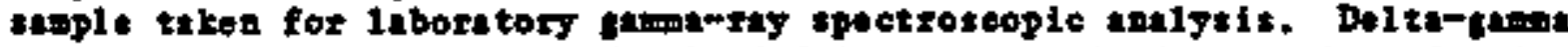

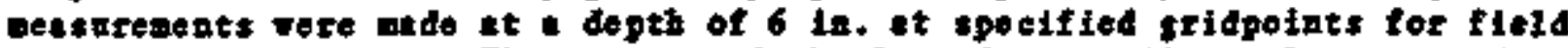

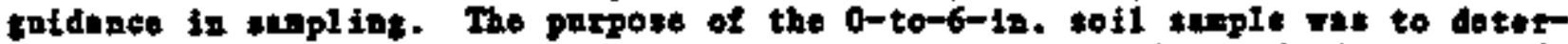
aine the extent of arfate contaninition exceodias the EPA trandezd of $\mathrm{s} \mathrm{pCH} / \mathrm{s}$

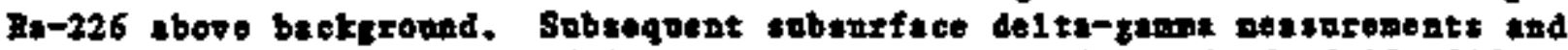

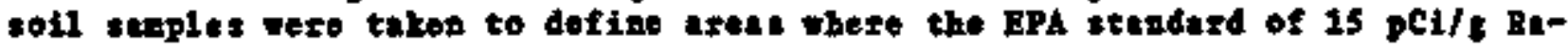




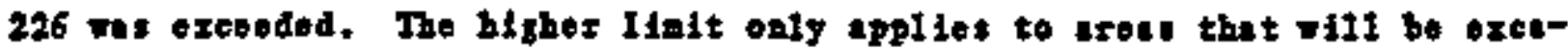
vated and then beckflled; the lowet 1 iait anst be appliod to uses thet any be

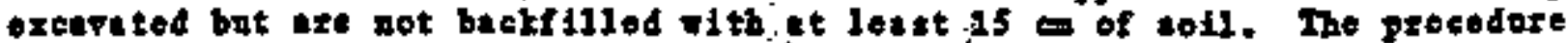

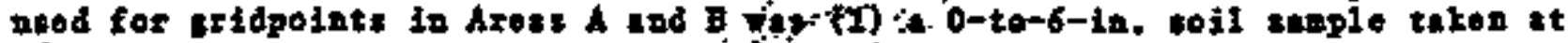

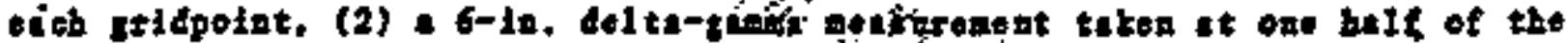
tridpoints, and (3) 6-to-12-in. solt sciple theze 6-ifi. deltu-gante

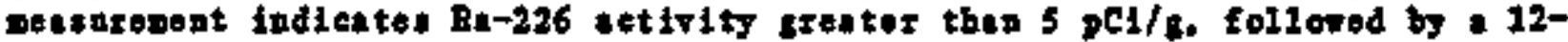

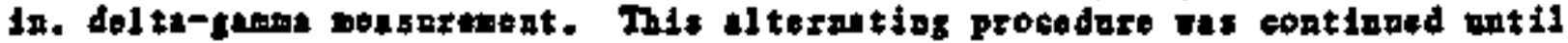

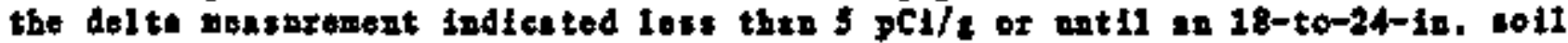
tample wat taken. In Areas $C$ and $D$ at every specifled eridpoiot this al terni-

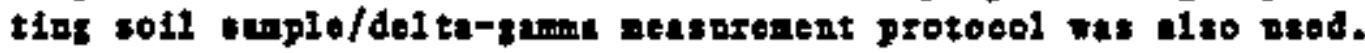

\subsubsection{Sol1_Strolins}

Sovernl steps were telen to ensure tho integrity of the soil sumples doring the

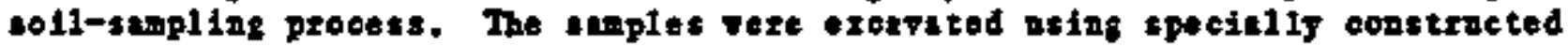
thovel. desiged to produce staples of equal sixo. Care was takn to collet

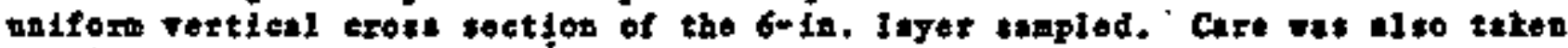

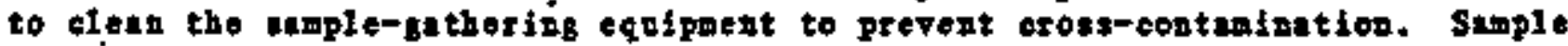

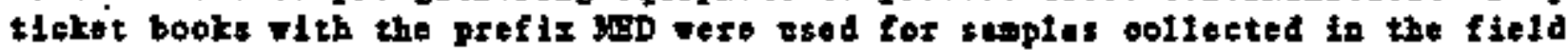
dpring this project. Pextixent toforation soch os sample type, grid lootion, taple depth, project oode, suplez's name, and soll conditions vere placed on oech suple ticket. A paper reoord of the supple-ticket otwber was plinced incide tho plistio bag boldisg the ataple and the top of the bag was then

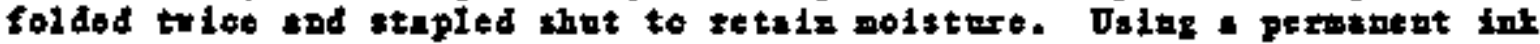

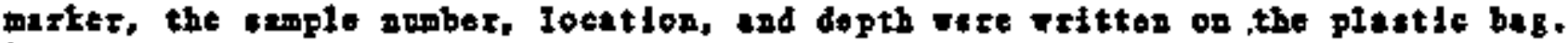
The plentit beg res then placed is deek-clotb bag ohieb wes ul so lebeled with

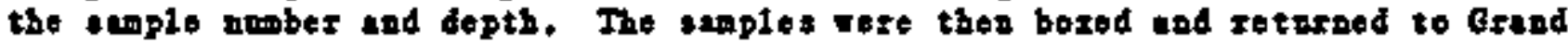
Jnetion for antlysis by the Bendis Antytienl Chenletry Leboratory.

Bollowing trenspot to Grand Junction, the soil sopits were weighed, driod, rewel thed, crushed ind pronid to -28 mesh, blended, and gealed in anple east. The difference in weight ifter drying. Or loss on drying (LOD), in pereent, was recordod for about 10 percent of the sumples subitited for andysts. The se Taloes were nsed leter to calcolete poistare correction factors. The theglos were stored for it Ionst 21 dogs to ellow the tudon and radon dughters to

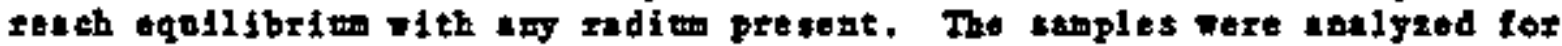

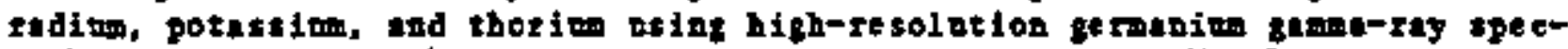

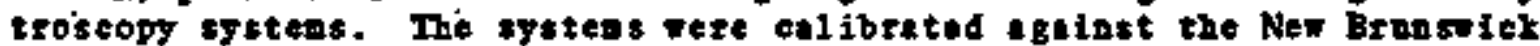
Leboratory (NBL) Soxies 100-A cortifiod gomerery cterdarde. All sapples were

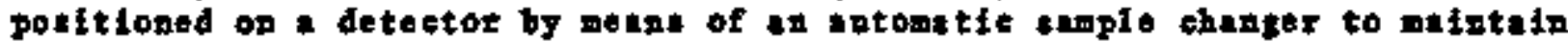
consiatent sample/detecter pootetry. The labortcory procedures tised axe

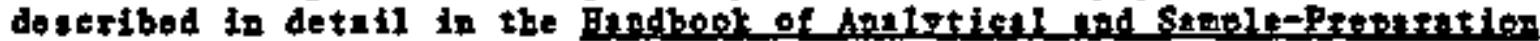
Whthods (Bordfx Field Entiventise Corporation, 1984C).

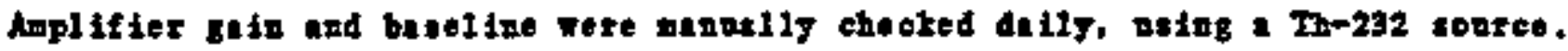
Two know control samples Tere and

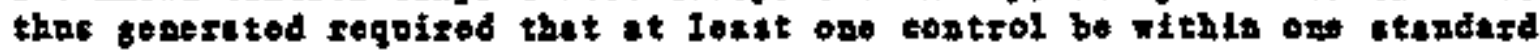
devietion and that both controls be Tithin to stinderd devintions of the

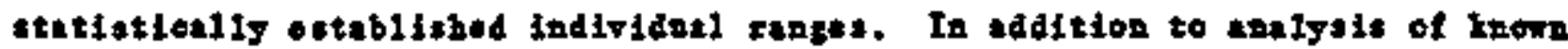
vontzols, 10 pexcent of the blended stmples wero spltt and antyzed for fadion

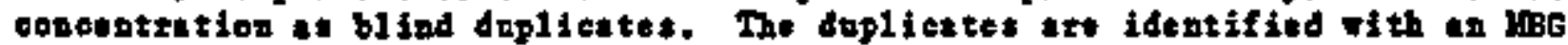
stoplo profix. 


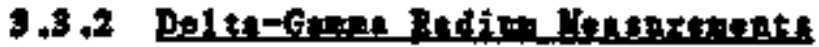

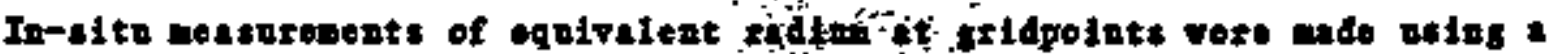

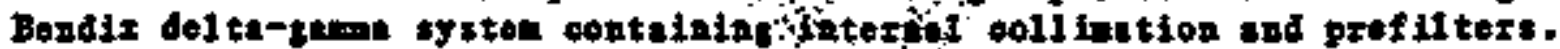
Bendix sytete vas operated on oomelpi pistion tuing a dolta (difference)

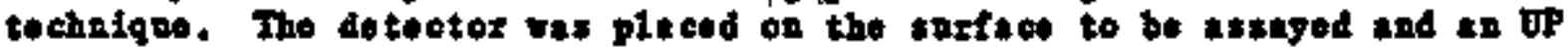

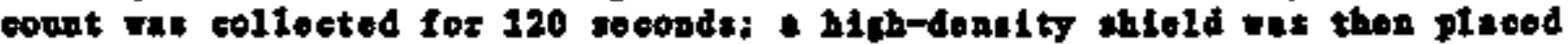
betwoen the detector and the surface boins assayed and a Dork count of 120

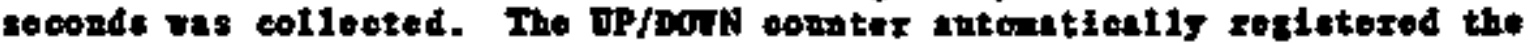
difference or delta eount.

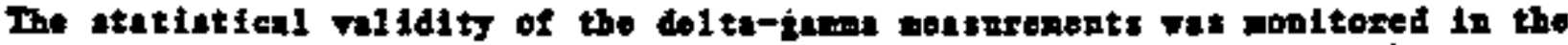

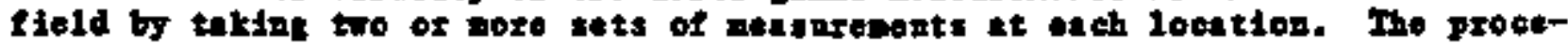
dores naod in doternining the stetistical ralldiby of the peturzonents and for

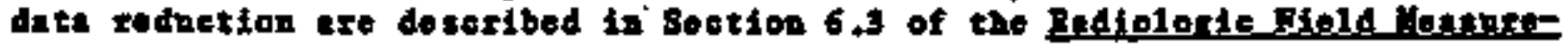

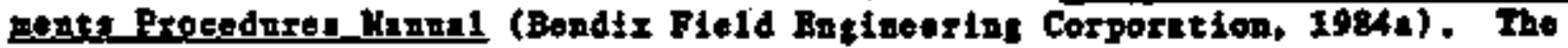

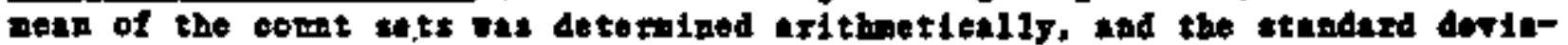
tion of each comt ist vat ealetiated risint the equation

$$
\sigma_{N_{d}}=\sqrt{2 N_{e}-N_{d}}
$$

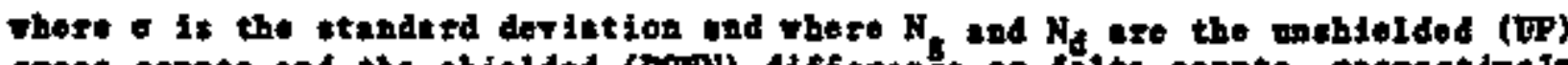

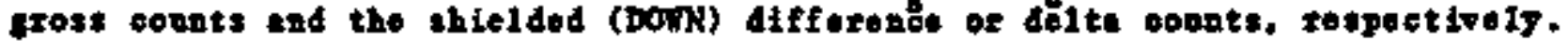

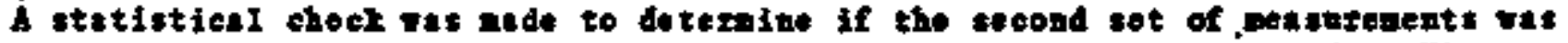

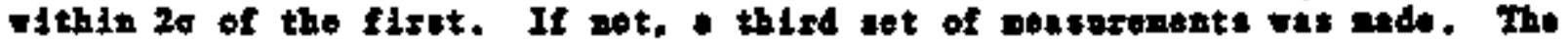

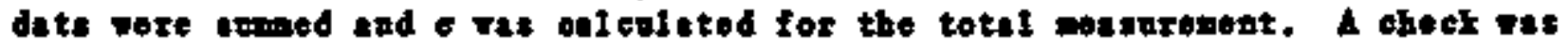
made to doterwine whether o is lese than 15 percent of the differtect cont thist the oquat 3 is

$$
\bar{J}_{d}=\frac{\sigma_{N_{d}}}{T_{d}}(100)
$$

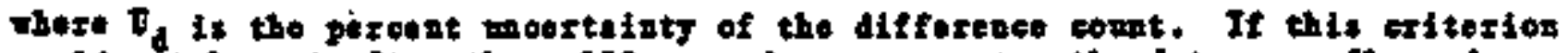

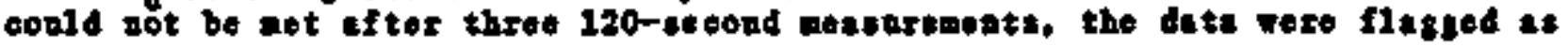
beint serf fquatitative.

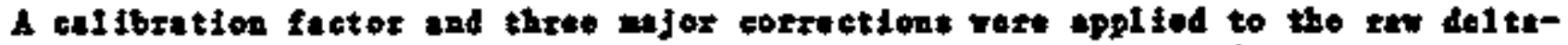

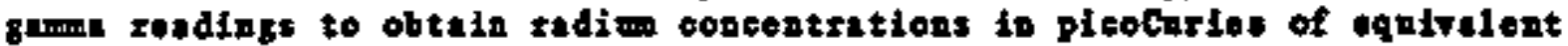

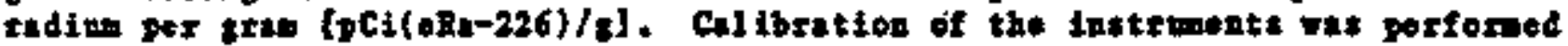
at the Depertaent of Erext (bOR) Falko: Field ond tbrition pads in Grand Junction, Colozado. The strippint fectors nocesery to oal enlete ran

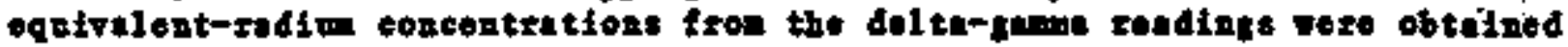
from tho callbretion data.

A correction for cortribetions frow tedioelemeats other than Ra-226 vet atde

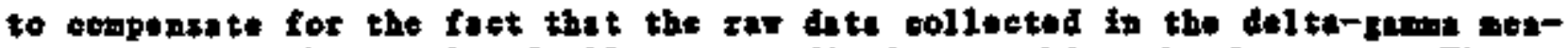
serowent: to the resolt of all camp redistion reaching the dotector. The

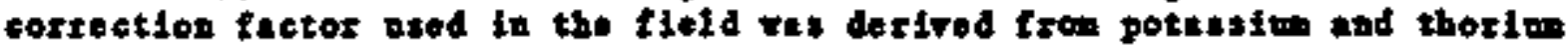

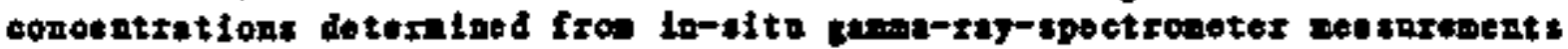

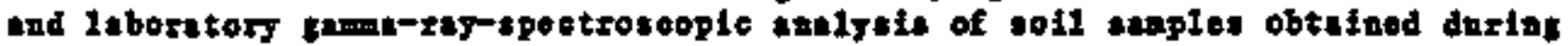
the orfeztation viste to the site. Tho obrzotion factor ased in this teport

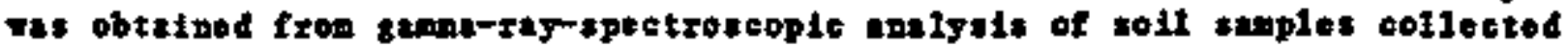
fertng the fluldwork phese of the project. 


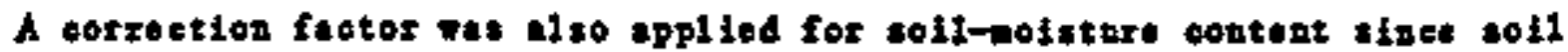

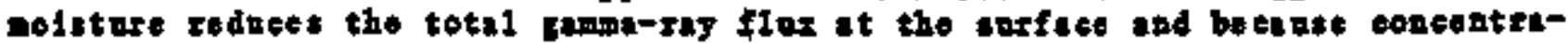

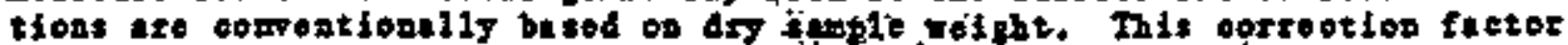

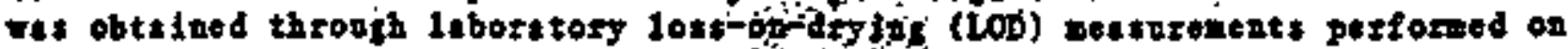
celocted soil inplos taken during the ffeldrork phs se of the peofoct. Dot to

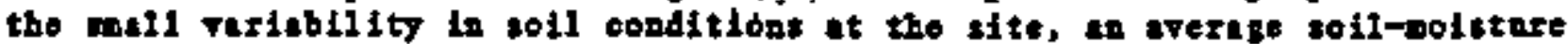

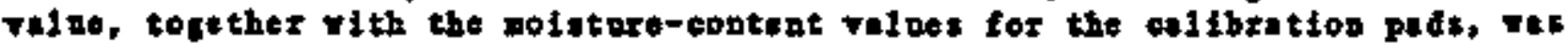
conferted to a single corfoction factor to bo applted to all of the del te-rama nessurenerte.

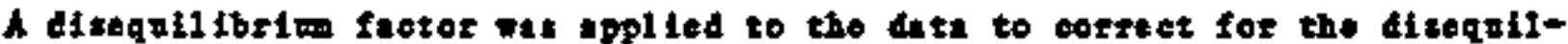

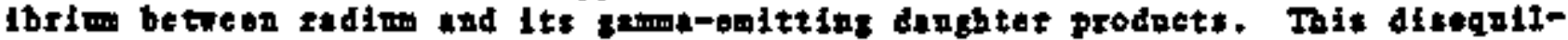

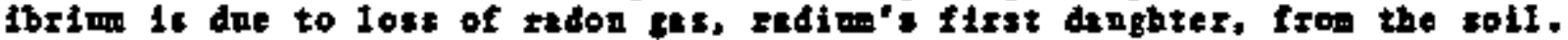

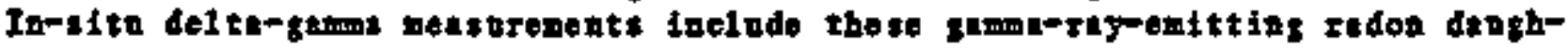
ters. Then the correction for fedon lost is applled, the epperent concentra-

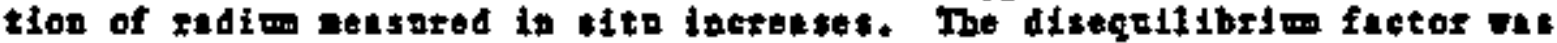

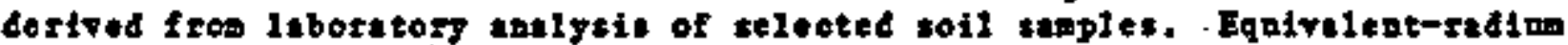

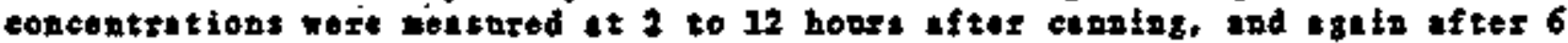

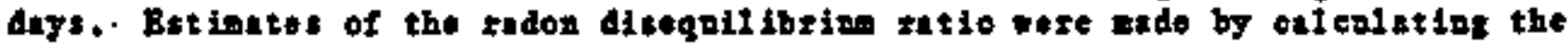

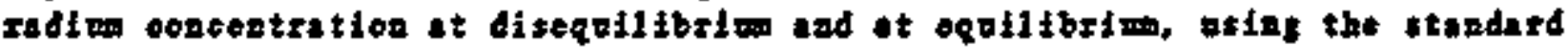

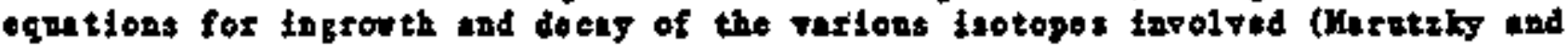
others, 2994; 8cott and Dodd, 1960).

\subsection{ABROYO DELTA-GAYMA UEASTREHFNOS}

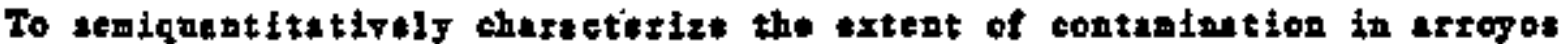

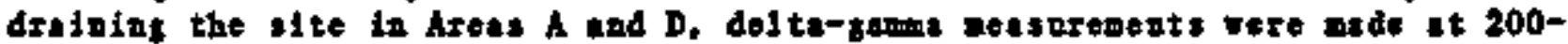

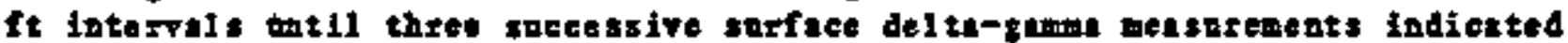

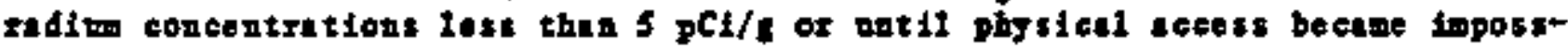
Ibis.

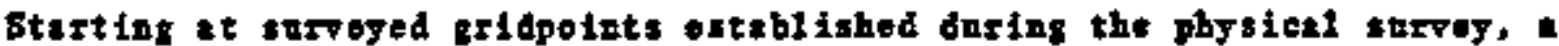
BOLLATAPE bf cyele whel distanct-aesering device vas ased to extablish del tatamin peasugenent locetione at approxisetely 200-ft interval along the bottons of the arrogos.

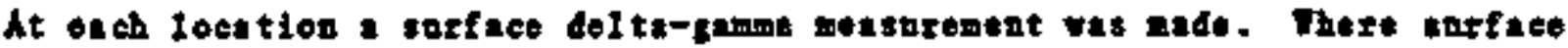

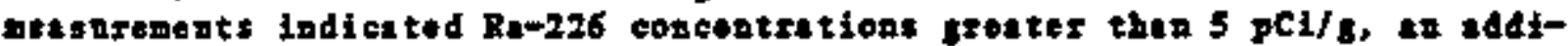
tional weescrement Fas mede at a depth of 6 in. This procedure ves followed

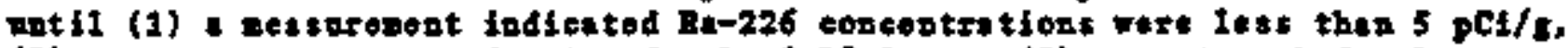

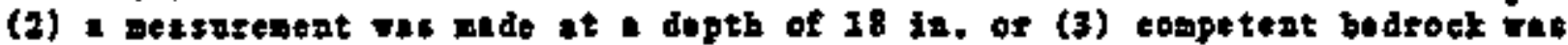
renthed.

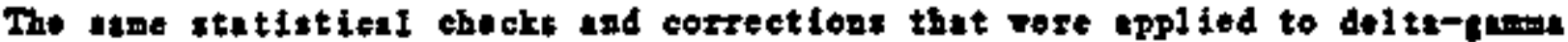

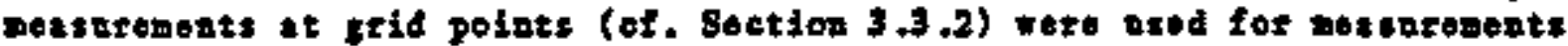

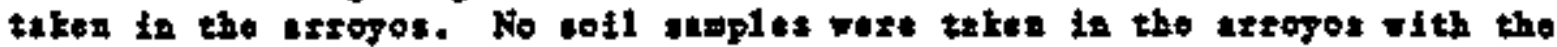

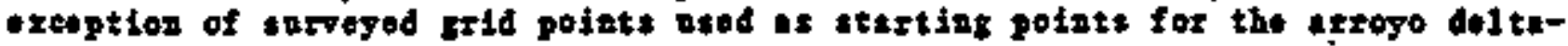
comina sertey. 


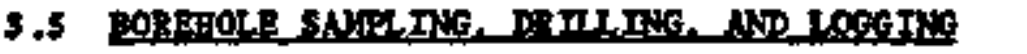

\section{$3 . \$$ Baplins}

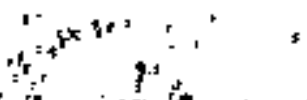

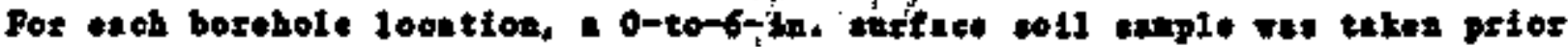

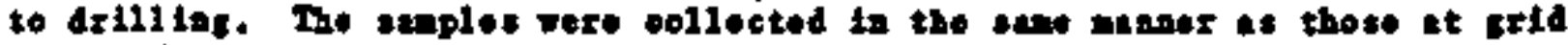

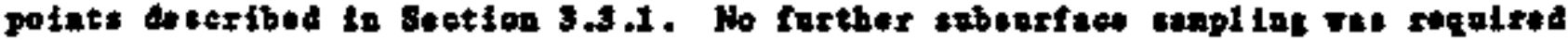
at borololo loetiont for tht: atedy.

\section{5 .2 Drdilies}

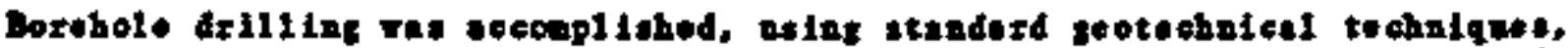

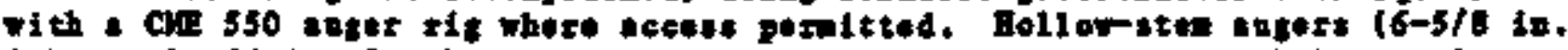
0.D. by 3-I/4 In. I.D.) vere used to drili to totel depth in weth hol o. Angers

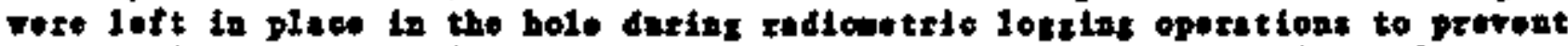

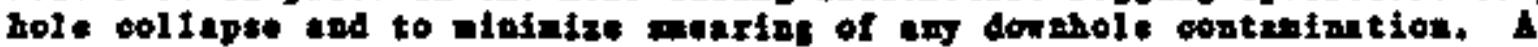

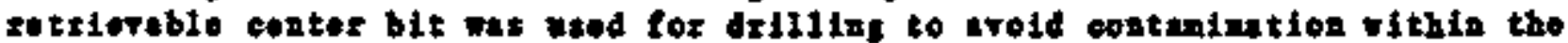

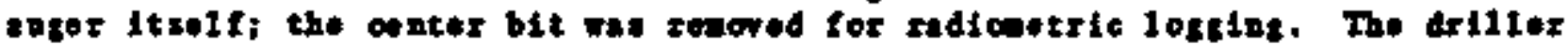

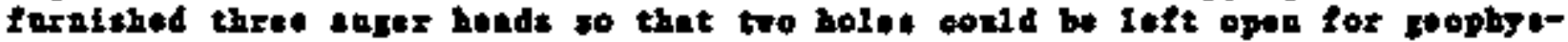
tosl losglat whtie the third hol wat bing atilted.

The bereholet Fero dxiled to olther the totel otineted fopth as epelfted In

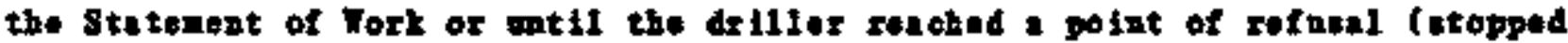

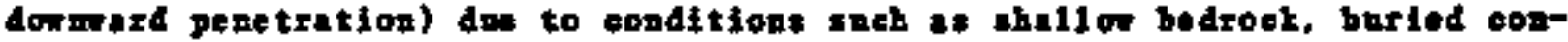
crote. large rocks. or other naturint thit rould stop poatration.

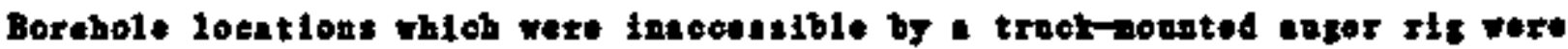

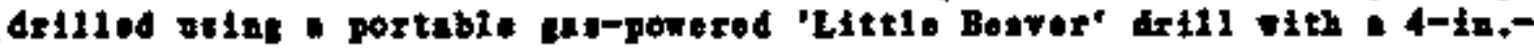

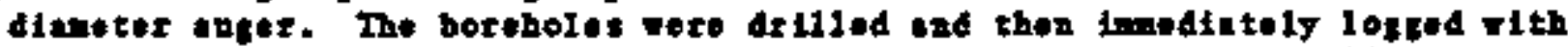

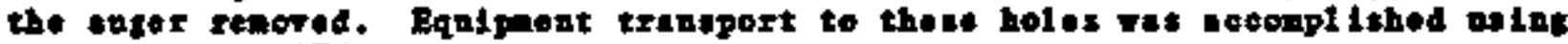
throe-rhos od ATCE.

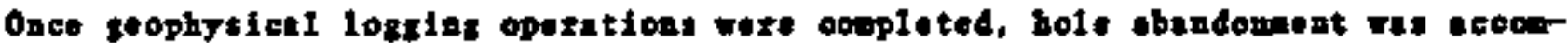

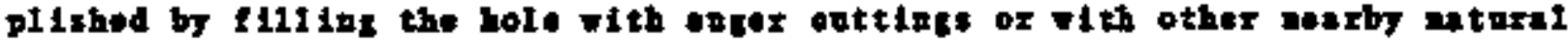

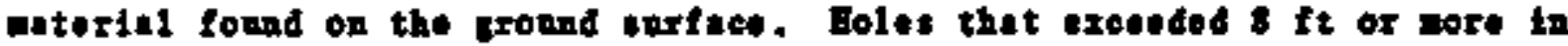

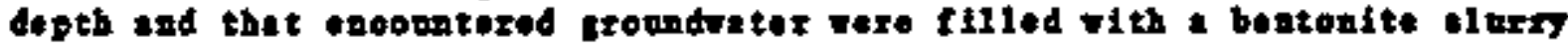
hrifin at 1ente a s5-socond Tiscosity.

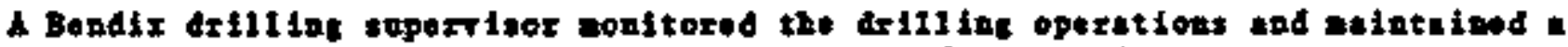

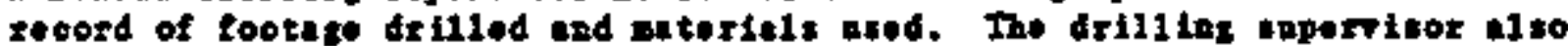

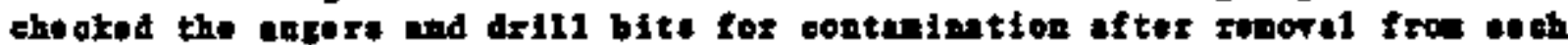

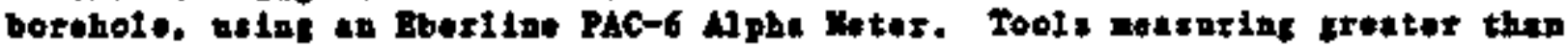

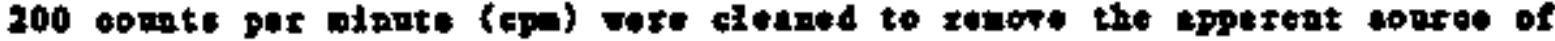

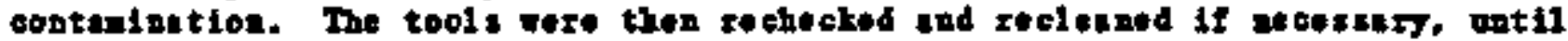
Dessorenente vere lose then $60 \mathrm{cos}$.

\subsubsection{Geophrelcat torting}

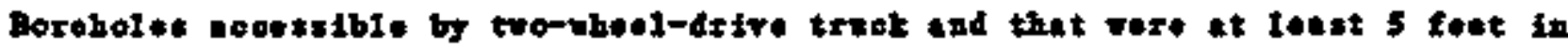

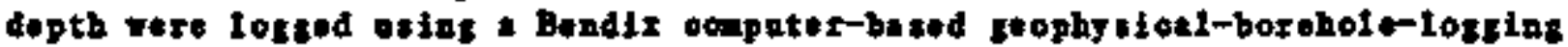

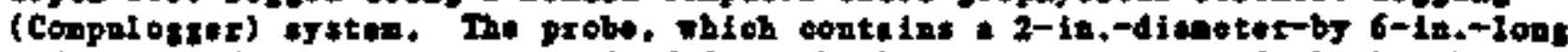

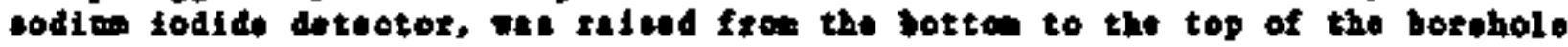




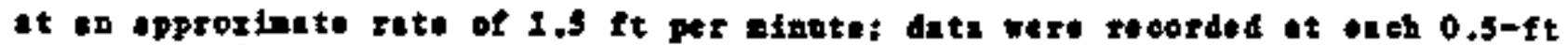

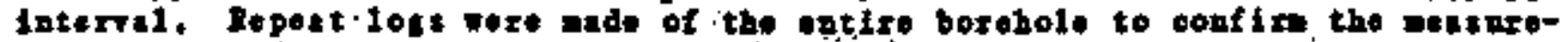

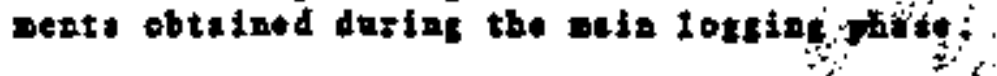

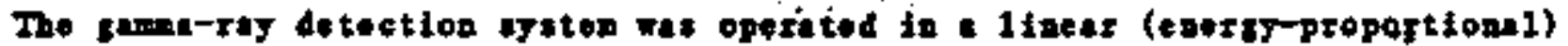

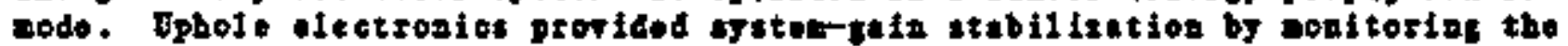

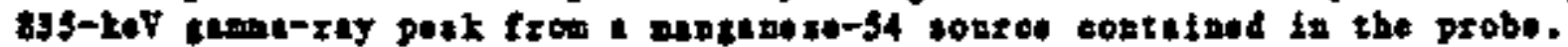

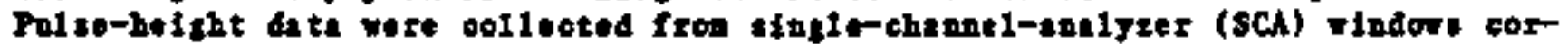

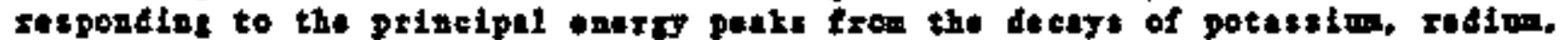
end thortu. Date frow fourth sCl vindow, the toteI-cont rindor (10s0 to 3000 zoV). Fexe collectad to provide statistically iproved rediw. eoncentrations.

Aftur raturalis to 6rand Janctlon, the date frow the Bendis eomputer-besed

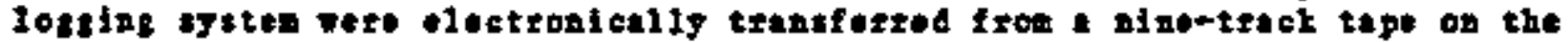
COC-815 coppter to a dick on the Zeaith Z-100 portuble cotspter agd rezified cecordins to epproved qualityasnrance grocodures, Date fros the total court

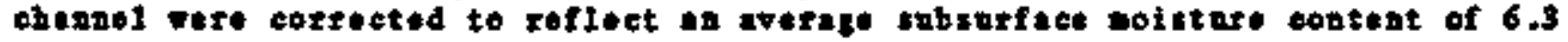
percest.

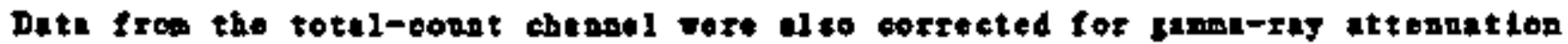

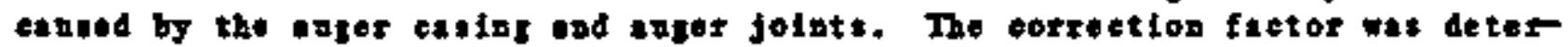

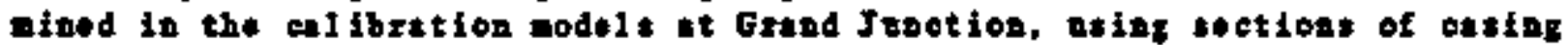
ldertieal to the cating osod at the fleld site. This fector to an oquetion

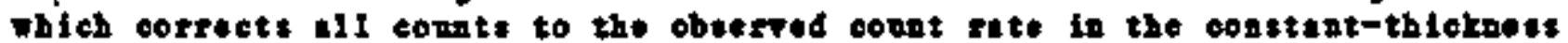

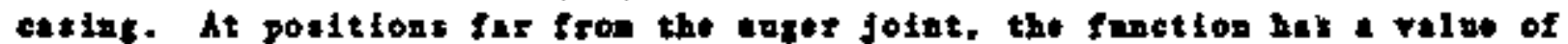
2.0. bet as the depth gensusad approschis tho conter of the jolnt, the value of

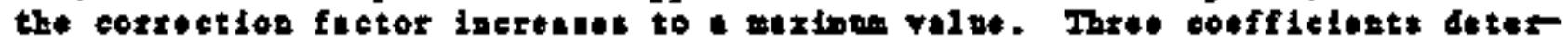
tino the corroction, one for the parimer value at the ootor, one for the

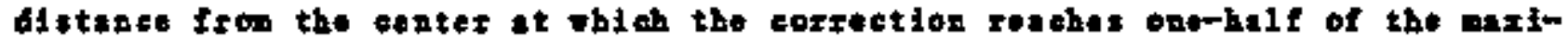

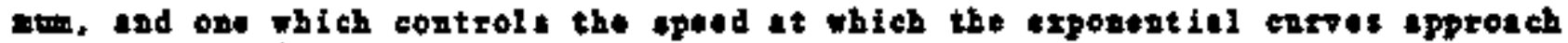

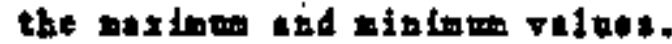

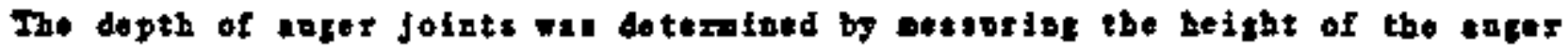

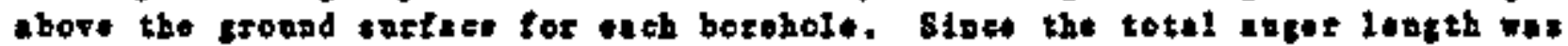

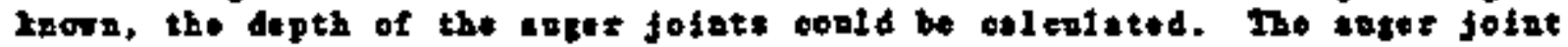
ootroction ves epplied onet the jolat dopth we loove.

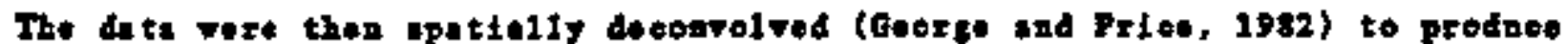

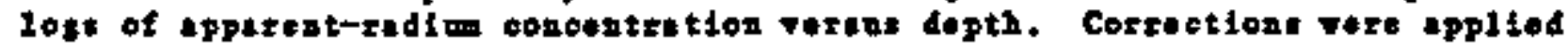

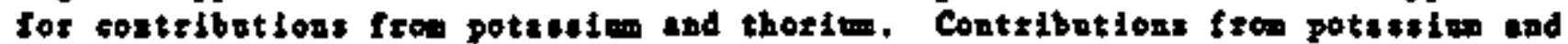

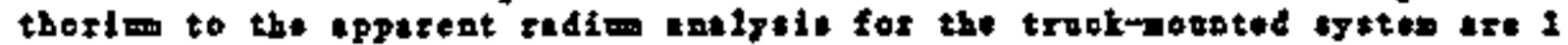

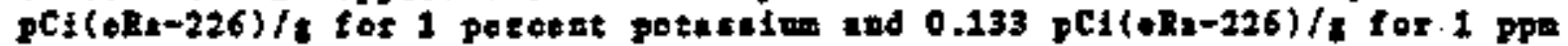

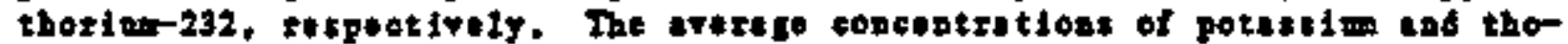

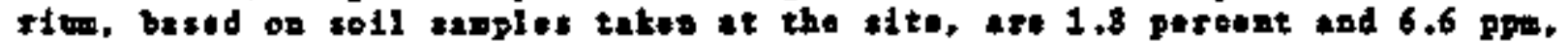
stopet ive 17.

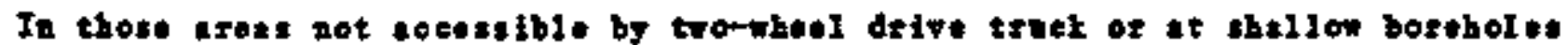

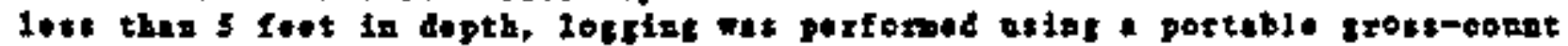

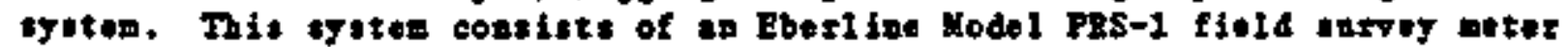

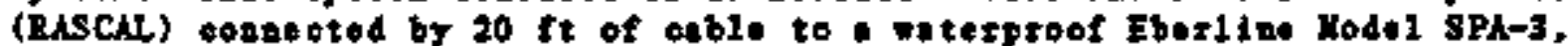
2-ig.-by-2-17. Hodim lodide detectos. The bytton is operated on conting

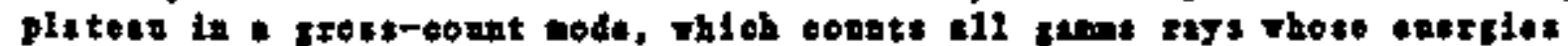
extest epproximately 30 ket. 


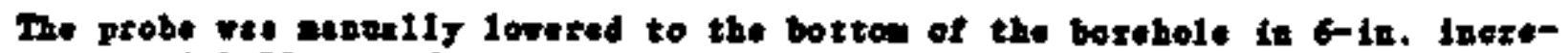

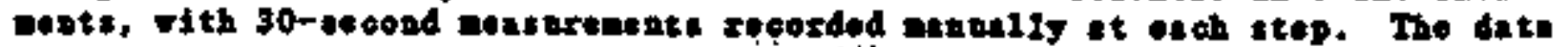

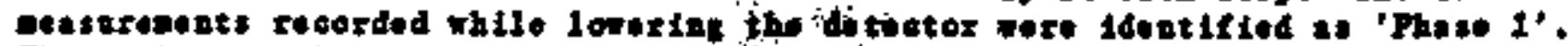
The probe was then raled to the top of the borehole in 6-12. 1merenents. Fith

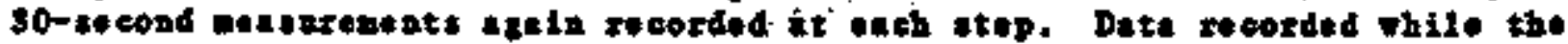

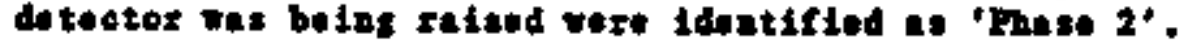

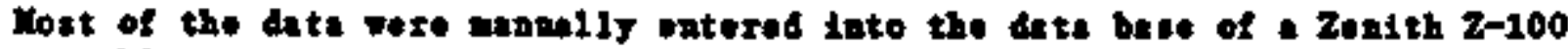

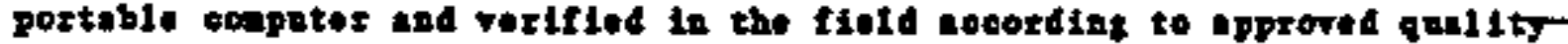

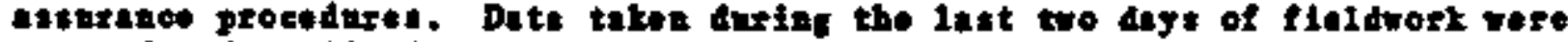

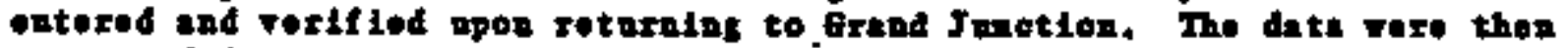

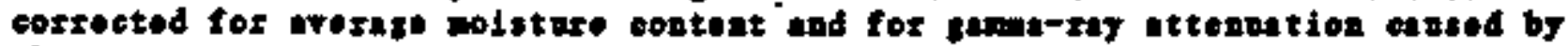

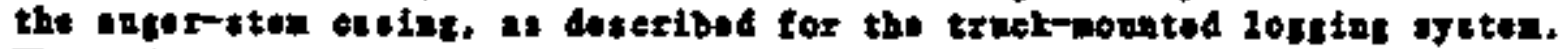

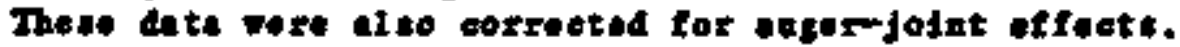

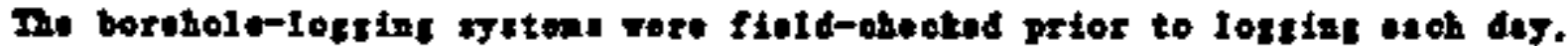

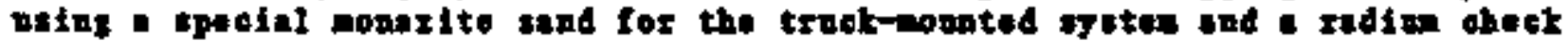
conres for the porteble issten. The reneltine dete fere reeordod and conpered

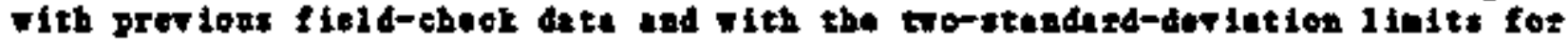

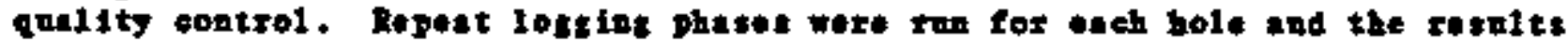
of both the wein and ropent phsent vere ohooked for agranent, Additionel

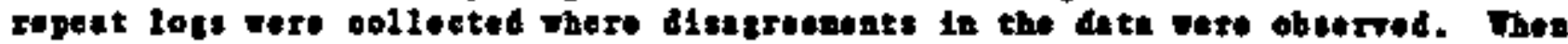
posaiblo, the apparent entet of the disegreanent vas noted.

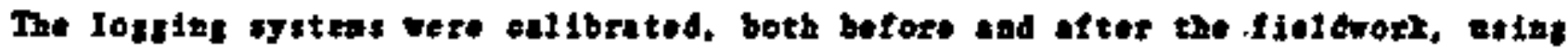

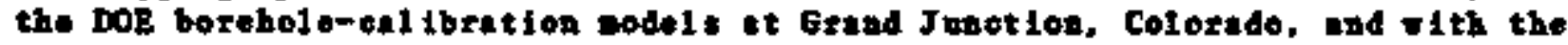

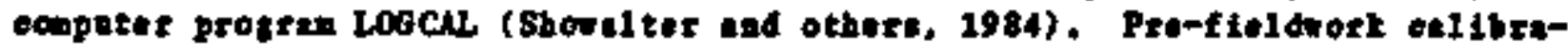

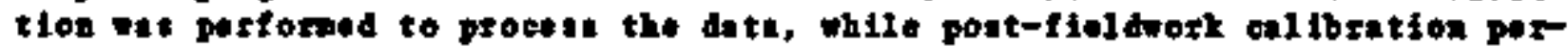

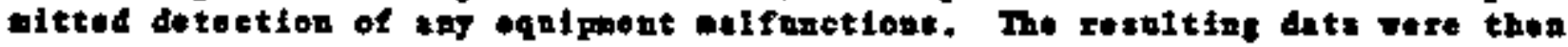
azehived in pornenont allibretion dete best.

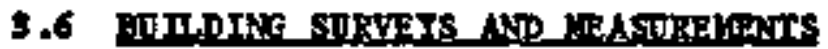

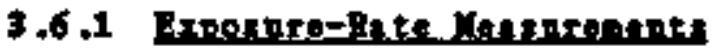

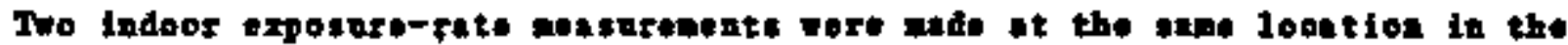

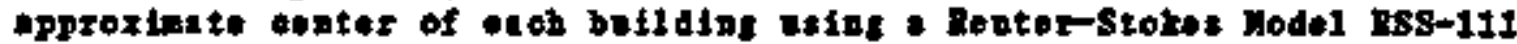

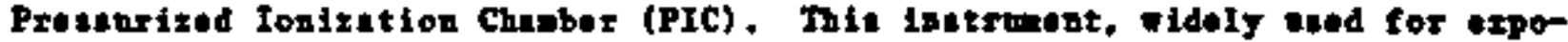

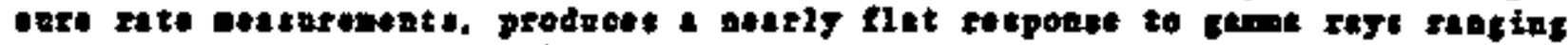

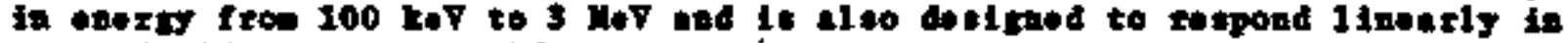
sening flolds op to $100 \mathrm{~m} / \mathrm{h}$.

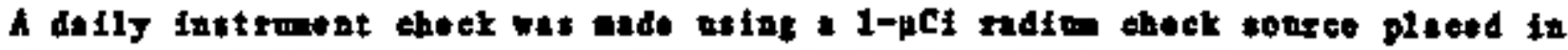
the conrce-holding flxter at the top of the lon ohaber. Fixte, with the

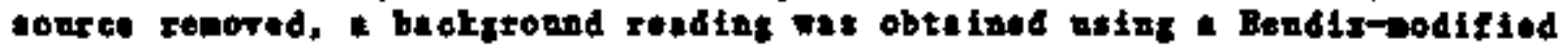

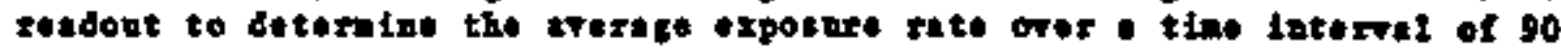

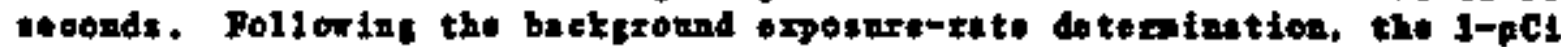

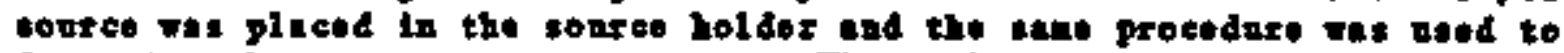

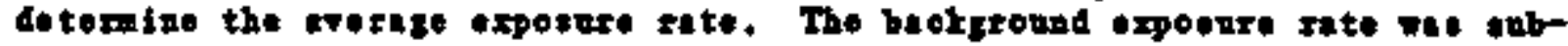

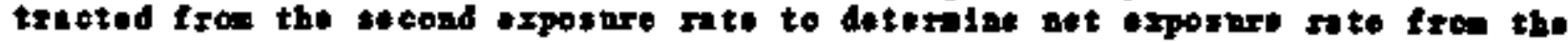

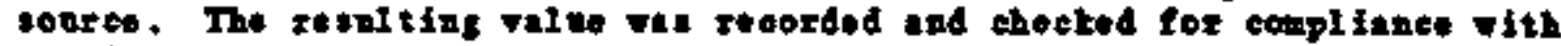

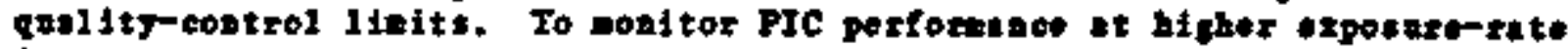

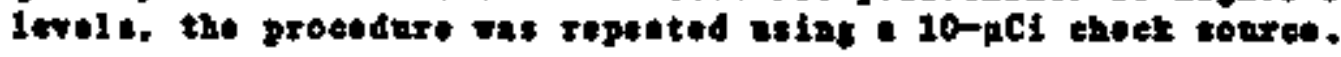




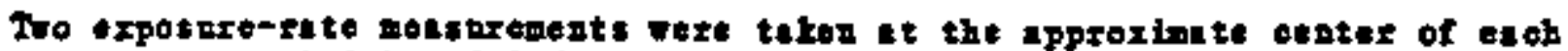

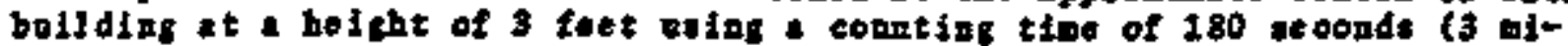

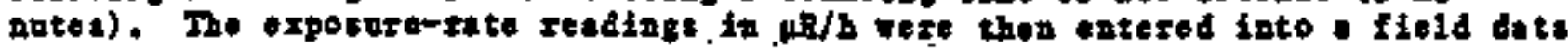
book.

\subsubsection{Hohs Hecsorements}

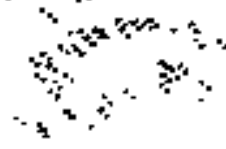

An Ebert toe Aif Proportzond Aphe Detector tas ased to obtain zrose-elphe

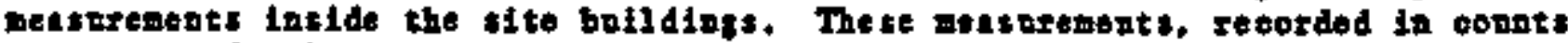
per vintite (cpm). Tere zede to doteraine ourfece contawintion lovel*. The overall objeotive of the meastexerts wis to determine the extent of decontamintion geoded in the baildings.

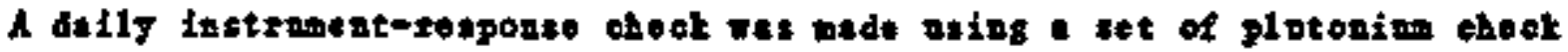
20750es. The detoctor was placed wer the nonzed and a reding wes rocordod. The dotector was rotated 180 degrets atd a tocond readfot wa recorded. The

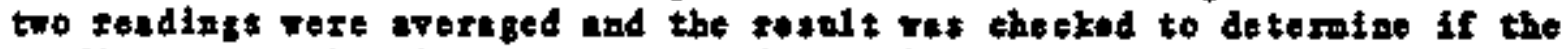

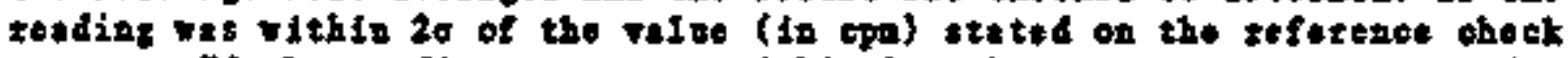

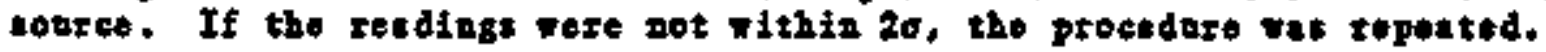

If the second tost fallod, the instrement was tented 'Do not USE' and sottrined to Gred Juption for repuix.

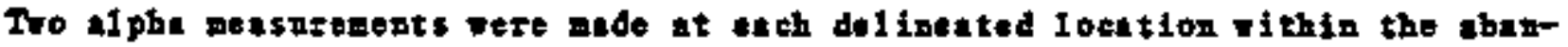

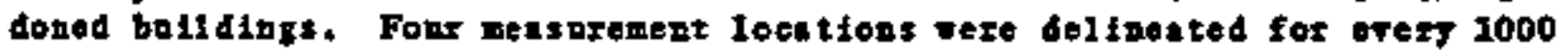
aquite feet of floor space. Fith a minimm of foer locktions per brildiag. The first reading was a direct anface roading. The jochtion was then wiped or broshed olens and a cond readigg ves taken. A parked decrouse in cont rate provided an indication thet tho contemintion ts romorablo. For tho tro bulldIngs sti11 in ose, the Hal chj ta Heal th Ceates and the looked atorage baj1ding, both olroet resdings and swears vere collectod. The smears rere taken at the

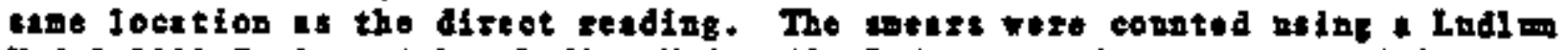

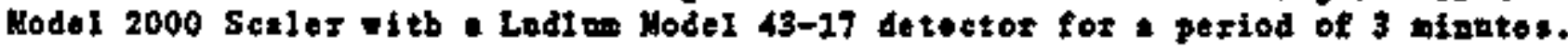

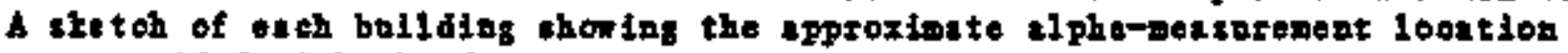
Tis proplded with the dete.

\subsection{BACXGROUND YEASUREKENIS}

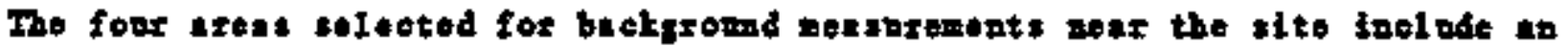
ared approsinetoly 3 ailes porthesst of the site neer the Hoxican But Rock, an

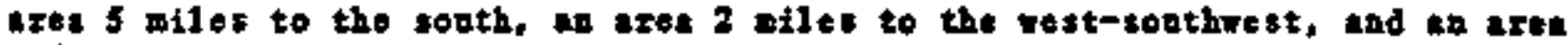
0.5 mile to the east. The beekrous aite to the ent rat lockted juat west of

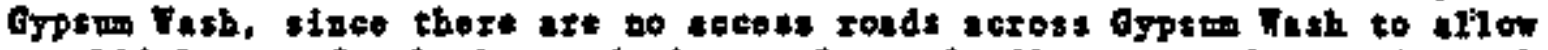
establishment of a bakfrond site at lenct 3 miles elst of the site. Thres

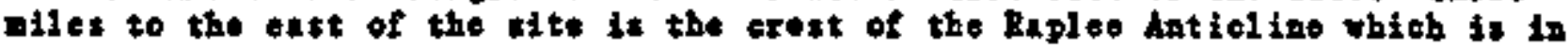

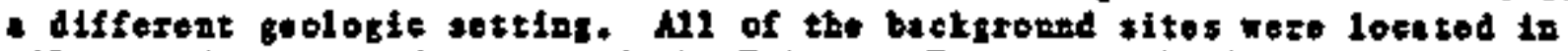
s11ty sadetonts and thales of the Balteito Torgye of the Cotier Forwation. s1011ar to the goologle settins at the villsito.

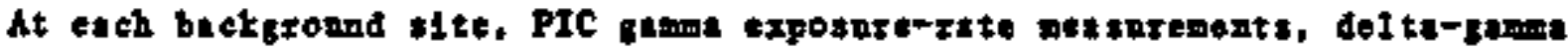
neasurements, and coll capies pere teten. Soll sables taka dortag the 
orientetlon vitit were and

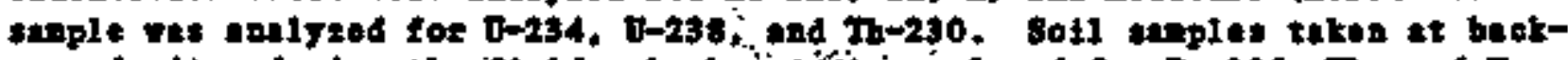

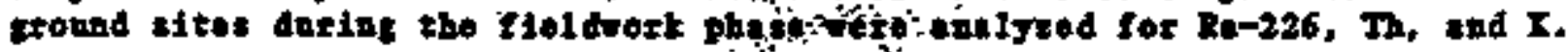

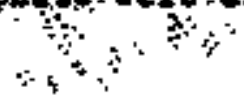

\section{4,0 MBSults}

Duto obteingd doelng the rloldworl phese of the projent aro proenrted in

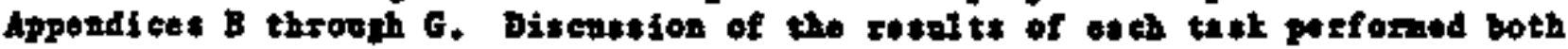

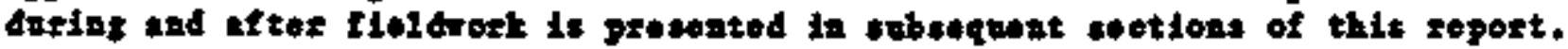

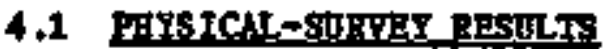

Bofore ostablithlag the baselines aspd in gridding the site, the Thoodol ito and

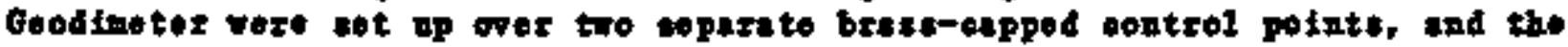

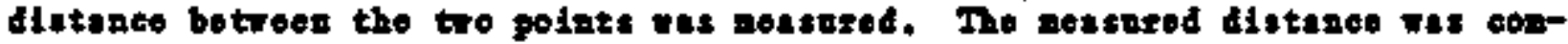
pared to the caloolated dintance ind zestits ladiested thet tho differenco

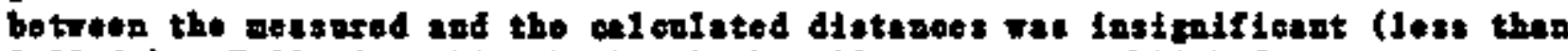
$0.30 \mathrm{ft}$. Followint thit theok, the batotlaes vexe established.

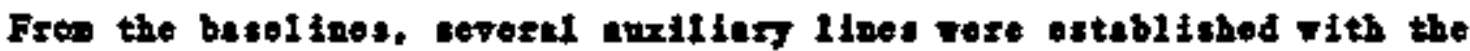

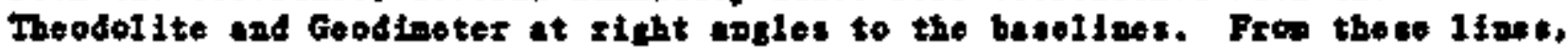
srfdpolnts were set by tape and 11 no of sitht. A total of 145 iridpoints for supl ing and wostarements Foro osteblichod.

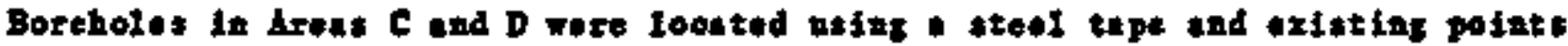
se Iine of aitht priot to drillins. Soverst boroholes In Arest $C$ and D Fere wored by the Bondix Site Hintgex to aroid powerlines, coneroto foundetions, totface bodrook, or other obsttuctions. These holes were escally nored only a

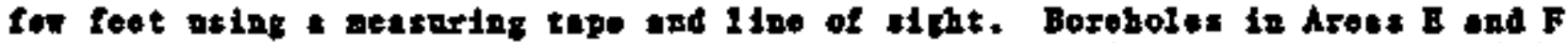
vere staked at approximete loontions, based on the semplo desibn anp prowldod

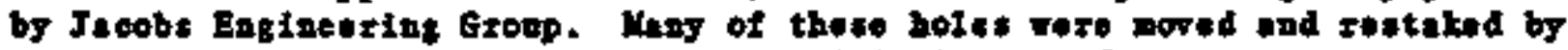

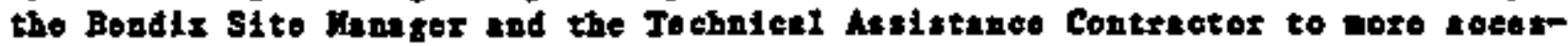
Ible locktions or to Iocktions that rould $y$ feld the bost cobereface inforst-

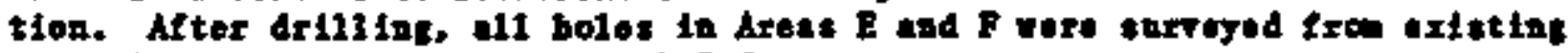
control stetions to tho netrust $0.5 \mathrm{It}$.

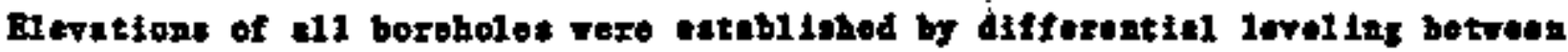

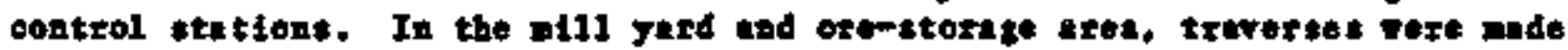
frow stetion 5 to station 7 to station 6 and beek to station 5 , and frol: Stetion 7 to stetion 8 . The differeaces 10 in levetion awroy closures noted at

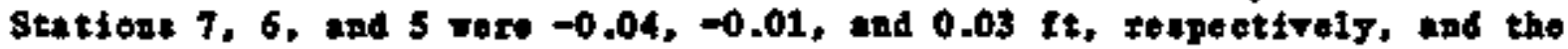

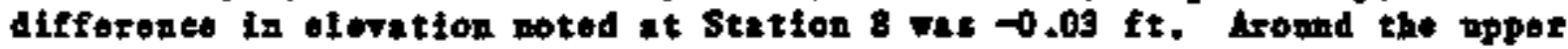

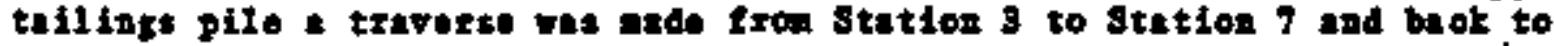
Station 3. No differuneo in elorition clopurb wat noted oithin this triverte. Aromf the lower talliogs pile a traverzo wat ande from station 2 to stition 1

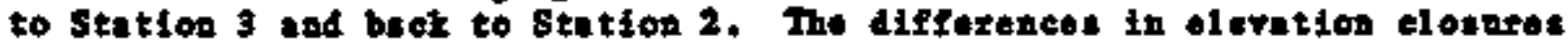

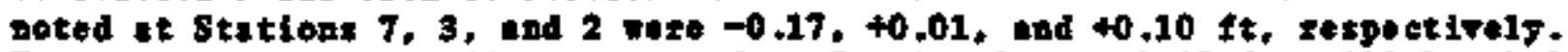
The oresall elosure difference noted at stetion 2 wes $-0.07 \mathrm{ft}$. All boreholo elepations Fore deternigud to the nearest $0.1 \mathrm{ft}$. 
The loettion of arevey control stations, establithod gridpotats, and botelole lootios: are thowh of Plite 1.

\subsection{SOIL, SALEING, BESULT}

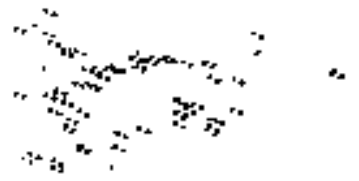

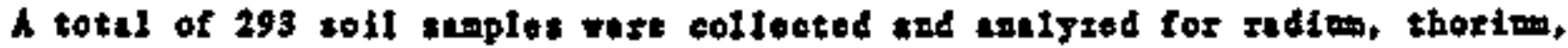
and potassinn; toonlts aro presented in Appondix B. Table B-1. Doplieste enti-

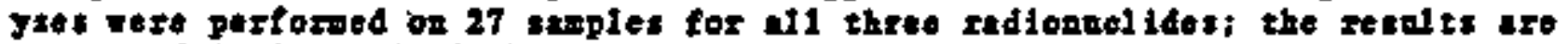

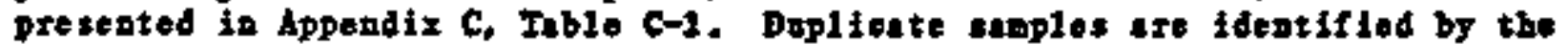
prefix IBG. Appendix C, Inble C-2, presents the rediw deta for tho orighal and dupileate enefytes to pertit epmpirison of the resolts,

The dffierins contamiation pattorns described in Soetion 9.1 of this report. - Ith the exception of arroyo contanination where soll seoples rere not teken, are indicated in the results of soil-sample avalyses. Flgte 2 fhors the dittribation of radim concentrations for soll sasples collected at 0 -to-6-in.

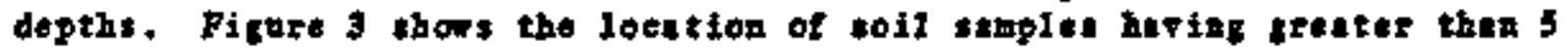

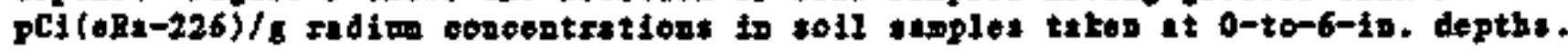

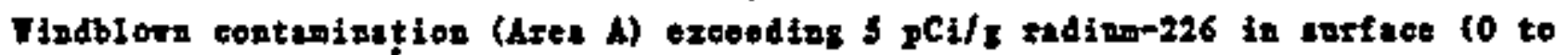

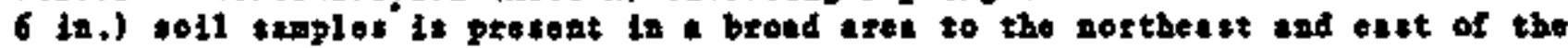
lower teilizes pile. Soll snmples taken at grid points N10252 and E9290, N10000 and E9200, N960 and E9246, and N10600 and E10400 contain radim conobDtrattons of $369,535,676$, and $100 \mathrm{pCi} / \mathrm{s}$, respect $1 \mathrm{v}+1 \mathrm{y}$. Tho first three was-

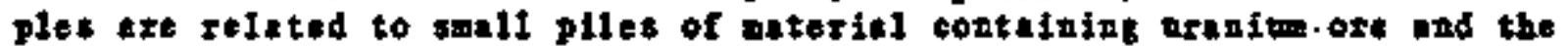
foorth wes take fros the botton of ar errogo coutainiag watertiensported

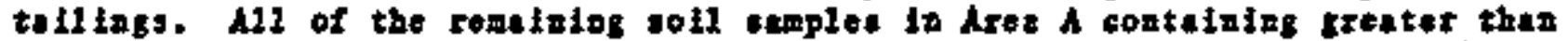

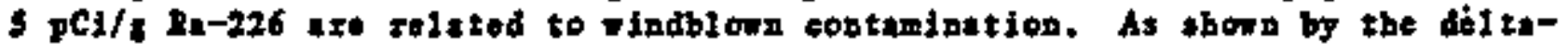

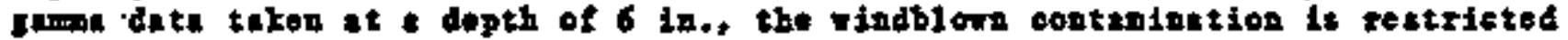
to tho npper $6 \mathrm{fn}$, of material prcept for so axta atong the oustern odge of the 10Ter pilo. Only one strface soil sumple in Area B has a concedration of radim-226 oxceeding $5 \mathrm{pCl} / \mathrm{g}$. This indicates thet wobblowe conteminetion is irsicalfictut south of the pile.

Contawiution in soll camplet taken from the mfIl and ore-storate aress (Area

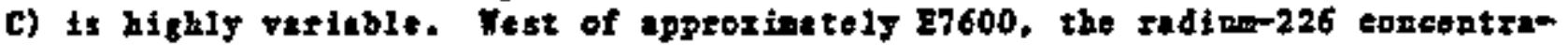

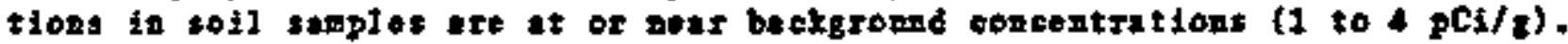
East of approriantely E7600 to the wpper tallings pile, the tadiun concentrutions rafy grently ranging from 3 to 363 pCl/s, reflectint the setetert ore, tallings, and contaminated debris found fo this aros. Weng of the soil sumples take $\mathrm{n}$ in Aree C corzespond to bortholo loet tioss.

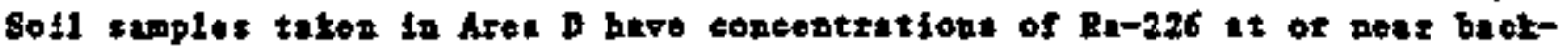
croond levels, escept those taken in the whsh of ersogo which dreles Aren $D$ to the nortb. The inples fros the wath have Bu-226 concentrations reng ing from 2

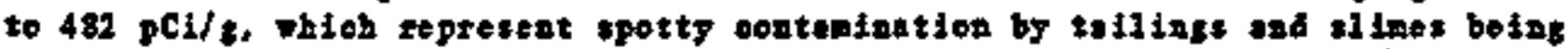

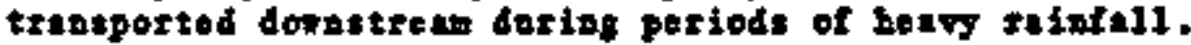

Equivaleat-thoritu coucestrations in soil iasples eze highly resiable, rangtag from 0 to $1384 \mathrm{ppm}$. Tht highest concontzations of thosiow are solatod to soll 

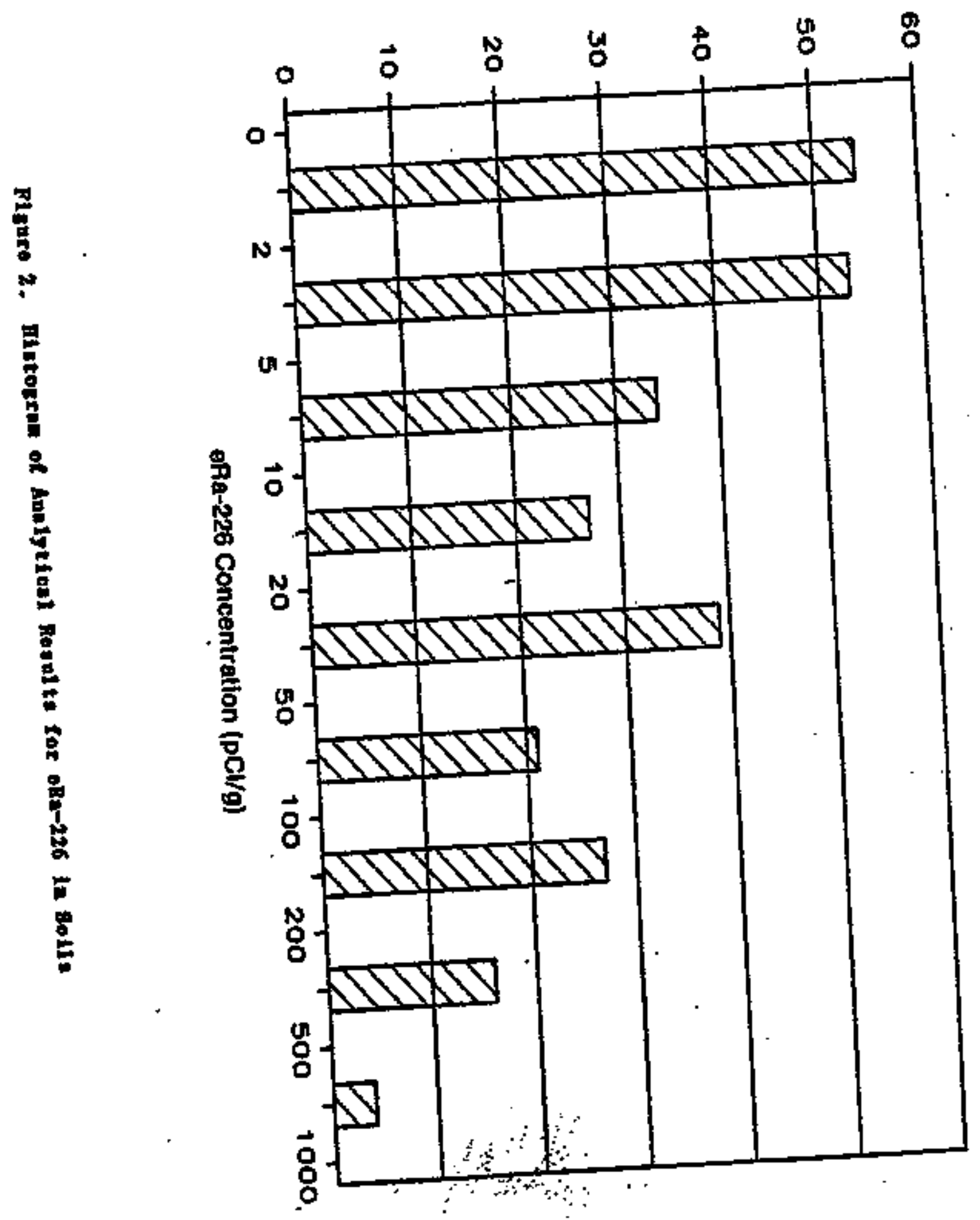


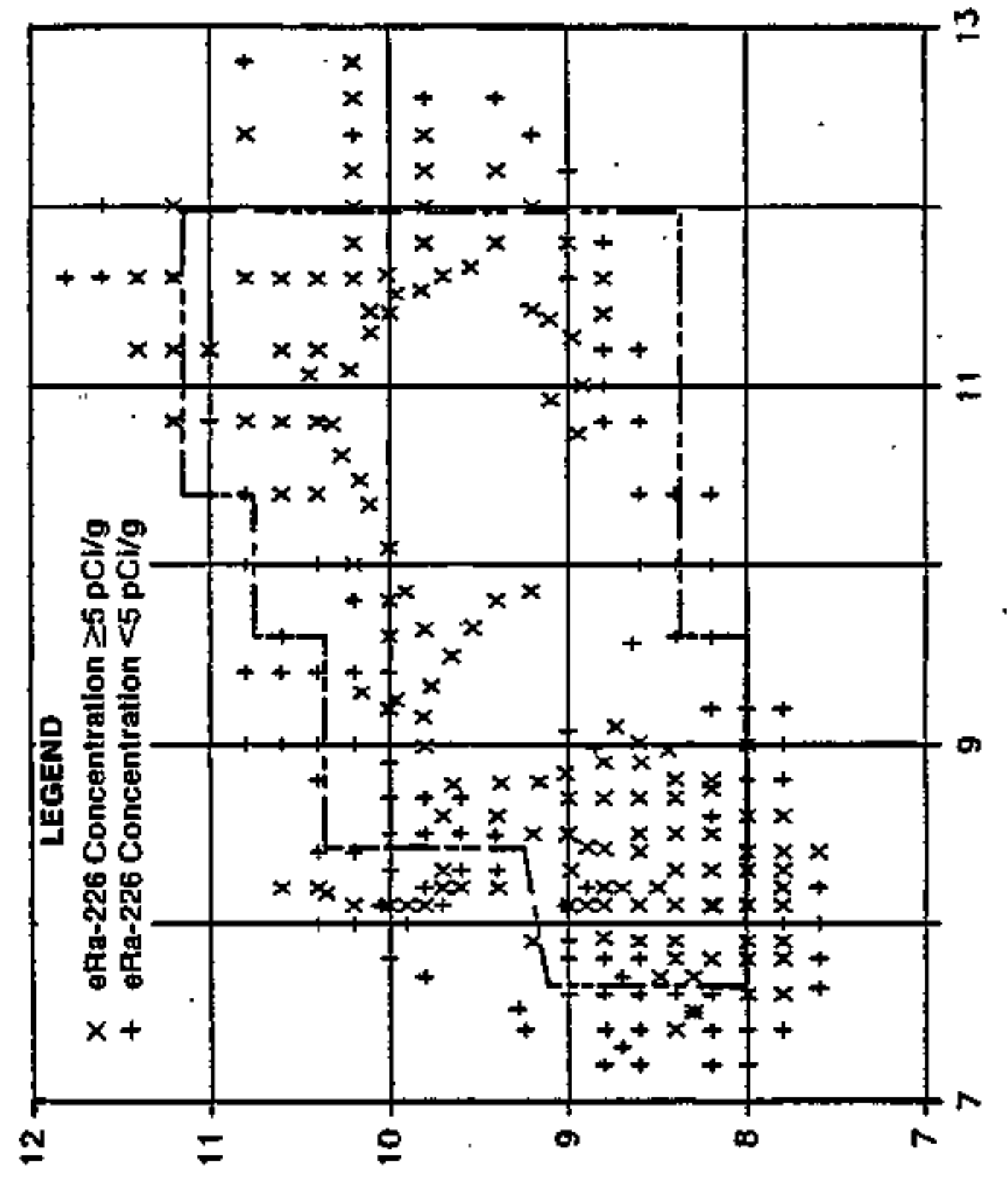

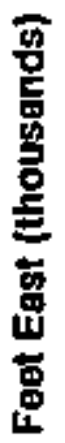

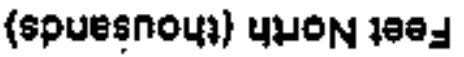




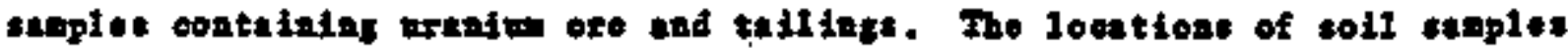

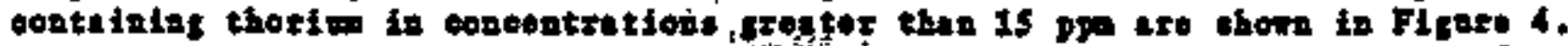

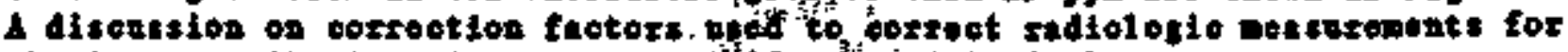

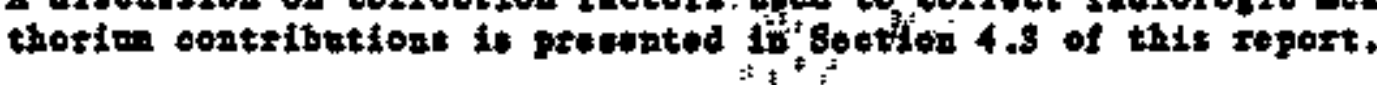

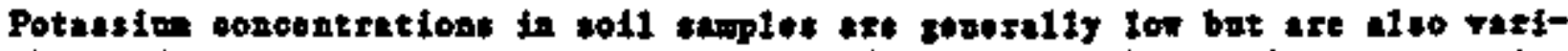
bl. and torge from bolon detection linit ( $<0,5$ percont) to 3.2 porcent. A

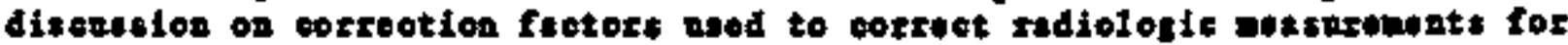
potestim epatcibations is prostated in the tollowing soction.

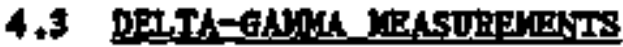

\subsubsection{Sol1-Wolntare Correction Fantort}

Lost-on-drytas (LOD) gecenresents were perforred on ter percent (28) of the soil sanplos oollected dnrint the flettuork phase of the stody and on 16 anplos tollected during tho itte-oriertetion piste, to dorivo en arerago

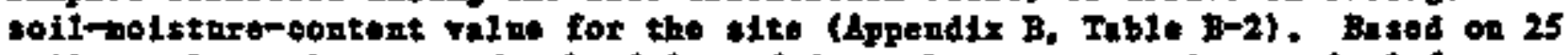

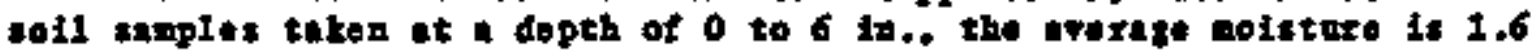
percont. Two samplos tukn at a dopth of 6 to $12 \mathrm{fm}$. and ono somple taker at a depth of 12 to $18 \mathrm{in}$. hed nolsture pontents of 1.13 peronet, 2.65 porcent, and 6.26 peroent, reepectrely.

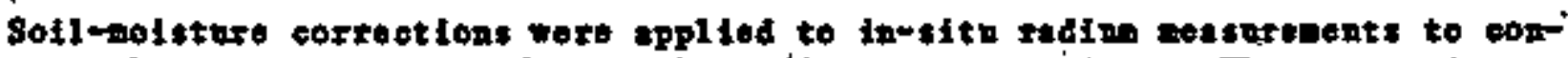

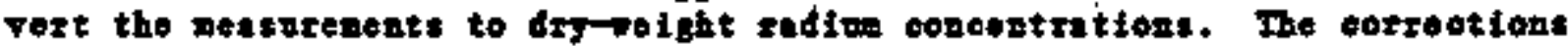

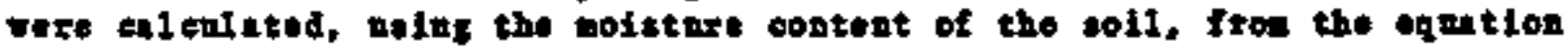

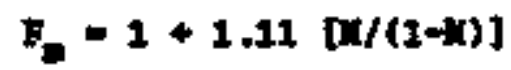

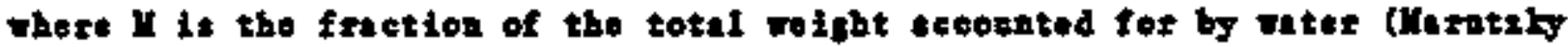
nind othert, 1984).

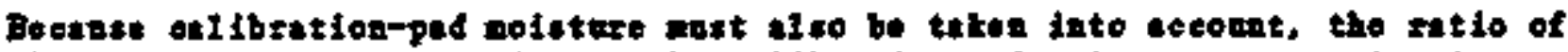
the noll-uoitture correction to the oslibration-pad-eolsture correction is applited to the date as tolions:

$$
F_{f}=\frac{1+1.21\left[\mu_{f} /\left(1-\mu_{p}\right)\right]}{1+1.21\left[\mu_{p} /\left(1-\mu_{p}\right)\right]}
$$

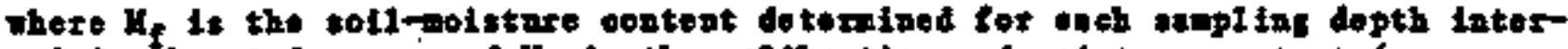

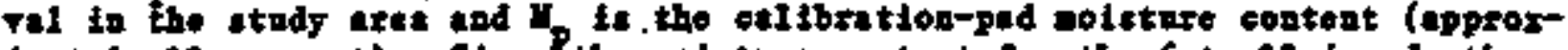
imtely 11 percent). Sines the solnture eontest for the 6-to-12-in. deptho was

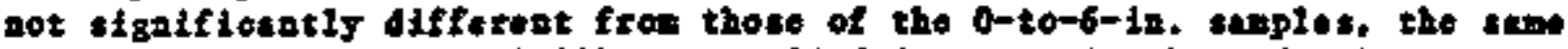
forrection factor (F: $=0.89$ ) was uplied for data in tiese depth rangos. Fot

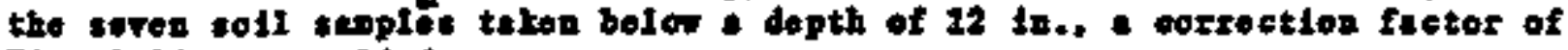
F: $=0.94$ vas epplited.

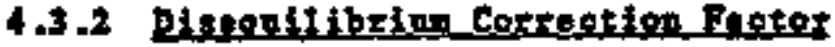

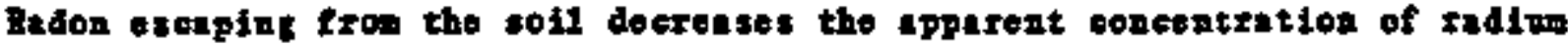

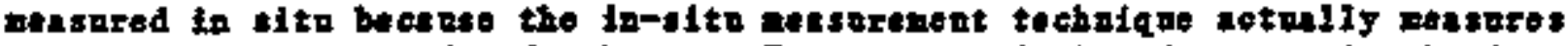
tame-rafomittint radon deghters. The wont of this docresse 1 the frecthan of radon thit esenpes 1 ron the 1011. 

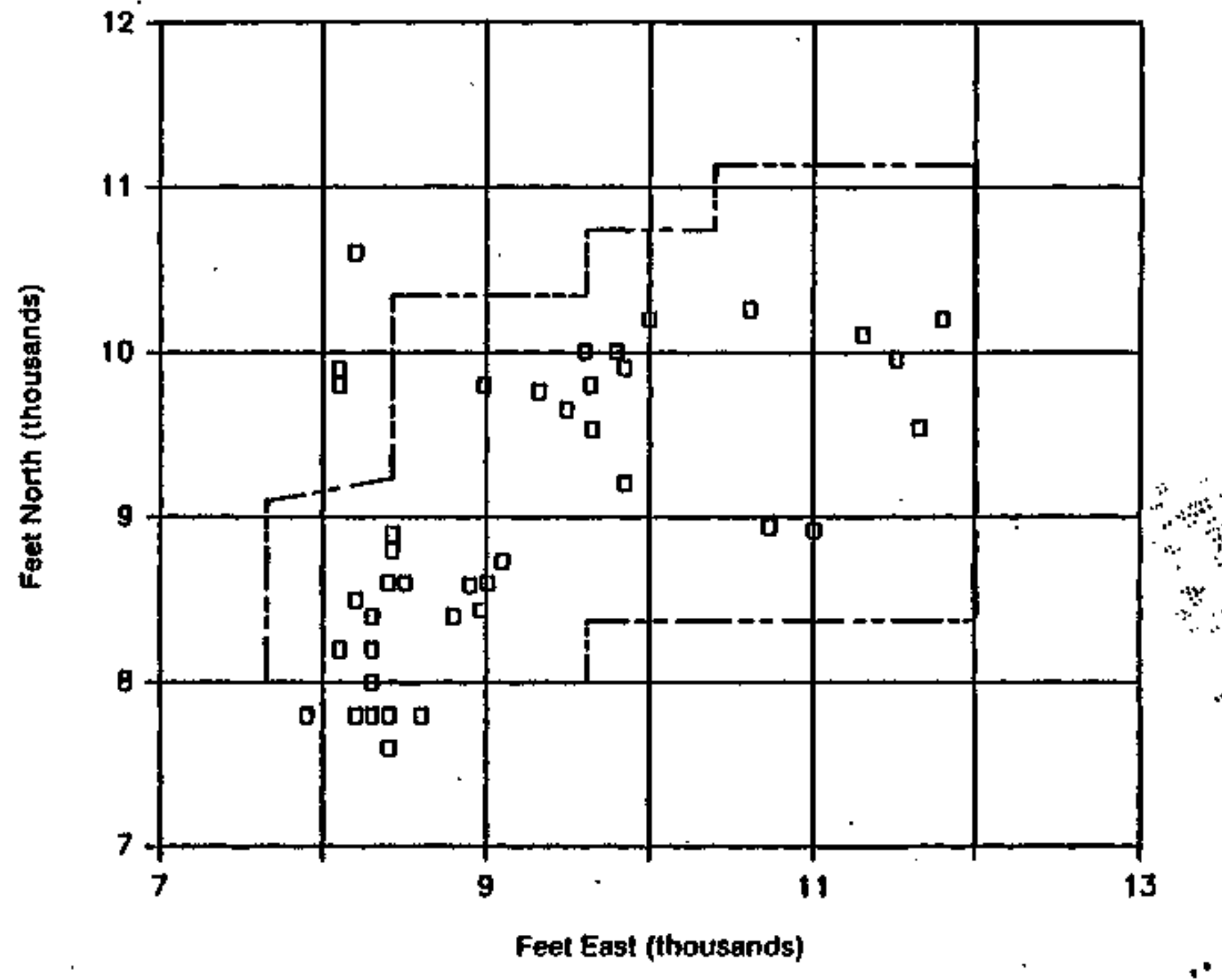

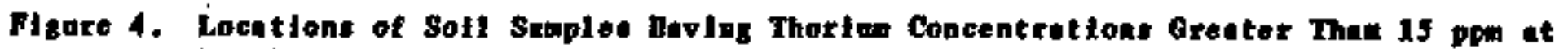
Dopths of 0 to 6 Inches. 


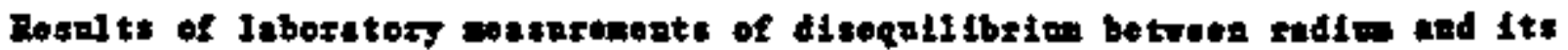

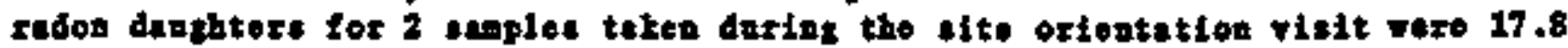
\pm 1.0 percent end $18.6 \pm 1.0$ percent; The eretege of the two val aet is $18.2 \pm$

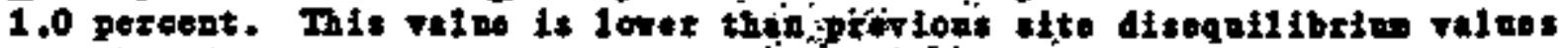

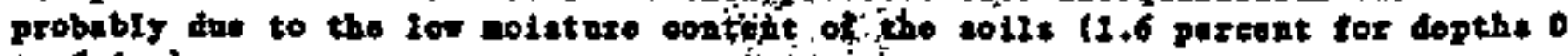
to 6 10.).

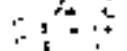

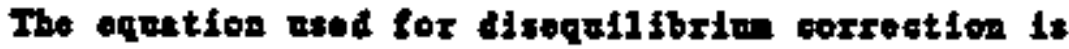

$$
F_{0}=1 /(1-B)
$$

where $F_{0}$ is the disequilibrim eorrection and $\mathrm{E}$ is the frecton of radion ascepini frow the coil. The apperent-rediw oopostratton eslauleted fron ir-

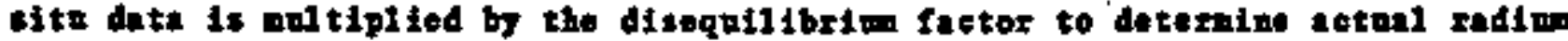

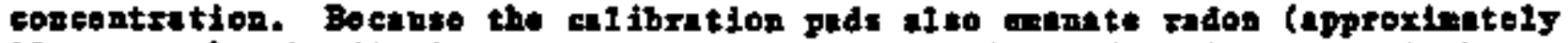

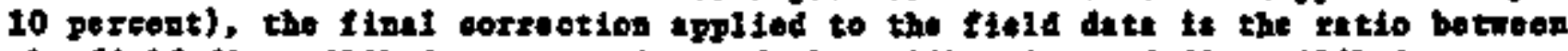
the field disegnilibrime correction and the cel fbratiog-pud diseguti lbrim porrẹetton, or

$$
F:=\frac{1 /\left(1-B_{f}\right)}{1 /\left(1-B_{p}\right)}=1.10
$$

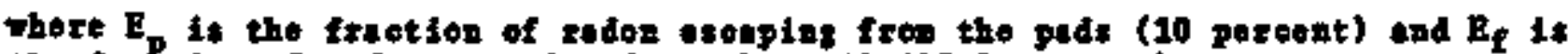
the frection of redou exceping tron the coll (18.2 pofeent).

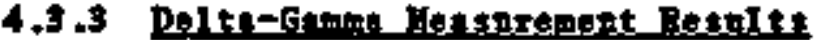

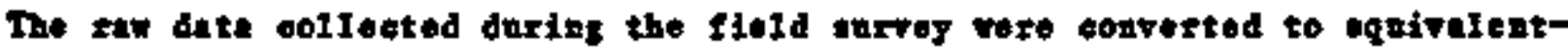

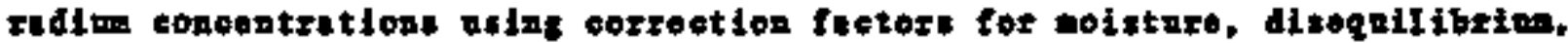

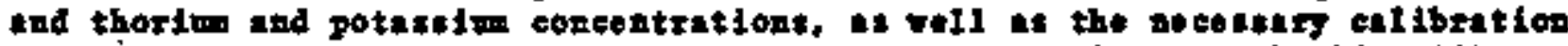

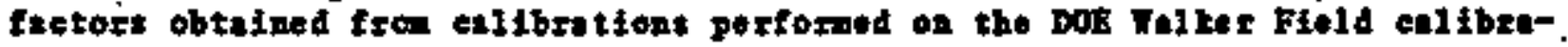
tion puds.

The ogation for date rodaction is

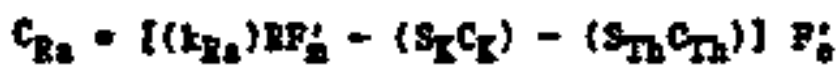

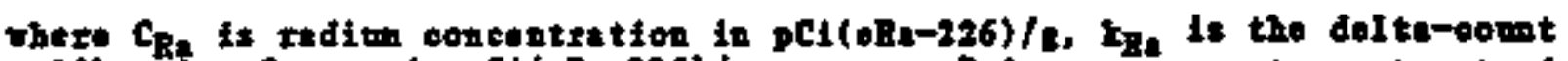

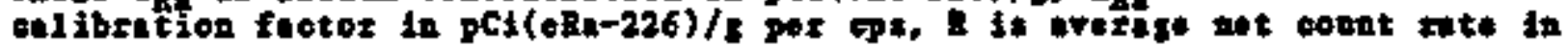

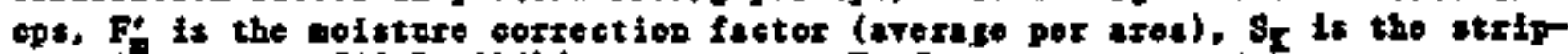

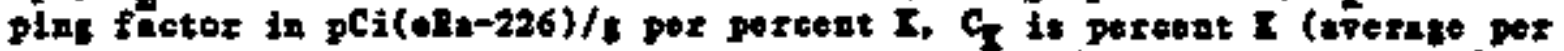

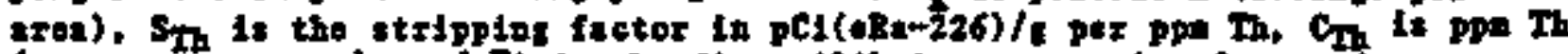
(avorage por area), and $\mathrm{F}$ ! is the disequilibritu corrootion factor (areztge per axes).

The disequilibfing coxtedtion fector (F!) detemined for this otrdy is 1.10

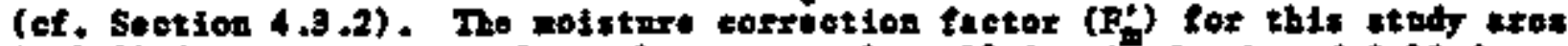

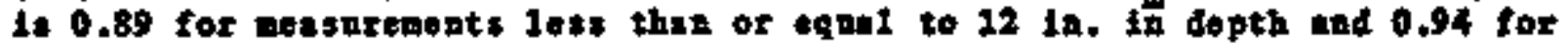

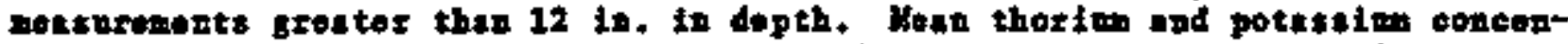

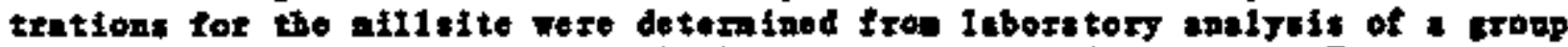

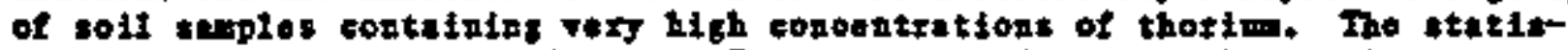

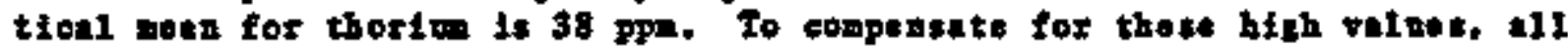

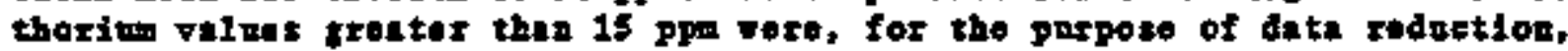

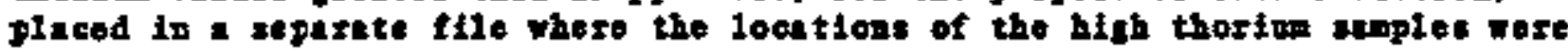




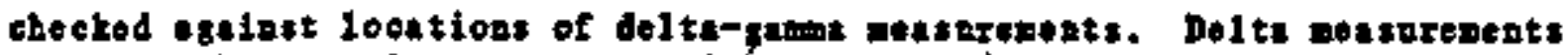

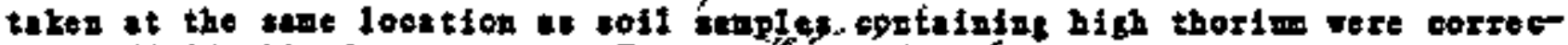

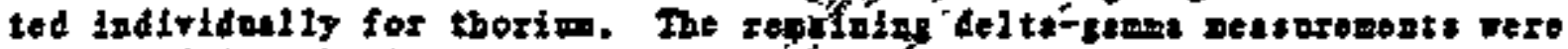

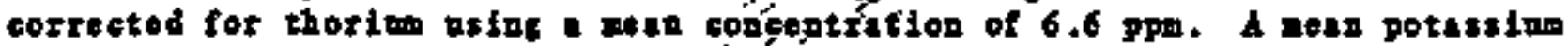

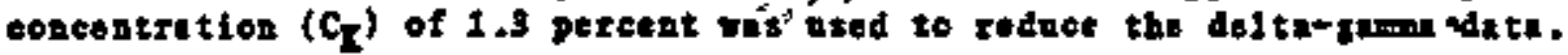

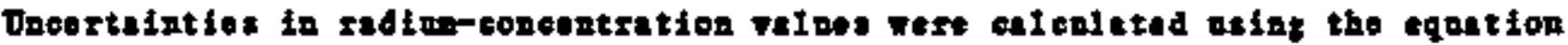

$$
\sigma=z_{B_{0}}\left[\left(2 N_{n}-N_{d}\right)^{2 / 2} / t\right]\left(F_{6}\right)\left(E_{m}^{\prime}\right)
$$

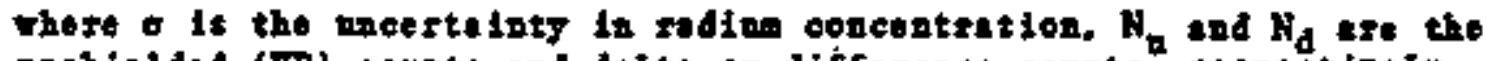
uashielded (UP) count: and doIte or differeace consts, tospetifoly, t is tho countins interval (time in acconds for the of coust). and the othor pirameter*

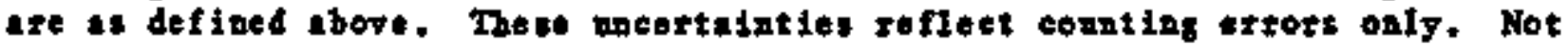

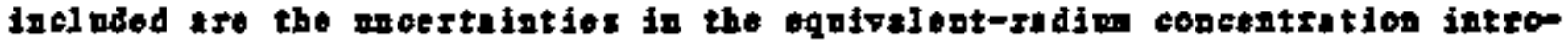
doted by the errora acsocieted ith each cerrection and ealibration factor.

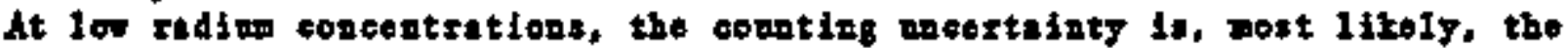

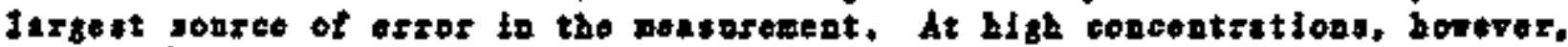

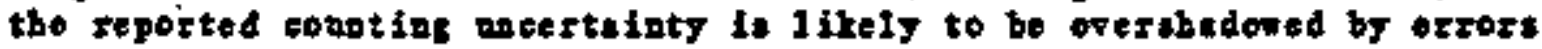

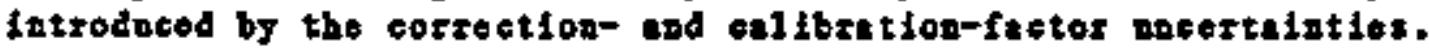

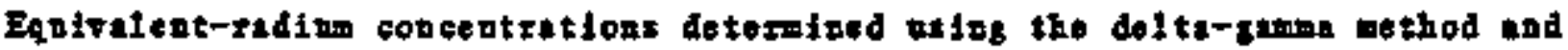

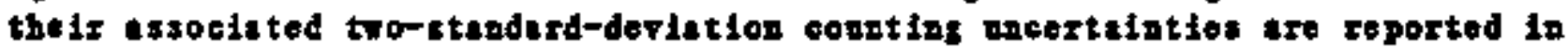

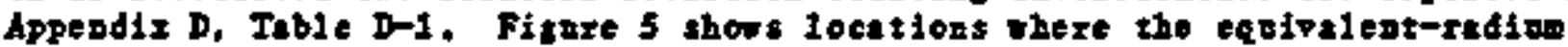
content of the soll exceods $5 \mathrm{pCl} / \mathrm{s}$ as deterwined by the delta-rama nethod.

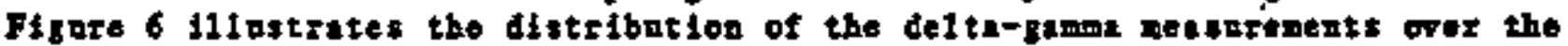
ruge of equipileat-radim copeegtrations deferminod for the tito.

Tith the exception of dolte-tames neasureserts in erroyes, nearis all of the

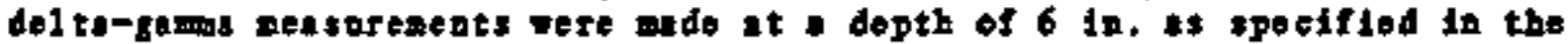

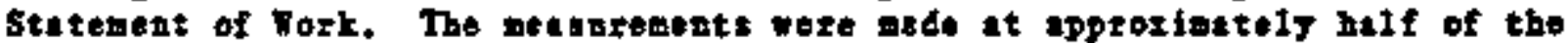
gridpotats in Arees $A$ ind $B$, and at all of the gridpolnts (exel ading borbhole loestions) tn Areas $C$ ind $\mathrm{p}$ following the coljection of a 0-to-6-in. soil simple. The alteratting soll semple/delte-gemen neasorement me thad deseribed In Sectson 3.3 wes red to dotermine the depth of contaminetton excediag EPh standards.

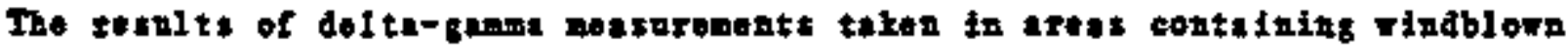

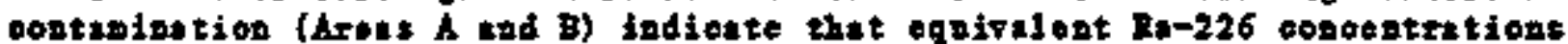

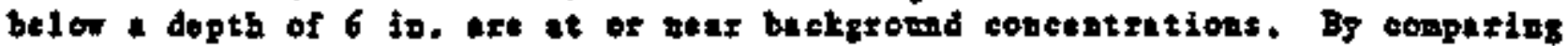

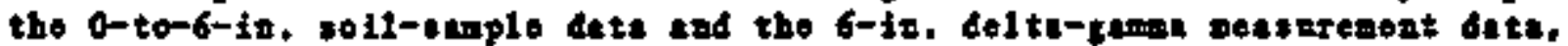

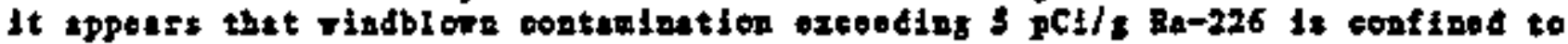
noar-arface ( 0 to 6 ind) beterials.

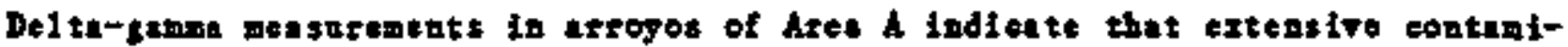

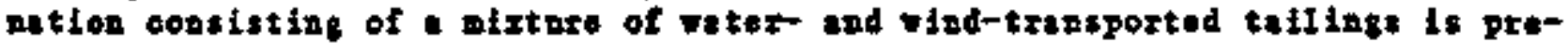
sent in scettered sand bers along anch of forx arrogot survtred, The extent of

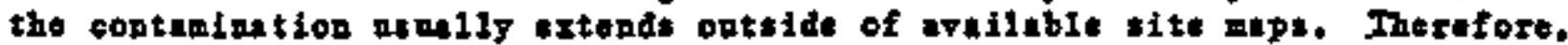
1n Appendis D. Table D-2, lostiog raluet of 99999 ise given for meatucemerts

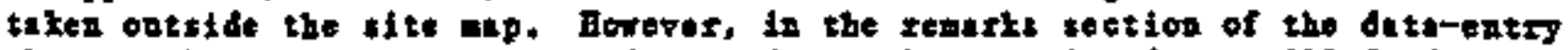
whets, tho measurements ere refezeneed to a born point $(0.8,400$ ft down-

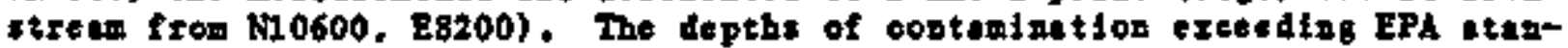
dards geveratly correspond to depths noeded to reich bodrock. The erroro

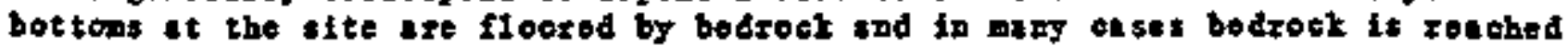



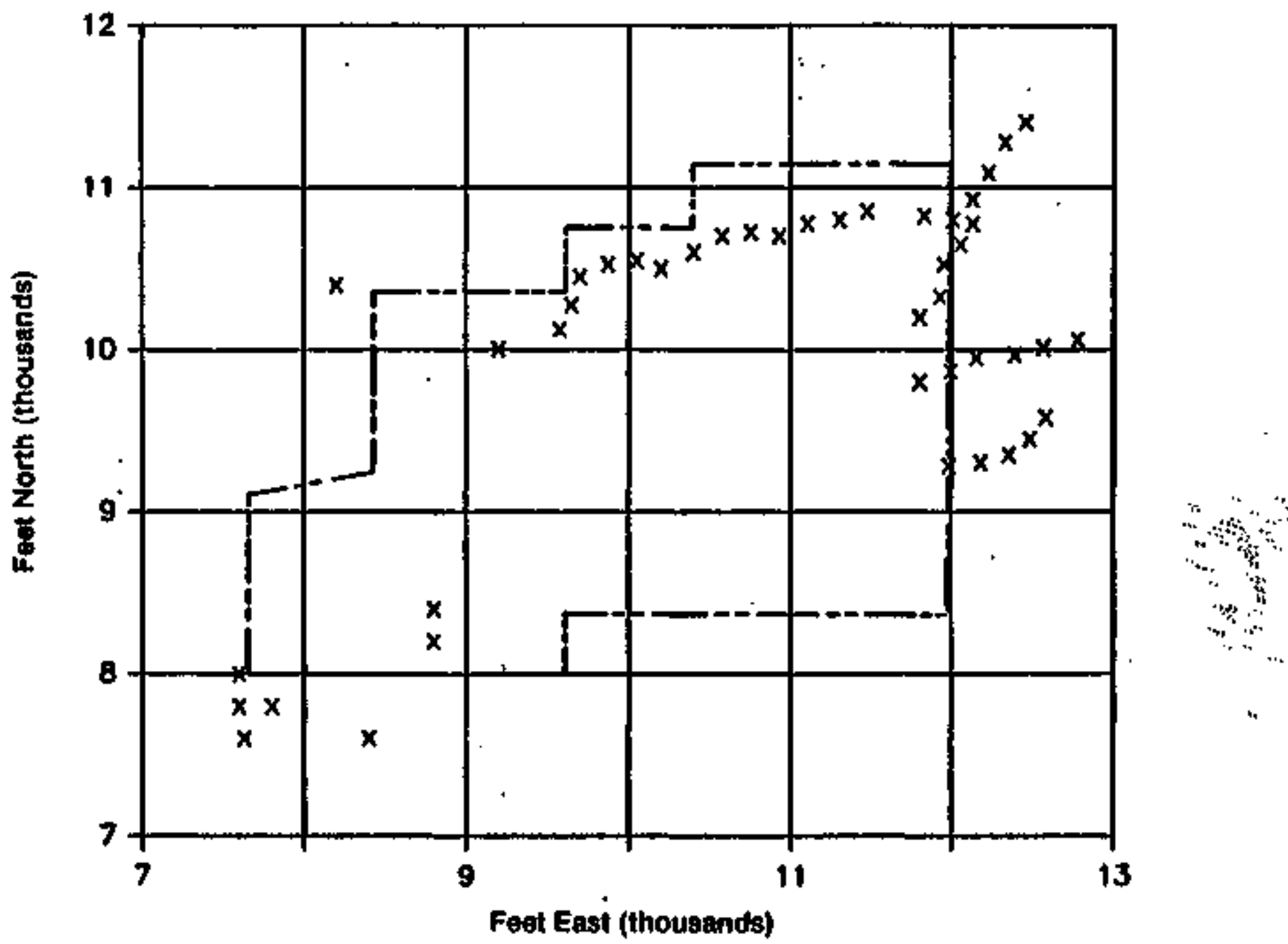

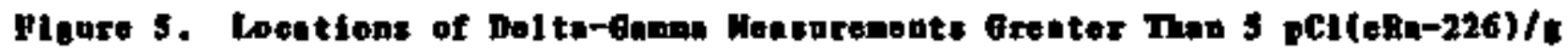




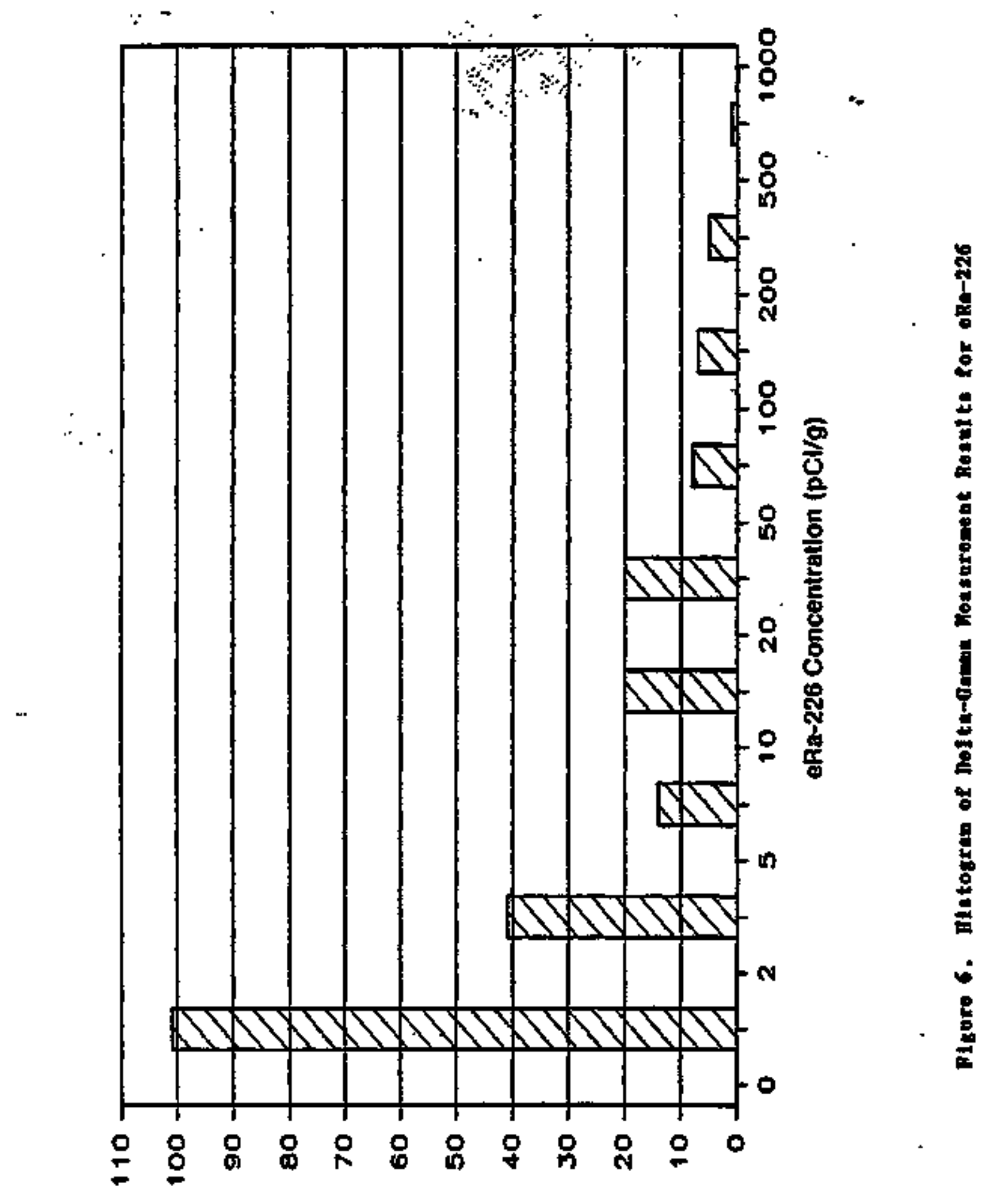

sluewesnseew to JequnN 
at a dogth of 3 to 4 ir. Once bodrock is reached, the ragiralobt-radiw

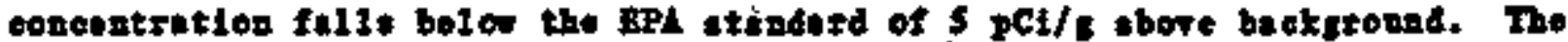

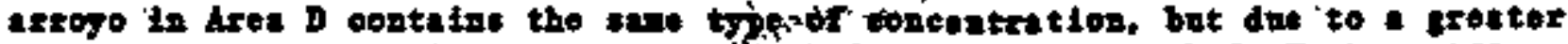

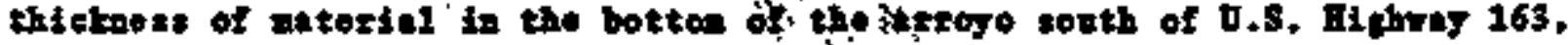

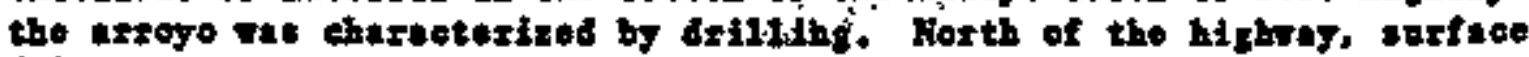

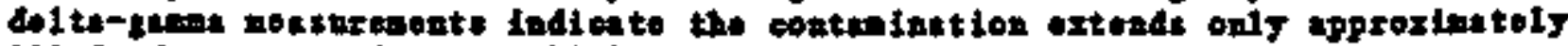

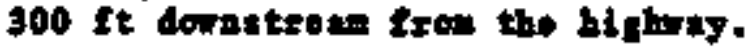

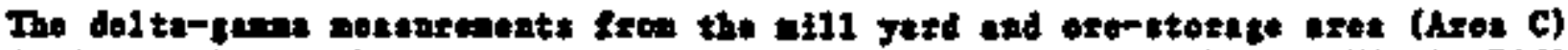
Indlot to thet of th the exerption of sis exidpoints (N8200, E7600; N8000, Bt600; 27800, E7600; M600, B7695; N7800, 28200; and H7600, B8400) the ofle-226 ooncen-

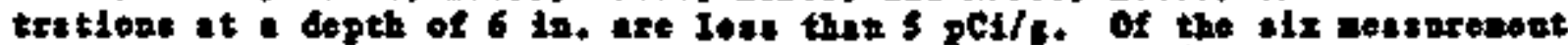

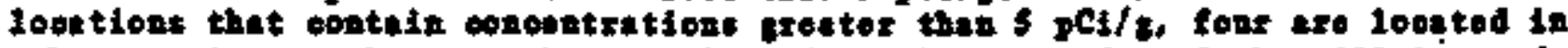
a borrow-pit ares between the grerol soud on the vest odge of the atI1 blte and

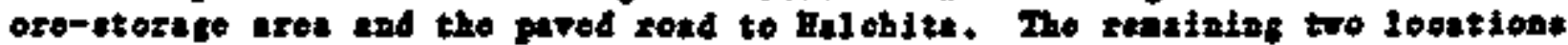

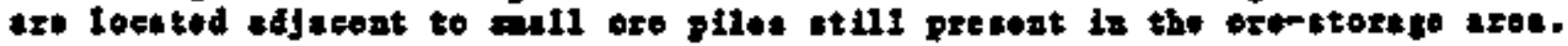

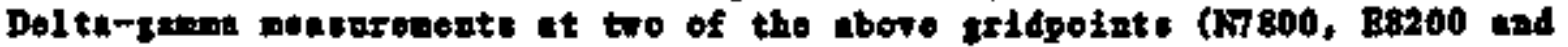

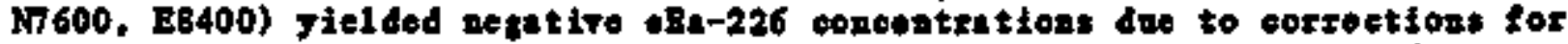

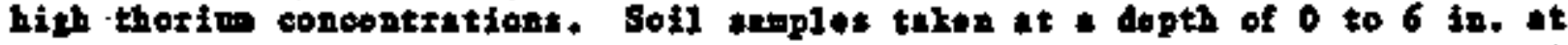

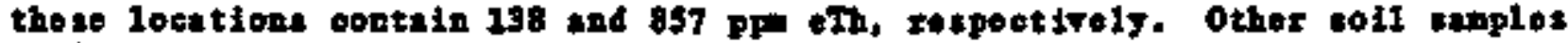

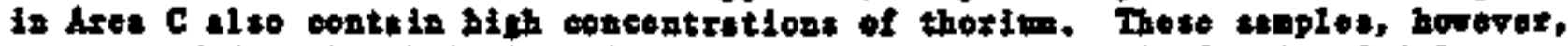

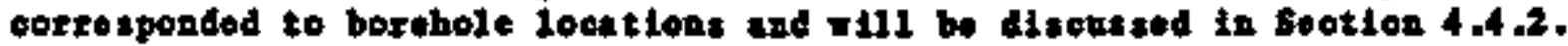

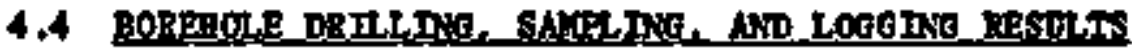

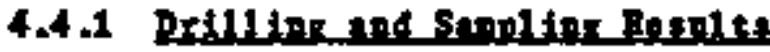

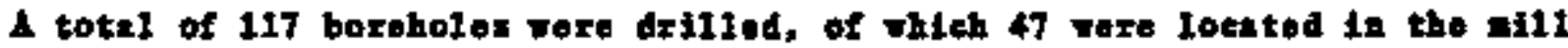

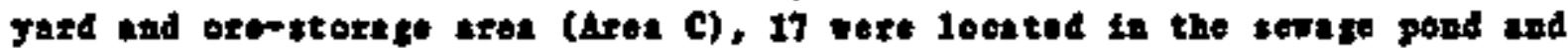
irfoyo ires in tho northwet portion of the tite (Area D), 23 Fore lockted along the periphery of the rpper gile (Ares B), 28 vero loented along the poriphery of the lower pile (hros F). end 2 hol ot rofe ldded to test piles of oonterinted weterint is Ares A. The borebolo nobera, coordinates, and elovetfons are ineluded in sppeadix B.

Boreholes in Area $C$ vere drilled by trat-moented anger eccordins to the

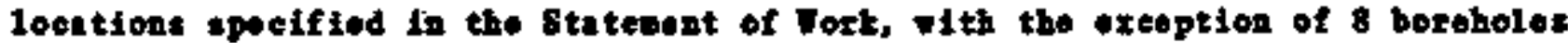
(HAT-013, 017, 022, 023, 026, 037, 048, and 051) that Foze worth to aroid otil1tios. exlstins strueturen, ind other obstruetions. Borohole BAT-057 was

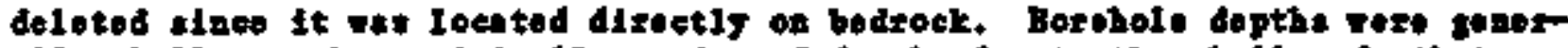
ol15 challomer than oziginally ontinated dopthe dat to the shellon depth to bodroek.

Boreholes in Ares D Fefe drilled vith trookgonted atger aceordist to

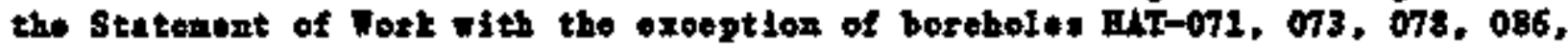
and 088 which were wored doe to difficult acoess or shillor dopth to bodrook.

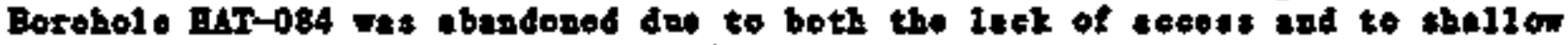
depth to bedxock. Borobole BAT-085 wes drillod asing a portablo argot.

Boreboles in Areas $E$ and $F$, Fith had approxtento loctilore spocif

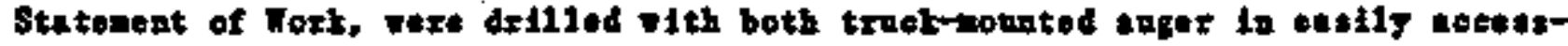
tble loottions and portable onger in diffient t-coess areas, Boreholes vere Iosted by the Toehnical Asivtance Contrecter and the Bendix alte meneger to 


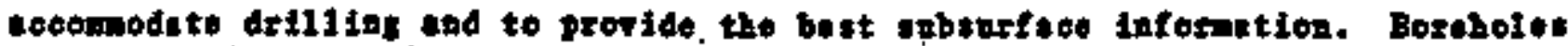

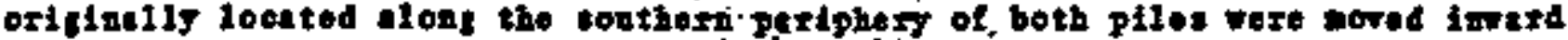

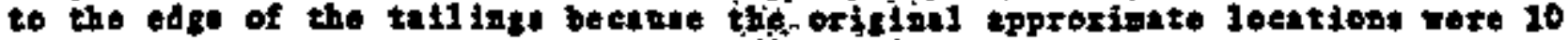
to 12 it in elevetion above the talligs pilit, whok wontd heve required

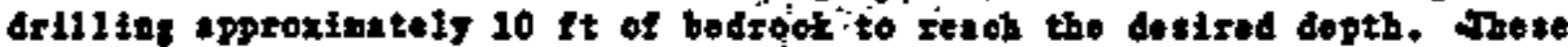

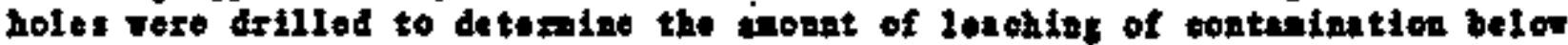
the tolt inta/bedrook interfaee.

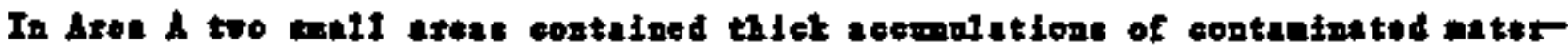

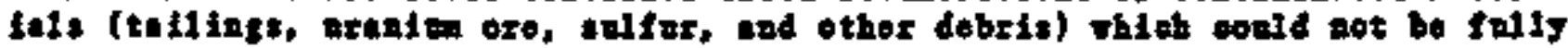

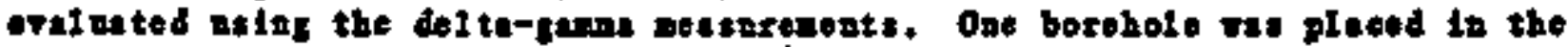

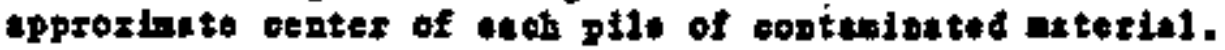

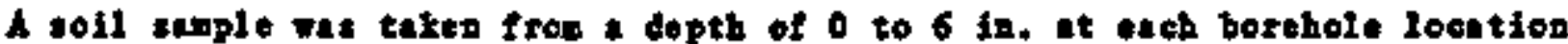

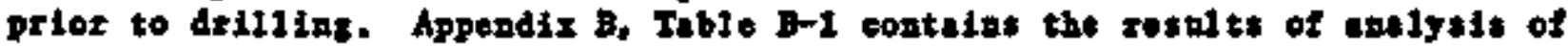
tho enimples.

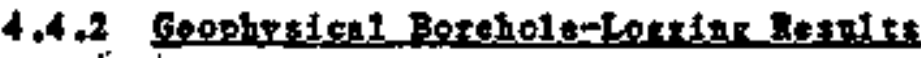

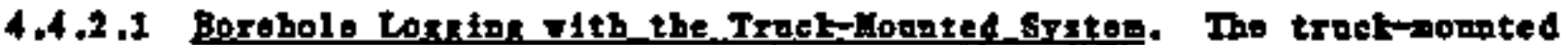

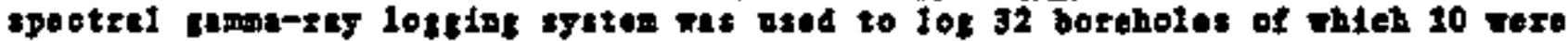
In Ares C, 9 were in Area D, 6 were In Arua $E$, and 7 vere in Area $F$. The

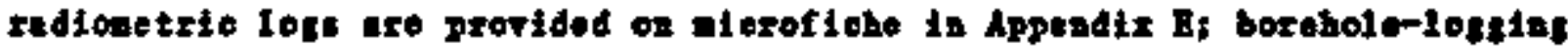
und equipuent dete ere presented la Tuble $B-1$.

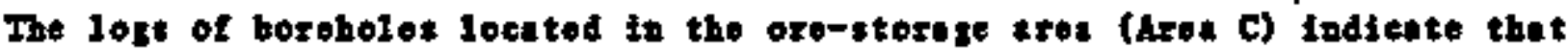

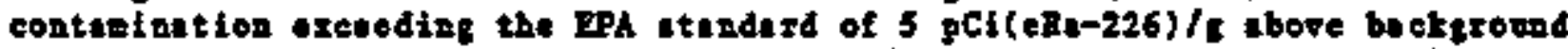
1. spotty and rangen froe no contamintion (BAT-021, 023, 029, and 045) to contiofintion at depth of 5 it (BWT-022). The doepert and uost eztersite

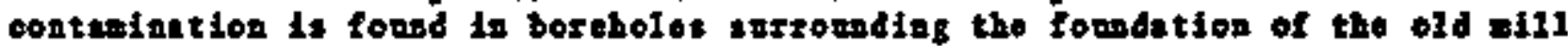
(BAT-022, 026, 031, and 035). The botehole del1Iod noxt to the track ocale

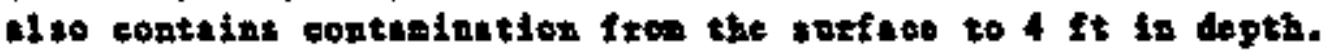

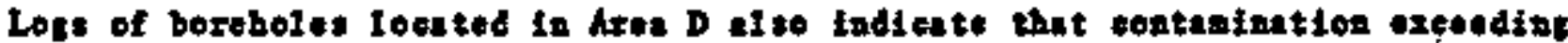
EPA standicds it spotty. In teaeral the copteisidetion is aballor (0 to 6 in. in BAT-073, 074, 080, 084, and 088) v1th the exception of boseboles FAT-071, 075, 081, 083, and 086, thiok havo spotty oogtetsintion to a depth of $4 \mathrm{ft}, 7.5$ ft, $10.5 \mathrm{ft}, 6.5 \mathrm{ft}$, and $2 \mathrm{ft}$, re epectively. Thene hol on aro 10eted in the crogo oostefing water-trtesported tallinge.

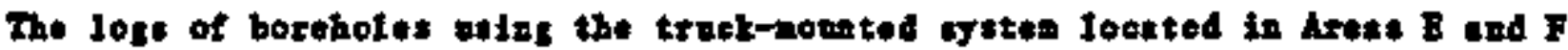
tro oonfined to boreholes located alone the noxthern perlphery of the ngpe: end Iower pilos with the oxception of borebolos EAT-120 and 131, locited on the west edge of the eppex plle ard BAT-110 loestod on the touth portion of

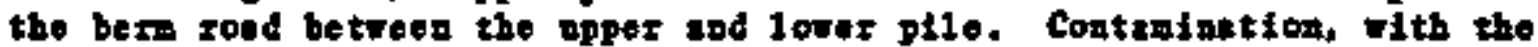
oxeption of bereholes BMT-102 and 103, is deep, ofter extending to the total degth of the hola.

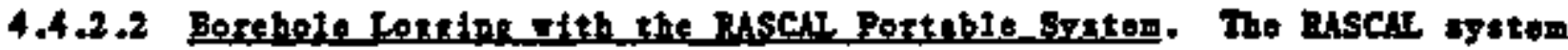

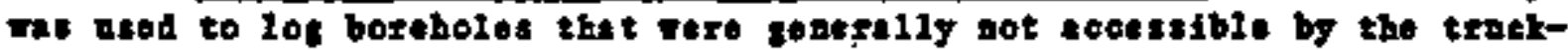

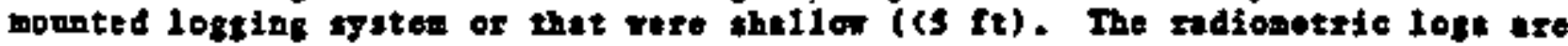

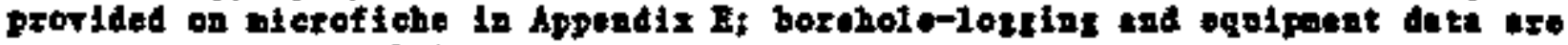
presented In IsbI $\mathrm{E}-2$. 


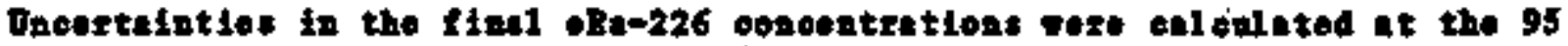

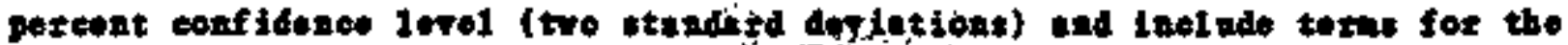
collorias perandet: :

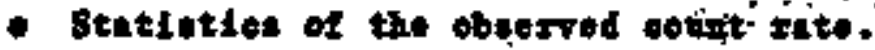

- The decontolntion itortth.

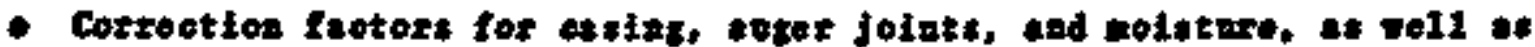

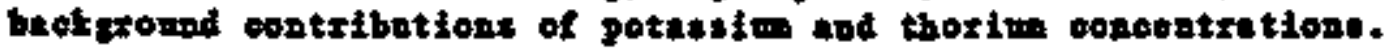

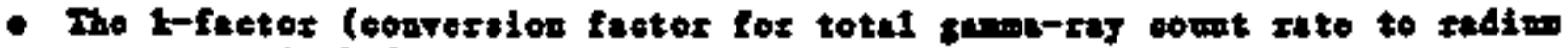
concontration) for the asten.

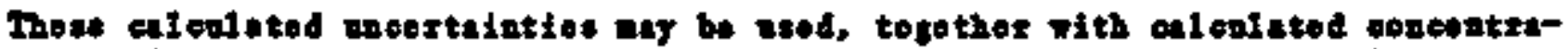

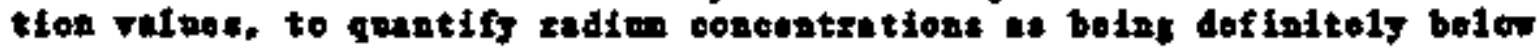
tpocifie 1 inite with of pozcont confidence.

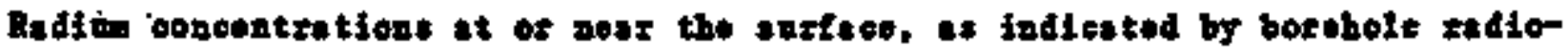

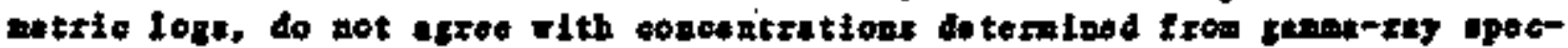

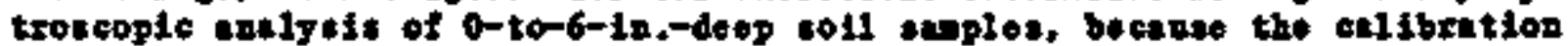
procedure does not siralste the alr/earth interfece at the top of the botebole.

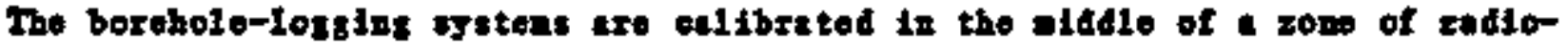

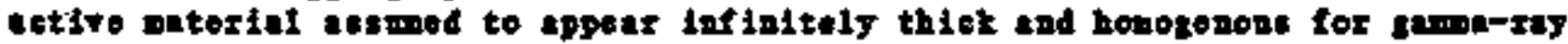
generetion and propagation. This sub arfmetion ft then wide, for the borehole When procestint the los dota, Fith eppropetete docorrolution to corrot for adjacent bod effects, bet aot insr-anface and sorfice measurenants for the ais/onrth jnterface offect:.

A totil of 82 boxtholes rexe logzed asing the pusclt bytom. Fonrtion of these

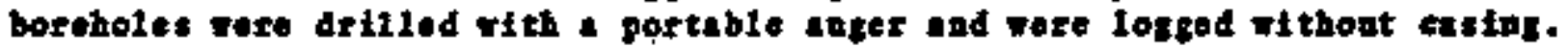
The tepiling boreholes were logged thropgh hollow-sten atgors.

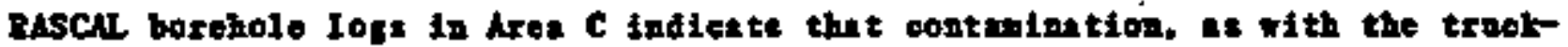

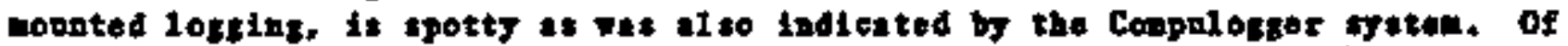

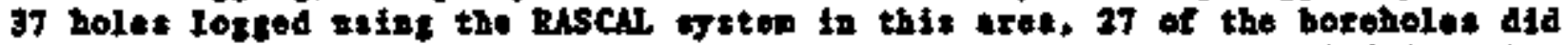

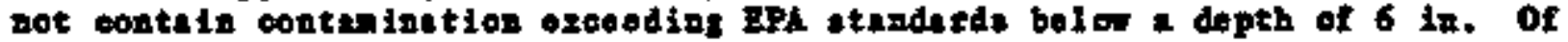
the 10 thet did contein sfestet than 5 pCi $(4 \mathrm{R} n-226) / \mathrm{s}$ betor e dopth of $6 \mathrm{ln}$. only 4 and conterinction belon a depth of $1 \mathrm{ft}$.

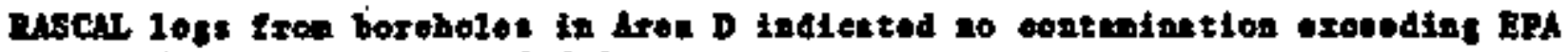
tandeds belor a dogth of $6 \mathrm{ln}$.

Lots of borebofes In Atons $\mathrm{E}$ and $F$ indies te thet conteination al ons the

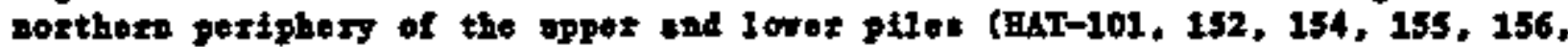

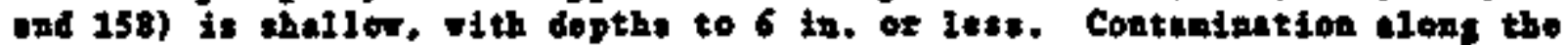

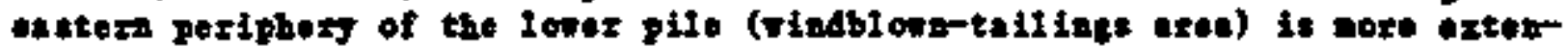
sire, fith depths to 12 ft. Bortholes on the southern geriphery wero drklled to bedroek on the edge of the talligz -1th a portable nefer. In most onces the contanlation exceods 3 pCi(alu-226)/s the botton of tho bolo. To

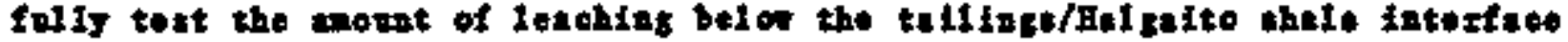

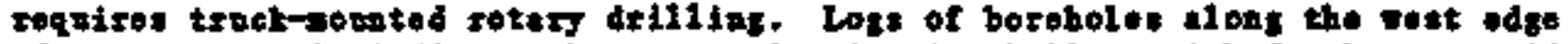

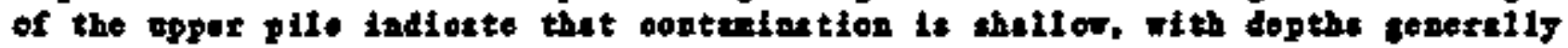
Iest thas $1 \mathrm{ft}$. 


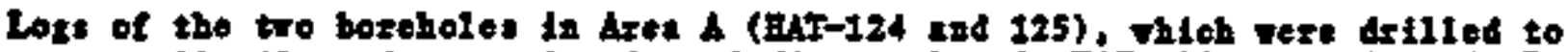

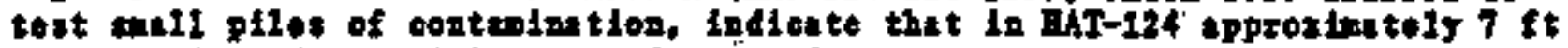

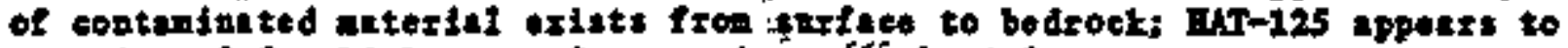

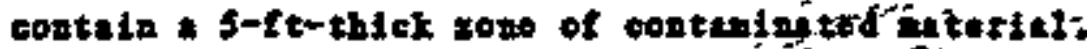

\subsection{BUIDINO_GDVET RESULTS}

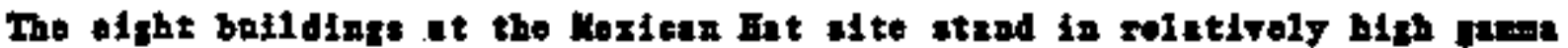
flel de (often rofored to as "pile ahine') dwe to thols proxivitg to the

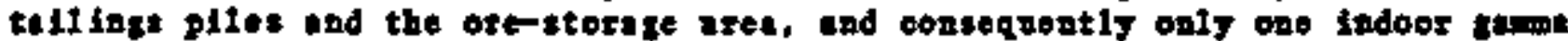

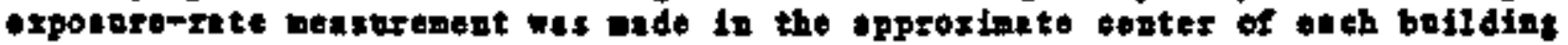

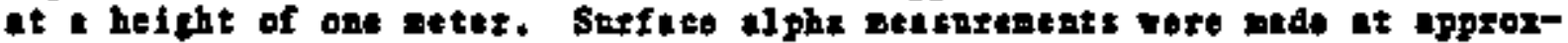

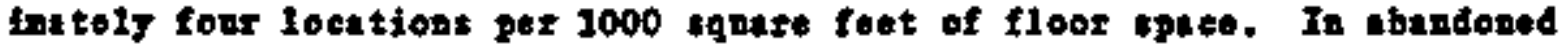

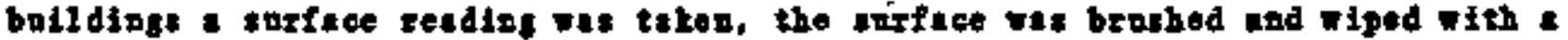

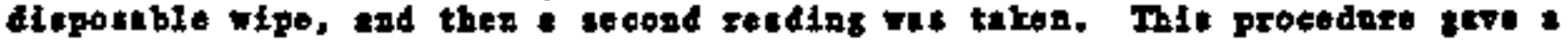

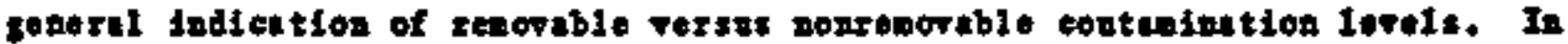
the two oocupled bofldiegt (tho Balchita Fealth cliale and tho shoet netal

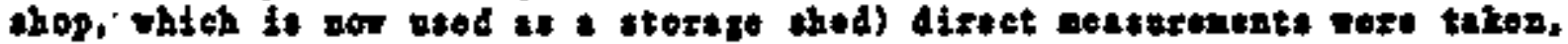
followed by toess on Aad-wipe filter.psper which vere then oouted. Appendix

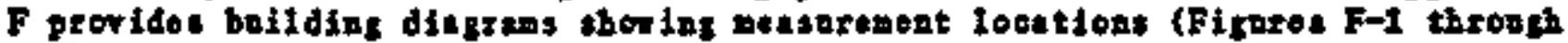

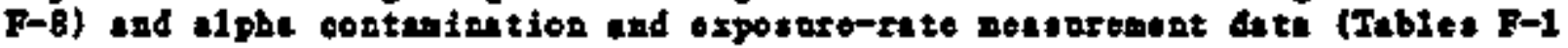
through $F-B$ ).

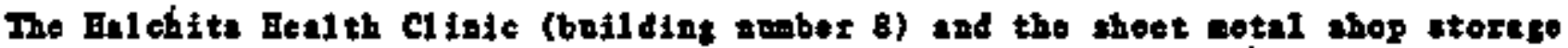

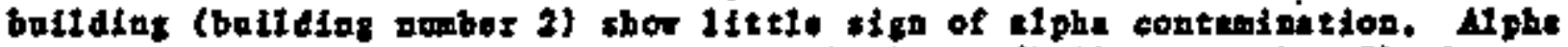
metsucement: in the former trede chool betlding (boilding nombet 5) also

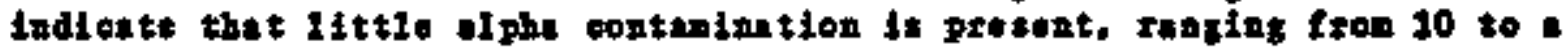

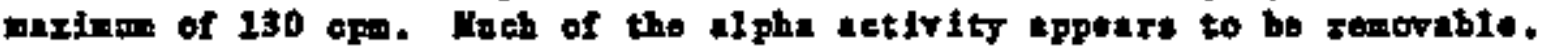

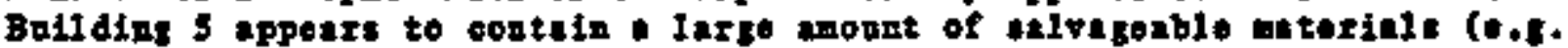
stroct oral steel). Another sall patel buifdins (building nombor 3) has higher

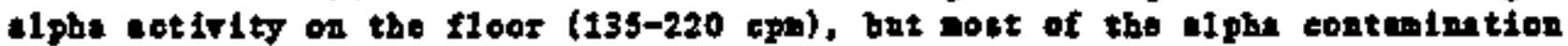
appears to be removable and the bnildies Itself appetre to bo in food condi-

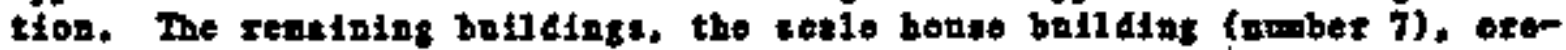

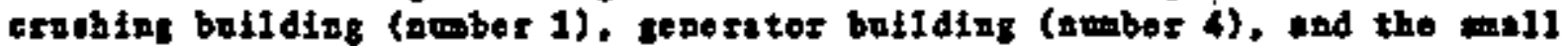

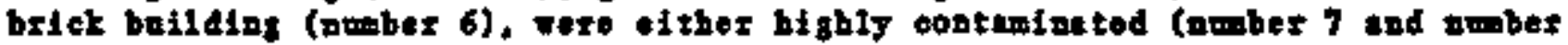
1) or had 1ftele talvage raloo (nmber 4 and nmber 6).

\section{4 .6 BACAGROOND GHARACTERFATION RESULTS}

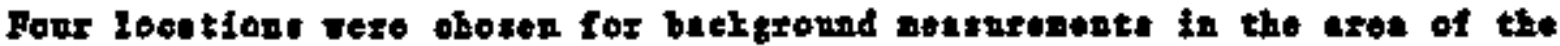

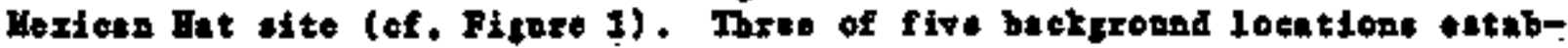

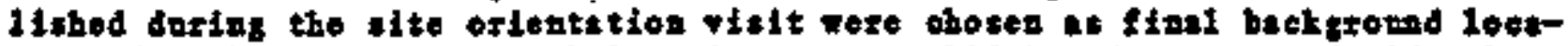
tiogs for the site. A forzth loostion ras oxtablished daring the floldroxk

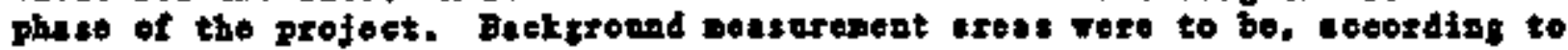
the 8twterent of Work, et toast 3 milos ecet ox northoset of the site and at

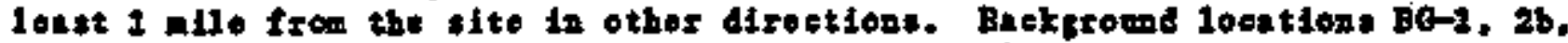

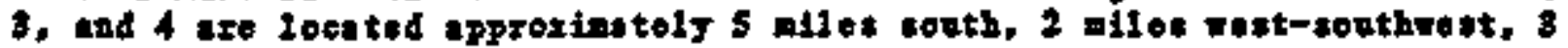

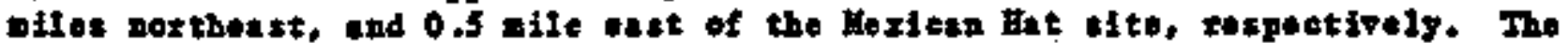

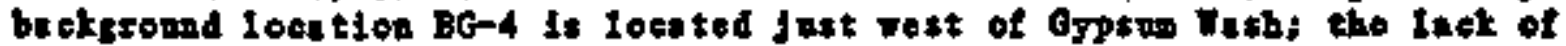

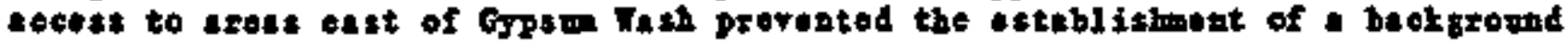

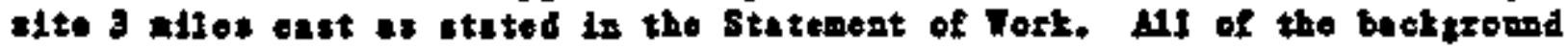




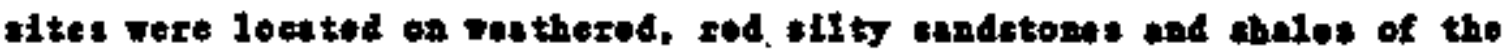

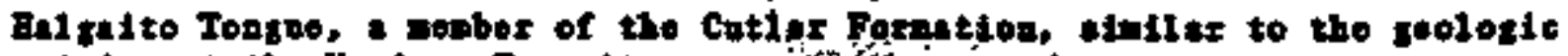

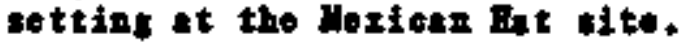

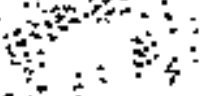

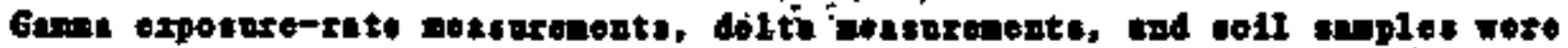

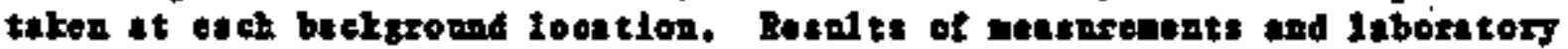

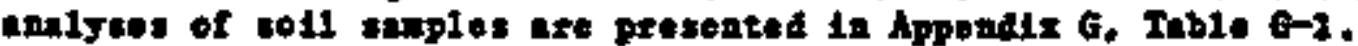

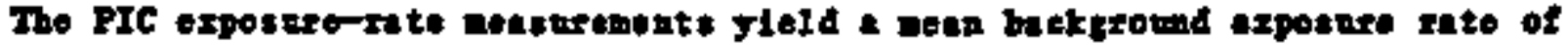

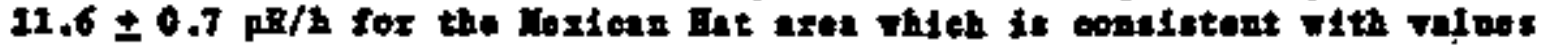
reported by prevloos stydiks.

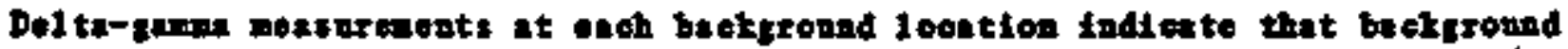

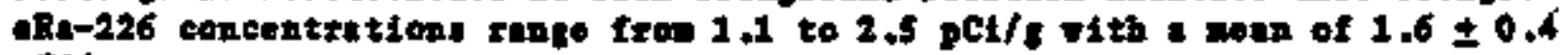
pcis.

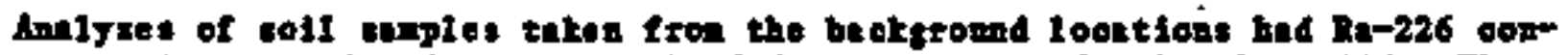

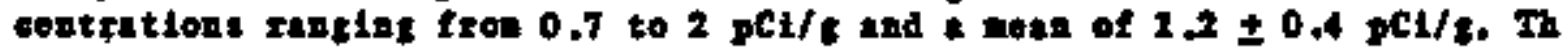

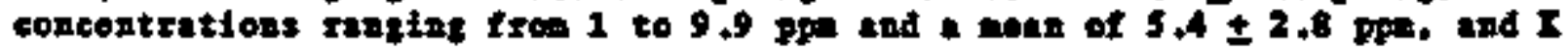

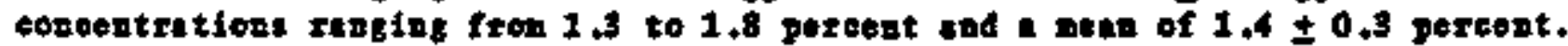

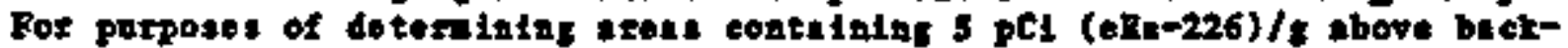

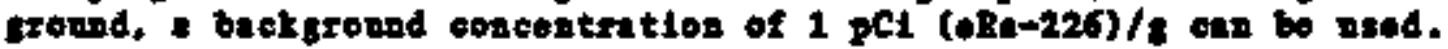

\subsection{SUDMRP}

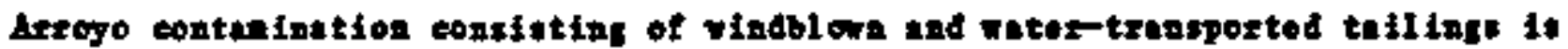

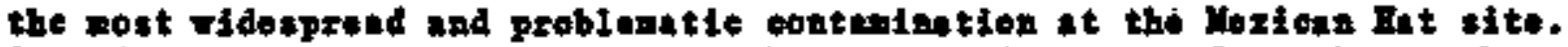

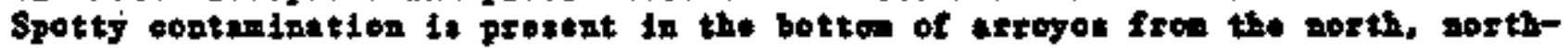

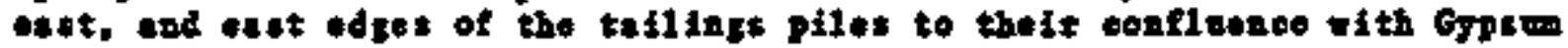

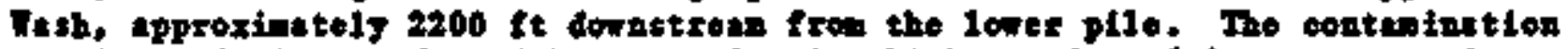
consists of elongated sand buts entige in thicknest frot 6 1n. to spprozi-

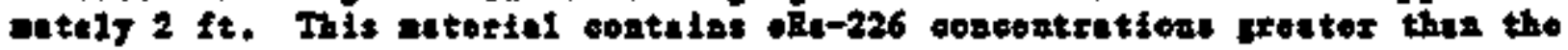
ERA standard of 5 pCl/s above betkgomd.

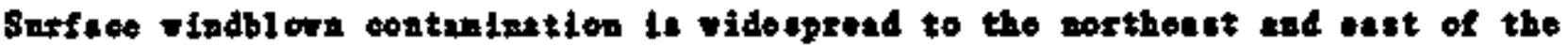

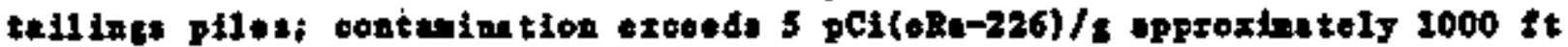
north and approximetely 800 ft eact of the Iower plle. Bentil thor that bost of this contesint ton doos not extend belor dopth of 6 10. To the soth and

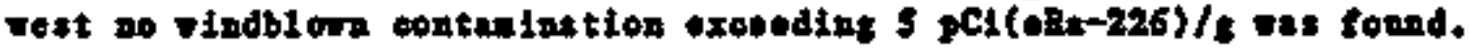

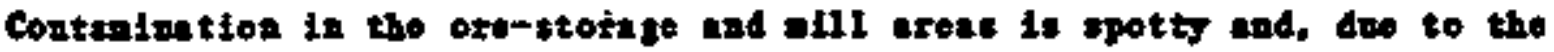
shat Io dopth to bedrook, is generally shetlow an vell. This contenintion

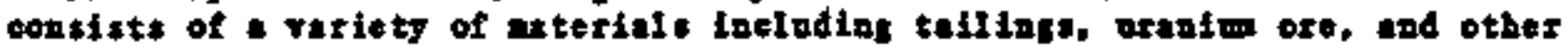
bexiod dobrft. The doepest contentertion oocurs in an eree suxronefint the lerge eoverete toundation of tho ofd aill.

The worrse pond and arroge sree in the northrest portion of the site do not eppear to eontain contanination exceodins 5 pCi(oRa-226)/s oxtept in and alont

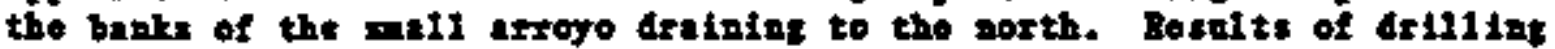
indicate thet noar the nead of the arroso (mear the opper plle) water-transported teilings contaninstion orceoding EPA stendards is present to as worh es

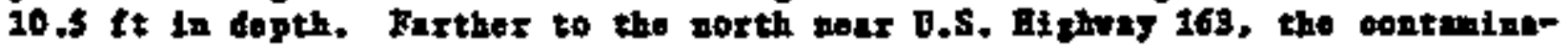




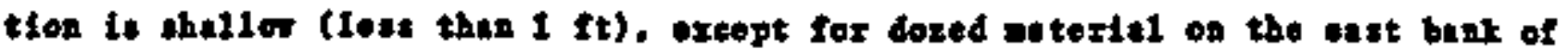

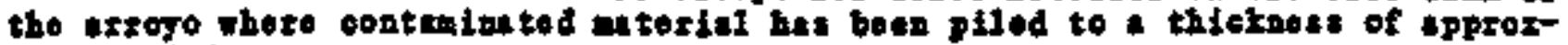
tontuly 4 it.

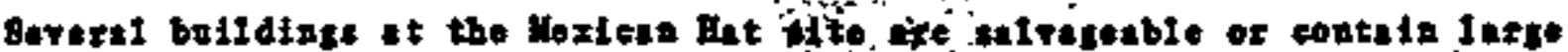

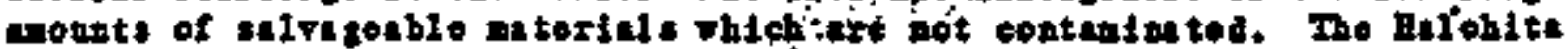

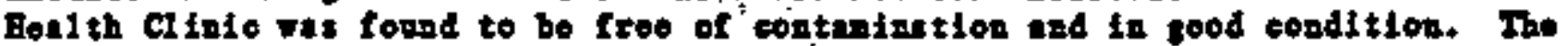

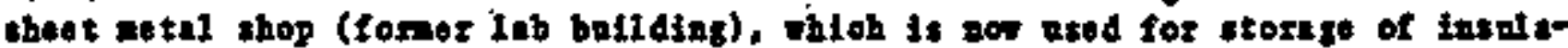

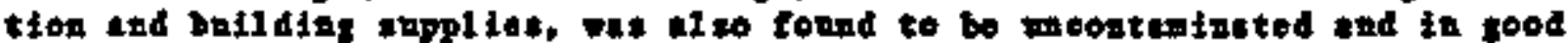

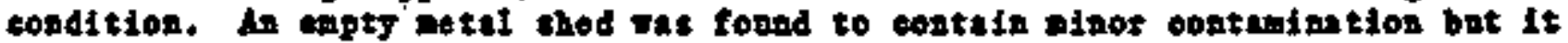

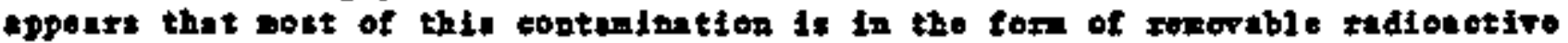
cost, and the bajldias Io la good condition. The long billdiss fornerly used

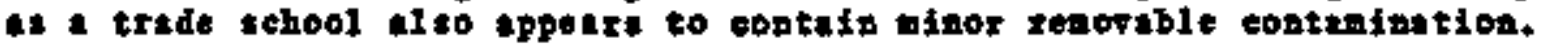

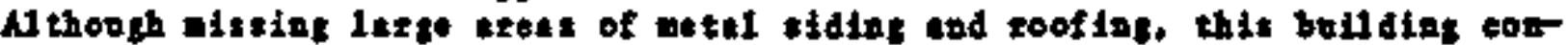

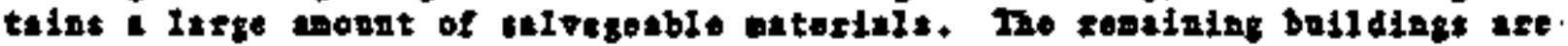

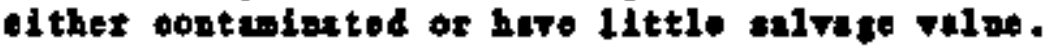

$\therefore$

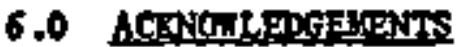

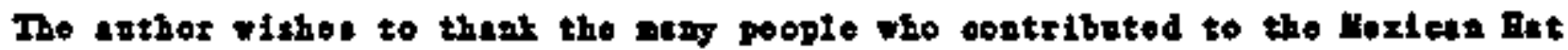

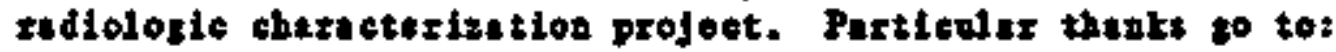

- Dontr Tooding and Phil Zelle, of the Jacobs-Toston team, for their ditection and atsistance with the flaldwork.

- The staff of the Balchita Ealth cinio for their cooporetion.

- Project Offiger Lerry Ball of the Depirtoent of Erorgy Grand Jnection Projets Offieo for his pobile relations wark at Maxien Bet.

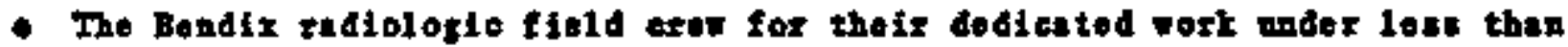
ideal rorking conditions. 


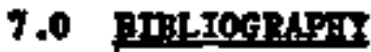

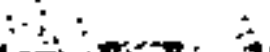

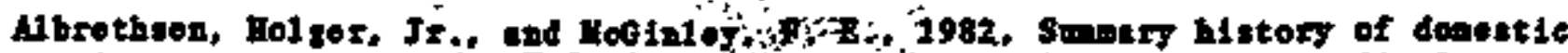

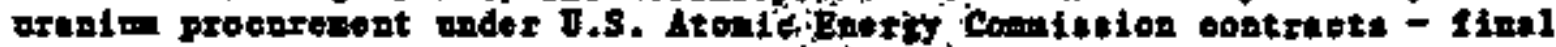

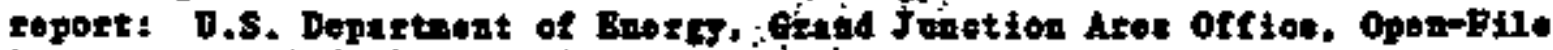
toport GJBT-220(82), P. A-83 to A-86!

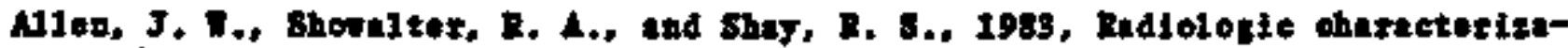

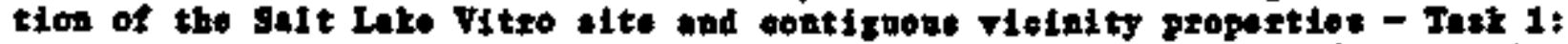

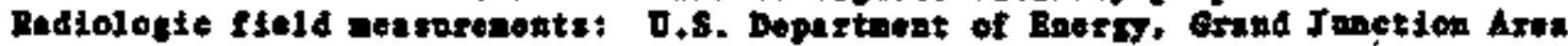
Offies, Moport $\mathrm{GJ}-0 \mathrm{~s}(23), 69 \mathrm{p}$.

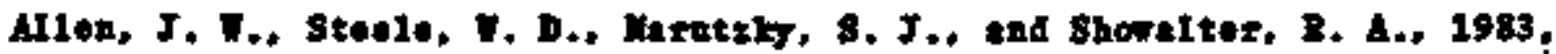

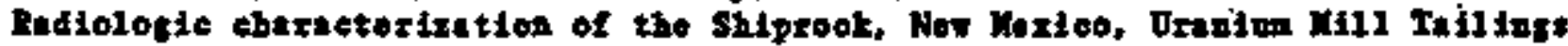

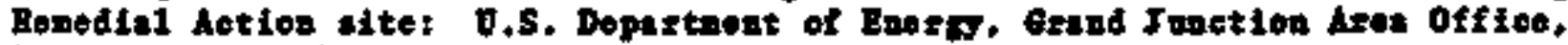
Peport 6J-11(93), 72 :-

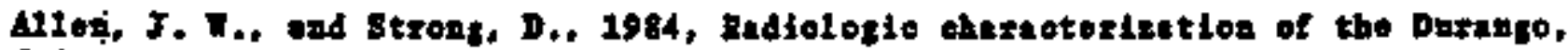

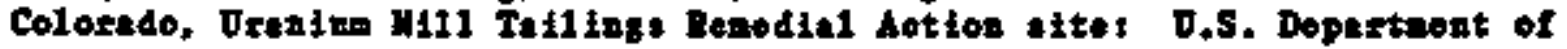
Enezby. Grand Jantion Axas Offlae, Report GJ-15, $58 \mathrm{p}$.

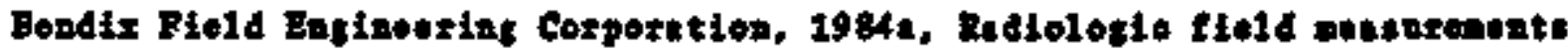
proeedures manel; Bondix Pleld Bnfineering Corporstion, Grend Jwetion Operstions, interasl docment, t5-09, Borlsion 02.

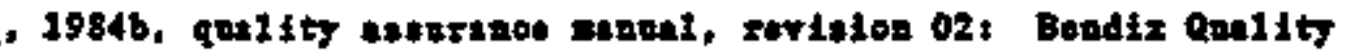

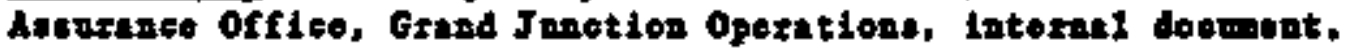
1984c, Handbooz of andytical and angle-proparation aothode:

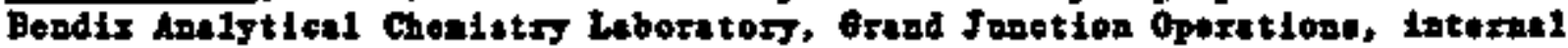
docmognt.

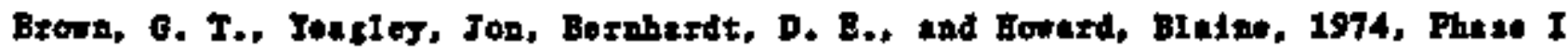

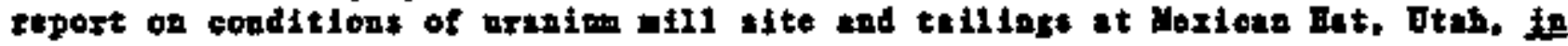

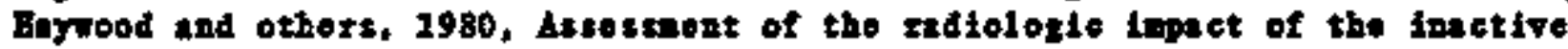

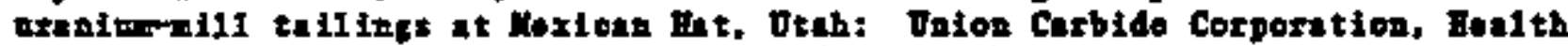
and Safety Resengeh Bitision, Oak Bidge Hetional Loboretory, Report Ond 5448, Apperdiz I.

Cooley, H, B., Earshbstcer, J. F., Arers, J. R., and Burdt, T. F., 1969,

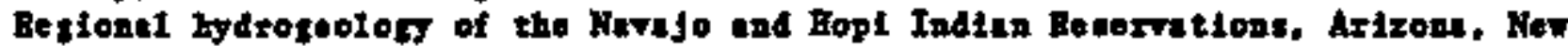

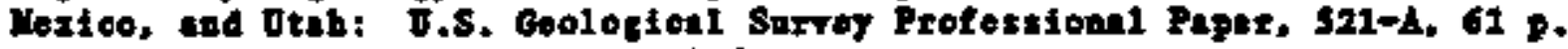

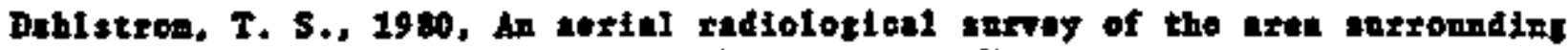

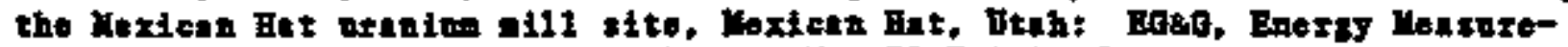
Dexts Grong, Las Veges, Herade, Report Ho. BP-B-009, 6 p.

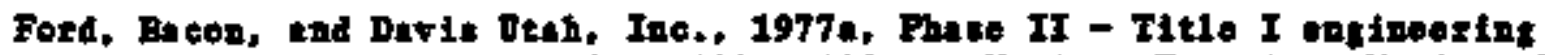

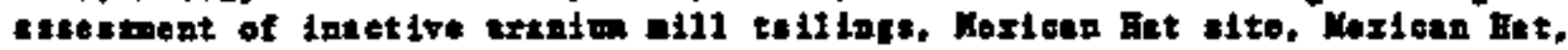

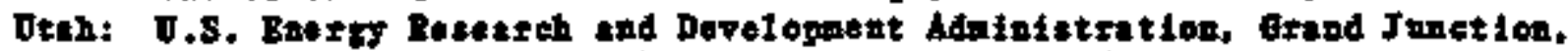
Coloredo, Contrect Ho. B (05-1) - 1658, Report GJT-3/6BDD 130-02. 


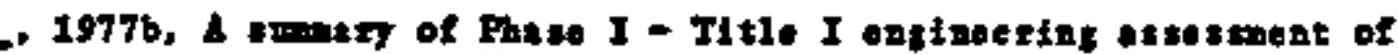

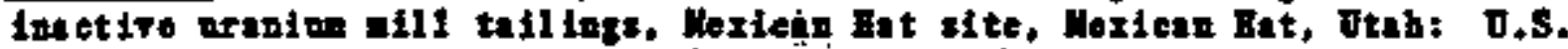

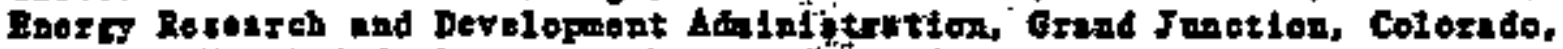

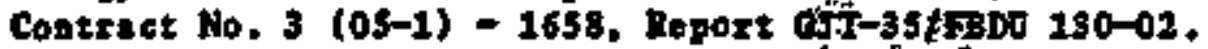

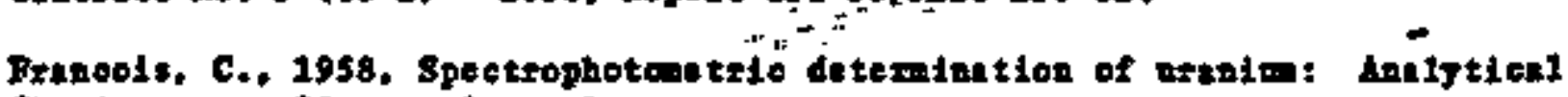
Chenistry, 7. 30, ao, 1 , p. 1 .

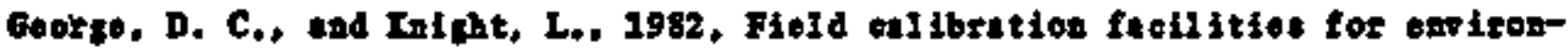

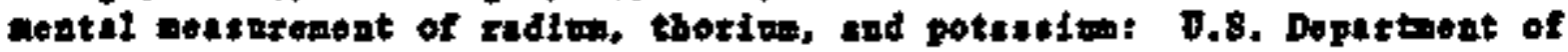

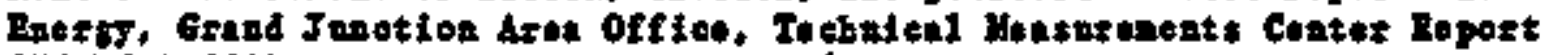
65/DMC-01 (82) .

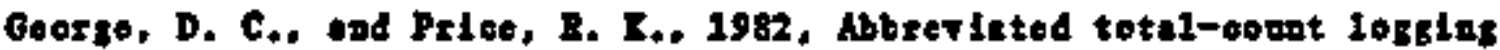

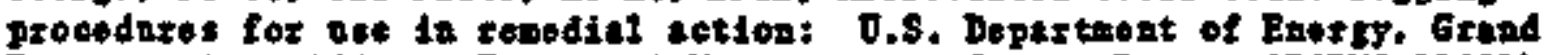

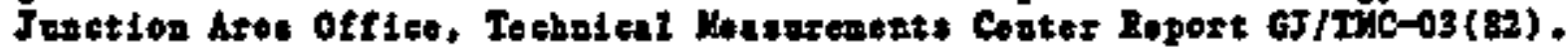

Goodkight, C, S., and Talker, B. W., 1984, Redloloste cherattezization of tho

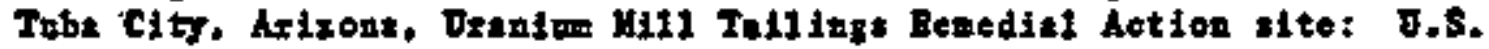

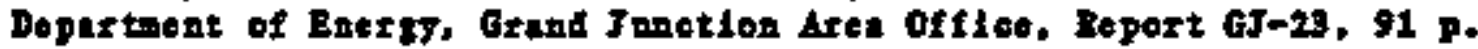

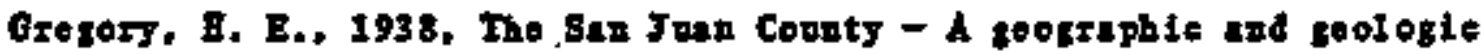

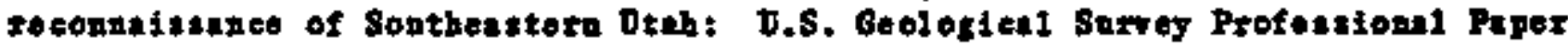
188.

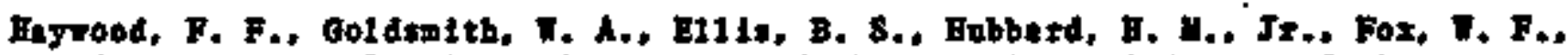
and shingatigh, W. H. 1980, Astassment of the tadiologlesl inpet of the

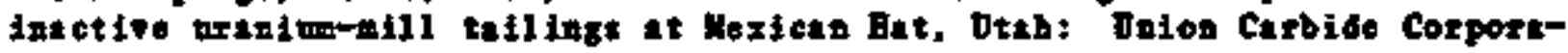

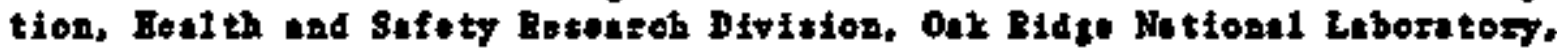
Roport Oknt 5448,86 .

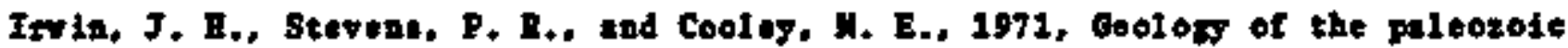

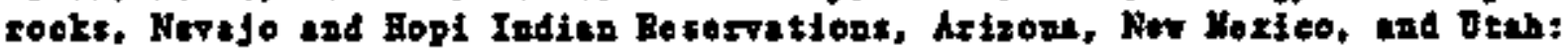
J.s. Goological Sarrey Professionel Peper 521-C.

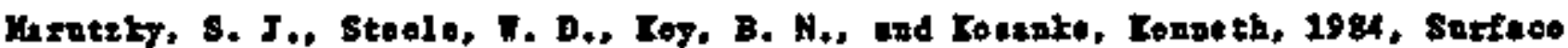

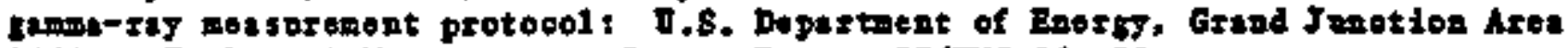

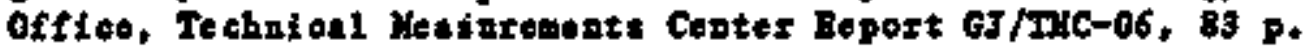

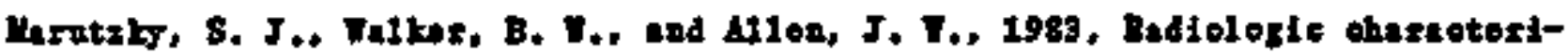

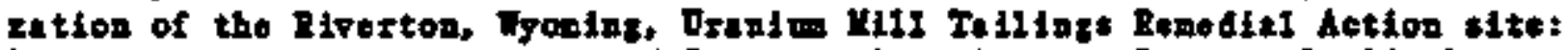

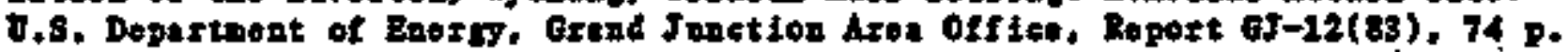

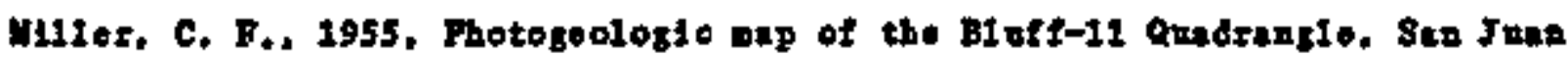

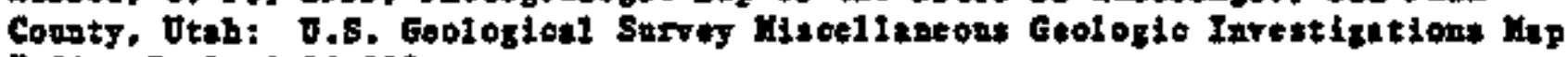
I-54. Setle $1: 24,000$.

0ril1d, P. P., 1955, Photogeotogle nap of the Blaff-12 ceadrestle, Sen Juan

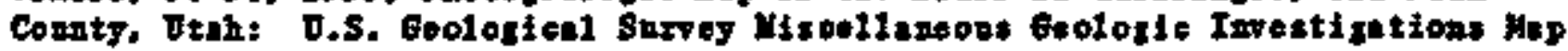
1-80. Sanle 1:24,000.

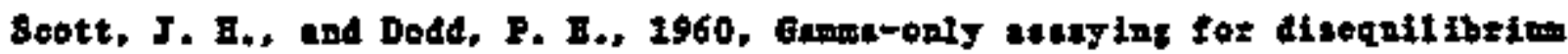

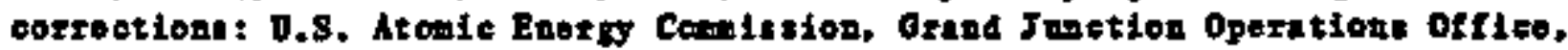
Opor-File Ropott BuE-13\$. 


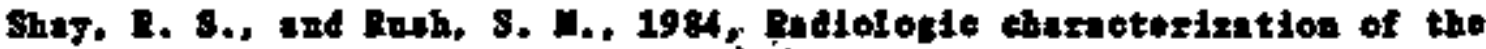

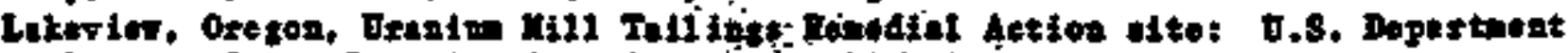

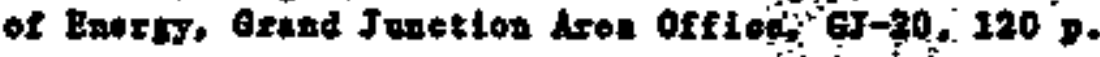

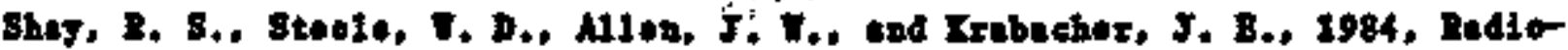

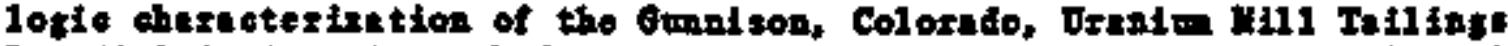

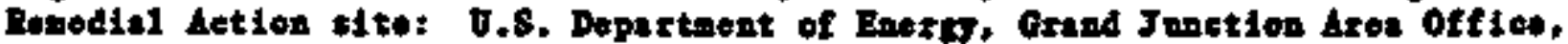
Popert 6J-34, $68 \mathrm{p}$.

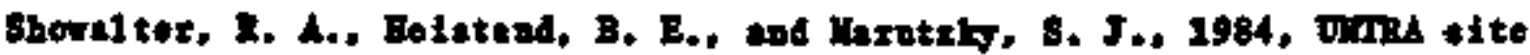

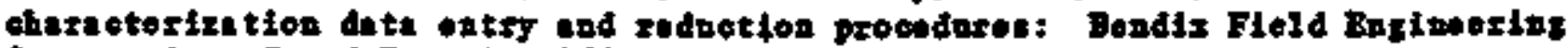
Corportelon, Grand Junction offies, Internil docment.

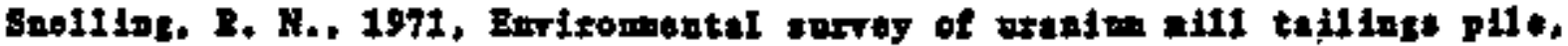

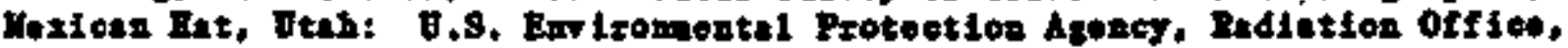
Radielogioal Feal th Dite and Roports, 7. 12, 20, 1, 2. I7-28.

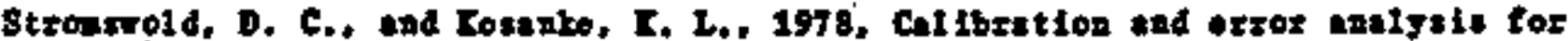
spectral redietion detecters: Ines Irinteotions on Noclear selonde, M5-25, no. 1. p. $782-786$.

Theder, B. E., Trttes, A, F., Jx,., and Finne11, T, L, 1964, Geolog and ore

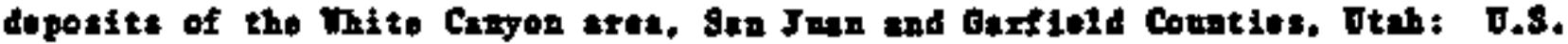
Ge0logial Snrog Bulletin 1125, $166 \mathrm{p}$.

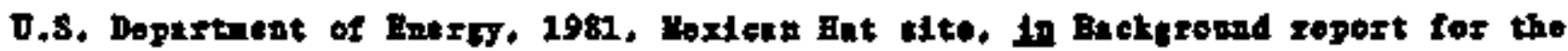
urantm all1 tat1 ings sites ronodint cetion progran: 0.5 . Departant of Enersy, Enviromentel and sefoty Englneering Division, Astiatent Sucrotary for

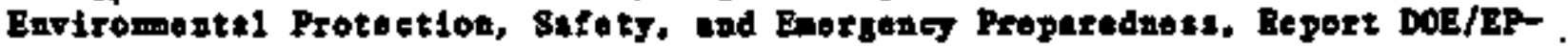
0011, p. 99-102.

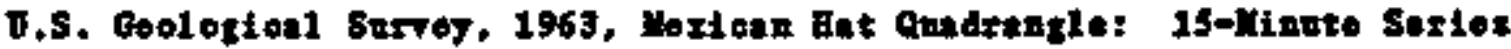
Topotraphic lep. Sesto 1: 62,5000.

1983, Bloff, Vtah and Colorado: 30 x 60 Minnte Aundragle

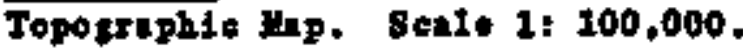


Append1x A

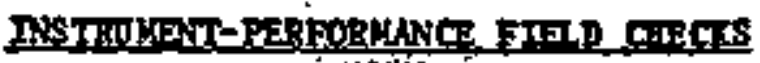

$\therefore$

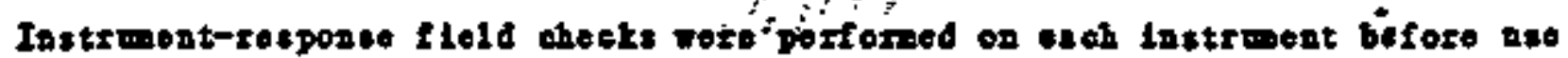

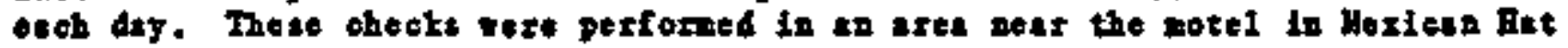

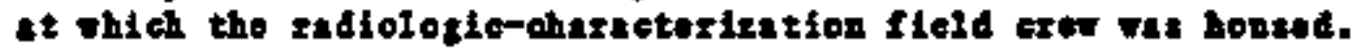

Bendinge vere recorded in the eppropedate fate book and plotted on a raph that displayed a history of provions tield-obeck resplts and speeffied quality-

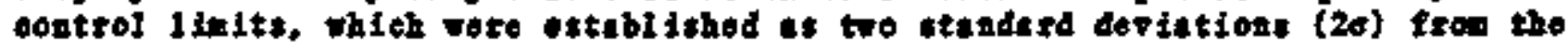

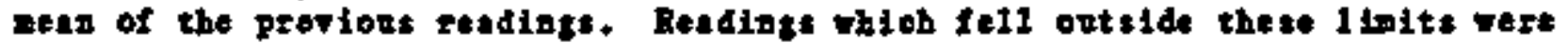
repostod to deterwine if they wero the resolt of an ibstrmentation problom or of the statistical probability that ipproximstig 5 pereont eoold be expected

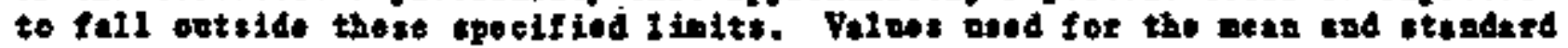

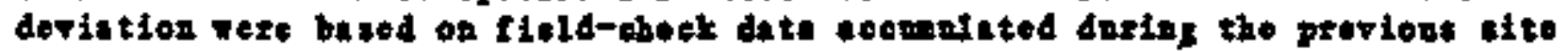
cheracterizations.

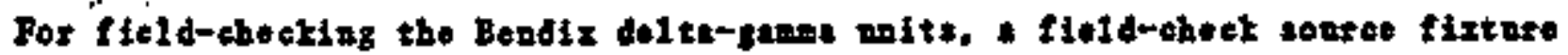

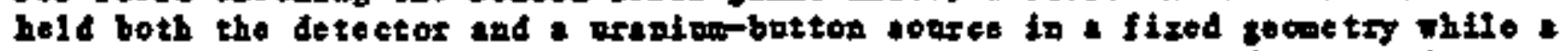
100-second UP cont vas collected. The source was thos tepored to point

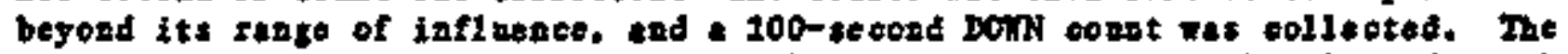
rosuting dolts cowt wes recorded in the aproprite dets book, plottel an the

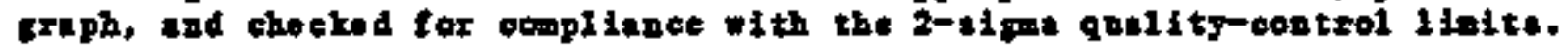

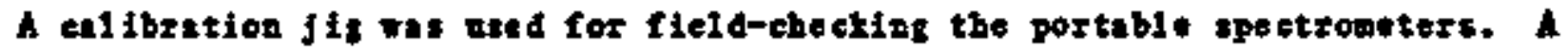
240-socond beckground cont ras followed by ancessive 240-second conts with a

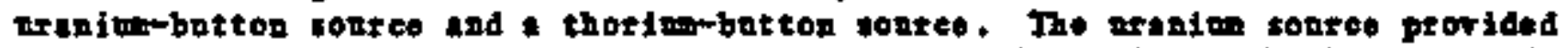

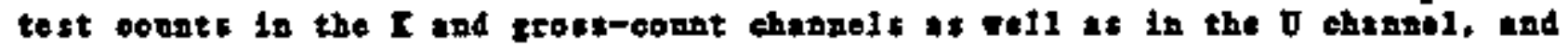
the thorf wo sorce propided a tett of the Th whinte. Becktround coonts in

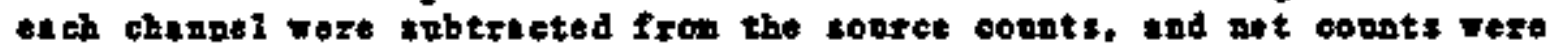
recorded In the approprinte datt book. Agtin, the conte vere plotted and wheked for compliage with gatity-eontrol 1 intes.

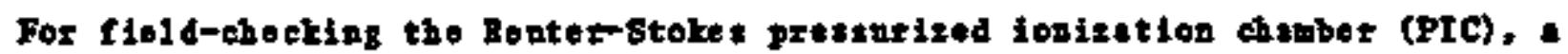

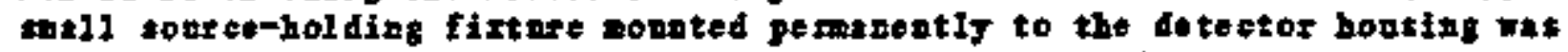

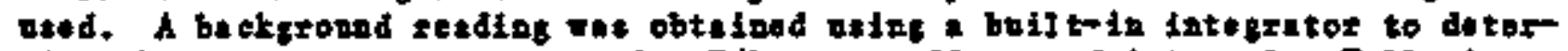

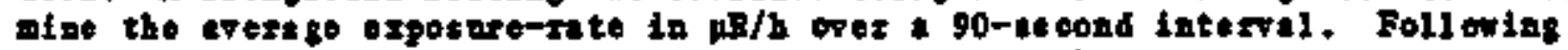

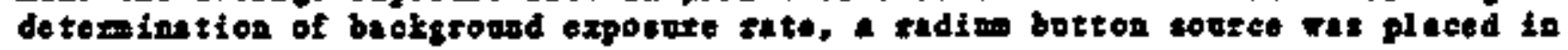
the soureo-holding fixtare and the verage ixposnet zato orer e so-aceond

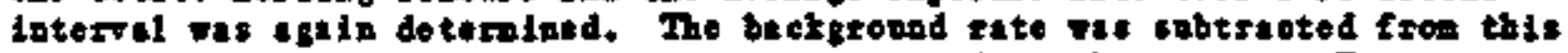

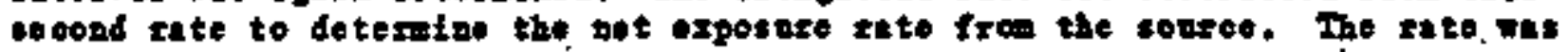

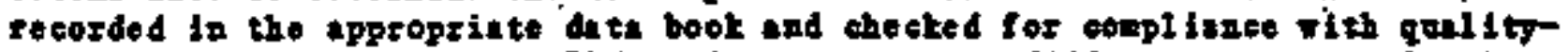
control 1 imits. To montfor PIC performenet to different oxposte ierelt,

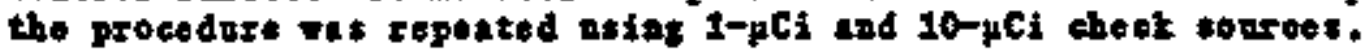




\section{ANAL FTCAT, DATA FOR SOT SAYPLES}

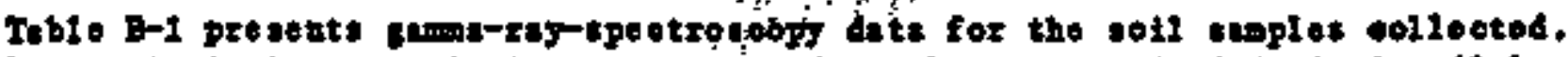

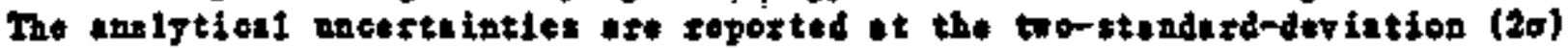

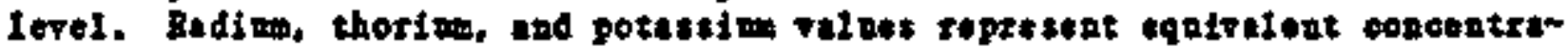

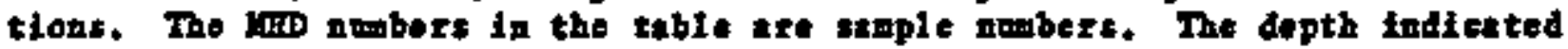
cefers to the top of 6-inch sanple intervat. The bor oholo gumber is ineluded

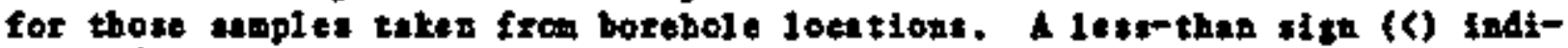

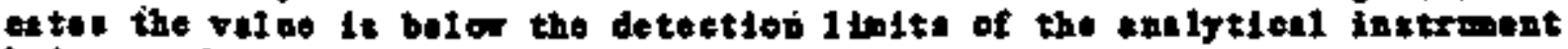
belis uted.

Iable $\mathrm{B}-1$

Game-Ray-8pottoncopy Data for Soll Saples

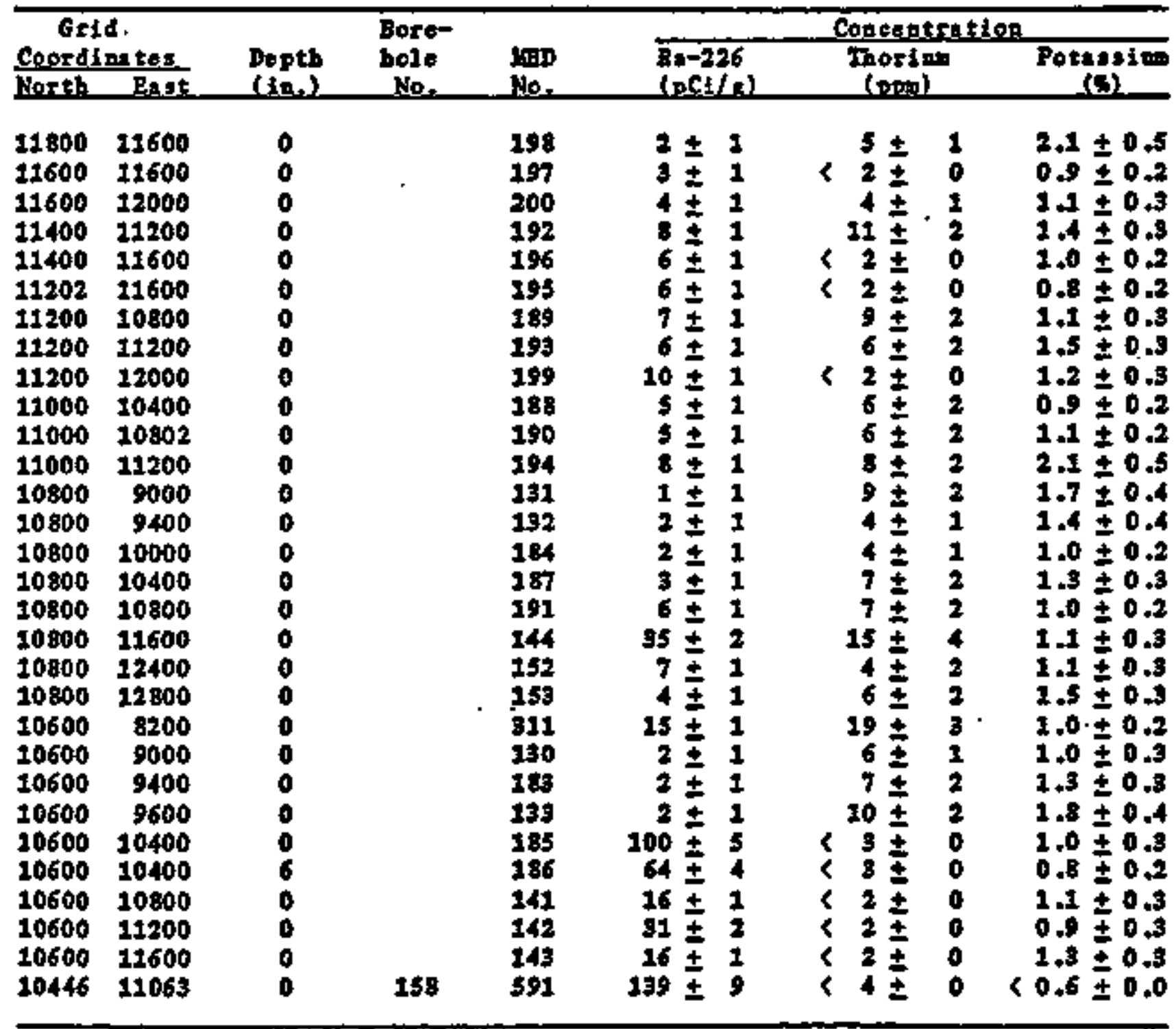


Table B-1 (contlunod)

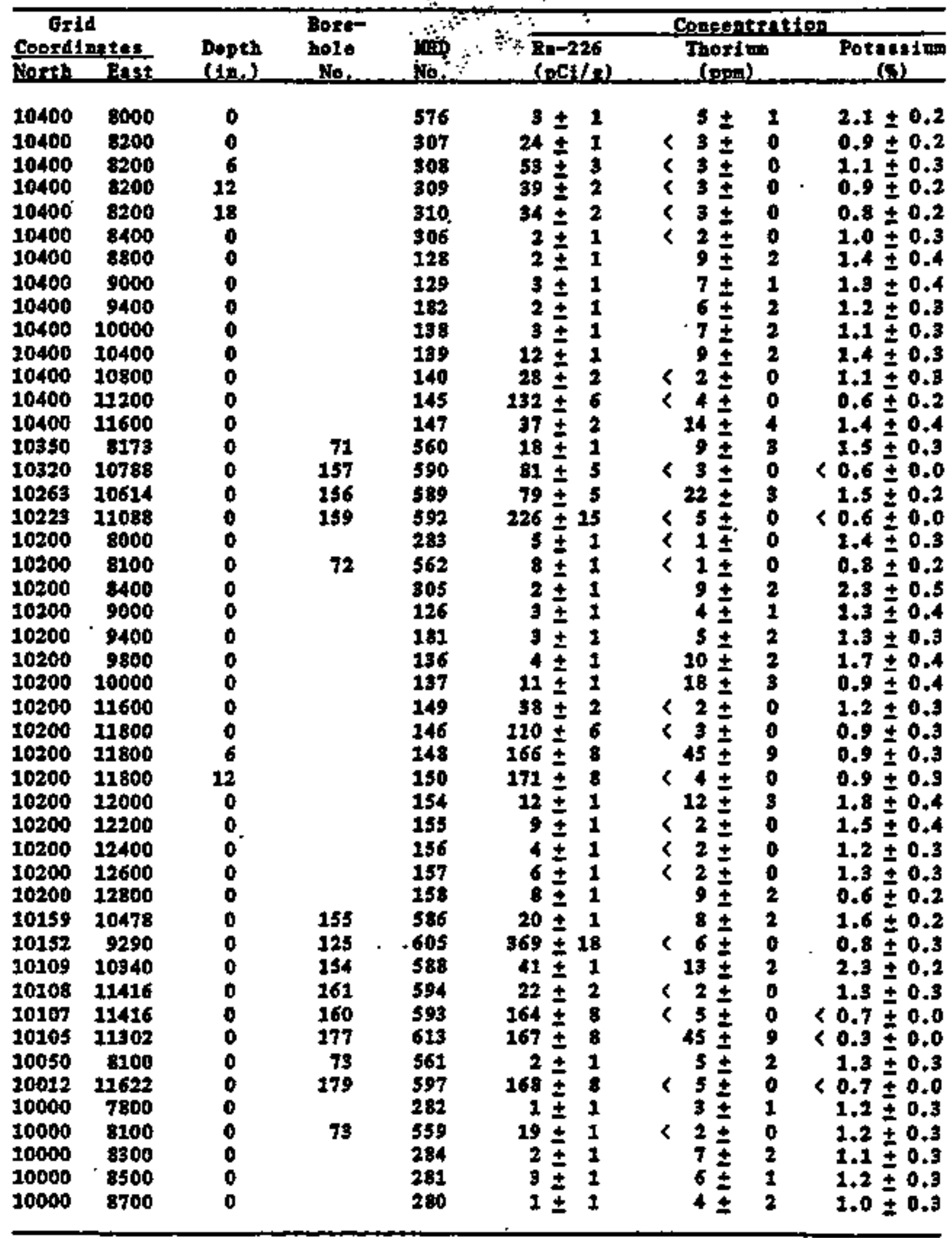


Table B-1 (oontinoed)

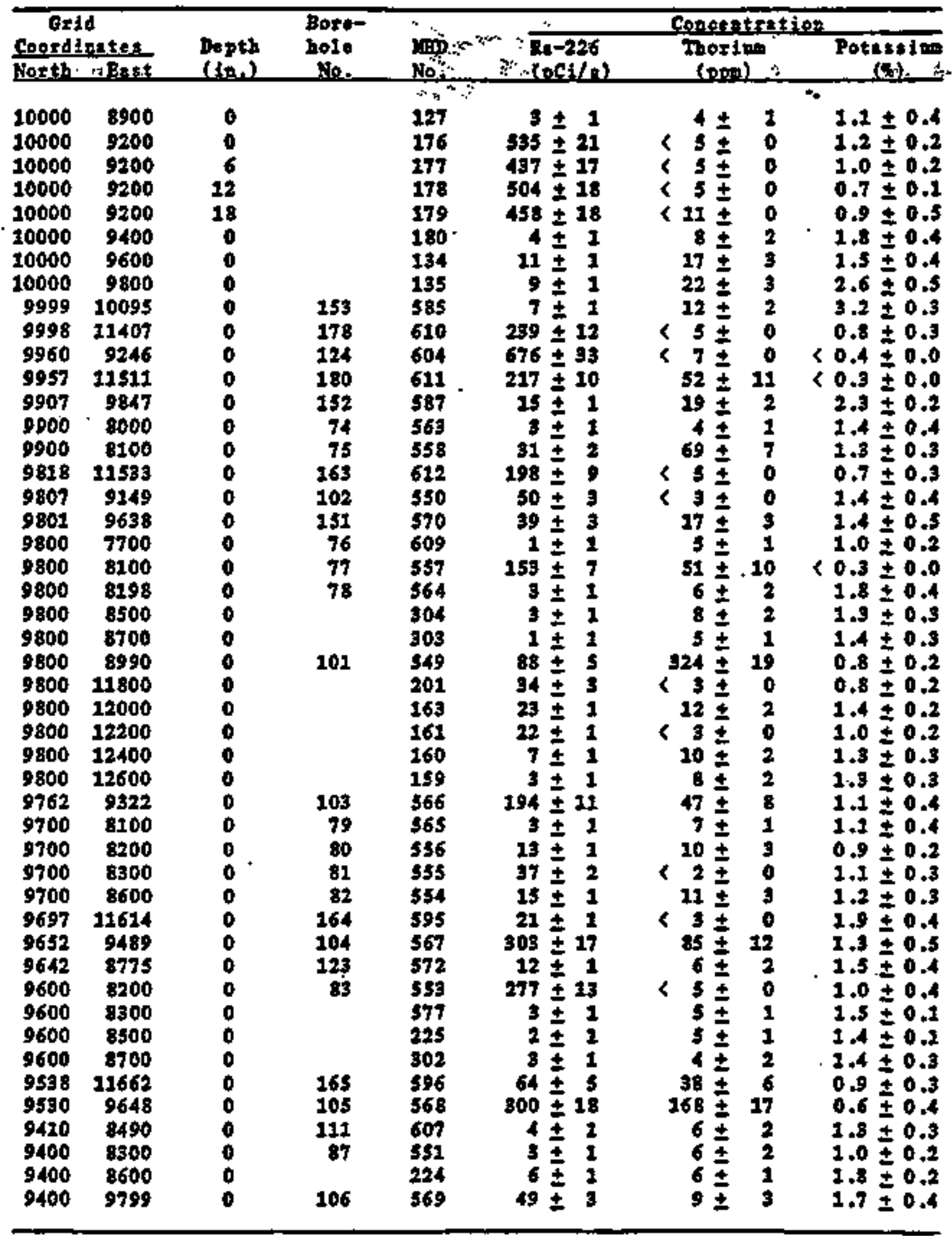


Tabl B-1 (eont1aned)

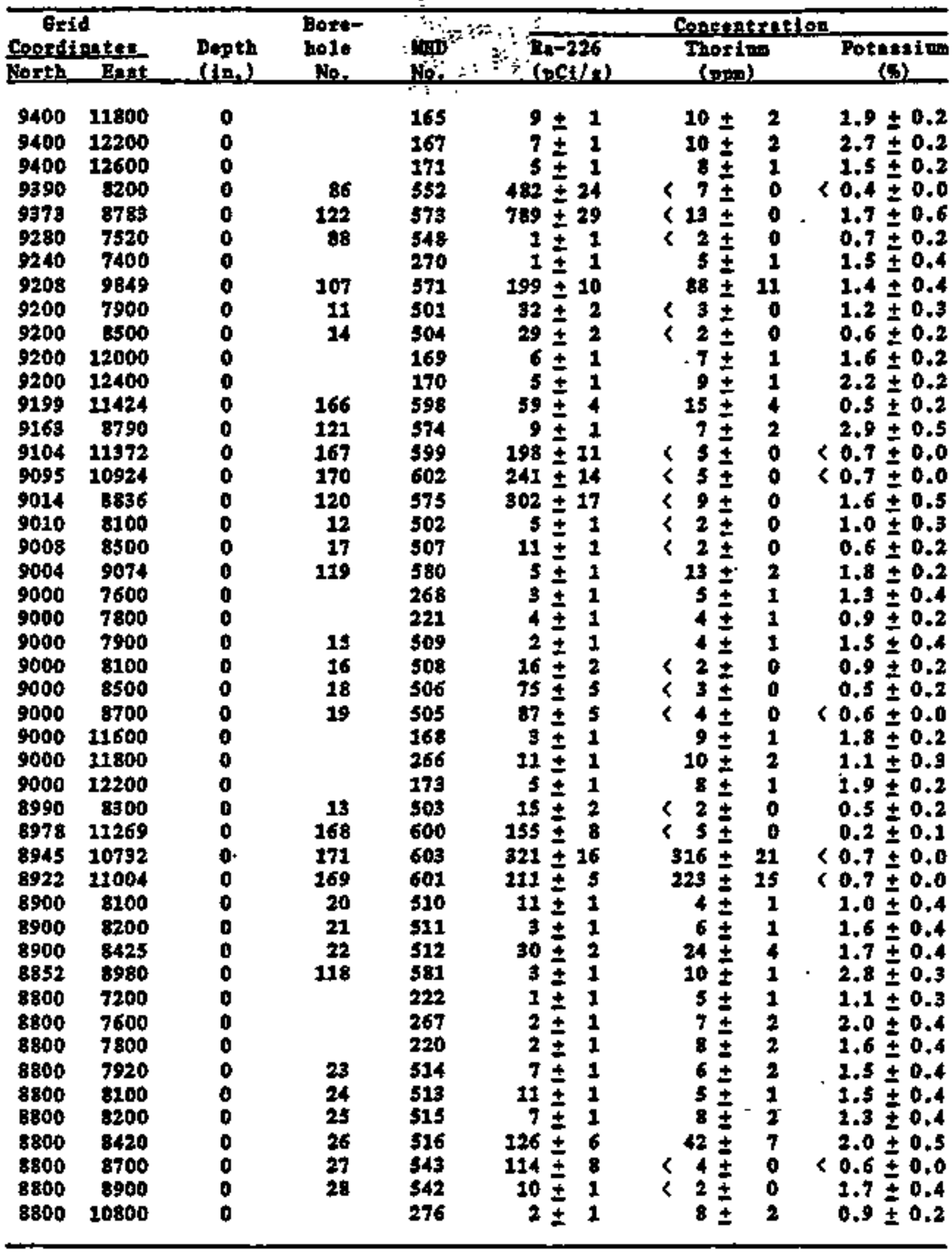


TabI E-I (continged)

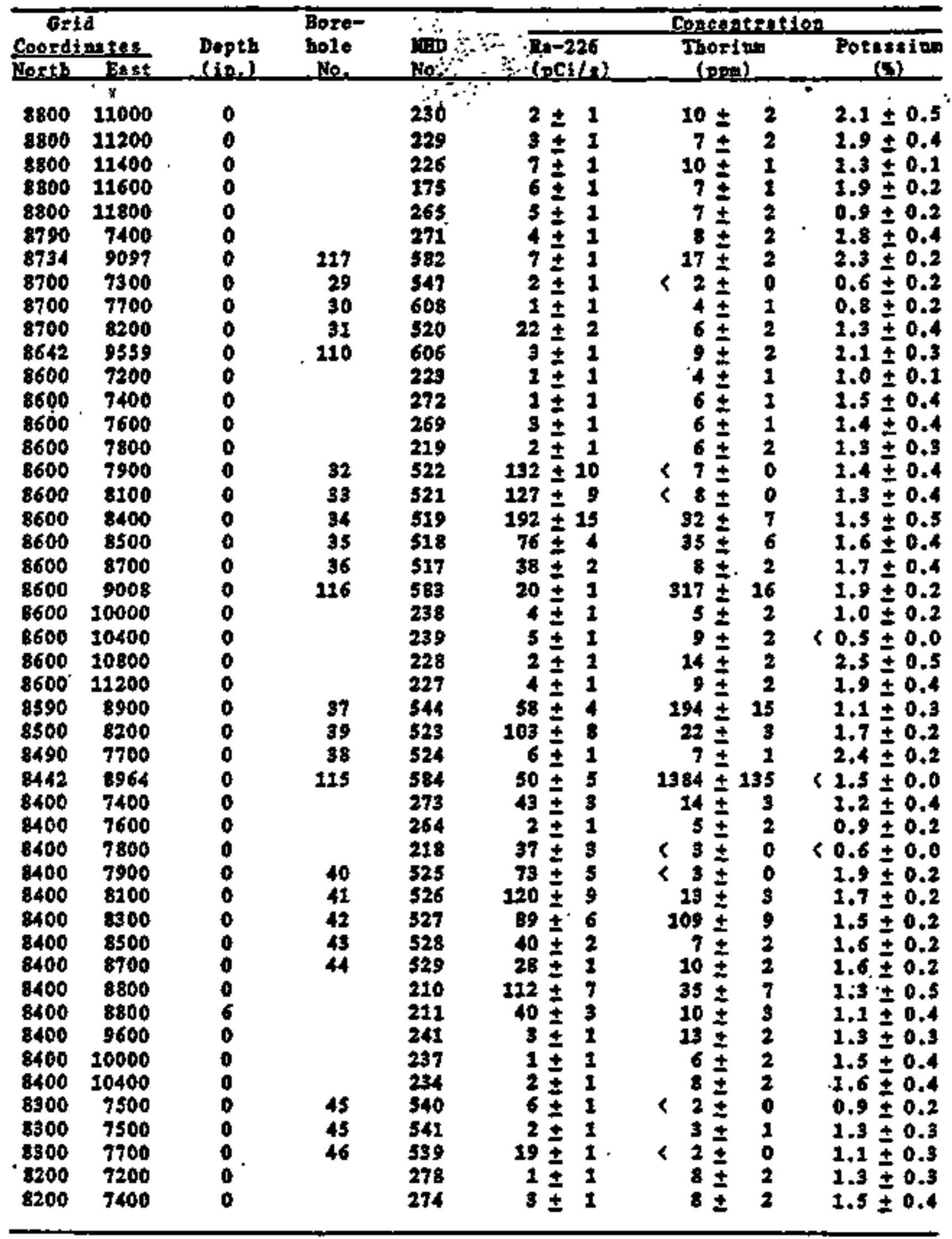


Table B-1 (costinned)

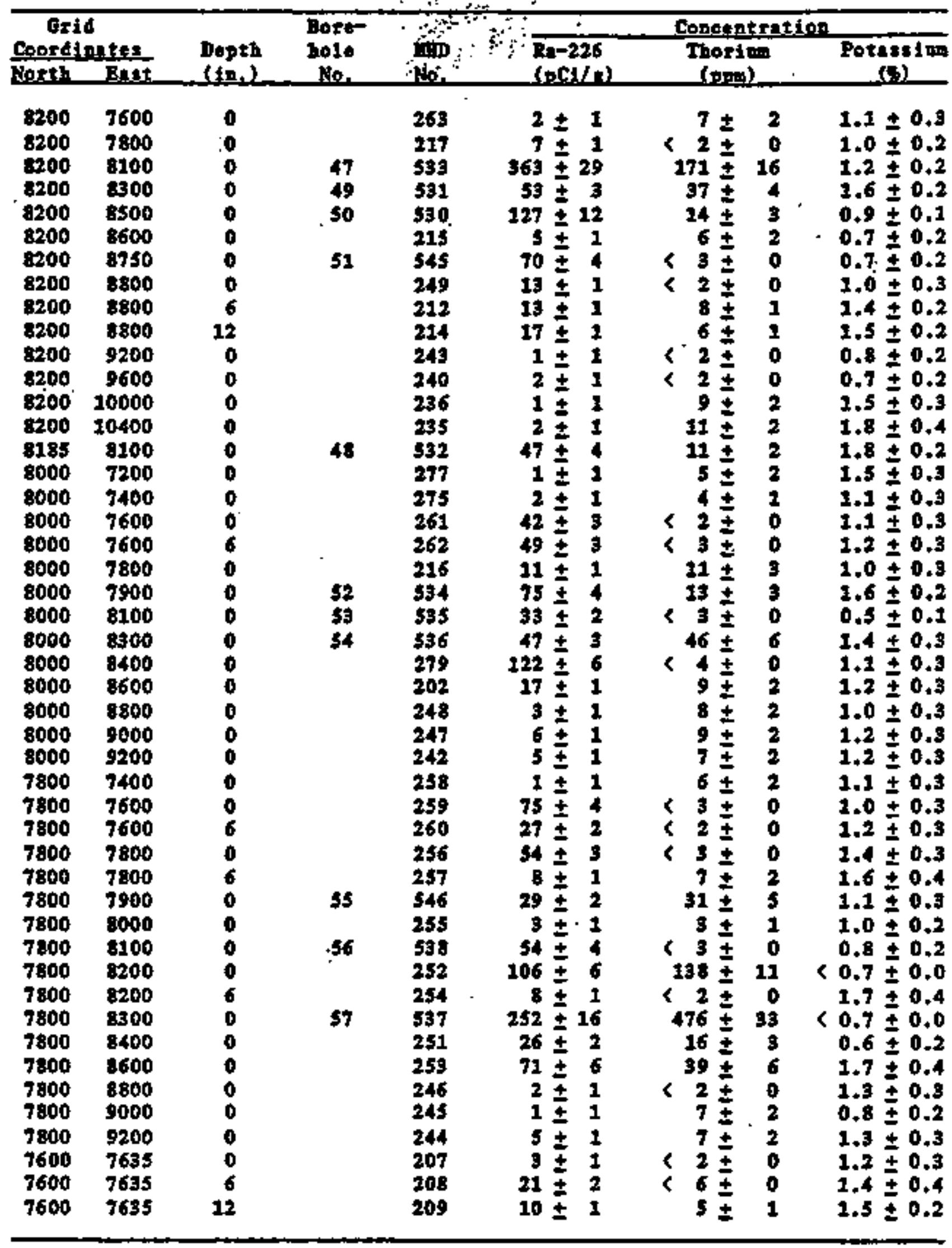


TEb1 B-2 (eontinos)

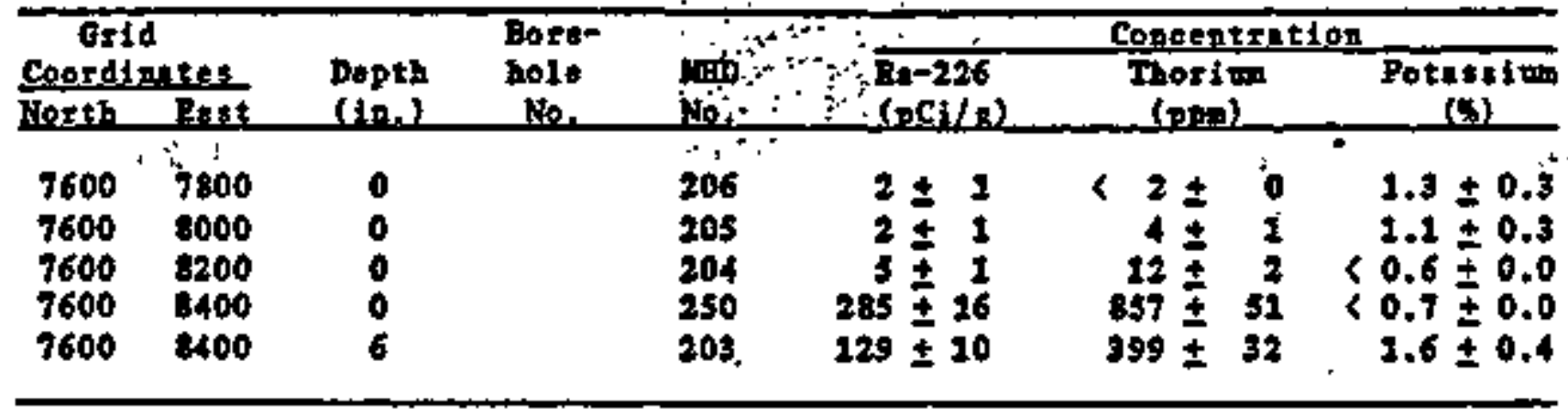


Tab1e B-2

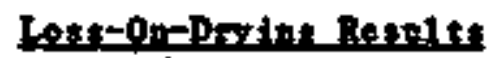

"ris

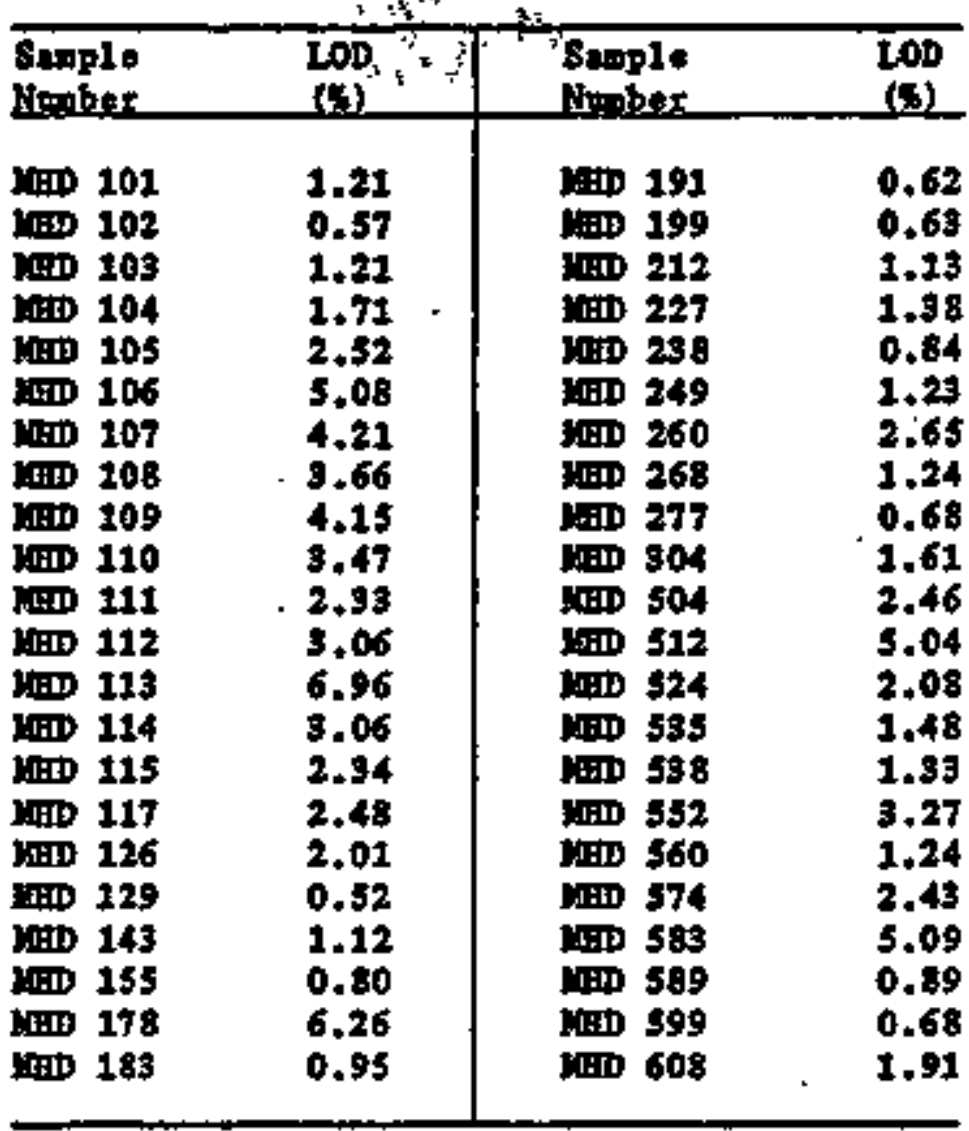




\section{APFENDI C}

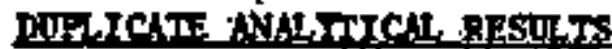

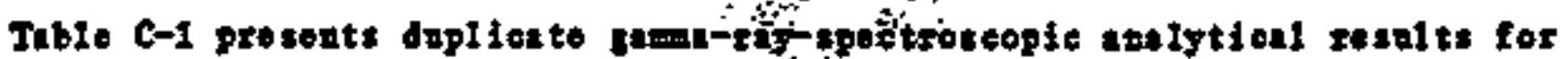

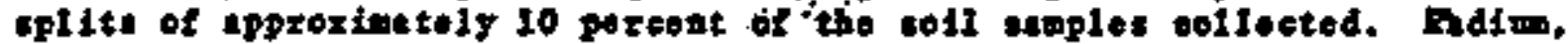

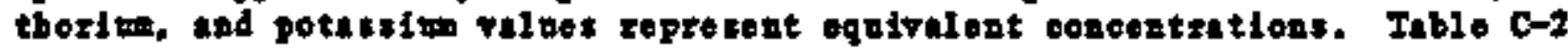

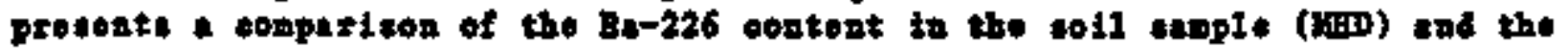

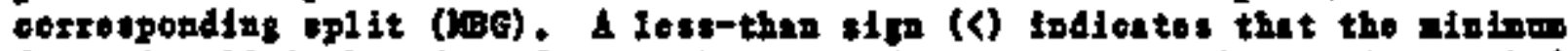

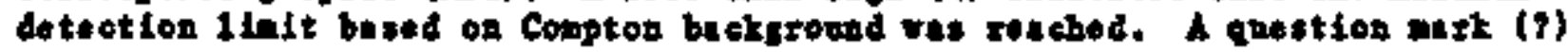

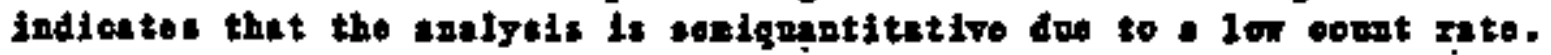

Tabte c-2

Dupl foete Gamas-Ray-8pectroscops Anatytical Besolts

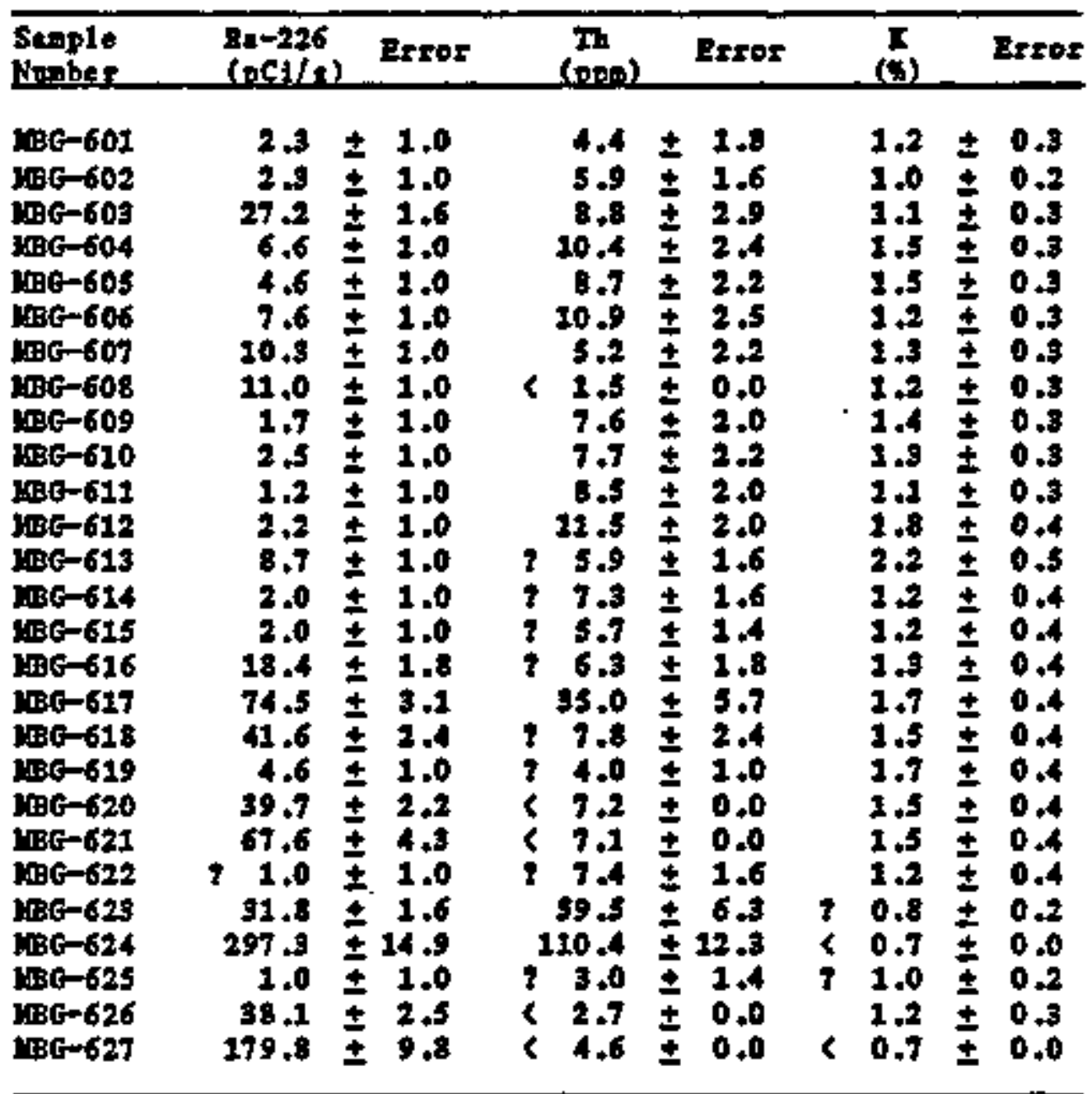




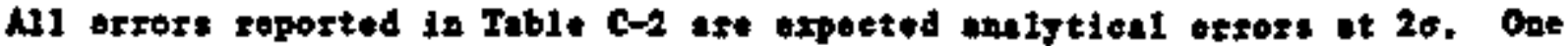

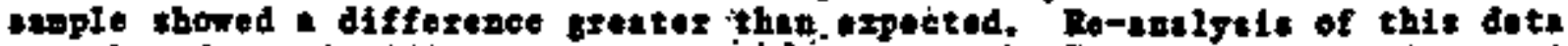

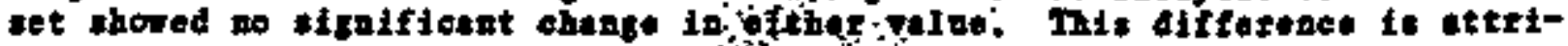
beted to camplo propertetion.

Tablo $\mathrm{C}-2$

Copperison of Ruding Renthe Betwees Samples and Coxteppondint 8plit:

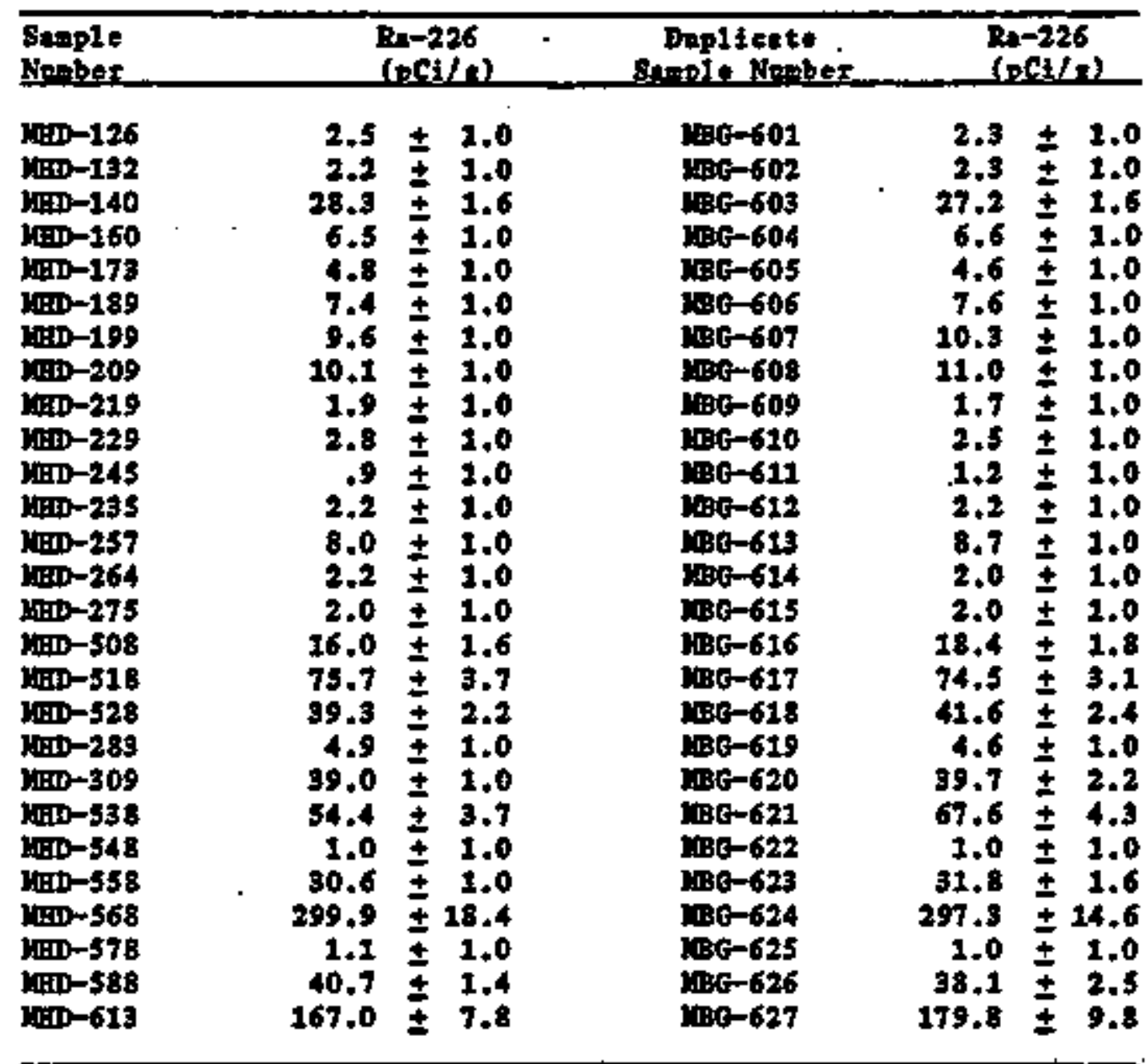




\section{Appead1 D

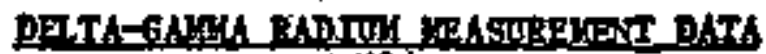

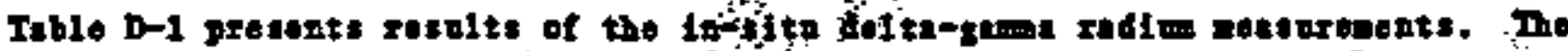

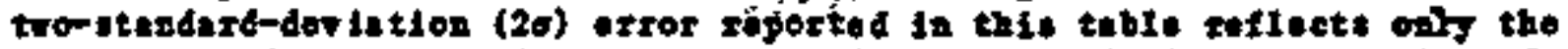

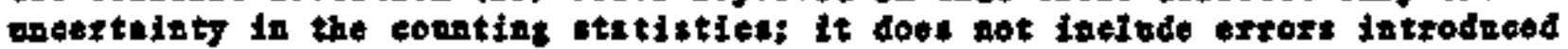
theough the wo of oslibration factor and throe corzection factors. These

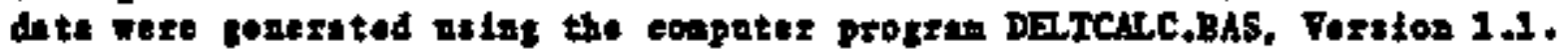

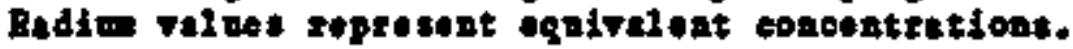

I.520 D-I

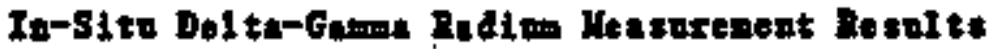

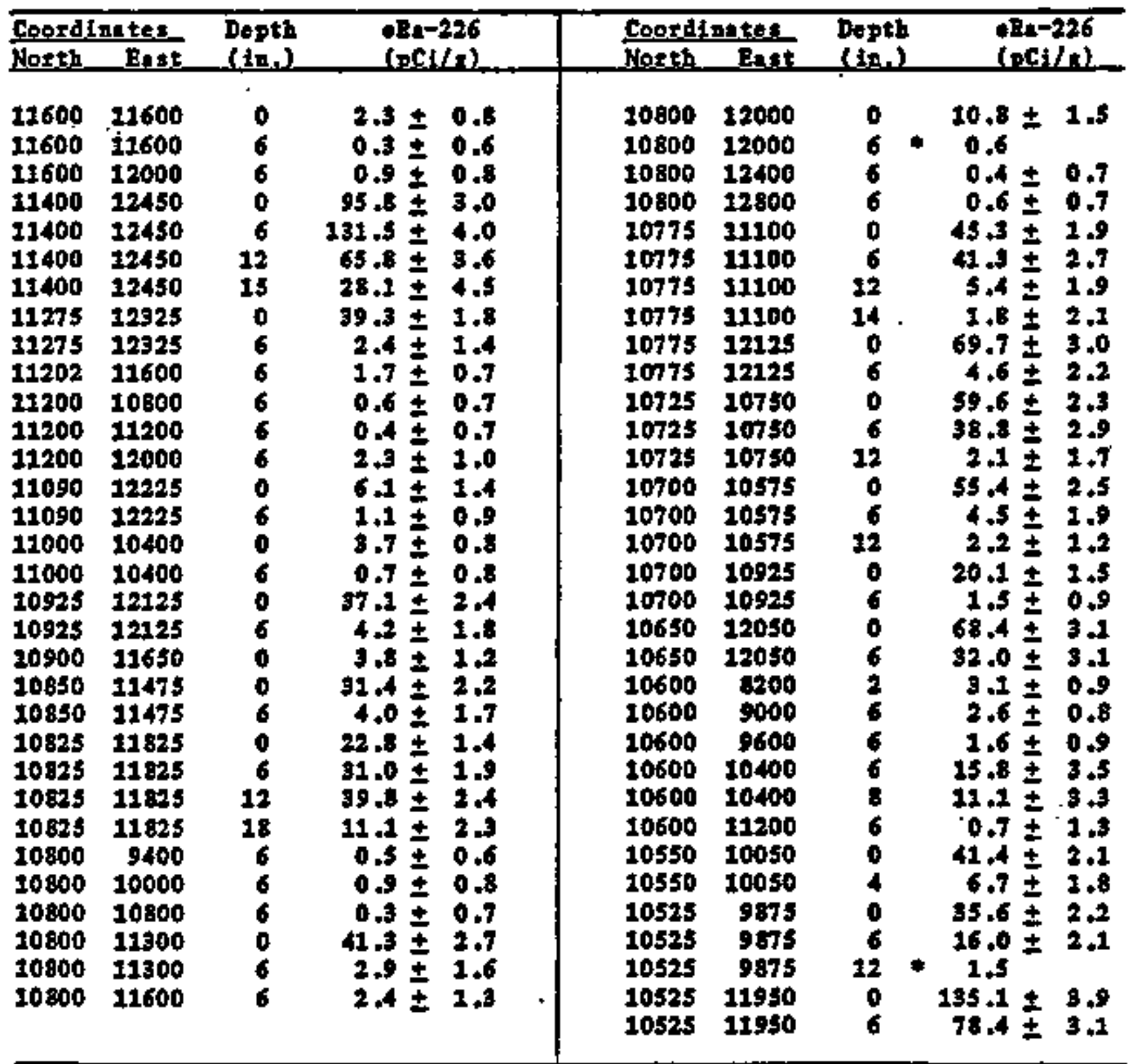

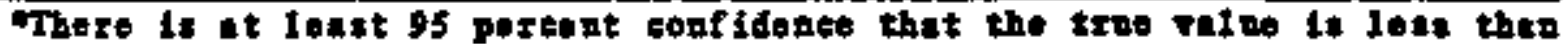
the mesureant reportod. 
Toble. D-1 (coatiswod)

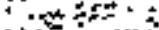

\begin{tabular}{|c|c|c|c|c|c|c|c|c|c|c|}
\hline leosd & $\begin{array}{l}\text { Lters } \\
\text { Eestat }\end{array}$ & $\begin{array}{l}\text { ept } \\
\text { in }\end{array}$ & $\cos$ & 1 & & Cogrd & $\frac{\text { thes }}{\text { Best }}$ & $\begin{array}{l}\text { Depth } \\
(1, n)\end{array}$ & & \\
\hline $\begin{array}{l}10525 \\
10500 \\
10500 \\
10500 \\
10450 \\
10450 \\
10400 \\
10400 \\
10400 \\
10400 \\
10400 \\
10400 \\
10400 \\
10400 \\
10400 \\
10400 \\
10400 \\
10325 \\
10325 \\
10325 \\
10275 \\
10275 \\
10200 \\
10200 \\
10200 \\
10200 \\
10200 \\
10200 \\
10200 \\
10200 \\
10200 \\
10200 \\
10200 \\
10125 \\
10125 \\
10125 \\
10059 \\
10059 \\
10010 \\
10010 \\
10000 \\
10000 \\
10000 \\
10000 \\
10000 \\
10000\end{array}$ & $\begin{array}{r}11950 \\
10200 \\
10200 \\
10200 \\
9700 \\
9700 \\
8000 \\
8200 \\
8200 \\
8200 \\
8200 \\
8400 \\
8800 \\
9400 \\
10400 \\
10800 \\
11600 \\
11925 \\
11925 \\
11925 \\
9650 \\
9650 \\
8000 \\
8400 \\
9000 \\
10000 \\
11600 \\
11800 \\
11800 \\
11800 \\
11800 \\
12200 \\
12600 \\
9575 \\
9575 \\
9575 \\
12775 \\
22775 \\
12565 \\
12565 \\
7800 \\
8900 \\
8500 \\
8700 \\
8900 \\
9200\end{array}$ & $\begin{array}{r}10 \\
0 \\
6 \\
12 \\
0 \\
4 \\
6 \\
6 \\
12 \\
18 \\
24 \\
6 \\
6 \\
6 \\
6 \\
6 \\
6 \\
0 \\
6 \\
10 \\
0 \\
6 \\
6 \\
6 \\
6 \\
6 \\
6 \\
6 \\
12 \\
15 \\
6 \\
6 \\
6 \\
6 \\
6 \\
6 \\
6 \\
6 \\
6 \\
6 \\
6 \\
6 \\
6 \\
6 \\
6 \\
6 \\
6\end{array}$ & $\begin{array}{r}10.8 \pm \\
240.2 \pm \\
278.1 \pm \\
2.2 \pm \\
12.8 \pm \\
1.1 \pm \\
0.7 \pm \\
45.4 \pm \\
24.1 \pm \\
15.9 \pm \\
4.1 \pm \\
0.9 \pm \\
0.7 \pm \\
0.9 \pm \\
1.5 \pm \\
2.3 \pm \\
3.6 \pm \\
44.2 \pm \\
59.0 \pm \\
9.4 \pm \\
143.6 \pm \\
19.1 \pm \\
0.8 \pm \\
1.8 \pm \\
1.0 \pm \\
0.9 \pm \\
2.3 \pm \\
124.8 \pm \\
224 . \pm \pm \\
194.4 \pm \\
115.8 \pm \\
1.9 \pm \\
0.3 \pm \\
155.9 \pm \\
8.2 \pm \\
5.4 \pm \\
5.9 \pm \\
0.7 \pm \\
5.3 \pm \\
0.6 \pm \\
0.9 \pm \\
2.2 \pm \\
1.3 \pm \\
1.1 \pm \\
1.7 \pm \\
510.8 \pm\end{array}$ & $\begin{array}{l}2.7 \\
4.5 \\
6.0 \\
4.1 \\
1.6 \\
1.2 \\
0.6 \\
2.1 \\
2.5 \\
2.6 \\
1.9 \\
0.8 \\
0.8 \\
0.7 \\
0.9 \\
1.3 \\
1.4 \\
2.5 \\
2.0 \\
2.3 \\
3.5 \\
2.5 \\
0.7 \\
0.9 \\
0.8 \\
1.0 \\
1.5 \\
3.8 \\
4.2 \\
5.4 \\
5.7 \\
1.0\end{array}$ & T & $\begin{array}{l}10000 \\
10000 \\
10000 \\
10000 \\
10000 \\
9967 \\
9967 \\
9950 \\
9990 \\
9866 \\
9866 \\
9800 \\
9800 \\
9800 \\
9800 \\
9800 \\
9800 \\
9600 \\
9600 \\
9600 \\
9600 \\
9580 \\
9580 \\
9590 \\
9450 \\
9450 \\
9400 \\
9400 \\
9400 \\
9950 \\
9950 \\
9900 \\
9900 \\
9280 \\
9280 \\
9275 \\
9240 \\
9200 \\
9200 \\
9000 \\
9000 \\
9000 \\
9000 \\
8800 \\
8800 \\
8800\end{array}$ & $\begin{array}{r}9200 \\
9200 \\
9400 \\
9500 \\
9800 \\
12990 \\
12390 \\
12150 \\
12150 \\
11994 \\
11994 \\
8500 \\
8700 \\
11800 \\
11800 \\
12200 \\
12200 \\
8300 \\
8500 \\
8700 \\
22000 \\
12580 \\
12580 \\
12580 \\
12480 \\
12480 \\
8600 \\
12000 \\
12600 \\
12350 \\
12350 \\
12175 \\
12175 \\
11980 \\
11980 \\
11800 \\
7400 \\
12000 \\
12400 \\
7600 \\
7800 \\
12200 \\
7200 \\
7600 \\
7800\end{array}$ & $\begin{array}{r}12 \\
18 \\
6 \\
6 \\
6 \\
0 \\
1 \\
0 \\
4 \\
0 \\
3 \\
6 \\
6 \\
0 \\
5 \\
5 \\
6 \\
2 \\
6 \\
6 \\
6 \\
0 \\
6 \\
8 \\
0 \\
6 \\
6 \\
6 \\
4 \\
0 \\
6 \\
6 \\
6 \\
5 \\
0 \\
6 \\
6 \\
6 \\
6 \\
6 \\
6 \\
6 \\
6 \\
6 \\
6 \\
6 \\
6 \\
6 \\
6 \\
6\end{array}$ & $\begin{array}{r}491.0 \pm \\
493.3 \pm \\
2.4 \pm \\
2.7 \pm \\
3.7 \pm \\
16.6 \pm \\
1.3 \pm \\
13.5 \pm \\
2.1 \pm \\
19.1 \pm \\
3.9 \pm \\
1.8 \pm \\
1.7 \pm \\
11.2 \pm \\
1.5 \pm \\
0.5 \pm \\
1.2 \pm \\
1.2 \pm \\
0.7 \pm \\
0.4 \pm \\
2.2 \pm \\
23.3 \pm \\
5.6 \pm \\
2.0 \pm \\
9.5 \pm \\
1.8 \pm \\
1.0 \pm \\
1.0 \pm \\
2.5 \pm \\
6.3 \pm \\
1.4 \pm \\
13.7 \pm \\
2.3 \pm \\
20.4 \pm \\
5.7 \pm \\
4.0 \pm \\
0.6 \pm \\
0.2 \pm \\
3.0 \pm \\
0.8 \pm \\
0.7 \pm \\
1.2 \pm \\
0.3 \pm \\
1.3 \pm \\
2.0 \pm \\
1.4 \pm\end{array}$ & $\begin{array}{l}7.2 \\
9.7 \\
0.8 \\
1.3 \\
1.4 \\
1.7 \\
1.3 \\
2.0 \\
1.2 \\
1.7 \\
1.5 \\
0.9 \\
0.9 \\
1.9 \\
1.1 \\
1.0 \\
1.0 \\
0.9 \\
0.7 \\
0.8 \\
1.2 \\
1.8 \\
1.4 \\
1.3 \\
1.3 \\
0.9 \\
0.9 \\
1.0 \\
0.8 \\
1.3 \\
0.8 \\
1.4 \\
1.2 \\
1.9 \\
1.3 \\
1.5 \\
0.7\end{array}$ \\
\hline
\end{tabular}

Where Is ot Iesst 95 percent corfiderce that the trae palus is loss than the veesuremet toported. 
Table D-1 (continged)

\begin{tabular}{|c|c|c|c|c|c|c|c|c|c|c|}
\hline $\begin{array}{l}\text { Copts } \\
\text { Noteth }\end{array}$ & Etes: & $\begin{array}{l}\text { Depth } \\
\text { (inc) }\end{array}$ & $\ln$ & & & Copots & 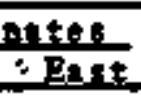 & $\begin{array}{l}\text { Depth } \\
\text { (tph) }\end{array}$ & $\begin{array}{l}\text { PRE- } \\
\text { CDCi }\end{array}$ & 226 \\
\hline $\begin{array}{l}8800 \\
8800 \\
8800 \\
8790 \\
8600 \\
8600 \\
8600 \\
8600 \\
8600 \\
8600 \\
8600 \\
8400 \\
8400 \\
8400 \\
8400 \\
8400 \\
8400 \\
8400 \\
8400 \\
8100 \\
8400 \\
8200 \\
8200 \\
8200 \\
8200 \\
8200 \\
8200 \\
8200 \\
8200 \\
8200\end{array}$ & $\begin{array}{r}11000 \\
11400 \\
11800 \\
7400 \\
7200 \\
7400 \\
7600 \\
7800 \\
10400 \\
10800 \\
11200 \\
7200 \\
7200 \\
7400 \\
7600 \\
7800 \\
8800 \\
8800 \\
9600 \\
10000 \\
10400 \\
7200 \\
7400 \\
7600 \\
7800 \\
8600 \\
8800 \\
8800 \\
8800 \\
9200\end{array}$ & $\begin{array}{r}6 \\
6 \\
6 \\
6 \\
6 \\
6 \\
6 \\
6 \\
6 \\
6 \\
6 \\
6 \\
6 \\
6 \\
6 \\
6 \\
8 \\
6 \\
6 \\
6 \\
6 \\
6 \\
6 \\
6 \\
4 \\
6 \\
12 \\
18 \\
6\end{array}$ & $\begin{array}{l}1.8 \pm \\
2.4 \pm \\
1.7 \pm \\
1.4 \pm \\
1.1 \pm \\
1.0 \pm \\
1.8 \pm \\
0.7 \pm \\
2.5 \pm \\
2.9 \pm \\
0.7 \pm \\
1.0 \pm \\
1.0 \pm \\
1.8 \pm \\
1.2 \pm \\
1.8 \pm \\
8.3 \pm \\
1.9 \pm \\
2.1 \pm \\
4.2 \pm \\
1.9 \pm \\
2.0 \pm \\
1.5 \pm \\
0.3 \pm \\
2.3 \pm \\
1.4 \pm \\
14.3 \pm \\
13.2 \pm \\
1.6 \pm \\
2.2 \pm\end{array}$ & $\begin{array}{l}0.9 \\
0.9 \\
0.9 \\
0.9 \\
0.7 \\
0.8 \\
0.9 \\
0.7 \\
1.3 \\
1.0 \\
0.7 \\
0.7 \\
0.8 \\
1.3 \\
0.8 \\
0.9 \\
1.7 \\
1.5 \\
1.0 \\
0.9 \\
0.7 \\
0.8 \\
0.9 \\
0.9 \\
0.8 \\
1.6 \\
1.6 \\
1.2 \\
1.0\end{array}$ & $\therefore 5_{3}=$ & $\begin{array}{l}8200 \\
7200 \\
8000 \\
6000 \\
8000 \\
8000 \\
8000 \\
8000 \\
8000 \\
8000 \\
8000 \\
7800 \\
7800 \\
7800 \\
7800 \\
7800 \\
7800 \\
7800 \\
7800 \\
7800 \\
7800 \\
7800 \\
7600 \\
7600 \\
7600 \\
7600 . \\
7600 \\
7600 \\
7600 \\
7600\end{array}$ & $\begin{array}{r}10000 \\
10400 \\
7200 \\
7400 \\
7600 \\
7600 \\
7800 \\
8400 \\
8600 \\
9000 \\
9600 \\
7400 \\
7600 \\
7600 \\
7800 \\
7800 \\
8000 \\
8200 \\
8200 \\
8400 \\
8800 \\
9200 \\
7695 \\
7695 \\
7695 \\
7800 \\
9000 \\
8200 \\
8400 \\
8400\end{array}$ & $\begin{array}{r}6 \\
6 \\
6 \\
6 \\
6 \\
12 \\
6 \\
6 \\
6 \\
6 \\
6 \\
6 \\
6 \\
12 \\
6 \\
12 \\
6 \\
6 \\
12 \\
6 \\
6 \\
6 \\
6 \\
12 \\
18 \\
6 \\
6 \\
6 \\
12 \\
12\end{array}$ & $\begin{array}{r}7.0 \pm \\
3.0 \pm \\
1.0 \pm \\
0.4 \pm \\
21.6 \pm \\
3.1 \pm \\
2.5 \pm \\
3.5 \pm \\
2.9 \pm \\
0.1 \pm \\
1.1 \pm \\
2.0 \pm \\
14.0 \pm \\
3.9 \pm \\
5.7 \pm \\
1.9 \pm \\
0.2 \pm \\
4.2 \pm \\
2.7 \pm \\
2.7 \pm \\
2.7 \pm \\
5.1 \pm \\
33.7 \pm \\
5.4 \pm \\
2.8 \pm \\
0.7 \pm \\
0.9 \pm \\
0.5 \pm \\
36.4 \pm \\
0.1\end{array}$ & $\begin{array}{l}0.8 \\
0.8 \\
0.7 \\
0.6 \\
2.1 \\
1.7 \\
0.9 \\
2.2 \\
1.3 \\
0.7 \\
0.8 \\
0.8 \\
2.1 \\
1.6 \\
1.8 \\
1.4 \\
0.7 \\
1.7 \\
1.2 \\
1.3 \\
0.8 \\
0.8 \\
1.1 \\
1.4 \\
1.4 \\
0.6 \\
0.8 \\
0.7 \\
4.2\end{array}$ \\
\hline
\end{tabular}

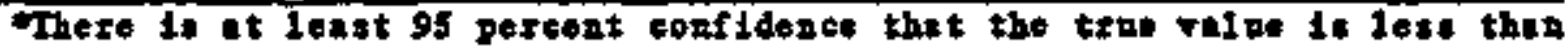
the moterremgat rapozted. 


\author{
Table D-2 \\ $\because 4 x+3$

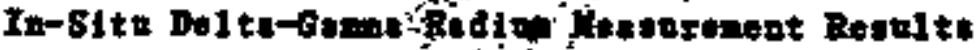 \\ for Apperiage Lochtions (N99999)
}

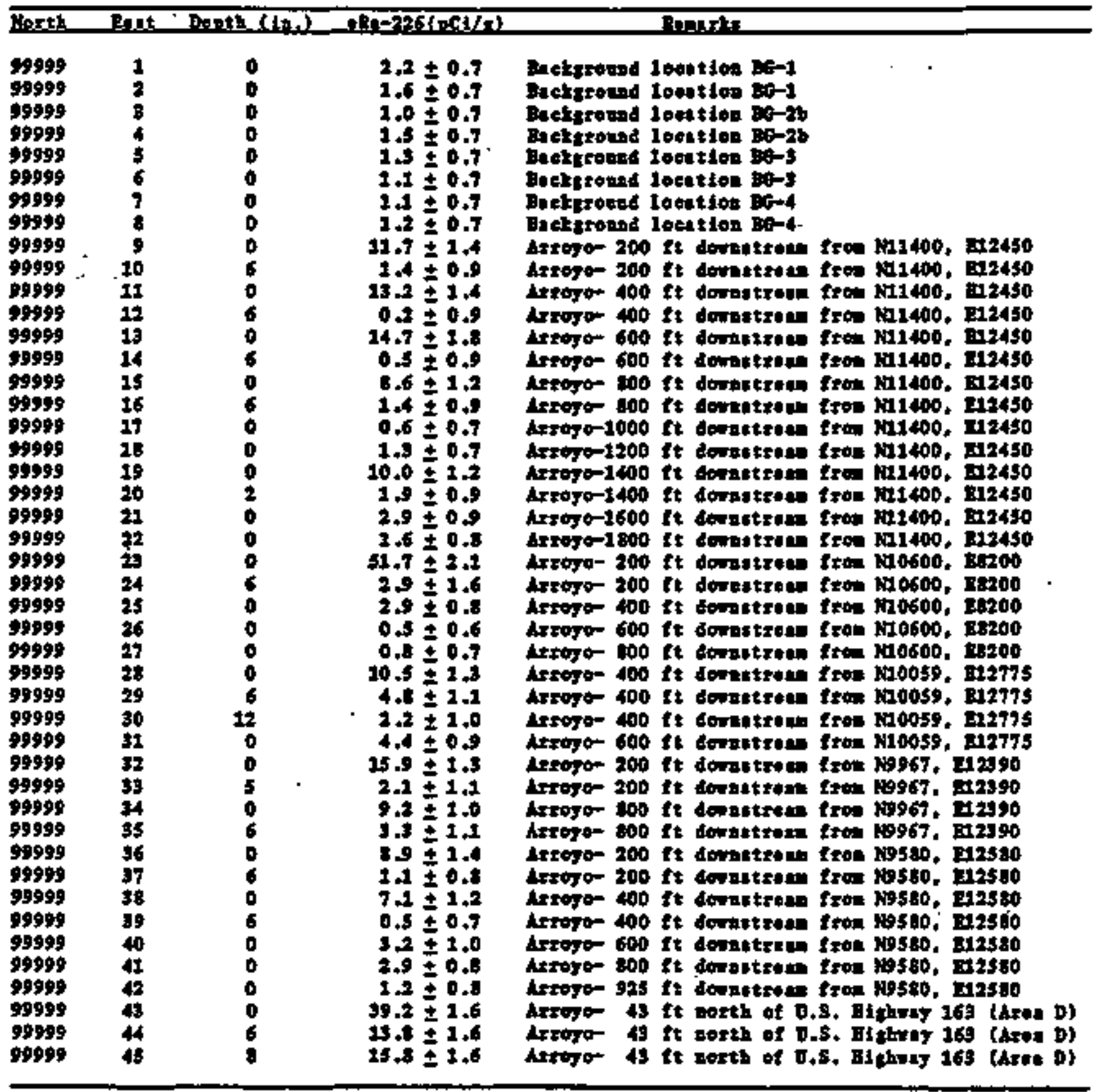




\section{Appudis $\mathrm{B}$}

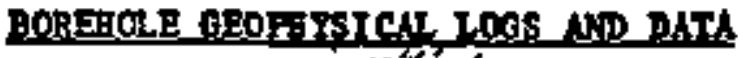

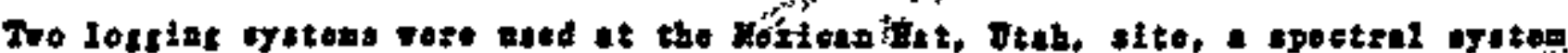

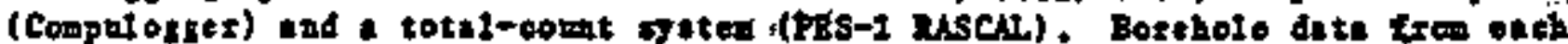

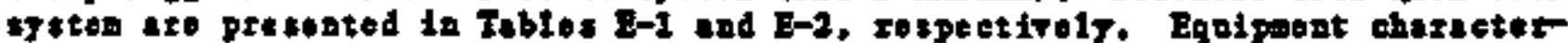

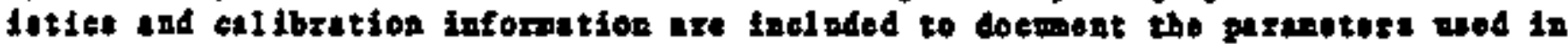
date rudeetion.

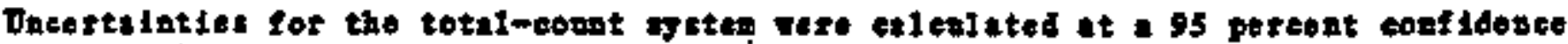
lerti (2o). Wette vas oneontered In eily tro boreholes. Lote for all other

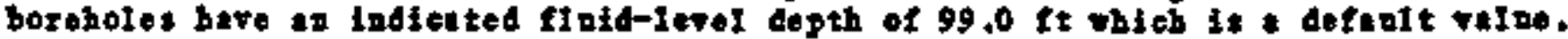

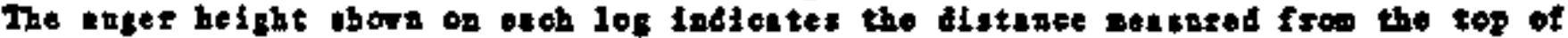
the enger to the groond stetince.

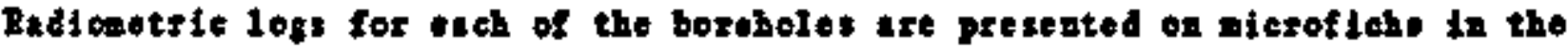

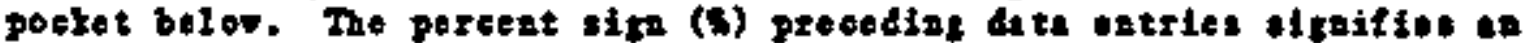
ovitflow 1n the dete field whith oceos after an epperent redim-226 concentration greater than 999,9 is zosched.

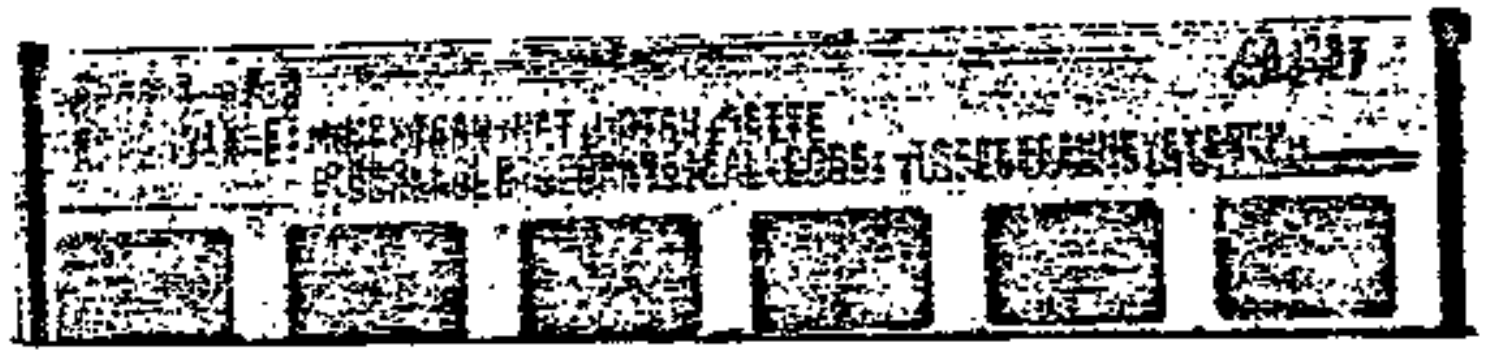




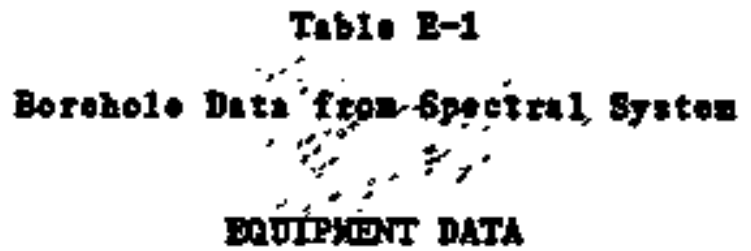

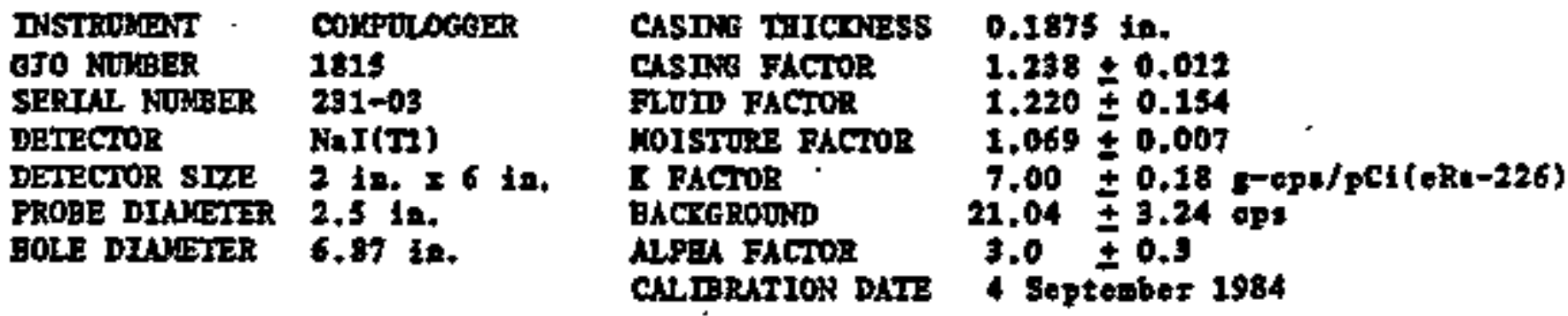

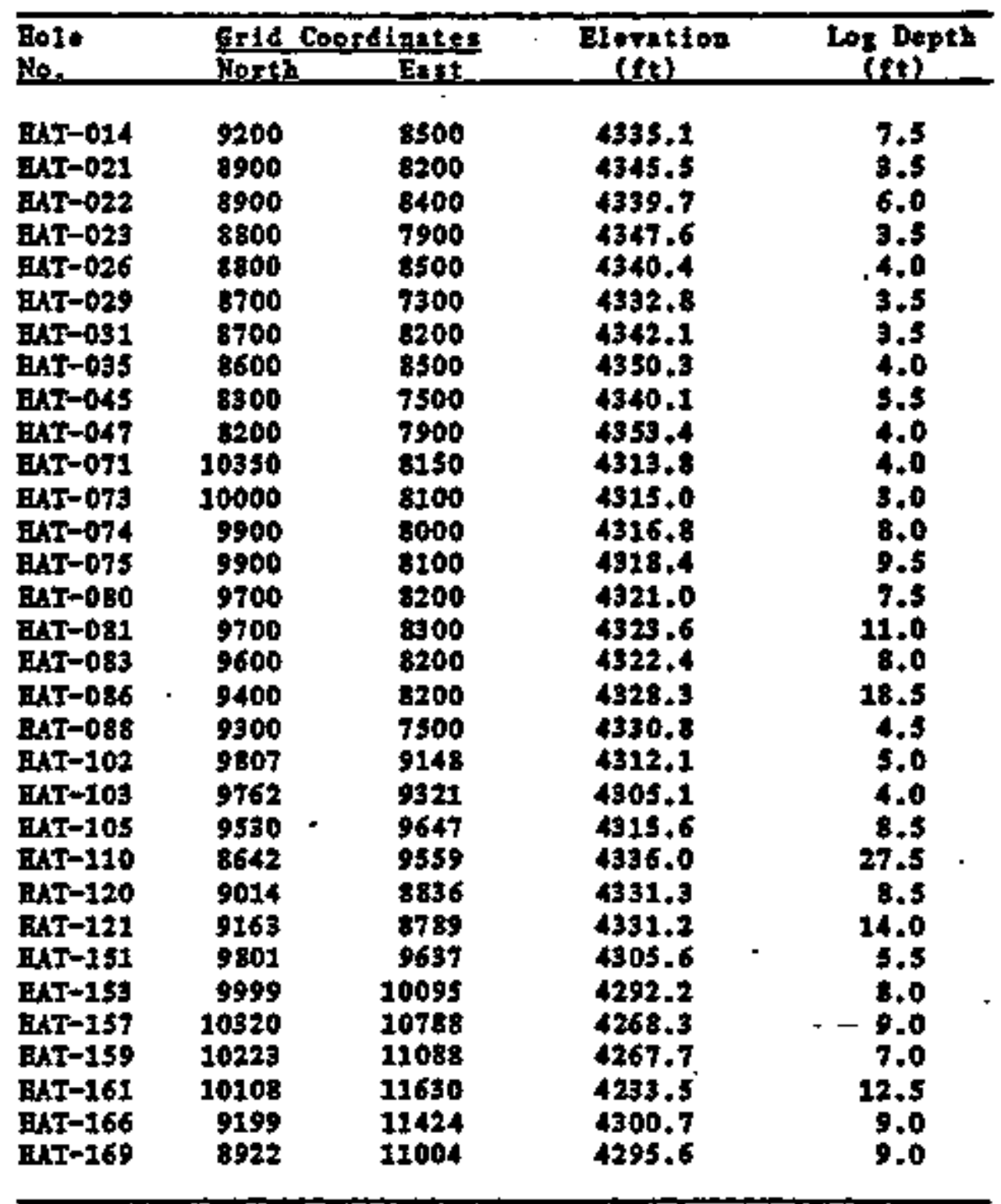




\section{Tab1 E-2}

Borabol, Date fitos Totul-Conet Srster.

\section{ERUIRIVIT DiTh}

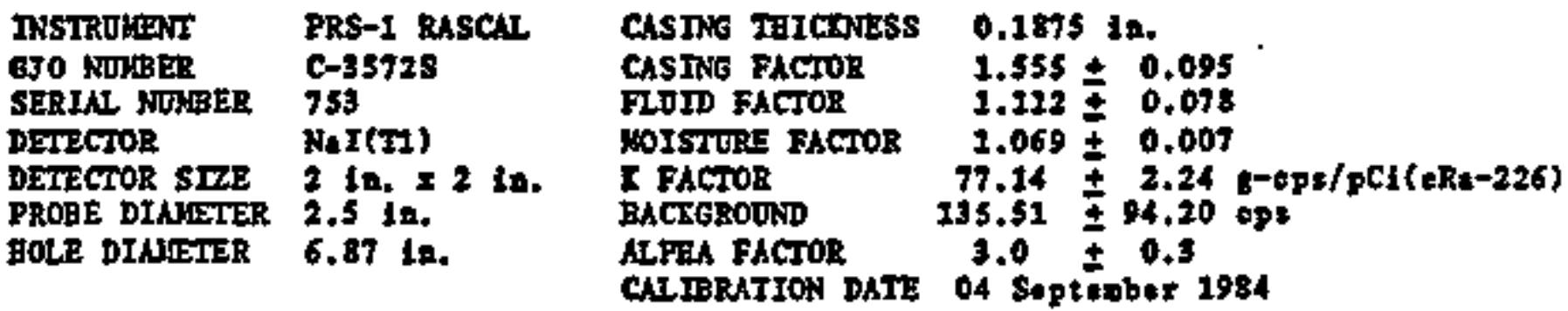

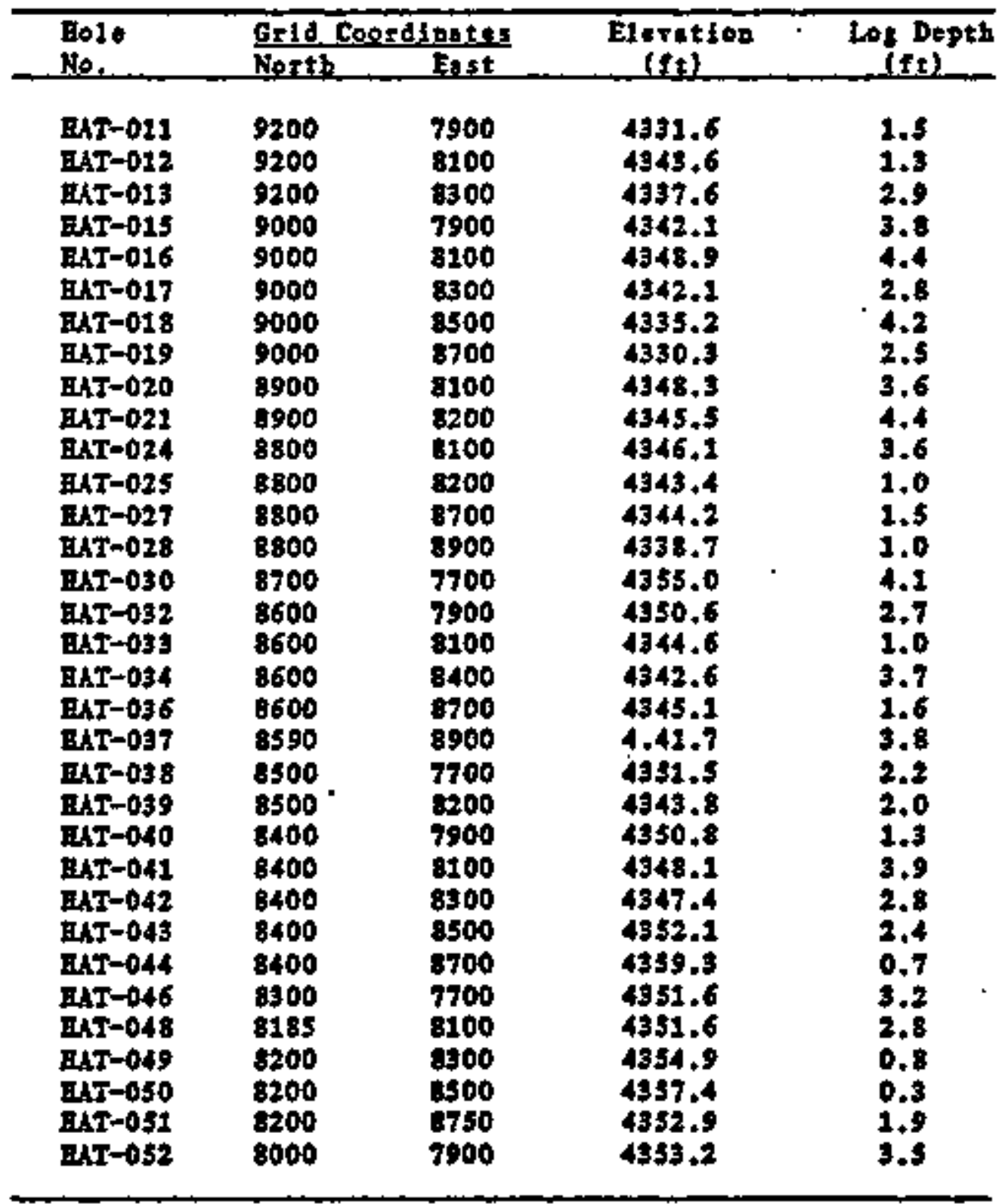


Tabl E-2 (eoptinged)

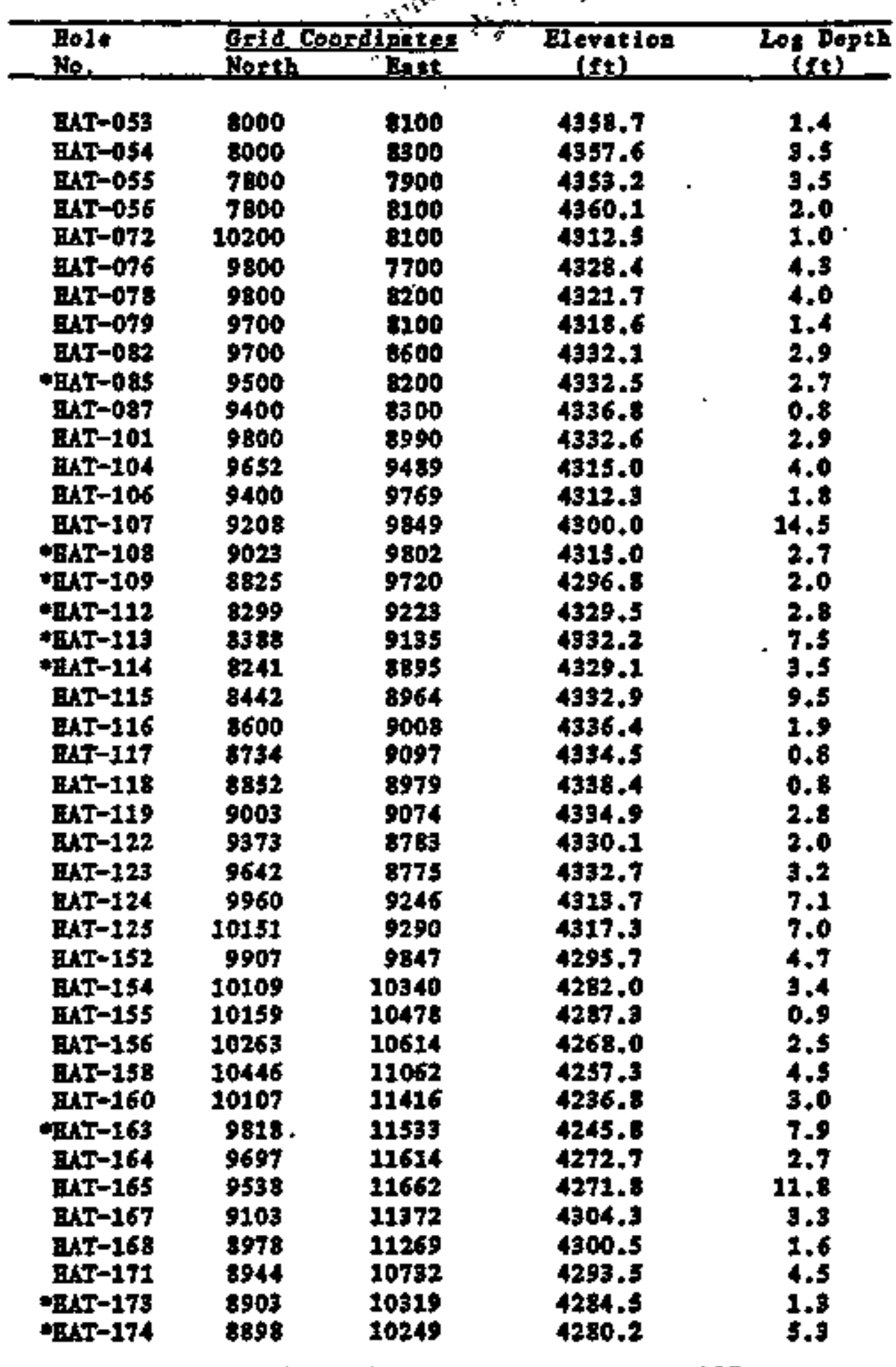

Boreholes Iogged Fithort onsing. Date wero redoed tor

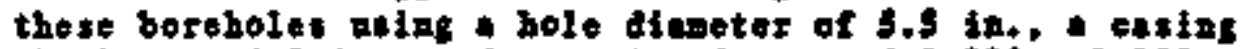
thicluess of $0 \mathrm{fn}$. . ond e estint factor of $1.000 \pm 0.000$. All other oqupment date vere tho same for all boreholes. 
Table E-2 (continood)

\begin{tabular}{|c|c|c|c|c|}
\hline $\begin{array}{l}\text { Hole } \\
\mathrm{No} \text {. }\end{array}$ & $\begin{array}{l}\text { Gxid } \\
\text { Notth }\end{array}$ & $\frac{d i d t e n}{\text { ditgit }}$ & $\begin{array}{l}\text { Elerption } \\
\text { (ft) }\end{array}$ & $\begin{array}{c}\log \text { Dogth } \\
\text { (ft) }\end{array}$ \\
\hline 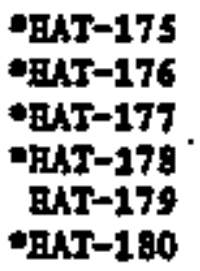 & $\begin{array}{r}8876 \\
8728 \\
10105 \\
9998 \\
10011 \\
9957\end{array}$ & $\begin{array}{r}10100 \\
9768 \\
11802 \\
11407 \\
11622 \\
11511\end{array}$ & $\begin{array}{l}4281.9 \\
4289.7 \\
4242.0 \\
4239.9 \\
4230.7 \\
4237.2\end{array}$ & $\begin{array}{l}1.9 \\
2.2 \\
2.5 \\
9.5 \\
2.7 \\
4.6\end{array}$ \\
\hline
\end{tabular}

Boreboles Iosged Fithort cesiog. Date whto toduced for these bortholes nsing bole dismeter of 5,5 in. a oasing thickess of 0 in. and cesing factor of $1.000 \pm 0.000$. AlI other equipuent data were the same for all borebolos. 


\section{Appendix F}

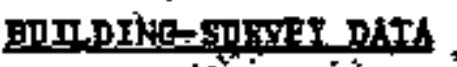

$$
i^{2}+s^{2}
$$

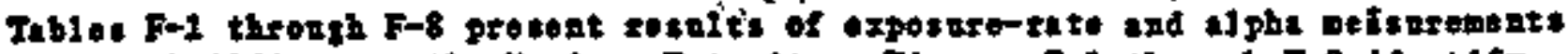

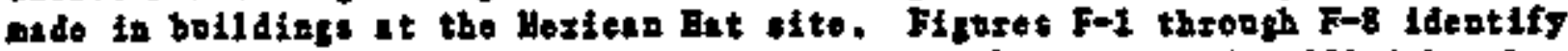

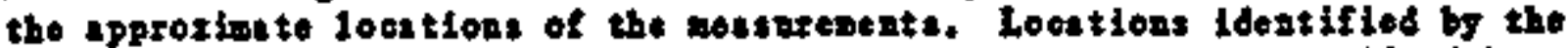

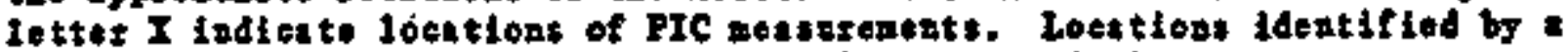

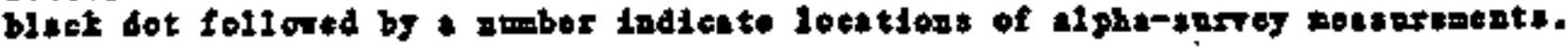


Tas: $\mathbf{F - 1}$

*:

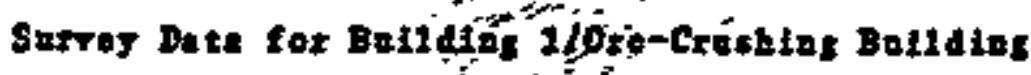

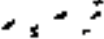

\begin{tabular}{|c|c|c|c|c|c|c|}
\hline \multirow{2}{*}{$\begin{array}{l}\text { Heatnzement } \\
\text { Lootition } \\
\text { No. }\end{array}$} & \multirow{2}{*}{\multicolumn{2}{|c|}{$\begin{array}{c}\text { Ares } \\
\text { serveged }\end{array}$}} & \multicolumn{2}{|c|}{ Athe sexter (ex) } & \multicolumn{2}{|c|}{ 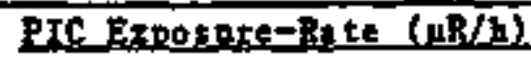 } \\
\hline & & & Dixot & $\begin{array}{l}\text { Clitan } \\
\text { Sinteres }\end{array}$ & $\begin{array}{l}\text { First } \\
\text { Rendints }\end{array}$ & $\begin{array}{l}\text { Seopnd } \\
\text { Rending }\end{array}$ \\
\hline $\begin{array}{l}x \\
1 \\
2 \\
3 \\
4 \\
5 \\
6 \\
7 \\
.8\end{array}$ & $\begin{array}{c}3 \text { ft/Cotter of } \\
\text { Floor } \\
\text { Floot } \\
\text { Floor } \\
\text { Floor } \\
\text { Floor } \\
\text { Floor } \\
\text { Footer } \\
\text { Plt }\end{array}$ & BIds. & $\begin{array}{r}570 \\
2000 \\
625 \\
395 \\
1400 \\
2100 \\
1100 \\
800\end{array}$ & $\begin{array}{r}345 \\
1030 \\
600 \\
320 \\
1900 \\
1400 \\
1100 \\
800\end{array}$ & 64.0 & $63+2$ \\
\hline
\end{tabular}
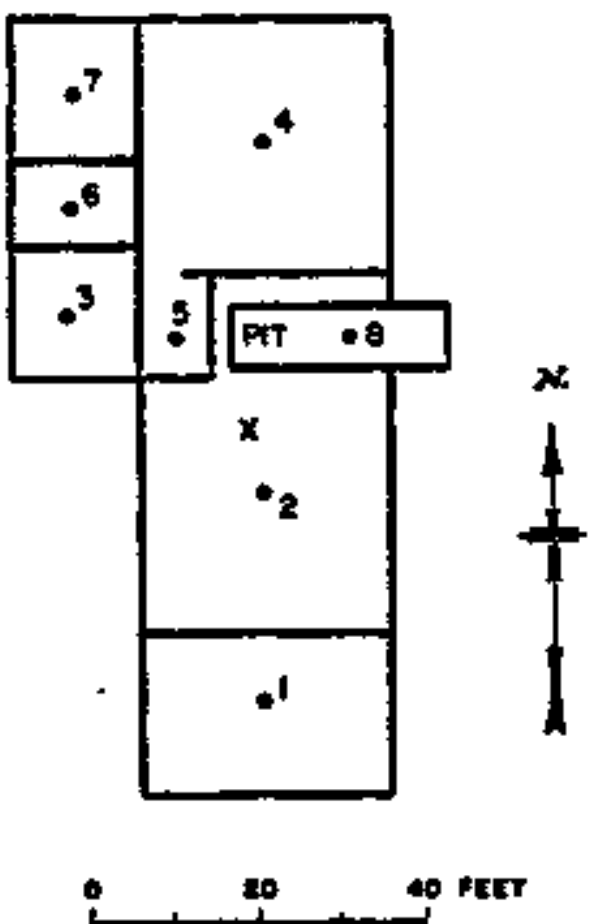

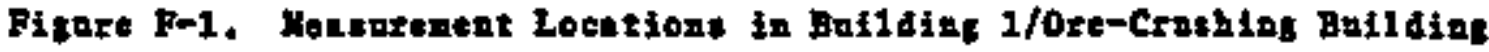




\section{Table F-2}

Sorrey Dete for soijding- 2/Sheet Wetal shop

\begin{tabular}{|c|c|c|c|c|c|}
\hline \multirow{2}{*}{$\begin{array}{l}\text { Hestorement } \\
\text { Loont10s } \\
\text { No. }\end{array}$} & \multirow{2}{*}{$\begin{array}{l}\text { Area } \\
\text { survered }\end{array}$} & \multicolumn{2}{|c|}{ 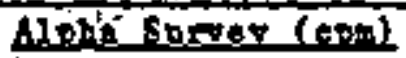 } & \multicolumn{2}{|c|}{ DIC Expostratsere $(1 \mathrm{R} / \mathrm{h})$} \\
\hline & & Distet & $\begin{array}{l}\text { Cloua } \\
\text { sinsties }\end{array}$ & $\begin{array}{l}\text { First } \\
\text { Beadiss }\end{array}$ & $\begin{array}{l}\text { Second } \\
\text { Reading: }\end{array}$ \\
\hline $\begin{array}{r}1 \\
1 \\
2 \\
3 \\
4 \\
5 \\
6 \\
7 \\
8 \\
9 \\
10\end{array}$ & 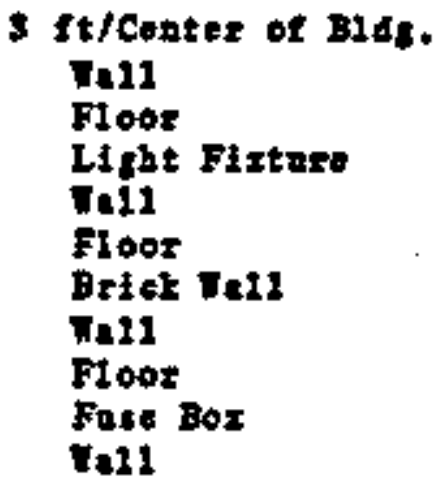 & $\begin{array}{l}20 \\
20 \\
25 \\
40 \\
20 \\
75\end{array}$ & $\begin{array}{l}0 \\
2 \\
5 \\
0 \\
4 \\
0 \\
2 \\
8 \\
8 \\
0\end{array}$ & 20.7 & 20.7 \\
\hline
\end{tabular}

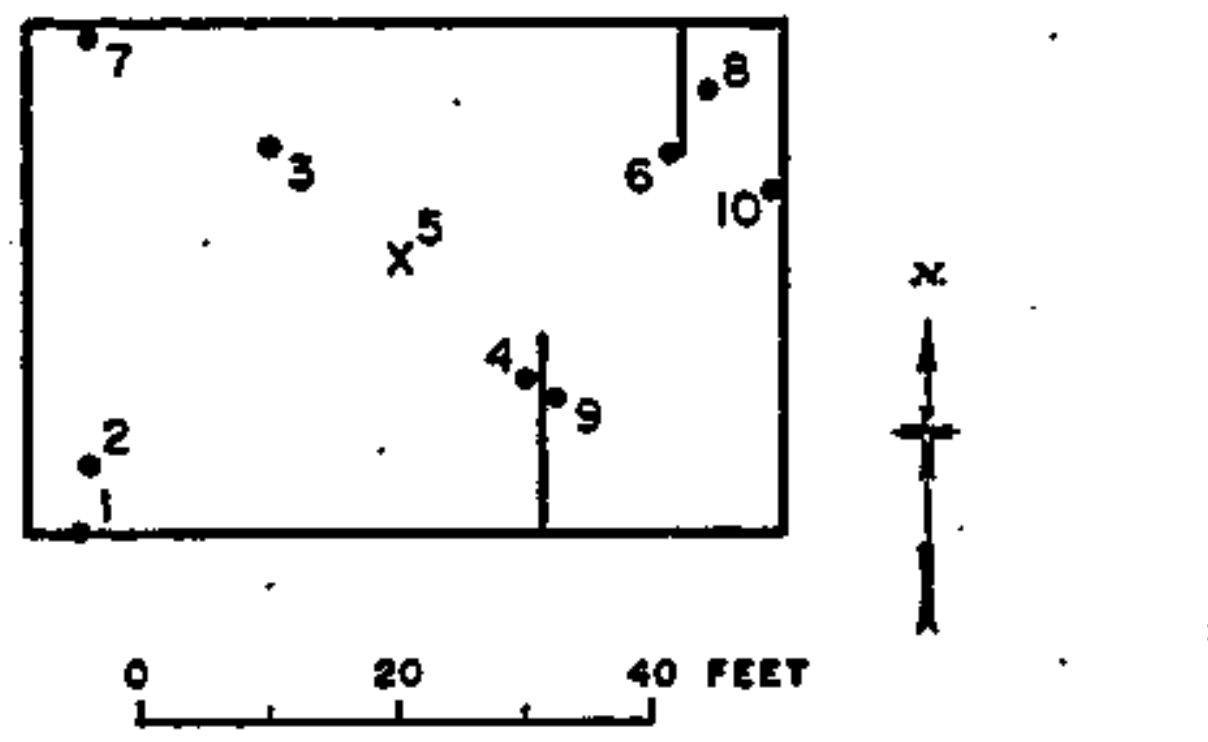

Pitare P-2. Wousurement Loctitons in Btildint 2/Stet Metel Shop 
Thb1: $\mathrm{p}-3$

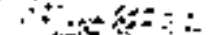

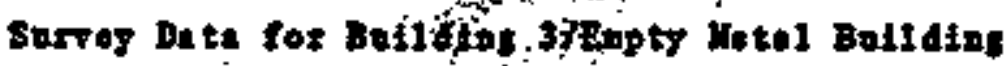

$\because=:$

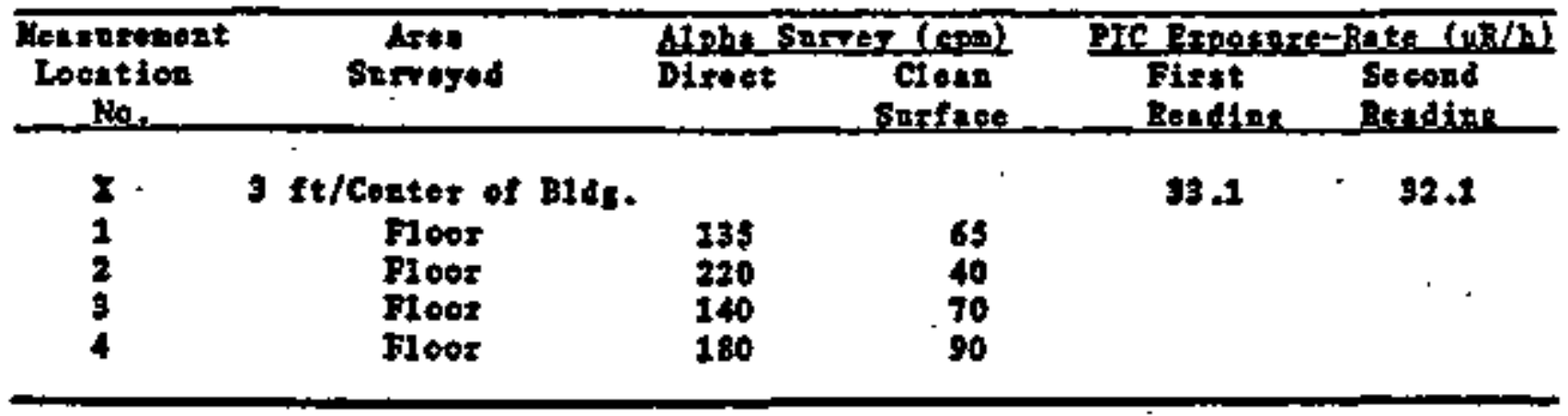
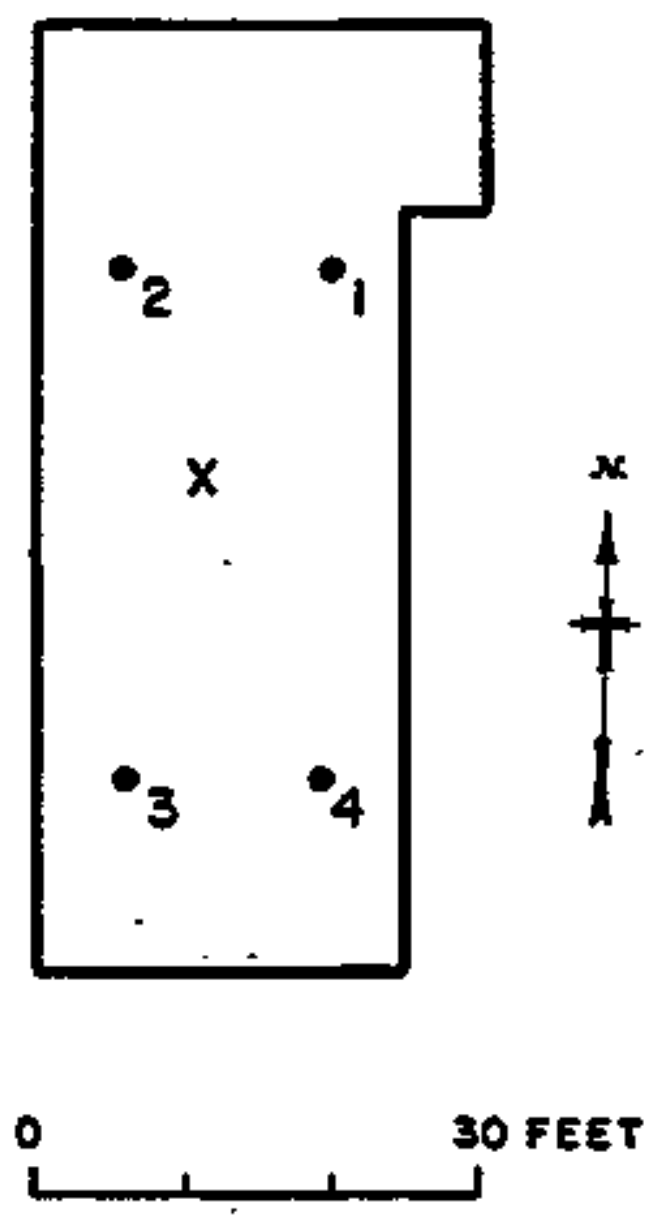

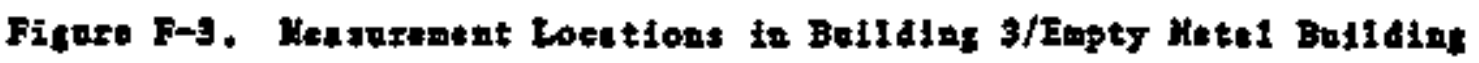


TrbI: $3-4$

Sorvey Date fot Bulldieg 4/Generstot Botldins

\begin{tabular}{|c|c|c|c|c|c|}
\hline $\begin{array}{c}\text { Hetstresent } \\
\text { Loentsos } \\
\text { No. }\end{array}$ & $\begin{array}{l}\text { Area } \\
\text { Stureyed }\end{array}$ & 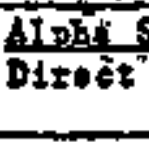 & 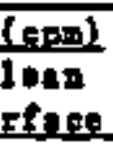 & $\begin{array}{c}\text { EIC Exposer } \\
\text { Fisto } \\
\text { Jinding }\end{array}$ & $\begin{array}{l}\text { Gite }(\mathrm{gR} / \mathrm{h}) \\
\text { second } \\
\text { Rendes. }\end{array}$ \\
\hline $\begin{array}{l}x \\
1 \\
2 \\
8 \\
4 \\
5 \\
6 \\
7 \\
7\end{array}$ & 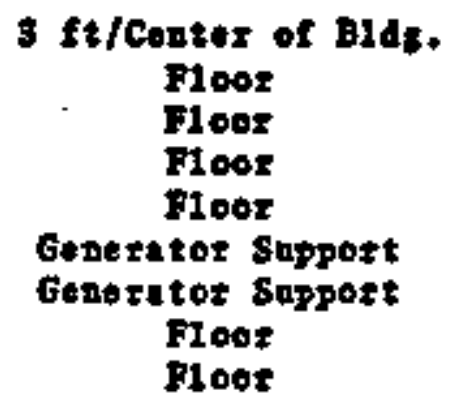 & $\begin{array}{r}100 \\
100 \\
50 \\
160 \\
120 \\
110 \\
75 \\
100\end{array}$ & $\begin{array}{r}95 \\
95 \\
110 \\
140 \\
140 \\
80 \\
75 \\
120\end{array}$ & 37.2 & 16.0 \\
\hline
\end{tabular}
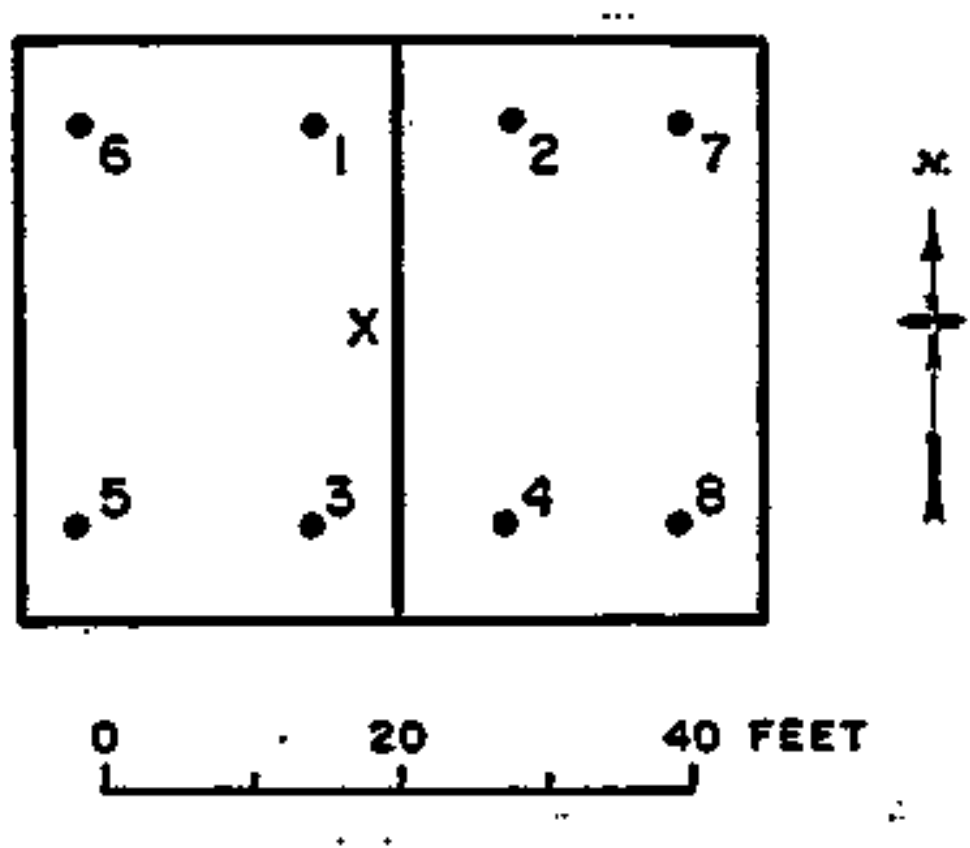

Figute F-4. Measerement Locktere in Building 4/Generator Bollding 


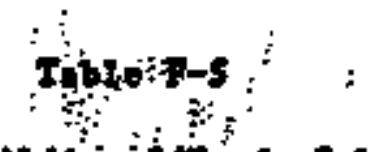

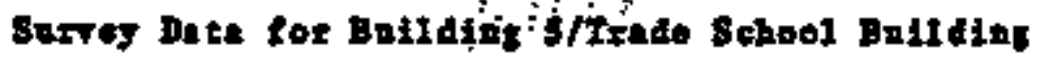

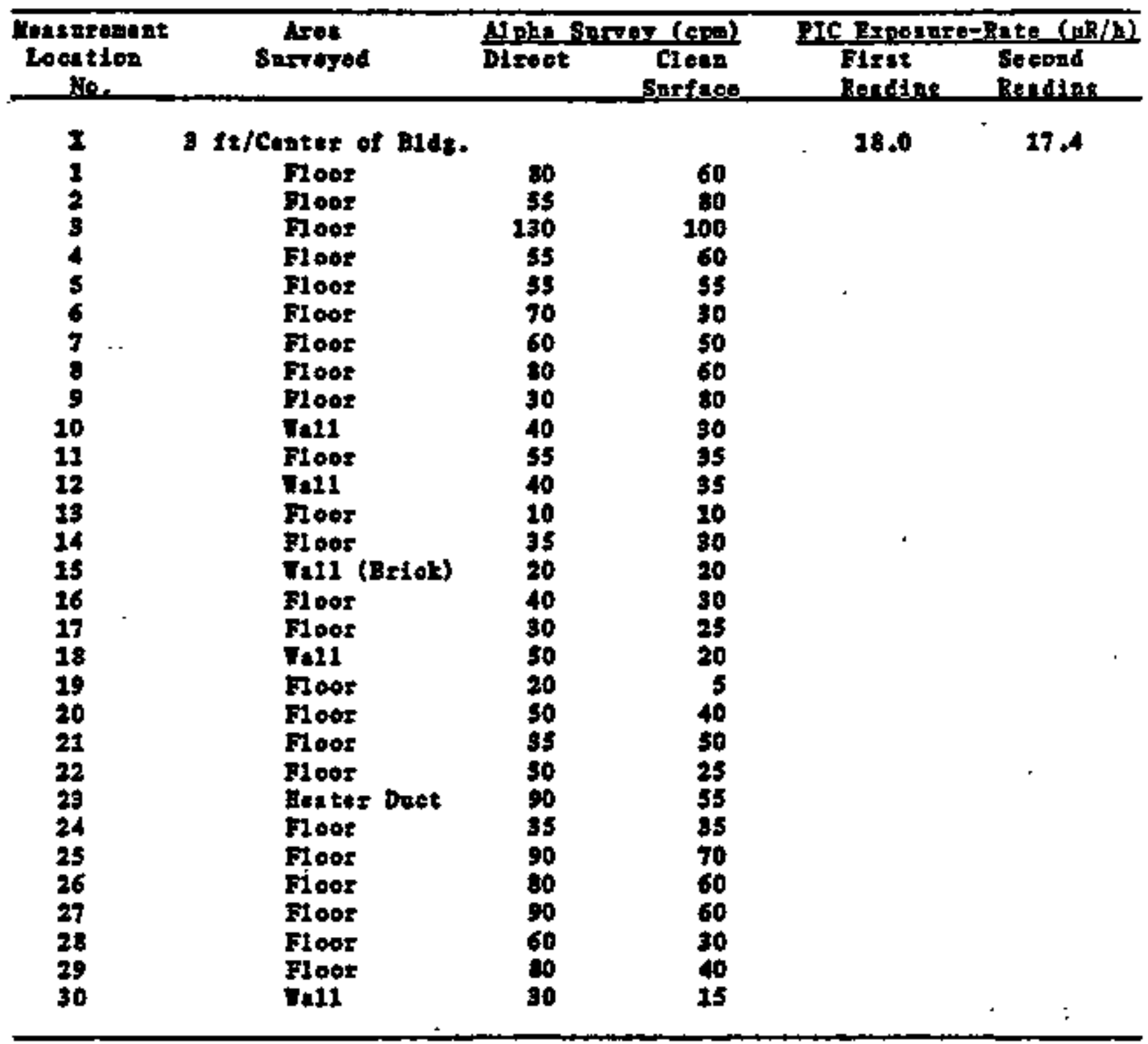




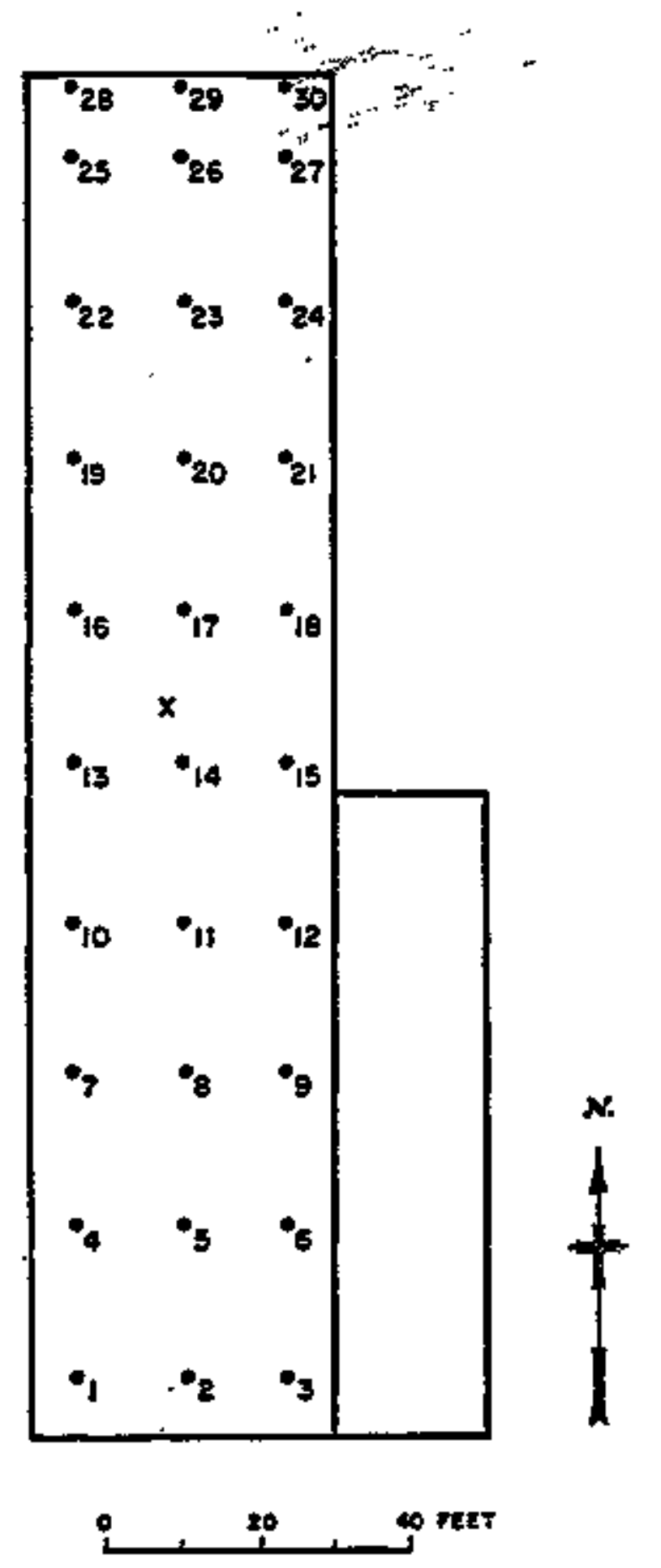

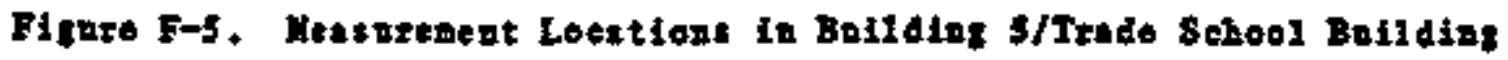




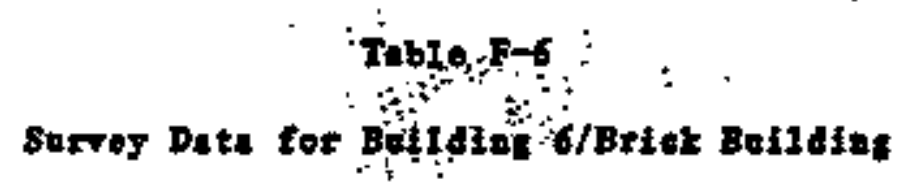

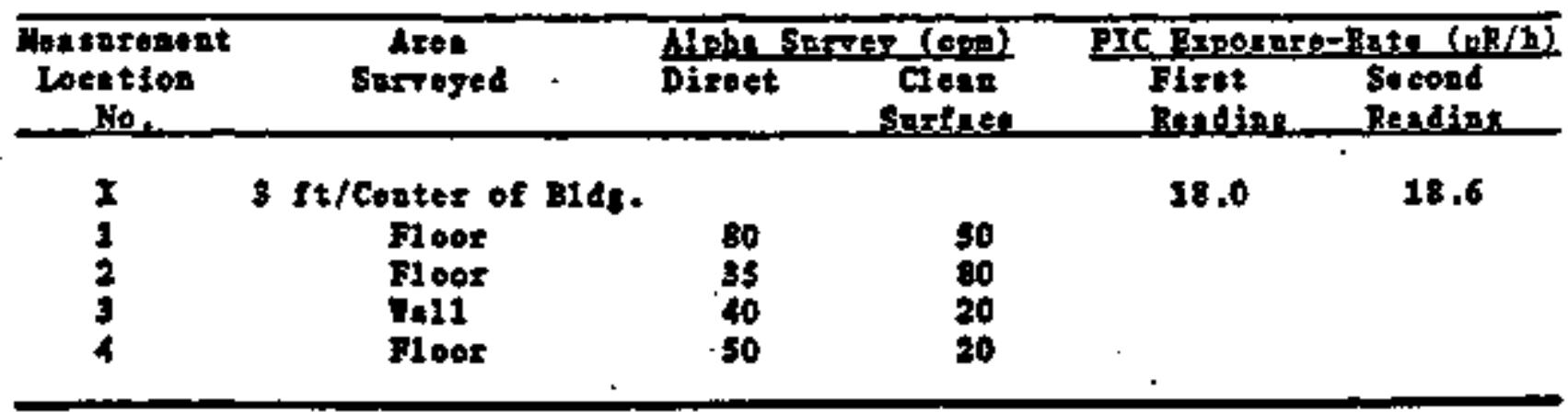

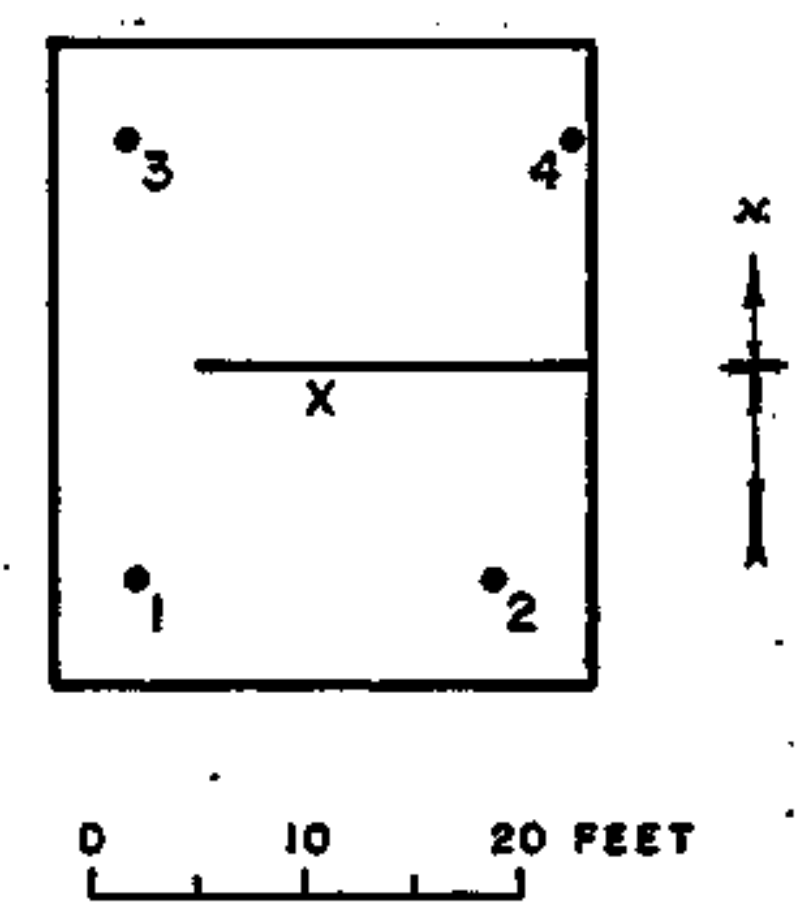

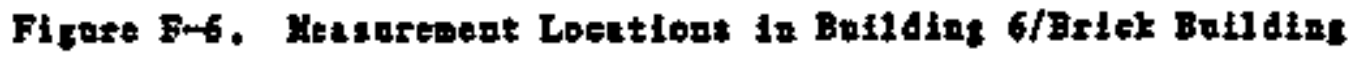


Iabie $8-7$

sertey Data for Bulldits 7/5eale Eouse

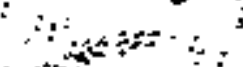

\begin{tabular}{|c|c|c|c|c|c|}
\hline $\begin{array}{c}\text { Mensurtaent } \\
\text { Doention } \\
\text { No. }\end{array}$ & $\begin{array}{c}\text { Area } \\
\text { Surresed }\end{array}$ & Mobtic & 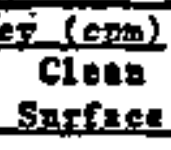 & 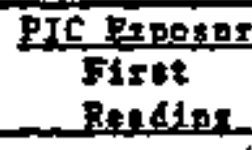 & $\begin{array}{l}\text { Pete }(\mathrm{d} / \mathrm{h}) \\
\text { Second } \\
\text { Reting }\end{array}$ \\
\hline $\begin{array}{l}x \\
2 \\
2 \\
3 \\
4\end{array}$ & $\begin{array}{l}\text { I fe/Center of Blds. } \\
\text { Floes } \\
\text { Floer } \\
\text { Bloot } \\
\text { Floor }\end{array}$ & $\begin{array}{l}330 \\
360 \\
235 \\
250\end{array}$ & $\begin{array}{l}280 \\
255 \\
400 \\
320\end{array}$ & 67.7 & 67.9 \\
\hline
\end{tabular}

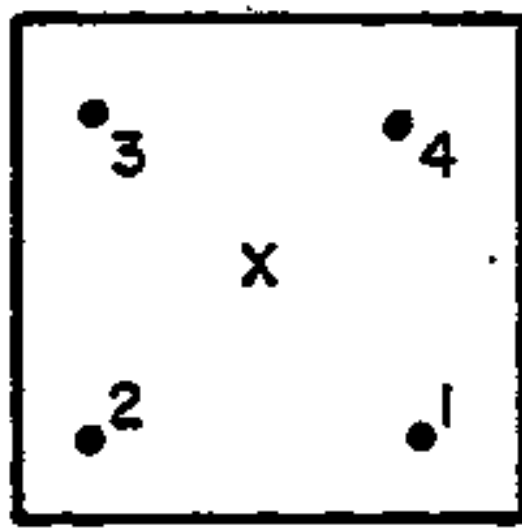

N.
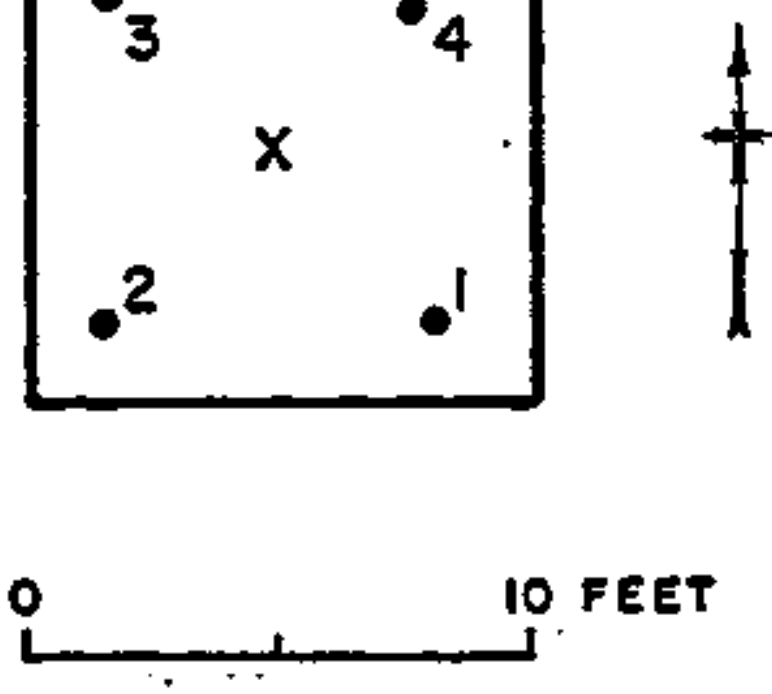

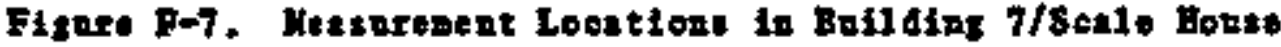


Iable F-8

$\therefore$

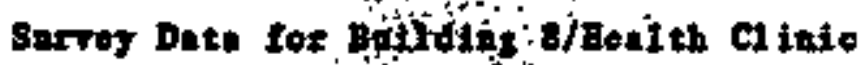

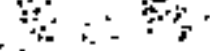

\begin{tabular}{|c|c|c|c|c|c|}
\hline $\begin{array}{l}\text { Mocsoresent } \\
\text { Doetetion } \\
\text { No. }\end{array}$ & $\begin{array}{c}\text { Ated } \\
\text { Sprezed }\end{array}$ & $\begin{array}{l}\text { Appe } \\
\text { Dizect }\end{array}$ & 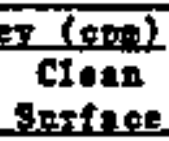 & $\begin{array}{c}\text { PIC Bxposts } \\
\text { First } \\
\text { Besditur }\end{array}$ & $\begin{array}{l}\text {-Rate }(\mathrm{g} R / \mathrm{h}) \\
\text { Second } \\
\text { Rendigh }\end{array}$ \\
\hline $\begin{array}{l}x \\
1 \\
2 \\
3 \\
4 \\
5 \\
6 \\
7 \\
8 \\
9 \\
10 \\
11 \\
12 \\
13 \\
14 \\
15 \\
16 \\
17 \\
18\end{array}$ & 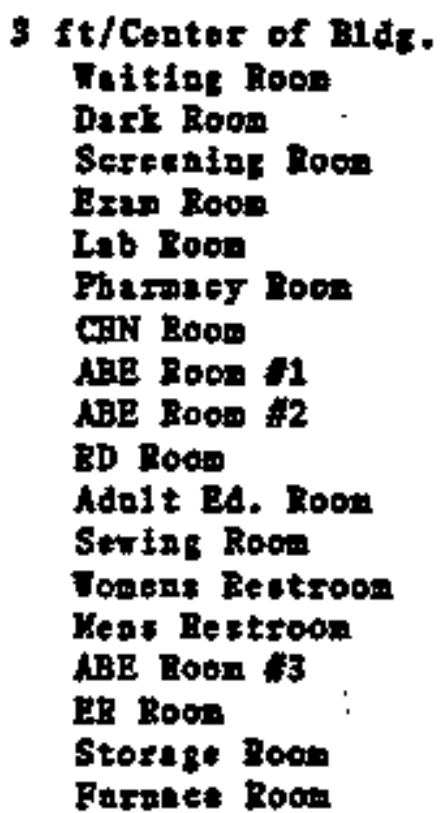 & $\begin{array}{l}40 \\
40 \\
40 \\
30 \\
30 \\
10 \\
20 \\
20 \\
10 \\
30 \\
20 \\
15 \\
40 \\
30 \\
30 \\
20 \\
25 \\
50\end{array}$ & $\begin{array}{l}0 \\
1 \\
1 \\
0 \\
0 \\
0 \\
1 \\
0 \\
0 \\
0 \\
0 \\
0 \\
0 \\
0 \\
0 \\
0 \\
0 \\
0\end{array}$ & $13+7$ & 13.5 \\
\hline
\end{tabular}




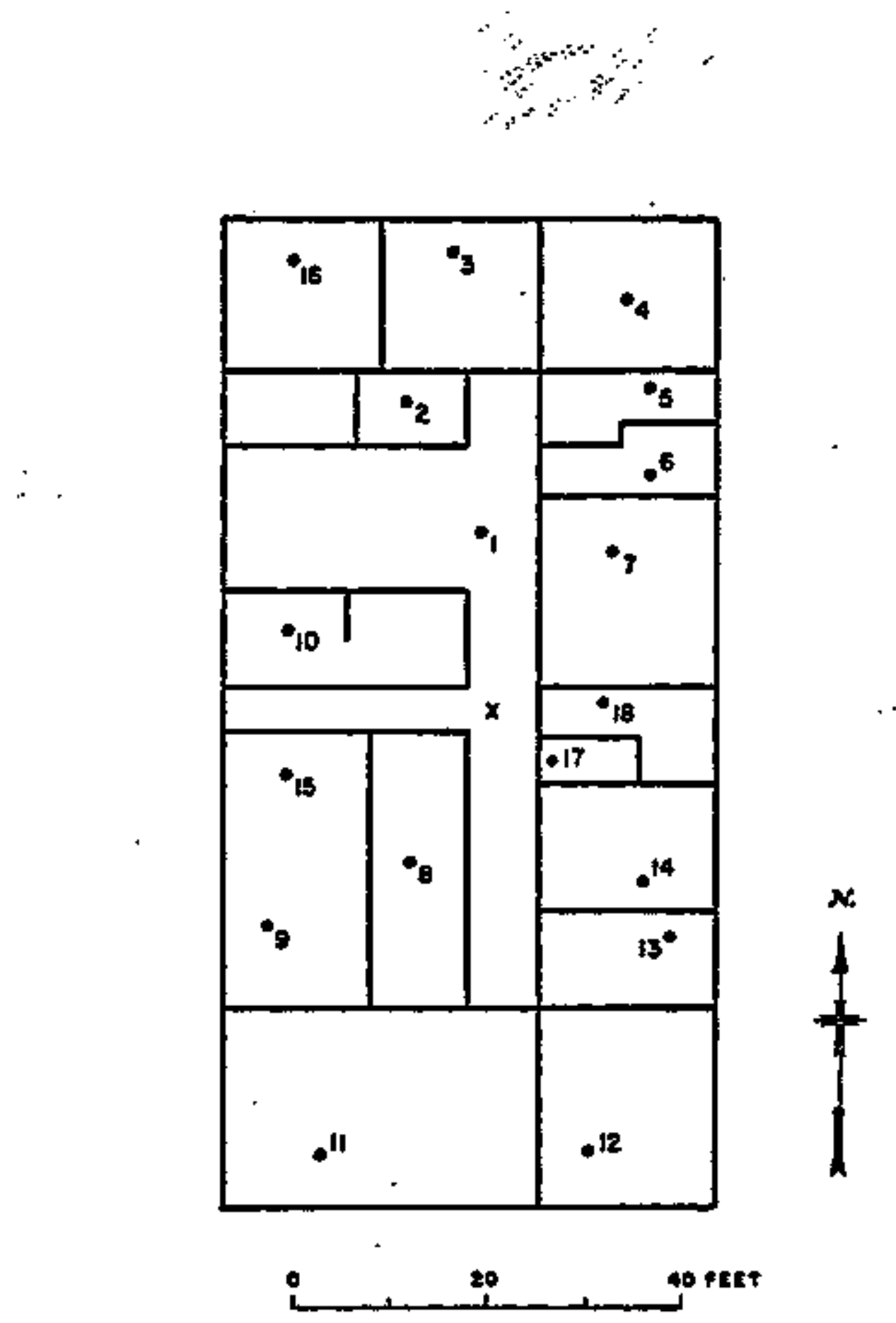

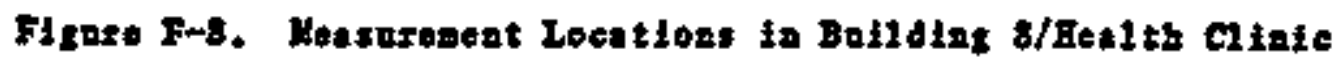




\section{Apperdix 6}

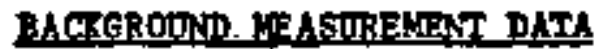

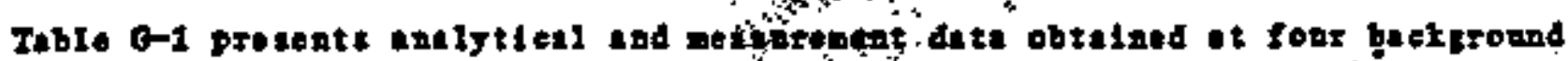

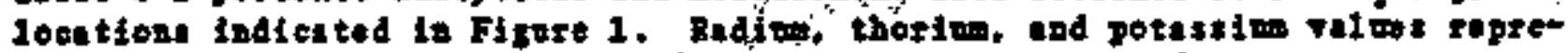

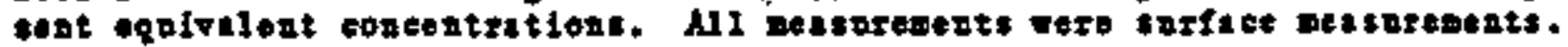

soll samples Fere ool lectod at a depts of 0 to 6 in. 
Tabte 0-1

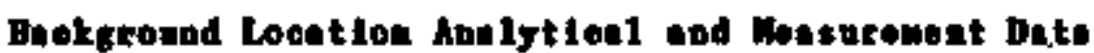

\begin{tabular}{|c|c|c|c|c|c|c|c|c|c|c|}
\hline \multirow{3}{*}{ Aren } & \multirow{2}{*}{\multicolumn{4}{|c|}{ 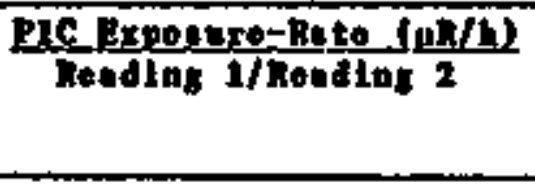 }} & \multirow{3}{*}{ 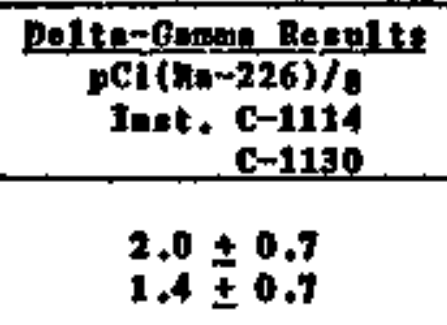 } & \multicolumn{5}{|c|}{ 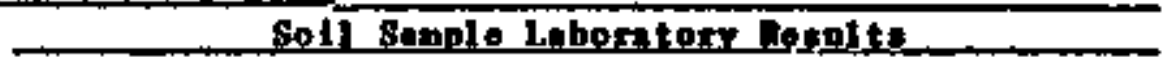 } \\
\hline & & & & & & $\begin{array}{l}\text { Soaple } \\
\text { Ho. }\end{array}$ & $\begin{array}{l}\text { Molsture } \\
\text { (s) }\end{array}$ & focluts & $\begin{array}{l}\text { Thorid: } \\
\text { fopas }\end{array}$ & $\begin{array}{c}\text { Potectits } \\
\text { (4i) }\end{array}$ \\
\hline & 11.5 & 1 & 11.4 & & & $\begin{array}{l}\text { Wh } 101 \\
\text { whin } 30 t\end{array}$ & 1.2 & $\begin{array}{l}1.0 \pm 1.0 \\
0.9 \pm 1.0\end{array}$ & $\begin{array}{l}6.0 \pm 2.0 \\
9.9 \pm 2.0\end{array}$ & \\
\hline$B 0-2 b$ & 11.7 & $t$ & 11.4 & * & $\begin{array}{l}1.3 \pm 0.7 \\
0.8 \pm 0.7\end{array}$ & $\begin{array}{l}\text { lind } 213 \\
\text { Ung } 625 \\
\text { ind } 578\end{array}$ & & $\begin{array}{l}1.4 \pm 1.0 \\
1.0 \pm 1.0 \\
1.1 \pm 1.0\end{array}$ & $\begin{array}{l}9.6 \pm 1.4 \\
3.0 \pm 1.4 \\
6.9 \pm 1.0\end{array}$ & $\begin{array}{l}1.0 \pm 0.4 \\
1.0 \pm 0.2 \\
1.0 \pm 0.2\end{array}$ \\
\hline$B 3-3$ & 10.7 & $\boldsymbol{I}$ & 10.9 & & $1.1 \pm 0.7$ & $\begin{array}{l}\text { hid } 103 \\
\text { hild } 151\end{array}$ & 1.2 & $\begin{array}{l}1.0 \pm 1.0 \\
0.7 \pm 1.0\end{array}$ & $\begin{array}{l}7.0 \pm 2.0 \\
1.7 \pm 0.0\end{array}$ & $\begin{array}{l}2.3 \pm 0.2 \\
1.4\end{array}$ \\
\hline lin-4 & 12.7 & 1 & $12+4$ & & $\begin{array}{l}1.0 \pm 0.7 \\
0.5 \pm 0.7\end{array}$ & $\begin{array}{l}\text { min } 114 \\
\text { lid } 579\end{array}$ & 3.0 & $\begin{array}{l}2.0 \pm 1.0 \\
1.3 \pm 1.0\end{array}$ & $\begin{array}{l}1.0 \pm 1.0 \\
5.6 \pm 1.4\end{array}$ & $\begin{array}{l}1.3 \div 64 \\
1,6 \pm 0\end{array}$ \\
\hline
\end{tabular}




$$
\text { by }
$$

ADOENDUM 02

FINAL RADIUG COHCEHTRATIOHS AND VOLUHES

OF TAILIHGS AT MEXICAN HAT 


\section{TABLE OF COHTENTS}

\section{Section}

D2.1 Final radium concentrations and volumes at the upper pile at Mexican Hat.................. D2-1

D2.2 Final radium concentrations and volumes at the lower pile at Mexican Hat .................... 02-10 
02.1 FIHAL RADIUM CONCEHTRATIONS AND VOLUMES AT THE UPPER PILE AT MEXICAN HAT

Pages 02-2 through 02-5 of . thit addendum present the concentrations of each grid cell by row, colum, and layer. Each page contains two rows and all columns and layers for those rows. The layer by layer concentrations are printed down the page, while the colums are organized across the page. Pages 02-6 and 02-7 give the depths to the . $75 \mathrm{pCl} / \mathrm{g}$ and physical interfaces, respectively. In these tables, the values are organized according to row and column. Page o2-8 presents the volune sumnary and the layer by layer averages with depth into the pile. 
FJLES: KTUPBI/DAT - ENTEAES S/21/O5 (RANEISON).

FILE DATA FROK ( 6 OY $\theta$ ) GRID - GRID SPACJHST $\$(160$ by 179 by 2.5 ) FEET

DATA FOR ROA : 1

\begin{tabular}{|c|c|c|c|c|}
\hline $0.0-2.3$ & 582 & 594. & 647 & 419 \\
\hline $2.5-5.0$ & 773 & 462 & 428 & 367 \\
\hline $5.5=7.5$ & 586 & $\operatorname{sez}$ & 947 & 326 \\
\hline $7.5-18.0$ & 435 & $5 B 5$ & 743 & 757 \\
\hline $8.8=12.5$ & 695 & 444 & 622 & 365 \\
\hline $2.5=15.8$ & is5 & 872 & 584 & 676 \\
\hline $5.0-17.5$ & 596 & 642 & 427 & $\$ 1$ \\
\hline $7.5-20.0$ & $98 \%$ & 1472 & 480 & 965 \\
\hline $0.0-22.5$ & 816 & 072 & 529 & 725 \\
\hline $2.5=25.6$ & 350 & 1800 & .662 & 941 \\
\hline $3.0-27.5$ & & & & 315 \\
\hline 38.6 & & & & 215 \\
\hline $0.0-32.5$ & & & & 200 \\
\hline 35.0 & & & & 777 \\
\hline & & & & \\
\hline
\end{tabular}

3.5 -

$40.9-42.8$

$42.3=43.6$

$45.0=47.5$

DATA FOR ROW : 2

\begin{tabular}{|c|c|c|c|c|c|c|c|}
\hline $0.0-2.5$ & 417 & 911 & 409 & 516 & 127 & 242 & 432 \\
\hline $2.5-5.6$ & 489 & 544 & 670 & 785 & 357 & 496 & 353 \\
\hline $5.0-7.5$ & 3 & 655 & 655 & 737 & 484 & 473 & 377 \\
\hline $7.5 * 18.0$ & & 522 & 287 & 699 & 232 & 636 & 6)3 \\
\hline $18.0-12.5$ & & 612 & 926 & 531 & $\$ 65$ & $7: 2$ & 581 \\
\hline $12,5=15,0$ & & 266 & 811 & SO4 & S4t & 5752 & 1268 \\
\hline $15.0-17.5$ & & & 30 & 575 & 704 & 957 & 677 \\
\hline $17.5=2.0$ & & & & EA9 & 43 & 717 & 442 \\
\hline $20.1=22.5$ & & & & 467 & 792 & $\$ 1$ & 1847 \\
\hline $22.5-25.0$ & & & & $\operatorname{taz}$ & ses & 1883 & 734 \\
\hline $25.8-27.5$ & & . & & ค1 & 242 & 874 & 377 \\
\hline $27.5-38.0$ & & & & 780 & 739 & 762 & 436 \\
\hline $38.0=32.3$ & & & & & 541 & S46 & - 396 \\
\hline $32.5=35.6$ & & & & & & 1715 & 239 \\
\hline $35.0-37,5$ & & & , & & & 1557 & 1205 \\
\hline $37.5=48.1$ & & & & & & 612 & 776 \\
\hline $40,0-42,5$ & & & & & & & \\
\hline $.5=45.0$ & & & & & & & \\
\hline $3.8=$ & & & & & & & \\
\hline
\end{tabular}


DATA FOR FOW : 3

$$
\text { DEPTH }
$$

Q.8 $-2,5$

$2.5-5.0$

$5.6-7.5$

$7.5=18.8$

$10.0-12.3$

$12.5-15.0$

$15.8-17.5$

$37.5=23.8$

$28.6-22.5$

$22.5-25.8$

$25.0-27.5$

$27.5-30.0$

$30.0-32.5$

$32.5-33.0$

$35.8-37.5$

$37.5-48.6$

$40.0-42.5$

$42.5=45.18$

$45.8-47.5$

$$
\begin{aligned}
& \mathrm{J}=1 \mathrm{~J}=2 \mathrm{~J}=3 \mathrm{~J}=4 \mathrm{~J} \cdot 5 \mathrm{~s}=6 \mathrm{~J}^{\circ} 7 \mathrm{~J}=8 \\
& 633 \quad 1634 \quad 327 \quad 312 \% 580212 \text { t018 } \\
& 470 \quad 1248 \quad 892 \cdot 299 \text { 746 } 384686 \\
& 455 \quad 1840 \quad 910 \quad 436 \quad 237 \text { 935 } 598 \\
& 141 \quad 1173 \quad 767 \quad 994 \text { ES6 } 1846 \quad 824 \\
& 699 \quad 1851 \quad 465 \quad 574 \quad 894 \quad 999 \\
& \begin{array}{llllll}
14 & 314 & 674 & 552 & 560 & 636
\end{array} \\
& \$ 52 \quad 515 \quad 757 \quad 693 \quad 771 \\
& 597 \quad 911 \quad 946 \quad 642 \\
& 342999 \\
& 1185 \quad 626 \quad 1824 \\
& 765 \quad 1871490 \\
& \text { B13 } 92 \\
& 795 \\
& 1597 \\
& 645
\end{aligned}
$$

MTA FOR HOH : 4

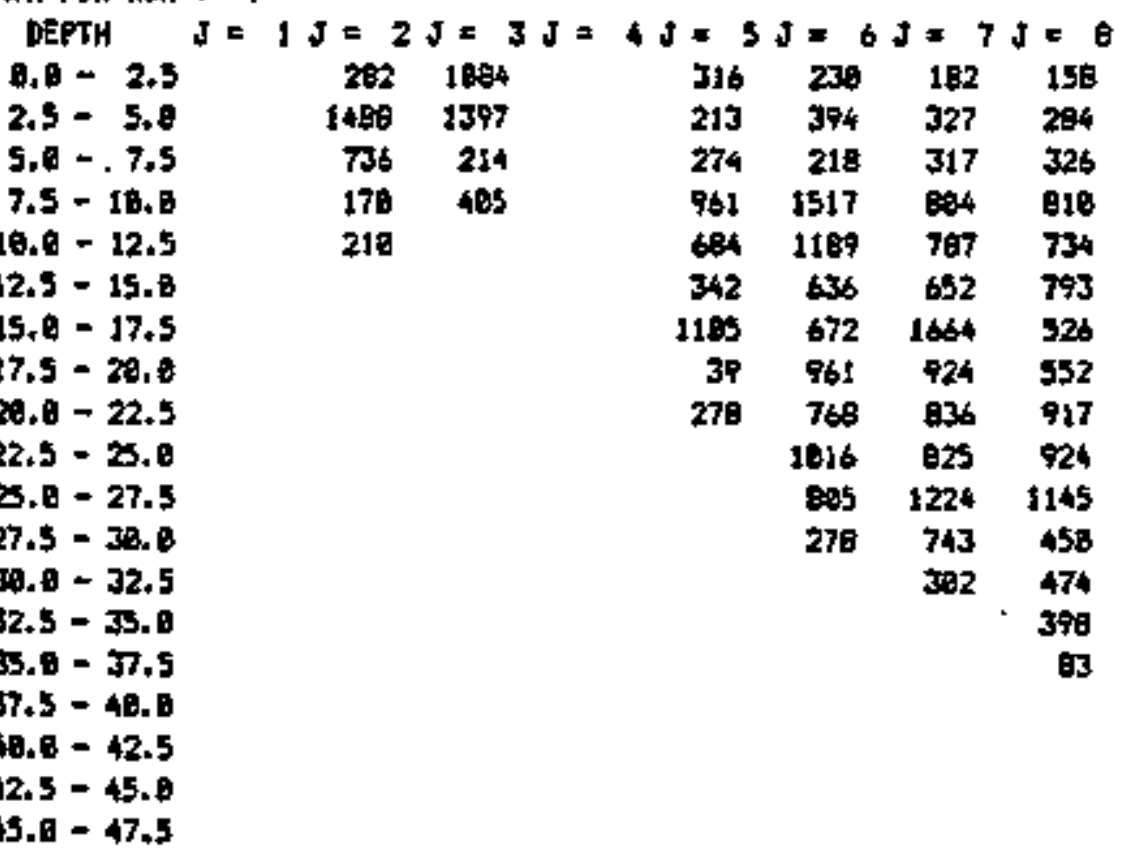


DATA FOR RON :

$$
\text { BEFTH }
$$

$$
0.0-2.5
$$

$2,3=5.0$

$5.8=7.5$

$7.5-10.0$

$10.0-12.5$

$12.5-15.0$

$55.6=17.5$

$17.5=20.6$

$20.0-22.5$

22.5 - 25.0

25. $0=27.5$

$27.5=35.8$

$30.0-33.5$

32.5 - 35.0

$35.6-37.5$

$37.5-40.0$

$46 . \theta=42.5$

$42.5-45.8$

$45.6=47.5$

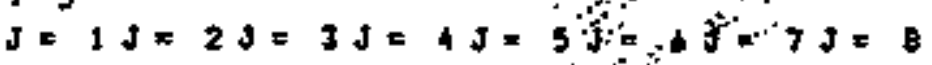

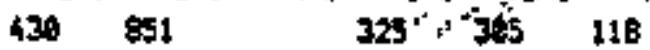

$236231 \quad+306249 \quad 239$

$485465 \quad 363214 \quad 26$

375664552

$373514 \quad 926$

$974 \quad 682924$

215 65s 1529

$10 \quad 1136 \quad 914$

391 1830

$11 \quad 1294$

DATA FOR ROW : 6

$$
\text { DEPTH }
$$

0.0- 2.5

$2.5=3.6$

$5.0=.7 .5$

$7.5-18.6$

$10.0-12.5$

$12.3-35.6$

15. $0=17.5$

$17.5=29.0$

$20.8-20.5$

22.5 - 25.9

$25.8-27.5$

$27.5=38.6$

39. $\mathrm{B}-32.5$

$32.5-35.0$

$75.9-37.5$

$37.5=49.1$

$48.6-43.5$

$42.5 * 45.0$

$45.0-47.5$

$$
J=1 J a 2 J \in 3 J=4 J=3 J=6 J=T J=B
$$

$\begin{array}{rrr}719 & 17 ! & 128 \\ 296 & 107 & 137 \\ 373 & 190 & 945 \\ 263 & 533 & 1353 \\ 133 & 596 & 1365 \\ 582 & 711 & 547 \\ 113 & 081 & \\ & 412 & \end{array}$


PAEE NO. 4

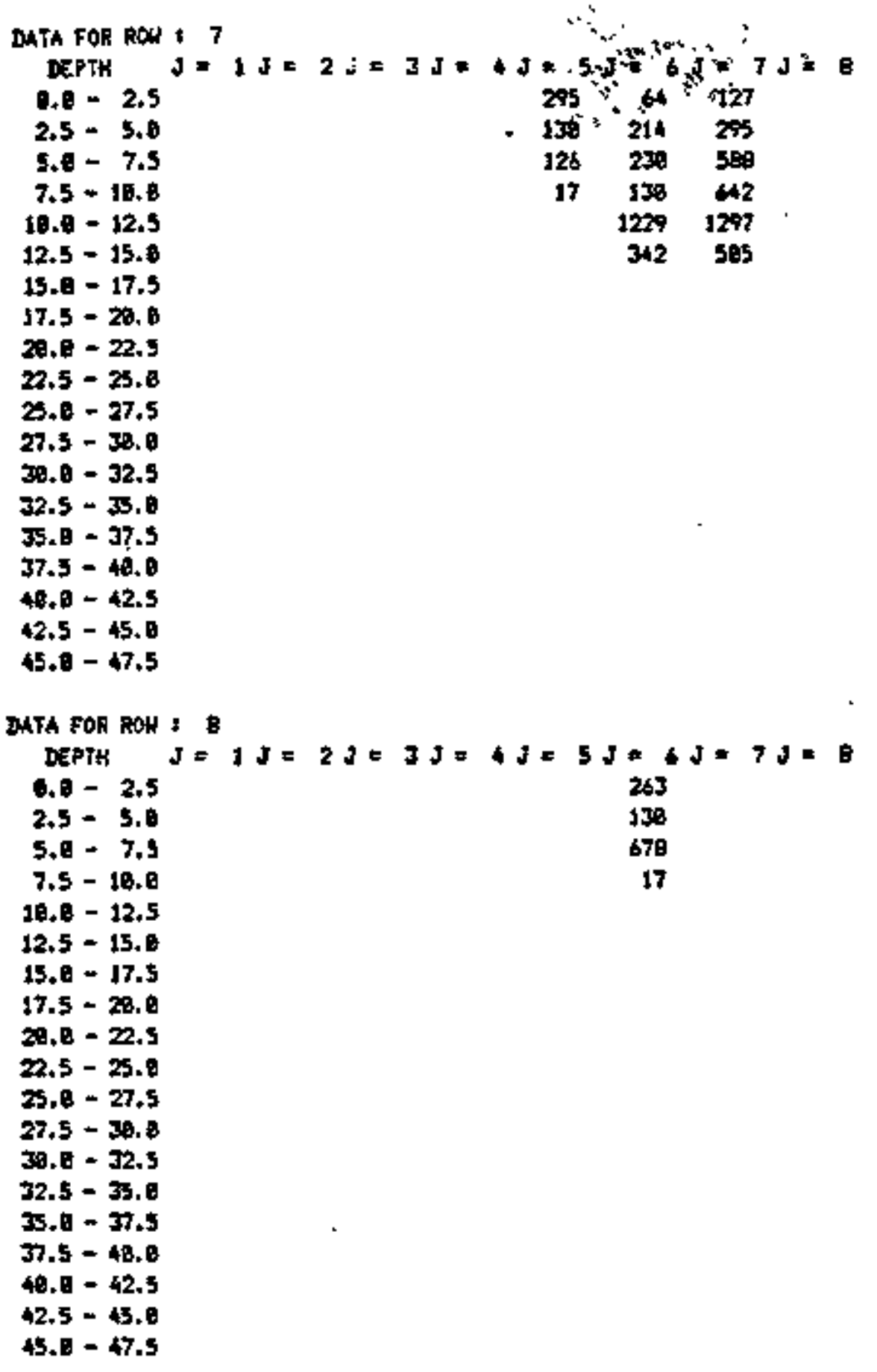


PAEE HO. 5

\section{DEPTKS TO 15 oCi/g INTEFFADE TABLE}

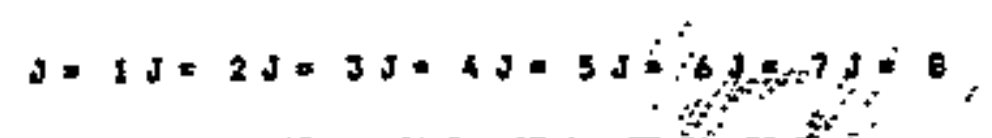

$1=1$

$1 * 2$

$1=3$

I $=4$

$1 * 5$

$I=6$

$1 \cdot 7$

I $\times$ B

$23.424 .6 \quad 23.1 \quad 3.1035 .5$

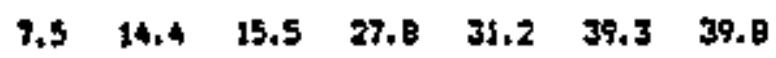

$\begin{array}{lllllll}0.9 & 13.7 & 17.5 & 18.3 & 29.5 & 55.6 & 25.6\end{array}$

10.0 $90.0 \quad 22.020 .6$ 31.日 36.8

6.96 .8

$19.8 \quad 24.8 \quad 25.0$

16.519 .514 .0

8.8 $13.6 \quad 14.2$

9.1 
DEPTHS TO MYSTCAL INTERFACE TABLE

\begin{tabular}{|c|c|c|c|c|c|c|c|c|}
\hline$I=$ & 1 & & 21.6 & 23.0 & 21.3 & 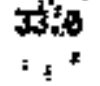 & $4 b_{2}$ & \\
\hline$I=$ & 2 & 5.5 & 12.6 & 25.0 & 26.0 & 29.4 & 37.5 & $39,0^{\circ}$ \\
\hline$I=$ & 3 & 7.1 & 12.2 & 15.7 & 16.5 & 27.7 & 33. & 25.6 \\
\hline$I=$ & 4 & 9.8 & B.5 & & 19.0 & 27.8 & 30.8 & 35.0 \\
\hline$I=$ & 5 & 5. 5 & 3.9 & & 17.8 & 22.0 & 24.0 & \\
\hline$I=$ & 6 & & & & 35.1 & 17.6 & 32.5 & \\
\hline$I=$ & 7 & & & & 7.0 & 12.0 & 12.4 & \\
\hline & 日 & & & & & 7,3 & & \\
\hline
\end{tabular}


DEPTHS FROK PHYSICAL TO 15 Pfi/S IMTERFAEE TARLE

$$
s=J=2 J \cdot 3 J \cdot 4 J \cdot 5 J=0 J=7 J=8
$$

\begin{tabular}{|c|c|c|c|c|c|c|}
\hline $1=2$ & 2.6 & 1.8 & e. 5 & 1.8 & I.e & 1.0 \\
\hline $1=3$ & 1.8 & 1.5 & 1.0 & 1.8 & 1.8 & 2.0 \\
\hline I. $=4$ & 萡 & 1.5 & & 3.0 & 1.0 & 1.8 \\
\hline$I=5$ & 1.8 & 2.1 & & 2.0 & 2.8 & 1.8 \\
\hline $1=6$ & & & & 1,8 & 1.9 & 1.5 \\
\hline $1=7$ & & & & 1.8 & 1.8 & 1.8 \\
\hline$I=\theta$ & & & & & J.8 & \\
\hline
\end{tabular}

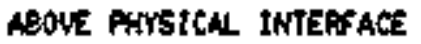

$7635=0$

2. $96167 E+87$

19.4556

561.697

AVEAAE ACTIVITY (pCi/9) $641.322 \quad 623.984$

ABOVE I5 pCI $/ 9$ INTEFFACE

B34528

$2.25323 x+67$

21.2633

577.203

AVEAAE ACTIVITY (pCi/9) $641.322 \quad 623.984$

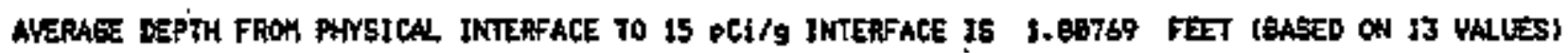

AVERAGE PJLE ACTIVITY PAOFILE

\begin{tabular}{|c|c|c|c|c|c|c|}
\hline \multirow{2}{*}{$\begin{array}{l}\text { DEFTH } \\
\text { (F;) }\end{array}$} & \multicolumn{2}{|c|}{ ABOVE PHKSICAL INTERTAEE } & \multicolumn{2}{|c|}{ NOOVE $15 \mathrm{pti} / \mathrm{g}$ INTERFACE } & \multicolumn{2}{|c|}{ TOTAL PILE } \\
\hline & vount & ACTIVITY & VocurE & Activity & WeclunE & ACTIVITY \\
\hline $\begin{array}{l}6.0-2.5 \\
2.5-5.6\end{array}$ & $\begin{array}{l}98,119 \\
96,952\end{array}$ & $\begin{array}{l}430 \\
479\end{array}$ & $\begin{array}{l}79.159 \\
90.159\end{array}$ & $\begin{array}{r}430 \\
476\end{array}$ & $\begin{array}{l}5,5119 \\
98,519\end{array}$ & $\begin{array}{l}430 \\
476\end{array}$ \\
\hline $5.6-7.5$ & 89.633 & 583 & 951099 & 487 & 90.119 & 485 \\
\hline $7.5-18.0$ & 79,556 & 712 & 6b, 7es & 665 & 90.119 & 392 \\
\hline $16.8-12.5$ & 73,696 & 719 & 77,760 & 710 & 98.115 & 576 \\
\hline $12.5-15.0$ & 63,751 & 720 & 71,678 & 697 & 98,119 & $\$ 23$ \\
\hline $15.8-17.5$ & $\$ 4,952$ & 775 & - 69,949 & 716 & 98,119 & 43 \\
\hline $17.5-28.8$ & 46,779 & 792 & 33,151 & 715 & 98.159 & AB7 \\
\hline $20.0-22.5$ & 42,324 & $77 \mathrm{~B}$ & 47,203 & 756 & 99,117 & 373 \\
\hline $22.5-25.0$ & 331944 & 869 & 40,520 & 837 & 99.119 & 372 \\
\hline $25,0-27.5$ & 271049 & 720 & 91,949 & 694 & 99,119 & 244 \\
\hline $27.5-38.0$ & 201791 & 577 & - & 594 & 96.119 & 184 \\
\hline $30.0-32.5$ & 15,511 & 503 & 19,118 & $\$ 12$ & 96,119 & 127 \\
\hline $32,5=35,0$ & 12,1052 & 669 & 15,911 & 693 & 98,519 & 137 \\
\hline $35,8-37.5$ & 5,504 & 1191 & E.3P6 & 670 & 961119 & 118 \\
\hline $37.5-40.0$ & 530 & 776 & $4+365$ & 284 & 78,119 & 52 \\
\hline $49.0=42.5$ & o & e & a & 酎 & 78,119 & 15 \\
\hline $42.5-45.0$ & - & D & 0 & o & 99,119 & 15 \\
\hline
\end{tabular}




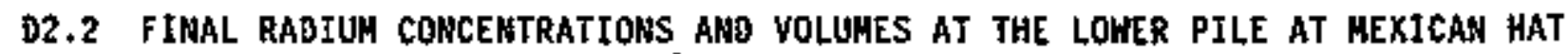

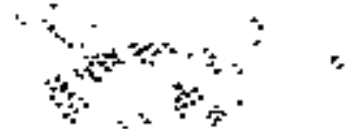

Pages 02-10 through 02-17 of , this addendum present the contentrations of each grid cell by roH, column, and layer. Each page contains one row and all columens and layers for that row. The jayer by layer concentrations are priated down the page, while the columns are organtzed across the page. Pages 02-18 and 02-19 give the depths to the $15 \mathrm{pct} / \mathrm{g}$ and phystcal interfaces, respectively. In these tables, the values are organized according to row and colutun. Page D2-20 presents the volume summary and the layer by layer averages with depth into the ptle. 
IEXJCAN HAT (LONER PILE) - HTL - WLFO \& SML DATA

EILES; HFLOO1/DAT - ENTERED S/22/OS IRANZLEON?

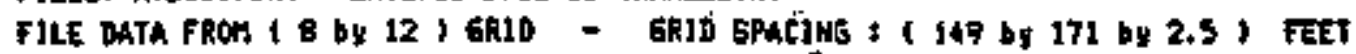

PAGE No. 1

DATA FOR RON : I

DEPTH.

$0.0-2.5$
$2.5-5.8$
$5.0-7.5$
$7.5-12.6$
$10.8-12.5$

$\$ 2.5=15.0$

$15.6-17.5$

$17.5-20.8$

$29.0-22.5$

$22.5=25.0$

$25.0-27.3$

$27.5=30.0$

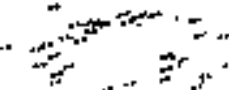

$30.0-32.5$

$32.5-35,0$

$35.0-37.5$

$\mathrm{J}=1 \mathrm{~J}=2 \mathrm{~J}=3 \mathrm{~J}=4 \mathrm{~d} * 5+5+\mathrm{J}^{2} \mathrm{~J}=7 \mathrm{~J}=0 \mathrm{~J}=8 \mathrm{~J}=10 \mathrm{~J}=11 \mathrm{~J}=12{ }^{\circ}$

$37.5=48.0$

263 668 $298 \quad 279 \quad 314 \quad 234$

$\begin{array}{llllll}671 & 482 & 277 & 252 & 932 & 213\end{array}$

$\begin{array}{llllll}269 & 263 & 218 & 261 & 265 & 298\end{array}$

$\begin{array}{llllll}379 & 671 & 278 & 278 & 379 & 244\end{array}$

$40.0-42.5$

$42.5=45,0$

$45.0-47.5$

$47.5-5 B, B$

$50.2-52.5$

$52.5-55.0$

$55.0-57.5$

$57.5=\$ 0.0$

$60.0-62.5$

$483 \quad 269 \quad 389 \quad 288 \quad 278$

$699 \quad 37 \% \quad 298 \quad 290194$

42

$704 \quad 323 \quad 314 \quad 314 \quad 260$

$1235 \quad 416 \quad 932 \quad 932 \quad 203$

163

$503 \quad 269 \quad 269 \quad 23$

$745 \quad 379 \quad 379$

242 264

223204

$221 \quad 247$

$254 \quad 233$

9 10 
DATA FOR RON : 2

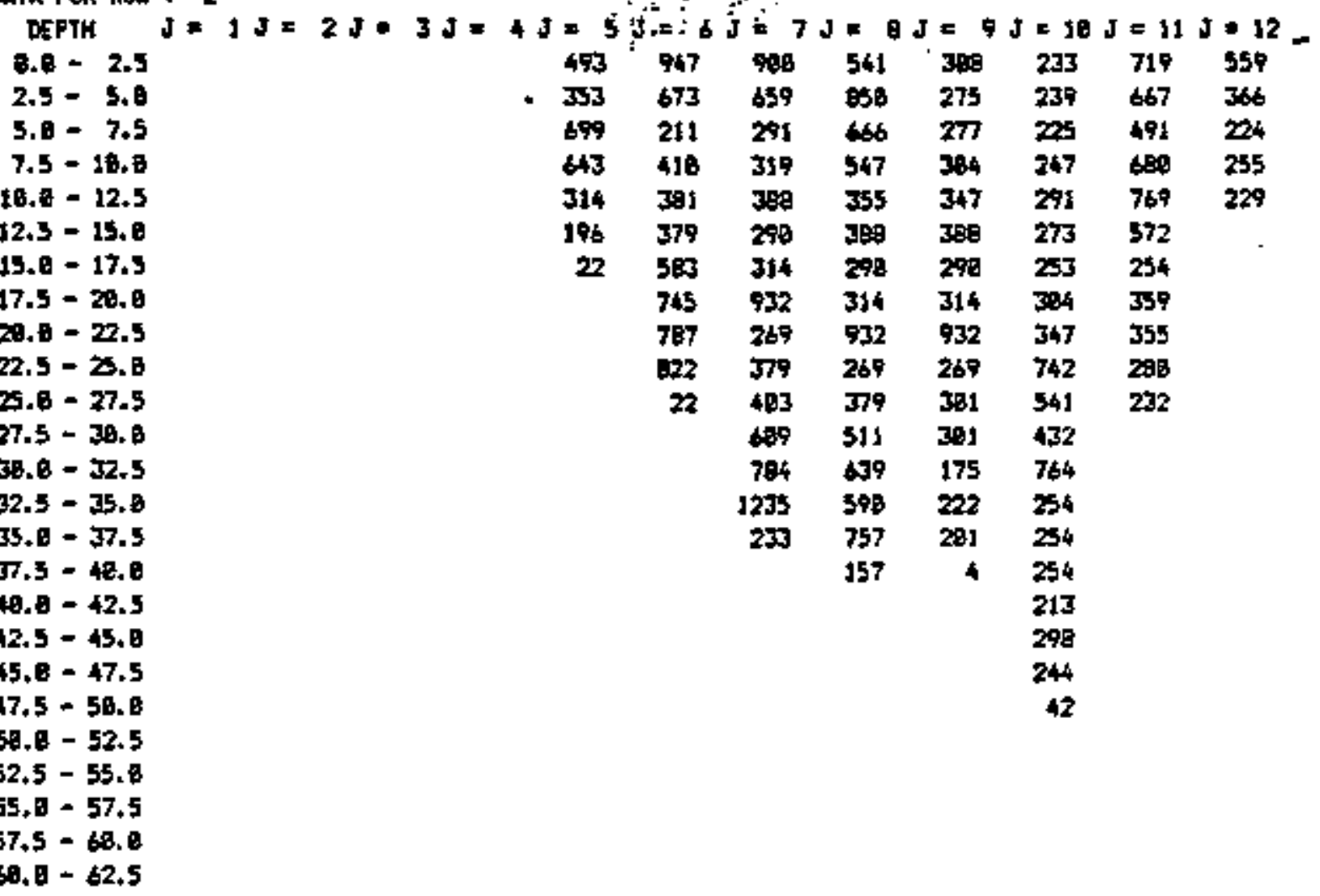




$$
\because \because \quad \therefore \text { FAEE NO. } 3
$$

AHTA FOR AOH : 3

\begin{tabular}{|c|c|c|c|c|c|c|c|c|c|c|}
\hline $0.0-2.5$ & b)1 & 763 & 762 & 1689 & 1926 & 1255 & 893 & Tes & $29 s$ & 571 \\
\hline $2.5=5.0$ & $\triangle B A$ & $443=$ & 977 & 505 & 1339 & 774 & $\Delta 63$ & 748 & 276 & 290 \\
\hline $3.0-7.5$ & 528 & 326 & 922 & 469 & 1329 & 668 & 460 & 679 & 274 & 203 \\
\hline $7.5=18.0$ & 232 & $18 p 9$ & 493 & 392 & 407 & 830 & $13: 2$ & 1061 & 270 & 350 \\
\hline $8.0=12.5$ & 523 & 1128 & 326 & 658 & 833 & 995. & 357 & 543 & 228 & 212 \\
\hline $2.5=15.6$ & & 90 & 582 & 767 & 530 & 416 & 599 & 397 & 228 & 245 \\
\hline $5.0-17,5$ & & $6=2$ & 1049 & $38 t$ & 263 & 398 & 746 & 490 & 367 & 219 \\
\hline $5-28.0$ & & 22 & 354 & 379 & 671 & 290 & 627 & 313 & 719 & \\
\hline $0-2.5$ & & & 196 & 679 & 269 & 314 & 647 & 233 & 667 & \\
\hline-28.0 & & & 22 & 643 & $\$ 79$ & 932 & 765 & 304 & 491 & \\
\hline $0-27.5$ & & & & 314 & 762 & 269 & 448 & 420 & 690 & \\
\hline $5-30.0$ & & & & 196 & $\Leftrightarrow$ & 379 & 416 & 719 & 769 & \\
\hline$\theta=32.5$ & & & & 22 & 1262 & 670 & 742 & 67 & 972 & \\
\hline$s=33.8$ & & & & & 1636 & 179 & S41 & 491 & 559 & \\
\hline $0-37.5$ & & & & & 457 & 71 & 432 & 680 & 366 & \\
\hline $.3=49.6$ & & & & & & 1414 & 764 & 769 & 226 & \\
\hline$\theta=42,5$ & & & & & & 457 & 682 & $\$ 72$ & 255 & \\
\hline $5=45.0$ & & & & & & & 849 & 765 & 229 & \\
\hline $0-47.5$ & & & & & & & 849 & 585 & & \\
\hline$-5 b_{1} 0$ & & & & & & & 649 & 542 & & \\
\hline-52.5 & & - & & & & & 286 & 31 & & \\
\hline$=55.0$ & & & & & & & 399 & 45 & & \\
\hline $.8-57.5$ & & & & & & & 232 & & & \\
\hline
\end{tabular}

57.5 - 69.8

$40.5-62.5$ 
PA6E nO.

DATA FOR FOW : 4

DEFTH

B. 0.2 .5460

$2,5-5.0 \quad 413$

$5.8-7.5123$

$7.5-10.0$

$10.8-12.5$

$12.5-15.6$

$15.0-17.5$

$17.5=28.6$

$20.1=2.5$

$22.5=25.0$

$25.0=27.5$

$27.5=38.0$

$37.0-32.5$

$32.5-35.8$

$75.8 \times 37.5$

$37.5=4 B . B$

$40.6-42.5$

$42.5-45.0$

$45.6-47.5$

$47.5-5 B_{4} 0$

$58.8-52.5$

$52.5-55.8$

$53.0-57.5$

$57.5-60.0$

$64.9=62.5$ $\therefore$

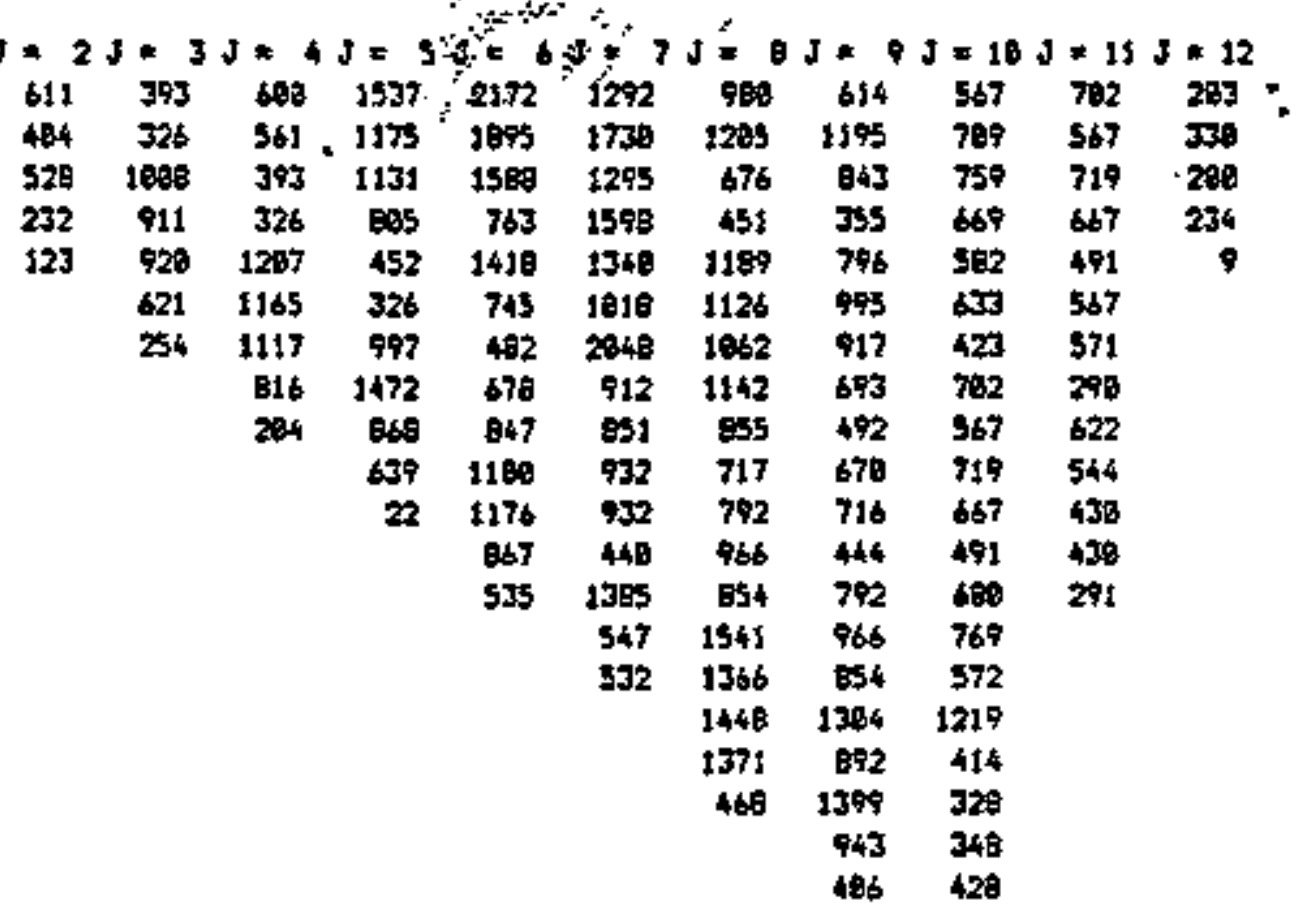


PASE NO. 5

DATA FOR RON : 5

\begin{tabular}{|c|c|c|c|c|c|c|c|c|c|c|c|}
\hline $6.8-2.5$ & 461 & I416 & 553 & 1261 & ís' & 1284 & 812 & 792 & 617 & 768 & 332 \\
\hline $.5-5.0$ & 123 & 1164 & • לים & 1317 & 2000 & 1631 & 619 & 966 & 782 & b) & 275 \\
\hline $0-7.5$ & & 1366 & 1166 & 509 & 1898 & 1625 & 1174 & $6 \$ 4$ & 567 & 339 & 259 \\
\hline $7.5=18.0$ & & 1263 & E\$7 & 471 & 2049 & 1142 & 1831 & 851 & 670 & 567 & $\mathbf{5 B}$ \\
\hline $0 . t=12.5$ & & 385 & 369 & 553 & 1031 & 1260 & $7 \mathrm{Bs}$ & 596 & 716 & 571 & B \\
\hline $2.5-15.6$ & & & 197 & 1365 & 987 & 1341 & 1712 & 1125 & 144 & 290 & \\
\hline $15.0-17.5$ & & & & 1456 & 627 & 1149 & 1142 & 1588 & $\$ 6$ & 732 & \\
\hline $7.5-20.0$ & & & & $\$ 264$ & $11+7$ & $1: 17$ & 340 & 273 & 747 & $m$ & \\
\hline $0.0-22.5$ & & & & 950 & 1778 & 93 & 633 & & 55s & 420 & \\
\hline $2.5-25.6$ & & & & 13 & 1424 & 1224 & 971 & & $\$ 13$ & 454 & ' \\
\hline $5.0-27.5$ & & & & & 1351 & 1303 & 1857 & & 1449 & 266 & \\
\hline $7.5-30.0$ & & & & & 531 & $\$ 493$ & 863 & & 5824 & & \\
\hline $0.0-32.5$ & & & & & & 1285 & 1369 & & 1141 & & \\
\hline $2.5-35.6$ & & & & & & $104 B$ & 14B\} & & 792 & & \\
\hline $5.0-37.5$ & & & & & & & 1270 & & & & \\
\hline$-40 . B$ & & & & & & & 1187 & & & & \\
\hline $0.0-42.5$ & & & & & & & 90 & & & & \\
\hline
\end{tabular}

$42.5=45.8$

$45.0-47.5$

$47.5-50.0$

$50.0-52.5$

$52.5-55.0$

$35.8-57.5$

57.5 - 60.8

6e. $6=-62.9$ 
PAEE HO. 6

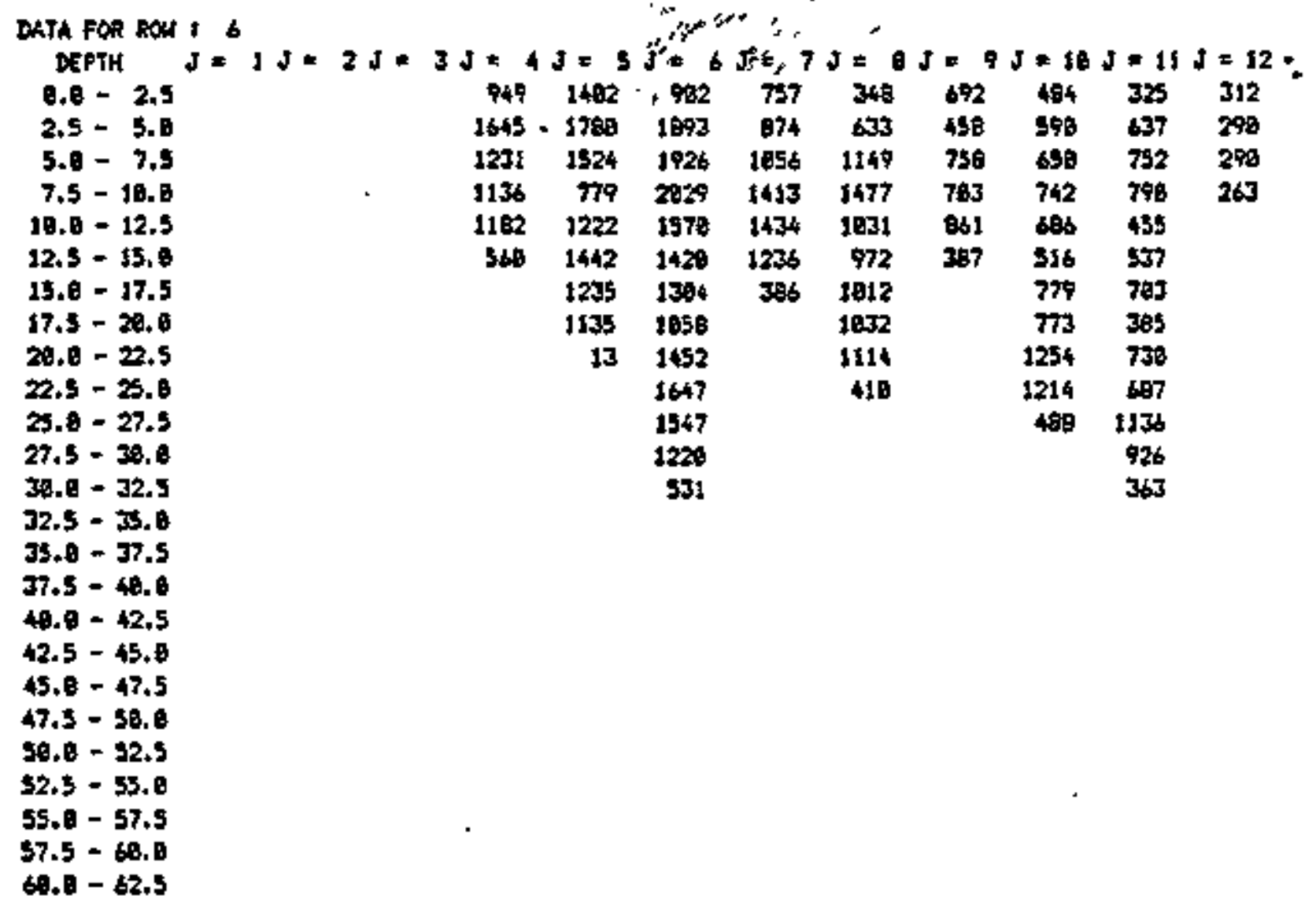




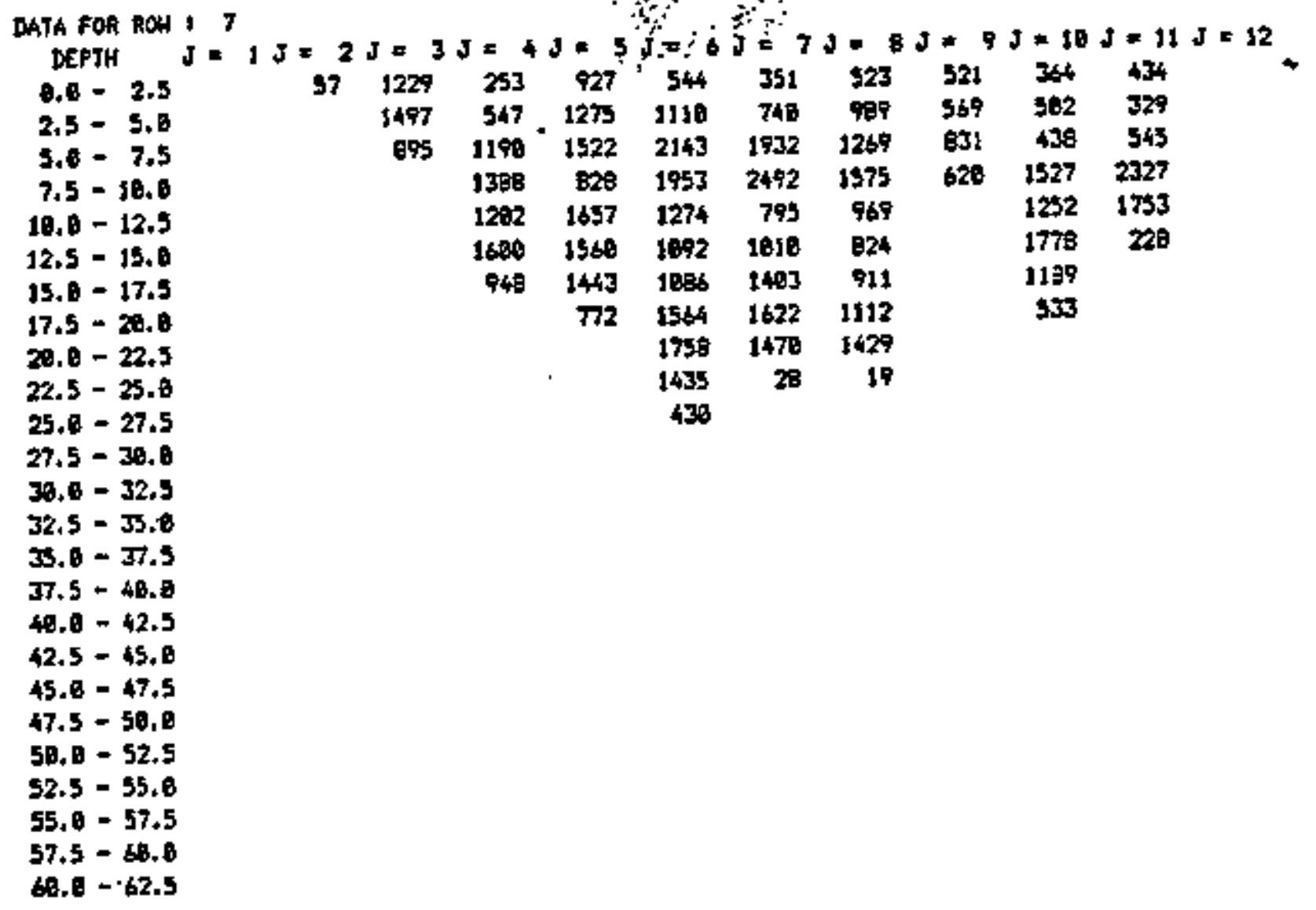

PAEE ND. T

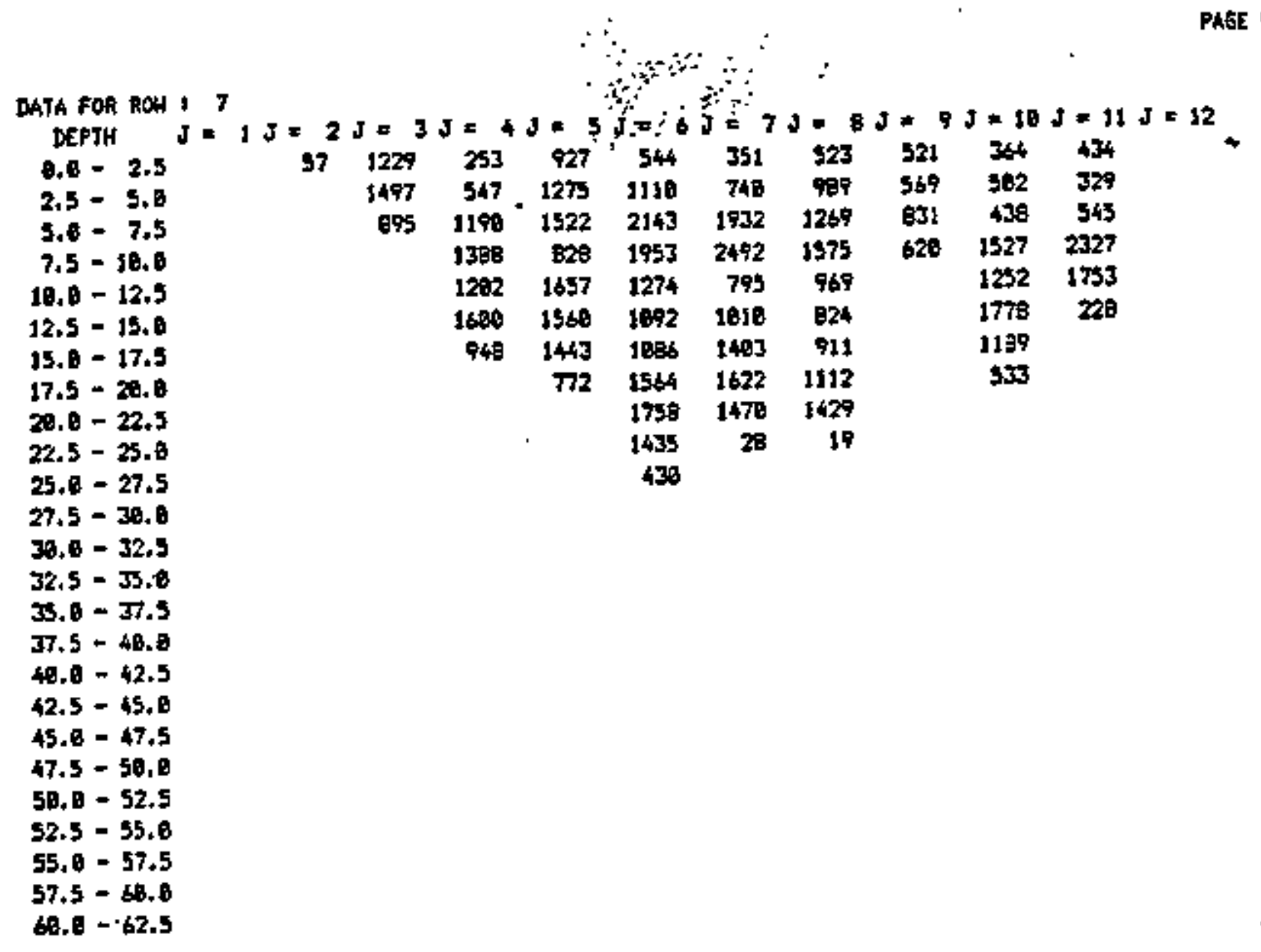


PASE WO. 5

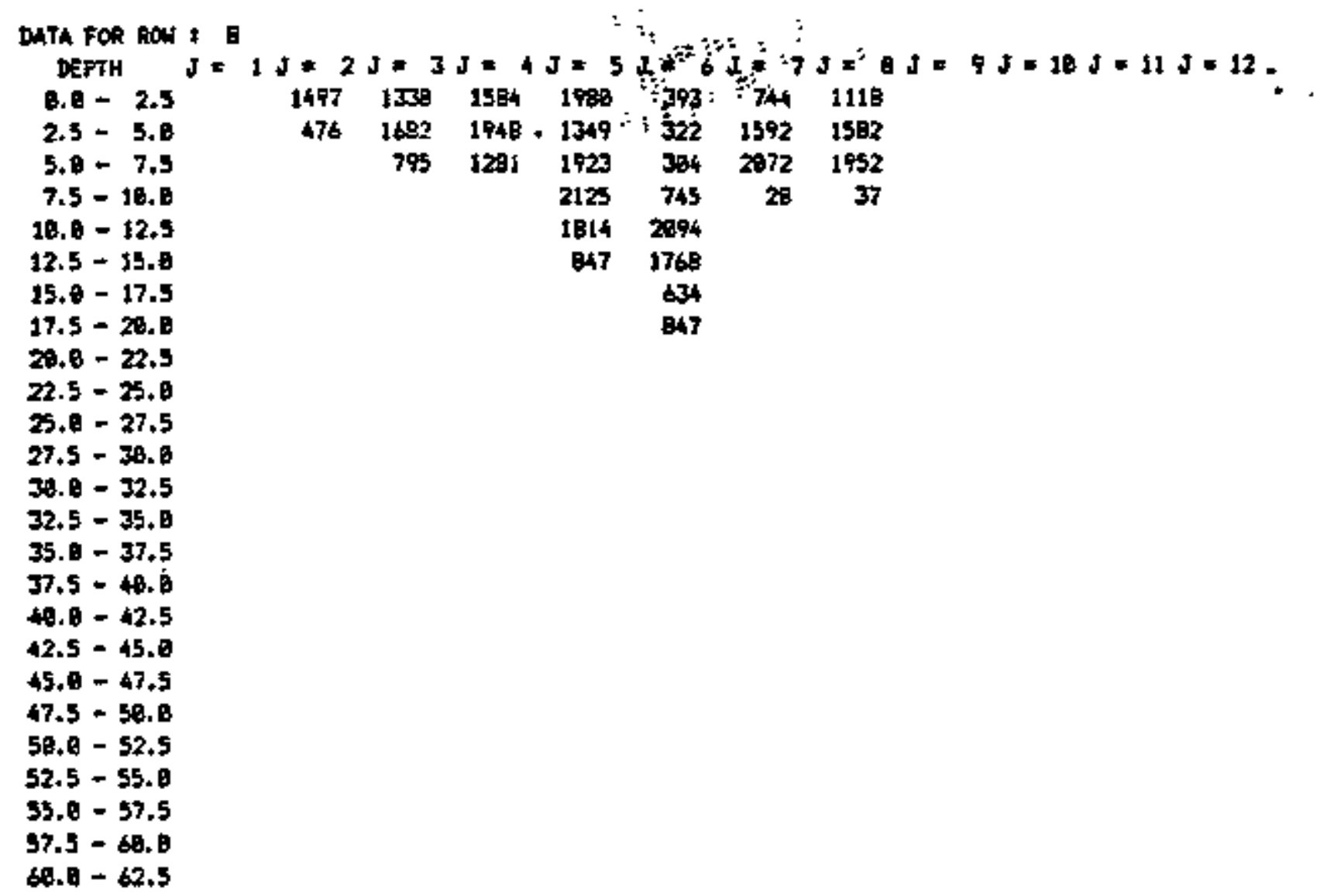


PASE W. $\%$

DEPTHS TO 15 DCI/S INTERFACE TABLE

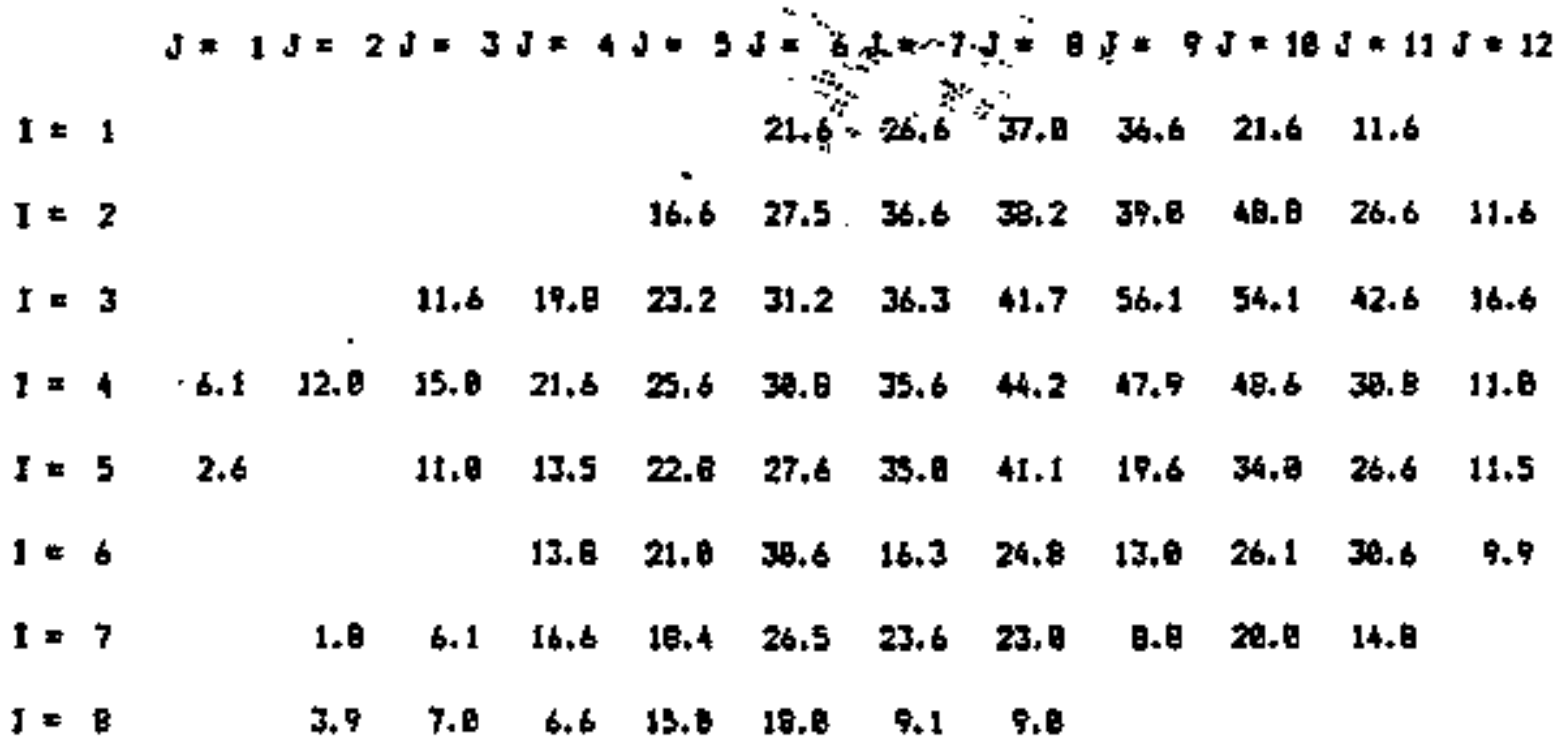

$\begin{array}{llllllllll}1.8 & 6.1 & 16.6 & 18.4 & 26.5 & 23.6 & 23.8 & 8.8 & 26.8 & 14.8\end{array}$$$
J=E
$$

$\begin{array}{lllllll}3.9 & 7.0 & 6.6 & 18.6 & 18.0 & 9.1 & 9.8\end{array}$

$02-18$ 
DEPTHE TO PAYSICOL INTERFACE THBLE

$$
J=J \mathrm{~J}=2 \mathrm{~J}=3 \mathrm{~J}=4 \mathrm{~J}=5 \mathrm{~J}=\mathrm{J}=7 \mathrm{~J}=0 \mathrm{~J}=0 \mathrm{~J}=18 \mathrm{~J}=1 \mathrm{~J}=12
$$

\begin{tabular}{|c|c|c|c|c|c|c|c|c|c|c|c|c|}
\hline$I=1$ & & & & & & 20 & 20 & 35.8 & 35.0 & 20.0 & 12.0 & \\
\hline$I=2$ & & & & & 15.0 & 25.9 & 35.0 & 36.6 & 37.8 & 47.0 & 25.0 & 18. $\theta$ \\
\hline$\pm=3$ & & & 10.0 & 16.3 & 22.0 & 27.6 & 34.7 & 48.1 & 54.5 & 52.5 & 42,0 & J5. \\
\hline $1=4$ & 4.5 & 31.6 & 13.9 & 20.0 & 24.0 & 29.2 & 34.0 & 42.6 & 46.3 & 47,0 & 29.2 & 20.0 \\
\hline $1=5$ & 1.0 & & 9.4 & 11.5 & 21.2 & 26.0 & 33.4 & 39.5 & 18.0 & 32.4 & 25.1 & SB.7 \\
\hline$I=6$ & & & & $\$ 2.2$ & 19,3 & 29.0 & 14.7 & 23.2 & 11.4 & 24.5 & 29.8 & 9.3 \\
\hline$I=7$ & & D. 2 & 4.5 & $\$ 5.9$ & 16.8 & 25.8 & 20.0 & 22.2 & 7.2 & 16.4 & 13.2 & \\
\hline $5 \propto 9$ & & 2.4 & 5.4 & 5.0 & 13.0 & 13.5 & 7.3 & 7.8 & & & & \\
\hline
\end{tabular}




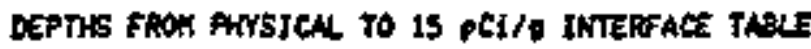

$$
J=1 J=2 J=J J=4 J=5 J * 6 J=7 J \cdot B J=9 J=18 J=11 J=12
$$

1 a I

$I=2$

$$
I=\mathbf{3}
$$

$1=4.6$

$I+5 \cdot 1.6$

】 6

$\mathbf{I}=\boldsymbol{7}$

$\mathbf{I}=\mathbf{\theta}$

B. 4

$1.61 .6 \quad 1.2$

:

$1.6 .54 .62,2.6,1.6 \quad 1.6 \quad 1.6$

裙,

$1.6 \quad 1.6 \div 1.6 \quad 1.6 \quad 2.8 \quad 1.8 \quad 1.6 \quad 1.8$

$\begin{array}{llllllll}1.2 & 1.6 & 1.6 & 1.6 & 1.6 & 1.6 & 1.6 & 1.6\end{array}$

$\begin{array}{llllllllllll}1.6 & 1.6 & 1.6 & 1.6 & 1.6 & 2.6 & 1.6 & 1.6 & 1.6 & 1.6\end{array}$

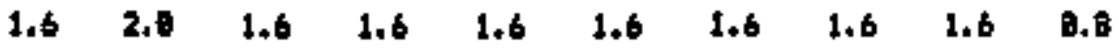

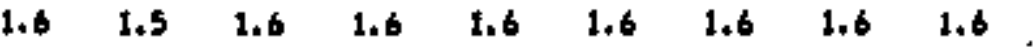

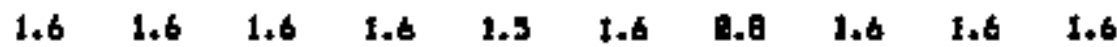

$\begin{array}{lllllll}1.6 & 5.6 & 9.6 & 2.6 & 2.9 & 1.6 & 2.0\end{array}$

AEOVE PHFSICAL INTERTACE

$1.51524 E+B S$

4. BP116E+07

21.5957

$\$ 366.29$

786.129
ABOVE 15 oCifo INTEAFACE

1.62JE+b6

4. $39211 E+07$

23.5601

1420.75

763.384

TOTHL ACTIVITY (Cj)
AVERAEE ACTIVITY (PCI/g)

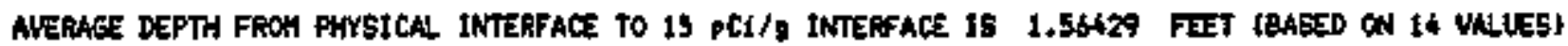

\begin{tabular}{|c|c|c|c|c|c|c|}
\hline \multirow{2}{*}{$\begin{array}{l}\text { REPTH } \\
\text { (FT) }\end{array}$} & \multicolumn{2}{|c|}{ AEOVE PAYSICAL INTERFACE } & \multicolumn{2}{|c|}{ ABOVE 15 \& $\mathrm{C} / \mathrm{g}$ JNTERFACE } & \multicolumn{2}{|c|}{ TOTAL PILE } \\
\hline & VOLUNE & ACTIVITY & WXuE & ACTIVITY & vocure & Attivitr \\
\hline $8.0-2.5$ & 168,492 & $7 \mathrm{Bg}$ & 171,525 & 780 & 572,259 & 777 \\
\hline $2.5=5.8$ & 164,190 & B66 & 166,337 & 9s7 & 172,219 & B37 \\
\hline $5.0-7.5$ & $\$ 55,337$ & B3s & 161,053 & Bas & 172.219 & Bes \\
\hline $7.5-10.8$ & 146,457 & 837 & $252,5 B 4$ & E1b & 172,219 & 729 \\
\hline $10.8-12.5$ & 129,660 & 898 & 539.139 & 78 & 172,219 & 631 \\
\hline $12.5-15.8$ & 117,345 & 795 & 525.629 & 771 & 172,219 & 573 \\
\hline $15.9-17.5$ & 103,237 & 764 & 511,799 & 764 & 172,719 & 512 \\
\hline $17.5-20.0$ & 95,877 & 751 & - $I 83,844$ & $7 \times 3$ & 572.219 & 457 \\
\hline $29.0-22.3$ & 92,476 & 753 & 90,392 & 721 & 172.259 & 청 \\
\hline $20.5-25.0$ & 721379 & 737 & 77,657 & 713 & 172,259 & 30 \\
\hline $25.8-27.5$ & 561 as4 & 796 & 66.326 & 654 & 172,215 & 270 \\
\hline $27.5=38.0$ & $50,40 \alpha$ & 671 & 34,321 & 643 & 172,219 & 200 \\
\hline $30.0-32.5$ & 42,371 & 799 & 46.271 & 761 & 172,219 & 231 \\
\hline $32.5-35.8$ & 37,369 & 761 & 45,454 & 769 & 172.219 & 201 \\
\hline $35.8-37.5$ & $24,63 t$ & 691 & 32,516 & $5 B 3$ & 172,219 & 132 \\
\hline $37.5-40.0$ & 201761 & 946 & 23.275 & B72 & 172.219 & 132 \\
\hline $48.0-42.5$ & 15,181 & 650 & 19,077 & 576 & 172.219 & 60 \\
\hline $42.5=43.6$ & 11,878 & 730 & 33,427 & 679 & 572,219 & 73 \\
\hline $45,0-47,5$ & 9,720 & $\$ 63$ & 11.776 & 576 & 172.259 & 57 \\
\hline $47.5=30.0$ & 4,718 & 996 & 7,293 & 470 & 172.219 & $4 \hat{2}$ \\
\hline $58.0-32.5$ & 4,718 & 311 & 4.718 & 311 & 172.219 & 23 \\
\hline
\end{tabular}

AVIRAEE PILE ACTJUITY PROFILE 


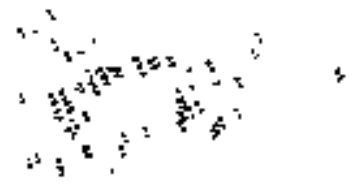

ADOENDUN D3

GEDLOGIC DATA 
Table

$$
\therefore \text { CONTENTS }
$$

Page

D3.1 Faults in the Mexican Hat site region, source, method of study, and comments................................ 03-1

03.2 NGQC earthquake data file, 200-km radius of Hextcan Hat, Utah. - 03-3

03.3 Field notes for ground reconnatssance. . . . . . . . 03-5 
Table 03.1 Faults in the Hexican Hat.site region, source, method of study, and comments

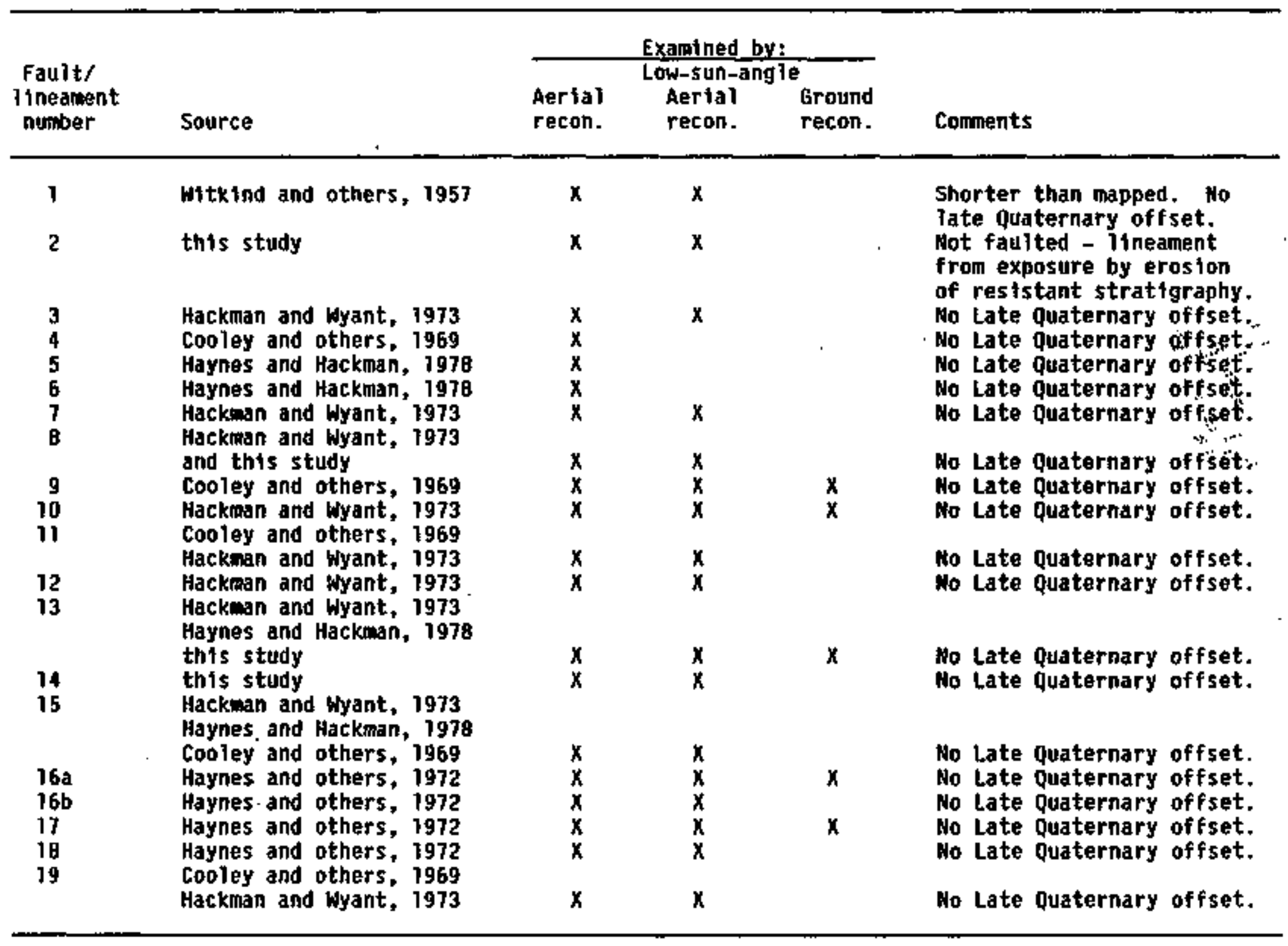


Table 03.1 Faults in the Mextcan Hat site regton, source, method of study, and comrents (Conctuded)

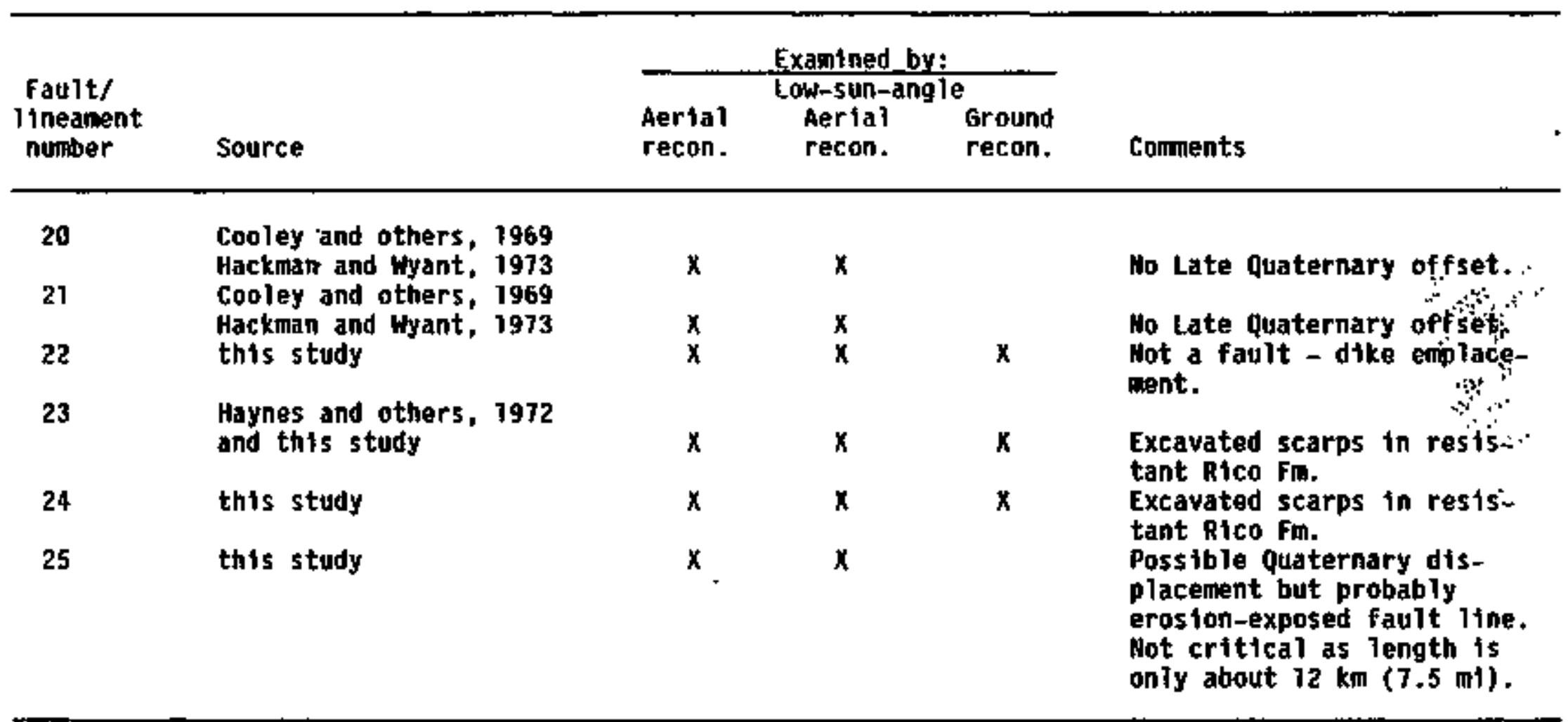


Table 03.2

-aul r. thtth

jeitent, nauskins t SECKuITH

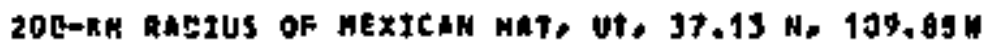

NGOC EARTHQUAKE DATA FILE

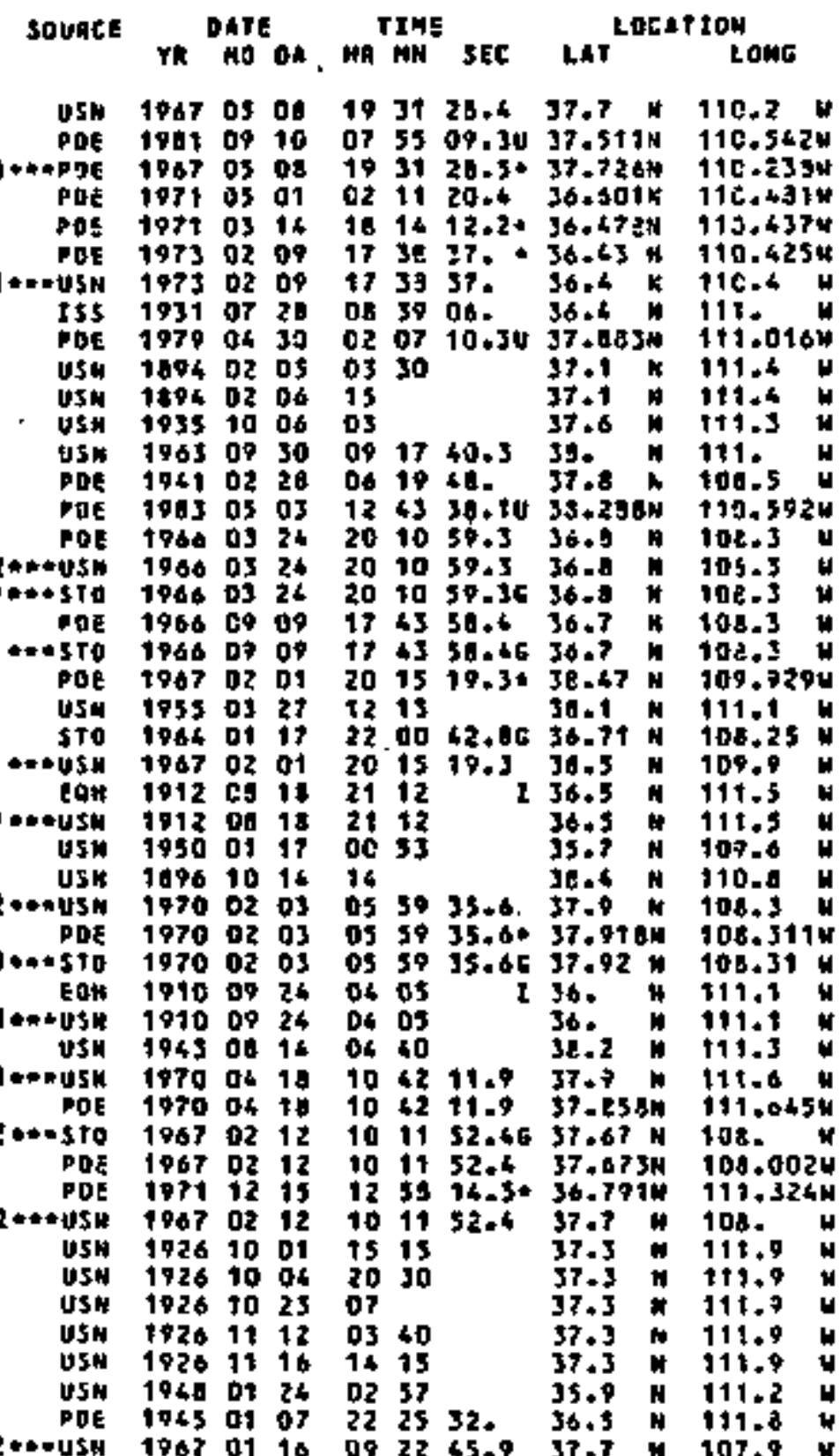

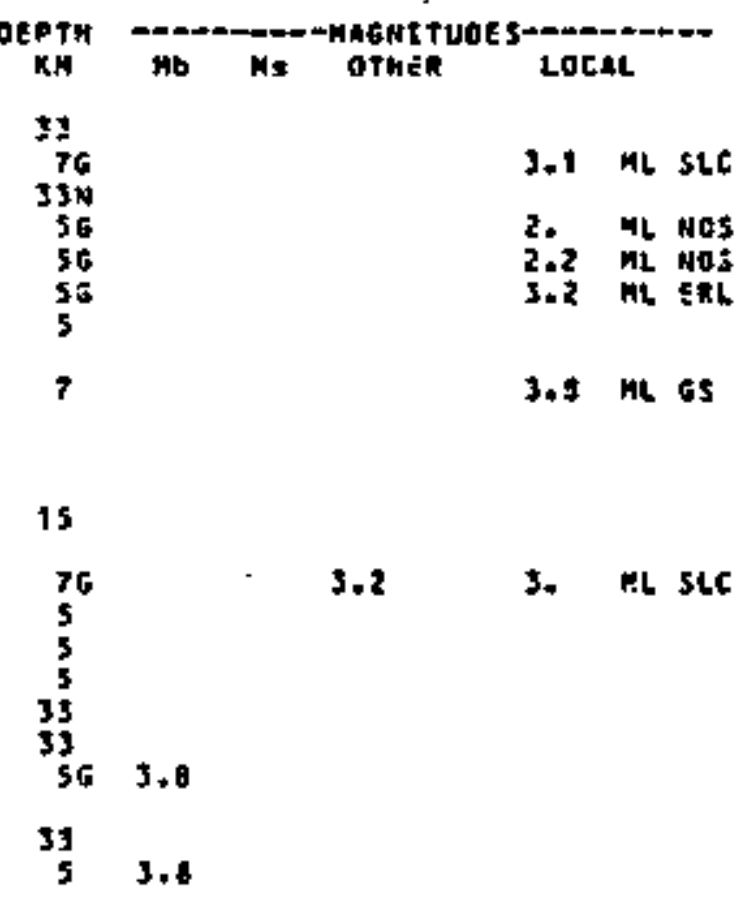

$334:$

334.

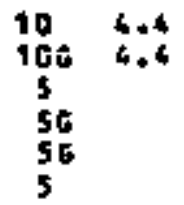

3. HL ERC. of to is

$0 \div \div 1 \div 50$

Int

max ETSUNO

P-s ce dra otstance

$\begin{array}{ll} & \\ & \\ \text { IV } & \\ \text { It: } & \end{array}$

4760

$\begin{array}{rrr}479 & 7 & 67 \\ 478 & -3 & 73\end{array}$

605

495

495

40

$48 \mathrm{~F} 2131$

470 $c$ 115

$47 \mathrm{a}$

478

470

479

479
478
696

406

496 है

$196 \cdots$. 3

$476 \div 80$

ty

It

III

II

478.

$400^{\circ}$

$670+0,1$

695010.1

495

495

47

479

in.

5

679

479
$695 \%$

v1

$t^{5}$

495

470

679

470

479

479

479

478.

473

475

$\geq 1$

11 :

5.1 Pls 
HGJC EARTHQuAXE Data pile

SOUACE

ta note

46? 0110 as 20

1967 o1 16

1965 os 10

19670904

$196709 \mathrm{DH}$

1841 of 04
1441 Do 29

19420623

$\begin{array}{lll}1477 & 05 & 05 \\ 1977 & 03 & 05\end{array}$

1976 ol os

1955 12 os

1976 01 05

1976 05 20

PDE
TINE

SEE

Id.3 4911.6 บ

$072245.9637 .67 \mathrm{~N} 107.06 \mathrm{~W}$

$092245.937 .672 \mathrm{~N} 107.06 \mathrm{~W}$

22511.9

32744.736 .2 i 111.7

1. 30

$13400.3 \%$

711 06. 67.3 127.7

D3 0354.7035 .91 M 105.27

030056.73 .9154 1C8.2064

06 2332.935 .0446103 .3644

21 is $37.4 \mathrm{H}$

462332,96 35.94 N

$1 \% 4321.96 \quad 35.47 \mathrm{~N}$

112.1

108. $\$ 4$

$109.04 \%$

194321.9635 .4761

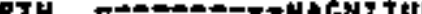

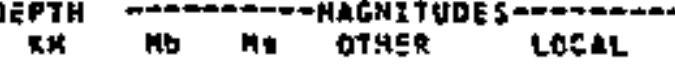

Ixt

334.1

3

3
3

2324.6

$22 \quad 4.6$

256 5.

255.

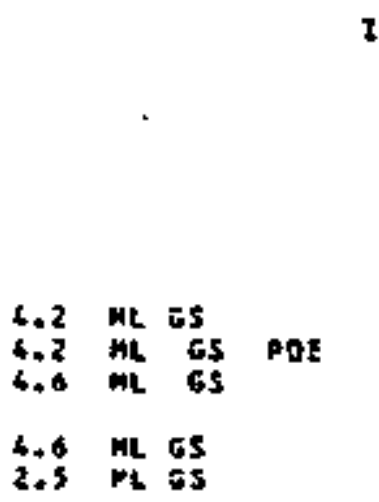

$4+6$ ML
$2+5$
II

vi

vI

vt
FEE CE TH DTStanCE

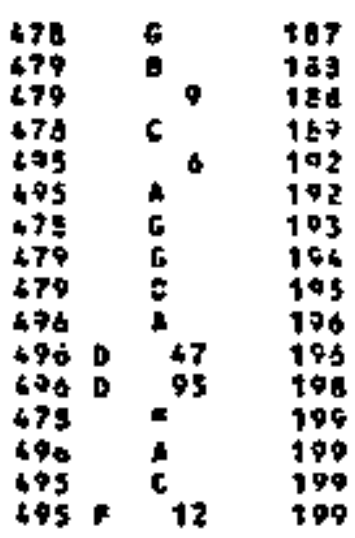

$\stackrel{:}{\stackrel{9}{0}}$

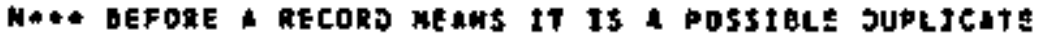

rotal HUHeER DF EVEMTs AETRIEVED is

TOTAL NUHJER OF SUSPEETED DUPLICATES IS

64

MATJOHAL GEOPHTSICAL ORTA CENTERIHOHA GOULOER, COLOHAOO OOSGJ 
Tabie D3.3 FIELD NOTES FOR GROUND RECONHAISSANEE 
$\forall$ N

so

ind

. 48

- की

की:

$x$, in $1<$

$x^{2} 1 y^{2}$

13

of sones

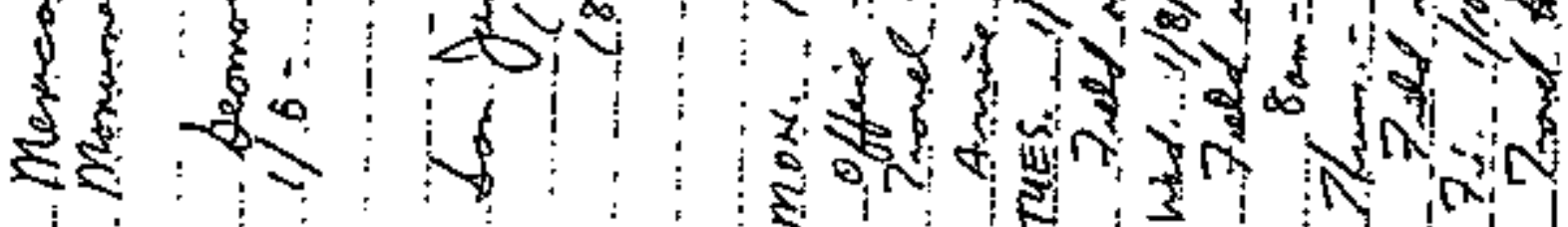

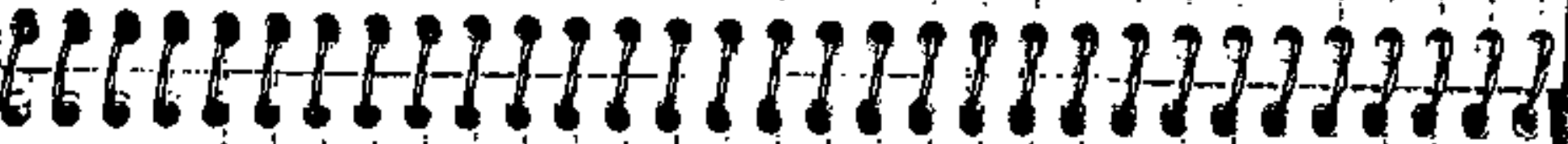

it

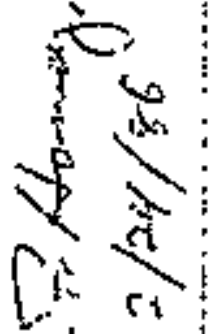

2 
na

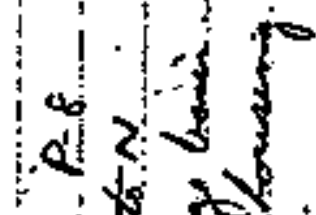

who sos tos

( )

H

$4+3$ s

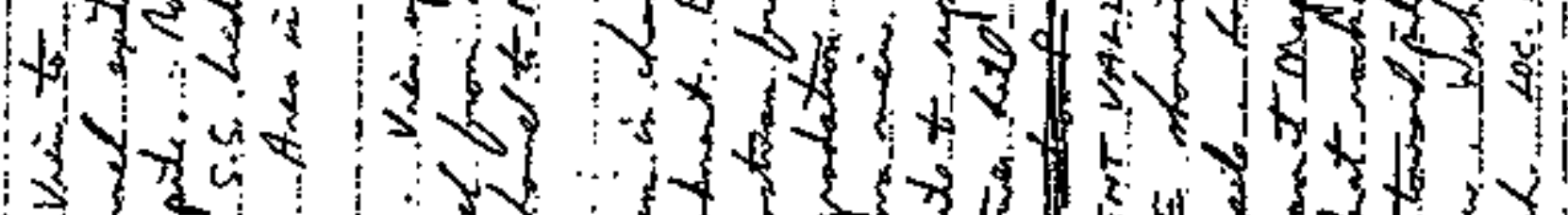

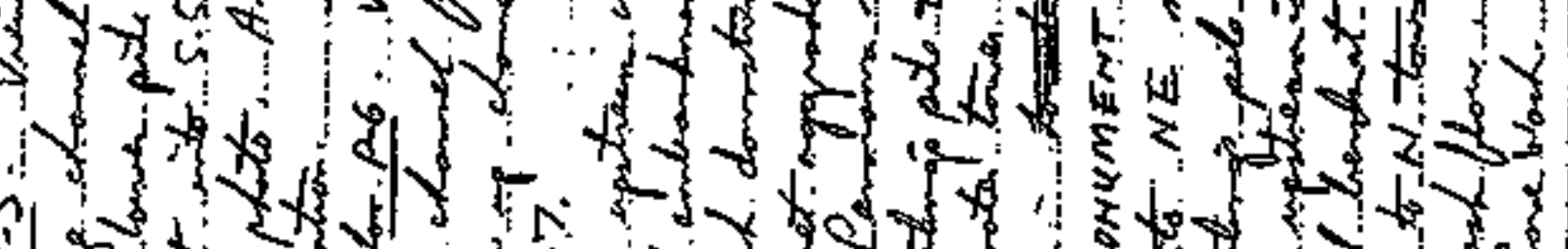

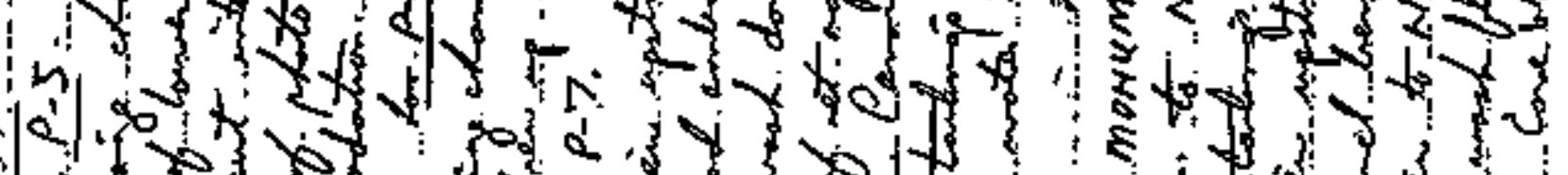

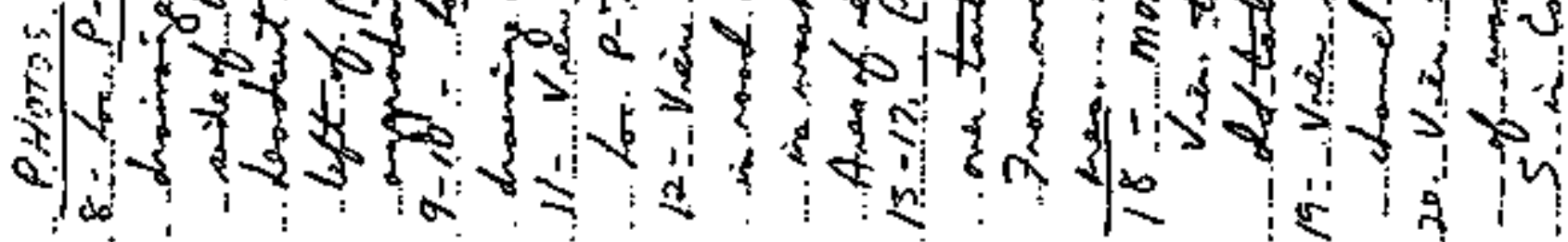

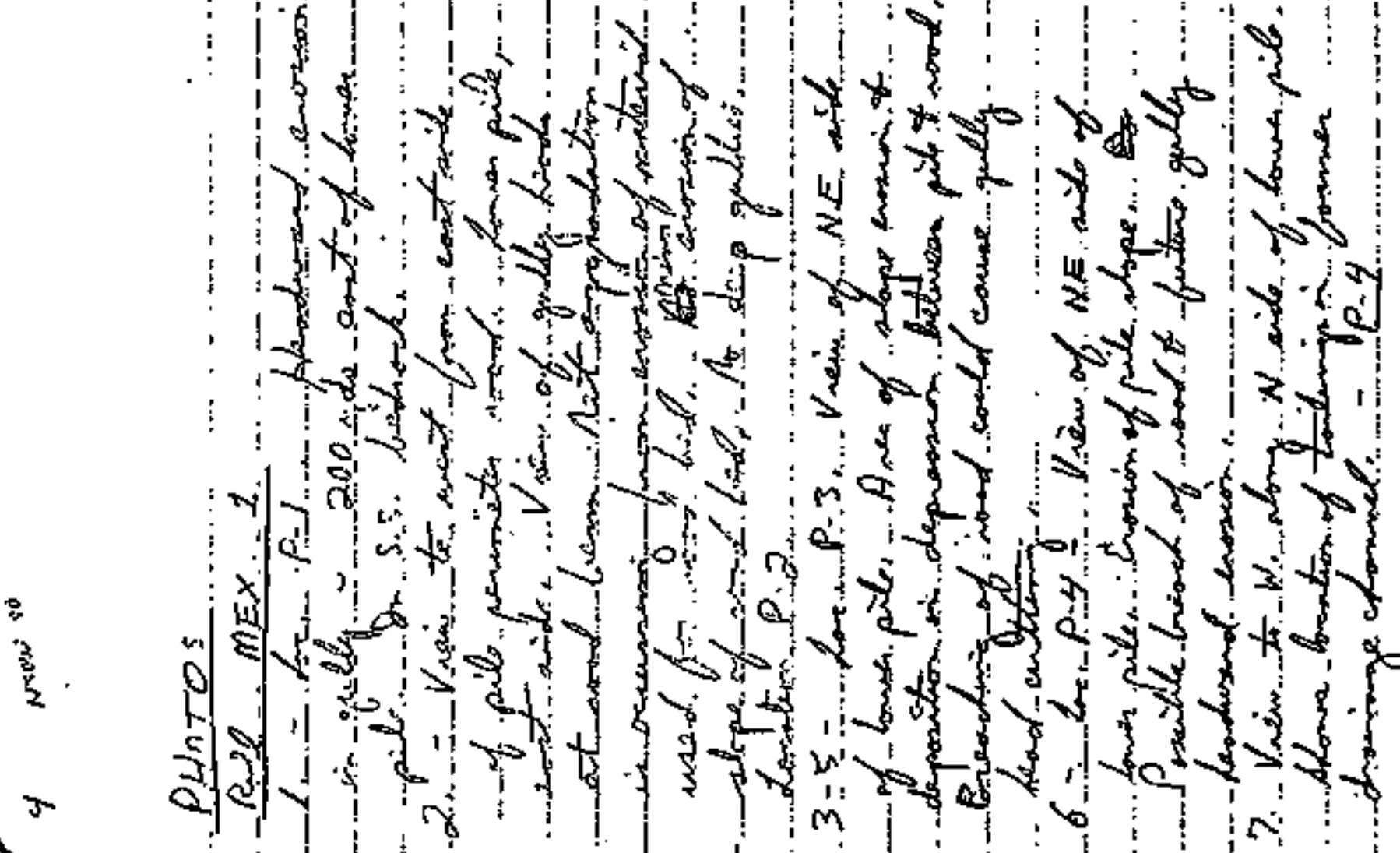


N

30

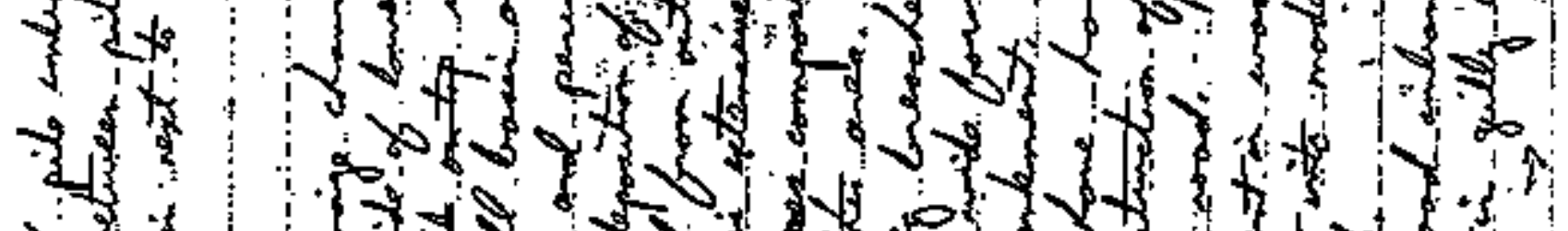

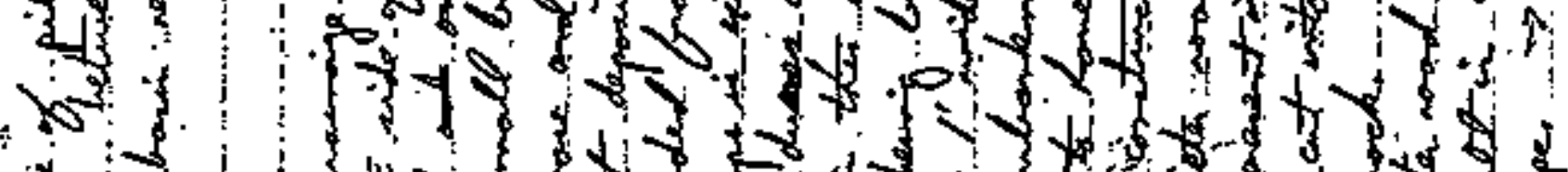
Jo, of और

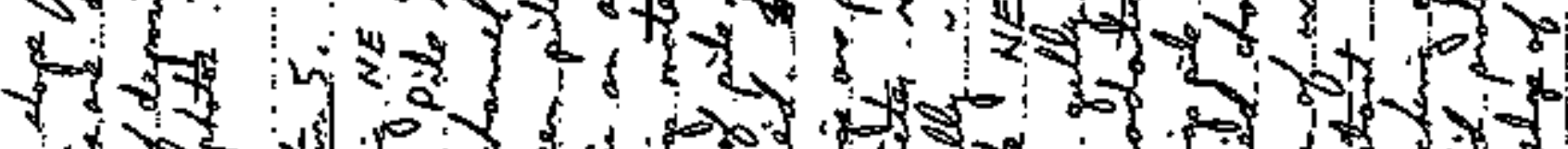

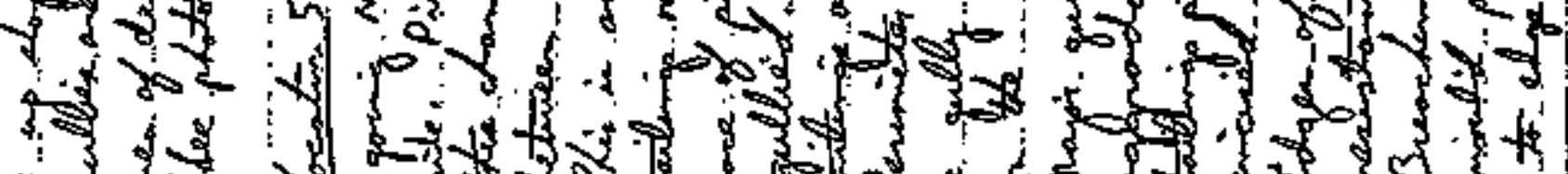

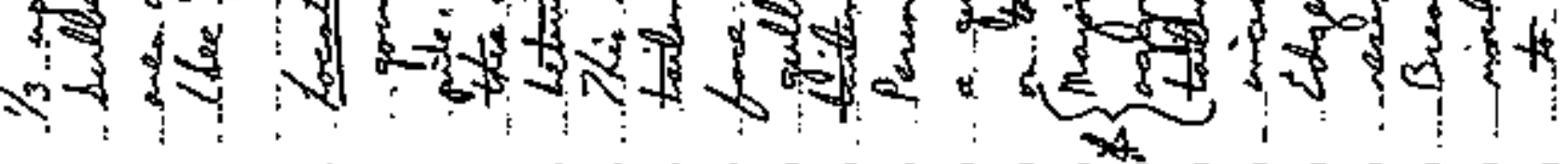

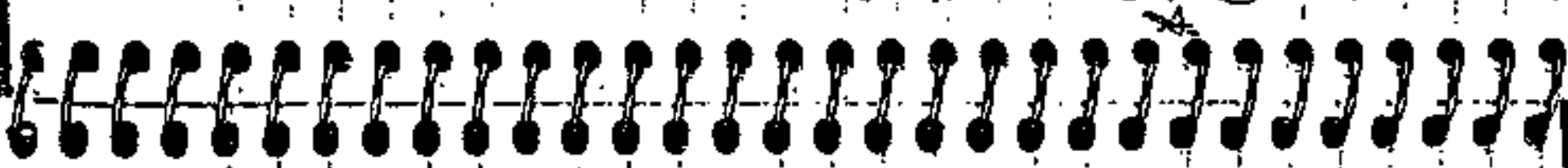

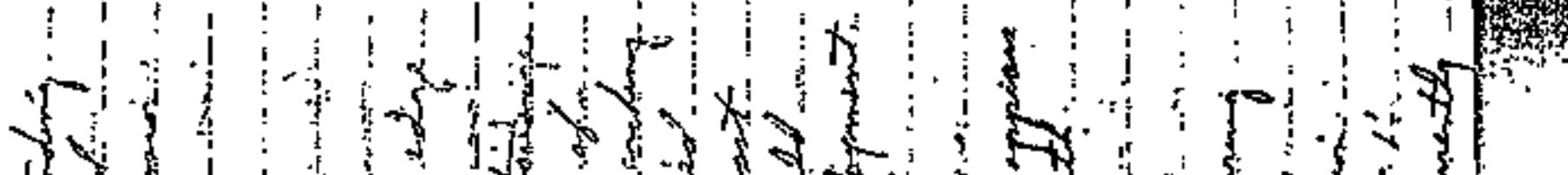
+5. s.

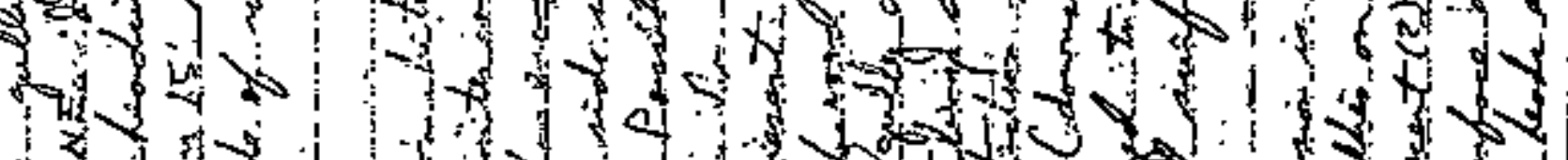

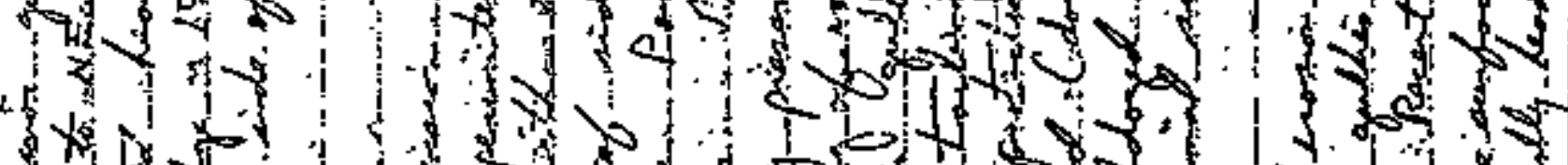

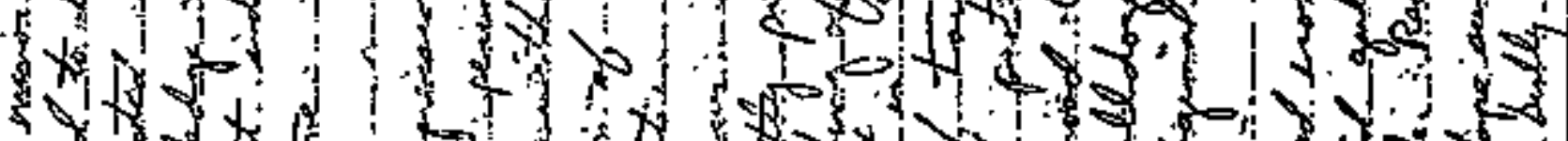

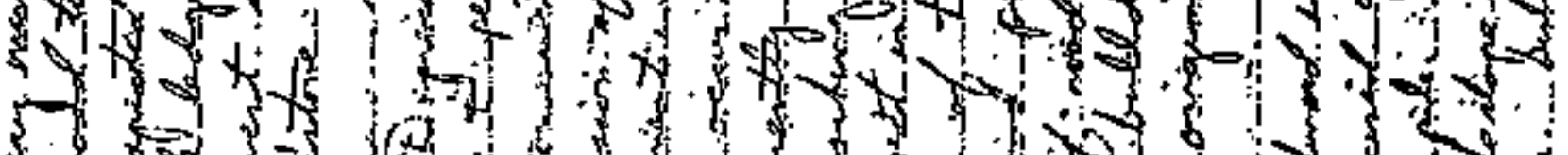

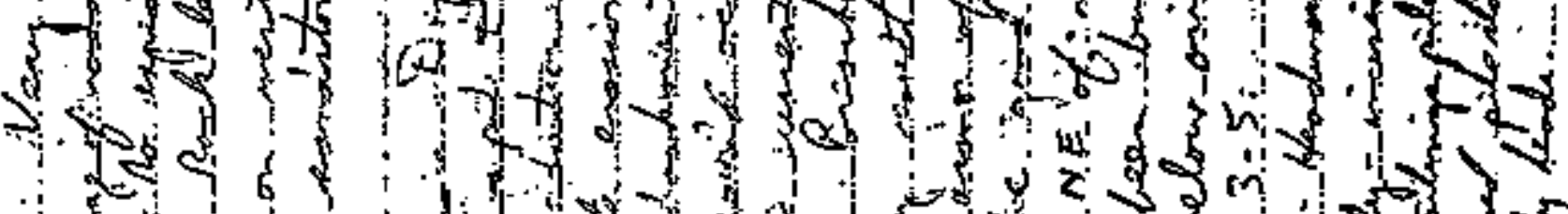

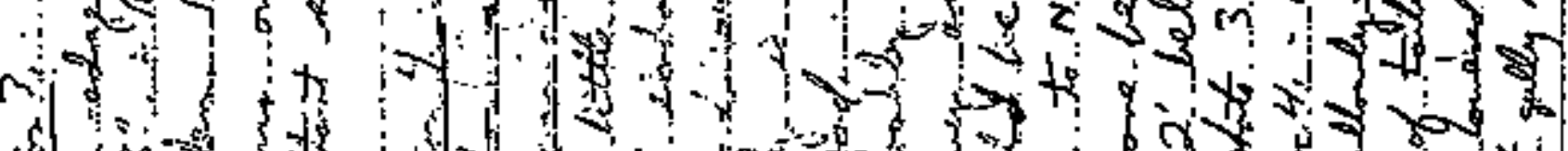
本

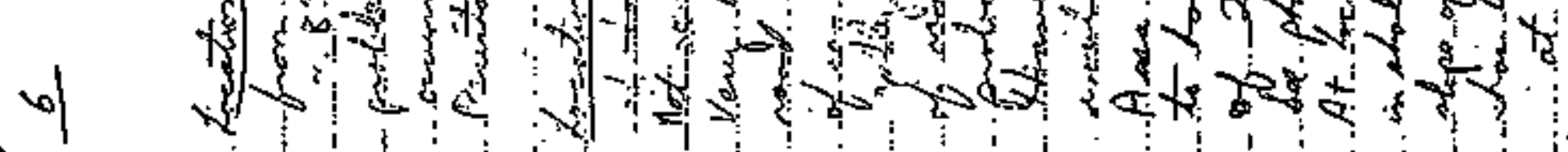


(1)

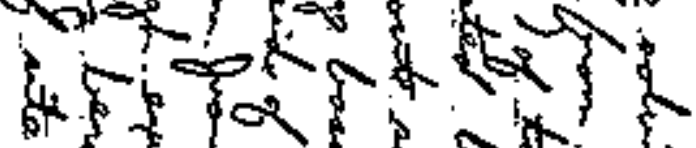
Stos T. 30.0 x? 5 ? $1 y^{2}+1$ Fond

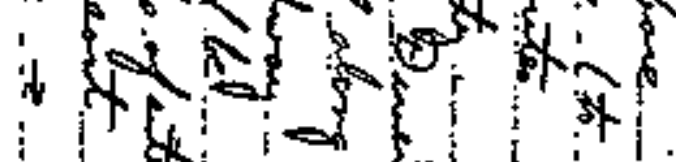
ats sis lo कI) bi $\rightarrow$ क 3. $x+200$

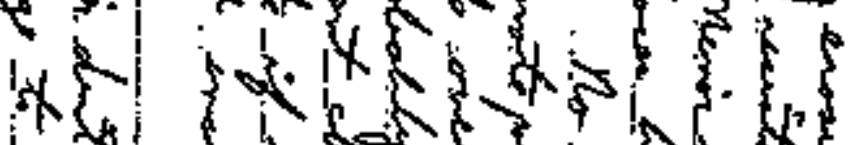

1 (1)

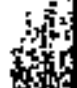

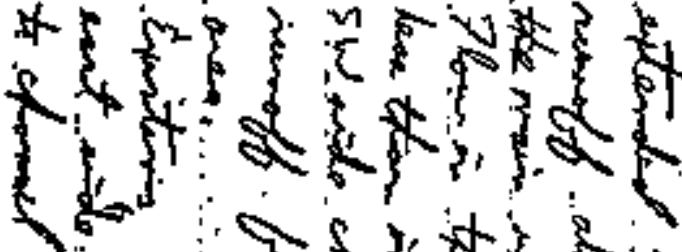

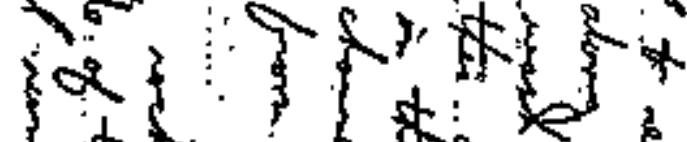
s.t. का

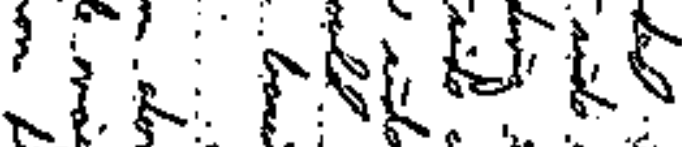
स. \$ कर ted Xद:

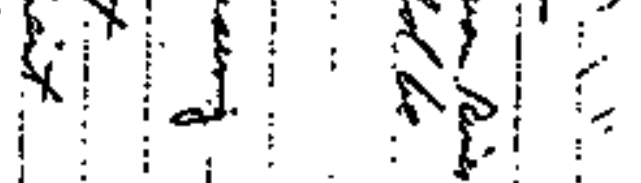


ADDENDLU D4

LITHOLOGIC LOGS 
This addendum contains borehole logs and test pit logs. When referring to the DOE boring and test pit ID niviber's; please note that the numbers used in the text refer to the numberfing, system as used in the DoE computerized Database Management System (DBMS):: The numbering system used on the field boring logs and tailings test pit logs ooes not match that used in the text. Therefore, when referring to the following DOE boring logs and test pit logs in conjunction with the text of this document it may be necessary to convert the field and laboratory numbers via Table 04.1. 


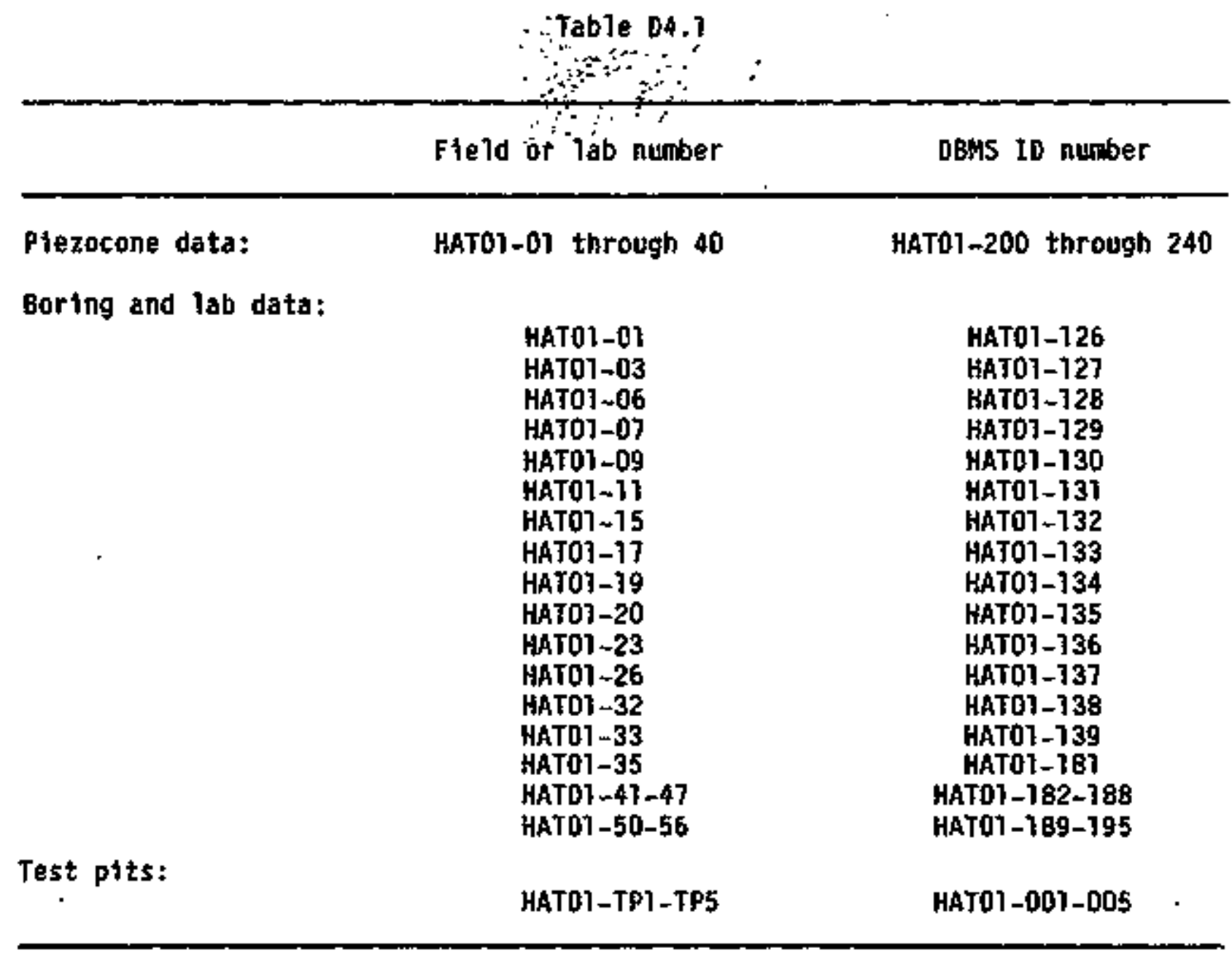


Table 04.2 Summary of geotechnical subsurface explorations at Mexican Hat, Jtah (DOE)

\begin{tabular}{|c|c|c|c|}
\hline Borling or test & Coord1nates. & Corpletion & Total depth \\
\hline plt numbera & Horthing Easting & date & (feet) \\
\hline
\end{tabular}

\section{Bor1ngs:}

\begin{tabular}{|c|c|c|c|c|}
\hline $\begin{array}{l}\text { (On the plle) } \\
\text { HATO1-126 } \\
\text { HATO1-127 } \\
\text { HATO1-128 } \\
\text { HAT01-129 } \\
\text { HATO1-130 } \\
\text { HATO1-131 } \\
\text { HATO1-132 } \\
\text { HATO1-133 } \\
\text { HATO1-134 } \\
\text { HATO1-135 } \\
\text { HATO1-136 } \\
\text { HATO1-137 } \\
\text { HATO1-138 } \\
\text { HAT01-139 } \\
\text { HATO1-183 } \\
\text { HATO1-911 } \\
\text { HATD1-912b }\end{array}$ & $\begin{array}{l}9711.3 \\
9310.0 \\
9710.0 \\
8516.0 \\
8910.5 \\
9570.0 \\
9770.6 \\
9770.0 \\
9708.5 \\
9909.5 \\
9508.7 \\
9310.0 \\
9510.9 \\
9511.1 \\
9310.3 \\
(9310) \\
(9520)\end{array}$ & $\begin{array}{l}9644.9 \\
9445.0 \\
9441.5 \\
9247.0 \\
9239.0 \\
9245.0 \\
10490.0 \\
10890.0 \\
11232.0 \\
10891.6 \\
10485.0 \\
10890.0 \\
10890.0 \\
11090.0 \\
11291.7 \\
(11090) \\
111160)\end{array}$ & $\begin{array}{l}10 / 11 / 84 \\
10 / 11 / 84 \\
10 / 11 / 84 \\
10 / 11 / 84 \\
10 / 11 / 84 \\
10 / 12 / 84 \\
10 / 12 / 84 \\
10 / 12 / 84 \\
10 / 12 / 84 \\
10 / 16 / 84 \\
10 / 16 / 84 \\
10 / 13 / 84 \\
10 / 16 / 84 \\
10 / 16 / 84 \\
10 / 15 / 84 \\
07 / 06 / 85 \\
03 / 28 / 85\end{array}$ & $\begin{array}{l}34.0 \\
27.0 \\
20.0 \\
12.5 \\
16.0 \\
17.0 \\
27.0 \\
45.0 \\
46.5 \\
41.0 \\
25.0 \\
22.5 \\
42.0 \\
34.0 \\
34.0 \\
98.0 \\
84.0\end{array}$ \\
\hline 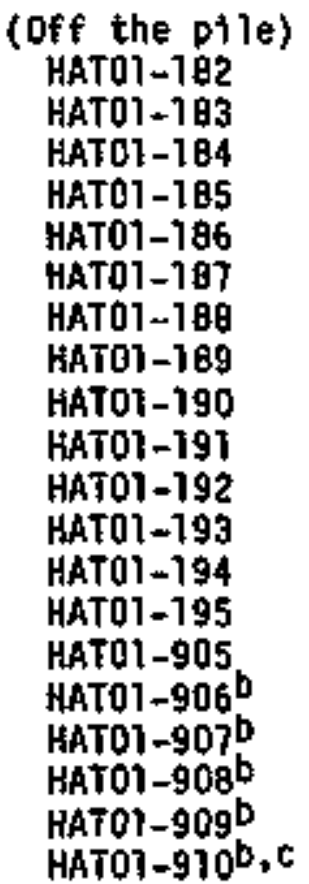 & $\begin{array}{r}8370.6 \\
8304.5 \\
8727.1 \\
9670.4 \\
10482.4 \\
10799.4 \\
9885.5 \\
(10950) \\
10791.3 \\
(11050) \\
11683.1 \\
10935.2 \\
10152.6 \\
10019.3 \\
10006.3 \\
10737.5 \\
10154.7 \\
(10000) \\
(7780) \\
(8400)\end{array}$ & $\begin{array}{r}9343.2 \\
10066.6 \\
10830.5 \\
11677.7 \\
11221.2 \\
10345.6 \\
9540.9 \\
(10930) \\
11109.9 \\
(72270) \\
72321.9 \\
12126.8 \\
11711.0 \\
9538.7 \\
9544.2 \\
11703.1 \\
31692.4 \\
(9580) \\
(7510) \\
(10400)\end{array}$ & $\begin{array}{l}10 / 10 / 84 \\
10 / 10 / 84 \\
10 / 10 / 84 \\
10 / 10 / 84 \\
10 / 10 / 84 \\
10 / 10 / 84 \\
10 / 10 / 84 \\
10 / 23 / 84 \\
10 / 23 / 84 \\
10 / 23 / 84 \\
10 / 23 / 84 \\
10 / 23 / 84 \\
10 / 23 / 84 \\
10 / 23 / 84 \\
12 / 11 / 84 \\
12 / 12 / 84 \\
12 / 19 / 84 \\
01 / 11 / 85 \\
01 / 19 / 85 \\
03 / 22 / 85\end{array}$ & $\begin{array}{r}5.0 \\
3.3 \\
12.0 \\
34.0 \\
3.0 \\
3.0 \\
6.0 \\
5.0 \\
5.0 \\
2.5 \\
2.5 \\
1.5 \\
2.0 \\
3.0^{0} \\
9.0^{d} \\
11.0^{d} \\
125.0^{d} \\
196.9^{d} \\
215.0^{d} \\
236.0^{d}\end{array}$ \\
\hline
\end{tabular}


Table 04.2 Sunmary of geotechnical subsurface explorations at Mexican Hat, Utah (DOE) (Concluded.)

\begin{tabular}{|c|c|c|c|}
\hline$y$, & $\therefore$ & $\therefore$ & $1: x$ \\
\hline $\begin{array}{l}\text { Boring or test } \\
\text { pit number }\end{array}$ & $\frac{\text { Coordinates }}{\text { Worthing }}$ & $\begin{array}{l}\text { Completion } \\
\text { date }\end{array}$ & $\begin{array}{l}\text { Totai depth } \\
\text { (feet) }\end{array}$ \\
\hline
\end{tabular}

Borings (Conit.):

(Off the pile) (Cont.)

$\begin{array}{lrrrr}\text { HATO1-930 } & 10550.337 & 7732.998 & 10 / 10 / 85 & .116 \\ \text { HATO1-931 } & 12083.029 & 9971.036 & 10 / 12 / 85 & 177 \\ \text { HATO1-932 } & 13767.679 & 12636.319 & 10 / 14 / 85 & 193 \\ \text { HATO1-933 } & 7782.264 & 8444.656 & 10 / 15 / 85 & 175 \\ \text { HATO1-934b } & 9223.312 & 11989.935 & 10 / 18 / 85 & 200 \\ \text { HATO1-935b } & 10023.747 & 12468.284 & 10 / 20 / 85 & 197 \\ \text { HATO1-936 } & 11397.741 & 10912.259 & 10 / 21 / 86 & 142\end{array}$

Test Pits:

(Ta111ngs)
HATO1-001
HATO1-002
. HATO1-003
HATO1-004
HATO1-005

(9710)

(10600)

(9510)

(9110)

(9310)

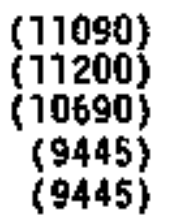

$10 / 17 / 84$

$10 / 23 / 84$

$10 / 23 / 84$

$10 / 23 / 84$

$10 / 23 / 84$
7.0

0.5

5.5

7.5

4.5

abBMS numbering system (See Table D4.1).

bThese borings were cased and completed as monitoring wells.

cTwo borings were advanced. One encountered hydrogen sulfide gas and was grouted to its futl depth and another drilled nearby.

Indicates that a f or part of the boring was advanced using rock corting techniques.

() Coordinates enclosed in parentheses are field estimates and were not surveyed. 
Table 94.3 Summary of barings at Mexitan Hat (MSRD/CSU)

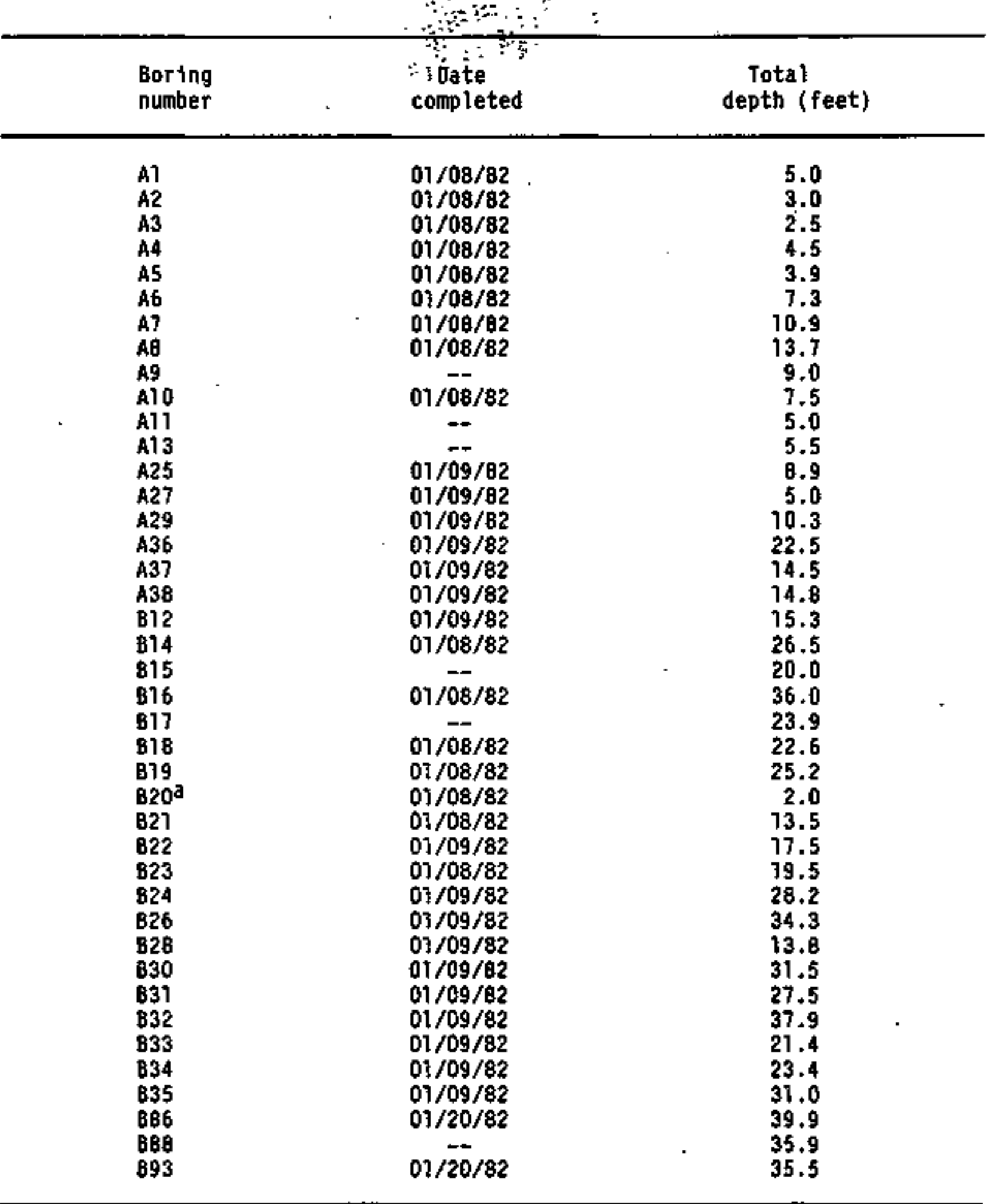


Table B4.3 Summary of borinģ at Mexican Hat (MSRD/CSU) (Contintued)

\begin{tabular}{|c|c|c|}
\hline $\begin{array}{l}\text { Boring } \\
\text { ntmber }\end{array}$ & $\begin{array}{l}\text { Date } \\
\text { completed }\end{array}$ & $\begin{array}{l}\text { Tota? } \\
\text { depth (feet) }\end{array}$ \\
\hline $\begin{array}{l}C 39 \\
C 40 \\
C 41 \\
C 42 \\
C 43 \\
C 44 \\
C 45 \\
C 46 \\
C 47 \\
C 48 \\
C 49 \\
C 54 \\
C 56 \\
C 58 \\
C 60 \\
C 62 \\
C 65 \\
C 67 \\
C 69 \\
C 71 \\
C 73 \\
C 75 \\
C 77 \\
C 79 \\
C 81 \\
C 83 \\
C B 7 \\
C 89 \\
C 90 \\
C 94 \\
C 95 \\
C 96 \\
C 97 \\
D 50 \\
D 51 \\
052 \\
053 \\
055 \\
057 \\
D 59 \\
061 \\
063 \\
064\end{array}$ & $\begin{array}{l}01 / 10 / 82 \\
01 / 10 / 82 \\
01 / 10 / 82 \\
01 / 10 / 82 \\
01 / 10 / 82 \\
01 / 10 / 82 \\
01 / 10 / 82 \\
01 / 10 / 82 \\
01 / 10 / 82 \\
01 / 10 / 82 \\
01 / 11 / 82 \\
01 / 11 / 82 \\
01 / 11 / 82 \\
01 / 11 / 82 \\
01 / 11 / 82 \\
01 / 11 / 82 \\
01 / 12 / 82 \\
01 / 12 / 82 \\
01 / 12 / 82 \\
01 / 12 / 82 \\
01 / 12 / 82 \\
01 / 12 / 82 \\
01 / 12 / 82 \\
01 / 12 / 82 \\
01 / 18 / 82 \\
01 / 18 / 82 \\
01 / 18 / 82 \\
01 / 19 / 82 \\
01 / 19 / 82 \\
01 / 20 / 82 \\
01 / 20 / 82 \\
01 / 20 / 82 \\
01 / 20 / 82 \\
01 / 10 / 82 \\
01 / 11 / 82 \\
01 / 11 / 82 \\
01 / 11 / 82 \\
01 / 12 / 82 \\
01 / 12 / 82 \\
01 / 12 / 82 \\
01 / 12 / 82 \\
01 / 12 / 82\end{array}$ & $\begin{array}{r}1.9 \\
4.0 \\
10.7 \\
12.0 \\
14.9 \\
23.2 \\
22.7 \\
9.7 \\
27.5 \\
35.4 \\
35.0 \\
34.8 \\
30.0 \\
24.4 \\
29.7 \\
34.4 \\
29.9 \\
22.5 \\
7.5 \\
3.4 \\
0.9 \\
6.1 \\
17.4 \\
79.8 \\
26.4 \\
24.9 \\
16.3 \\
14.5 \\
6.0 \\
21.0 b \\
26.0 b \\
19.0 b \\
7.5 \\
54.8 \\
42.4 \\
41.3 \\
18.4 \\
33.9 \\
26.1 \\
29.3 \\
13.6 \\
24.1 \\
46.9\end{array}$ \\
\hline
\end{tabular}


Table DA.3 Summary of borings at Mexican Hat (HSRO/CSU) (Continued)

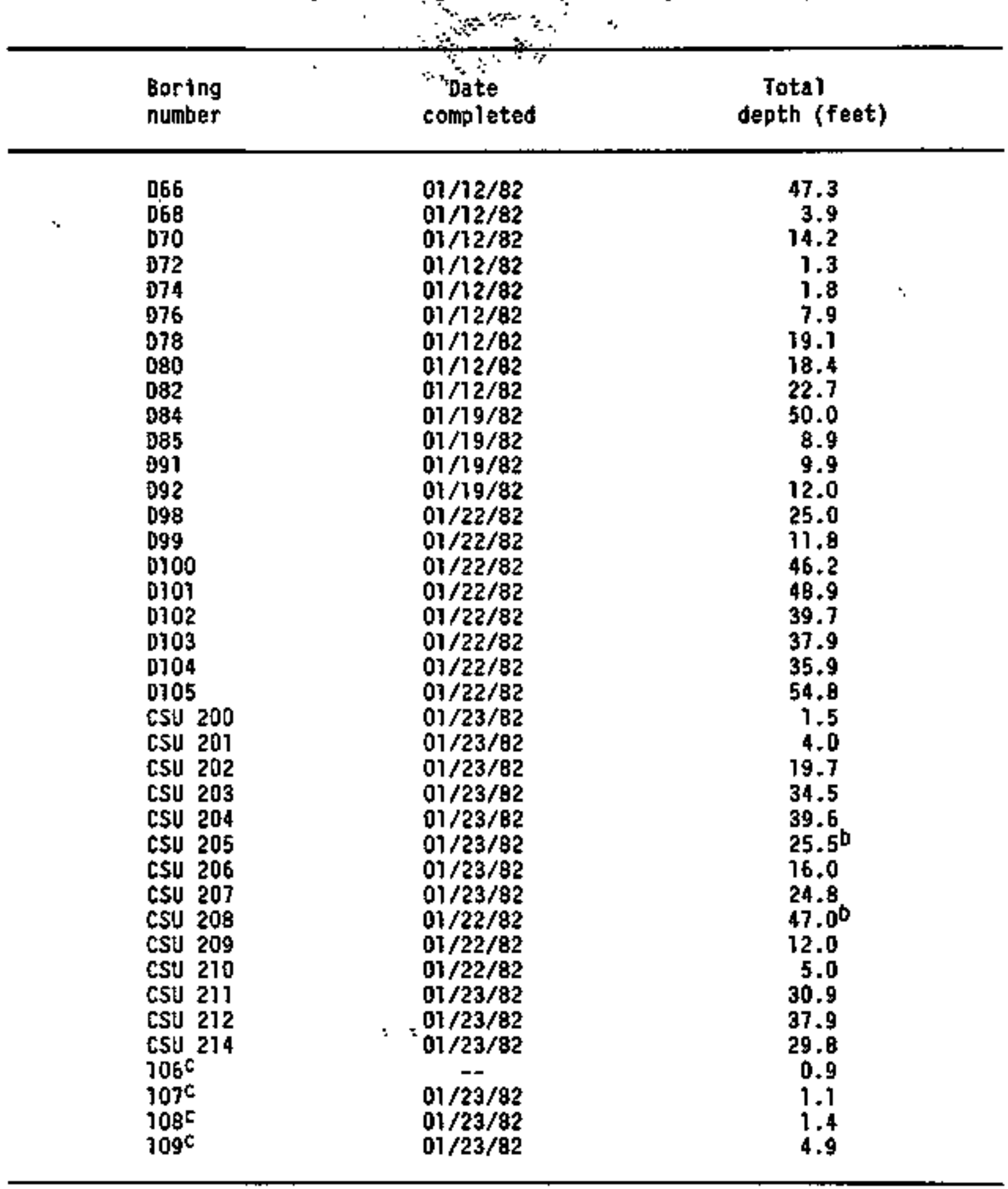


Table D4.3 Sumary of boringes at Mexican Hat (MSRD/CSU) (Concluded)

\begin{tabular}{|c|c|c|}
\hline $\begin{array}{l}\text { Boring } \\
\text { number }\end{array}$ & $\begin{array}{l}\text { "Date } \\
\text { completed }\end{array}$ & $\begin{array}{c}\text { Total } \\
\text { depth (feet) }\end{array}$ \\
\hline
\end{tabular}

\section{Piezometers}

$\begin{array}{lll}H G 2^{d} & 01 / 23 / 92 & 20.0^{b} \\ H G 3^{d} & 01 / 23 / 82 & 60.0^{b}\end{array}$

"This boring is marked as A2O on original maps but appears as B20 in borehole logs.

DAll ar part of the boring was advanced using rock coring techniques.

coff-pile holes (not shown on location map).

dBoring logs missing. 


$$
\begin{aligned}
& a \\
& -4
\end{aligned}
$$

DOE BORING LOGS 


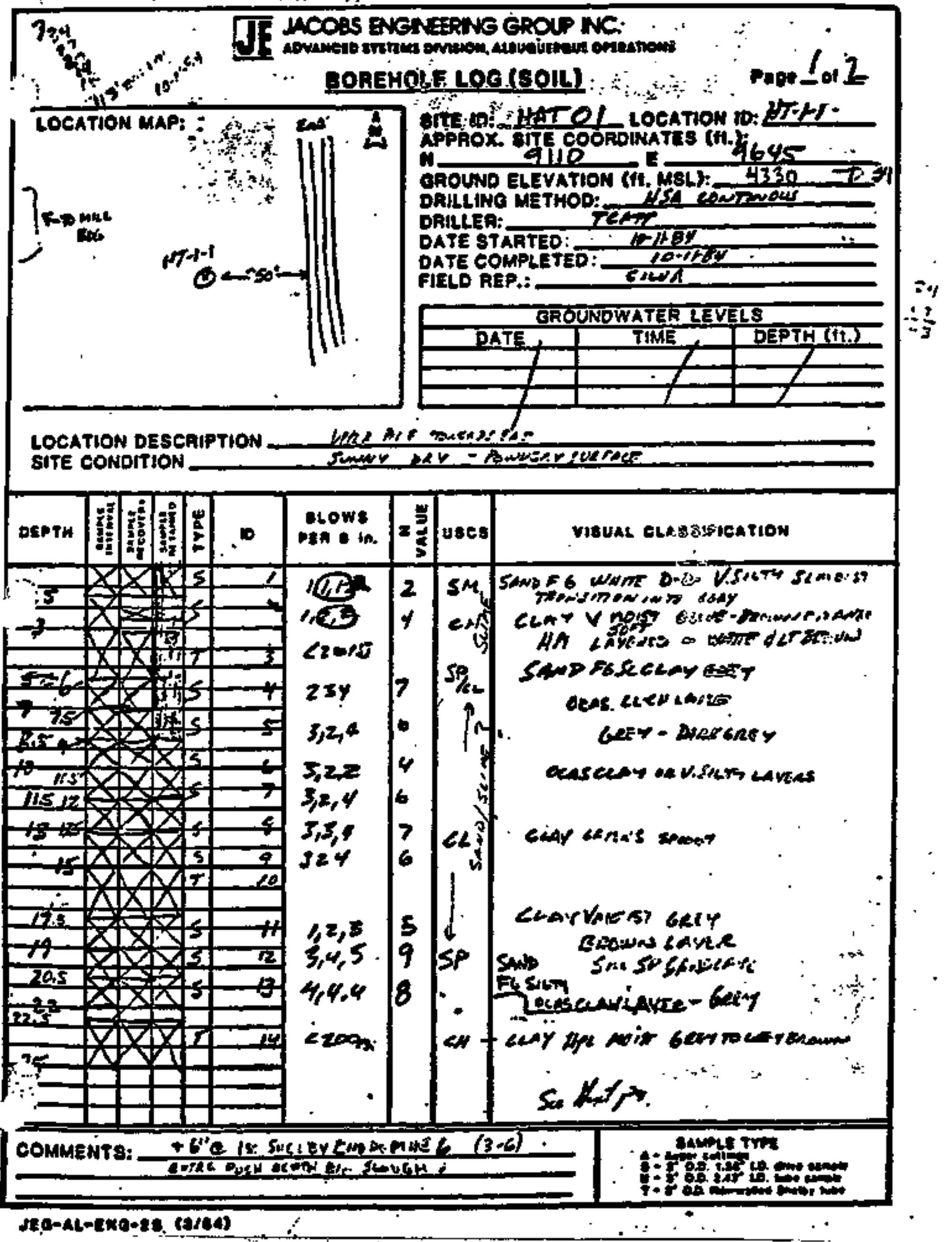




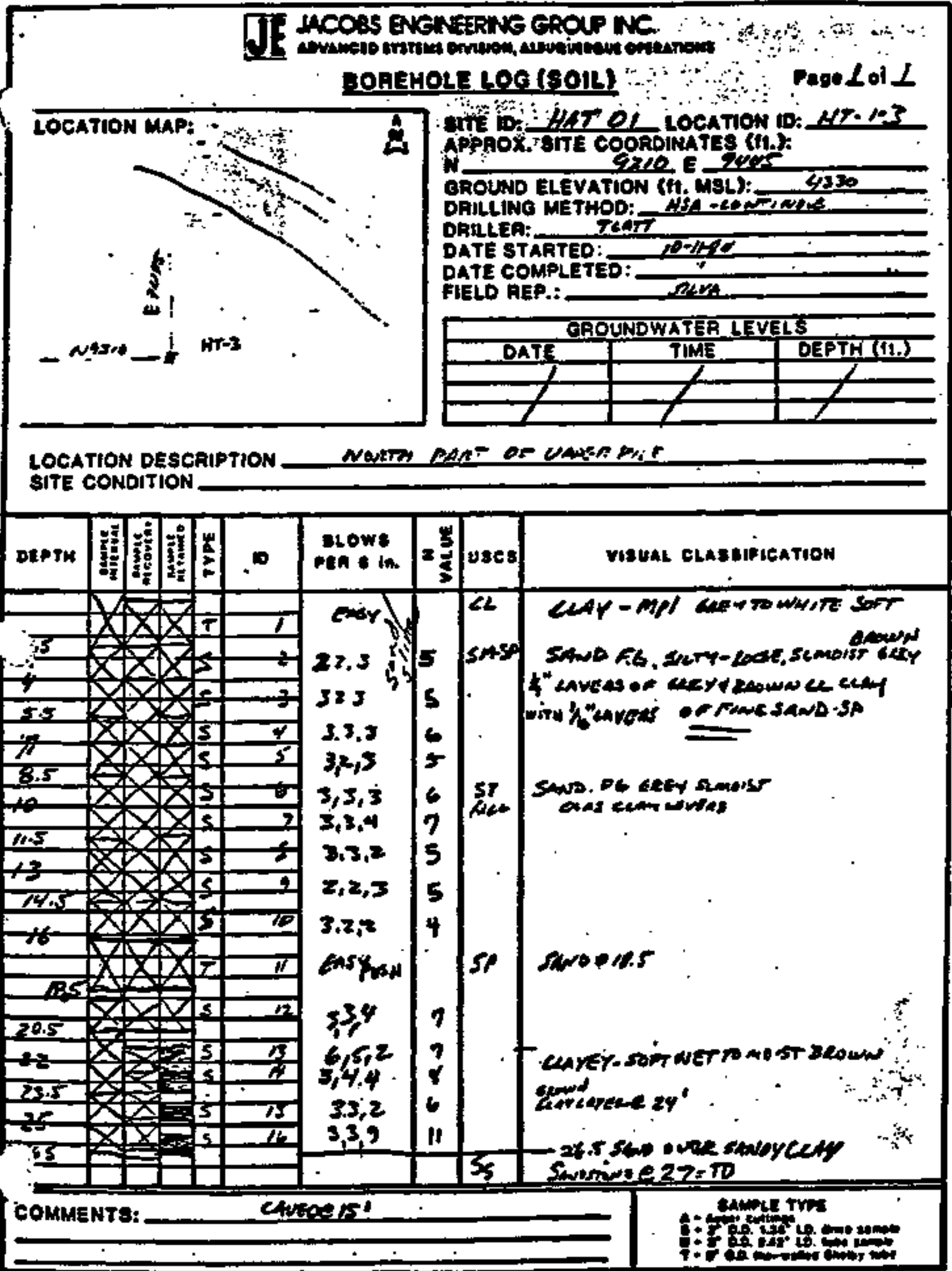




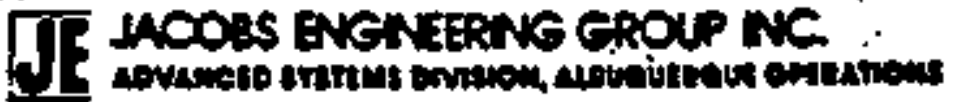

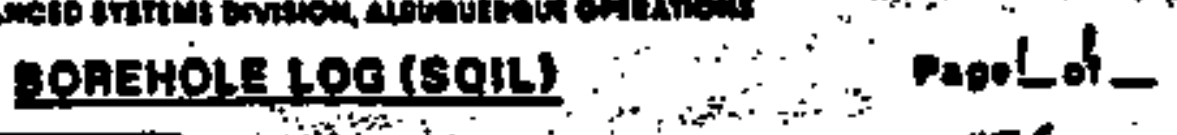

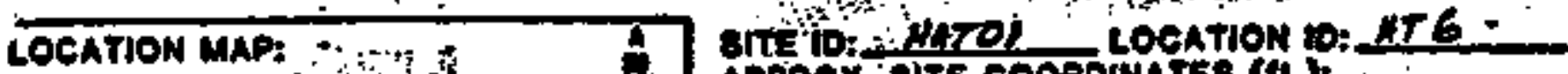

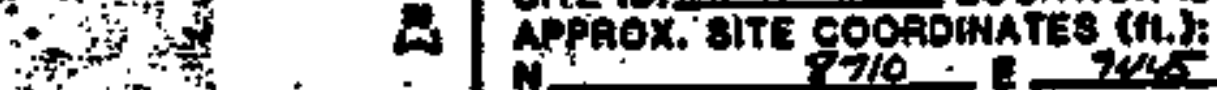

in

4 vmerpiét-

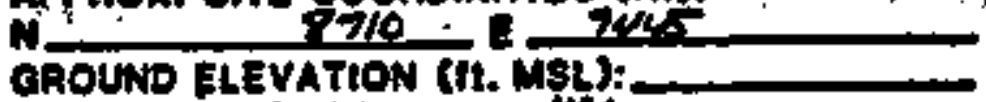

DRIUNO METHOD (11. MSL):

DRILLER:

DATE STAATED: 10-HLS

DATE COMPLETED: $10-7 \mathrm{~m}$

FIELO REP.:

a Nro

\begin{tabular}{|l|l|l|}
\hline GROUNDWATER LEVELS \\
\hline DAIE & TMME & DEPTH (II.) \\
\hline & & \\
\hline
\end{tabular}

LOCATION DESCAIPTION SOUTH SIOE JPAEA BICE

SITE CONDITION

winoy swewY

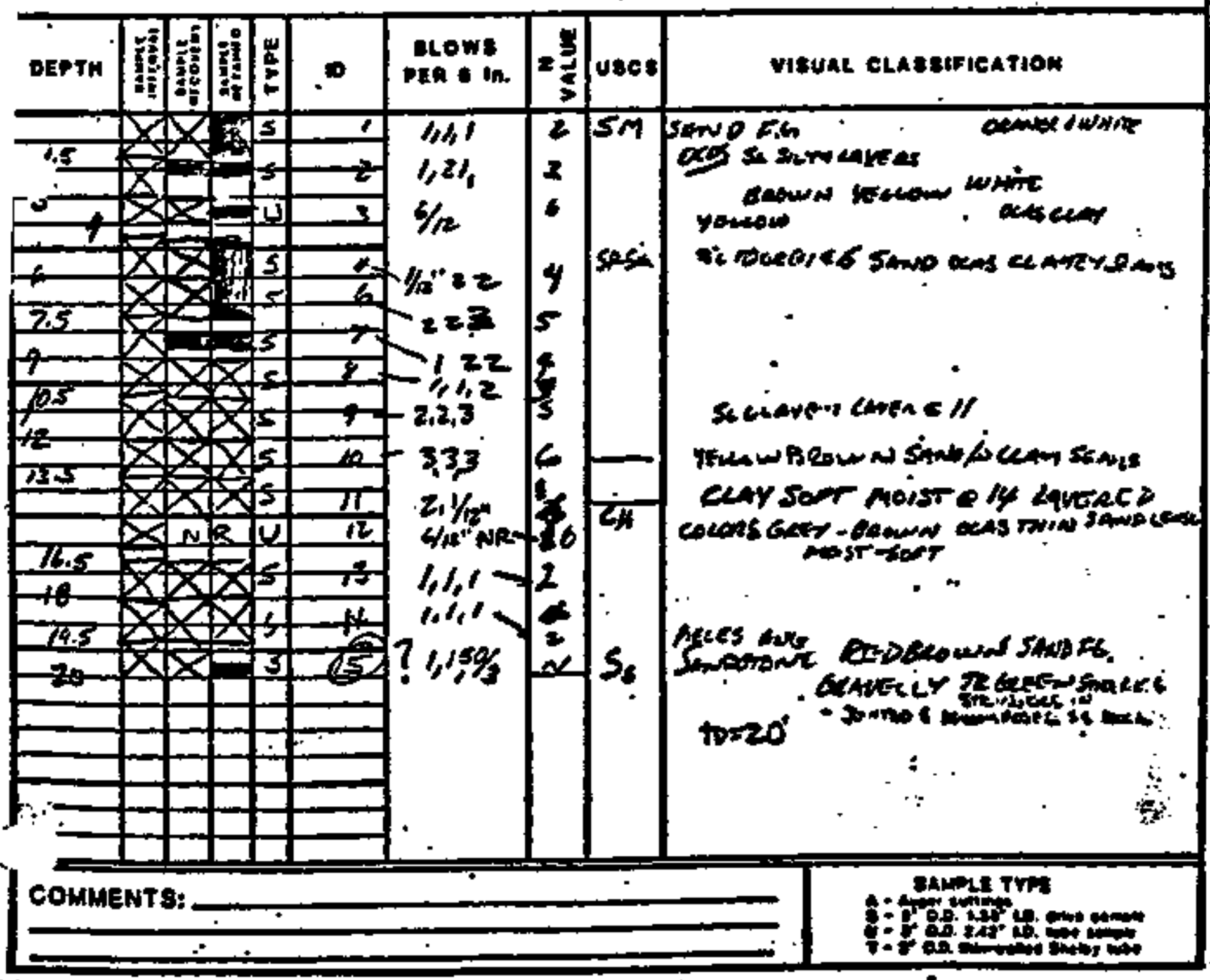




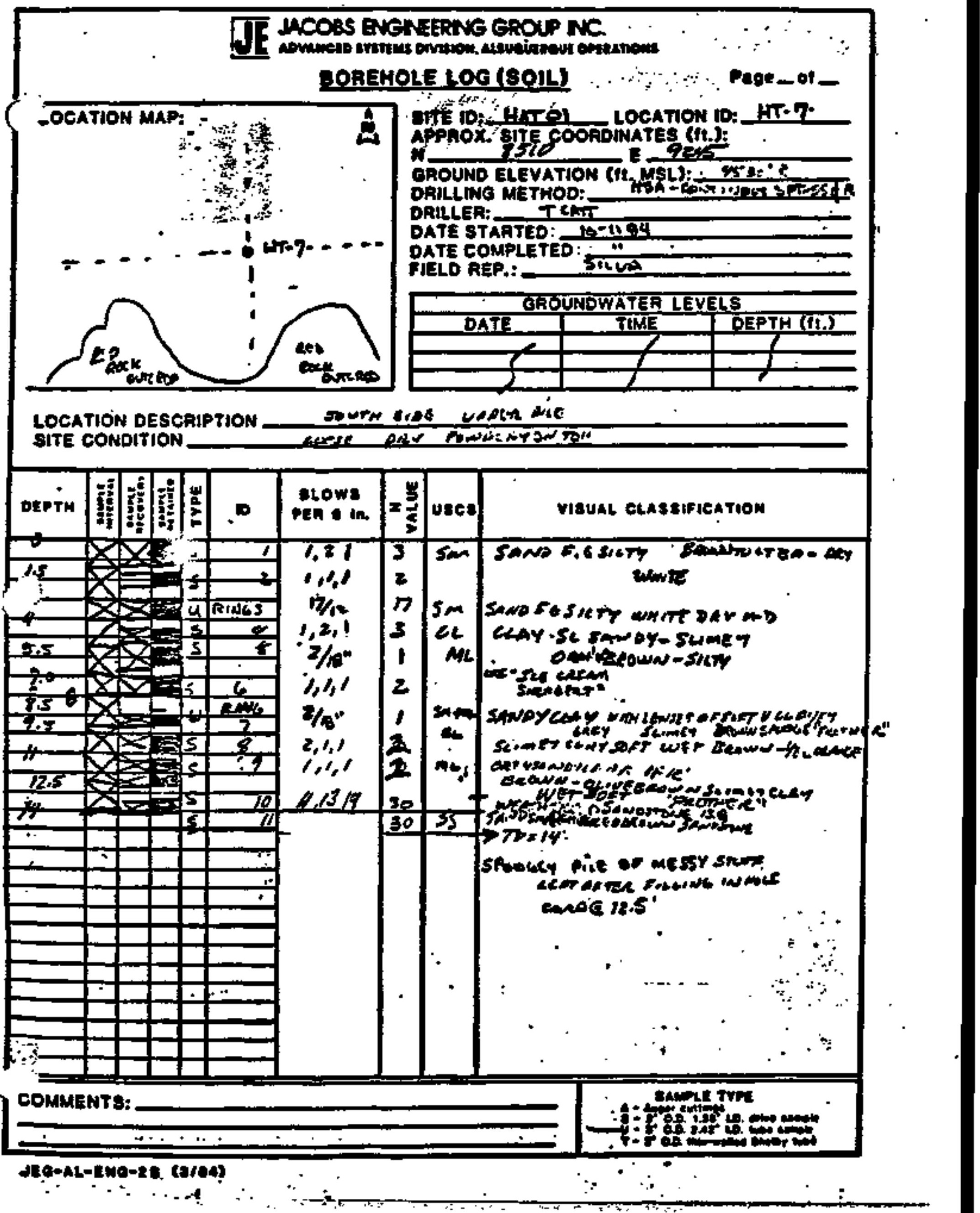




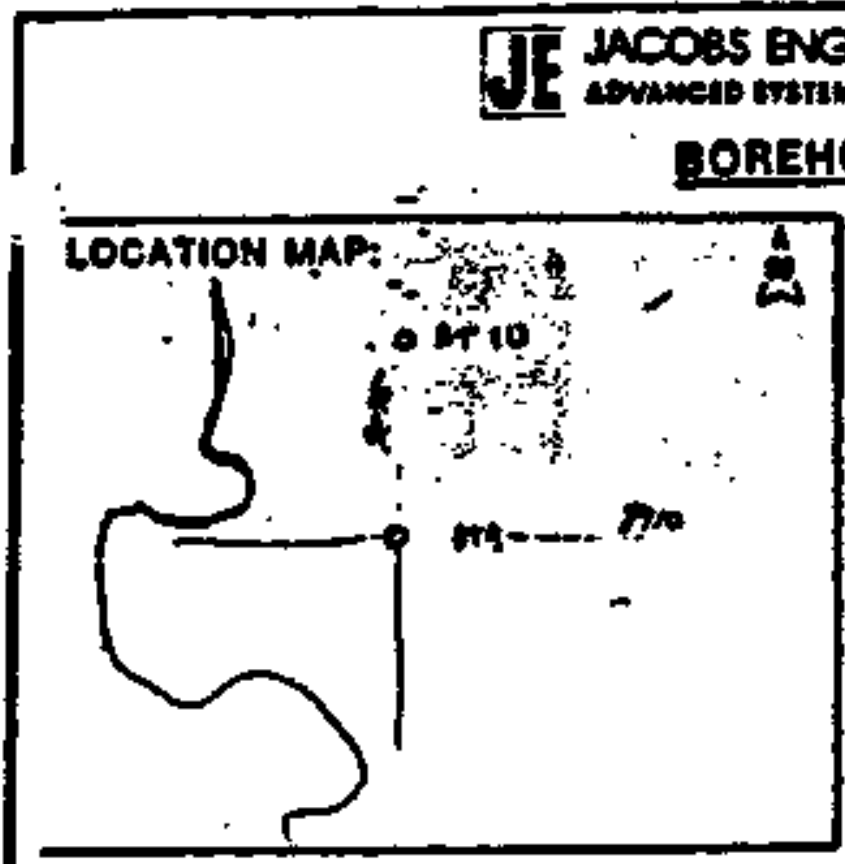

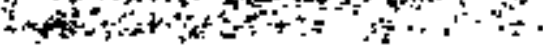

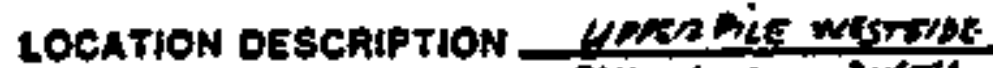

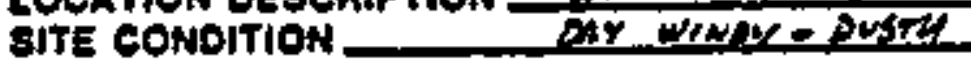

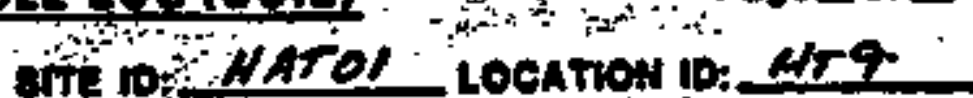

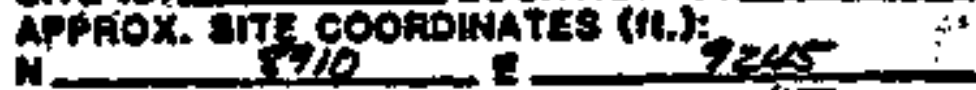

RROUNO ELEVATION (ft, MSL): VFa

DAILLNA METHOD: Us

DRILLER: Fun

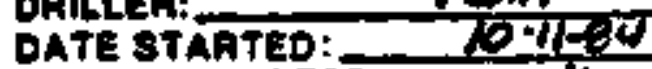

DATE COMPLETED:

FIELO REP.:

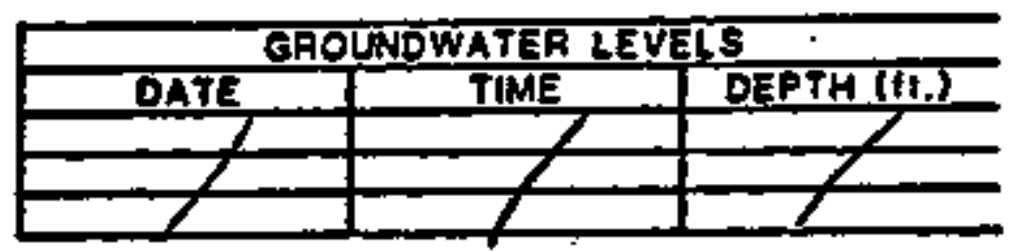

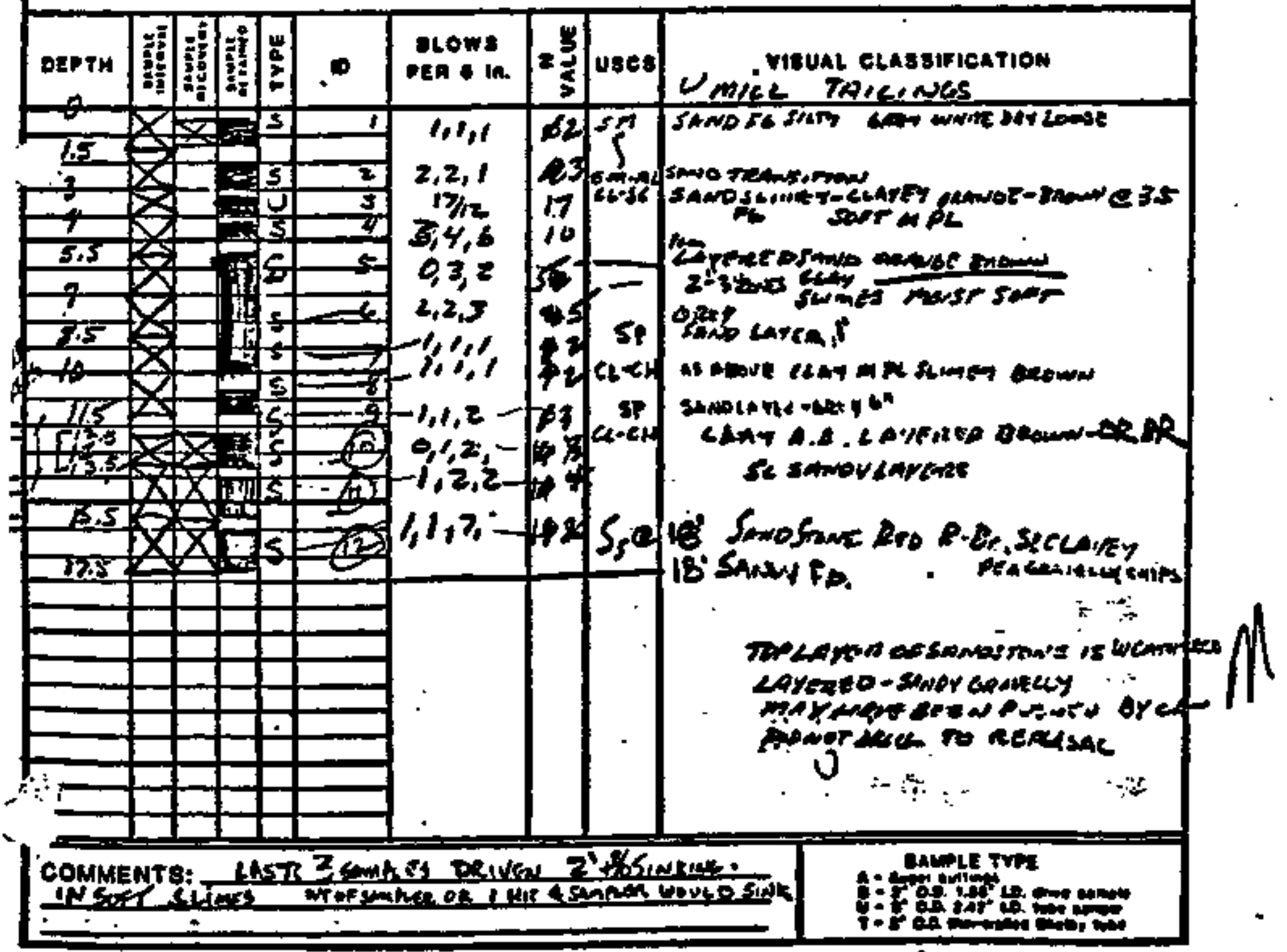




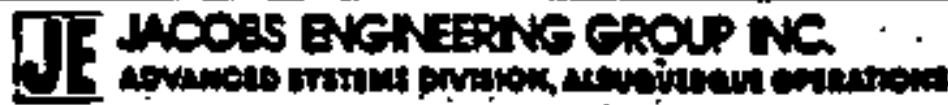

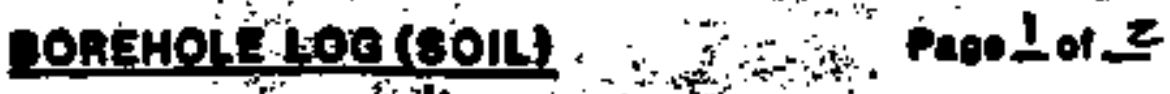

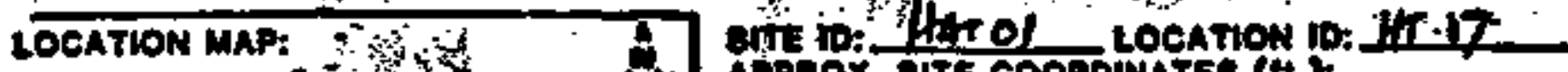
- D. A. APROX. BITE COORDWNATES (tt.)

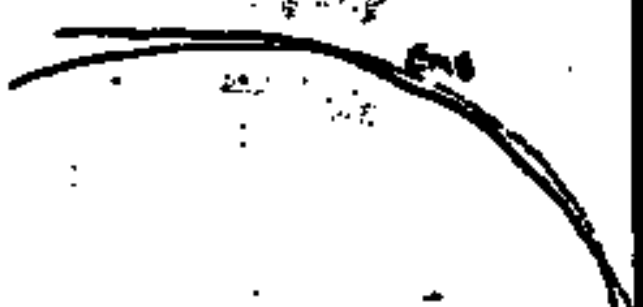

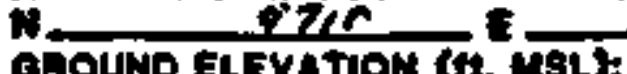

GAOUND ELEVATION (th, MBL):

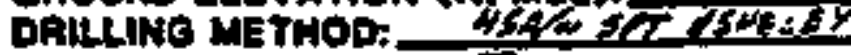

OAlLLER: TETT

DATE BTARTED: $\frac{10-\sqrt{2}-14}{2}$

PATE COMPLETRD: $\frac{h}{\operatorname{Sinh}}$

Q 4T 17 GOUONDWATER LEVEIS

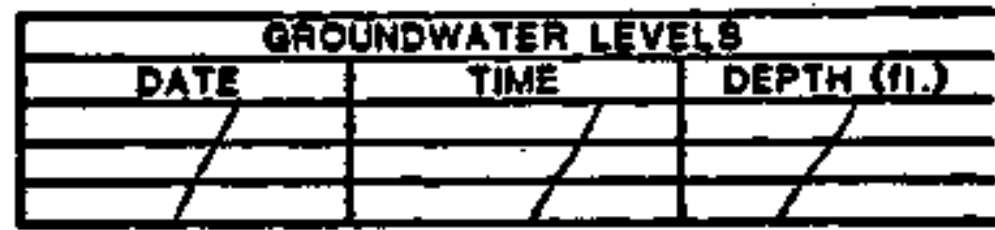

LOCATION DESCRIPTION

Antidetat if SITE CONDITTON

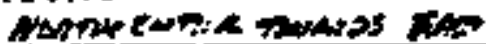

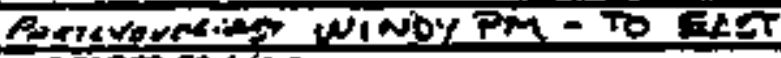

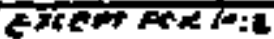

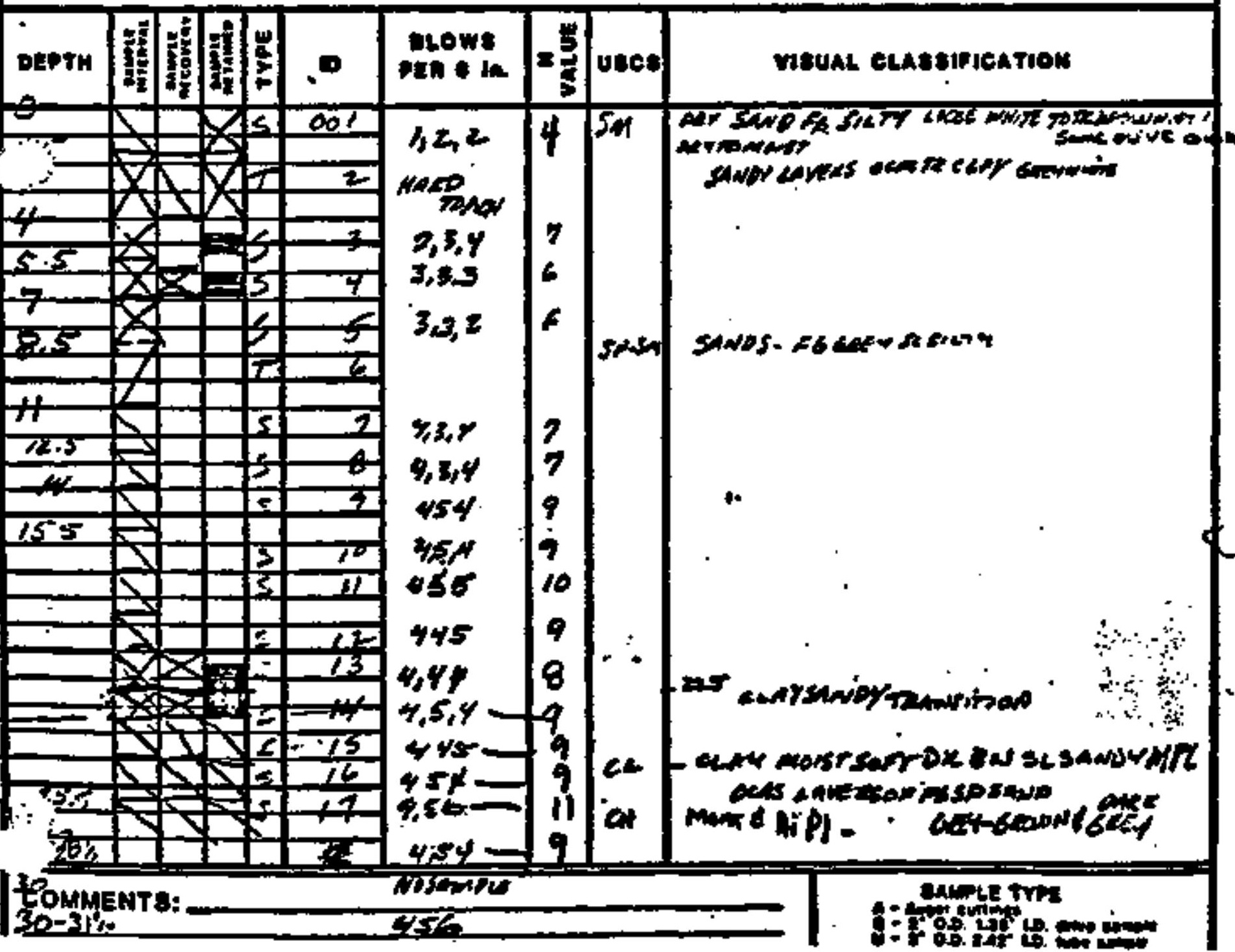




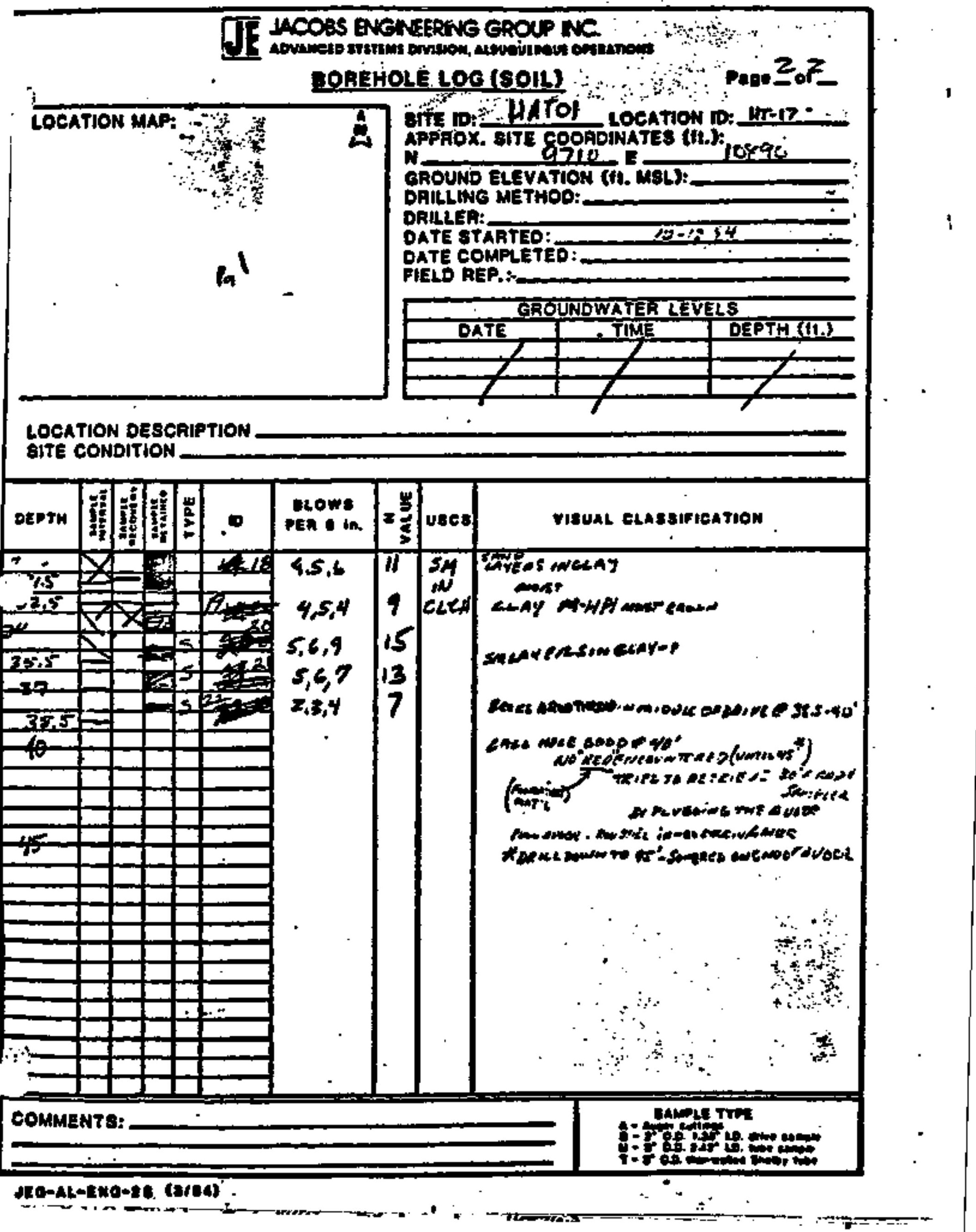




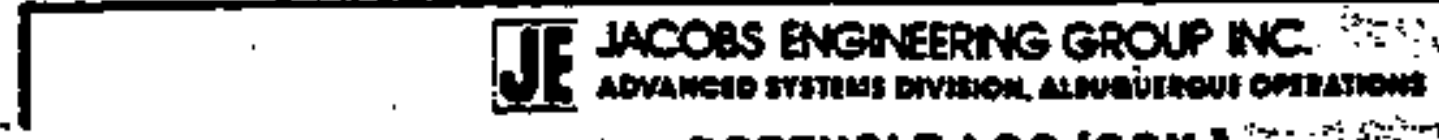

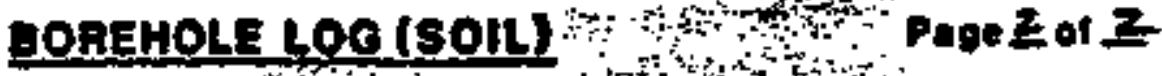

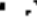

LOCATION MAP:

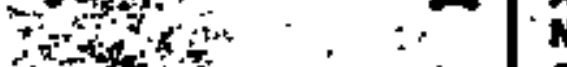

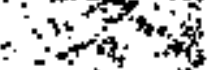

;

GTE TOS AATOL LOCATION ID: HT2A

APPROX BATE COORDIMATES (it.):

N o

GROUND ELEVATION (t). MSL\}:

DALLINO METHOD:

DAILLER:

DATE STAATED:

DATE COMPLETED:

FIELD REP.:

\begin{tabular}{|c|c|c|}
\hline \multicolumn{3}{|c|}{ GHONDWATER LEVELS } \\
\hline DATE & TIME & DEPTH TI. \\
\hline & & \\
\hline & & \\
\hline
\end{tabular}

LOCATION OESCAIPTION SITE CONDITION

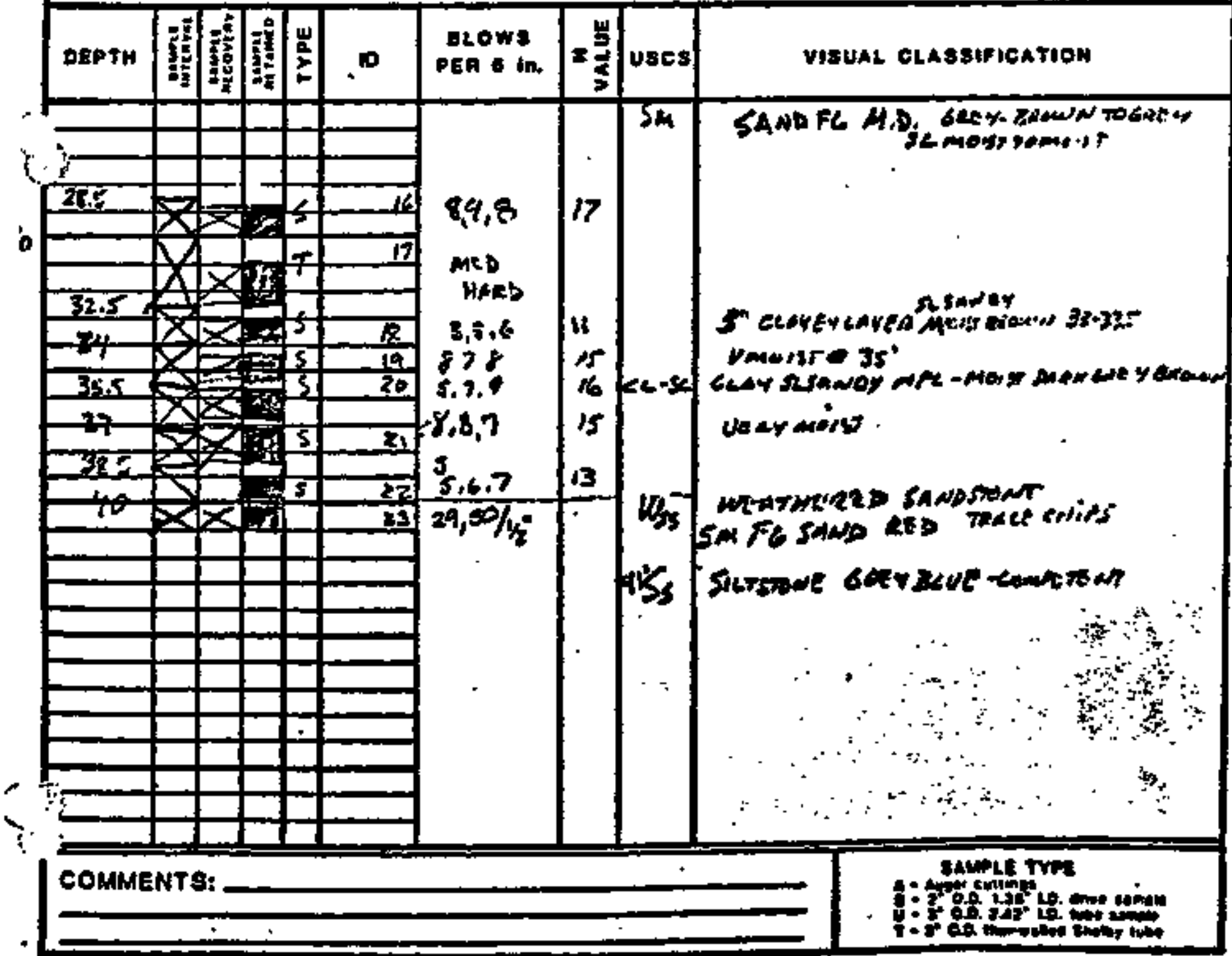




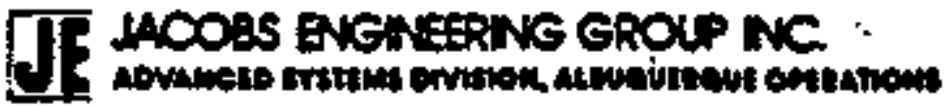

BOREHOLE LOQ(SOIL)

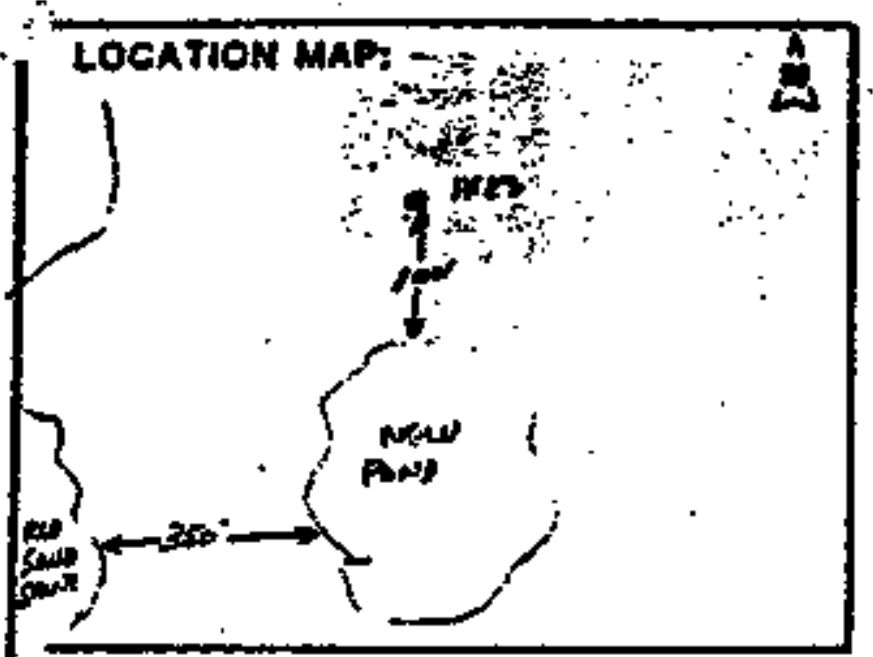

Lochton do: HTZ3

a APAOX.+\$ITE COORONATES (ti)
M.
otos

CAOUND ELEVATtON (ft. MSL):

DAILLING METHOD: WA

DAILLEA: TRAT

DATE STAATED:

DATE COMPLETEO:

FIELD REP.: SHLL

\begin{tabular}{|c|c|c|c|}
\hline \multicolumn{3}{|c|}{ CAOUNDWATER LEVELS } \\
\hline DATE & IIME & DEPTH (1.) \\
\hline & & & \\
\hline & & & \\
\hline
\end{tabular}

LOCATION DESCAIPTION SITE CONDITION

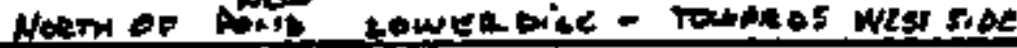

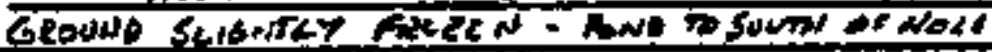

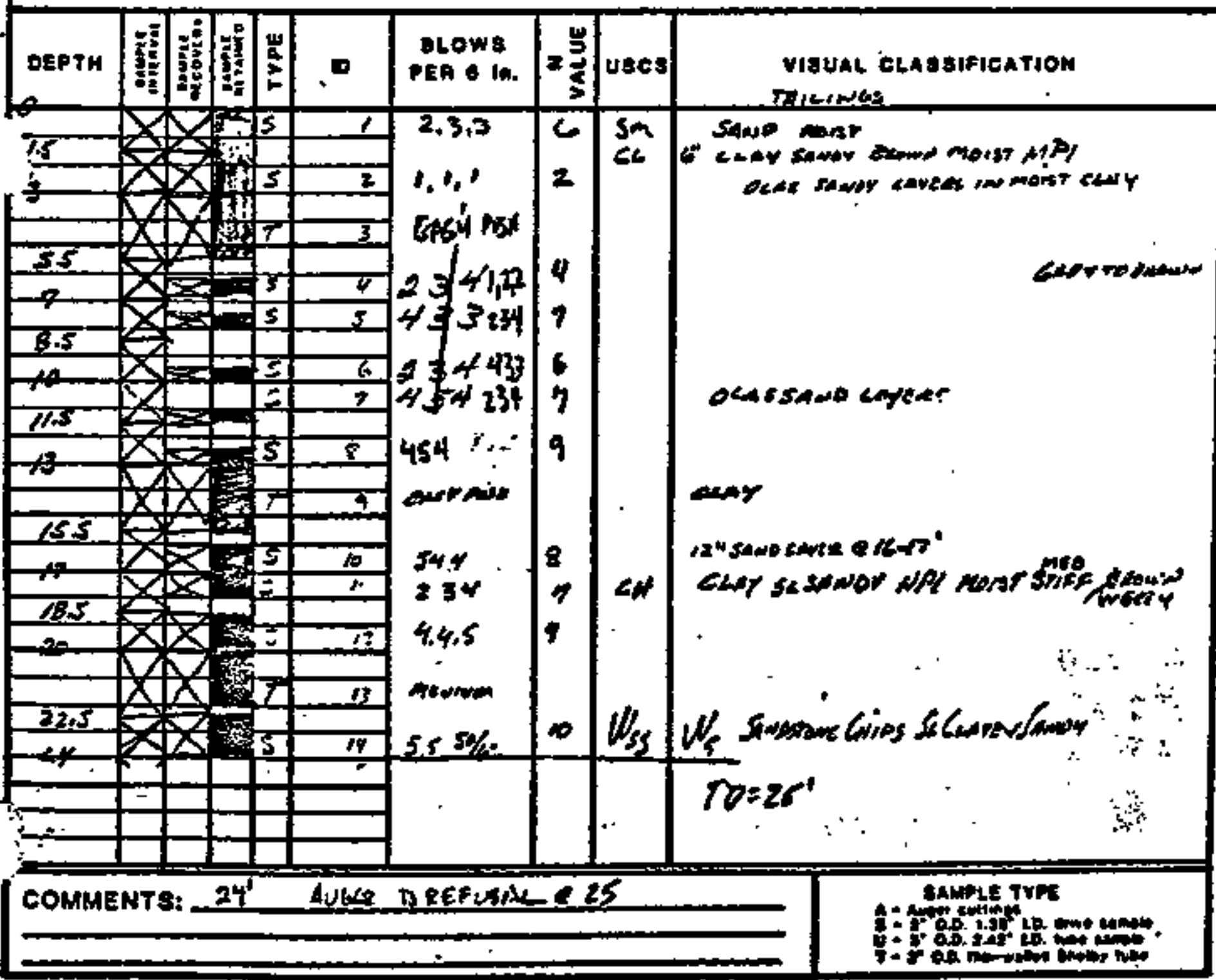

dRQ-AL-EWG-ge (9/BA) 


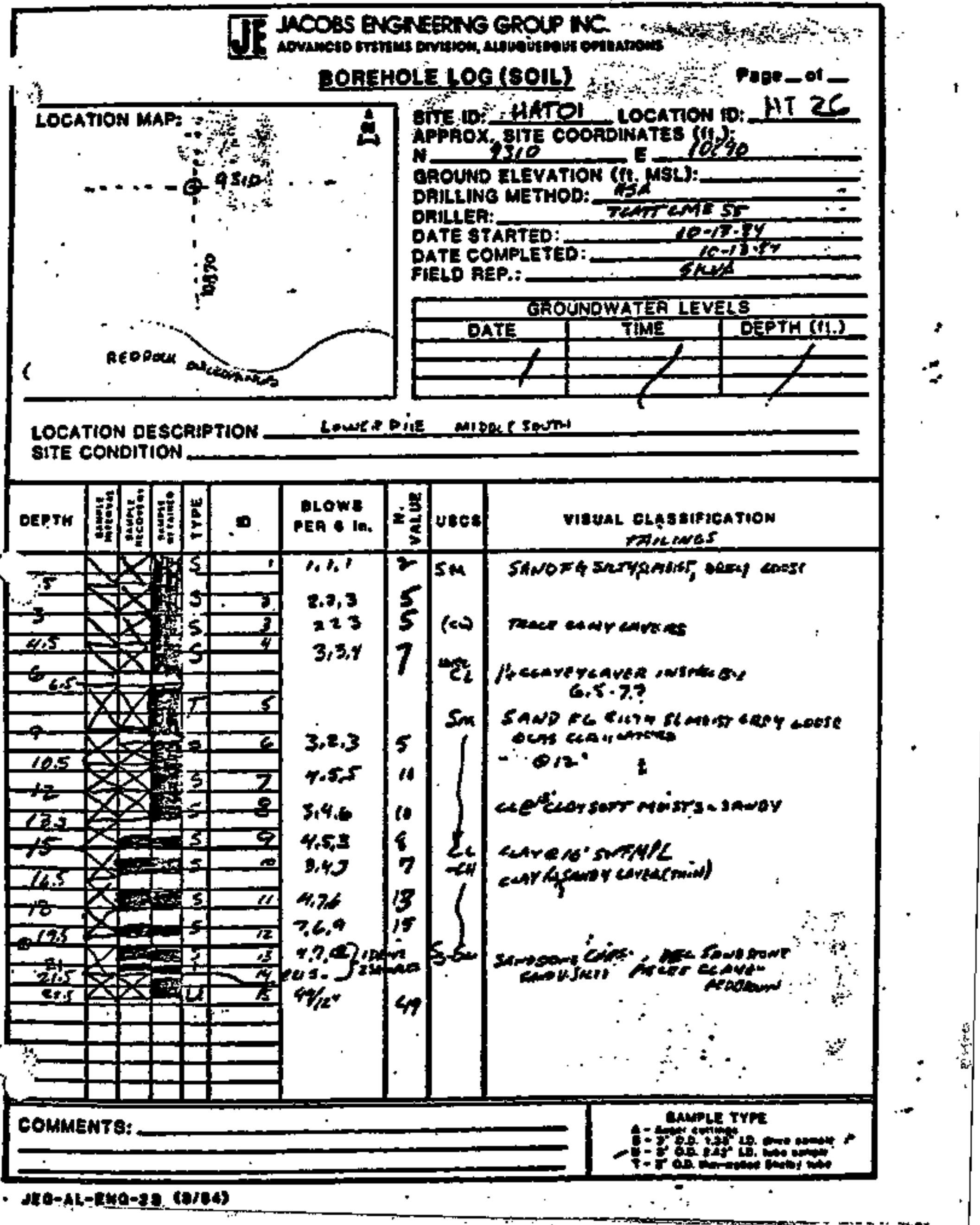




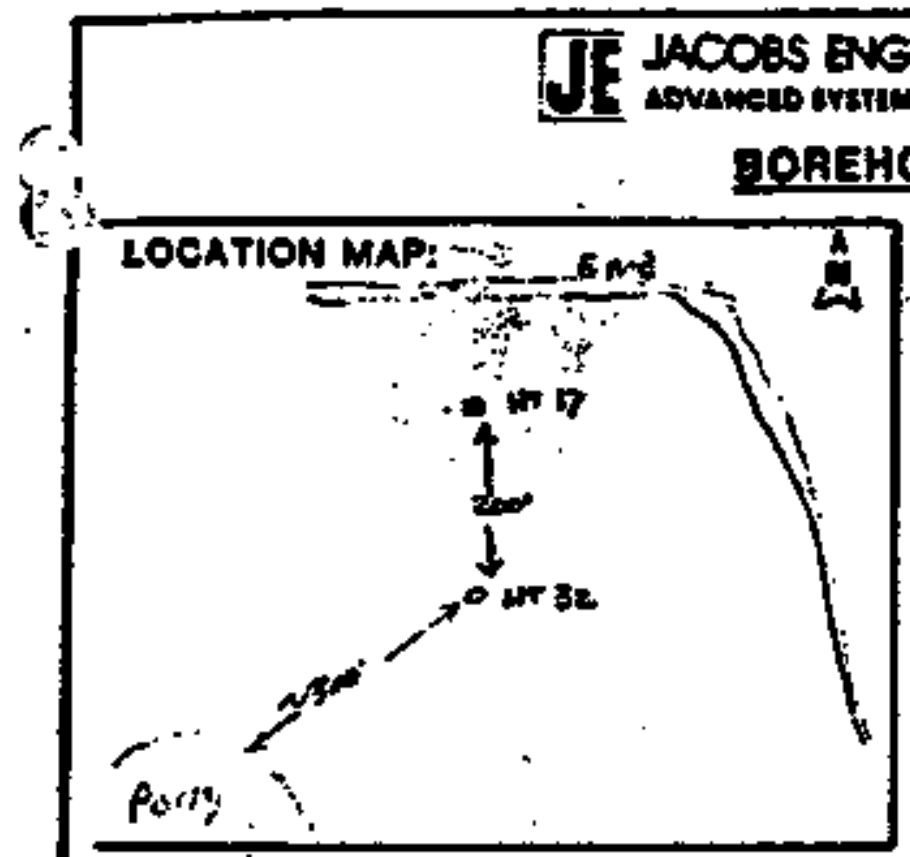

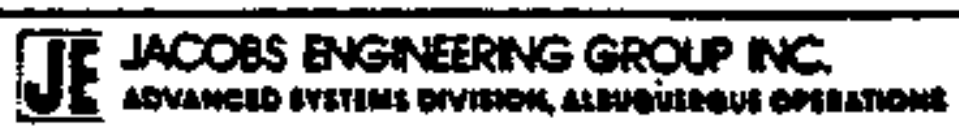

BOREHOLE LOO (8OIL)

Pooe 1 of $z$

\section{ifon}

Location 10 HT32 APPROX. BITE COORDWATES (ft.).

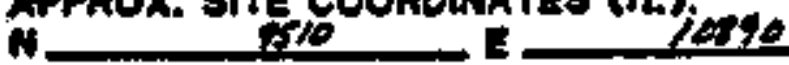

GAOUND ELEVATION (tt. MSL):

DAILLING METHOO:

DAILLEA: Ar:A.

DATE STAATED:

DATE COMPLETEO:

FIELD REP.:

STiva

\begin{tabular}{|l|l|l|l|}
\hline \multicolumn{2}{|c|}{ GEOUNDWATER LEVELS } \\
\hline OLTE & TIME & \\
\hline & & \\
\hline
\end{tabular}

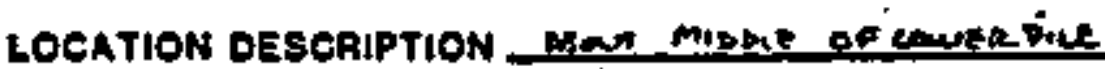

SITE CONDITION

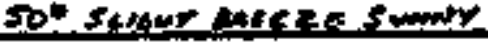

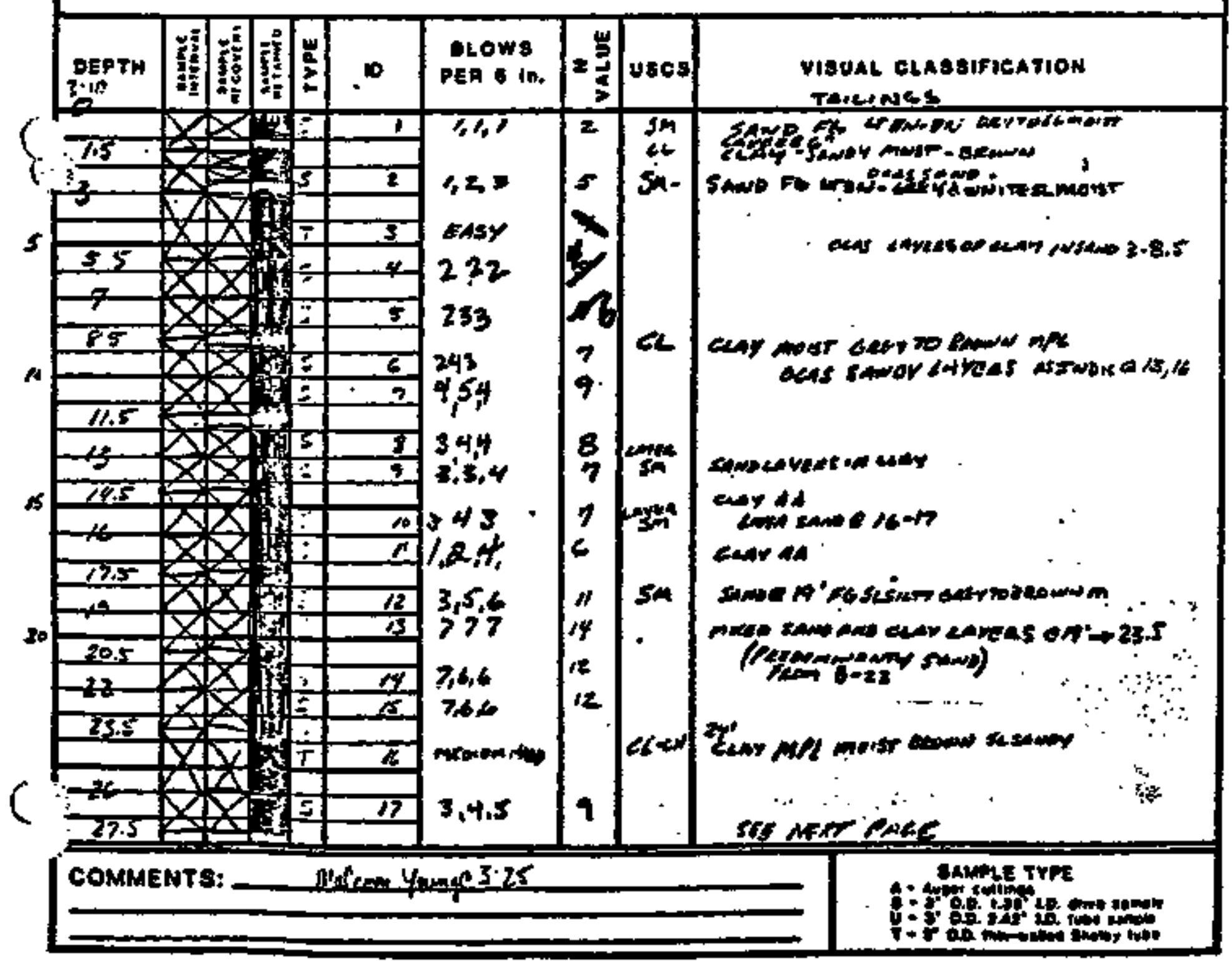

t(to-AL-Ewa-se (ajab) 
BOREHOL LOE (SOLL)

LOCATION MAP:

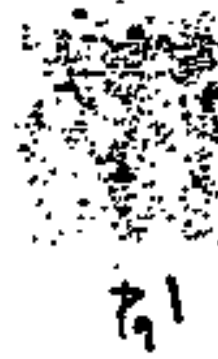

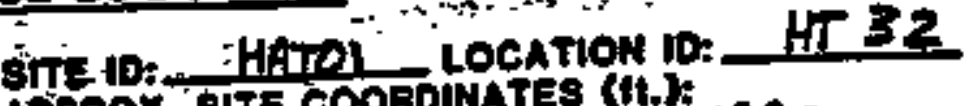

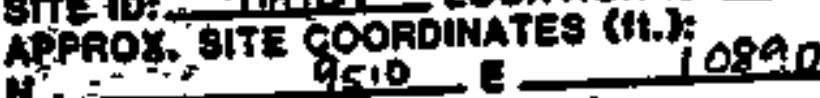
HOUND ELEVATION (tt. MSL): DRILLINO METHOD:

DAILLEA:

DATE STARTEO:

DATE COMPLETED:

FIELD REP.:

\begin{tabular}{|c|c|c|}
\hline \multicolumn{2}{|c|}{ GROUNDWATER LEVELS } \\
\hline DATE & TIME & DEPTH (it.3 \\
\hline & & \\
\hline & \\
\hline
\end{tabular}

\section{LOCATSON DESCRIPTION}

SITE CONDITION

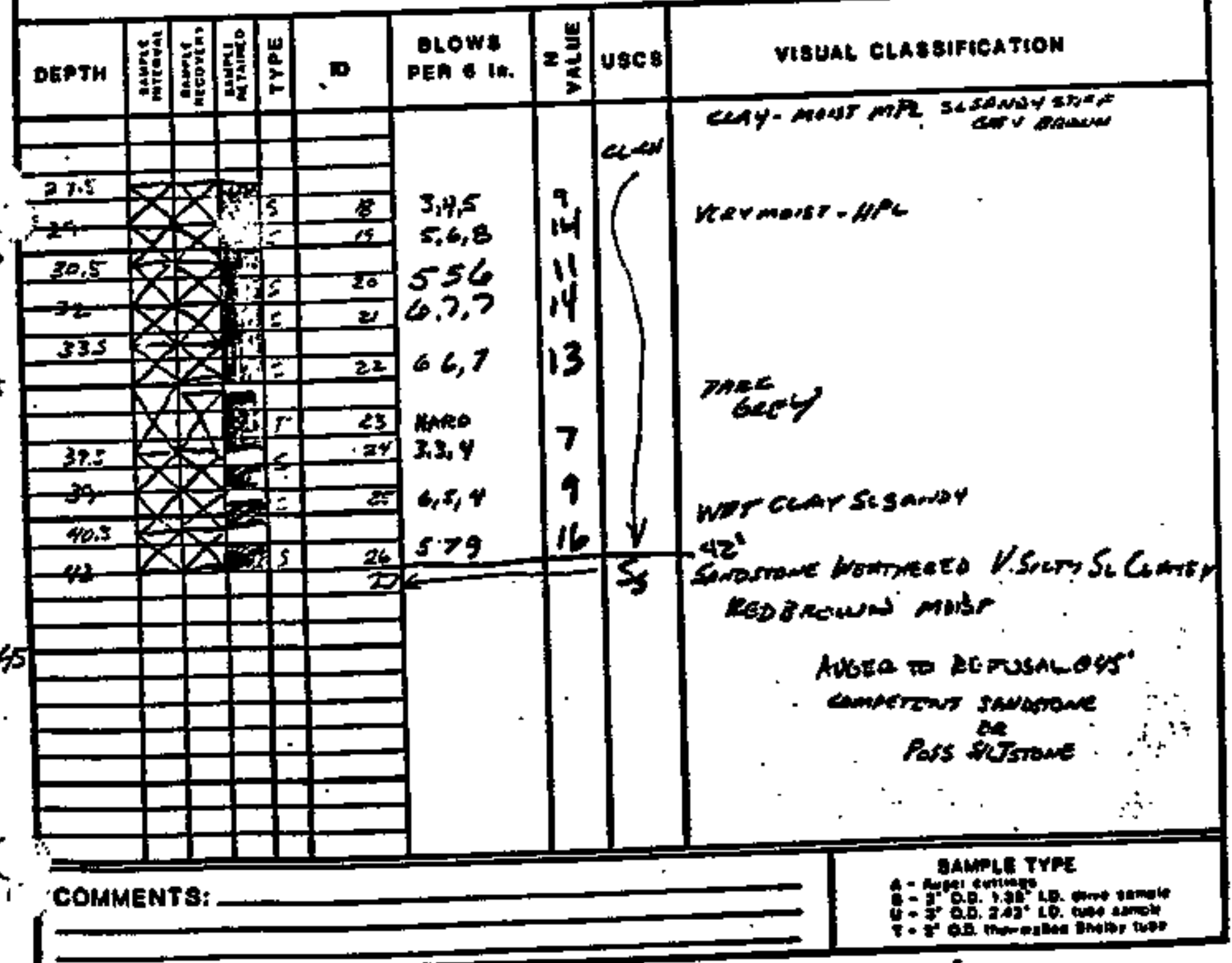




\section{DE LCOAS ANCWEERNG GROUP NC. $\therefore$}

BOREHOLE lOQ (8O14) $\cdots$ Page tof 1

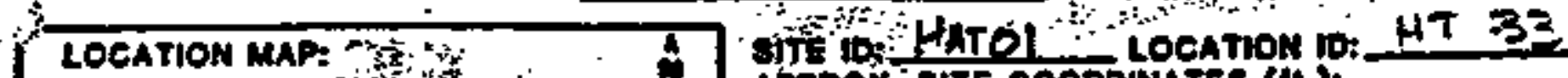

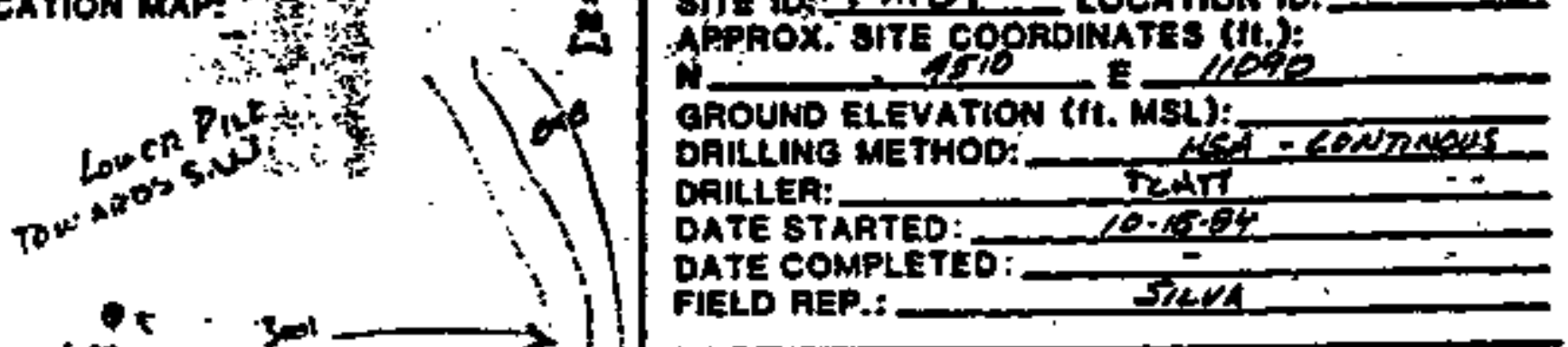

H 33

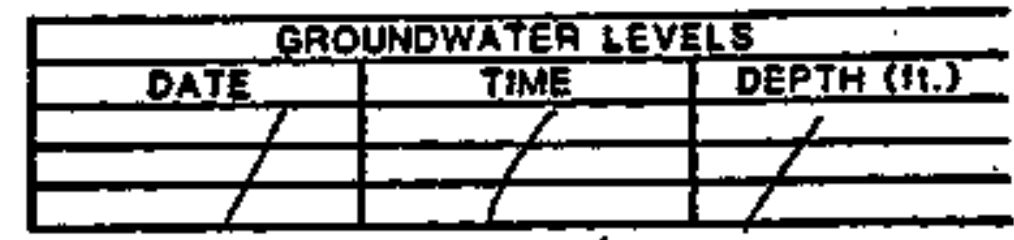

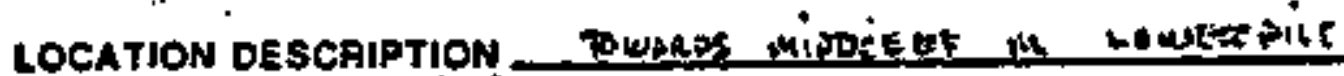

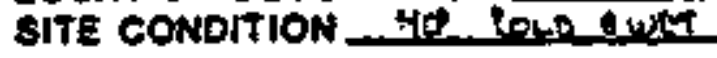

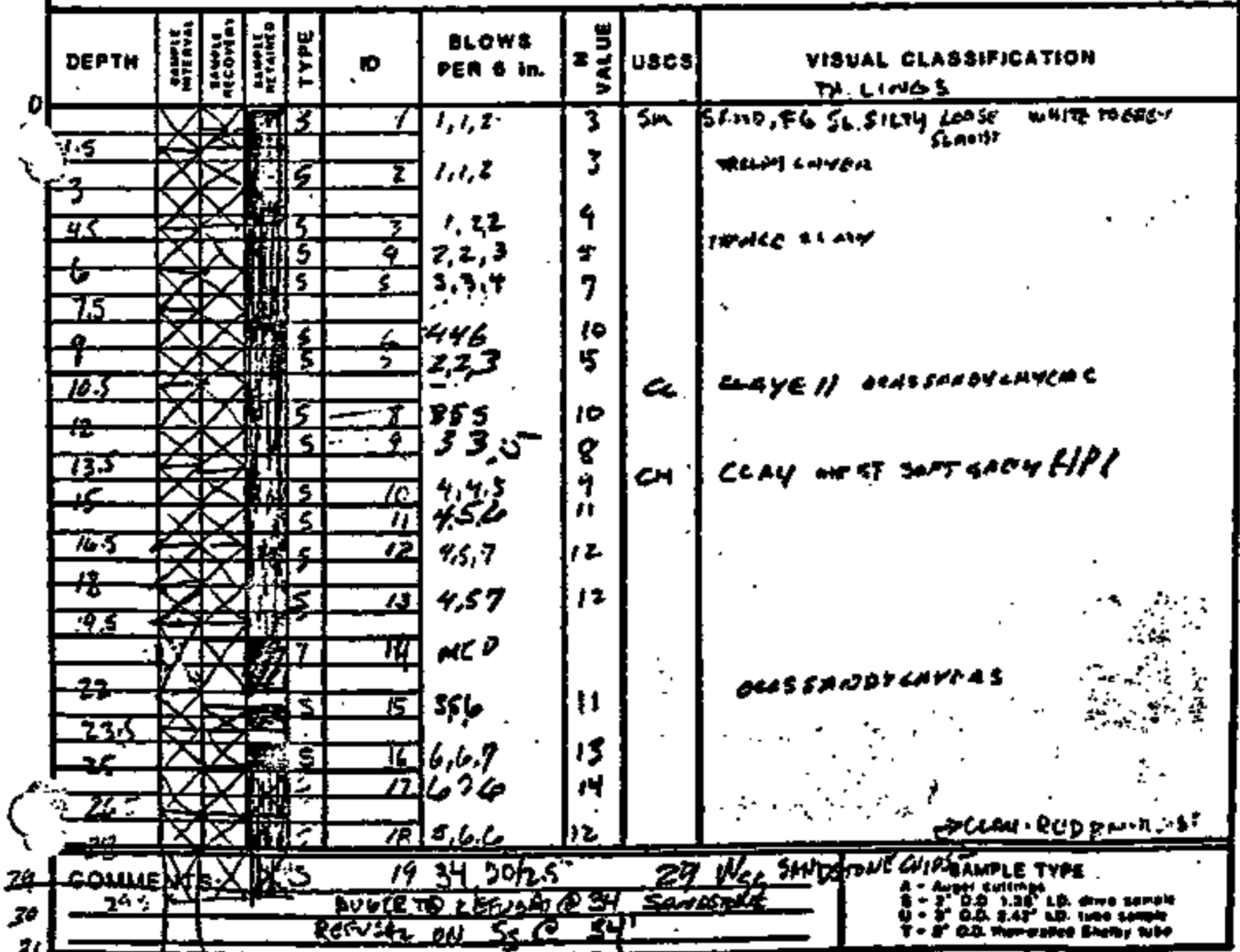

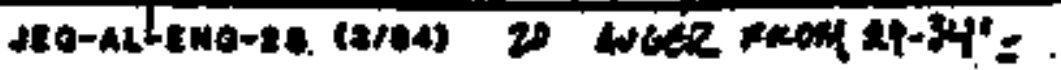




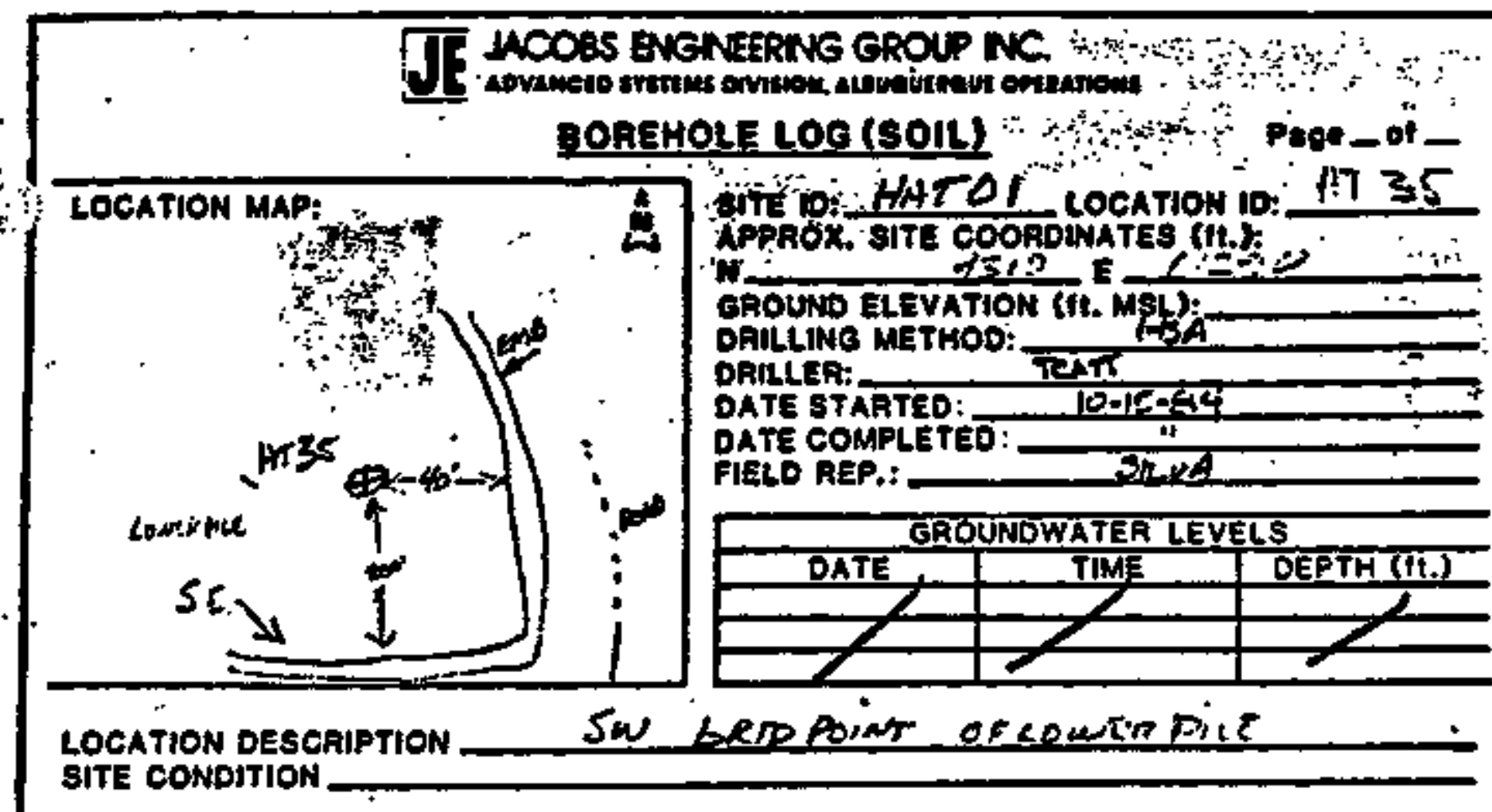

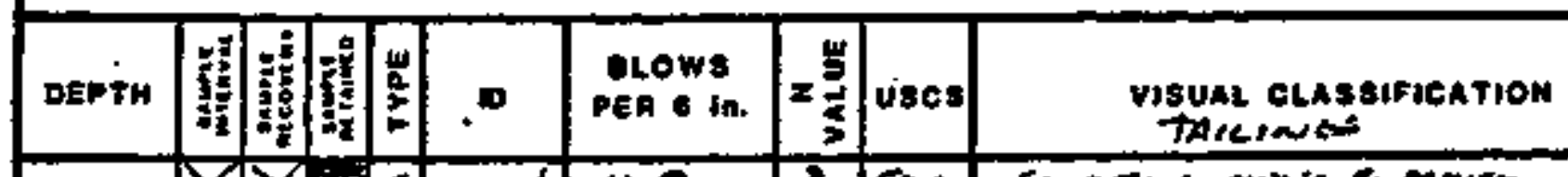

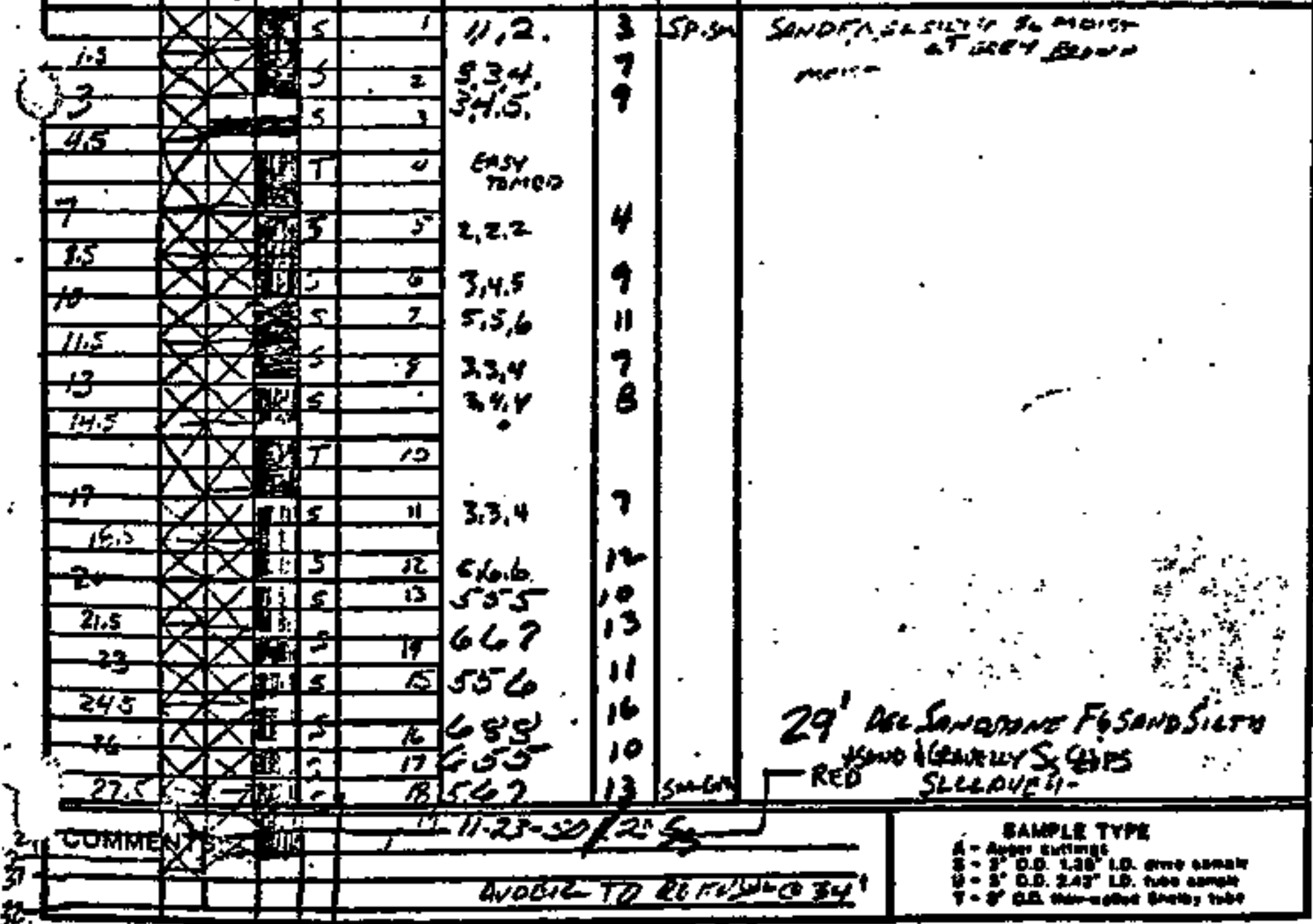

JEO-AL-Eue-z). (F/4) 
TF HCOES ENGNARG GROAP NC.

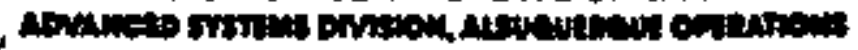

\section{OOREHOLE LOC (SOH)}

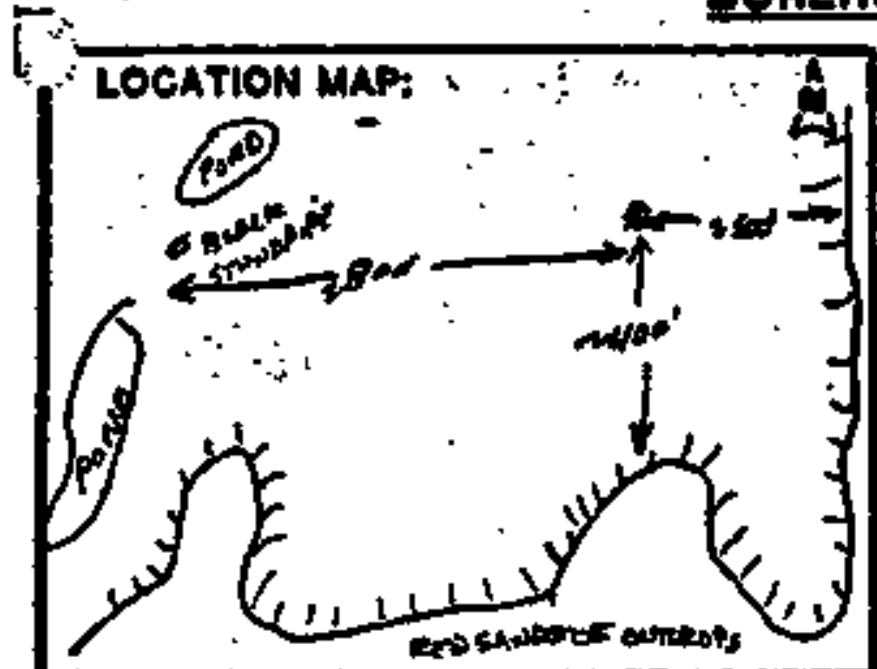

Give Hatiol

Locatron ID: 911 .

al APPAOX OTE COOADINATES (t.):

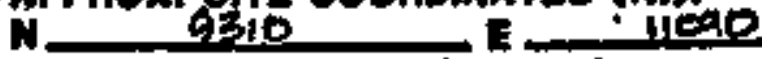
GROUND ELEVATION (t. MBL).

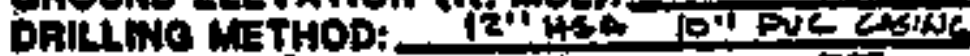

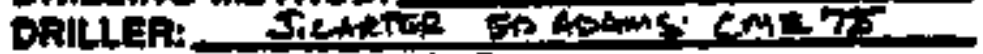
DATE BTARTED: $1-5+5$

DATE COMPLETED: GROATDAN T-GFF

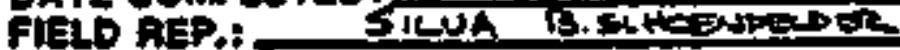

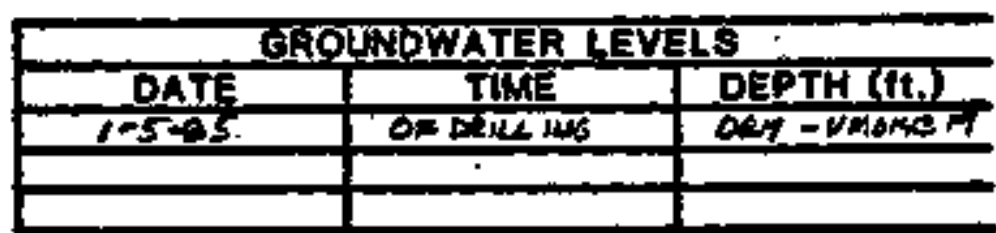

LOCATION DESCFIPTION

IAT ONPILE IO" LASING INSTALGRONON SITE CONDITION ON TALING TOWTL DILE TOWARD EASTE-SOLTA

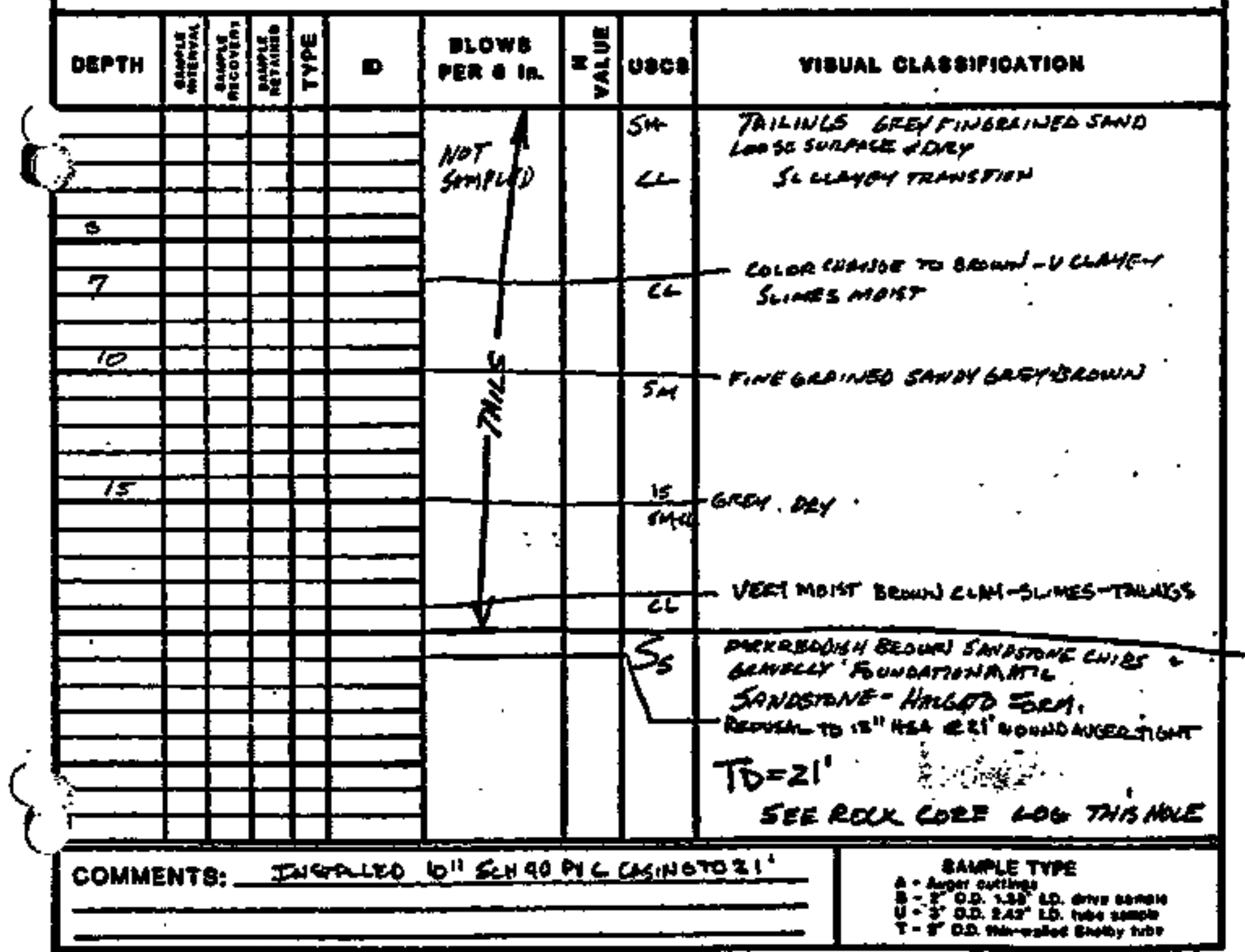

JEe-AL-IMe-2t. (s/tu) 


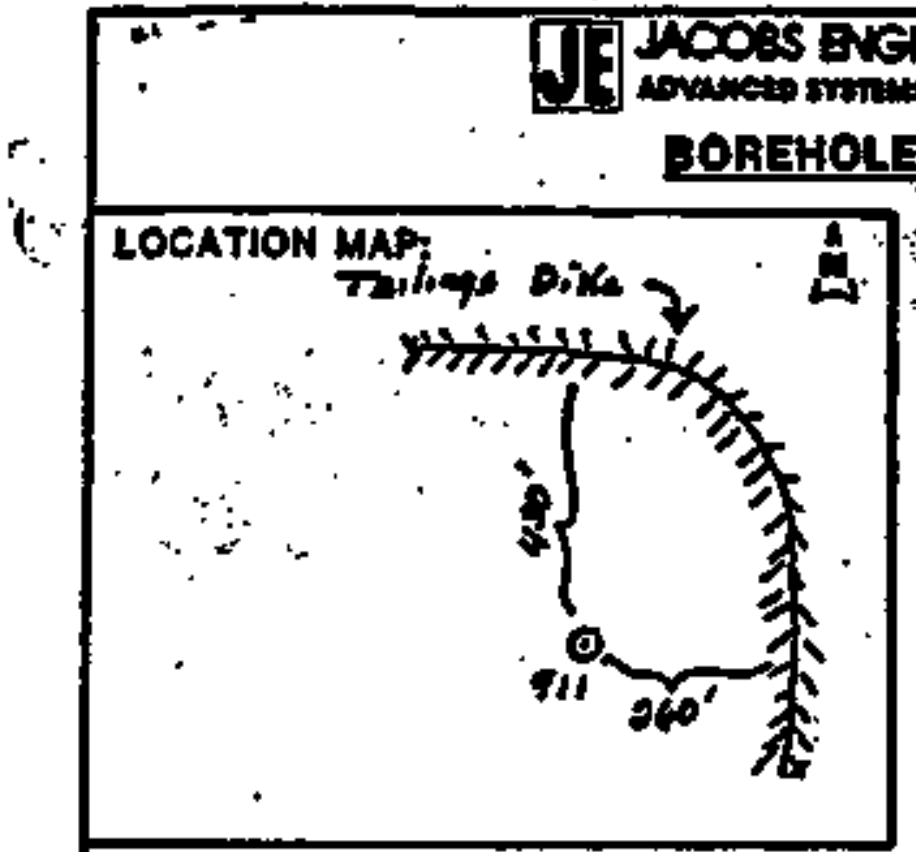

\section{Fave CRolp are

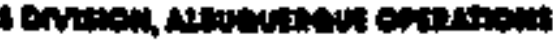

Page $<\mathrm{ol}$

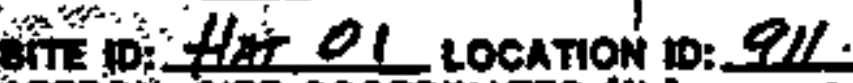
APPROX . BITE COOADINATES (t.):

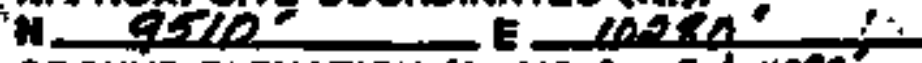

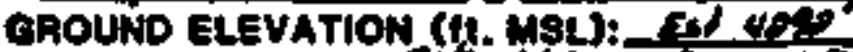

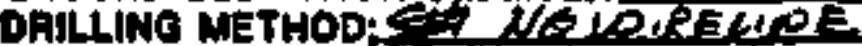

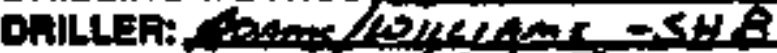
DATE STARTED: 214 LEL DATE COMPLETED: FIELO REP.: TA Machars

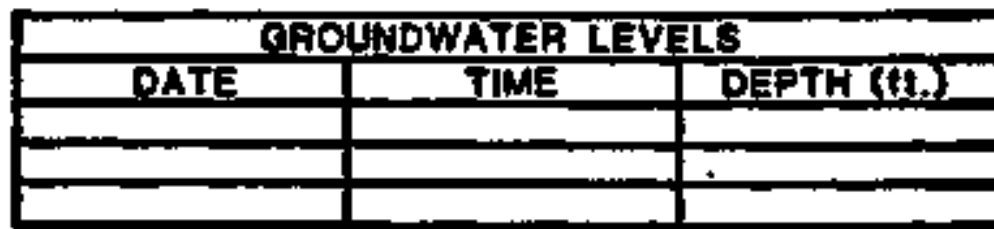

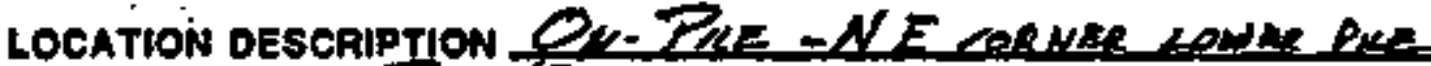
SITE CONDITION $72 z y, 70.72$

$*$
8

$$
1
$$
(1) wibs anche

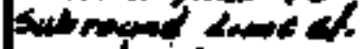
aref, $>$ rant. wy miner ater. ation ategles $46 \div$ oults hene ar as oury cofte and

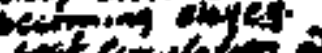

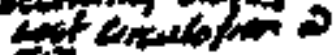
soft to dord

8 vater lavels.

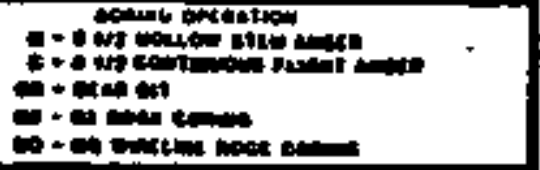




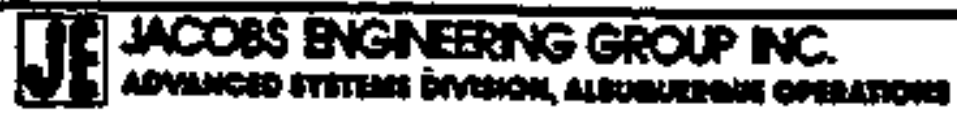

BOREHOLE LOO (BOCK) : = O

LOCATLON MAP: $\because$ D

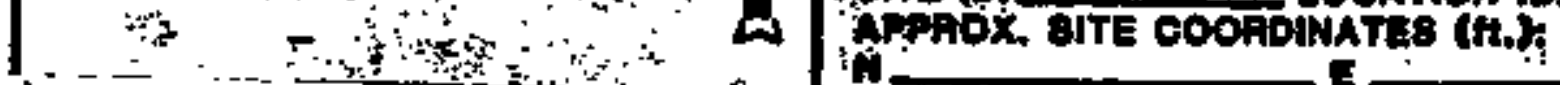

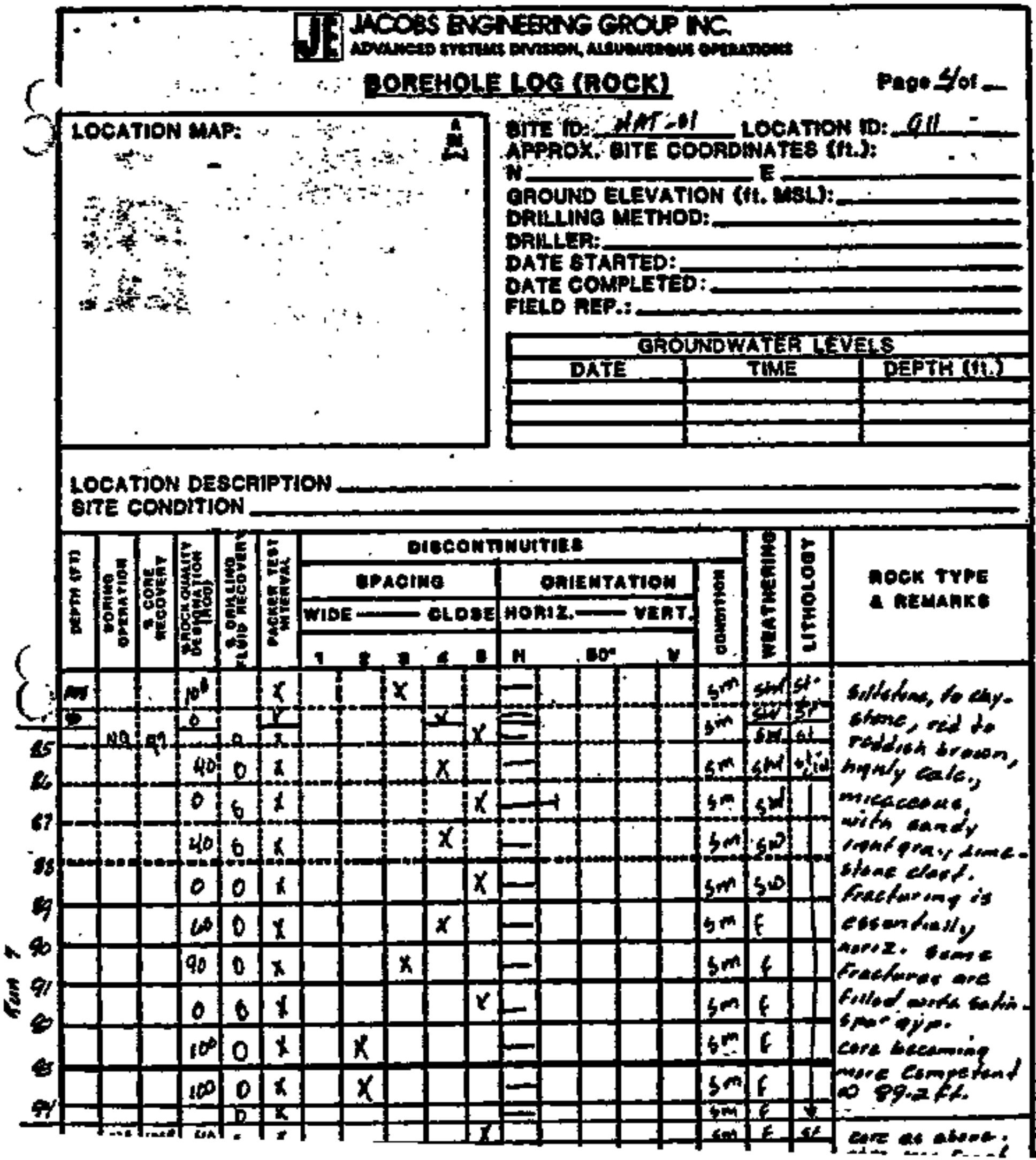




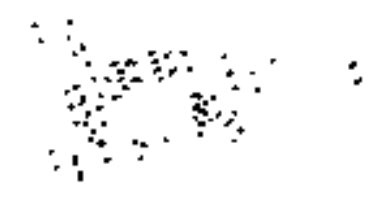

TAC TEST PITS:

ON PILE

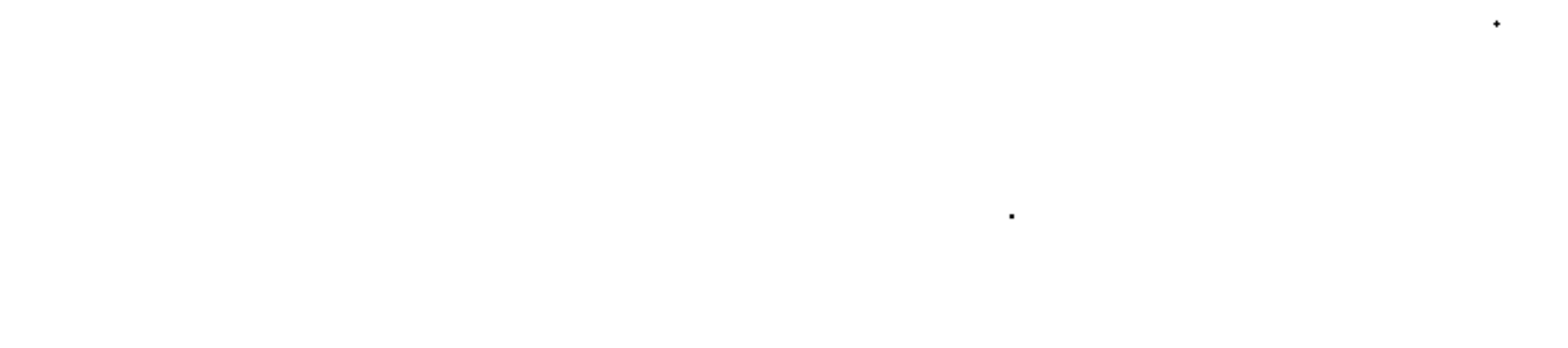



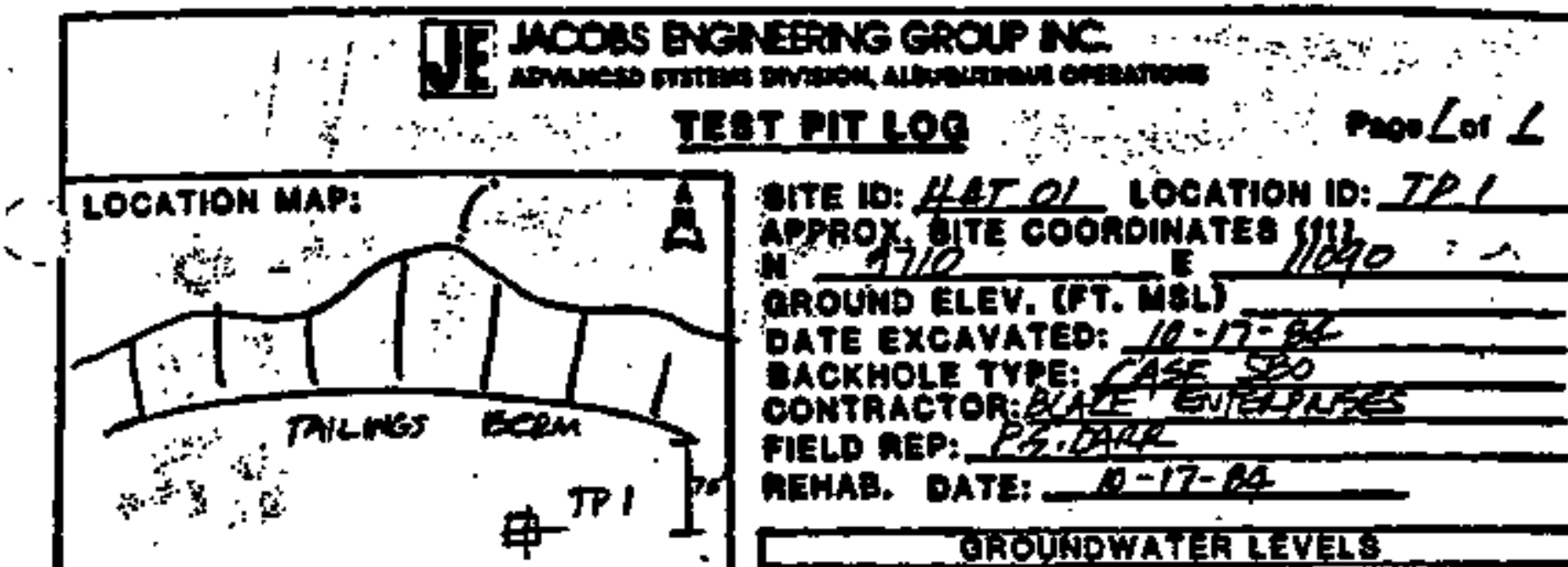

ATE D: $\angle 4 T$ of LOCATIOH ID: 72

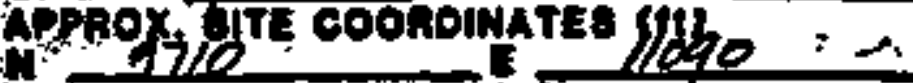
CRound ELEV, (TT. MS Date excaVATED: $10-17=2$ BACKHOLE TYPE; CASE $\$$ CONTRACTOR Q FIELD AEP: EZT:LFCL

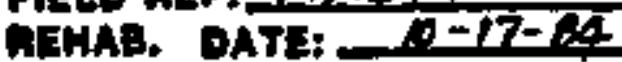

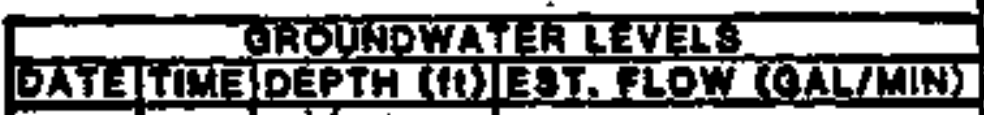

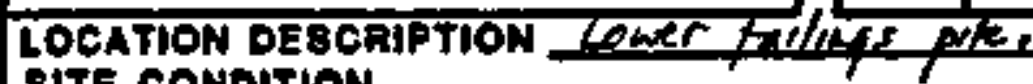

SITE CONDITION

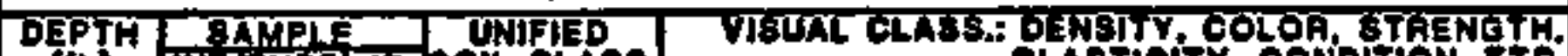
(4.) UNh TYSETD SOLL CLA8S.

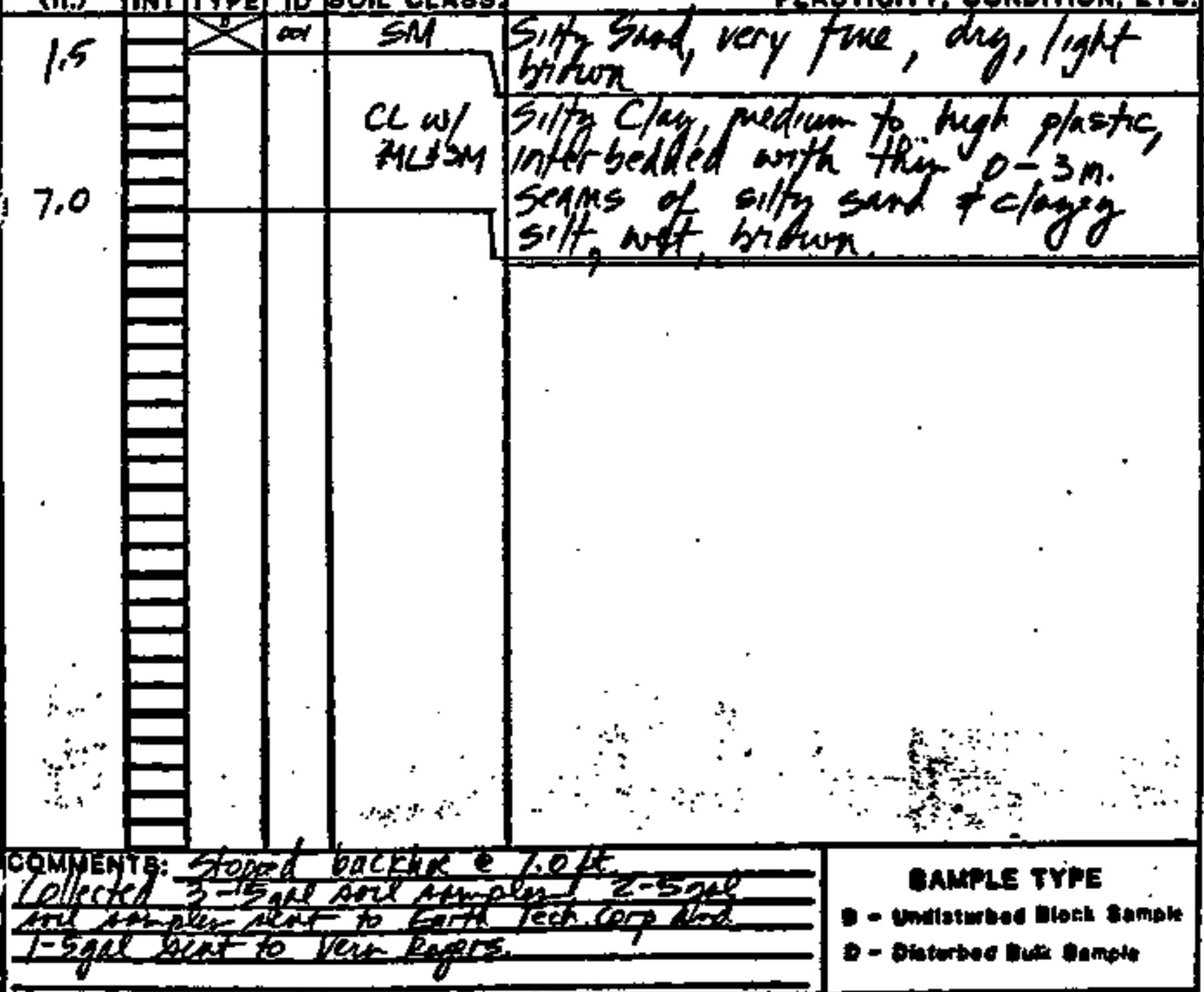

JEO-AL-ENG-T (SHA) 


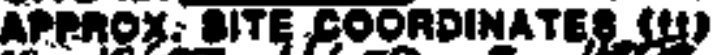

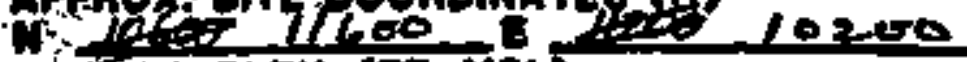

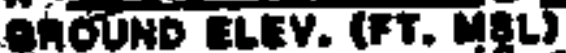

DATE EXCAVATED: $D-2 F E$

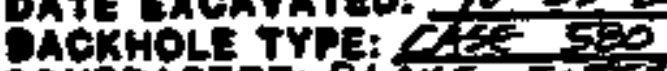

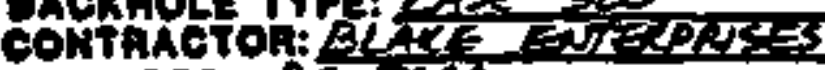

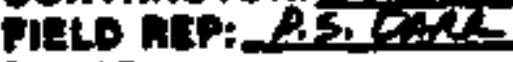

AEHA. PATE;

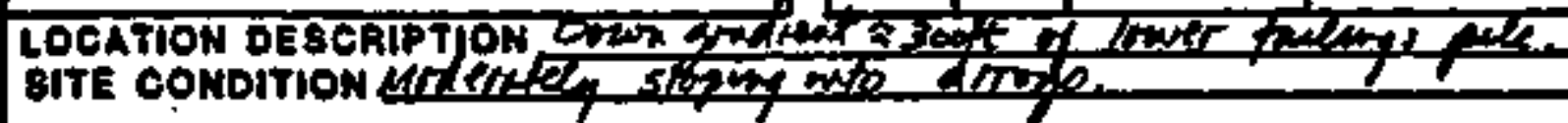

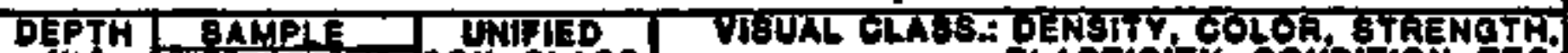

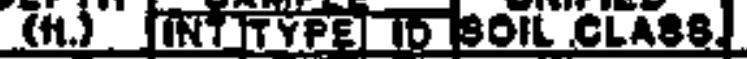

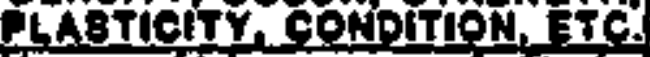
0.5 IXT TYPE ID BOIL,CLAB.

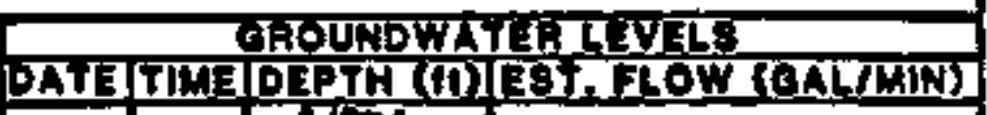

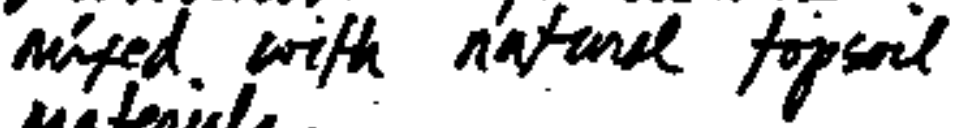
Mafoidas 


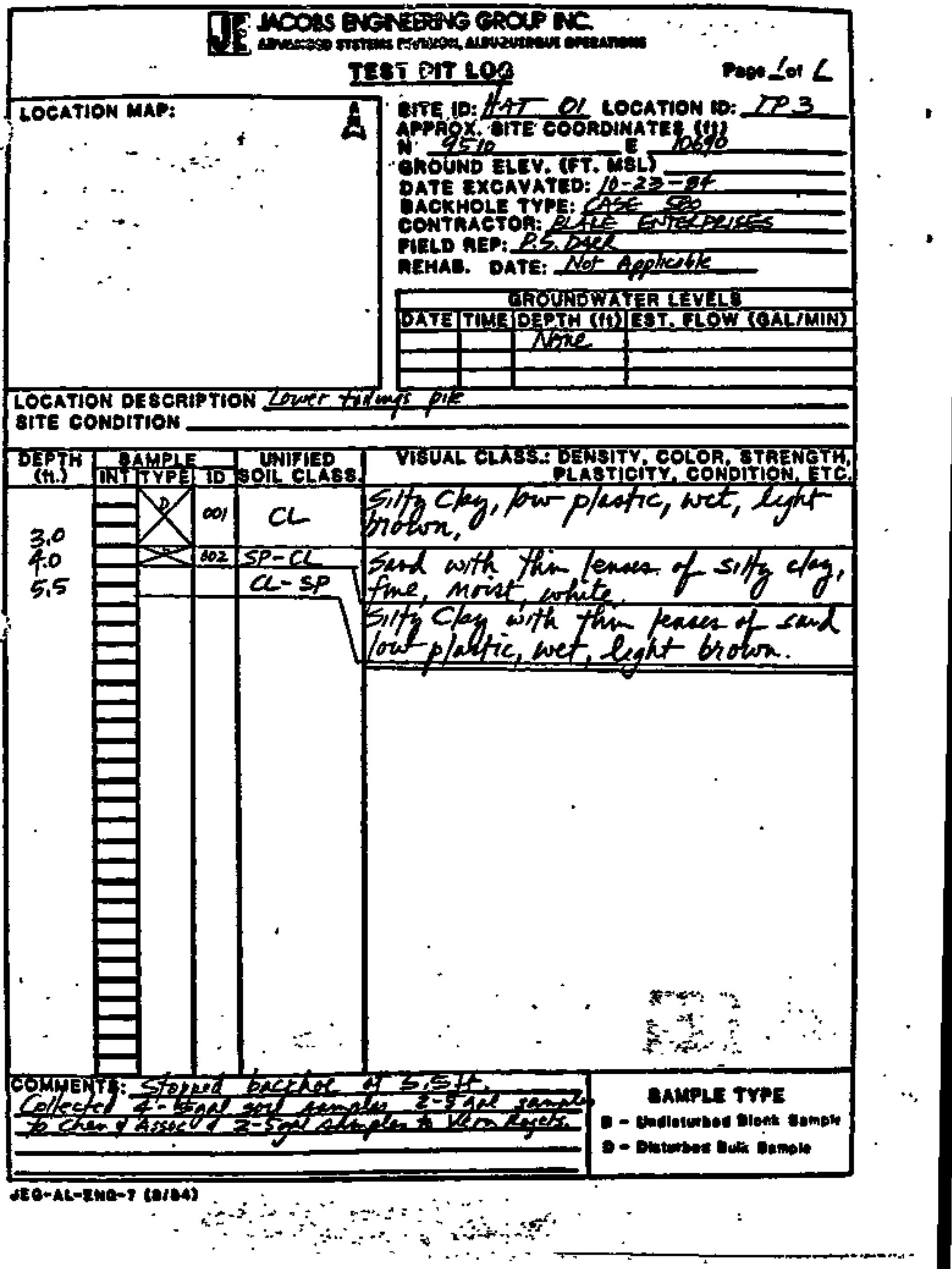




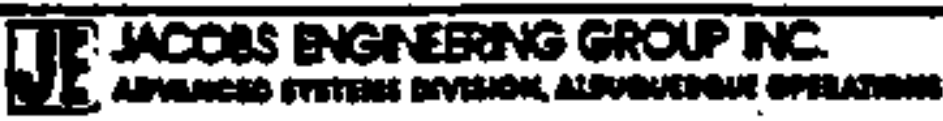

TEST NT 1.09

$\lim \angle$ or $\angle$

\section{LOCATION MAP:}

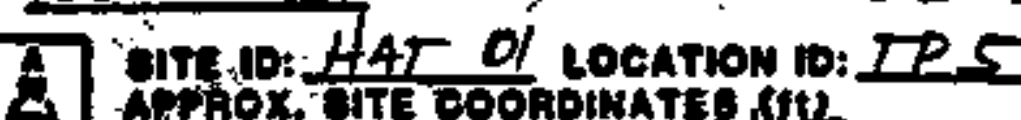

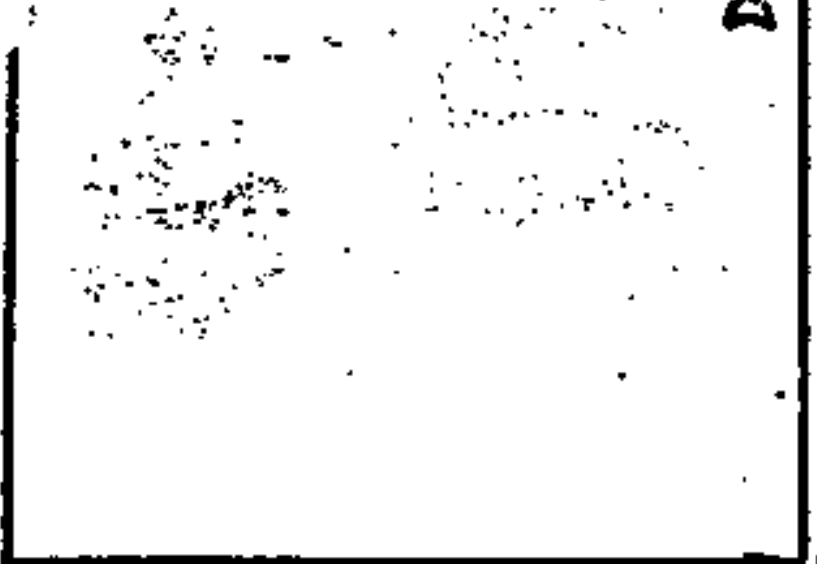

(a)

GOUND ELEV. (FF. Dht

DATE EXCAVATED: $10-23-84$

GACKHOLE TYPE: CACE 530

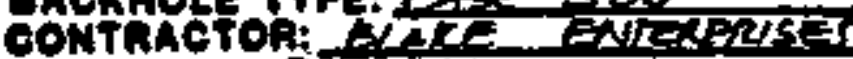

FIELD REP: $A 5, D$.

REWAB. DATE: STE Aptholle

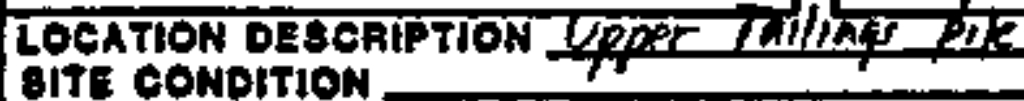

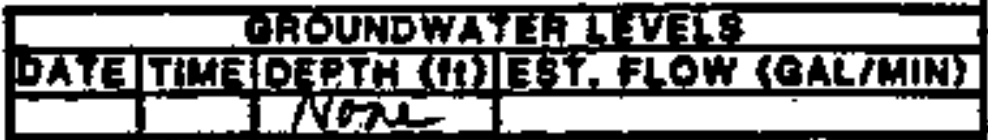

DEFTH BAMPLE $]$ UNIFED

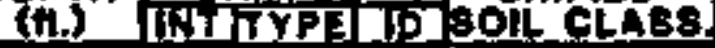

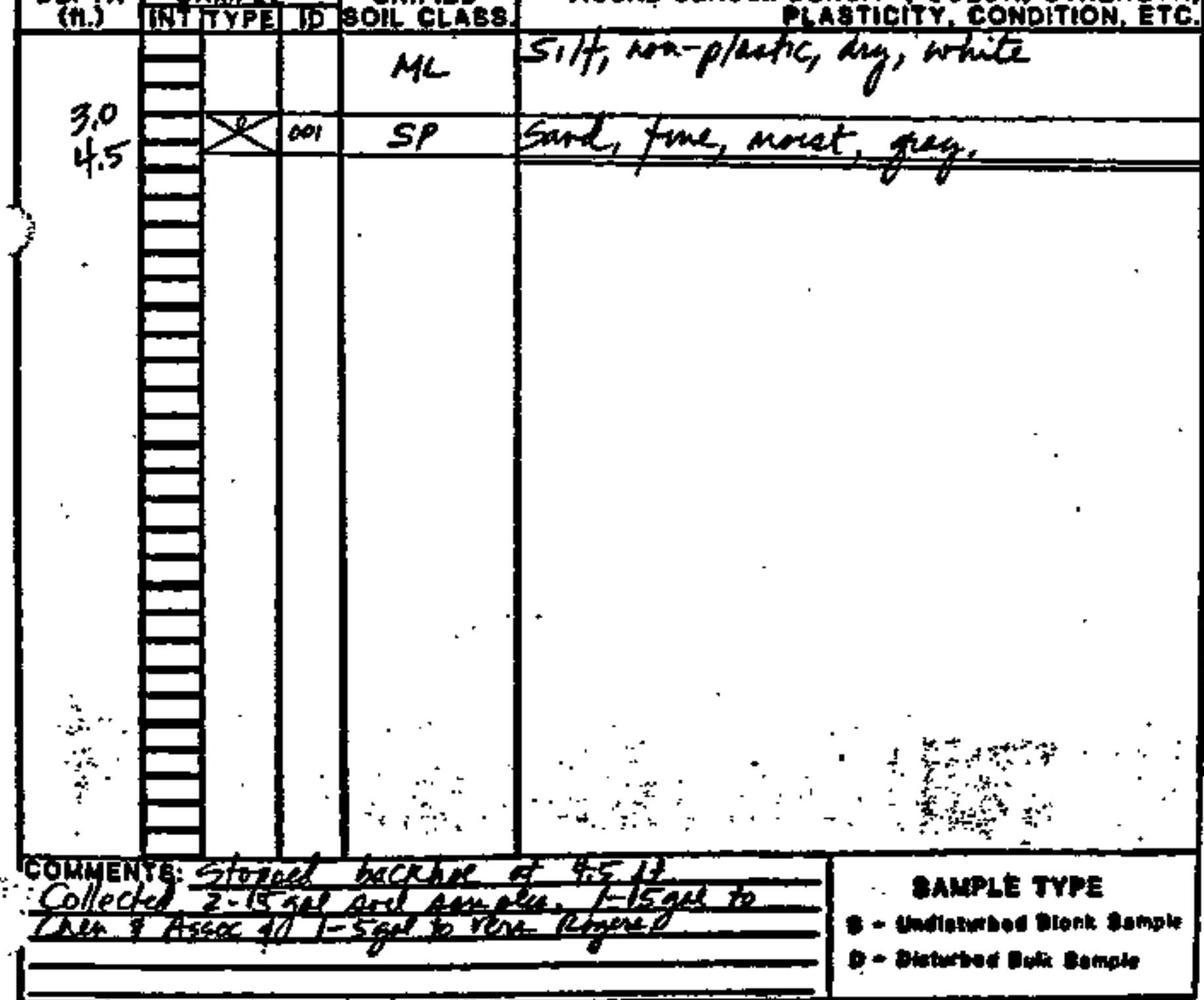

dto-At-tue-s (t)ist औt 


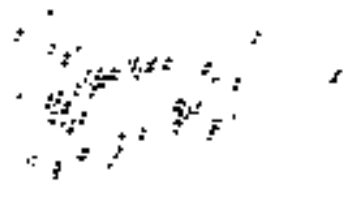

TAC BDRING LOGS:

OFF PILE 


\section{AT MCOAS ENen

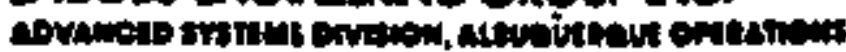

BOAEHOLE 1OC(BOIL)

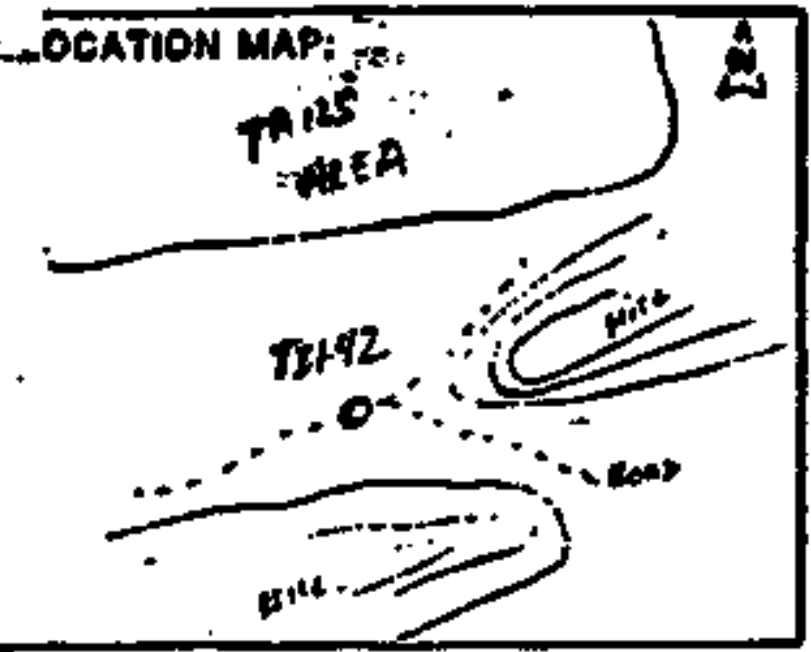

81T to Hat APPROX. BITE COOROINATES (tt.):

GAOUND ELEVATLON (H. MSL)

DRILLING METHOD: HSA

DAILLER: TeCATt

DAJE STARTED: UAOST

DATE COMPLETED:

FIELD WEP.: SIL-WA

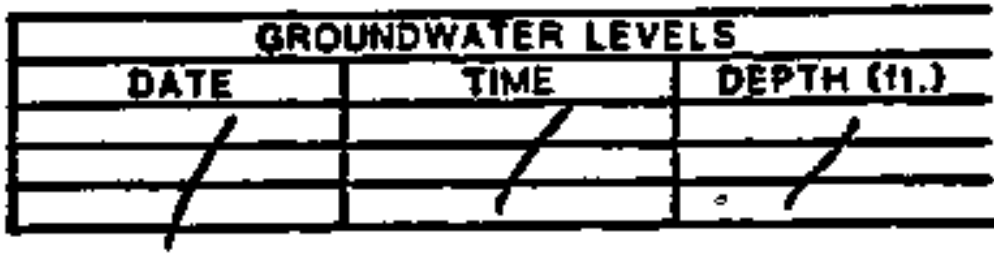

LOCATION DESCAIPTION BITE CONDITION.

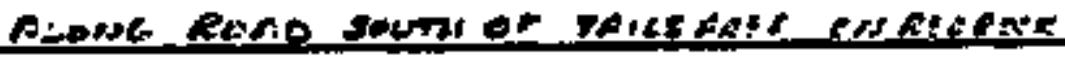
may

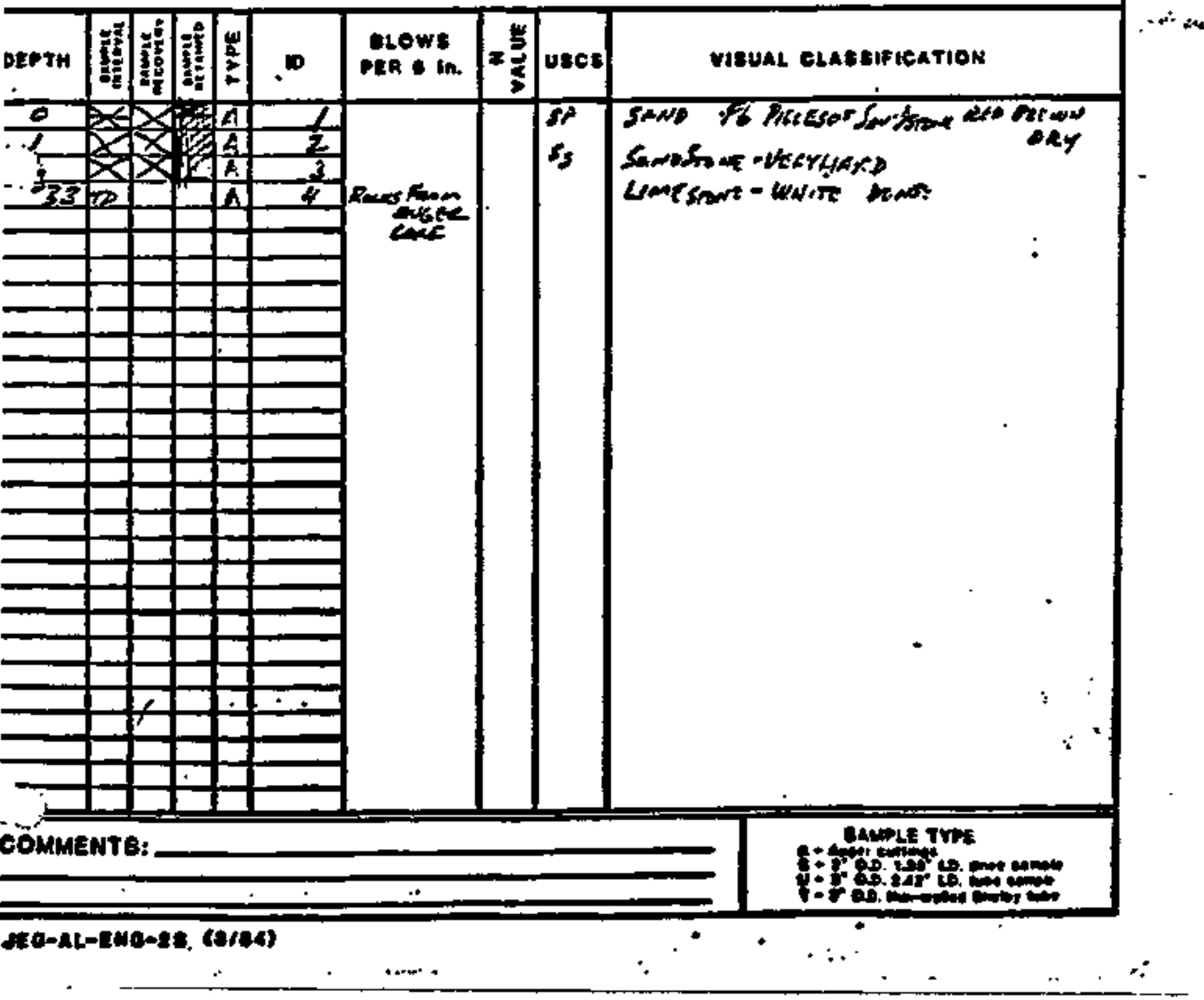




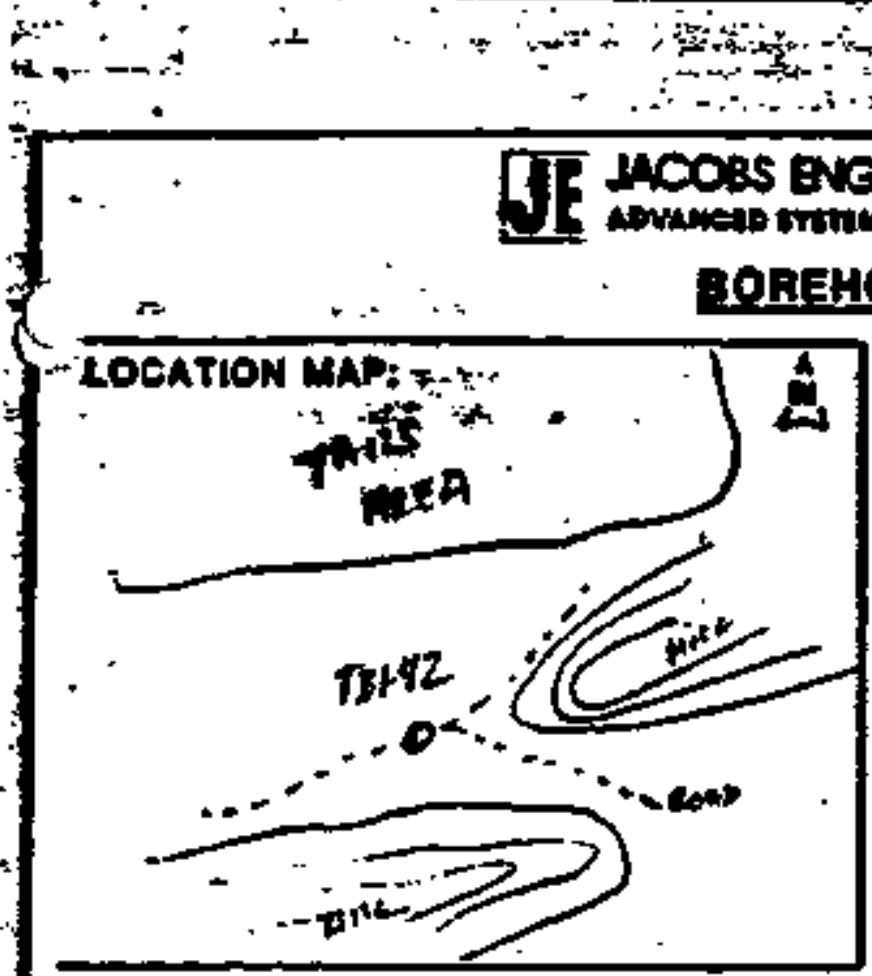

1 1 t

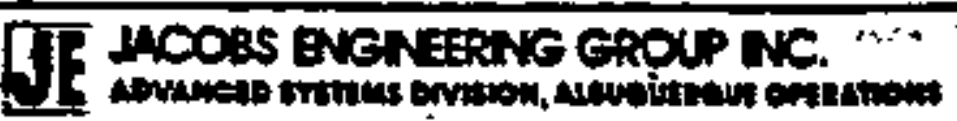

8OREHOL_ LOC (8OH)

LOCATION MAP: z-r.

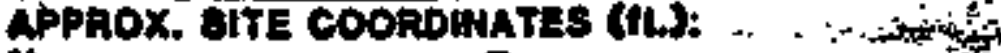

N.

GAOLNO gLEVATION (ft. MSL):

DALLLNG METHOD: usA

DRILLEA: TCAII

DATE STARTED: $20+O=\bar{C}$

DATE COMPLETED: $1 \%+10$ PQ

FIELO REP.: THL

\begin{tabular}{|r|r|r|}
\hline \multicolumn{3}{|c|}{ OROONDWATER LEVELS } \\
\hline DATE & TIME & DEPTH (1.) \\
\hline & & \\
\hline & & \\
\hline
\end{tabular}

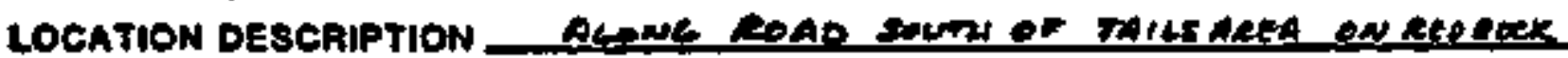
BITE CONDITION__ rey

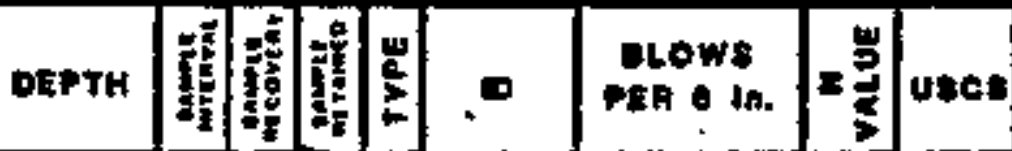

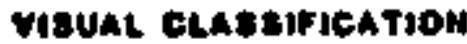

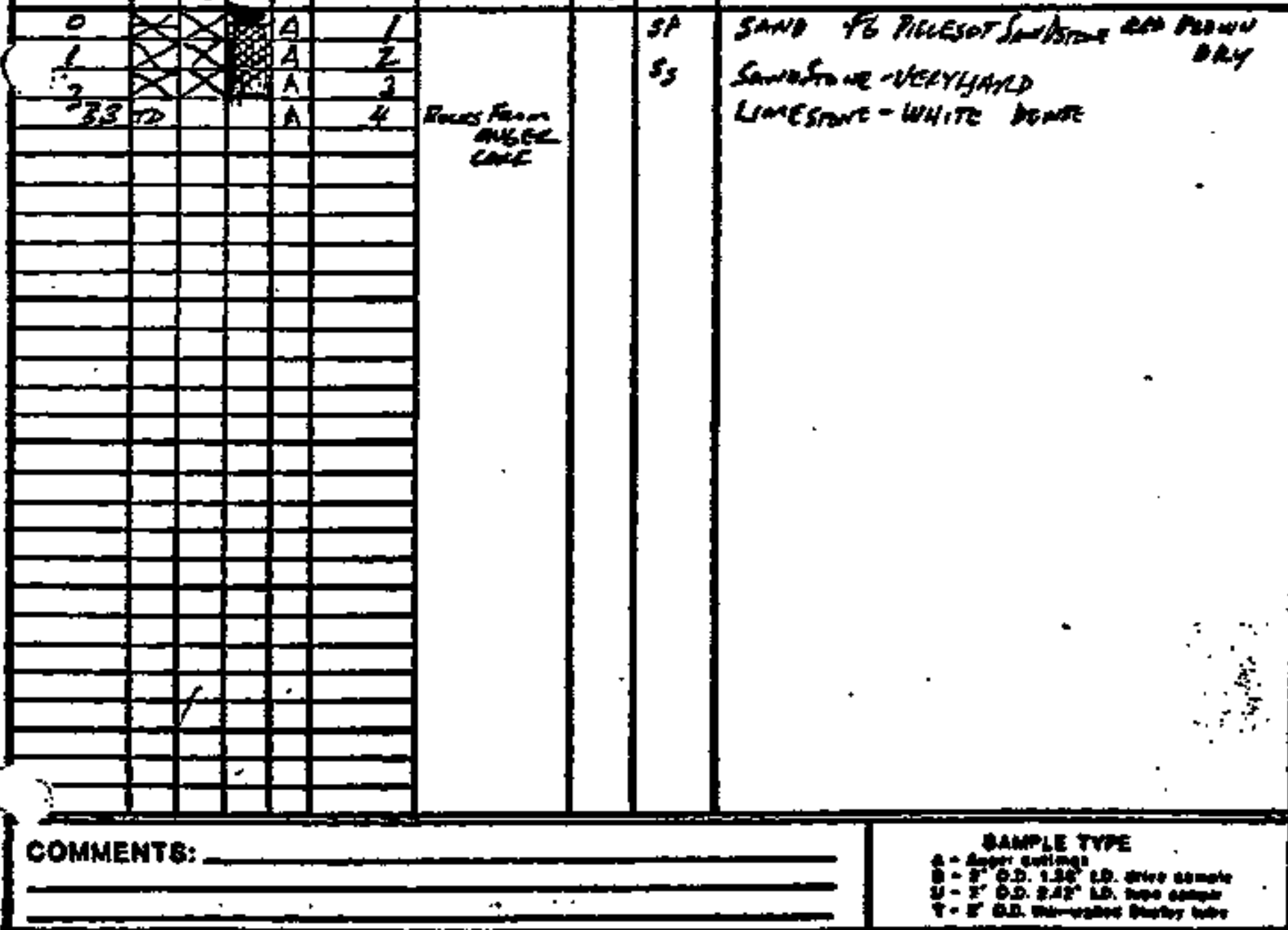




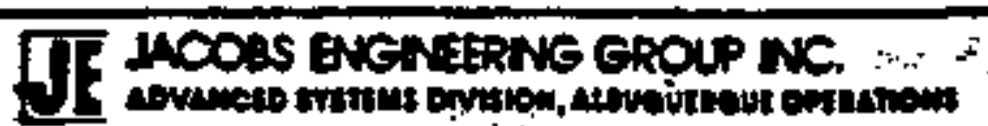

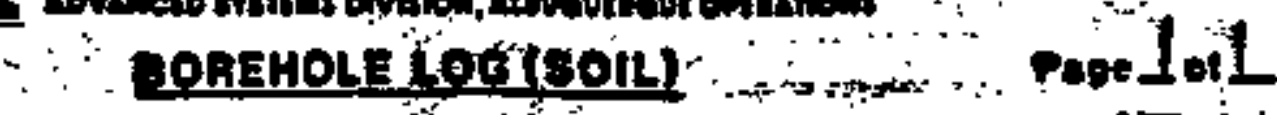
LOCATION MAP:

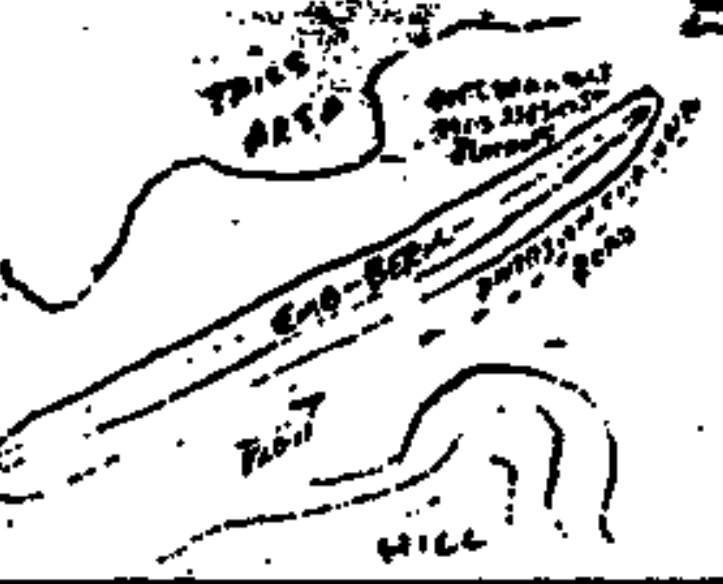

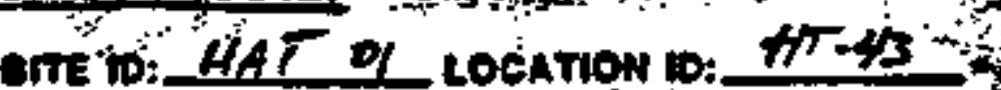
APPROX. MITE COORDINATES (Ii.):

W 8730 E 100302

tistion

Tyerts.t.

AROUN口 ELEVATION (ft. MSL):

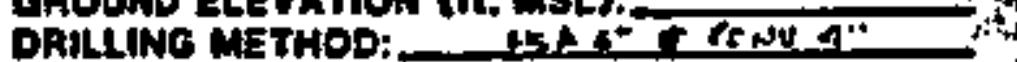

DAtLLER: Tretrat

DATE STARTED:_ $10-10.50$

DATE COMPLETED:

FIELD REP.:

$\operatorname{crs}$

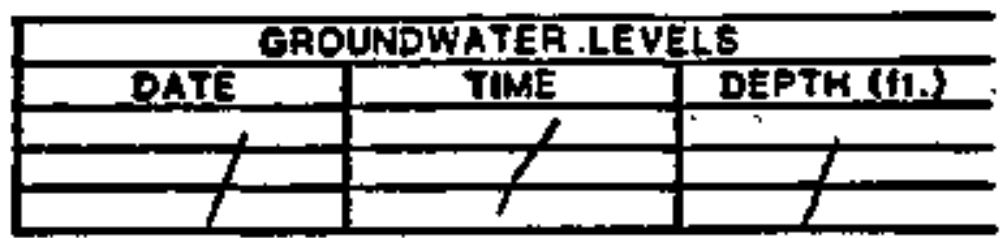

LOCATION DESCRIPTION

Gurer Sire ondis

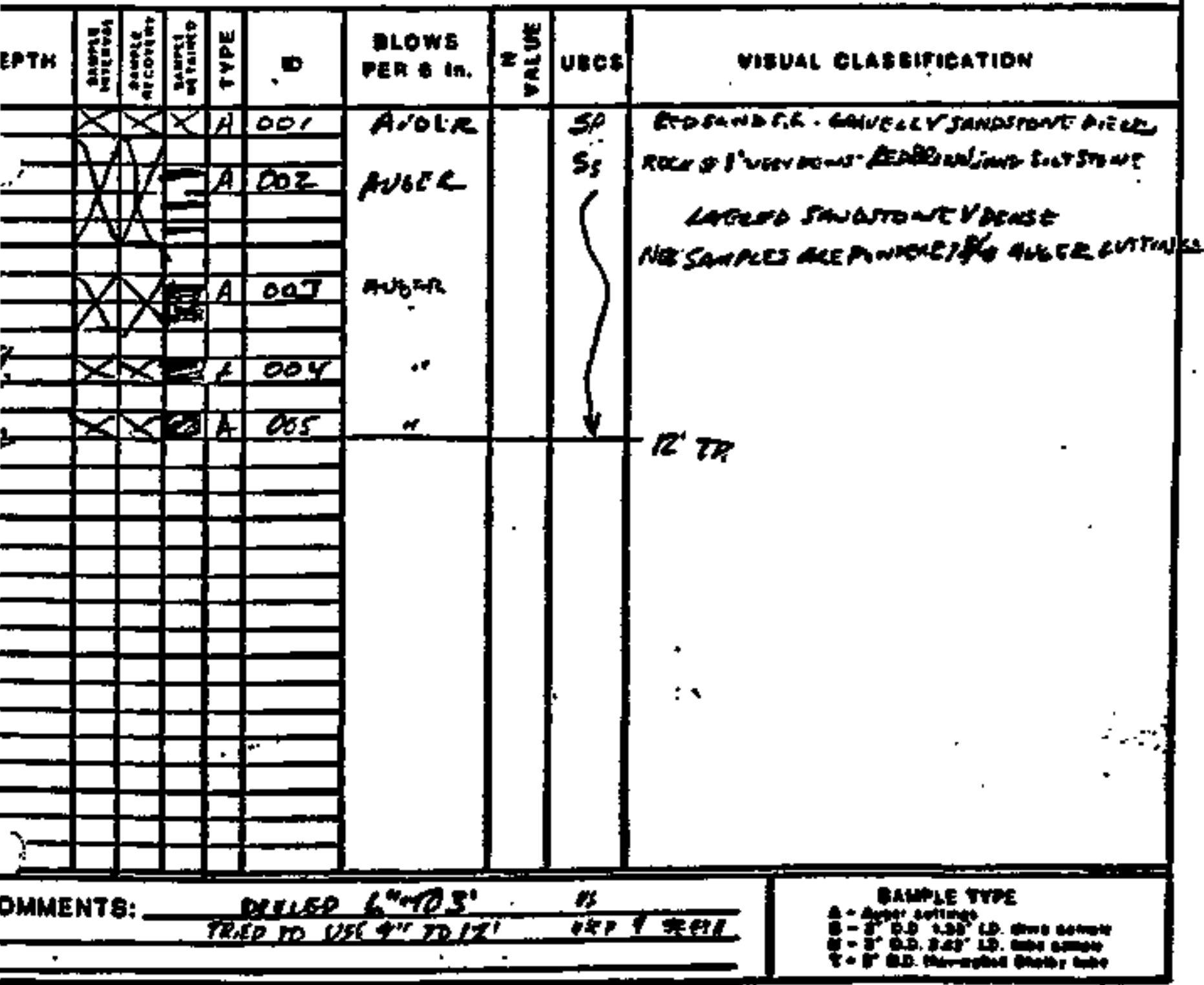





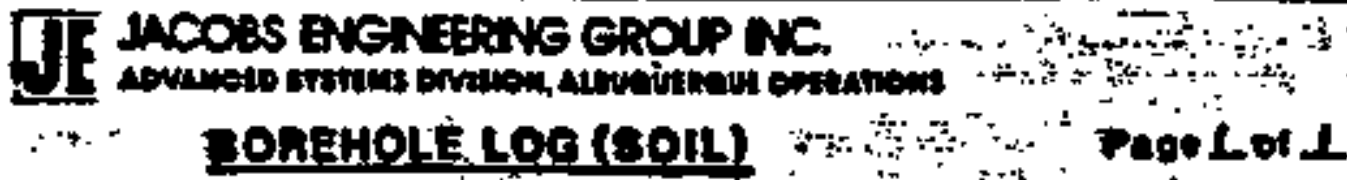

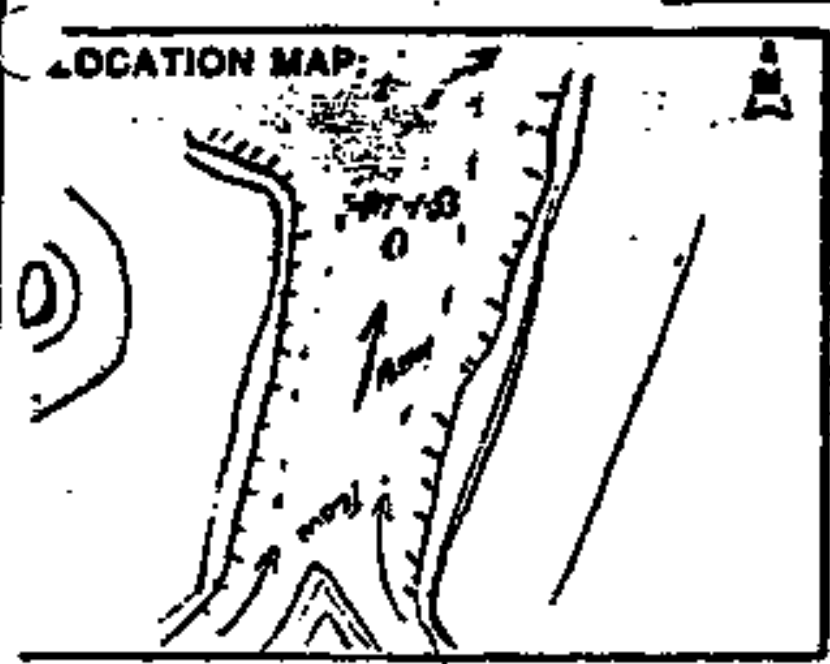

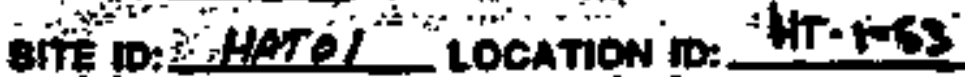
ARPAOX. BTE COORDINATES (It.):

N noro E erte

OAOLND ELEYATION (11. MLSL): REE

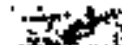

DALLINS METHOD:

DAILEER: onth

DATE STARTED:

PATE COMPLETEO:

FIELO GEP.:

singer.

\begin{tabular}{|c|c|c|}
\hline \multicolumn{3}{|c|}{ GROUNOWATER LEVELS } \\
\hline DATE & THME & DEPTH (it.) \\
\hline & & \\
\hline & & \\
\hline
\end{tabular}

LOCATION DESCAIPTION SITE CONDITION

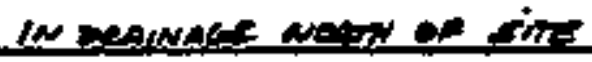

ser exts

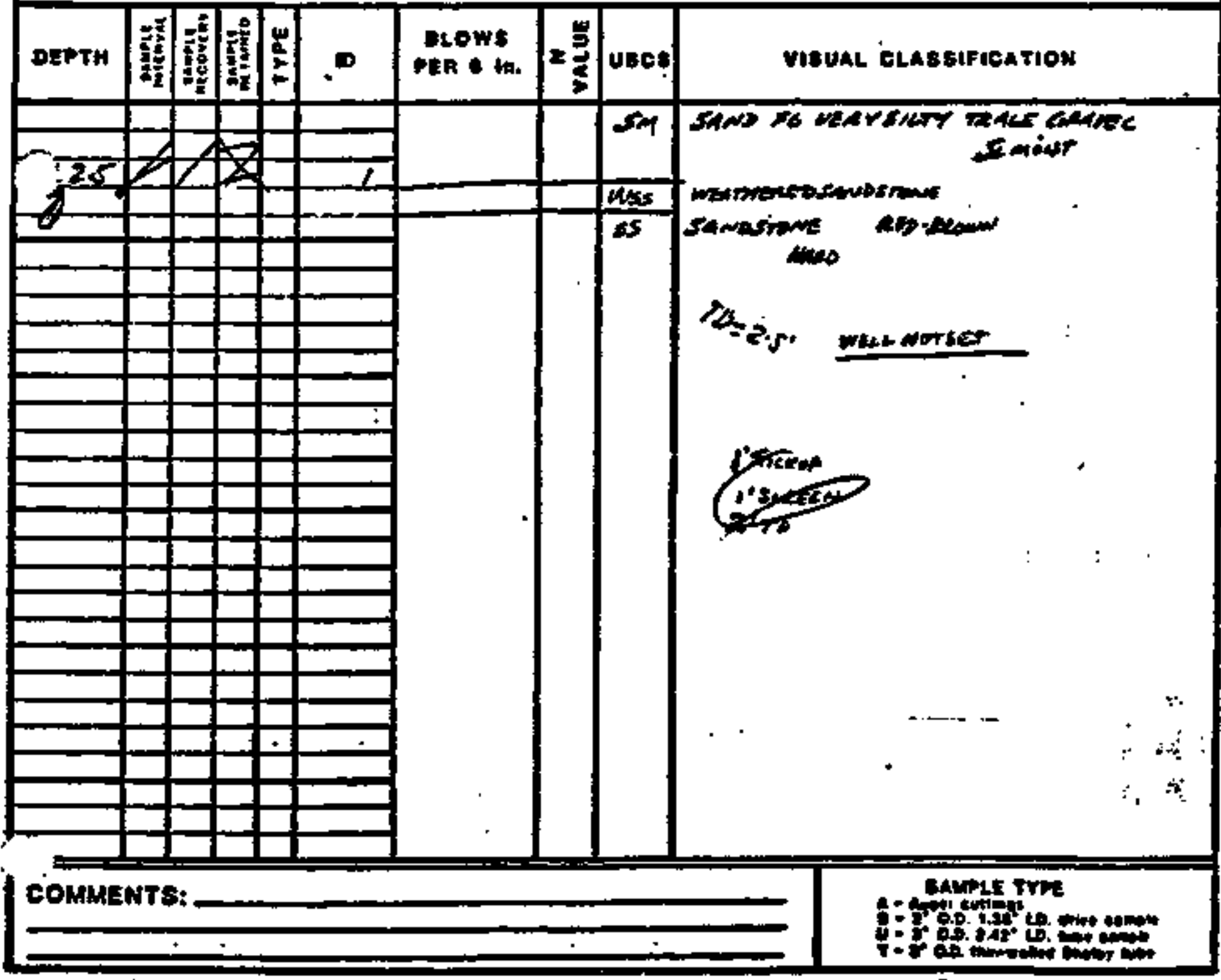




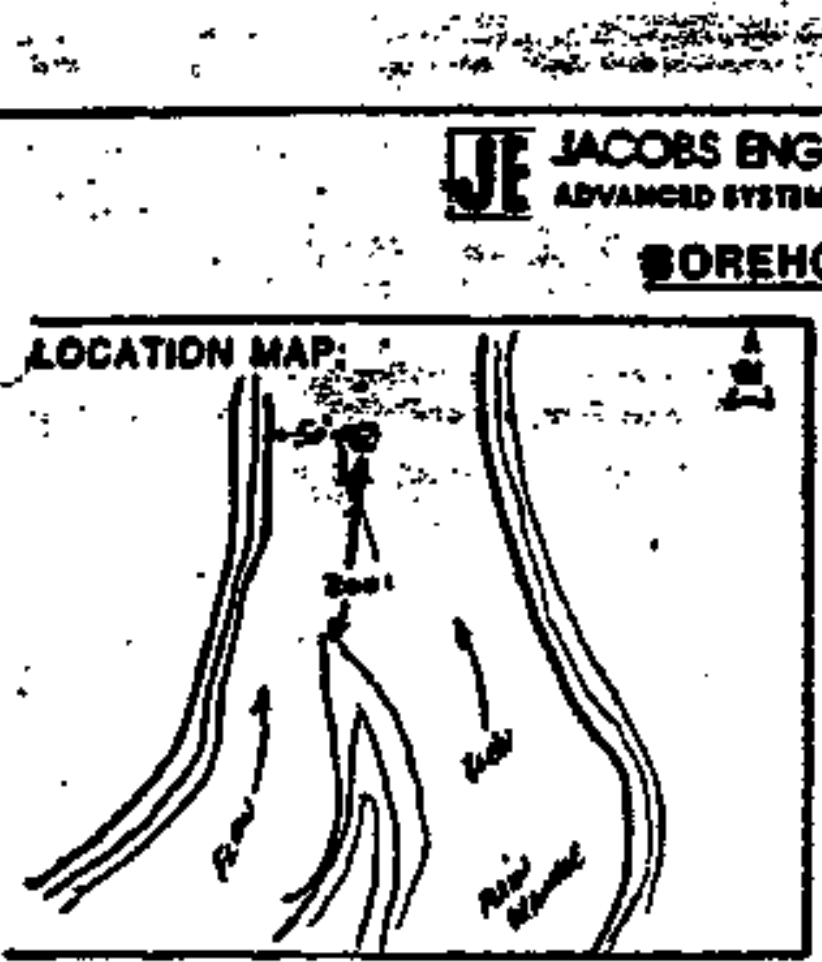

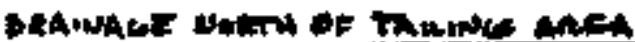

LOCATION DESCAIPTION BITE CONDITION

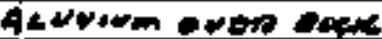

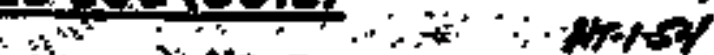

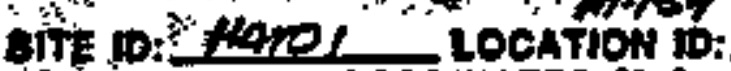
Approx. 8ITE COORDINATTS (h.): N Mes e max gROUND ELEVATION (ft. MSL): DAILLNG METHOD: G HS

DAILLEA:

DATE STARTED:

$10-25+8$

DATE COMPLETED:

FIELO AEP.:

\begin{tabular}{|c|c|c|}
\hline \multicolumn{3}{|c|}{ GEOUNDWATER LEVELS } \\
\hline DATE & TIME & DEPTH (f.) \\
\hline & & \\
\hline & & \\
\hline
\end{tabular}

rage Lol t t $4+53$. ninto Trito

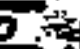

$\sin 3$

VIBUAL CLABBification

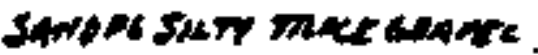

s.

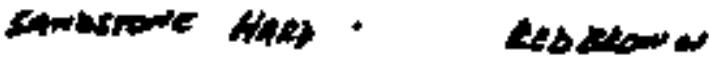

$1+2+2$

wroster

werestarezt

Antout of"

1 suector

lensils

$m=25$

T. 3.7

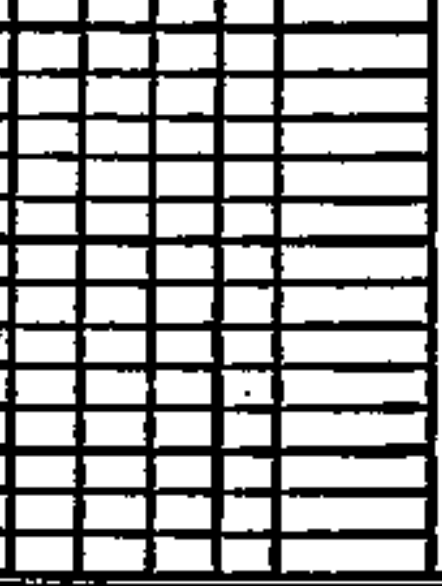

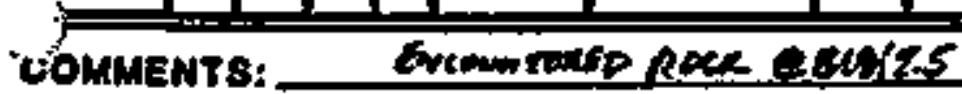

calpre Trwi

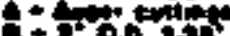

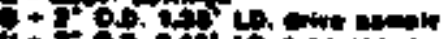

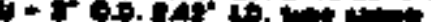

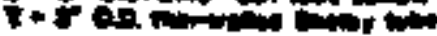




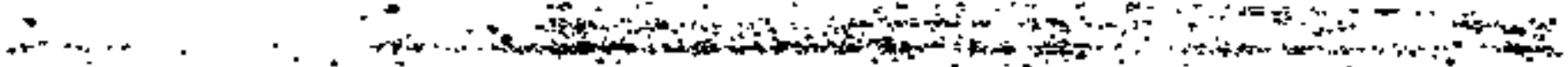

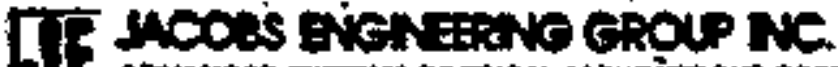

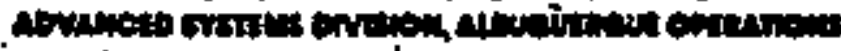

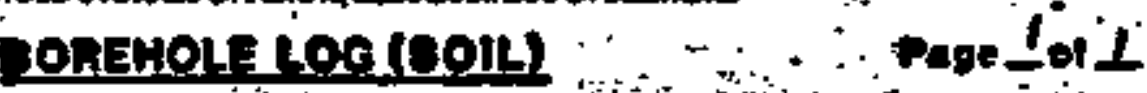

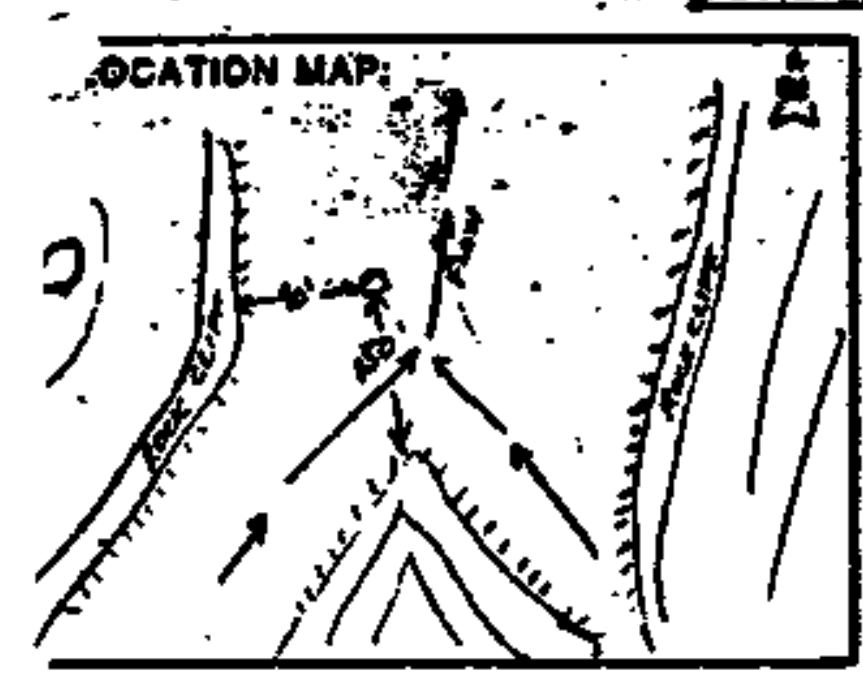

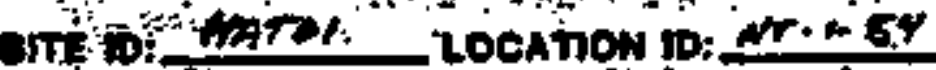
Appox. TITE COORpwattes (th.):

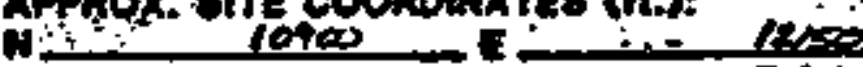

GROUND ELEVATION (ft. ysL):_ . T45

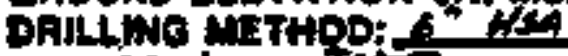

Prilues:

DATE BTARTED: PFFE+CT

DATE COMPLETED:

16

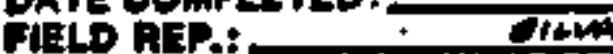

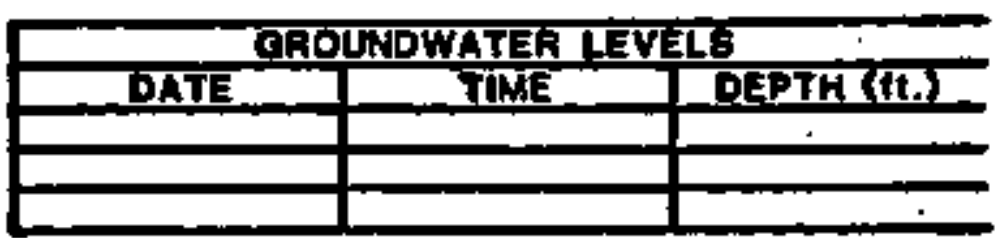

LOCATION DESCRAPTION

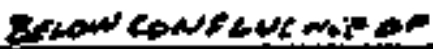

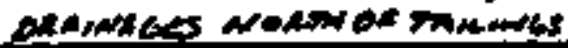
BITE CONDITION

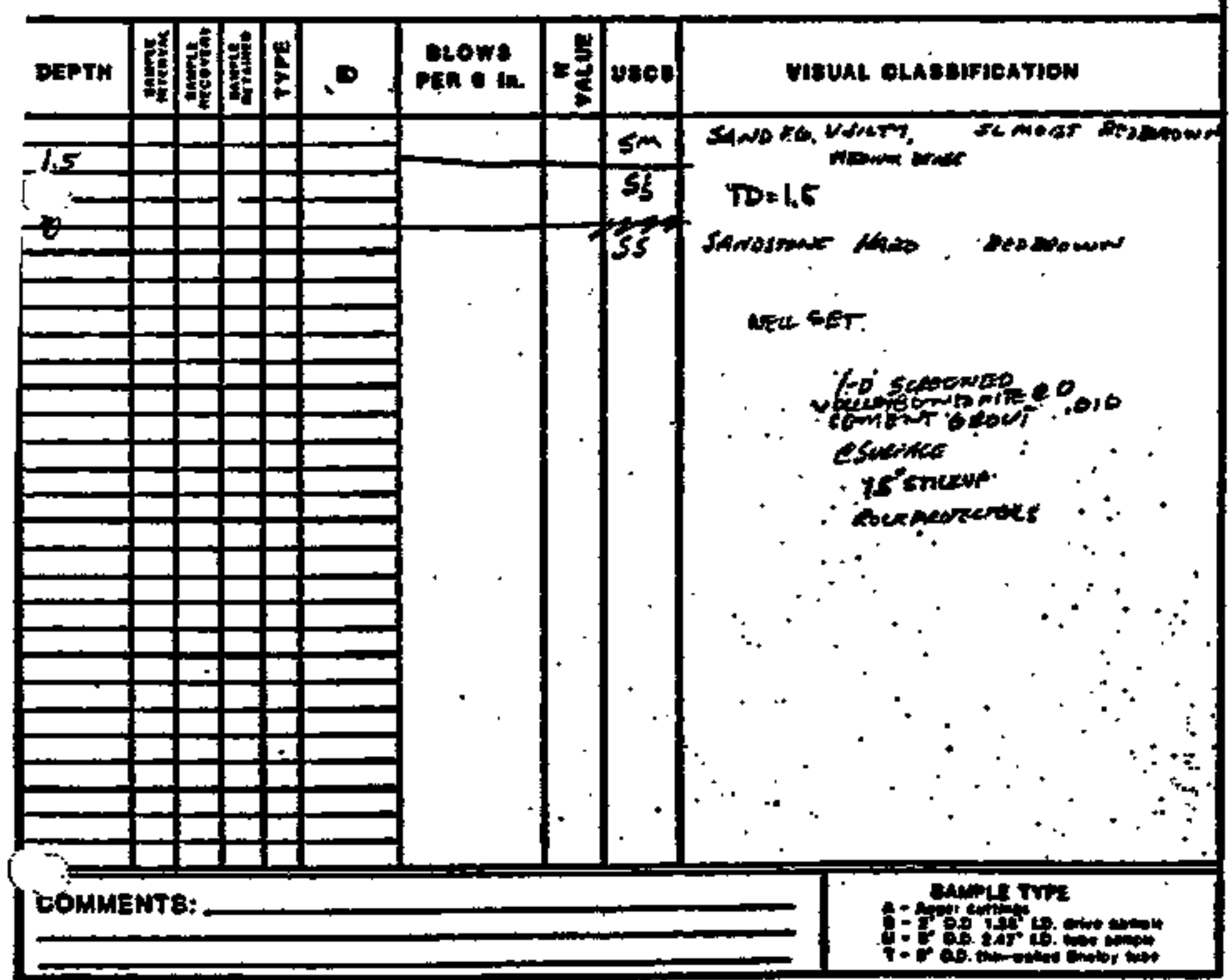




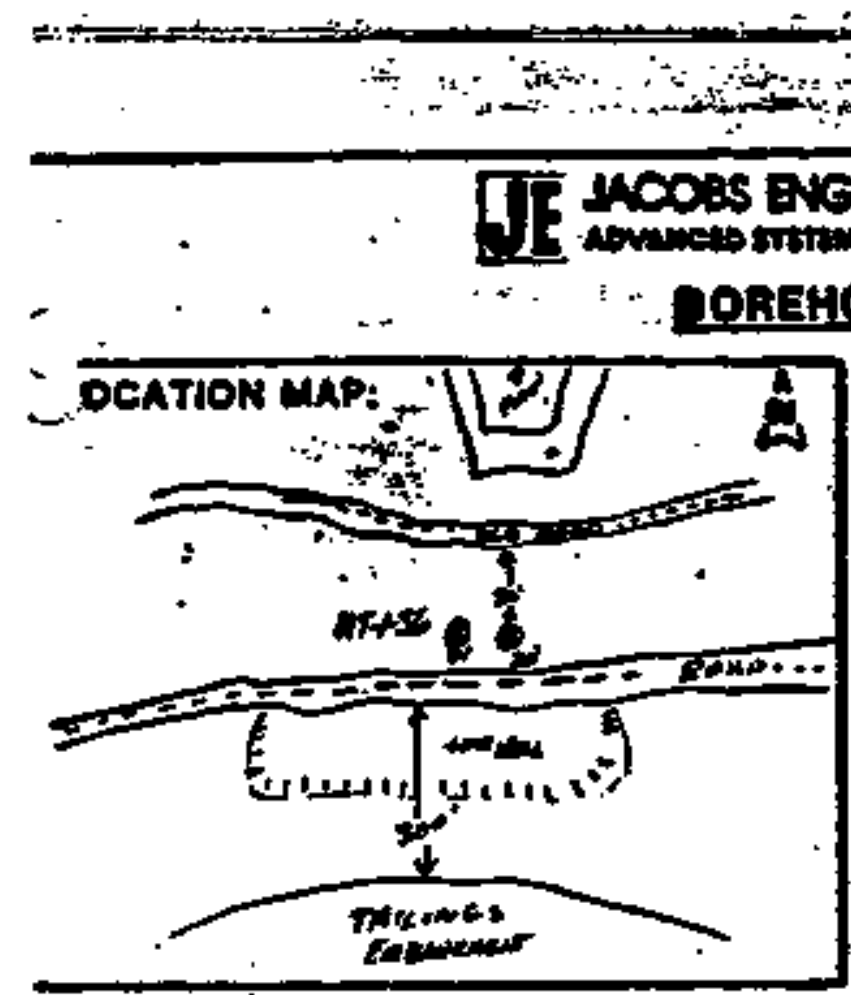

.

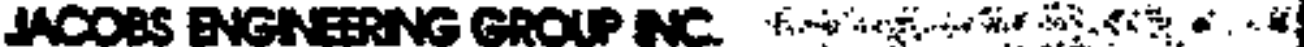
(1)

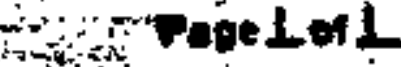

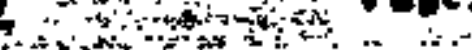
$+1$

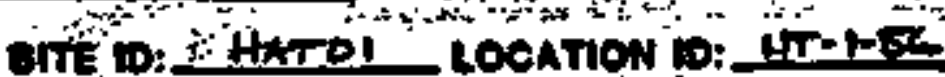

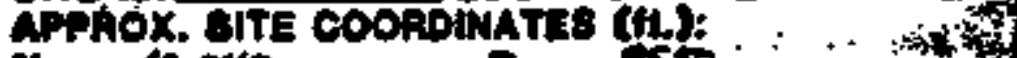

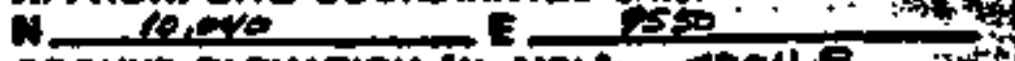

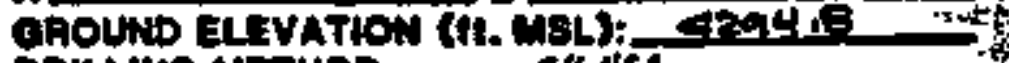
DRILHA MTHOD: DRHLER: DATE STAFTEO:

DATE CONPLETED: FIELO REP.:

$$
\text { rent }
$$
cents

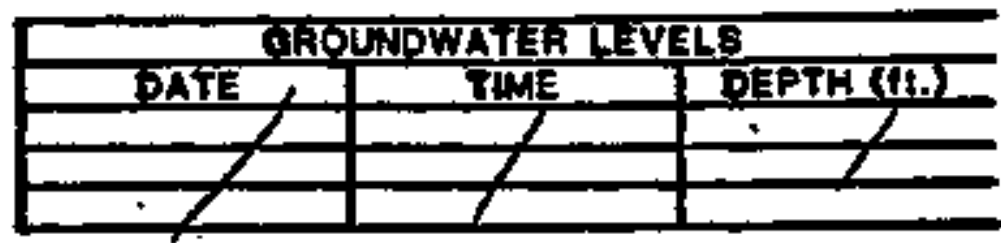

LOCATION DÉseartion

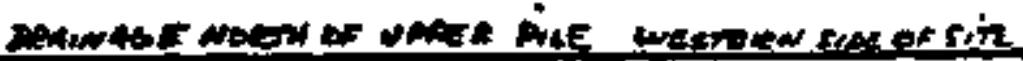
DITE CONDITION

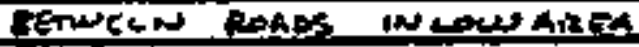

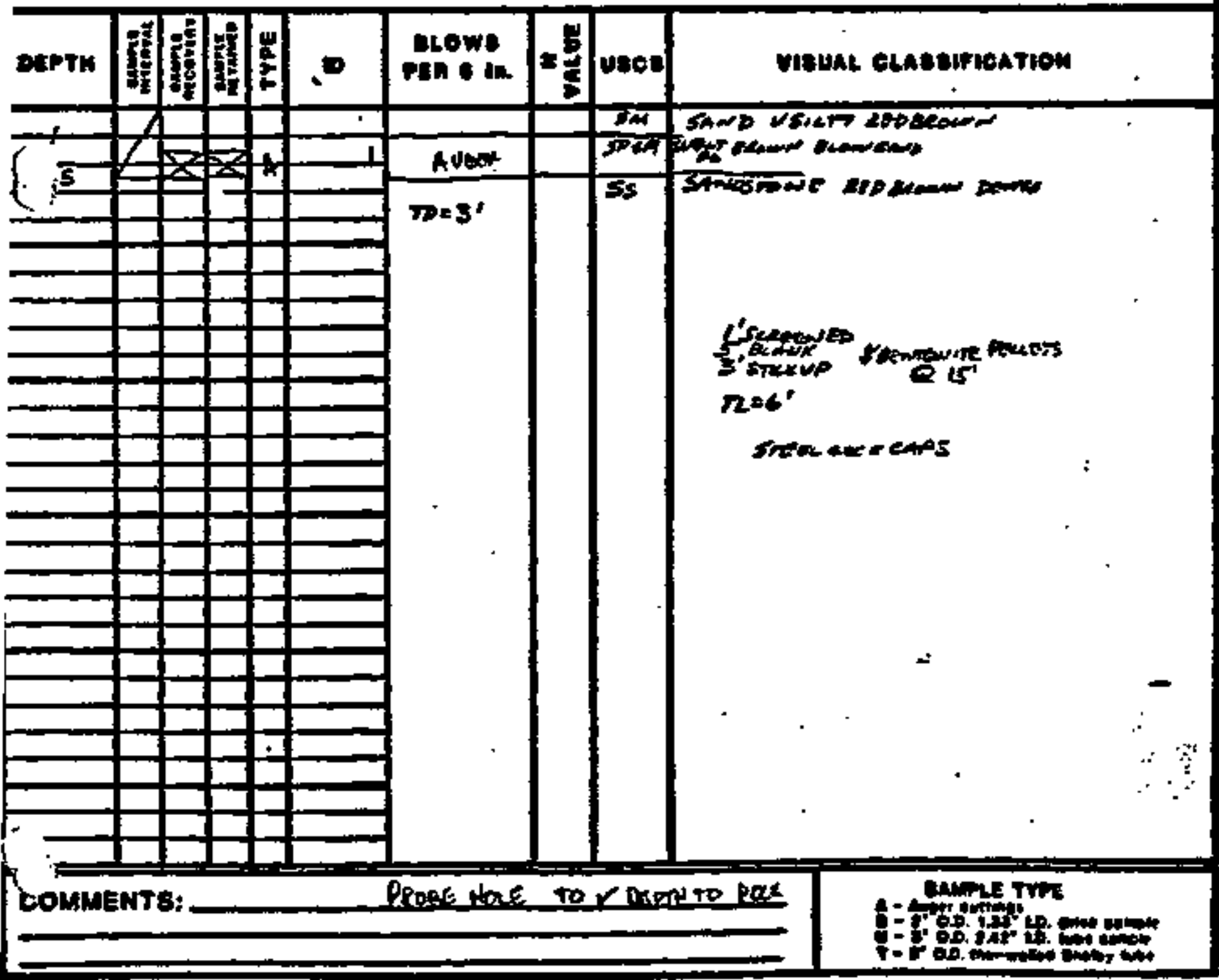




\section{BOREHOLE LOC (ROCK) PADEZ OI Z}

LOCATION MAP:

sce P. I
A BITE ID: HAT- OI LOCATION ID: 905

a APFROX. SITE COORDINATES (tt.):

N. GROUND ELEVATION (tt, MSL):

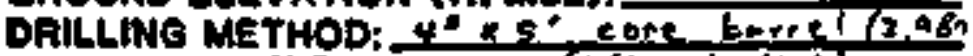
DAILLER: $S A B$

DATE 8TARTED: $12 / 10 / 64$

DATE COMPLETED: $21 / 2184$

FUELD REP.: F. Savd kets

\begin{tabular}{|c|c|c|}
\hline \multicolumn{2}{|c|}{ BROUNDWATER LEVELS } \\
\hline DATE & TIME & DEPTH (fI.) \\
\hline & & \\
\hline & & \\
\hline & & \\
\hline
\end{tabular}

LOCATION DESCRIPTION

SITE CONDITION (5)

$=$
$\mathrm{z}$

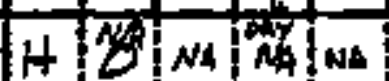
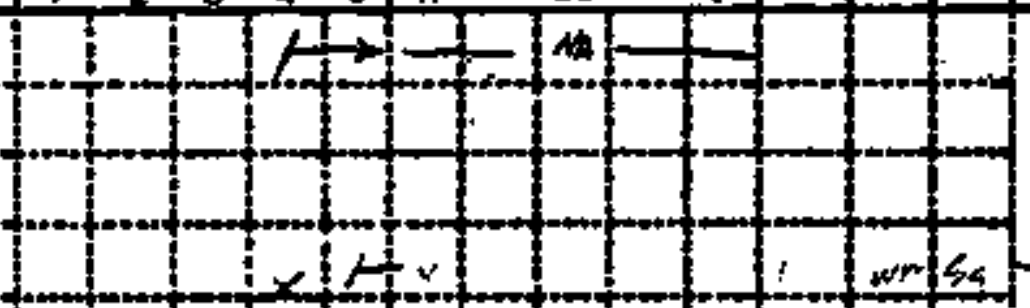

Stes Son bearioles AN POI

Sinds filk

406Ad Ajta

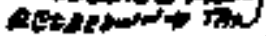

sner anamezlos

\section{.}

:

5
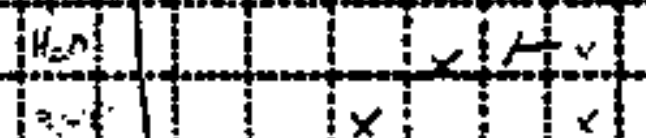

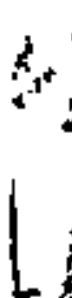
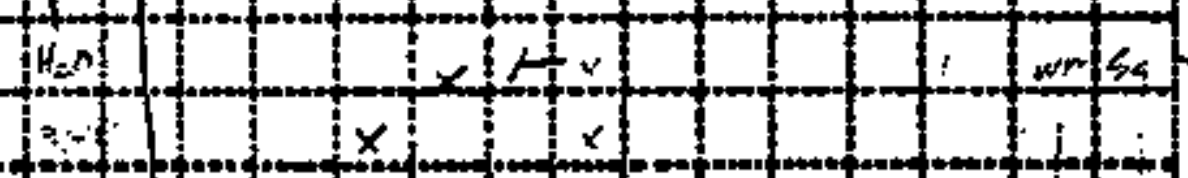

whises

ROCK TYPE

a namaks

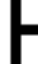

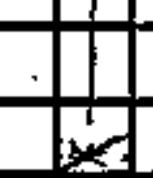

$y^{2}$

9.

(N) $\cos \%$ क

r. $r a^{\prime}$

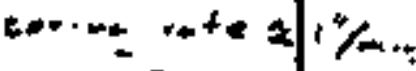

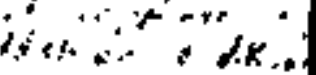

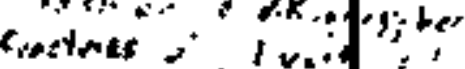

ro

$$
\text { GB. }
$$

$<10$

$+$

$\therefore 0$ v 10

c.

$7+2=x$

$x+\frac{x}{x} x$

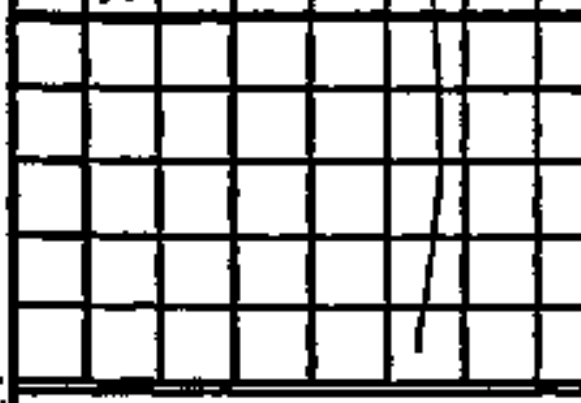

\footnotetext{
com
}

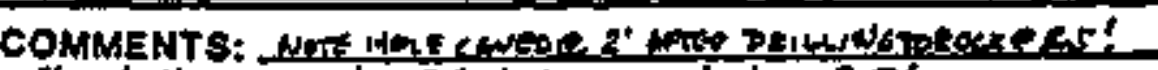

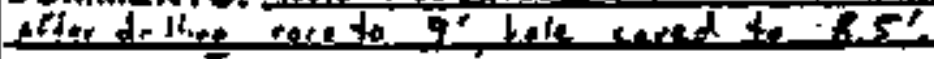

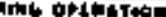

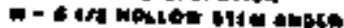

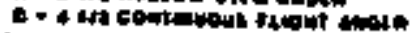

corat ot

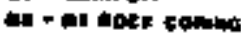

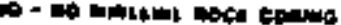




\section{IT JACOBS ENENEERING EROUP NKC.}

LOCATION MAP;

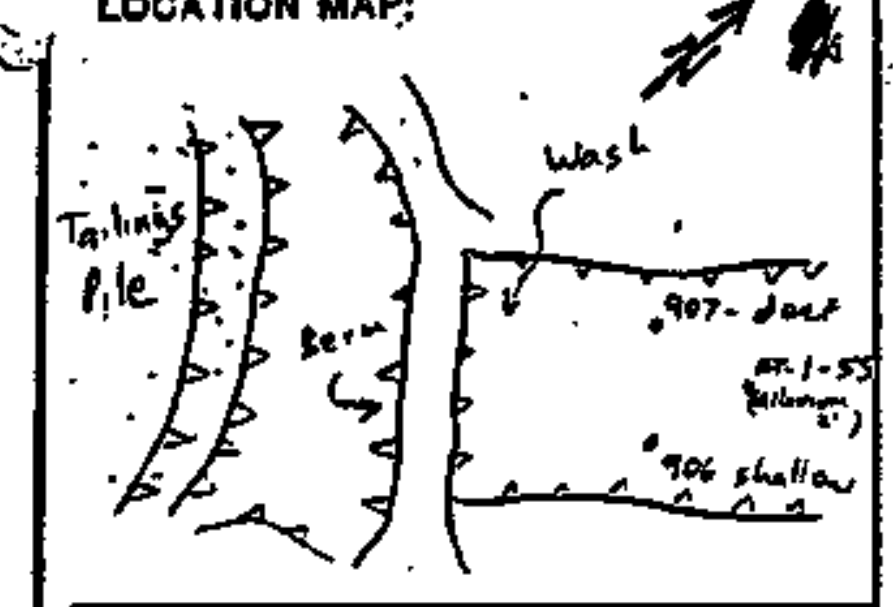

BHE ID JNAT: OI LOCATION ID: $906^{\circ}$ HPPAOX. BITE COORDINATES (th.):

wi E

CROUND ELEVATION (ft. MSL):

DRILLINE METHOD: $4 \& A$

DRILLER: SHA = Jin Corter

DATE STARTED: $12 / 12 / 84$

DATE COMPLETED : $12 / 12160$

FIELO AEP.: D.D. D.

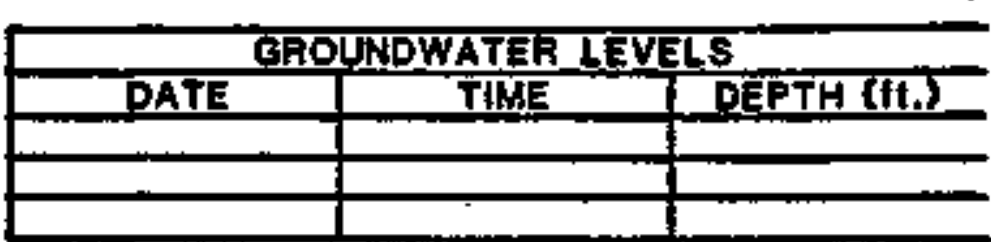

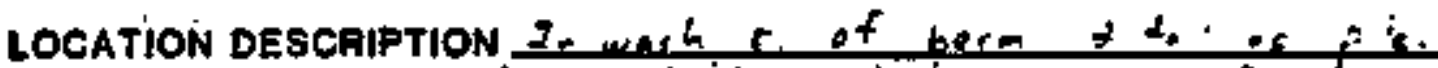

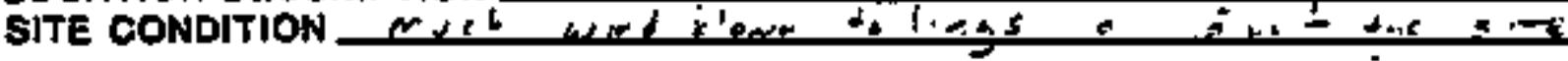

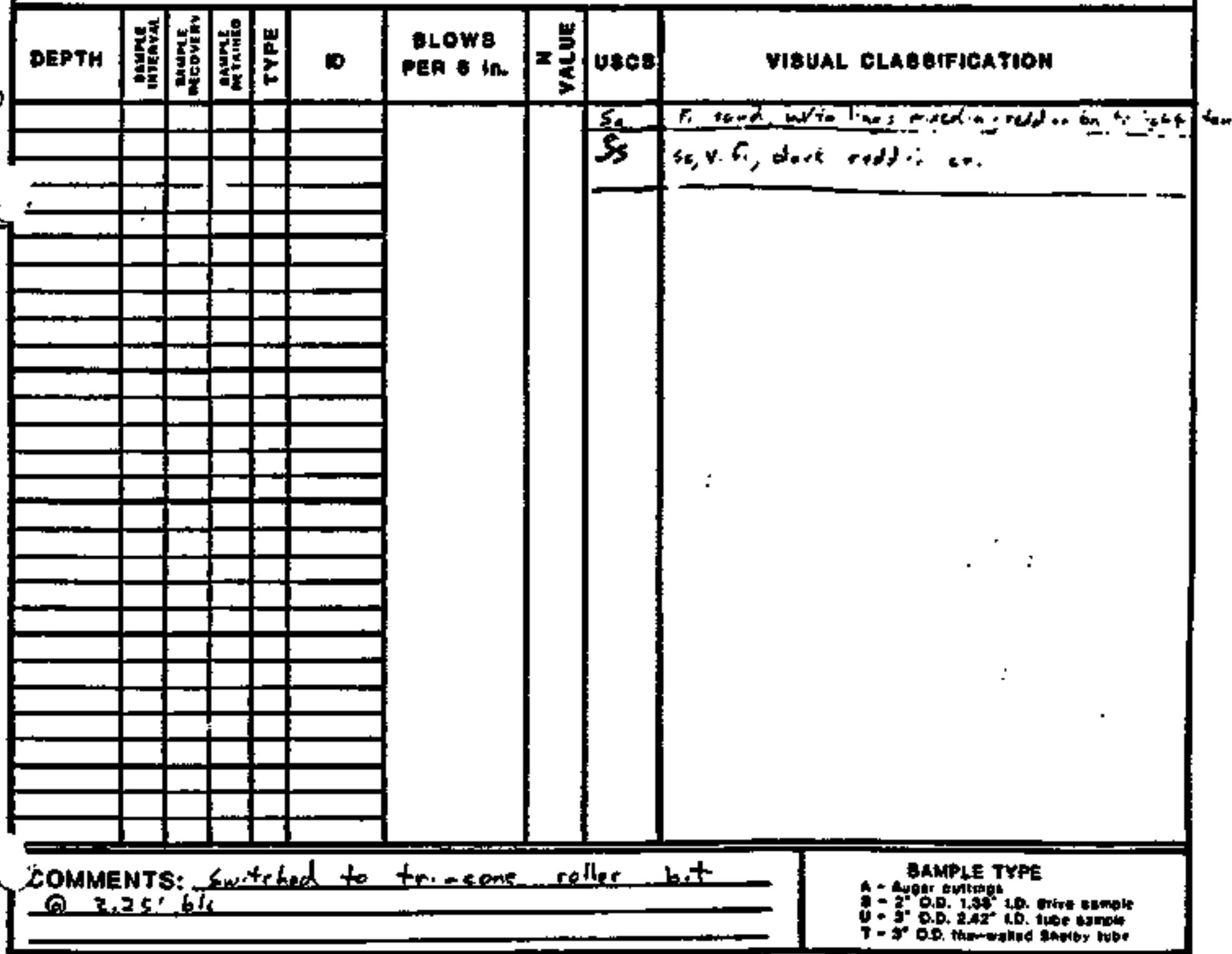




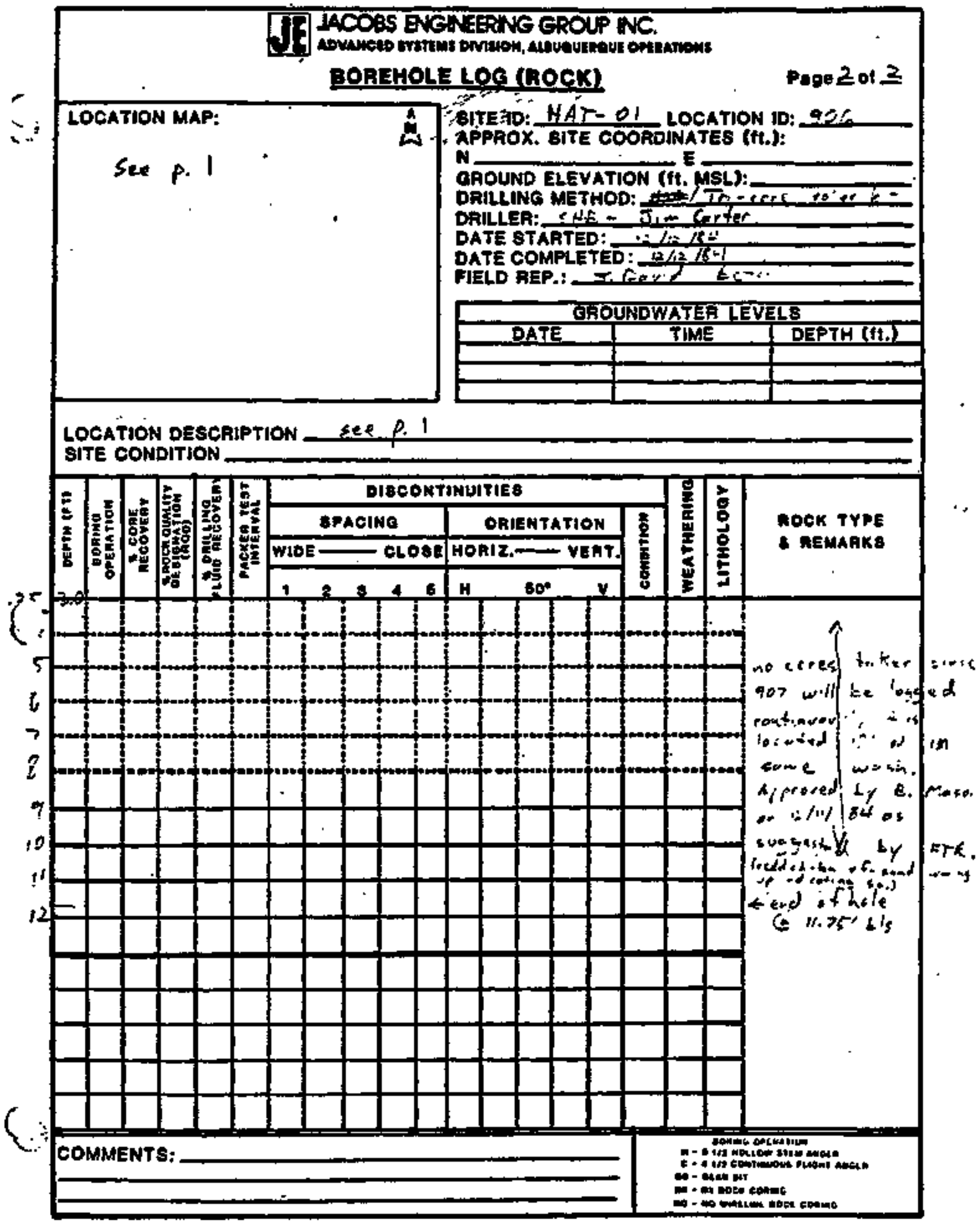




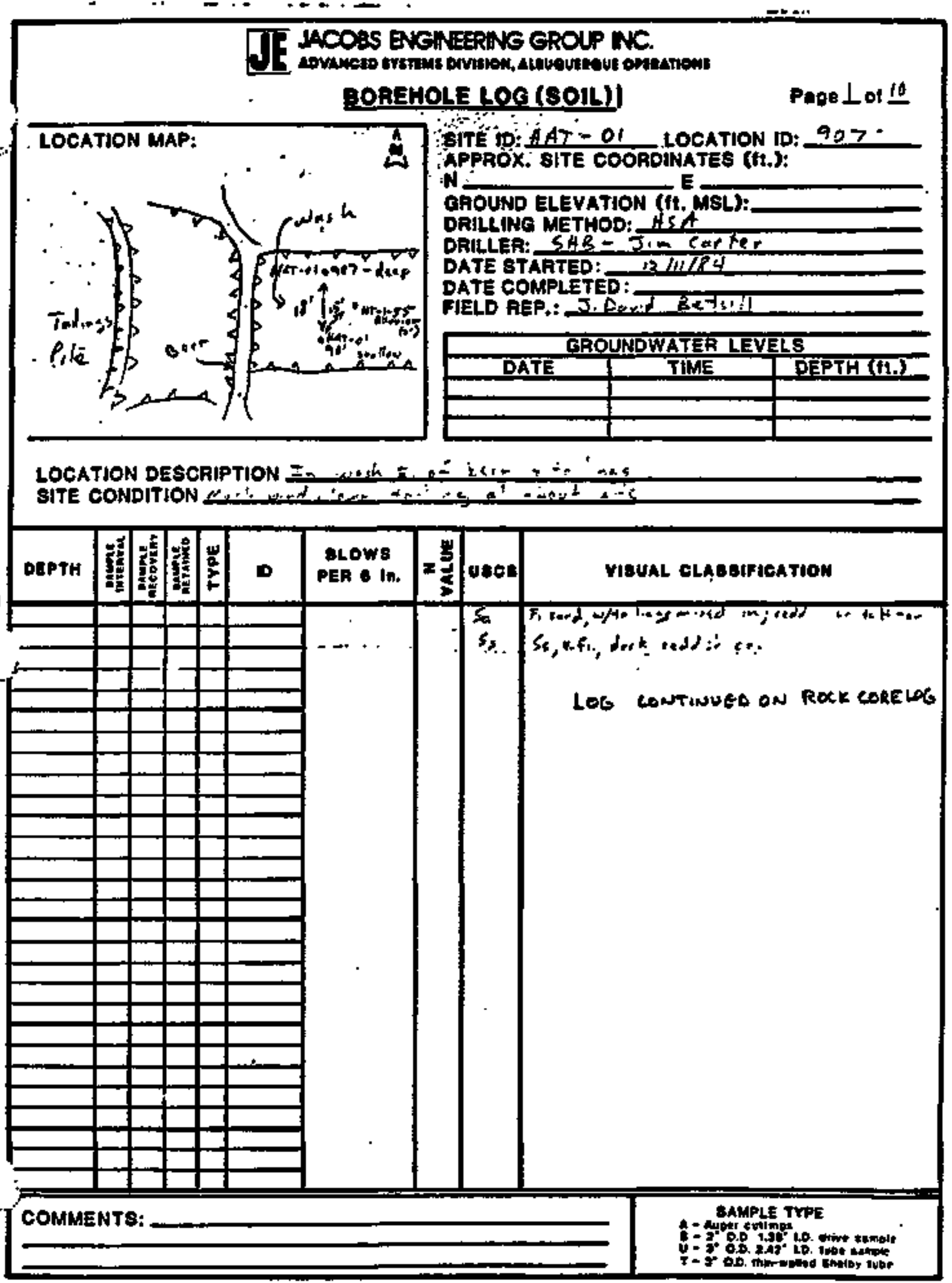




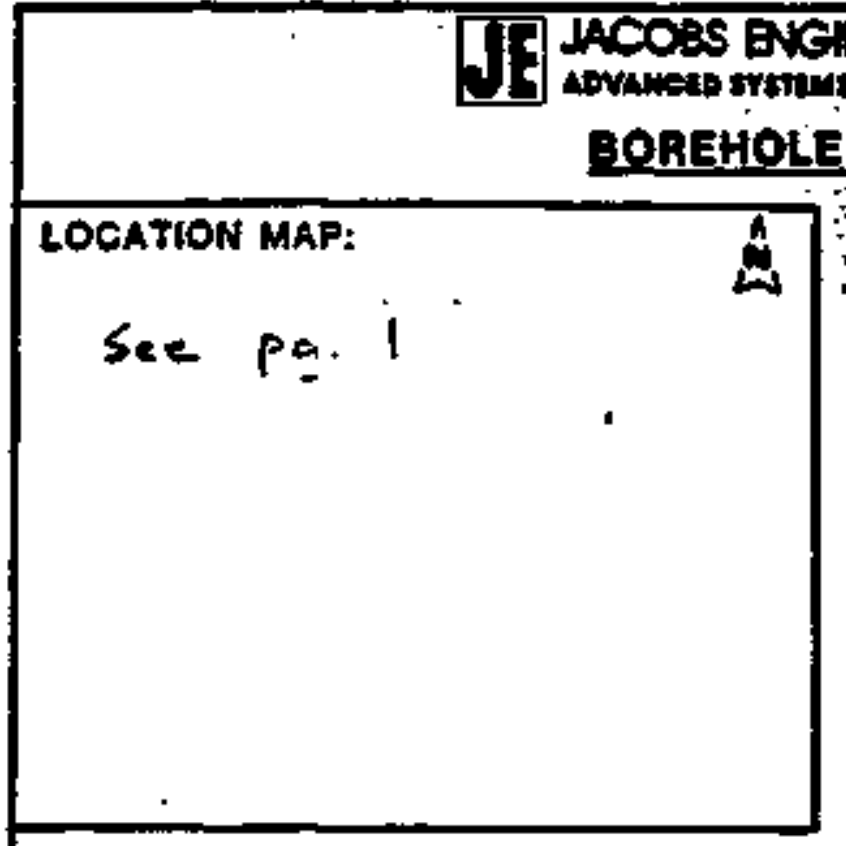
LOCATION DESCRIPTION SES AO I
SITE CONDITION

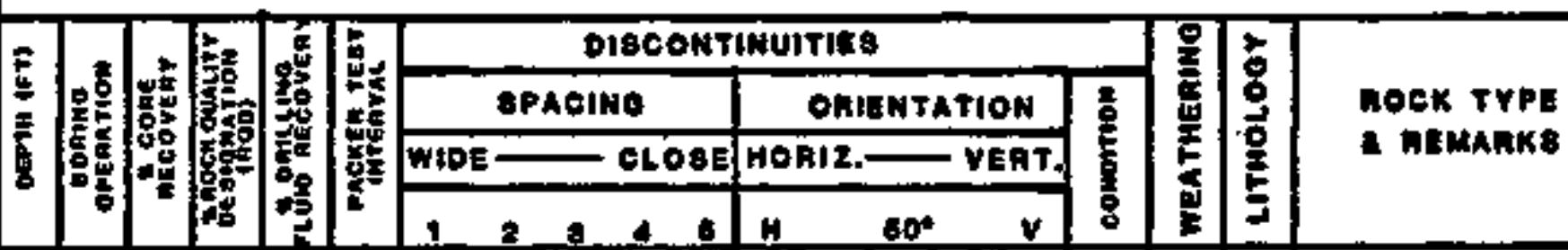

6

$4,3100125 \div 0$

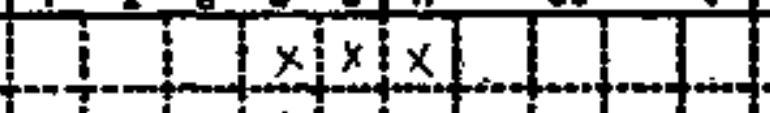

$x$

1 302,390

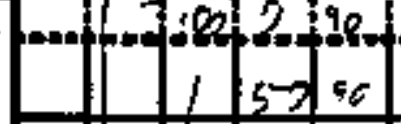

I

$+12$

B

14

14

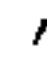

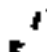

1

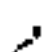

\section{$110 *$}

?

(1)

$100 \pi$

ix

$\left\{\begin{array}{l|l|l|}x & x \\ x & x & x\end{array}\right.$

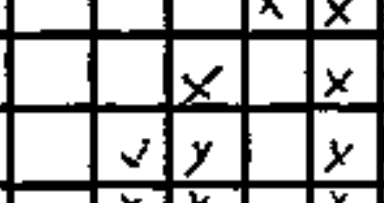

$+1$

$\cdot-\frac{x}{y}$

$+\quad \sqrt{ } \quad x$
1) 75 in

WH. $s_{\text {S }}$

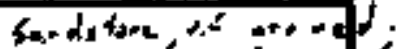

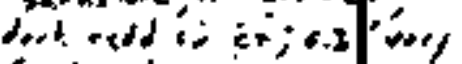

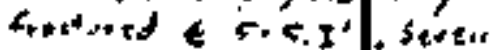

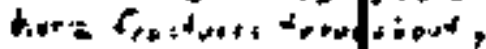

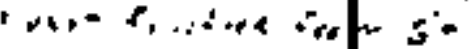

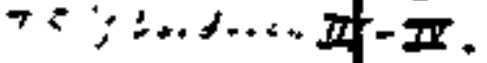

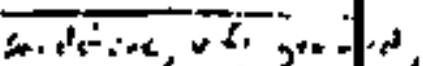

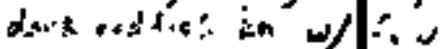

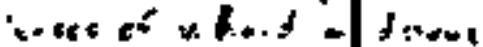

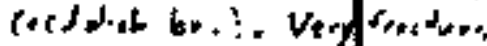

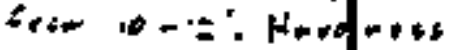

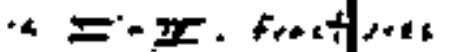

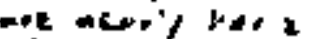

a.tenthat.

saldatane os

jo+t +bove.

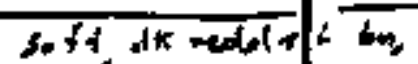

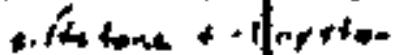
$2 x^{t+}-v \infty t+(x-2)$

17

$\sqrt{4} s_{5}$

fondrtone at ateor with linht sey

ent

Nâ 100 in

COMMENTS: 
LOCATION MAP:

sec $\mathrm{Pg} \cdot 1$
A. SITEËH HAT OI LOCATION ID: 907

N.

CROUND ELEVATION (ft. MSL):

DRILLING METHOD:

DRILLER: Dim. Carter (SHB)

DATE STARTED: $t 2 / 1 / 84$

DATE COMPLETED:

FIELD REP.: T. Beved Bargi il

\begin{tabular}{|l|l|l|}
\hline \multicolumn{3}{|c|}{ GROUNDWATER LEVELS } \\
\hline OATE & TIME & DEPTH (fi) \\
\hline & & \\
\hline & & \\
\hline
\end{tabular}

LOCATION DESCRIPTION SES ME L 1
SITE CONDITION

SITE condolTion

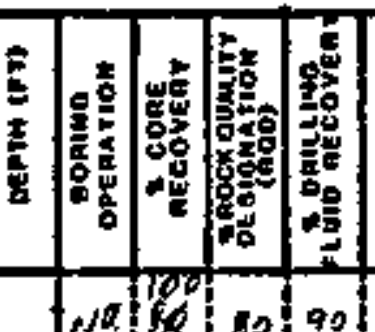

(c)
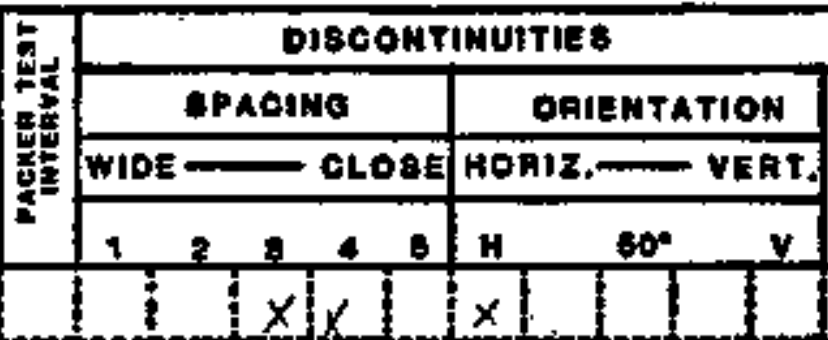

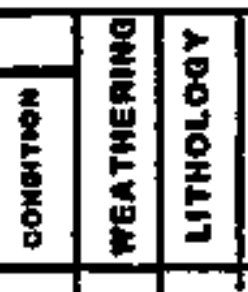

$=2$

2?

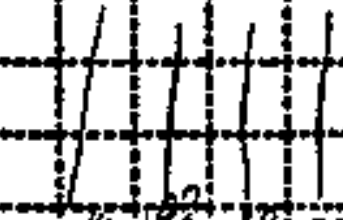

$+\infty$

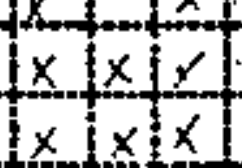

$x+x$

paticis

$x+x$

ont this os

ton.t.

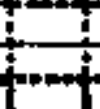

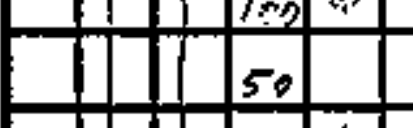

$=0$

$x$

$x y$

$\mid x$

$x+\frac{x}{x}$

1130

$x$

$\checkmark|x|$

$y$

\begin{tabular}{l|l|l|l|}
\hline$y$ & $x$ & \\
\hline
\end{tabular}

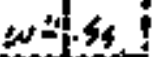
lathiez cent atof hKC

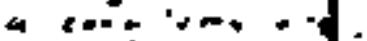

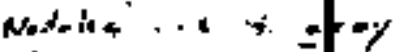
4. 0.42 .

$40 \mid$

$x|x|$

1
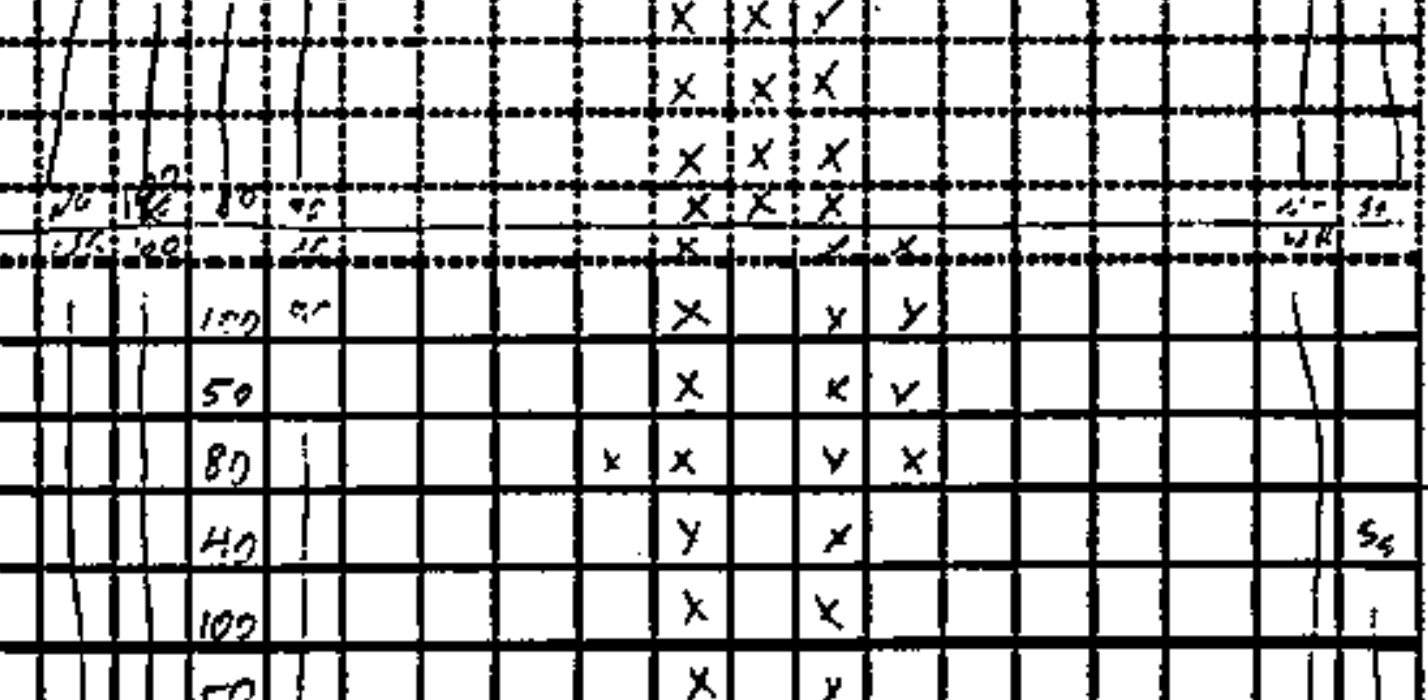

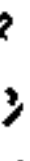

\begin{tabular}{|l|l|l|l|}
\hline & $x$ & & $y$ \\
\hline$x$ & $y$ & & $x$ \\
\hline$x$ & $y$ & & $x$ \\
\hline$y$ & & $x$ \\
\hline & $x$ & & $x$ \\
\hline
\end{tabular}

Les $\frac{100}{0}$

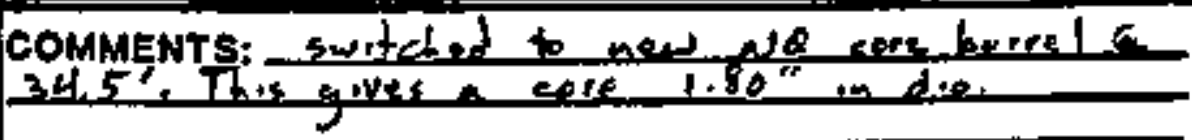




\section{1.] IACOES ENGNEERNG EROOP NC.}

\section{BOBEHAOLE LOG (BOCK)}

LOCATION MAP:

$$
\text { see p. } 1 \text {. }
$$

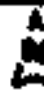

BITE ID: $A 4 T$ OI APPAOX. SITE COORDINATES (ft.):

N GROUND ELEVATION (ft. MSL): DRILLINB METHOD:

DRILLER: SHA - 5 - Lerter DATE STARTED: $12 / 11 / 84$ DATE COMPLETED:

FIELD AEP.: J. Oavd Et. ${ }^{\prime \prime}$

\begin{tabular}{|c|c|c|}
\hline \multicolumn{3}{|c|}{ GROUNDWATER LEVELS } \\
\hline DATE & TIME & DEPTH (II.) \\
\hline & & \\
\hline & & \\
\hline
\end{tabular}

\section{LOCATION DESCRIPTION Ste Pg 1
SITE CONDITION}

\section{F \\ DIscontikuit:E}

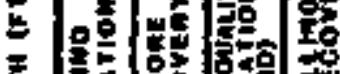

E

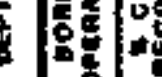

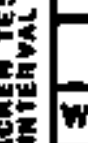

$=$ cive

-
WIDE
37

3

37

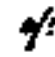

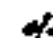

43

$12 \% 100170: 20$

11.100

100

0

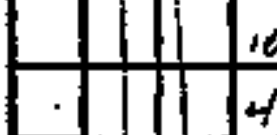

109

4

(1)

ORIENTATION

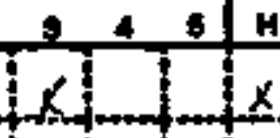

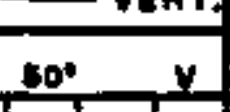

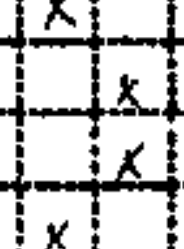

ix!

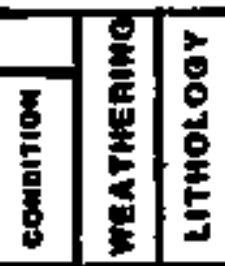

AOCK TYPE

a REMAKs

X

$+x$

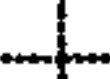

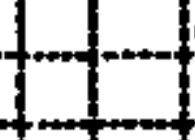

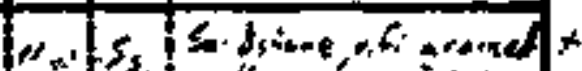

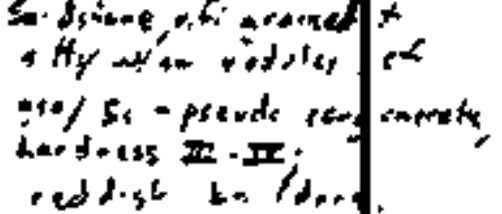

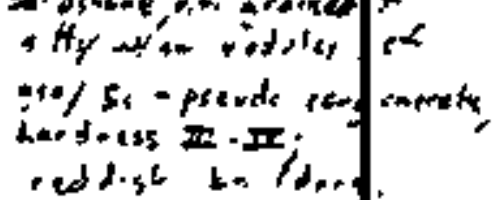

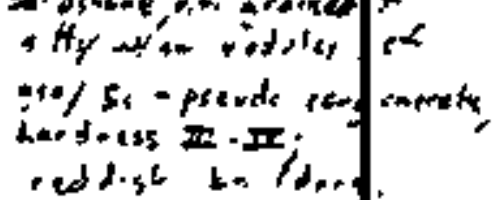

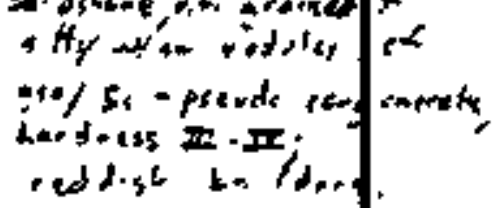

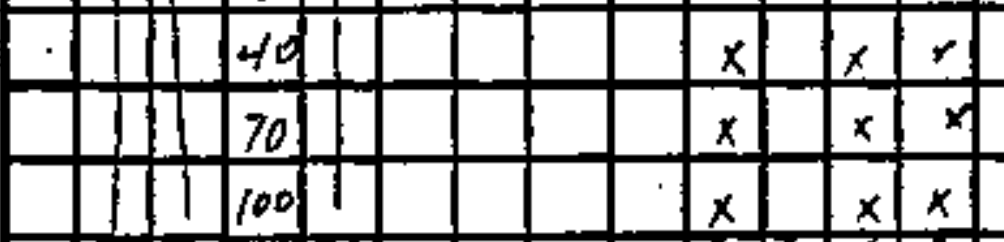

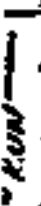

45

7 \begin{tabular}{|l|l|l|l|l|}
\hline NQ & 100 & 20 & 20 & \\
\hline
\end{tabular}

$x \times x|x| x$

.

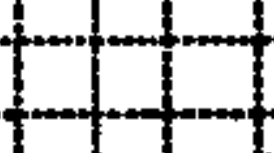

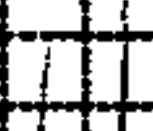

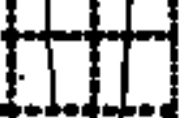

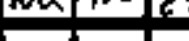

\begin{tabular}{|l|l|l}
\hline$x$ & $y$ & \\
\hline$x$ & $y$ & \\
\hline
\end{tabular}

$x \times$

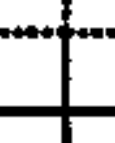

-

\begin{tabular}{|l|l|l|l|}
\hline & & & \\
\hline & & & \\
\hline
\end{tabular}

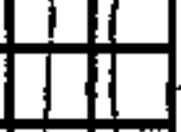

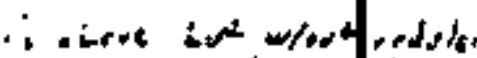

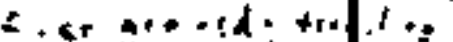

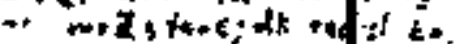

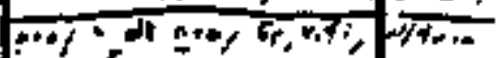

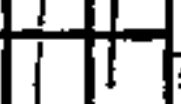

iersof gray fos. 042.2

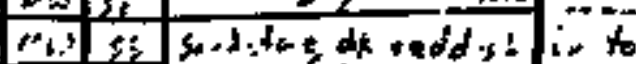

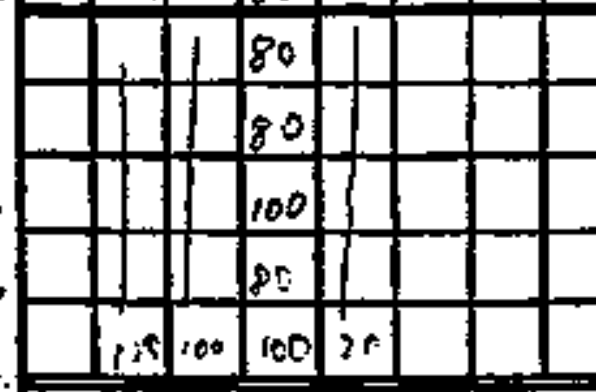

COMMENTS:

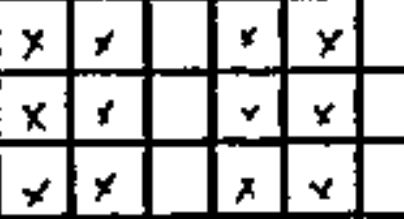

H. ted.sh to, v.f hate (ar)

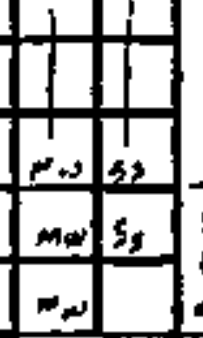

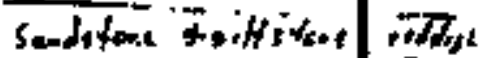
ba 


\section{JACOAS ENENEERNG GROUP NC.

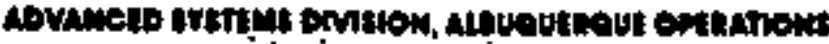

BOREHOLE:LOR (ROCX)

Page $\sum$ of $\frac{10}{2}$

LOCATION MAP:

see p. 1
4

BITE ID:A4T - DI LOCATION ID: 907

4 'APPAOX. SITE COORDINATES (H.):

it CAOUND ELEVATION (tt. MSL):

DAILLINS METHOD:

DRILLEA: $S H B-5$ S Cater

DATE STARTED: $12 / 11 / 84$

DATE COMPLETED:

FIELD REP.; S. DEY I BETs./t

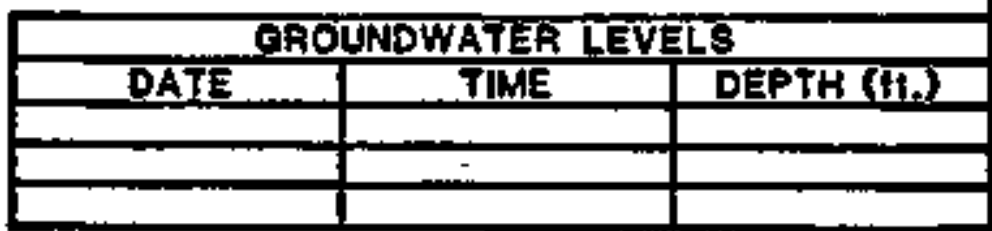

\section{LOCATION DESCAIPTION SEe f.I SITE CONDHTION}

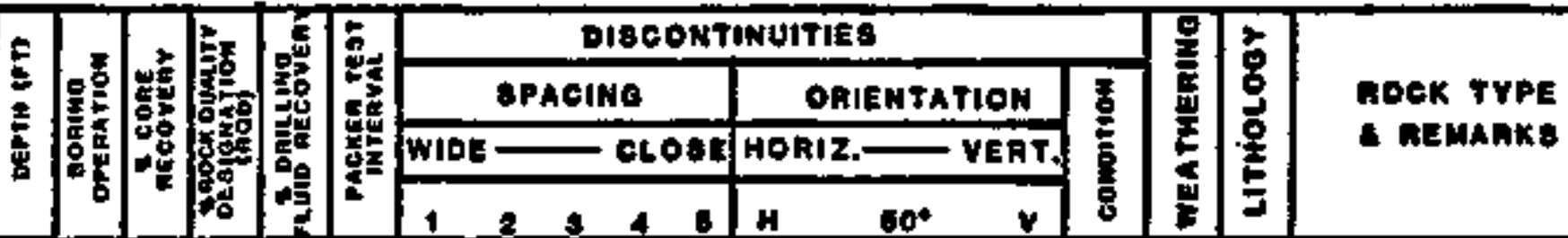

\section{Natiogot 20}

52

$1+70$

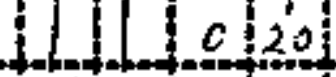

Libical o 601

56
56
57
58
57
66
61
63
64
$N \rightarrow 100$

110000

10060

50 50

115060

10100

62
64
3 $x^{2}$

$x$
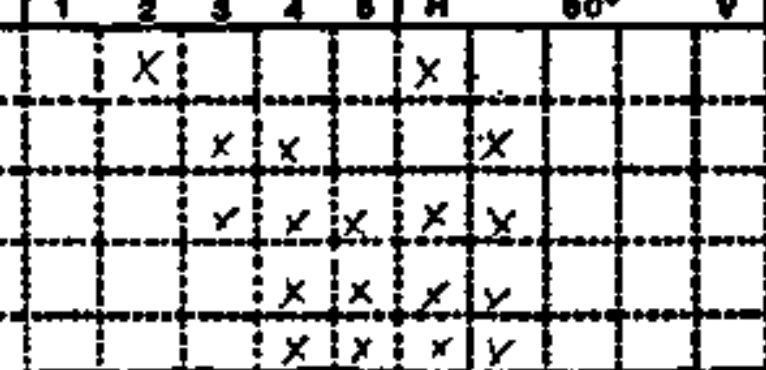

twe

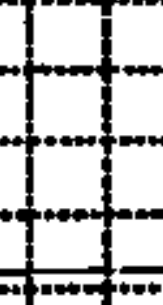

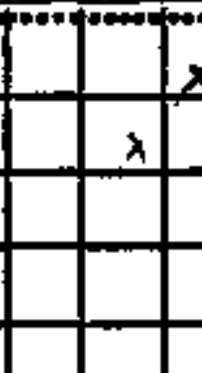

$x \times x|x|$

$x|x| x$

$x+x \mid x$

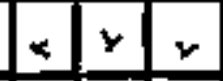

saditanc, v. dark retat

le to redd sin and

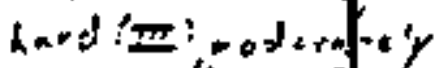
veditict, Jeform

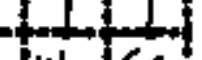

five $\leq \leq$

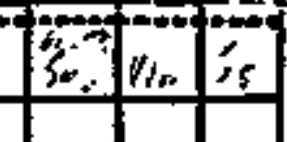

Shigrestests

rasenitis

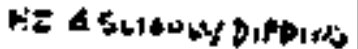

tewerters

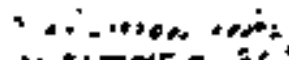

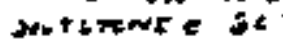

45

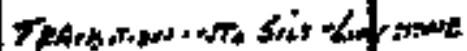

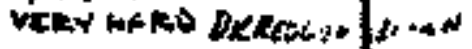

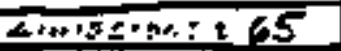




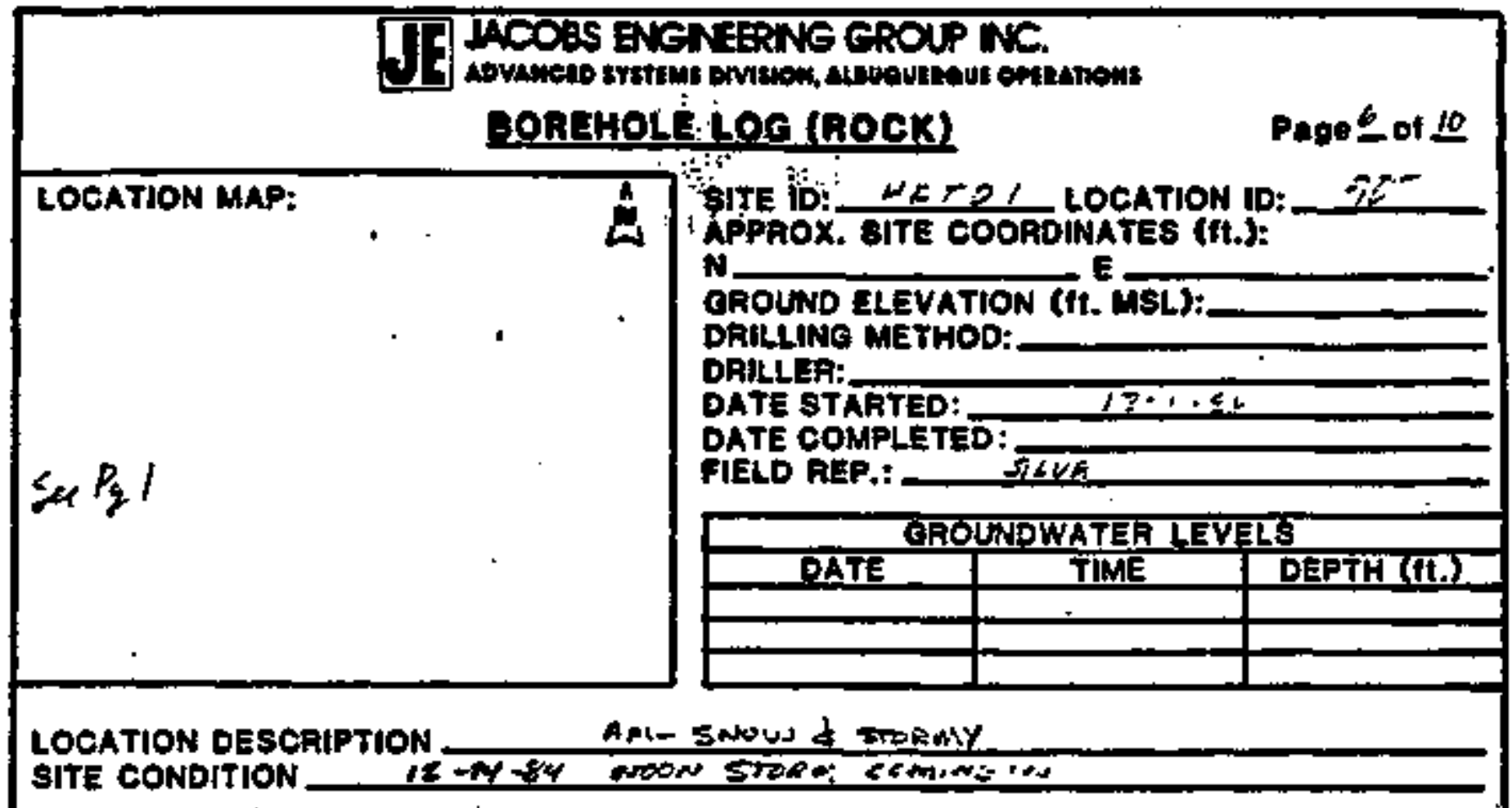

\begin{tabular}{|c|c|c|c|c|c|c|c|c|c|c|c|}
\hline \multirow{4}{*}{ E } & \multirow{4}{*}{ 望 } & \multirow{4}{*}{\multicolumn{2}{|c|}{ 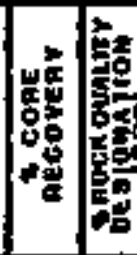 }} & \multirow{4}{*}{ 哭 } & \multirow{4}{*}{ 䇾 } & \multicolumn{3}{|c|}{ DI8CONTINUITIEs } & \multirow{4}{*}{. } & \multirow{4}{*}{ 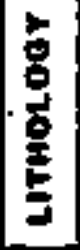 } & \multirow{4}{*}{ 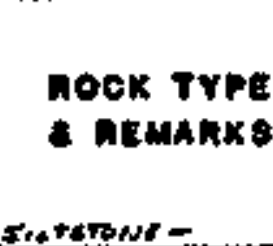 } \\
\hline & & & & & & DPaCIMe & OAIENTATION & $\mathbf{z}$ & & & \\
\hline & & & & & & WIDE - CLOEE & MORIZ, — VERT. & E & & & \\
\hline & & & & & & 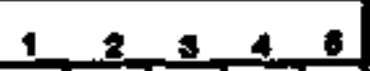 & $60^{\circ}$ & 8 & & & \\
\hline
\end{tabular}
NG 100

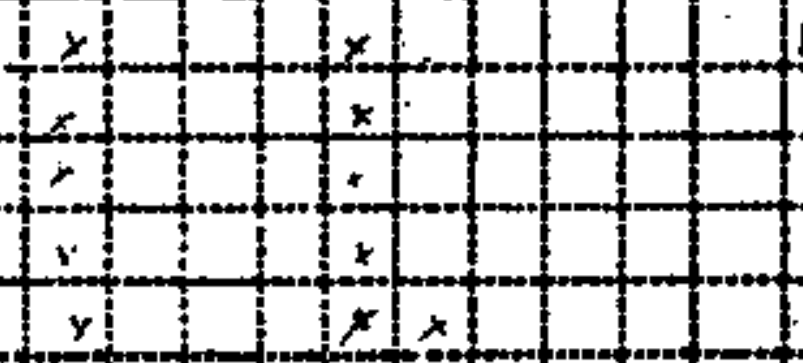
$10: \$ 50$ (1) fats $+1+9$

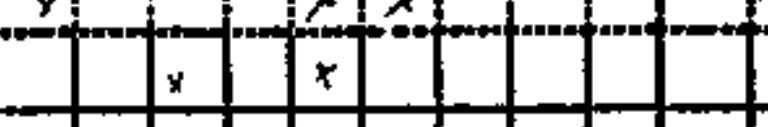
NS Ls 0

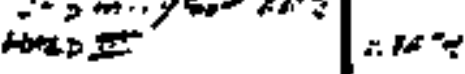
WNisem N!" +.. .

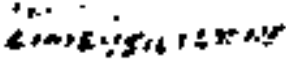

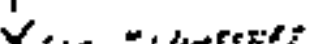

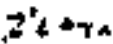
No

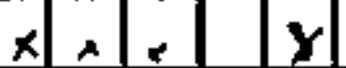

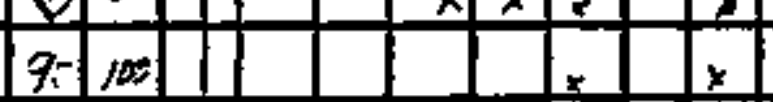
$x=t, \quad, 7 k$ T

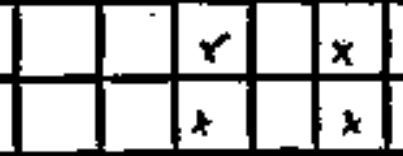

.

6.3. 66:45:. $x^{2}$

mapertidstate vorernmot

oventesto mentation $3 / 4$

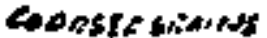
Thatement peps $\sin =* 1]$ 
17 ACOBS ENGMNESRING GROUP NC.

BOREHOLE LOC (ROCK)

LOCATION MAP:

S. 学' A BITE TD: HATOJ LOCATION ID: 907 .

N: GROUND ELEVATION ( $(1, \mathrm{MSL})$

DRILLING METHOD: ${ }_{0}$ W Uagh DRILLER:

DATE BTARTED:

DATE COMPLETED:

FIELD AEP.: B5r $12 \cdot 11=74$

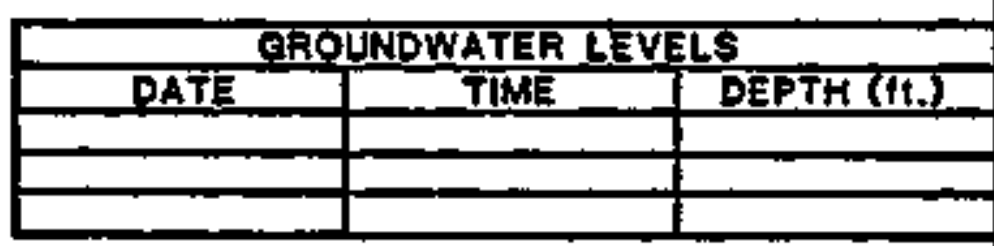

LOCATION DESCRAPTION

SITE CONDITION

conb-CNONH4

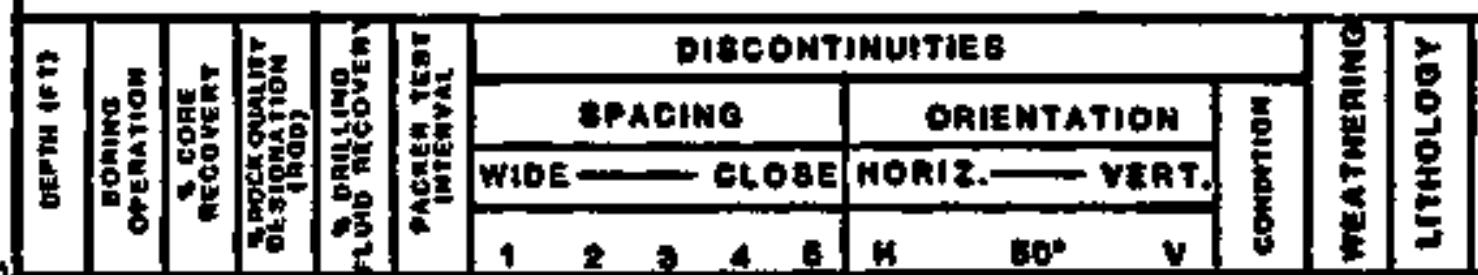

Na 100 100 10

H

\begin{tabular}{ll|l|l|l|} 
& \\
\hline & & & \\
\hline
\end{tabular}

1 10 Ws ${ }^{5}$

$11010 x$

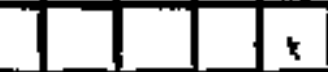

11011

\begin{tabular}{|l|l|}
\hline$x$ & \\
$x$ &
\end{tabular}

$\sqrt{4} \sqrt{ }$

$+$

$+$

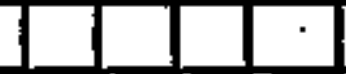

15: 10010090

r

$v_{3} \leqslant$

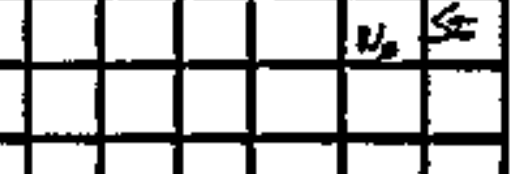

$x$

fwis

$r$

$\because+$

$r$

n.

$+$

111

$+5 \times x$

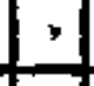

1

Siarsmat - thertsost

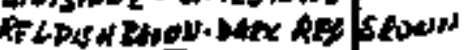

veryetest

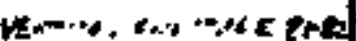

sis rester

covenea

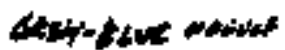

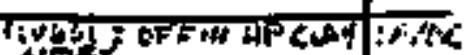

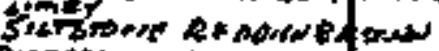

pancitit os virtwest on mose

$r$

$10+v=x+x$

$x$
$x$

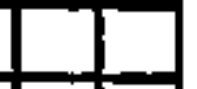

1

Gue beer $y=0$

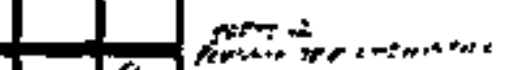

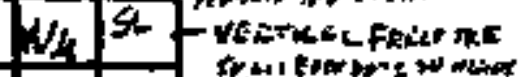

q0.5-94:

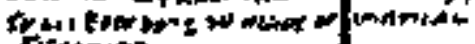
risarnere

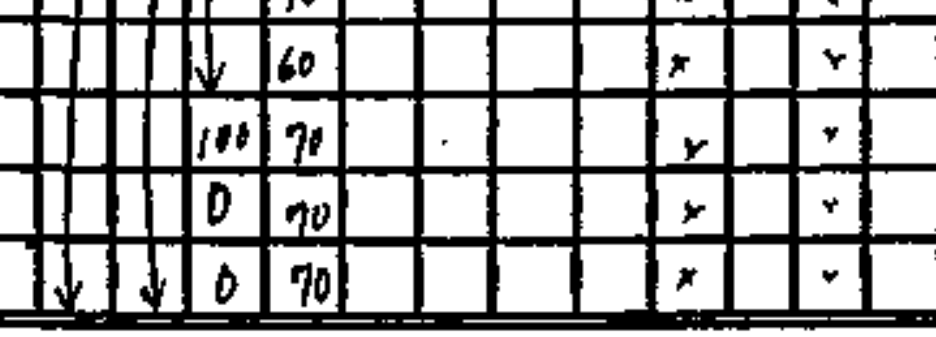

12

W)

anime -

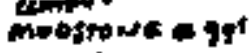

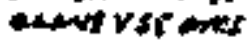

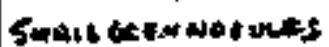

CONMENT 8:

$+$ 


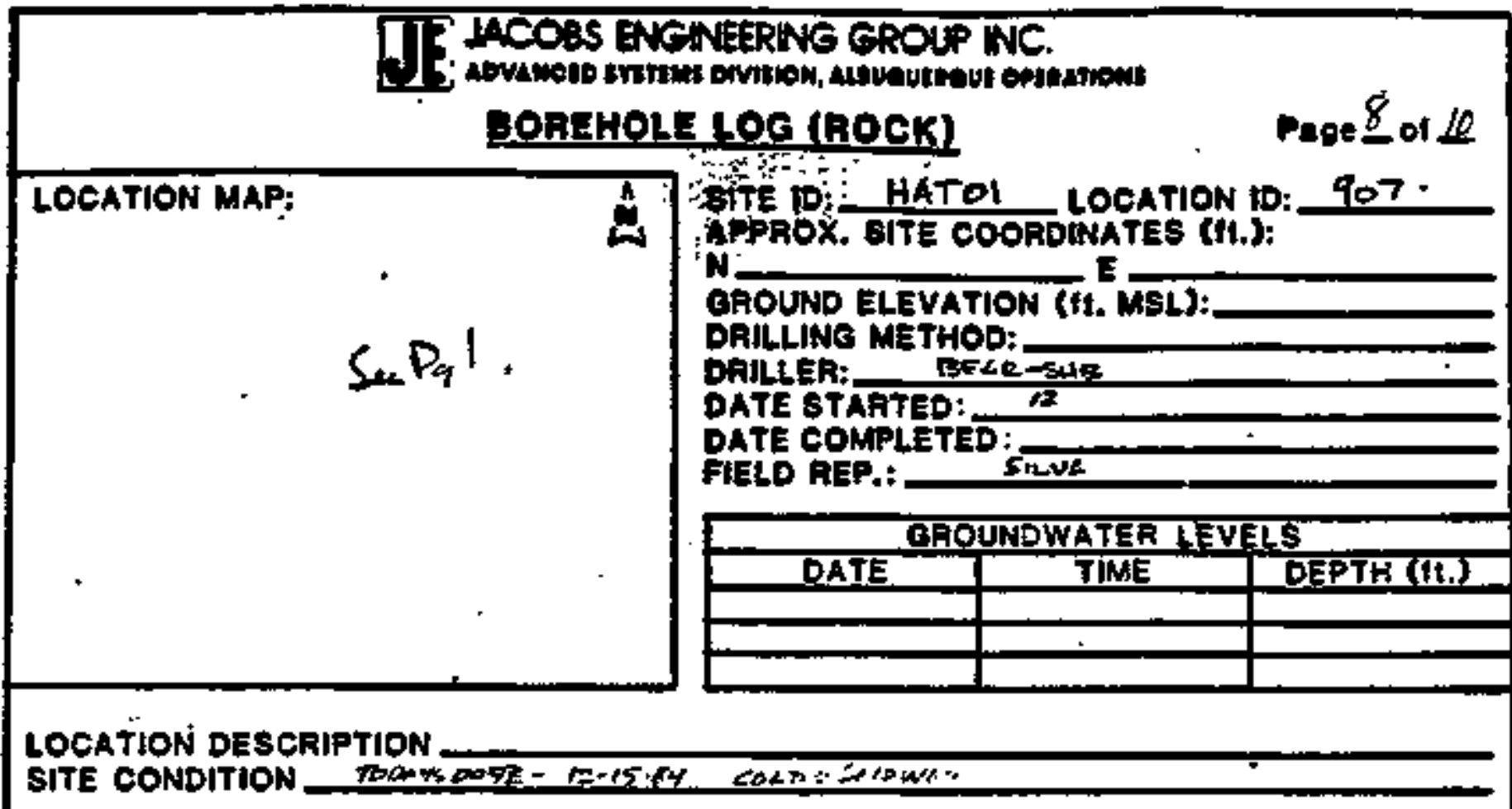

MOEK TYPE

a AEMAAKS

8520

$x$

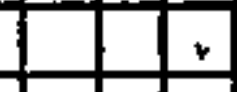

$\begin{array}{lllll} & & & & \end{array}$

in 50

$+$

$\checkmark v$

S.

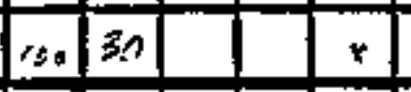

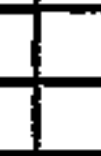

\begin{tabular}{|l|l|l|l|l|l}
\hline$r$ & & & & & \\
\hline$r$ & & & & &
\end{tabular}

\begin{tabular}{l|l|l|l|l|}
\hline & & & \\
\hline & & & & \\
\hline & & & & \\
\hline
\end{tabular}

CZAYSDNA-MUADONE

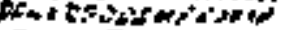

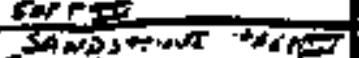

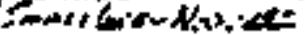

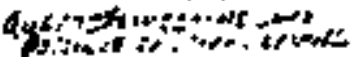

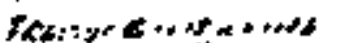

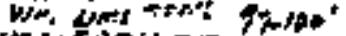

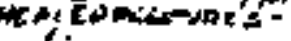
t...

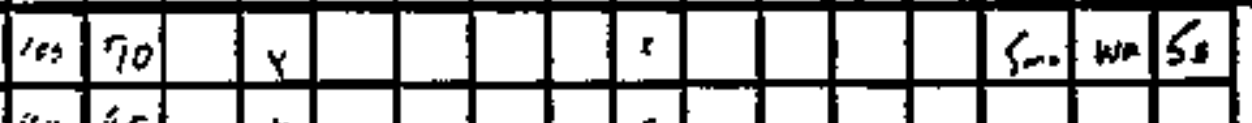

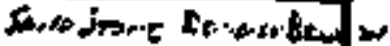

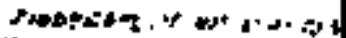

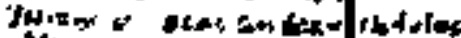

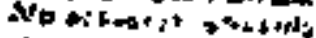

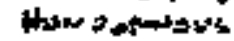




\section{LOCATION MAP:}

$\operatorname{sen} 1$
A. a. APPROX, SITE COOROINATES (th.) N GROUND ELEVATION (tt. MSL): DALLLING METHOD: DRLLEF: DATE STARTED: DATE COMPLETED:

FIELD REP.: Actor/ay

\begin{tabular}{|c|c|c|}
\hline \multicolumn{3}{|c|}{ GHOUNADWATER LEVELS } \\
\hline DATE & TIME & DEPTH (t.t.) \\
\hline estip & S.0089 & Som $x 5^{\prime}$ \\
\hline $245-A$ & $y=b_{n}$ & $\angle A \otimes C^{\prime}$ \\
\hline $2.15+4$ & $10 \mathrm{Fr}$ & $16 e m+6$ \\
\hline$\sqrt{2 \cdot 16 \cdot \theta}$ & $10.15+4$ & $26 \mathrm{dn} \& 15$ \\
\hline
\end{tabular}

LOCATION DESCRIPTION SITE CONDITION

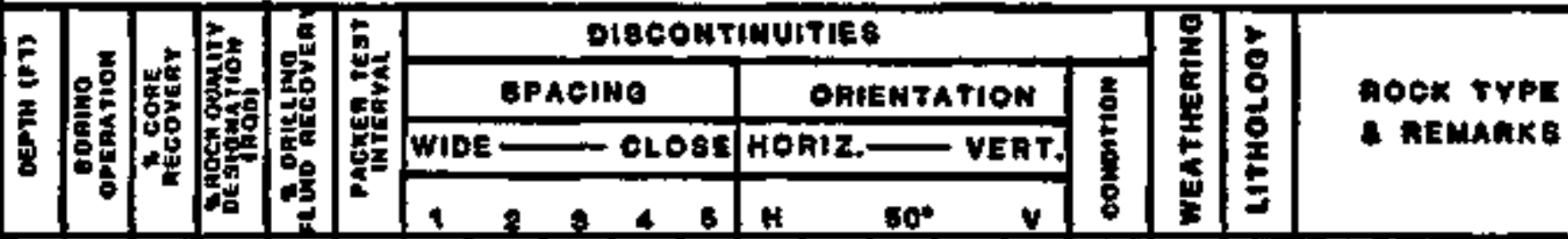

$11_{100} 1_{100}$

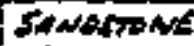
:

Nous weAnteres

Tequnted ato.s 


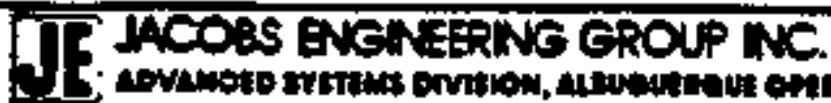

BOAEHOLE LOG (AOCK)

Poge $\frac{10}{6}$ or 10

LOCATION MAP:

A

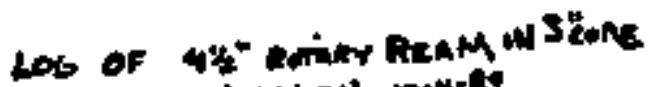

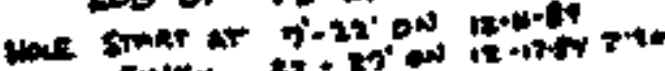

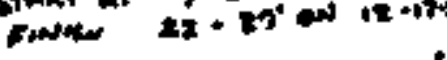

BITE, 10: MEFOL LOCATION 10: 907. APPADX: SITE COOROLNATES (fI,):

Ni: $\rightarrow$ maton (th

BROUND ELEVATION (H. MSL):

DRILLINE METHOD:

DRILLER:

DATE 8TAMTED:

$\pi-1 / 14$

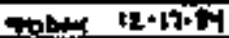

DATE COMPLETED:

FIELO REP.:

\begin{tabular}{|c|c|c|}
\hline \multicolumn{3}{|c|}{ BAOUNDWATERLEVEIS } \\
\hline DATE & TME & DEPTH (tt.) \\
\hline 19.06 .84 & $2: 10+4$ & $2 \leq / 4$ \\
\hline 12.17 .7 & $5.30 \mathrm{Ar}$ & $56 \mathrm{Pm}$ \\
\hline $18+\beta-18 ;$ & way & $1.5122 .54 p$ \\
\hline
\end{tabular}

insareve

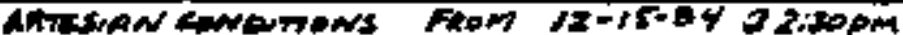

LOEATION DESCRIPTION

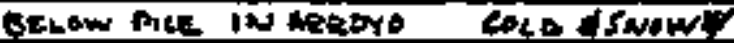

SITE CONDITION

DtaCONTINUITIE 6

\begin{tabular}{|c|c|c|c|c|c|c|}
\hline \multirow{3}{*}{ 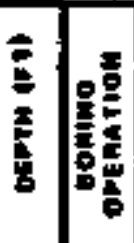 } & \multirow{3}{*}{ 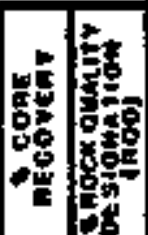 } & \multirow{3}{*}{\multicolumn{2}{|c|}{ 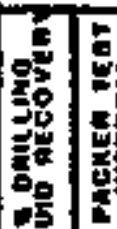 }} & \multicolumn{2}{|c|}{ DtacontINUITIE $B$} & \multirow{3}{*}{$\frac{5}{5}$} \\
\hline & & & & EPACINo & OAIENTATION & \\
\hline & & & & WDE $\longrightarrow$ CLO8E & HOAIZ. & \\
\hline
\end{tabular}

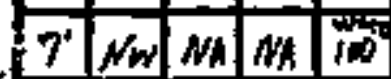

1

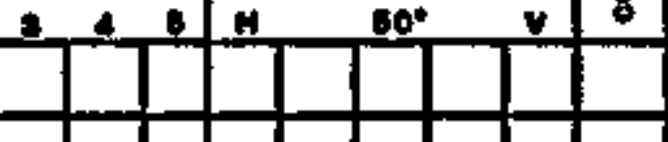

3 10 434 ritate 110

$2=22$

37)

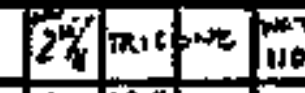

等

11

(4.).

as

1223

\begin{tabular}{llllll}
\hline & & & & \\
\hline & & & &
\end{tabular}

$9 n$

$+$

1

167

$+1$

18

AotK TYPE

- hemaks

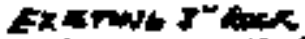

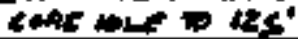

LOAFin otromxy

Nowe totaming

miteng

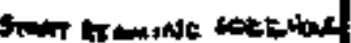
aisen

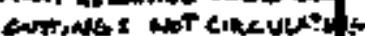

Machere Hate

$1 / 2 z$

Rate rinuthas

1

19

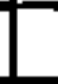

I

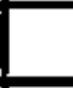

$+$

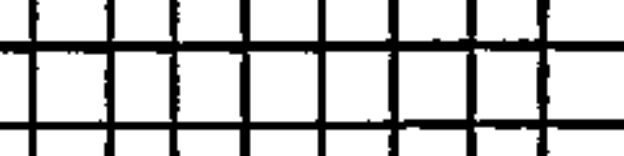

+ ב

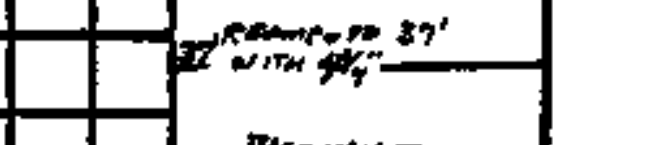

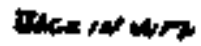

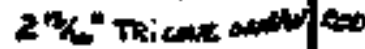

r. $8 \mathbf{F}^{*}$

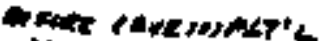

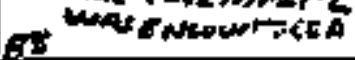
$9+2$ tas

77- and mater! ret $\operatorname{los} 6 .+\infty$

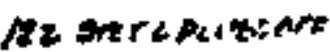
倡会:

1

(1)

$+2$
1

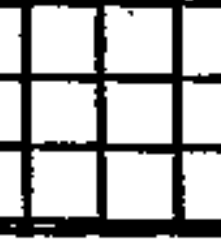

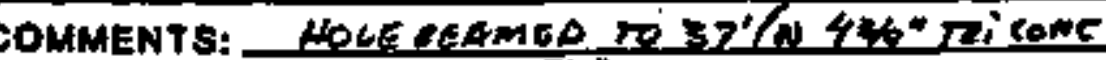

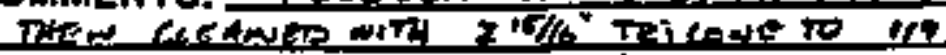
$7 \pi 0$ $445+6 A$ Ta $100^{\circ}+1 r^{\prime}$ werst

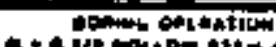

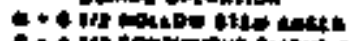

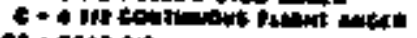

ctectot on

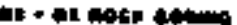

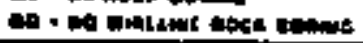




\section{THCOBS ENGNEERING CROUP NC.

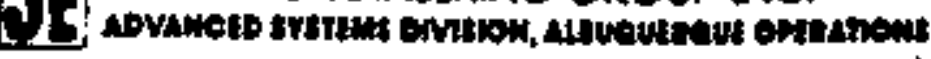

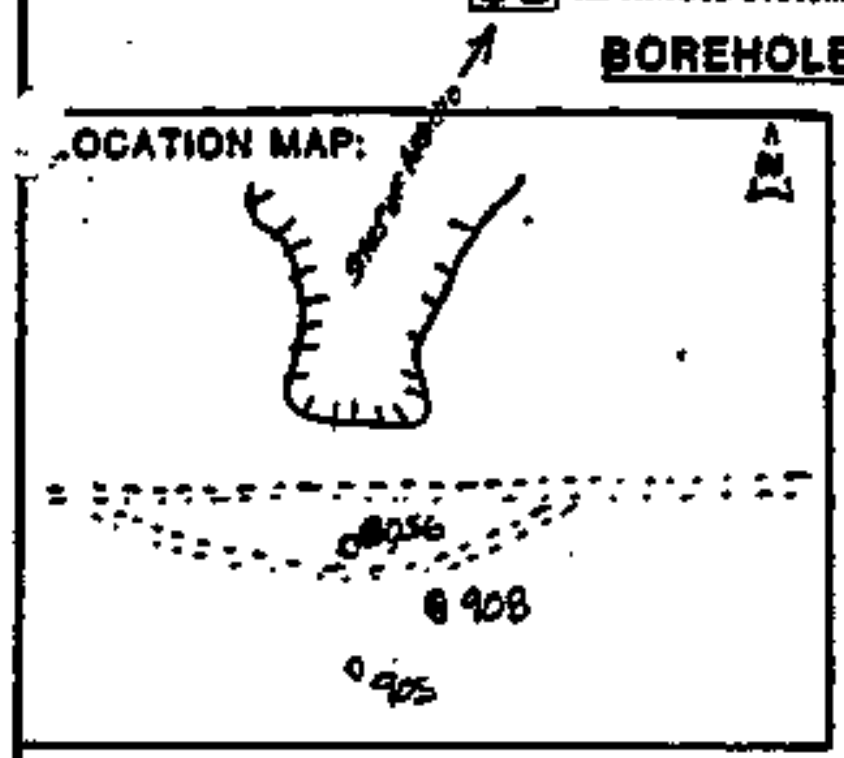

\section{QG (ROCK)}

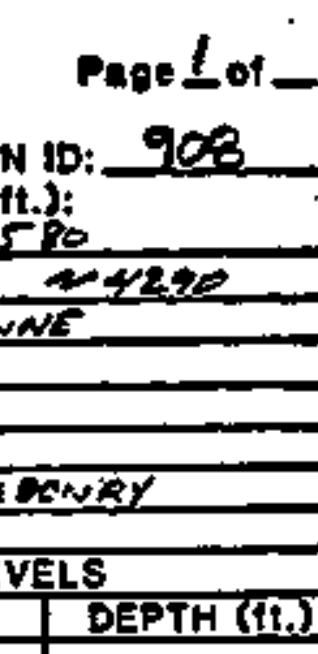

OCATION MAP:

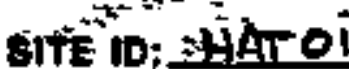

tockTION ID: 903

APPROX. 8ITE COORDINATES (ft.):

N roo o E 5 ro

CROUND ELEVATION (ft. MSL): 4 Y2220

DAILLINO METHOD: NAMRPLNE

DRILLEP: E CAER:

DATE STARTEO: 1025

DATE COMPLETED:

FIELD REP.:

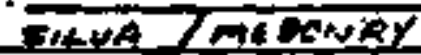

\begin{tabular}{|c|c|c|}
\hline \multicolumn{2}{|c|}{ BAOLNDWATER LEVELS } \\
\hline DATE & TIME & DEPTH (II.) \\
\hline & & \\
\hline & & \\
\hline
\end{tabular}

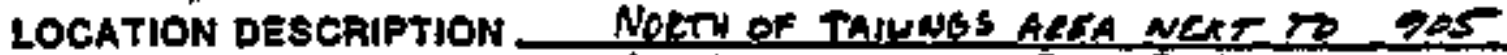

SITE CONDITION

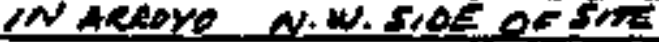

\begin{tabular}{l|l|l|l|l|l|l|l|l|l|l|}
\hline \\
$z$
\end{tabular}

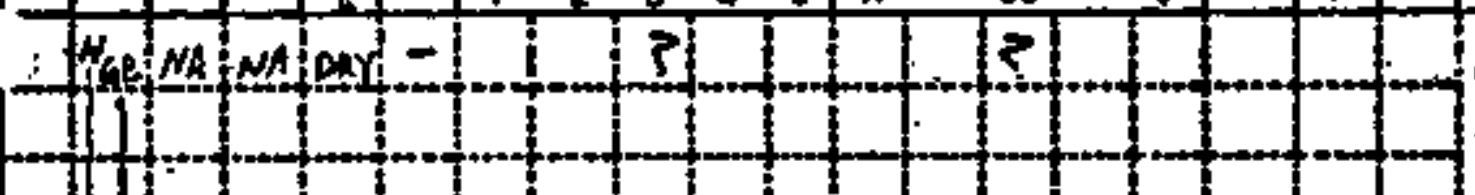

1

,

1

NA 100

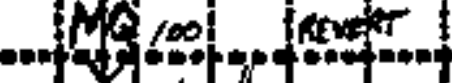

No 1 100

$x$

$+6$

1) 0

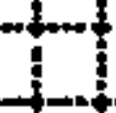

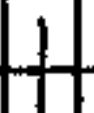

$x$

$\times{ }^{-1}$

160

0

1

-

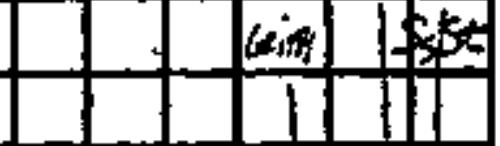

1000,

$\sin _{0} n_{s}$

190180

\begin{tabular}{ll|l|l|l|l|}
\hline & & & & & \\
\hline & & & & &
\end{tabular}

1 N/folo

$\sqrt{1}$

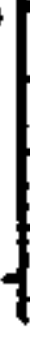

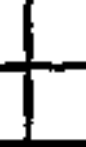

1

Hz ramentas

entopor

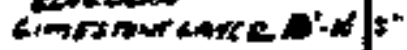

Ans:

$2 \pi n$ brectoror

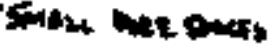

1

COMMENTS:

4 orcasion

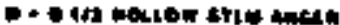

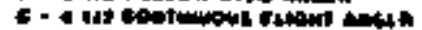

c - ctat at

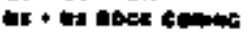

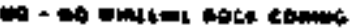




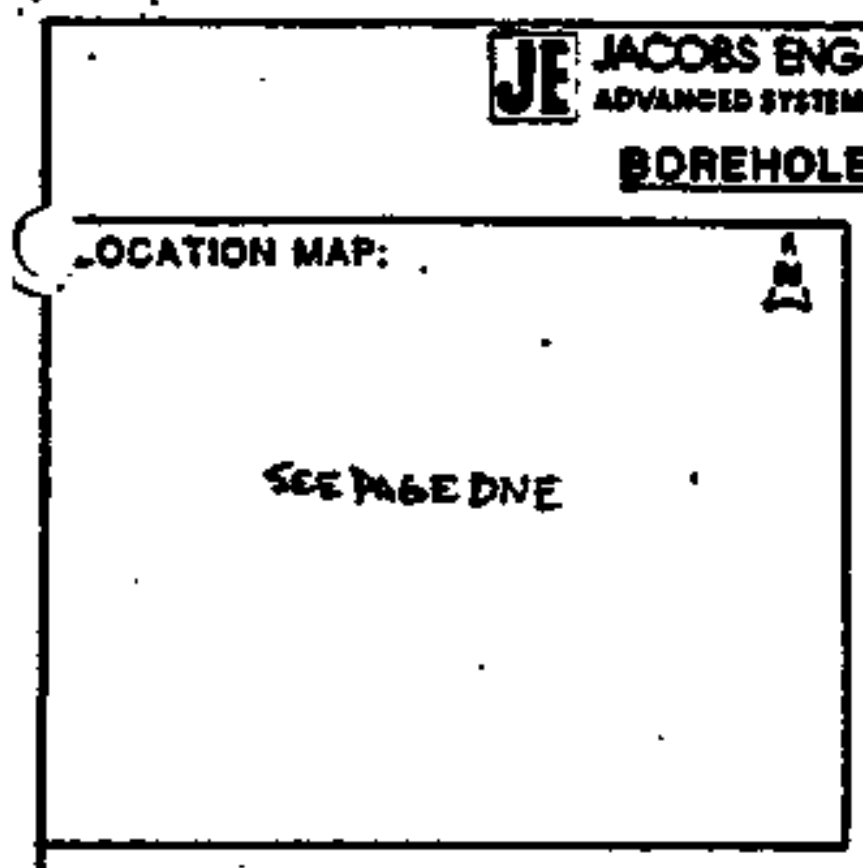

LOCATION DESCAIPTION SITE CONDITION

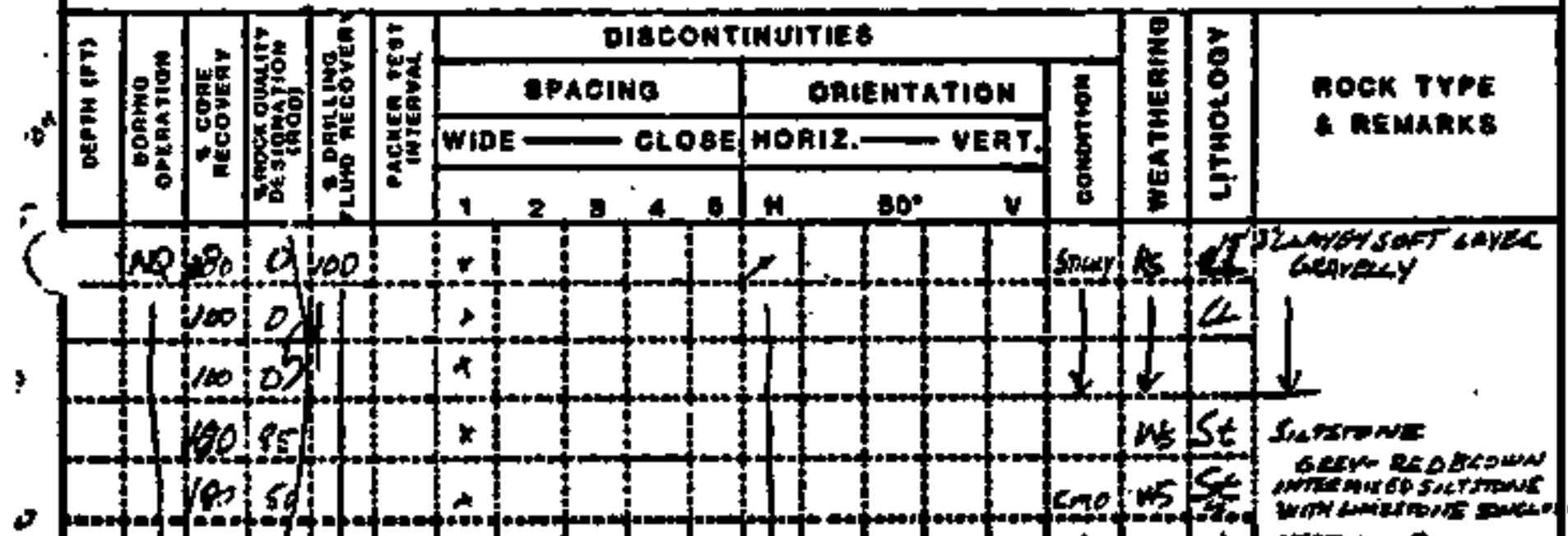

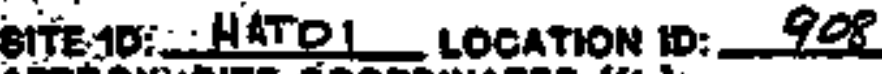

AdPAOX:StrE COOHDUNATES (ft.):

$N \because:-$ :

gROUND ELEVATION (t. MSL):

DRILLING METHOD:

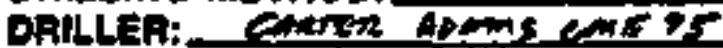

DATE STAATED: ARTS

DATE COMPLETED:

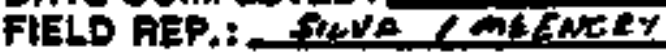

\begin{tabular}{|c|c|c|}
\hline & GAOUNDWATER LEVELS \\
\hline DATE & TIME & DEFTH (TI) \\
\hline & & \\
\hline & & \\
\hline & & \\
\hline
\end{tabular}

$100 \mid$, $100 \mid$

$\mid x$

$x$

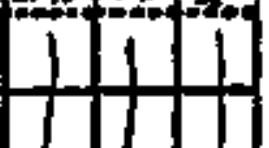

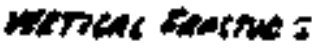

(1)

1

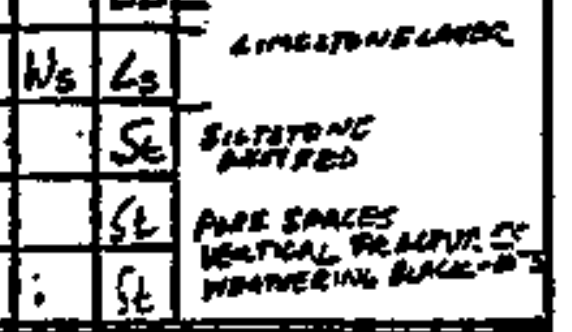


DE TACOBS ENGNEERRV GROUP NC.

BOREHOLE LOG (HOCK)

Papelot -

LOCATION MAP:

$\frac{1}{5}$

Sul pose one
A sition:m:

$+4$

8ITE

APPAOX: SITE COOADINATES (tt.):

$\mathrm{N}_{\mathrm{i}}: \mathrm{i}_{\mathrm{O}} \mathrm{E}$

GROUND ELEVATION (tt. MSL): $\angle 4290$

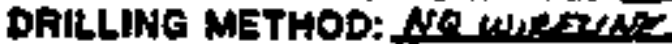

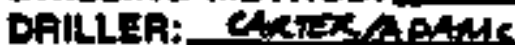

DATE 8TARTED: d/T/T5

DATE COMPLETED:

FIELD AEP.: AKSTAC

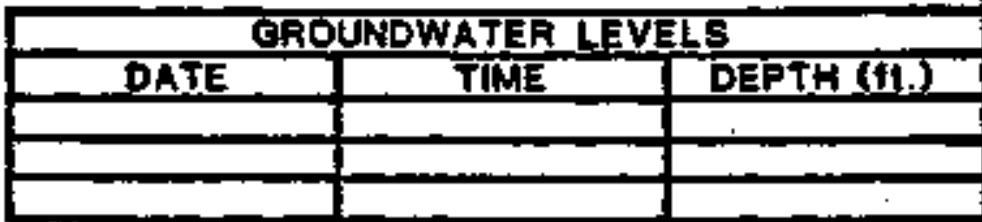

LOCATION DESEAIPTION

SITE CONDITION

ह

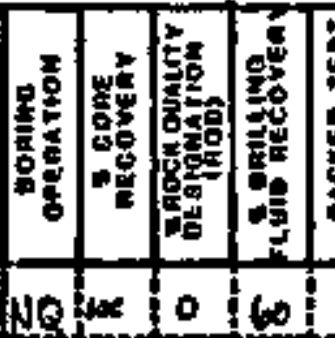

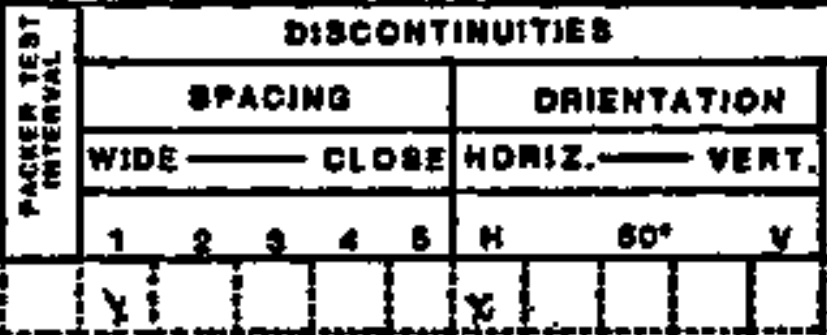

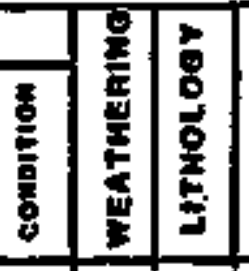

nOCK TYPE

- REMARK

, $\sum_{0} 1$

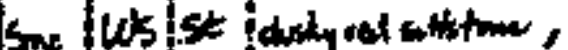

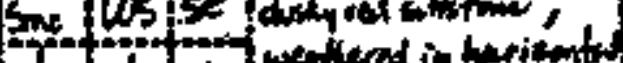
50

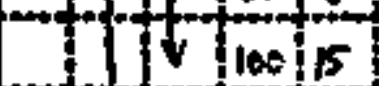
4 $y$ 1 1.

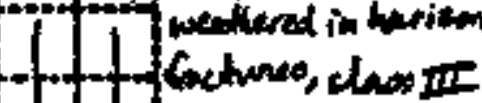
1. 1. [a barches jordes 170 to astimatures or

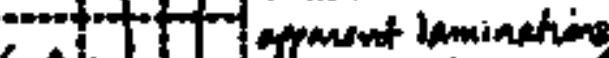

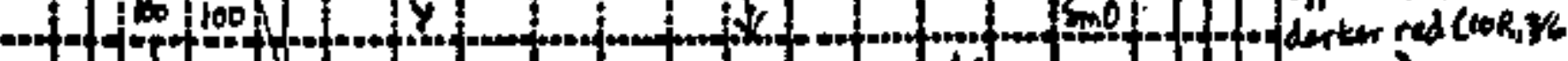

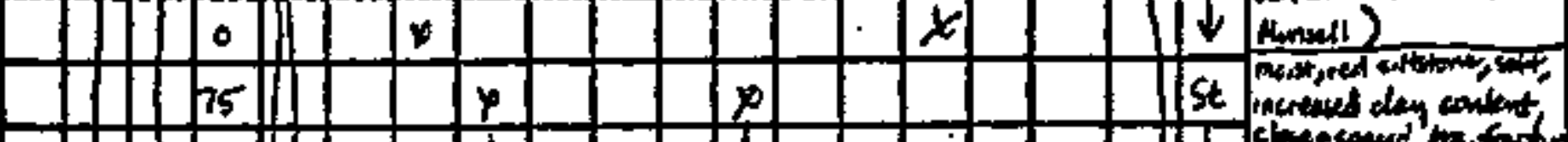
$\prod \mathrm{son}$ 1724 1150 1 11 1

$11+$

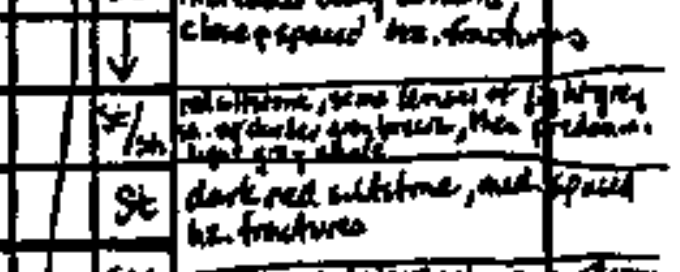

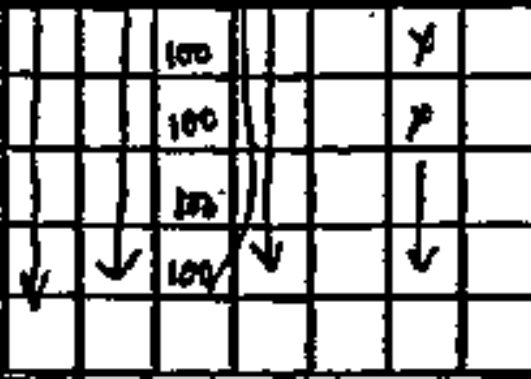
AOMMENTS: 


\section{T5 JACOAS ENGENEERNE CROUP NC.

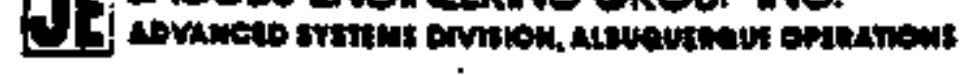

\section{BOREHOLE LOQ (ROCK).

HOCATION MAP:

4 SITE

LOCATION ID: 908

a APPROX. BITE COORDWNATES (tt.):

Nt

GROUND ELEVATION (ft. MSL): 4ZZGO

DRILLING METHOD: NQ WIREAST

Seepastoner

DAILLER: CARTAR GQAMS

DATE STARTED: W755

DATE COMPLETED:

FIELD REP.: MCEN

\begin{tabular}{|l|l|l|}
\hline GROUNDWATEA LEVELS \\
\hline DATE & TIME & DEPTH (t.) \\
\hline & & \\
\hline & \\
\hline
\end{tabular}

LOCATION DESCAIPTION

SITE CONDITJON

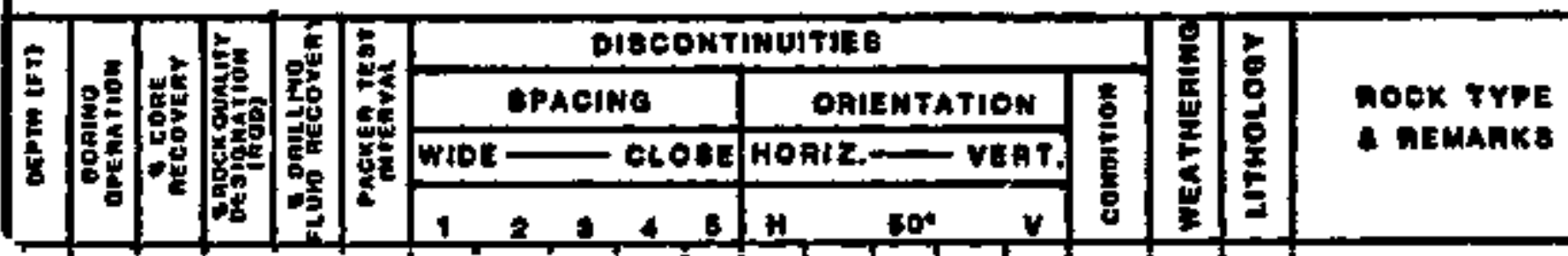

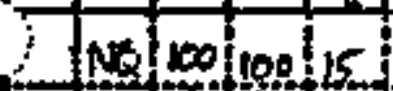

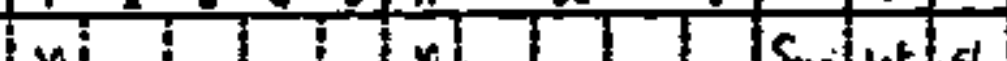

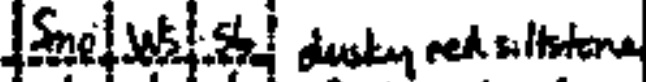

140 yi

4.

onfractives, handment

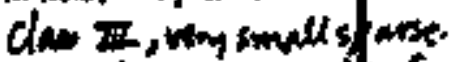

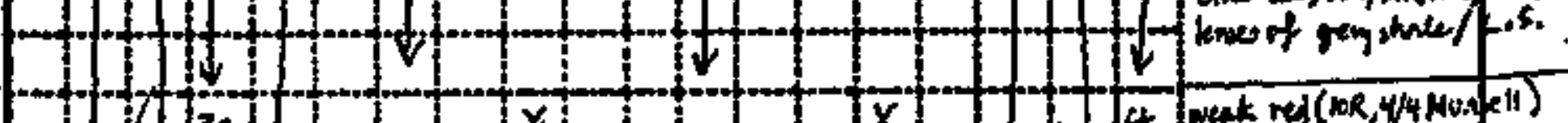

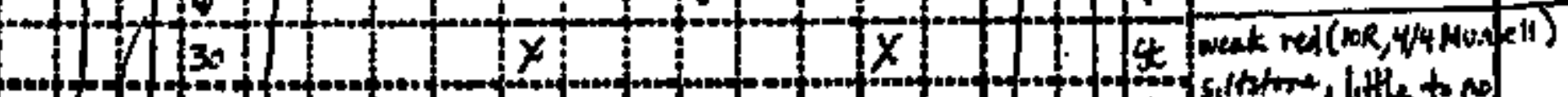

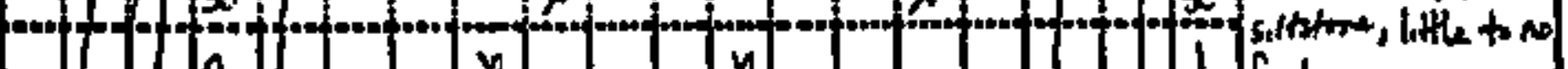
175 $x$ $x$ fructures fretures, weat nod siltation stacel:

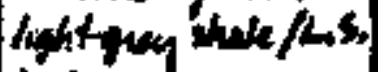


D3.

ROREEHOLE LOO (ROCK)

Page ef $_{\text {_ }}$

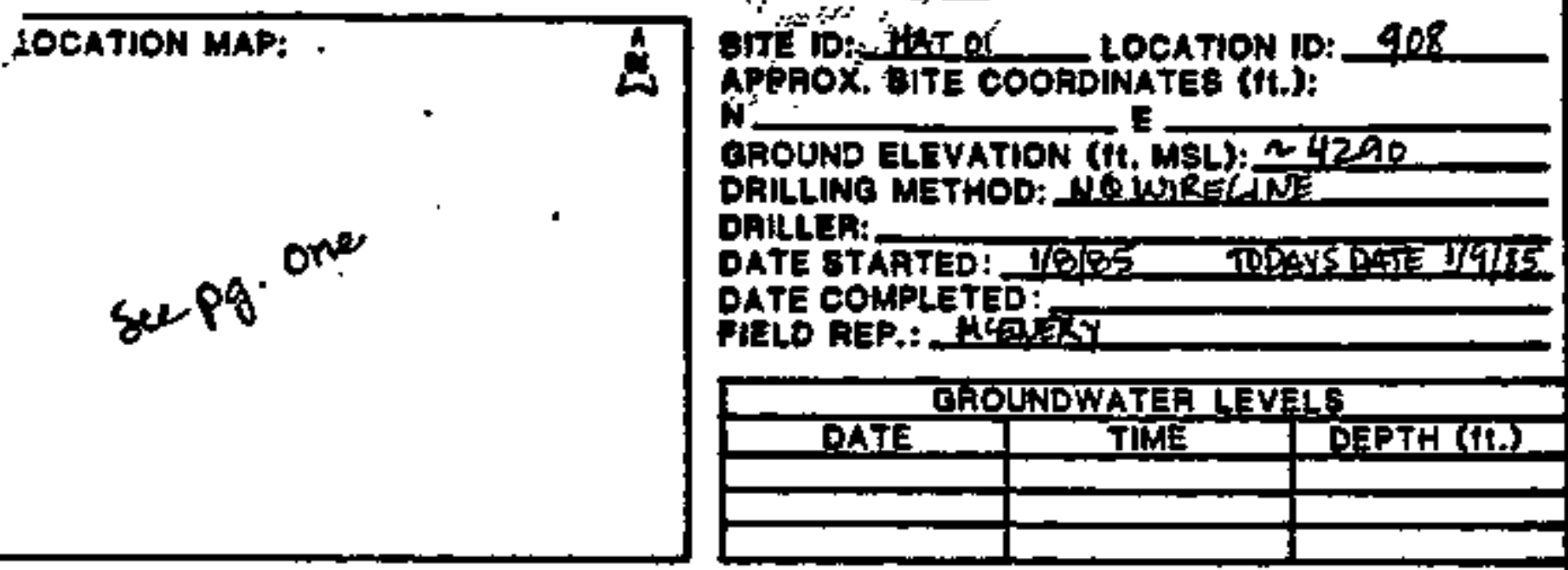

LOCATION DESCRIPTION

SITE CONDITION

\begin{tabular}{|c|c|c|c|c|c|c|c|c|c|c|}
\hline \multirow{3}{*}{\multicolumn{2}{|c|}{ 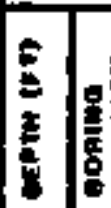 }} & \multirow{3}{*}{ 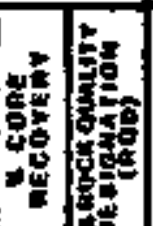 } & \multirow{3}{*}{ 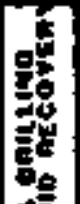 } & \multirow{3}{*}{ 数 } & \multicolumn{2}{|c|}{ DIseontantutes } & \multirow{3}{*}{$\frac{5}{5}$} & \multirow{3}{*}{ 量 } & \multirow{3}{*}{$\begin{array}{l}5 \\
8 \\
8 \\
\end{array}$} & \multirow{3}{*}{$\begin{array}{l}\text { nook Troe } \\
\text { e nemanke }\end{array}$} \\
\hline & & & & & epacing & OHIENTATION & & & & \\
\hline & 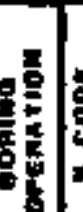 & & & & WTDE - CLOHE & HORIZ.- VEAT, & & & & \\
\hline
\end{tabular}

1

m

-

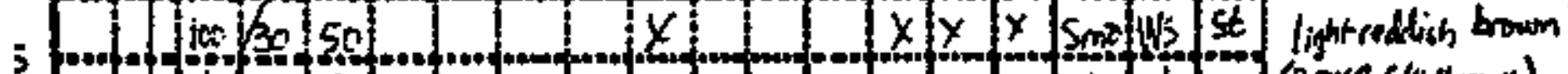

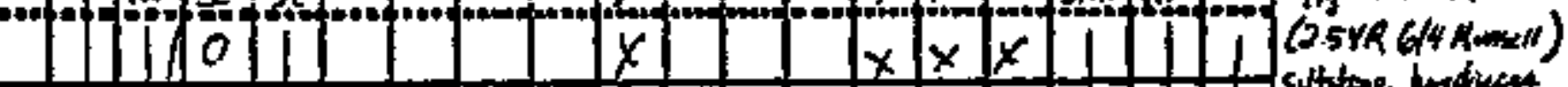

$113 c$

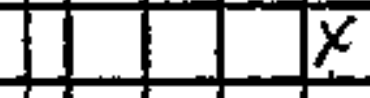
suthome, hadiwent closs $\pi$, ter bed

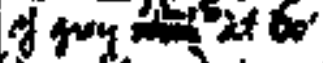
130 $x+\frac{x}{x}$ kindery), thends 100 $x$ 63' ven def uest to

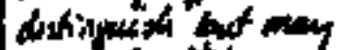

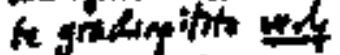




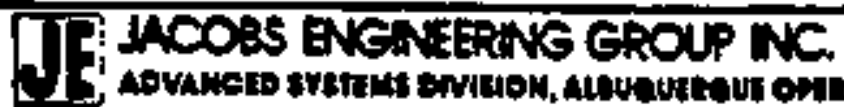

BOREHOLELOO (ROCK) Page 6 of -

LOCATION MAP:

$A$ sine tDitat of

LOCATION ID: 908

- $A$ APPROX. BITE COORDIMATES (ft.):

स: E

GROUND ELEVATION (1T. MSL): 4290

sepgore.

DAILLING METHOD: WQWhR LINE

DAILLER: CARTER LOAMS

DATE STARTED: VAlS FOAY:DGIS

DATE COMPLETED:

FIELD REP.: MLEYEXY

\begin{tabular}{|l|l|l|}
\hline \multicolumn{3}{|c|}{ GROUNDWATER LEVELS } \\
\hline DATE & TIME & DEPTH (ft) \\
\hline & & \\
\hline & & \\
\hline
\end{tabular}

LOCATION DESCRTPTION

SITE CONOITION

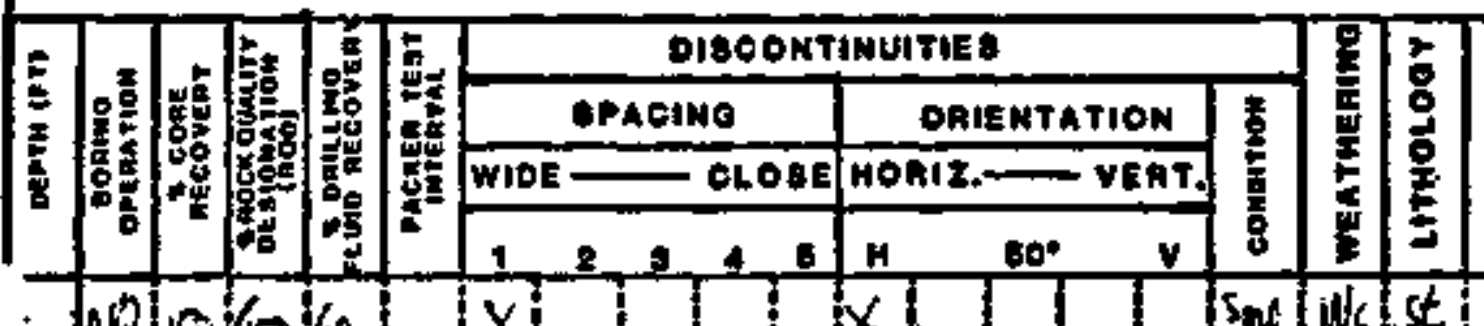

3

$60^{\circ} \quad v \quad 0$

nock Troe

- nemanks

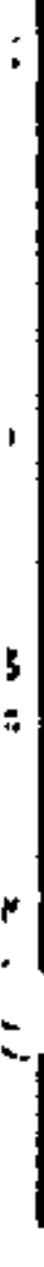

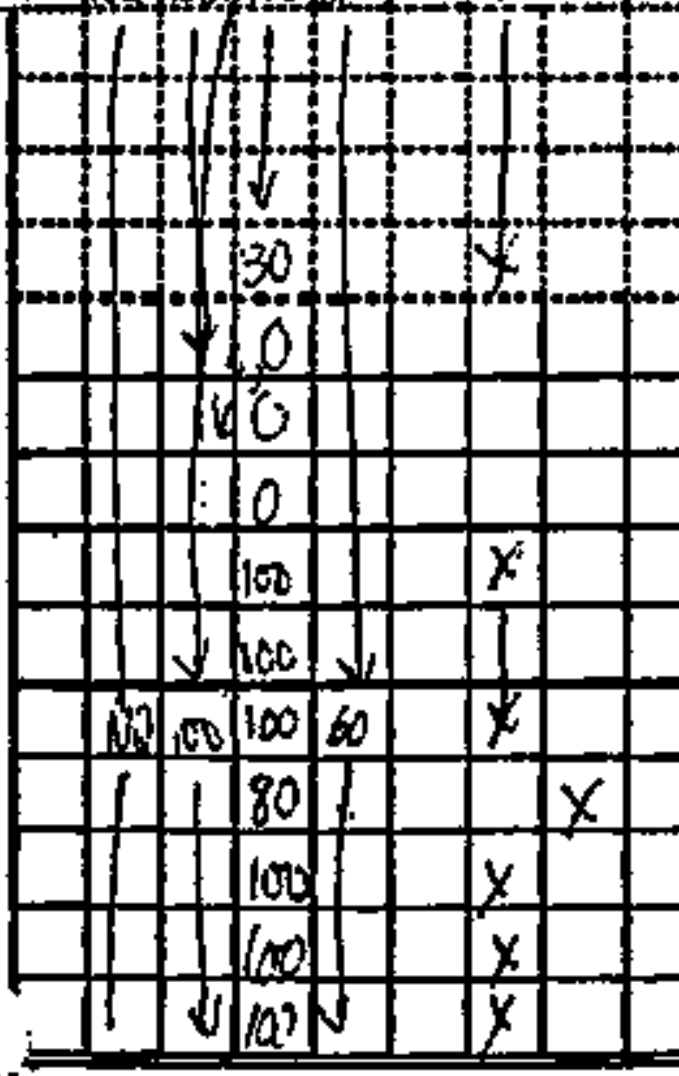

COMMENT \&:

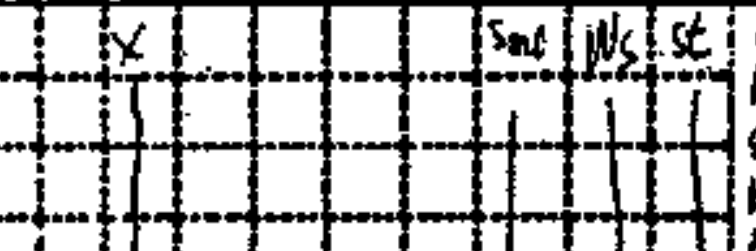

$1,+1+101$

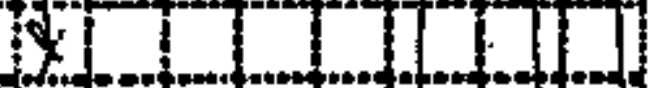

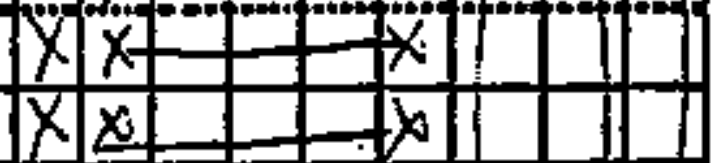

$x \times-x \mid$

$y$

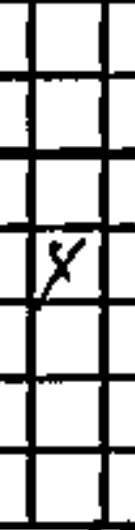

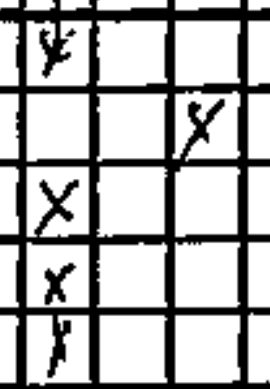

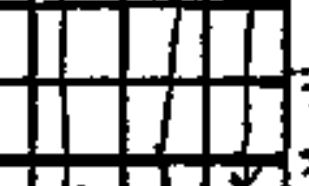

Thin teat ofjen 4 .s. at at $73^{\circ}-73 / 2$ conpreationg wht raldsh bromen silfitort come chancientitics as the $77^{2}$ contorm. 


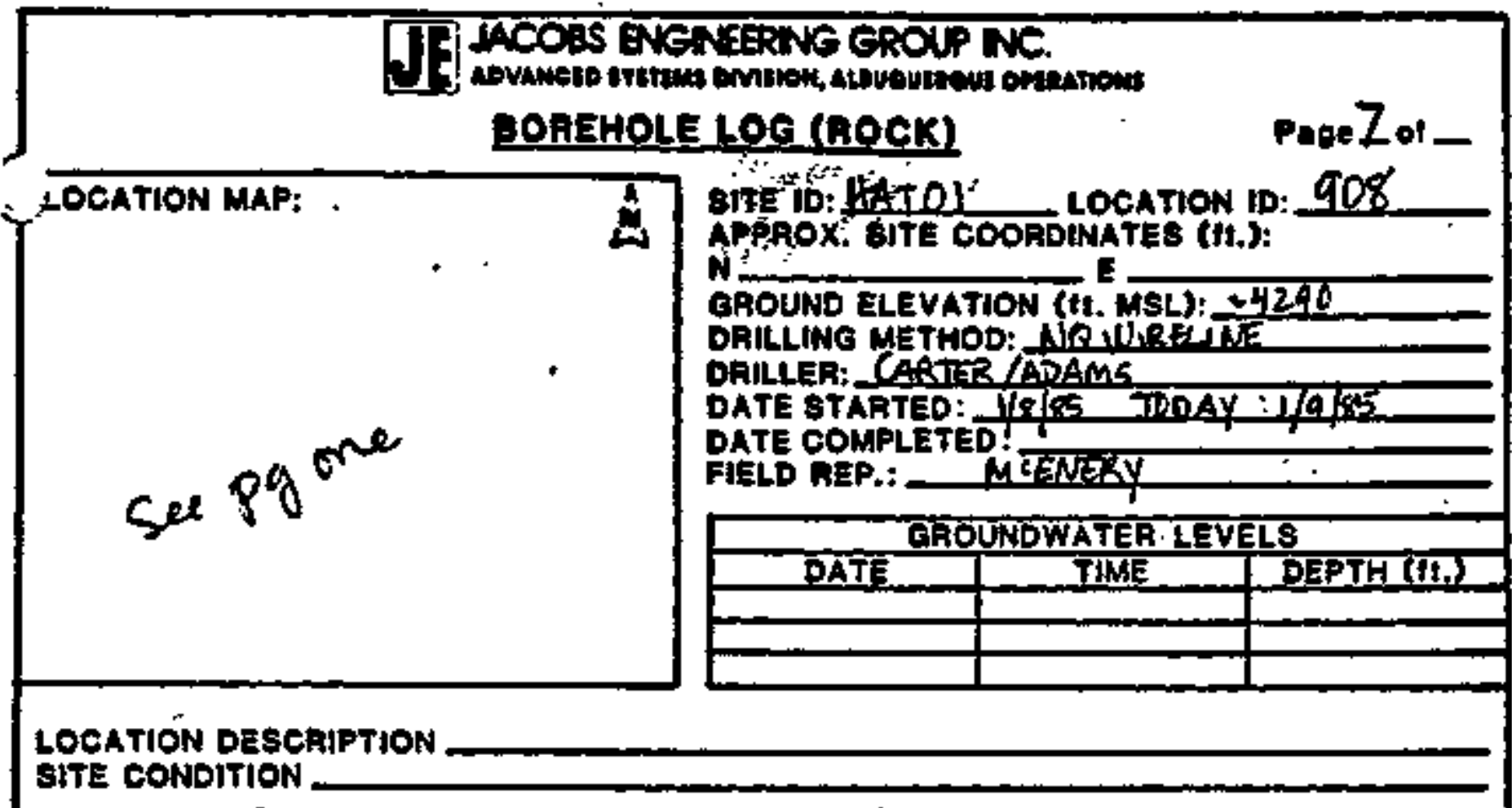

\begin{tabular}{|c|c|c|c|c|c|c|c|c|c|c|}
\hline \multirow{4}{*}{ E } & \multirow{4}{*}{ 资 } & \multirow{4}{*}{ 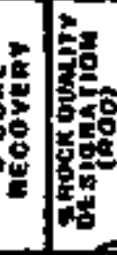 } & \multirow{4}{*}{ 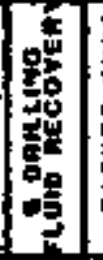 } & \multirow{4}{*}{ 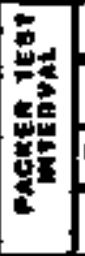 } & \multicolumn{2}{|c|}{ DIsCONTINUITIE: } & & \multirow{4}{*}{$\mid$} & \multirow{4}{*}{$\begin{array}{l}5 \\
8 \\
0 \\
0 \\
0 \\
5\end{array}$} & \multirow{4}{*}{$\begin{array}{l}\text { nock TYHE } \\
\text { e REmAKKo }\end{array}$} \\
\hline & & & & & SPACING & OnIENTATION & है & & & \\
\hline & & & & & WIOE $\longrightarrow$ CLOBE & HORIZ, - VEAT. & & & & \\
\hline & & & & & +2 & $4 \quad 60^{\circ}$ & $\overline{8}$ & & & \\
\hline
\end{tabular}

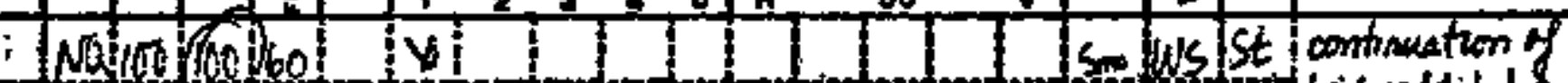

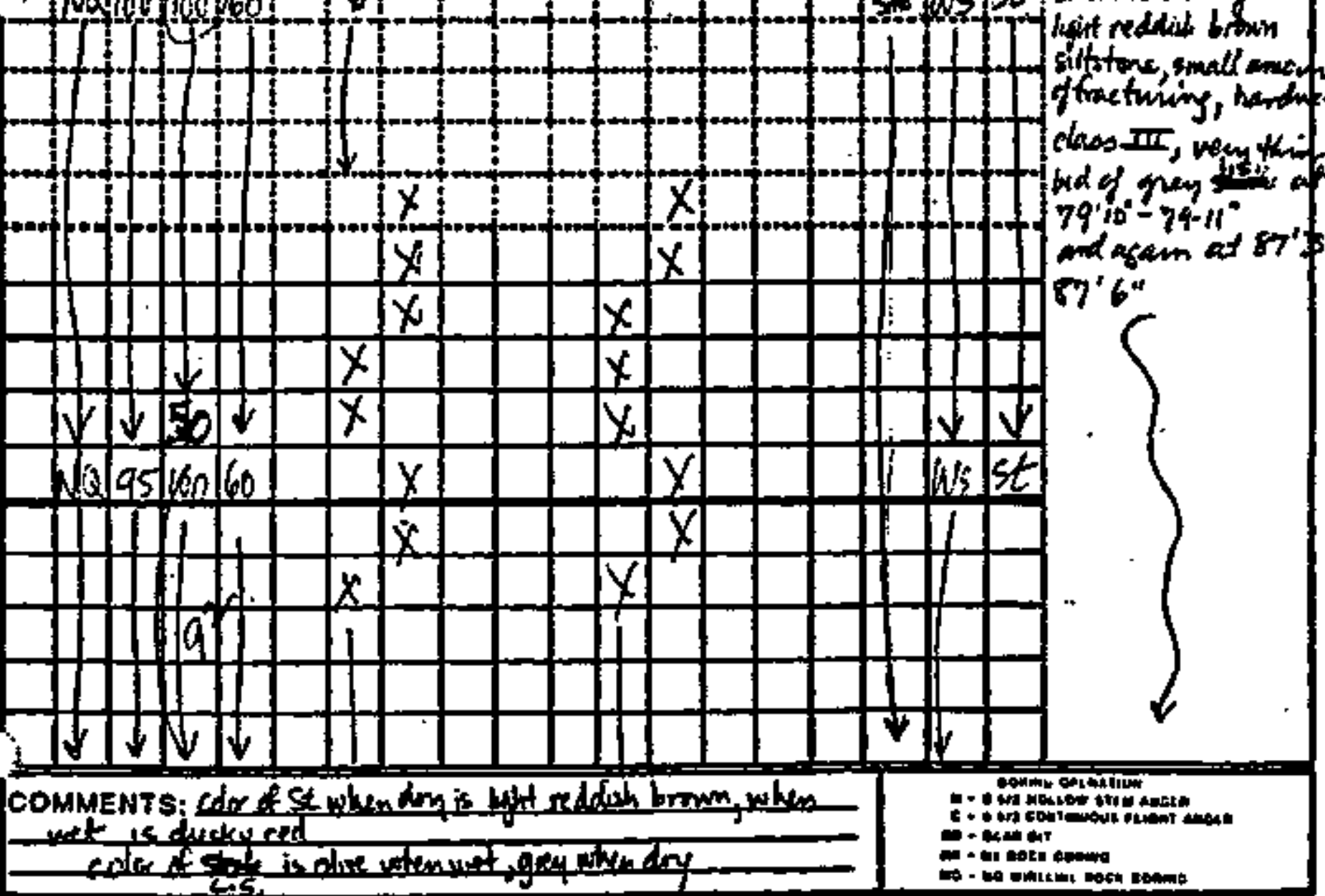




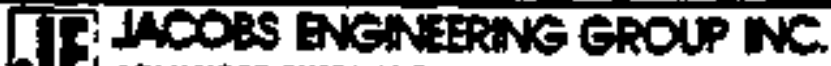

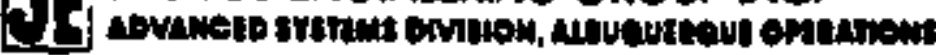

ROREHOLE LOQ (ROCK)

Page 8 of

LOCATION MAP:

is

serpgone

Bitevititiol : Location to: 903

APHTOX. TETE COORDINATES (4.):

$N:$ : : E

CROUND ELEVATION (4t. MSL): 4 (2SO

DAILLNO METHOD: NR WHELANE

DAILLER: CAKTRRLOAMS

DATE BTARTED: WLS 7DDAY JWIS

DATE COMPLETED:

FIELO REP.: $M(Q) \overline{A B}$

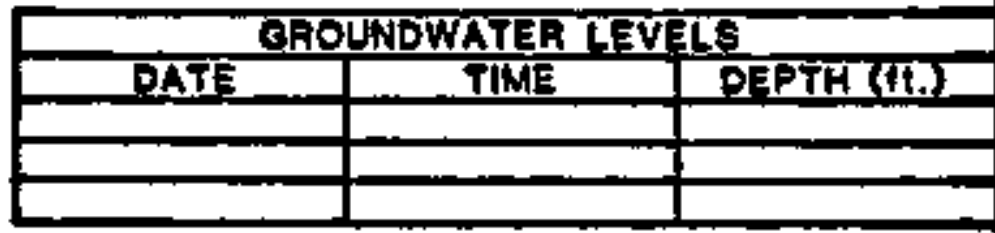

LOCATION DESCFIPTION

8ITE GONDITION

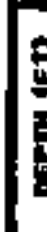

\begin{tabular}{|c|c|c|c|c|c|c|c|c|}
\hline \multirow{3}{*}{ 㩆 } & \multirow{3}{*}{ 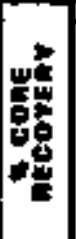 } & \multirow{3}{*}{ 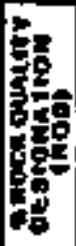 } & \multirow{3}{*}{ 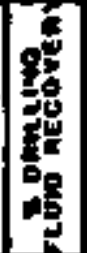 } & \multirow{3}{*}{ 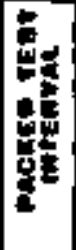 } & \multicolumn{2}{|c|}{ DIESORTINUIFIE } & \multirow{3}{*}{$\begin{array}{l}8 \\
8 \\
8 \\
8\end{array}$} & \multirow{3}{*}{ 衰 } \\
\hline & & & & & opacino & OnILNTATION & & \\
\hline & & & & & WIDE $=$ CLOSE & MORIz.— VEAT. & & \\
\hline
\end{tabular}

nOEK TYOF

e n:manke

wo $\operatorname{las} / 10 \mathrm{tan}$

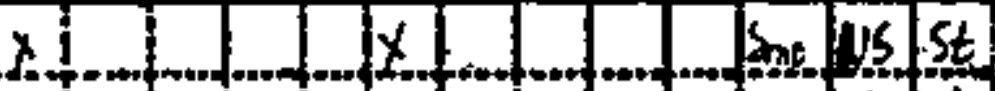

1.1. 1004.1 .1$.

X.

1010

10.160

.y.

$x$

$\sqrt{2} 160+1$

$495 / 60125$

$\mathrm{X}$

(x)

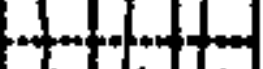

ompinceation 7

light tedduch brom

stitore, small

ancount of

fucturning,

$1=1801111$

$1]$ 5c

$1]$ ing

$\mathbf{x}$

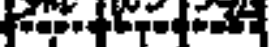

huiner ctaos

III, sligitth

witakend in

fordures germ

$\prod_{1}+\frac{x}{1}$

homogeneme whit

11150

1

trom $93^{3}$ is

sone inclutusis

divetsos the

groterat, then

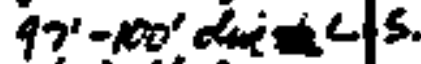

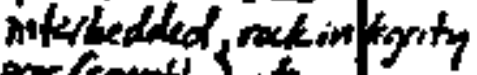

$\sqrt{4}$

$\sqrt{1}$

por (combly) atas inthere bam. As Ist.

$\therefore \sqrt{ }$

s.

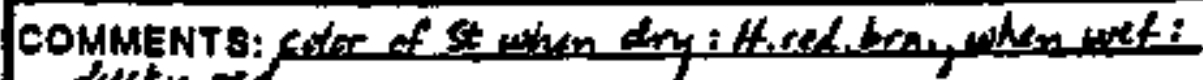
tustey $\mathrm{N}$

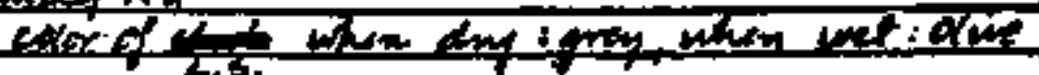

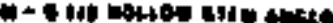

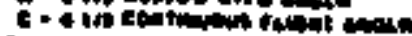

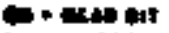

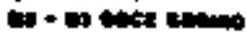

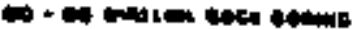




\section{TF ACOBS ENGNEERING GROUP NC.}

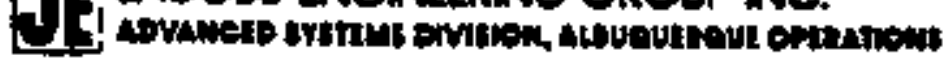

BOREHOLE LOG (ROCK)

Pageqor_

LOCATION MAP:

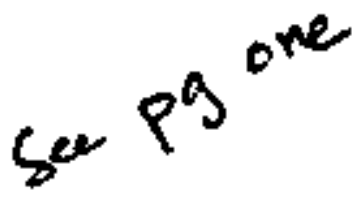

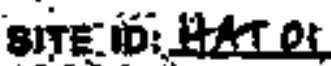

N:

GROUND ELEVATION (t1. MSL): $\sim 4290$

ORILLINO METHOD: HOWRTAN

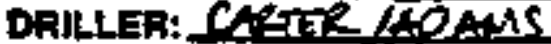

DATE STARTEO: HeVOS TMOAY IETS

DATE COMPLETED:

FIELD REP.: HERNERY

\begin{tabular}{|l|l|l|}
\hline \multicolumn{3}{|c|}{ CROUNDWATER LEVELS } \\
\hline DATE & TIME & DEPTH (IL.) \\
\hline & & \\
\hline & & \\
\hline
\end{tabular}

\section{LOCATION DESCAIPTION}

SITE CONDITION

告

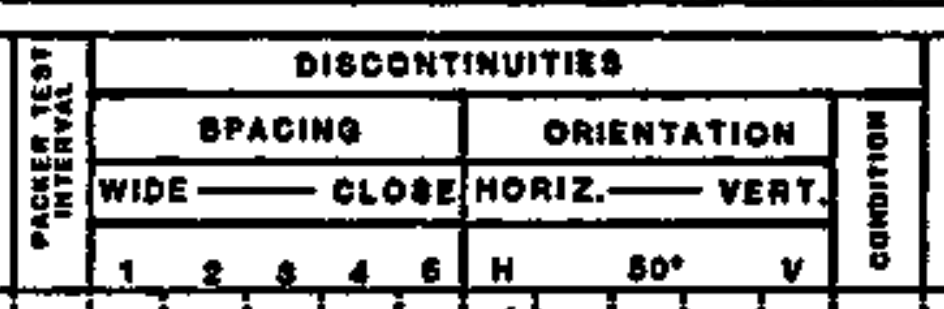

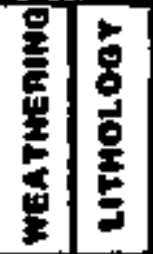

notK TYPE

a Atranks

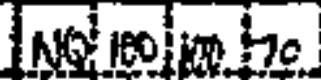

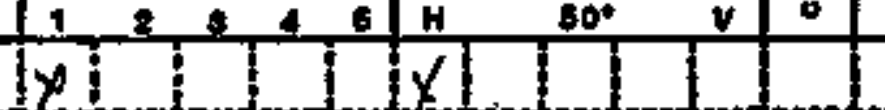

Ist loght reddish brom

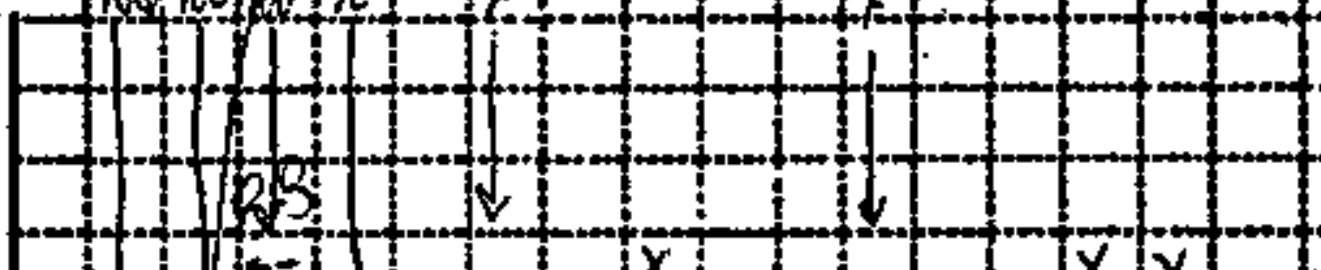

71 siltowe with trace of diex she/s, tows interkedded

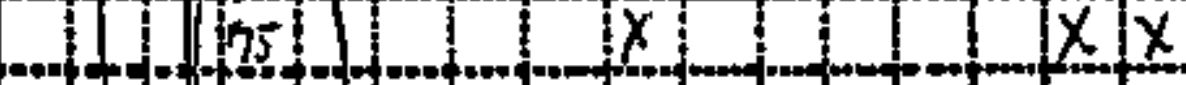

$\frac{3}{5}$

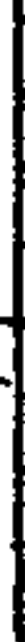

1100

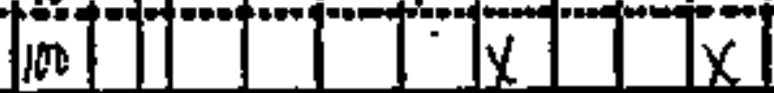

1150

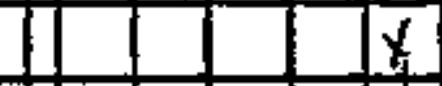

1

$\sqrt{ }$

$x+1$

vertieal fructuring of 108

CL moist dution end

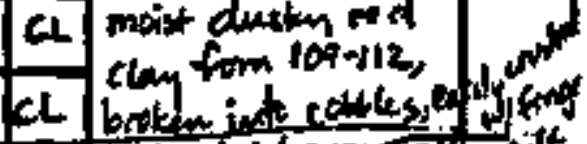

$\rightarrow 110$

$1 \sqrt{1} \downarrow$

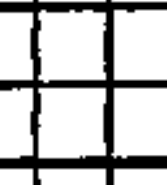

1

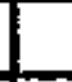

$1 \mathrm{cL}$

4.

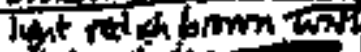

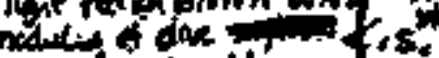

side of minest

$V$

and. fuctives of 113

\section{COMMENTS:}




\section{DE ACCOBS ENGA EERTNG GROPP NC.}

BOREHOLE LOO (ROCK)

Pagellot-

LOCATION MAP:

$\dot{\Delta}$

ing

BIFE Ho: Harel LOCation ID: 708 APPROX, gITE COORDINATES (tt.):

N 10.00 E E Es:

GROUND ELEVATION (tT. MBL):

Pl

DRILLINO METHOD:

DAILLER: S.C. $5 \mathrm{NA}$

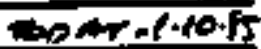

DATE 8TAATED: WO/OS

DATE COMPLETED:

FIELD REP,: \&NAT

\begin{tabular}{|l|l|l|}
\hline \multicolumn{3}{|c|}{ GODONDWATEA LEVELS } \\
\hline OATE & TIME & DEPTH(it.) \\
\hline & & \\
\hline & & \\
\hline & & \\
\hline
\end{tabular}

LOCATION DESCRIPTION SITE CONDITION

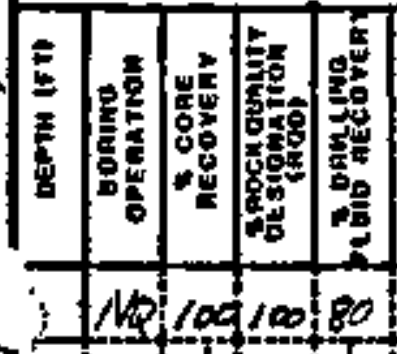

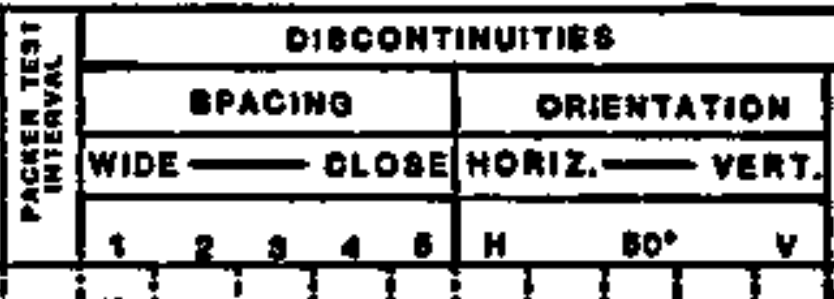

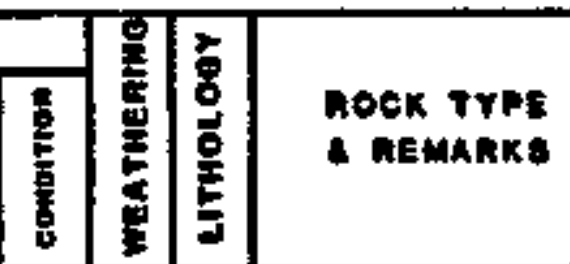

5

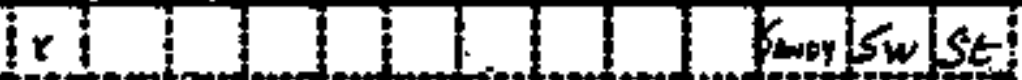
1111 1.

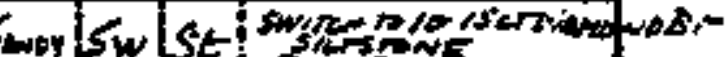
$1+\infty$ 1. 13

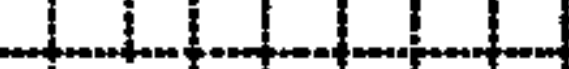
(1) Haseas.nos VNAT III DoweES $\frac{5}{4}$ 11 lentes 146 $1]$

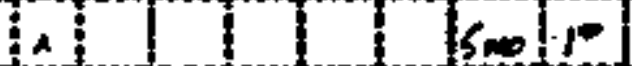
stersong comernowe tare

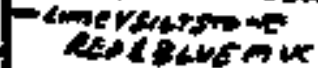
$x+r$

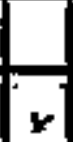

stist Lingen 4 Riander avedeans \begin{tabular}{lllllll|l|l|} 
& & & & & & & & $x$ \\
\hline
\end{tabular} venespe chem

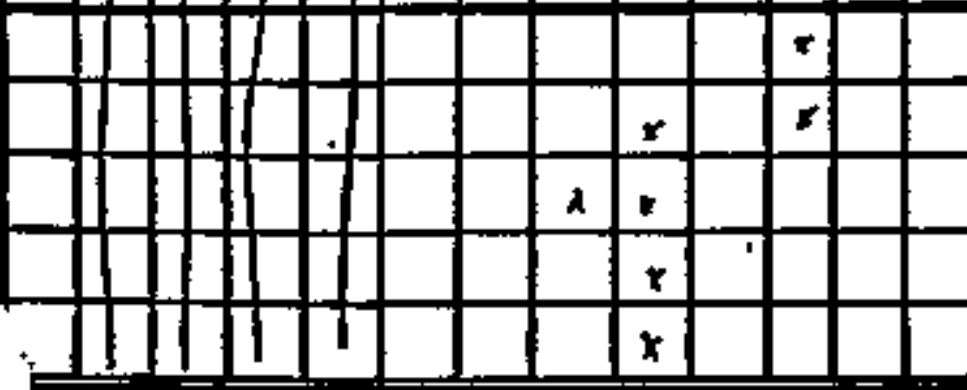
s.

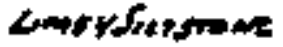
JCOMMENTS: 


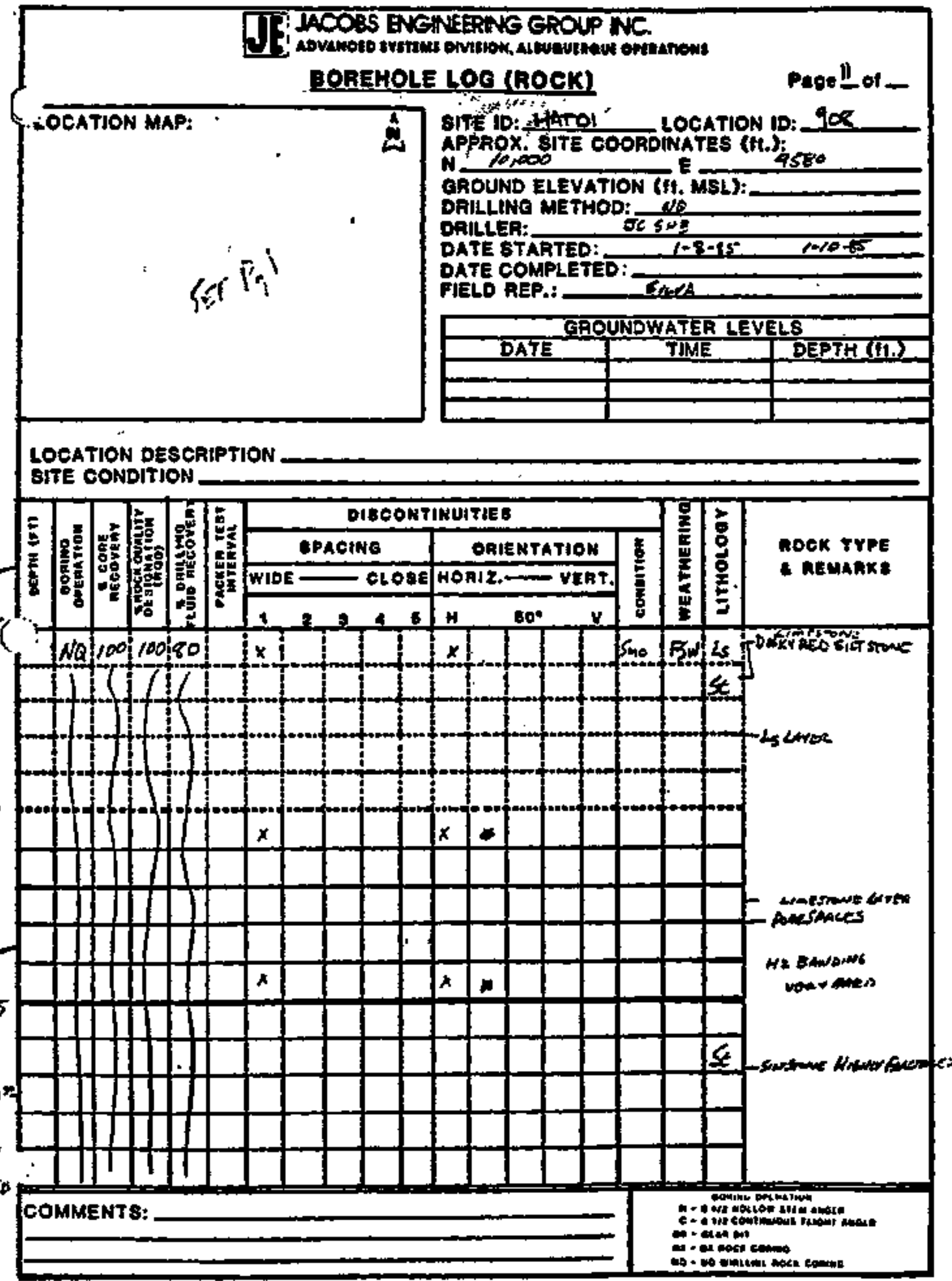




\section{DE LACOBS ENGNEERNG GROVP NC.}

\section{BOREHOLELOG (BOCK) "PAPE'L of -}

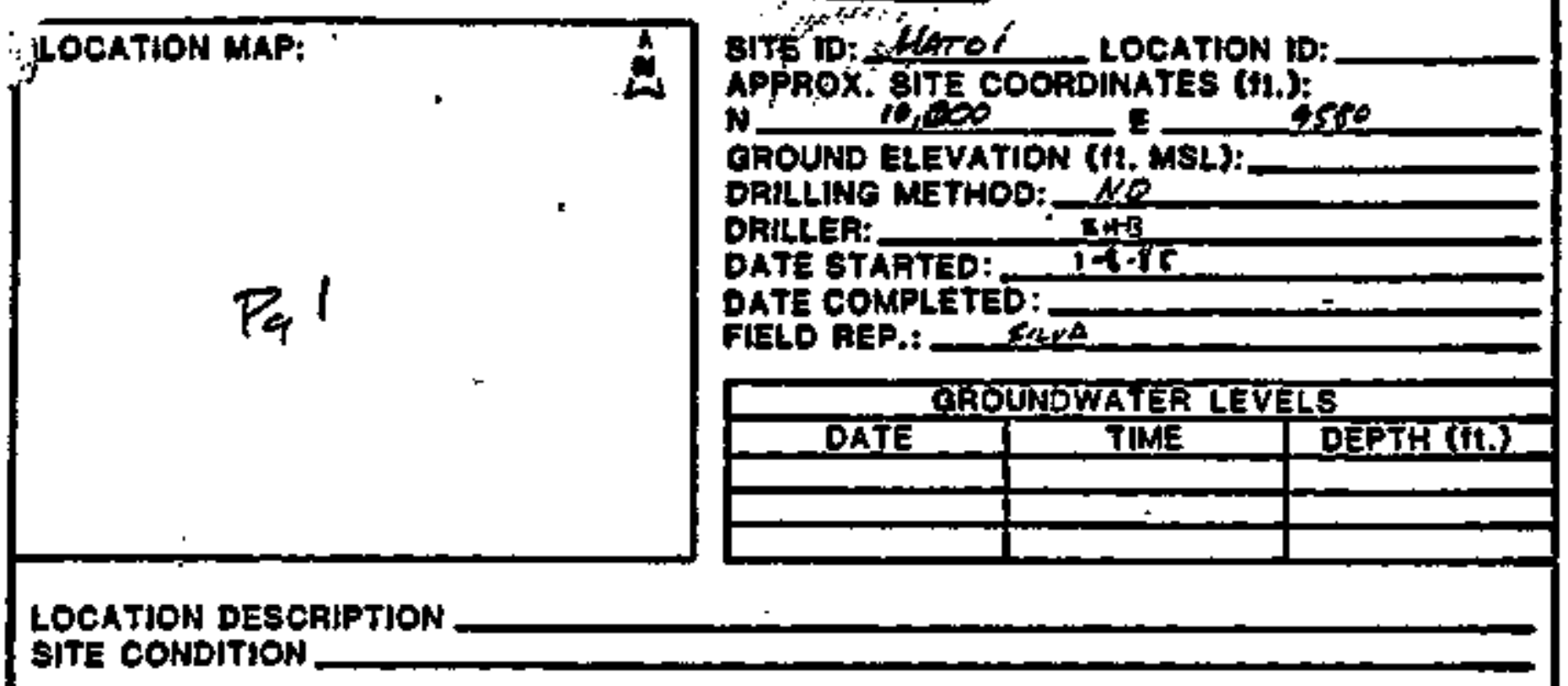

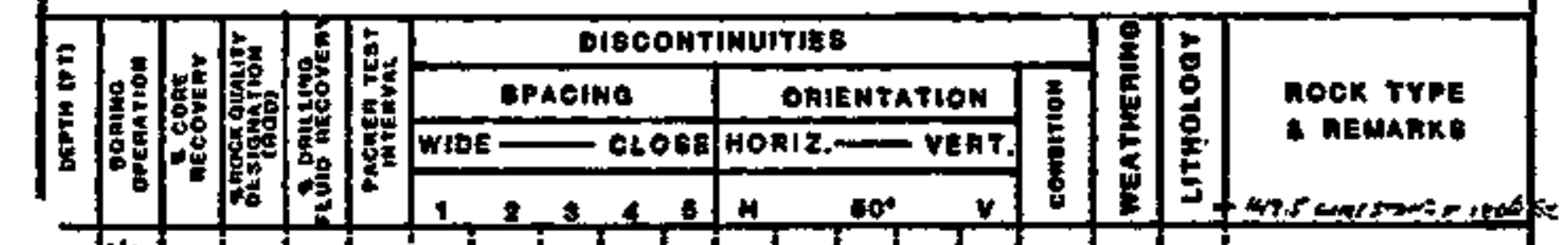

1 Wa 100100180
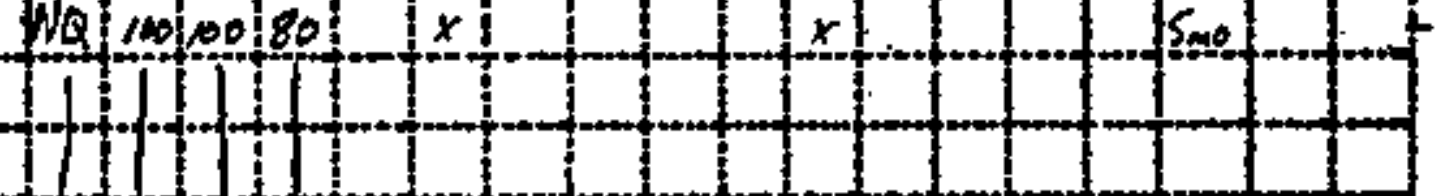

Persaming zis

win aponame?

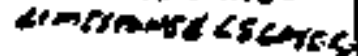

(1)

his wetring

Linat bitrsemente

Sury Liserone

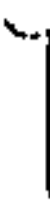




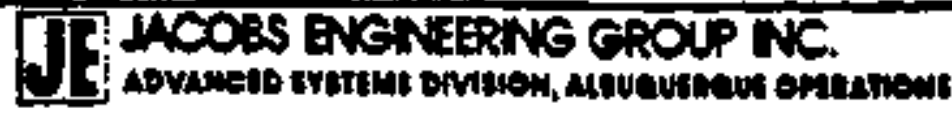

BOREHOLE LOQ (ROCK) PAOOLf Ot -

ZOCATION MAP:

4

81TE ID: AATTOC LOCATION ID: 908

APPROX. EITE COORONATES (tt.):

GROUND ELEVATION (ft, MSL): +4290

DRILLINO METHOD: NOW WE ANE

DRILLER: CAEERLOSAMS

sore pare
DATE 8TARTED: WOES
DATE COMPLETED?
FIELD REP.: MCGNEY

\begin{tabular}{|l|l|l|}
\hline OAROUKOWATE LEVELS \\
\hline TIME & DEPTH (1t.) \\
\hline & & \\
\hline & & \\
\hline
\end{tabular}

LOCATION DESCRIPTION

SITE CONDITION

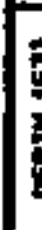

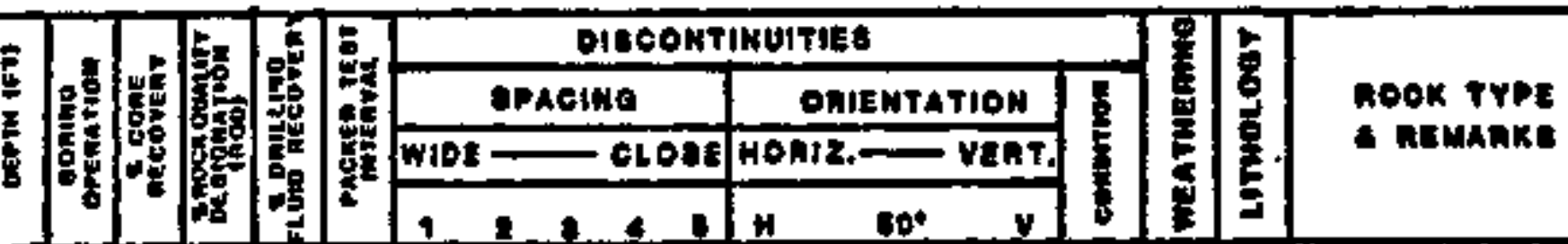

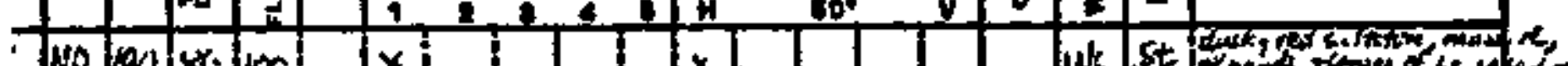

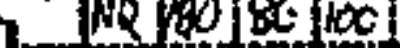
(1)

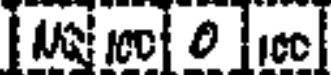
and

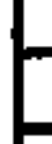
o) 11 $+$

$$
1 x
$$
.

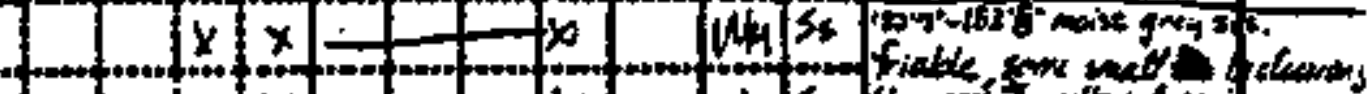
, 190 $|x| x|x|+x \mid x$

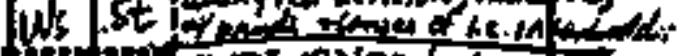
X

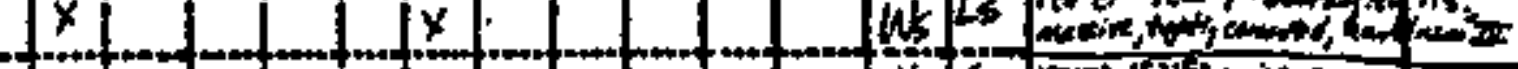

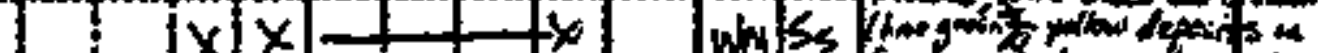

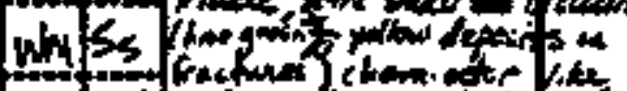

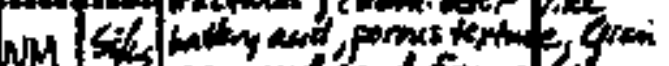

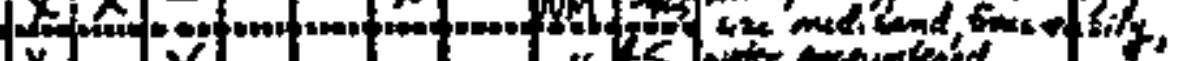

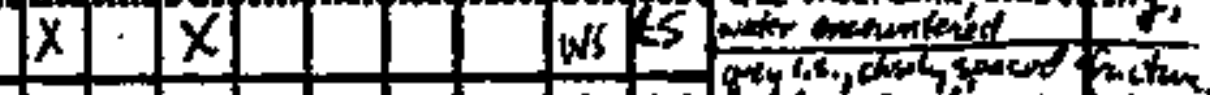

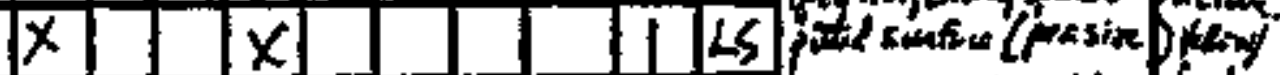

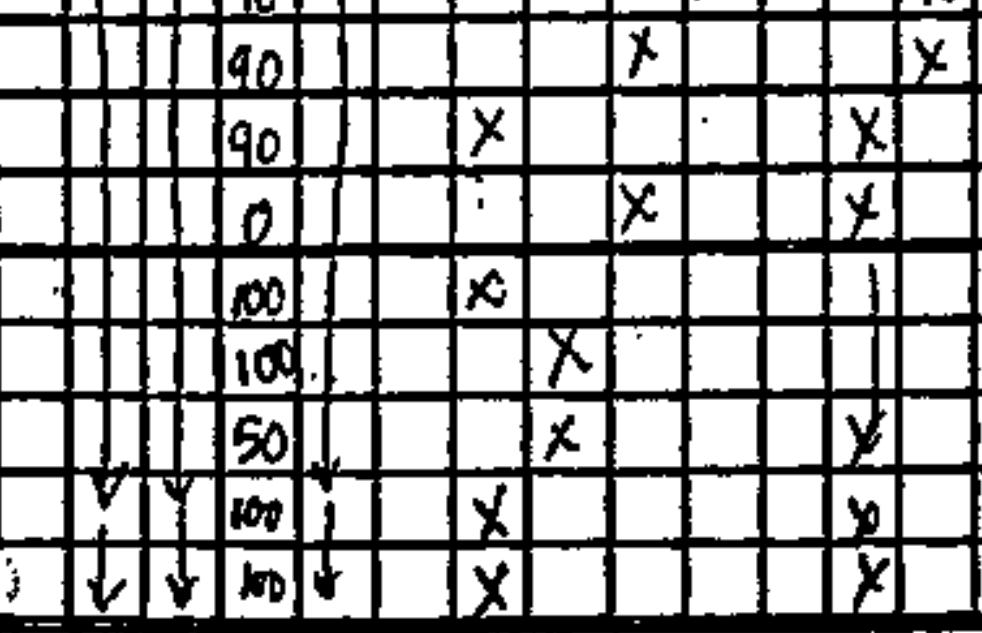

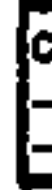




\section{IT HCOBS ENGNEERNG GROUP NC.

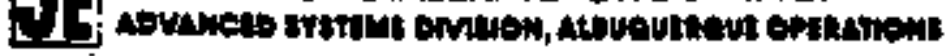

DOREHOLE LOG (ROCK)

Page of -

SOCATION MAP:

STE bo HLT al LOCATION ID: 1905 APPAOX. BITE COORDINATES (ft.):

$N$ : i

GHOUND ELEVATION (H1. MSL):

DRILLINS METHOD:

Sra $p_{1} 1$

DRILLEA: Brak Befo

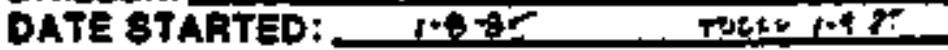

DATE COMPLETED:

FIELD REP.:

\begin{tabular}{|l|l|l|}
\hline DATE & \multicolumn{3}{|c|}{ GROUNOWATER LEVELS } \\
\hline & JIME & DEPTH (II) \\
\hline & & \\
\hline & & \\
\hline
\end{tabular}

LOCATION DESCRIPTION

SITE CONDITION

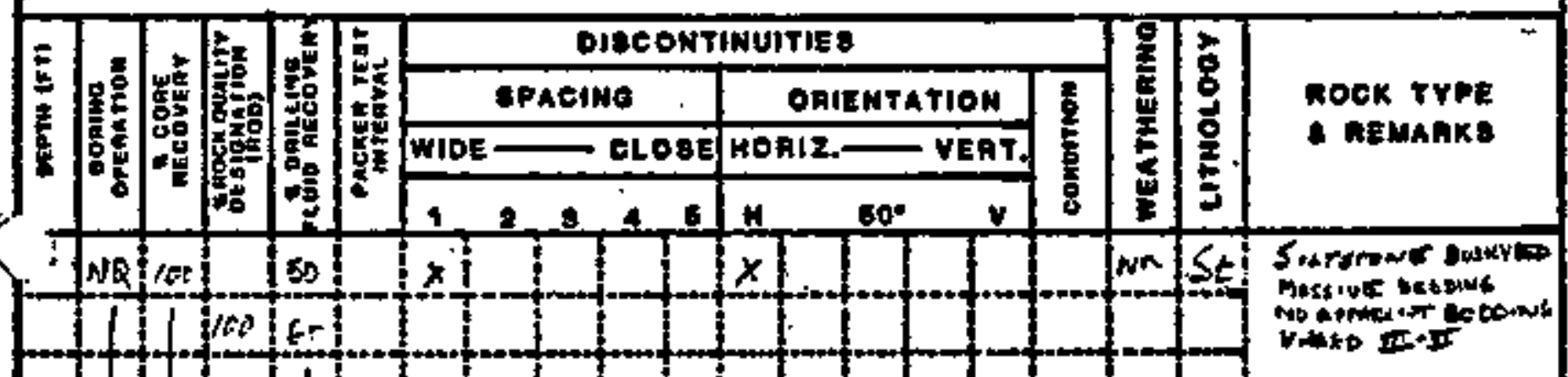

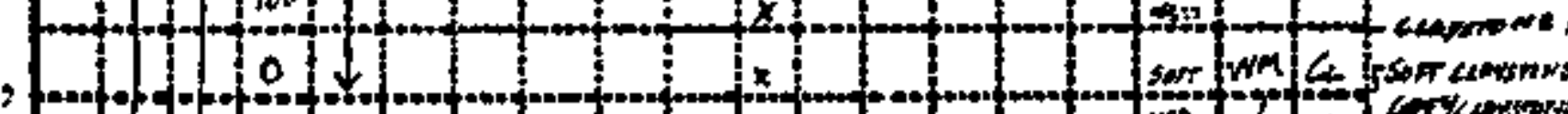

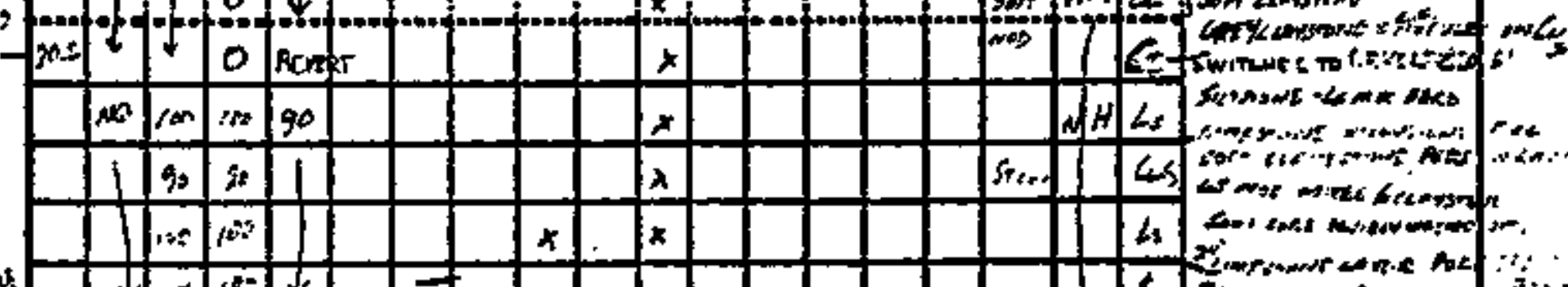

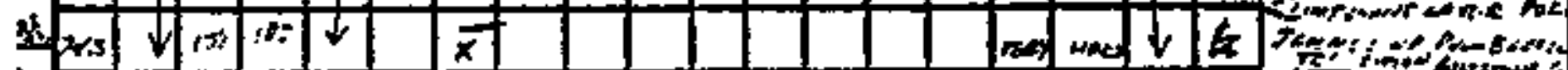

Na $100018001, x$

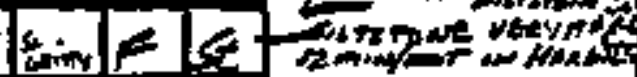

1111.1

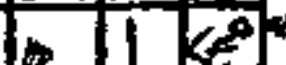

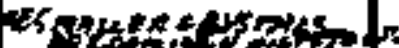

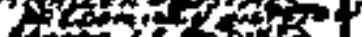
Tarrents satarome 20

\section{COMMENTS:}
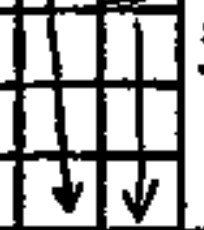

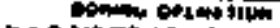

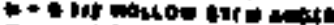

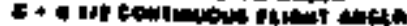

Citan at

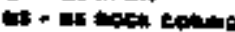

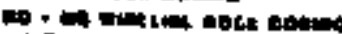




\section{6] TACOBS ENGNEEANG CROUP NC.}

\section{BOREHOLE LOG (ROCK)}

Page E

JOCATION MAP:

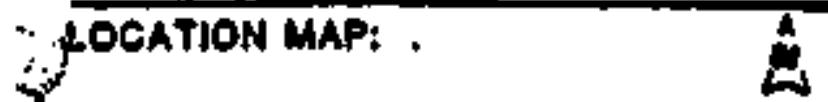

Sest

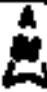

sitol

BIFE DD: HATOI LOCATION D: GOSGIOG

APAROX, GITE COORDINATES (H.):

N?

GROUND ELEVATION (ft. MSL)

DRILLINO METHOD:

DAILLER:

DATE 8TARTED: $\angle P$-PS

$7 \cdot 7+d=$

DATE COMPLETED:

FIELD REP.:

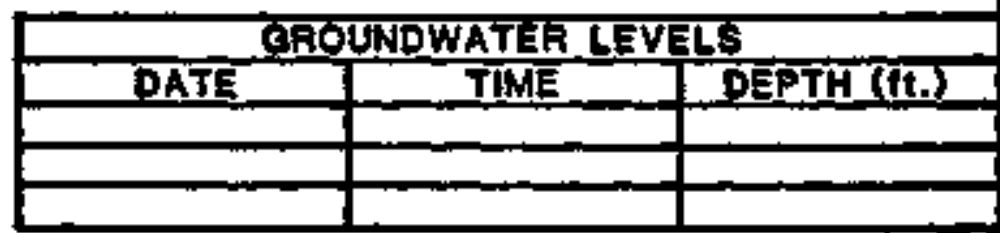

LOCATION DESCRHPTION

SITE CONDITION

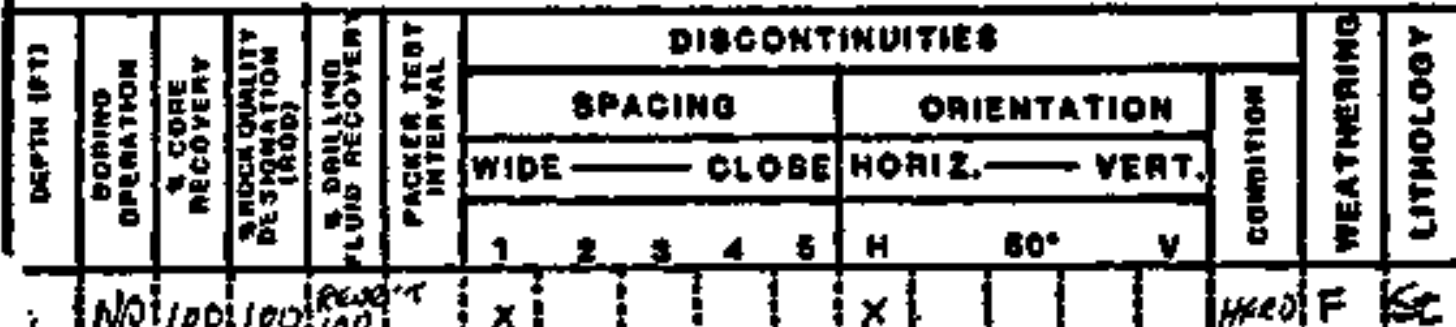

nock troe

- nemalos

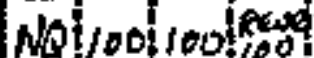
$x$ $x$

$x$

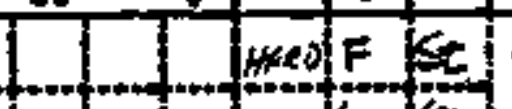

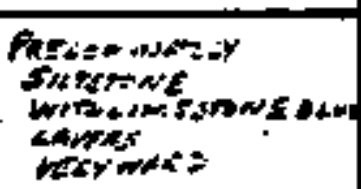

$n$ 10 +....(1)

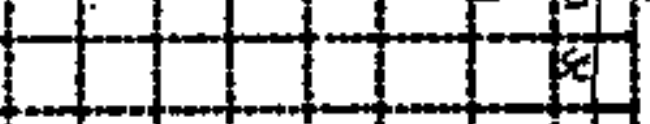

necrintat:

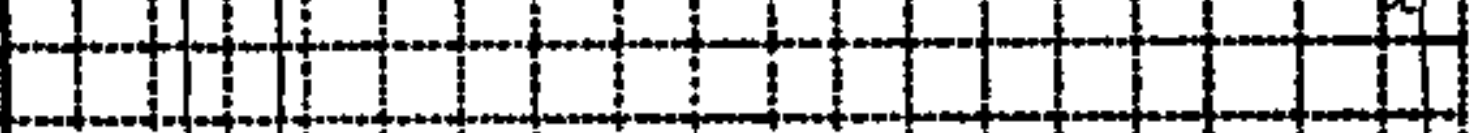

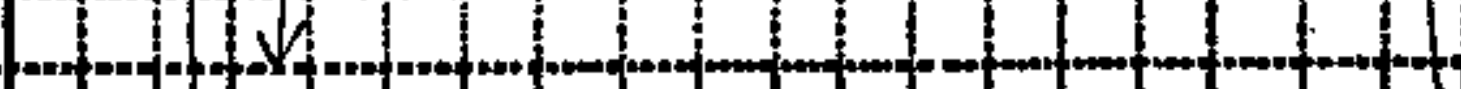




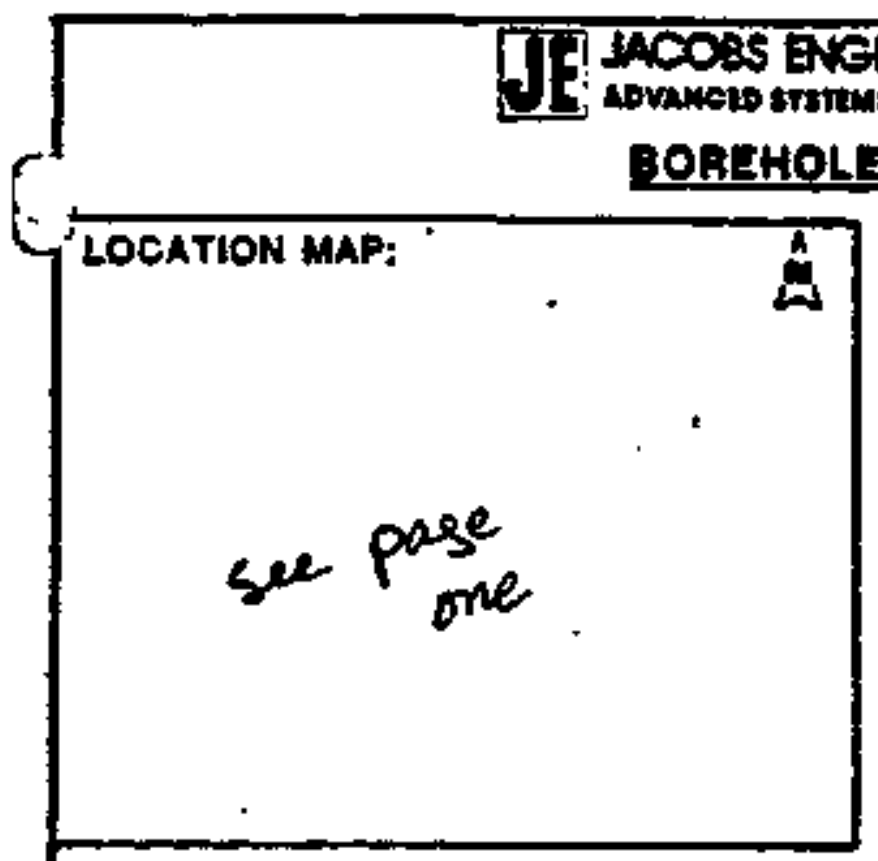

\section{DE] SACOSS ENGNEERTNG EROOP NC.}

BOREHOLE LOCAROCK) PAgELOA_-

s.

LOCATION ID: APPROX. 8ITE COOFDINATES (ft.):

GROUND ELEVATION (tt. MSL):

DRLLLNO METHOD: NOW WIRE TASE

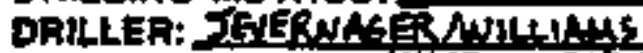

OATE STARTED: $-1 / 185$ JOLAY J110/85

DATE COMPLETED:

FIELO REP:: HLi N

\begin{tabular}{|l|l|l|}
\hline \multicolumn{2}{|c|}{ GROUNOWATER LEVELS } \\
\hline DATE & TWME & DEPTH (II \\
\hline & & \\
\hline & & \\
\hline
\end{tabular}

\section{LOCATION DESCRIPTION}

SITE CONDITION

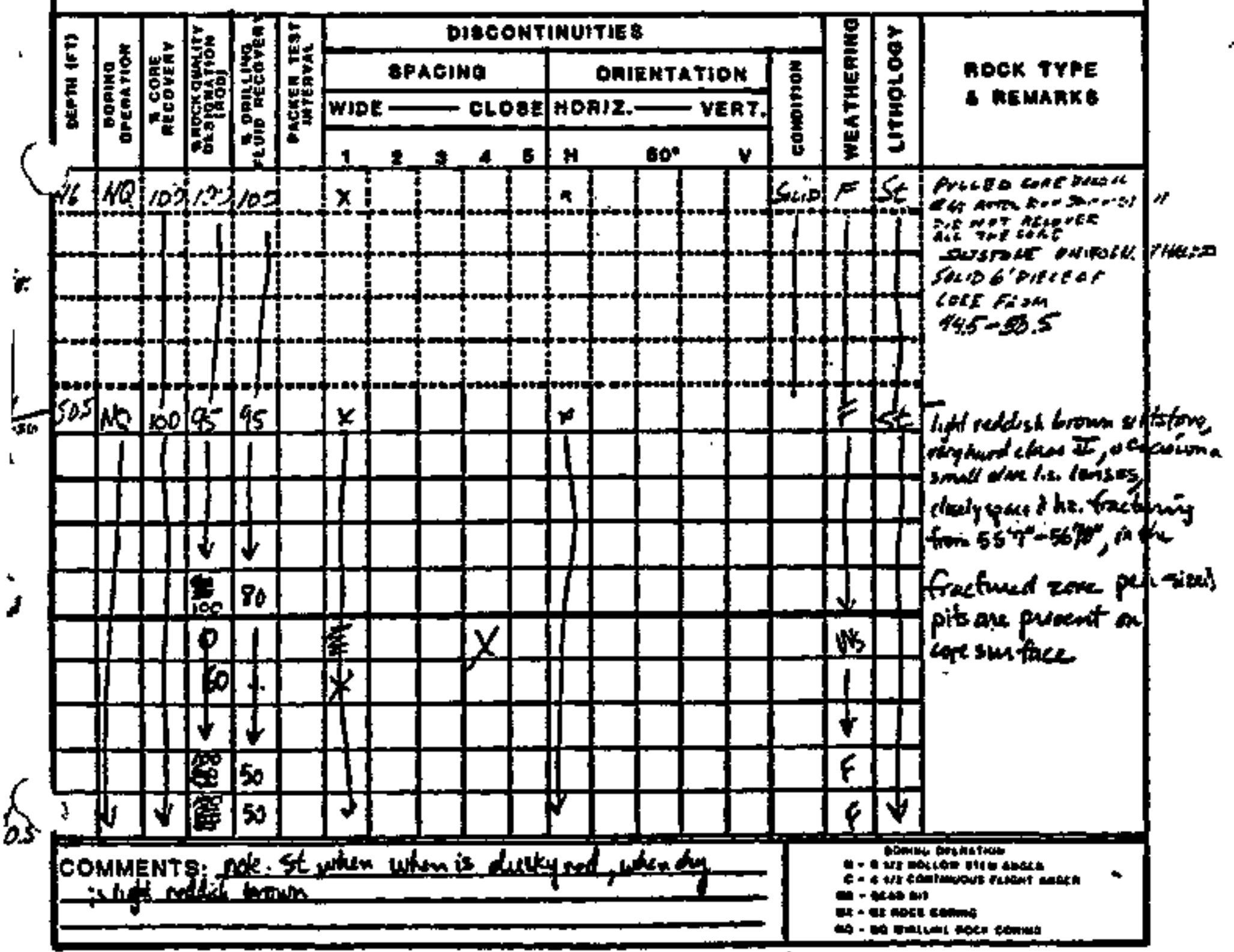




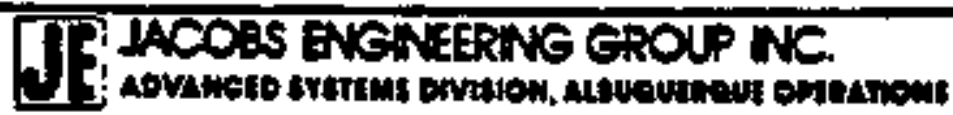

\section{BOREHOLE LOC (AOCK) PAPESOI -}

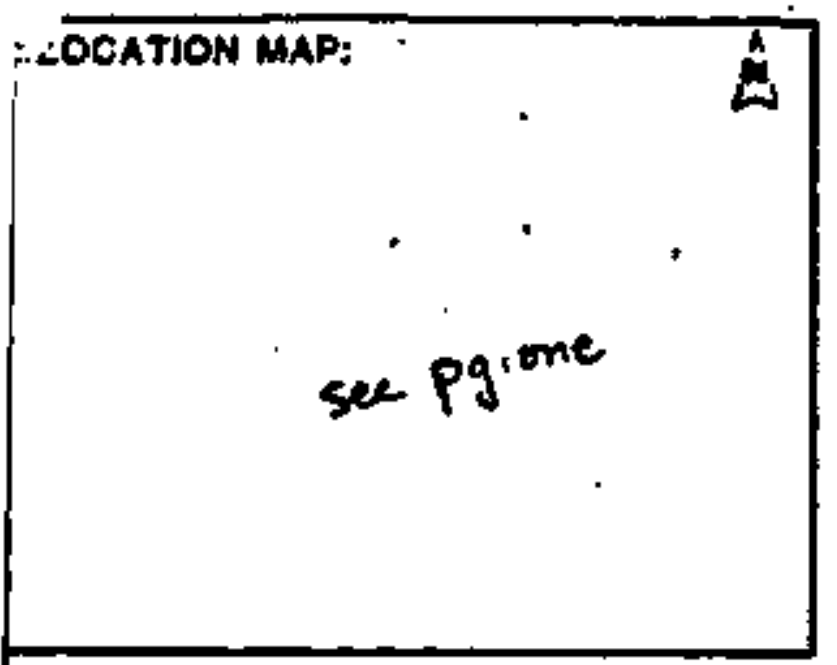

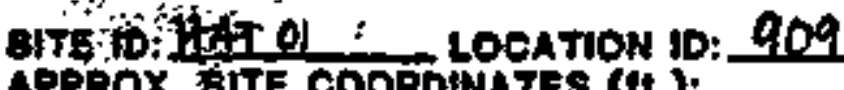
APPROX. BITE COORDINATES (ft.):

GAOUND ELEVATION (ft. MSL):

DATLLING METHOD: NQ WiRgANE

DRILLER: TAVRNAGERNULLAMS

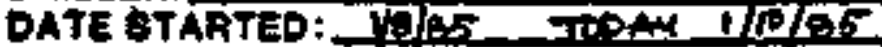

OATE COMPLETED:

FIELD REP.: NER:

\begin{tabular}{|l|l|l|}
\hline DATE OROUNDWATER LEVELS \\
\hline DATEE & TIME & DEPTH (At.) \\
\hline & & \\
\hline
\end{tabular}

LOCATION DESCAIPTION

SITE COHDITION

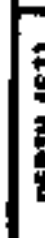

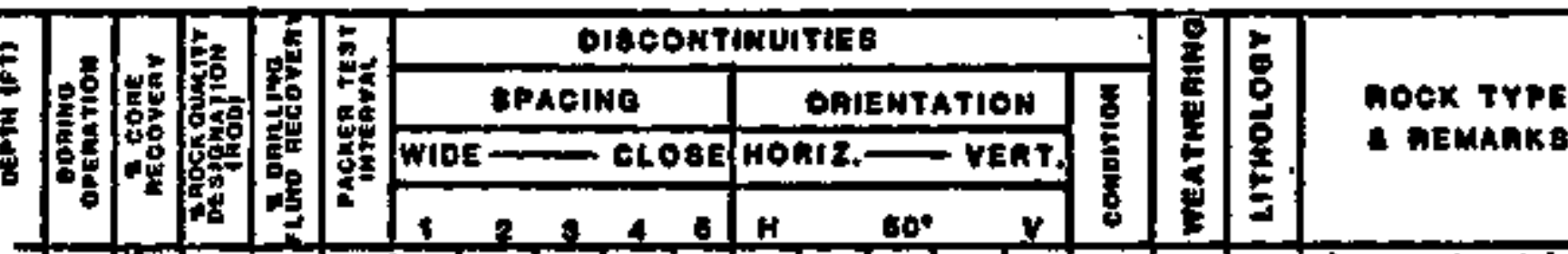
Apot

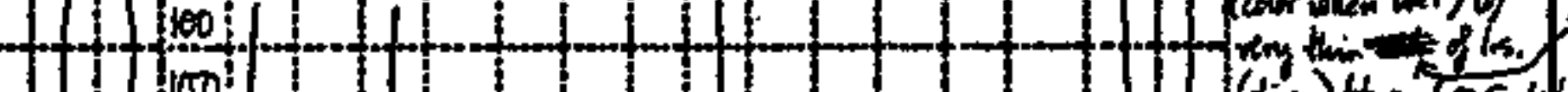

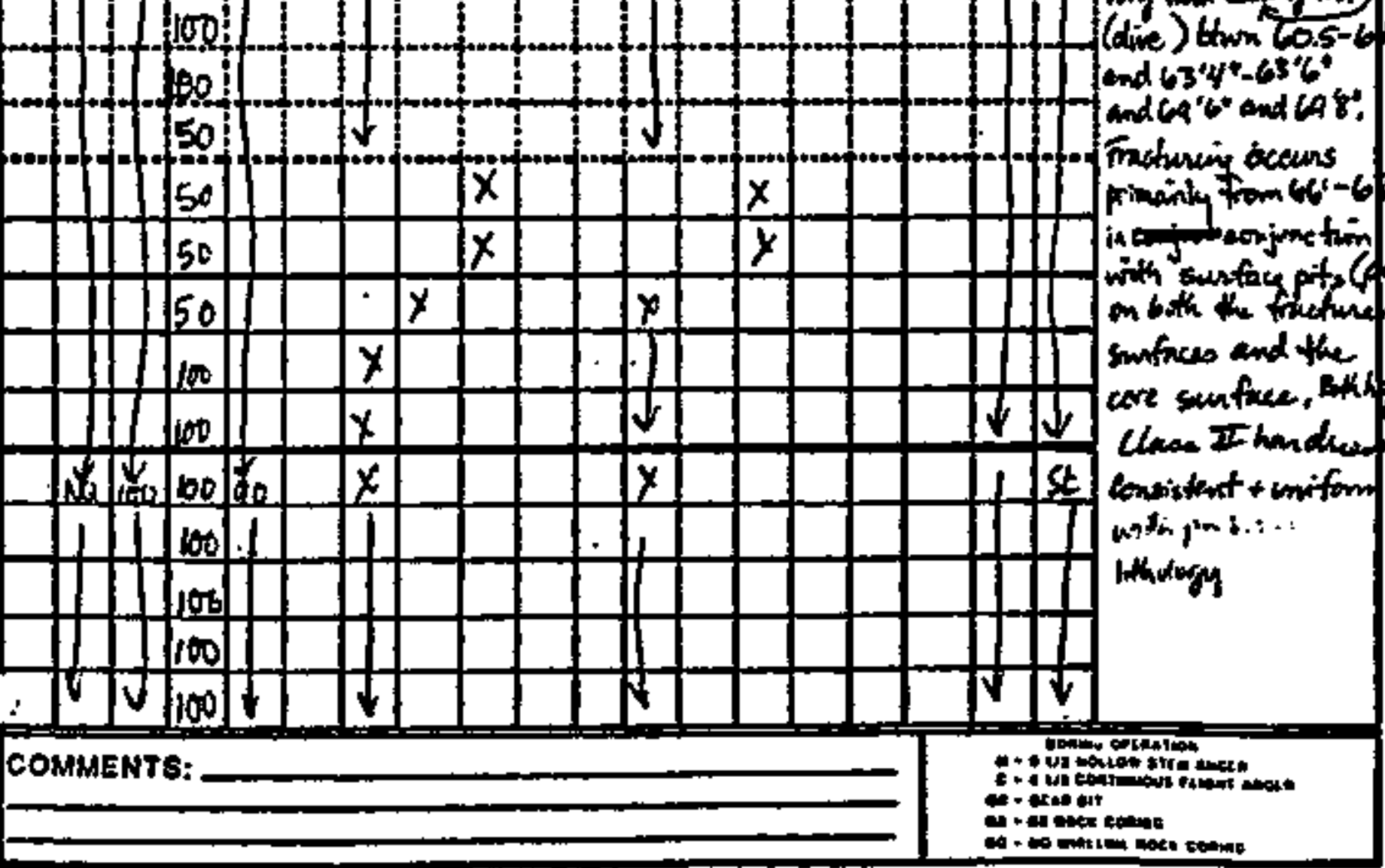




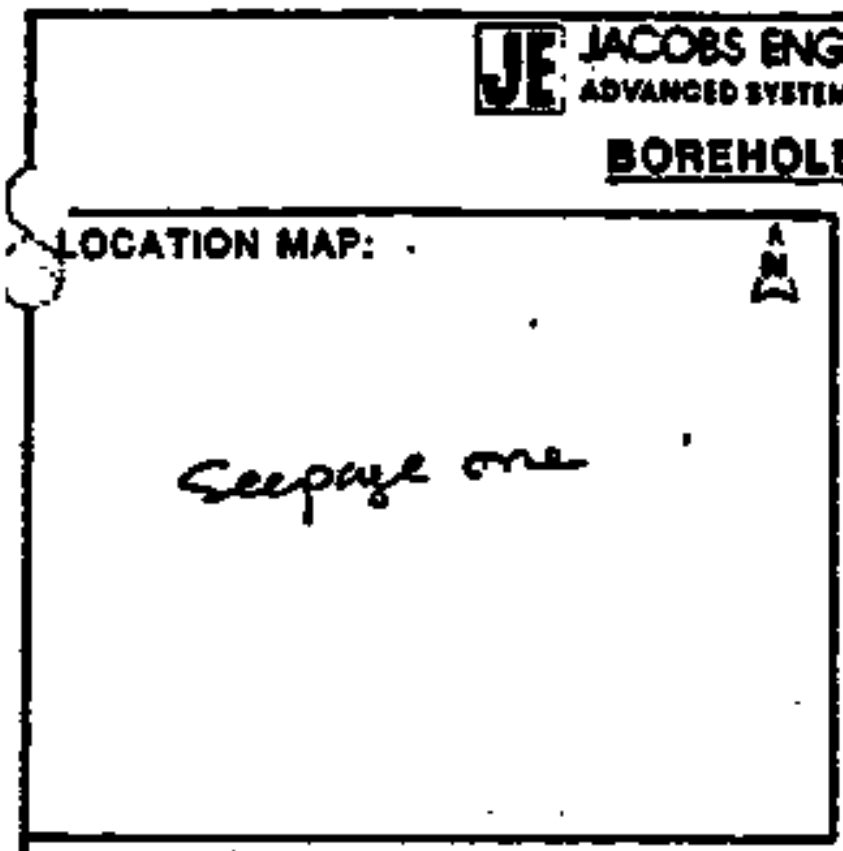

NEFRING CROUP NC.

\section{thatrous}

Page 6 of

HOCATION MAP: .

t
SITE 1D: HET oI LOCATION ID: 909 APPAOX. BITE COORDINATES (ft.):

$N$

GROUND ELEVATION (ft. MSL):

DAHLINO METHOO: RO WURELUN

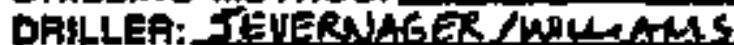

DATE STARTED: I/S/OS TDAY IPES

DATE COMPLETED!

FIELD REP.: AM SENERY

\begin{tabular}{|c|c|c|}
\hline \multicolumn{3}{|c|}{ GROUNDWATER LEVELS } \\
\hline DATE & TIME & DEPTH $(\mathrm{ft})$ \\
\hline & & \\
\hline & & \\
\hline & & \\
\hline
\end{tabular}

\section{LOCATION DESCRIPTION}

SITE CONDITION

\begin{tabular}{l}
\hline \\
\hline
\end{tabular}

TQ: $100: 0: 50$

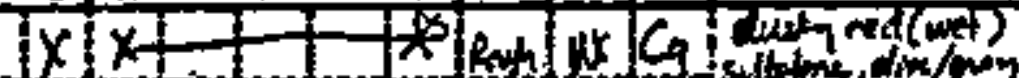

1] $] 1]$ I

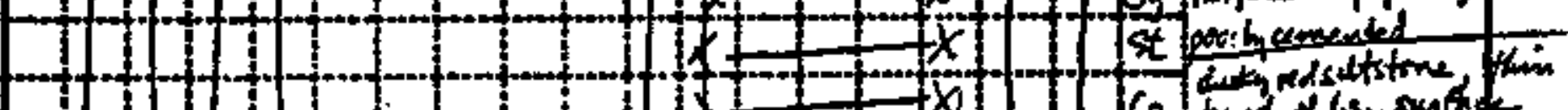

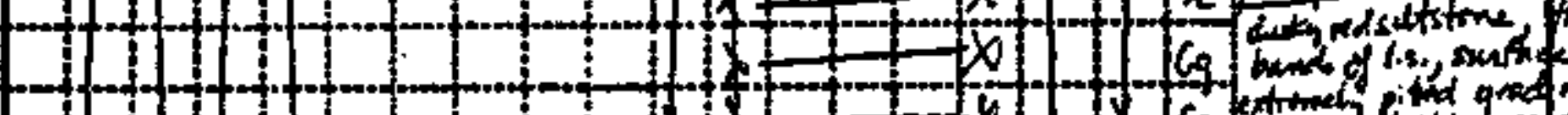

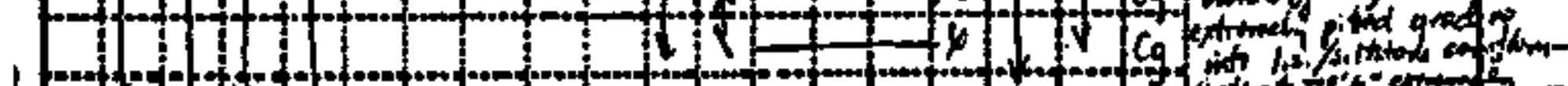

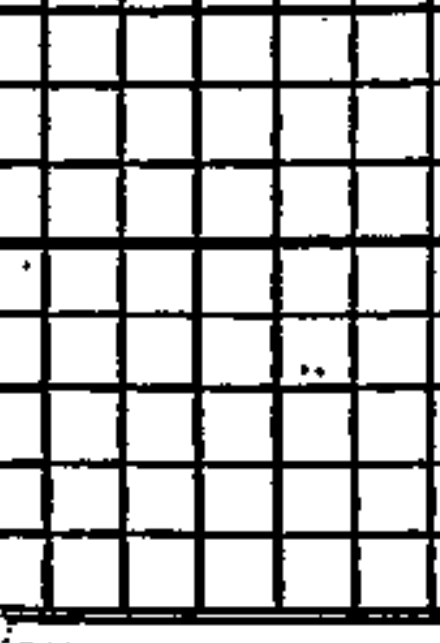

JOMMENTS: 


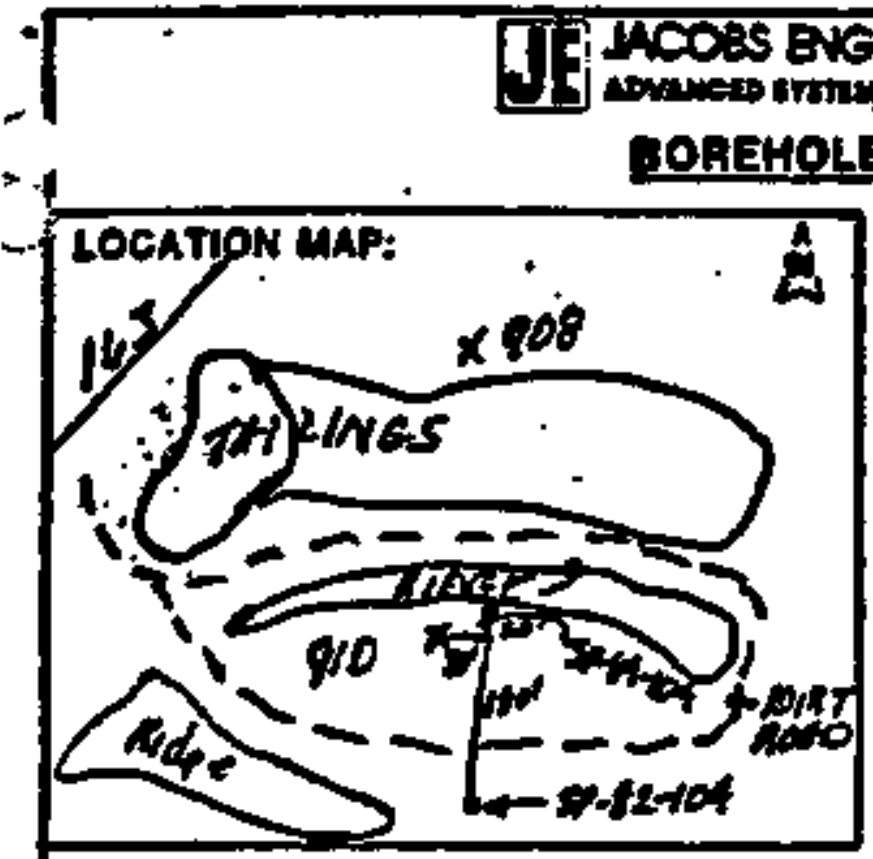
LOCATION DESCRAPTION
SITE CONDITION

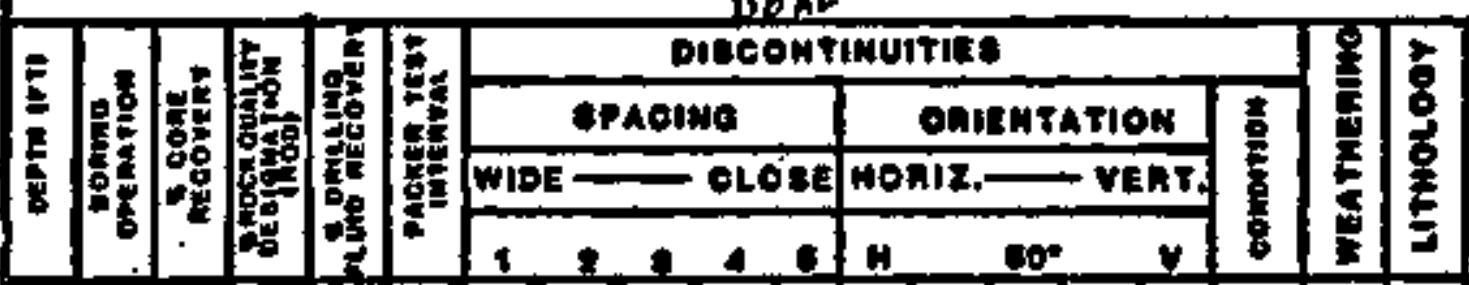

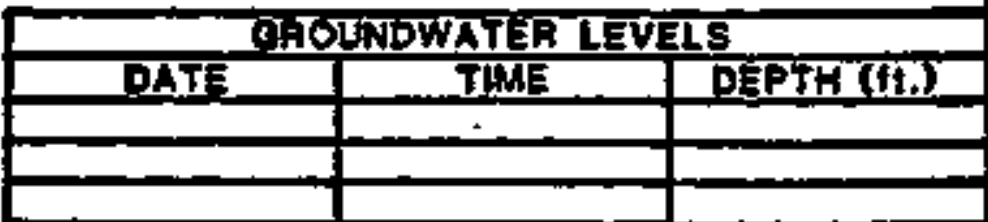
GTE ID: $\$$ ATOL LOCATION D: 9.D APPROX. glTE COORDNATES (ft): N 400 E 100 EROUND ELEVATION (4. MSL) CISOO DAILLINS GETHOD: NQ UNPEUNE

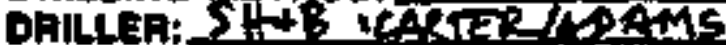
DATE STARTED: 114185 DATE COMPLETED:

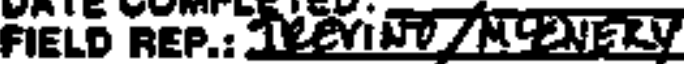

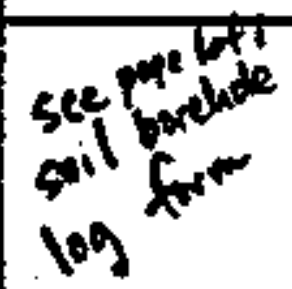
$\begin{array}{llll}0 & & & \\ & & & \end{array}$ 8 50 द noek Troe a heuank: 171 $x$ (1) 1
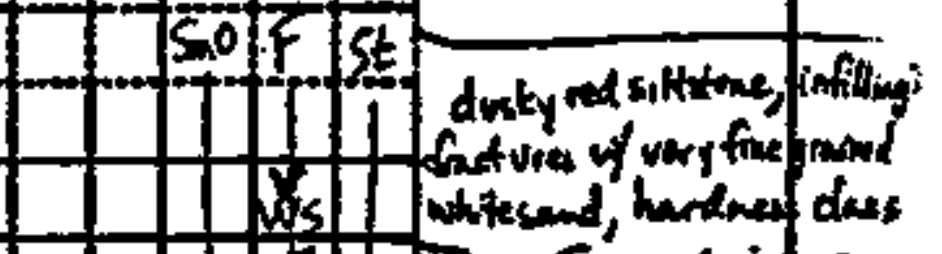
100

$x+1$.

17

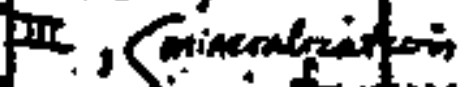
11180 x $1+2$ onemfin in frocture 


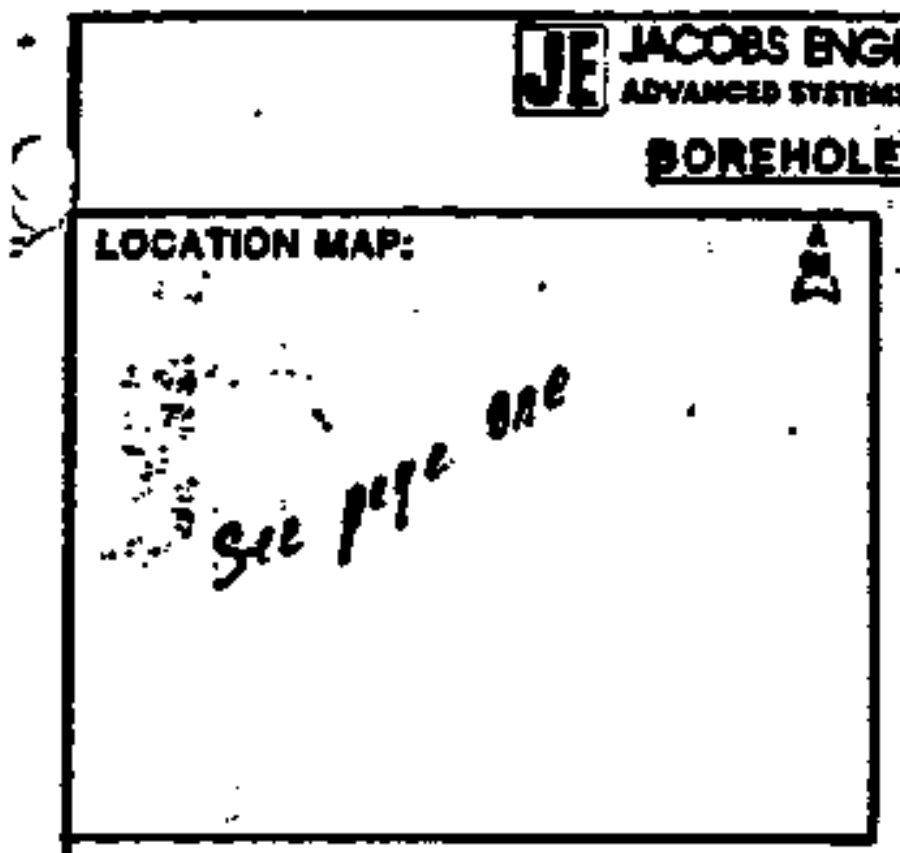

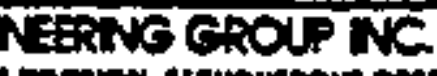

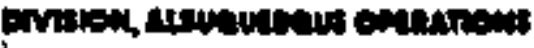

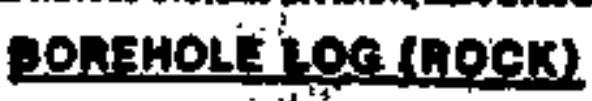

Page 2or -

(n)

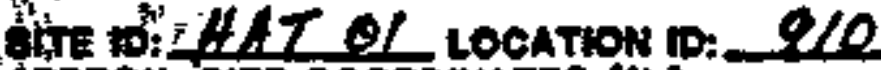

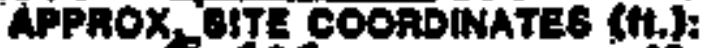

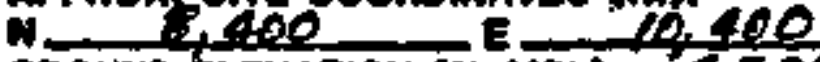

GROUND ELEVATION (t. Mst): 4700

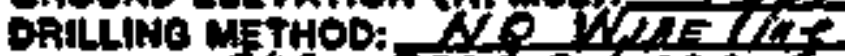

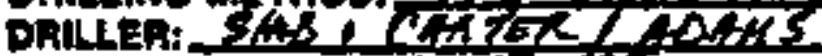

DATE BTAATEO: $Y=Z 7-7$,

DATE CONPLEIED:

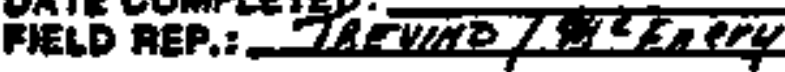

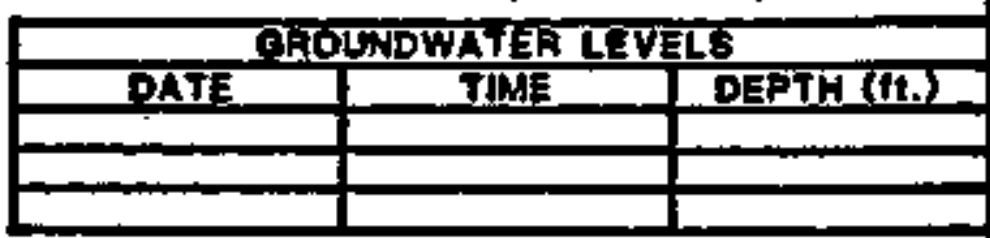

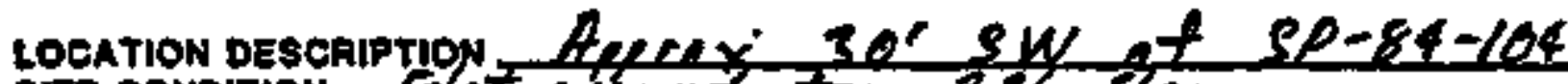

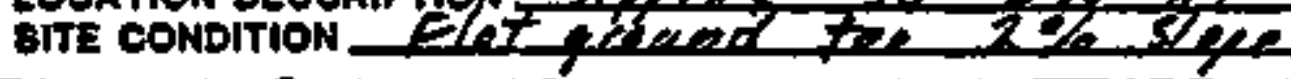

$*$
$*$

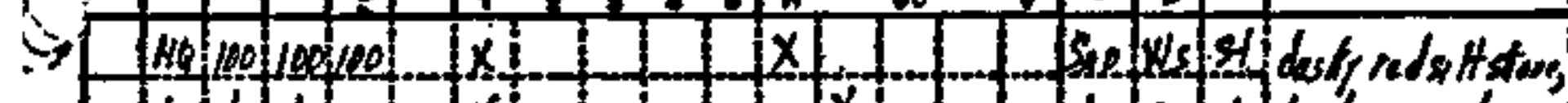

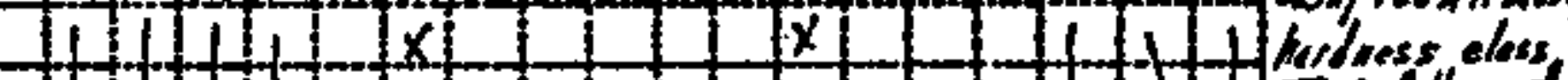
is $8 \times$

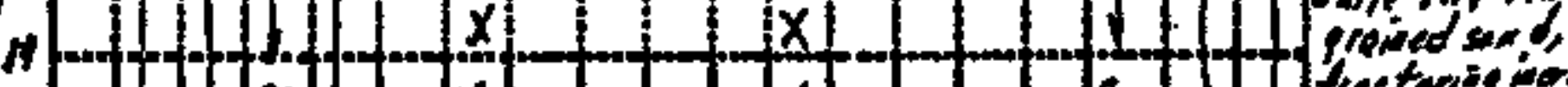

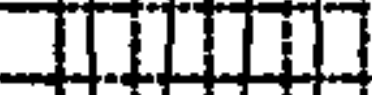

\section{d} d H

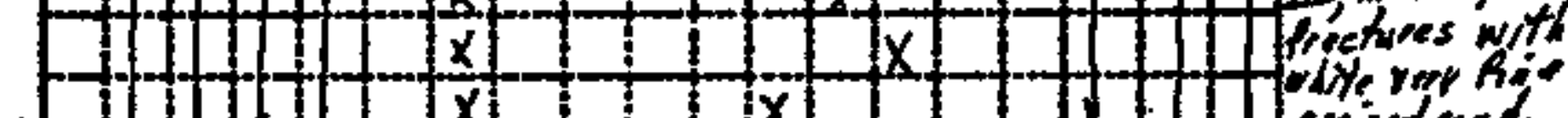

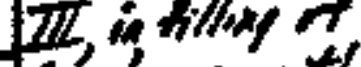

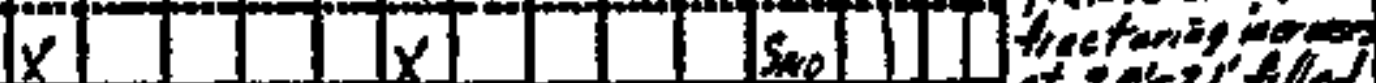

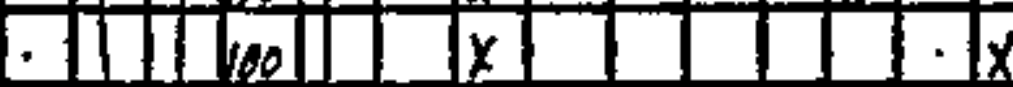

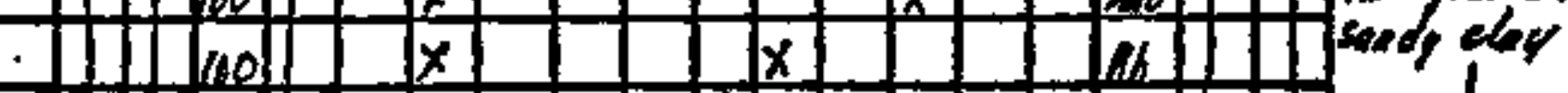

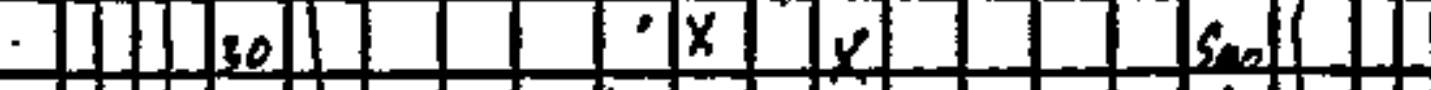
II $\mid$ ind $x|\times|$

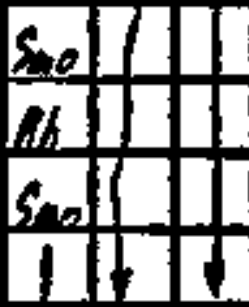

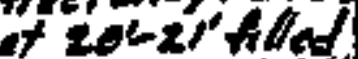
tis weiperis
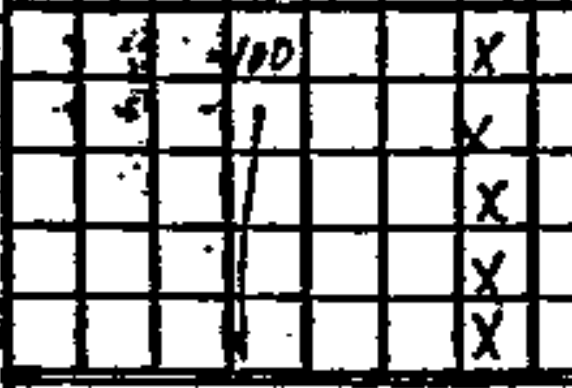

COMAENT 8:

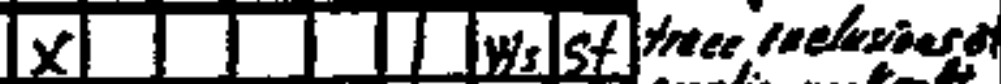

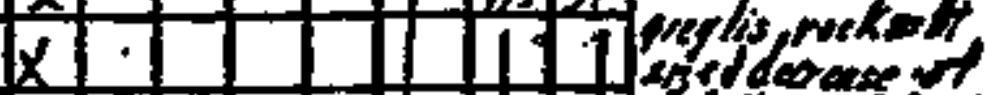

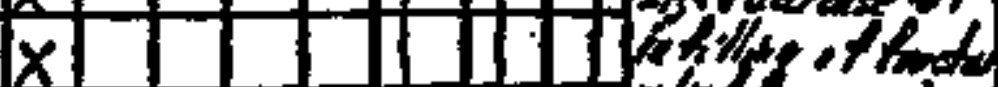

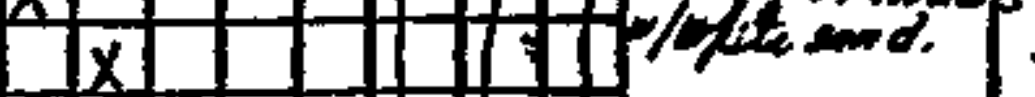
$|x|$

Cominents: 
$\bullet$

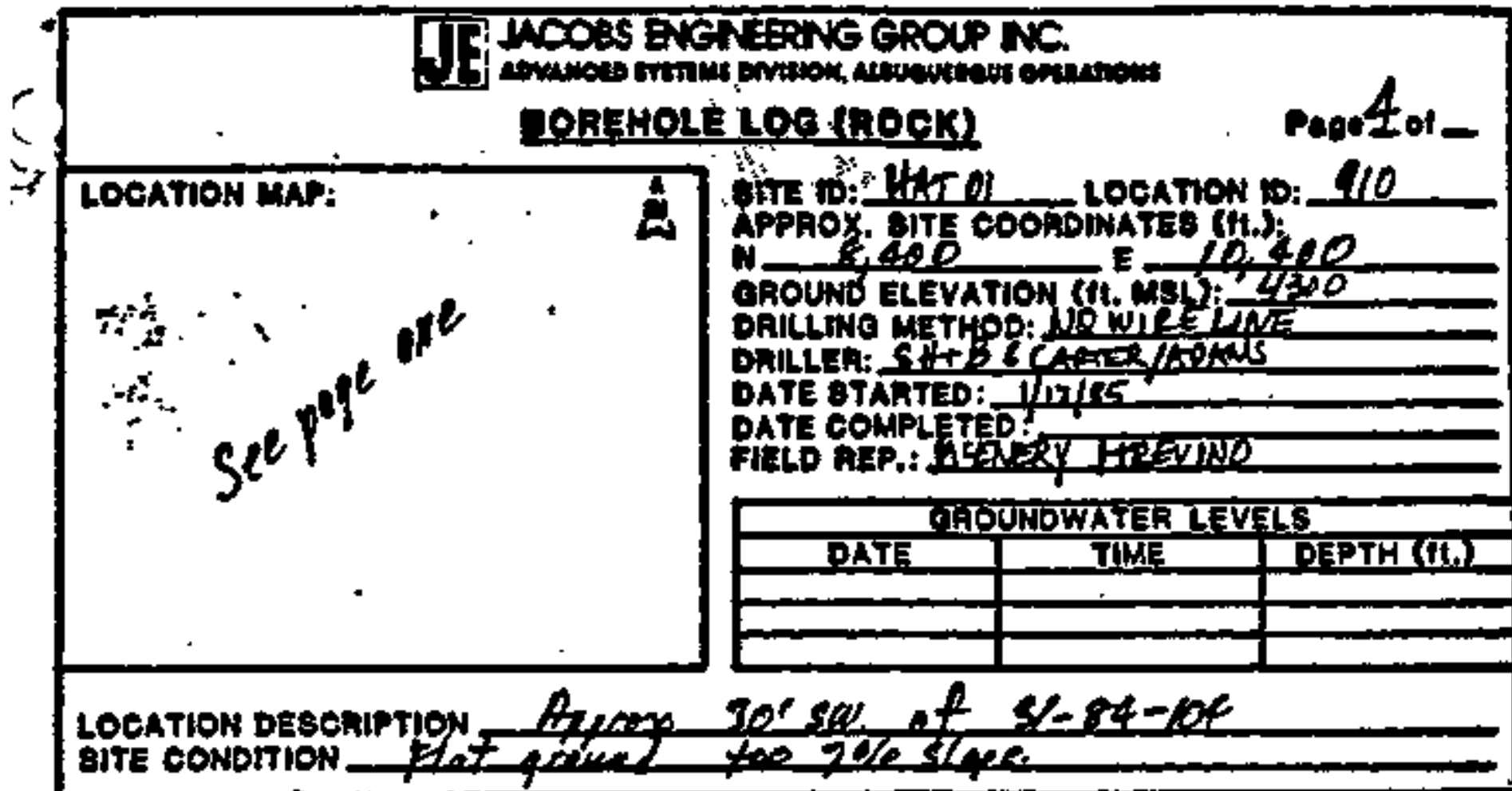

8
8

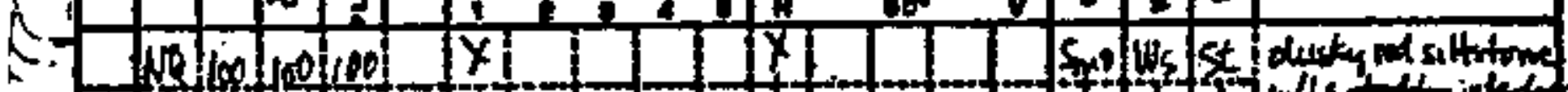




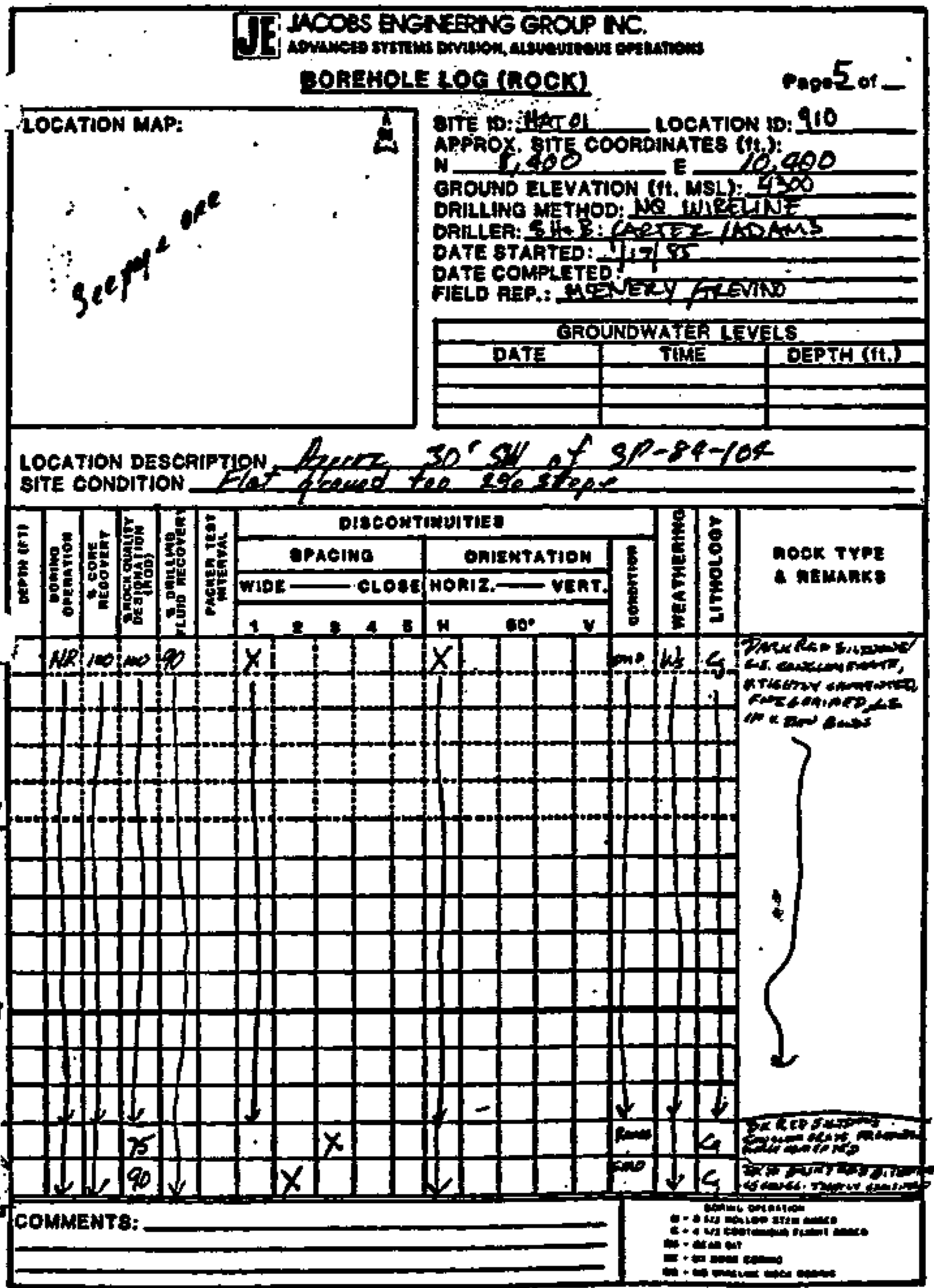




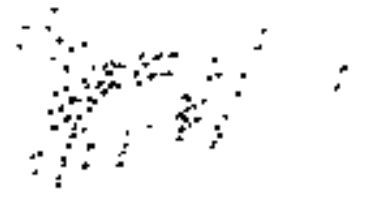

TAC TEST PITS:

ON PILE 


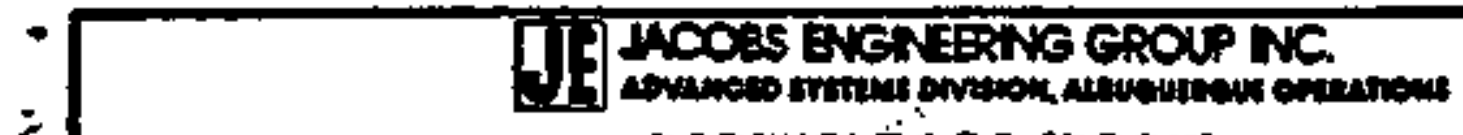

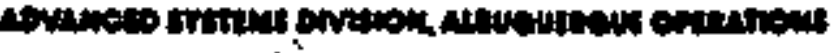

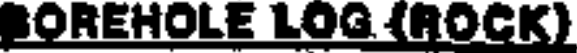
LOATION MAP:

$\lambda$

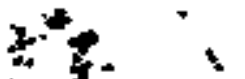

$\because 2 y=0$ Doe 1

.

$\because$
4

4 suter

CokTON D: $\%$

APPROX, BITE COOROHATES (ft.):

Nenes

E- -420

GROUND ELEVATION (tt. ASL F FreA

DRtLLiNo METHOD: Ne Maryer

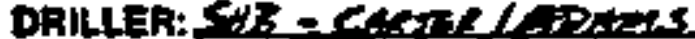

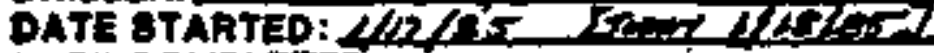

OATE COMMLETED:

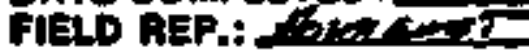

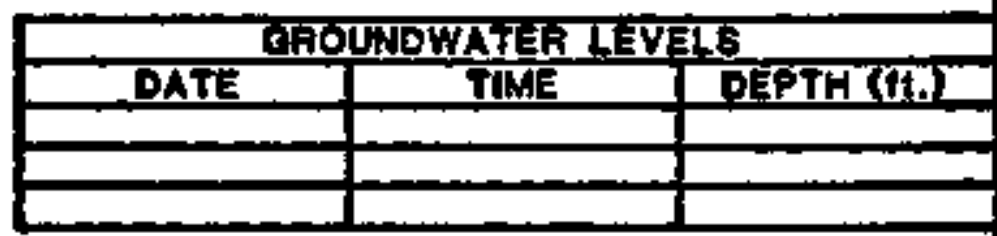

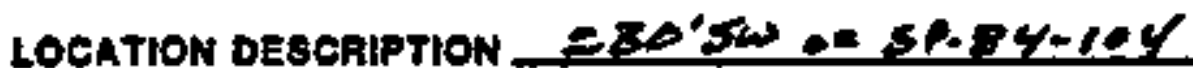

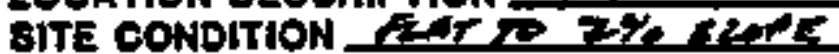

\begin{tabular}{|c|c|c|c|c|c|c|c|c|}
\hline \multirow{3}{*}{8} & \multirow{3}{*}{ 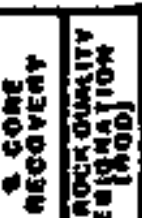 } & \multirow{3}{*}{\multicolumn{2}{|c|}{ 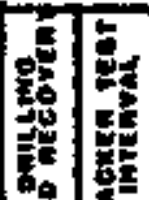 }} & \multicolumn{3}{|c|}{ Diseontunutrite } & \multirow{3}{*}{ 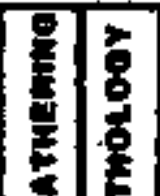 } & \multirow{3}{*}{$\begin{array}{l}\text { work Trot } \\
\text { o minkmk }\end{array}$} \\
\hline & & & & BPACHe & PAIEnTATIOn & & & \\
\hline & & & & - $620 \mathrm{et}$ & HOAIZ.- VEAT, & 8 & & \\
\hline
\end{tabular}

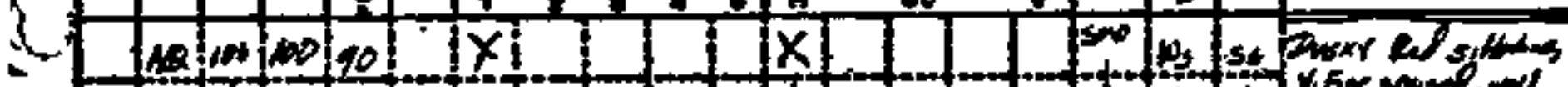

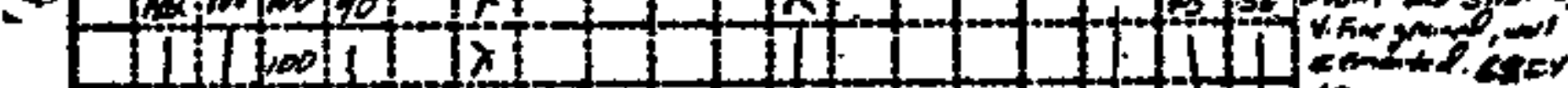

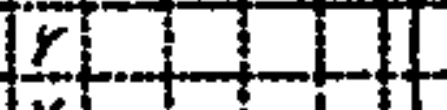

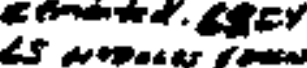

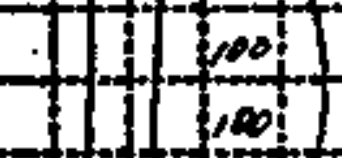

$\mathbf{T r}$

110 Y)

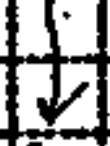

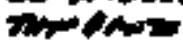

6x:

$.11010 \times 1 \times$

1100

k.

1] $\Leftrightarrow$

$\mathbf{x}$

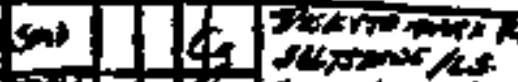

1912

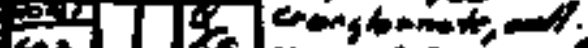

1]

$\alpha$

50

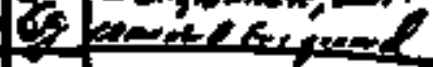

$\||x \rightarrow| x$

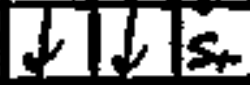

Chatronsons-

twe $7:-\infty$,

$\mathbf{x}$

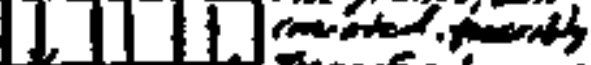

t.

$\because 1:$ sol

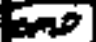

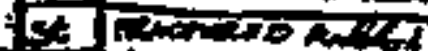

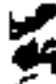

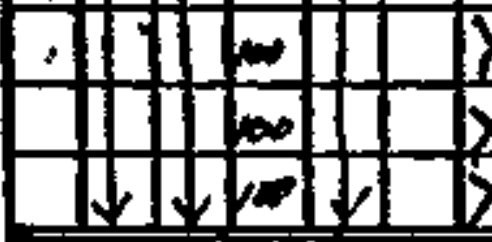

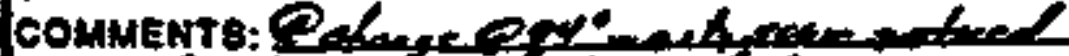

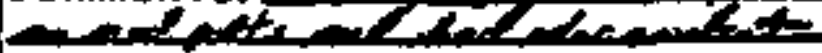

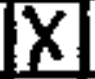

Tा:?

Mron stithe.

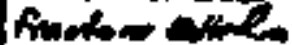

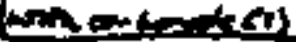

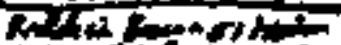

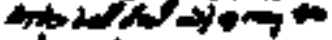
otse 4 


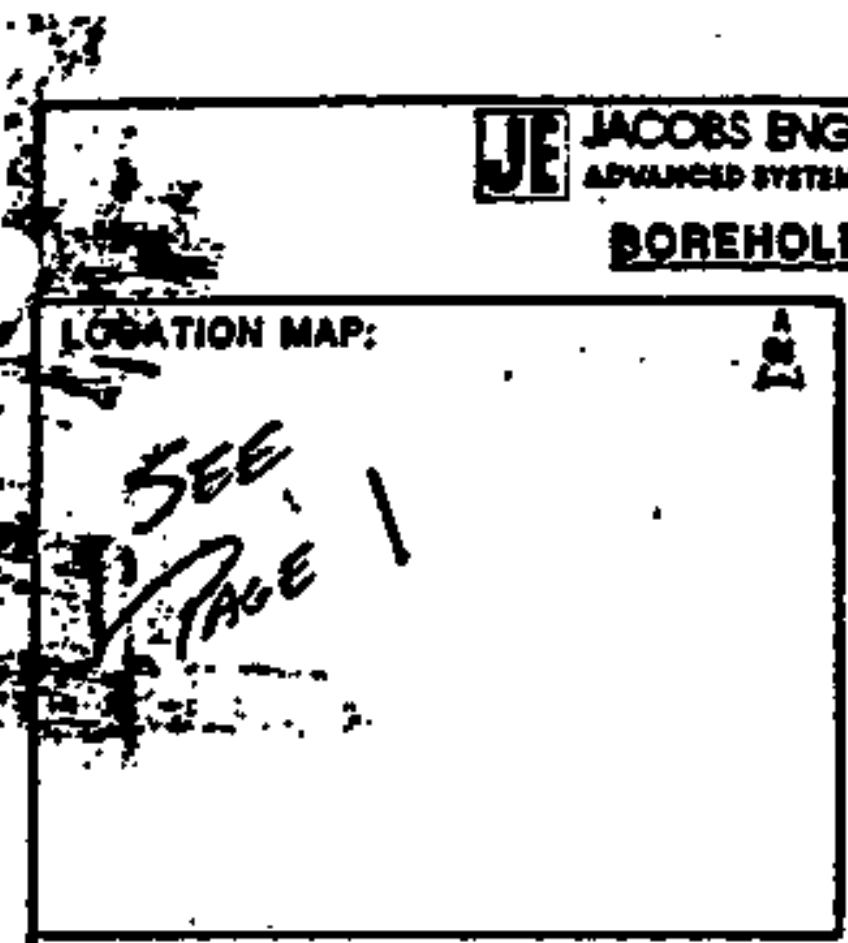

ENETE EROU NC.

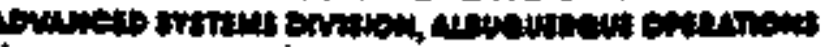

POREHOLEL LOC (AOCK)

Paget of -

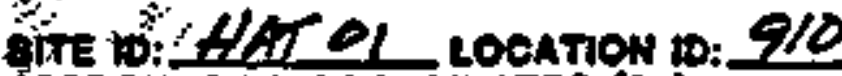

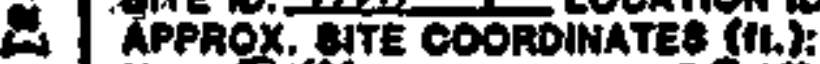

M sime

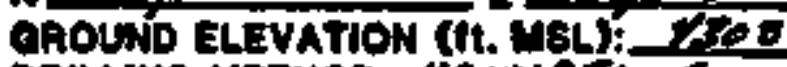

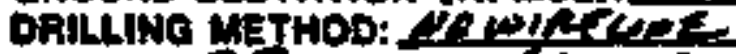

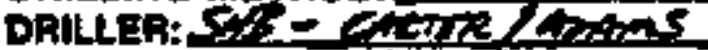

DATE 8TARTED:WUL GrN

DATE COMPLETED:

FIELO REP.:

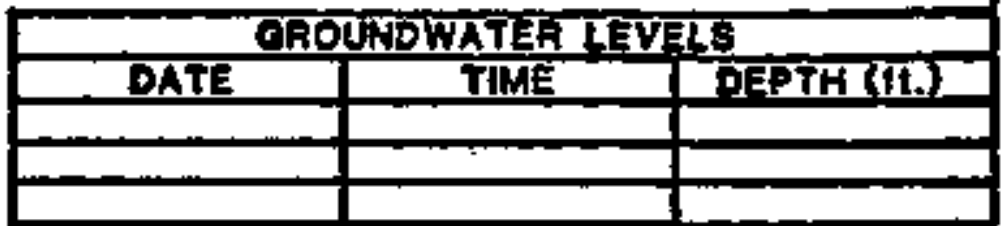

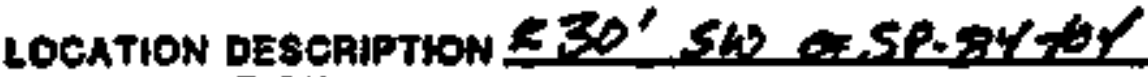
BITE CONDITION

8 E

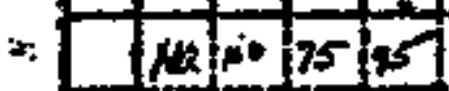

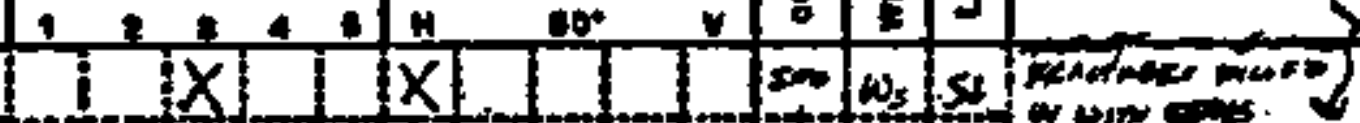

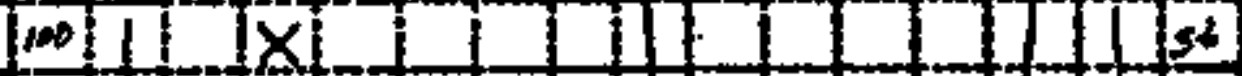

(1)


-

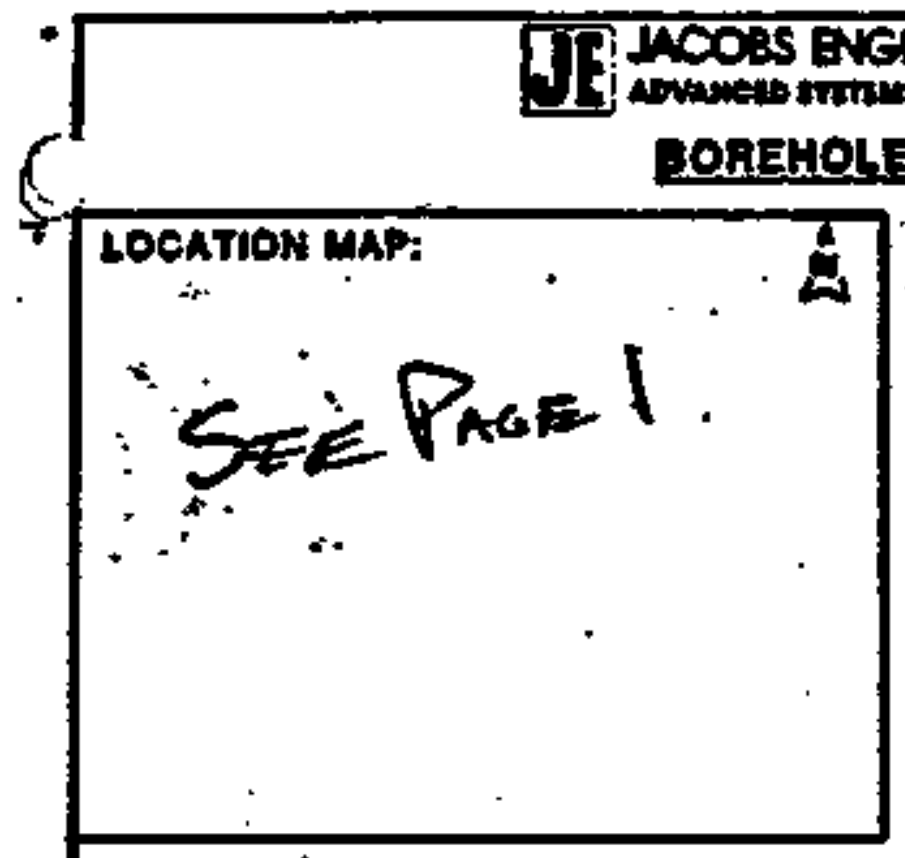

NARE CAOP NE

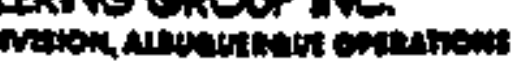

ROREHOLE Loo (RoOX) Papo Iot -

at?

GTE WH:STOL LOCATION DD: 910

APPAOX. OITE COOADINATES (tt.):

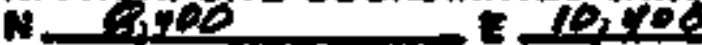

GAOUND ELEVATION (11. MSL):_LTOD

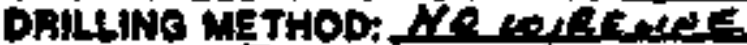

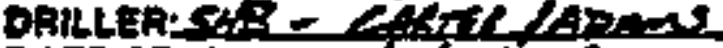

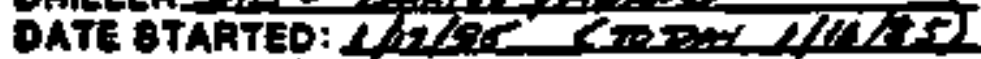

DATE EOMPLETED:

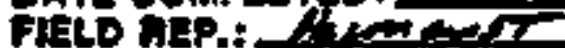

\begin{tabular}{|c|c|c|}
\hline CROUNDWATER LEVELS \\
\hline DATE & TLME & DEPTH (IT) \\
\hline & & \\
\hline & & \\
\hline
\end{tabular}

LOCATION DESERIMTION

SITE CONDTION

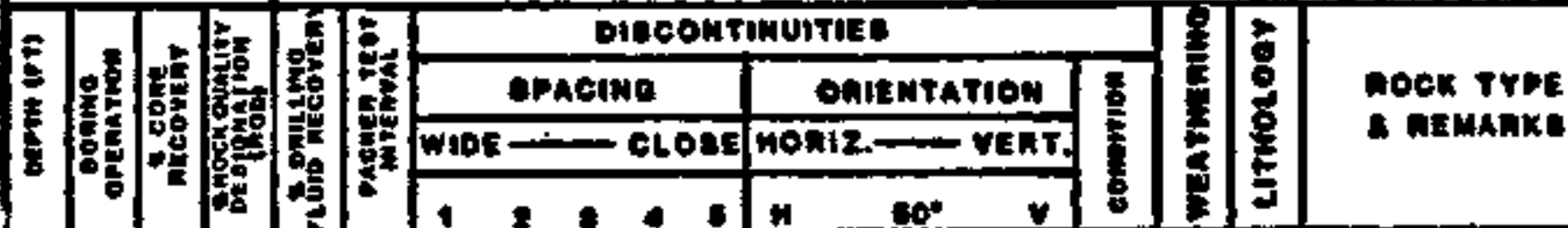

(n)

$x: 19 x$

cov $v$

$10+100$

$1 \frac{100}{100}+\cdots \frac{X}{x}$

ix

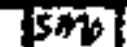

$1435 \sin ^{3}$

PowAltED.

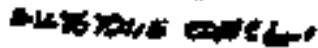

Hacstat.

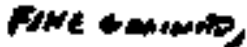

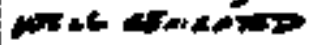

13

100

$x$

$1 ?$

$+$

3

11110

$x$

Than atosingont Var fis maly onsmof that of armate m nothes Prant-

99

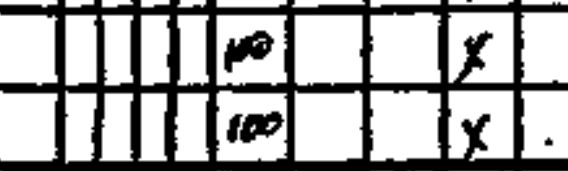

IfTlor

7. 125

$\times \times$

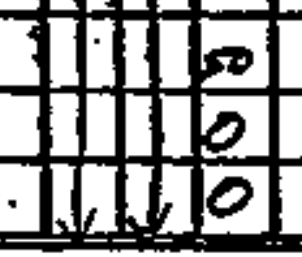

X
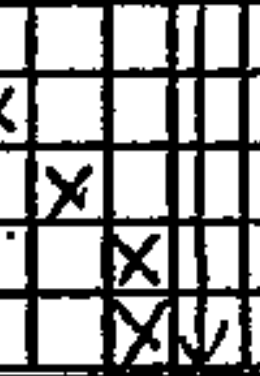

$\infty$

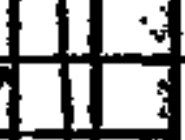

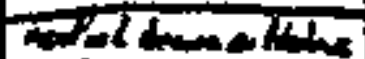
anst atus Angm

ancenten. int otent?

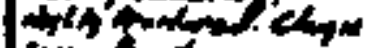

COMMENT: 


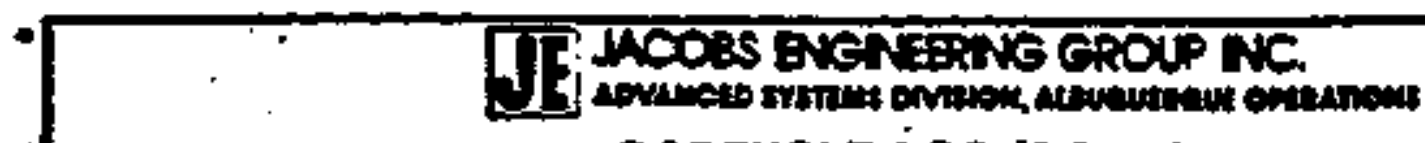

\section{LOAEHOLE IOR (BOCK)}

ROCATION MAP:

4

intithat

LOCATION ID: 910

APPAOX. gITE COORDLWATES (H.):

$N$ Stos E 10,400

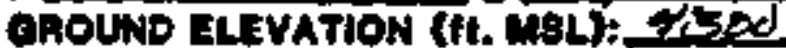

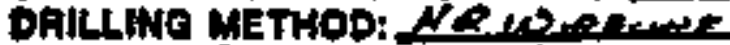

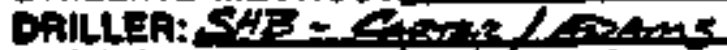

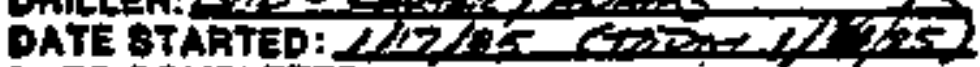

DATE COMPLETEP:

FIELD REP.: D O

\begin{tabular}{|c|c|c|}
\hline ER & OUNDWATER LEV & ILS \\
\hline DATE & TUME & DEPTH (G) \\
\hline & & \\
\hline
\end{tabular}

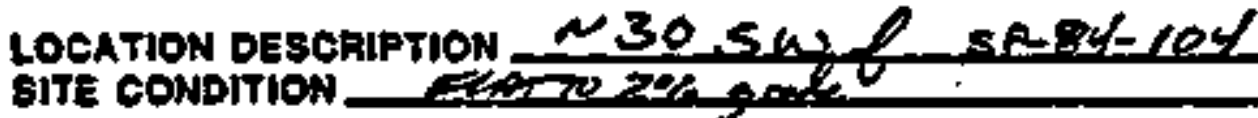
Encozen som

If

$\because$ matolo

$m$ 11100 $+\frac{|x|}{|x|}$ s.m. 175. $x$ Iftos so: s.
$\therefore$ 260 X 711256 $x+1$
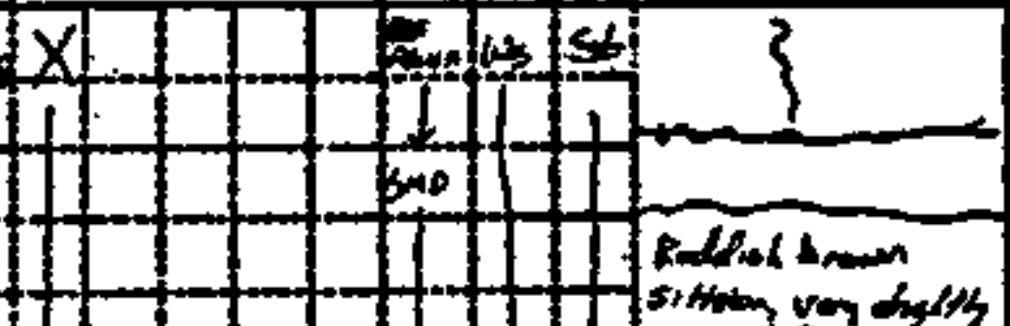


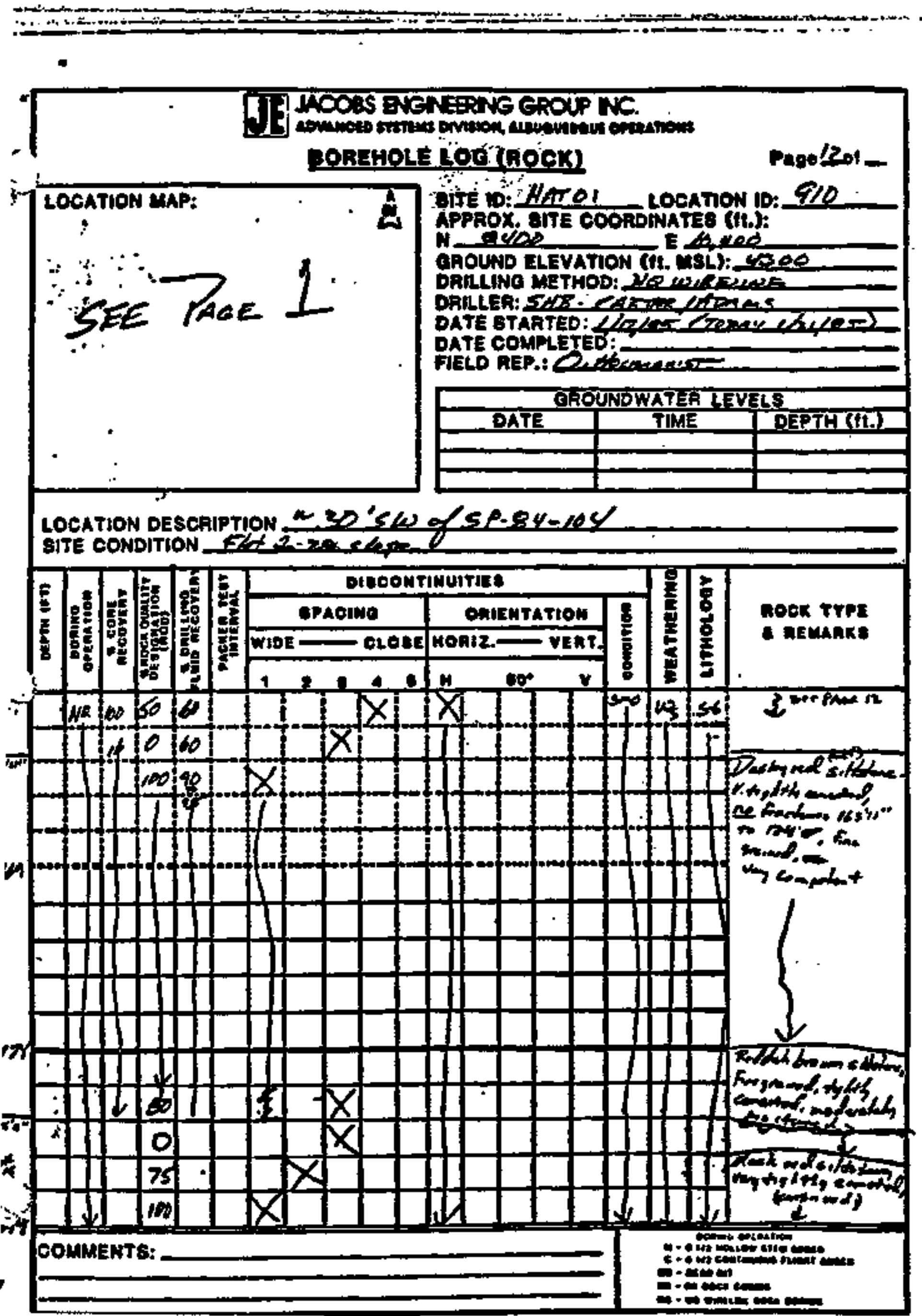




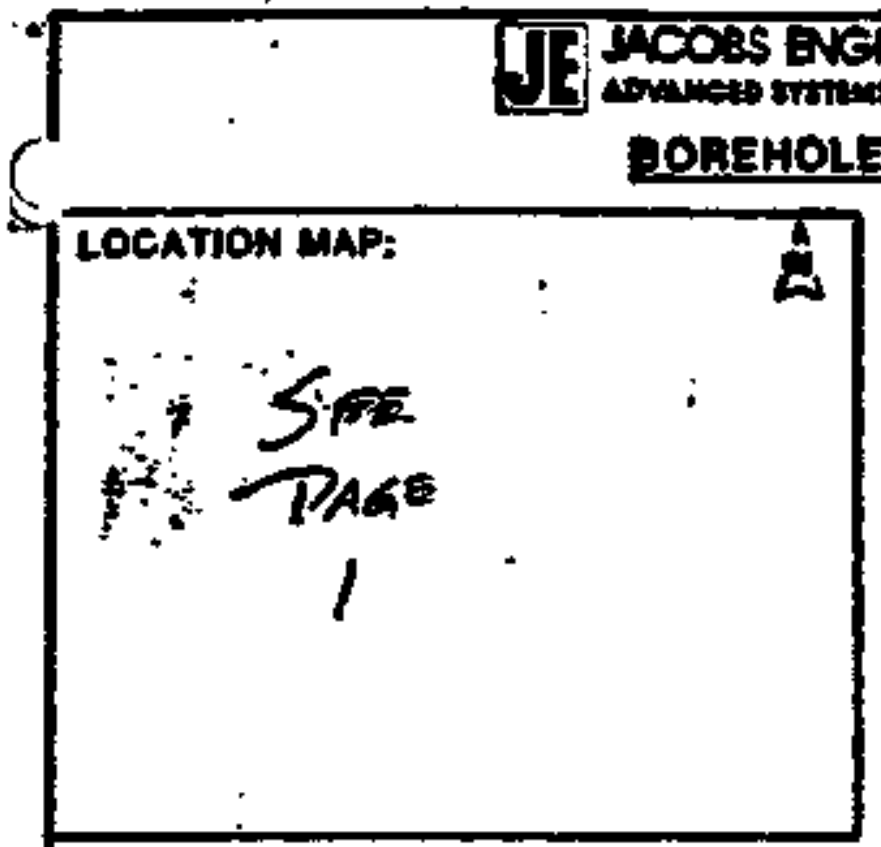

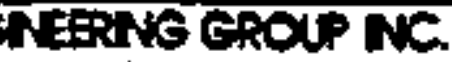

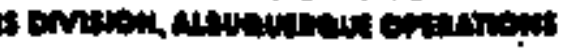

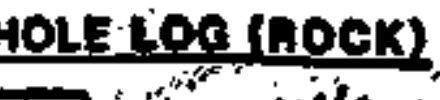

Page 26 i

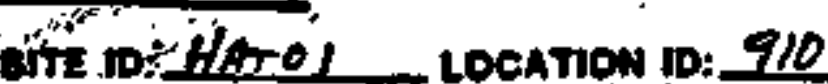

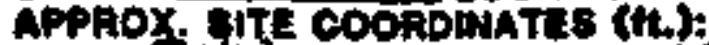

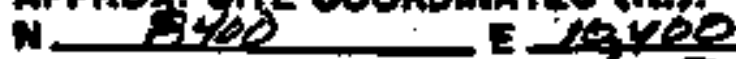

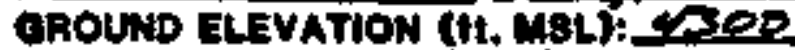

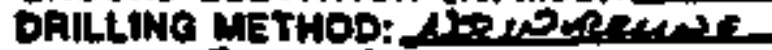

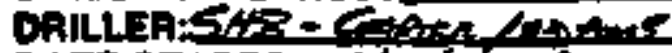

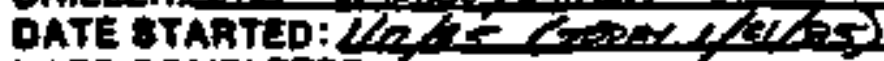

DATE COMPLETED:

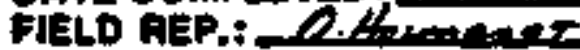

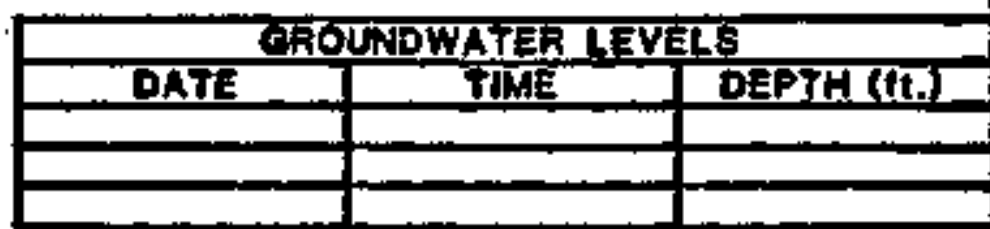

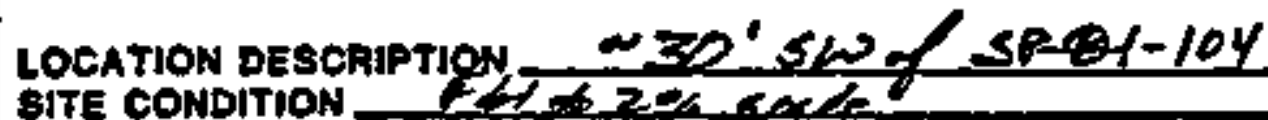

A

8 8

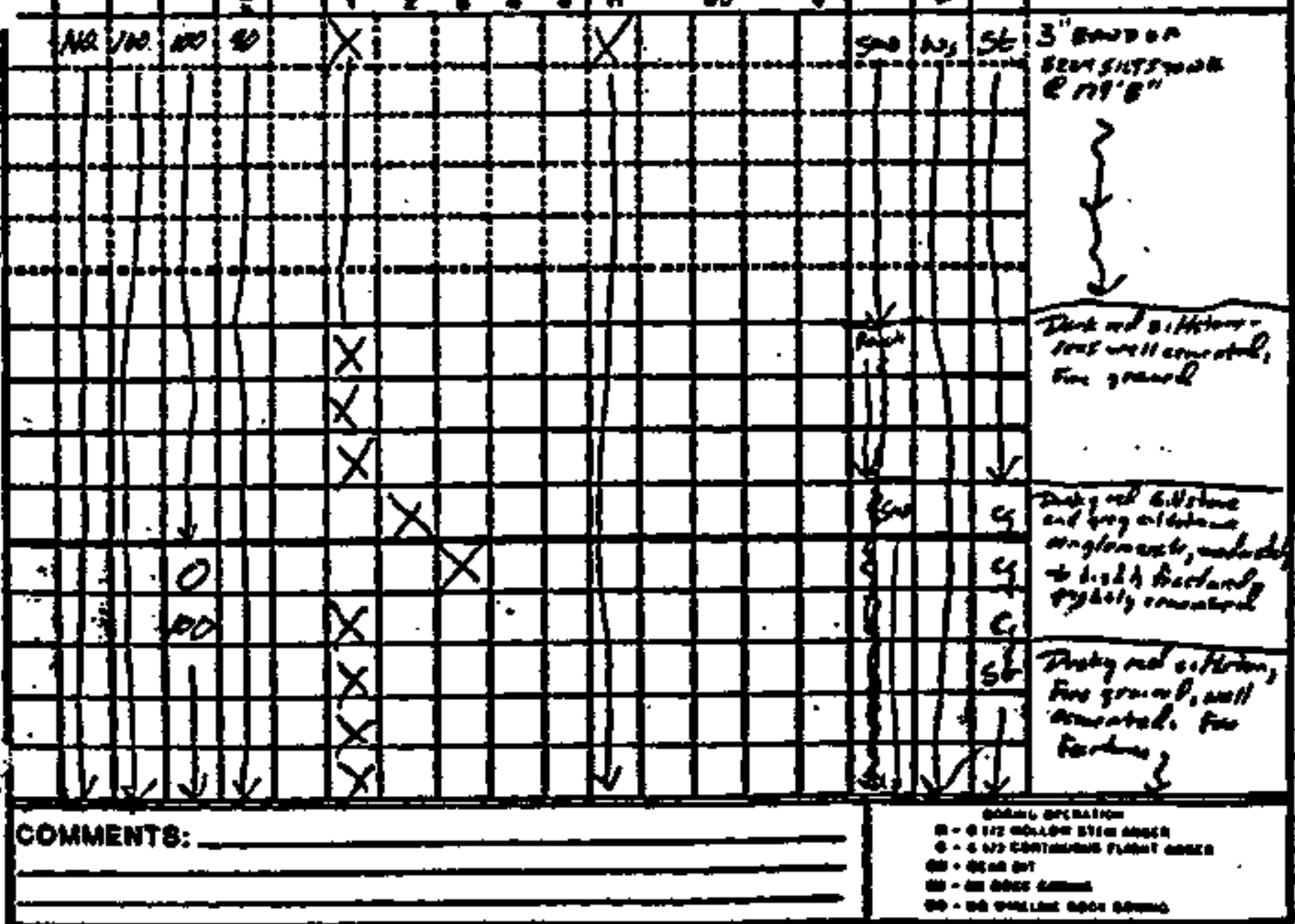




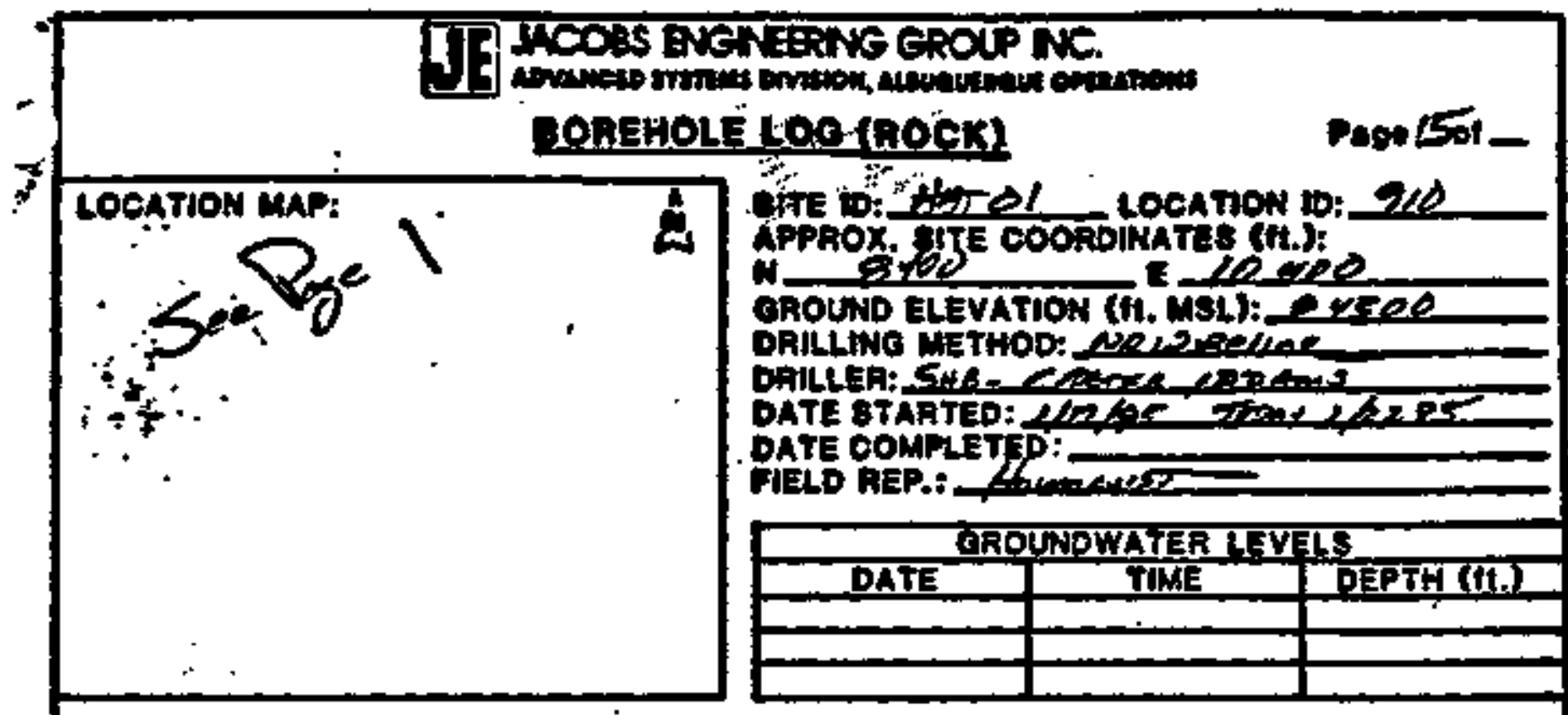

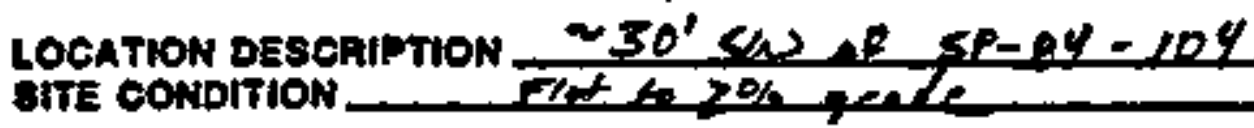

.

.

(x) X:

X)

(1)

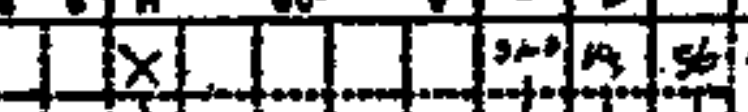

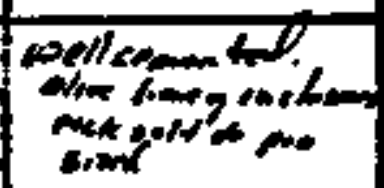

$+1 .+1+1+1$

noek Tres

- newanke

$\therefore$

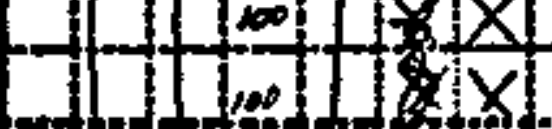

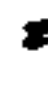

24

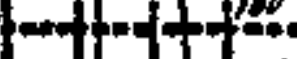

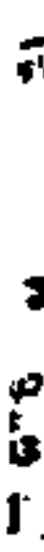

100

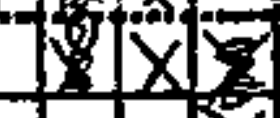

$1 \rightarrow$

$1 \mathrm{x}$
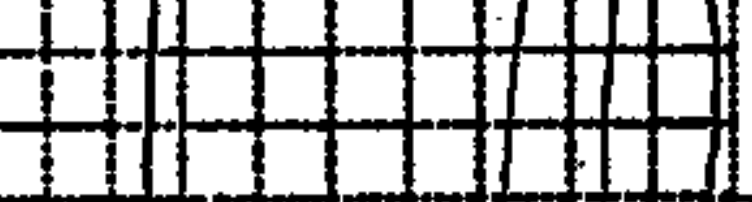

4

117

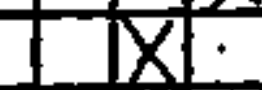

$+1$
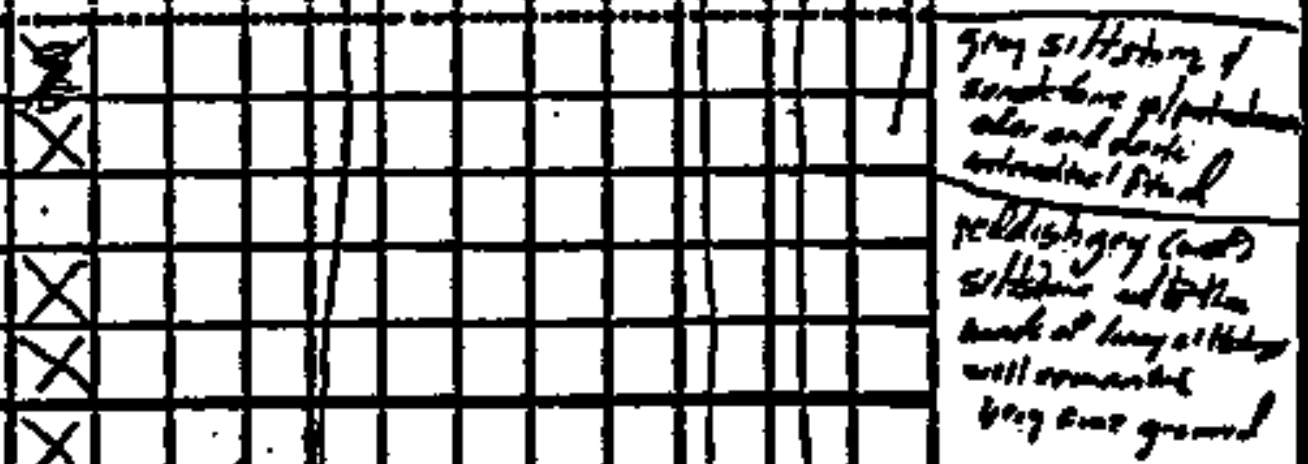

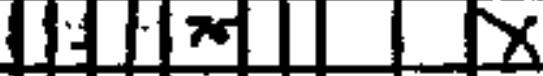

111.25

X

$\sqrt{1}, \mathrm{X} \times$

$\frac{x}{x}$

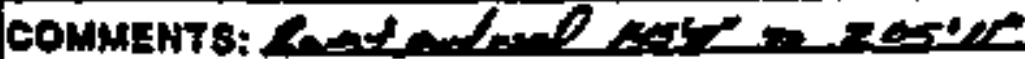




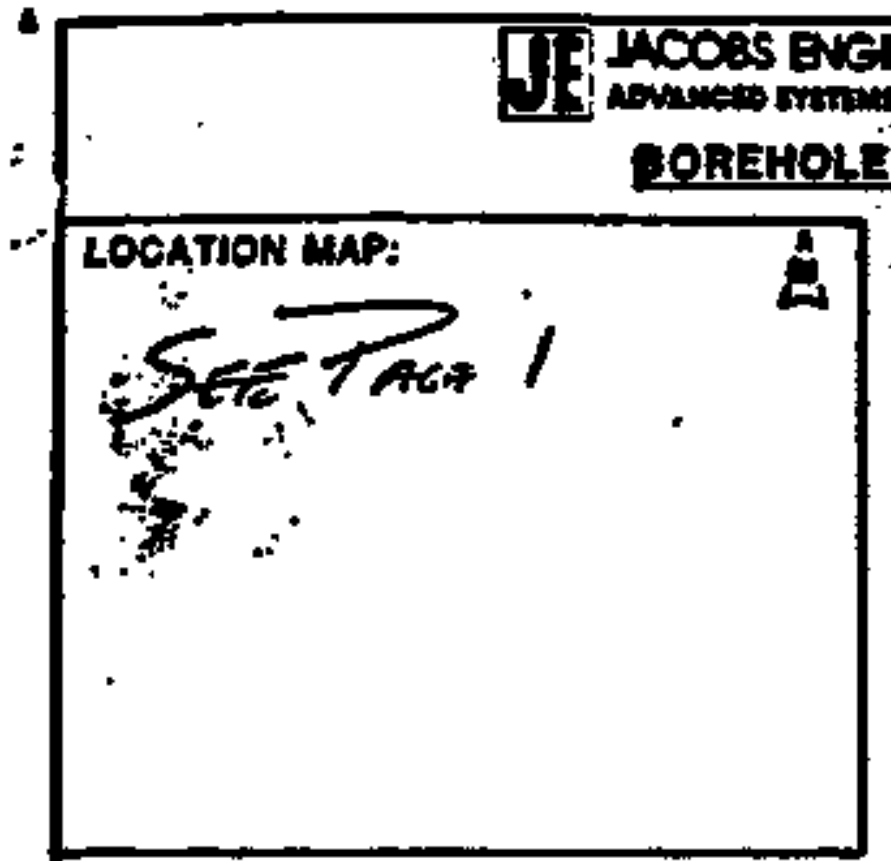

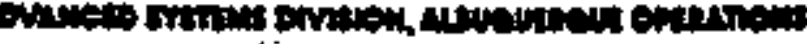

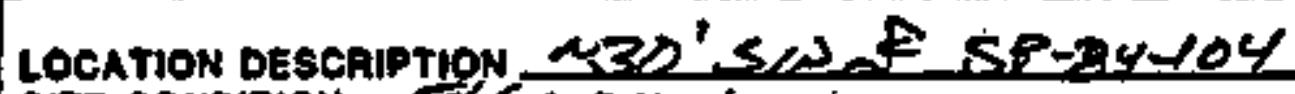
SITE CONOITION EL

\begin{tabular}{l}
\hline \\
\hline
\end{tabular}

\begin{tabular}{|l|l|l|}
\hline & GROLNDWATER LEVTS \\
\hline DATE & TIME & DEPTH (It) \\
\hline & & \\
\hline & & \\
\hline
\end{tabular}

$=1 / 2 \operatorname{sos} 20$

$\pi$ 1. 


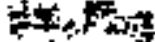

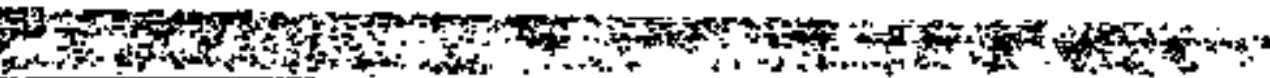

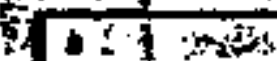

is

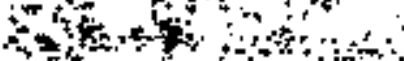

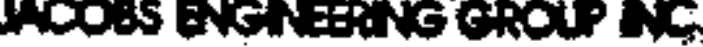

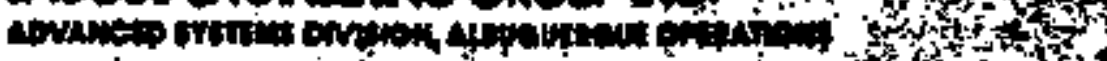

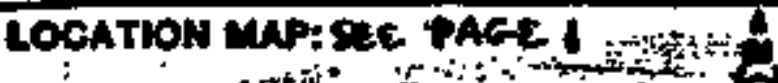

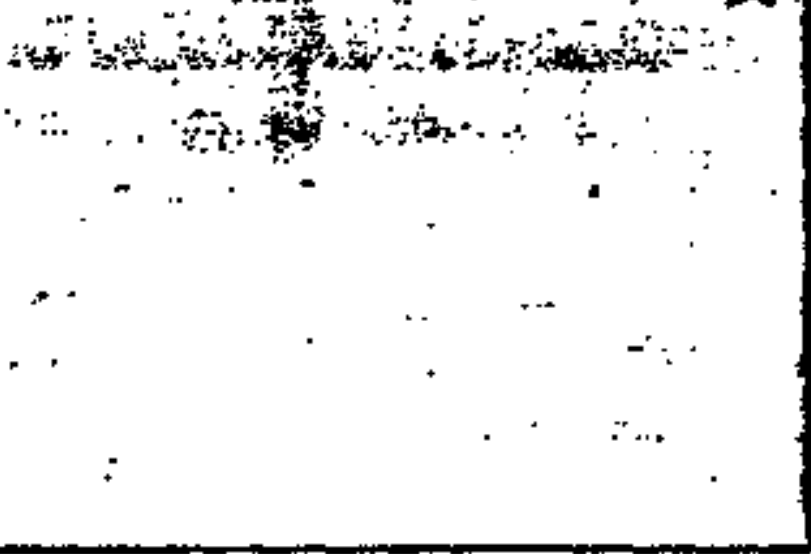

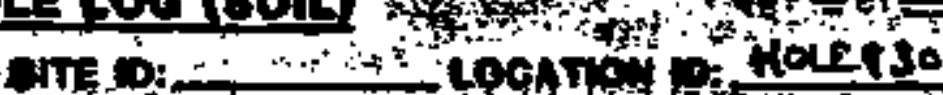

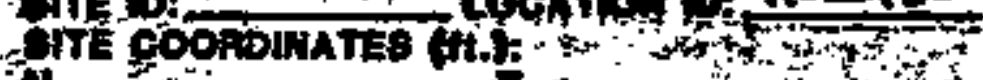

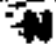

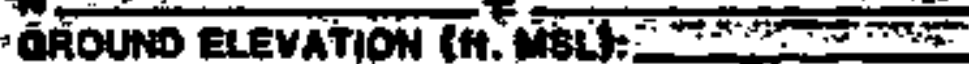

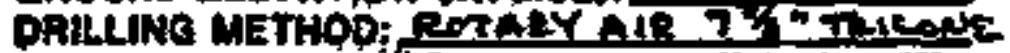

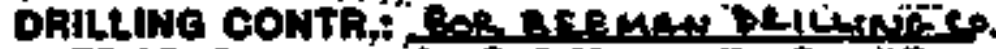

DATE BTARTED: TO-9-95

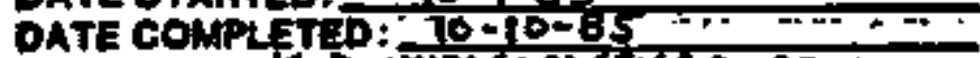

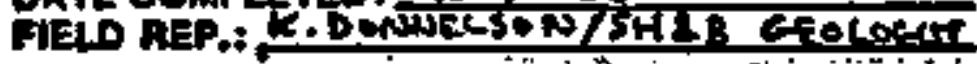

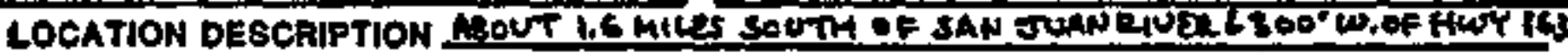

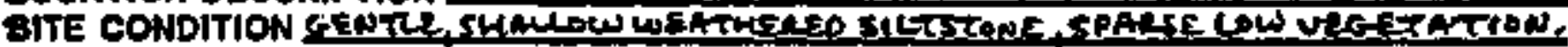

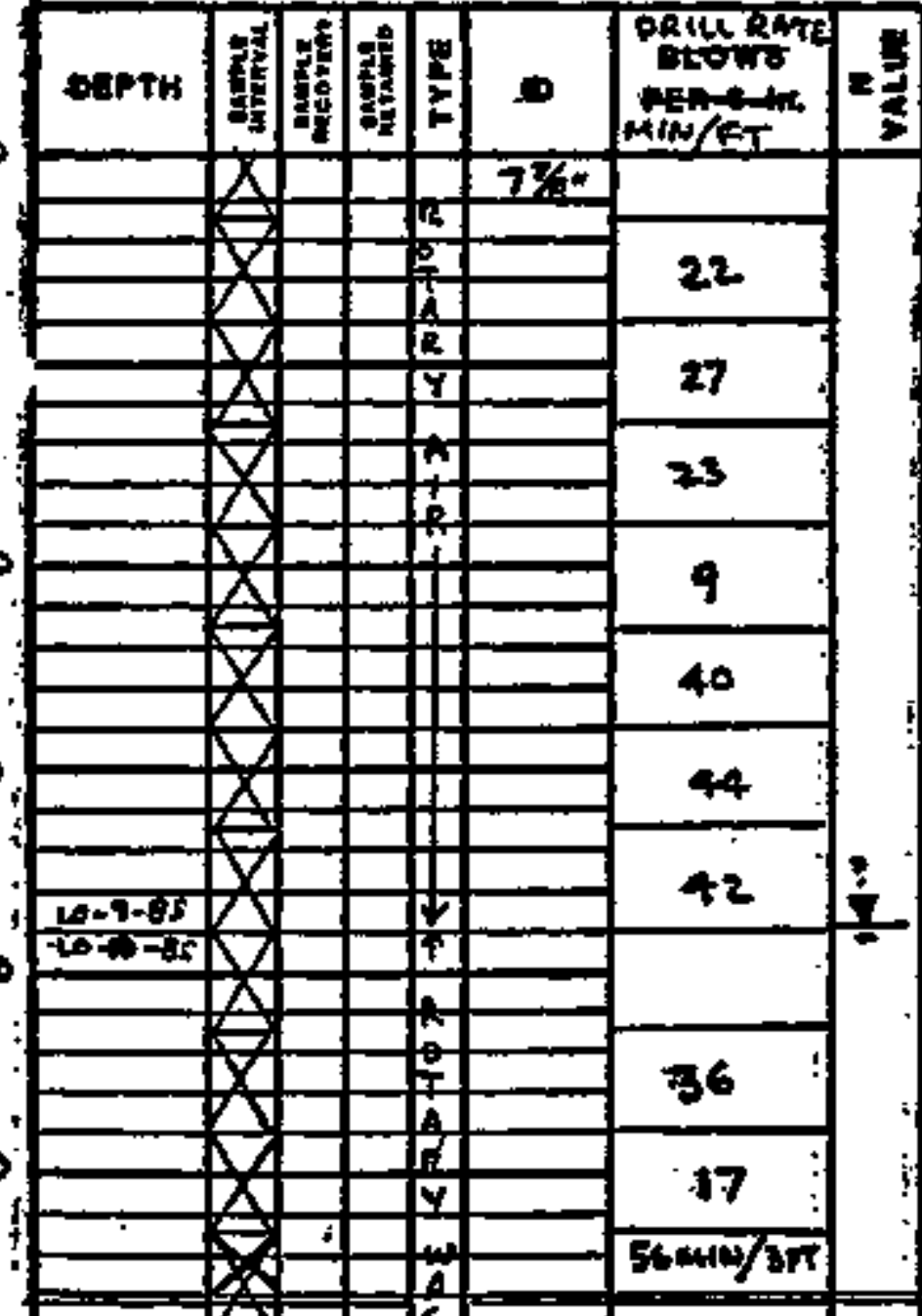

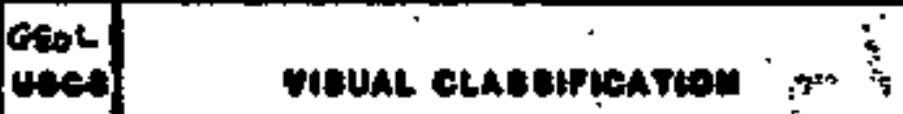

18

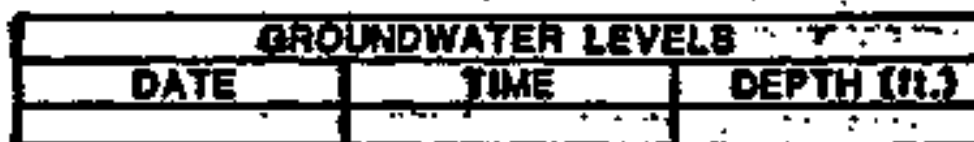

COMNENTS: EOTAY $A I R$ O $=9 B$,

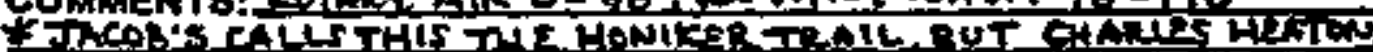

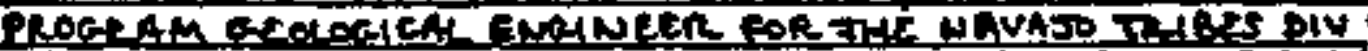

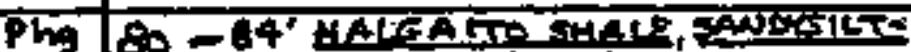

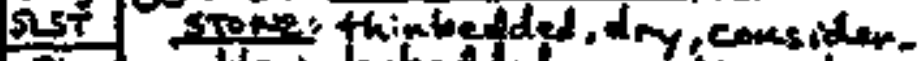

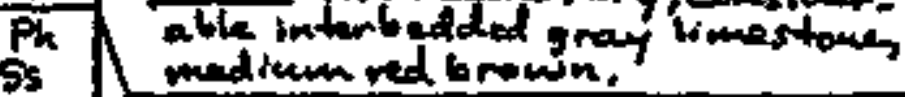

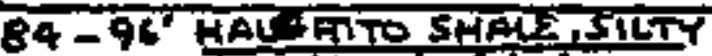
ravpstonse thim bediled, sema

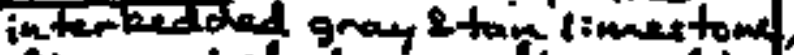
sive-rvained dry, medium ad bn

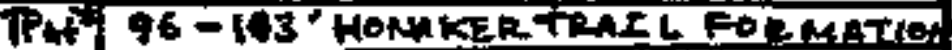
Is LimesteruE Lisht -madium gray srating to light blue greens s: is $k$ t purple

ares sons idencble intented id tive-grained bru sactstind

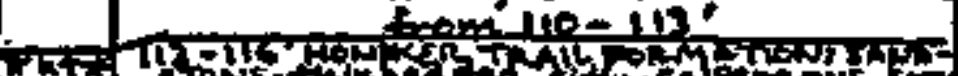

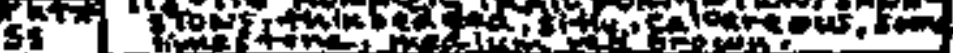
T. $0,116,9158 \mathrm{Am}+10-10+8 \mathrm{~S}$

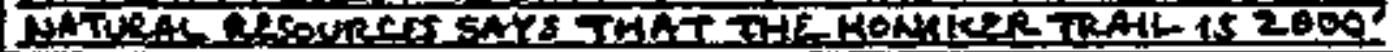


然 \& an

Boshton MAP:

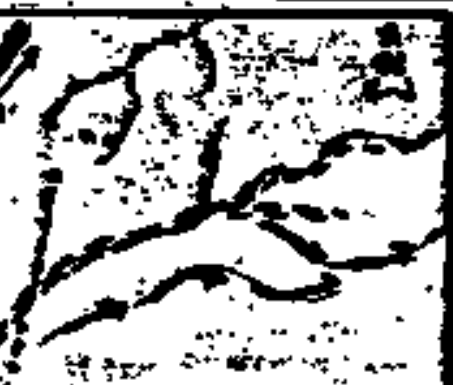

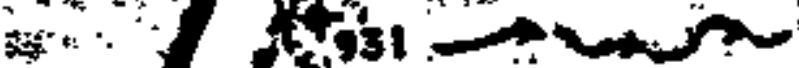
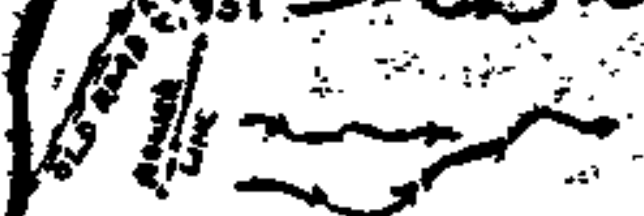

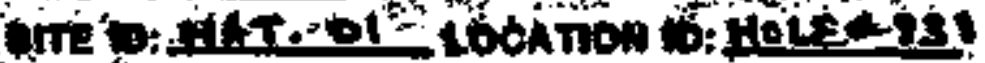

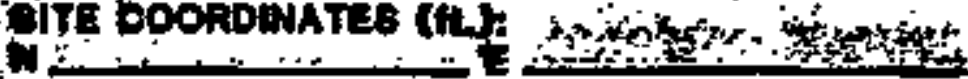

BROLND ELEVATION (ft. WBL)

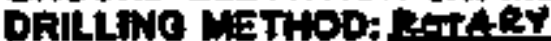

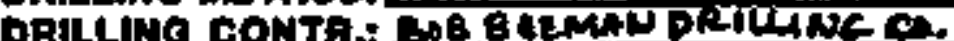

GATE 8TARTEO: $10-100.5$

DATE COMPLETEO: $\left(6-r_{2}=\mathrm{BS}\right.$

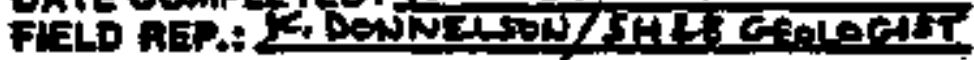

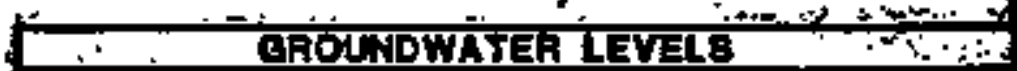

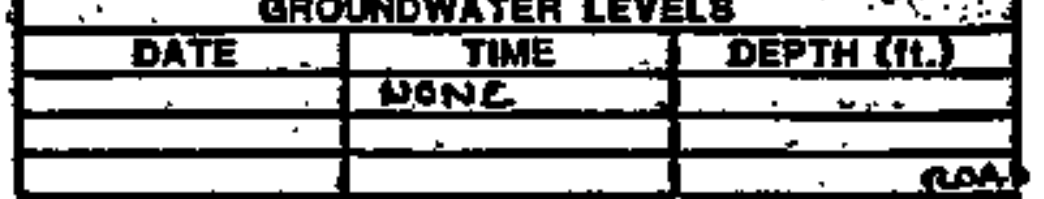

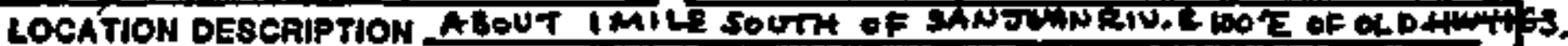

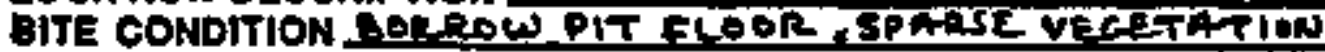

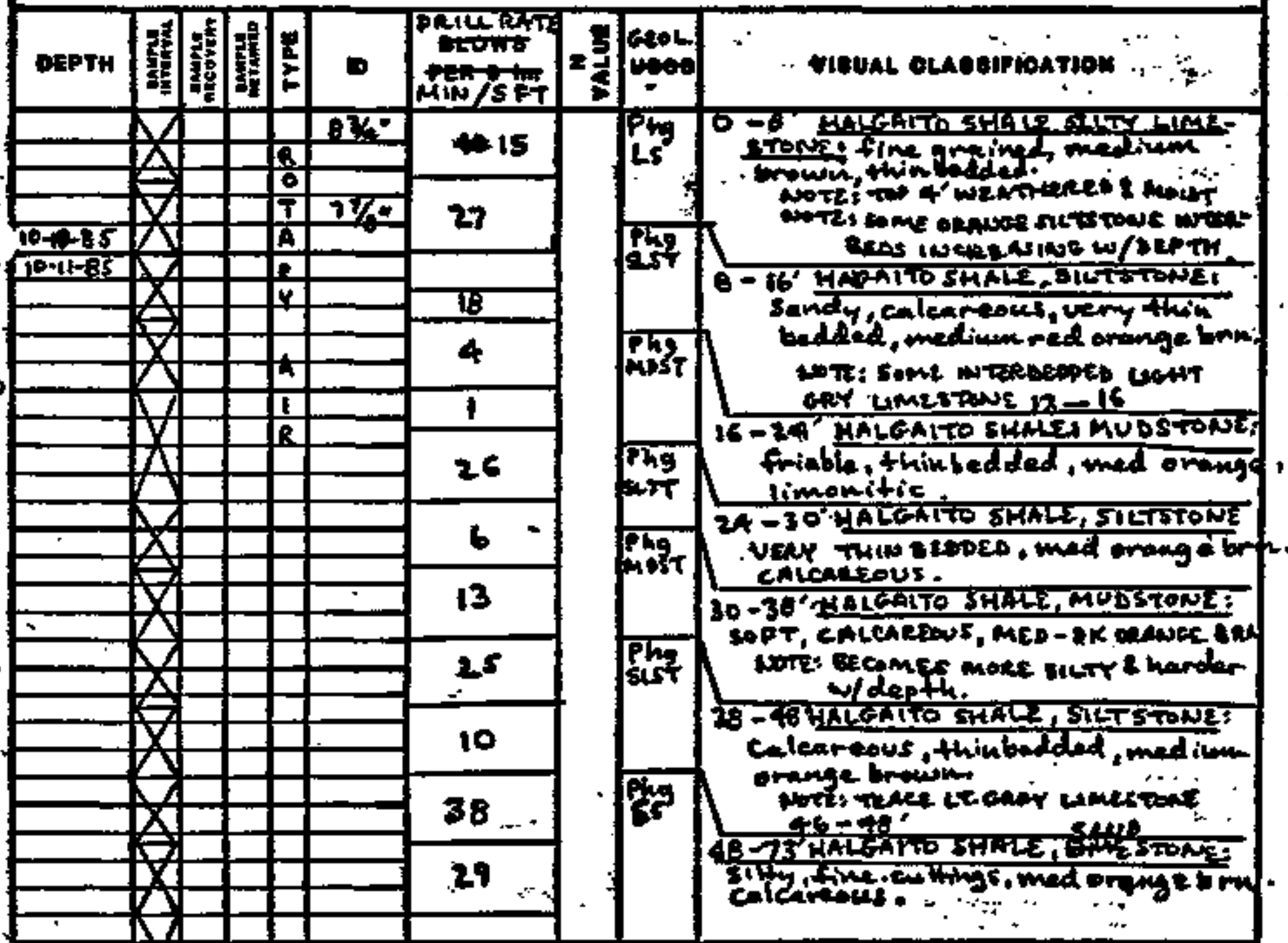

OOMMENT 8: HOLE ABAWDONED AT A BSPTH OF 172FEET?

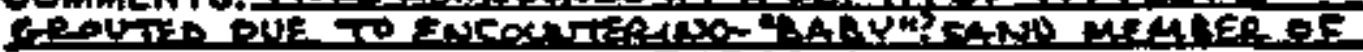

Hance Tris :

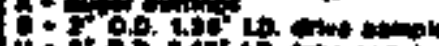

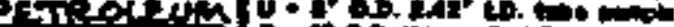

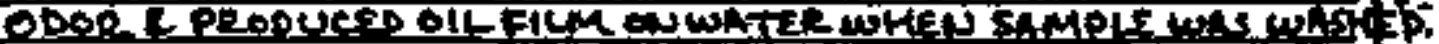




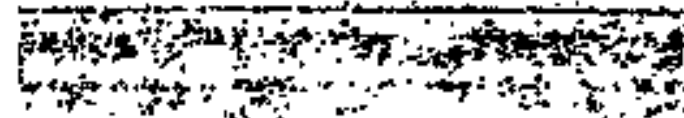

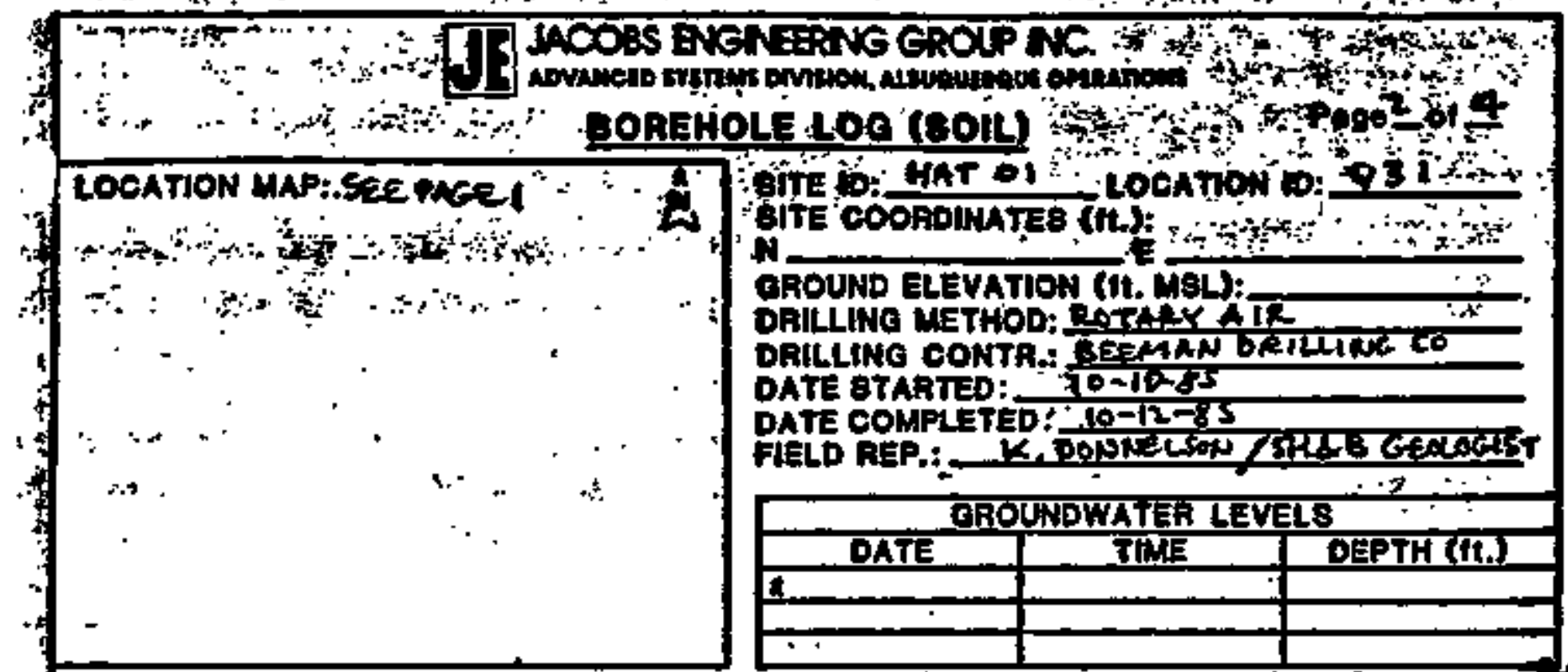

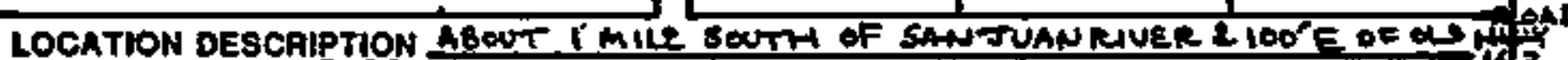

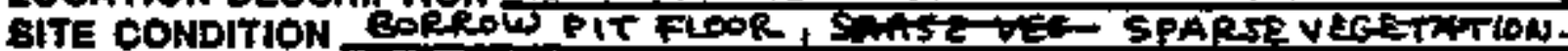

10

10

to

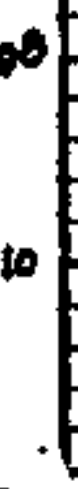

\footnotetext{
(1)

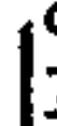

西 oerm te=

MUBUAL GLABEIFLATION

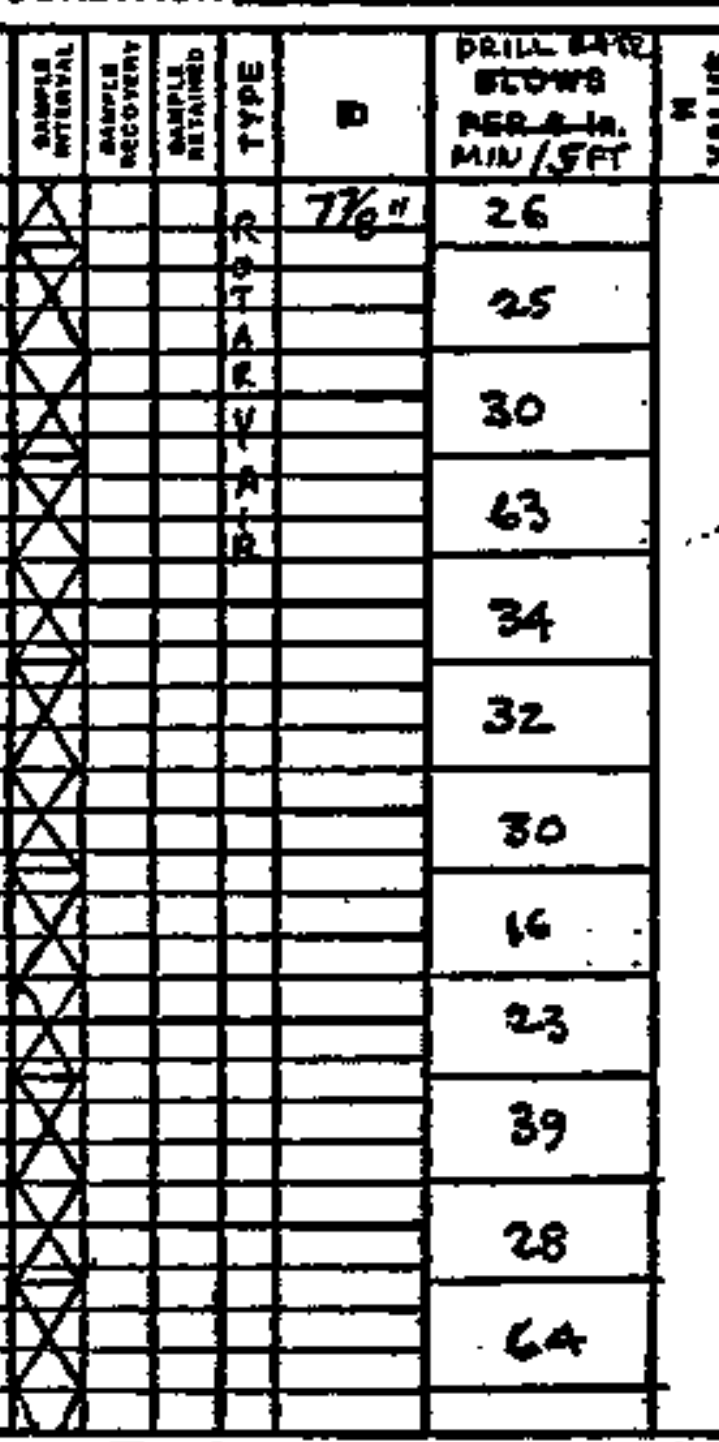

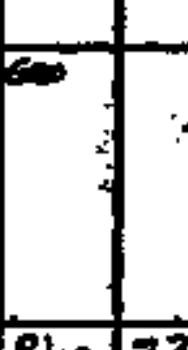

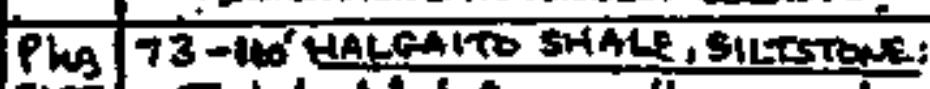
sust

Thimbedded, fime cutting s, calear-

cous, light-medium bown.

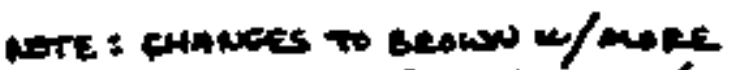

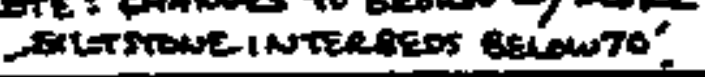
1

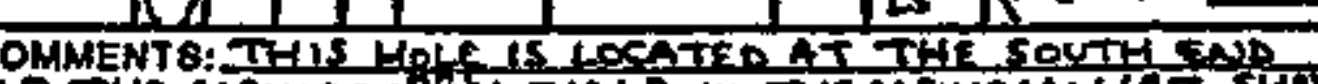
fiche ic

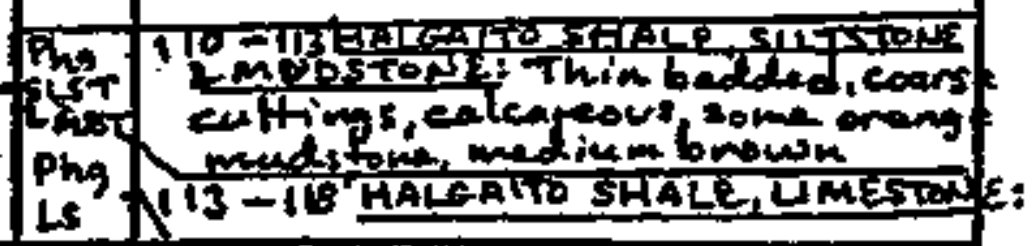

Note: some inturbads of light erange bonom mudstone $98^{\prime}-100^{\circ}$

mistim Ls N13-13 HALAtro SHALE, LIMESTE . 


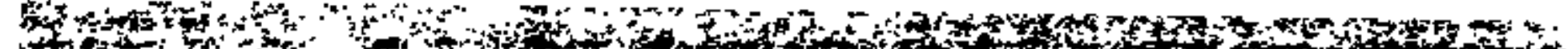
H.

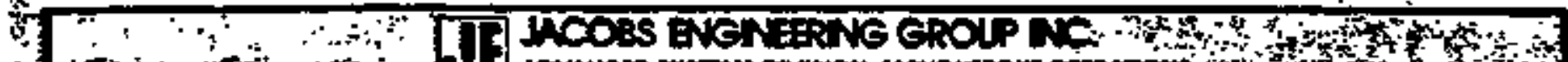
it 4.

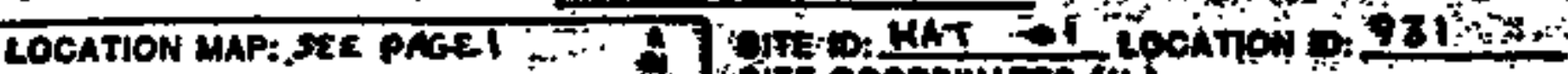

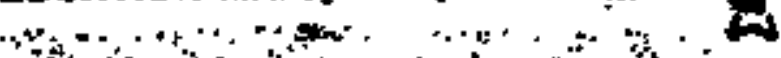
$\ddot{z}$

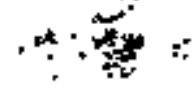

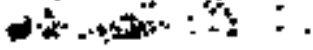

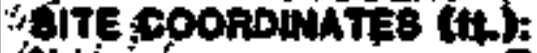

Ni:

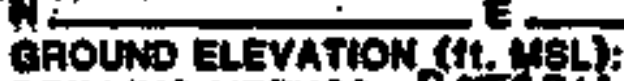

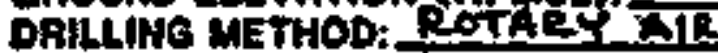

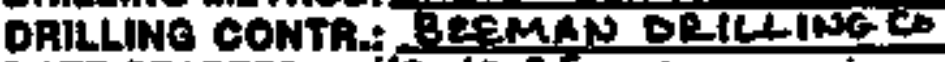

DATE OTARTED: $18-10-35$

DATE QONPLETE: 10-10-35

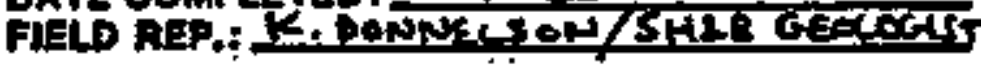

$+4+4$

$\therefore$.

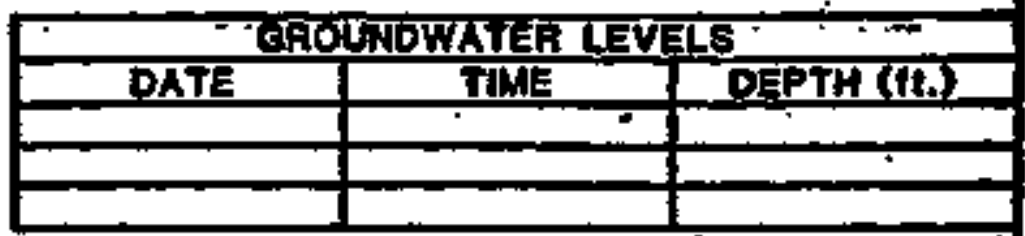

LOCATION DESCAIPTION ABOST IMIL SOUTH OF SAN THAW RIVER \& $100^{\prime}$. OF"

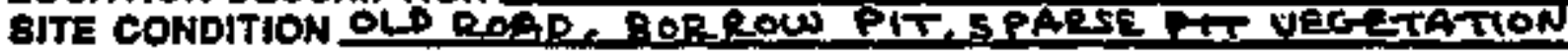

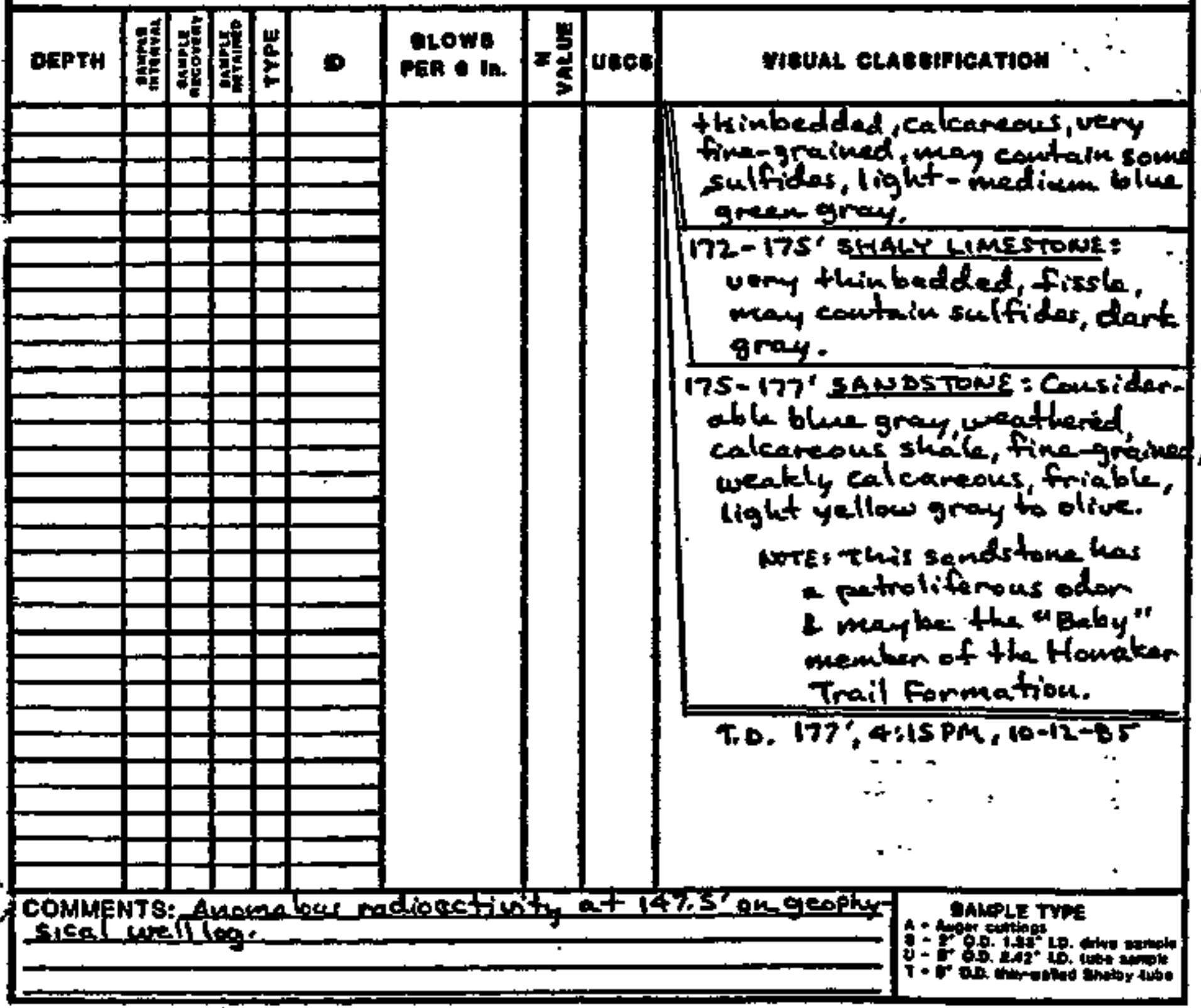




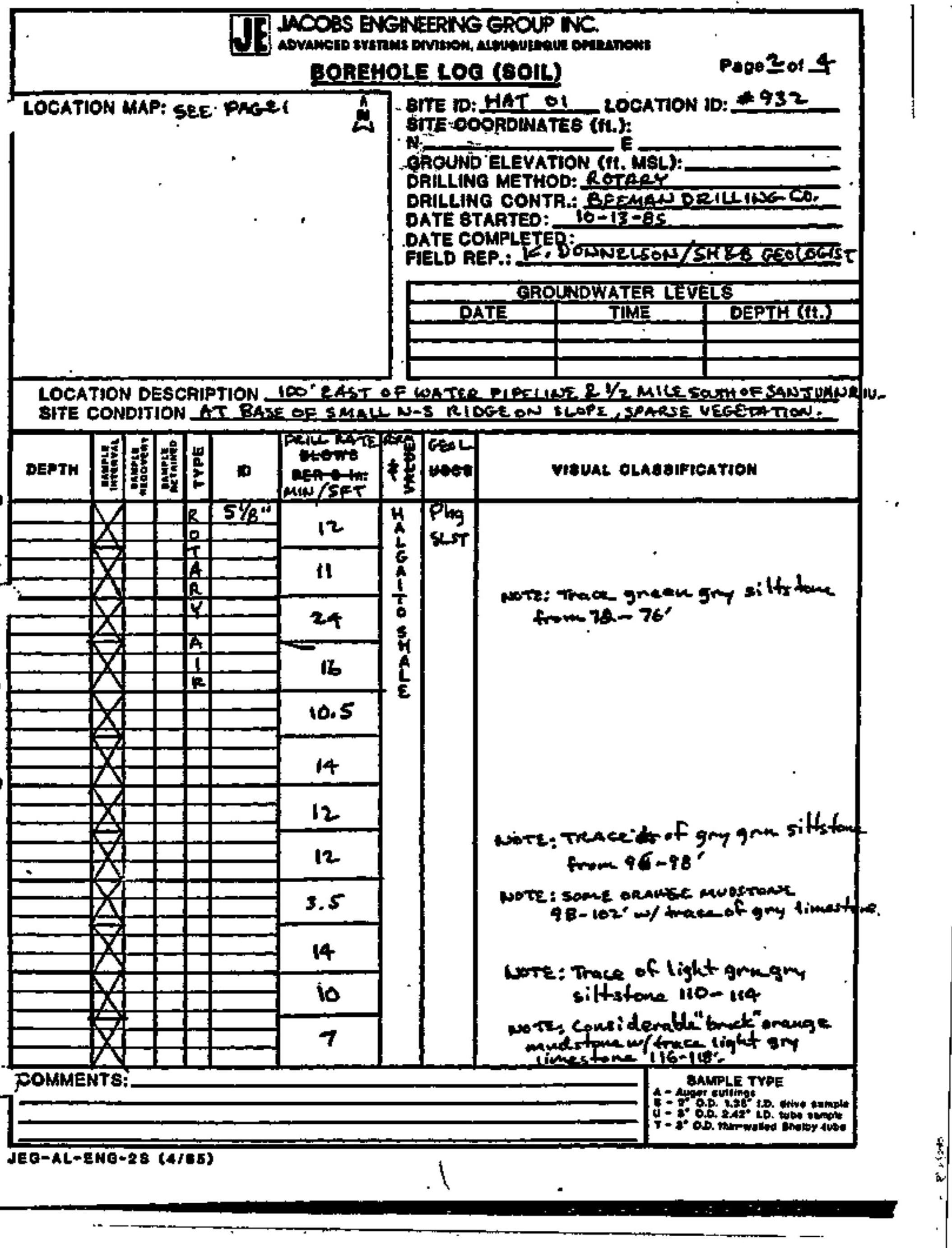




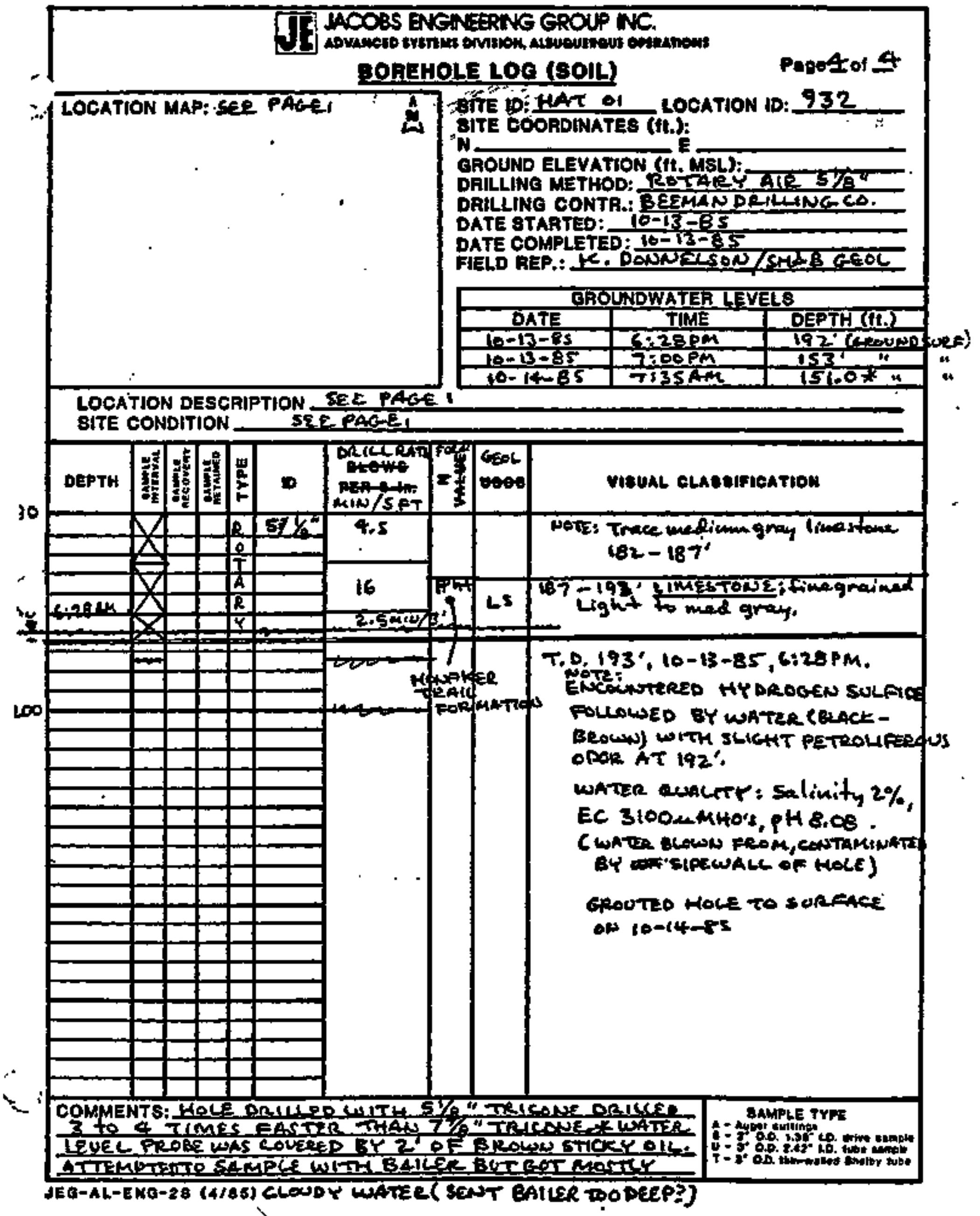




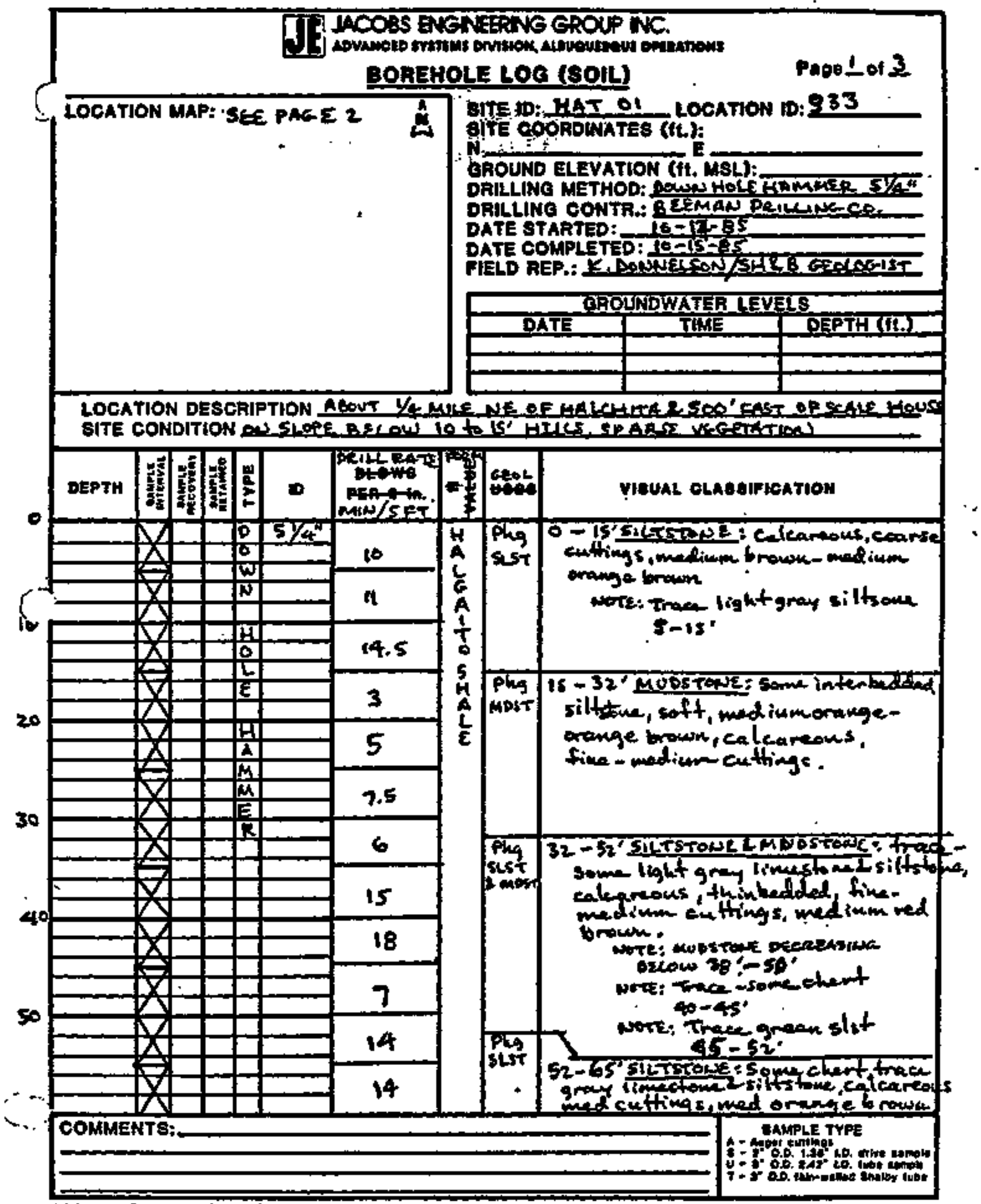

JEO-AL-ENB-2 E (4JEB) 
TS HOONAS GNGNEERNG EROUP WC.

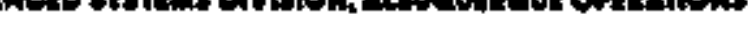

BOREHOL 200 (BOIL)

Pagezot 3

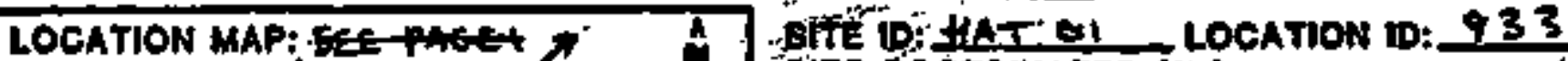

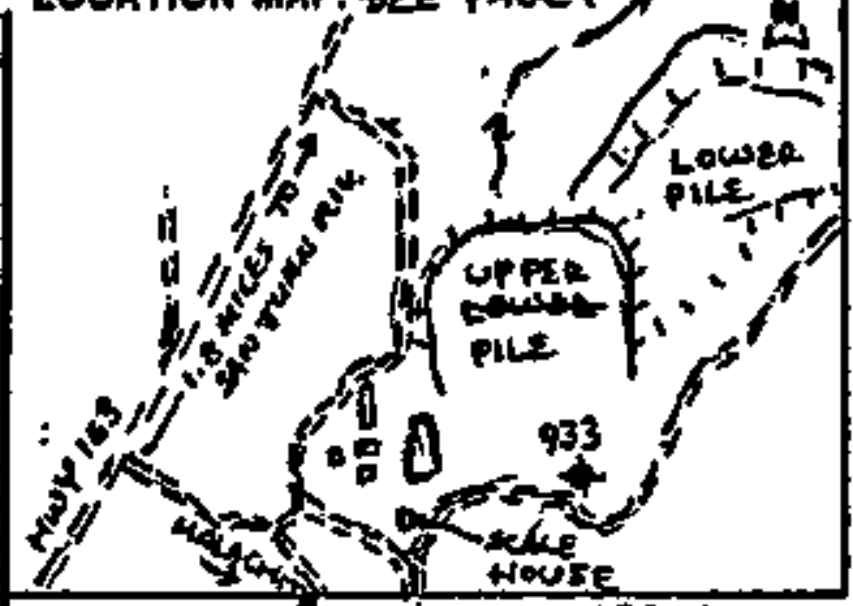

GITE COOHDINATES (tt.):

is

CROLND ELEVATION (ft. MBL):

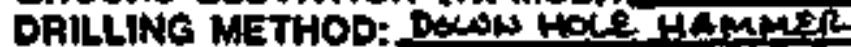

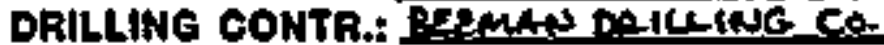

DATE STARTED: $10-14-85$

DATE CONPLETED: $10=1 \$-6 S$

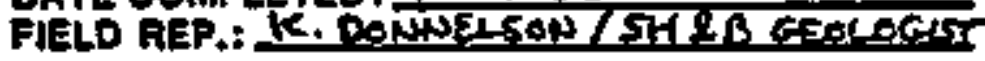

\begin{tabular}{|c|c|c|}
\hline \multicolumn{2}{|c|}{ GFOUNDWATER LEVELS } \\
\hline DATE & TIME & DEPTH (ft.) \\
\hline & & \\
\hline & & \\
\hline
\end{tabular}

LOCATION DESCAIPTION SES PAEEI

SITE CONDITION

SEE PNGE।

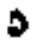

0

0

$\infty$

10

oter

0

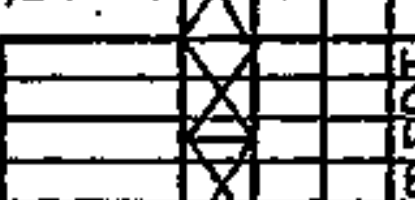

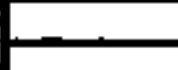

\begin{tabular}{|l|l|l|l|}
\hline 1 & 0 & $51 / 4$ \\
\hline 7 & & 0 & \\
\hline & &
\end{tabular}

7

N 19

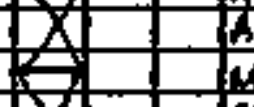

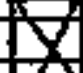

$17+2:$

X:-

COMMENTS

A

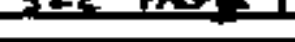

\begin{abstract}
-
\end{abstract}
tion
H pote: limestone \& gray siltsouch
absent 55-160

\title{
visedal clasatfication
}

pote: sone araceje mudstoned. ingt grem gray siltstome

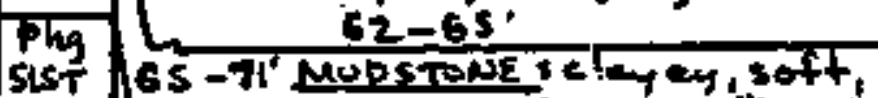
fint entims, celcareous, brick" crange

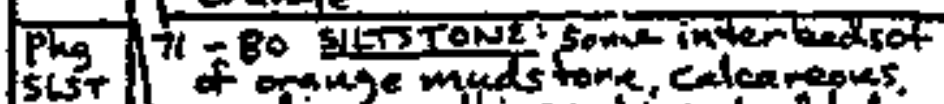

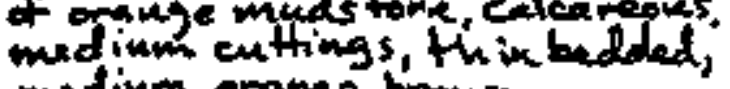

Phy medium orange brown.

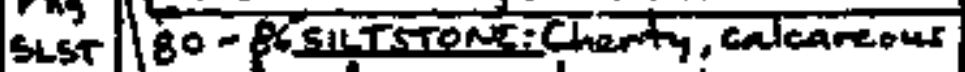
med ved orange broum:

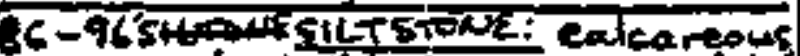

some interbidid orunge mudstom th

Aing

$-23.5$

205

SLST

med red brown.

Nere t Trace light ony limes tone

Egru gry siltsone 90-76'

$96-169^{\prime} 511$ Ts Tont : Calcareous, champ,

ined rid orange bri. trace gry grt sithstame to 100: -

poz: sove onange medstome.

$106-106$

NATE: TeACE - SEMES LT GAMY

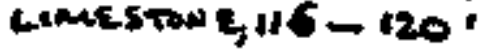




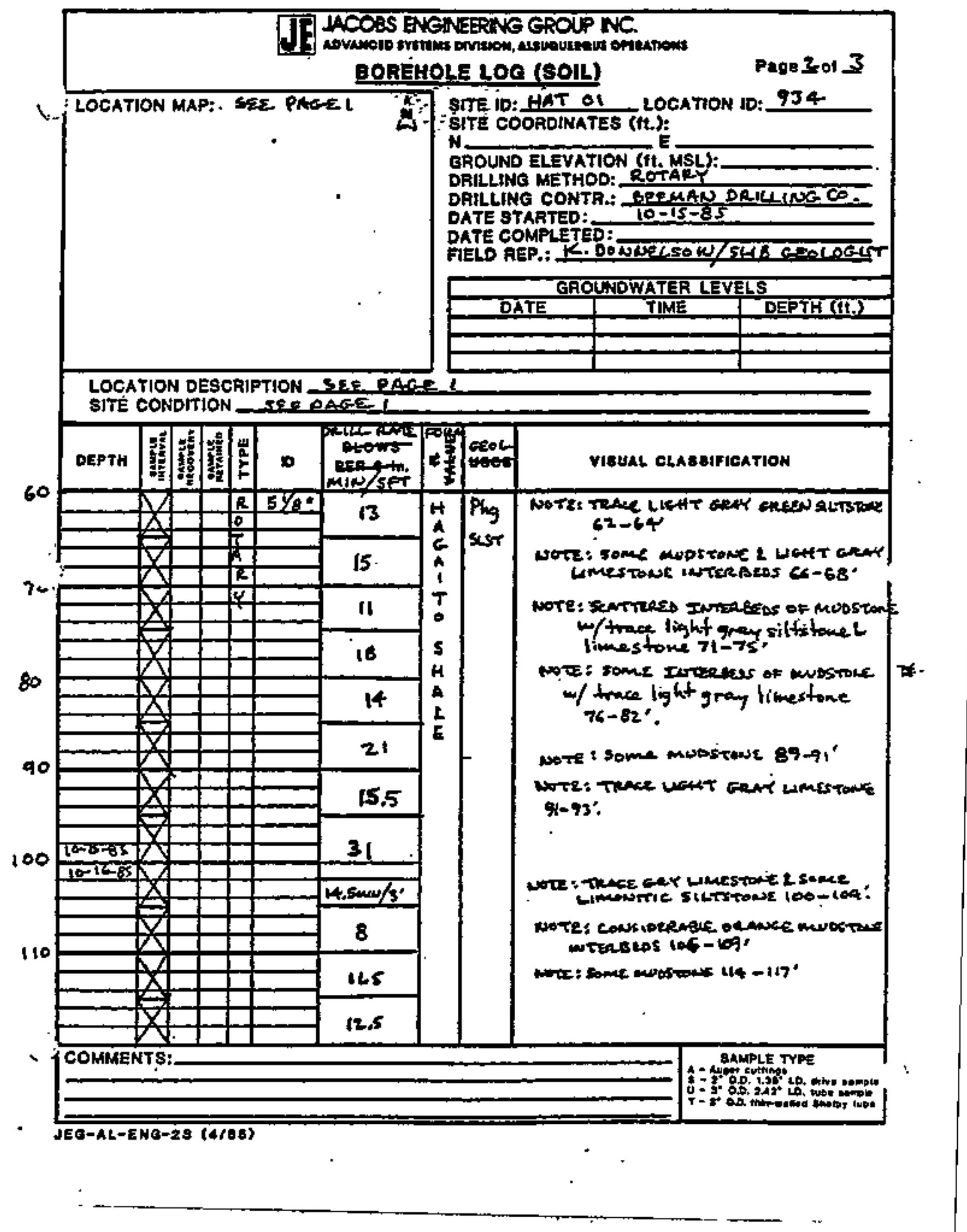




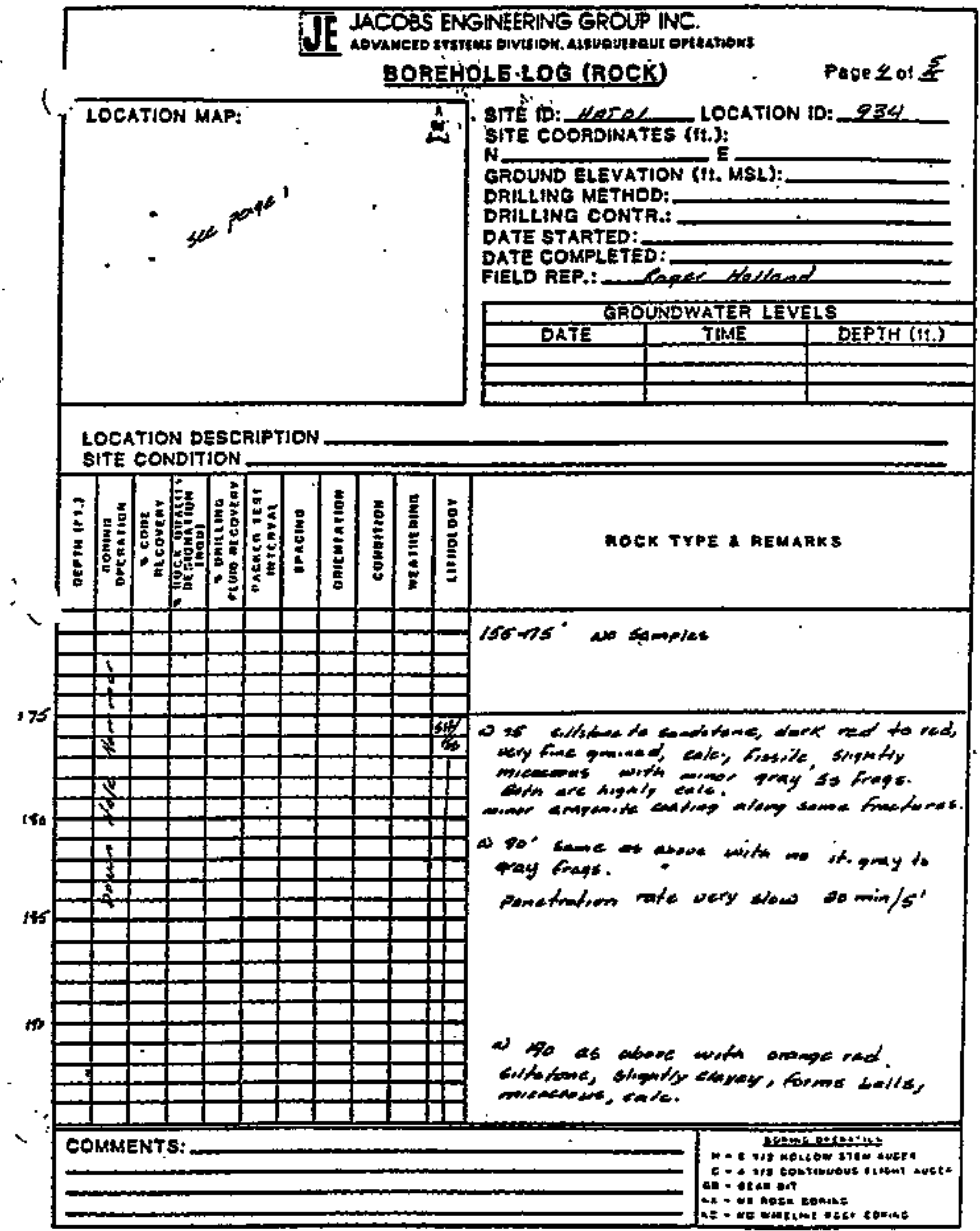

JES-AL-ENO-2A (4/SS) 


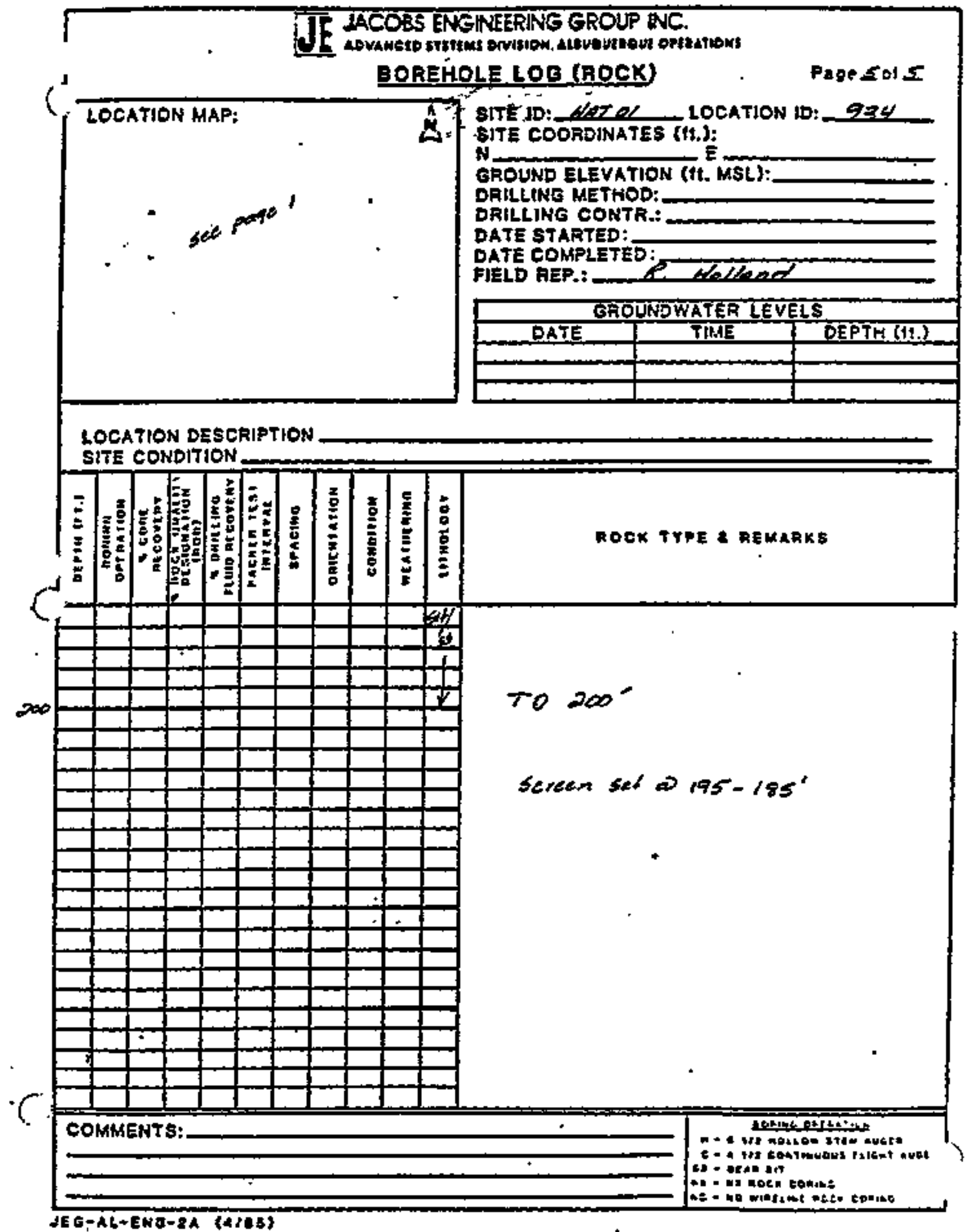


. . TE JACOBS ENGINEERING GROUP INC.

BOREHOLE LOG (ROCK)

Pape $\angle$ or $\mathcal{B}$

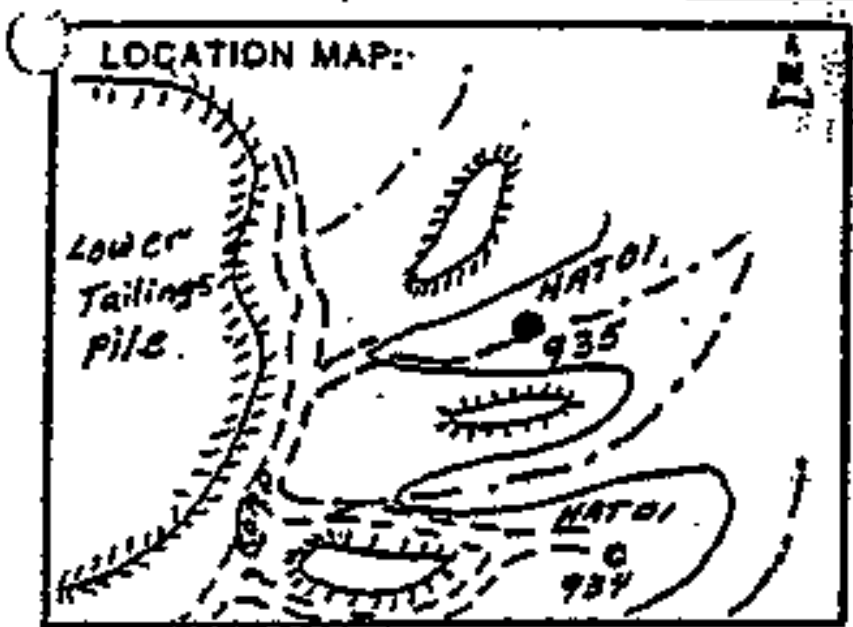
SIIE TD: HATOL
LOCATION ID:-45:
SITE COORDtMATES (11.)
N SR
GROUND ELEVATION (4t. MSL):UK
DRILLING METHOD:

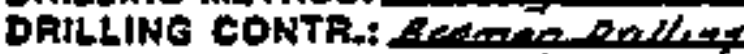
DATE STARTED: LOLPLT:
OATE COMPLETED: MleQRES
FIELD REP.: R Allinel

\begin{tabular}{|c|c|c|}
\hline \multicolumn{3}{|c|}{ GROUNDWATER LEVELS } \\
\hline SIME & DEPTH (tt.) \\
\hline & & \\
\hline & & \\
\hline
\end{tabular}

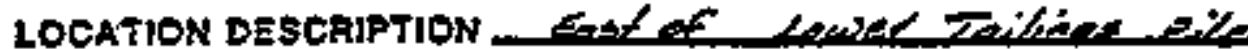

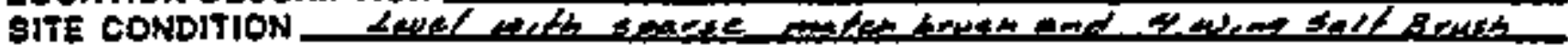

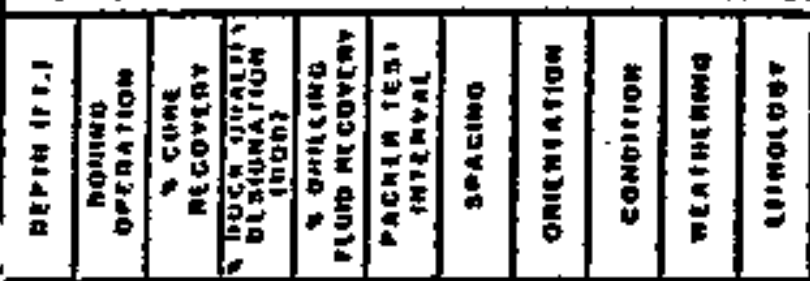

RDER TYOE 2 REMARKS

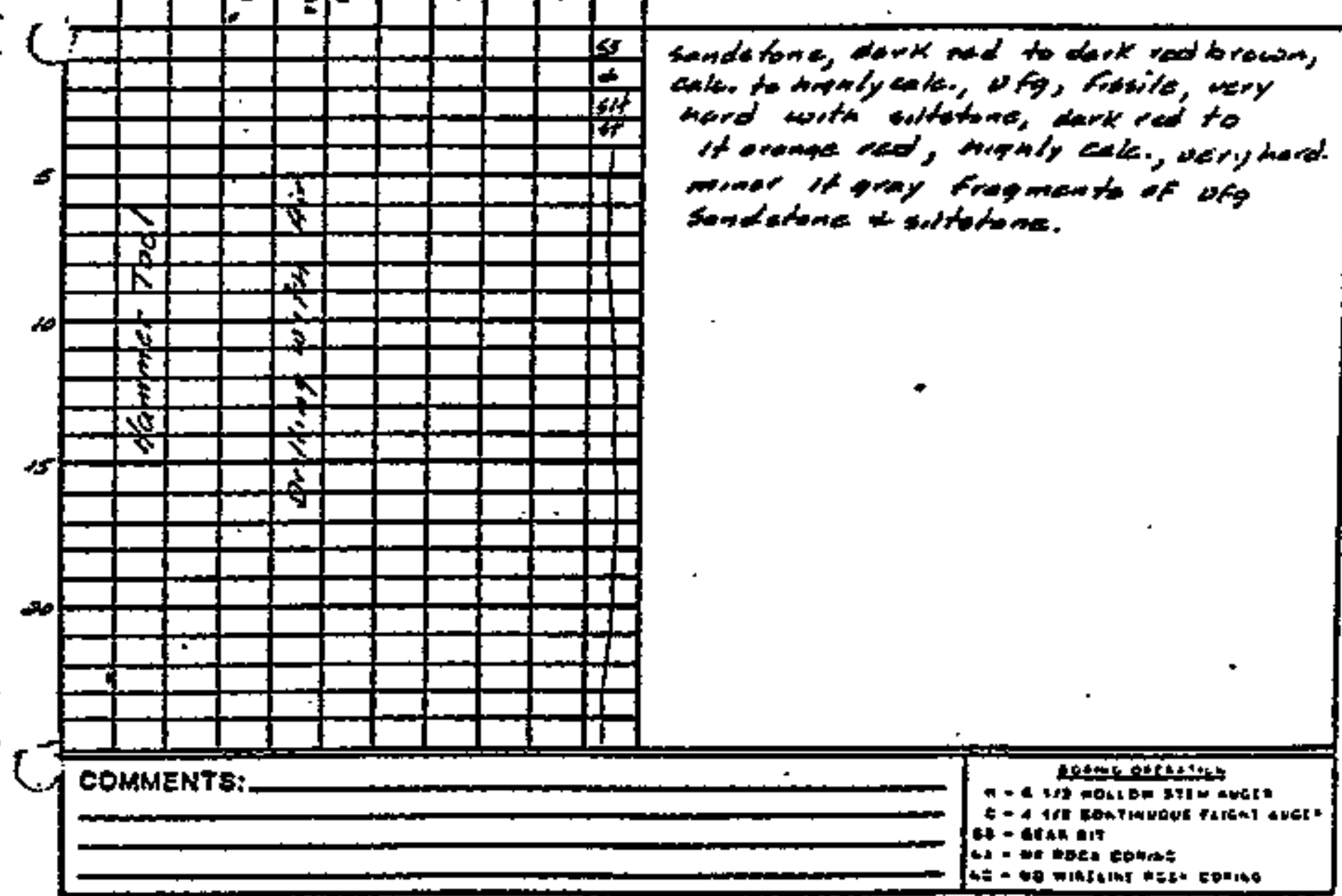

SEO-AL-ENO-3A (St:\$) 


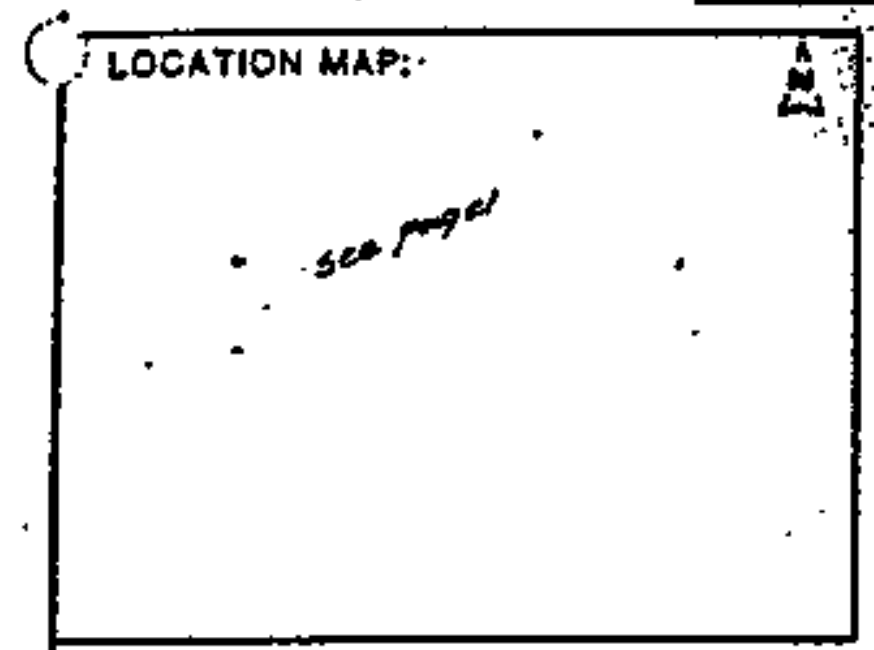

LOCATION ID: 9S5

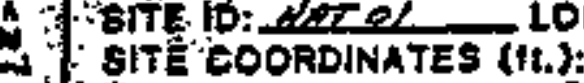

A

E

GROUND ELEVATION (II. MSL):

DRILLING METHOD:

DRILLING CONTR.:

DATE STARTED:

DATE COMPLETED:

FIELD AEP.:

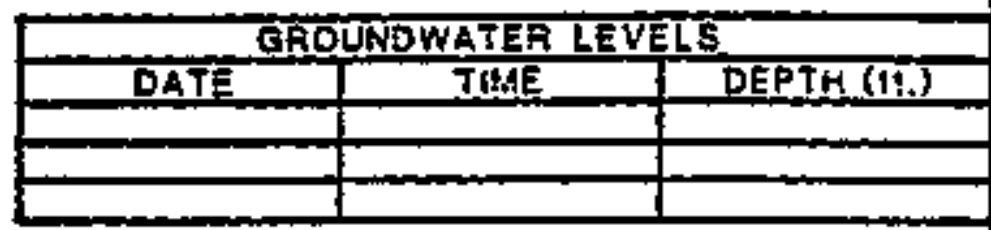

LOCATION DESCAIPTION

SITE CONDITION

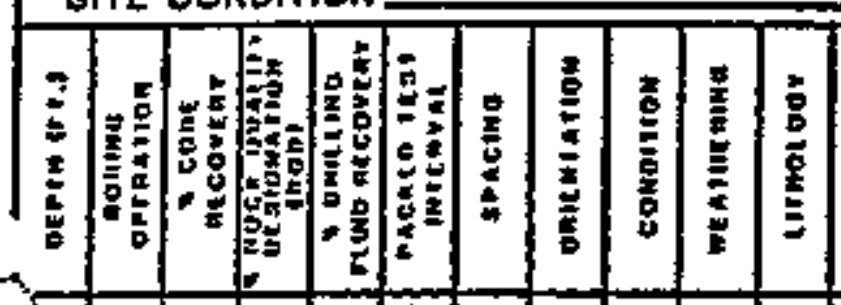

ROEK TYPE $\boldsymbol{B}$ REMARKS

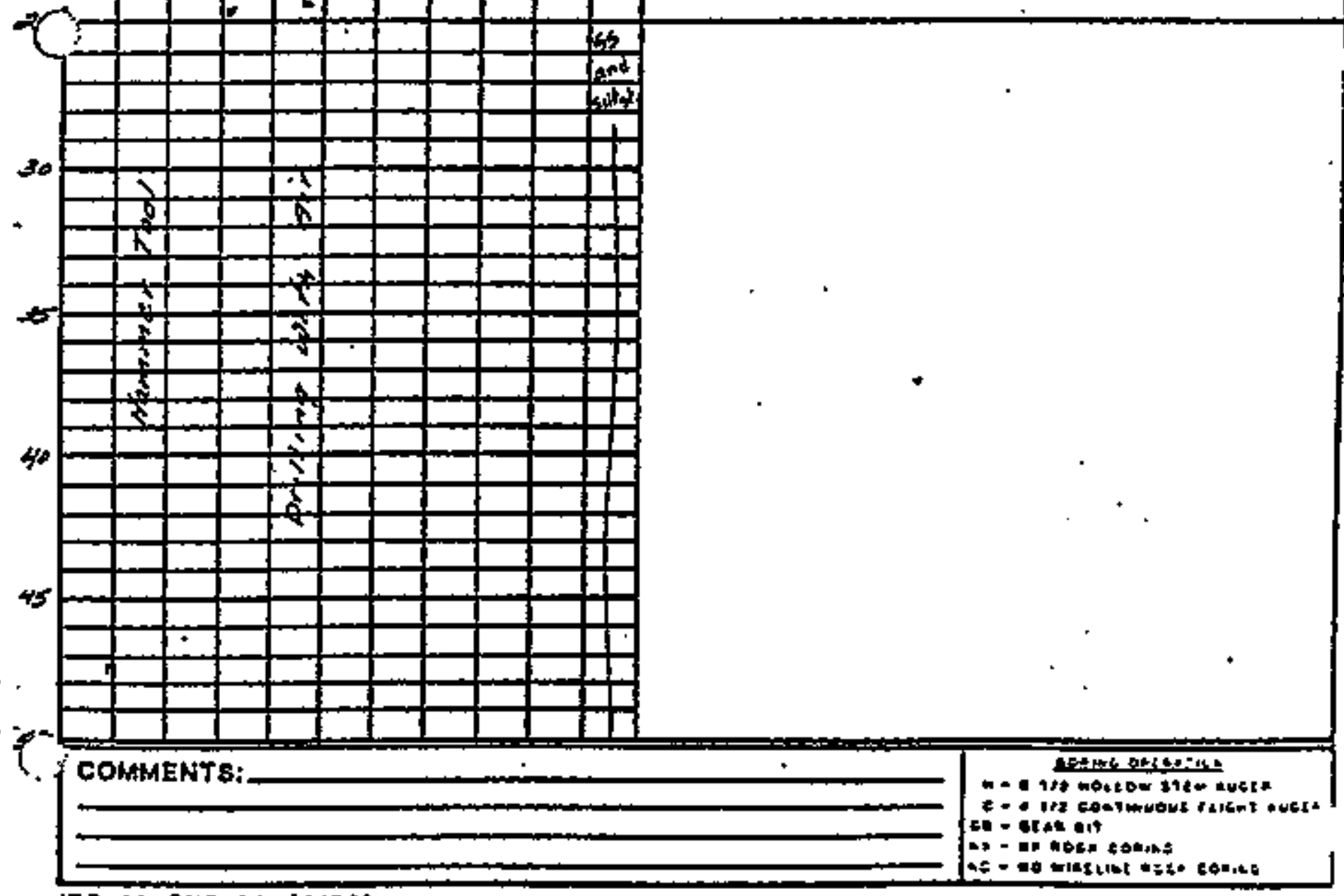

JEG-AL-FWO-2A (4/A) 

$\sqrt{\text { JE IACOBS ENKSNEERING GROUP INC. }}$

BORE⿱⺌⺝LLE LOG (ROCK)

Page $A$ or $\&$

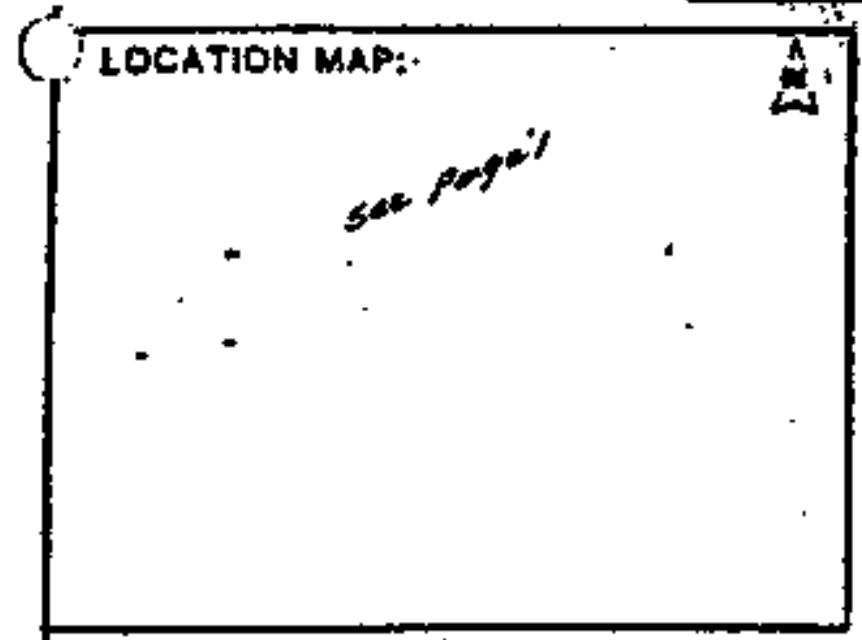

EITE ID: vetes

LOCATION ID: 25s

STTE COORDINATES (i1.):

GROUND ELEVATION (It. MSL)

DRILLING NETHOD:

DRILLING CONTR.:

DATE STARTED:

DATE COMPLETED:

FIELD REP.: A whllad

\begin{tabular}{|l|l|l|}
\hline \multicolumn{3}{|c|}{ GEOUNDWATER LEVELS } \\
\hline DATE & IIISE & DEPTH (II) \\
\hline & & \\
\hline
\end{tabular}

LOCATION DESCRIPTION

SITE CONDITION

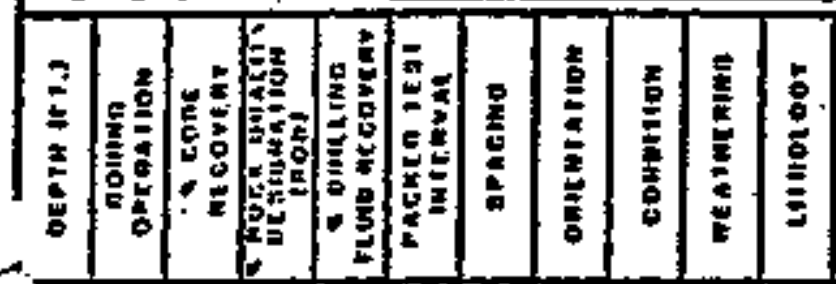

ROCK TYPE I REMAAKS

$\because$

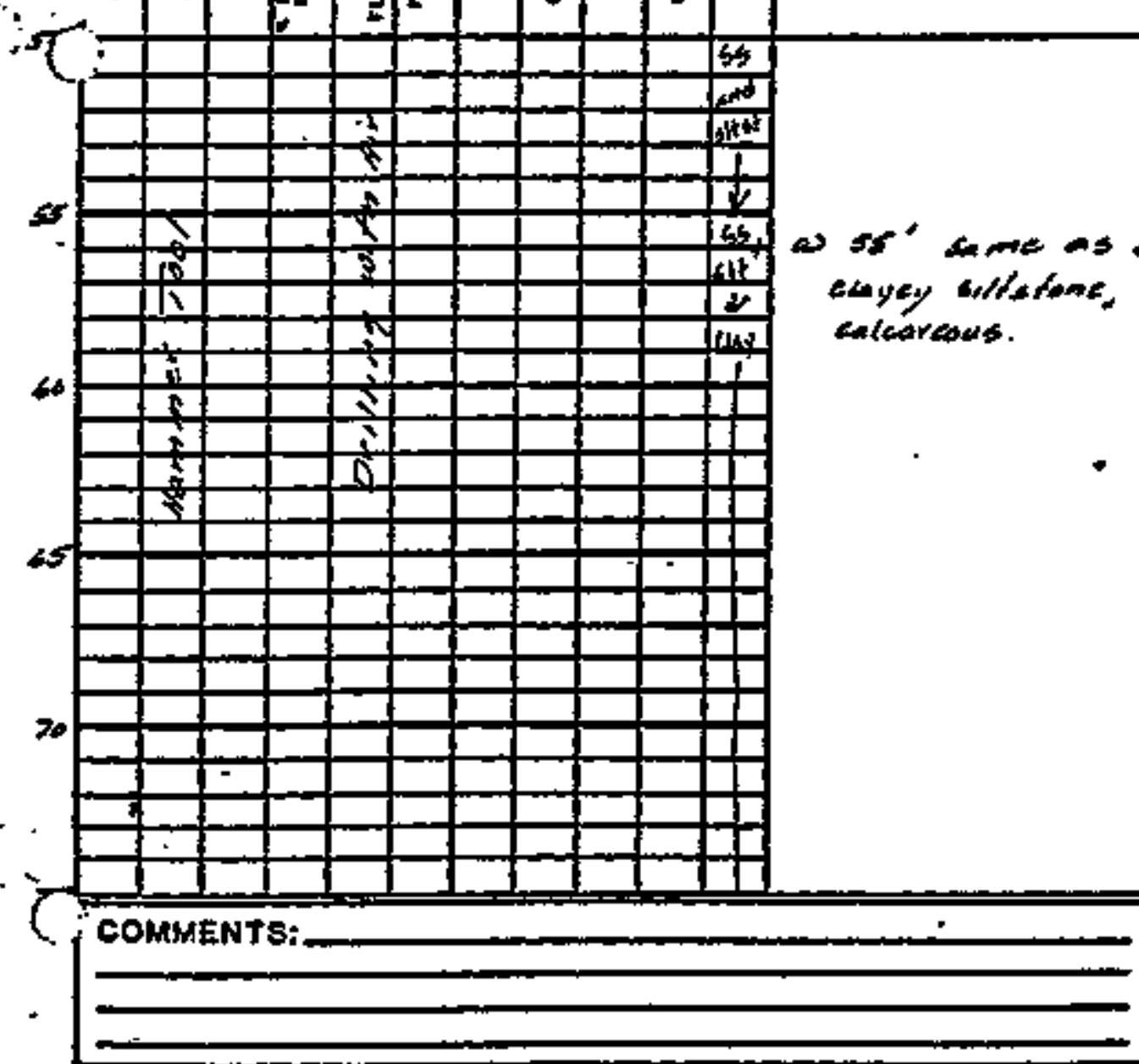

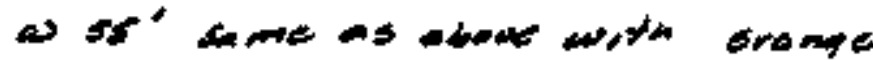
exyey wltetone, soft, formt belle.,

JEG-AL-ENA-2A (AKB)

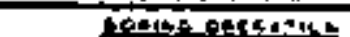

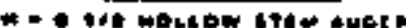

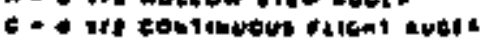

A* - arat in

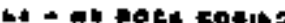

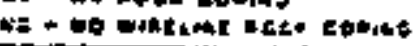




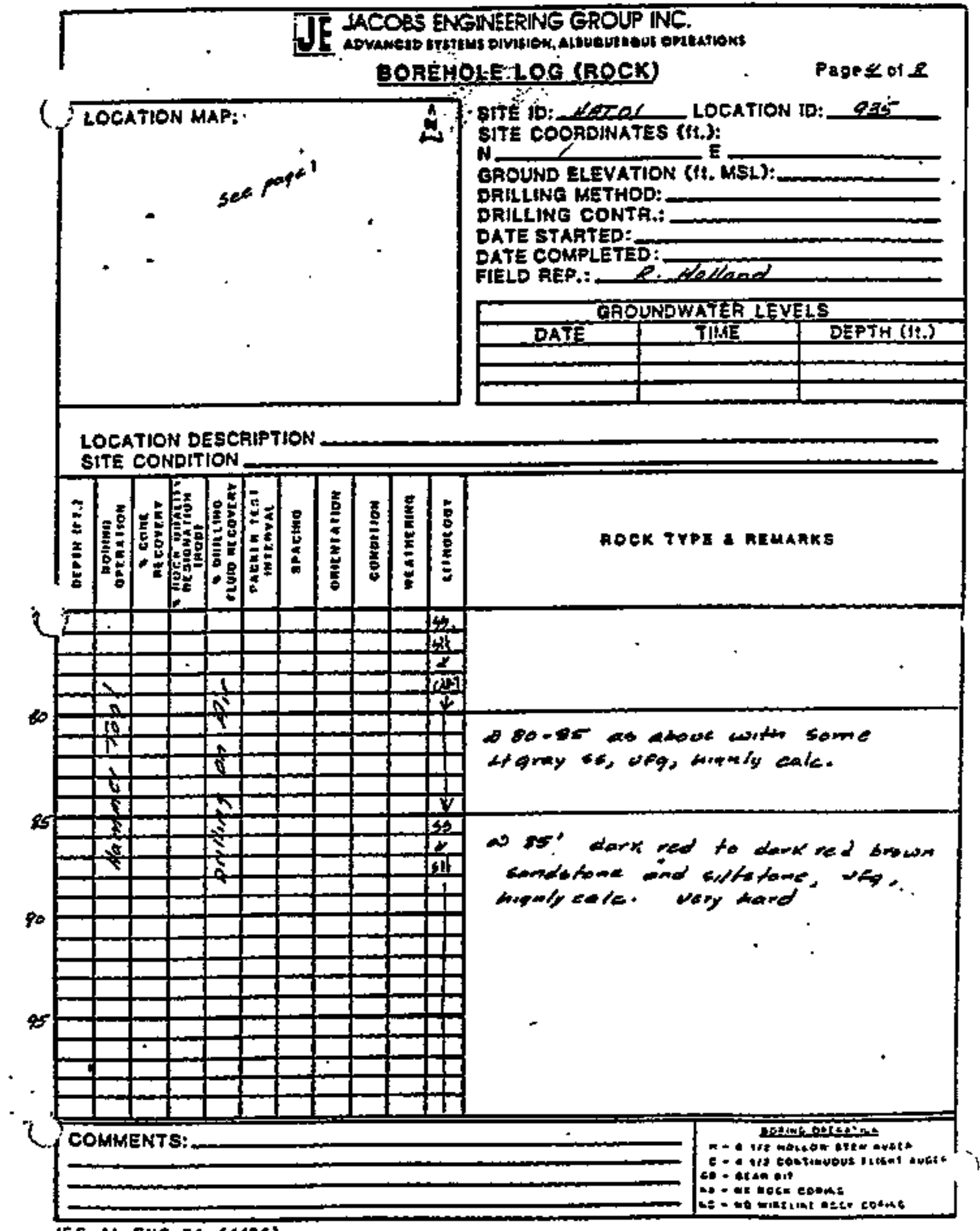




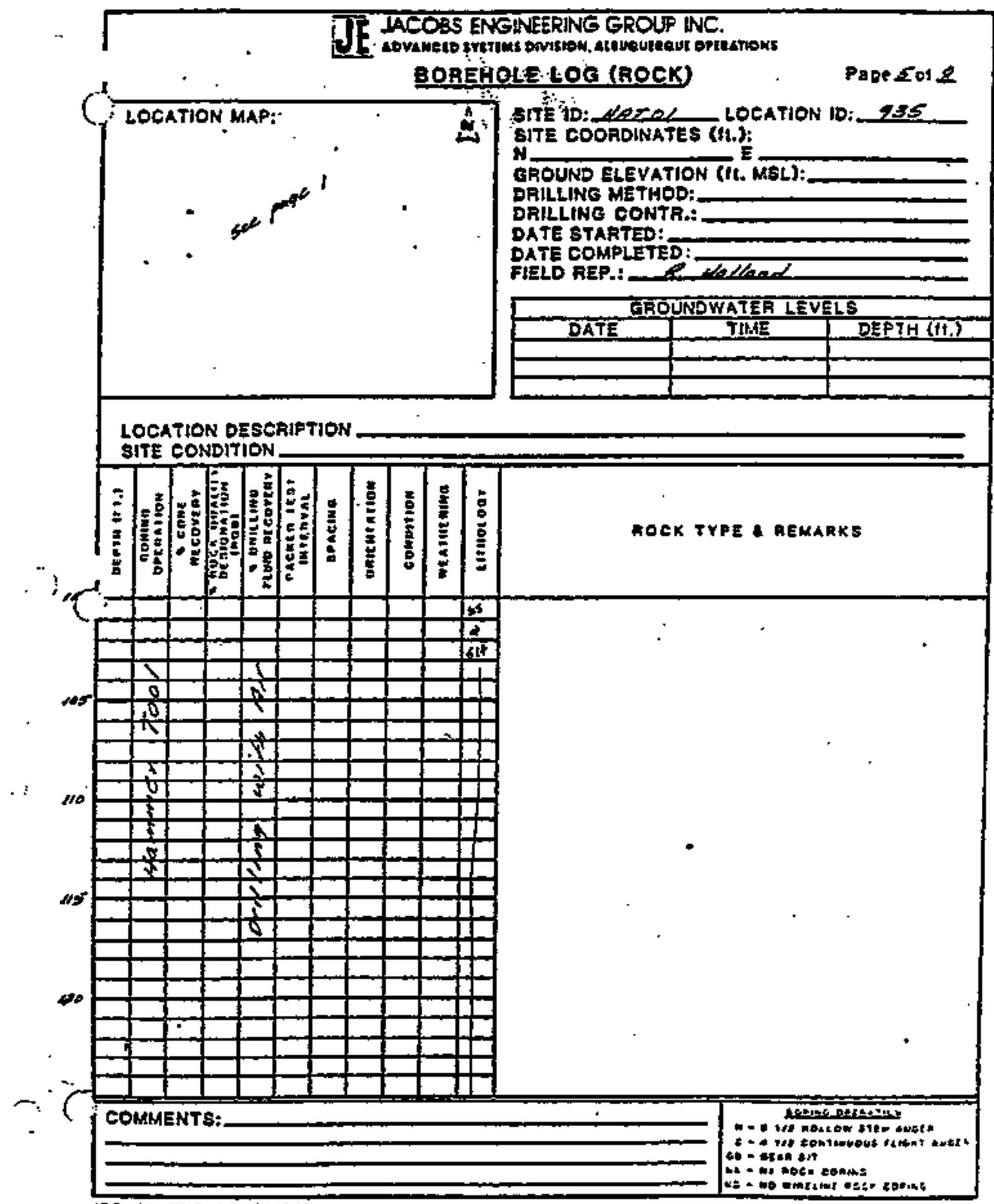




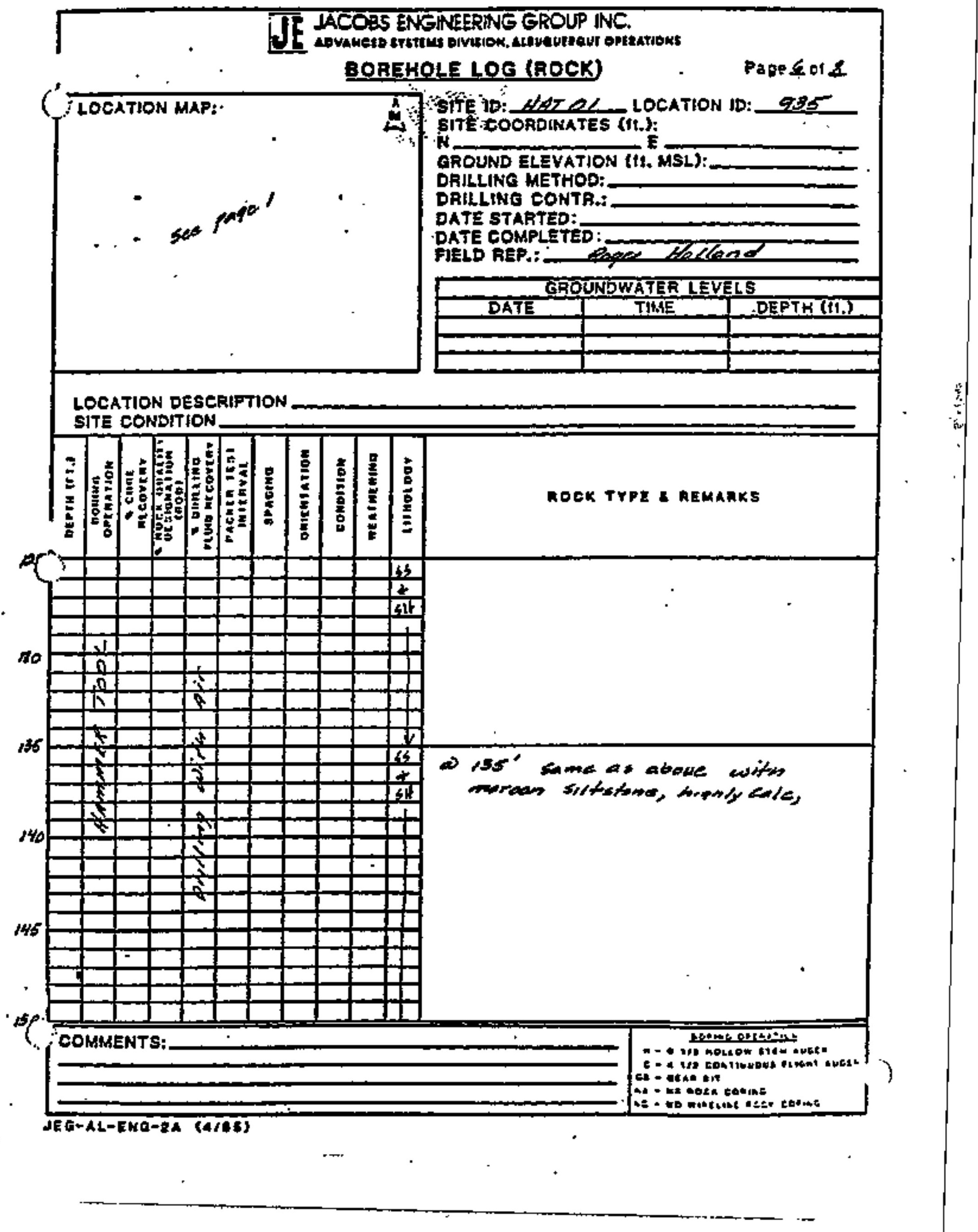




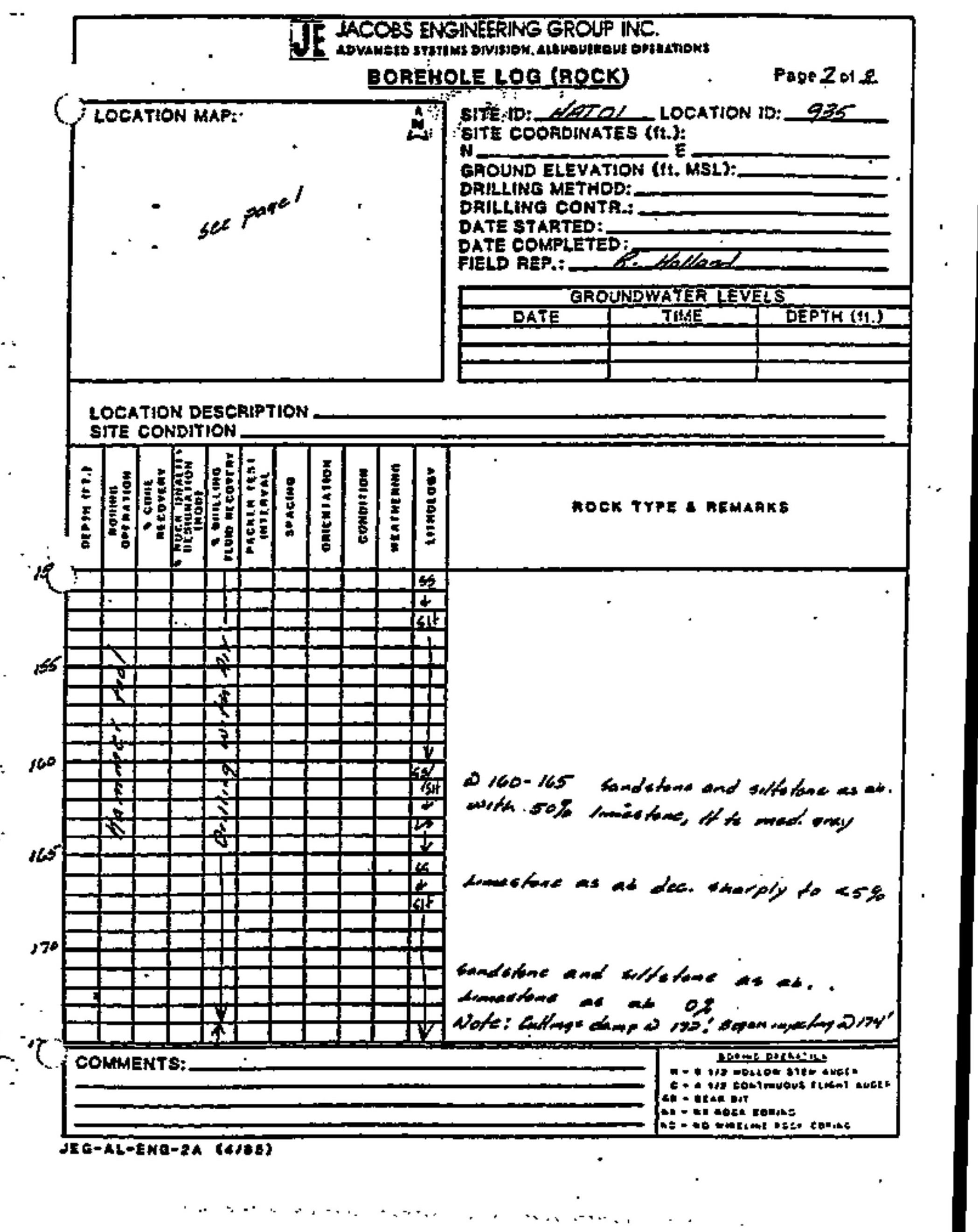




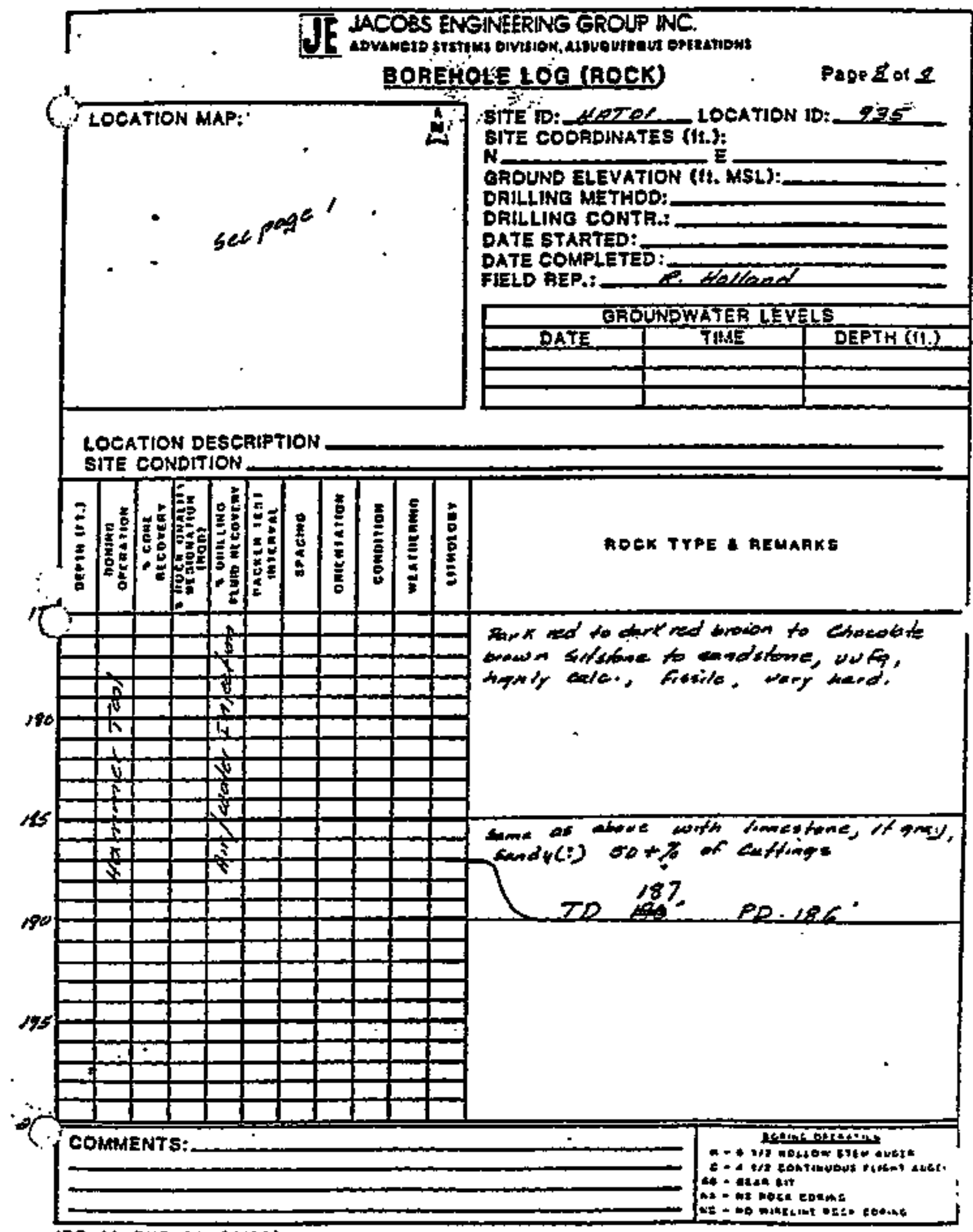




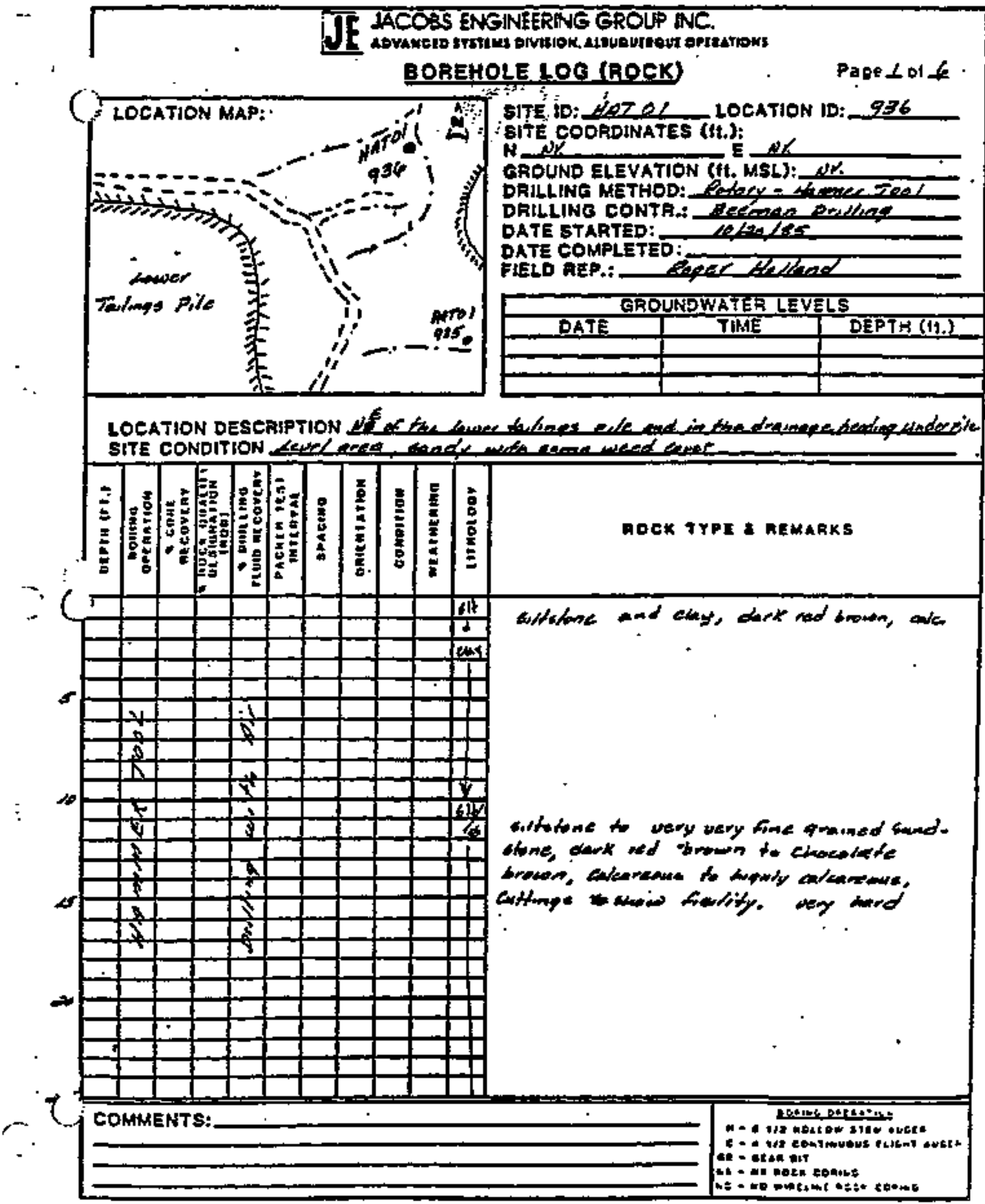

JETFALENE-2A (1/35) 


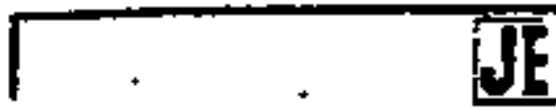
IACOBS ENGINEERING GROUP INC.

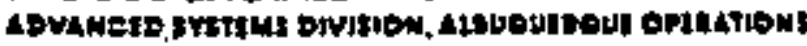

BOREFiOLE LOG (ROCX)

Page르 의 6

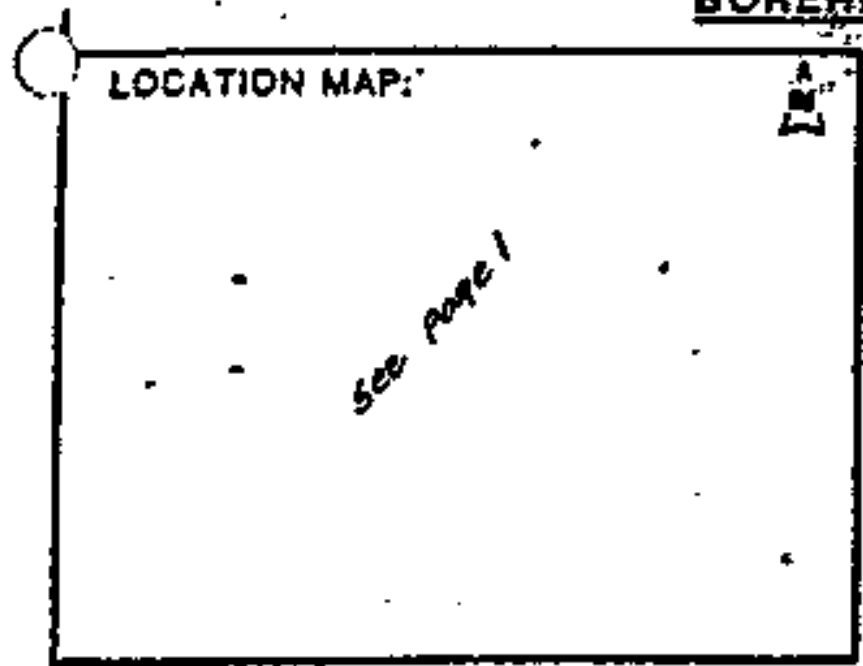 SITE ID:-COTOL LOCATION ID: 936 SITE CODRDINATES (tL):

N GAOUND ELEVATJON (ft. MSL):

DAJLLWE METHOD:

DAILLINO CONTR.:

DATE STAATED:

DATE COMPLETED

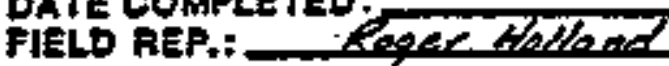

LOCATION DESCRIPTION

SITE CONDITION
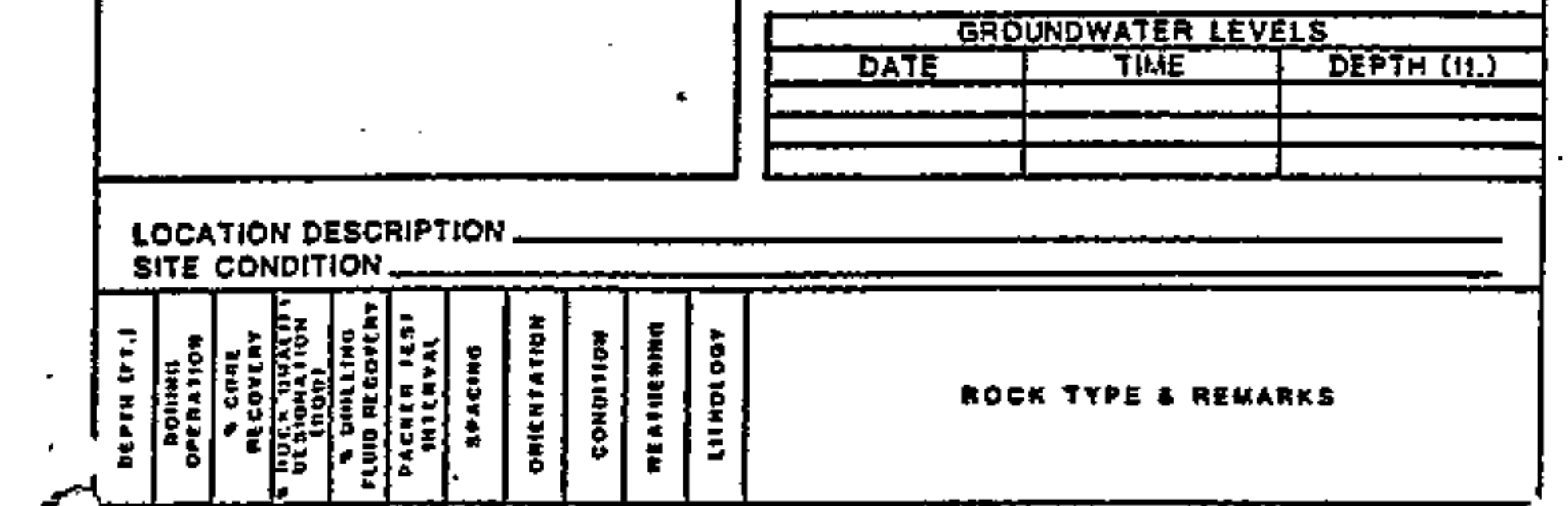

20

4

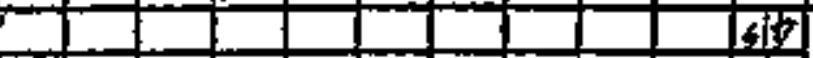

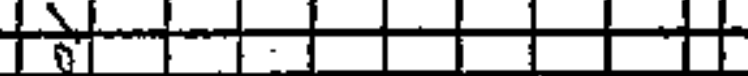

$+-1$

我

$\rightarrow$

$+2$

N 12

I

$x^{3}$

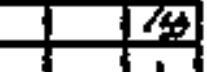

s

14

1

$+$

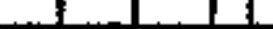

th

$+$

it

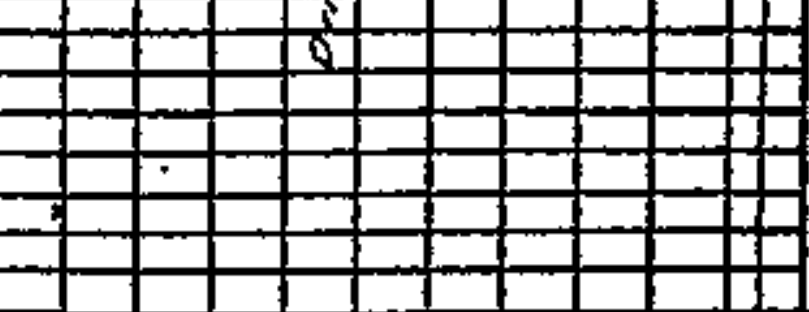

C

COMHENT \$:

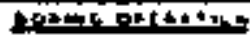

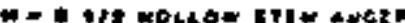

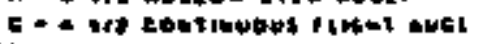

at - arth on?

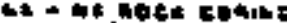

JEG-AL-ENG-2A (4/E)

F + to 


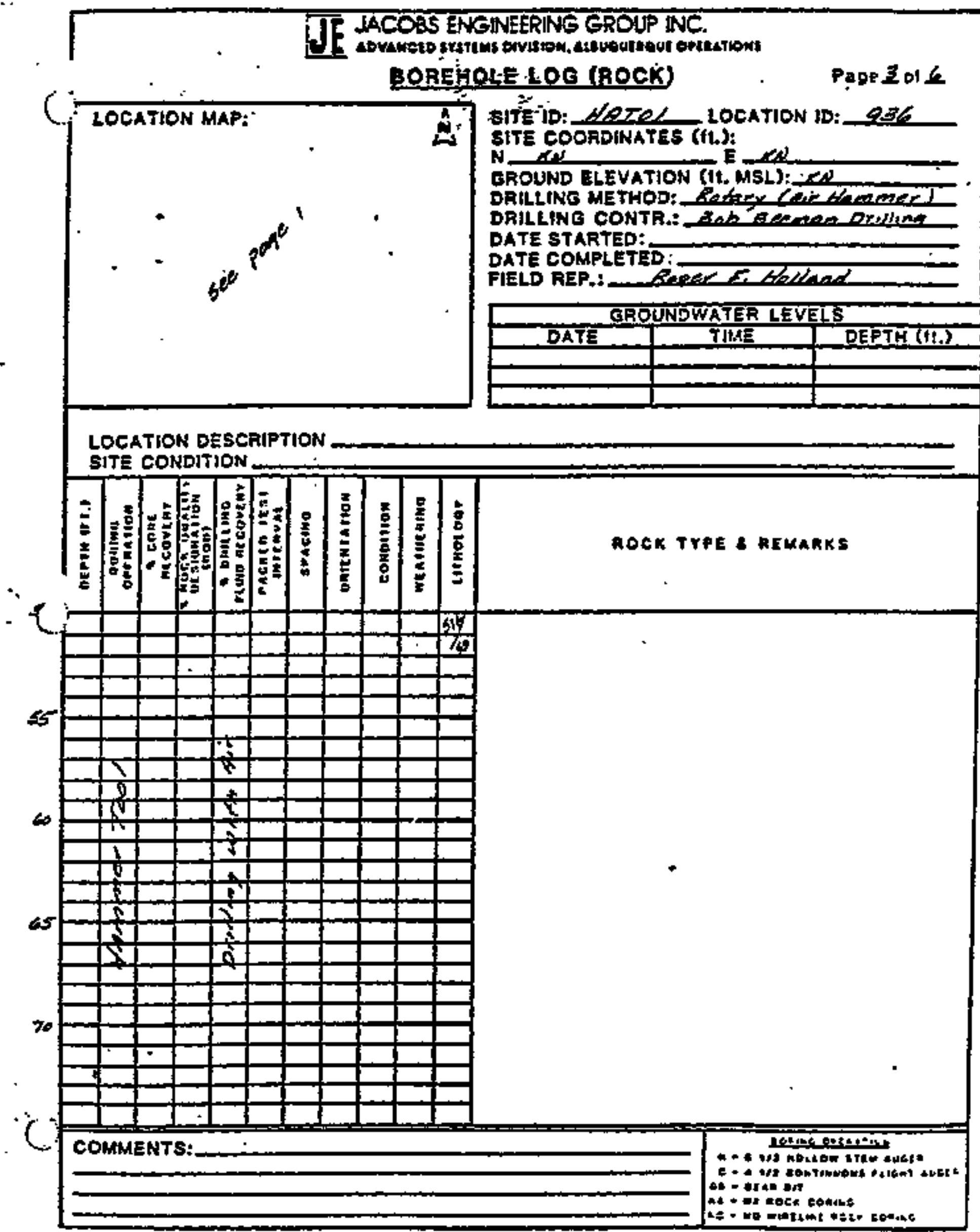

dEG-AL-EN(S-2A (4tas) 


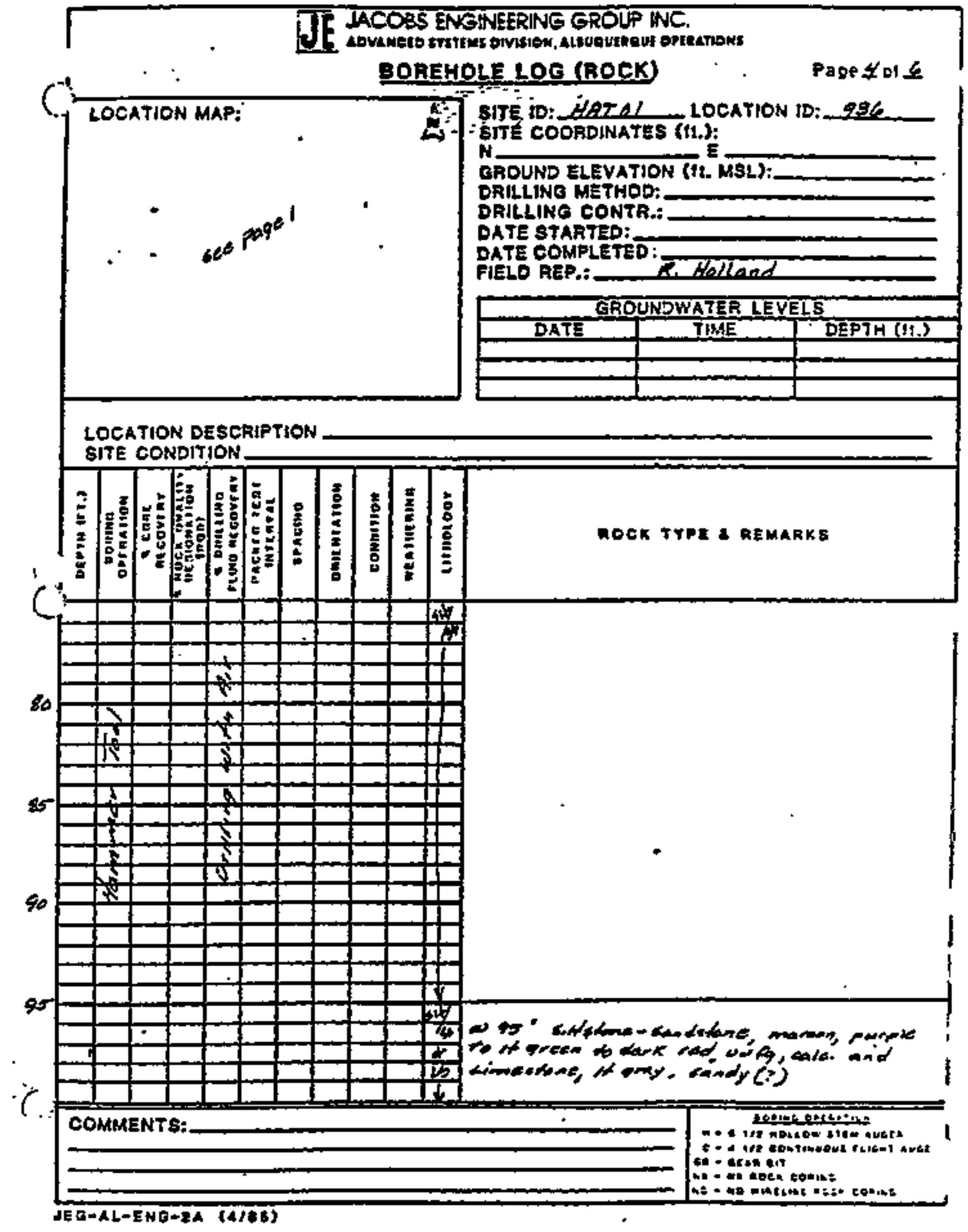


PROJECT Mexican Hat Tailies

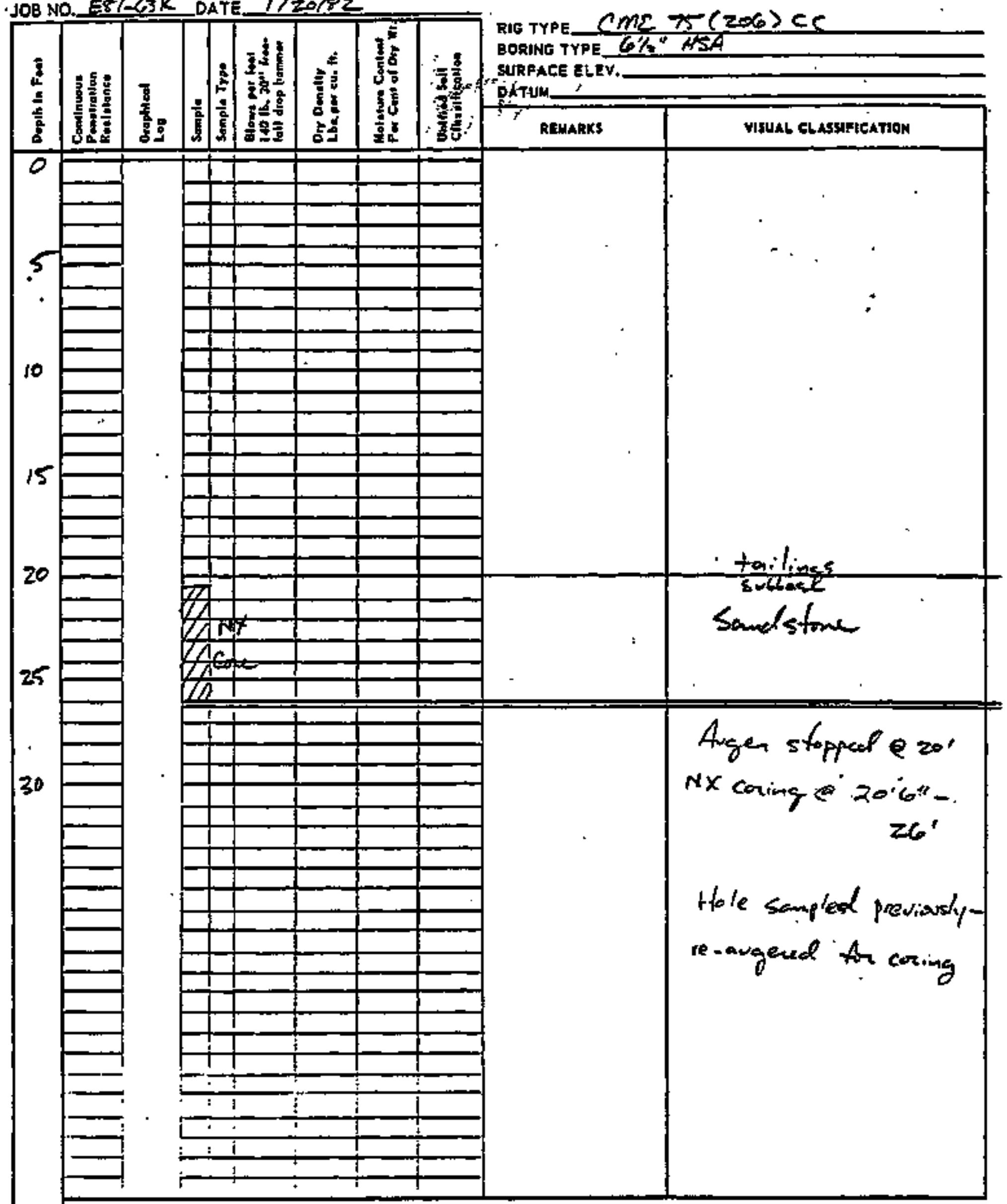

LOO OF TEST DORINO NO. 995

$75(2+6) c$
GROUND WATER

\begin{tabular}{|l|l|l|}
\hline DEPTH & HOUA & OATE \\
\hline WlONSE & & \\
\hline & & \\
\hline
\end{tabular}

\section{SAMPLE TYPE}

A - Auger euringl. $B$ - bleck semple

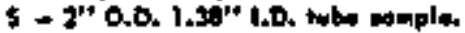

U - J" 0.0. 2.42" H.D. tube stomple.

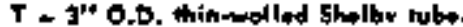


PROJECT Mexichw - the -

JOB NO.E\& -67K DATE $1 / 20 / 6 \mathrm{Z}$

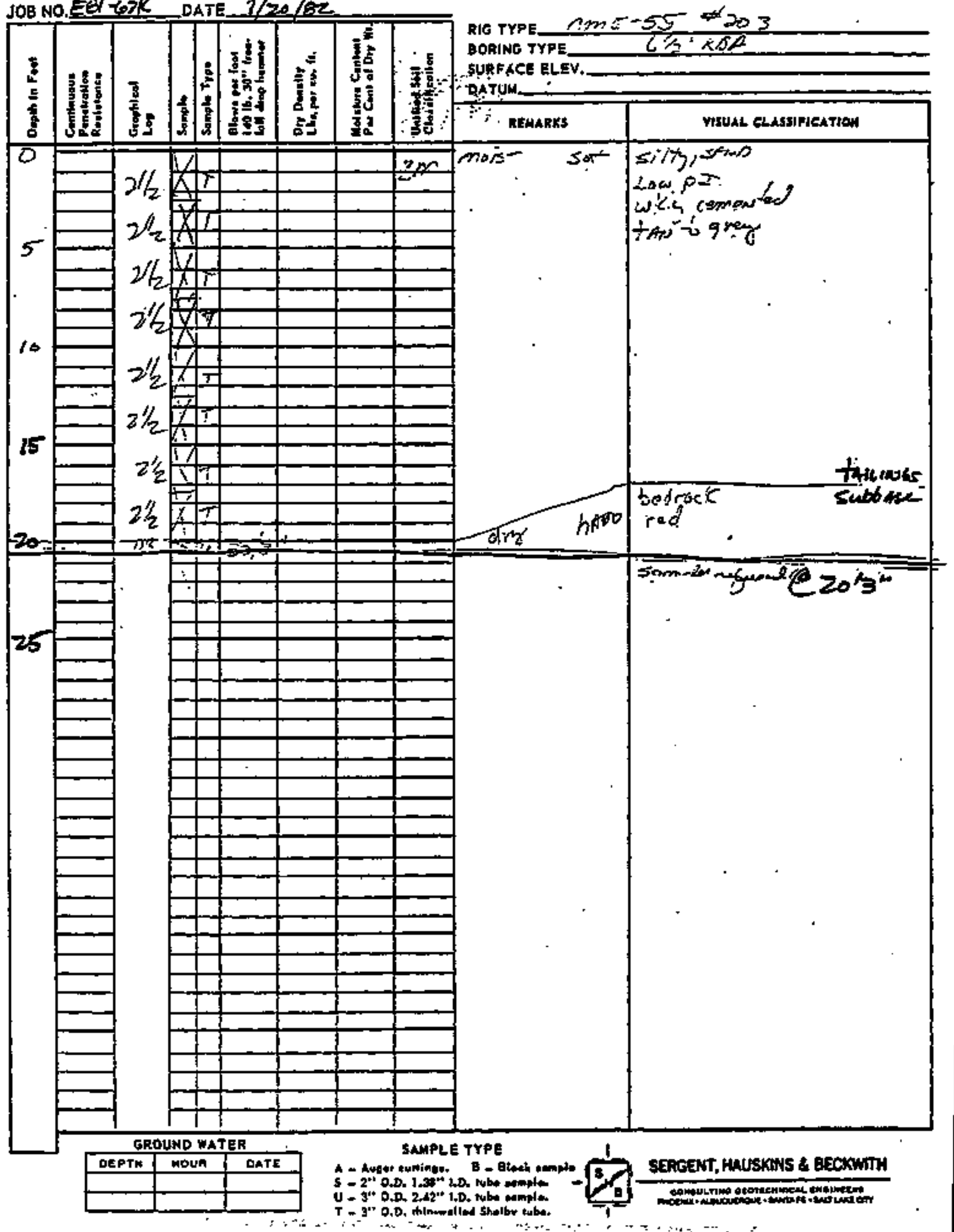


PROJECT Mexicm Hat Tailing: Lo' of test DORINe no.C94 JOB NO.EAT-63K DATE $1 / 20 / 8 Z$

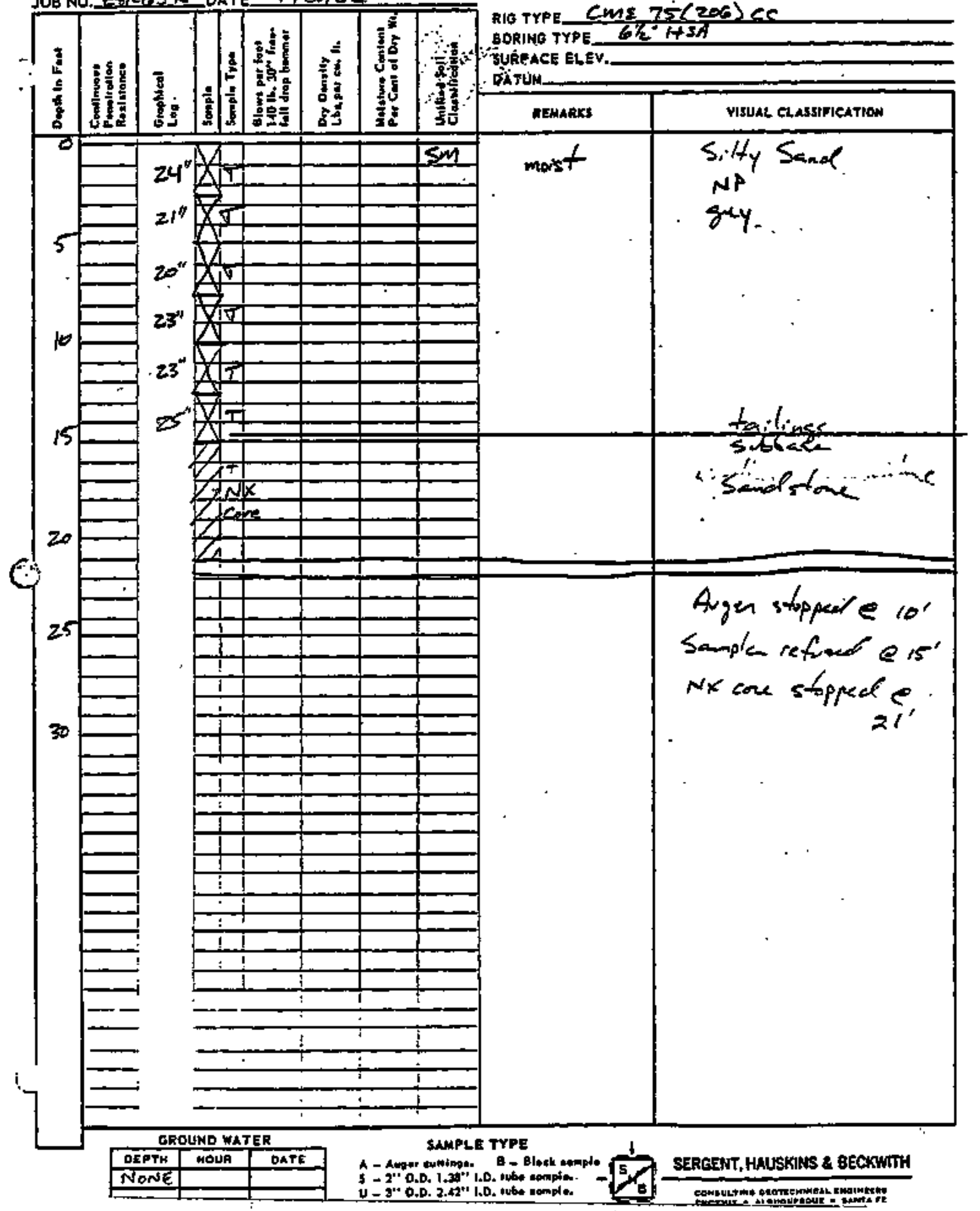


PRoJect Mexican Hat

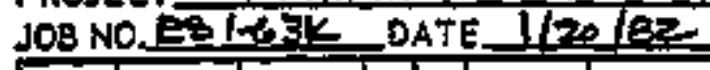

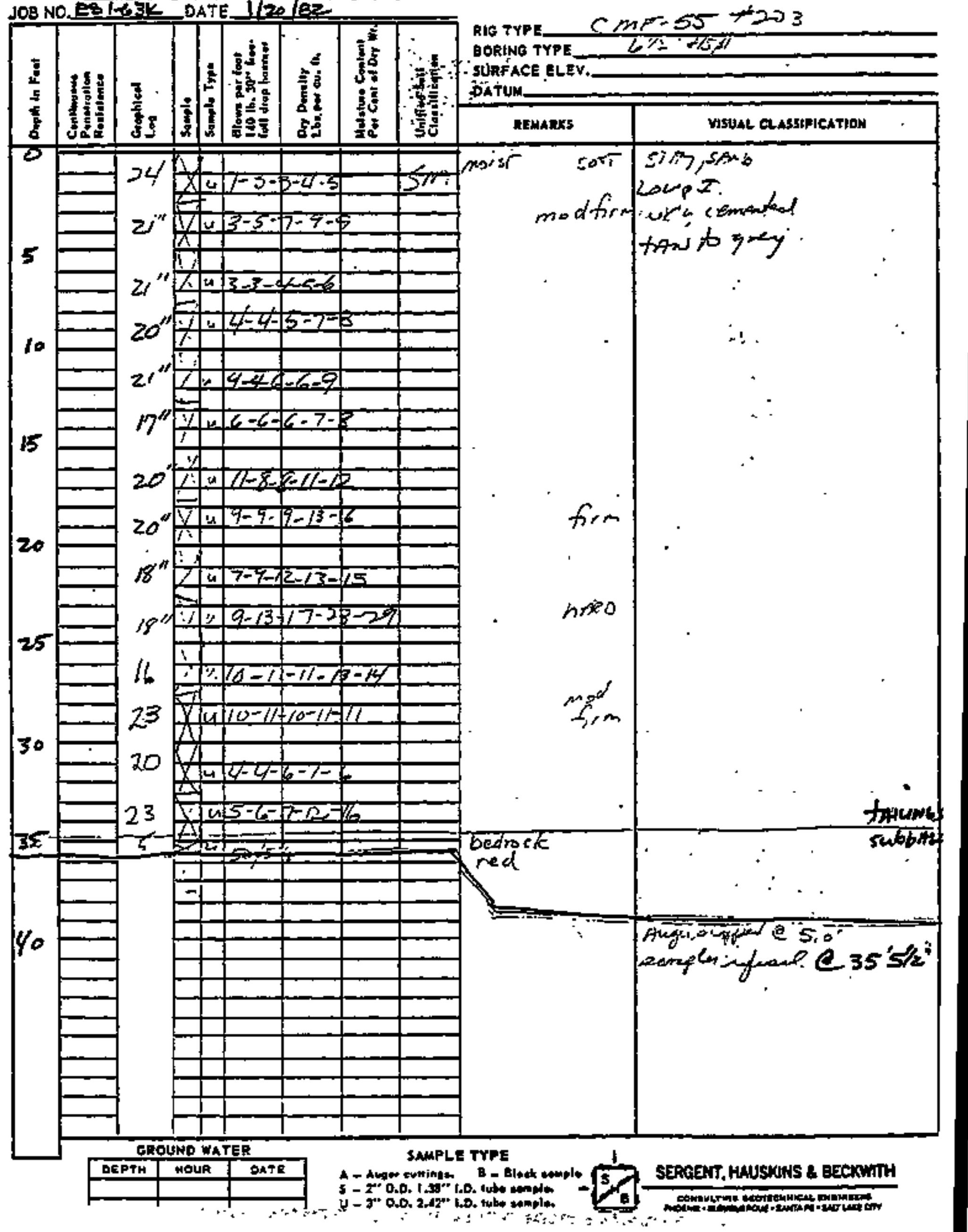


PROJECT MéXICAN: HoT:

LOe OF TEST BORINO NO.DQZ

JOB NO ERTAK DATE HIV/EZ

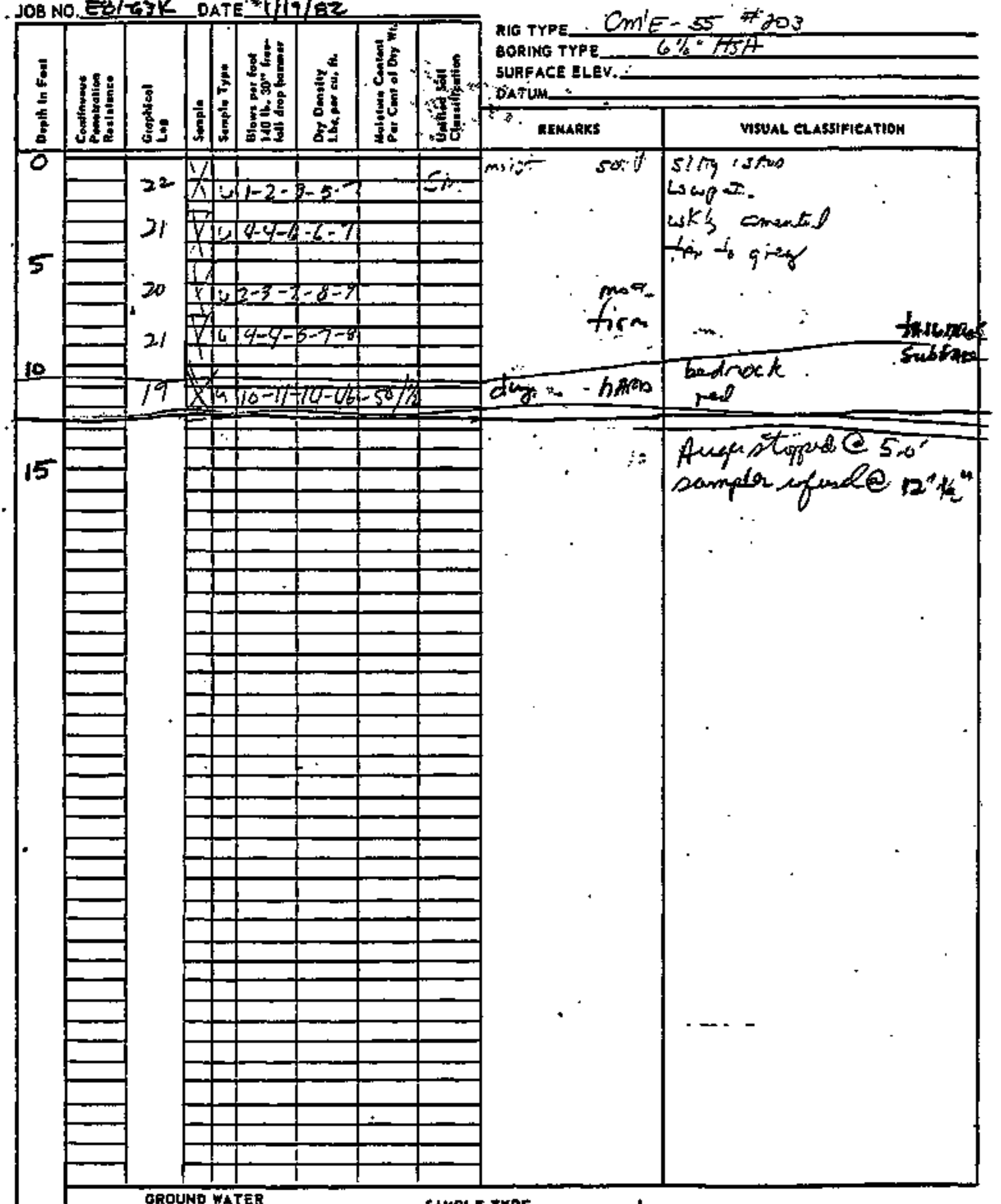

\section{\$AMPLE TYPE}

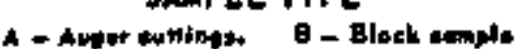

5 - 2'0.0. 1.30' 1.0. teb mole.

U - $7^{14}$ 0.0. 2.42'1 1.0. Nubs oninole.

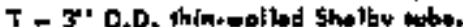

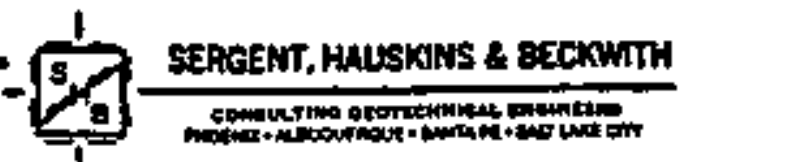


'FROJECT Nhexirin' thit

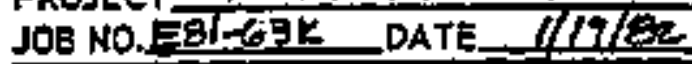

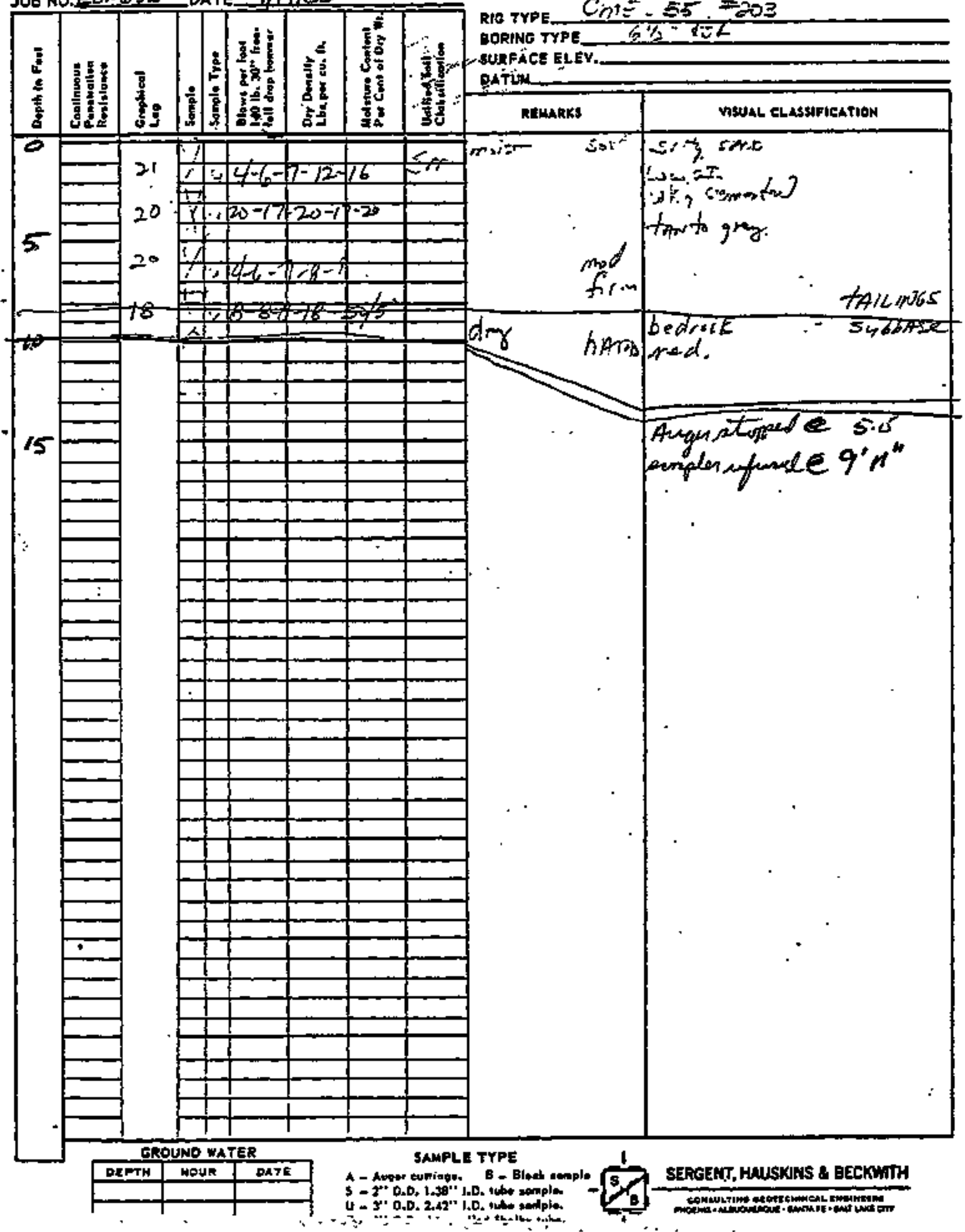




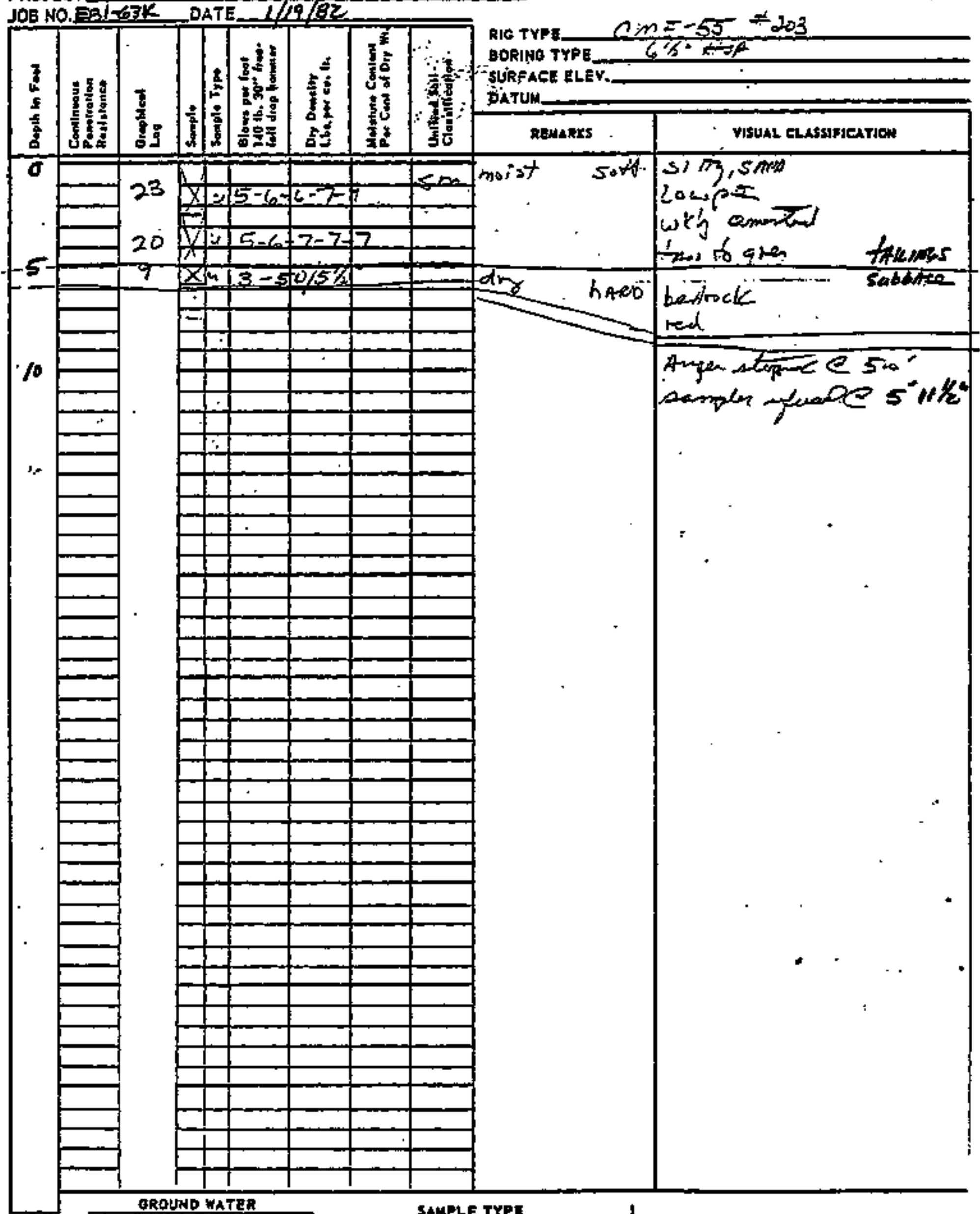

log of thet sokine no.c.90 
PROJECT If

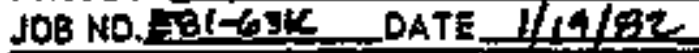

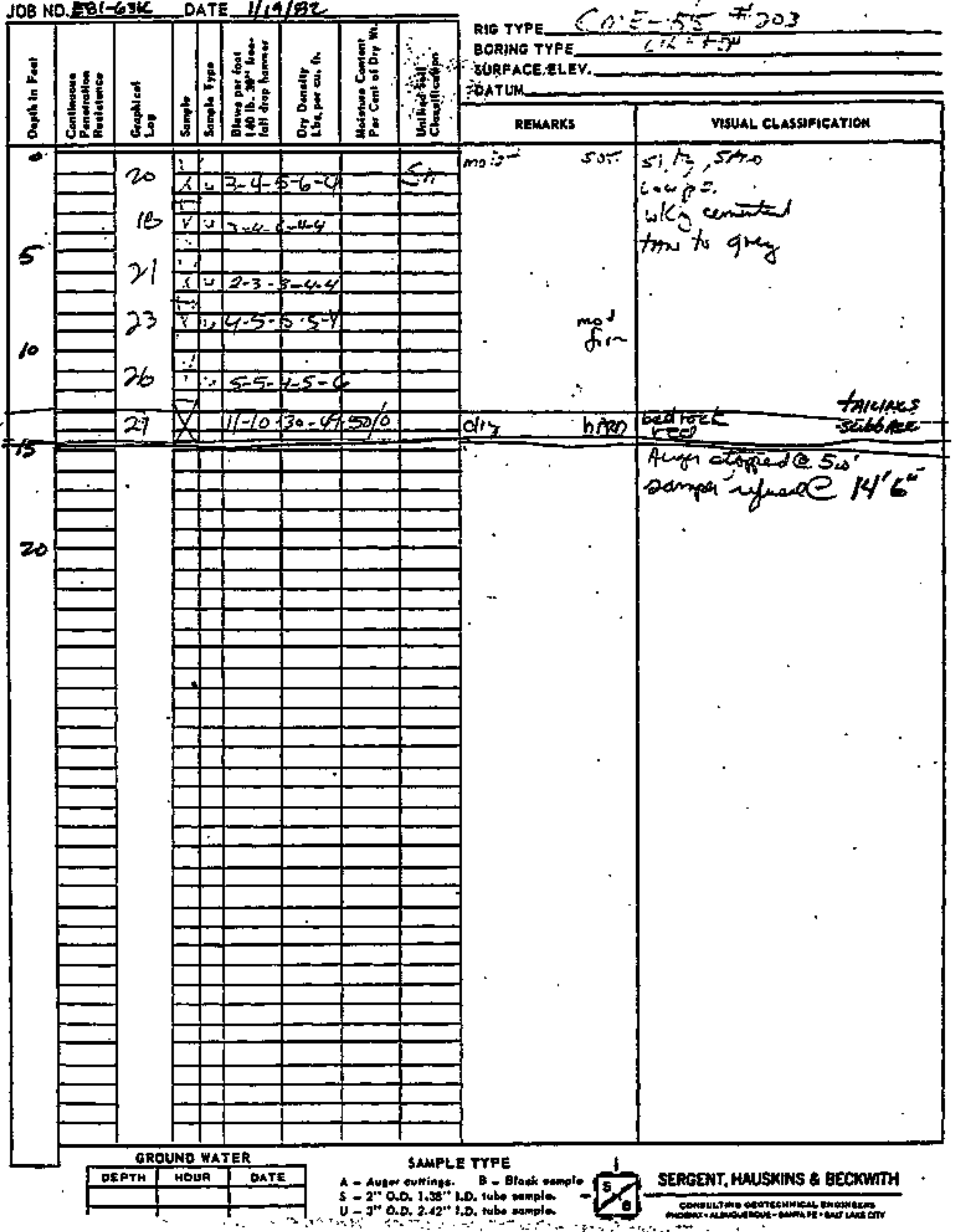

Lo of ratr nomine no. .39

CA.5-.5 क 303

BORJNG TYPE.

teteV.

REMARKS

VISULLL CLASSIFIEATION 
PROJECT OHOVIA., , :-FT

loe of tast nonine No.B88

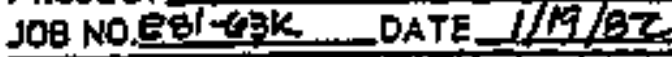

cms:- 55 सd

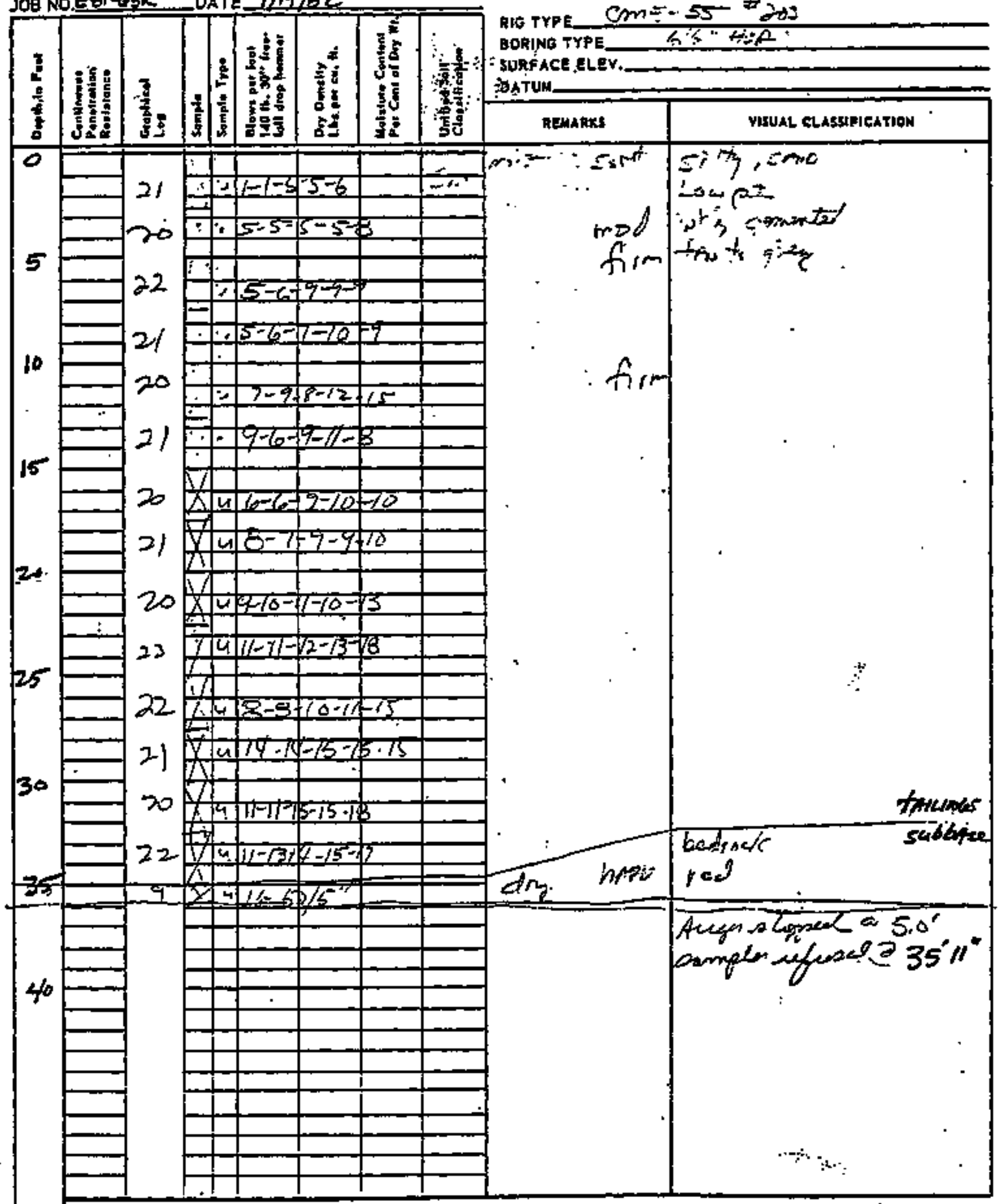




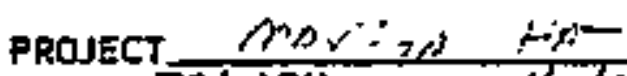

JOB NO EST-GiE DATE / / $/$ / $/ \mathrm{BZ}$

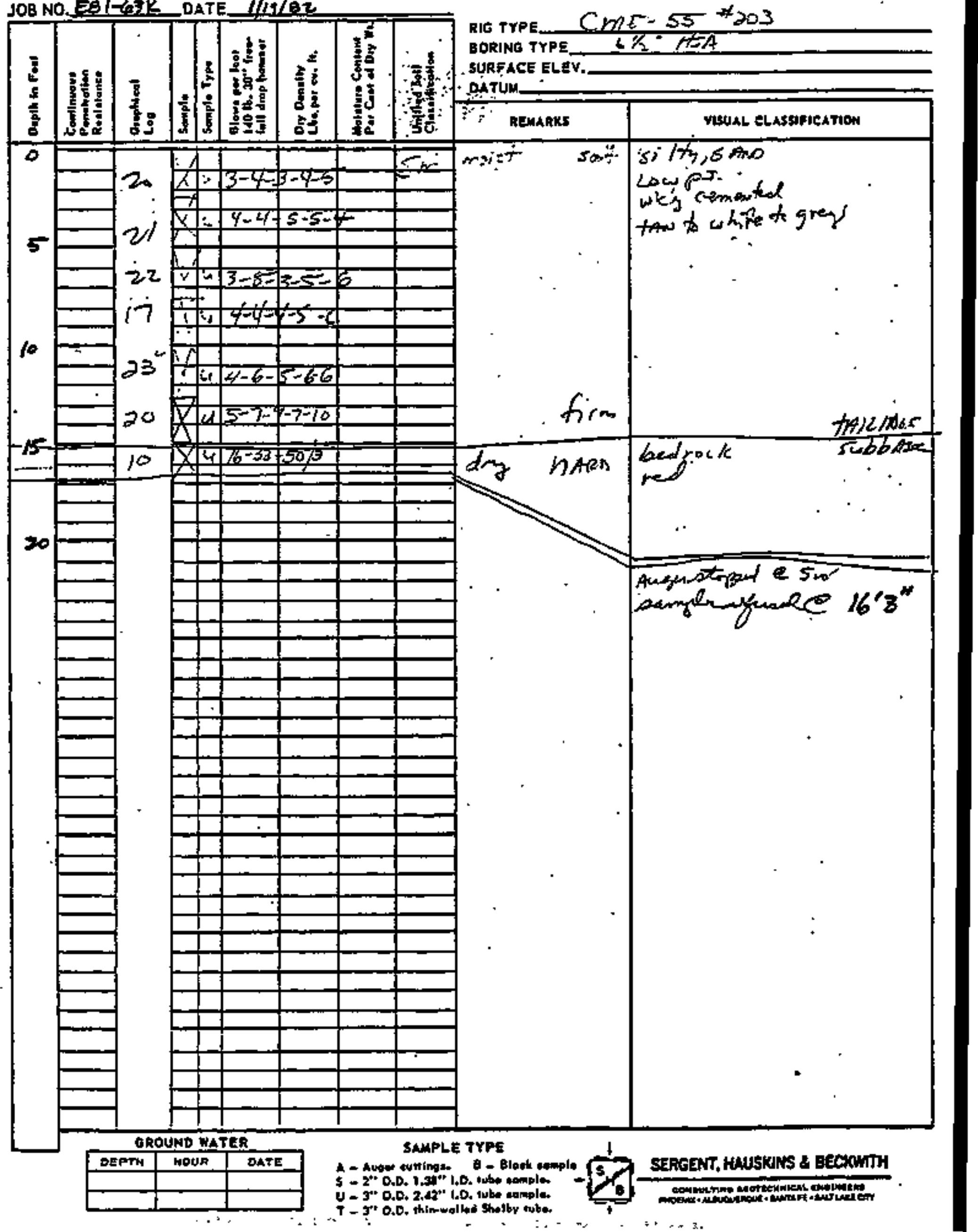


PROJECT MEXiCA) $\angle P A T$ Jos NO. B8T-63K DATE $1 / 20 / e z$

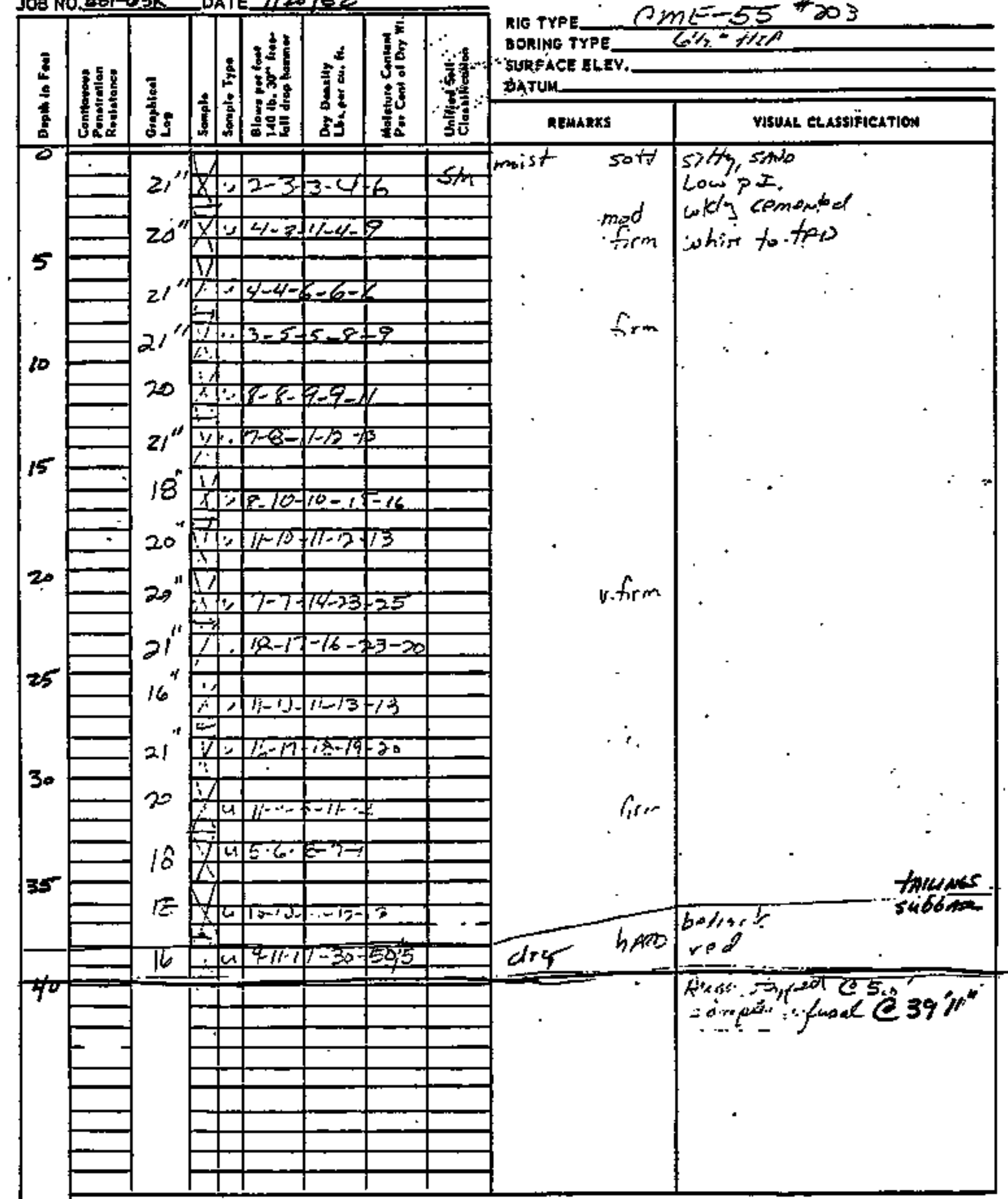




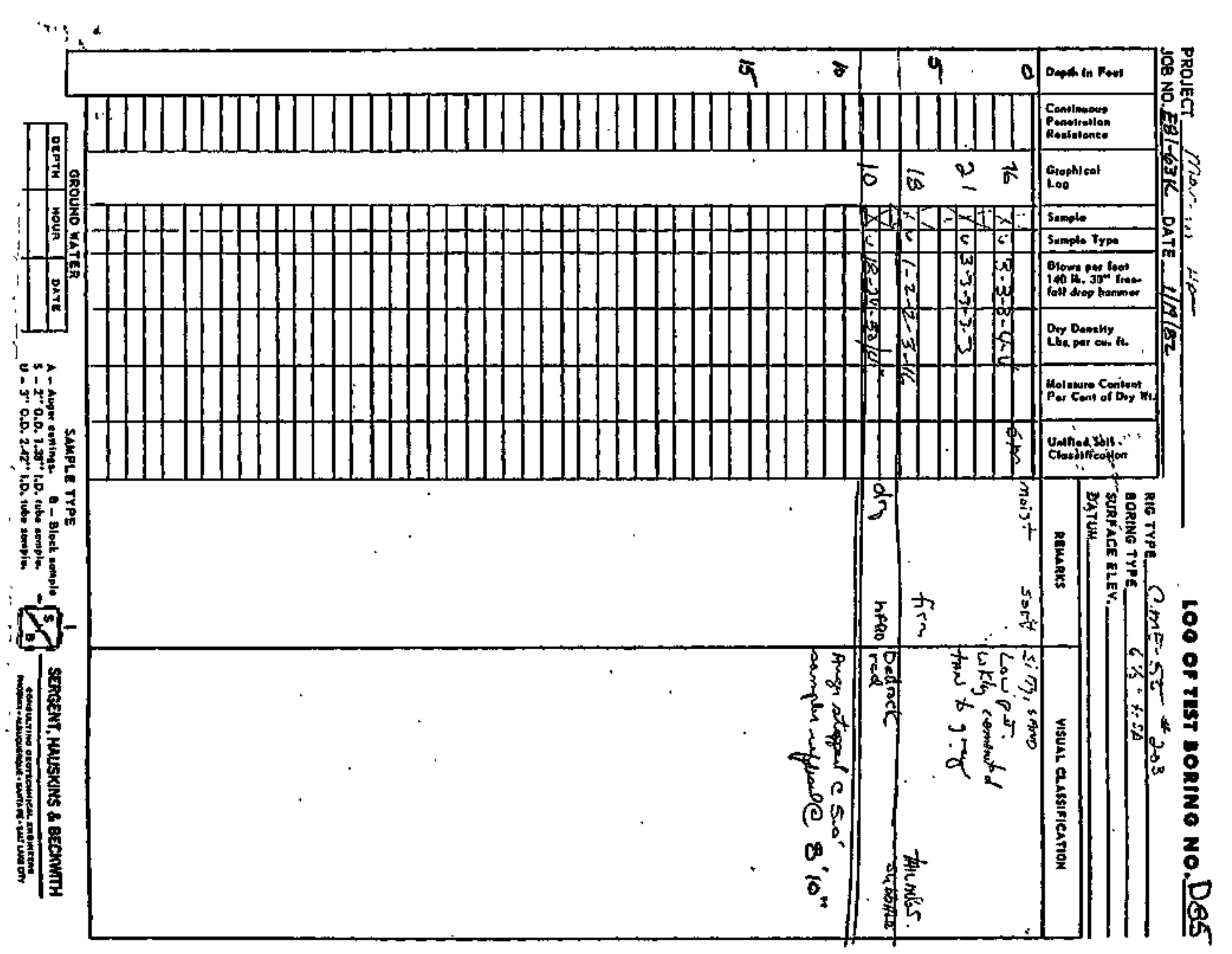


PROJECT MeXicen Hat Tailings

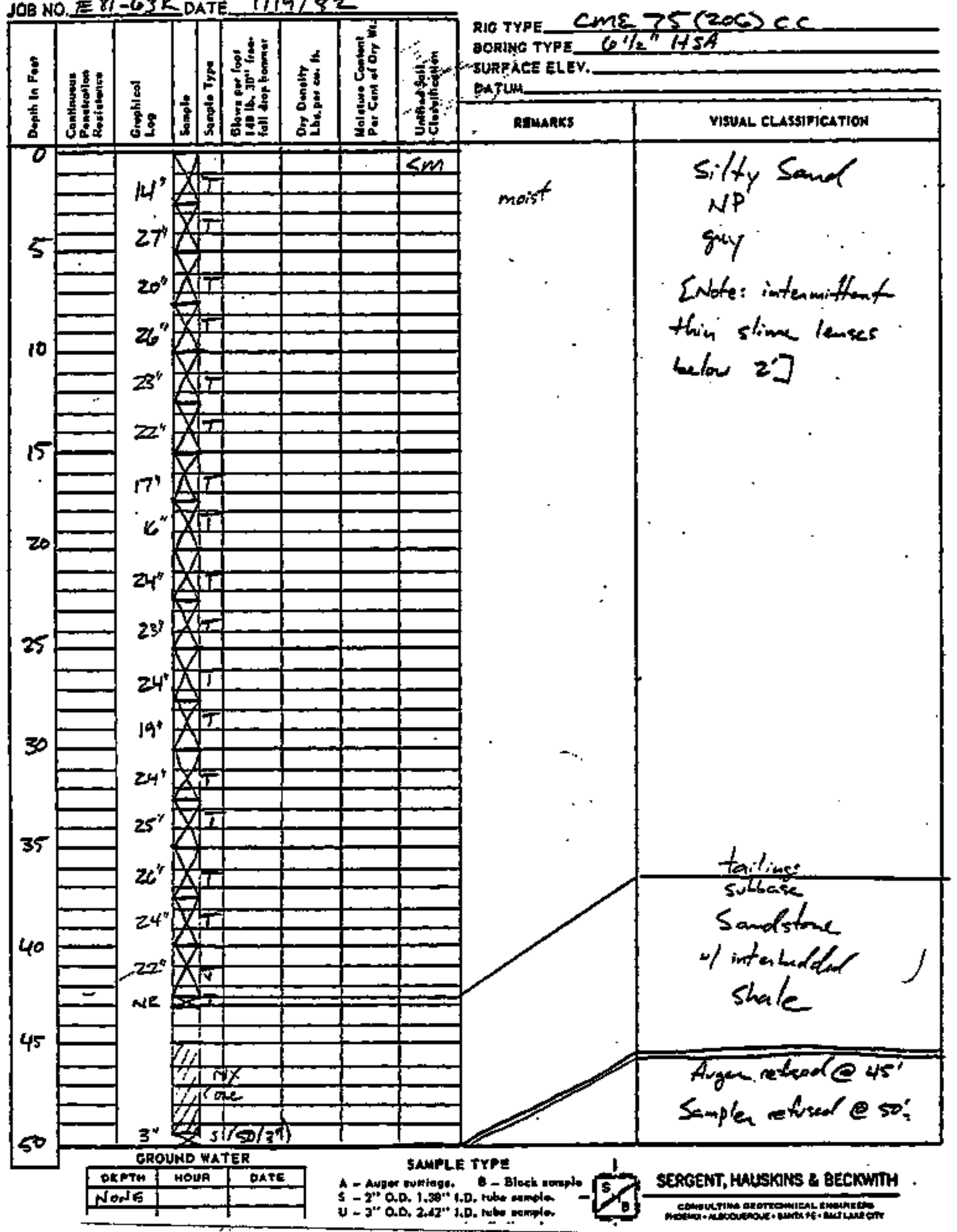


PROJECT mor

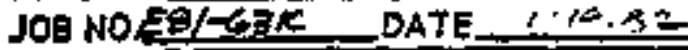

Loe of rest sorine No.CB3

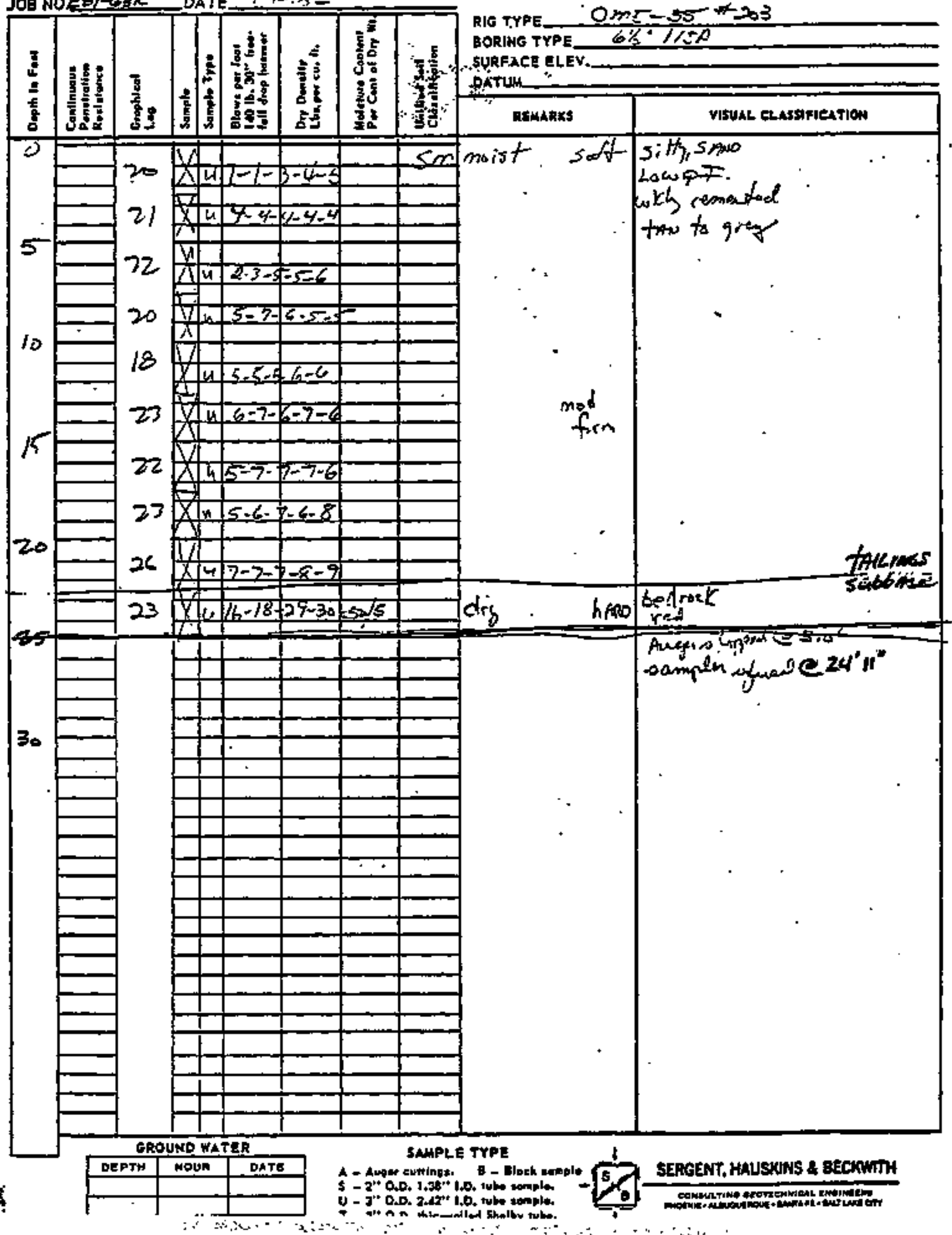


PROJECT Mexicam Hat Tailings JOB NO. ER/-6/3K,DATE $I / / 2 / 82$

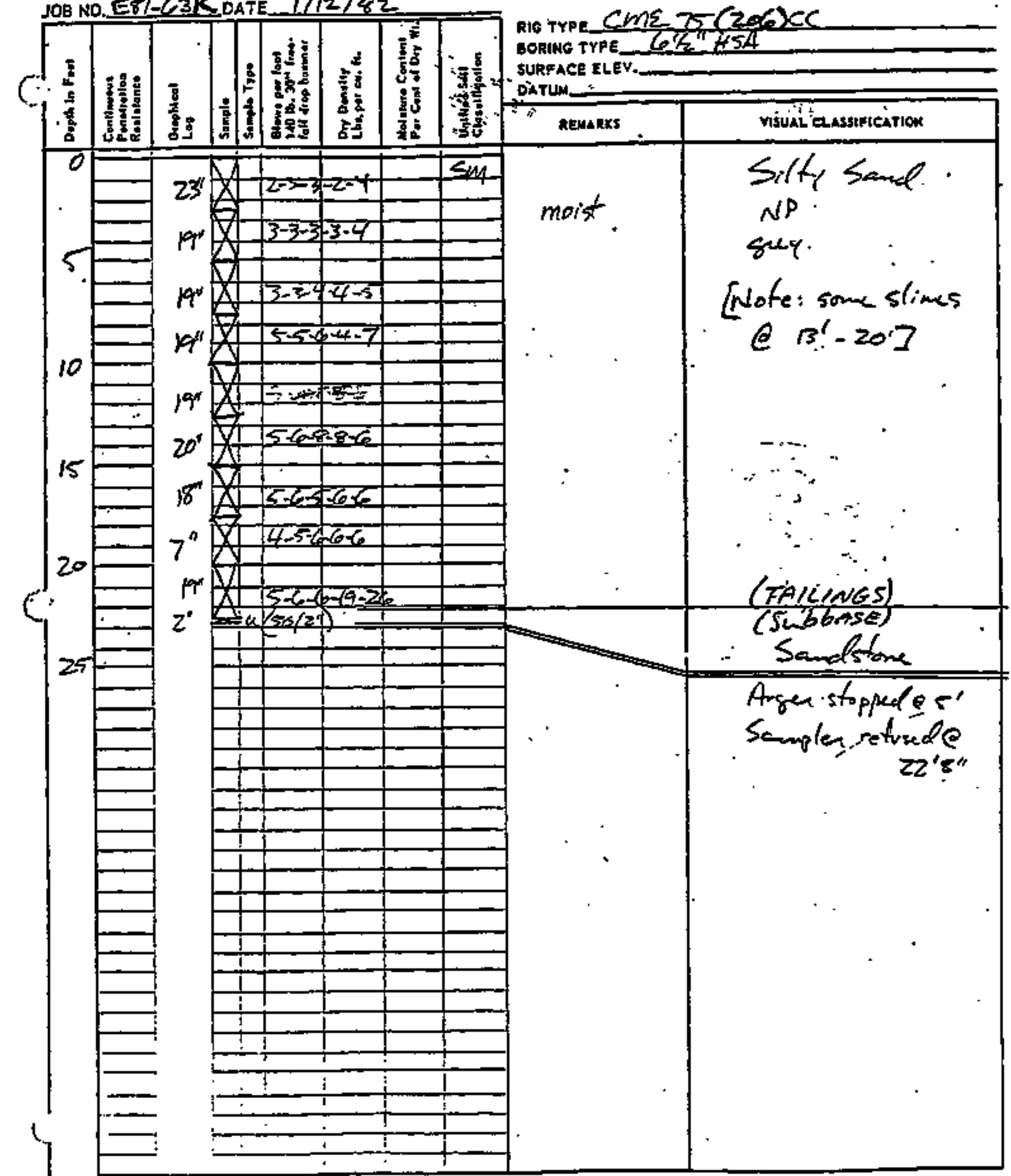


PROJECT MaviEHat Hat JOB NO.E E

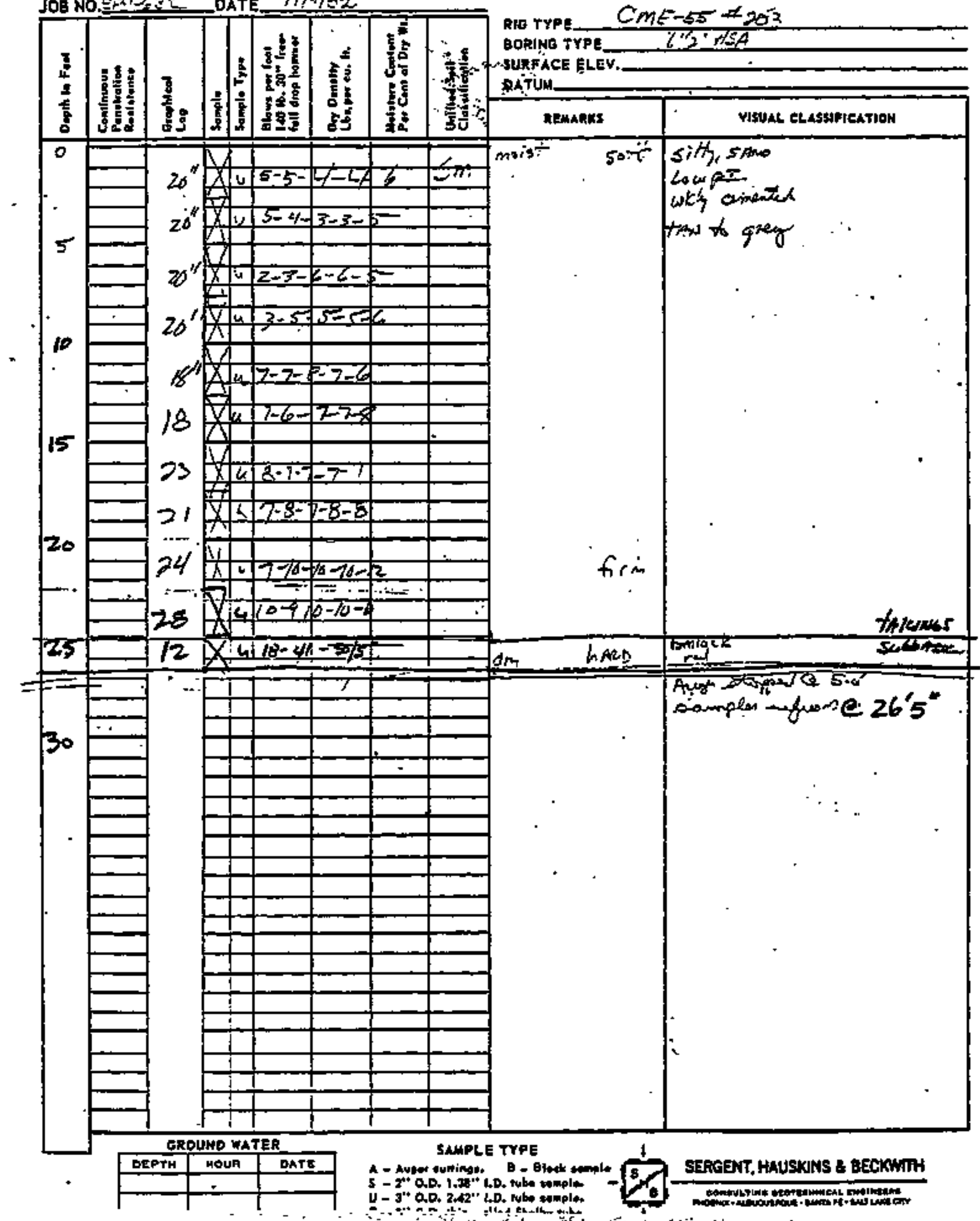


PROJECT Mexicen Hat Talinss JOB NO ESIF-3K DATE . $1 / 12 B 2$

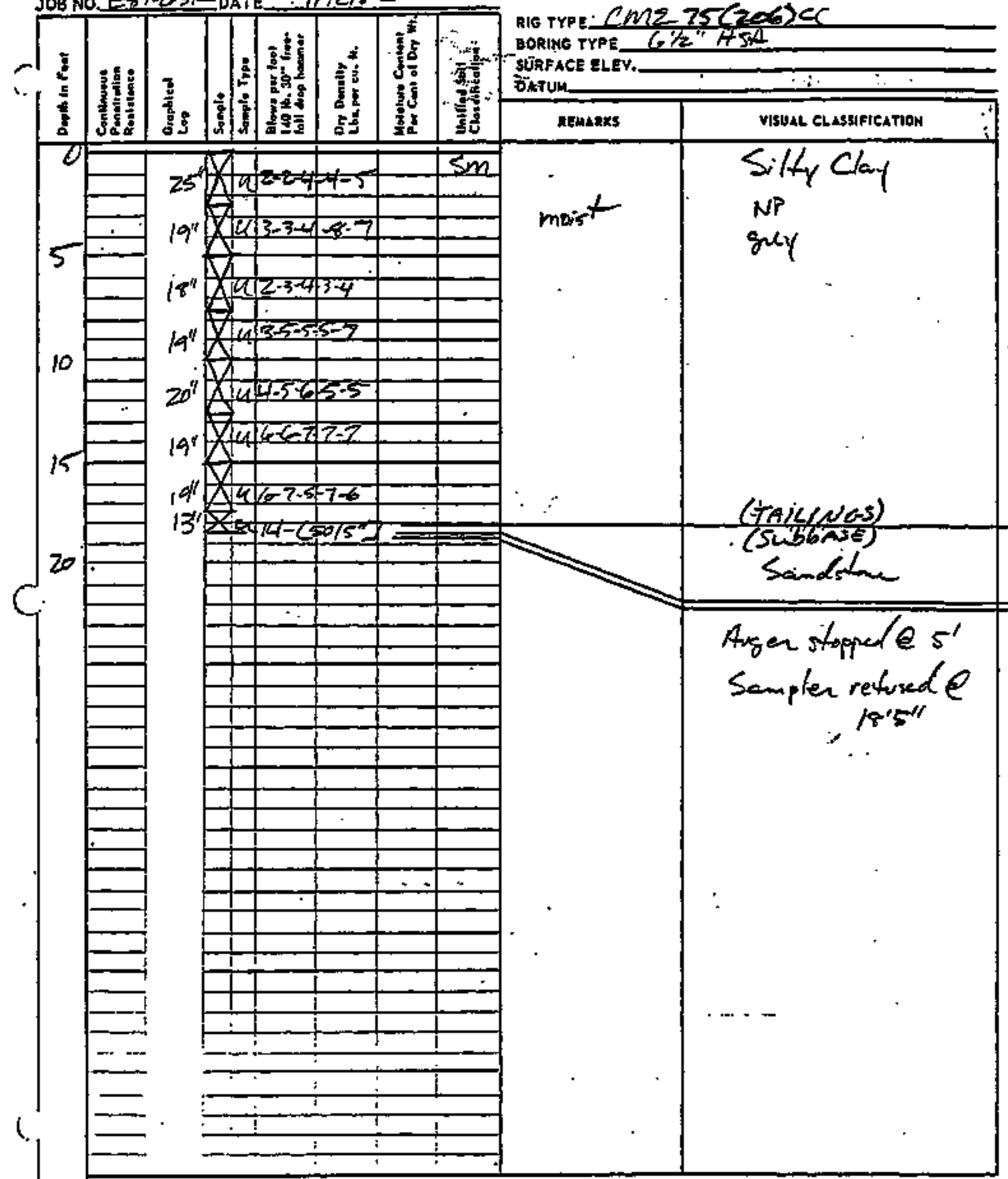

LOE OF Tast DORING NO.D8O 


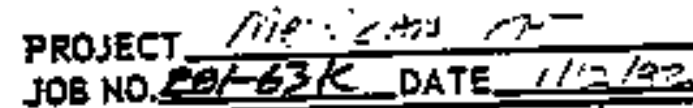

Loo of test nontwo no. $67^{9}$ \begin{tabular}{|c|}
\hline \\
$\mathbf{z}$ \\
$\frac{2}{2}$ \\
$\bar{z}$ \\
\hline \\
\hline
\end{tabular}

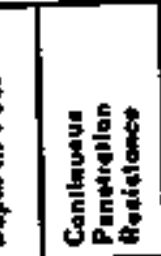

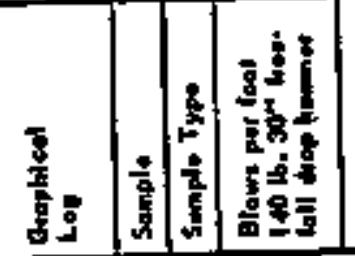

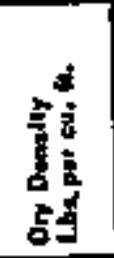

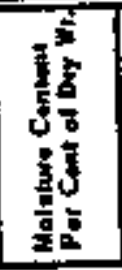

NIG TYPE CANE- $-53 \quad(203)$

$\circ$ C

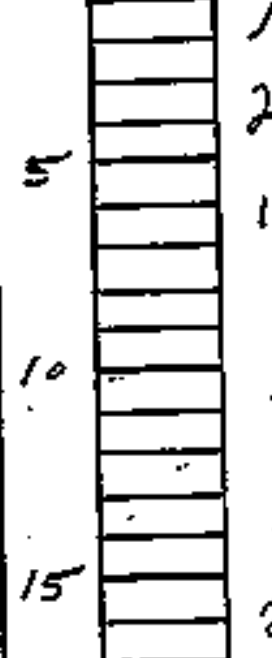

$18^{\prime} \times 4$ :

$26 \mathrm{y} \cos 20$

$$
+
$$

5

BDRINGG TYPE

$6 \%+\%$

18 in $-2=4-3$

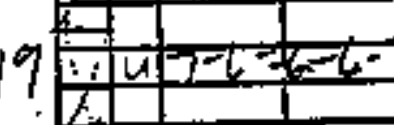

$18:-2+2-5=27$

20

in $u+3-2, y=6$

23

\begin{tabular}{lll}
\hline M. & \\
\hline
\end{tabular} moist 50\%: 5it: 5 500

YLUAL CLASEIFICATION

low $p=$.

iskly ceinertark

then $\frac{1}{10} 5,-x$

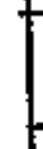

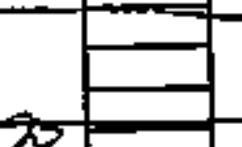

$$
d x+8-9-19-503^{1 / 2}=0
$$

$d x \quad . \quad h$

Damescoly

(TAL/NGS) bedrocic (SubGaSE)

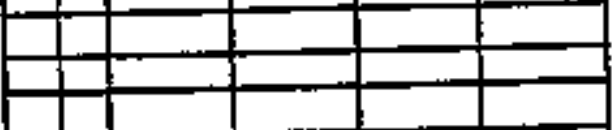

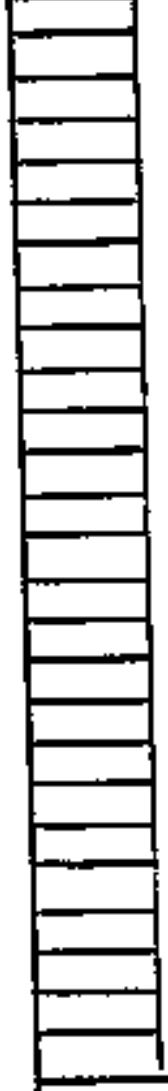

\begin{tabular}{|l|l|l|l|l|}
\hline & & & & \\
\hline
\end{tabular}

\begin{tabular}{|l|l|l|l|l|}
\hline & & -1 & & \\
\hline & & & & \\
\hline
\end{tabular}

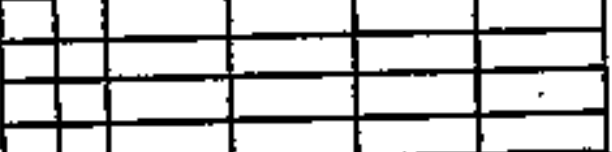

\begin{tabular}{|llllll|}
\hline & & & & & \\
\hline & & & & & \\
\hline
\end{tabular}

\begin{tabular}{|l|l|l|l|l|}
\hline & & & & \\
\hline & & & & \\
\hline
\end{tabular}

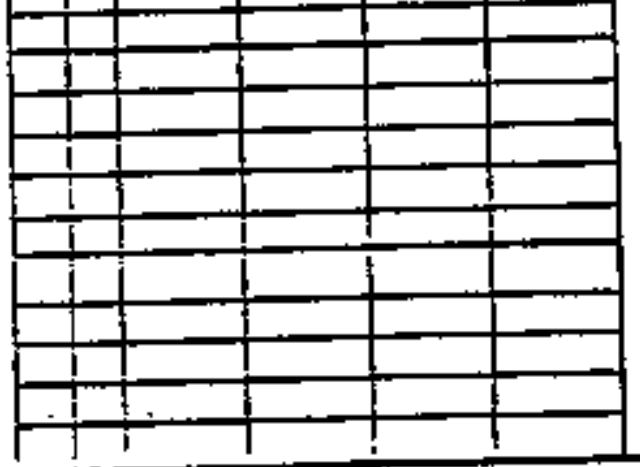


PROJECT Mexican Hat Tadines JOB NO. E81-G3 L DATE $\mid / / 2 / 81$

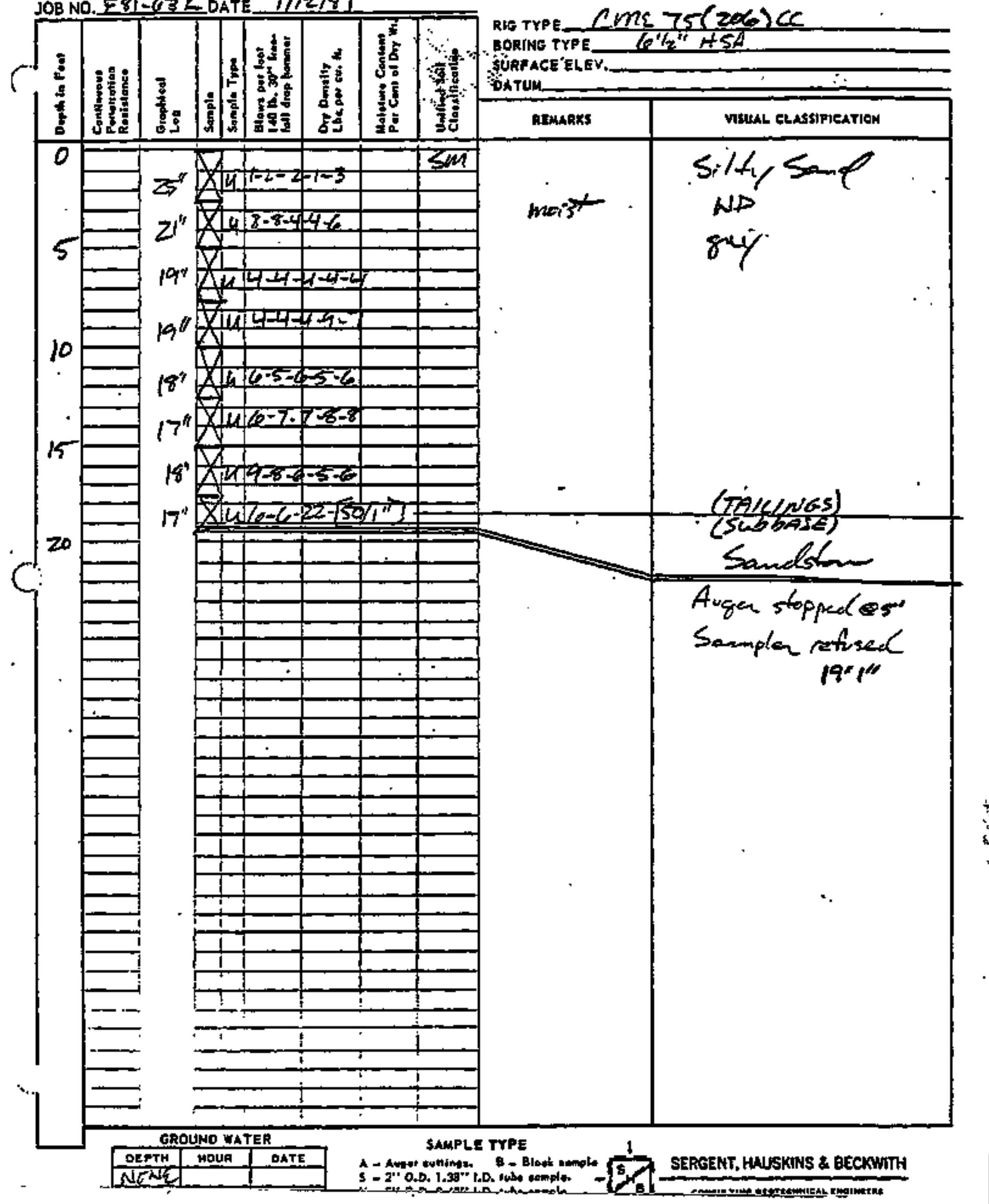


PRoJect Mexican HAT JOB NO BSACEK DATE $1 / 12 / 32:$

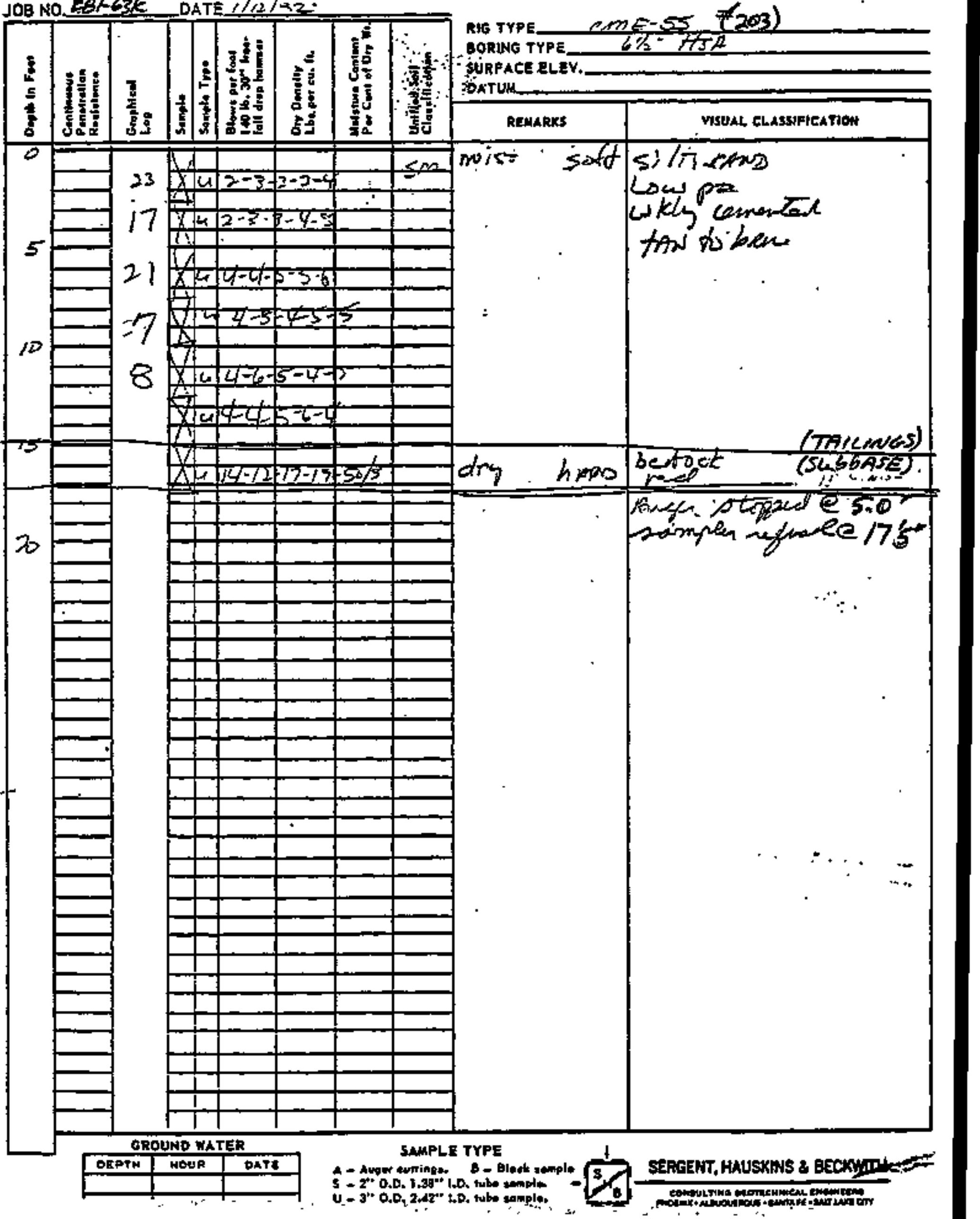




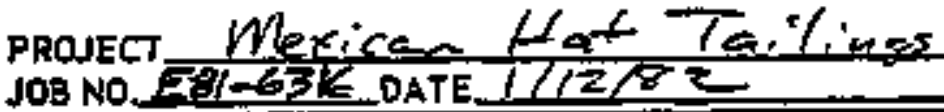

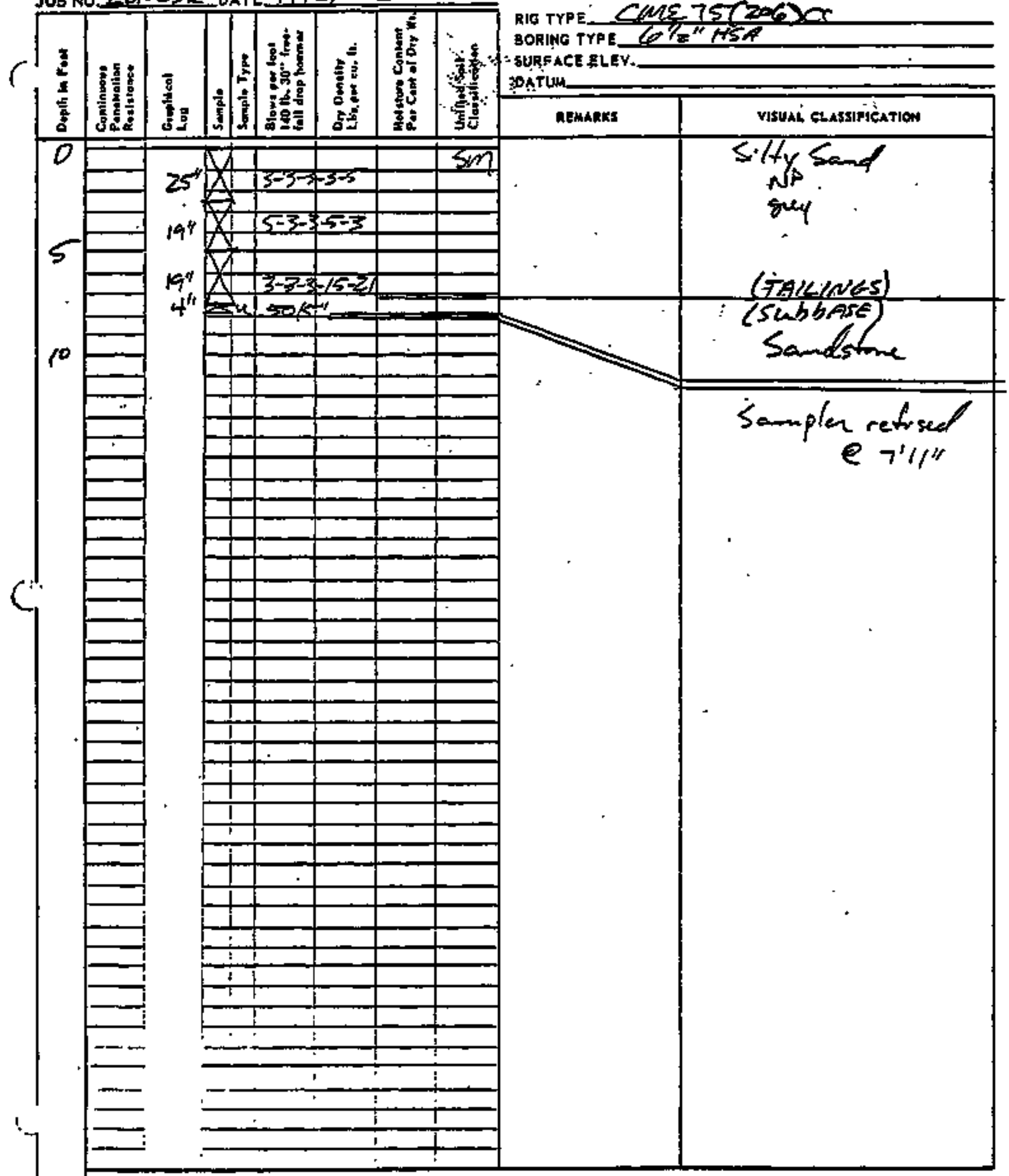

GROLNND WATER

\begin{tabular}{|l|l|l|}
\hline DEPTH & HOUN \\
\hline
\end{tabular}

Nonst
SAMPLE TYPE

4- Auger turinga, $\theta$ - Block nopplo

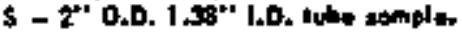

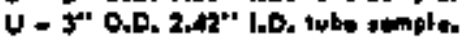

LOe OF TESt DORINe no.DZ

to sand

finy
(GALINLS)

Subbare

Sandstime 


$$
\text { tr? }
$$




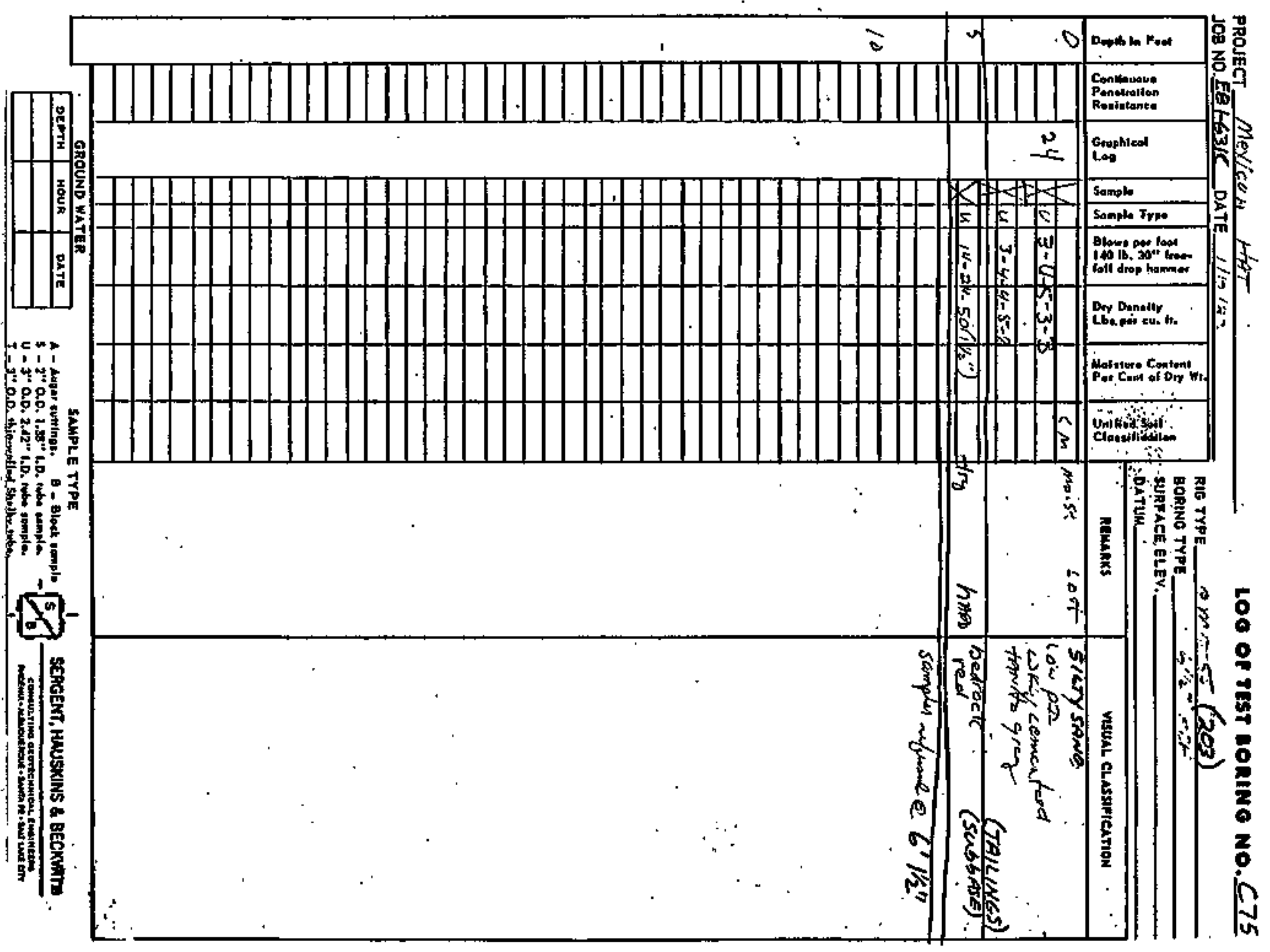




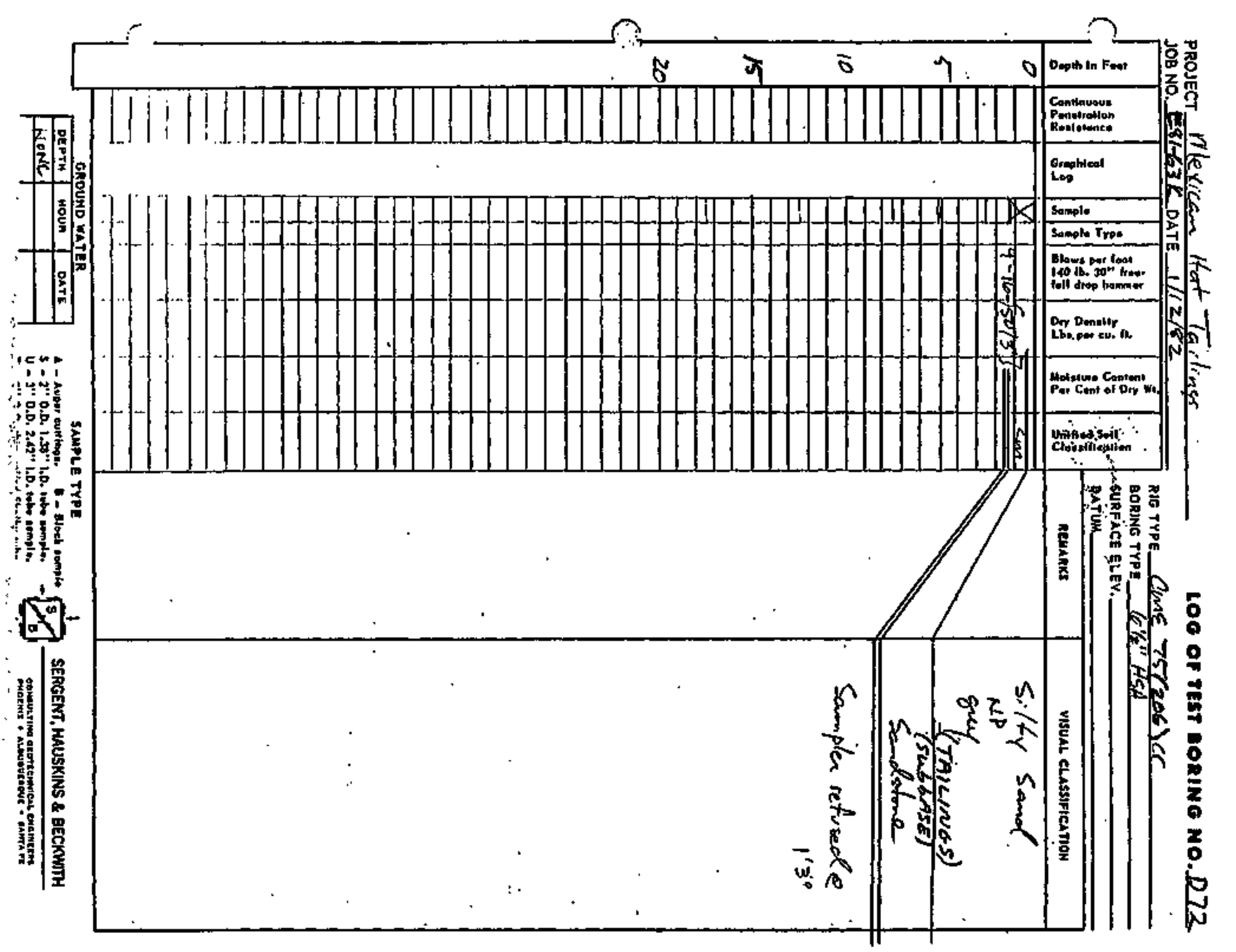


PROAECT (is., CA, $\rightarrow \mathrm{N}^{-}$ JOB NOEQT-63K DDTE $1,2 \%$

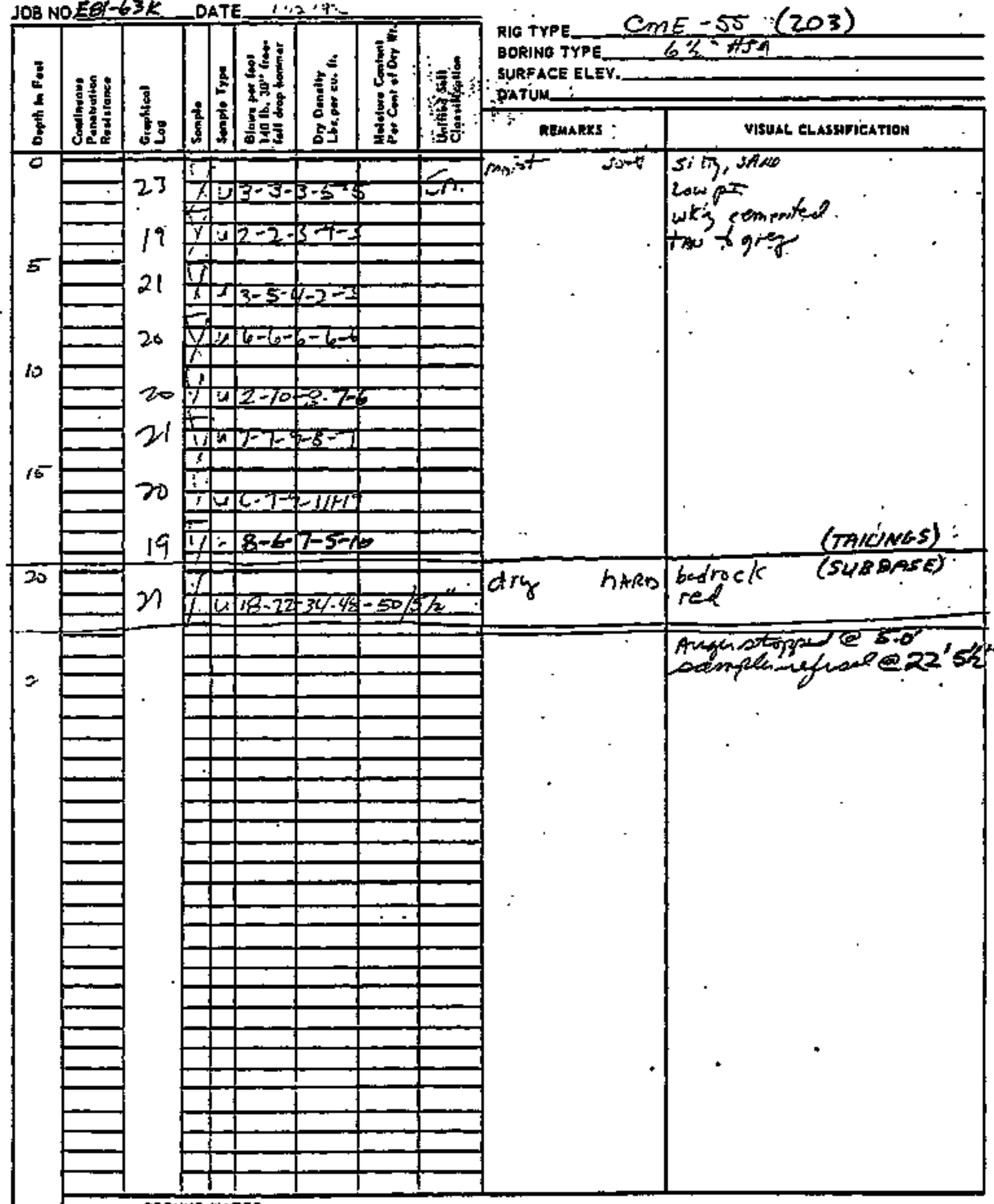

GROUNO WATER \begin{tabular}{|l|l|l|}
\hline OEPTH & WOUN \\
\hline
\end{tabular}

\section{SAMPLE TYPE}

- Auger cumlngh. $=$ Blod traplo

5 - 2'0 0.5. 1.38" L.D. thlo tanale.

Y = 3" 0.0. 2.42" L.D. tubt tomple.

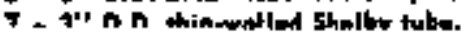

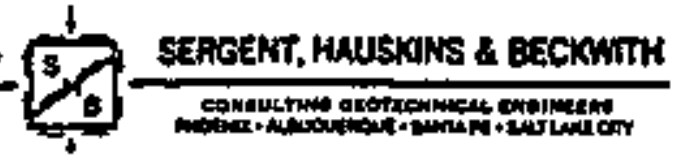

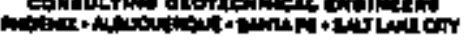




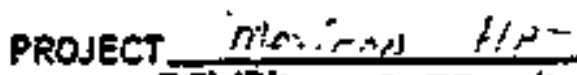

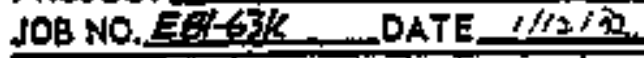

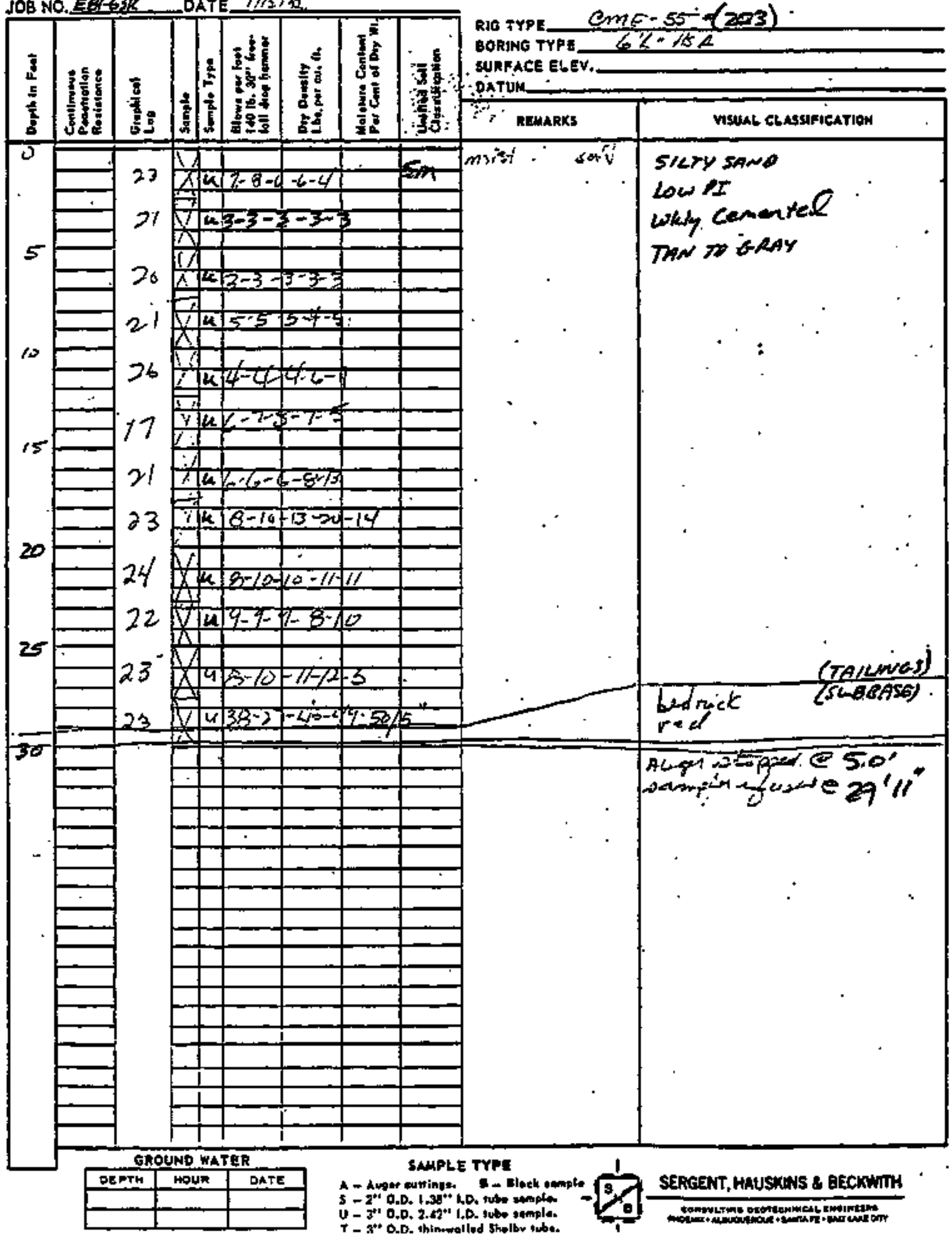


PROJECT Maxiean HAT JOB NO.ES1-GKK DATE / $12 . / 62$

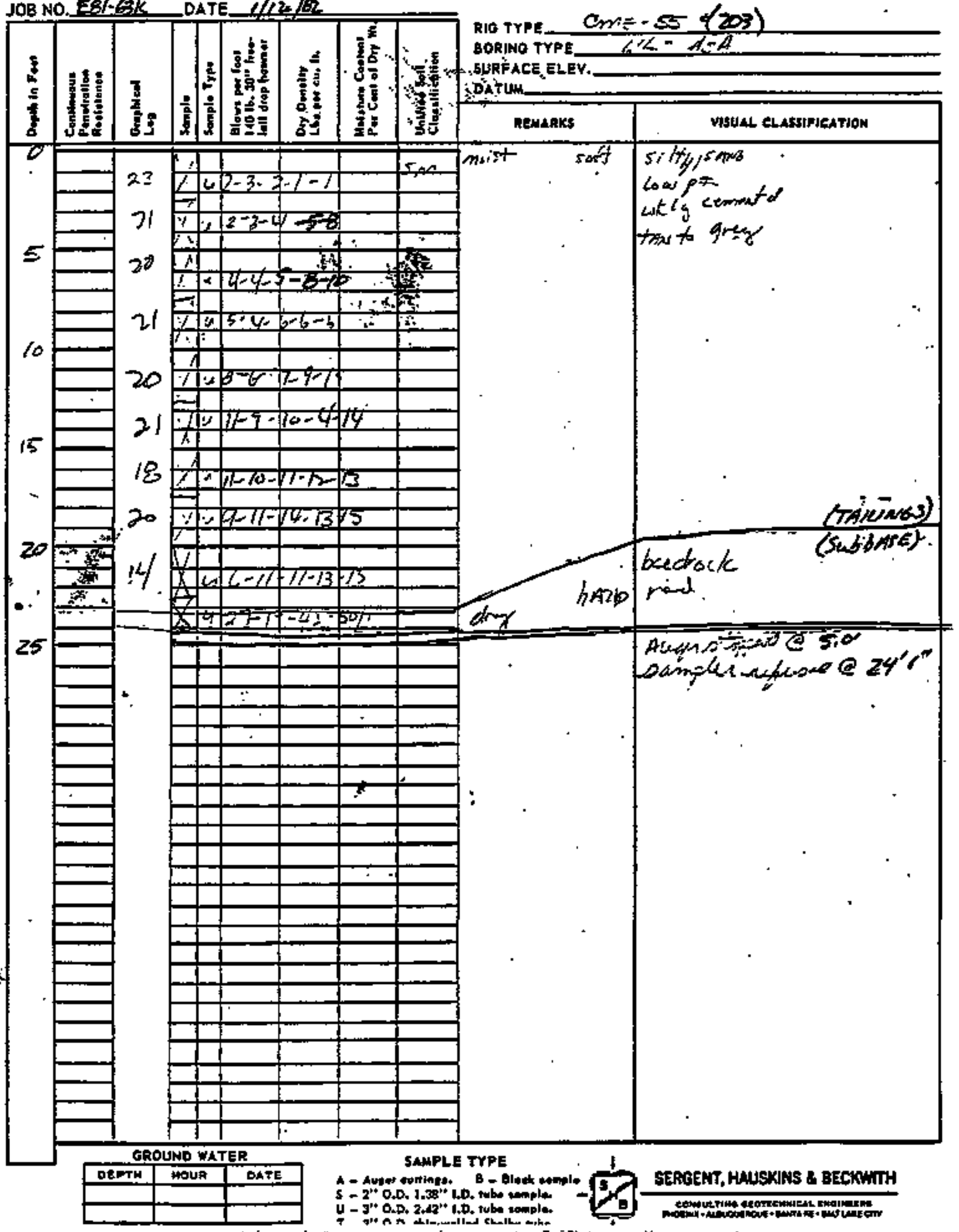




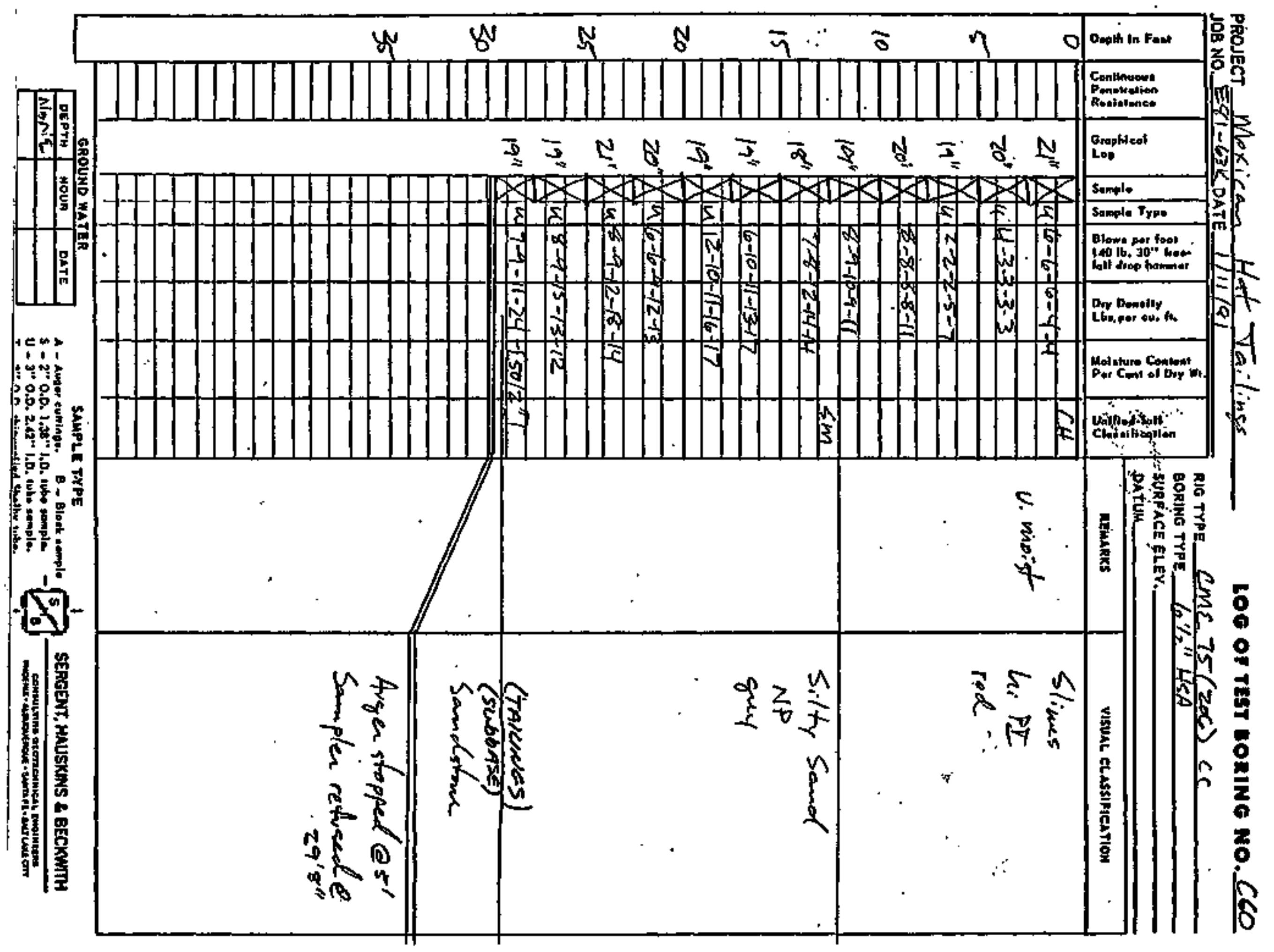




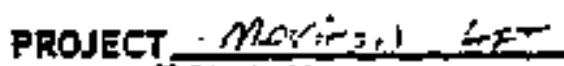

coc of rast nontas no.D6/i

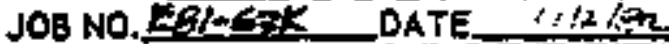

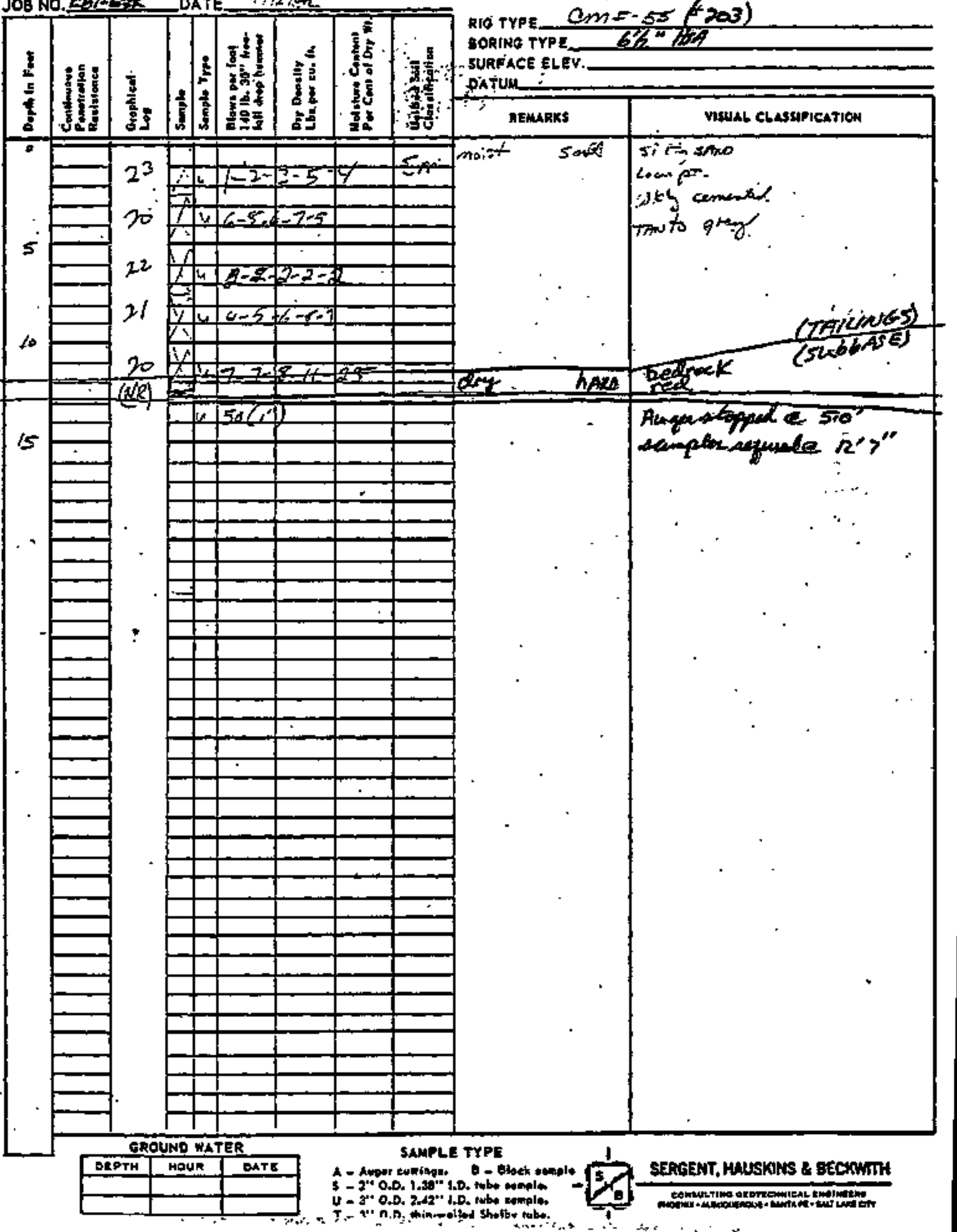




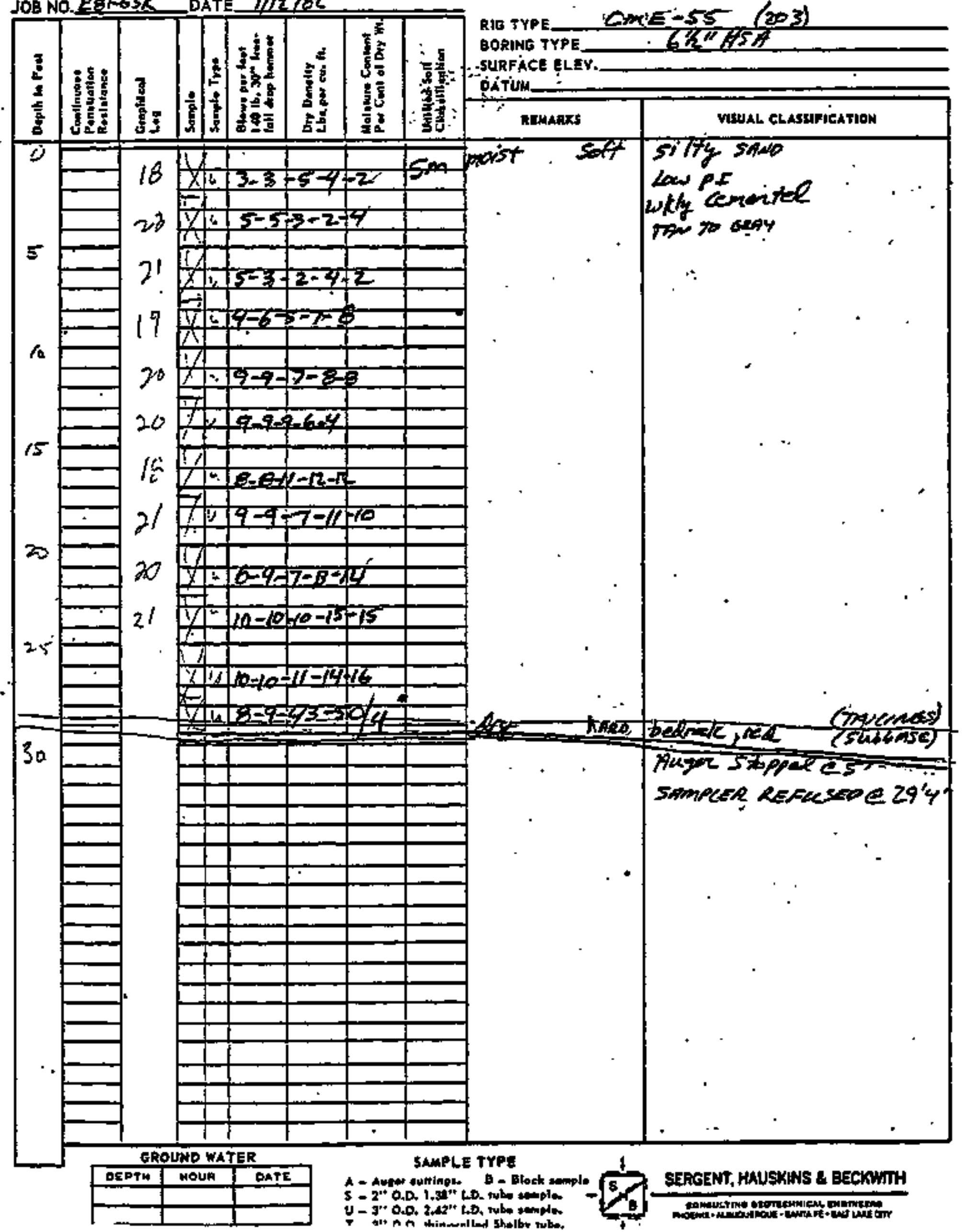




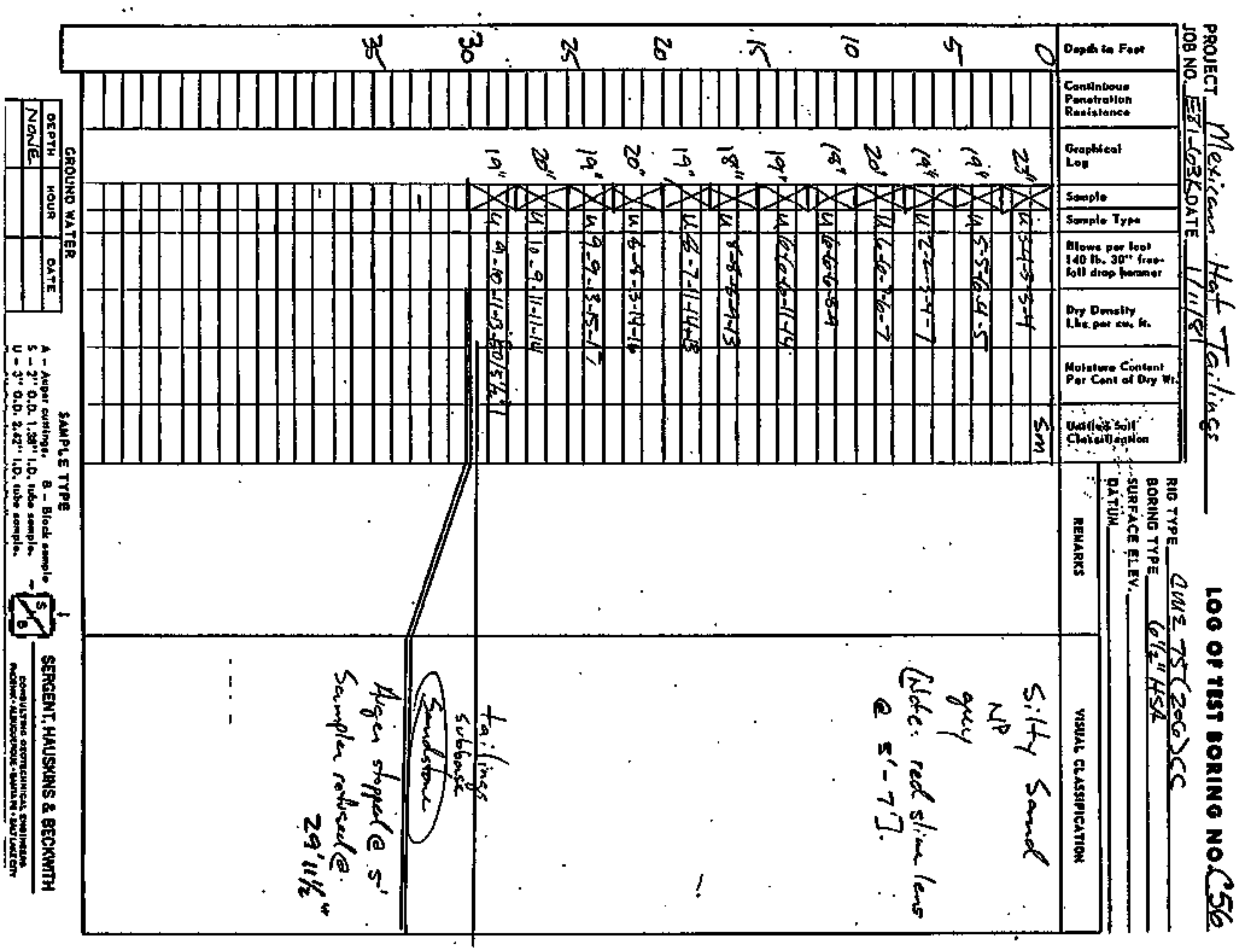


PROJECT MEXEAA ALT

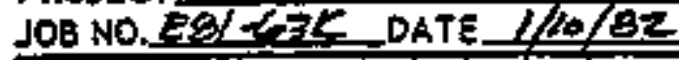

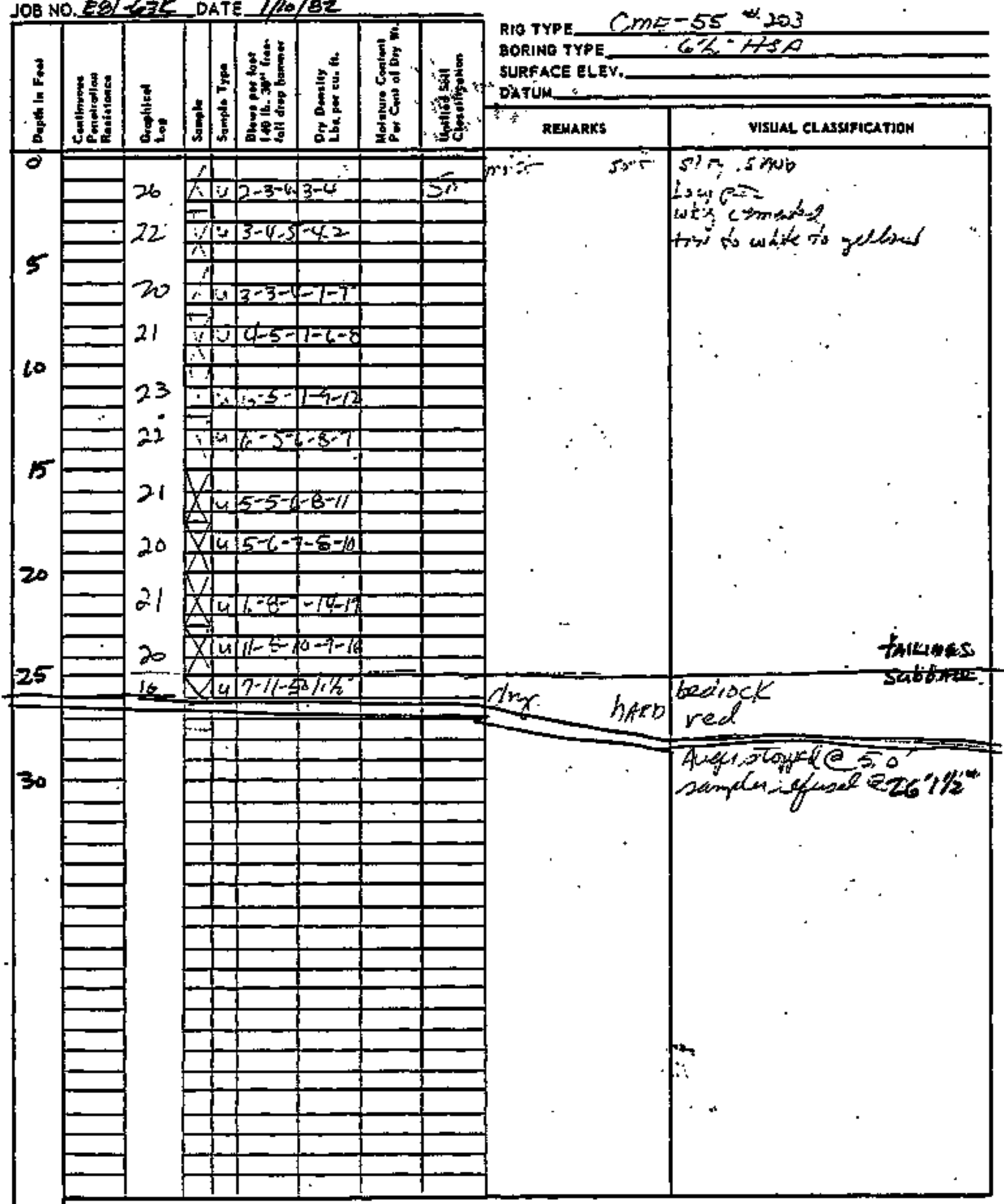


PROJECT Mexican Hat Tailings " 100 of TEST JOB NO. EST-SOKK DATE I IIIRZ?

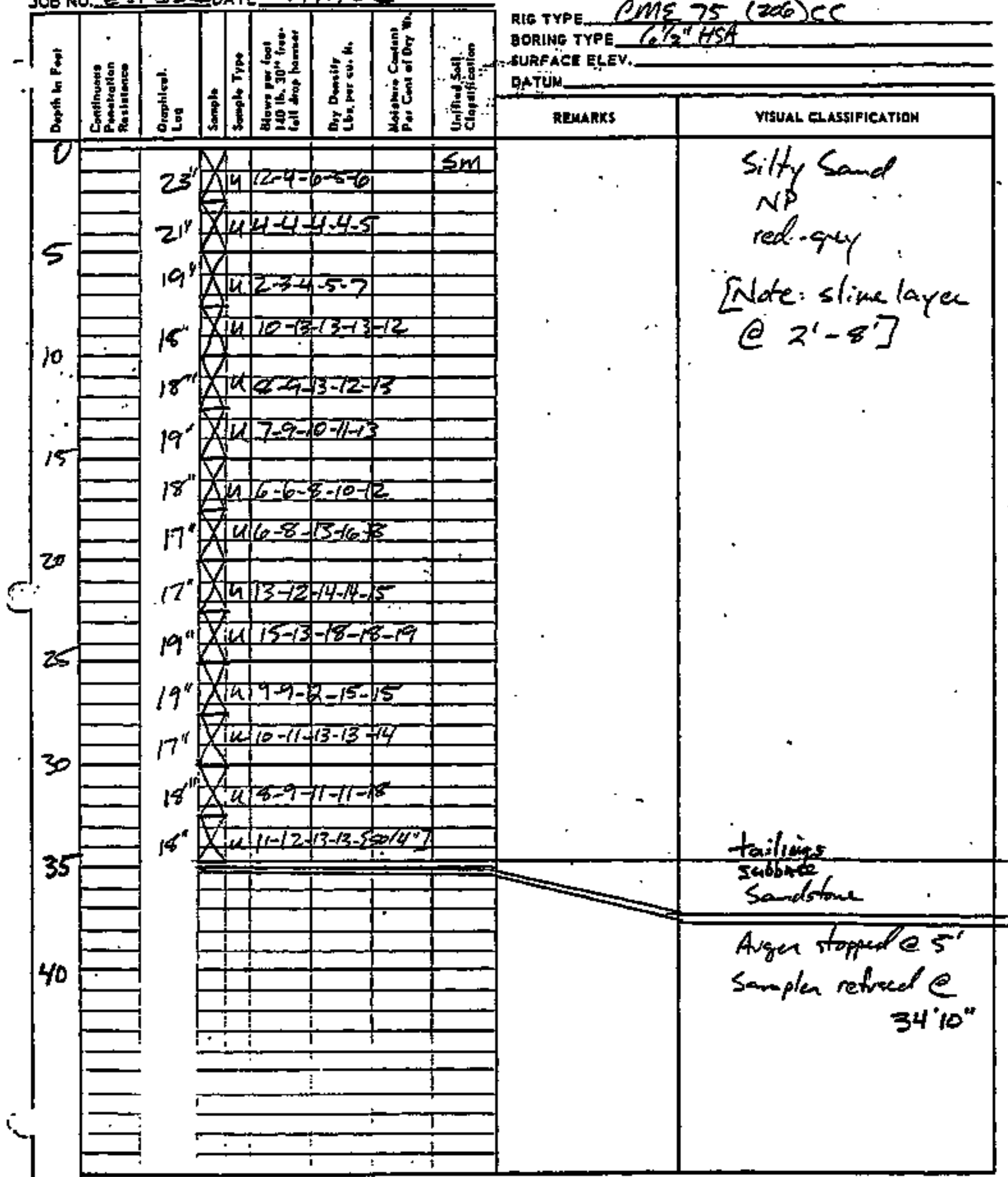

GROUND WATER

\begin{tabular}{|l|l|l|}
\hline DEPTH & HoUk & OAFE \\
\hline Nexis & & \\
\hline & & \\
\hline
\end{tabular}

\section{SAMPLE TYPE}

A - Awar coninge $\mathrm{Q}$ - Bleak sempla to

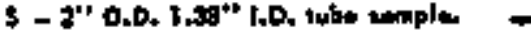

U - 3"' O.D. 2.42" I.D. tute aqmpie.

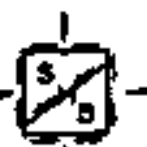

SERGENT, HAUSSINS \& BECKWTH 
PROJECT Alevicted - HAT.-

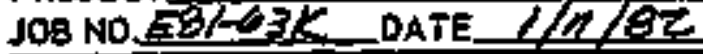

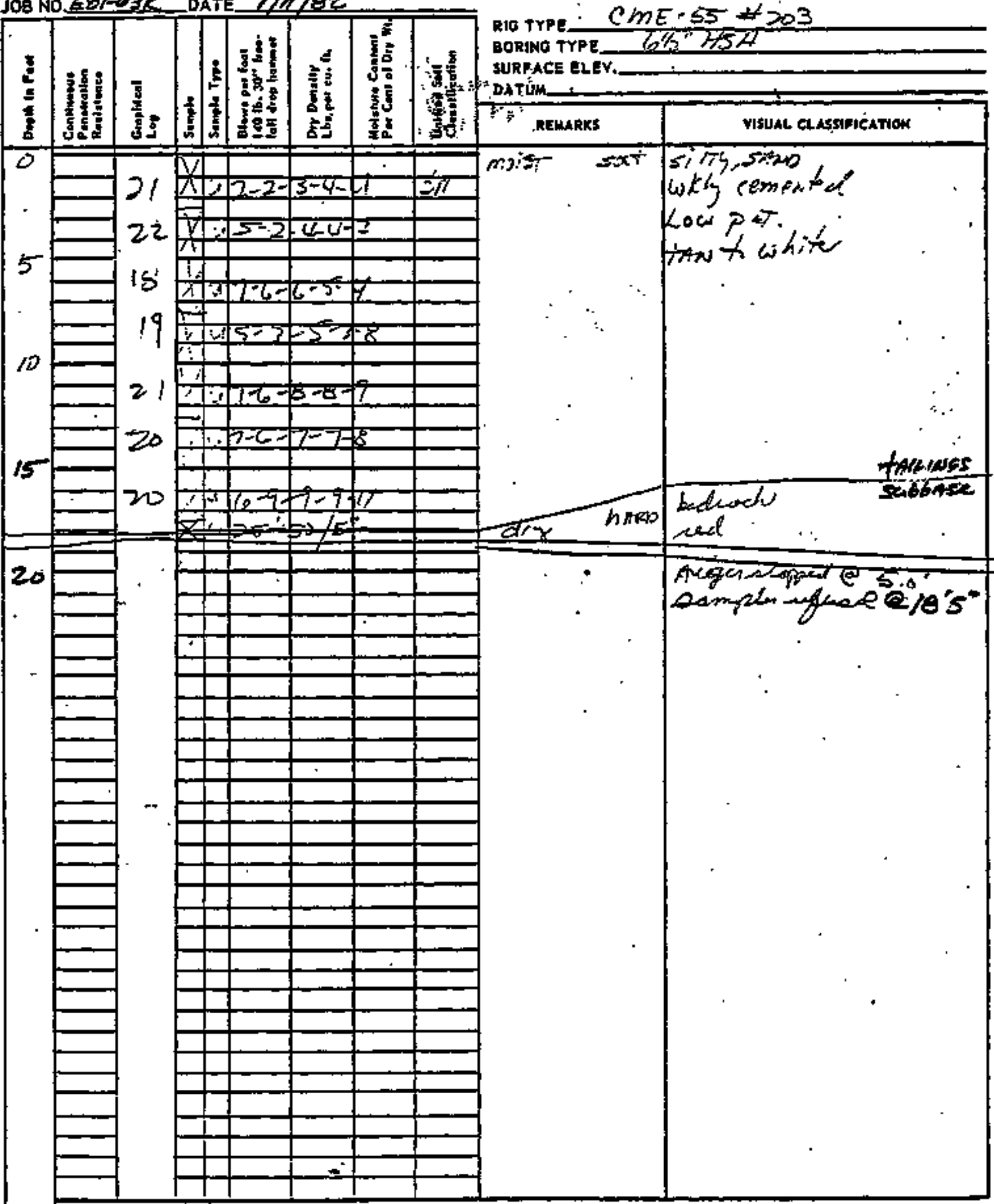




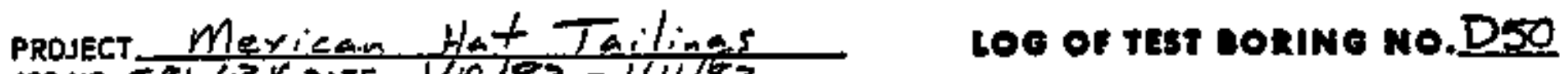
LOB NO.EPI-63KDATE $1 / 10 / 82-111 / 182$

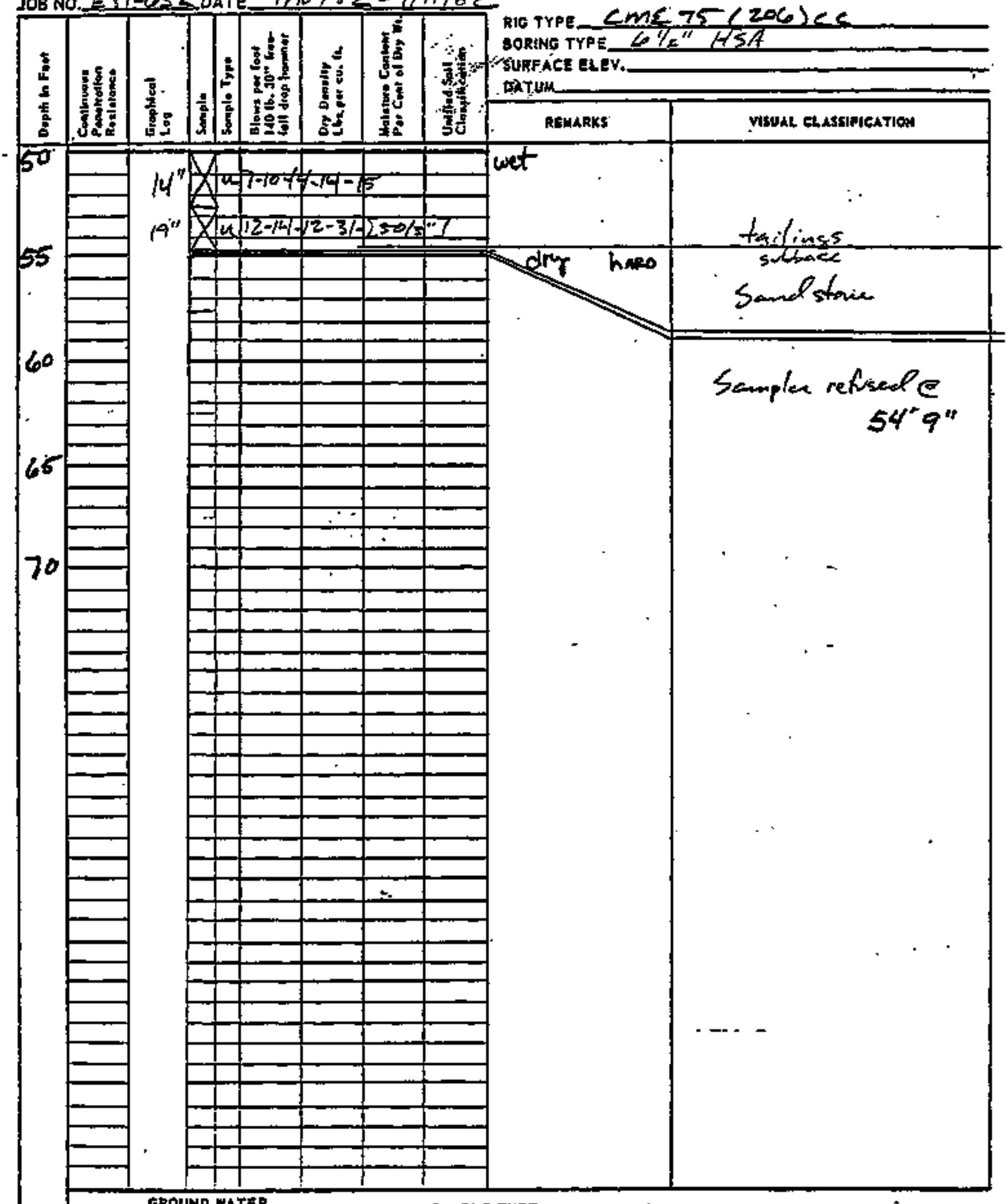

GROUND WATER

\begin{tabular}{|c|c|c|}
\hline DEPTH & HOUR & DATE \\
\hline$+1 \times 50^{\prime}$ & $a: a t$ & 111768 \\
\hline
\end{tabular}

SAMPLE TYPE

A - Auger euthtage. 0 - Block inmple

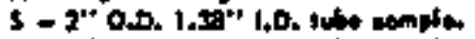

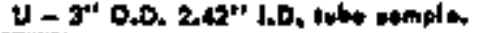

SERGENT, MAUSKINS \& BECKWTH

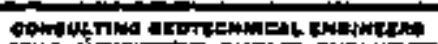

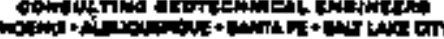




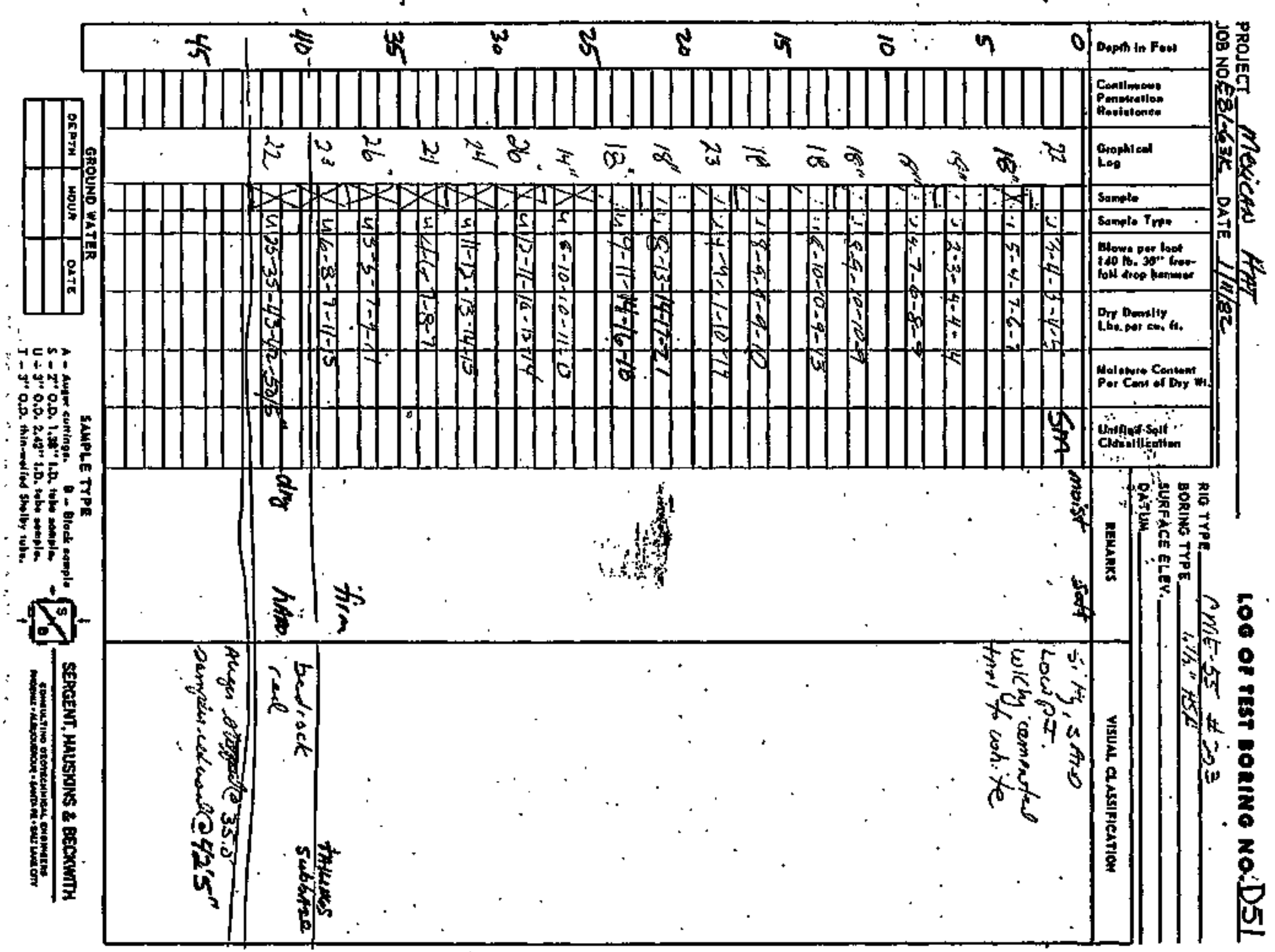


PROJECT MEXICAN HAT JOB NO. EQT-6.JK DATE JWIIEZ

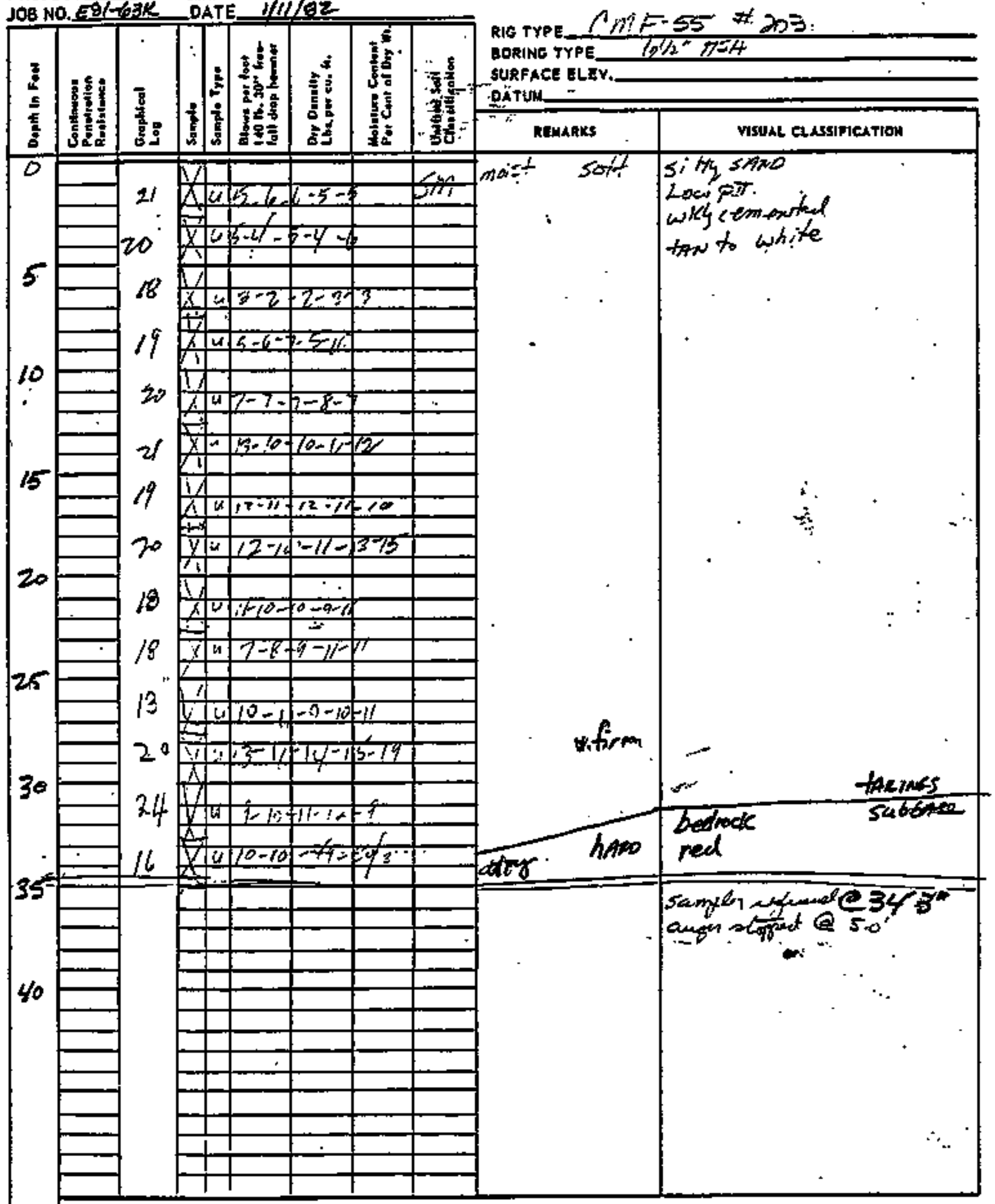

GROUND WATER DEPTM HOUP DATE

\section{SAMPLE TYPE}

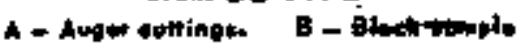

S - Z* O.D. J.3." H.D. tube vamile.

U = अ" O.D, 2,42" 1.D. mba somile.

T - 3" O.D, thinemelled Shelto lube.

Loo or rist borine no. 449

Cnif-ss \# 205:

$7=55$ 20

prt

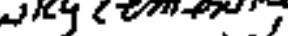

tan to white 
PROJECT MEYICAN AKT JOB NO.EB-G3K DATE_Llo/

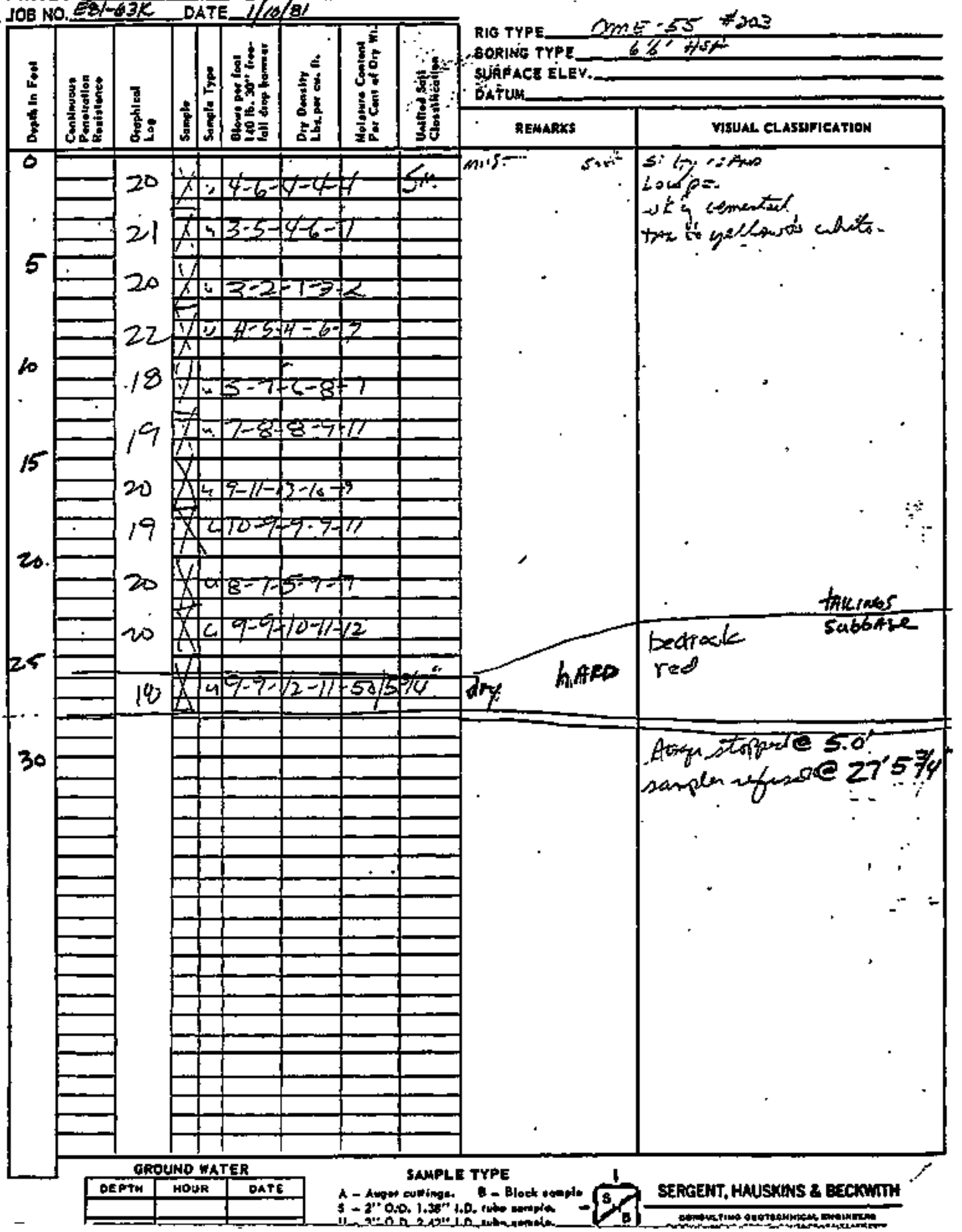




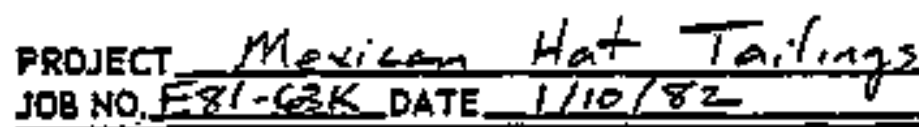

Los of tat nentne no: 448

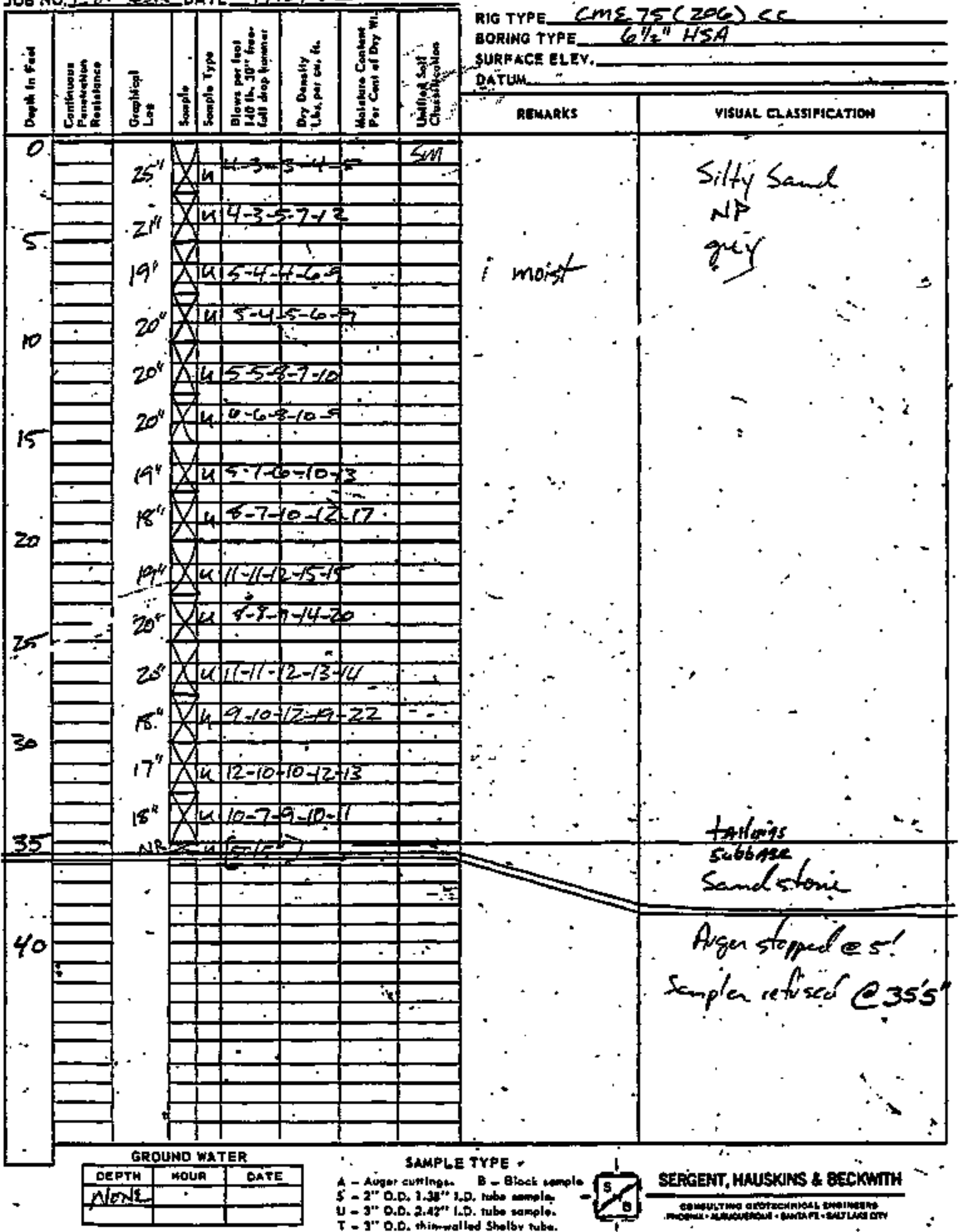




\section{PRDJECT MeXican HWT}

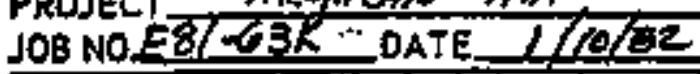

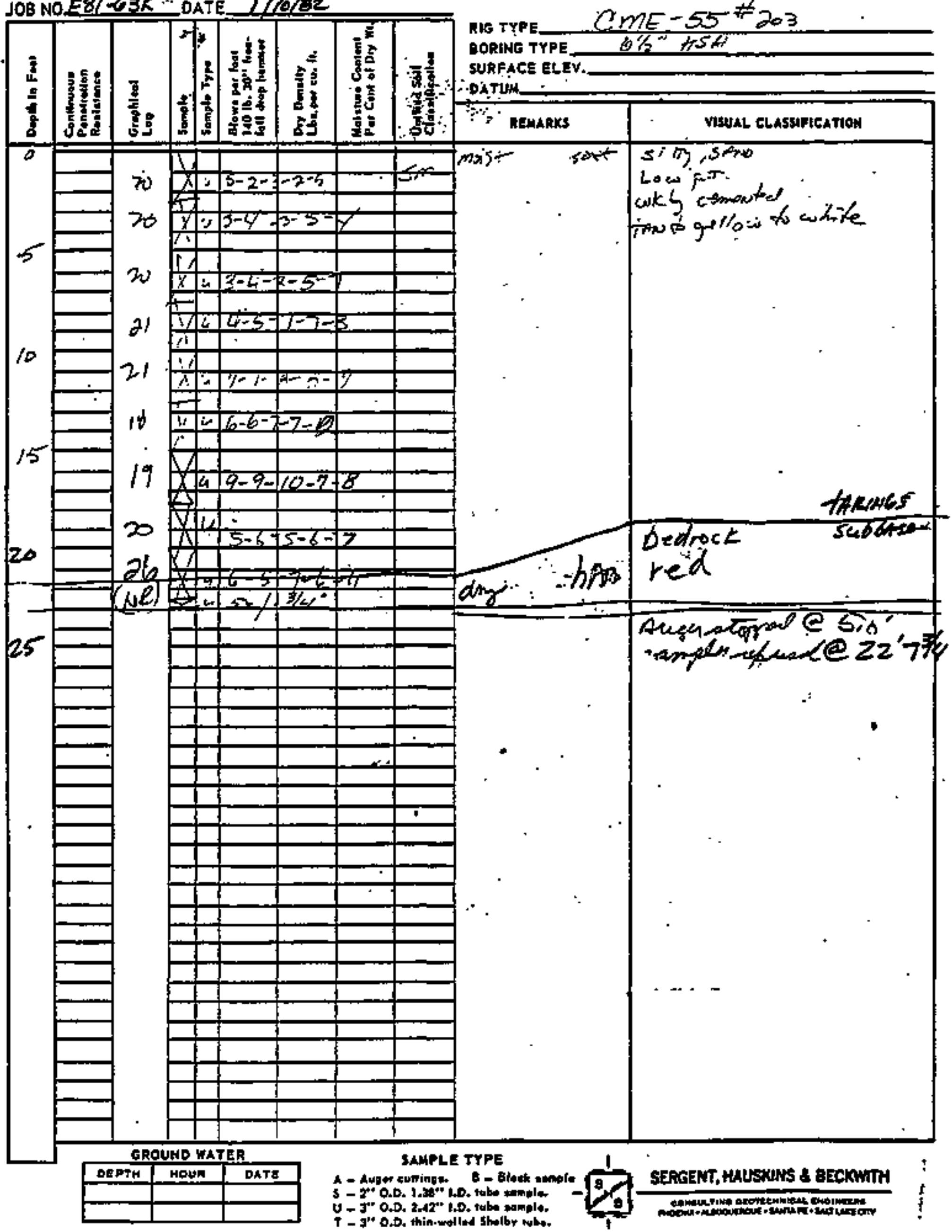


PROJECT Mexican Itat Tilinas JO3 NO. EST-63K DATE $1 / 10 / 72$

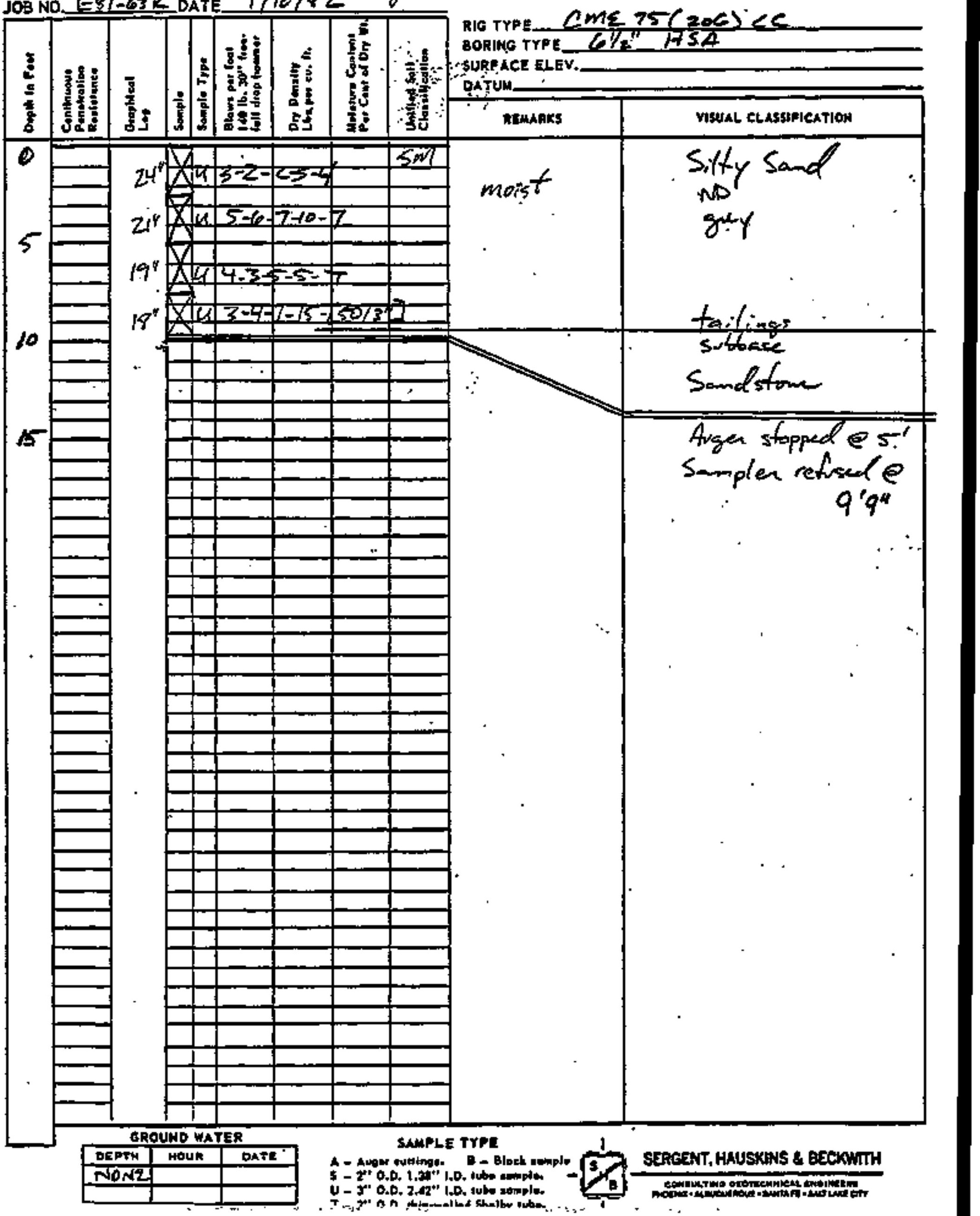


Project Mixican Hat Tailimes JOB NO. FEI 6 KL DATE $1 / 10 / 9 Z$

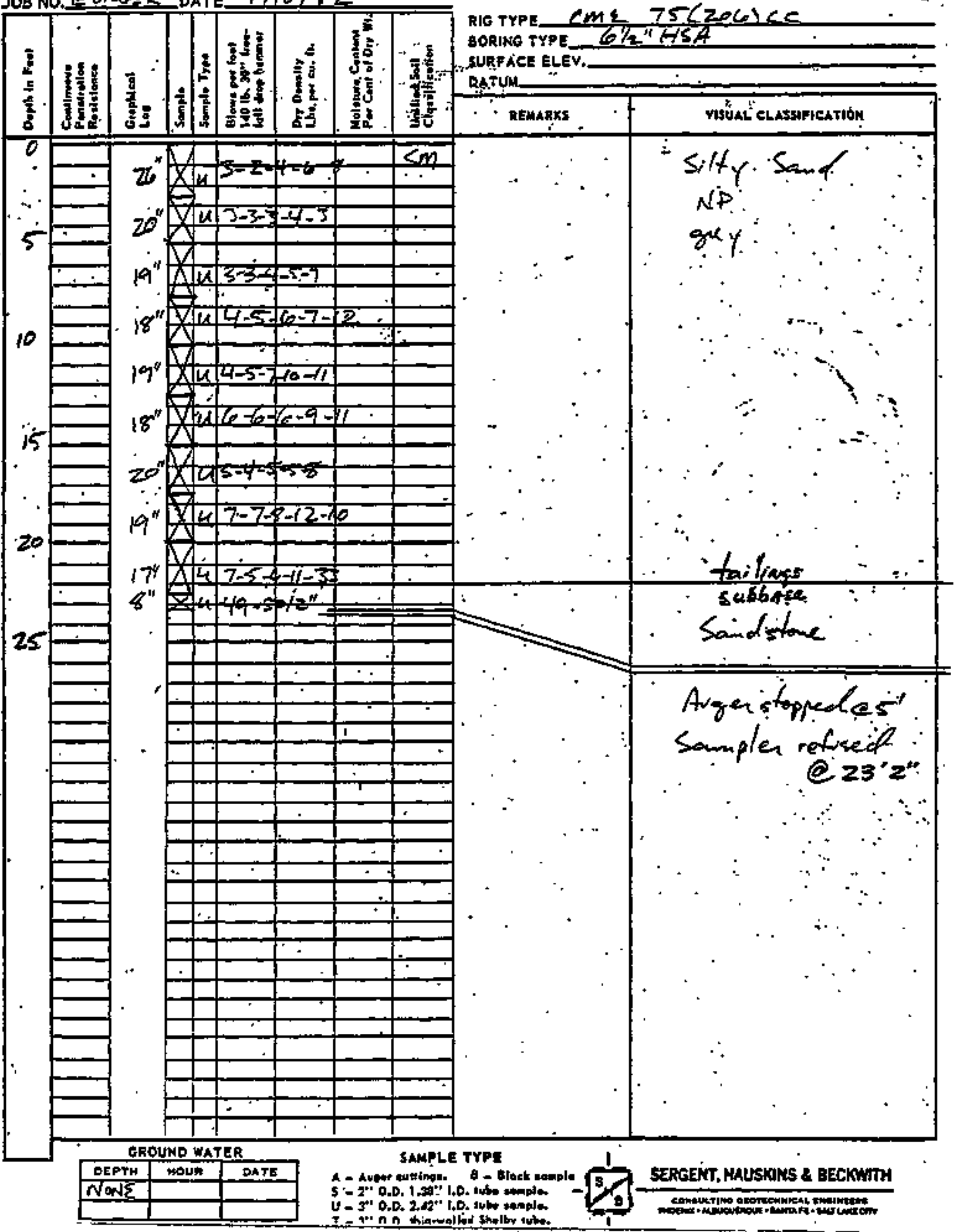

5
$\vdots$
$\vdots$
$\vdots$
0 


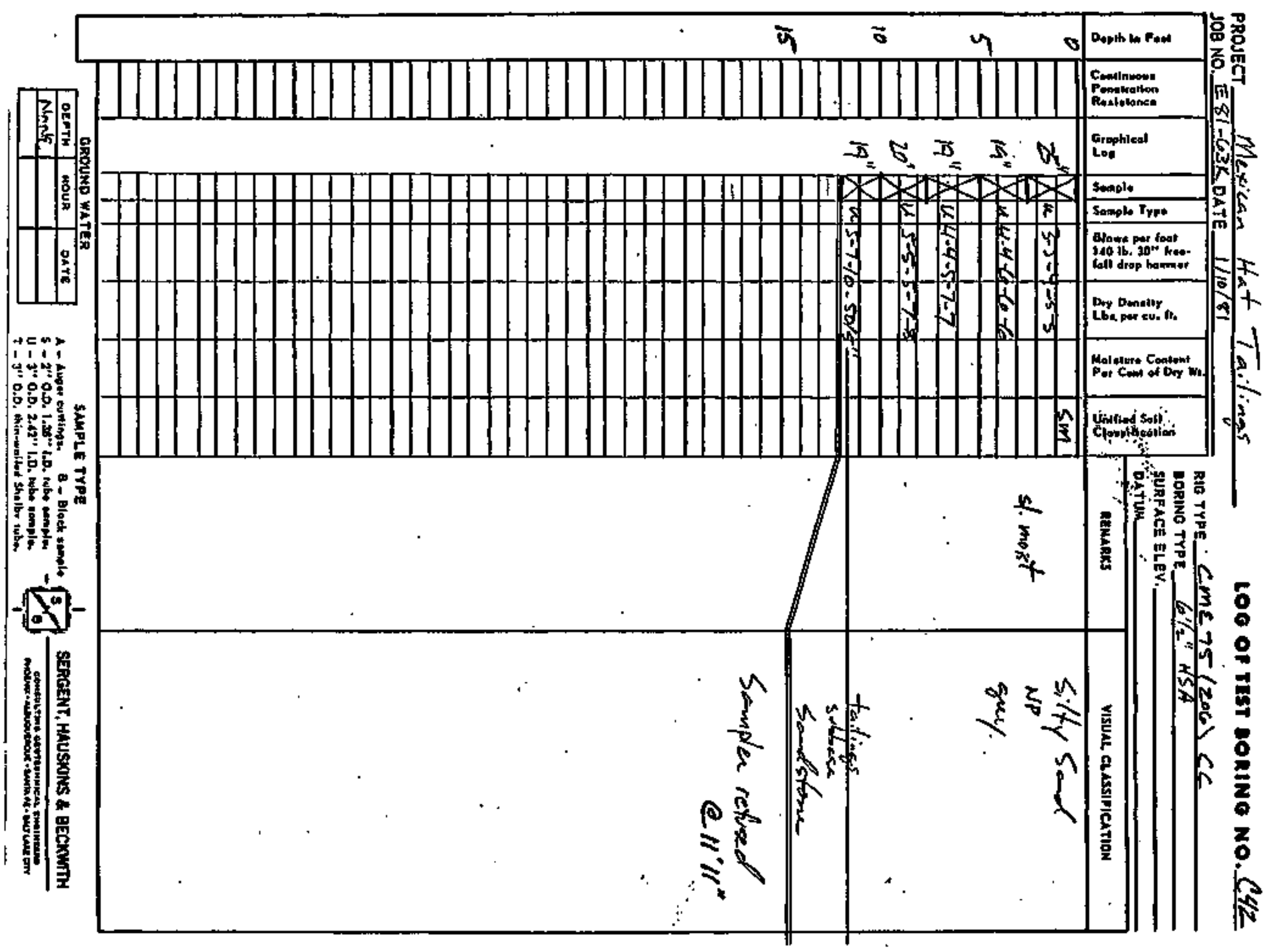




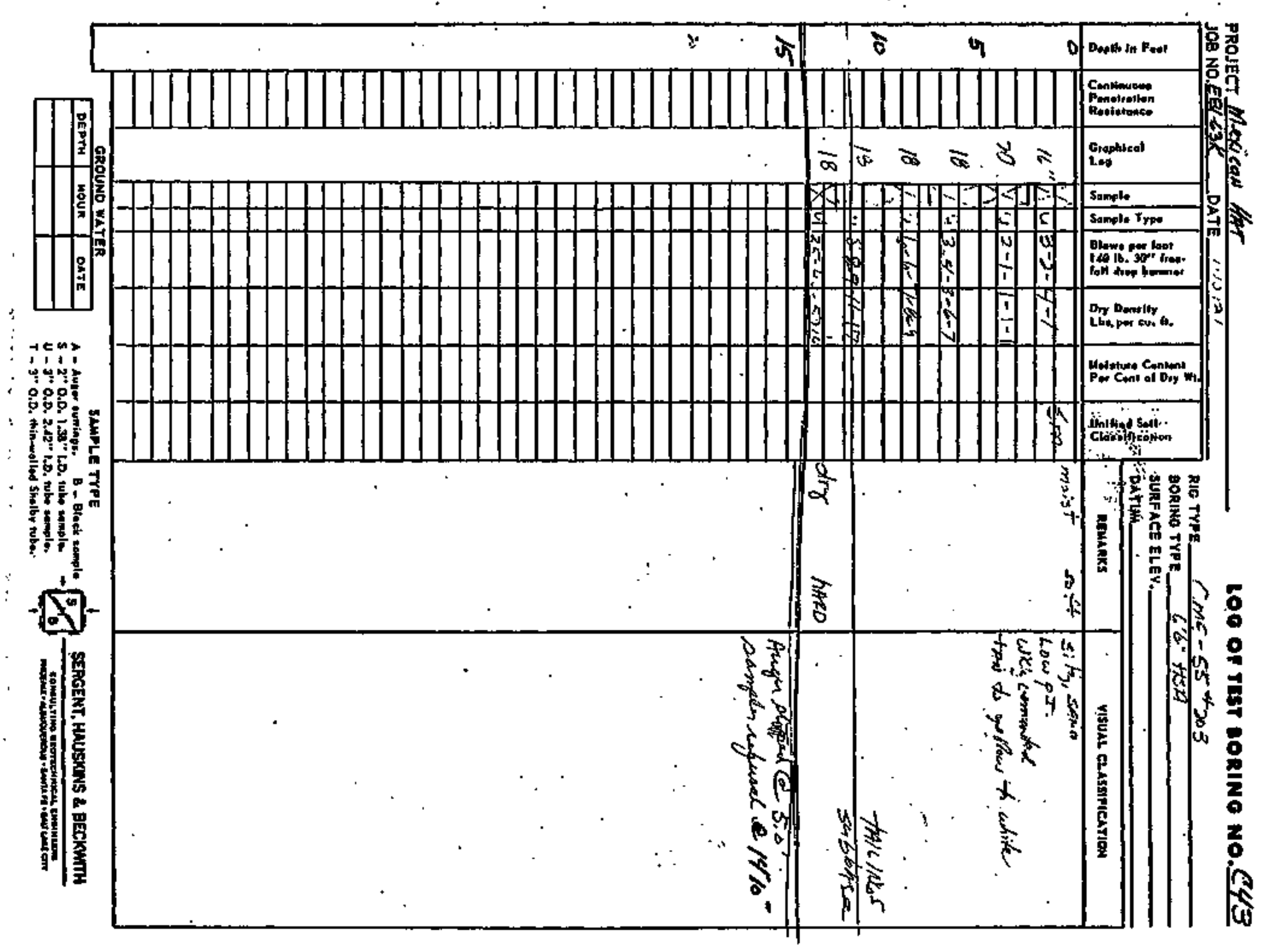




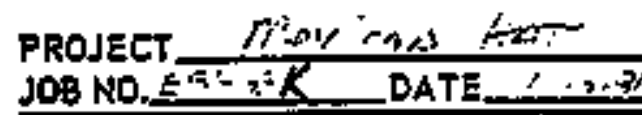

100 of rest BORINe no. C./.

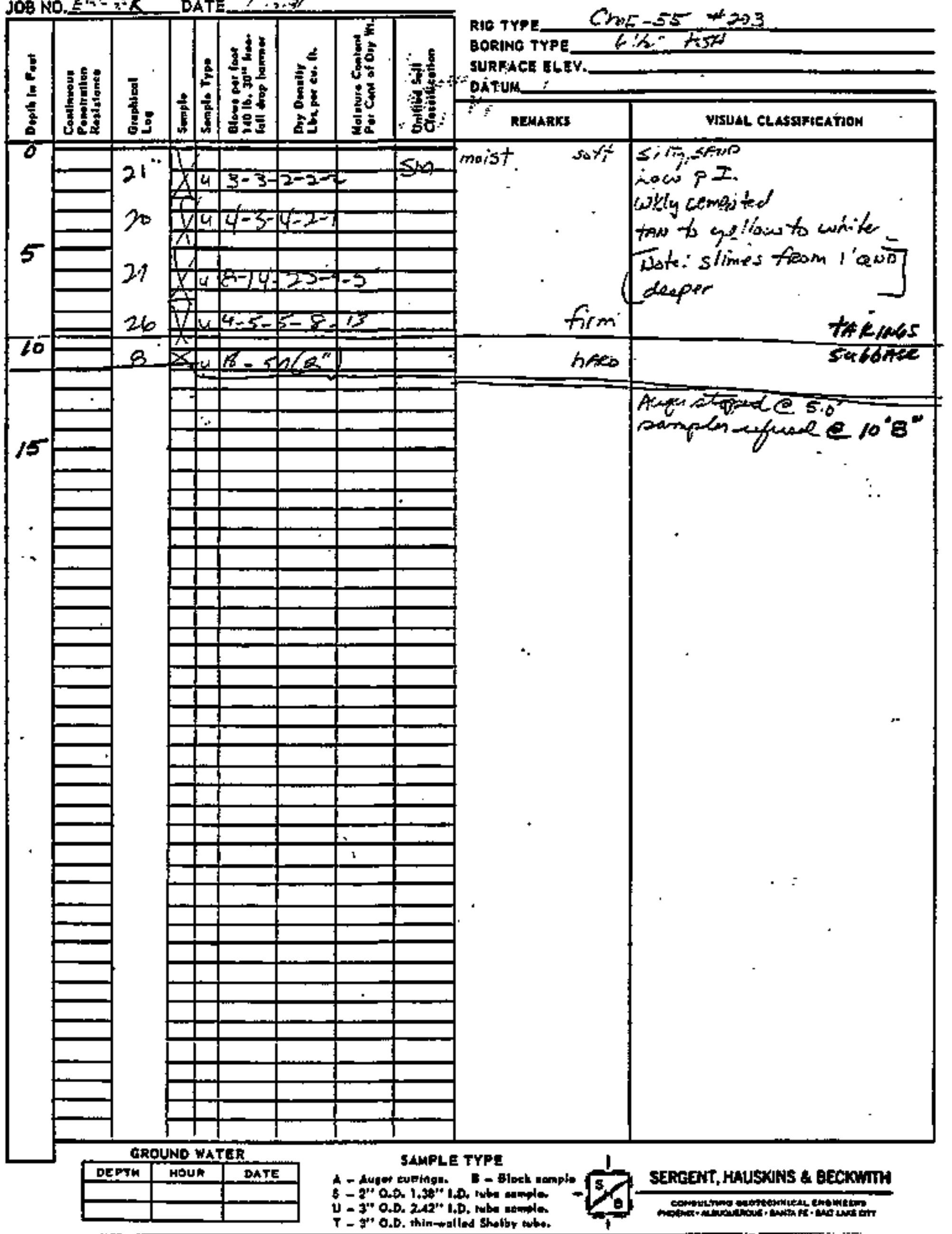


PROJECT Mexican Hat Ta.lings

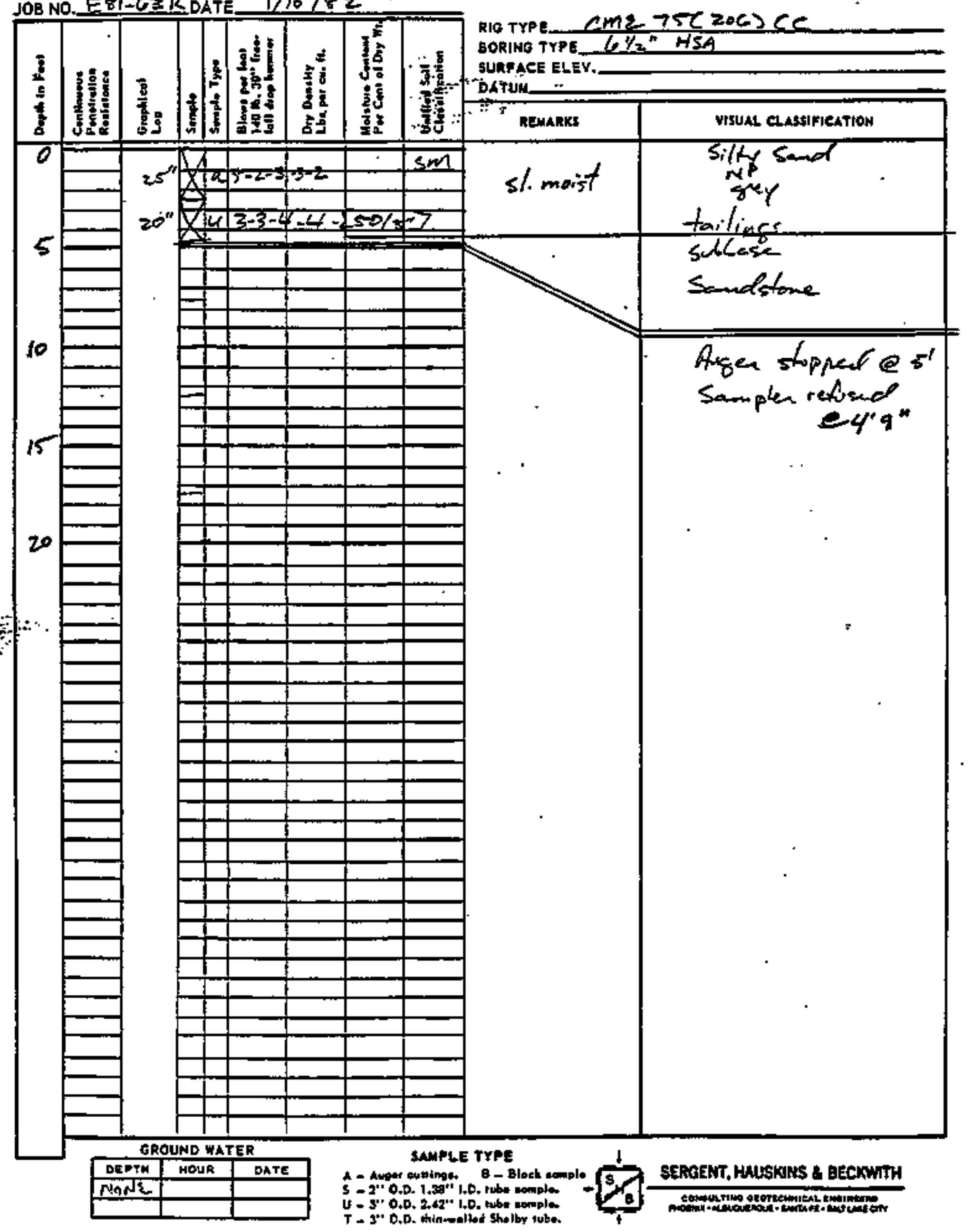


PROJECT MEXiCAN HAT JOB NOESTESK DATE

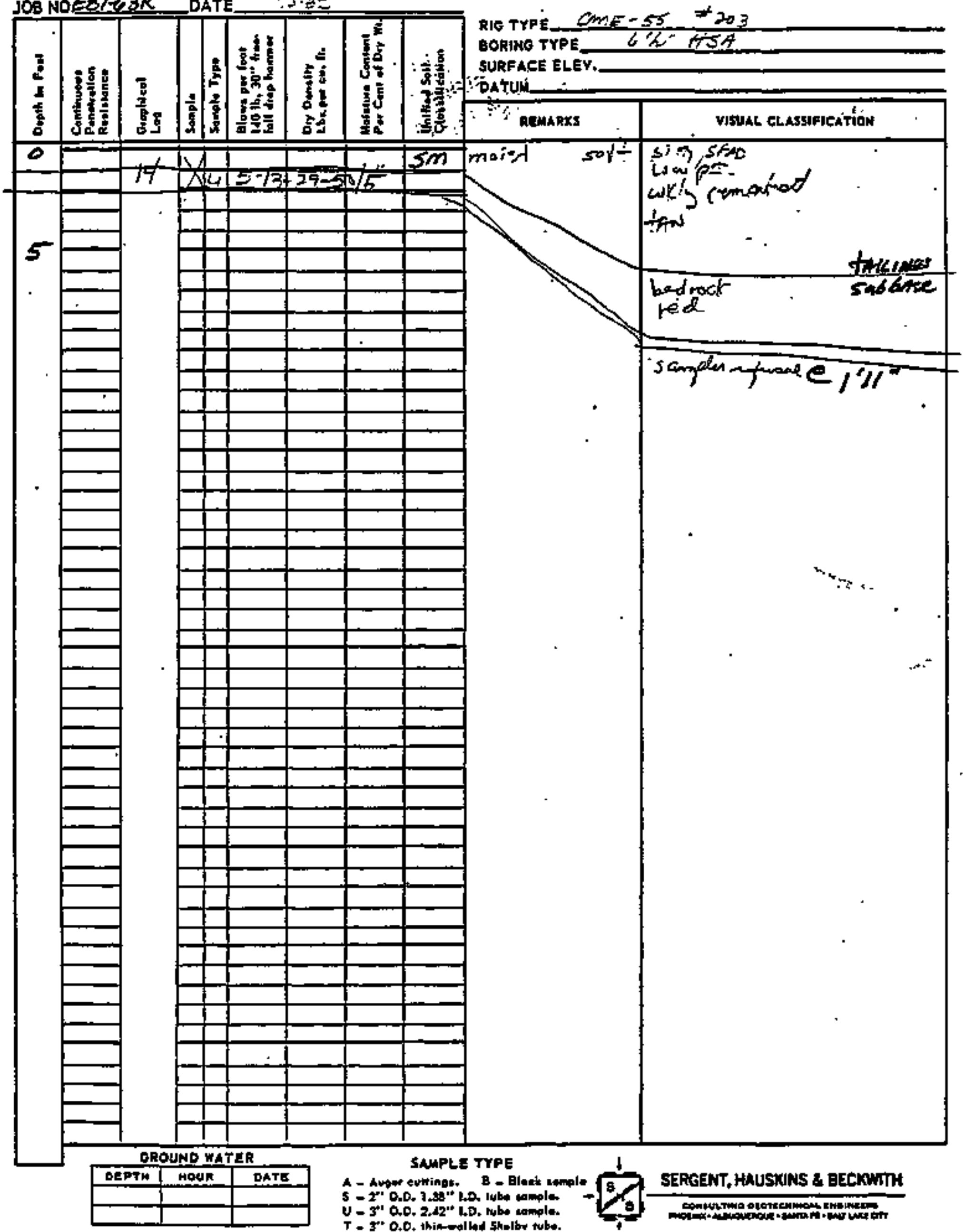


PROJECT Mexicsun Het Tailinger

LOG OI TEST BORING NO.A38 JOB NO. EES/ UAZCLDATE I/A/QI

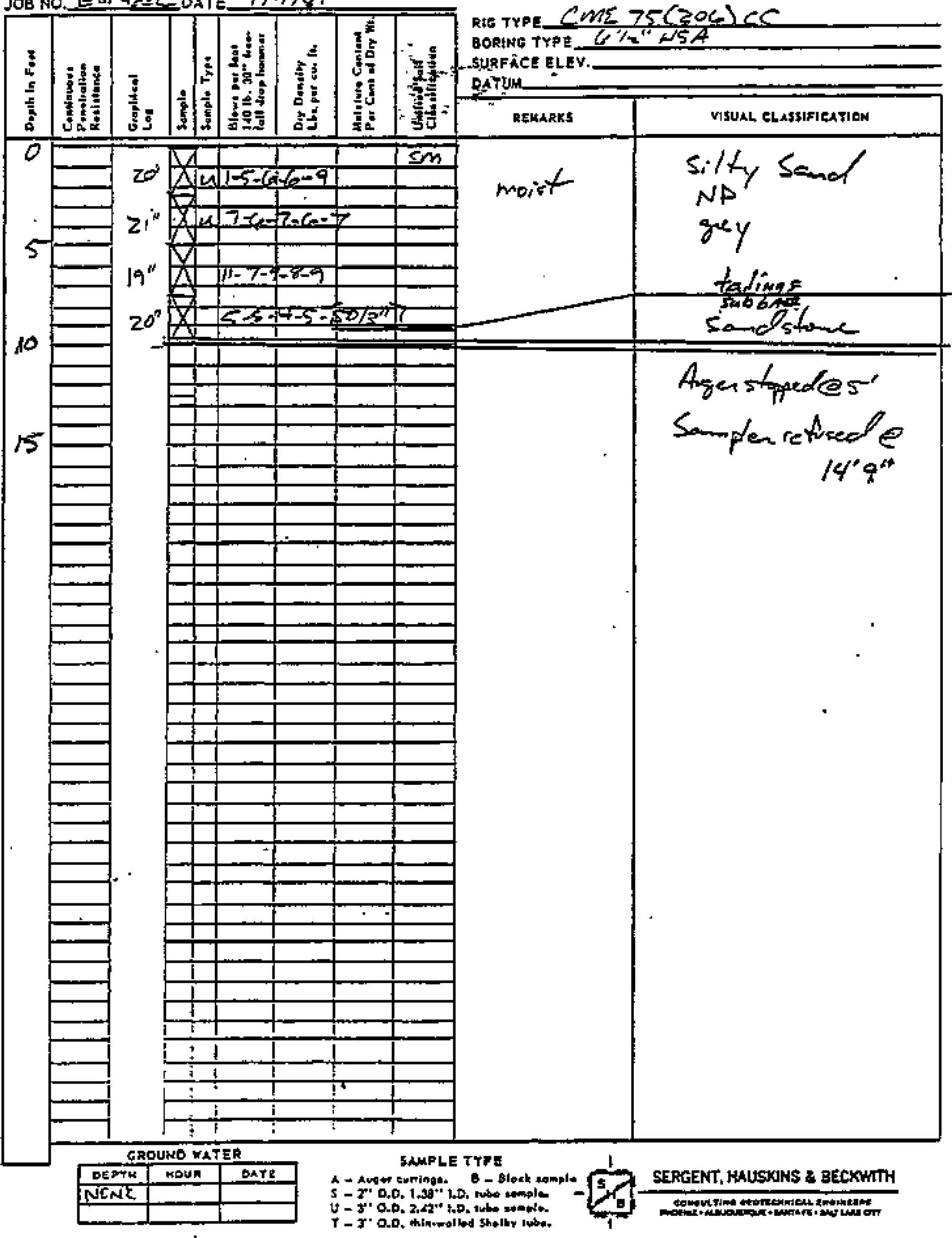


PROJECT Aex HAT

JOB NO.EBTESK DATE $\perp 19 L B Z$

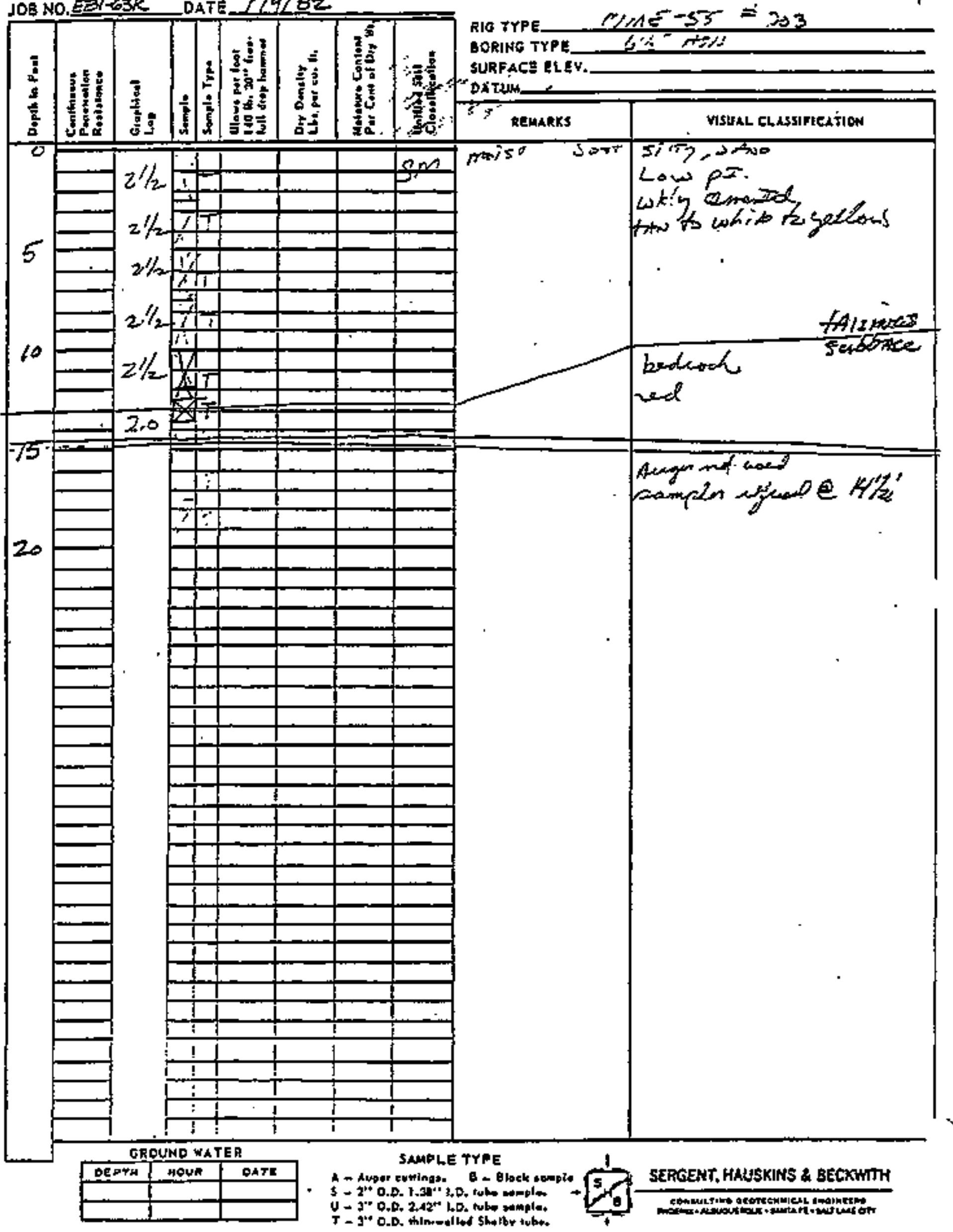

LOC OR TEST DORING NO. $\frac{.37}{6}$ RIG TYPE_LCOAS-55 $=203$ SURFACE ELEY.

Dxinu 


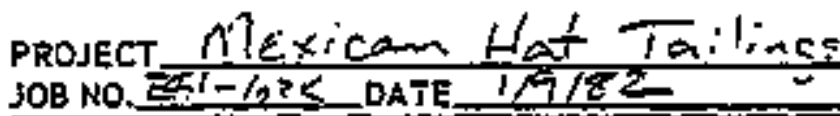

Loe OF TEST DORING No.A36

ms $75(206) \mathrm{CC}$

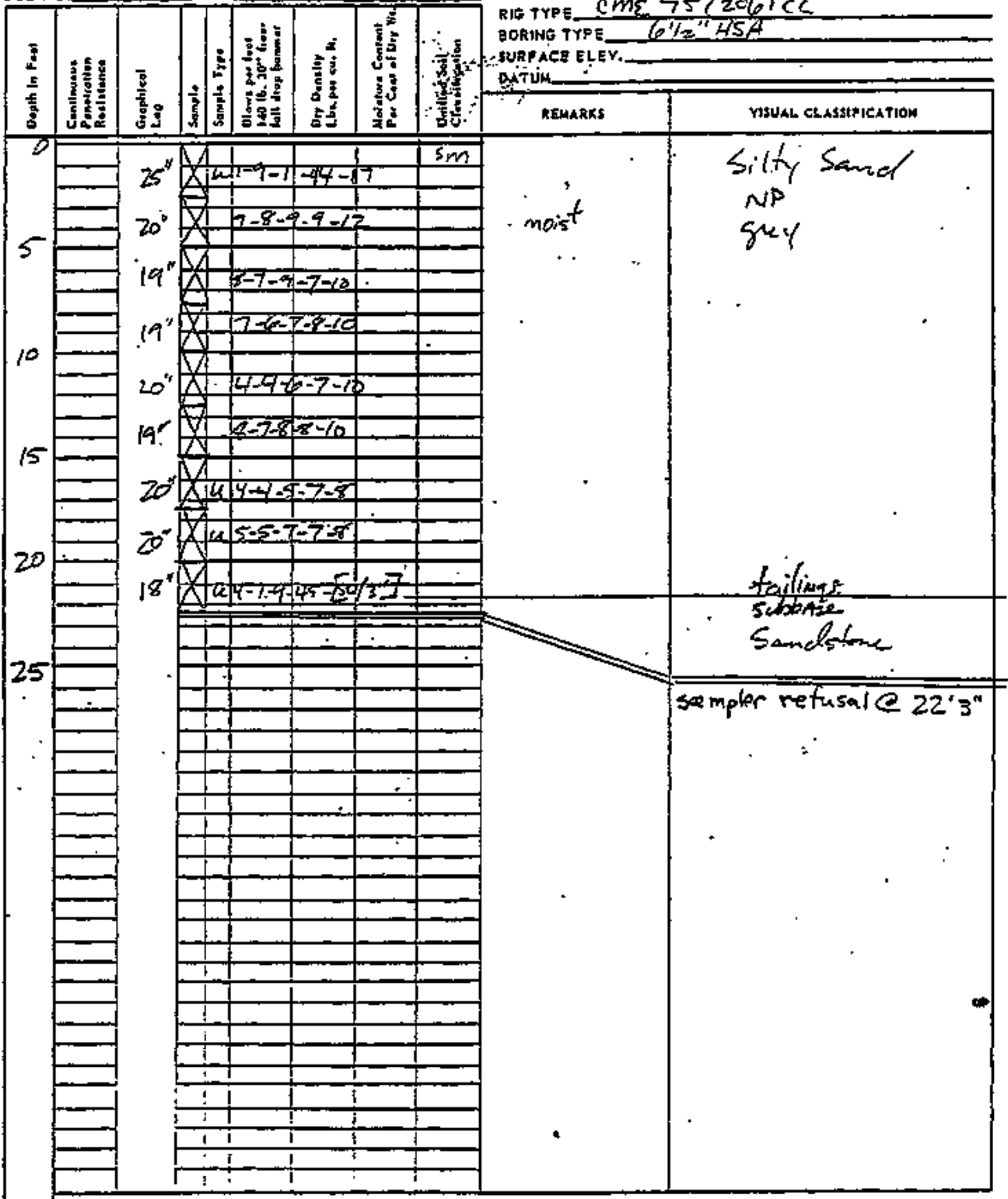

GROUNO WATER

\begin{tabular}{|c|c|c|}
\hline DEPTM & MOLA & DATE \\
\hline NEMF & & \\
\hline & & \\
\hline
\end{tabular}

5AAPLE TYPE

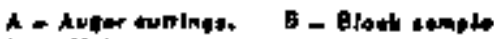

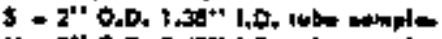

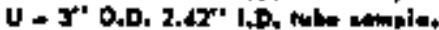

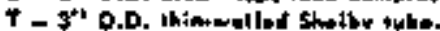

SERGENT, HAUSKINS \& BECKWTH

ب

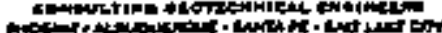




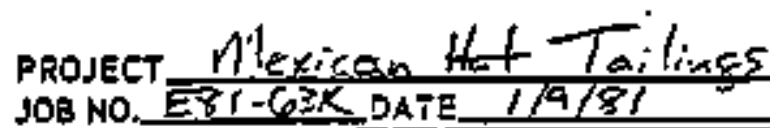

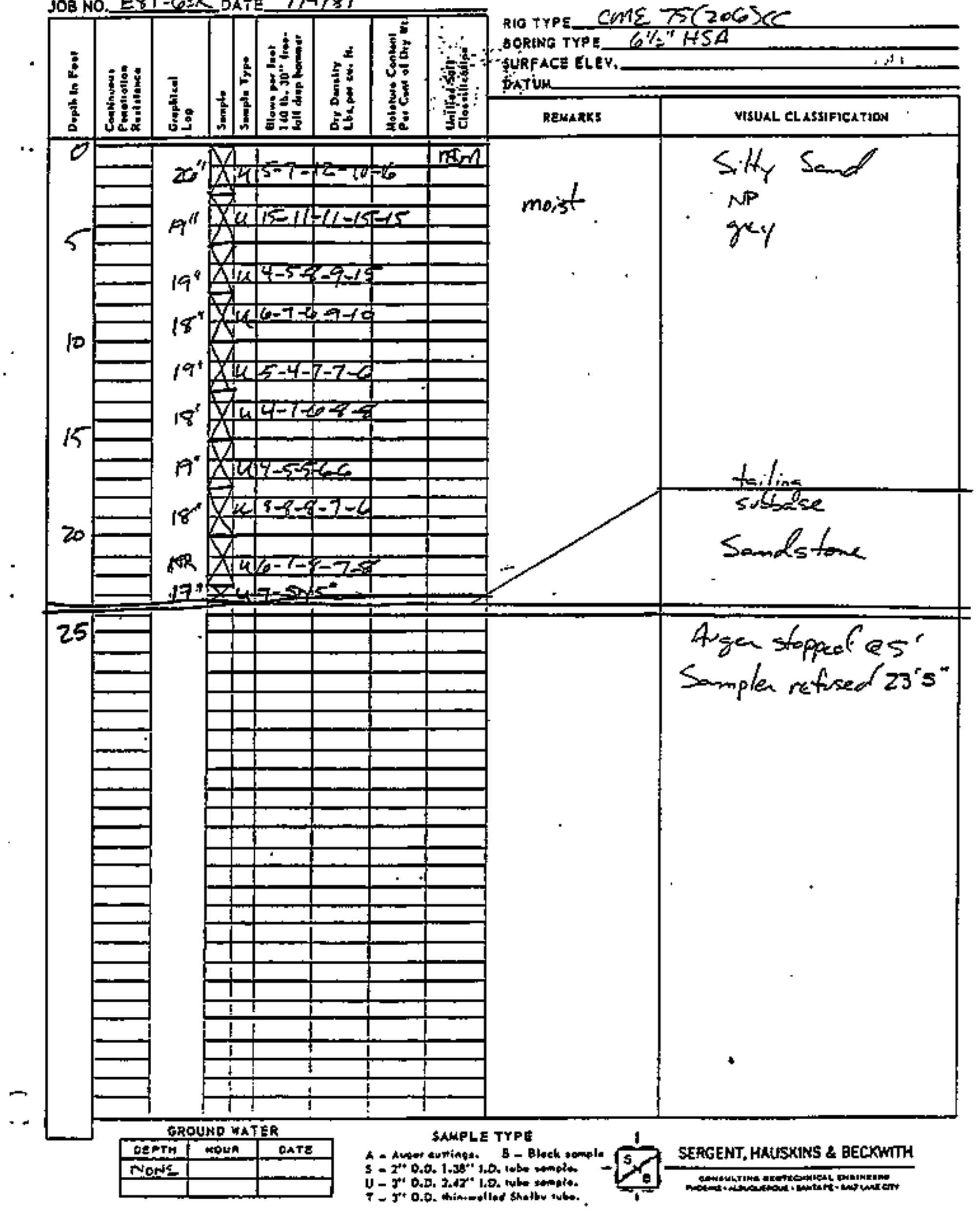


FRONECT MleXiCAN Vtat JOB NDEET-GBK DATE / / 192

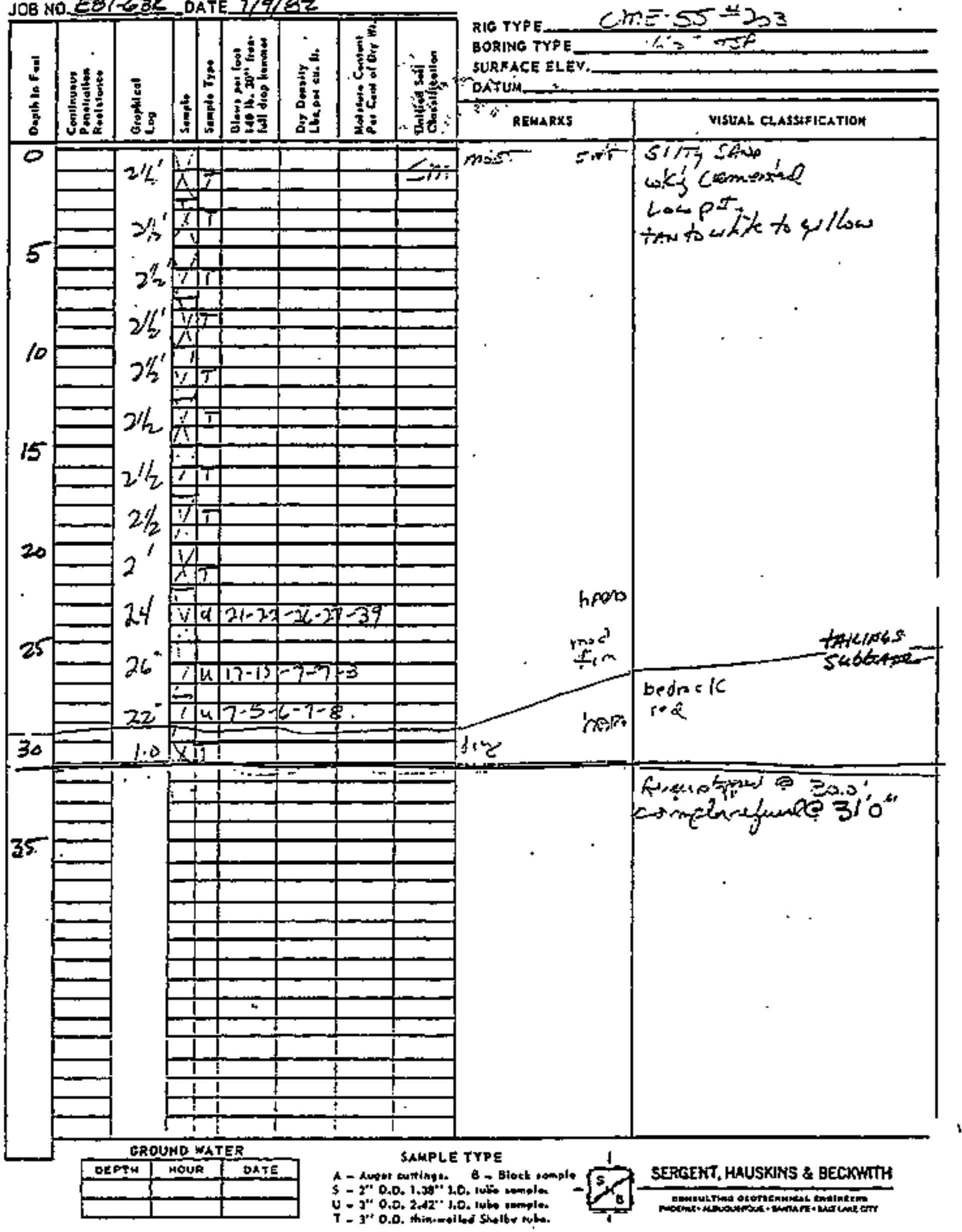

LOQ OF TEST SORINe NOB35 ch:-5S $=23$ 


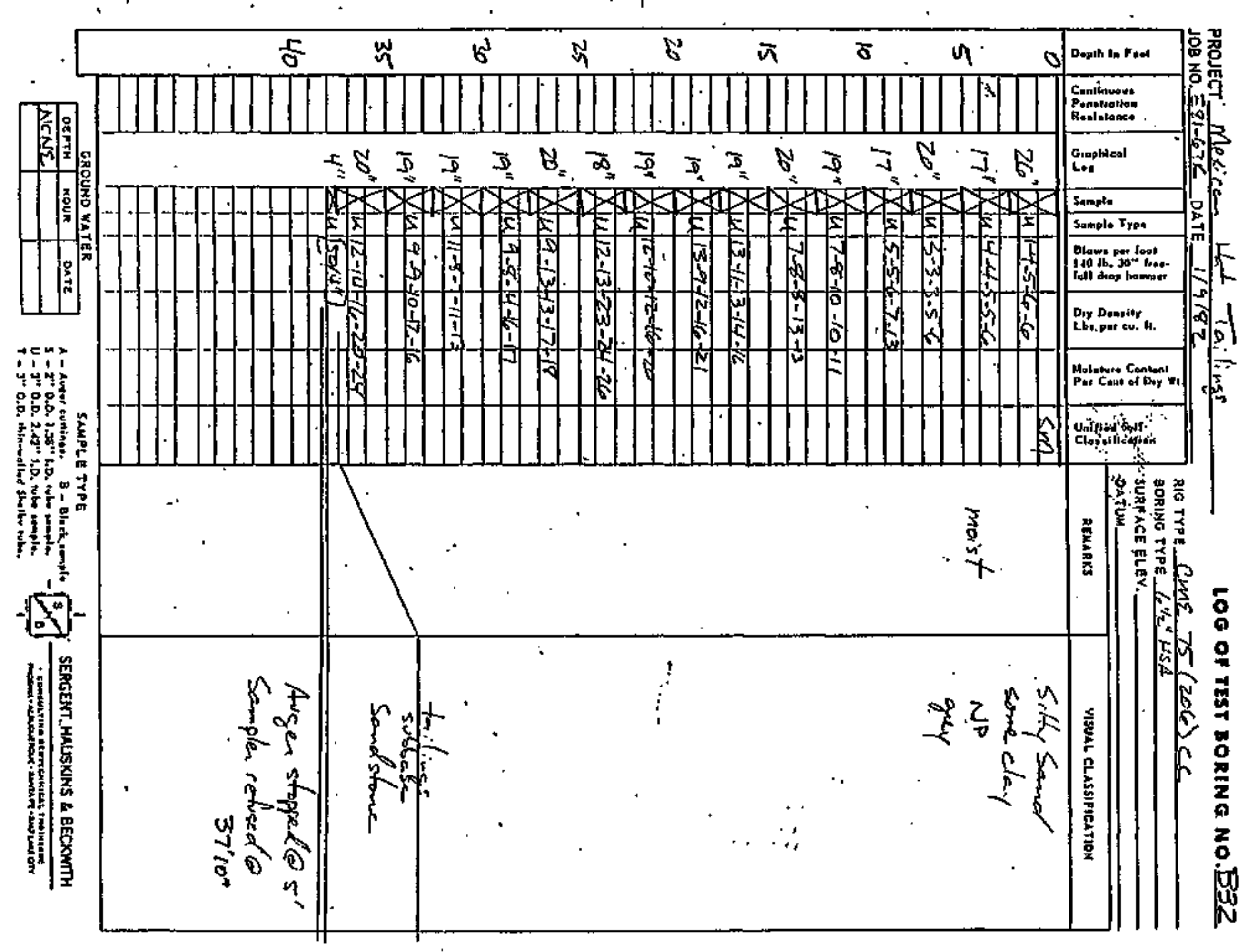


PROJECT MEXiCAN HAT JOB NO ERTKGK DATE I/G/E2

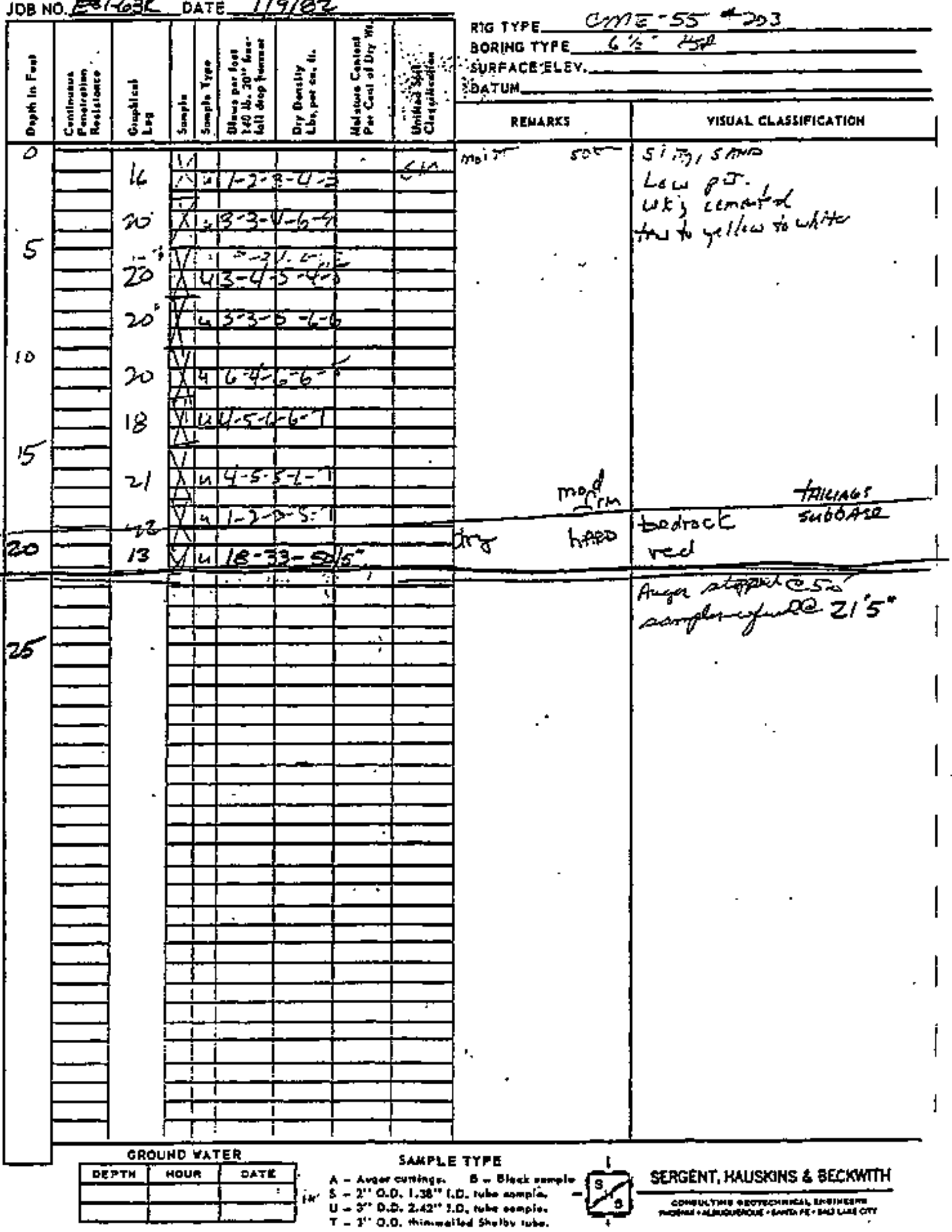


PROJECT $\quad \gamma^{\circ} 0: \quad \cdots \%^{-*}$

JOB NO.EEKS $K$ DATE_.?.'

LOG OF TKST BORLNG NO.B3O

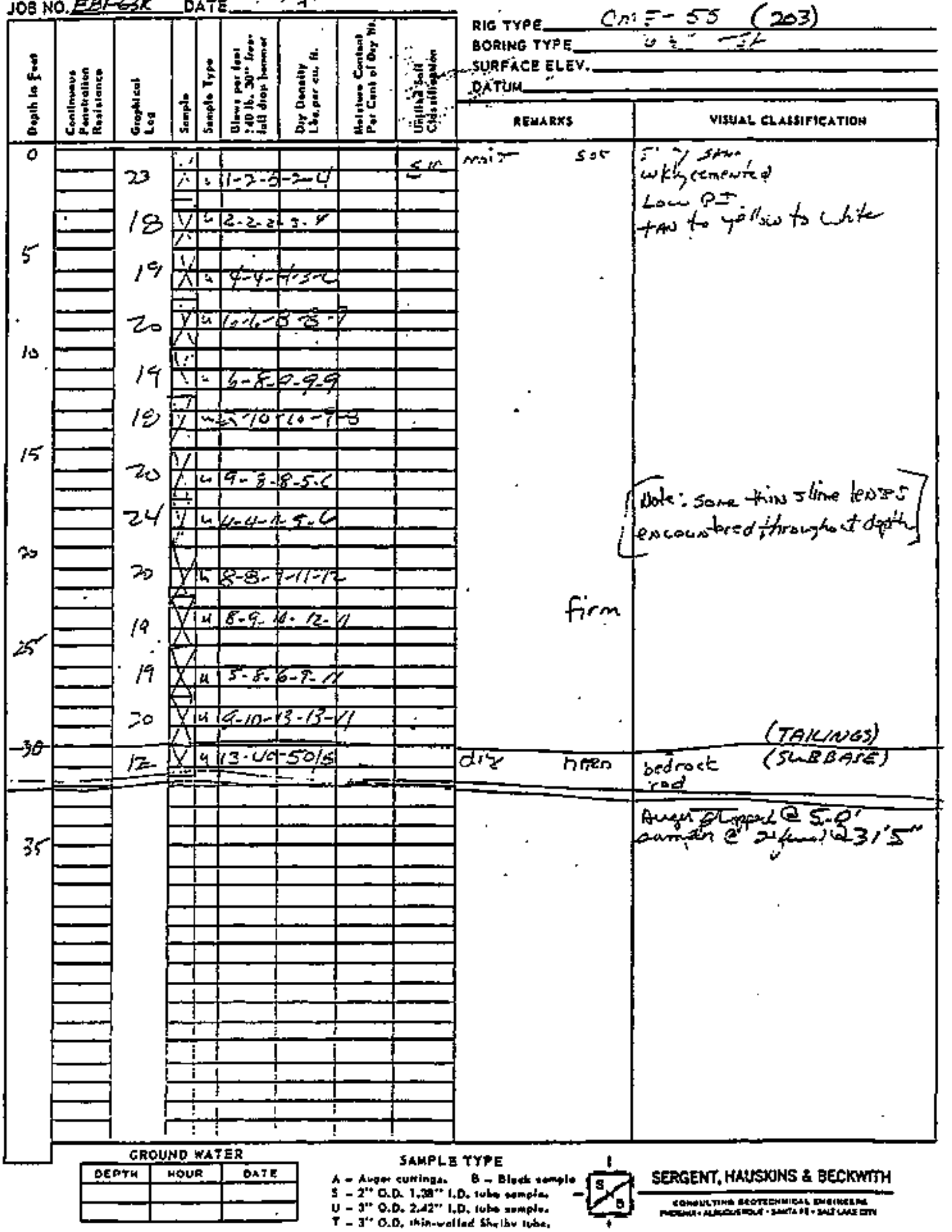




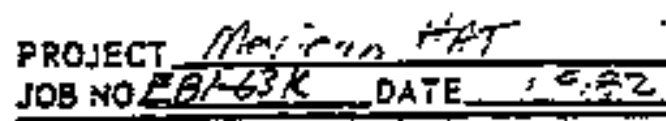

LOC OF REST BORINO NO.EZI

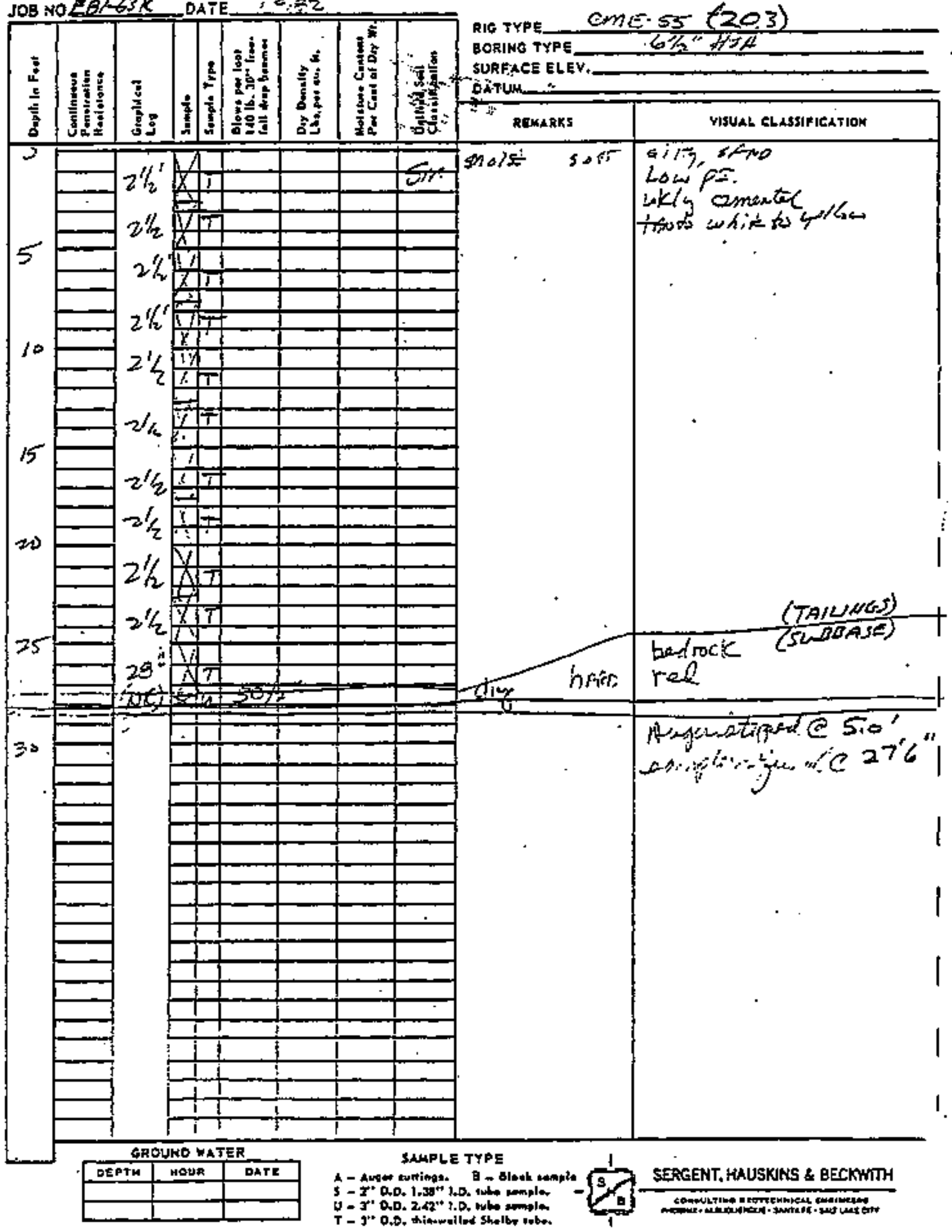



PROJECT Mexican Hat Talings

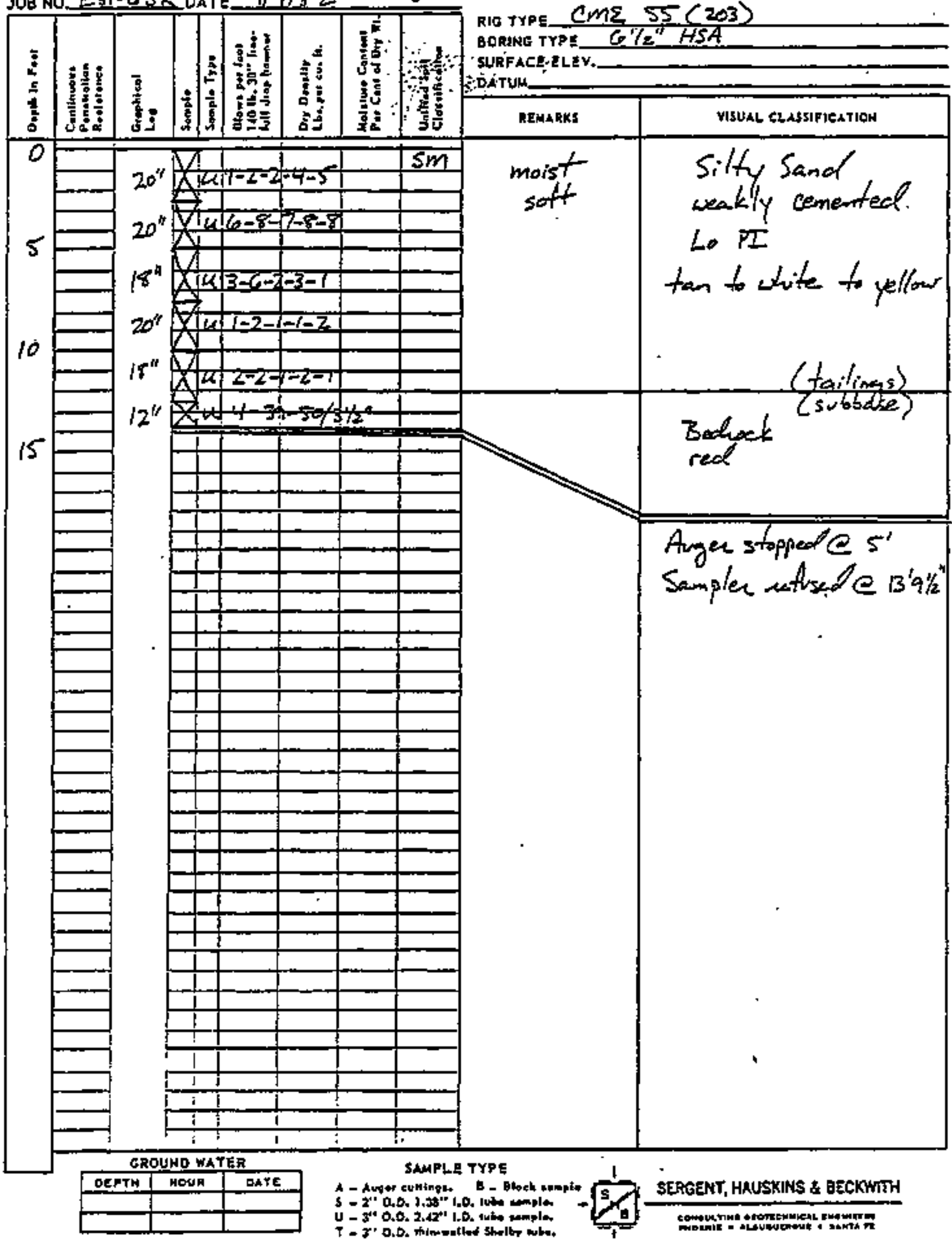

LOO OR TIST BORING NO.B- -28 RIG TYPE CME $55(203)$

THNG TYPE CO 


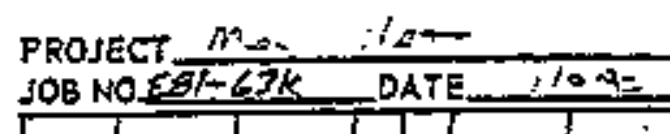

LOG OF TIST DORING NO.A29

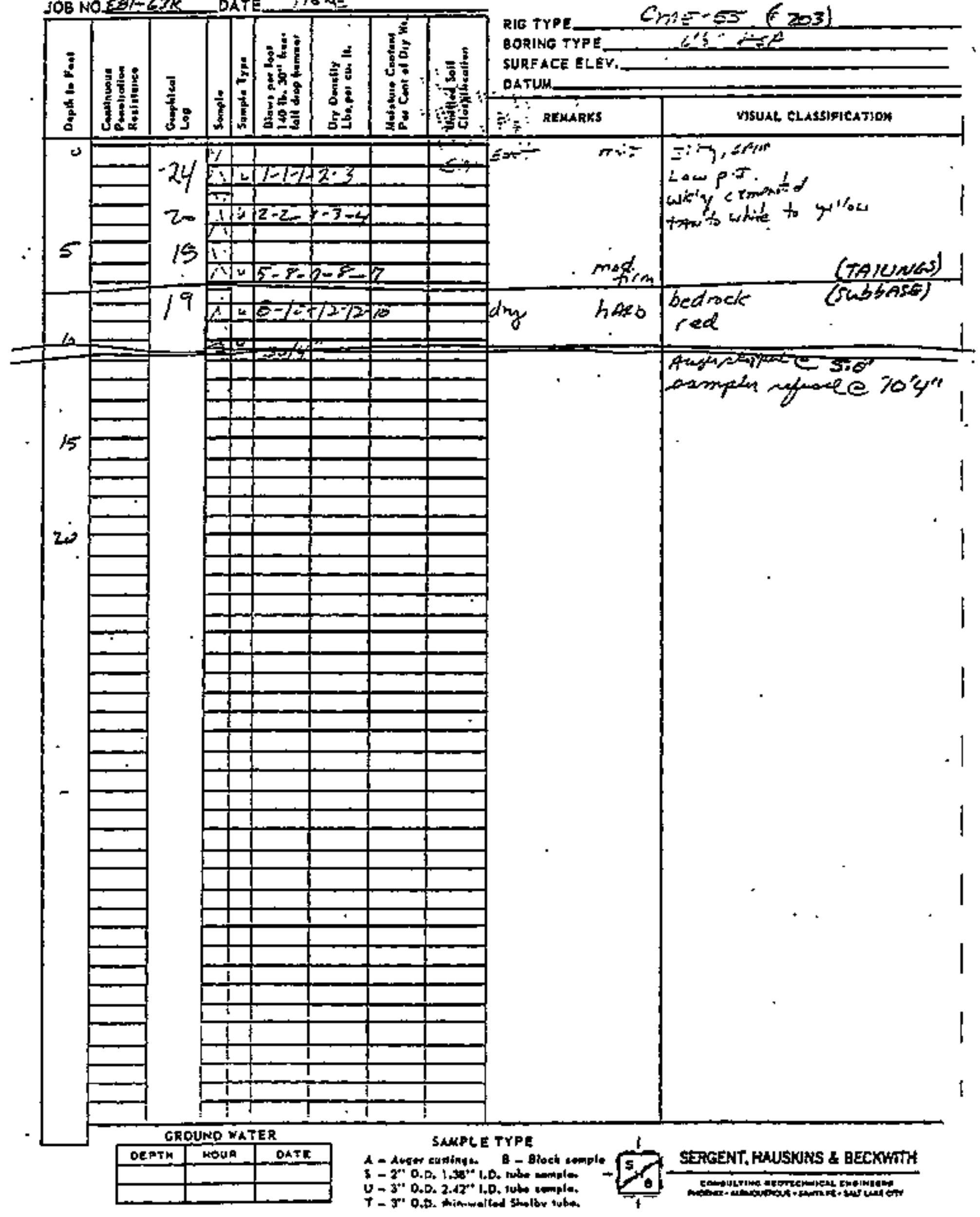




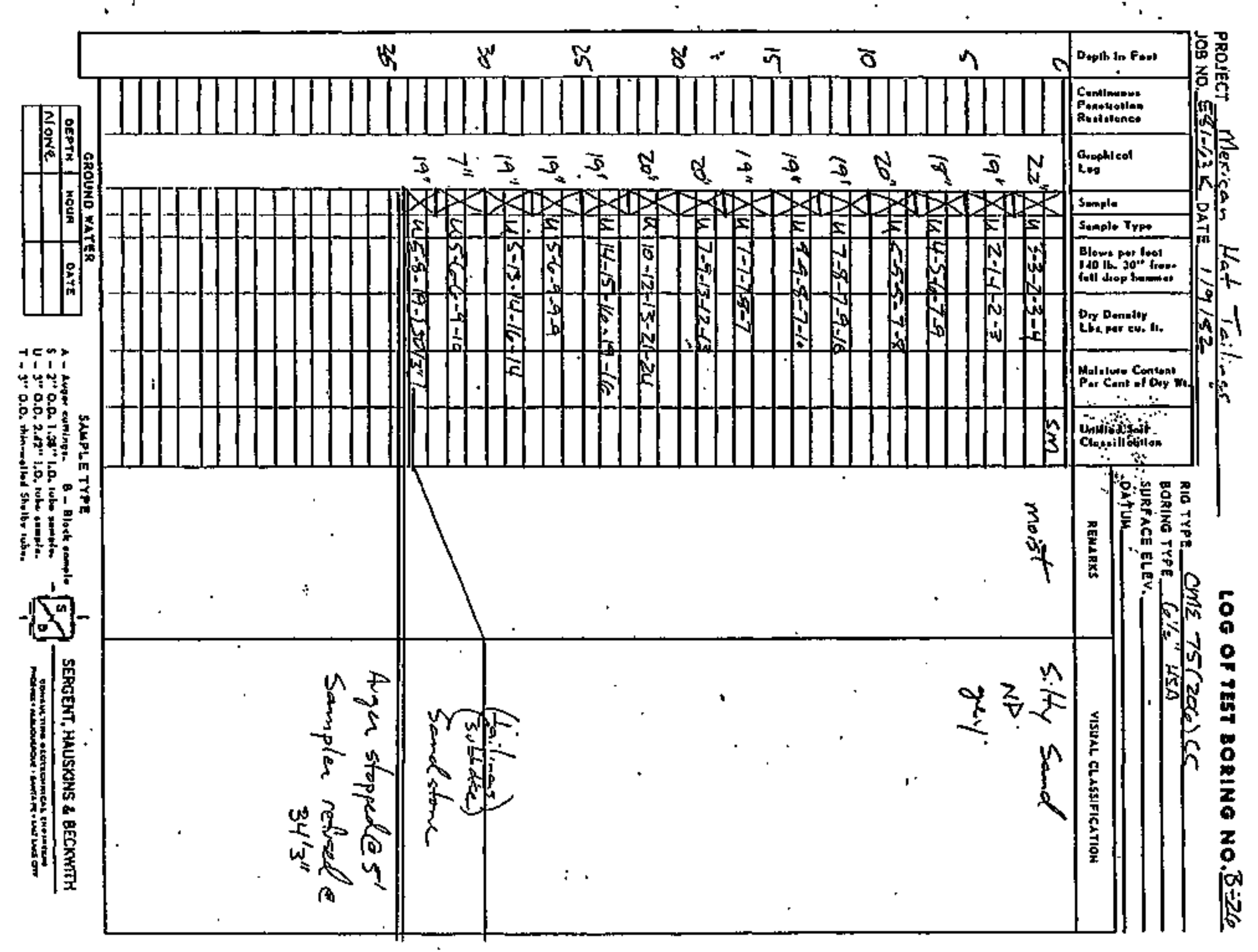


PROJECT rilexican that Tailings JOB NO. ES/-636DATE $/ / 9 / 82$ i)

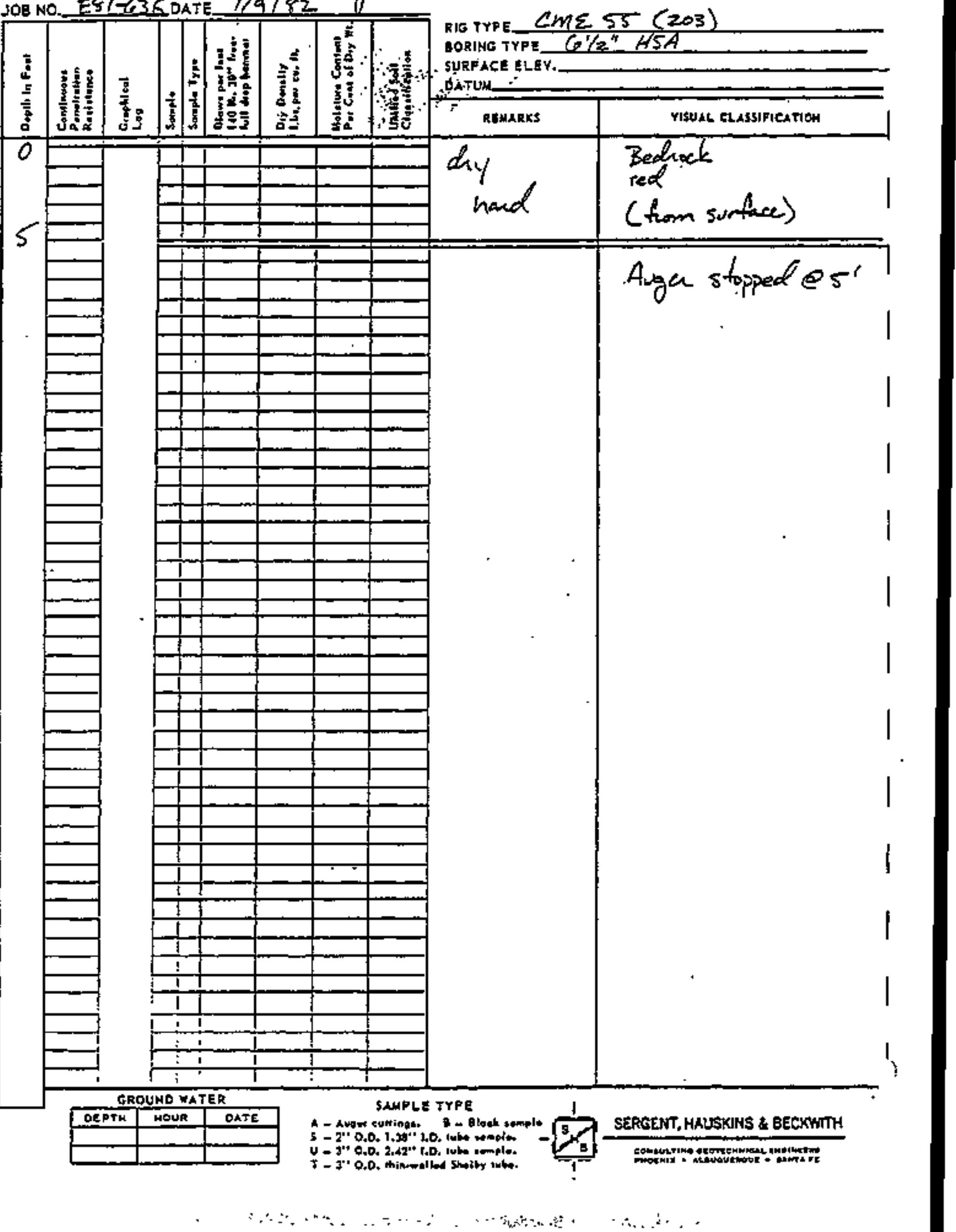


PROJECT Mexican Hat Tailines

LOO OF TESt BORINO NO.B-24

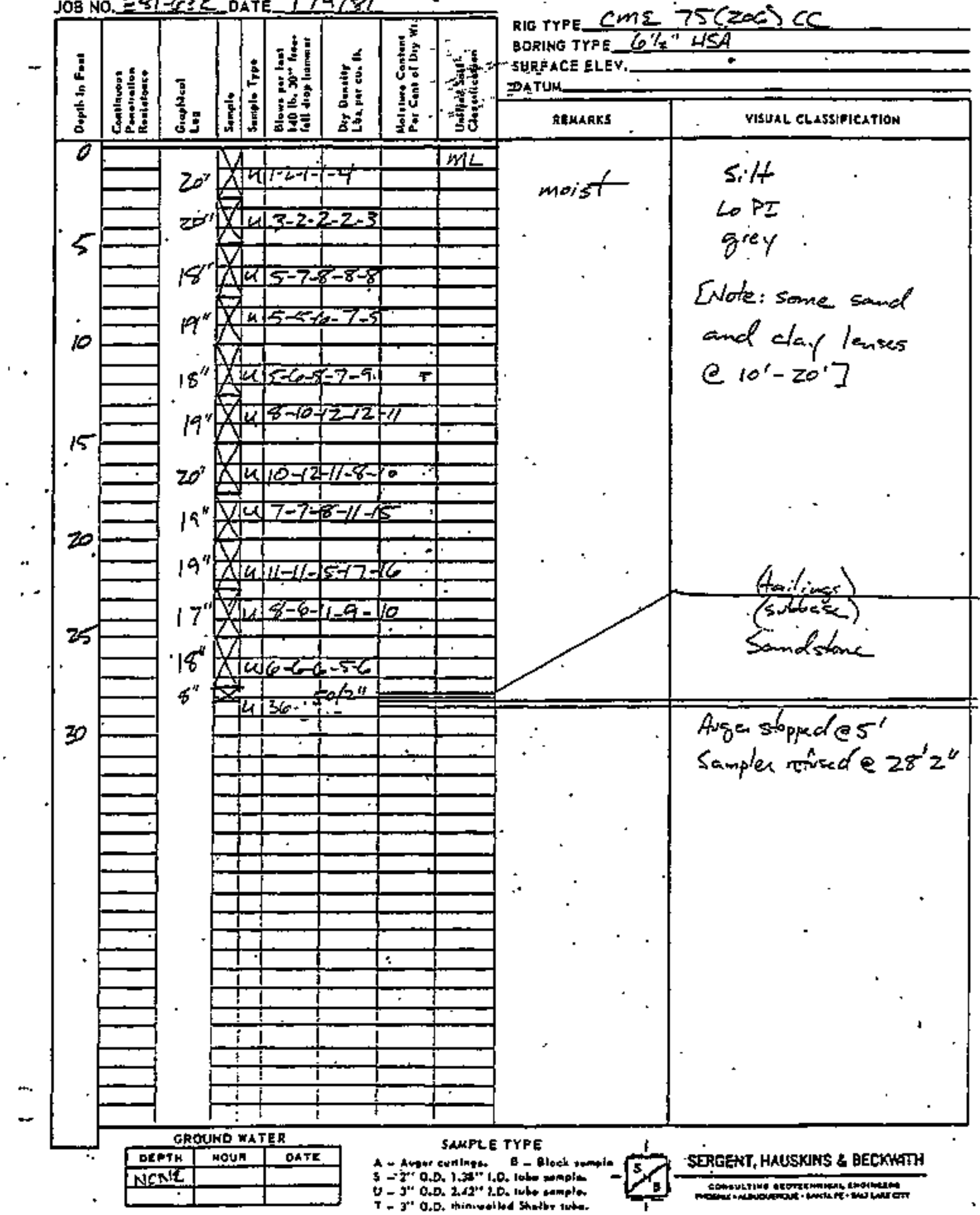



PROJECT Mexican Hat Tailings
JOB NO.ES1-63K DATE $1 / 9 / 22$ o

LOE OF TEST DORING NO.A-ZS

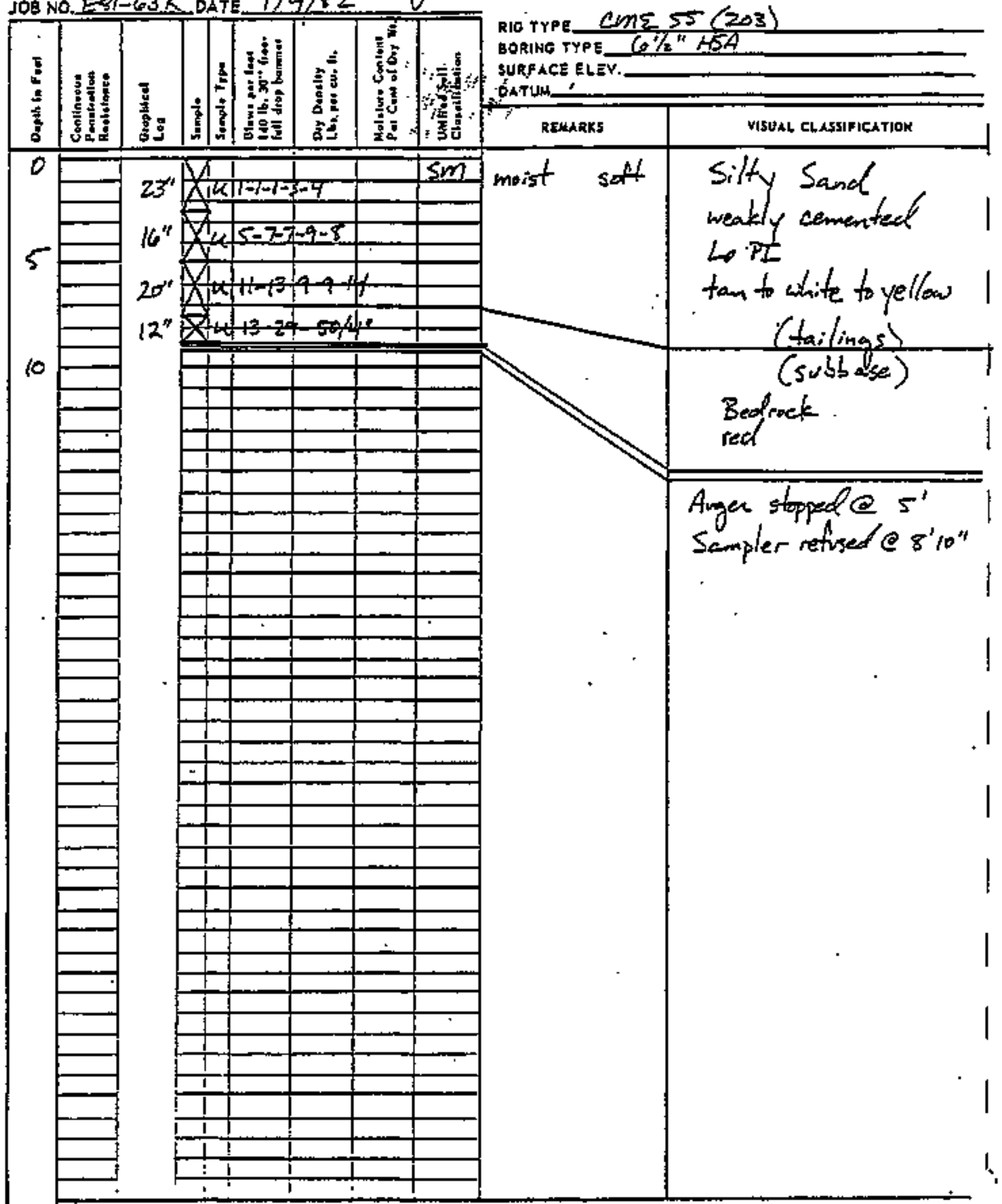

GRDUHD WATER

\begin{tabular}{|l|l|l|}
\hline DEPTH & MOUN & DATE \\
\hline & & \\
\hline & & \\
\hline
\end{tabular}

\section{SNAPLE TYPE}

A - Awhor curtingat B - Bloch somplo

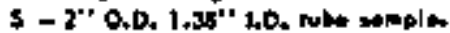

U - 9" O.D. 2.42" J.0. Iut cenmple.

7 - 3" O.D. Hinemalled Shetby inte.

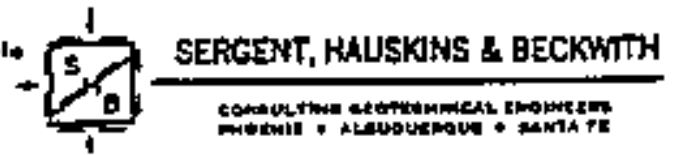




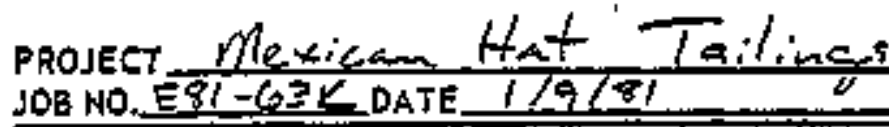

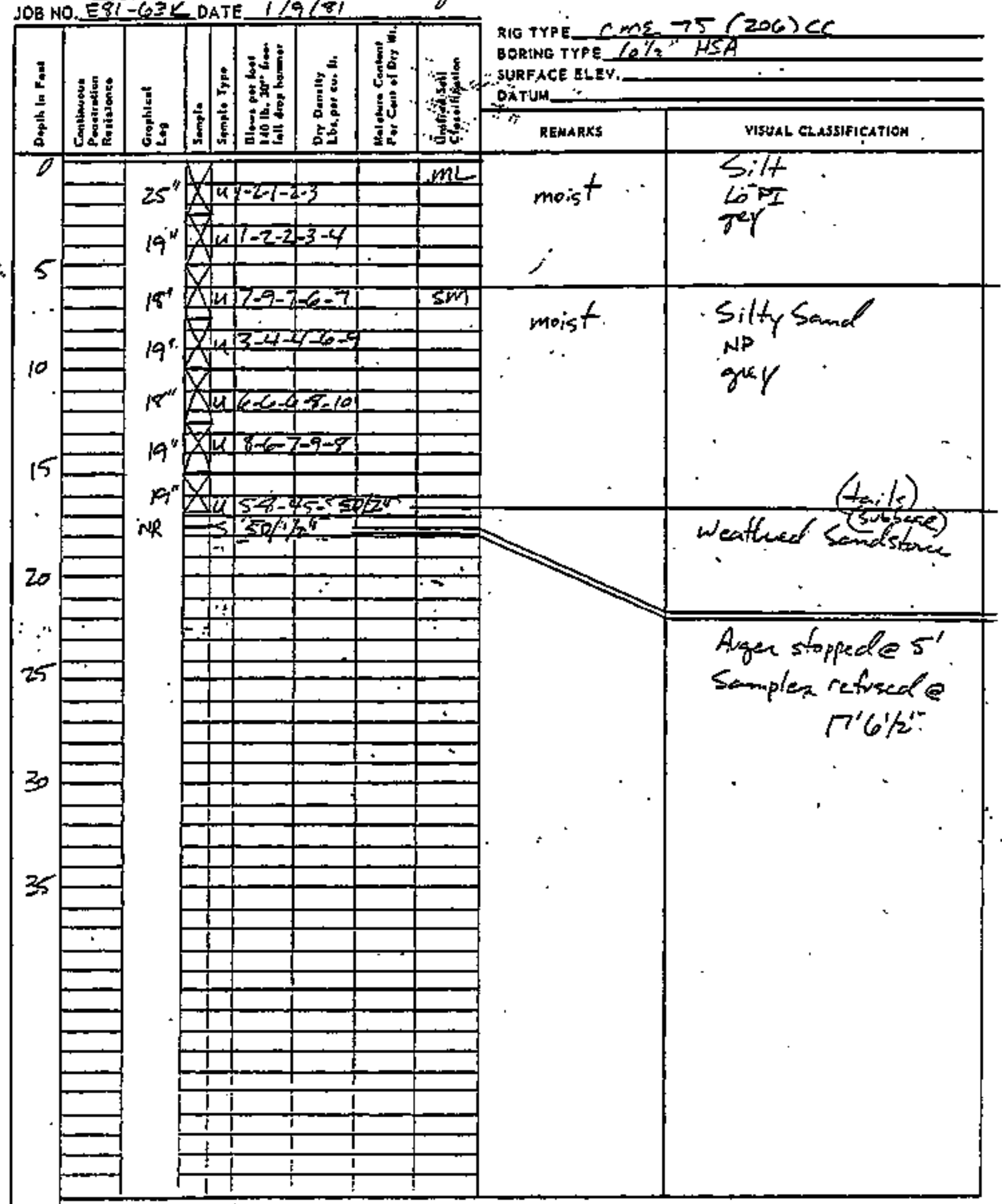

GROUHE YATER

\begin{tabular}{|l|l|l|}
\hline DEPTH & MOUN & DATE \\
\hline NONLE & & \\
\hline & & \\
\hline
\end{tabular}

SAMPLE TYPE

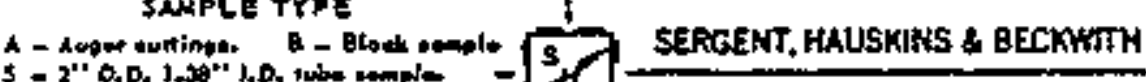

U - J"T O.D. 2.42" 1.D. 14k momple.

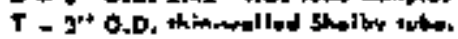


PROJECT Mexican Hat Tailings

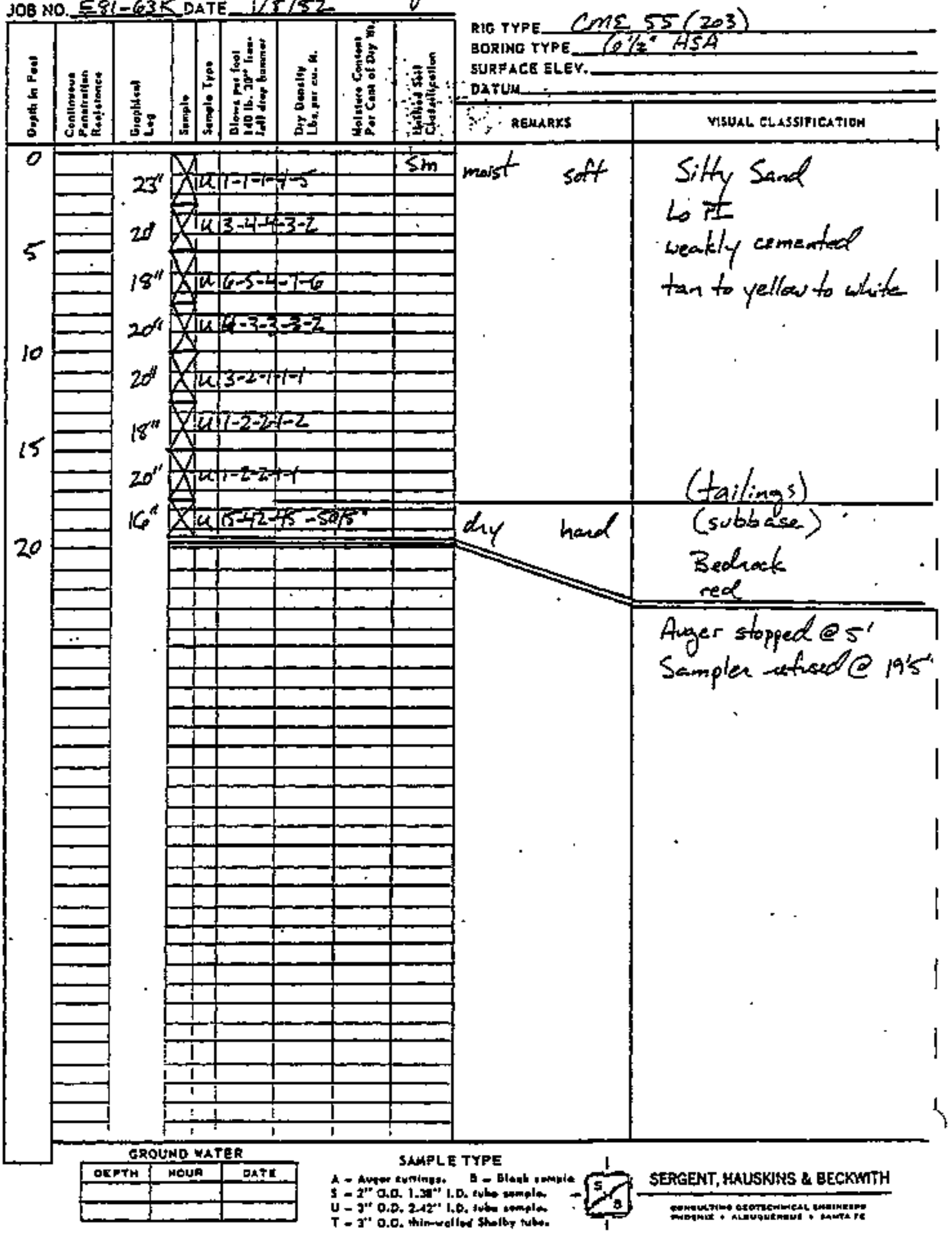




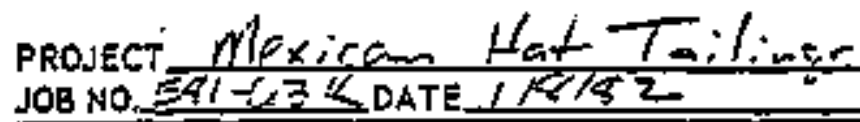

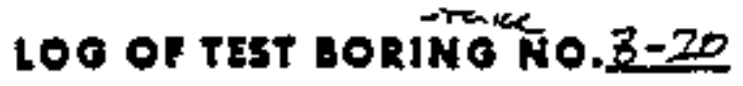

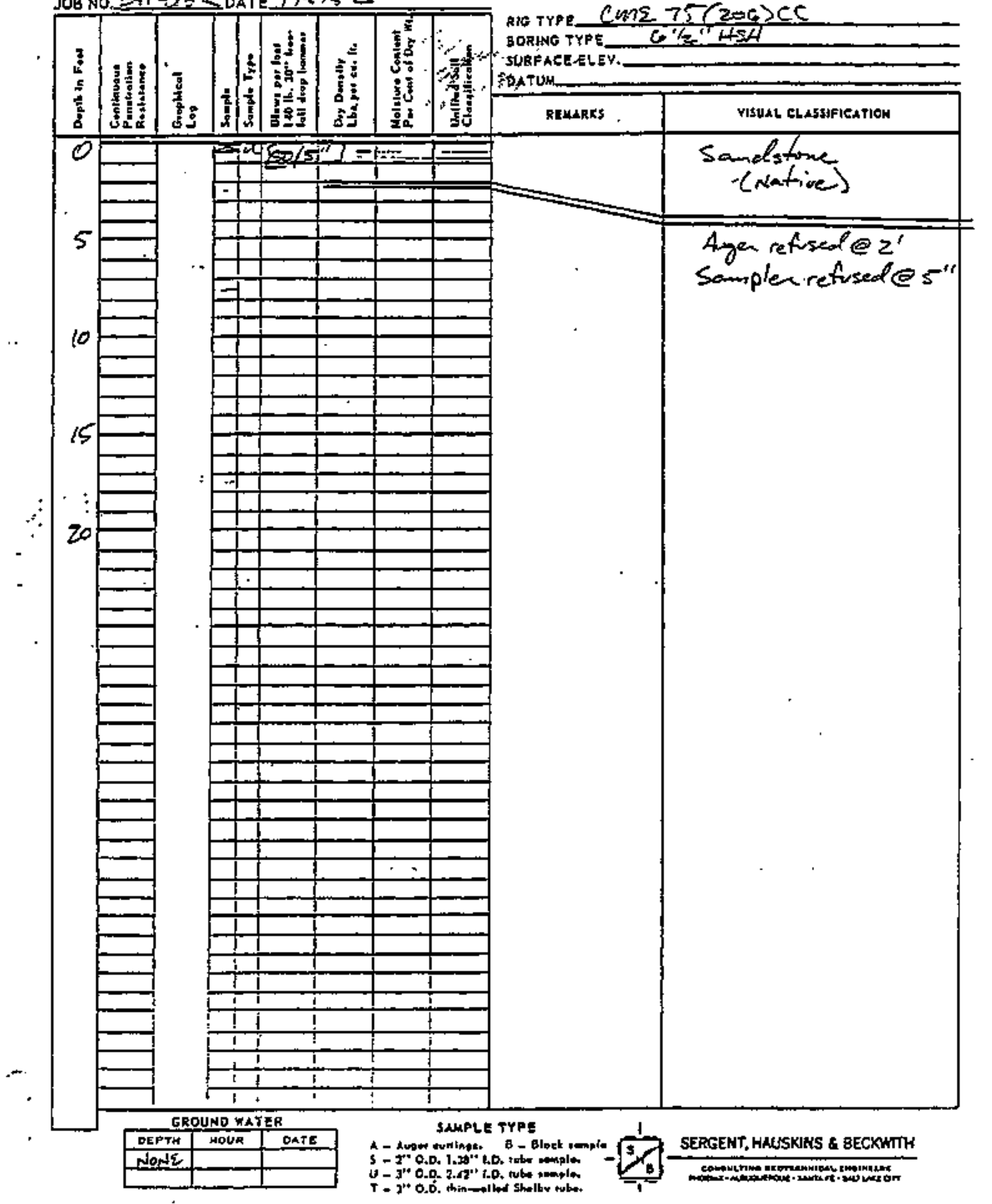


PROJECT Mexican Hat Tailings

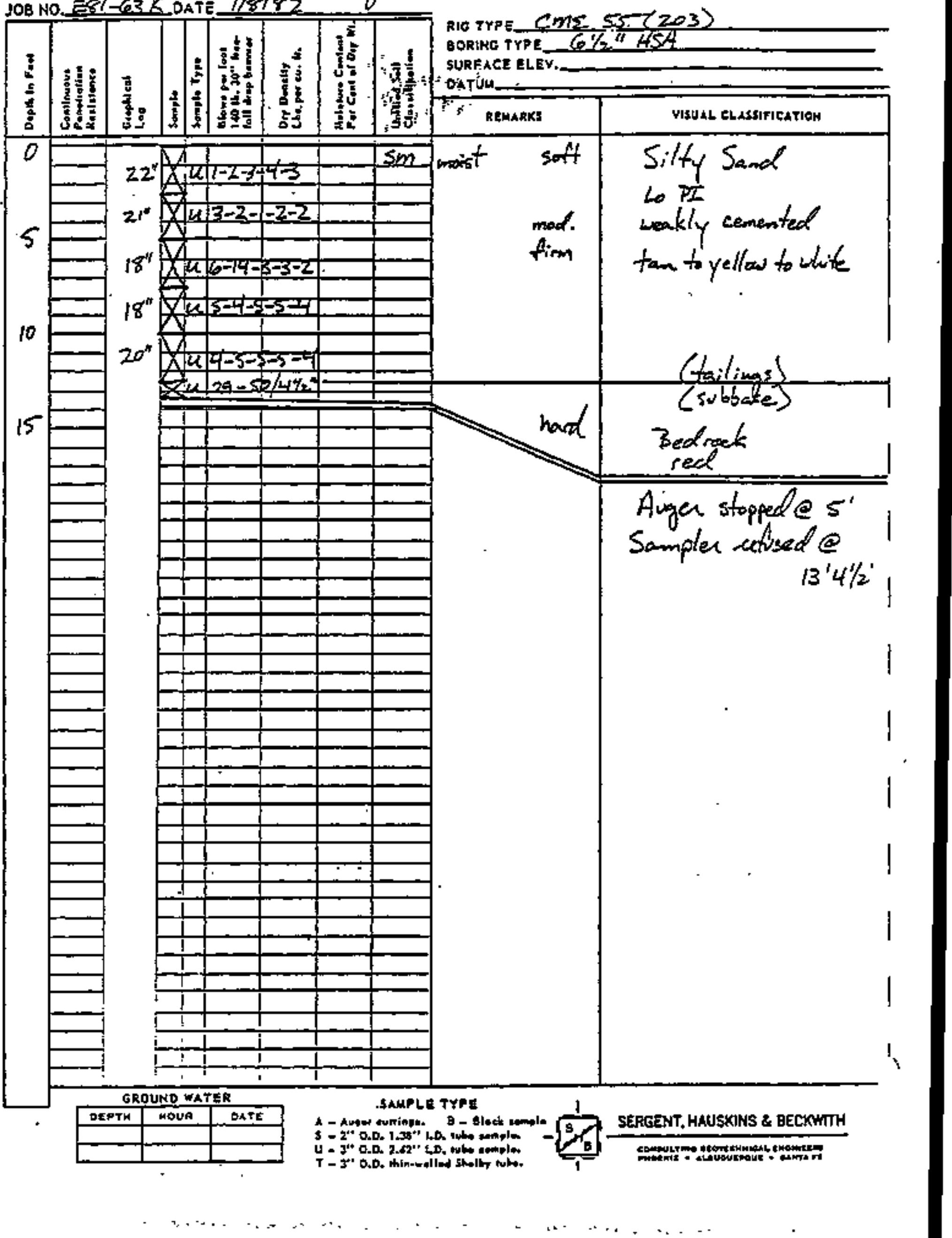




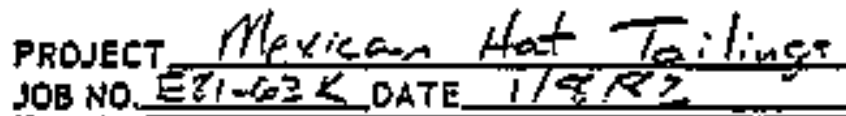

LOC OF TEST DORING NO.B-IS

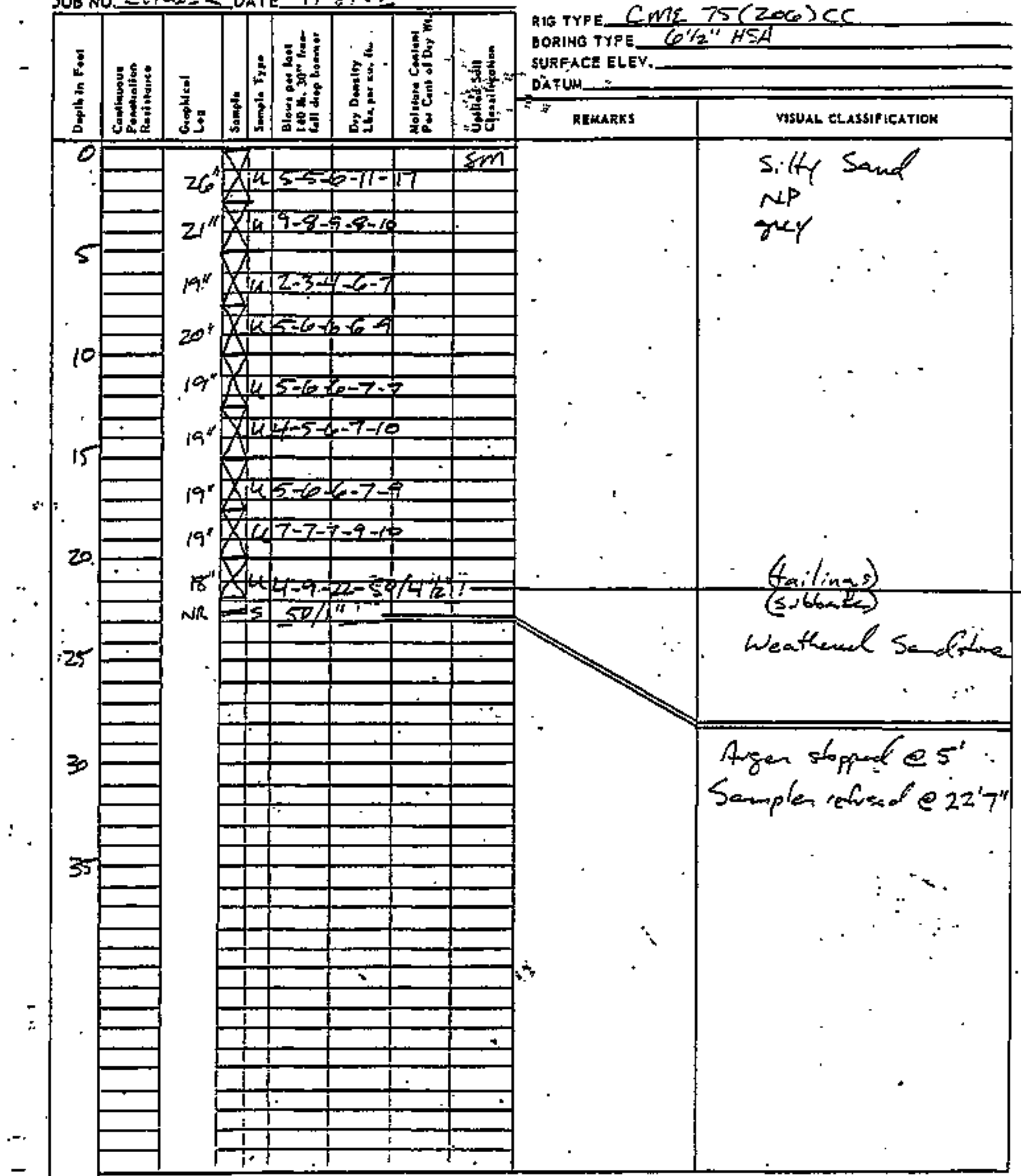


PROJECT Mefican that trailings JOB NO. ESI-G3K DATE 1/8182

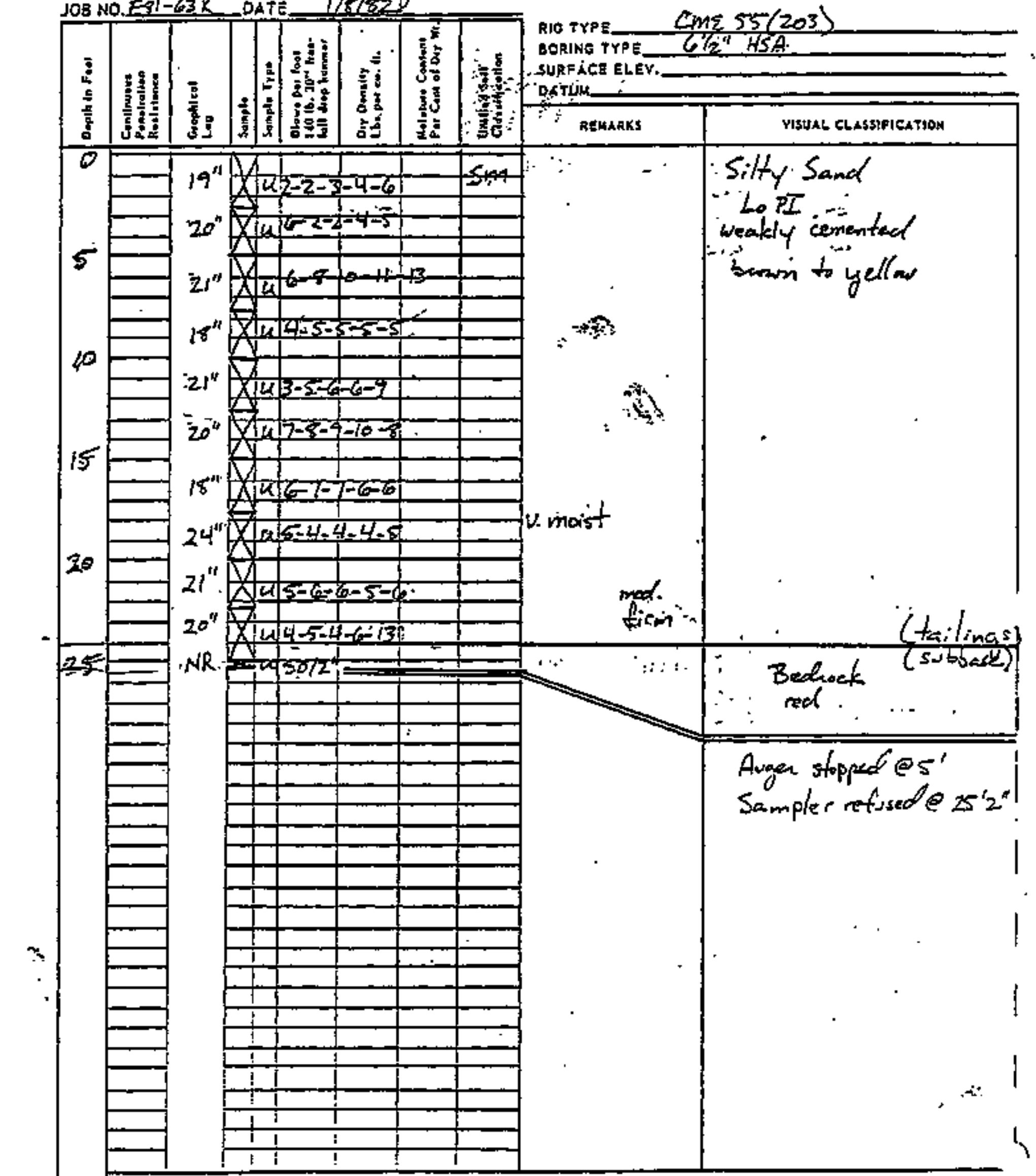

GROURD WATER \begin{tabular}{|l|l|l|}
\hline DEPTM & MOUA & DATE \\
\hline
\end{tabular}

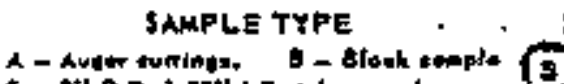

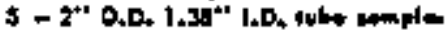
L $-3^{+4}$ O.D. Z.A2" I,D, tobs temole.

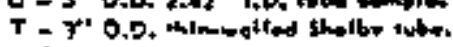

LOG OF TEST BORING NO.B-19. $\operatorname{cins} 55(203)$ RIC TYPE SURFACE ELEV.

\section{weakly cemented sormin to yellow}


PRoject . Mexicen 1 tat Tiril: JOB NO. EQ $1-143 \% 0 A T E$ I/87/52

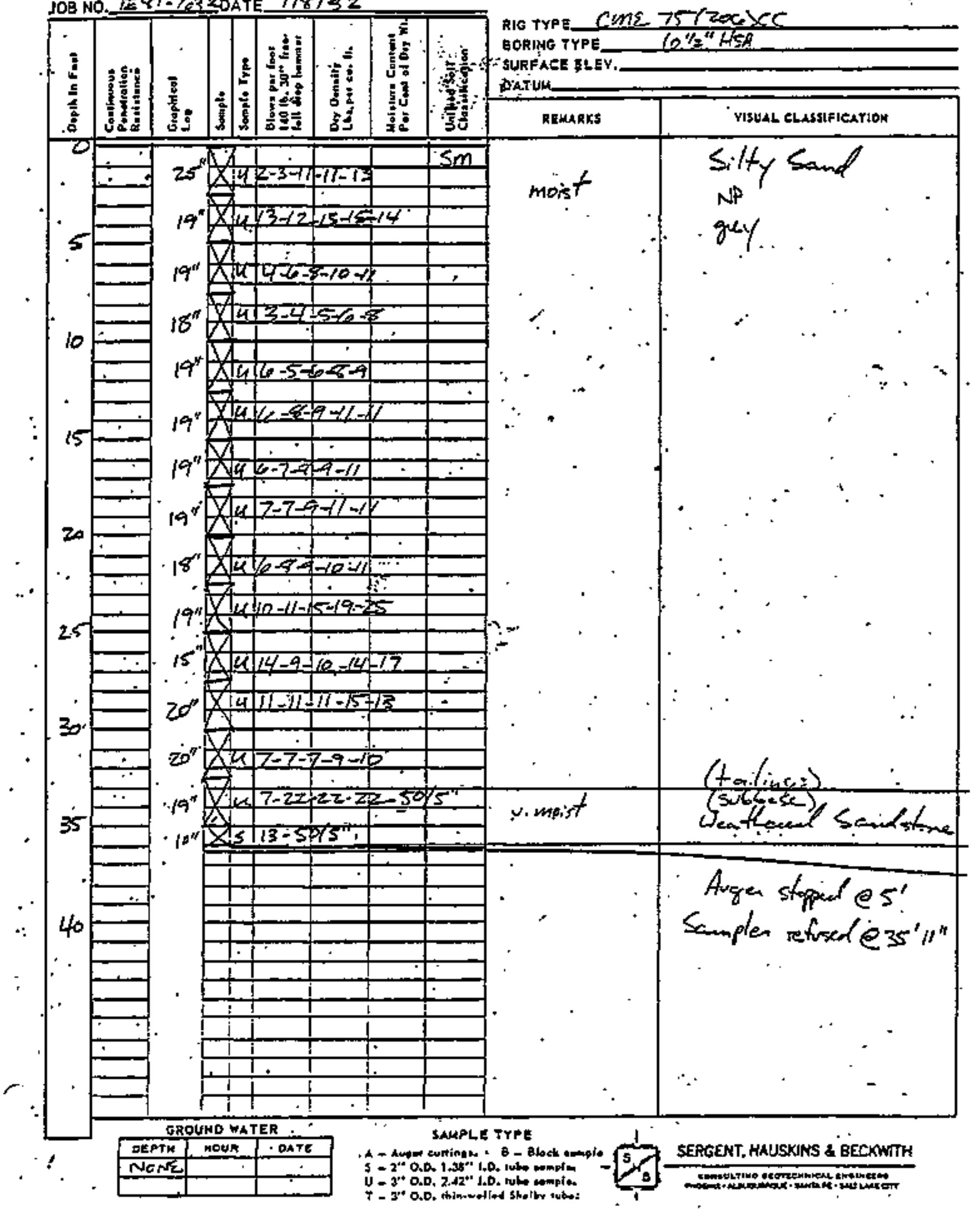

LOE OR TIST LORINO NO. 
PROJECT Mexican that Tailings

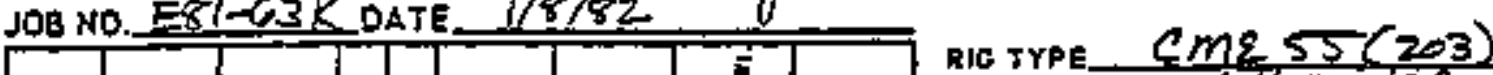

\begin{tabular}{|c|c|c|c|c|c|c|c|c|c|}
\hline \multirow{2}{*}{ 竞 } & \multirow{2}{*}{ 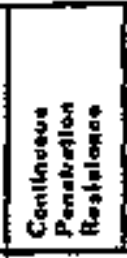 } & \multirow{2}{*}{ 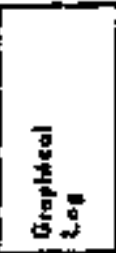 } & & \multirow{2}{*}{ 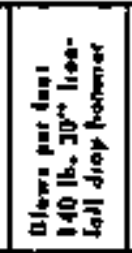 } & \multirow{2}{*}{ 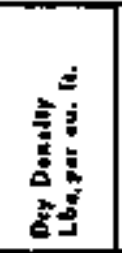 } & \multirow{2}{*}{ 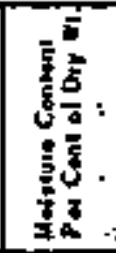 } & \multirow{2}{*}{ 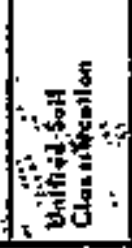 } & \multicolumn{2}{|c|}{ 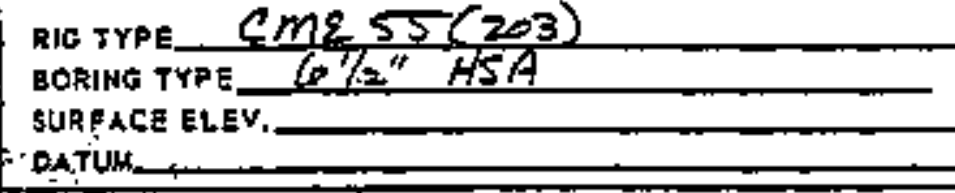 } \\
\hline & & & & & & & & REMARKS & ViSUAL ELASSSFIEATION \\
\hline$\overline{0}$ & & $\mathrm{Z}^{\prime \prime}$ & $\sqrt{1 \infty}$ & 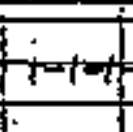 & $2-3-$ & 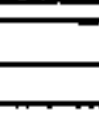 & $5 \mathrm{sm}$ & moist soft & Silty Sand \\
\hline 5 & & $2 i^{*}$ & Xlat & $5=-4$ & $-3-2$ & & $=$ & & Lo PI \\
\hline & & & $x_{1}^{x}$ & $x-3=$ & $-3-6$ & & $E$ & & tan to brown to yellow \\
\hline 10 & & 181 & 大 & & & 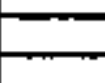 & & & \\
\hline & & $18^{\prime \prime}$ & $\Delta \mu$ & $3-3-4$ & $-2-2$ & & & & \\
\hline 15 & & $15^{\circ}$ & Xik & $\mid-1-1+4$ & $2-4$ & & - & & \\
\hline & & $18^{\prime \prime}$ & $\frac{4}{\Delta x}$ & $4-4-2$ & $z=2-1$ & & & & \\
\hline & $\overline{-}$ & $2 t^{\prime \prime}$ & $\sqrt{|\bar{x}|}$ & $\mid \frac{1-1-T_{4}}{4}$ & $2-1$ & $=$ & E & & \\
\hline 20 & & 20 & पे & 1 & 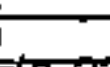 & $=$ & E & & 11 \\
\hline & & $16 \%$ & $\overrightarrow{1}+\infty$ & $y-2+2$ & $-50 / 5$ & & & hand & (subbase) \\
\hline & & & & & & & & & Bedrack \\
\hline & & & 1 & & & $E$ & 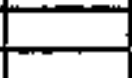 & & \\
\hline & E- & & $T$ & & 二 & & 二 & & Auger stoped es' \\
\hline & & & + & & & & & & Samsler refusad e \\
\hline & & & 1 & & - & - & - & & $23^{\prime} l^{\prime \prime}$ \\
\hline & & & I & $m$ & 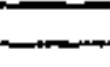 & 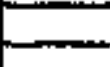 & - & & \\
\hline & & & & & - & $=$ & - & . & \\
\hline & & & & & & - & 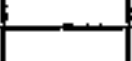 & & \\
\hline & & & $i$ & & & & E & & $\cdots$ \\
\hline & & & - & & & - & Z & & \\
\hline & & & & & & & 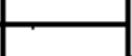 & & \\
\hline & & & $-\frac{1}{1}$ & & & & & & \\
\hline & & & 1 & & & & 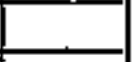 & & \\
\hline & $=$ & & & & & & - & & \\
\hline & & & سب! & & & & $=$ & & \\
\hline & & & $40 \mathrm{WA}$ & TER & & & & & \\
\hline & & & . & & & & 1.07 & mansion & SERGENT, HAUSKONS \& BECKWTH \\
\hline
\end{tabular}


FROJEC: Mexicam Hat Tarliate

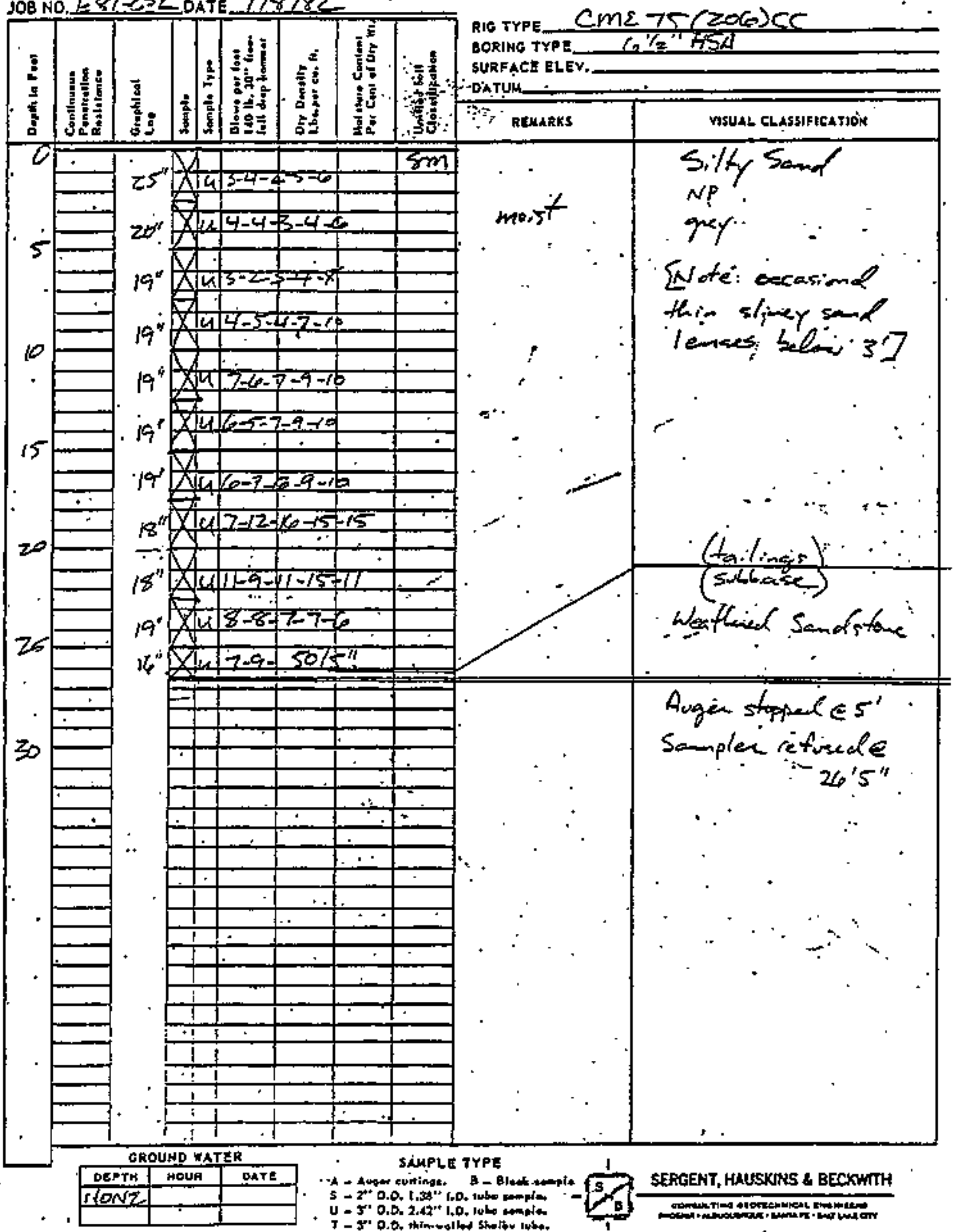

LOO OP TEST BORINO NO. B-14 RIG TYPE $C m E-75(206) C$ RING TYP

$\because y_{7}$ RLIMAkKS
S. $/ 4$ Sampo
NP.
gney. Hit stger somal

Wexflide Sandstare
Sote: accasimol

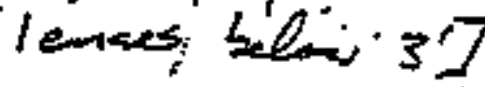



PROJECT Mexican Hat Tailings
JOS NO. ES/-63KOATE I/8/82

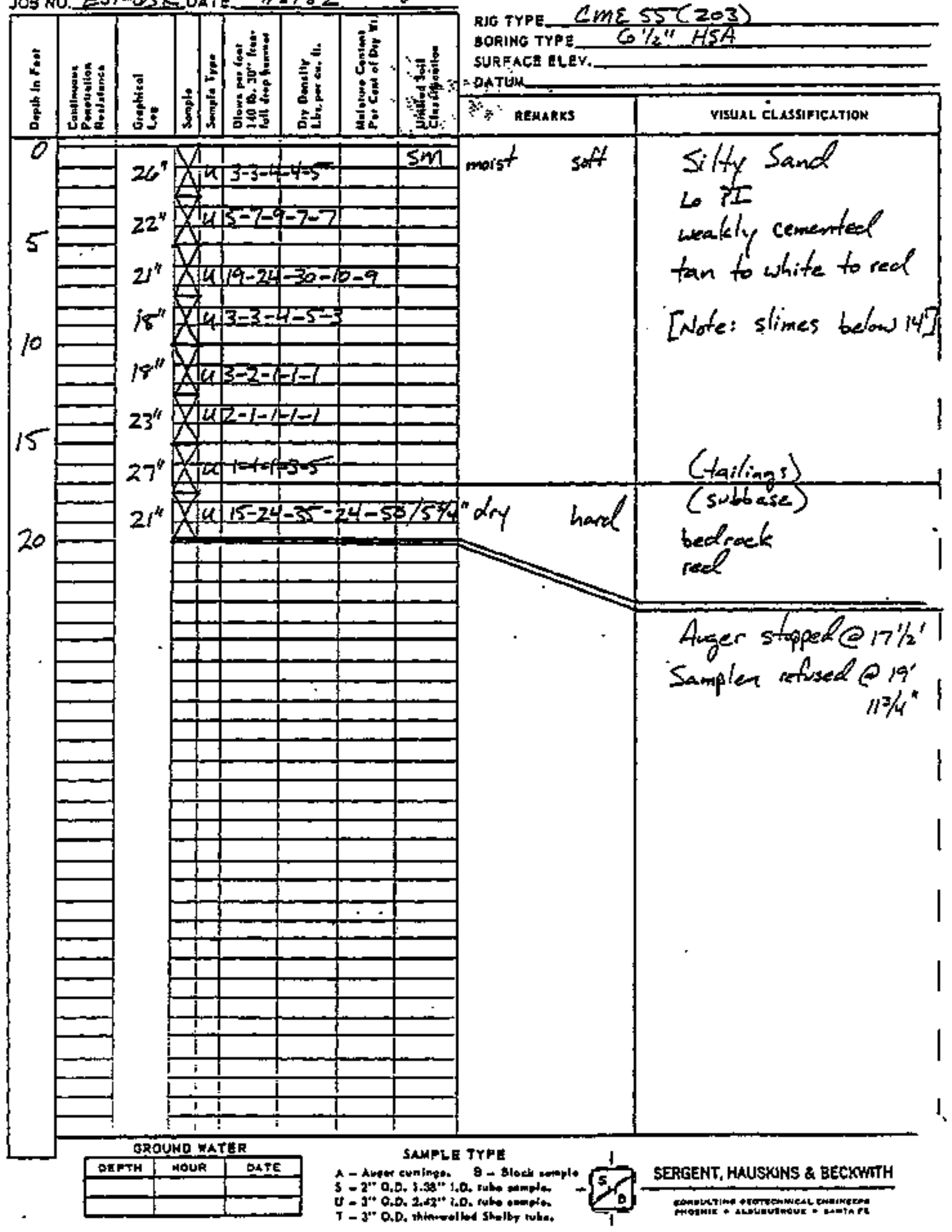

LOE OF TEST DORING NO. $B-15$ RIO TYPE $\quad C m E S 5(203)$ DORING TYPE Go " HSA DATUM

2 邽 weakly cementel $\tan$ to white to red [Note: slimes below i4] 


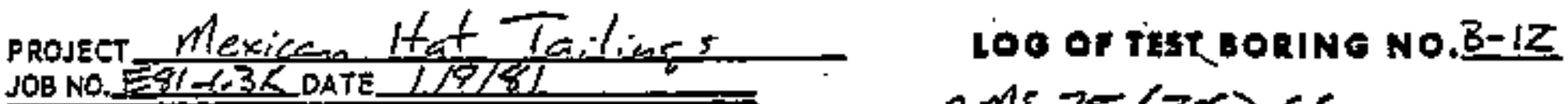

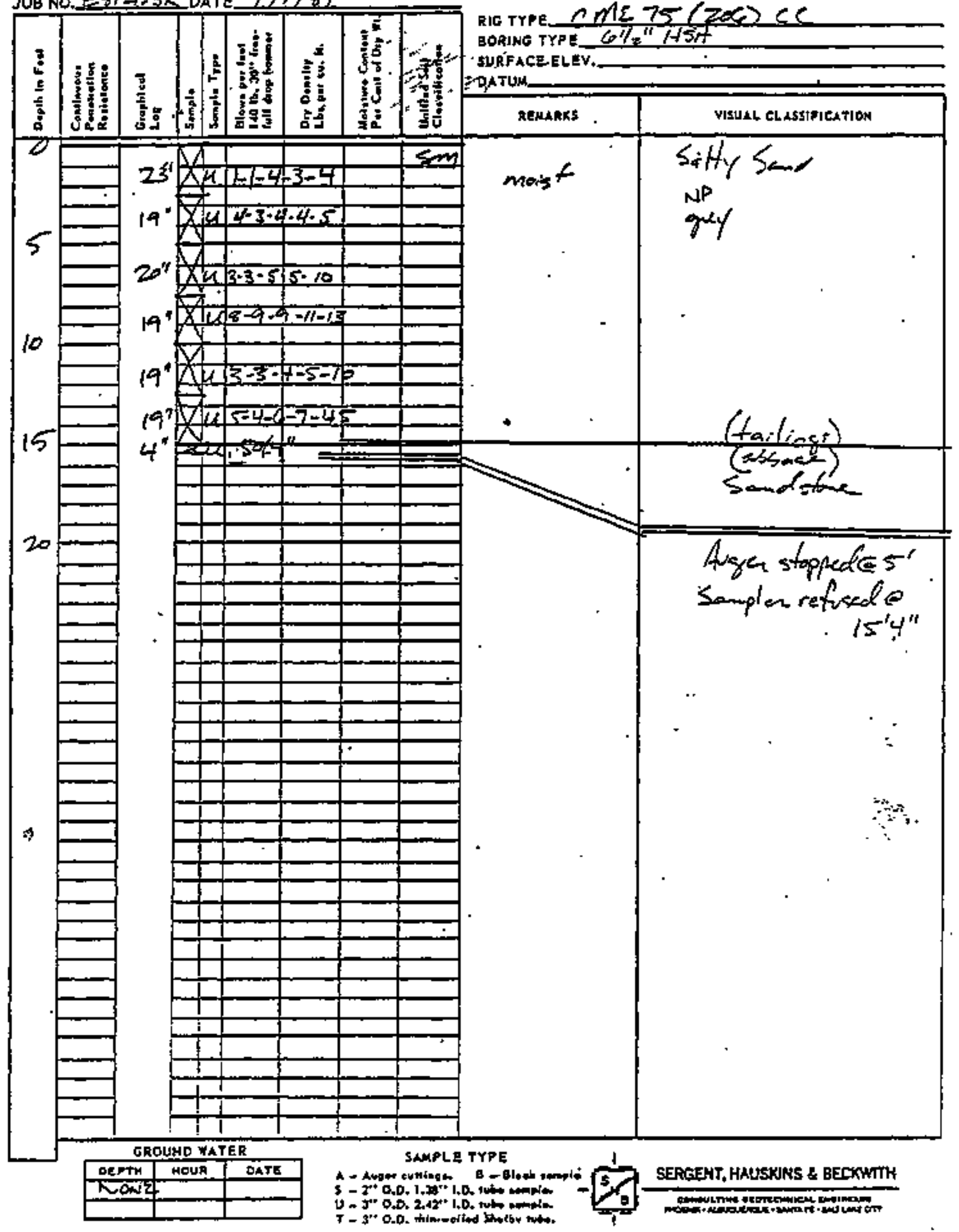


PROJECT Mexican Hat Tailings
JOB NO.E81-63ROATE $1 / 5 / 82$

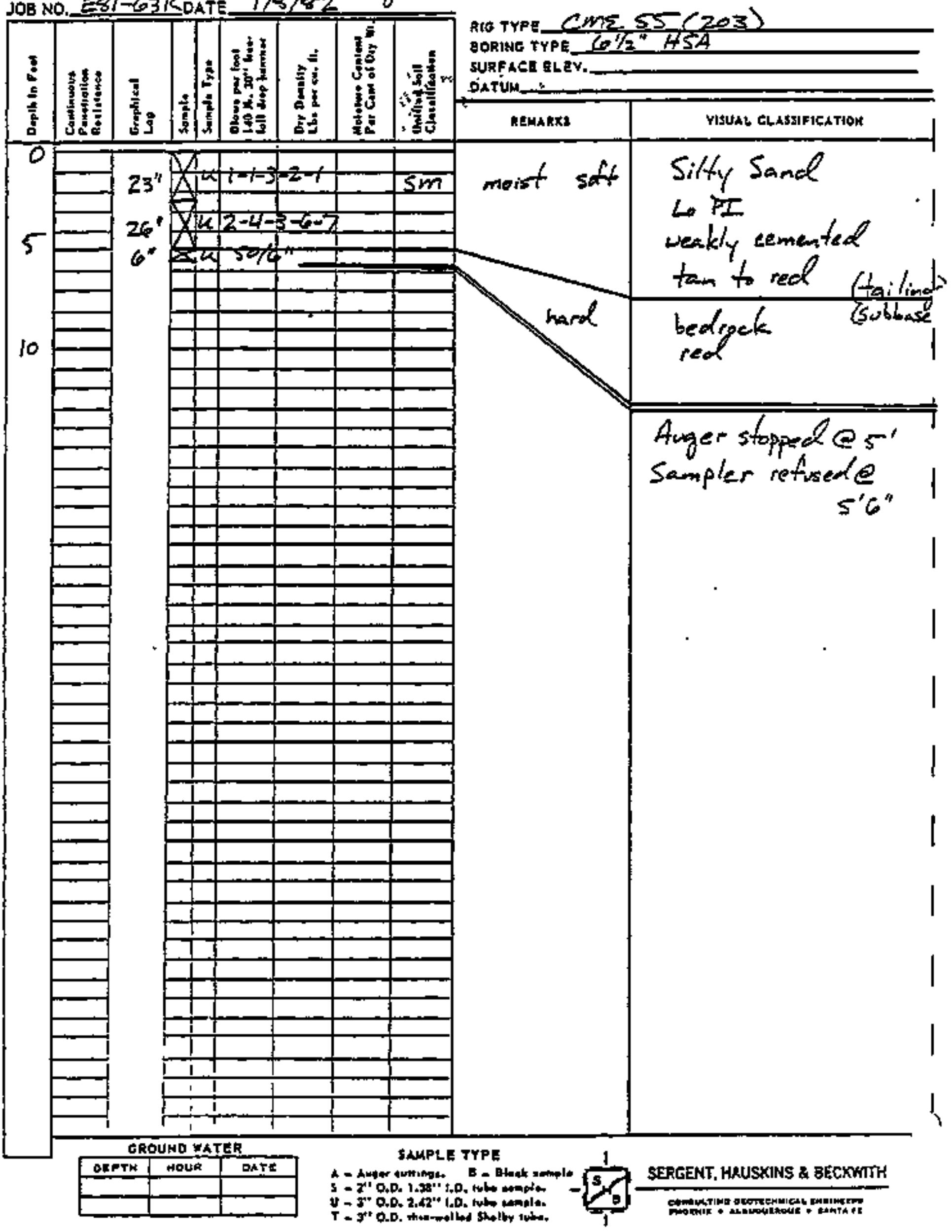

LOC OF THST LORINO NO.A-13

(2) 
PRoJeCT Mexicen Hat Tigines JOB NO. $P 1 / 03 K$ DATE $1 / 8 / 87$

LOO OF TEST BORING NO.A-10

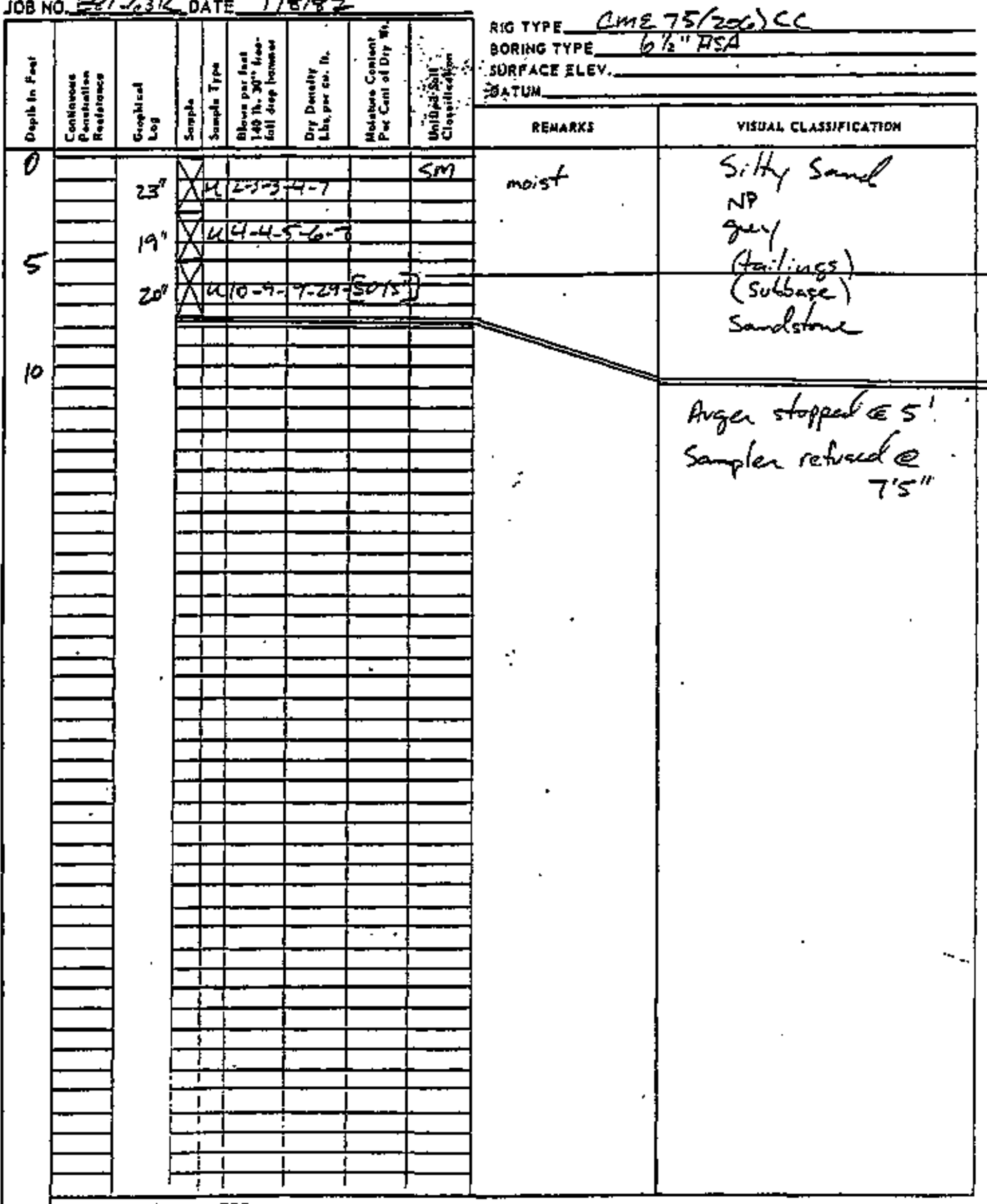

GROUND WATER

\begin{tabular}{|c|c|c|}
\hline PEFTH & HOUR & OATE \\
\hline NENE & & \\
\hline & & \\
\hline
\end{tabular}

\section{SAMPLE TYPE}

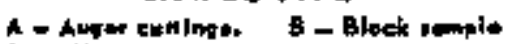

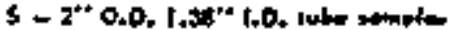

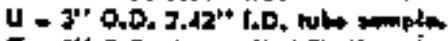

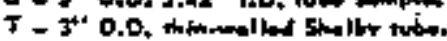

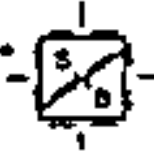

SERGENT, HAUSKLNS \& BECKATTH

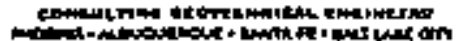


PROJECT Mexican Hat Tailings

LOE OF TEST DORINe NO. A-ll JOB NO EBI-C3KDATE WBTEZ

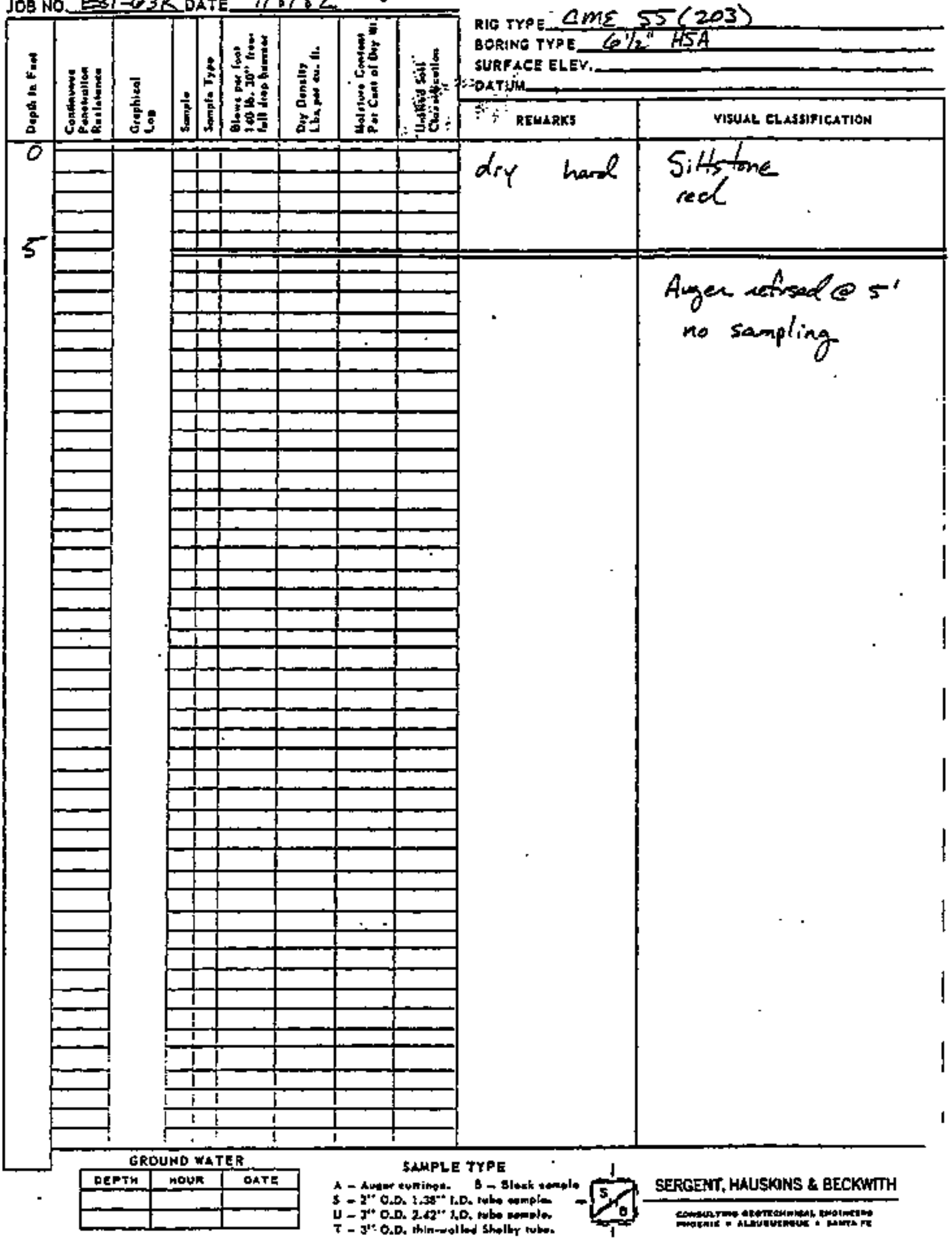




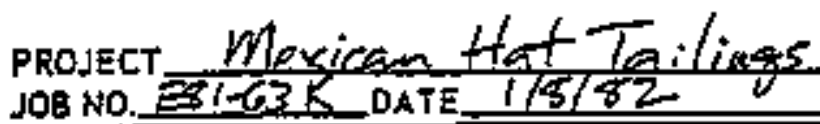

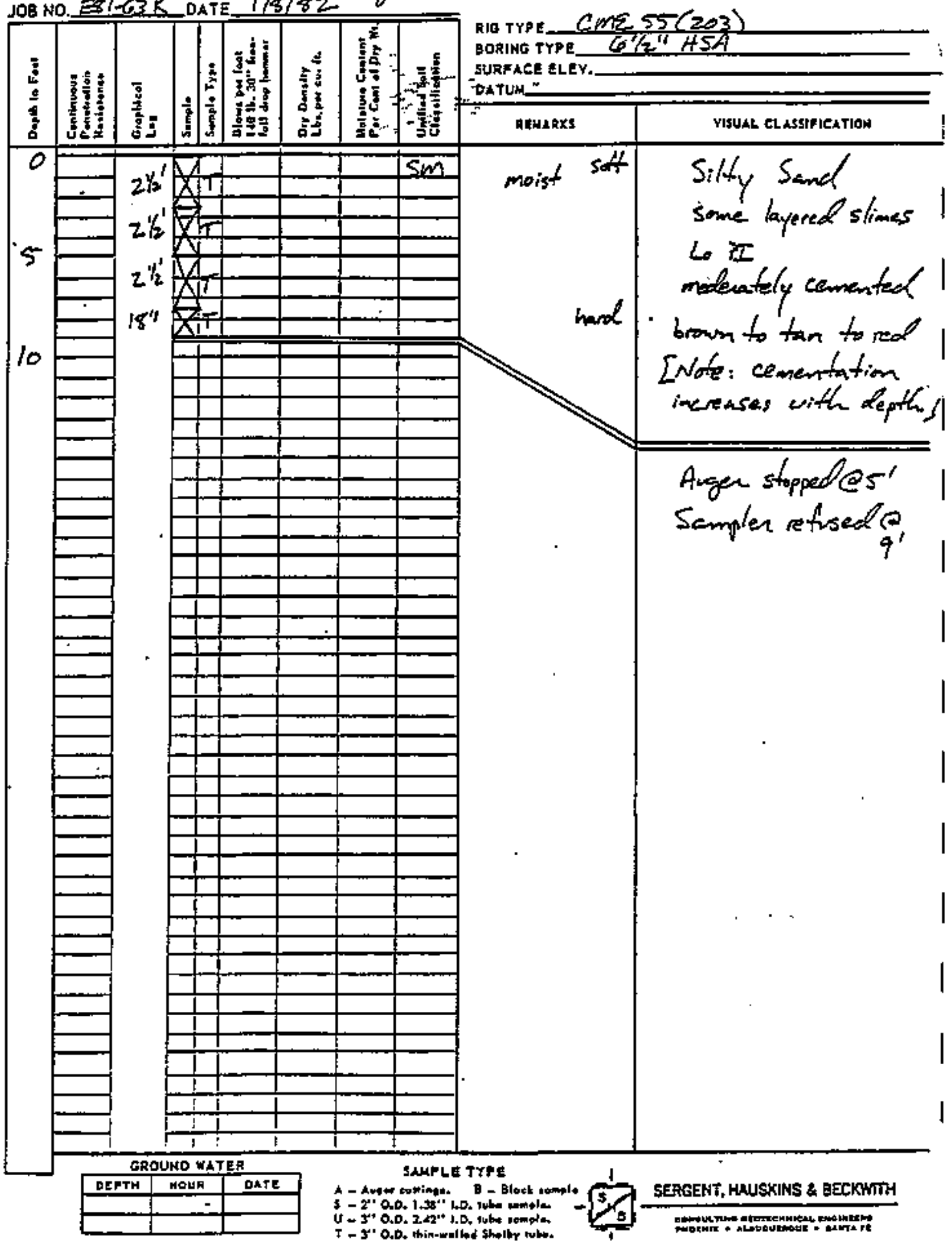


PROJECT Mexican Hat Tailings LOS OF test BORING No.A-7 JOB NO. E8/G $3 K$ DATE $1 / 8 / 82$

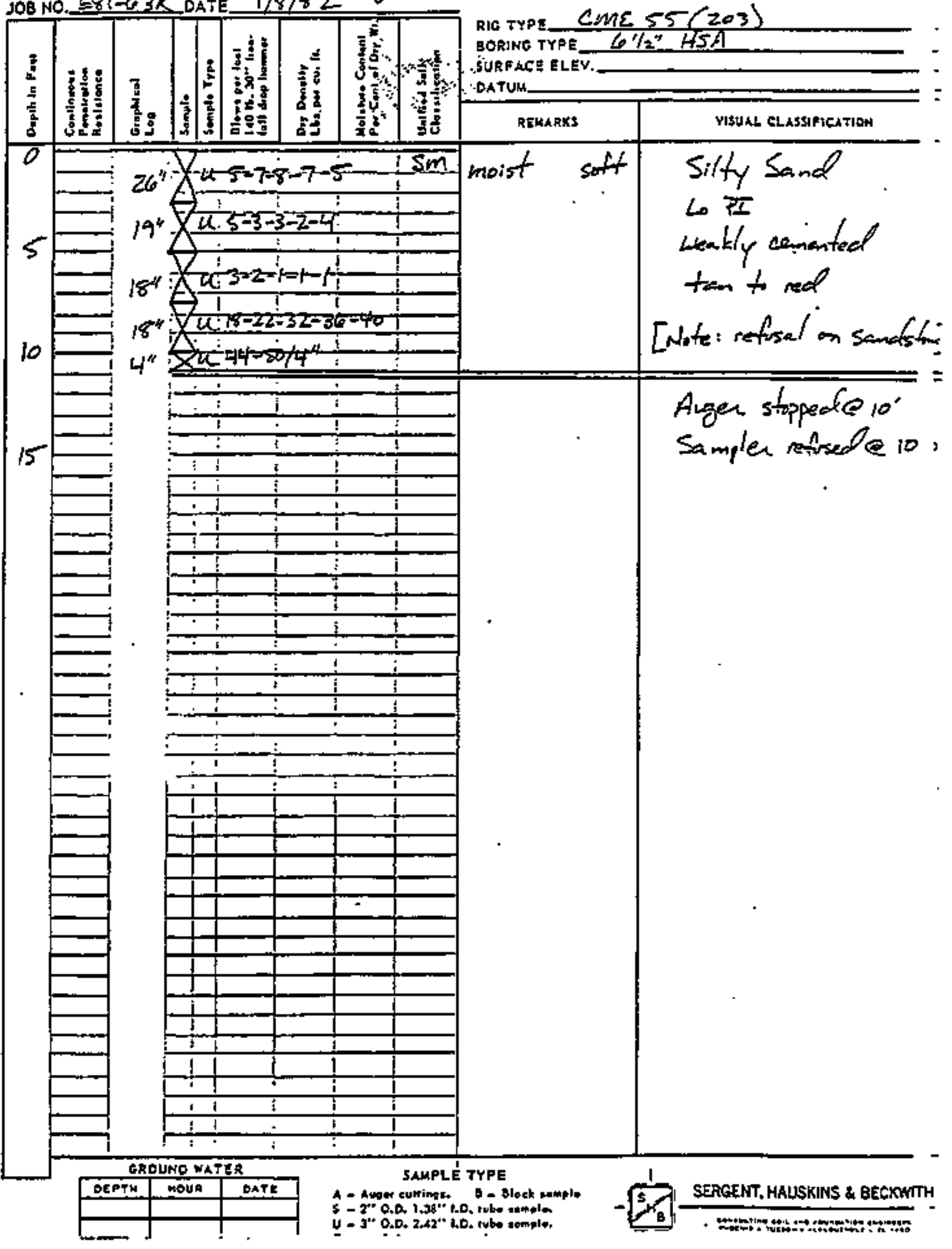




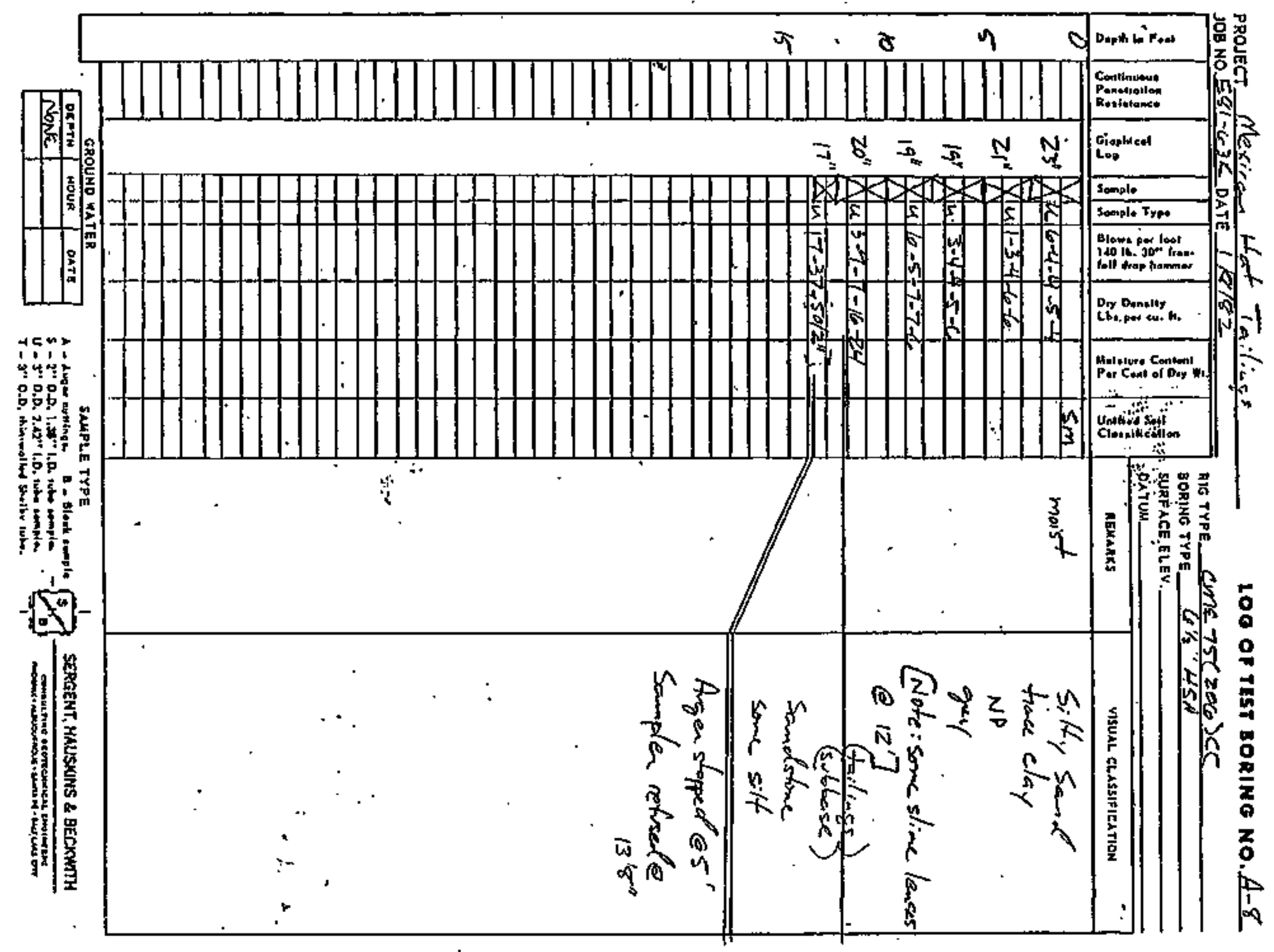


PROJECT MeXican Hat Tailings
JOB NO ESI-63K DATE I/8/8Z

100 OP TEST BORIN O NO. $\$-5$

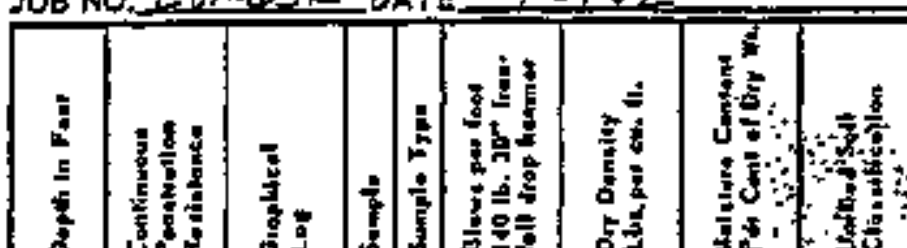

RIC TYPE__CME 55 (203)

BORING TYPE $61 / 2^{4}$ HSA

SURFACE ELEY.

DÁtum

$\theta$

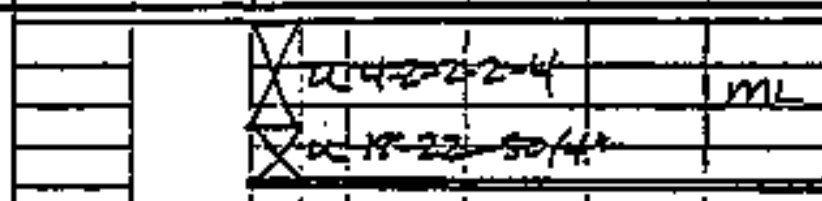

5

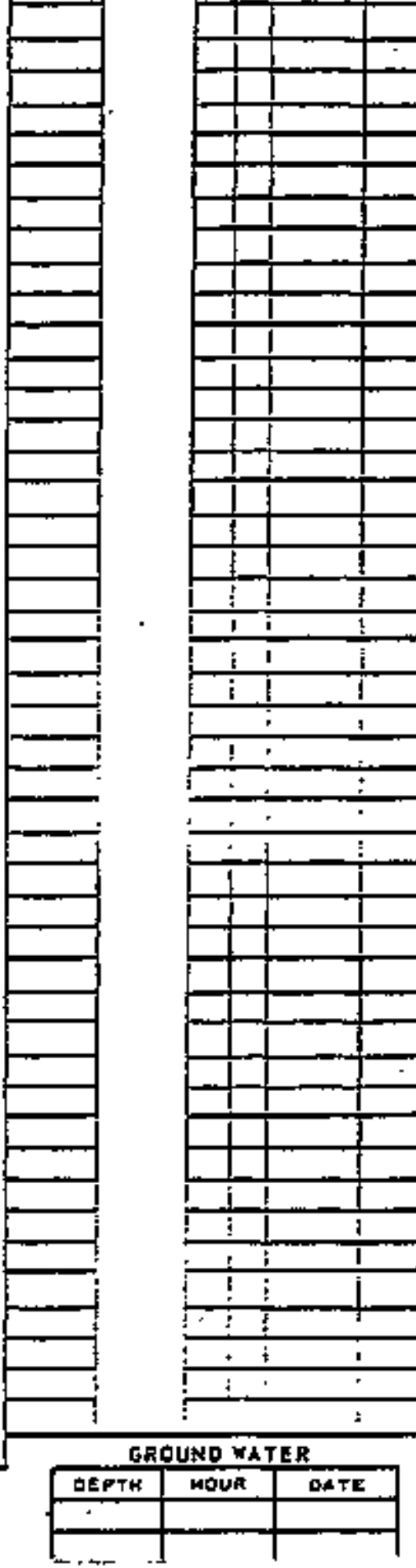

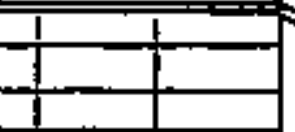

I

$+2$

$+$

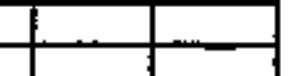

$-1$

$+$$$
+
$$$$
1
$$$$
+
$$$$
+
$$

\begin{tabular}{|l|l|}
\hline 1 & 1 \\
\hline 1 & 1 \\
\hline
\end{tabular}

" REkanxs most soit $5 i l$ hare
VISULL CL A5SFIEATIOH

weakly cemental

NP

$\tan$ to red

refrell on tebrock

Auger stoppede $212^{\prime \prime}$

Sempler retrsed $@$
$310^{\prime \prime}$. 


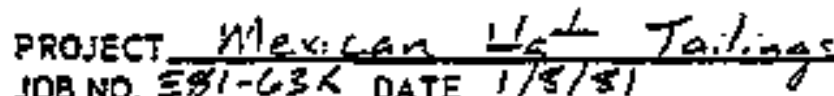

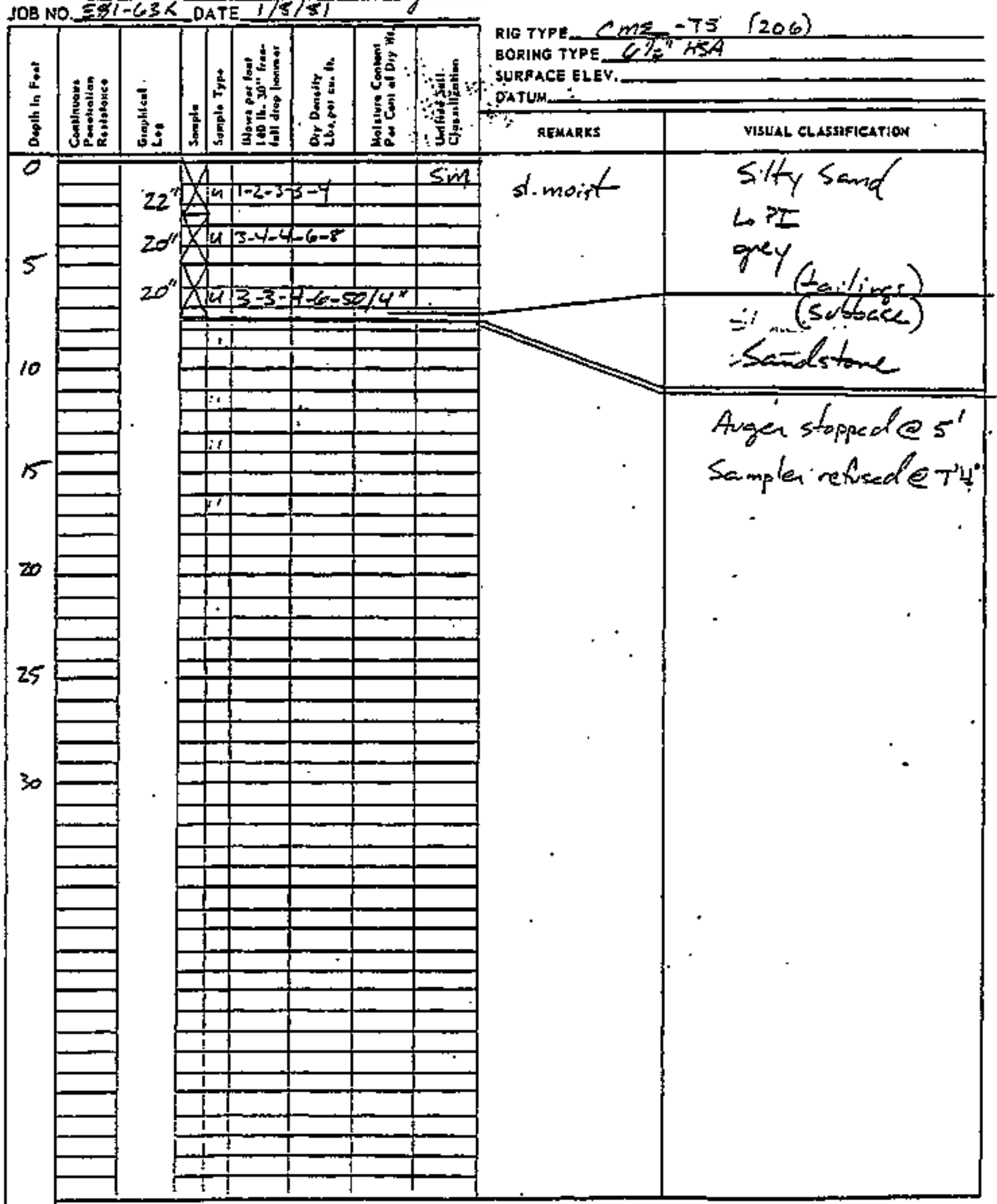

GROUHD WATER

\begin{tabular}{|c|c|c|}
\hline AEPYM & KDUR & OATE \\
\hline NEMG & & \\
\hline
\end{tabular}

5MPLE TYPE

A - Auper swingh. - Block somple

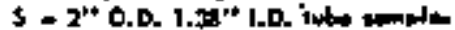

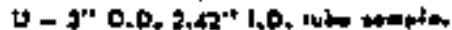

$T=J^{\prime *}$ O.D. Ihinforlited sholler tubt.

LOO OF TEST BORING NO.A-6

Lo PI

grey

$\because$ (solisak)

Standstome

Augen stoped $@ 5^{\prime}$.

Samplen refued $\tau^{\prime u}$ " 


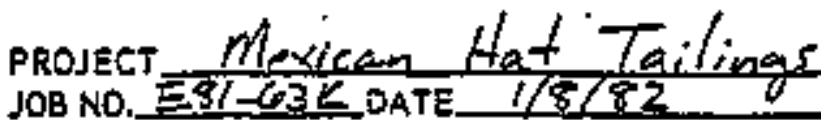

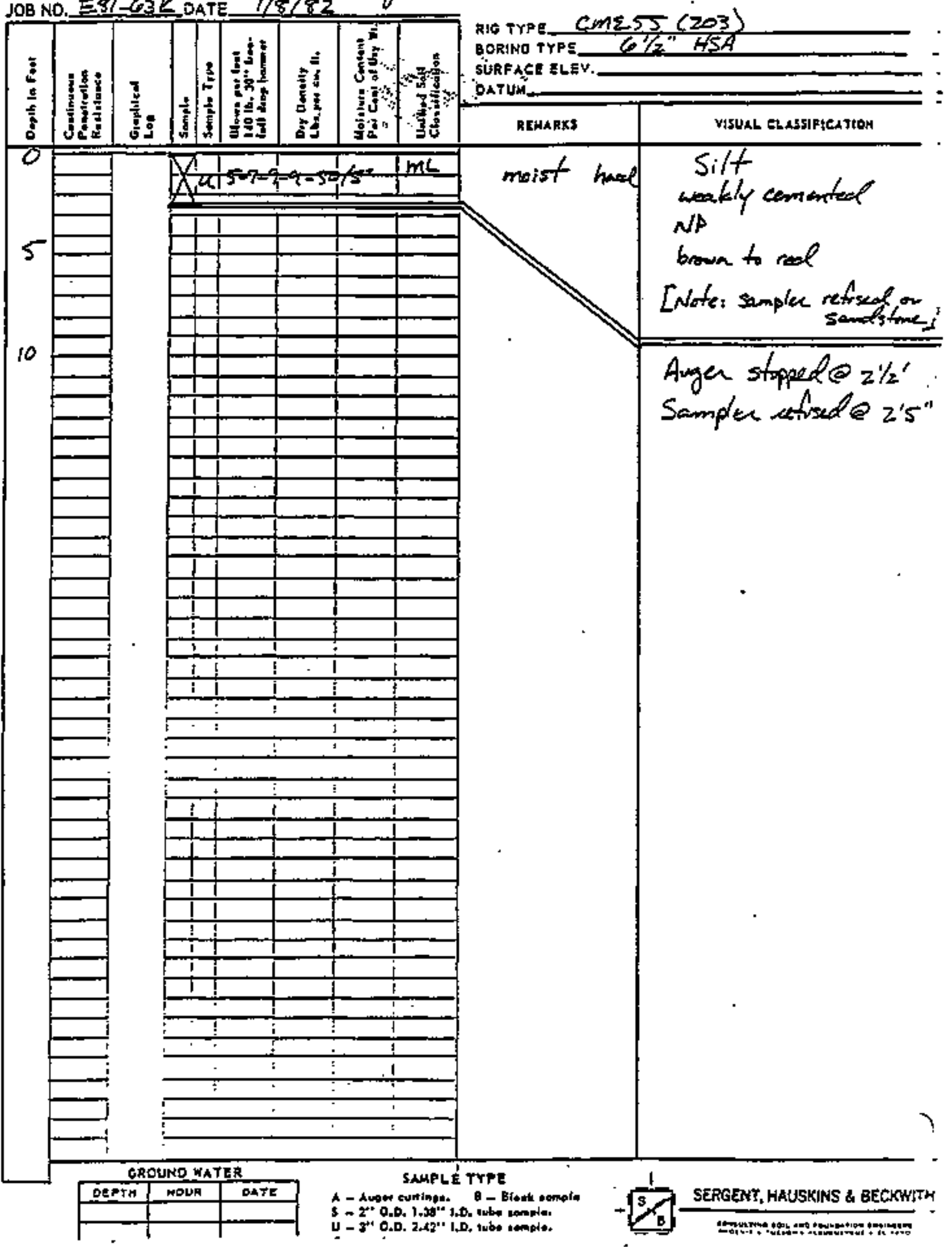

LOG of tist dontNo No. A-3 


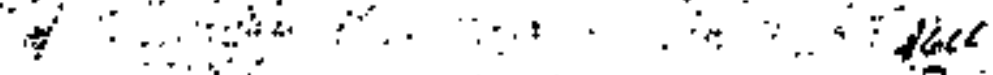

WELT DR PIEZONOTER NTMBER 3

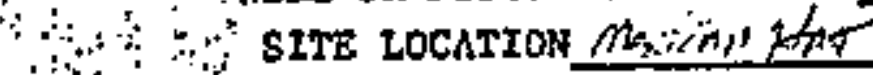

if $\because: \because$ GROUNDWATER LOCATION
DATE $1 / 25 / 62$ is JOB No. $E 8 / 63 K$. P1 $\therefore$. 14:

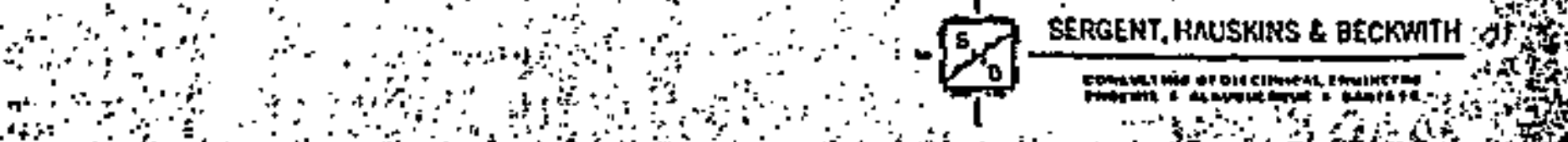

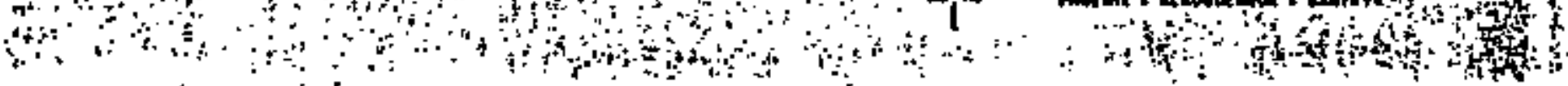




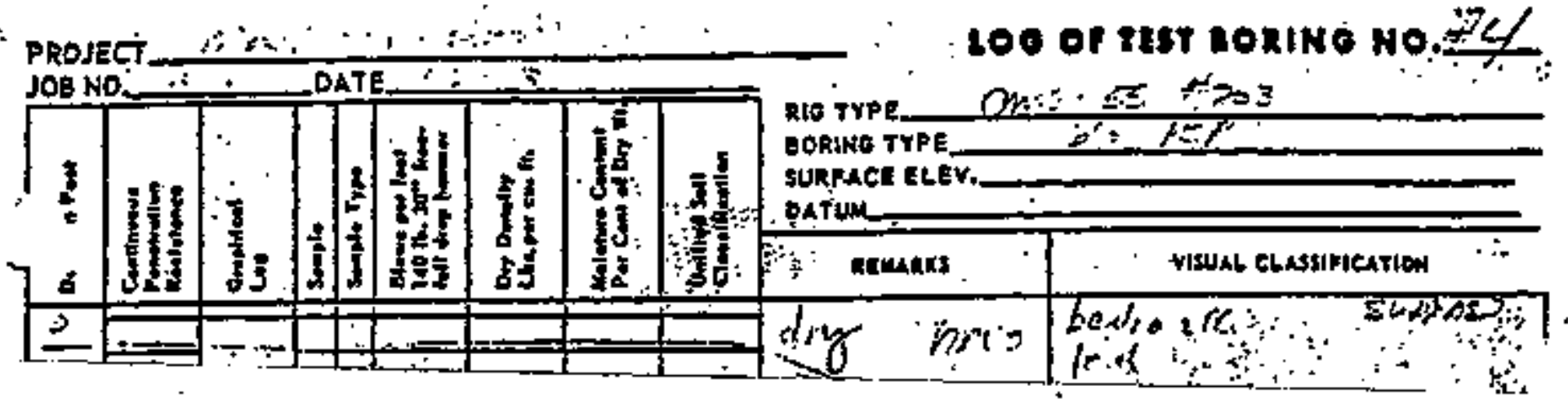

\section{PIEZOMETER. OR WELL COMPIETION RECORD}

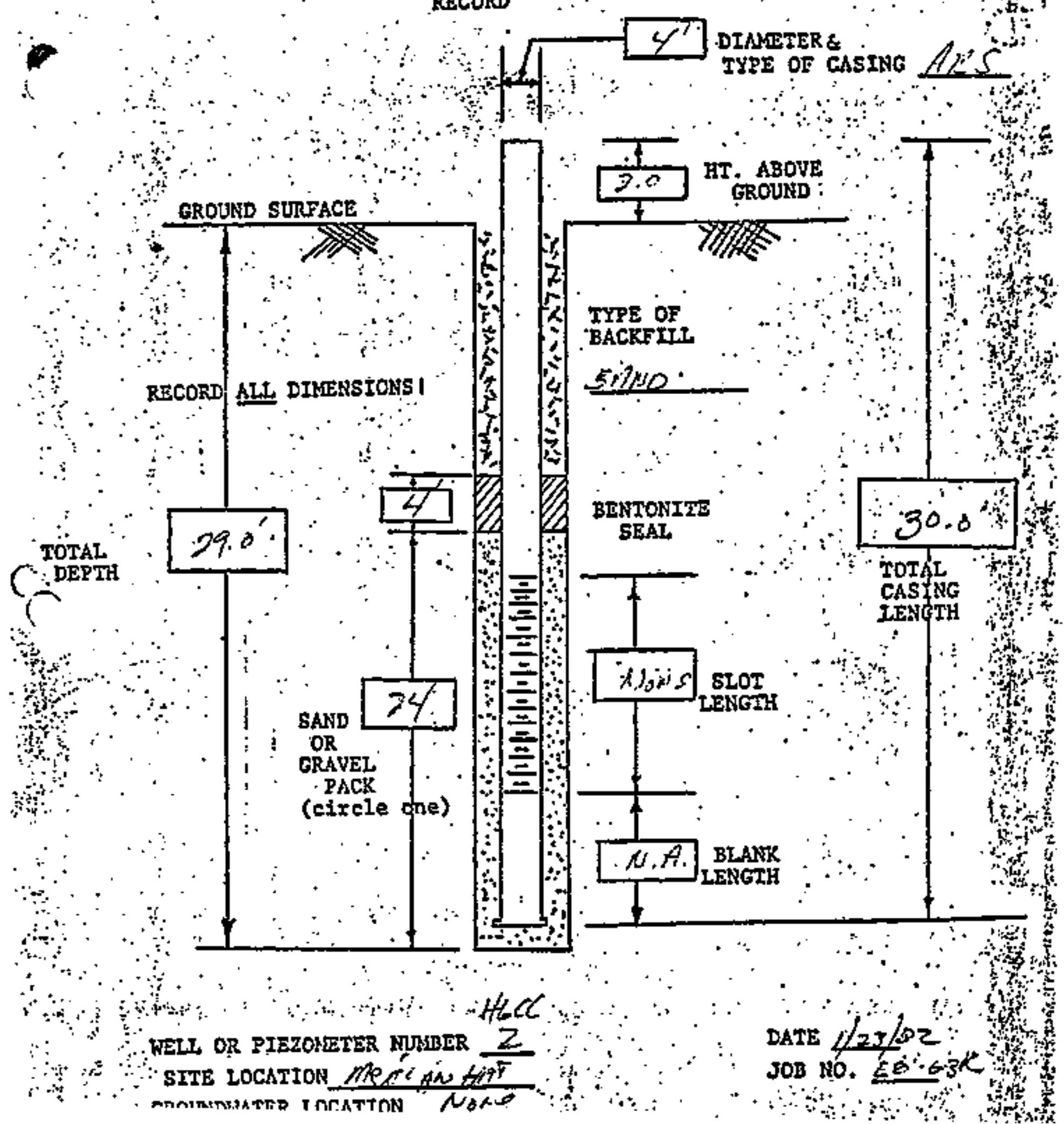




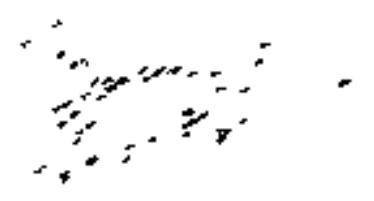

MSRD BORING LOGS 


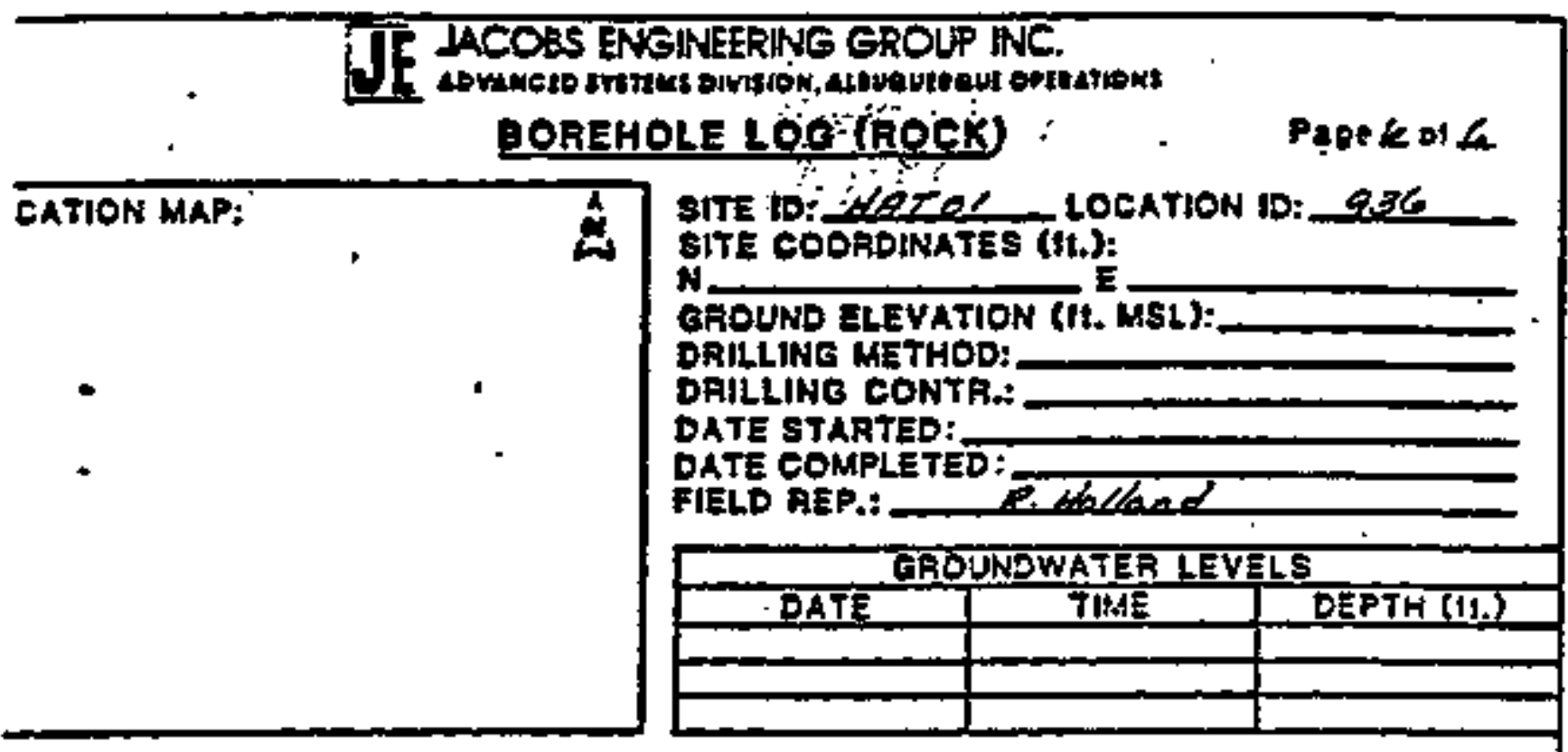

CATION DESCAIPTION

IE CONDITION

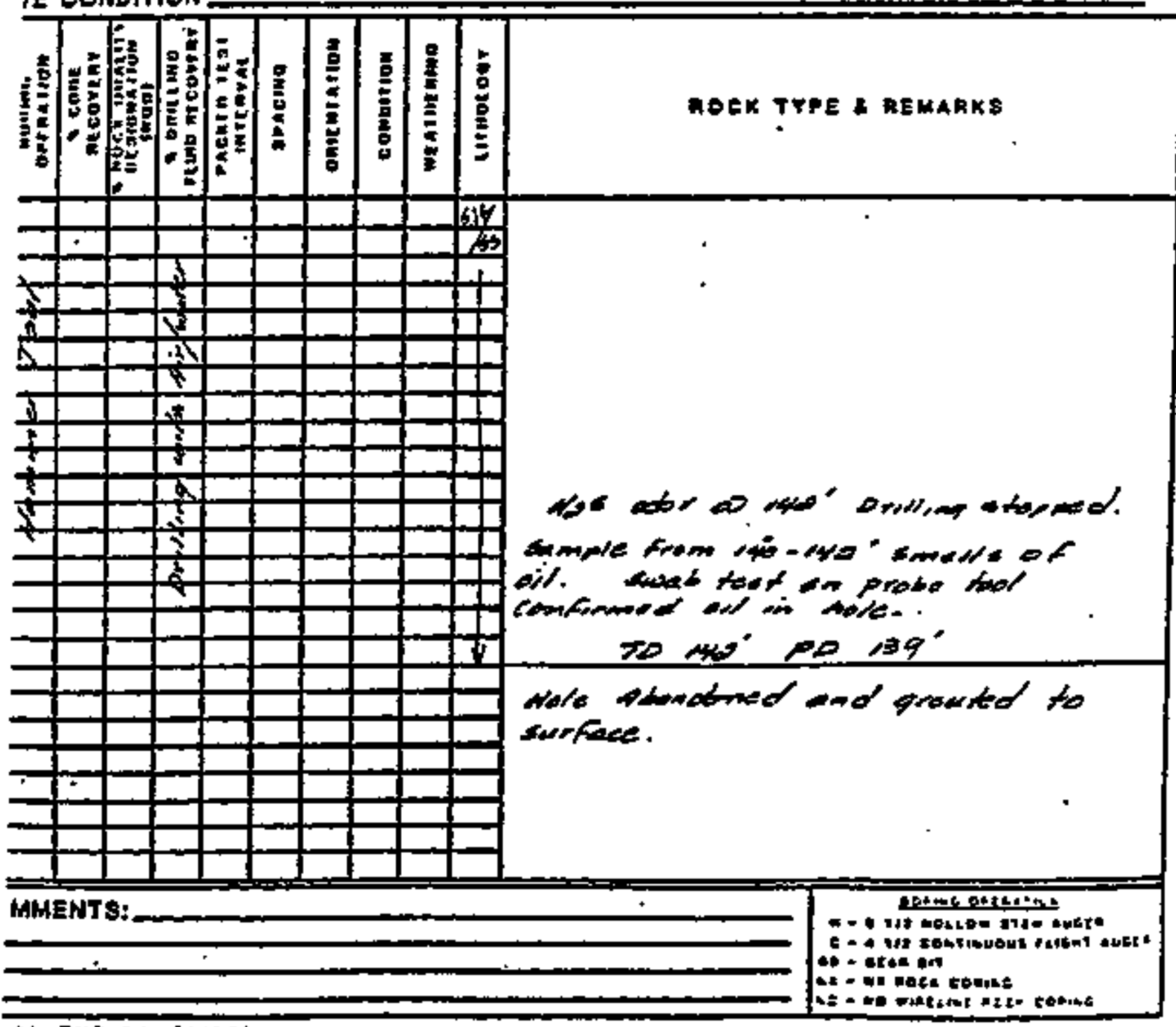


PROJECT MEXICh HAT

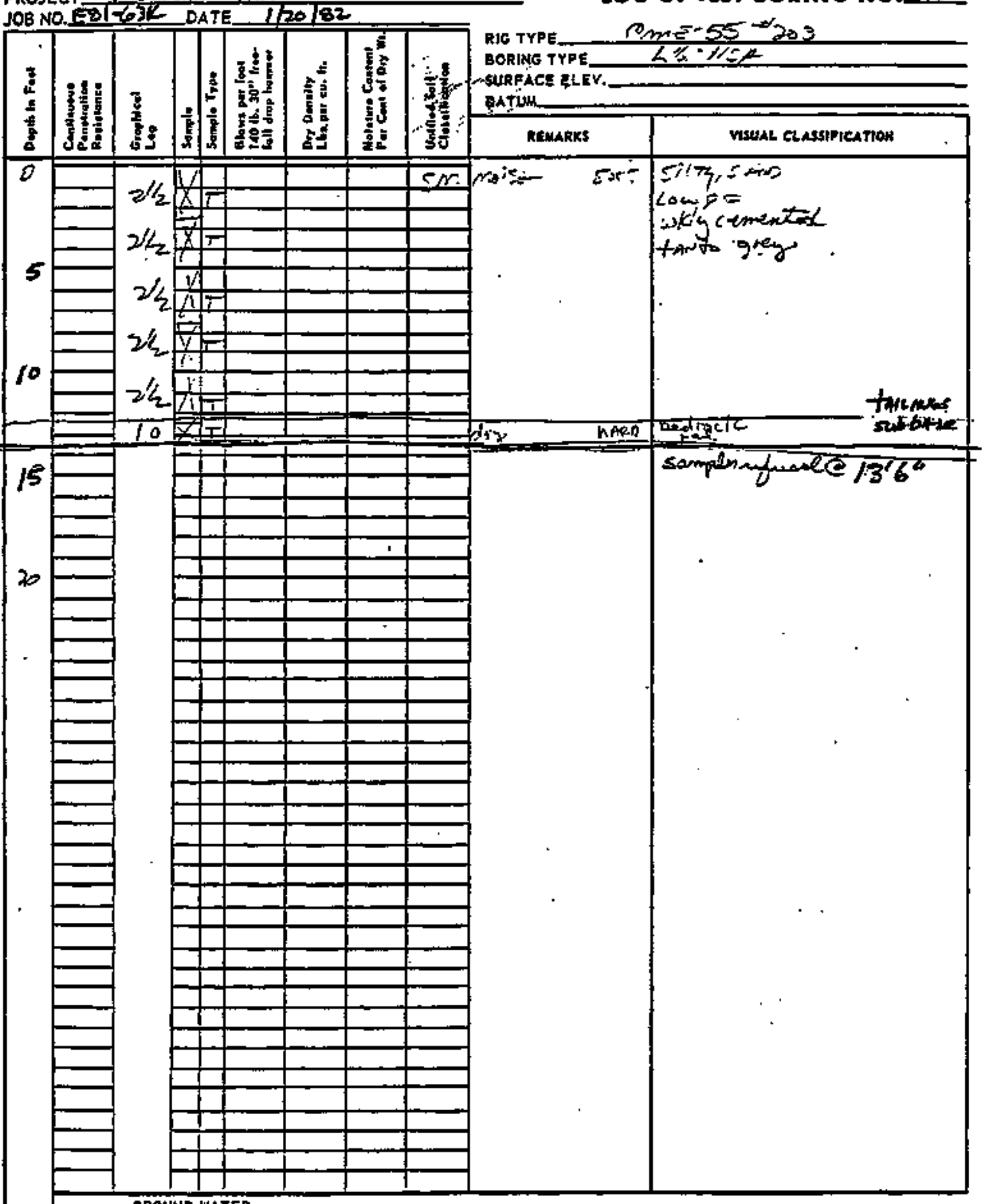

LOe of tLSt HORINe no 196 


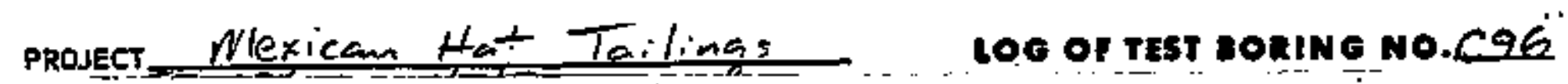

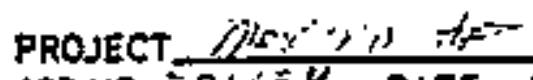

JOB NO. $=2 A K X$ DATE 1025

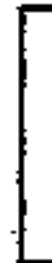

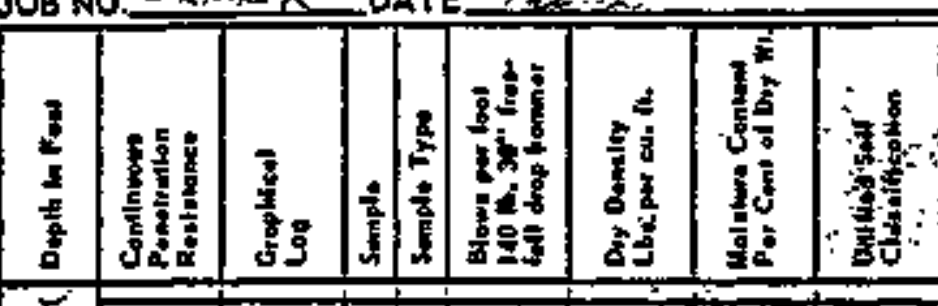

is

24

$\square$

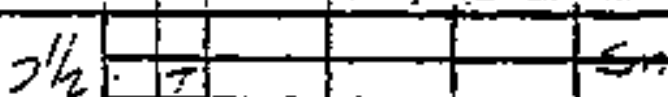

$2 \%$

7

Si

$2 t$

$\stackrel{1}{*}$

$\pi$

$\mathrm{X} 7$

$\div 7$

$= 2 \longdiv { 1 }$

$2 \% \sqrt{1-1}$

$\rightarrow 1 /$

$32 \frac{1}{2}$

$v_{2}^{\prime \prime}$

$\because z$

$2 i_{2}=1$

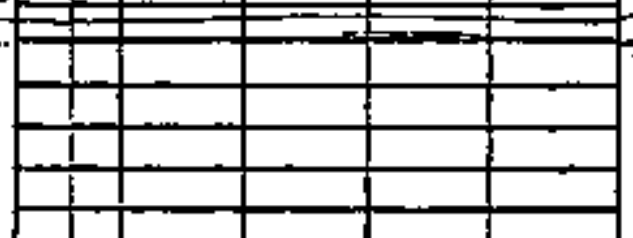
RIG TreE (mF-55 (703)

BORINO TYPE.

$4 \%+\pi S A$

SURFACE ELLEV.

patum

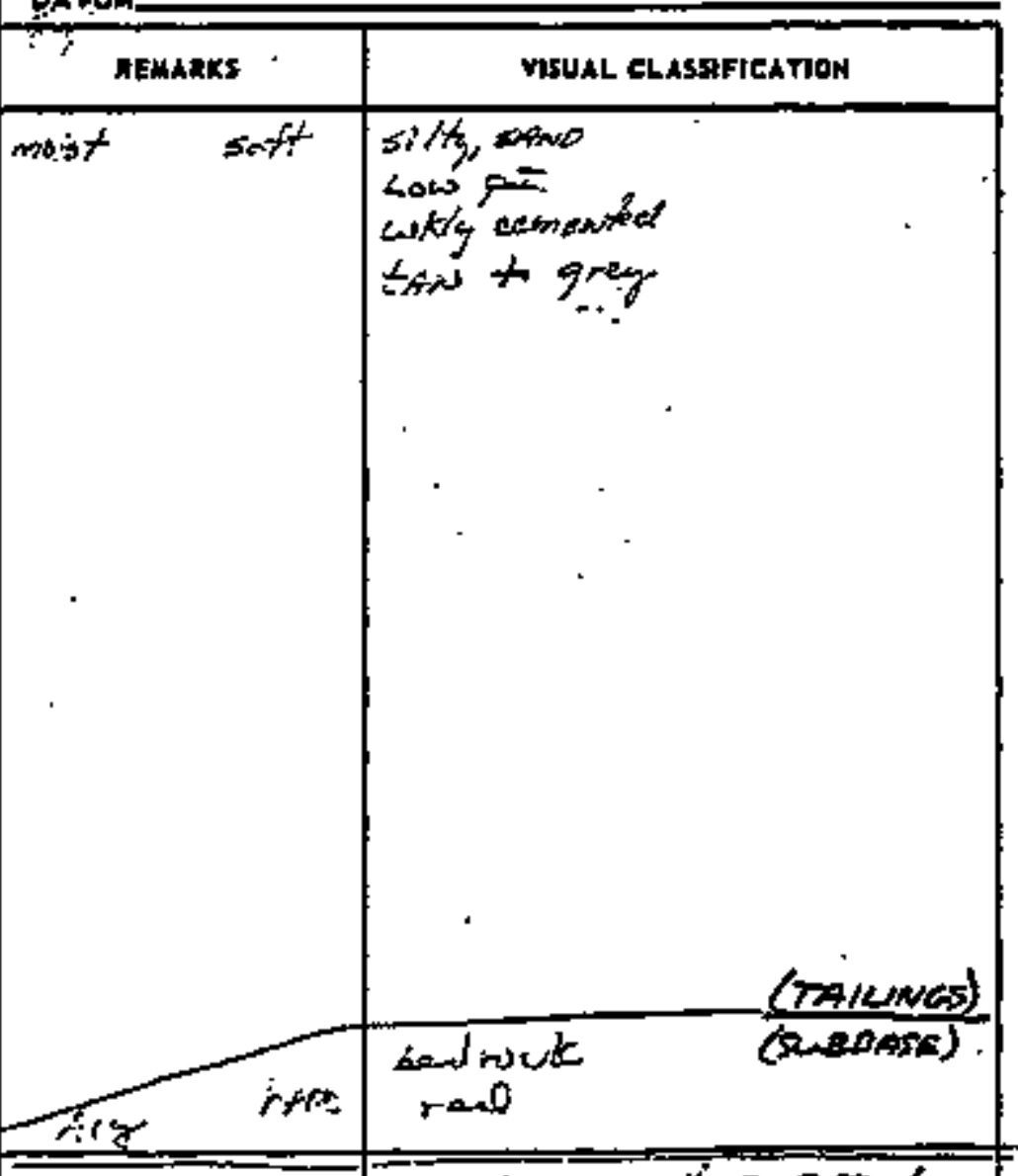

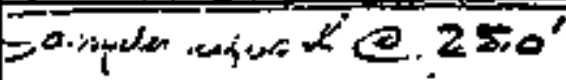

LOe OF JAST DORING NO.D98

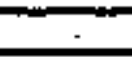

\begin{tabular}{|l|l|l|l|l|}
\hline 1 & & & & \\
\hline 1 & & & & \\
\hline 1 & & & & \\
\hline
\end{tabular}

\begin{tabular}{|l|l|l|l|}
\hline 1 & 1 & & \\
\hline 1 & 1 & & \\
\hline 1 & 1 & & \\
\hline
\end{tabular}

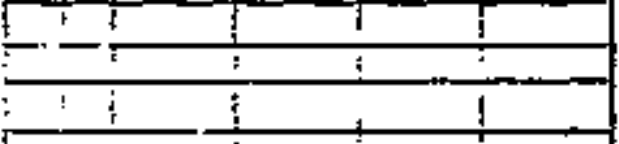


PROJECT MEXich HAT JOB NOEB 6 JK OATE 1 FolB2

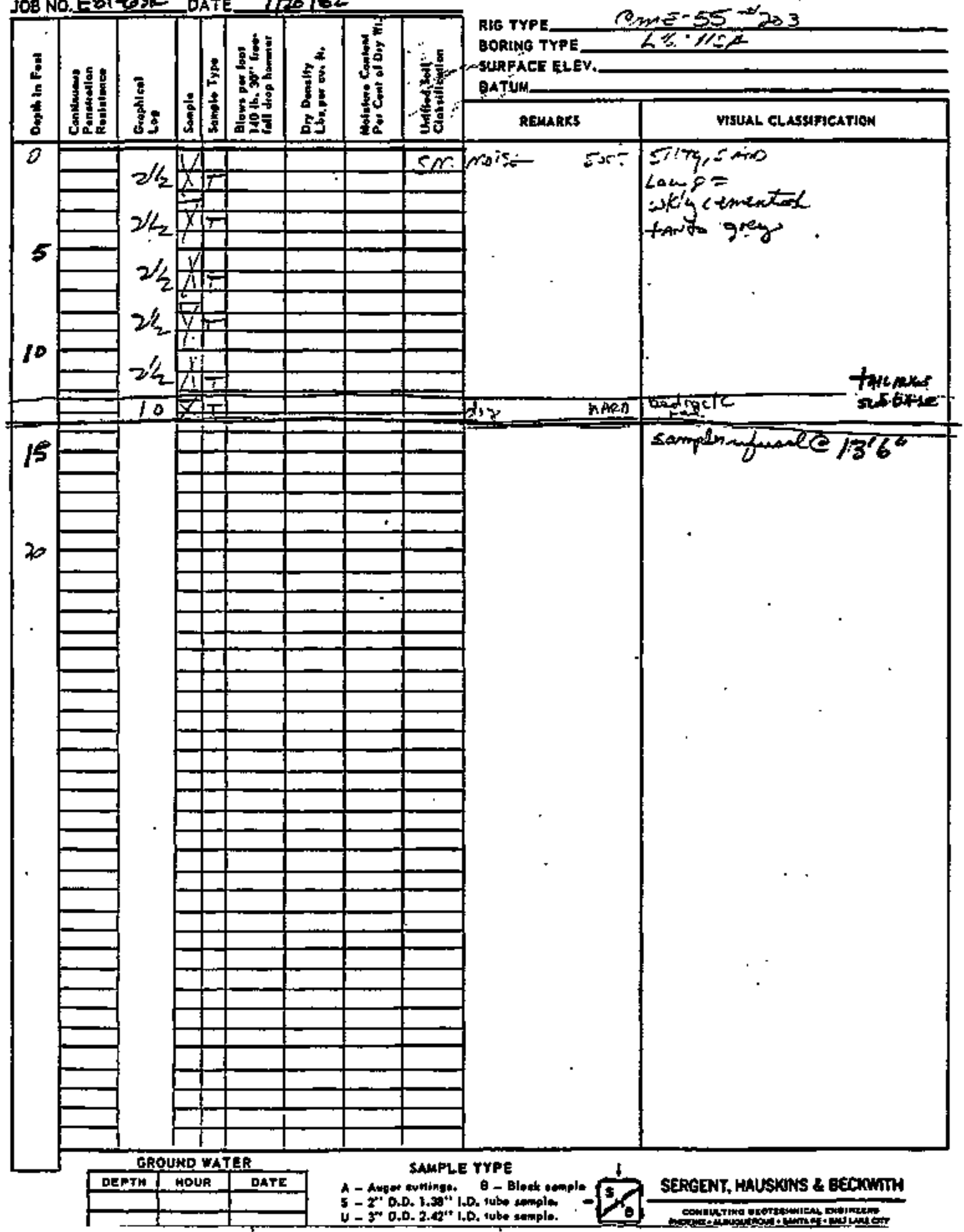


PRoJect Nlexican Hat Tallimas JOB NO. E8/-63R DATE $1 / 228 z$

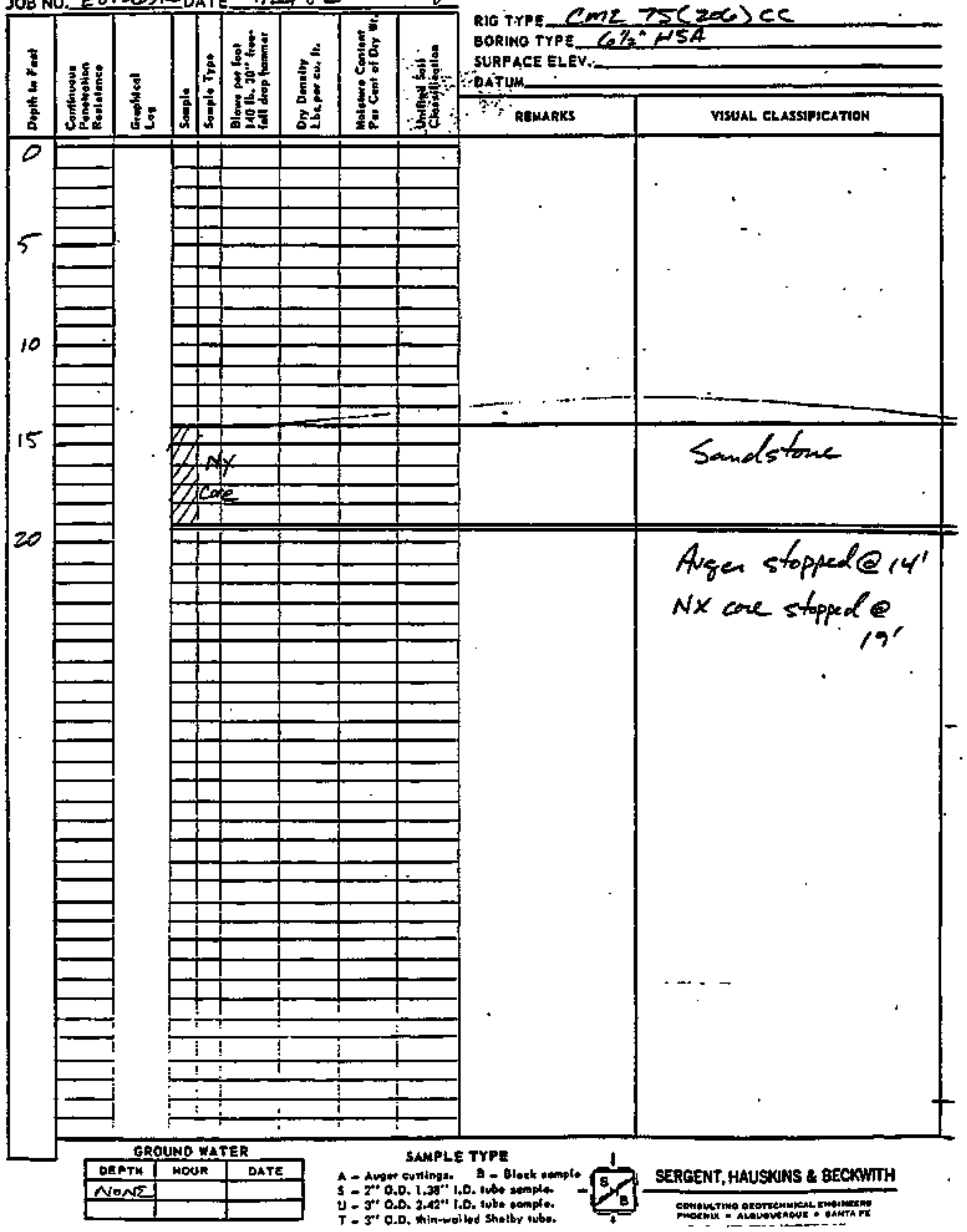




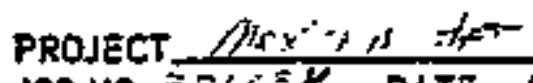

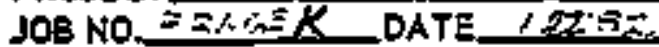

\begin{tabular}{|c|c|c|c|c|c|c|c|c|}
\hline 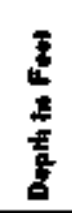 & 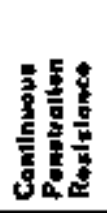 & $\begin{array}{l}\overline{8} \\
\overline{8} \\
\overline{8} \\
5\end{array}$ & 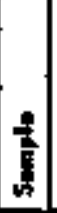 & 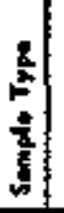 & 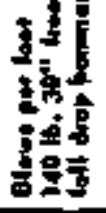 & 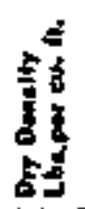 & 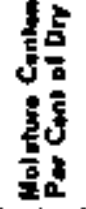 & 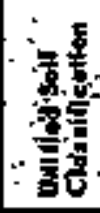 \\
\hline
\end{tabular}

15

24

10

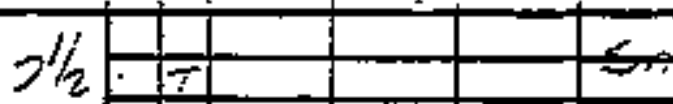

$-$

$2 \%$

viन

$2^{\prime}$

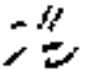

A

7

$x_{7}$

$\because 1$

$=27$

1 I

$7 / 2 \sqrt{1}$

$+1$

IT

$+2 \mathrm{~A}=$

$2 t$

$+2$

$\because \frac{1}{7}$

$-1$

\begin{tabular}{|l|l|}
\hline 7 & \\
\hline \hline & \\
\hline
\end{tabular}

$2 i$

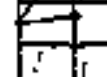

! i

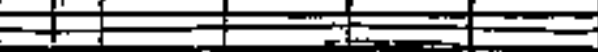

RIG TYPE (ME-5S (203)

BORINE TYPE, $6 / 3^{*}$ TSA

SLRFACE ELEVV.

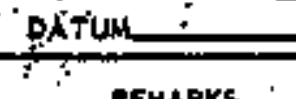

REMARKS

most sett

sithy, struo

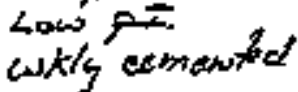

IAfit t gray

VISUAL CLASSIFICATIOH

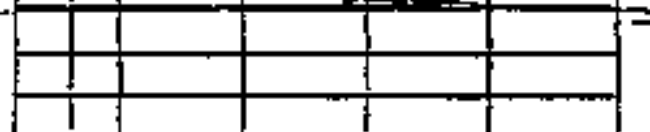

Pry rifs:

bisck

(7a10wos)

Dمer (suatass).

Fo "ither wive

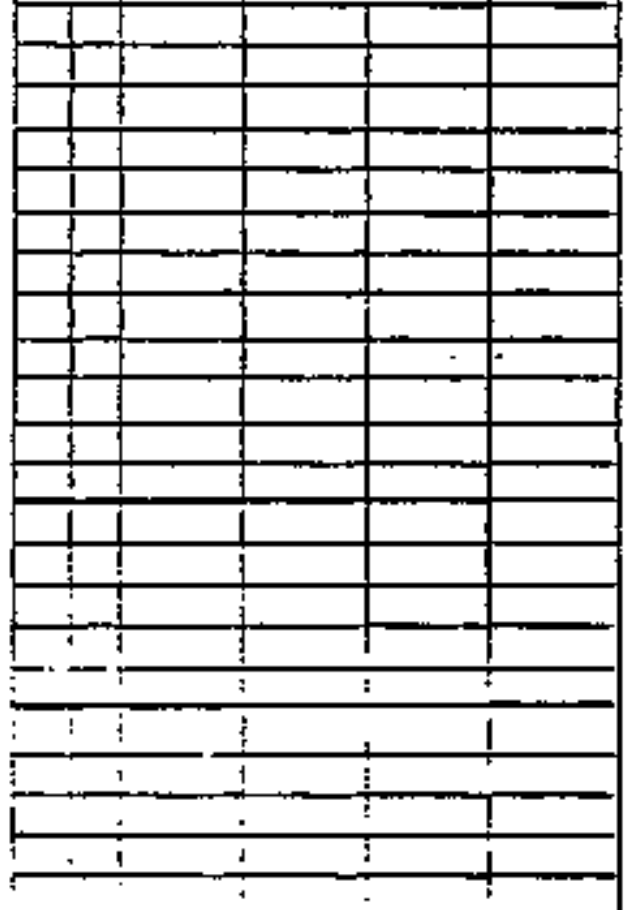

GROUNO WATER

\begin{tabular}{|c|c|c|}
\hline SEPTH & HOUN & OATE \\
\hline & & \\
\hline & & \\
\hline
\end{tabular}

SAMPLE TYPE

A- Awow artinja. $\mathrm{O}$ - Black nomplo

S - 2*t 0.D. 1.39" J.0. Rube amole.

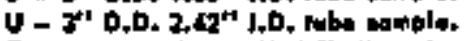

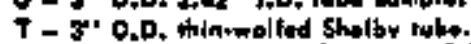

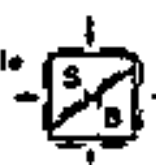

SERECNT, HAUSNNS \& BECKWTH

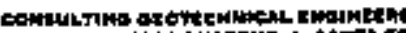

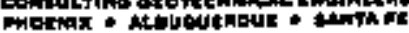




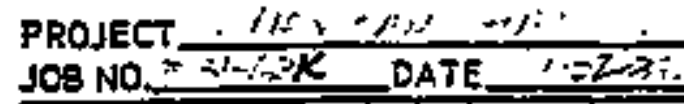

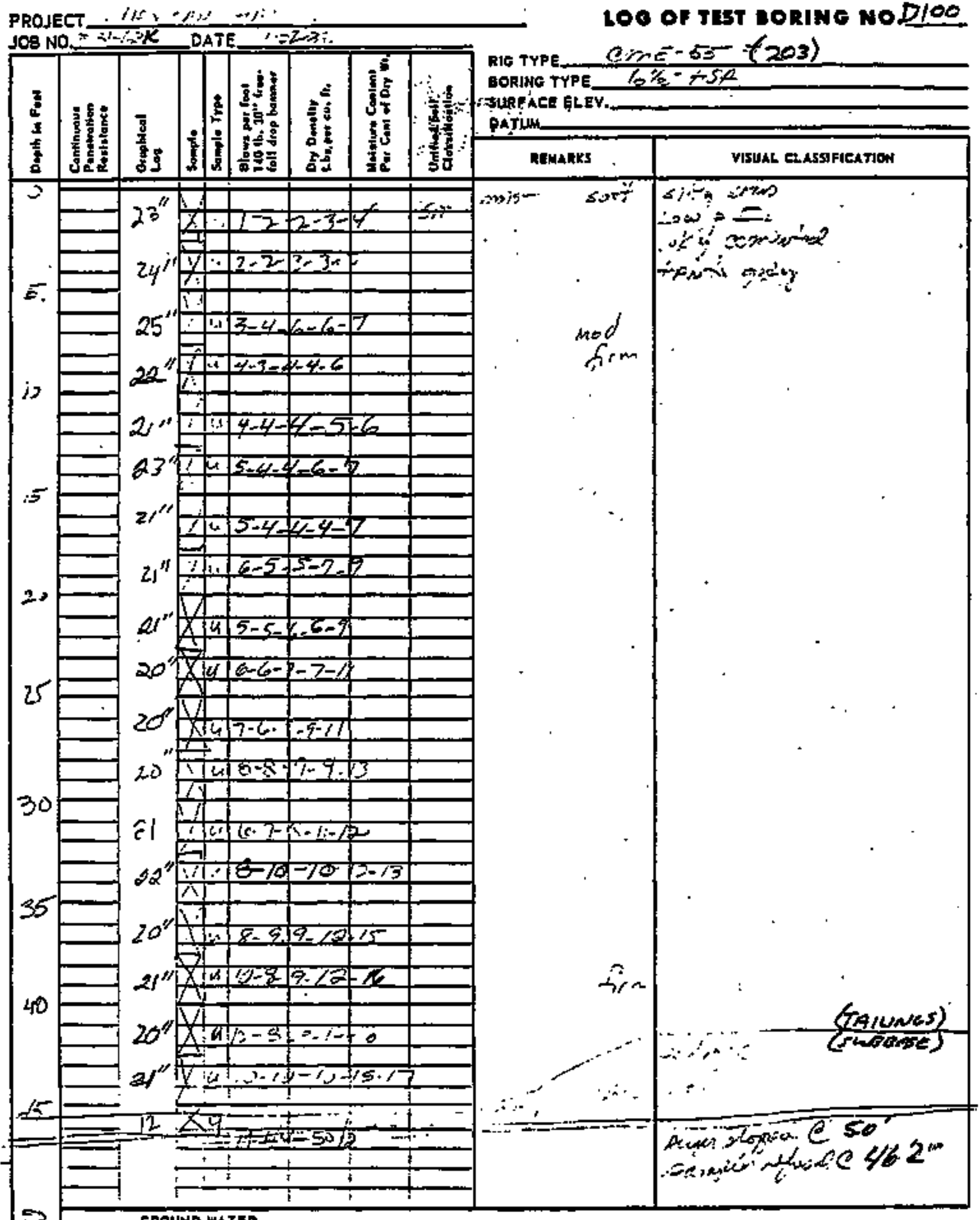




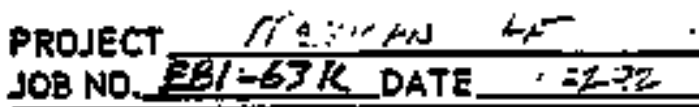

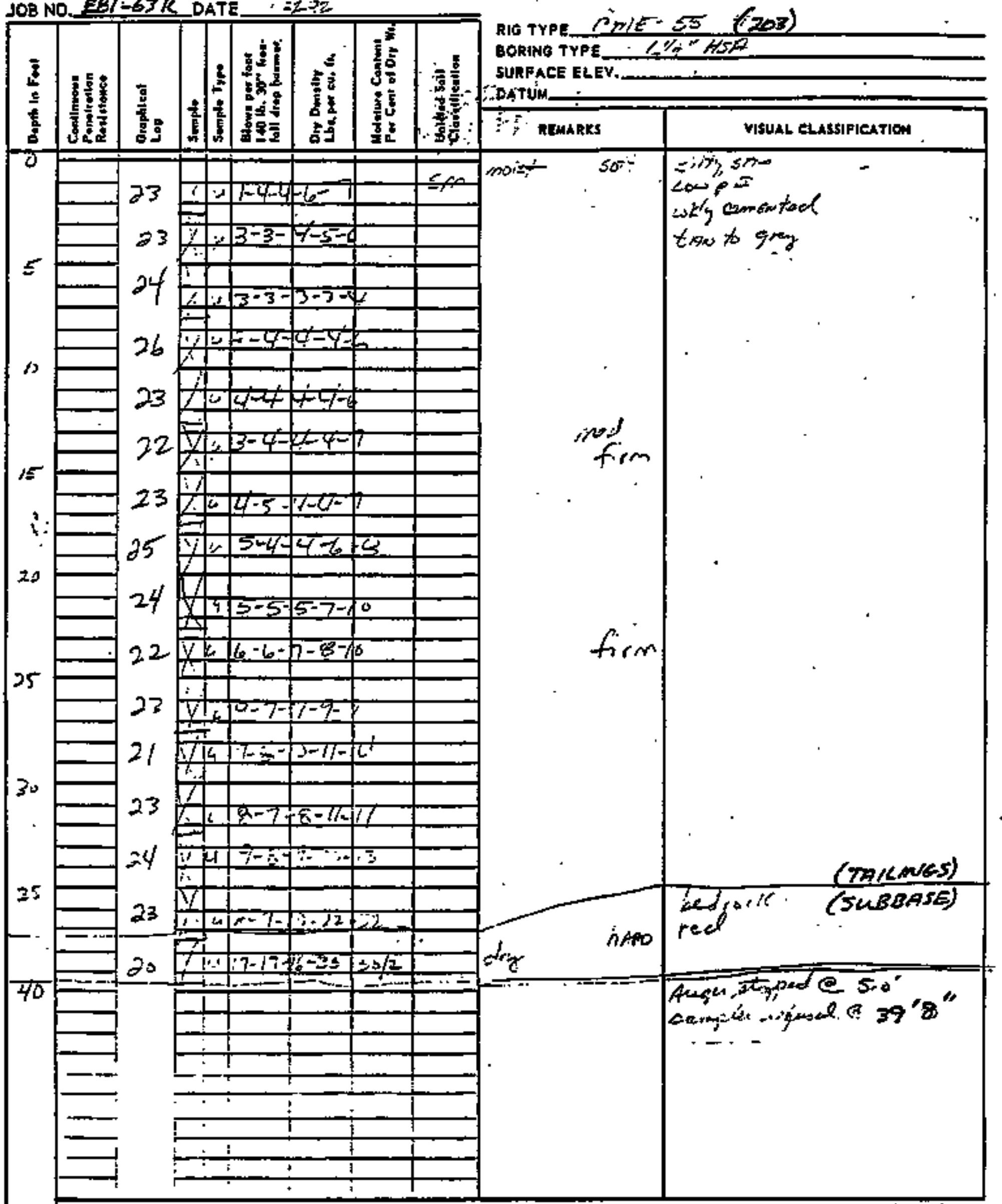

GROUND WATER

\begin{tabular}{|l|l|l|}
\hline DEPTH & HOUR & OATE \\
\hline & & \\
\hline & & \\
\hline
\end{tabular}

\section{SAMPLE TYPE}

A - Augw suttinga. B - Bloak namplo

\$ - z' O.D. 1.39"* 1.0. 1ule romple.

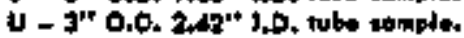

$T$ - 3" 0.0, thinmalled Sholby iubs.
LOE OF TEST CORJNG NO.DIOZ

inte ss (20B)

Low

w2ty amatod

the to sing 
PROJECT

LOG OF test dorine no.ploz

JOB NOE $E$ - $63 K$ DATE $/ 2.2 \xi$

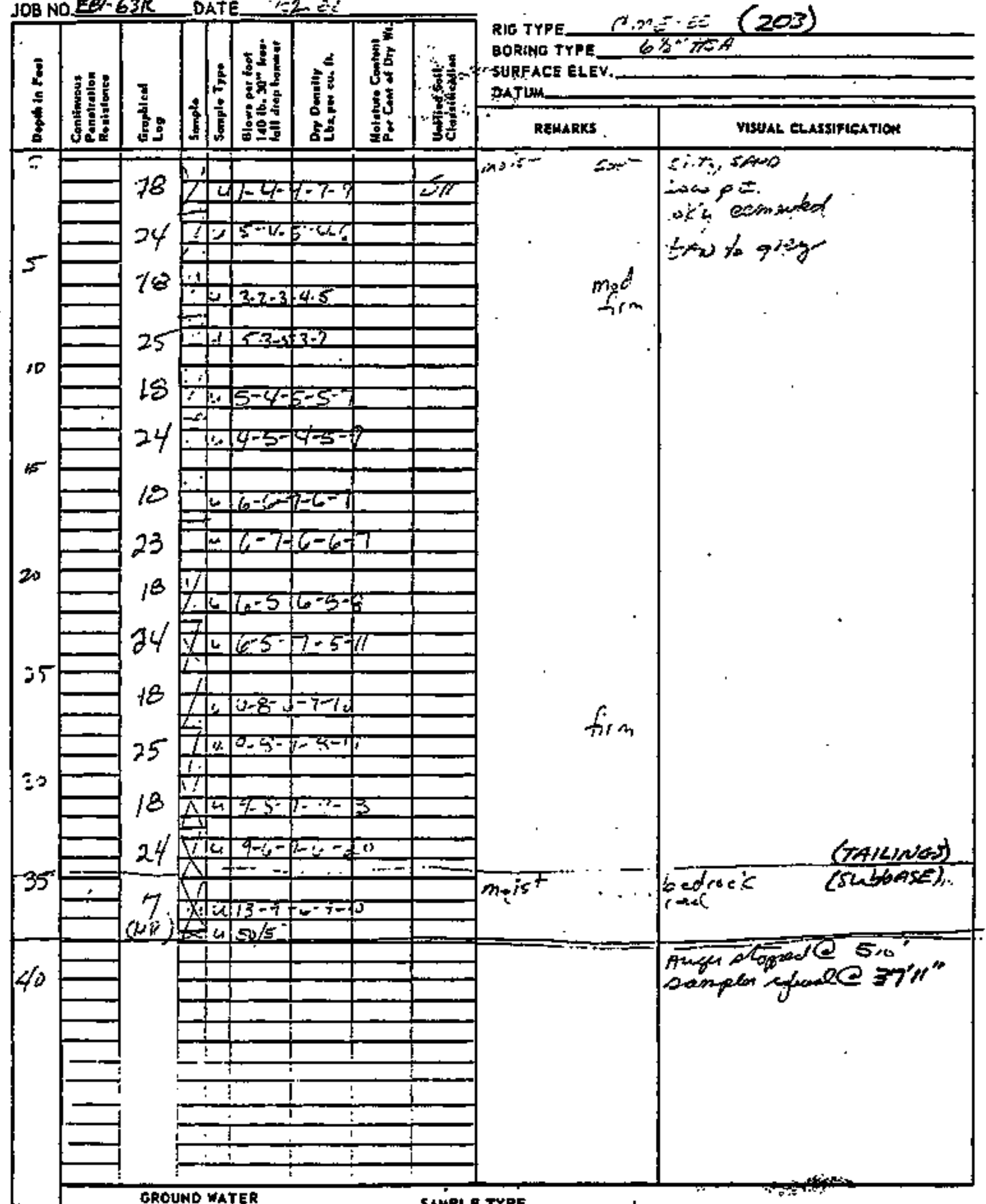

GROUND WATER

\begin{tabular}{|l|l|l|}
\hline DEPTH & MOUA & DRTE \\
\hline & & \\
\hline & & \\
\hline
\end{tabular}

\section{SAMPLE TYPE}

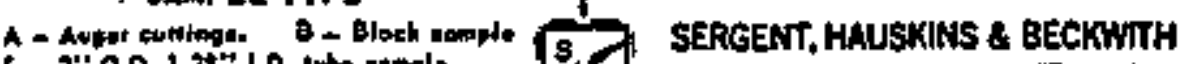

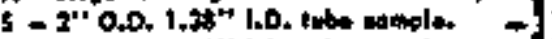

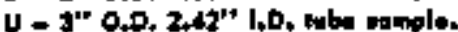




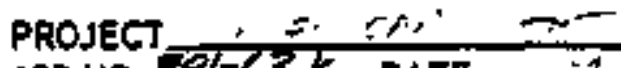
JOB NO. BOF-GK_DATE_ $=2-9$

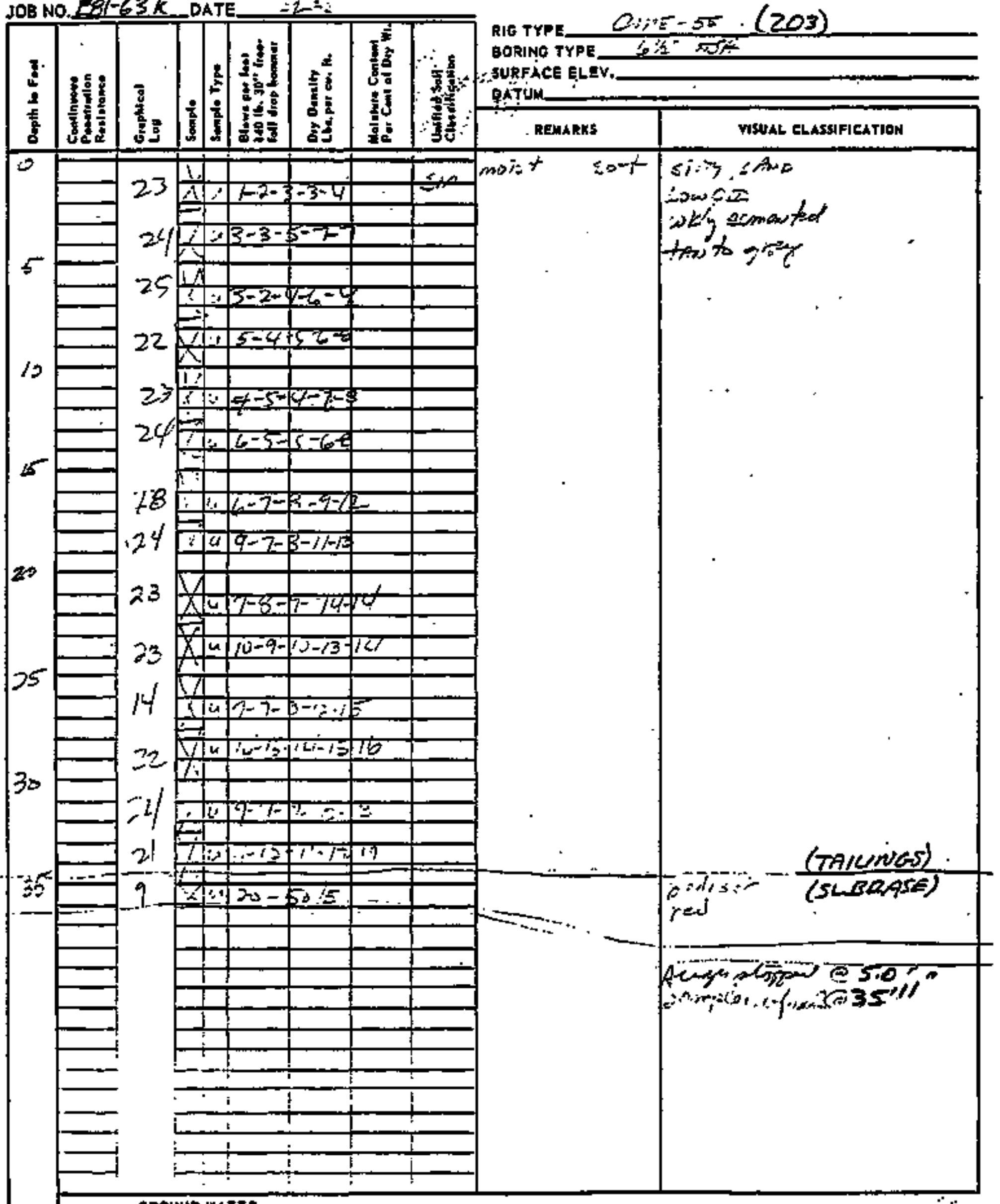

GROUKO WATER

\begin{tabular}{|l|l|l|}
\hline DEPTH & MOUR & DATE \\
\hline
\end{tabular}
SAMPLE TYPE

A - Avpw cuwing.

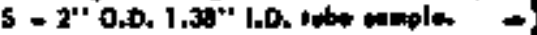

U - 3*' D.D. 2.4Z" I.D. 1ube complo.

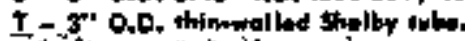

inswa

sky acmanted

this $\rightarrow>-2$ 


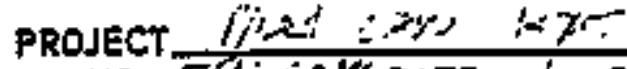

Jos No. $=4 \% 3 K$ DATE $1,72,32$

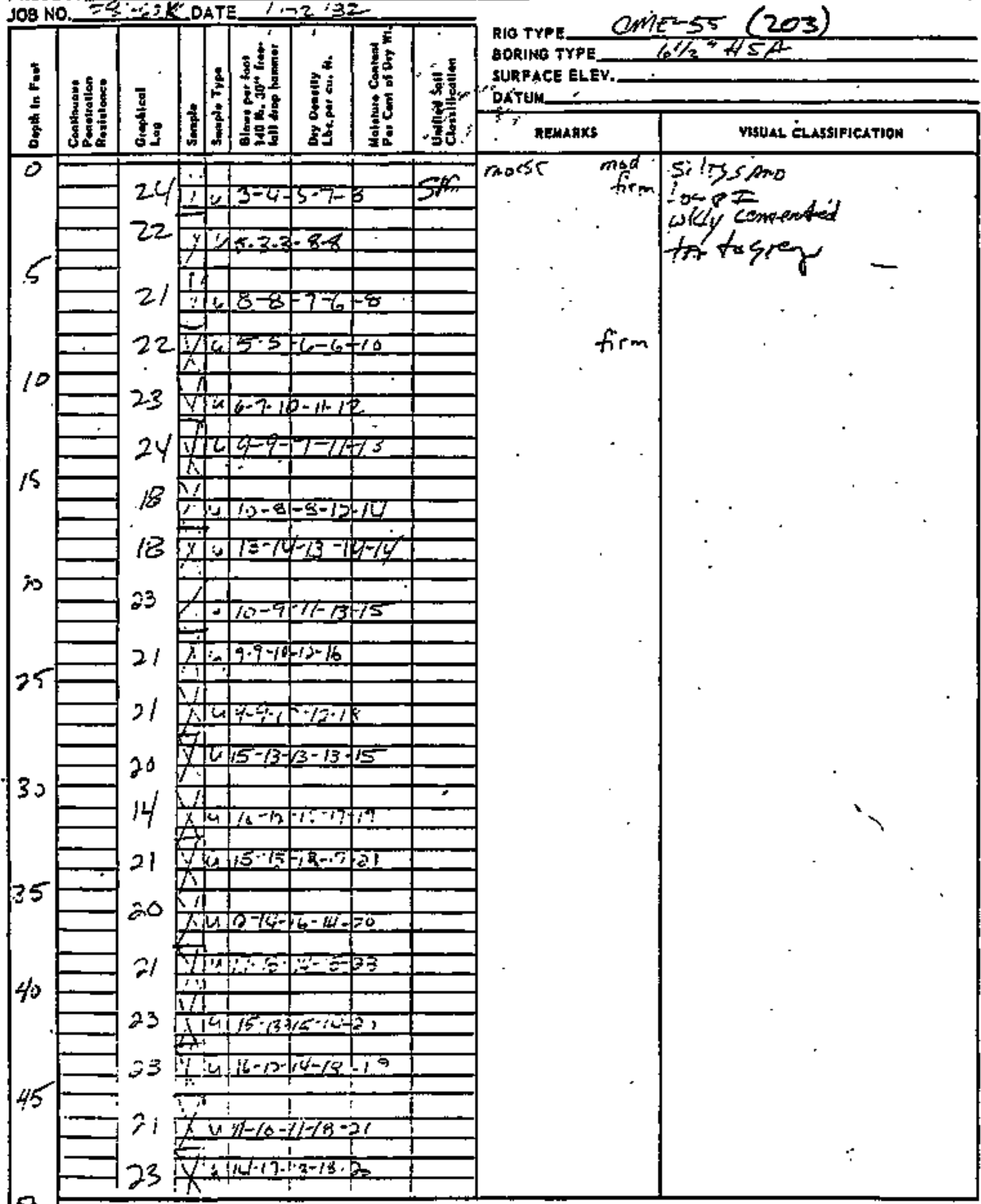

53

GROUMO WATER

\begin{tabular}{|l|l|l|}
\hline DEPTH & MOUF & DATE \\
\hline & & \\
\hline & & \\
\hline
\end{tabular}

\section{SAMPLE TYPE}

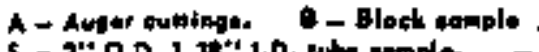

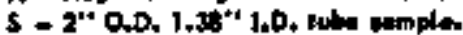

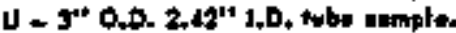

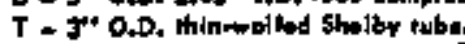

SERGENT, HAUUSKINS \& BECKWITH

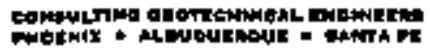




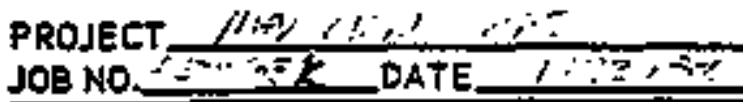

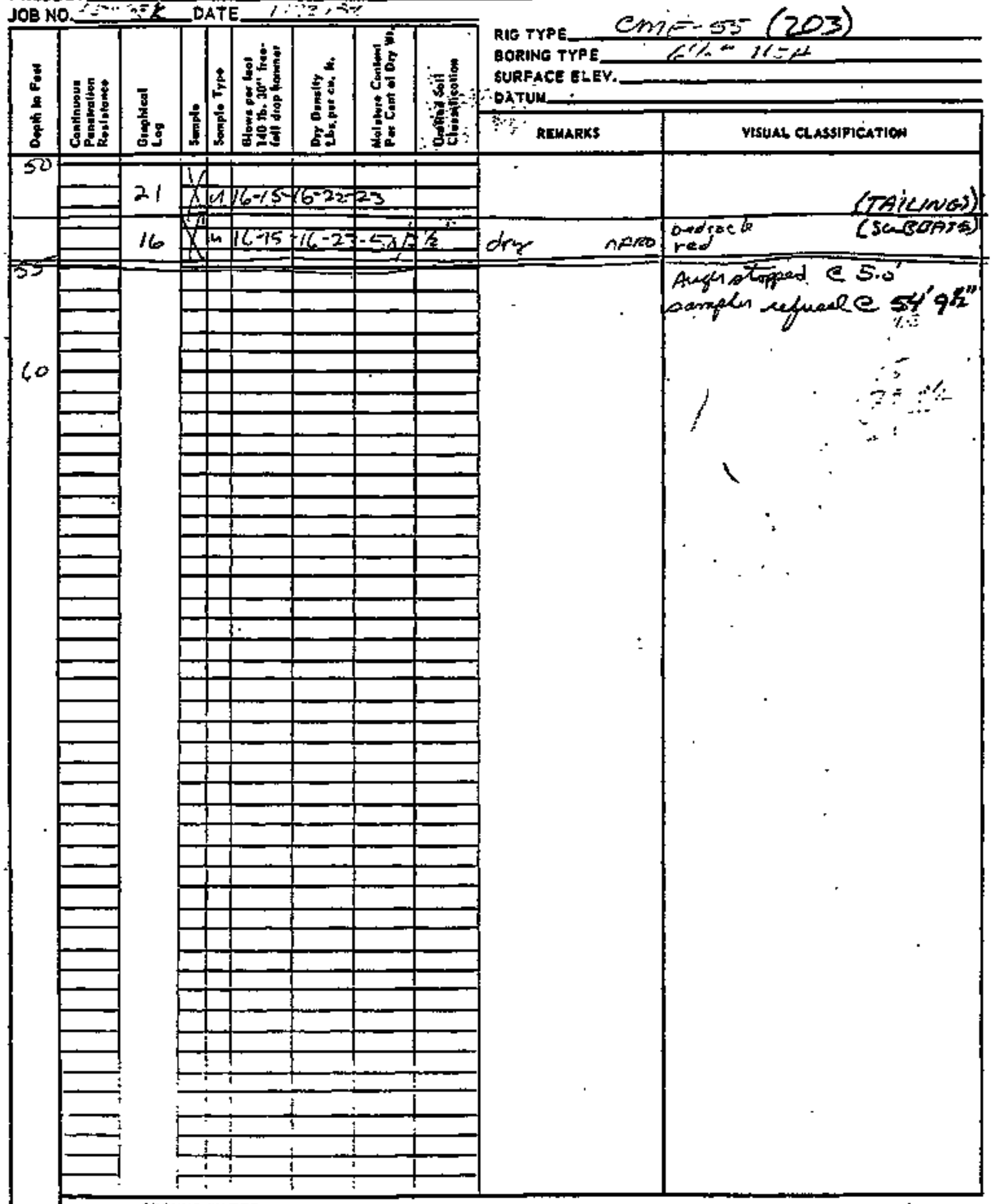
$2 n 7=53$ (203) 
PROJECT Hin JOB NO,ESt $63 \mathrm{~K}$. DATE I: :

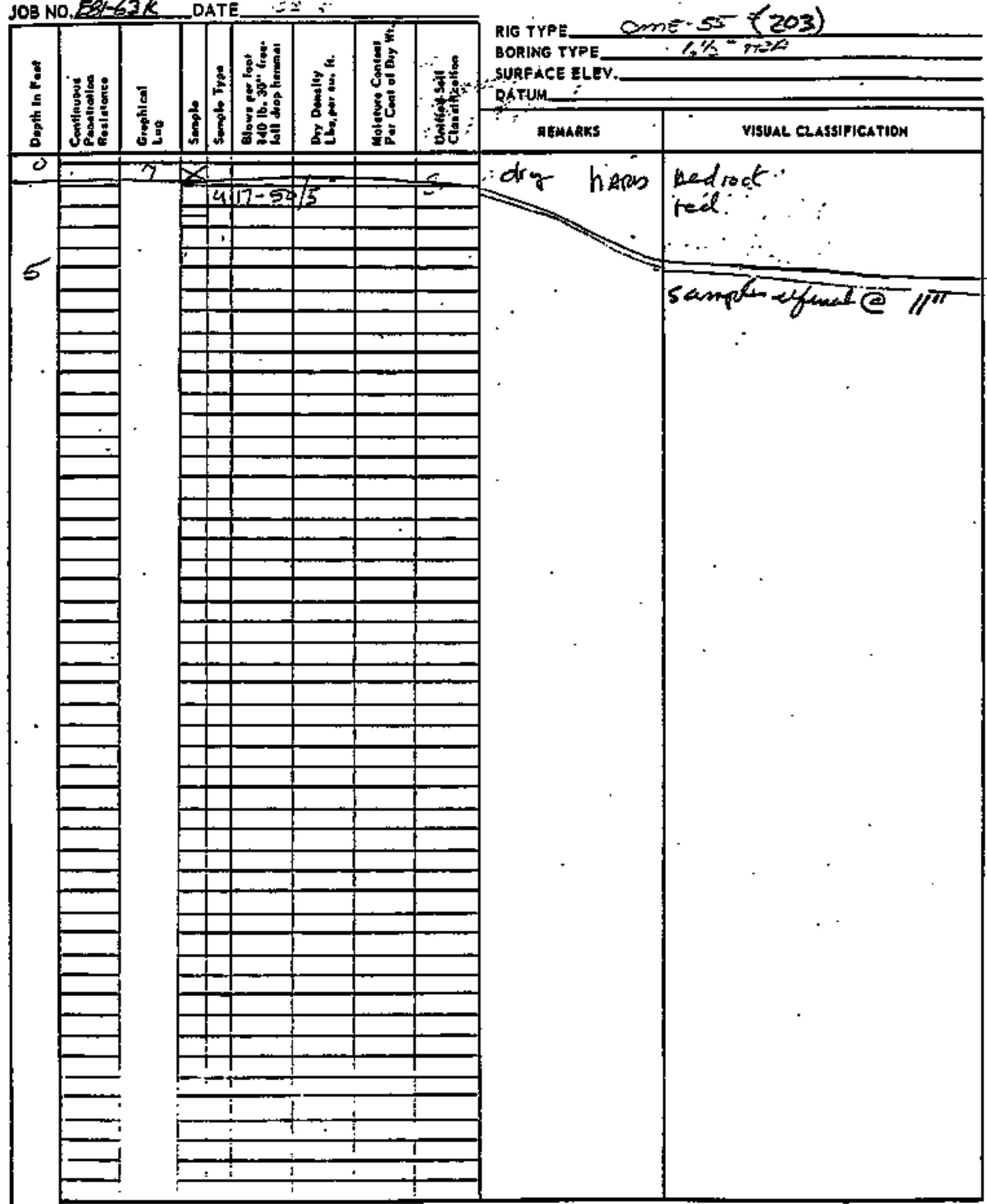

GROUND WATER

\begin{tabular}{|l|l|l|}
\hline DEPTH & HOUH & DATE \\
\hline & & \\
\hline & & \\
\hline
\end{tabular}

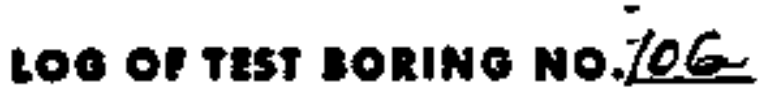
$\cos \sin ^{-5}(203)$

BORING TYPE $11 \%{ }^{2}+2 \%$ SUURFACE ELEV.

\section{SAMPLE TYPE}

5 - z+O. O. 1.38 " T.D. tube romple

$\mathrm{U}=\mathrm{J}^{+1}$ O.D. 2.42" t. D. tube somela.

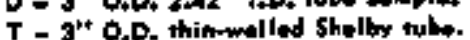

A - Awo curingl. B - Btack vemple
SERGENT, HAUSKINS \& BECKWITH

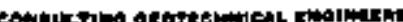

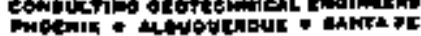


PROJECT
JOB NOE E $/ 7 /-63 K$ DATE

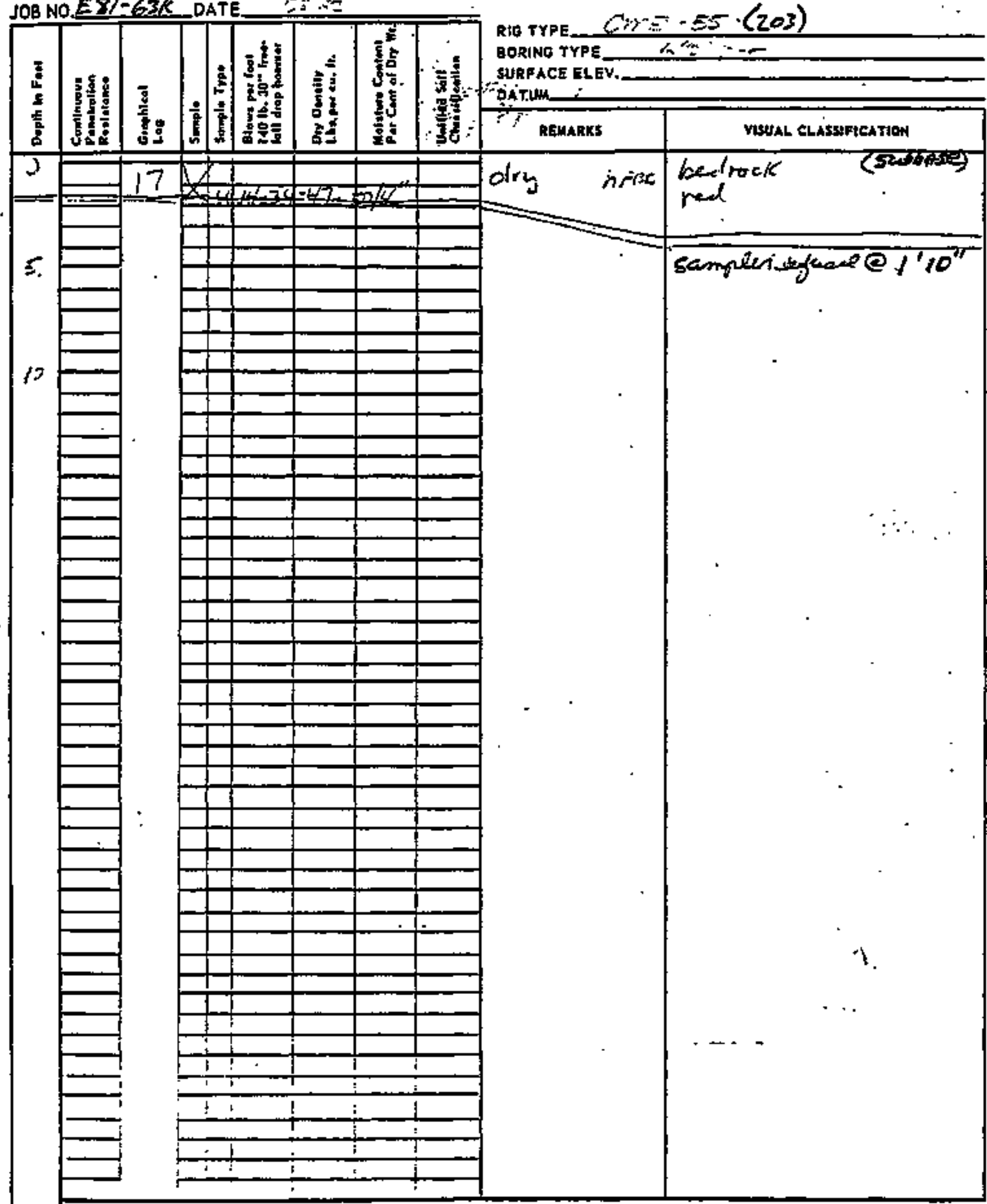

$\cos =-55 \cdot(203)$

LOE OF THST DORINO NO. 107 
PROJECT MleYirind hintJOB ND.ES/-63k DATE / $123 / \mathrm{AT}$

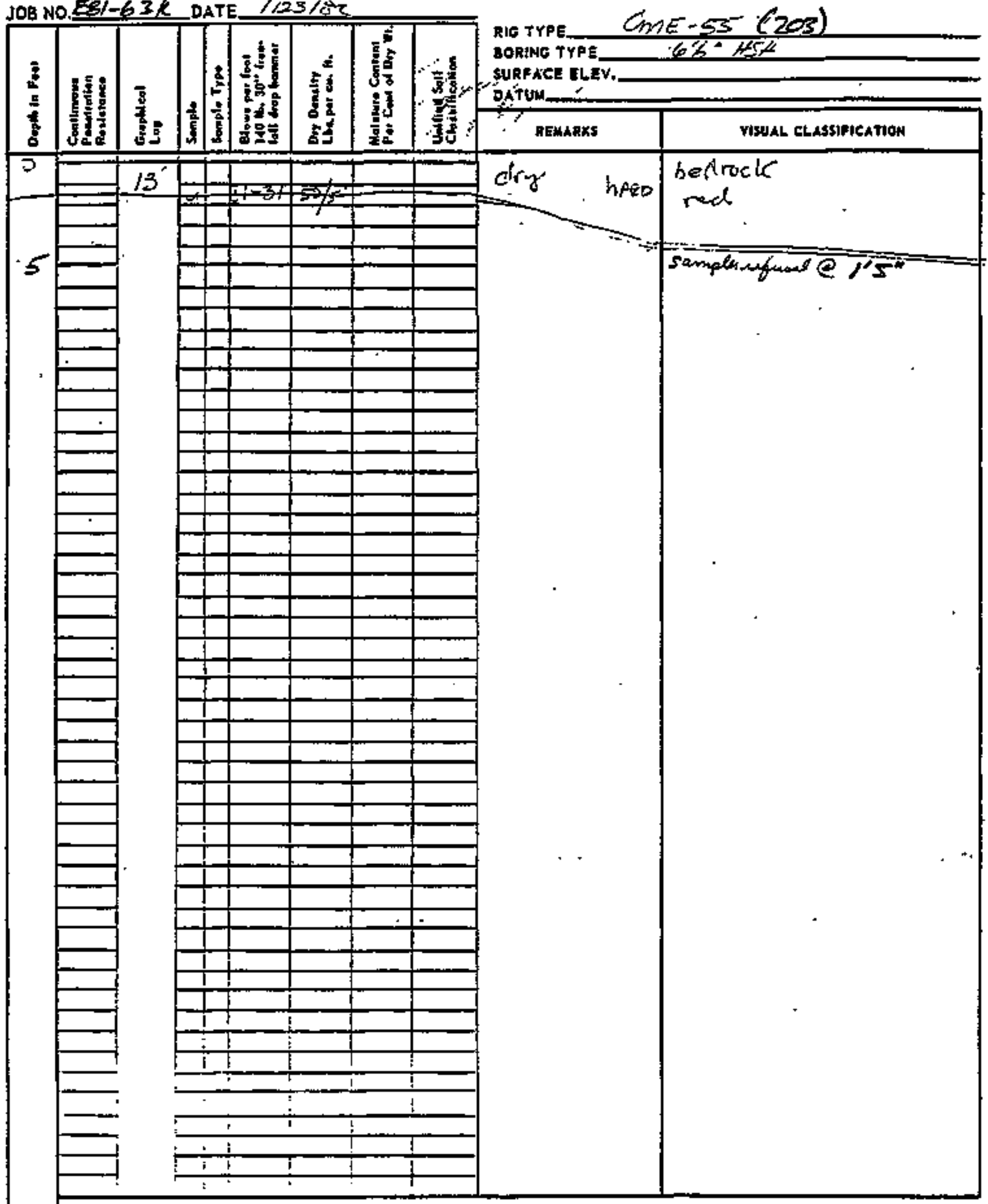




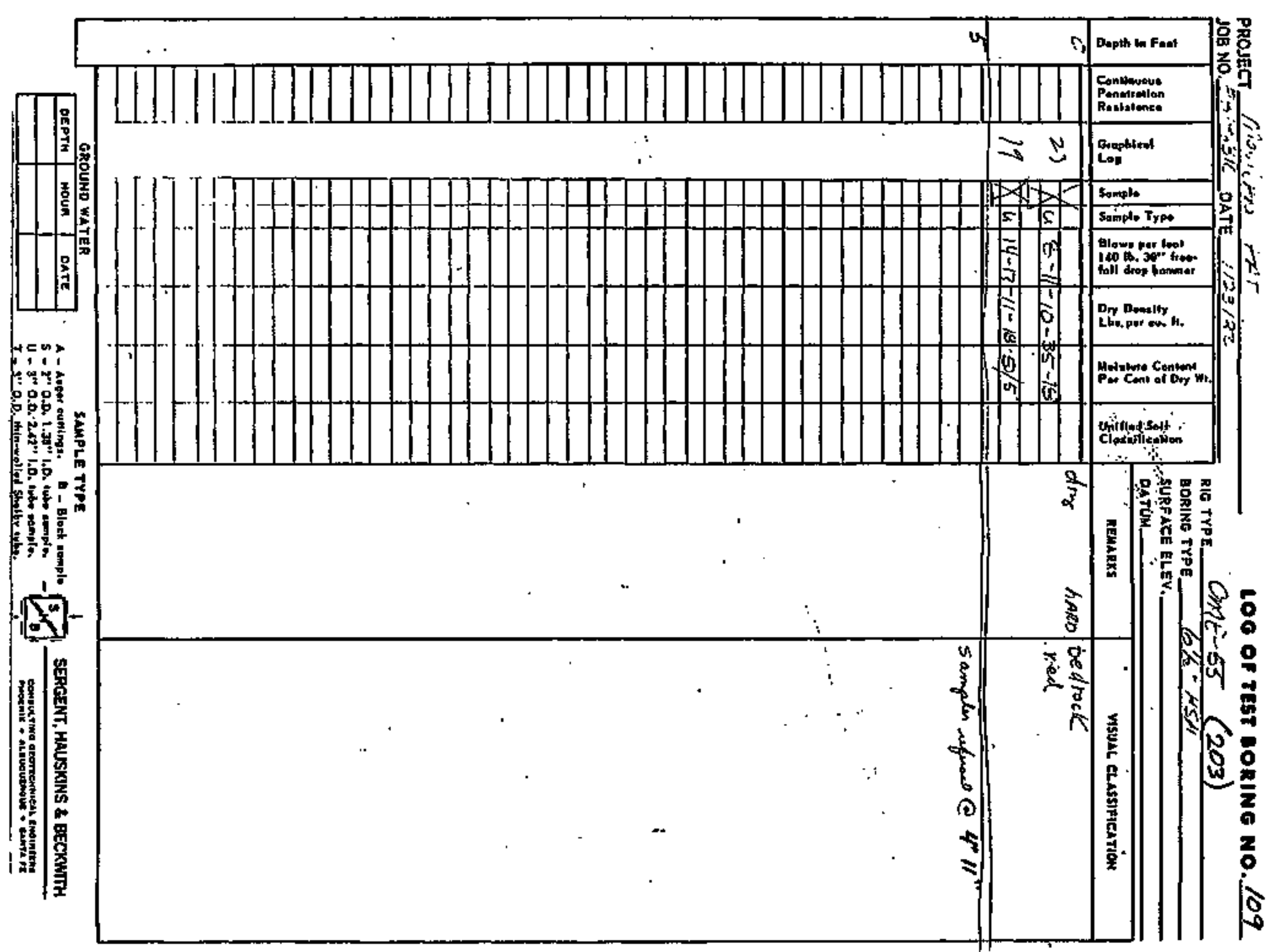




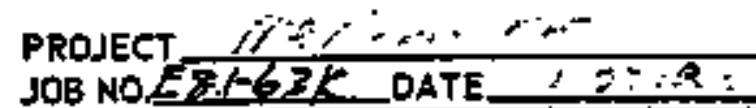

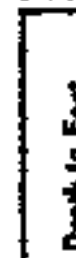

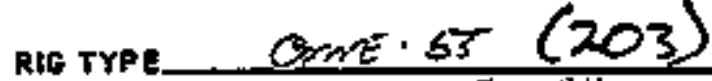

BORING TYPE

SURFACE ELEV.

F" ACHARKS

VISHAL CLASIFFICATION

dry intion $\begin{aligned} & \text { bedion } \\ & \text { red }\end{aligned}$

10

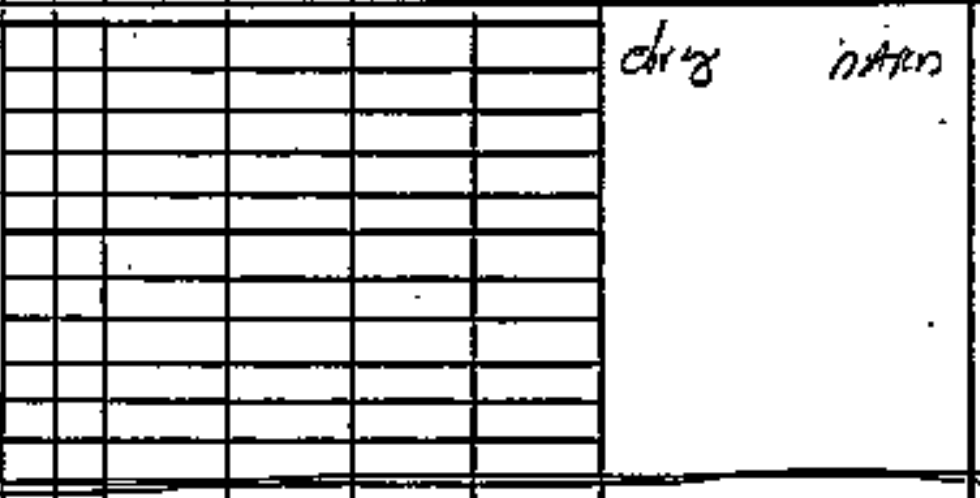

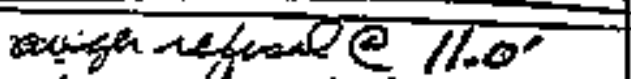

piezometh h/e-

Hos piec sat)

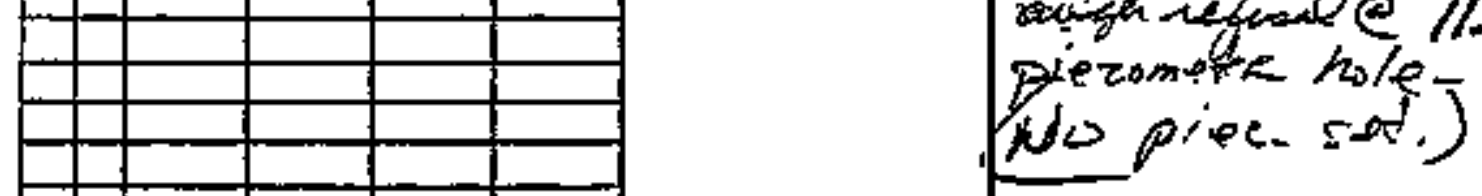

\begin{tabular}{|}
\hline \\
\hline \\
\hline \\
\hline \\
\hline \\
\hline \\
\hline \\
\hline
\end{tabular}

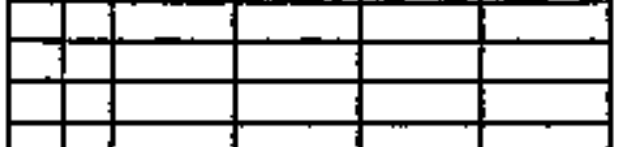

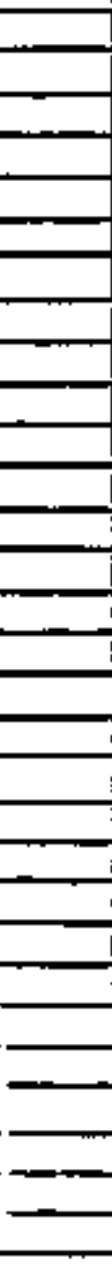

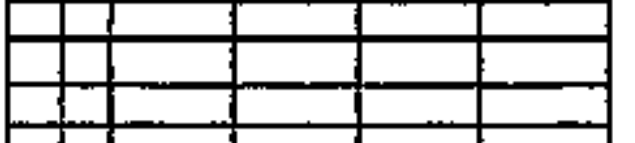

\begin{tabular}{|l|l|l|l|l|}
\hline 1 & & & & \\
\hline 1 & & & & \\
\hline & & & \\
\hline
\end{tabular}

\begin{tabular}{|l|l|l|l|}
\hline & & & \\
\hline
\end{tabular}

\begin{tabular}{|l|l|l|l|l|}
\hline 1 & & & & \\
\hline 1 & & & & \\
\hline
\end{tabular}

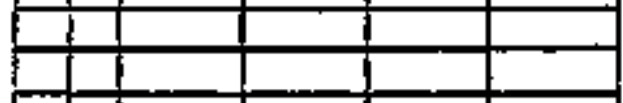

\begin{tabular}{|l|l|l|l|l|}
\hline & & & & \\
\hline & & & & \\
\hline
\end{tabular}

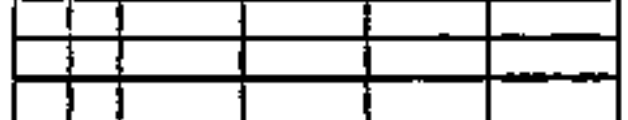

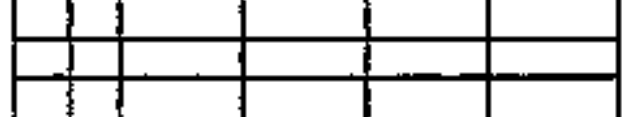

\begin{tabular}{|l|l|l|l|}
\hline 1 & & & \\
\hline 1 & & & \\
\hline
\end{tabular}

\begin{tabular}{|l|l|l|l|l|}
\hline 1 & & & & \\
\hline 1 & & & & \\
\hline
\end{tabular}

\begin{tabular}{|l|l|l|l|}
\hline 1 & & & \\
\hline 1 & & & \\
\hline
\end{tabular}

\begin{tabular}{c|c|c|}
\hline$\vdots$ & $\vdots$ & $\vdots$ \\
\hline
\end{tabular}

\begin{tabular}{|llll}
\hline & & & 1 \\
\hline & 1 & 1 & 1 \\
\hline
\end{tabular}

\section{SAMPLE TYPE}

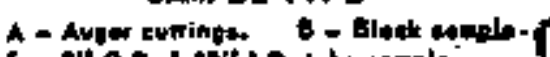

S = 2" 0.0. 1.35" 1.0. 1ubt temple.

$U$ = S" 0.\$, 2.42" 1.0. 14te comple.

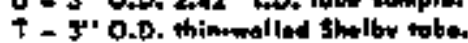

SERGENT, HAUSKINS \& BECKWITH

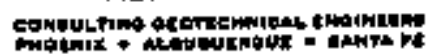




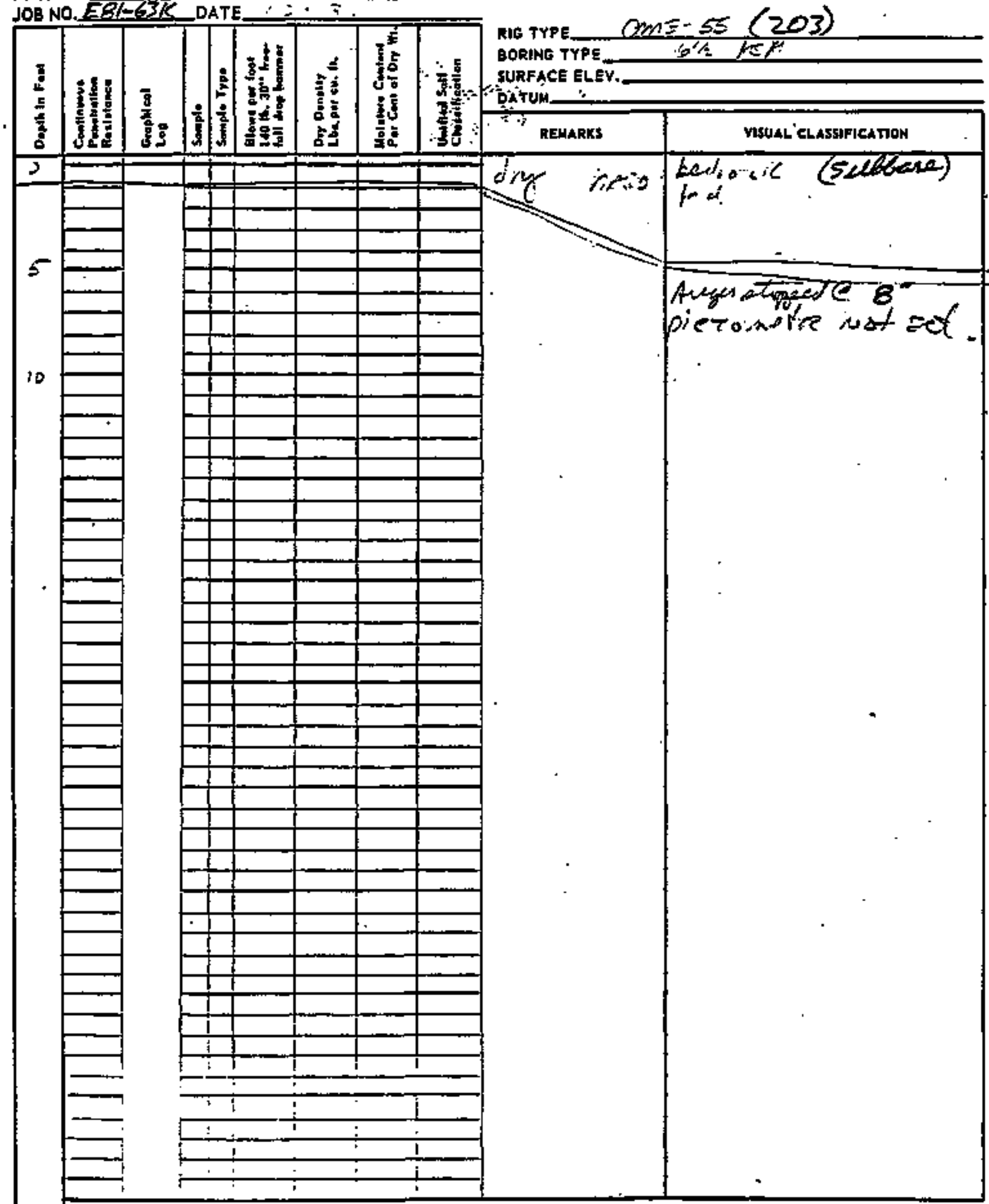




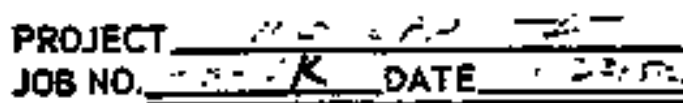

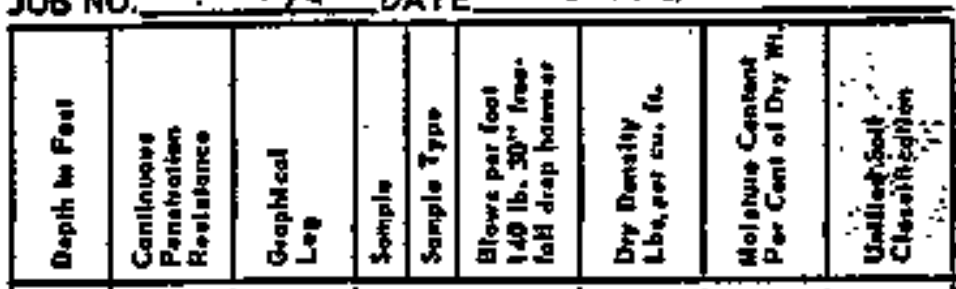

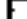




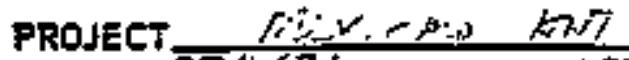
JOBNO. ED/ $63 k$ DATE $1 / 27 / 2$

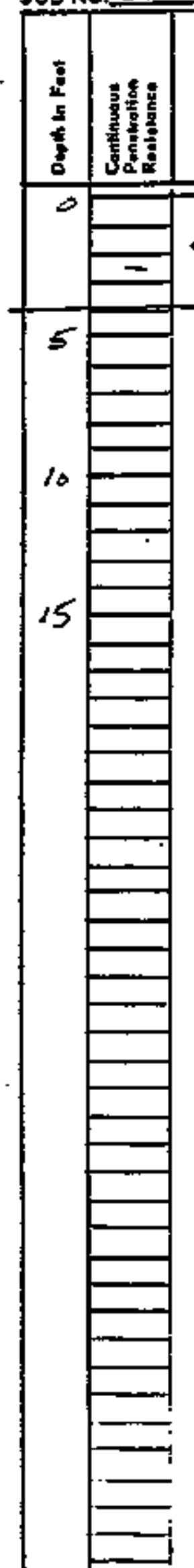

LOO OF TEST MORING NO.2OI RIS TYPE _. emente-s- (203)

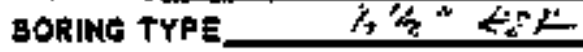

SOUBF ACE ELEV.

QATUM

Rlumaks

VIOAL CLASSIFICATION bedrack red

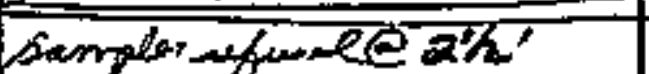

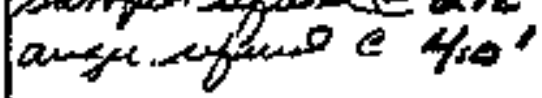




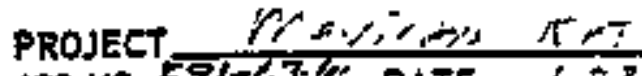
JOB NO.ERI-G3K OATE I TRAA

GROUND WATER \begin{tabular}{|l|l|l|}
\hline DEPTH & HOUP & DATE \\
\hline
\end{tabular}

\section{SAMPLE TYPE}

A - Avear putringa. 0 - Hoch notaplo

\$ - 2"' O.D. 1.3" 1.0. Pube mample.

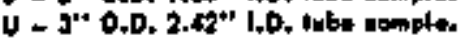

T - '" O.D. *tan-wollod Shelly tube.
Loe of rest tontNe no.20? cmet $55-7(203)$

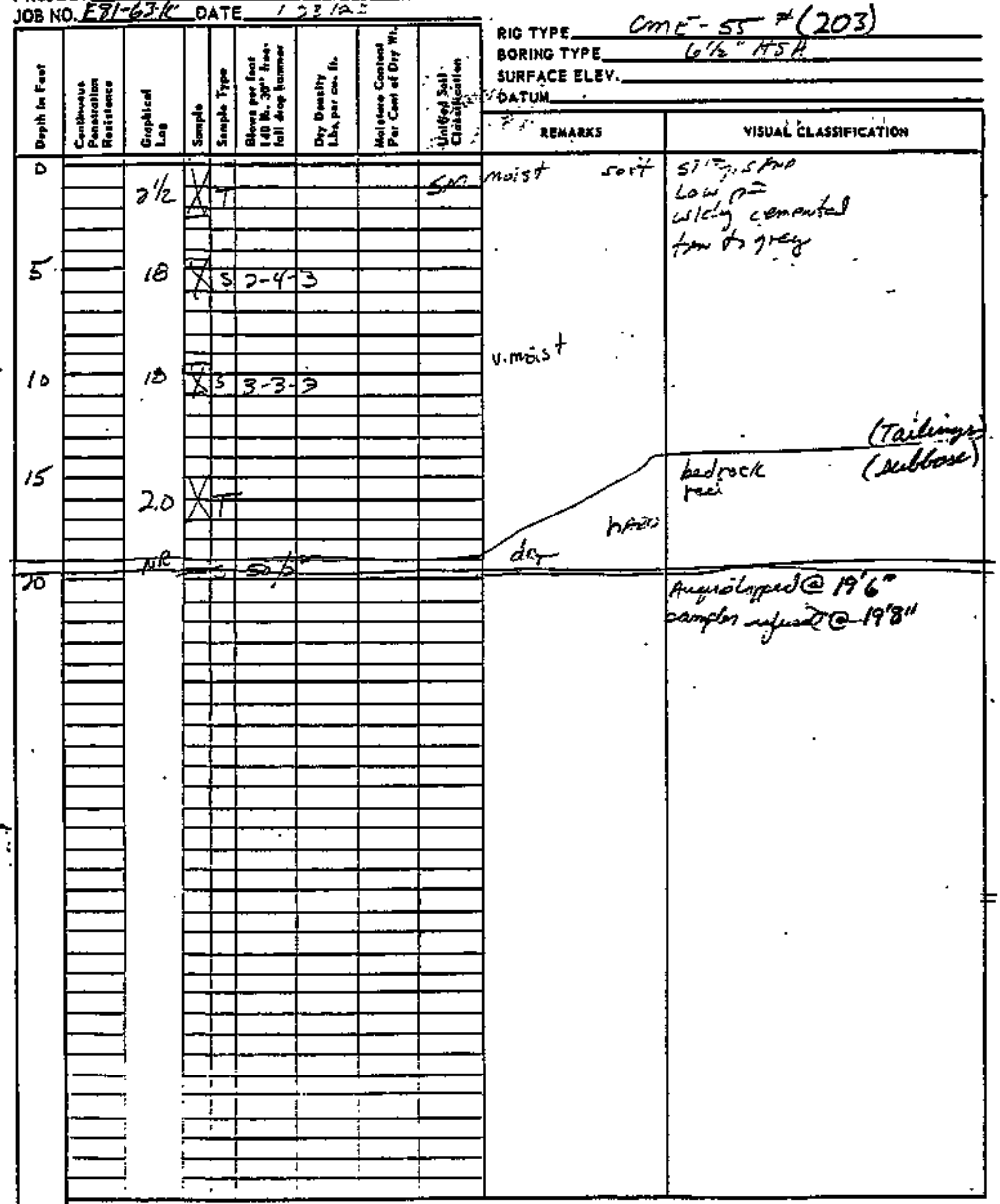

\section{VISUAL CLASSIFIEATIOA}

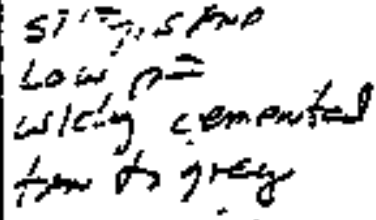

bedreck (sectrose)

Anutityod@ 196

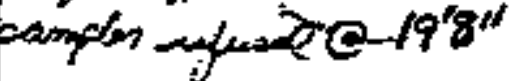

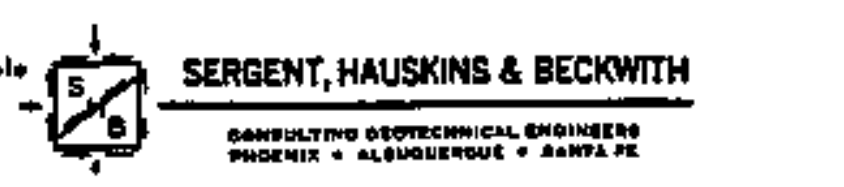




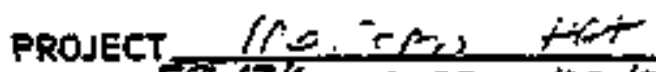

JOB NO E 6 KK DATE $1 / 2=192$

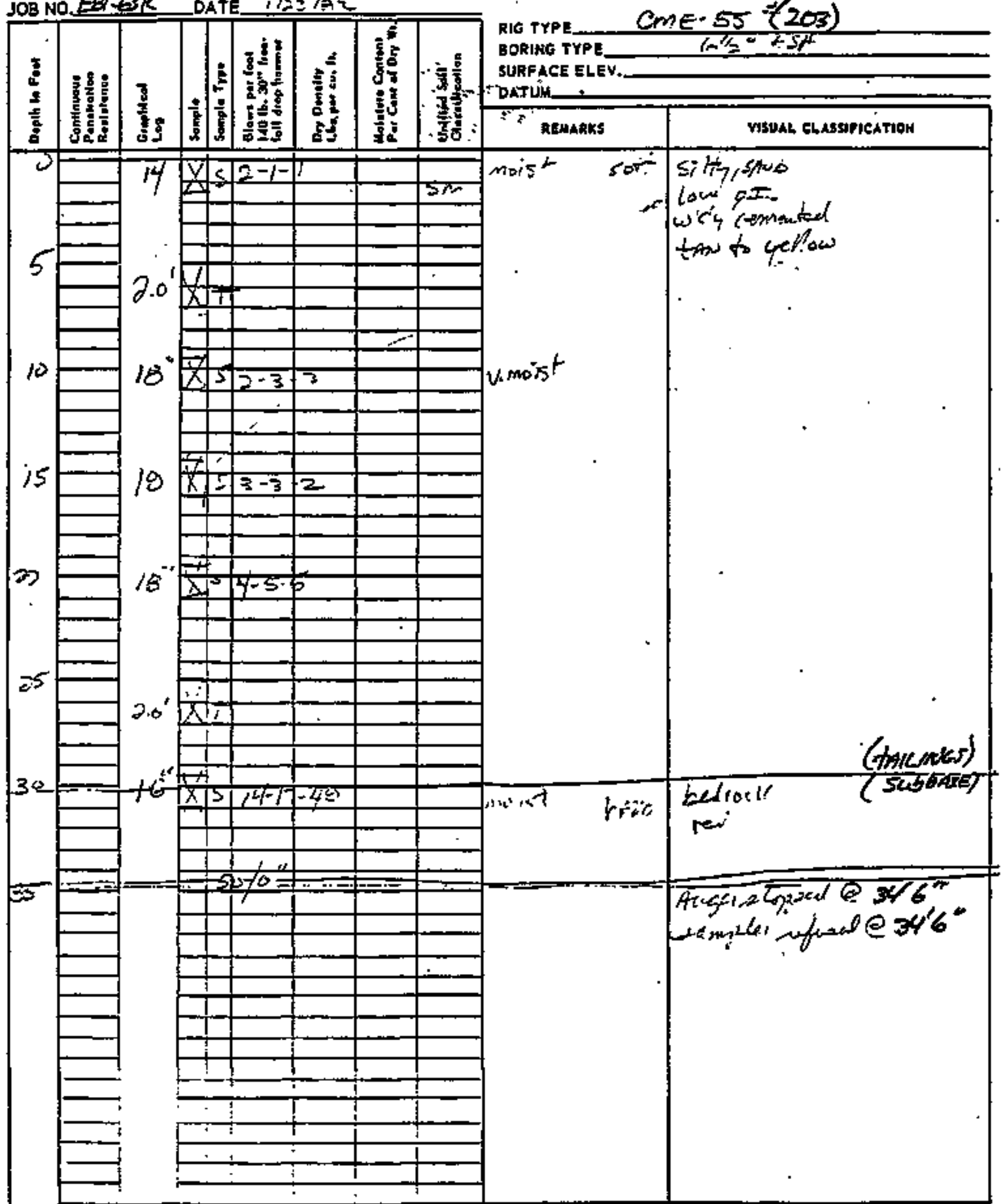

VHSAL ELASSIFICATIOH

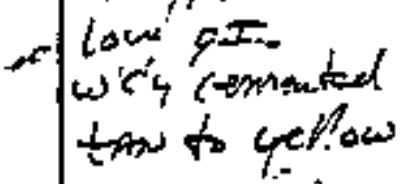

GROUND WATER

\begin{tabular}{|l|l|l|}
\hline DEPTH & HOUN & OAFE \\
\hline & & \\
\hline & & \\
\hline
\end{tabular}

SAMPLE TYFE

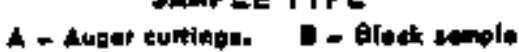

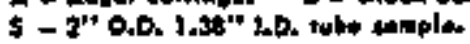

U = 3" 0.0. 2.42" H.D. rube tample.

$T=3^{\prime+}$ 0.0, thinmellod sholby twber
Los of ths conine No. 203 CME.55 (203) 


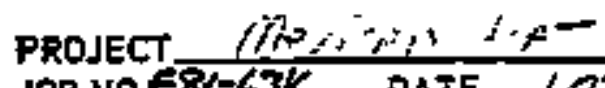

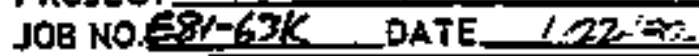

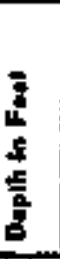

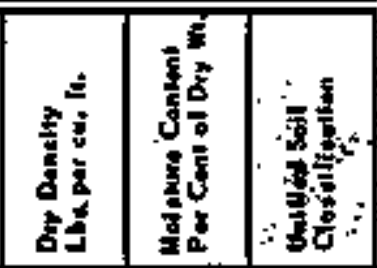

R16 TYPE.

LOG OF TEST BORING No. 205 EORJNO TYPE

imE-SE (203)

SURFACE ELEV.

"oiTty :

?

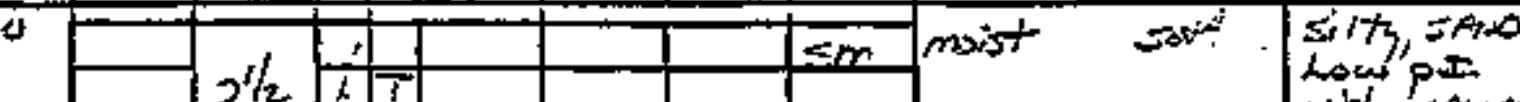

widu cemented

th $=$

5

10

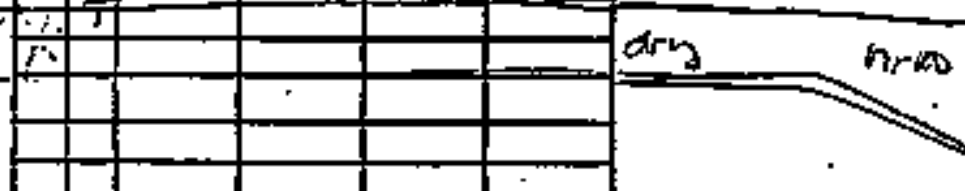

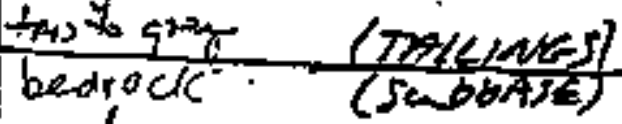
reel

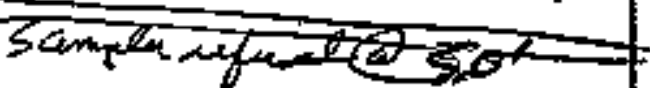




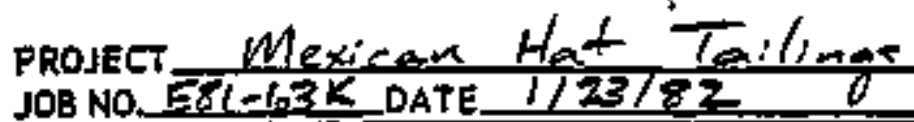

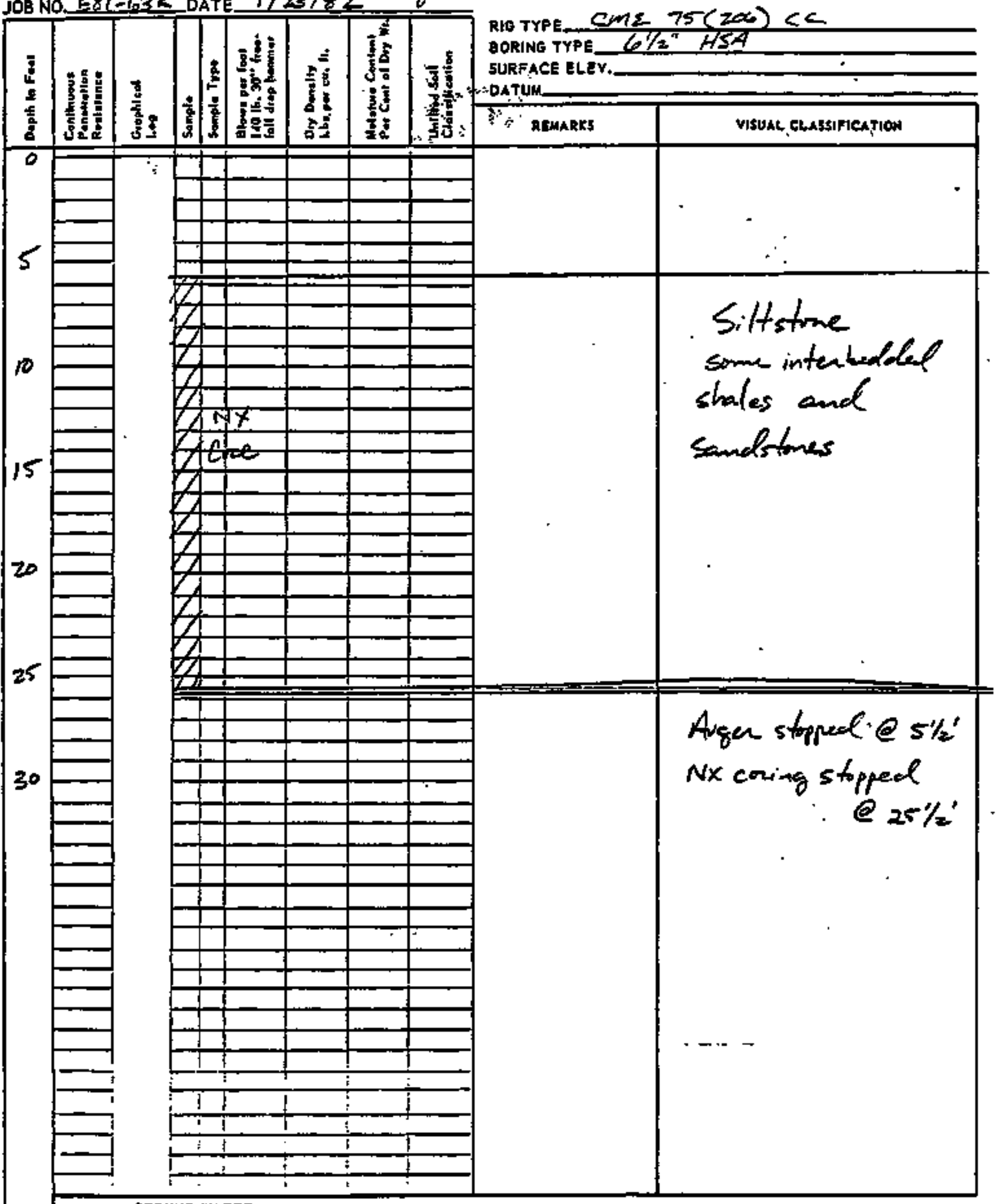

GROUND WATER

\begin{tabular}{|l|l|l|}
\hline OEPTH & HOUR & DATE \\
\hline NEN/F & & \\
\hline & & \\
\hline
\end{tabular}

SAMPLE TYPE

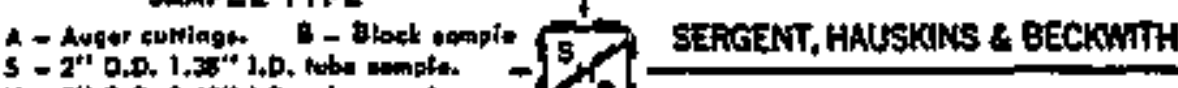

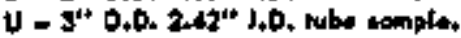

T = 3" 0.D. thinemalled shelbr tube.

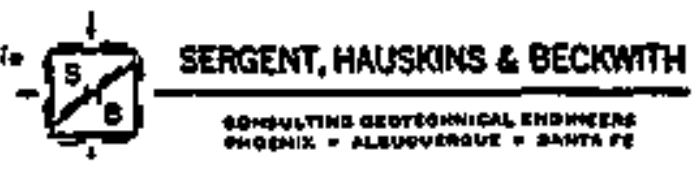




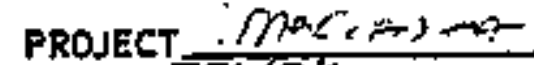

JOQ NO.EFGSK DATE 1 ZZ\&

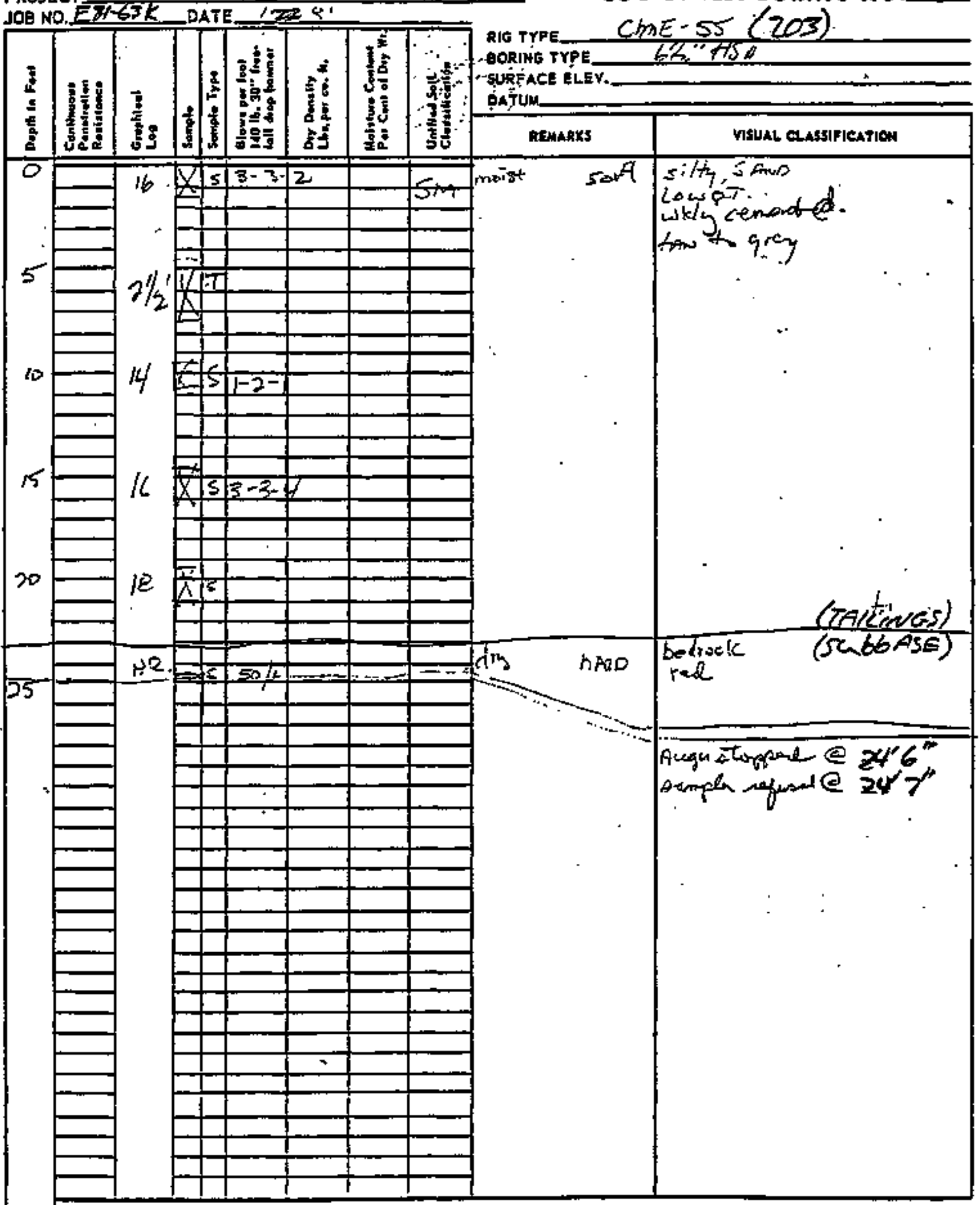




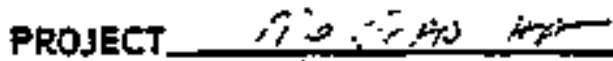

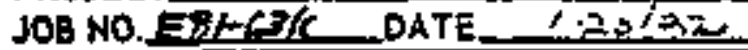

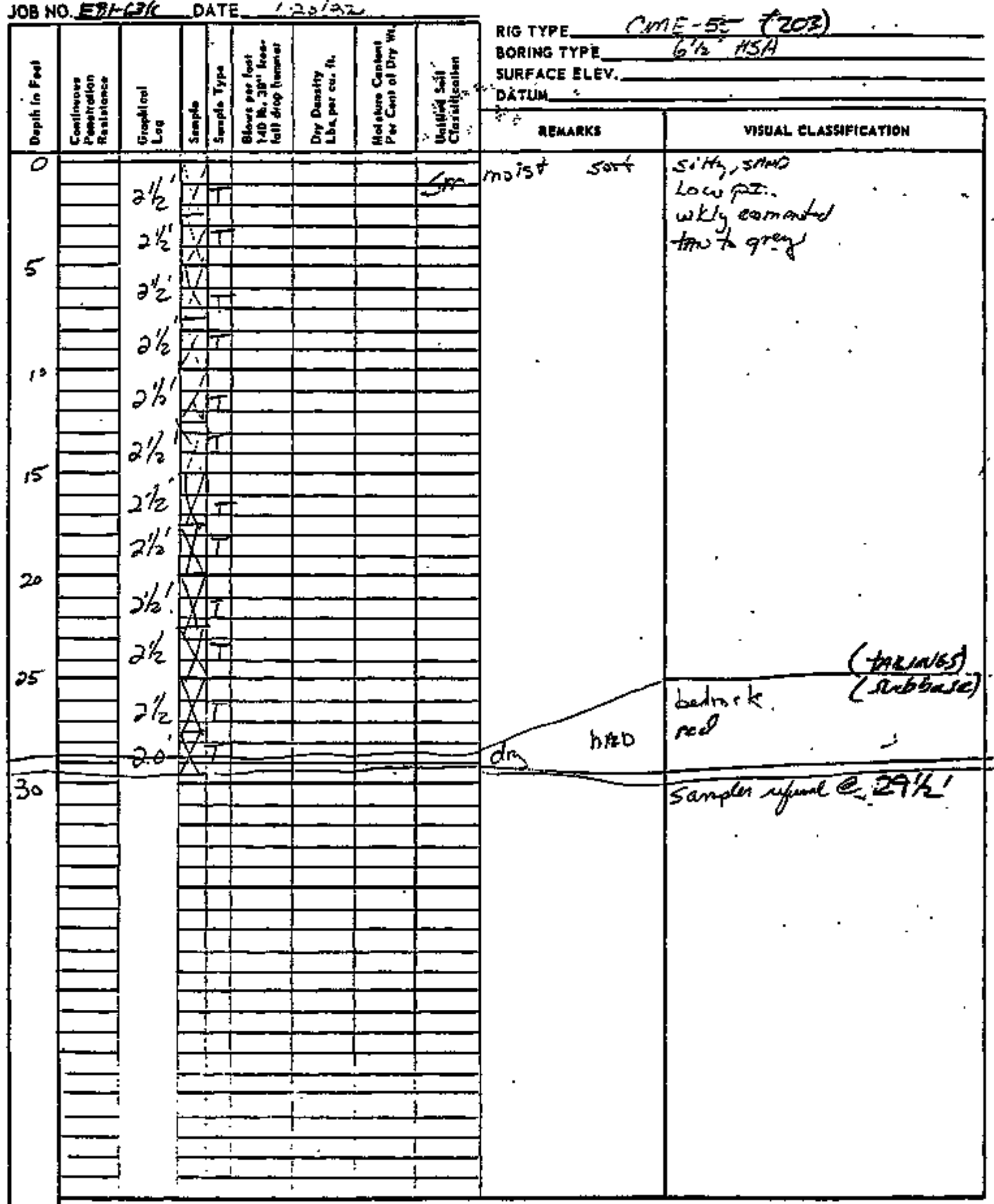

\section{GROUND WATER}

\begin{tabular}{|l|l|l|}
\hline OEPTH & HOUA & OATE \\
\hline & & \\
\hline & & \\
\hline
\end{tabular}

\section{SAMPLE TYPE}

A - Auger turingl.

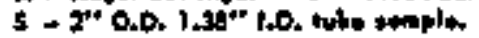

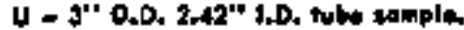

$T$ - 9" O.O. thin-mallad shalby tuber.
LOG of ths torine Mo.20\%

$\operatorname{Con}=-5^{2}(20)$

VISUAL ELASAFFEATION

$5 \cdot A_{3}, 5$ stmos

Lo4 fr:

$\omega k / y \cos a x-d$

$\tan 7 q^{2}=$

bedrot $k$

rell

sampln ryme $27 \%$ 
PRONECT $\therefore$, $\therefore$ - -2

JOB NO.EDF-63K DATE $1,222^{2}$

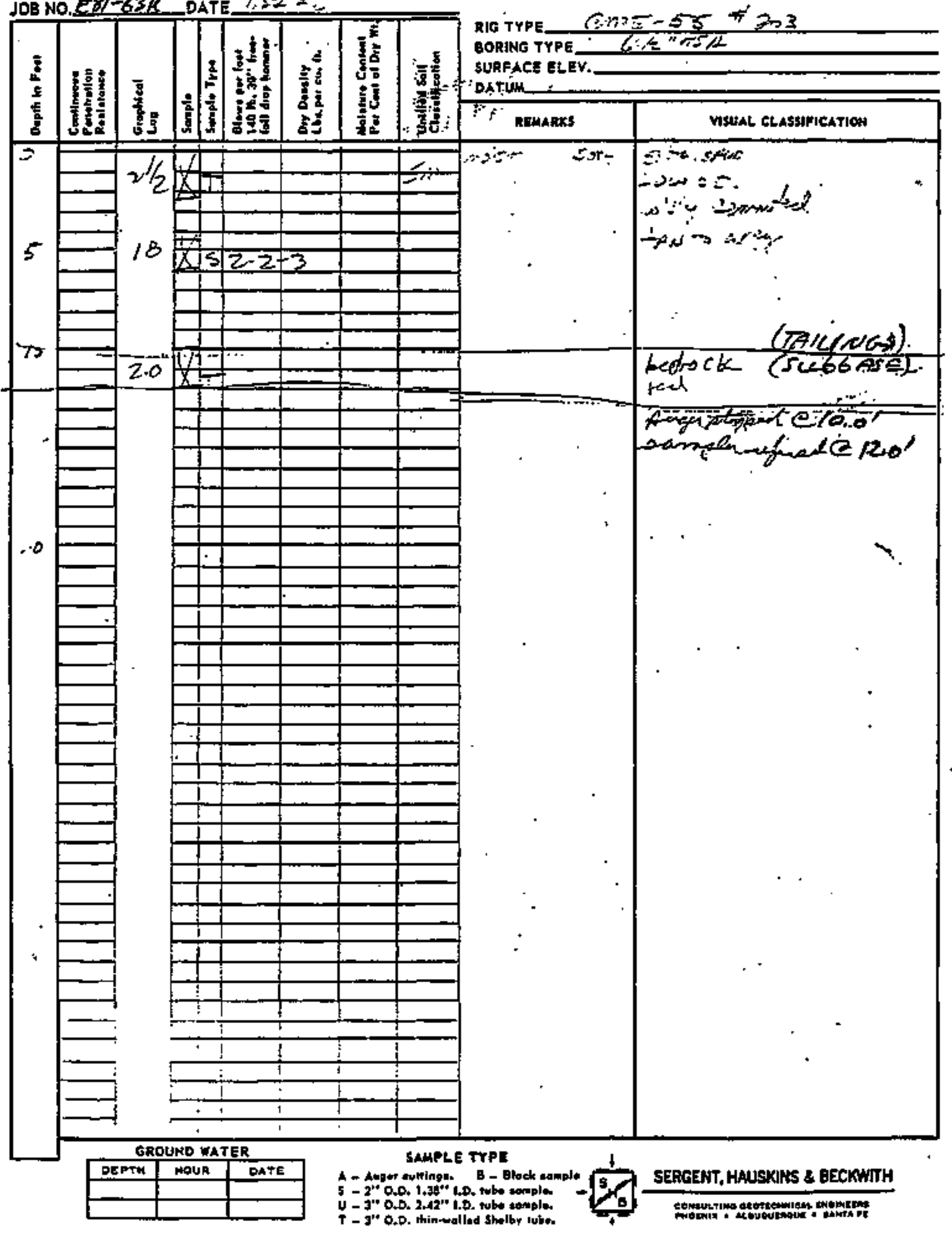




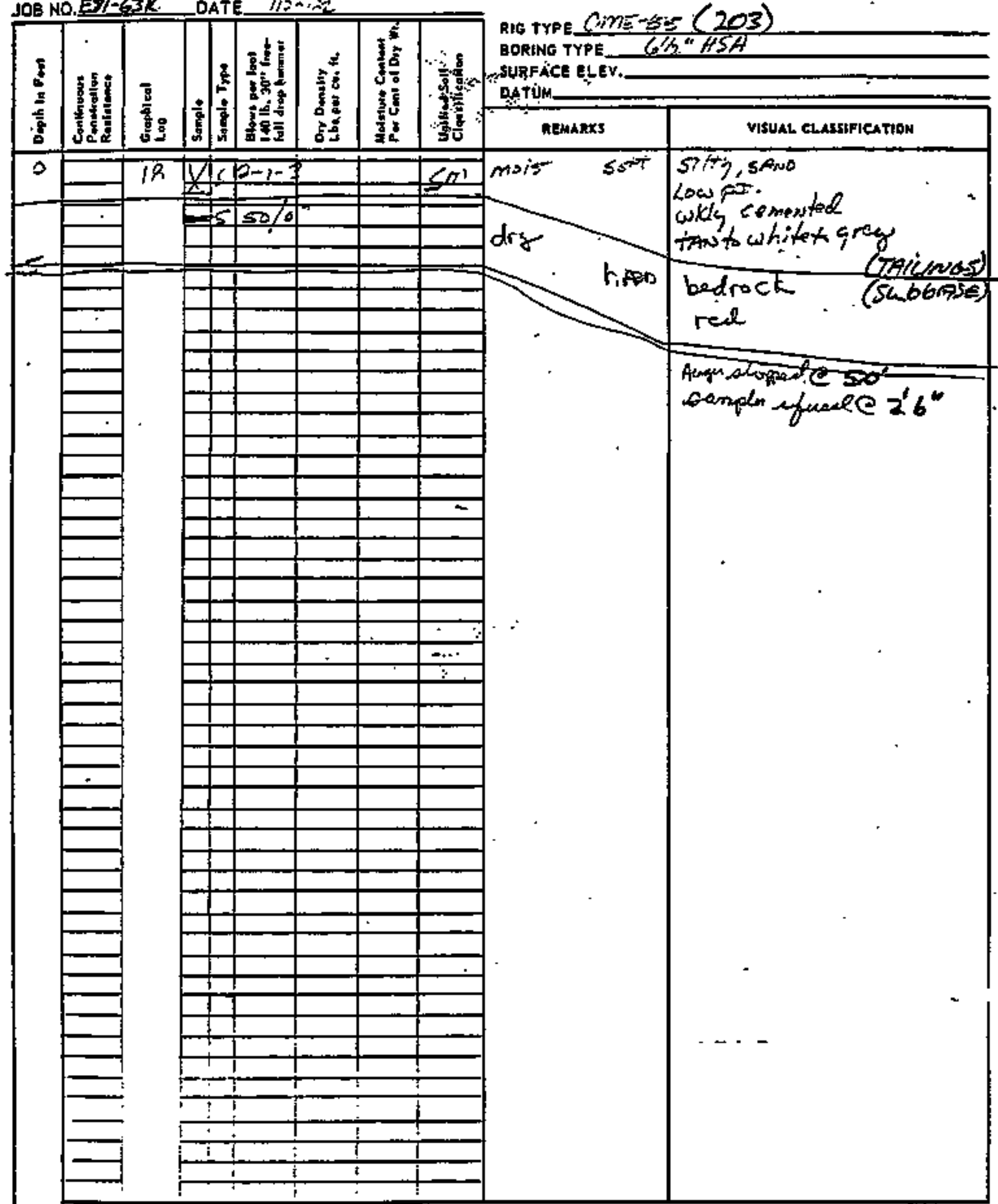

GROUNO WATER

\begin{tabular}{|l|l|l|}
\hline DEPTH & HOUA & DATE \\
\hline & & \\
\hline & & \\
\hline
\end{tabular}

\section{SAMPLE TYPE}

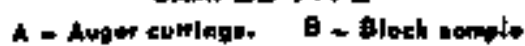

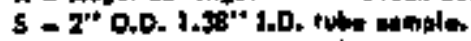

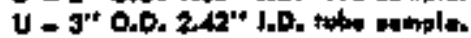

$T$ - T" O,B. thinumeltae sholke nule.

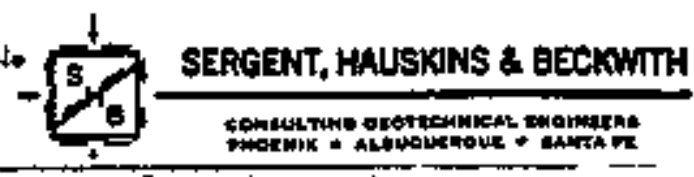




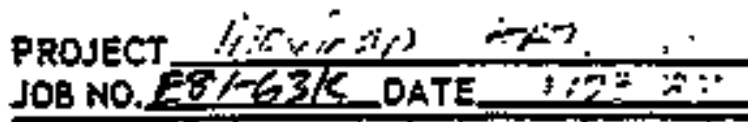

Loe of tist conime no.2/l

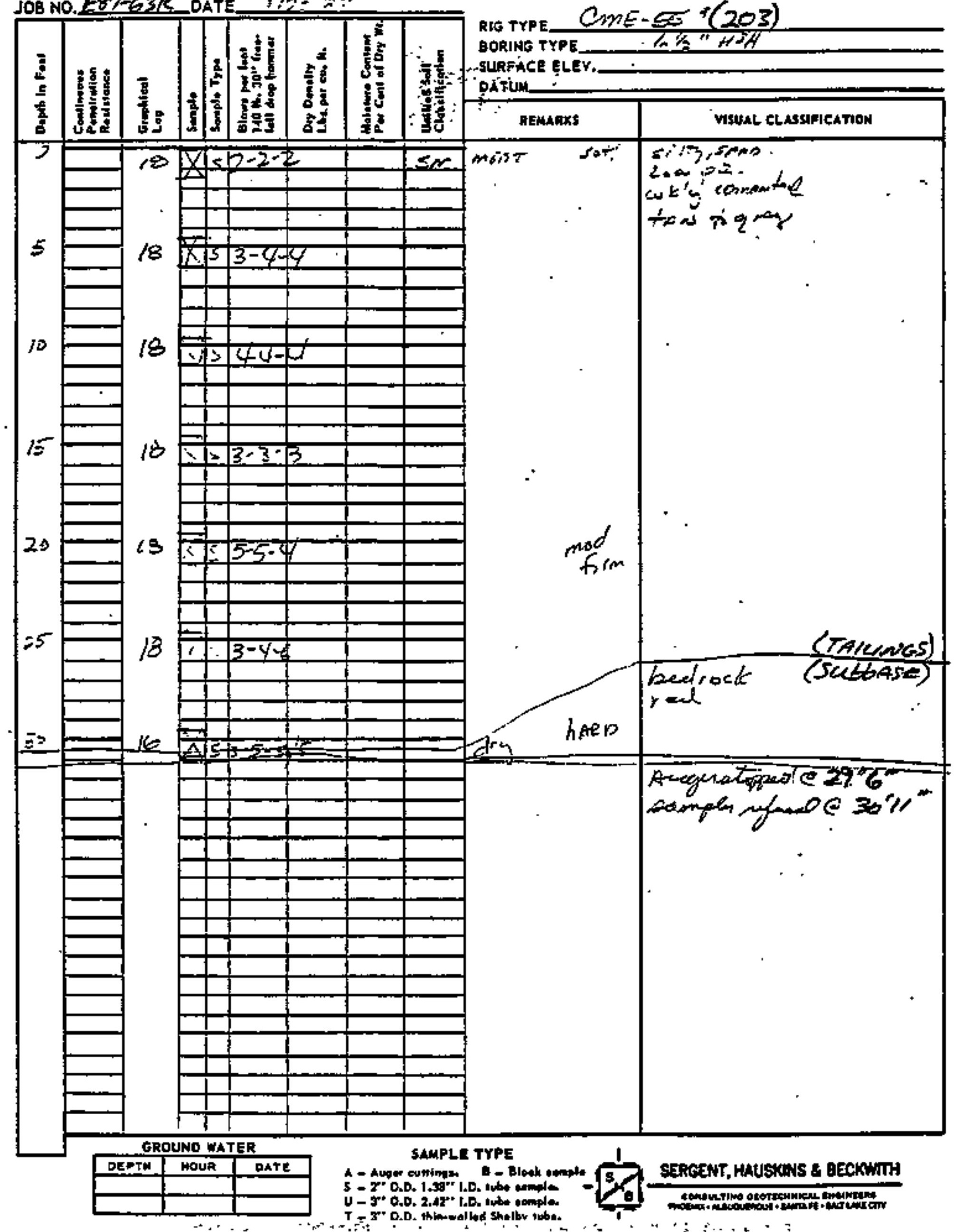


PROJECT. if $, \dot{v}, u, \therefore-$

JOE NO. EDI-6K DATE_ $1 / 23 / 82$

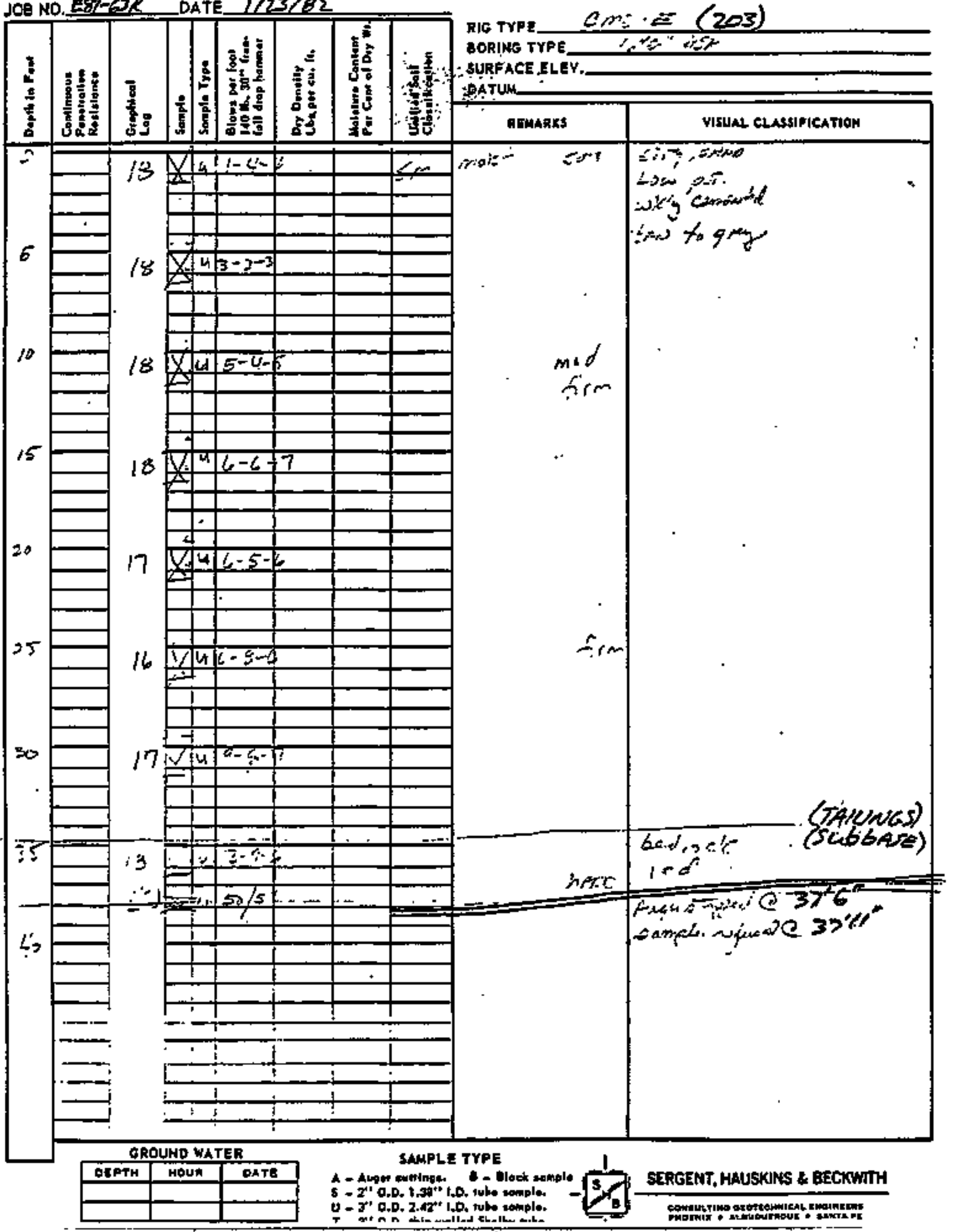

LOO Of TEST DORINO NO.Z2: $c m_{-}=(203)$

BORING TYPE SURFACE ELEY.

patur 


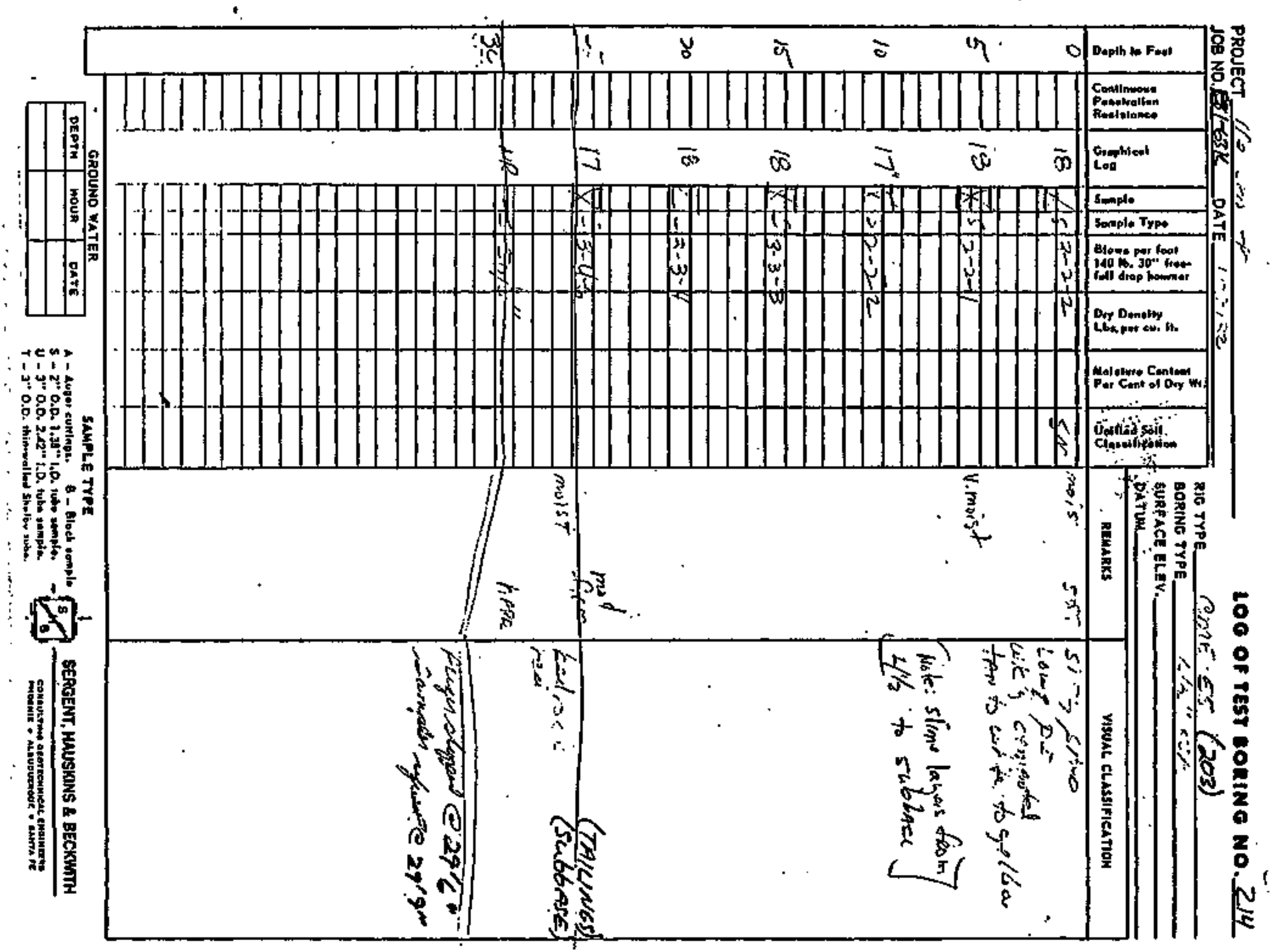




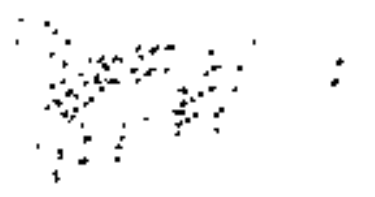

TEST PIT LOGS OF COVER BORRON AREA 


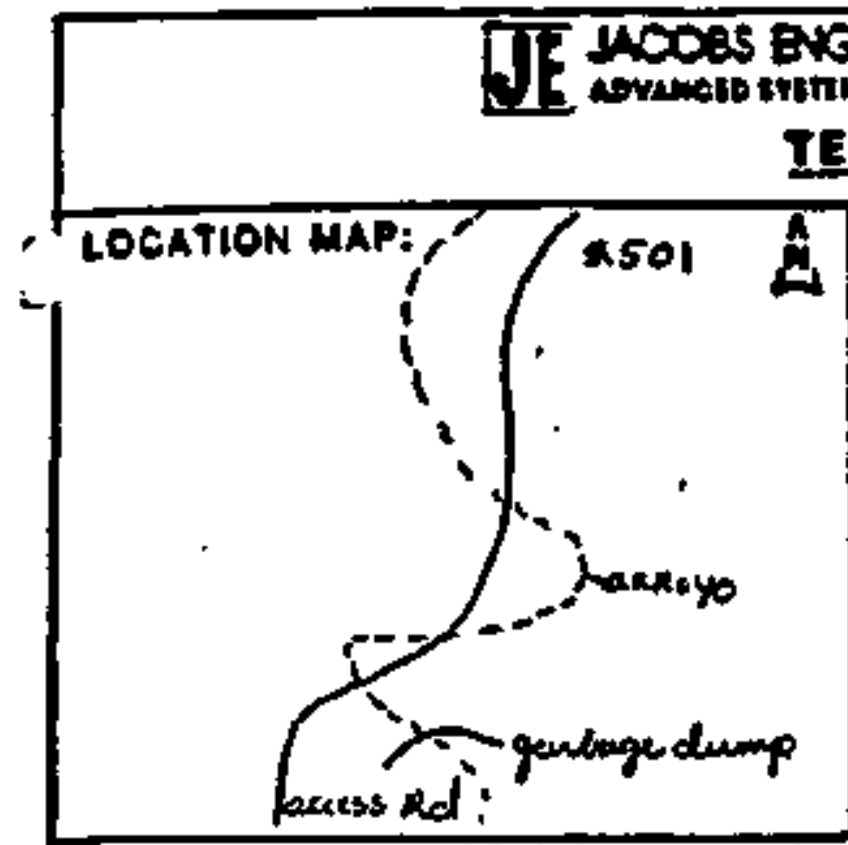

Texting

IEST PIT LOE

pope Lot 1

LOCATION DESCRIPTION Shat

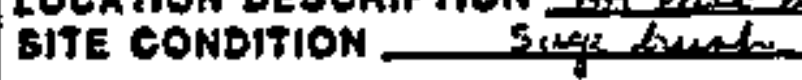

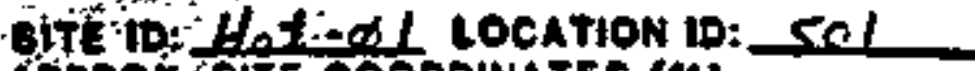
APpgox. BtTE CoOROINATES (11)

N. F

CROUND ELEV. (FT. MSL)

DATE EXCAvated $85757 / 4$

DACKHOLE TYPE: LES SAO

contractor: blate cinz

FIELO REP: - DRibrent:

REHAB. DATE:

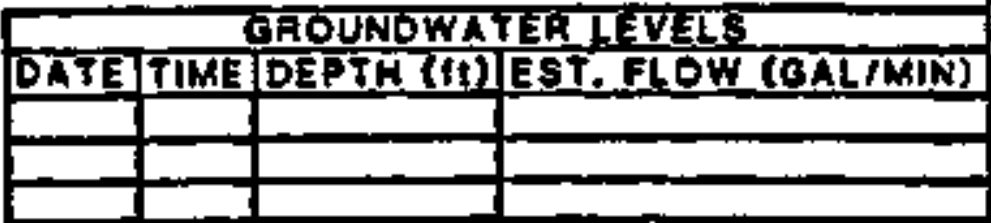

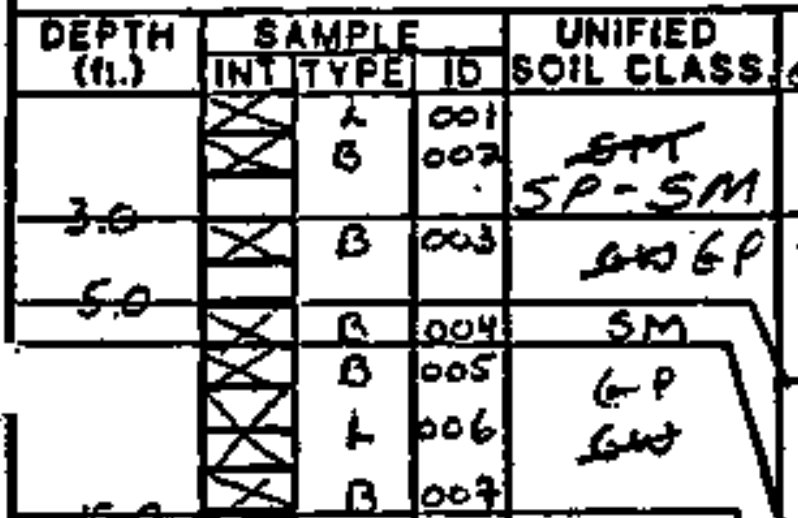

VISULL CLASE: DENSTY, COLOA, STRENGTH, Gy Fito Cs PLASTICITY, CONDITION, ETC.

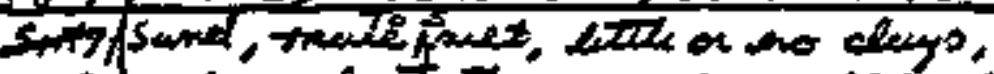

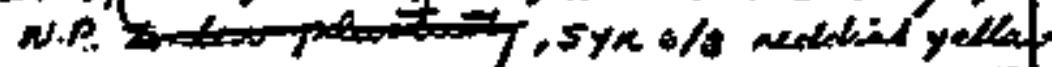

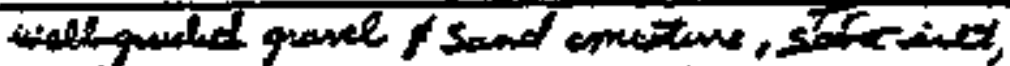

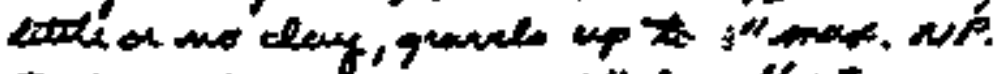

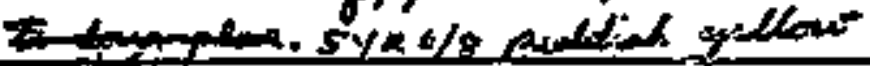

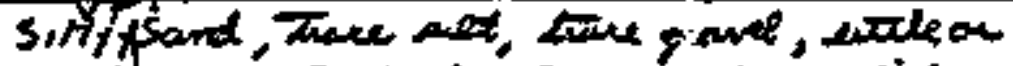
wo devo, U.P.

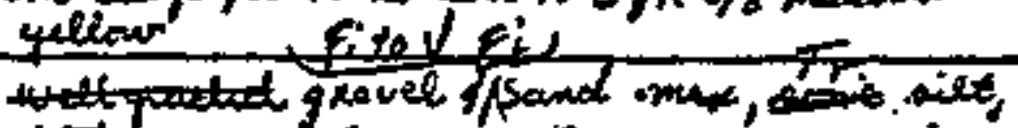

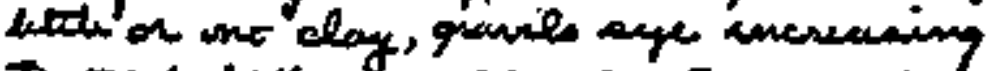

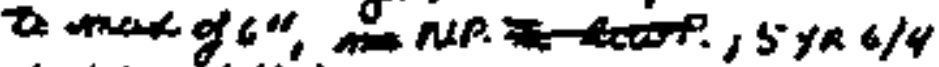
Latit redelinh sucus stopdiging backhoe extenaled

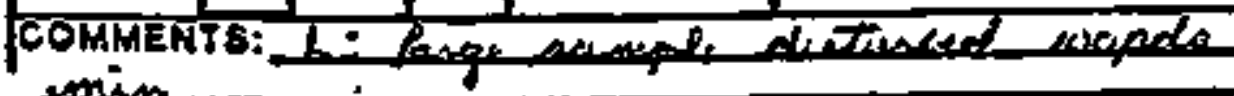
(1)

BAMPEE TYPE

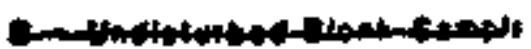
D - Bisiurbed Dula stampit: 


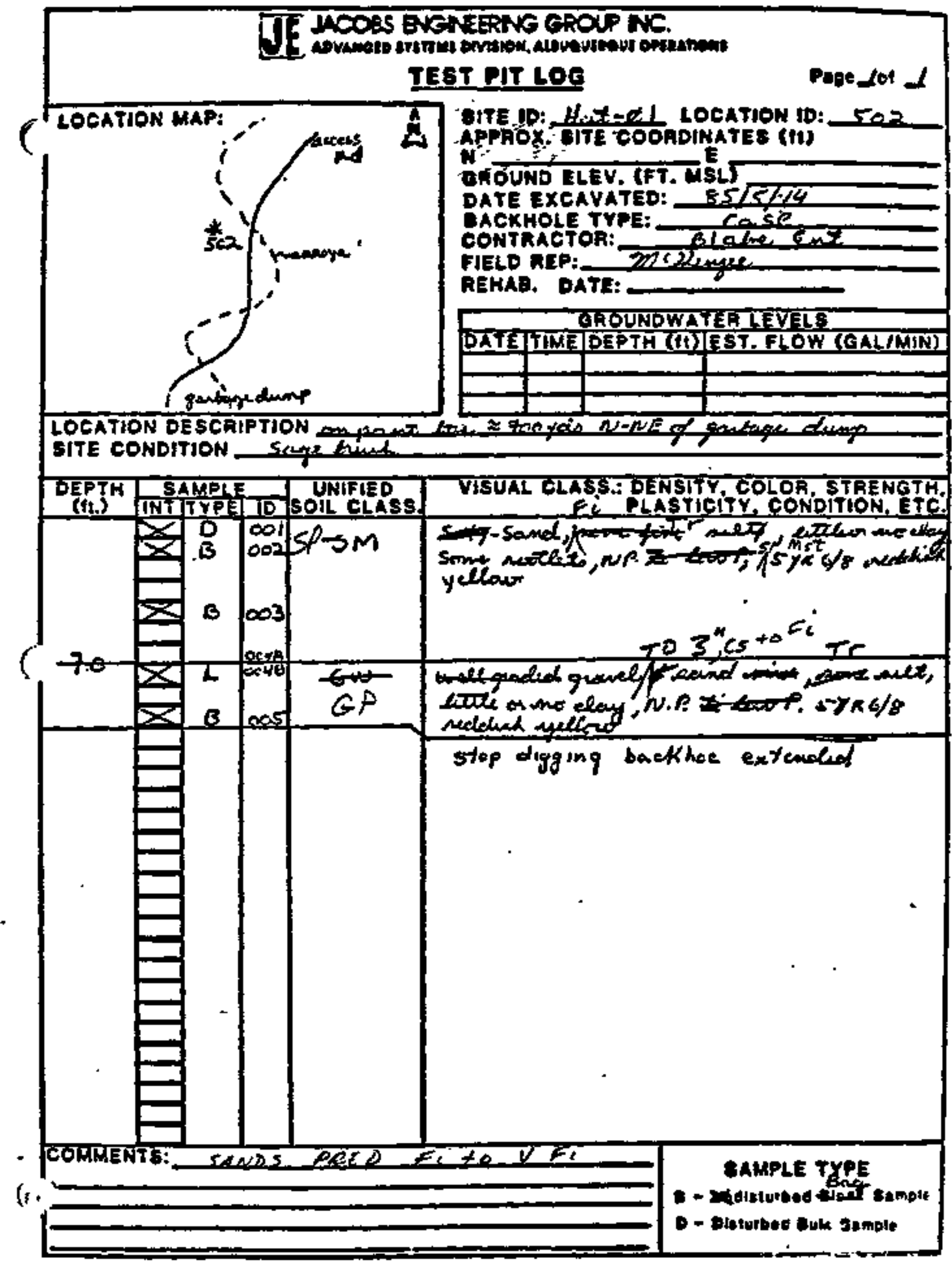



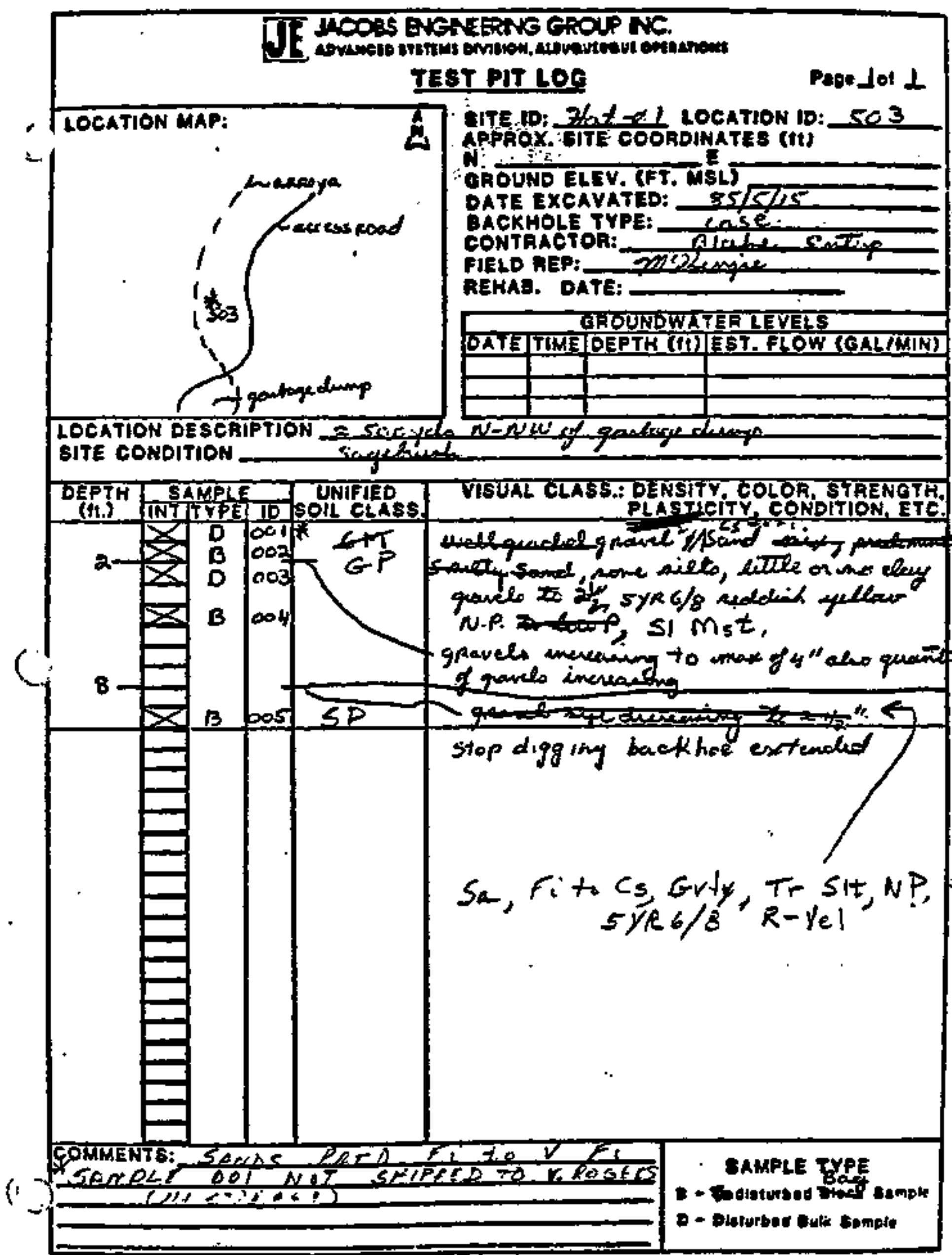


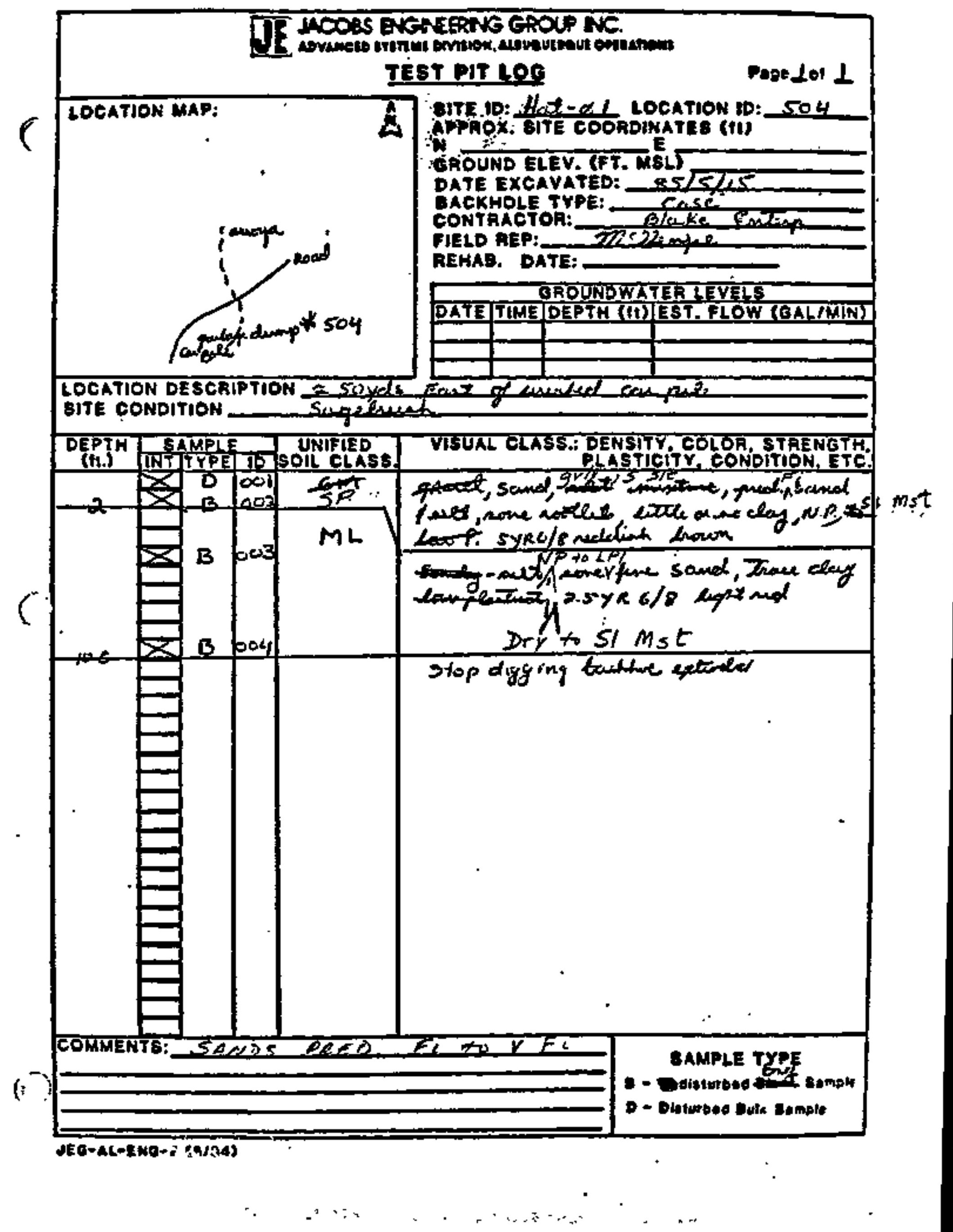




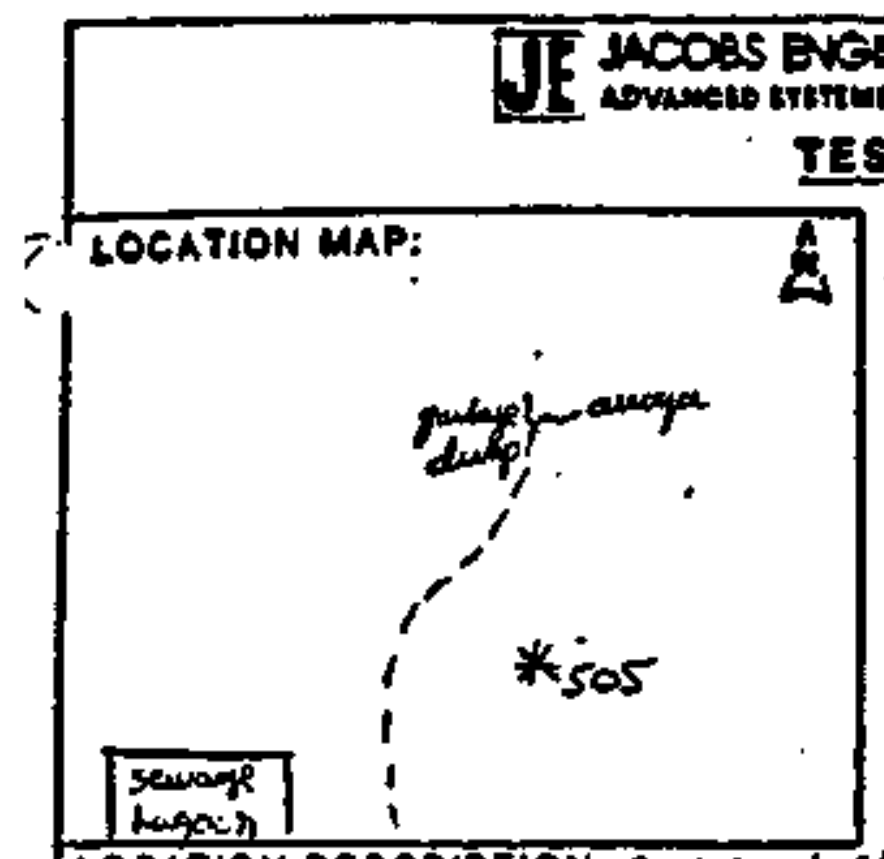

TEST PIT LOR Pape for $L$

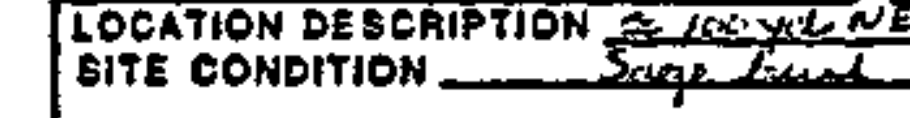

GITE ID: HeC OS LOCATION HD: 505 APPROX SITE COORDIXATES ( $t$ t)

GROUND ELEV. (FT.MEL)

DATE EXEAVATED: RS/FTh

BACKHOLE TYPE:

CONTRACTOR:

FIELD REP:

REHAB. OATE:

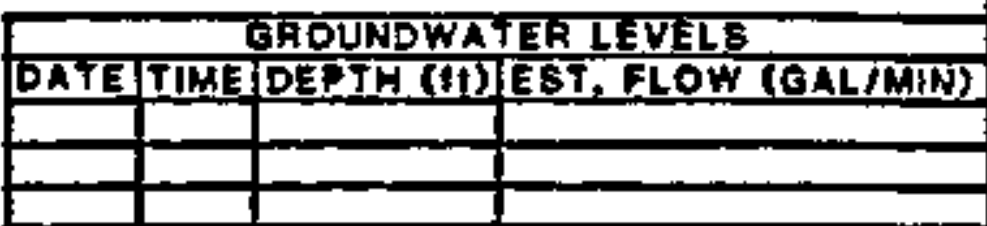

E asicy batan

DEPTH SAMPLE T UNIFIED VISUAL CLASS. DENSITY, COLOR, STAENG TA,

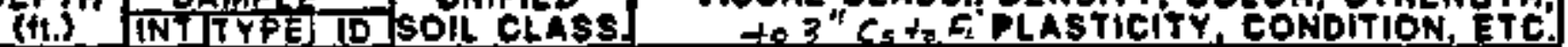

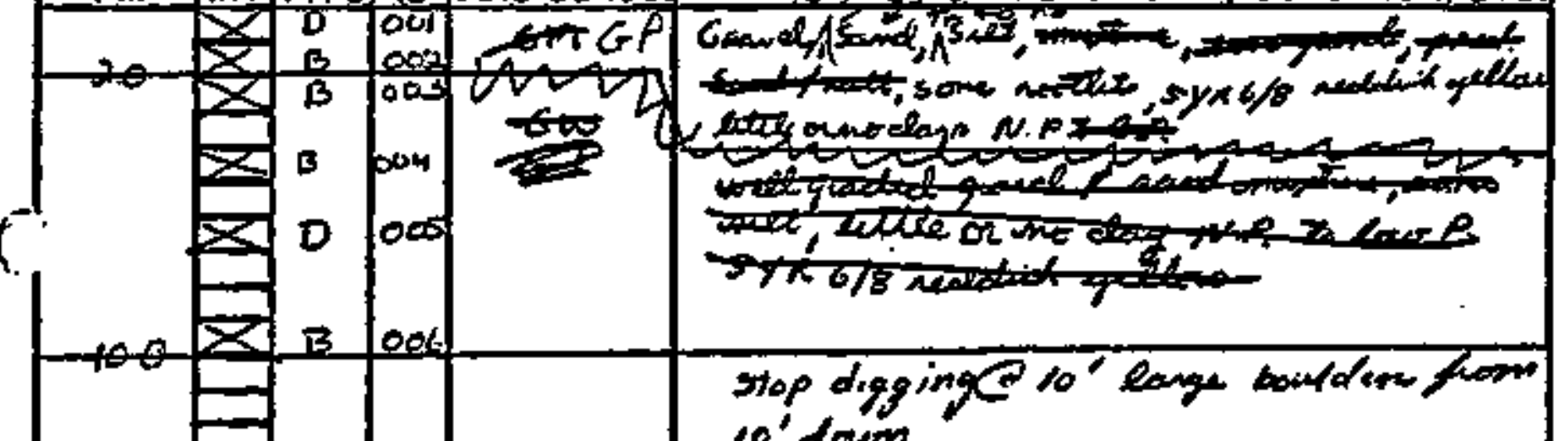

$(8$

COMMENTE: SANDS ERED E: Fo $V F_{4}$

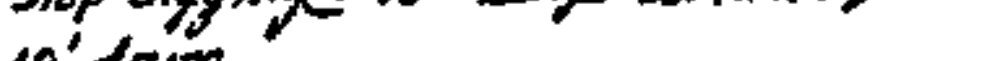

10 down

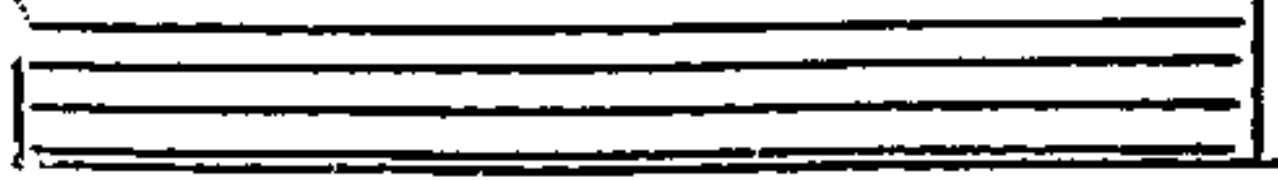

SAMPLE TYPE

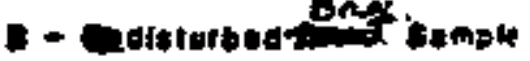

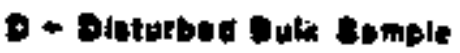




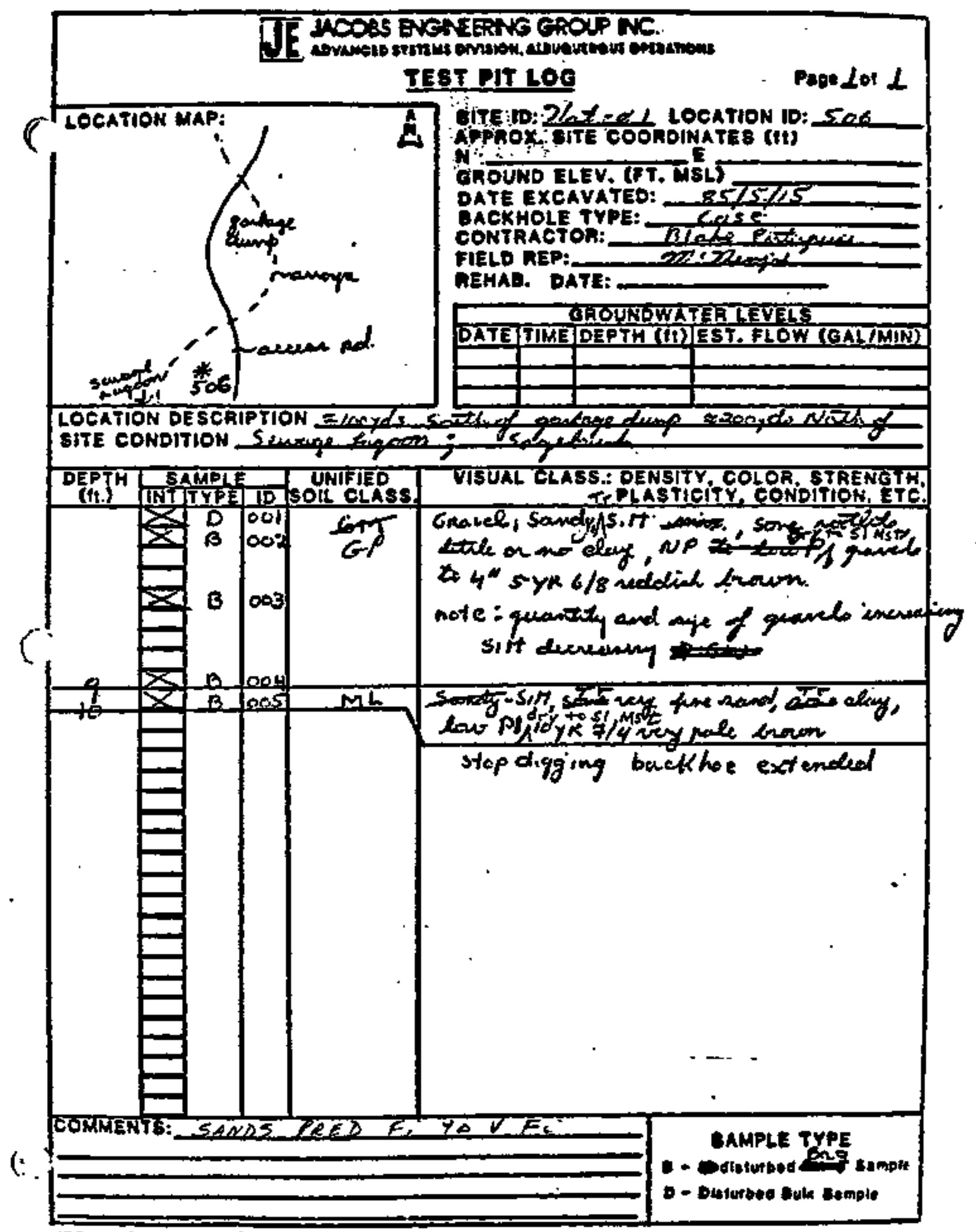

iEC-AL-ENQ-7 (BIOA) 


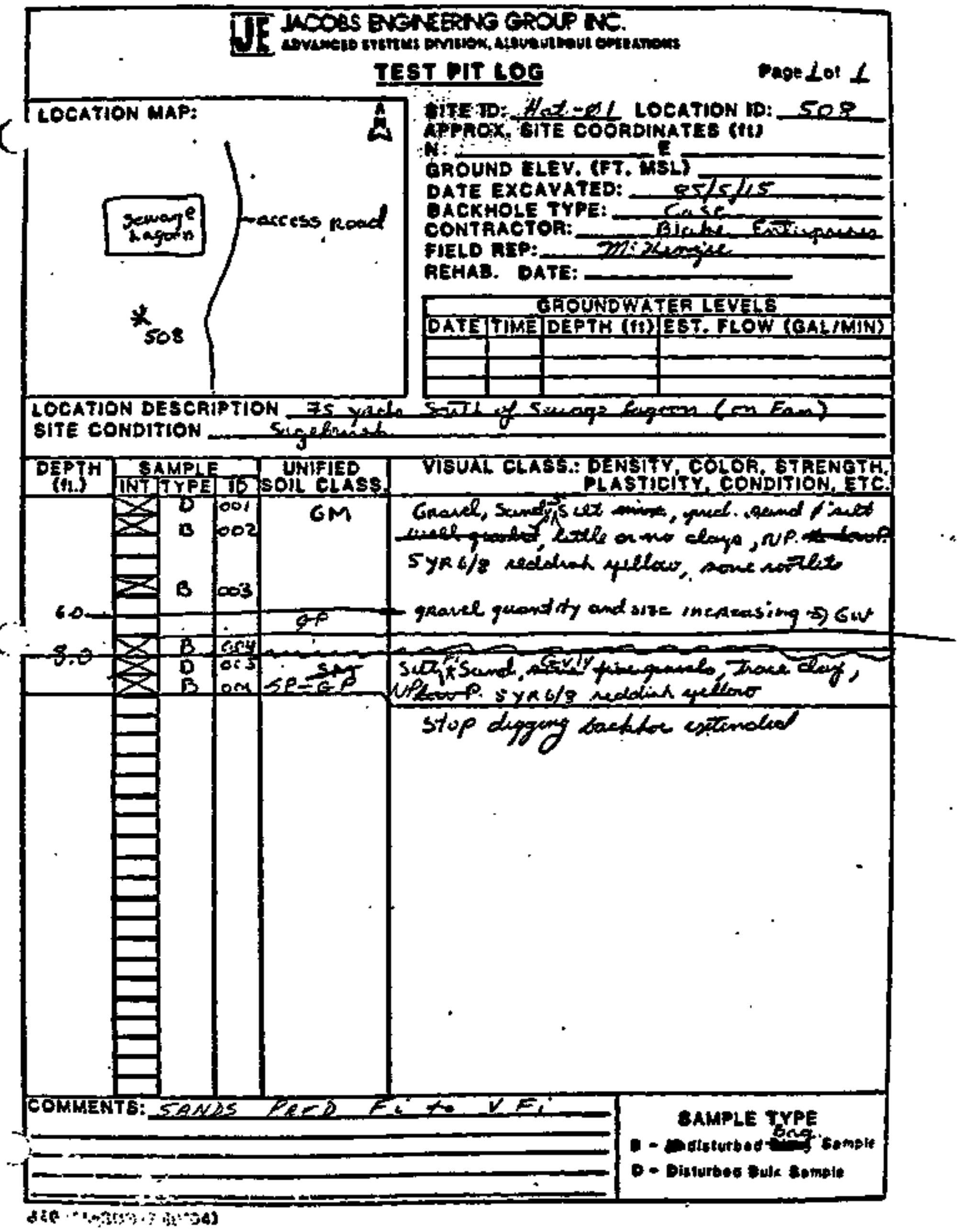




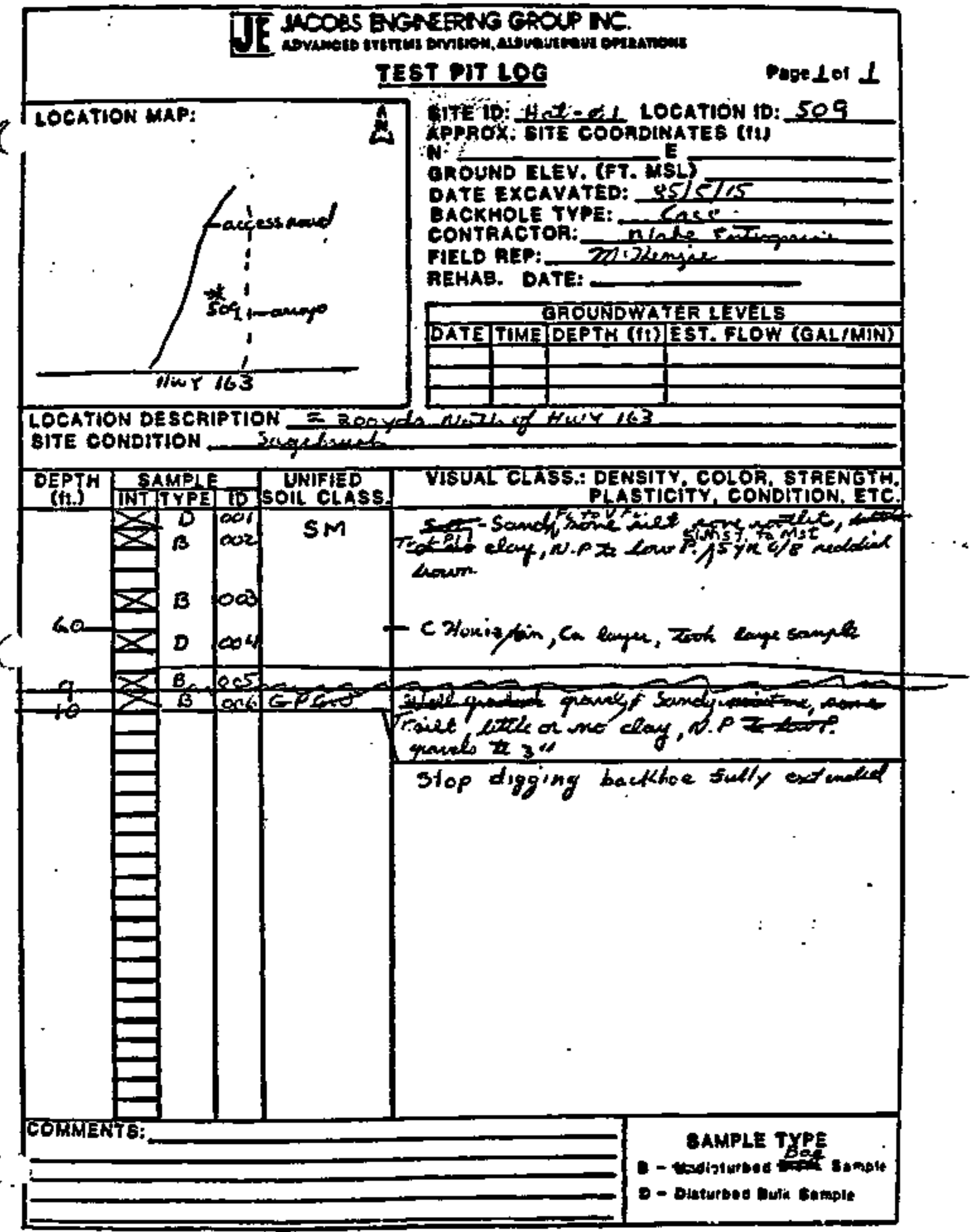




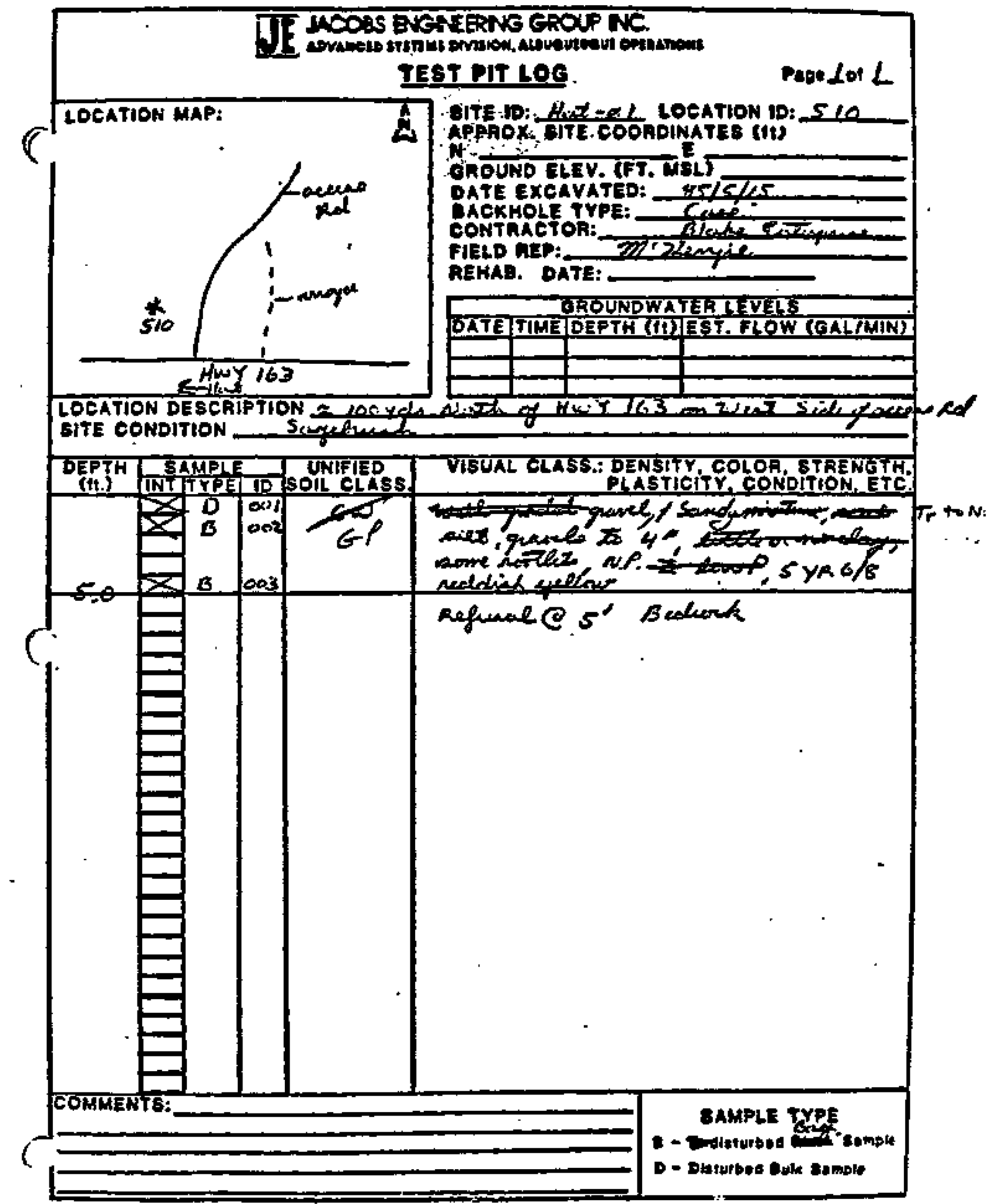




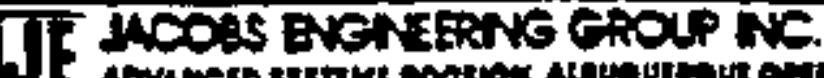

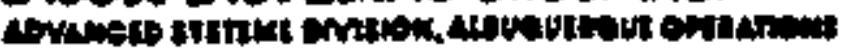

TEST PIT 200

Page Lot L

I LOCATION MAP:

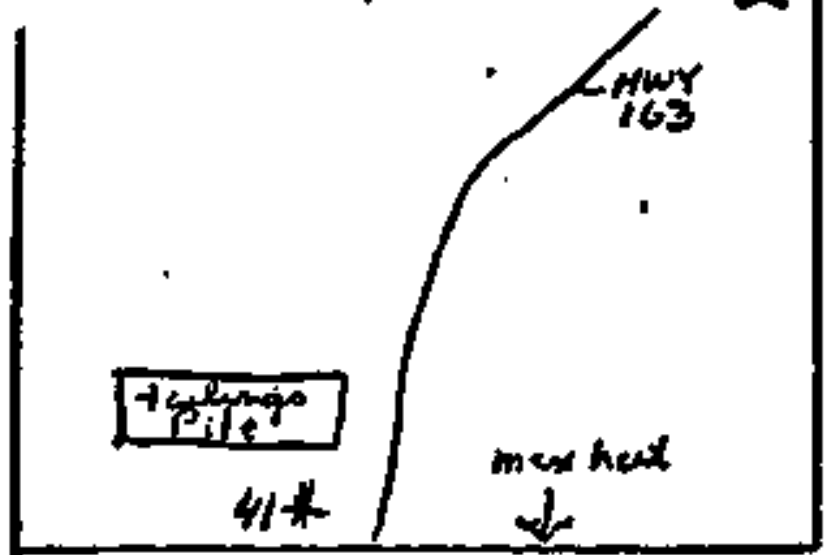

OITE:JD: APROK BITE COORDINATES (it)

$\mathrm{A} ; \mathrm{i}, \mathrm{s}$

6ROUND ELEV. (FT. MSL)

DATE EXCAYATED: $8 5 \longdiv { 5 1 6 }$

OACKHOLE TYPE:

CONTRACTOR:

FIELO REP:

NA

REHAB. DATE:

GROUNDWATERTEVELS

DATE TIME]DEPTH (II)EST, FLOW (CAL/MIN)

LOCATION DESCRJPTION

SITE CONDITION

DEPTH SAMPLE UHIFIED (ti) INT TYPE ID SOIL CLASS.

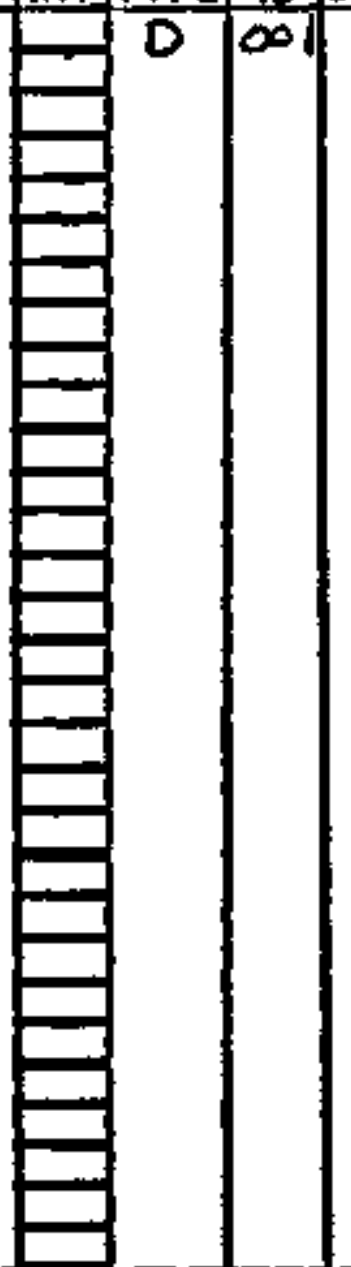

COMMENTS:

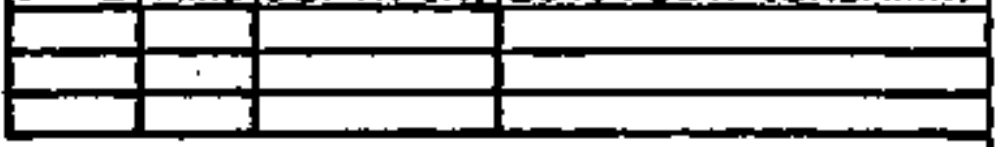

DAMPLE TYPE

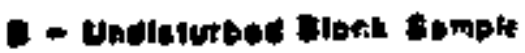
D - Dlsiurboe Sula fompito

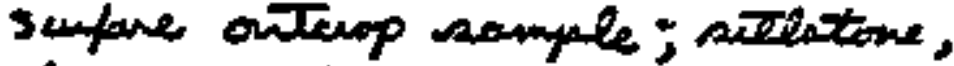
the graind $\neq 100$ pelonetamied 


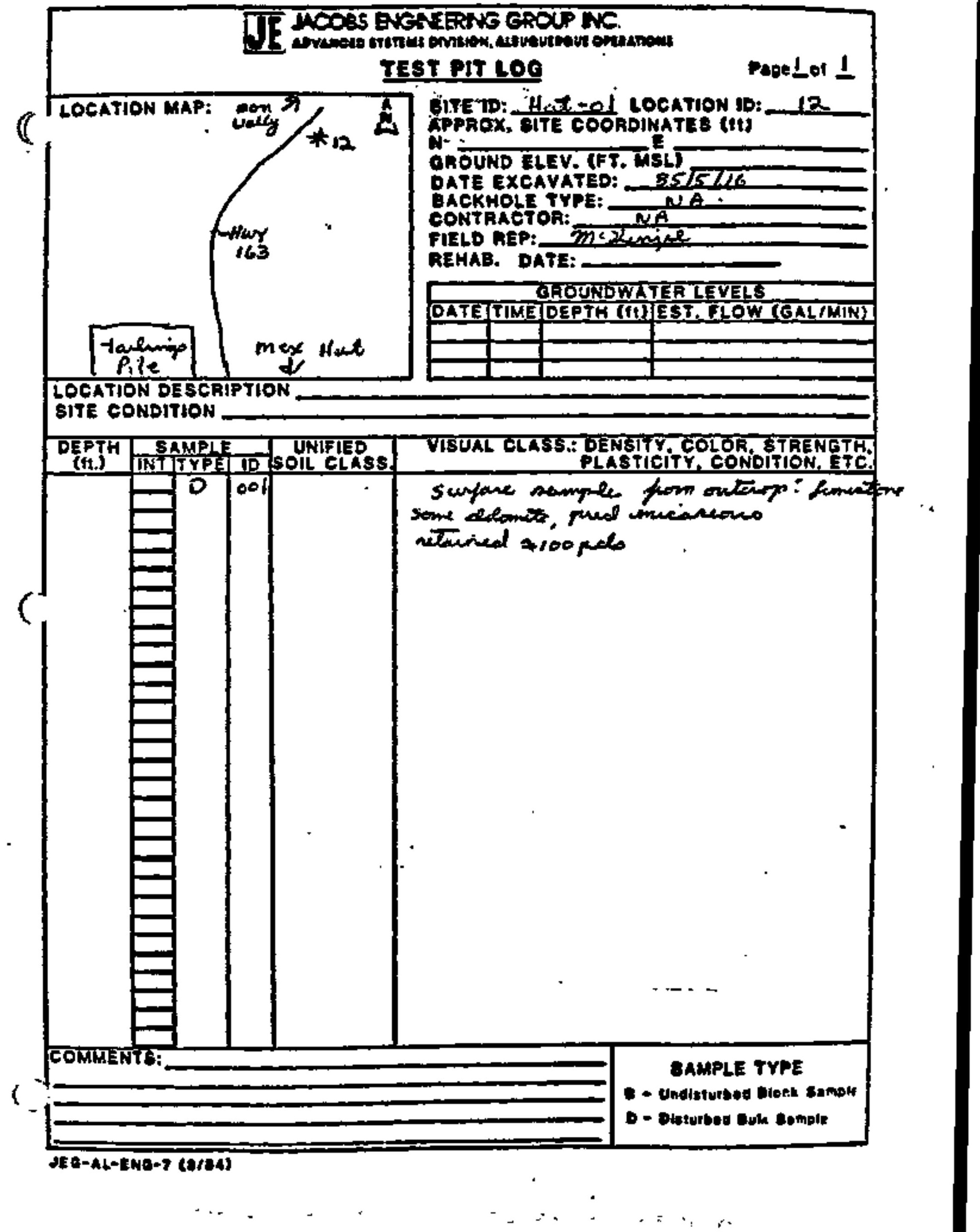




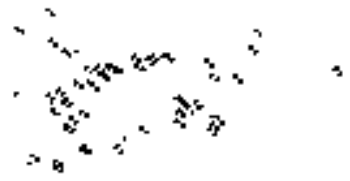

ADOEHOUM 05

\section{PARTICLE SIZE OISTRIBUTION CURVES}




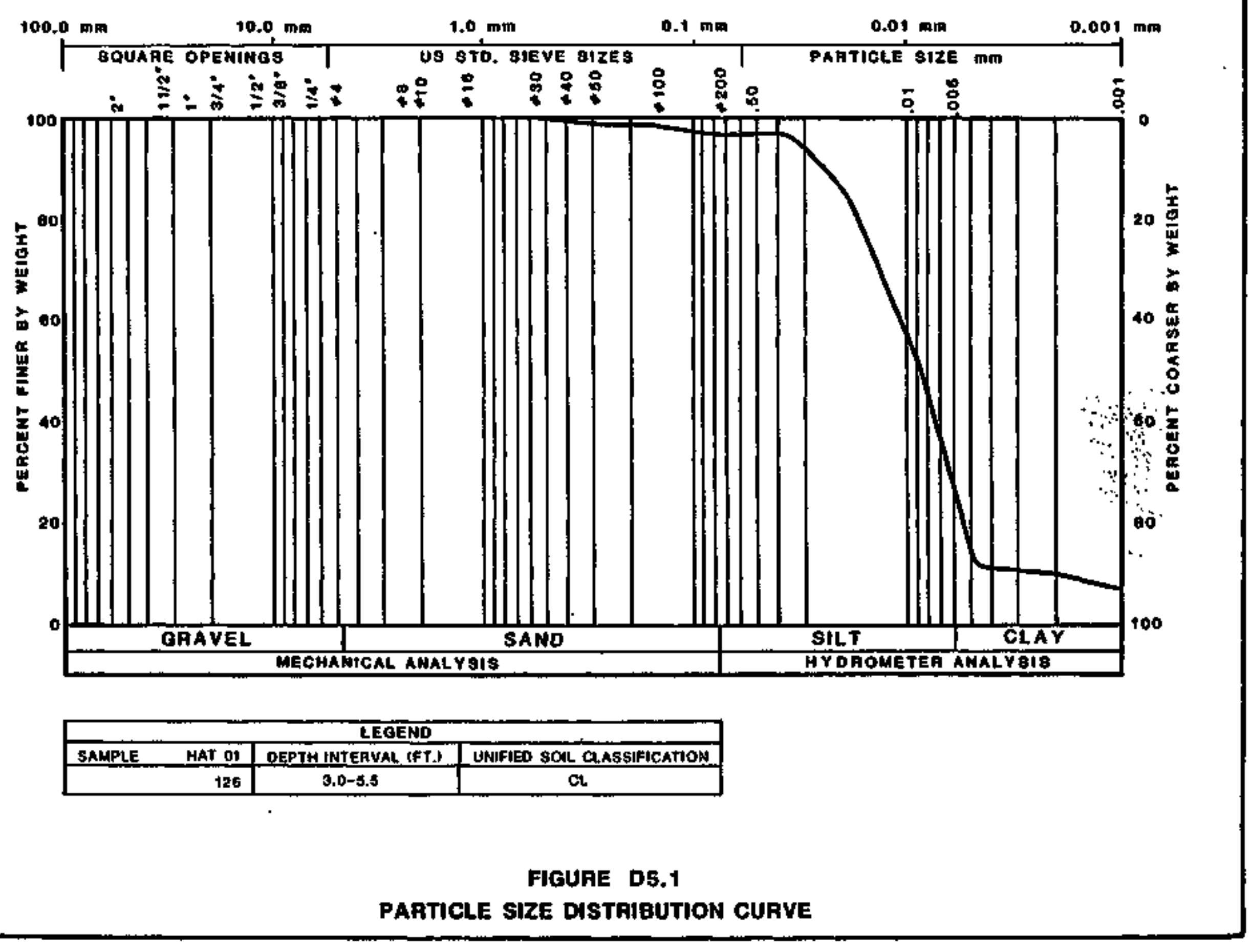




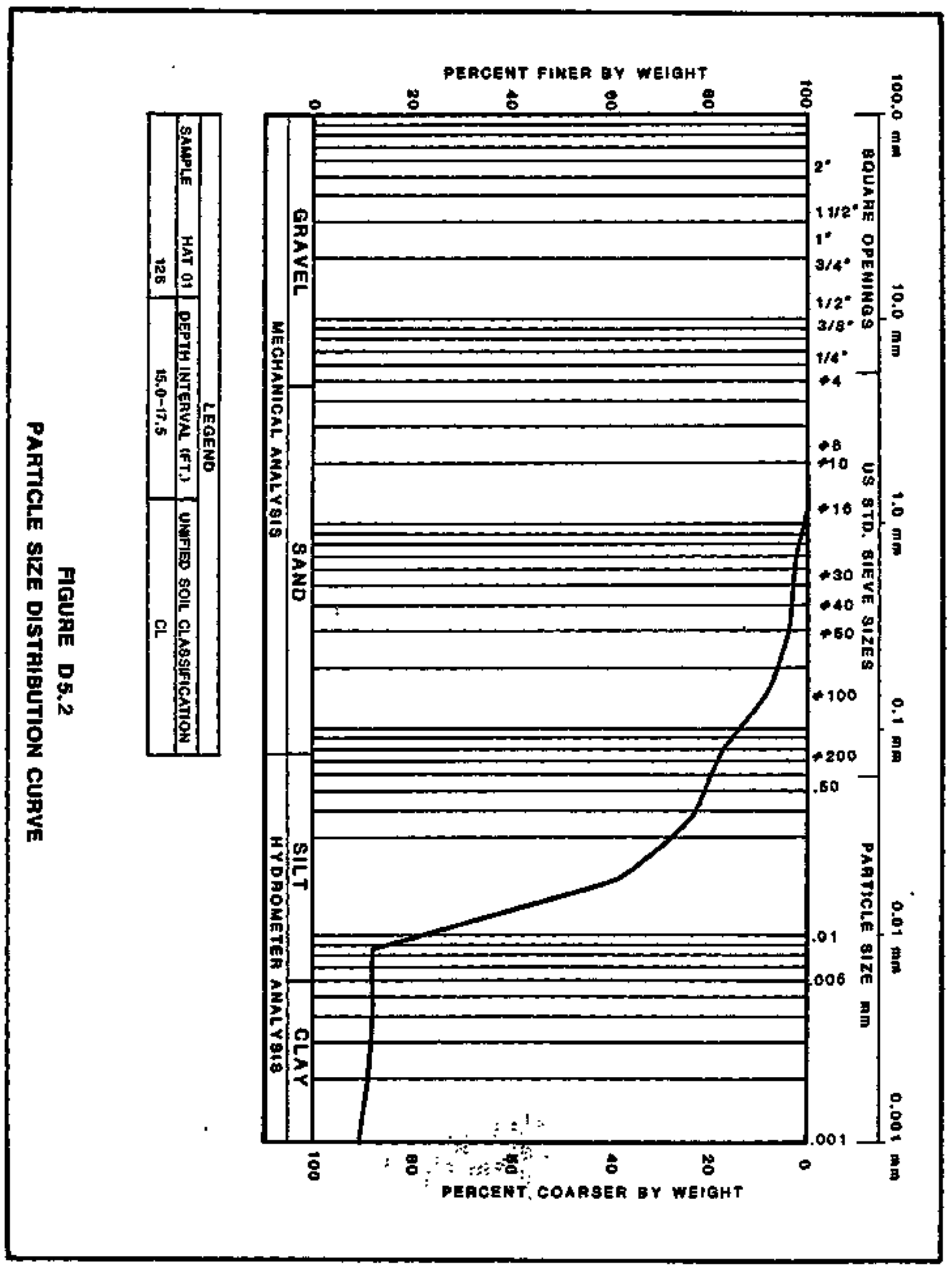




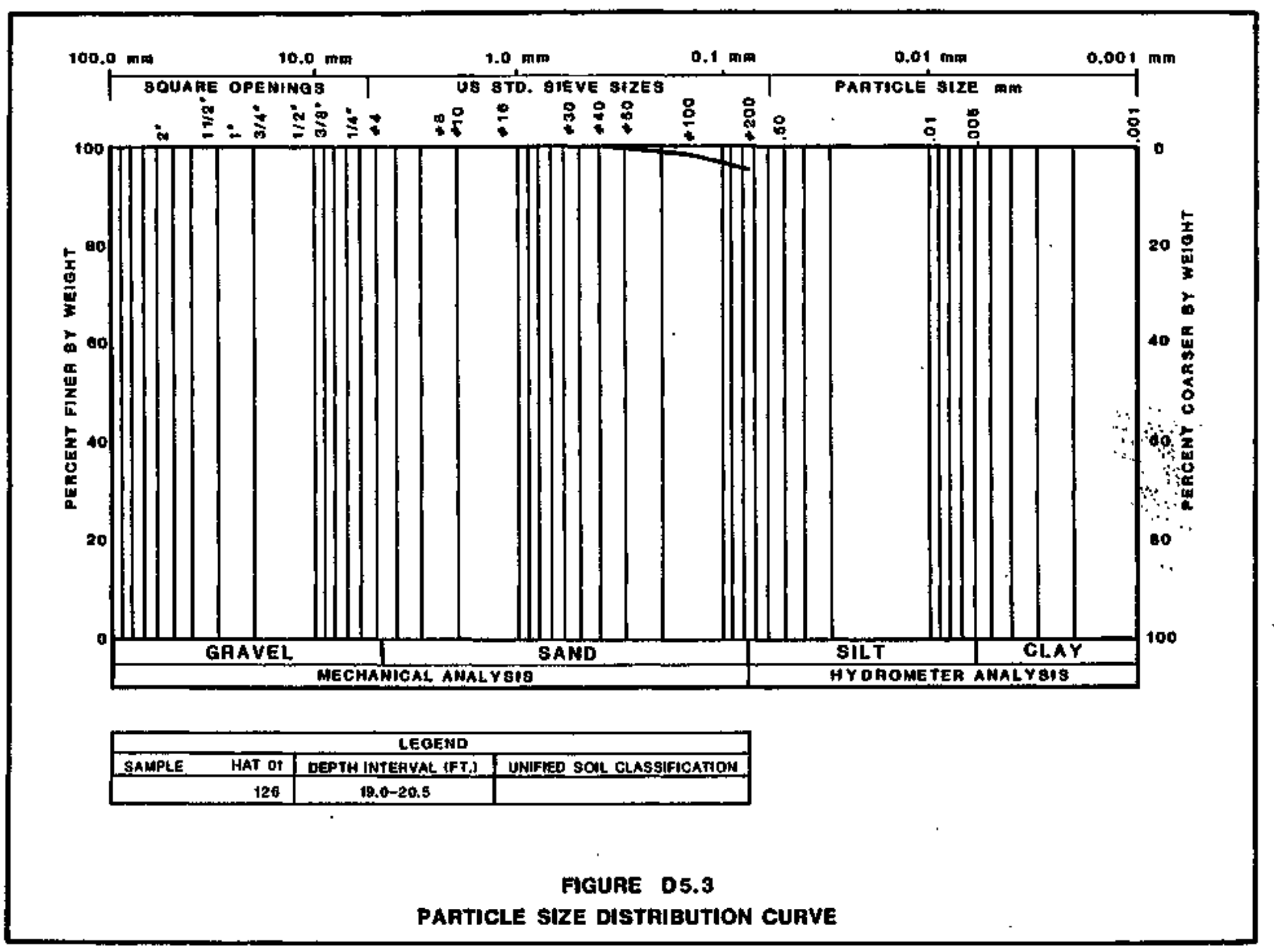



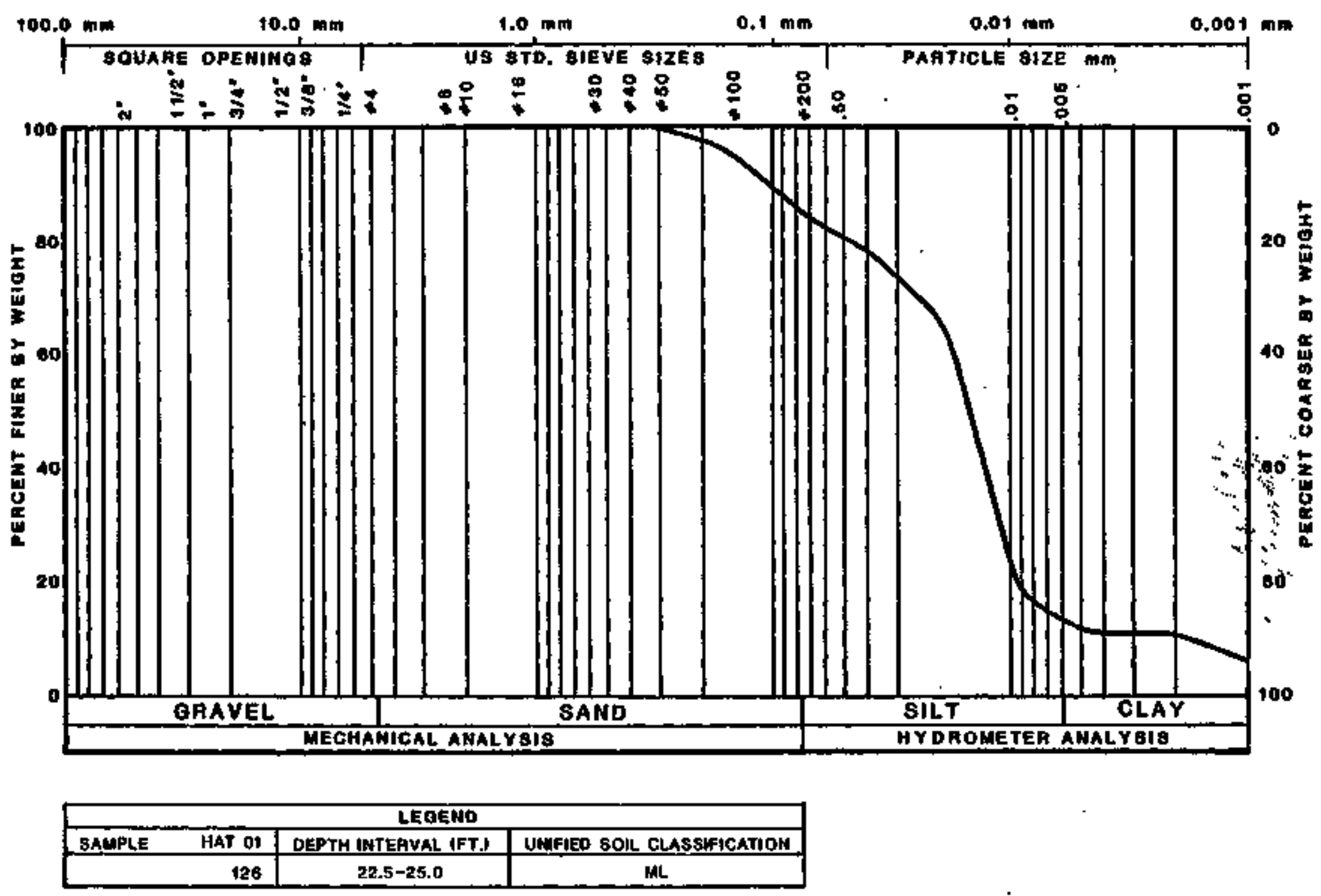

FIGUAE 05.4

PARTICle SIZE DISTRIBUTION CURVE 


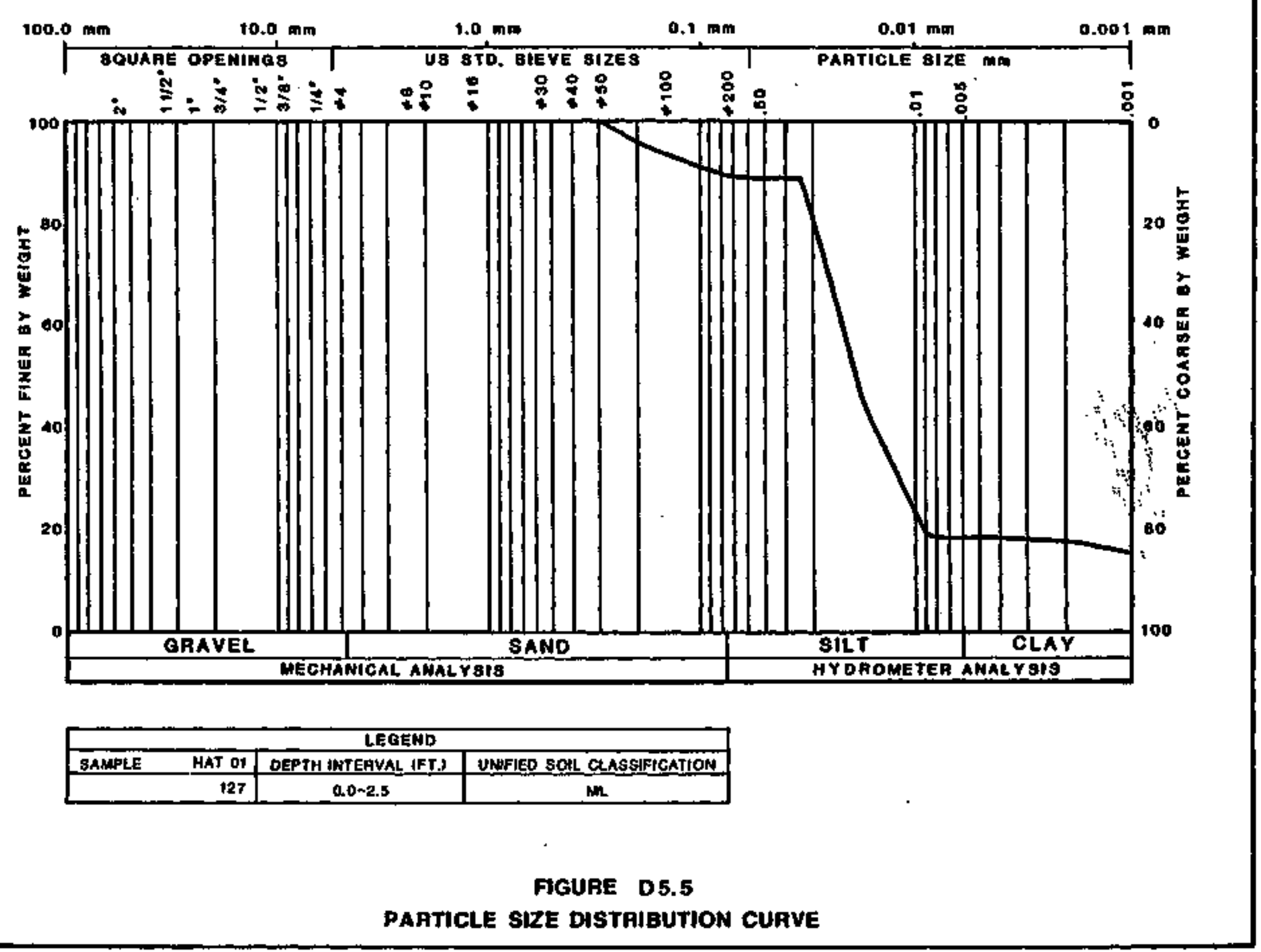




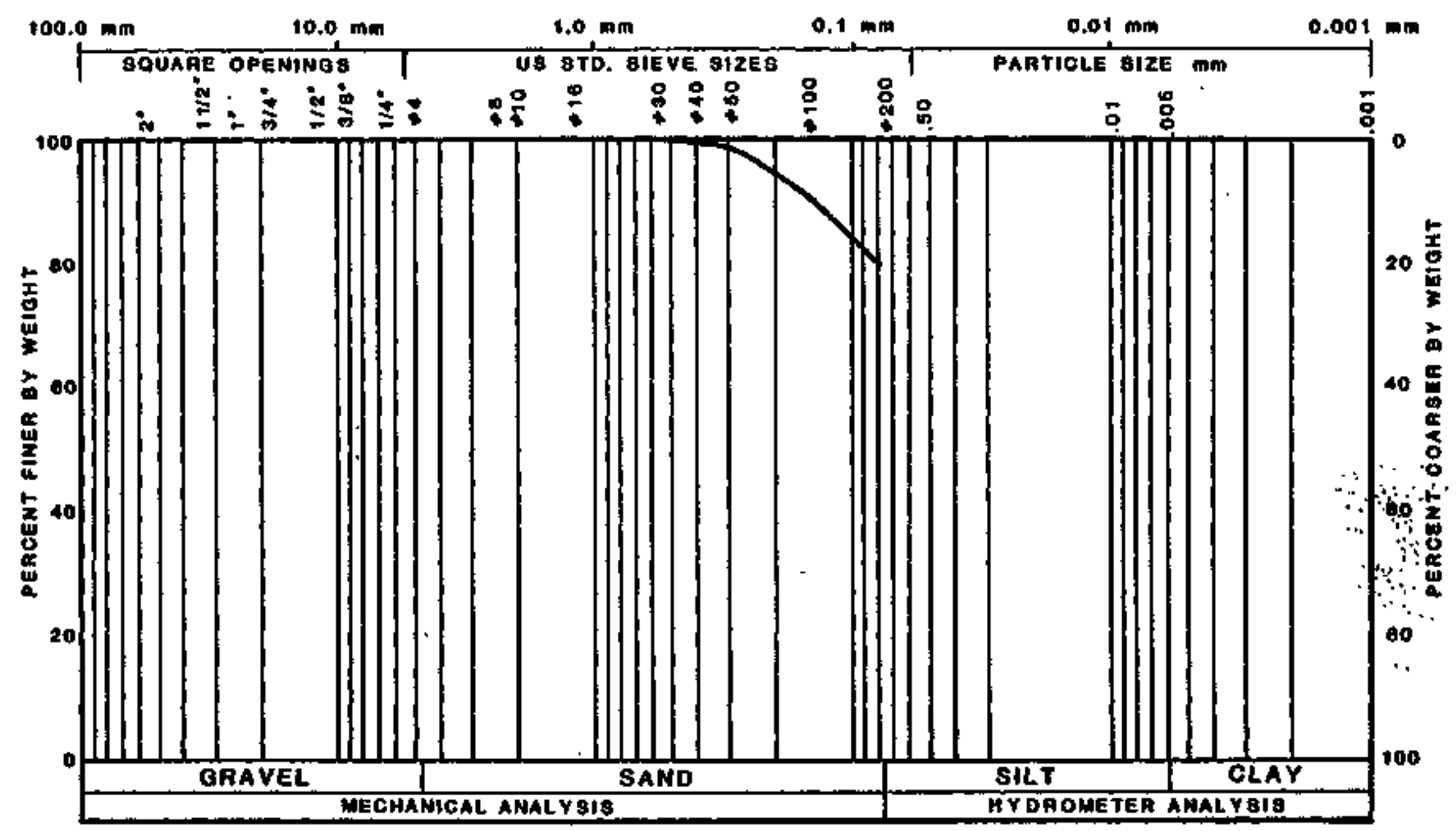

\begin{tabular}{|c|c|c|c|}
\hline \multicolumn{4}{|c|}{ LEOENO } \\
\hline SAMPLE & HAT o & DEPTH IAITEAVAL IFT.] & UMMFIEO SOIL GLASGIFIGATION \\
\hline & 127 & $. \quad+4.5-18.0$ & CL \\
\hline
\end{tabular}

FIGUAE D 5.6

particle size distaibution curve 


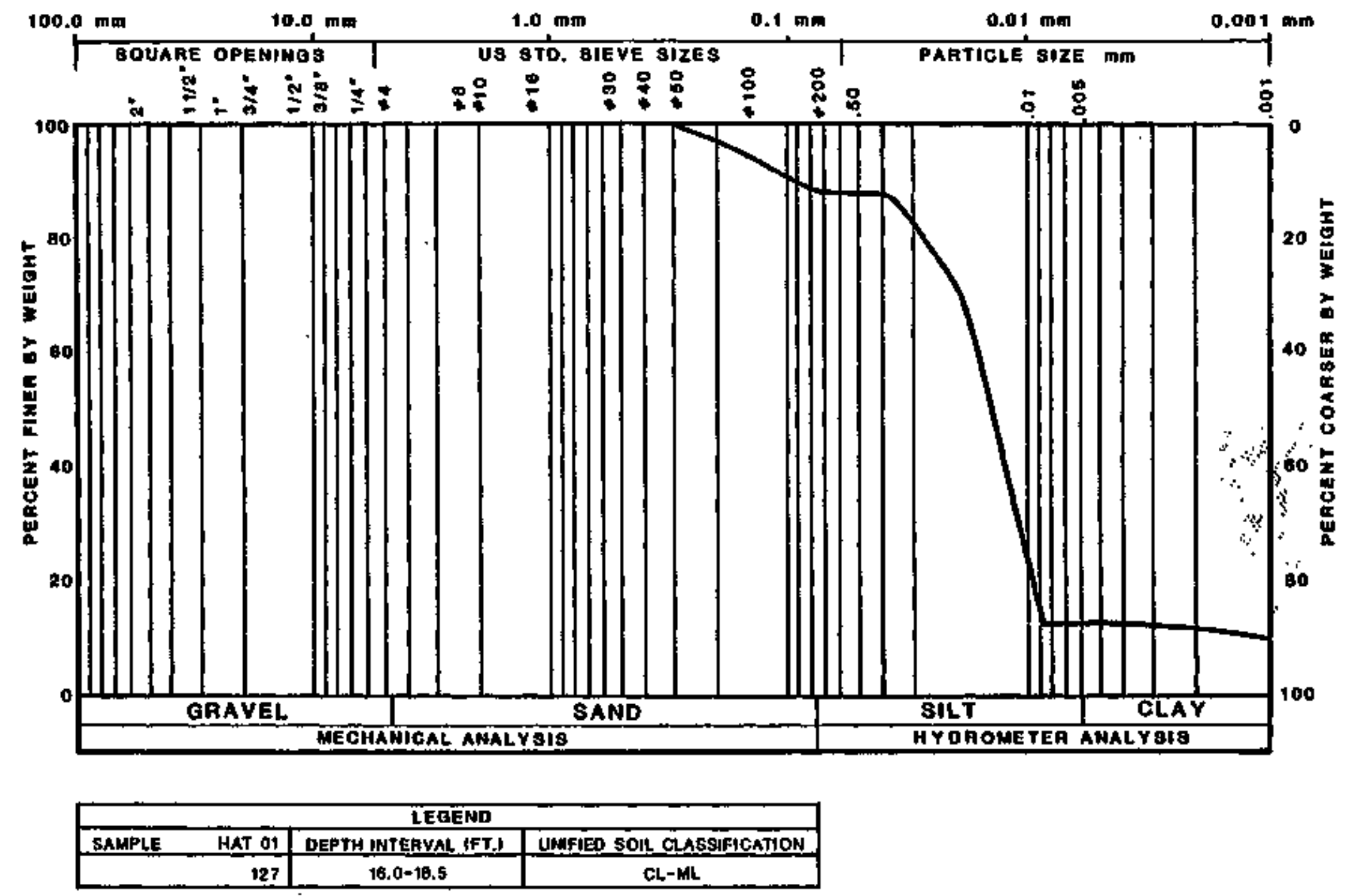

FIGURE D5.7

PARTICle size distaibution cuRVE 


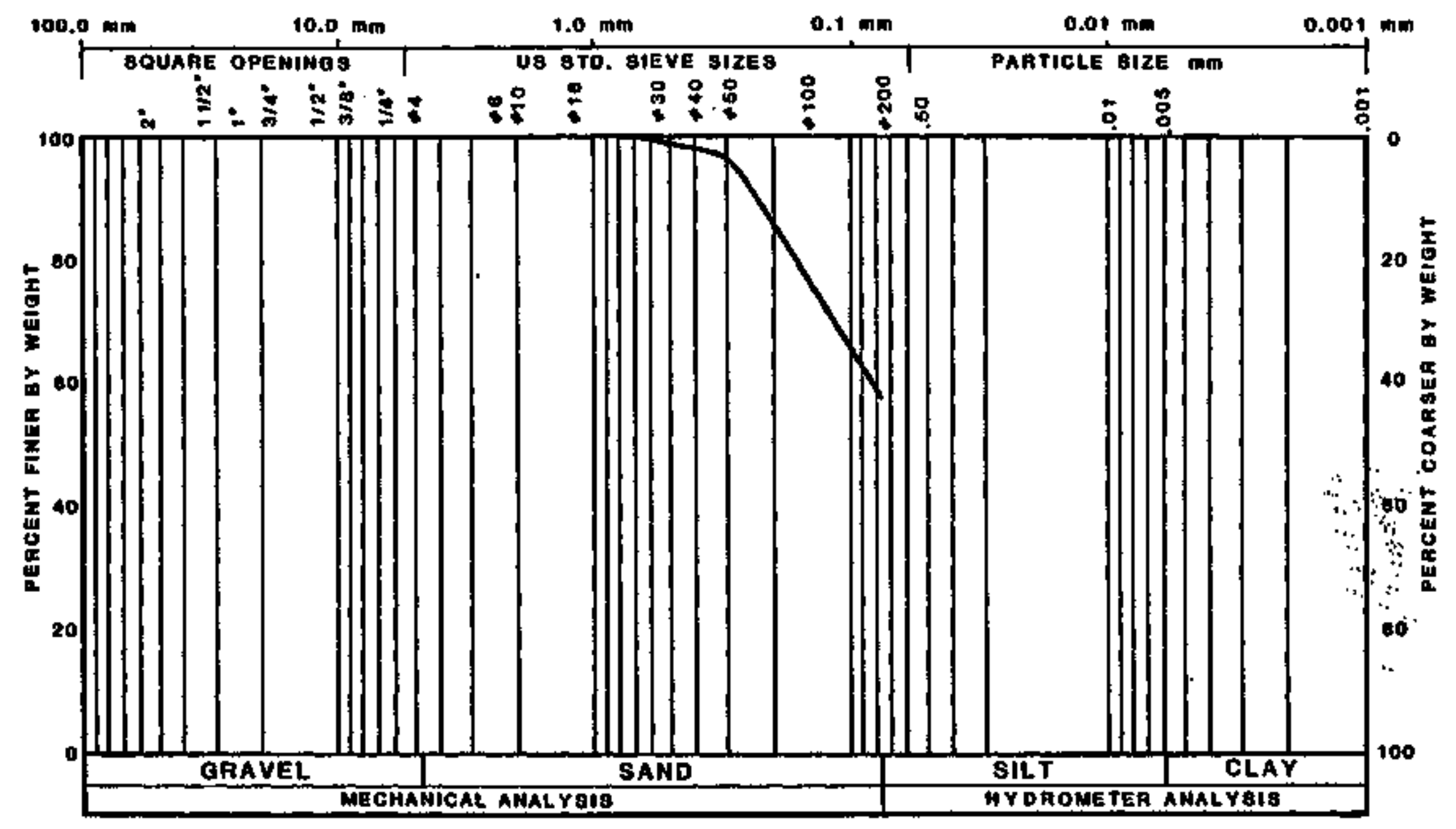

\begin{tabular}{|r|c|c|}
\hline \multicolumn{3}{|c|}{ LEGEND } \\
\hline SALPLLE_ HAT 01 & DEPTH INTERYAL (FT) & UNIFIED SOHL CLASSIFICAMON \\
\hline 127 & $19.0-20.5$ & ML \\
\hline
\end{tabular}

FIGUAE D5.6

particle size distriBution curve 


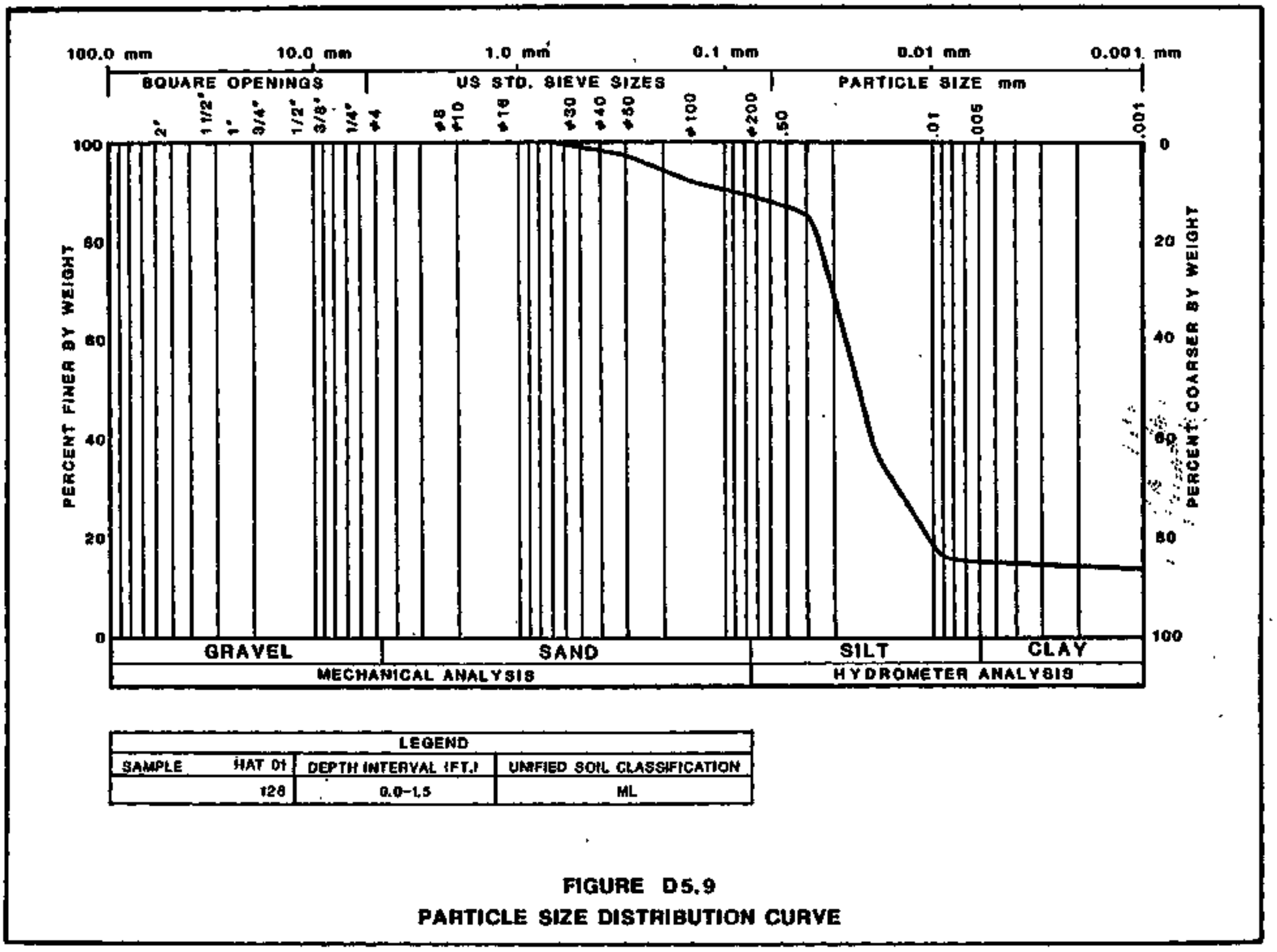




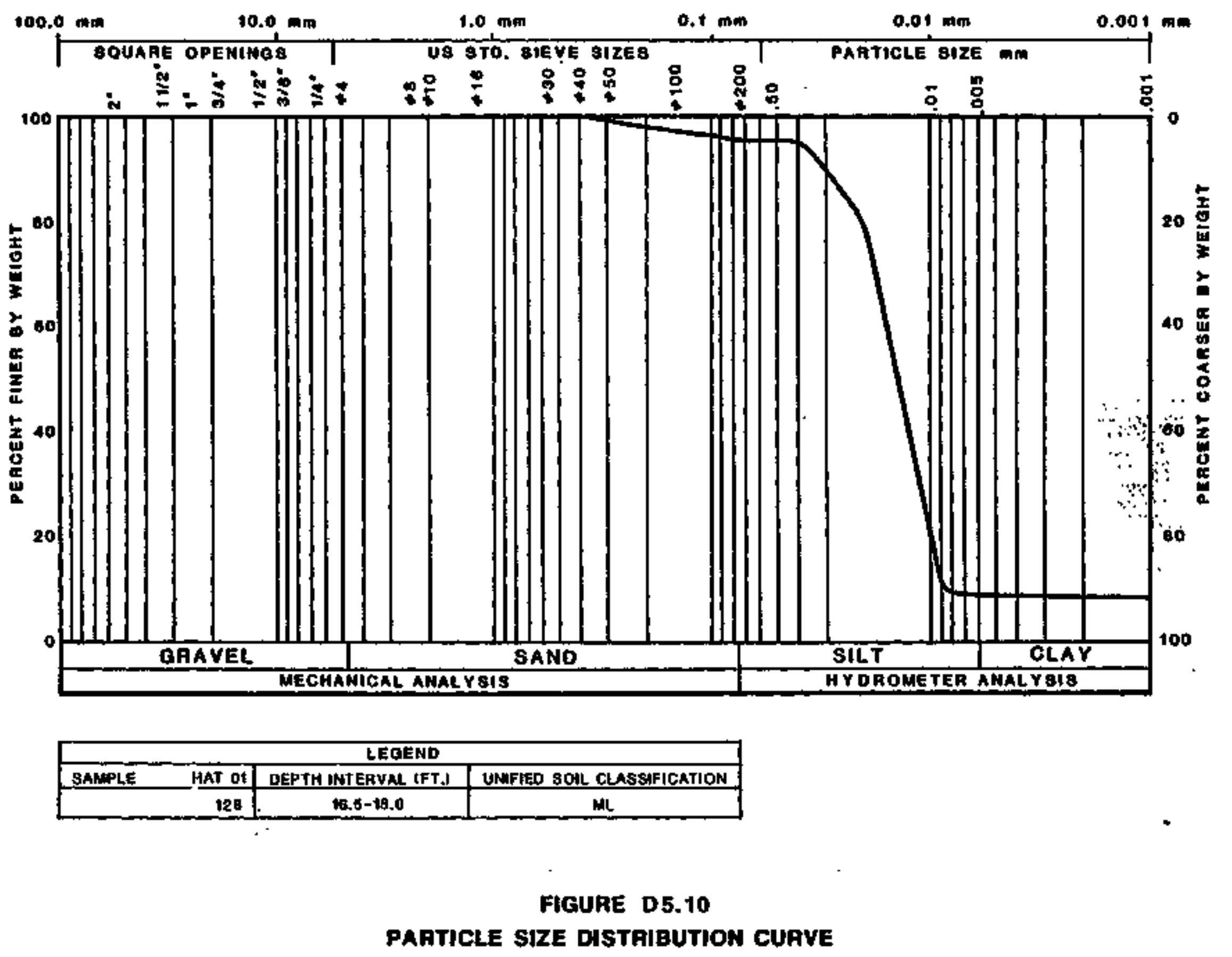




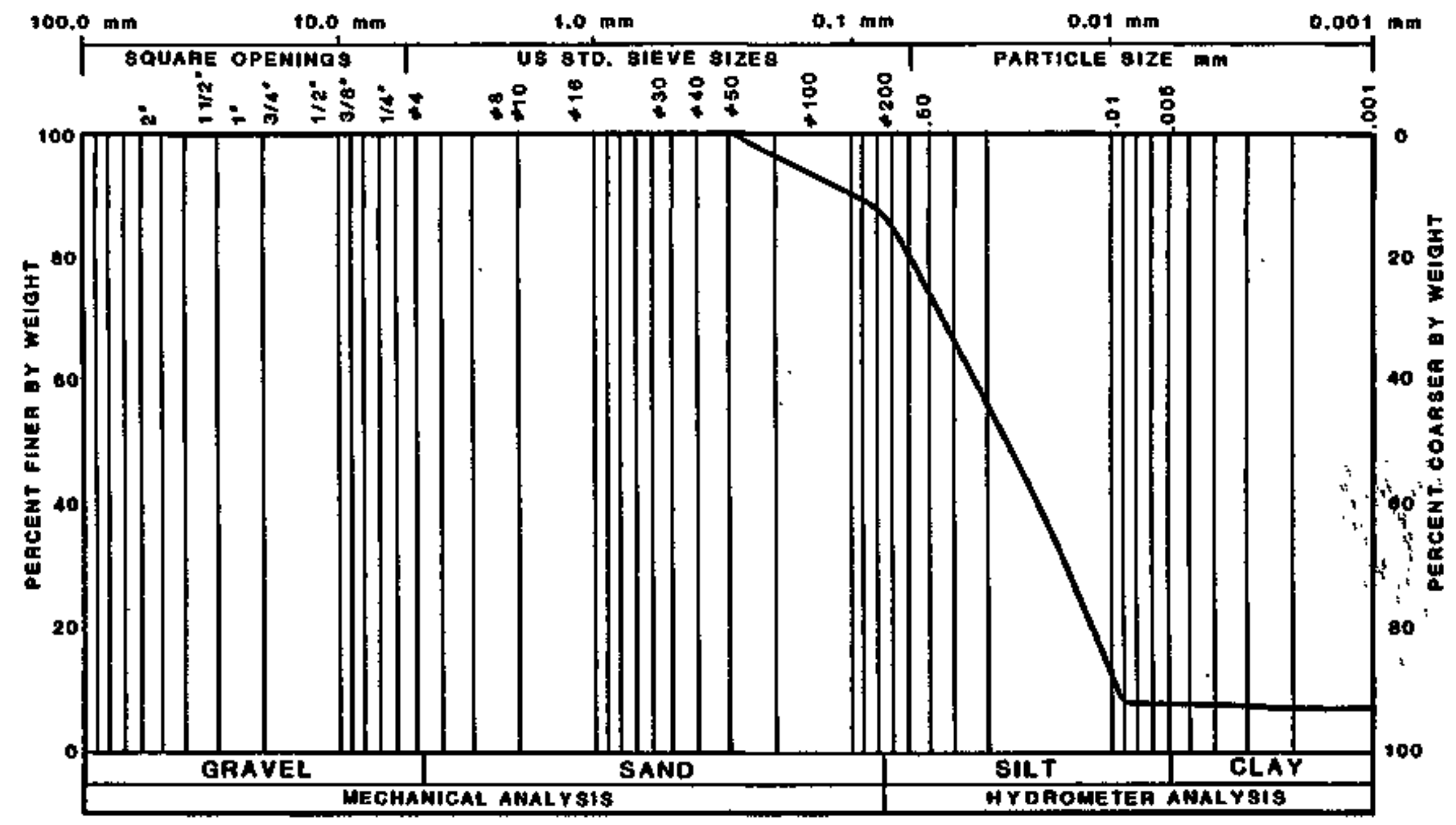

FIGUAE D 5.11

particle size dStaleution curve 


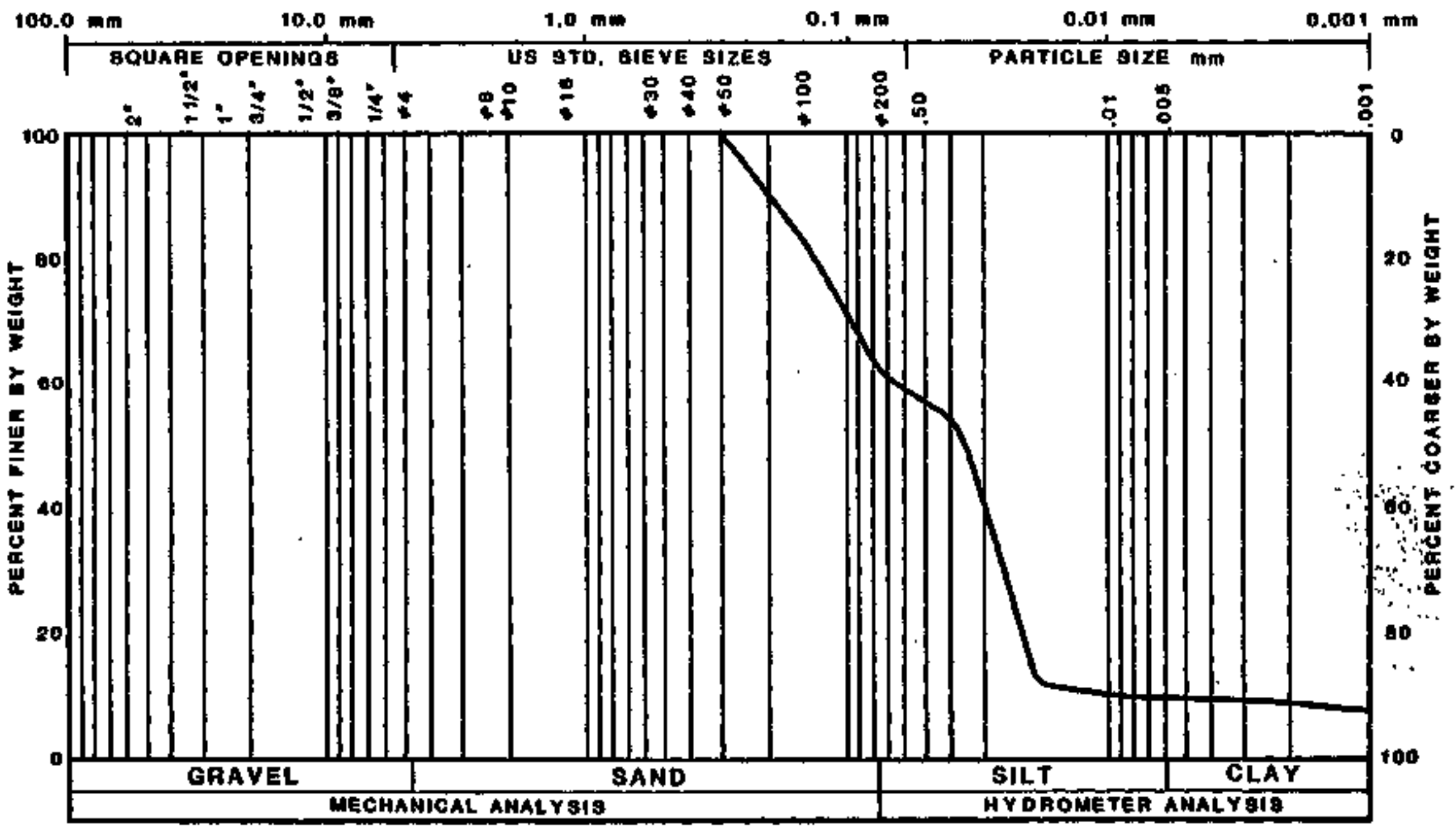

FIGURE 05.12

PARTICLE SIZE DISTAIBUTION CURVE 


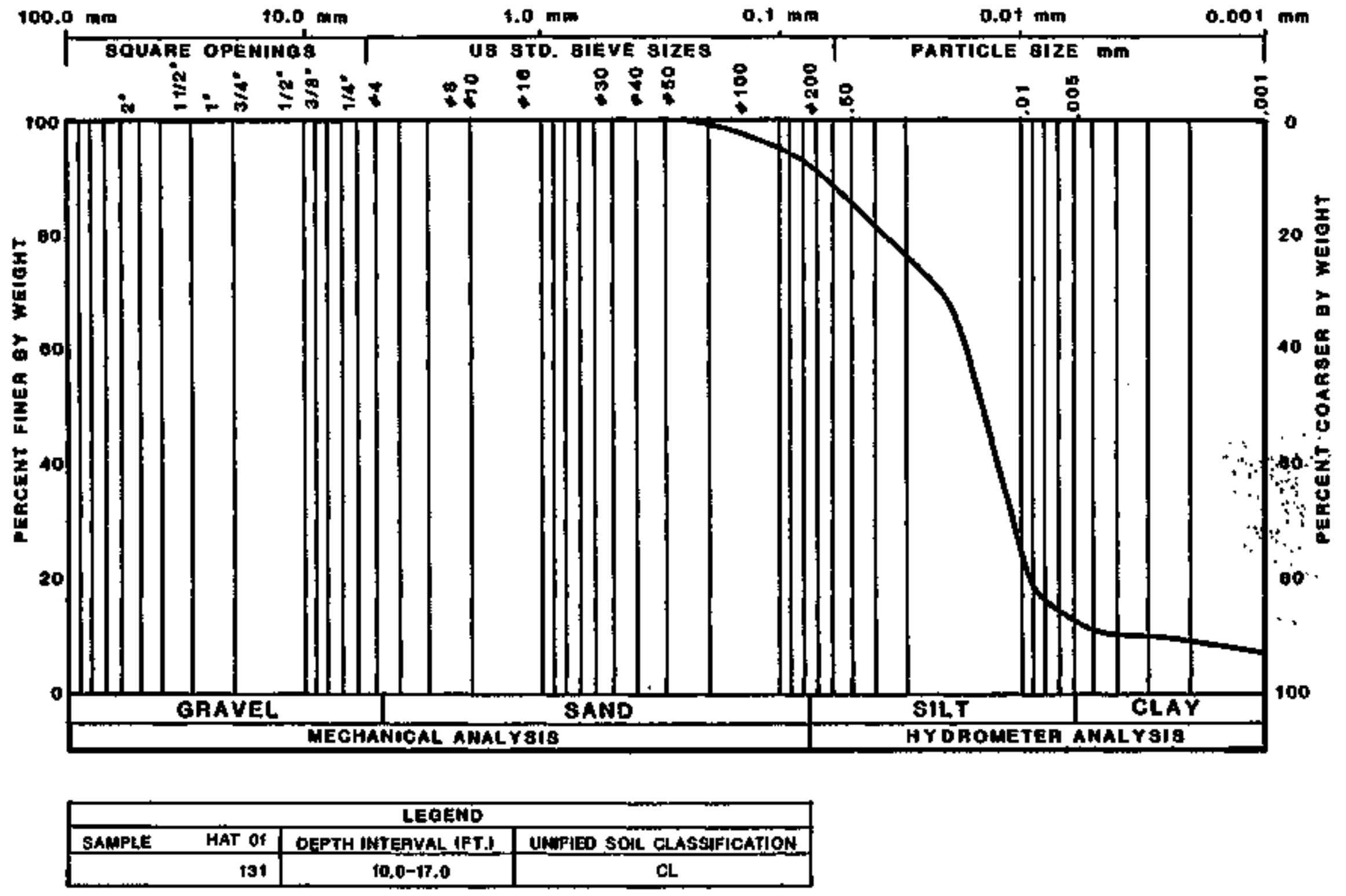

FIGURE D5.13

particle sIze distribution CURVE 


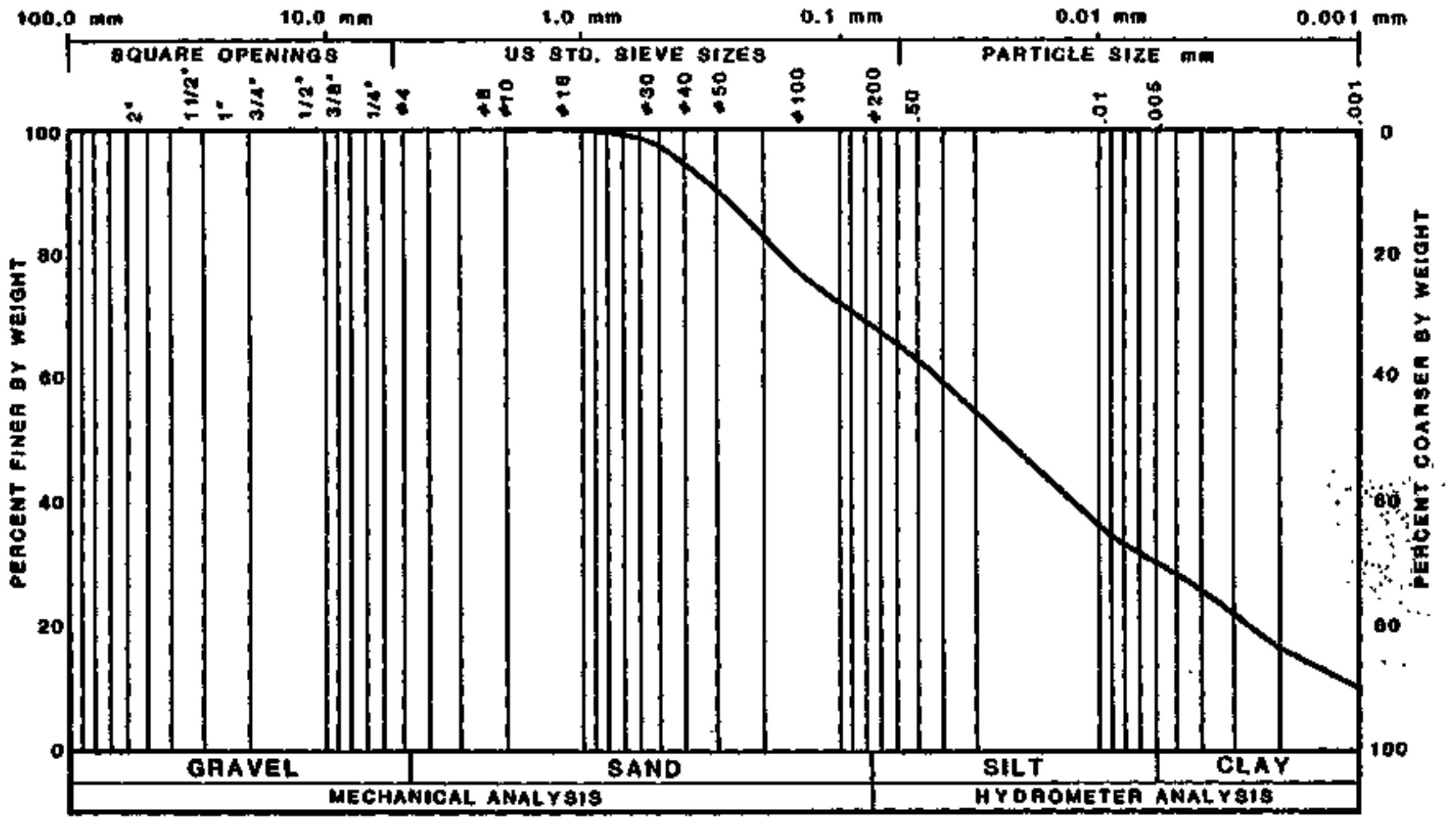

\begin{tabular}{|c|c|c|c|}
\hline \multicolumn{4}{|c|}{ LEGEMD } \\
\hline SAMPLE & HAT OA & OEPIH IWTERAVAL IFTJ & UNIFIED SORL CLASSUFICATION \\
\hline & 131 & $16.5-17.0$ & Mh \\
\hline
\end{tabular}

FIGUAE D5.14

PAaticle size distaigution curve 


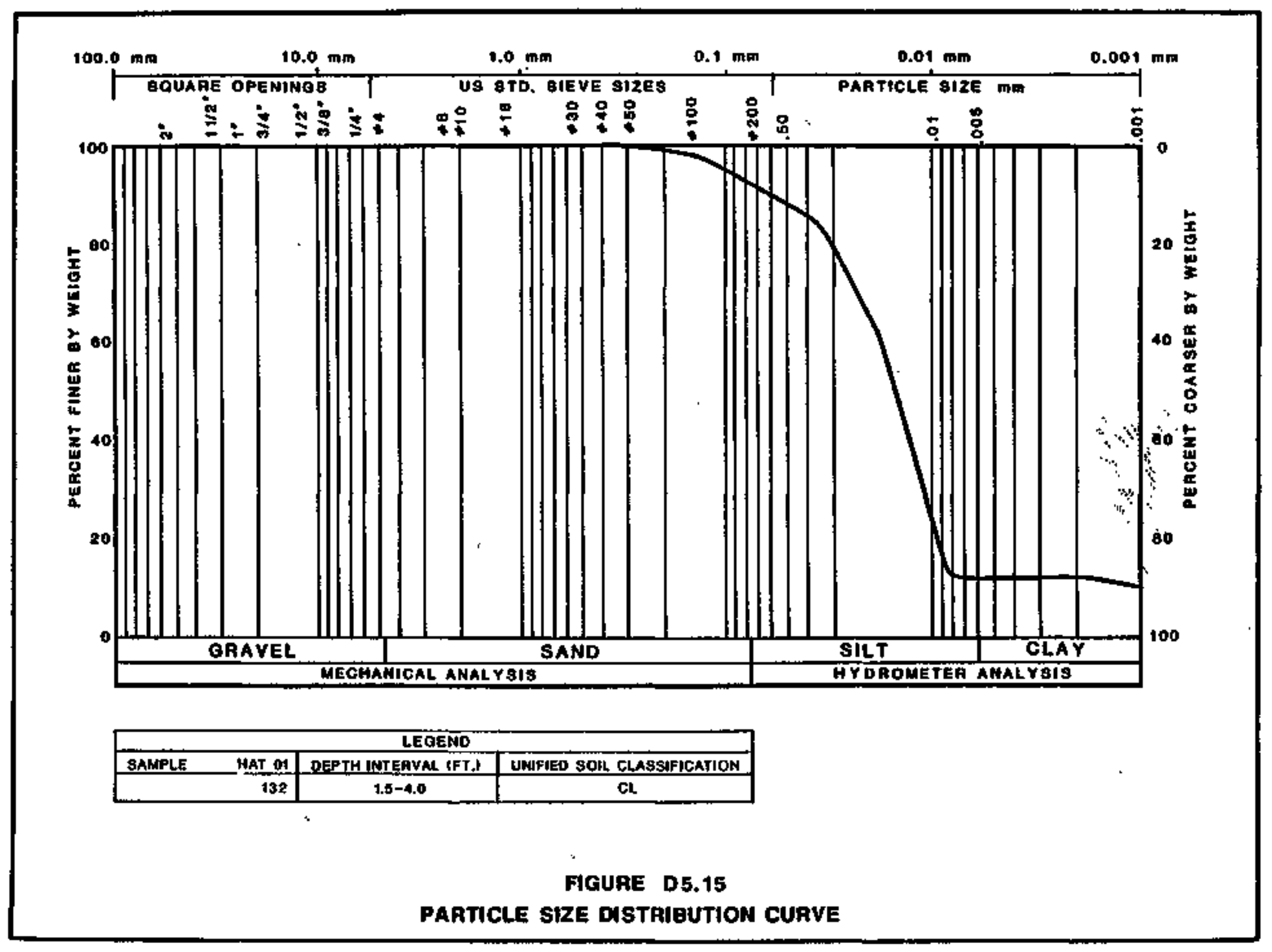




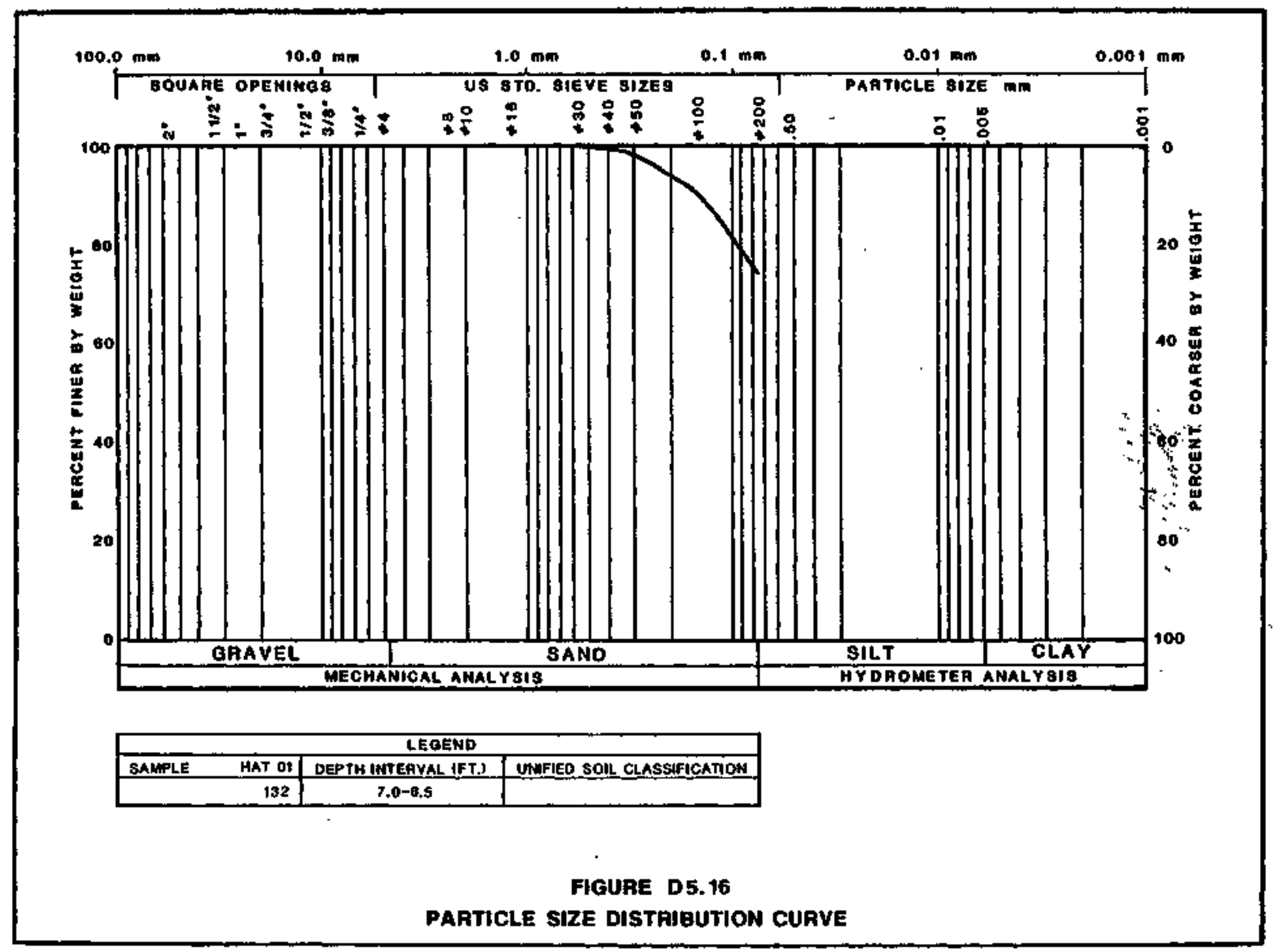




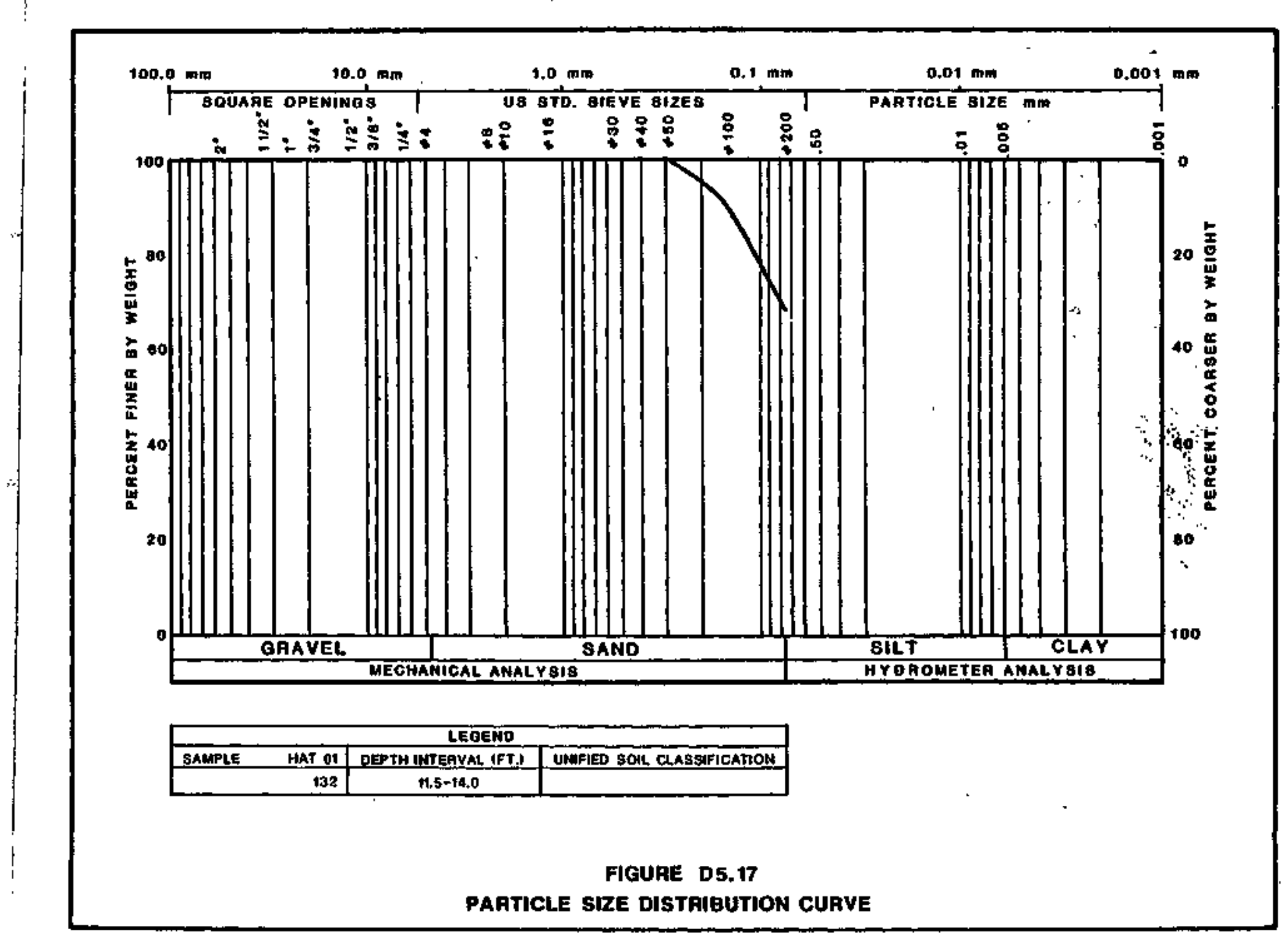




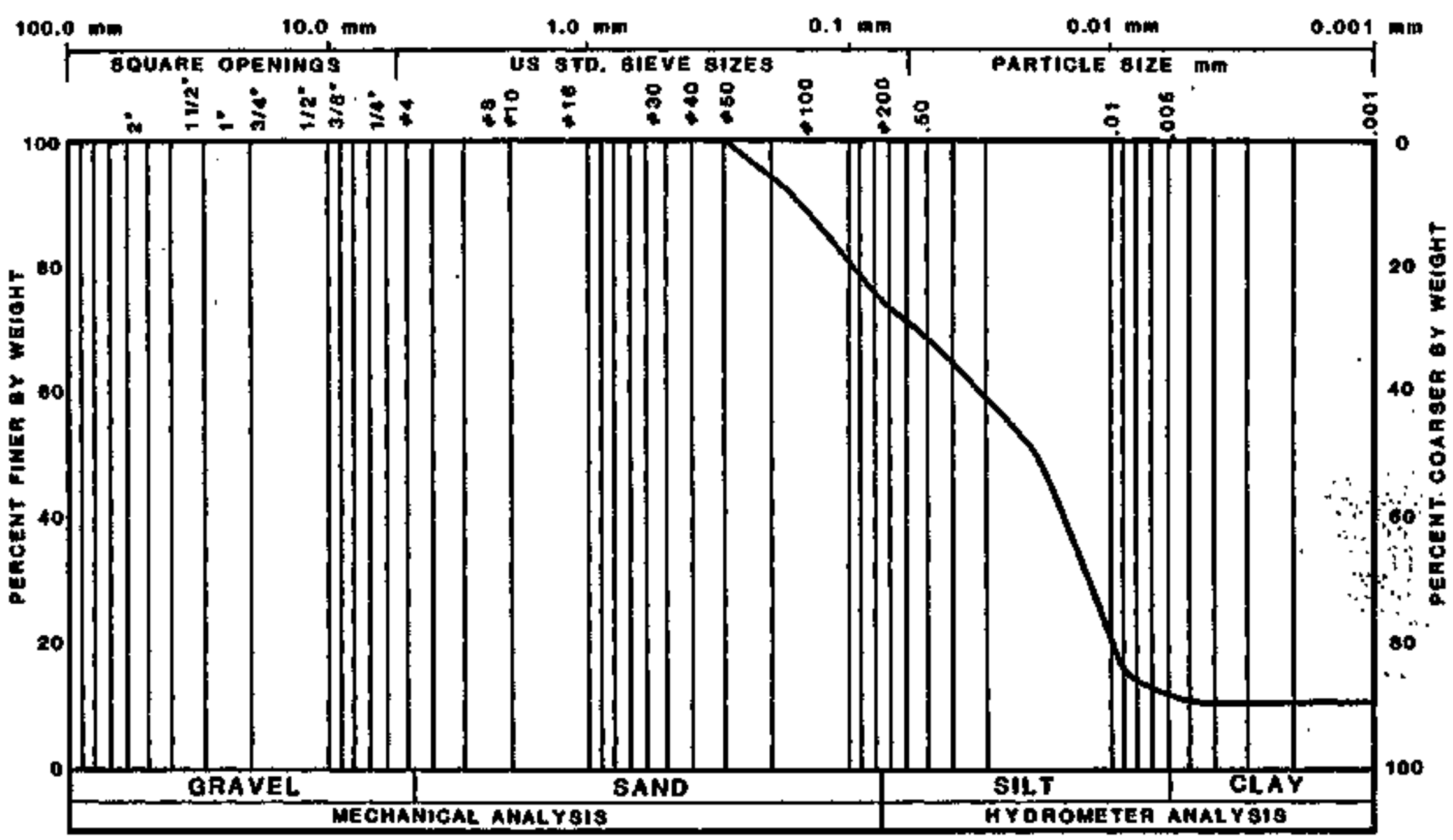

\begin{tabular}{|c|c|c|c|}
\hline \multicolumn{4}{|c|}{ LEÄEND } \\
\hline SAMPLE & HAT Ot & GEPTH TNTEHVAL (FT.) & UMUFIEO SOIL CLAGSIFICATION \\
\hline & 132 & $24.5-28.5$ & sh. \\
\hline
\end{tabular}

FIGURE D5.18

PARTICLE SIZE DISTAIBUTION CUAVE 


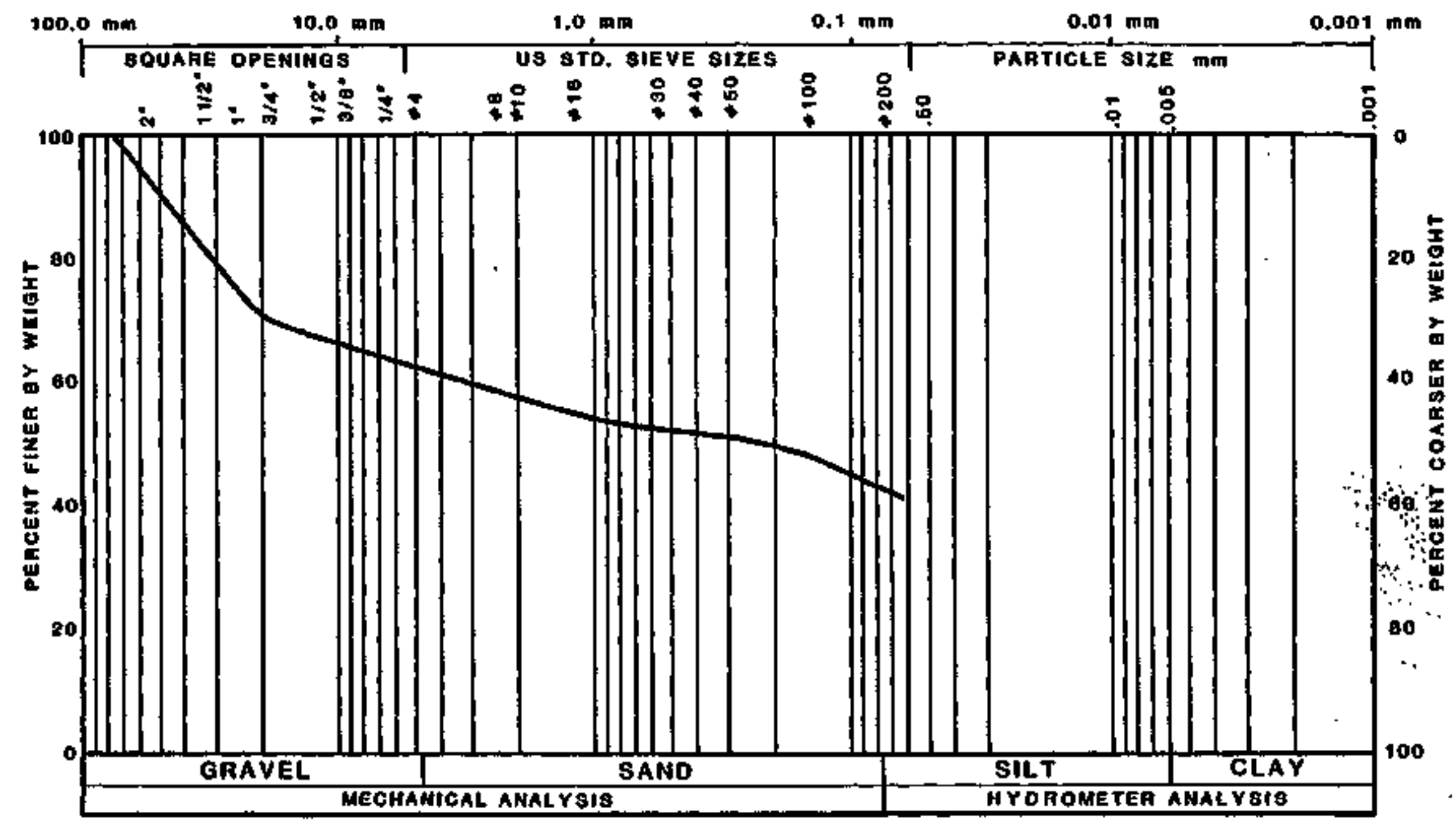

FIGURE D5.19

PARTICLE STZE DISTRIBUTION CURVE 


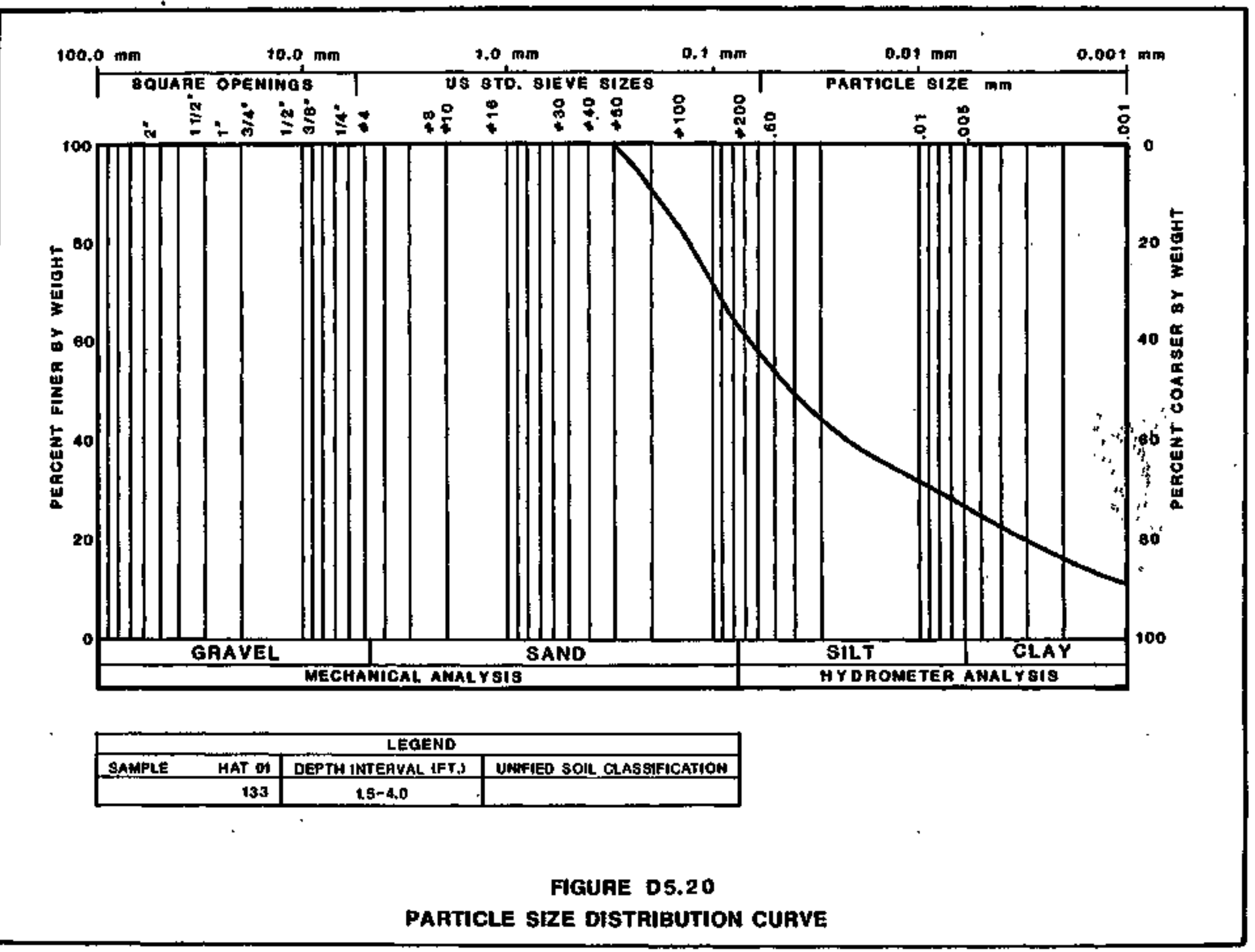




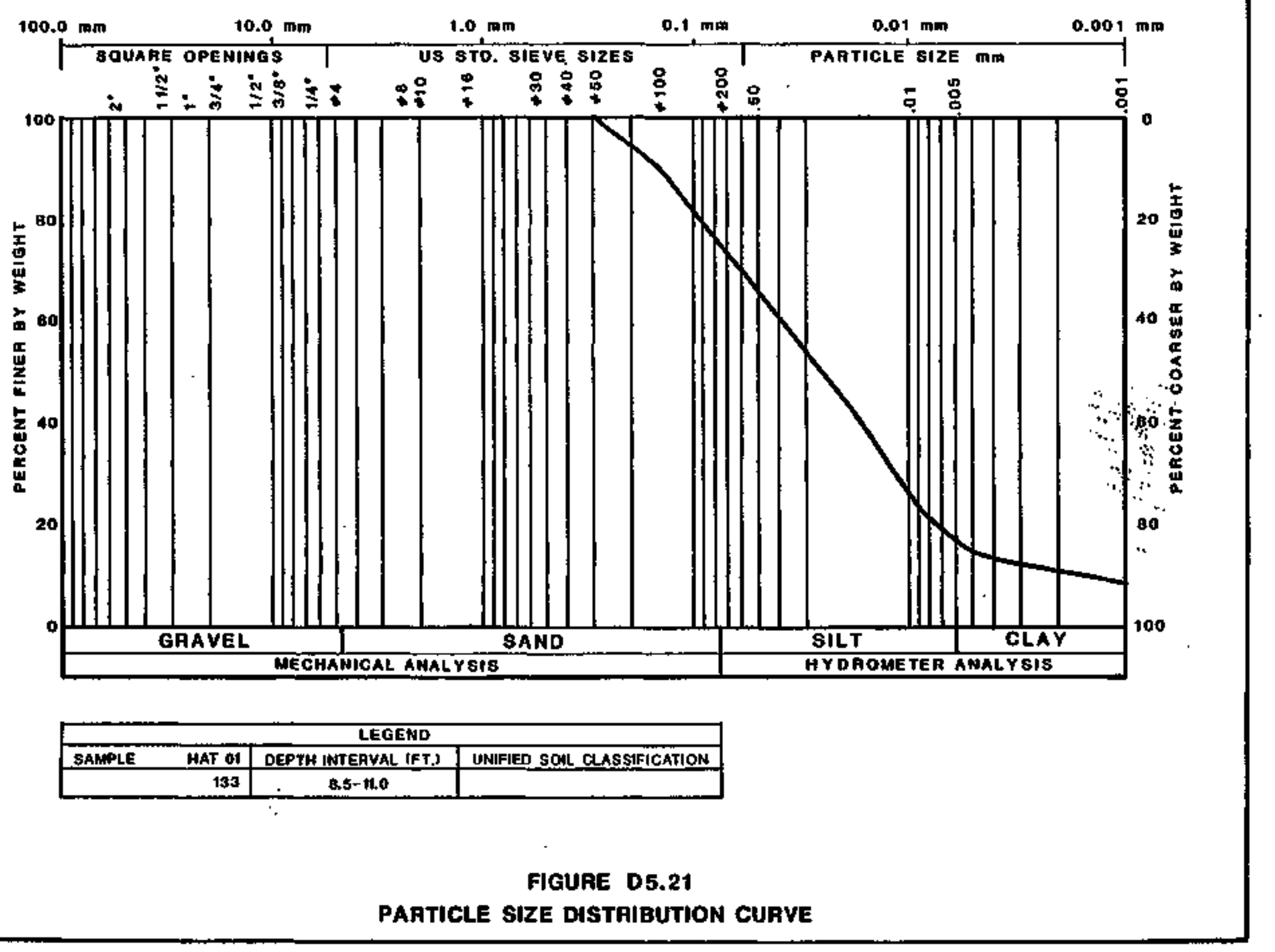




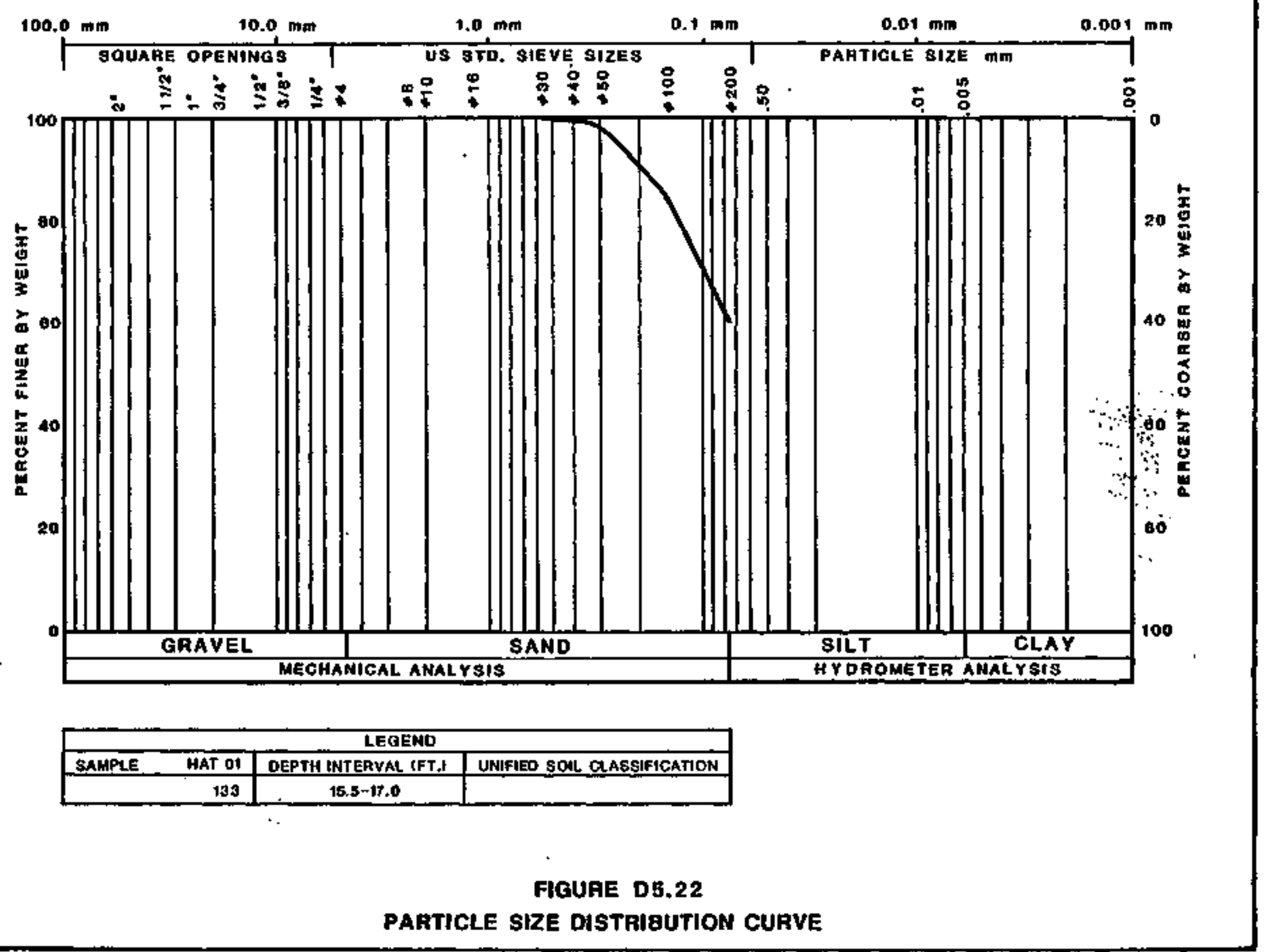




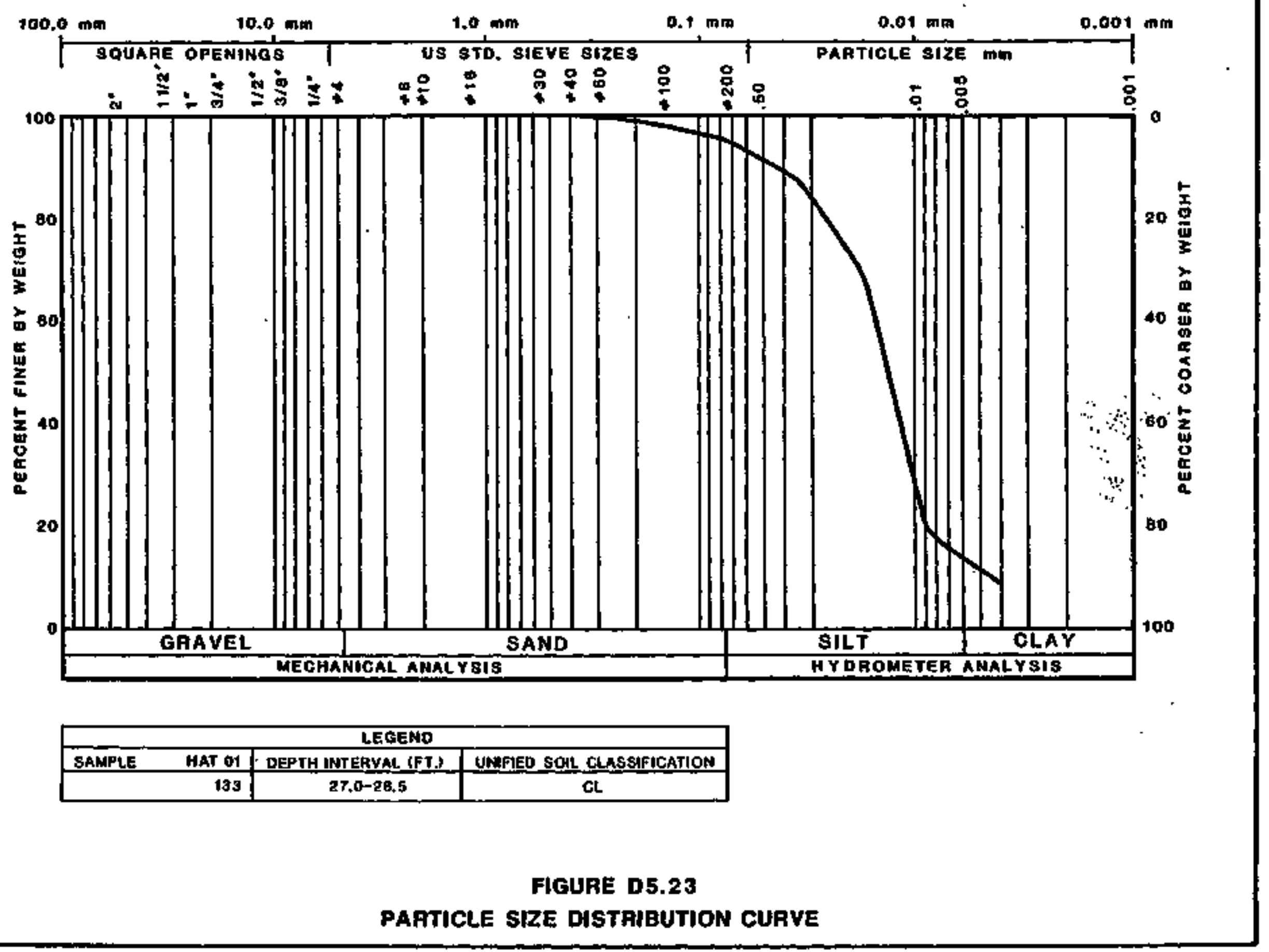




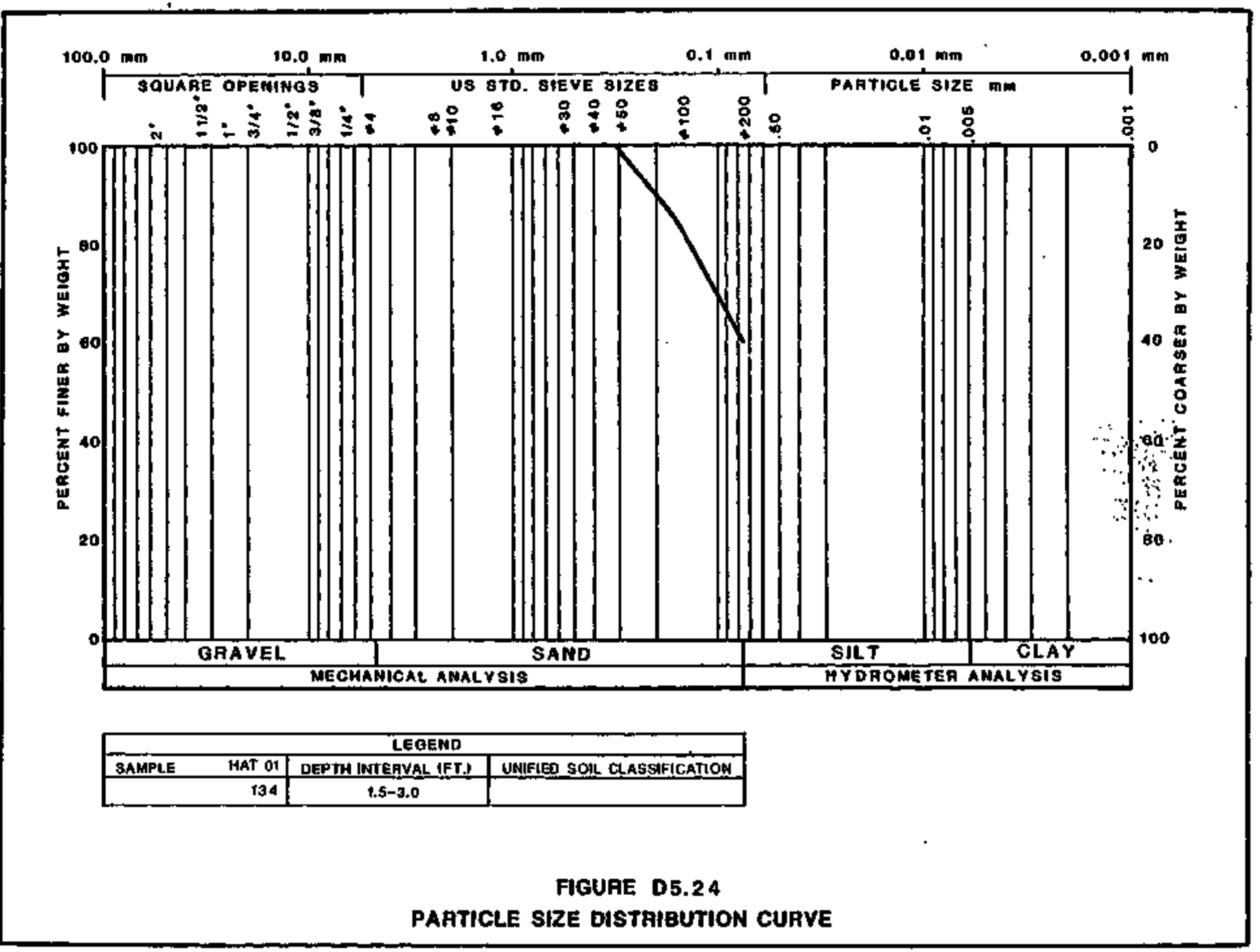




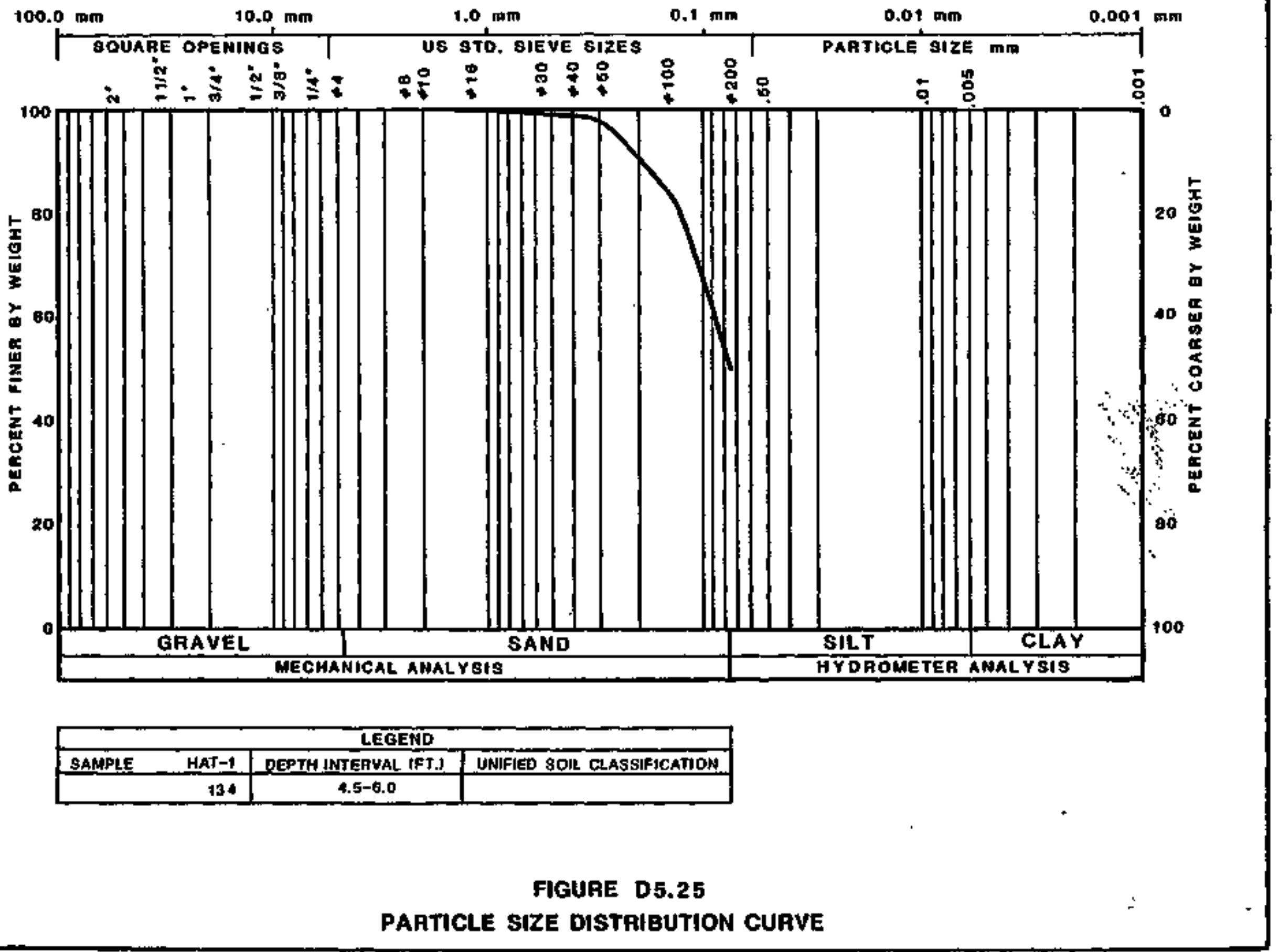




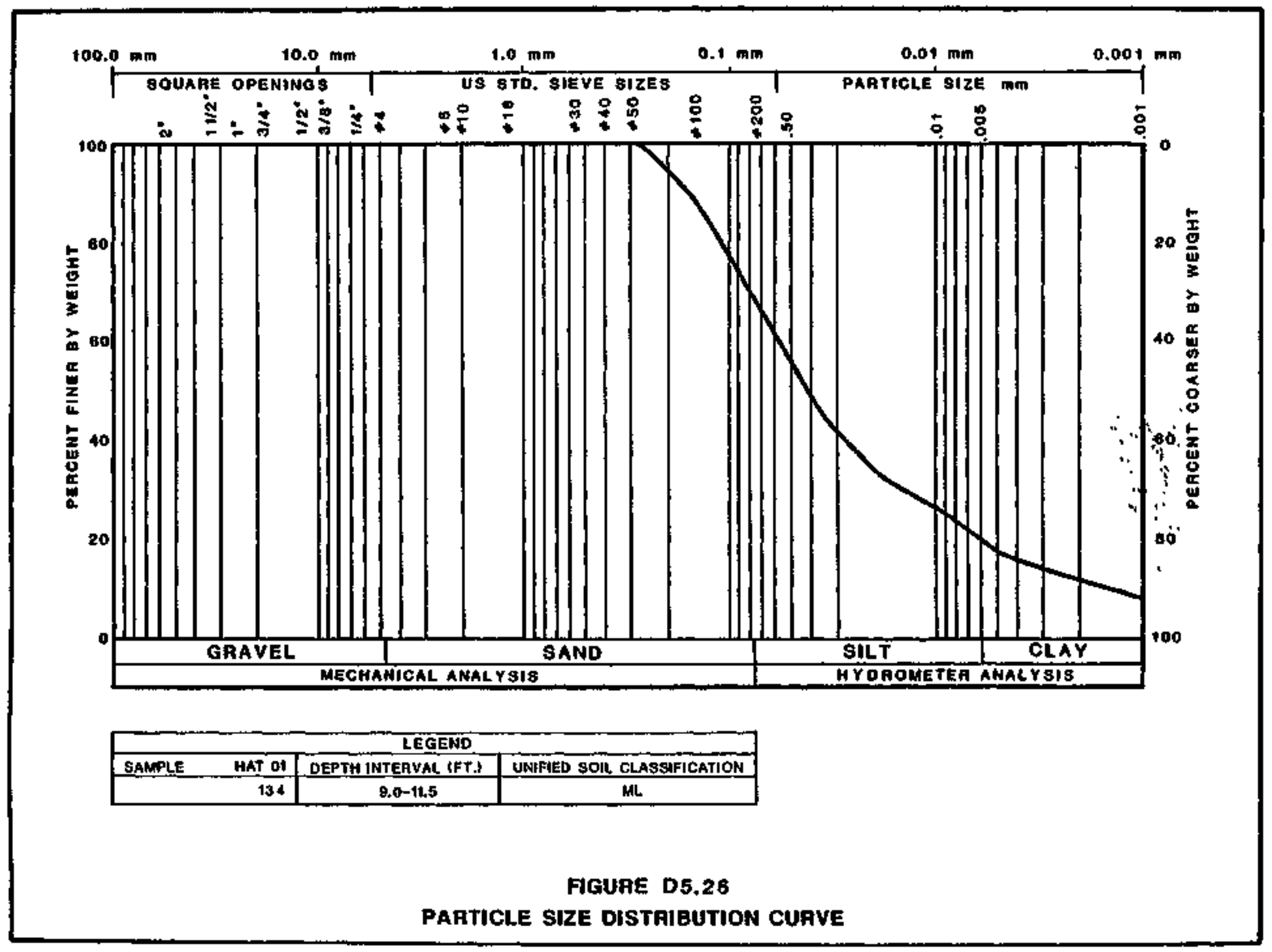




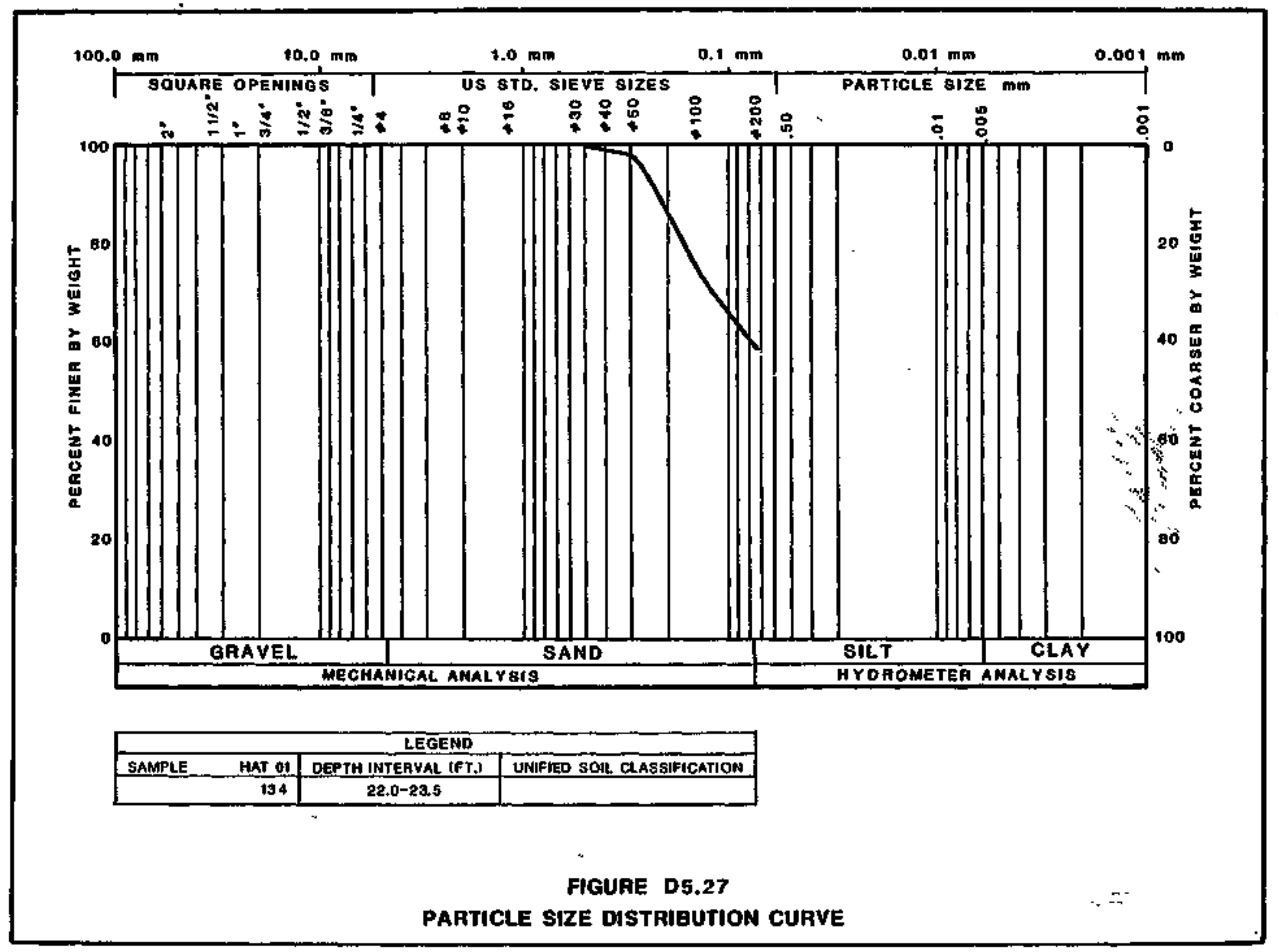




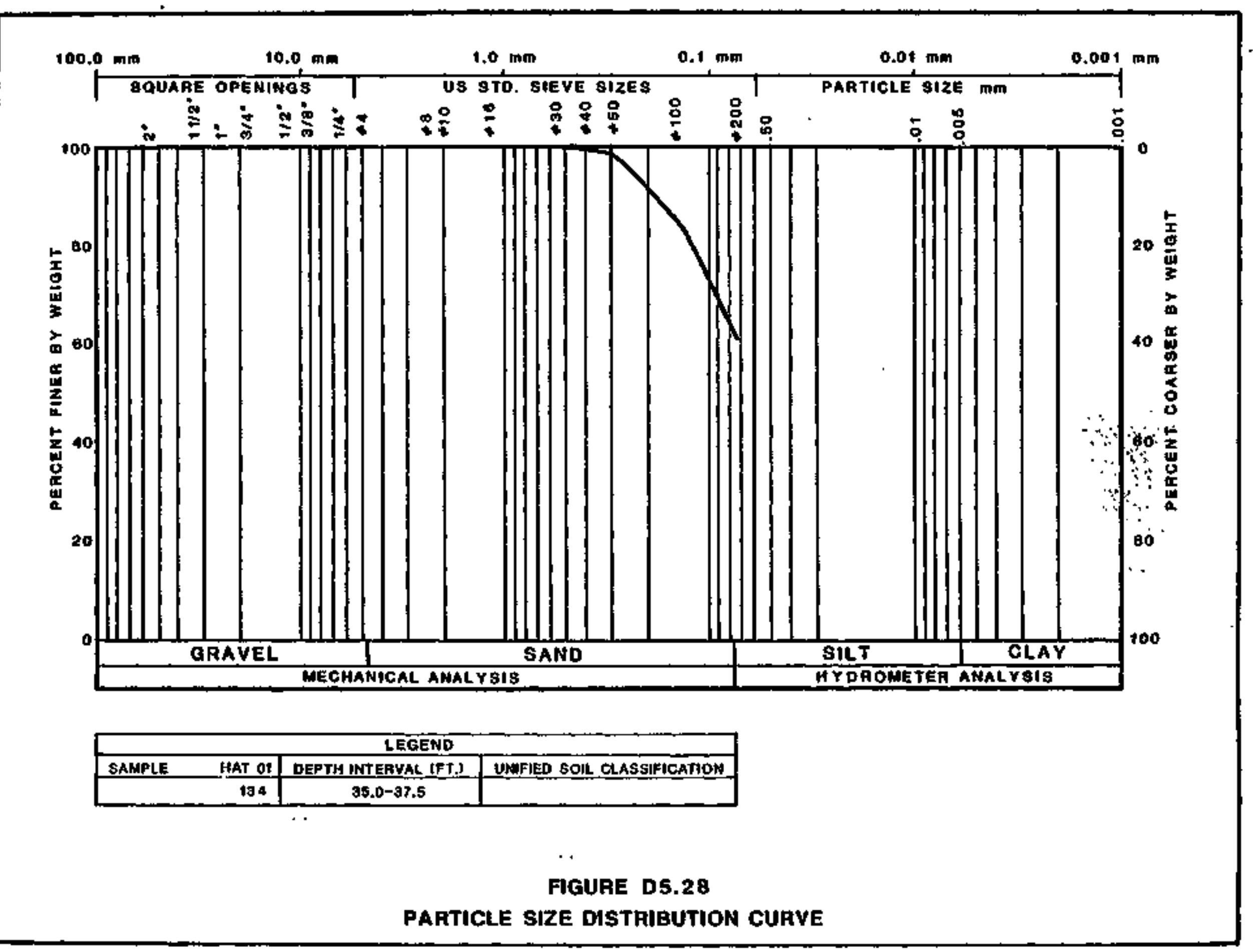




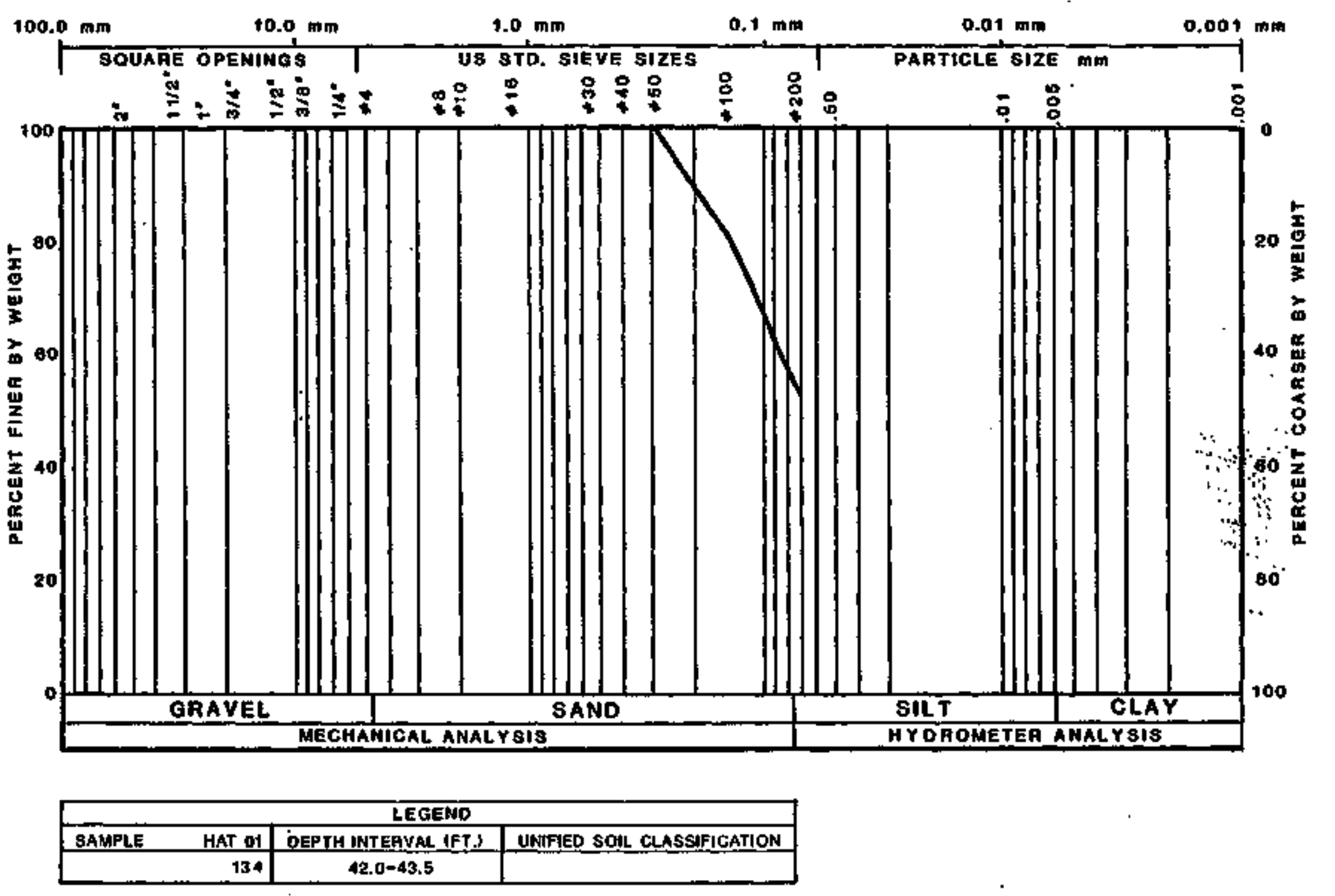

FGURE D5.29

PARTICle sIzE DISTRIBUTION CURVE 


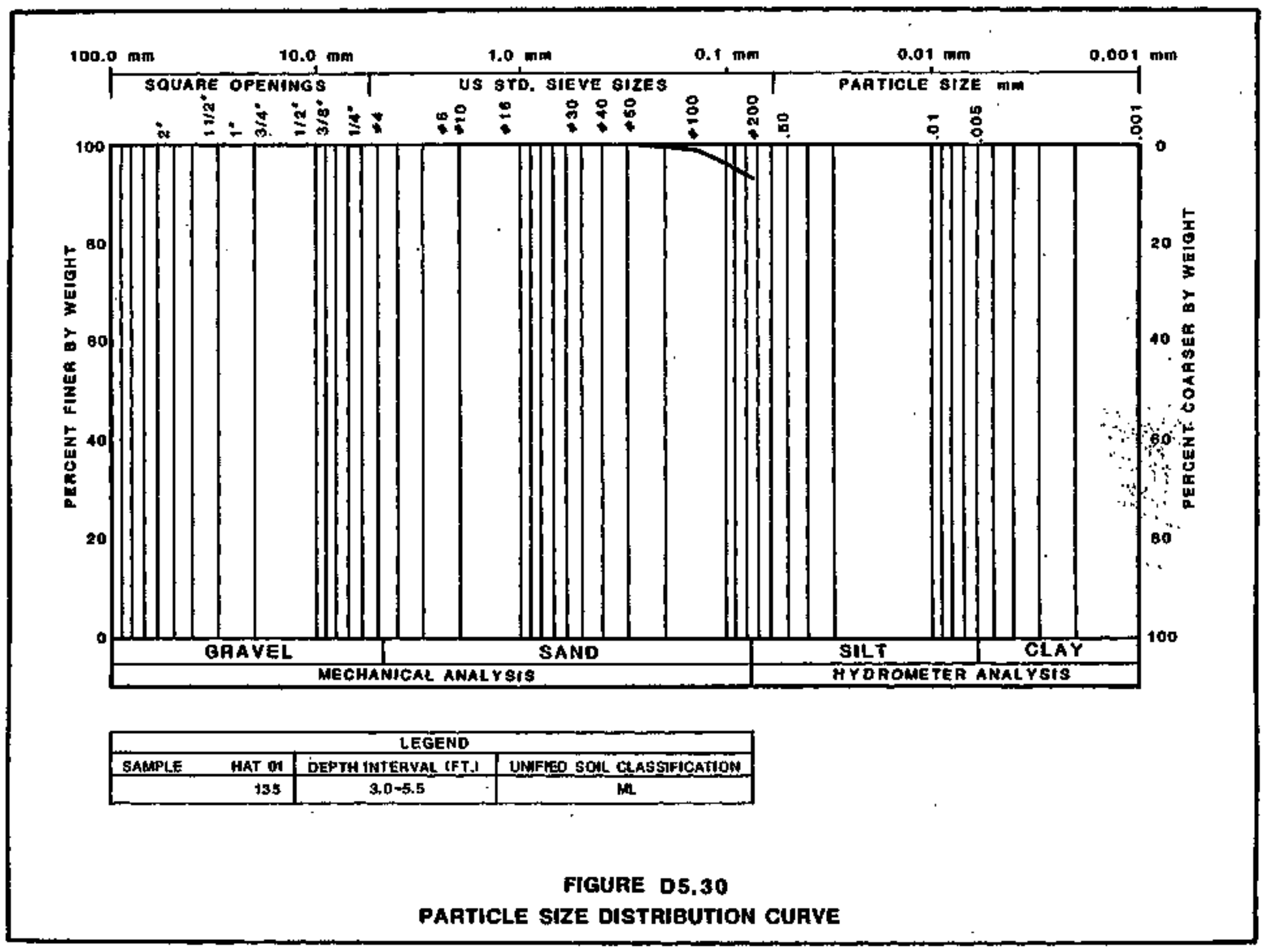




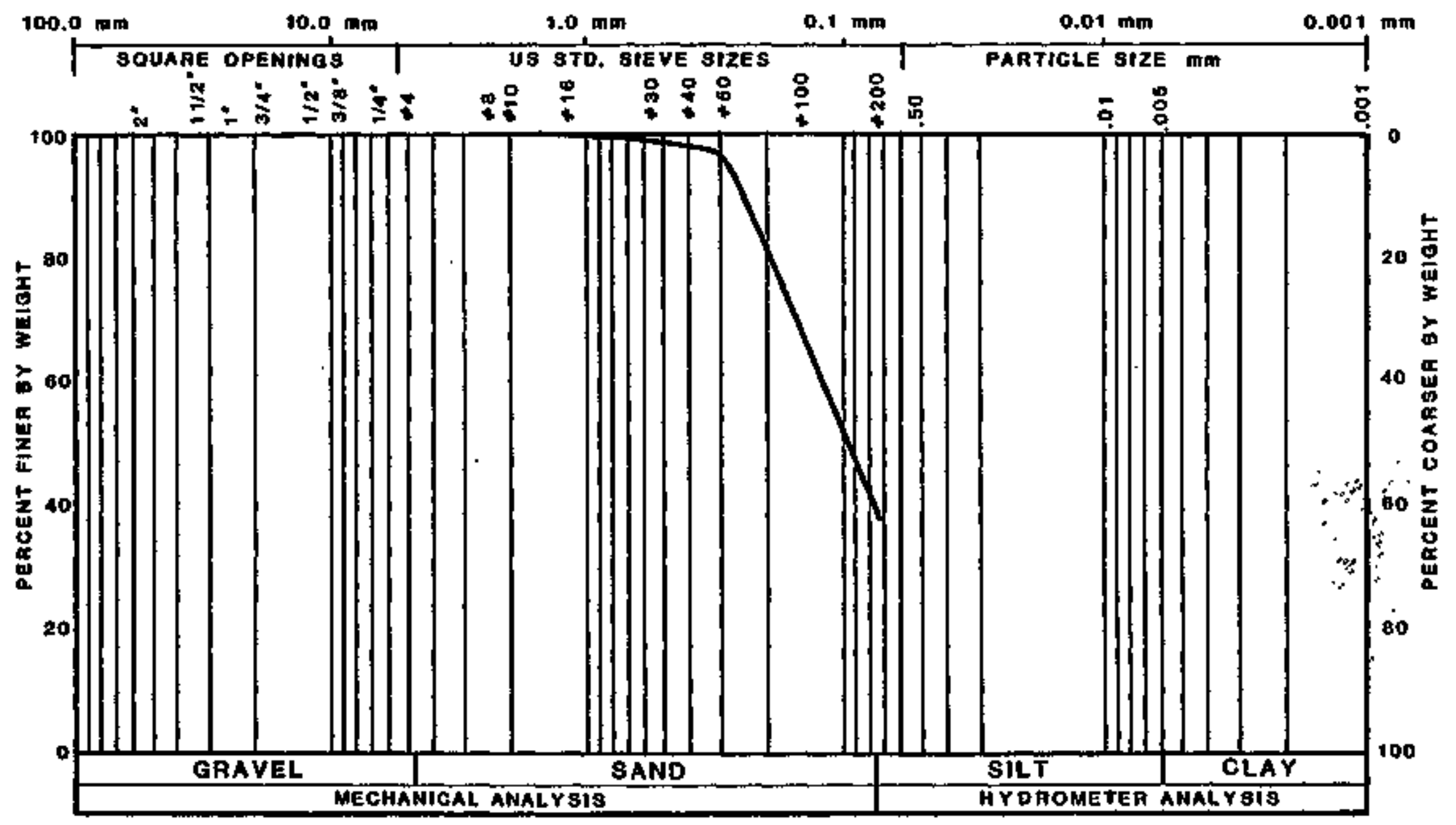

FIGUAE D5.31

PARTICLE SIZE DISTRIBUtion CuRVE 


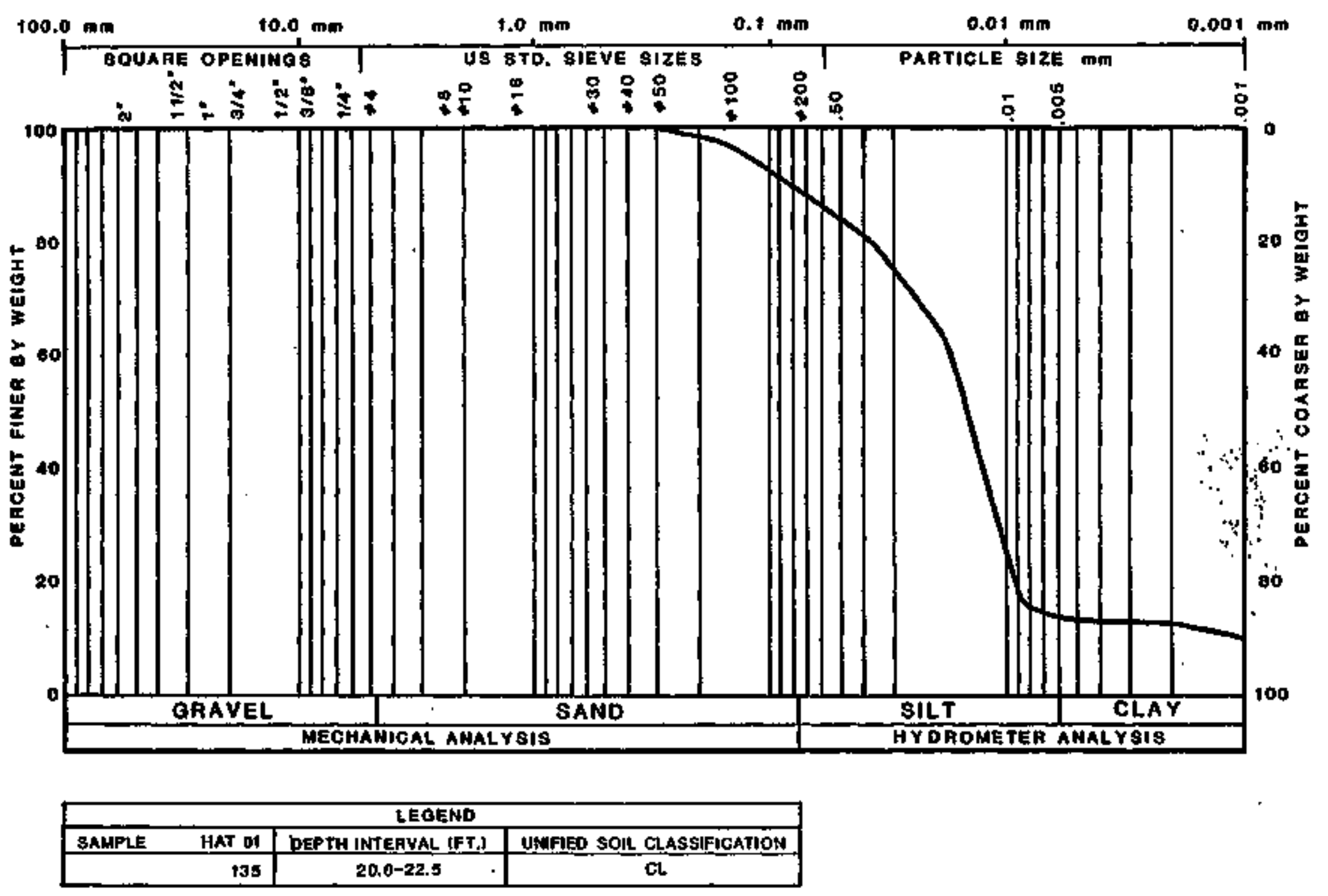

FIGUAE D5.32

PARTICLE SIZE DISTAIBUTION CURVE 


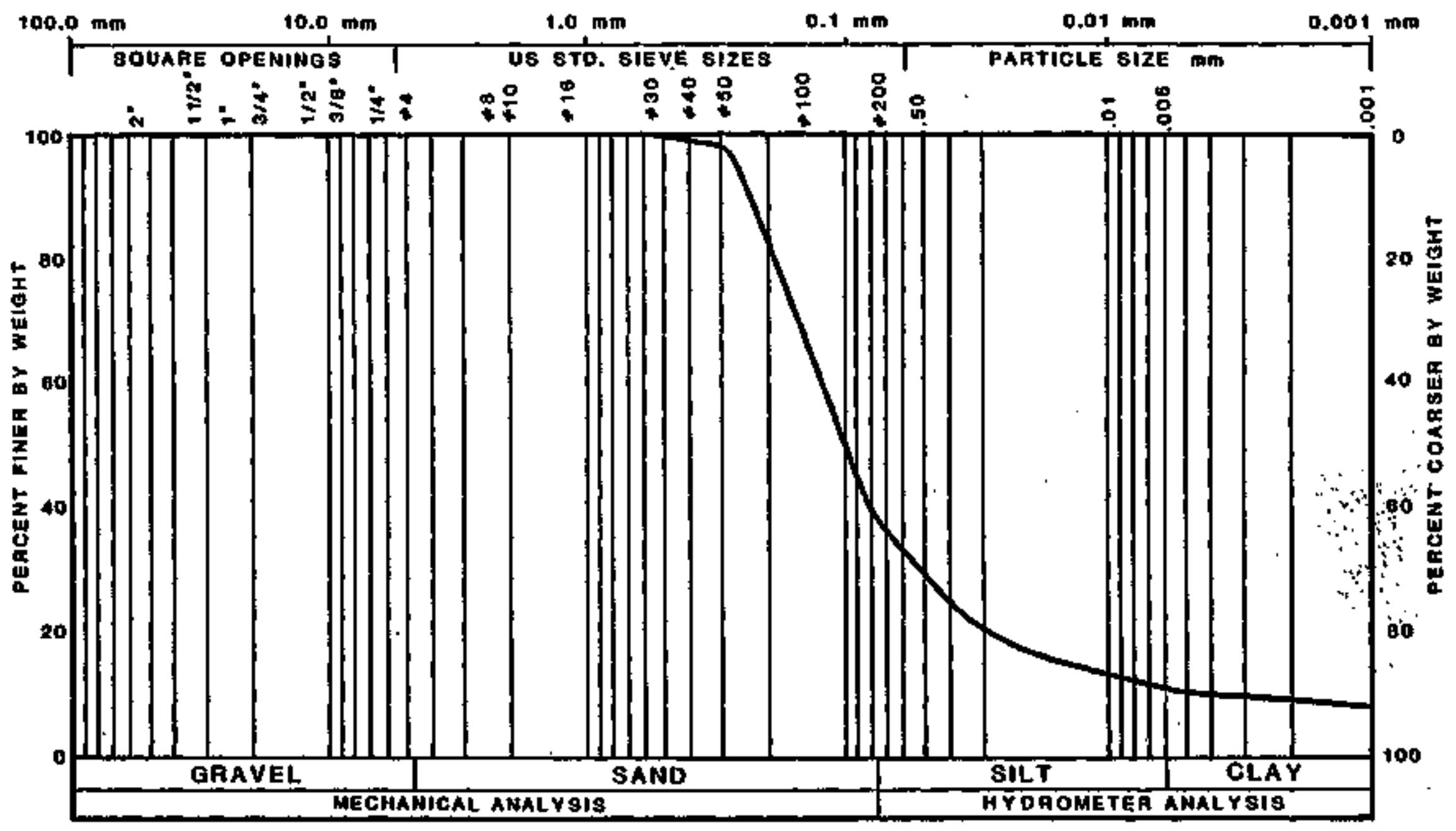

\begin{tabular}{|r|c|c|}
\hline SAMPLE_ HAT O1 & OEPTH INTERVAL (FT, & UNIFIEO SOIL CLASSIFICATION \\
\hline 138 & $30.0-32.5$ & SH \\
\hline
\end{tabular}

FIGUAE D5.33

particle size distaigution CuRVE 

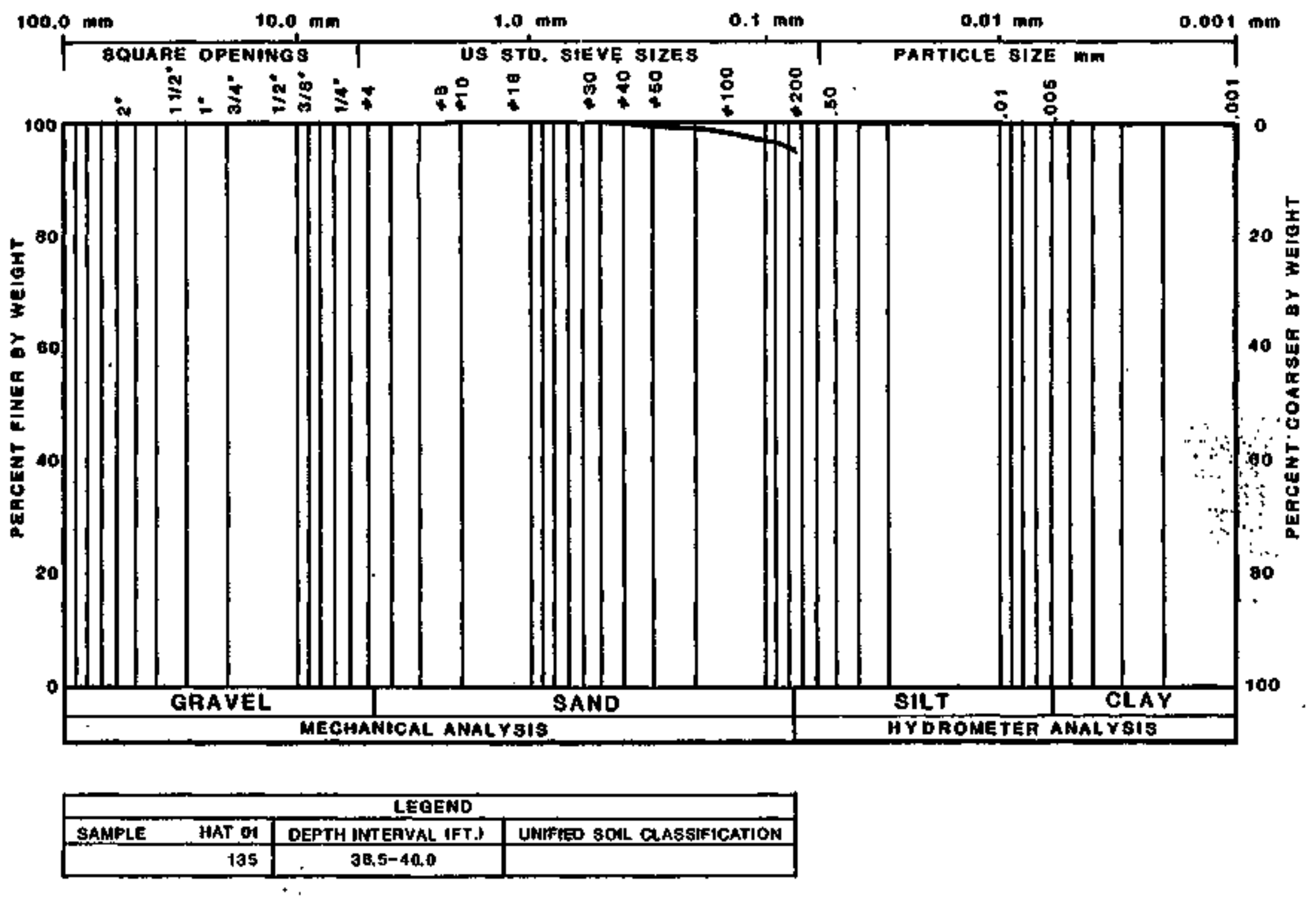

FIGUAE D5.34

PAATICLE SIZE DISTRIBUTION CURVE 


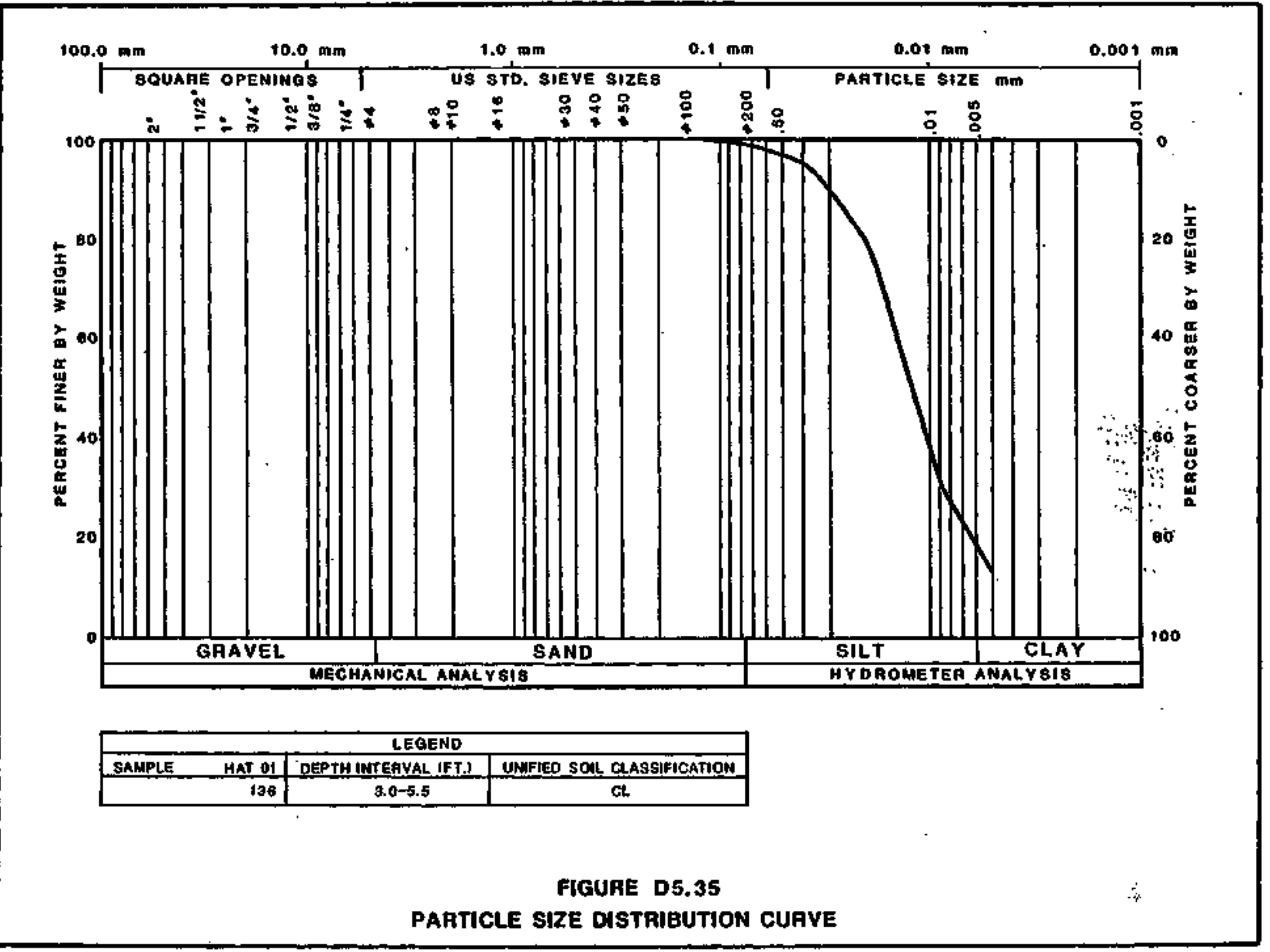




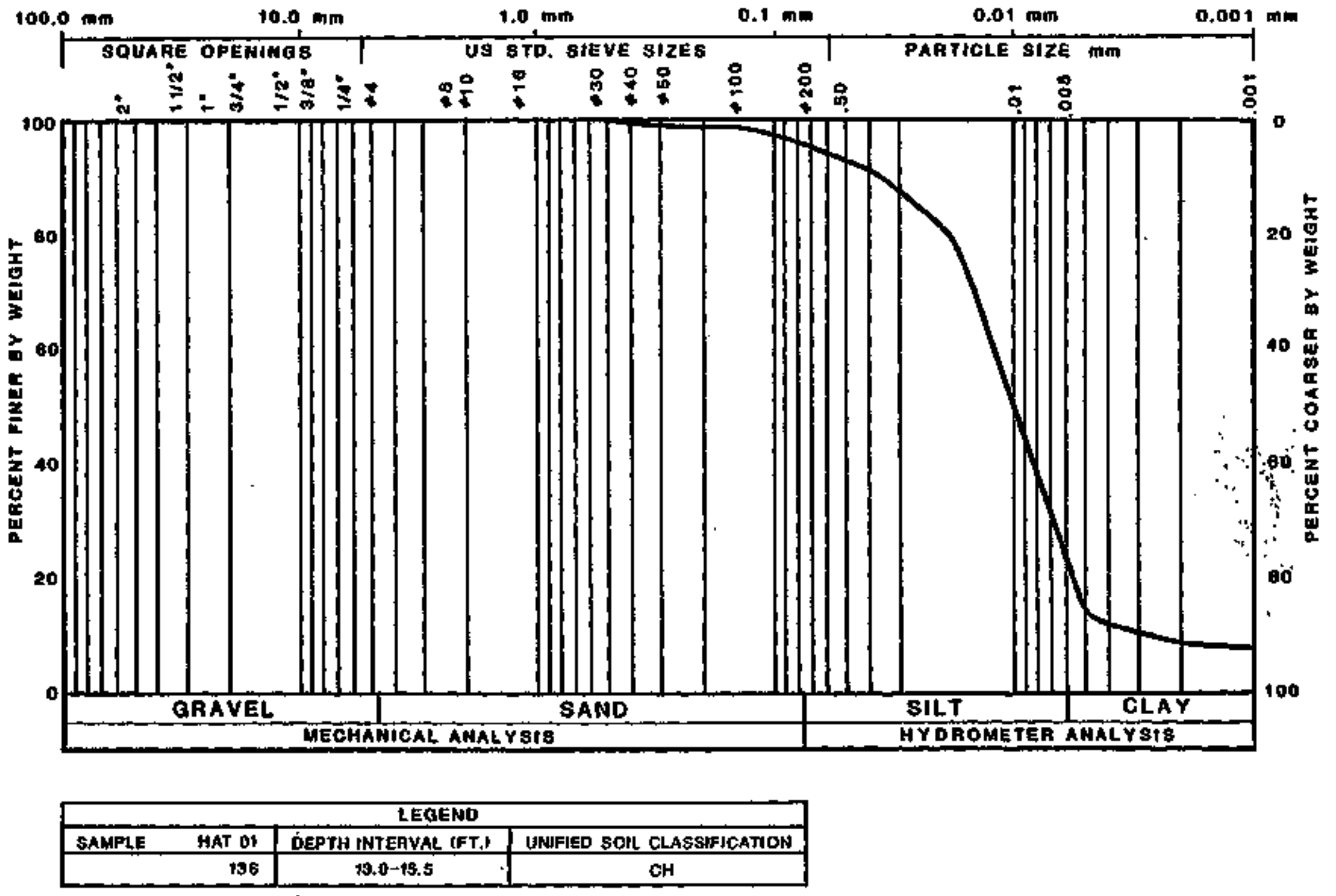

FIGUAE 05.36

PARTICLE SIZE DISTRIBUTION CURVE 


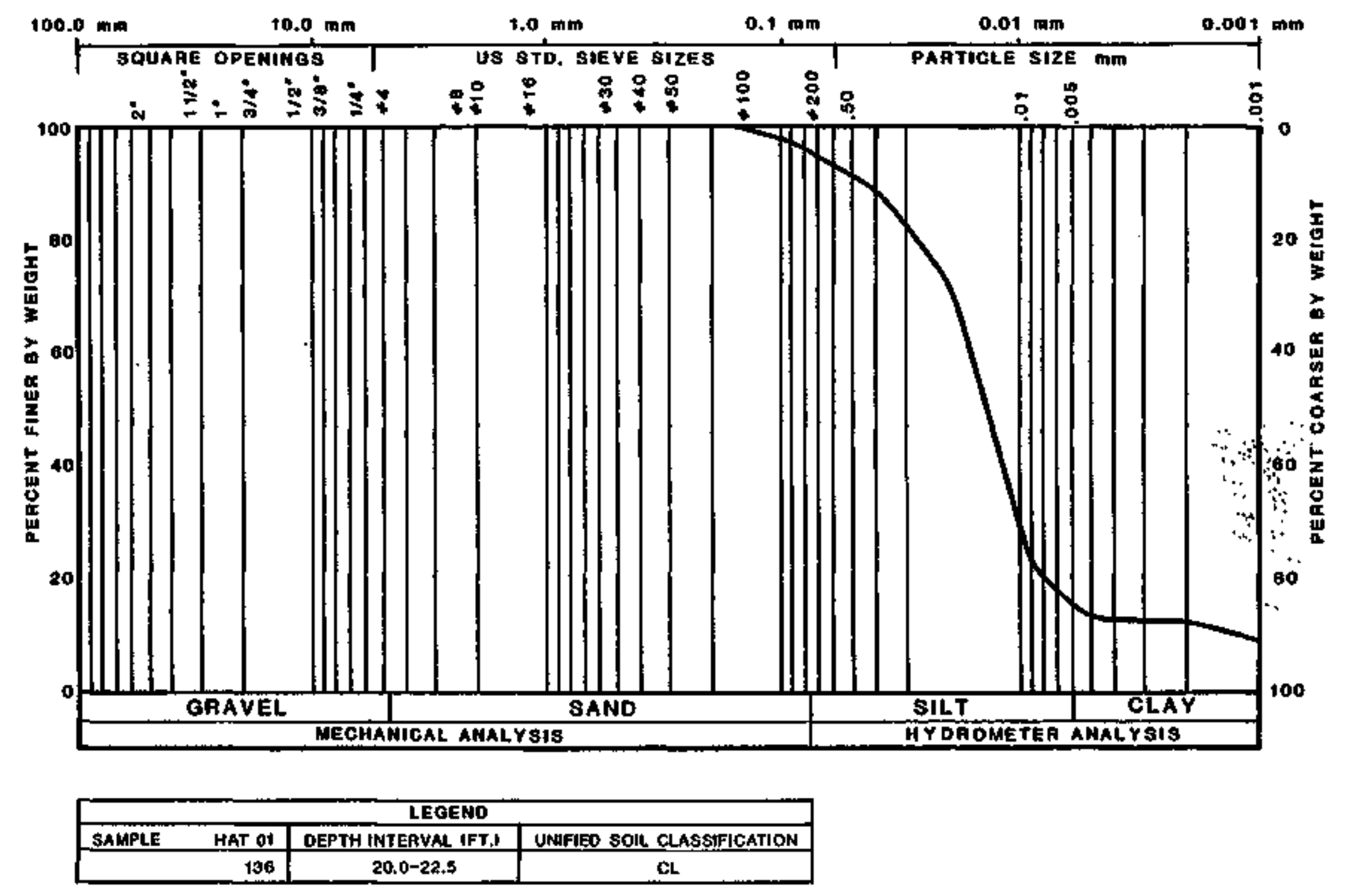

FIGUAE D5.37

particle size distaibution cuave 


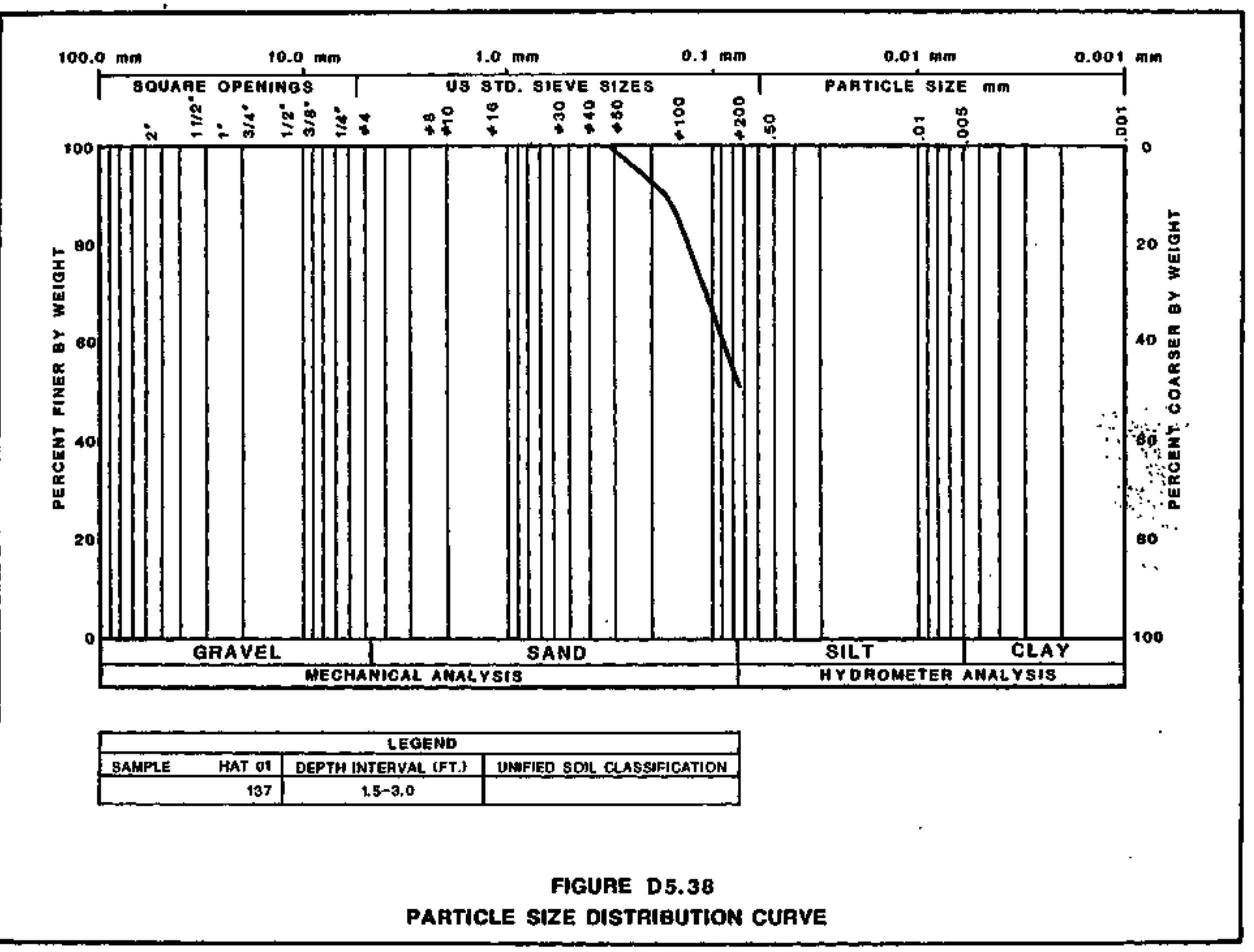




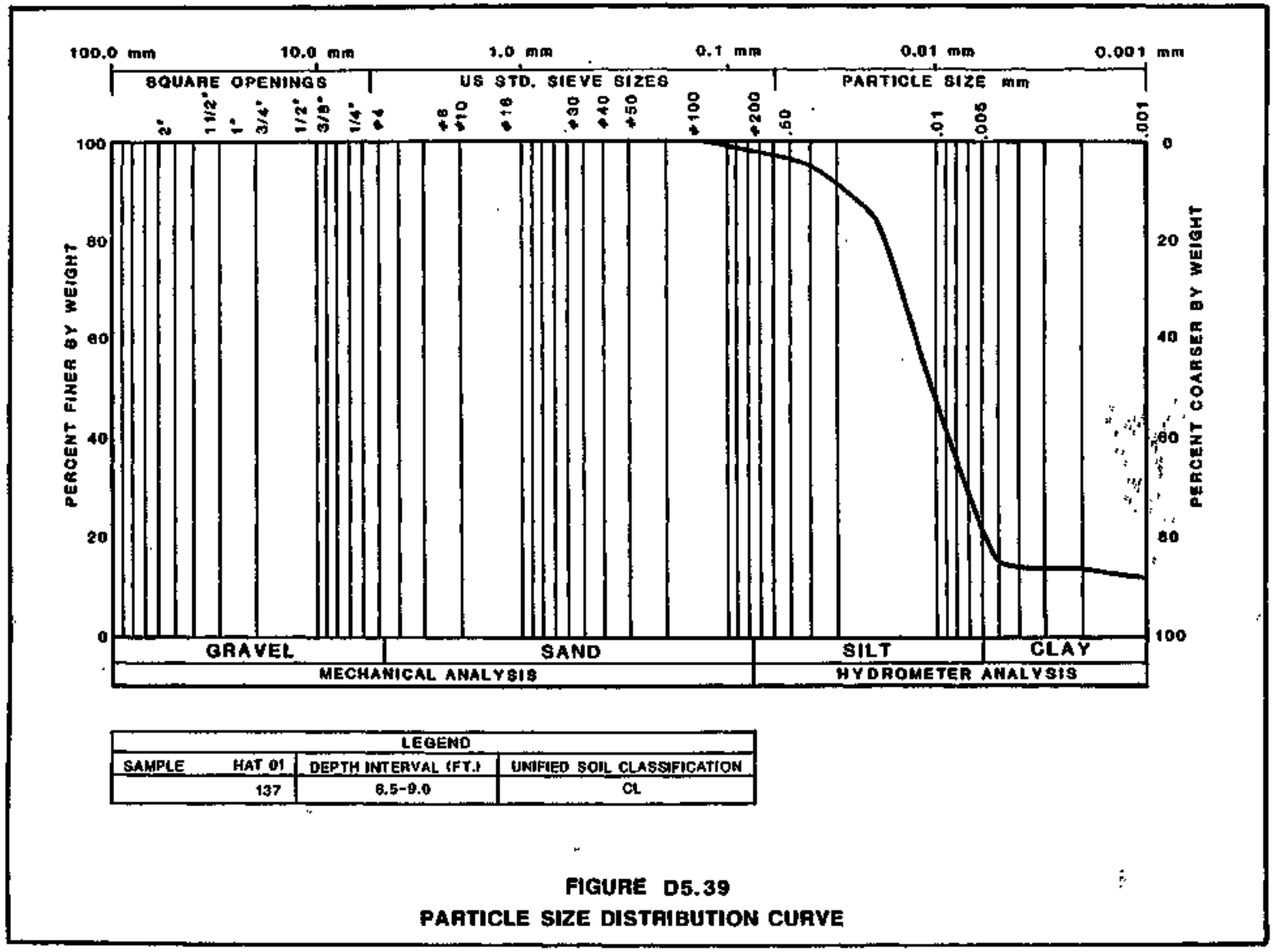




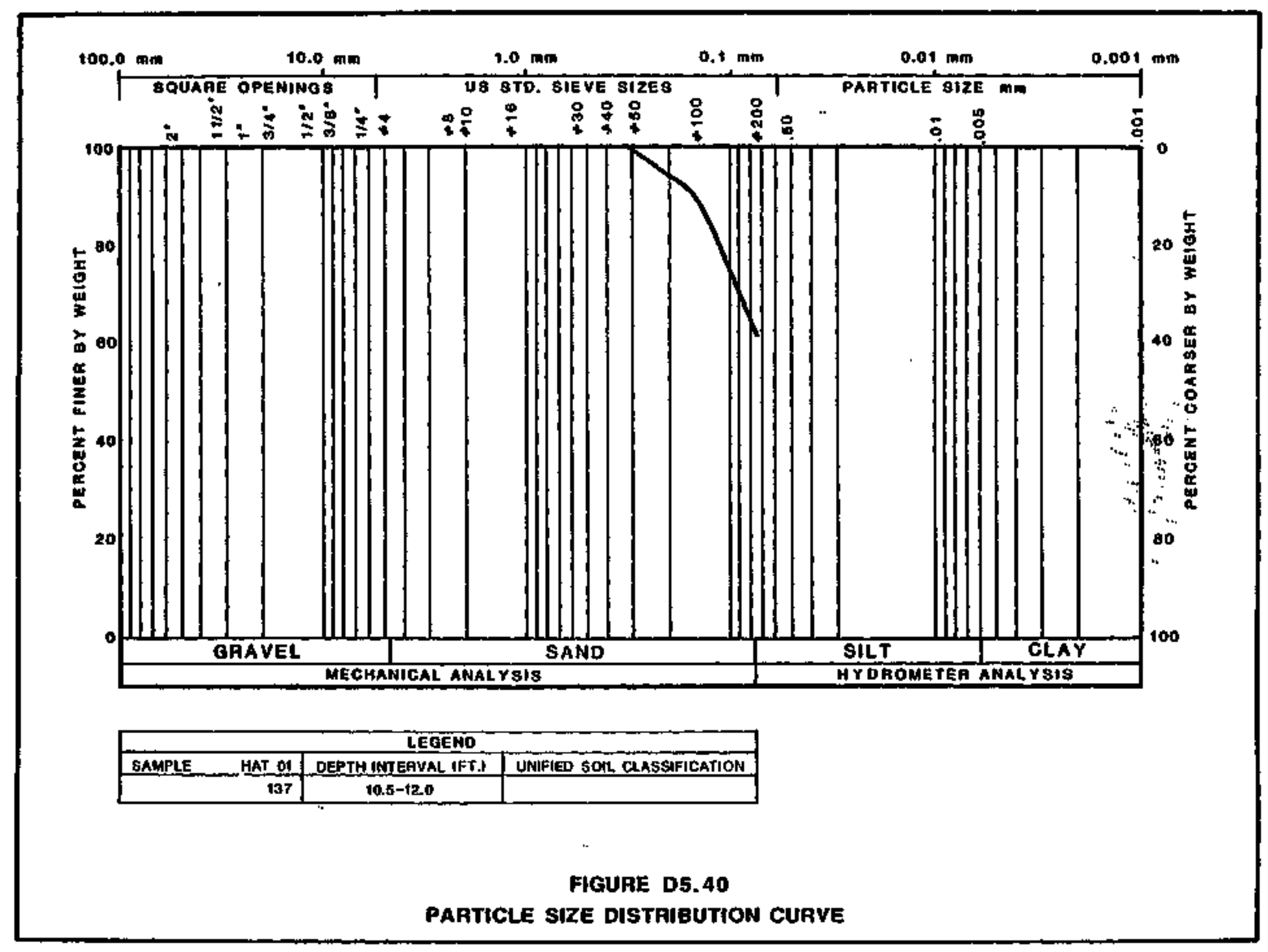




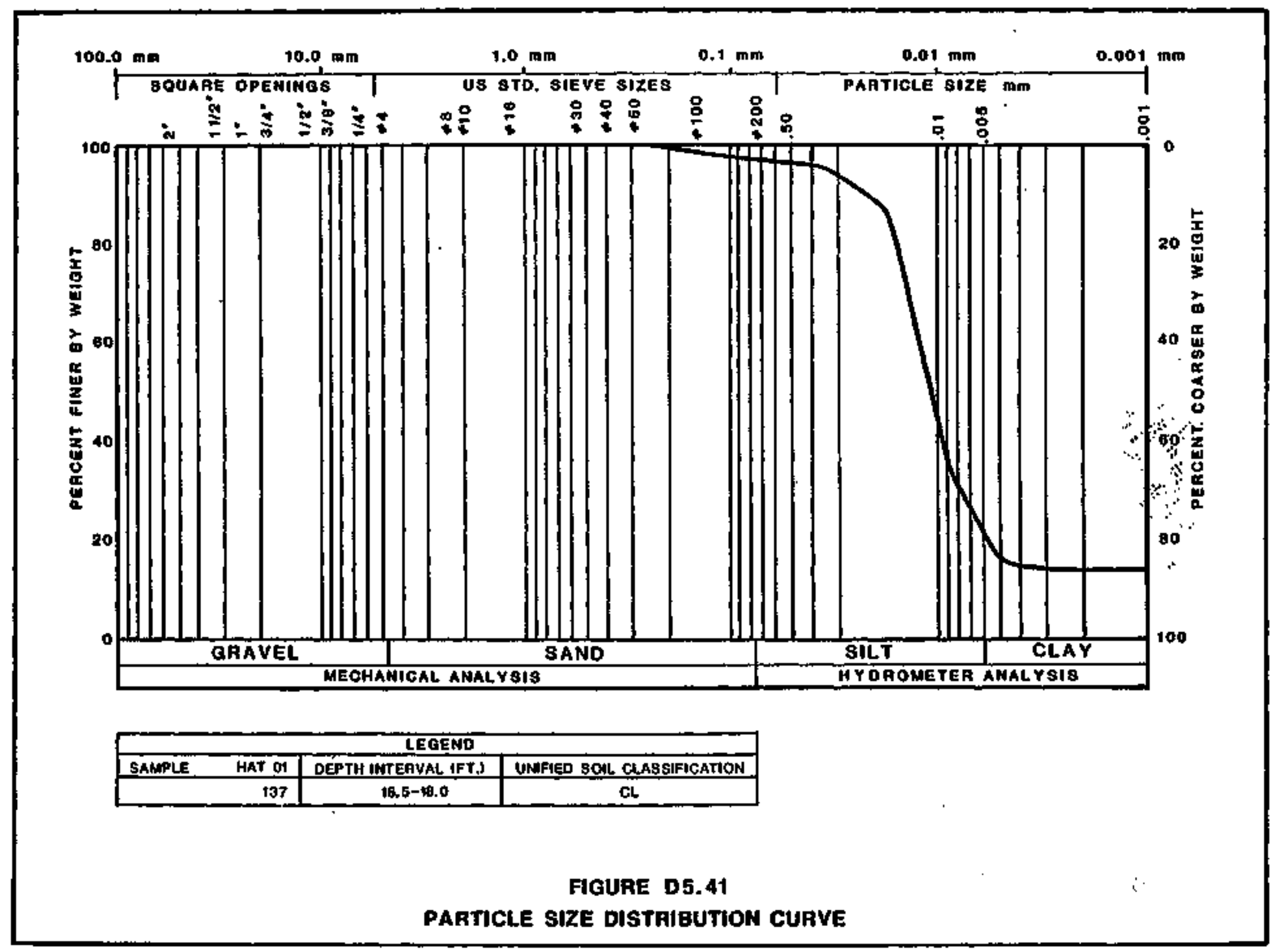




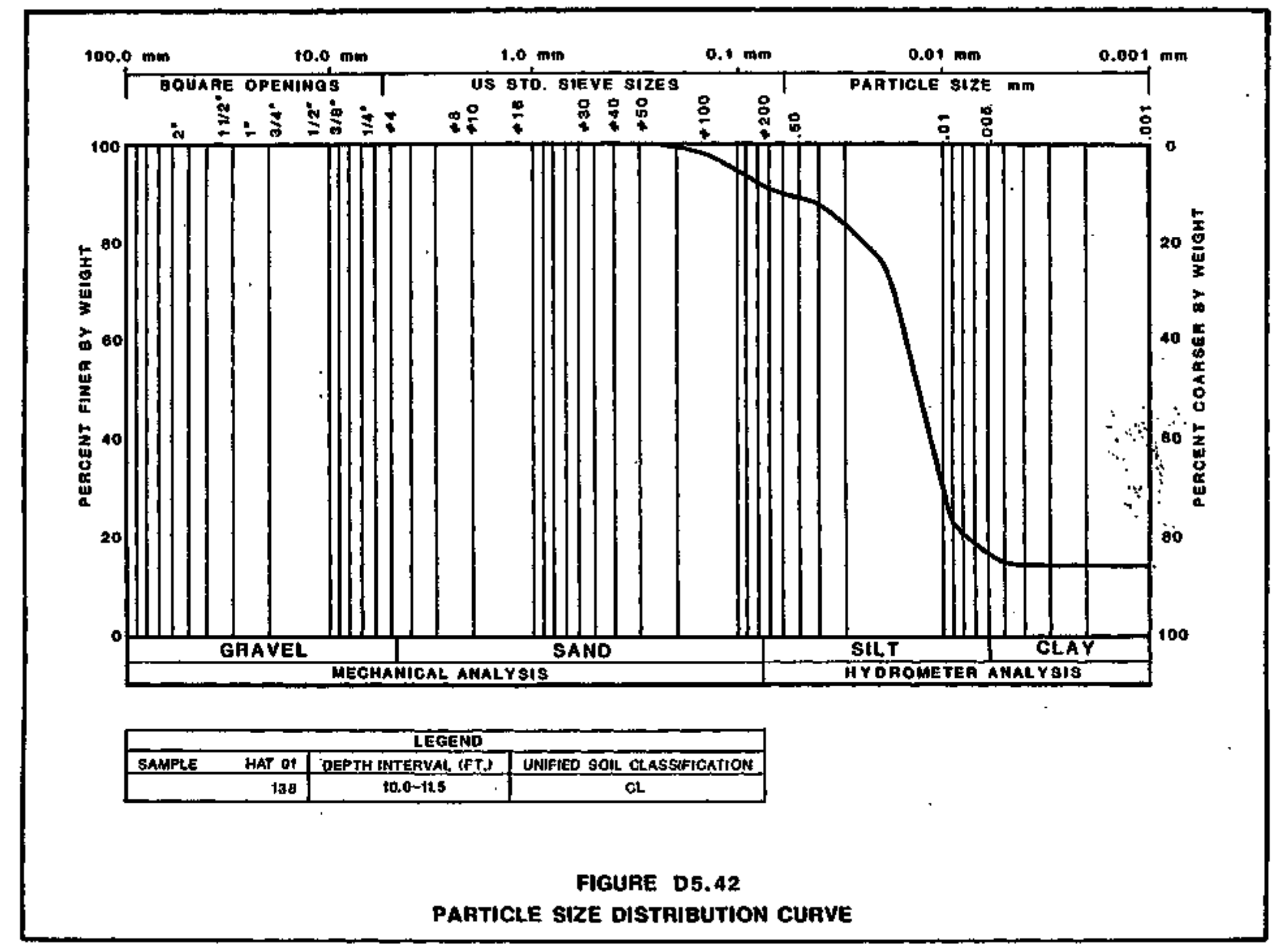




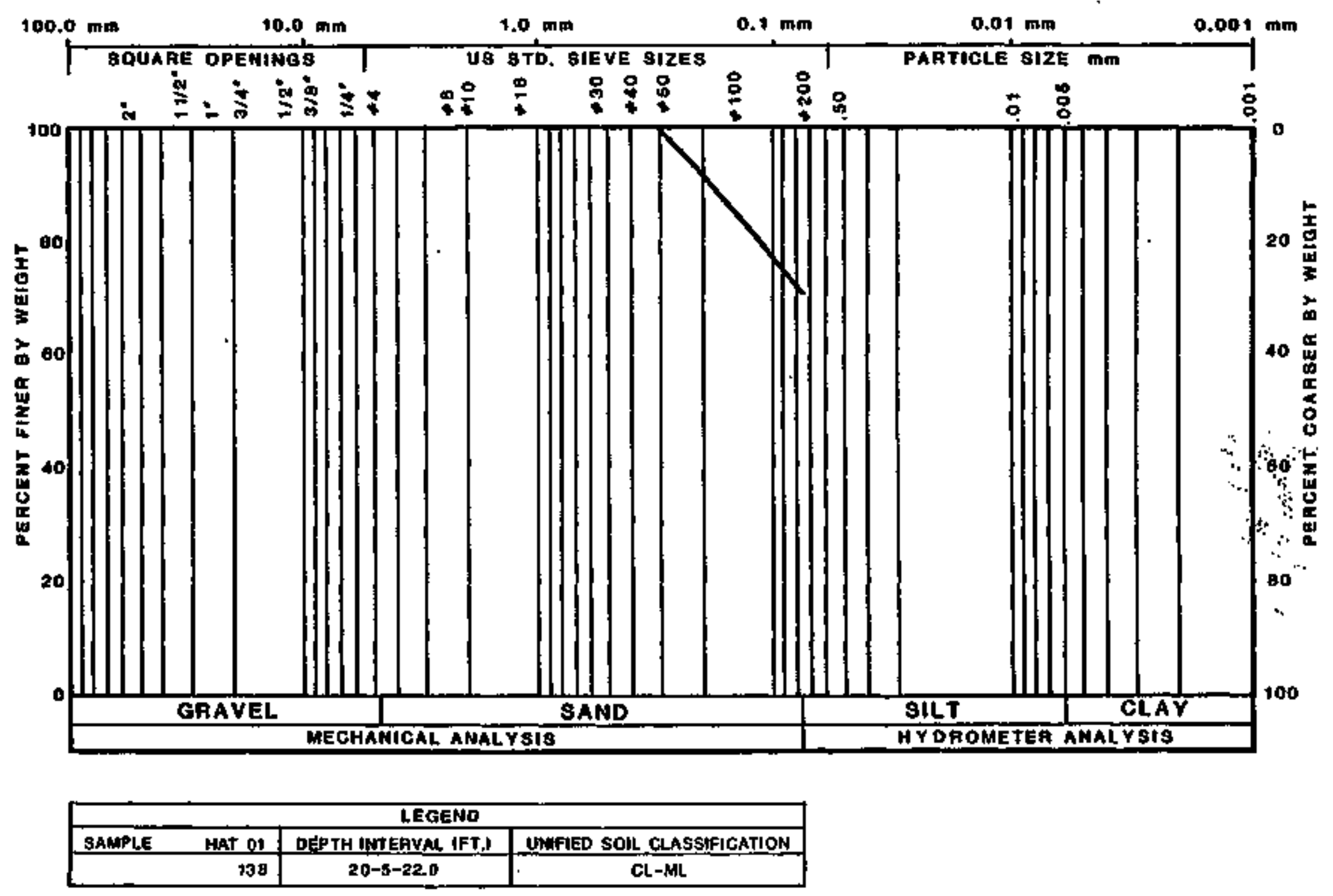

FIGURE D5.43

PARTICLE SIZE DISTAIBUTION CURVE 


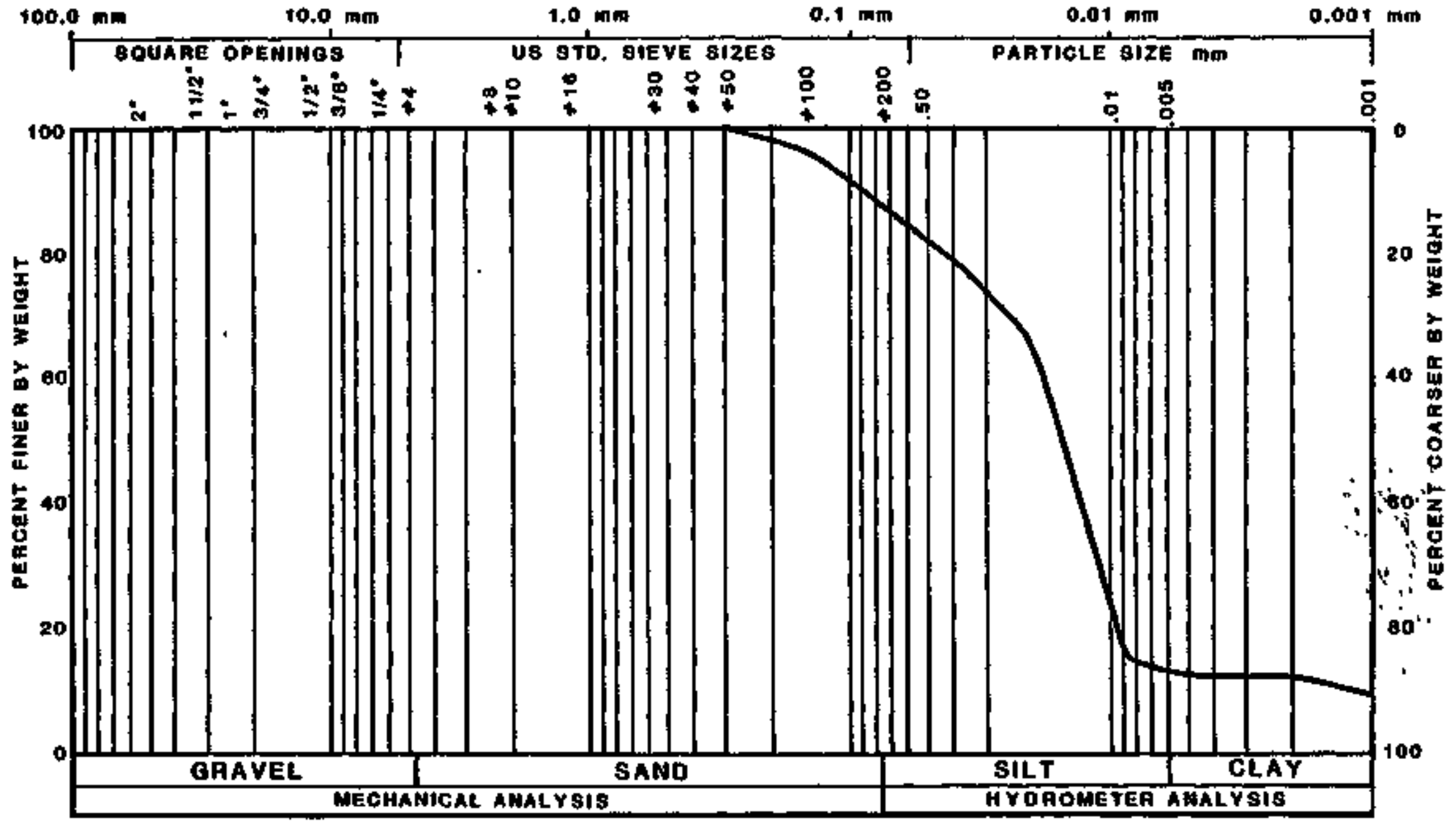

\begin{tabular}{|rr|c|c|}
\hline \multicolumn{3}{|c|}{ LEGEND } \\
\hline SAMPLE & HAT O1 & DEPTH 1NIERVAL IF T.I & UMWFIED SOIL GLASSIFICATION \\
\hline & 138 & $35.0-97.5$ & CL \\
\hline
\end{tabular}

FIGUAE D\$.44

PARTICLE SIZE DISTRIBUTION CURVE 


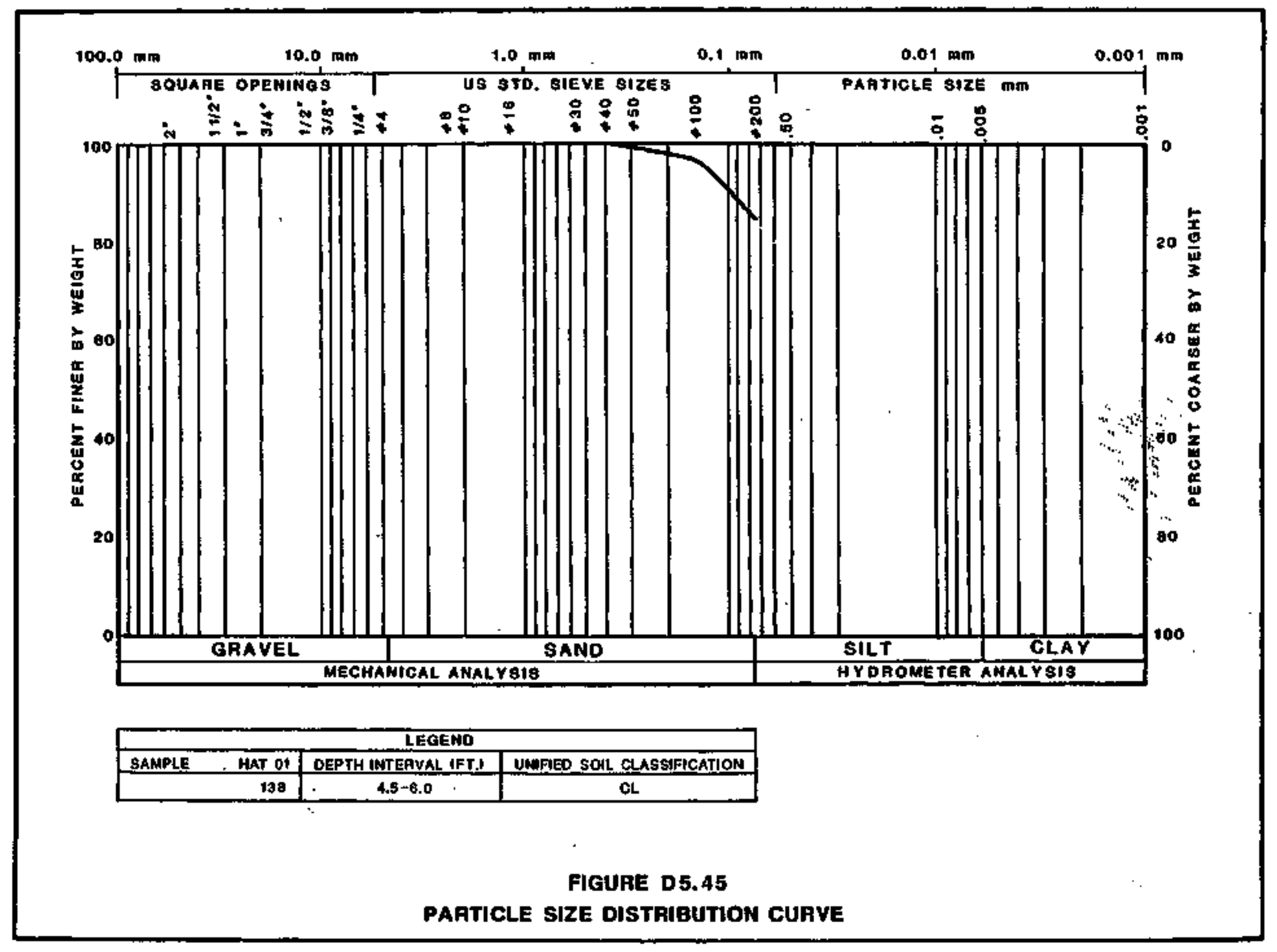




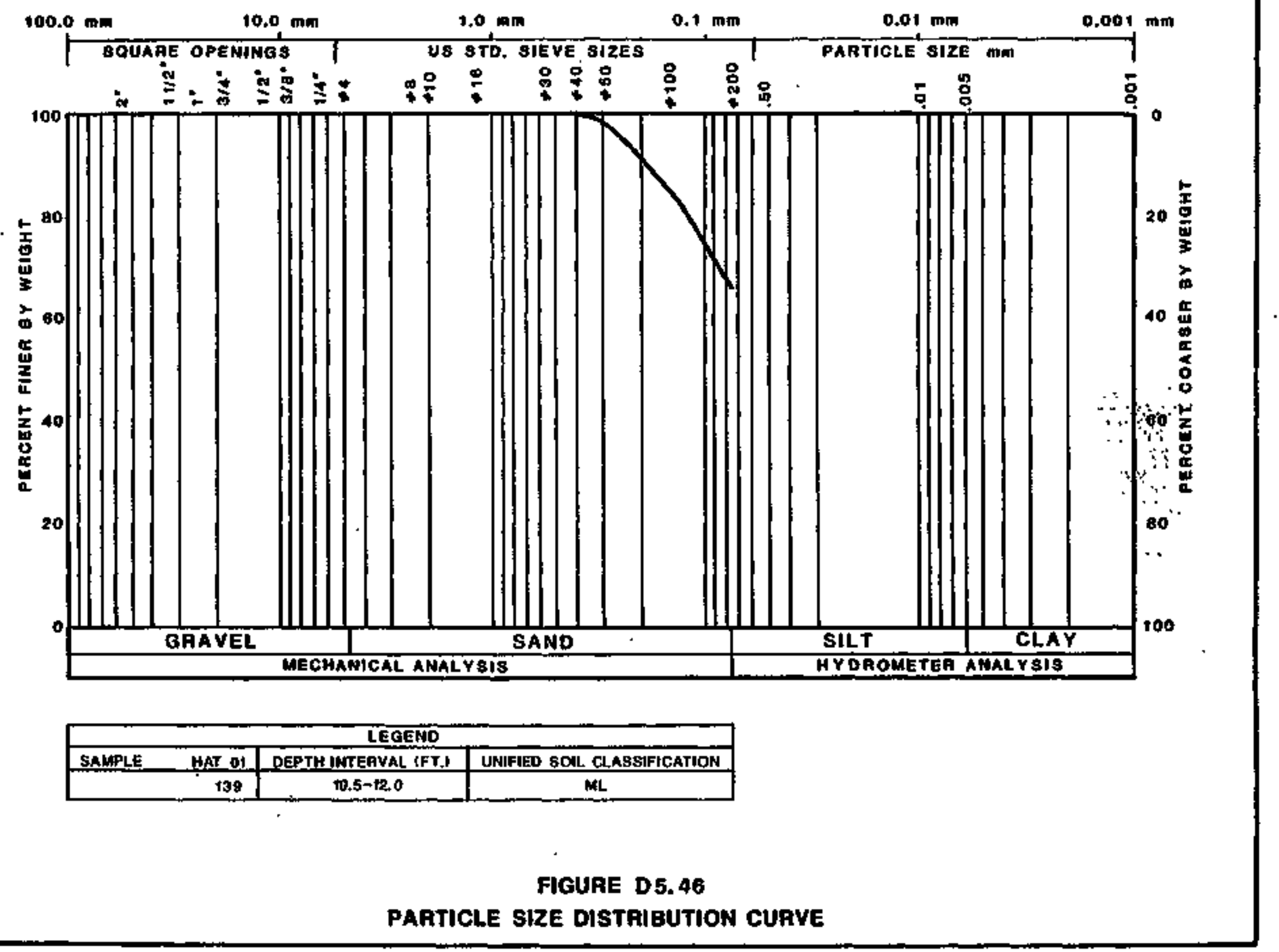




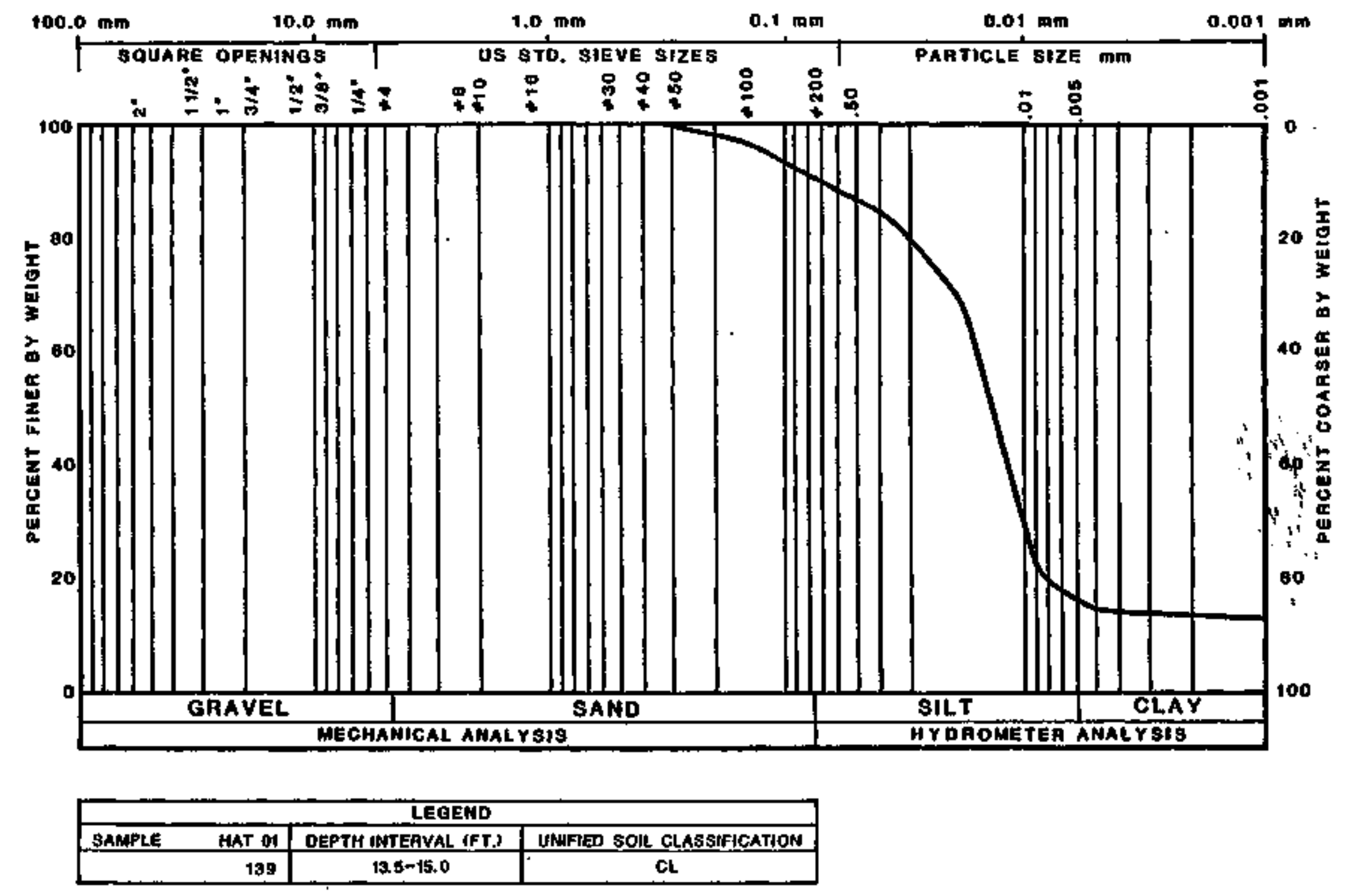

FIGUAE D5.47

PARTICLE SIZE DISTFIBUTION CURVE 


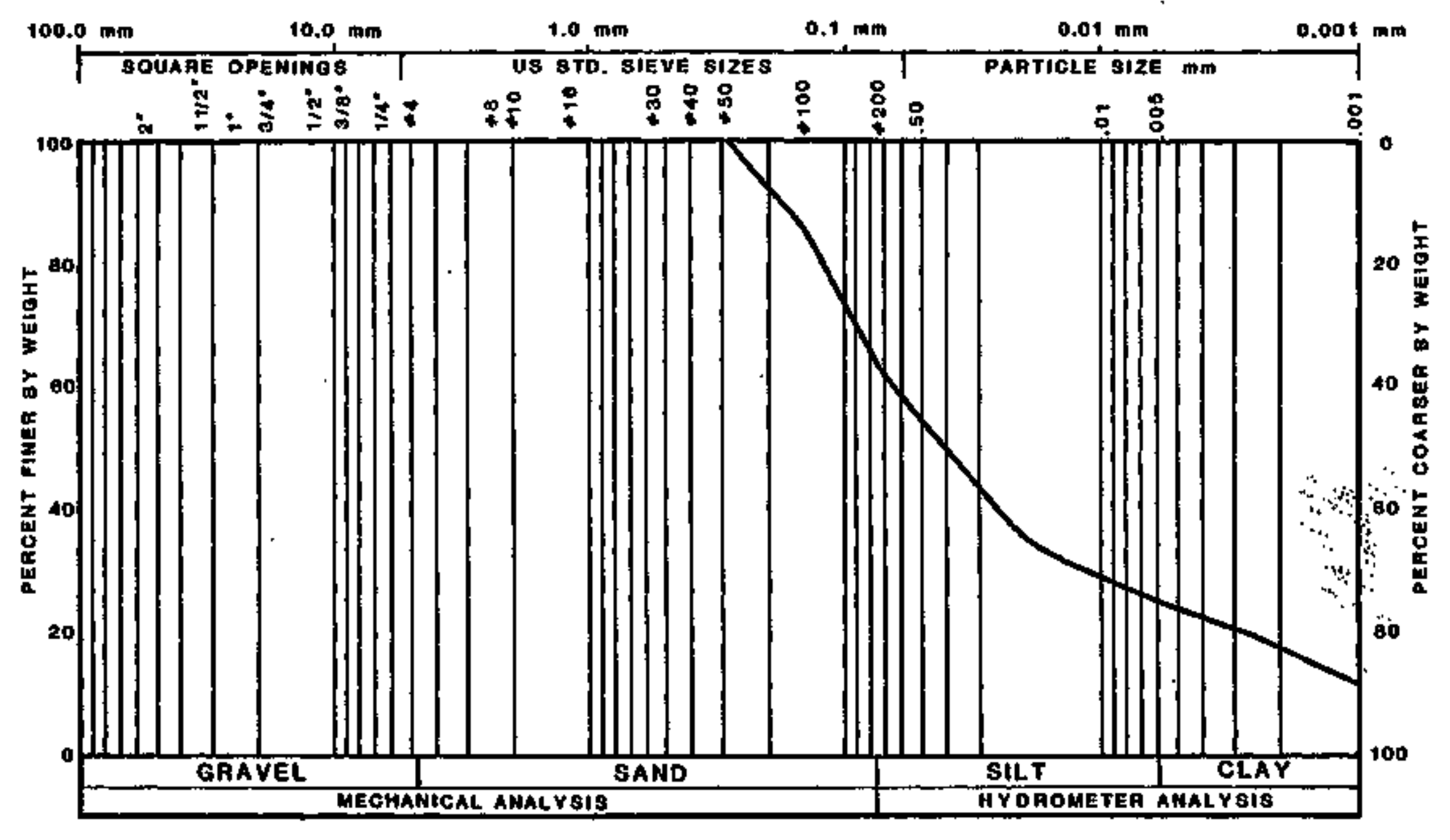

\begin{tabular}{|c|c|c|c|}
\hline \multicolumn{4}{|c|}{ LEGEMO } \\
\hline SAMPPLE & HAT O1 & DEPTH IGTERVAL IFT, & UMFIED GOTL CLASSIFIGATION \\
\hline & 139 & $19.5-22.0$ & ML \\
\hline
\end{tabular}




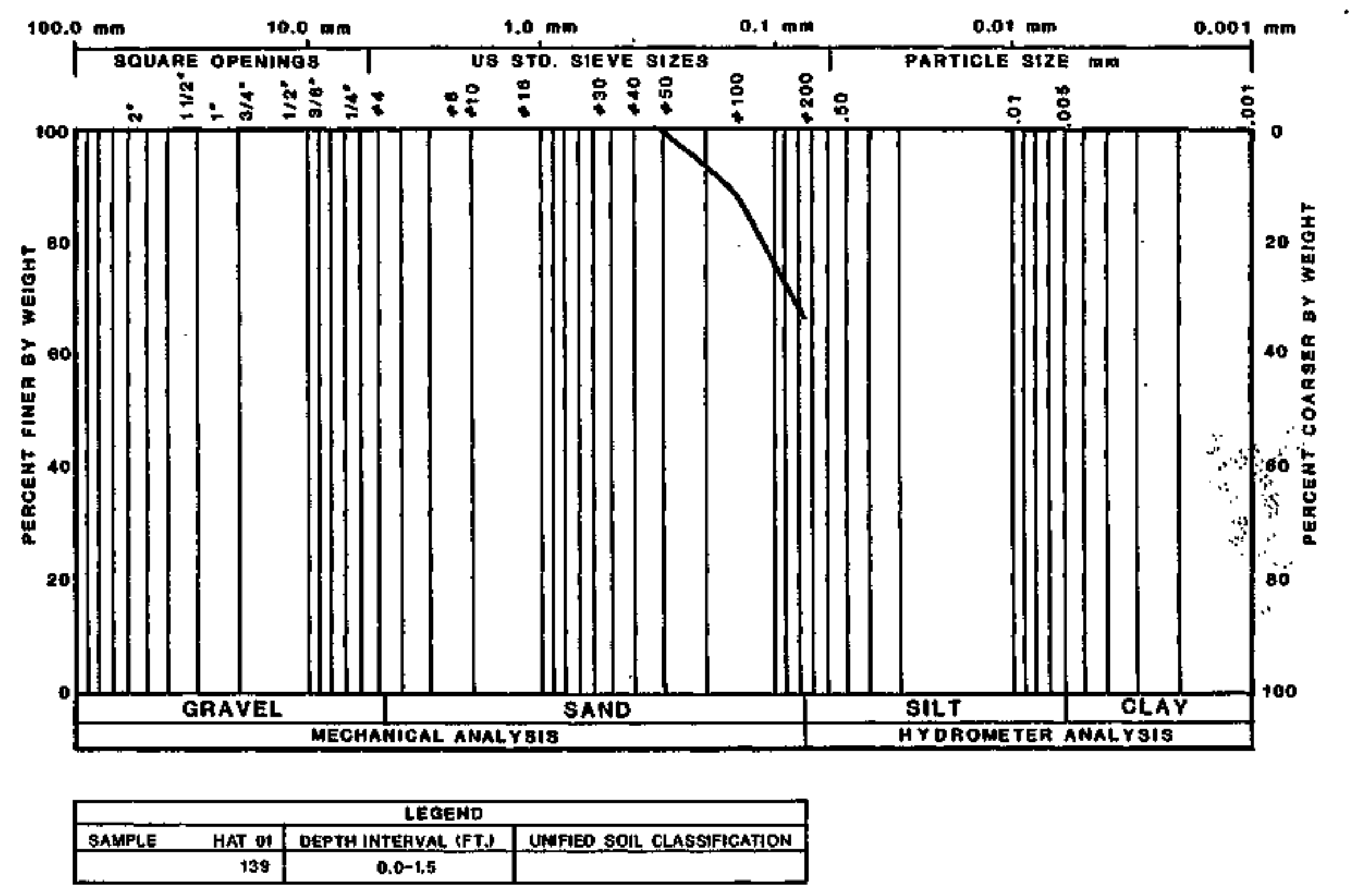

FIGUAE D5.49

PARTICLE SIZE DISTRIBUTION CURVE 

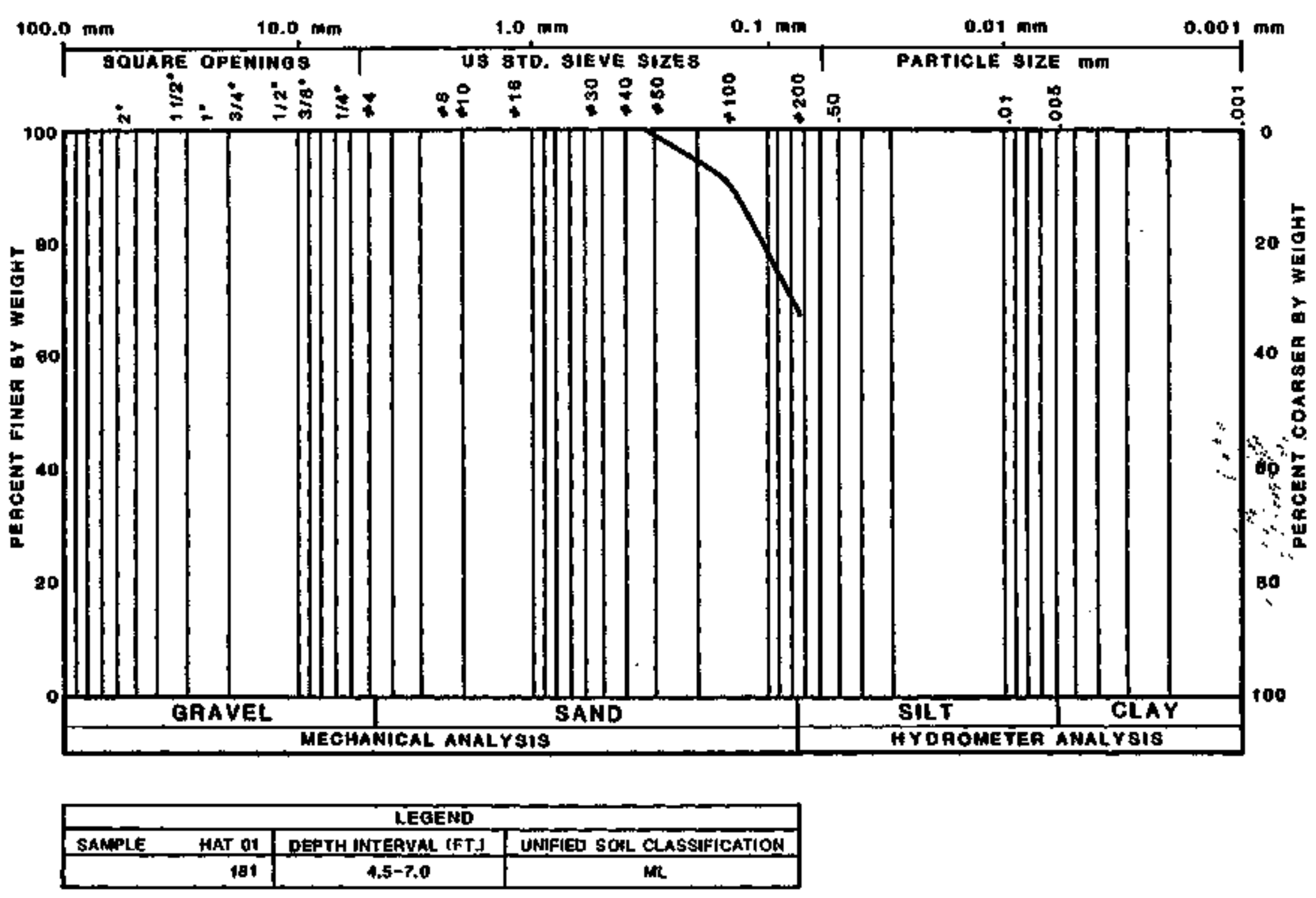

FIGUAE D5.50

PARTICLE SIZE DISTAIBUTION CURYE 


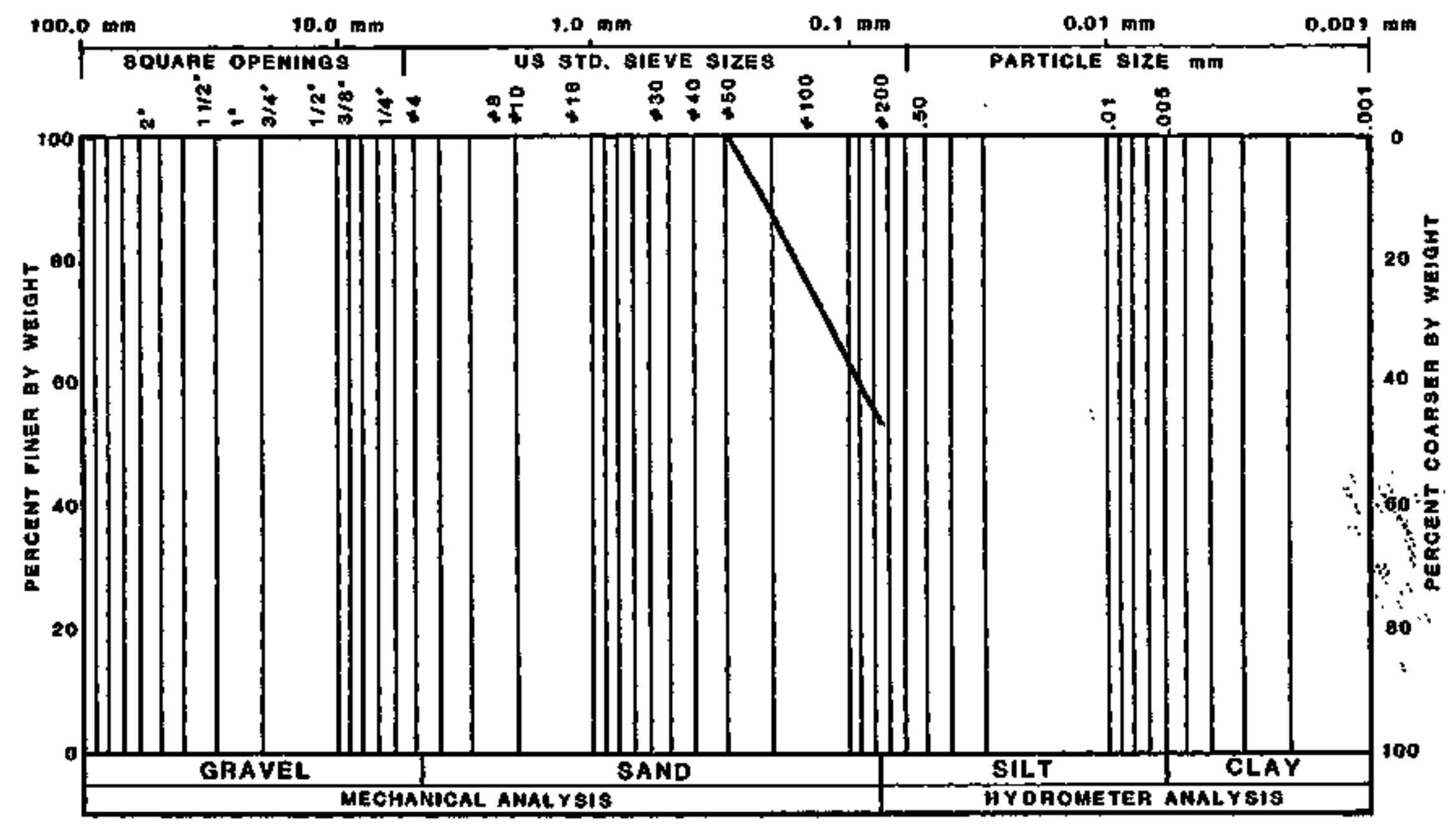

\begin{tabular}{|rr|c|c|}
\hline SAMPLE & HAT O1 & DEPTH INTEAVAL IFT, & UNAFIED 8OAL Q QASSIFICATION \\
\hline & 161 & $0.5-10,0$ & \\
\hline
\end{tabular}

FICURE D5.51

PARTICle size dSTAIBUTION CuAve 

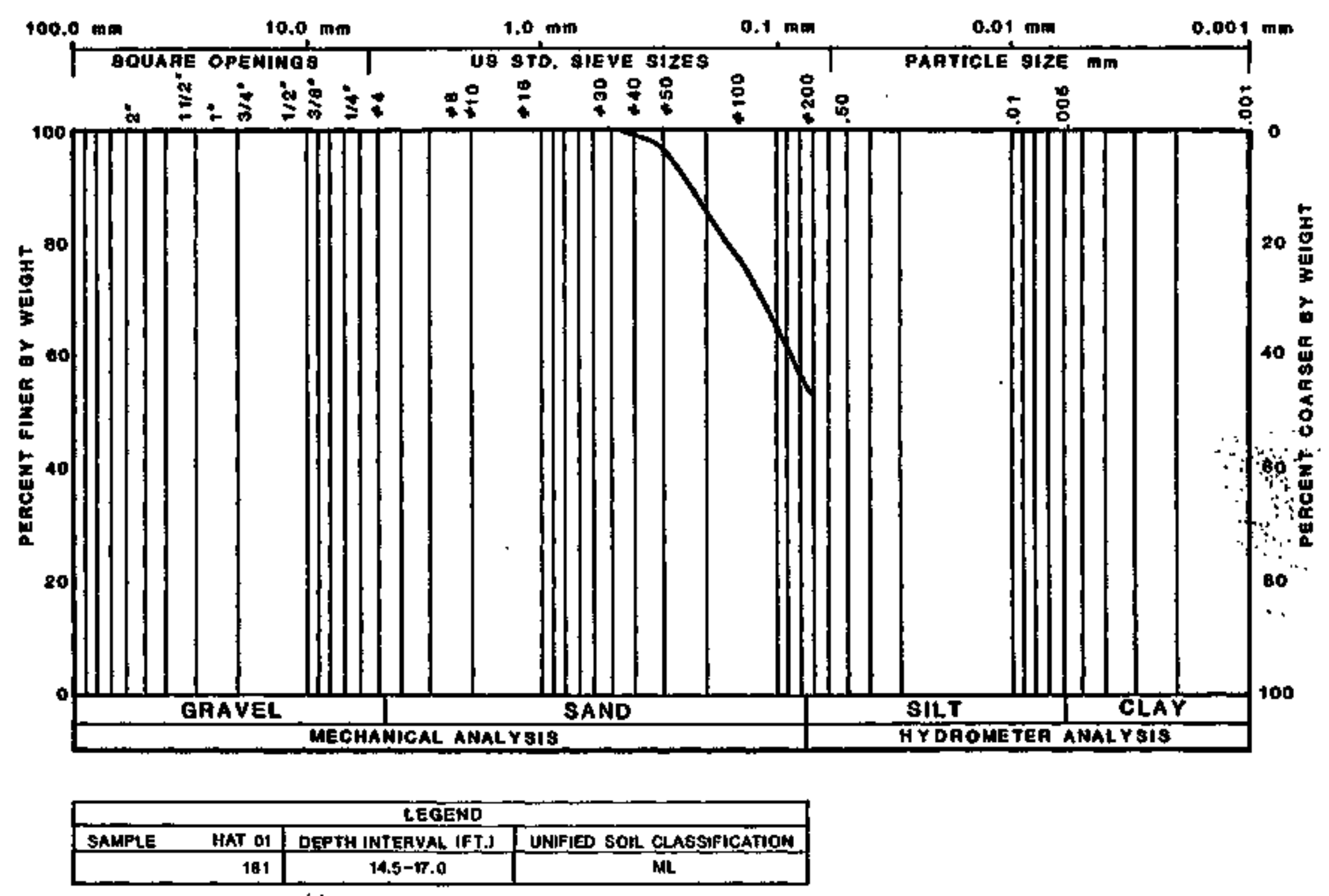

FIGUAE D5.52

PARTICLE SIZE DISTAIBUTION CUAVE 


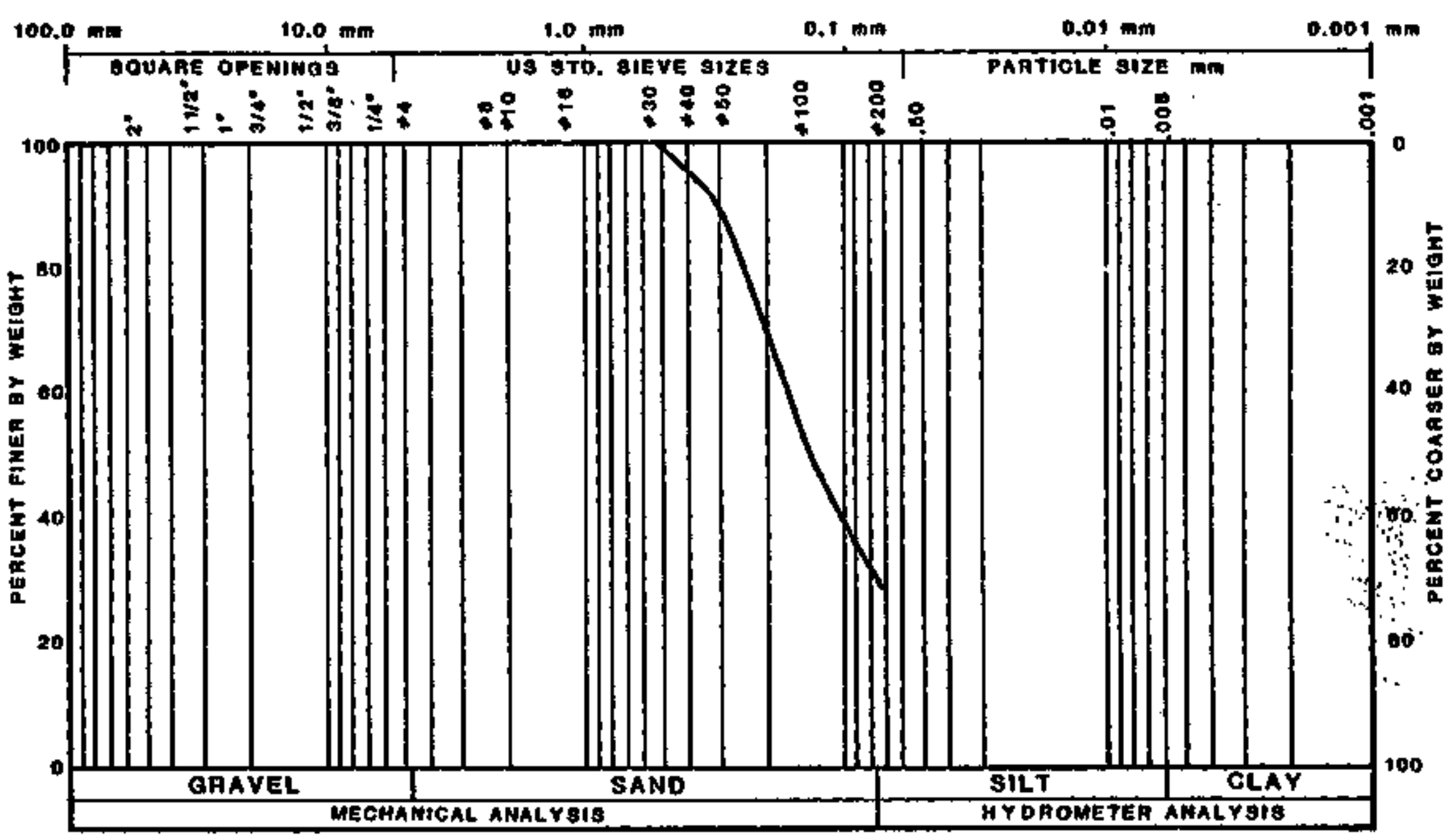

\begin{tabular}{|c|c|c|c|}
\hline \multicolumn{4}{|c|}{ LEGEND } \\
\hline SAMPLE & HAT Ó1 & DEPTH INTEAVAL IFT. & UMMFIED SOL CLASSIFICATION \\
\hline & 101 & $20,0-215$ & Sh" \\
\hline
\end{tabular}

FIGUAE DS.53

PARTICLE SIZE DISTRIBUTION CURVE 


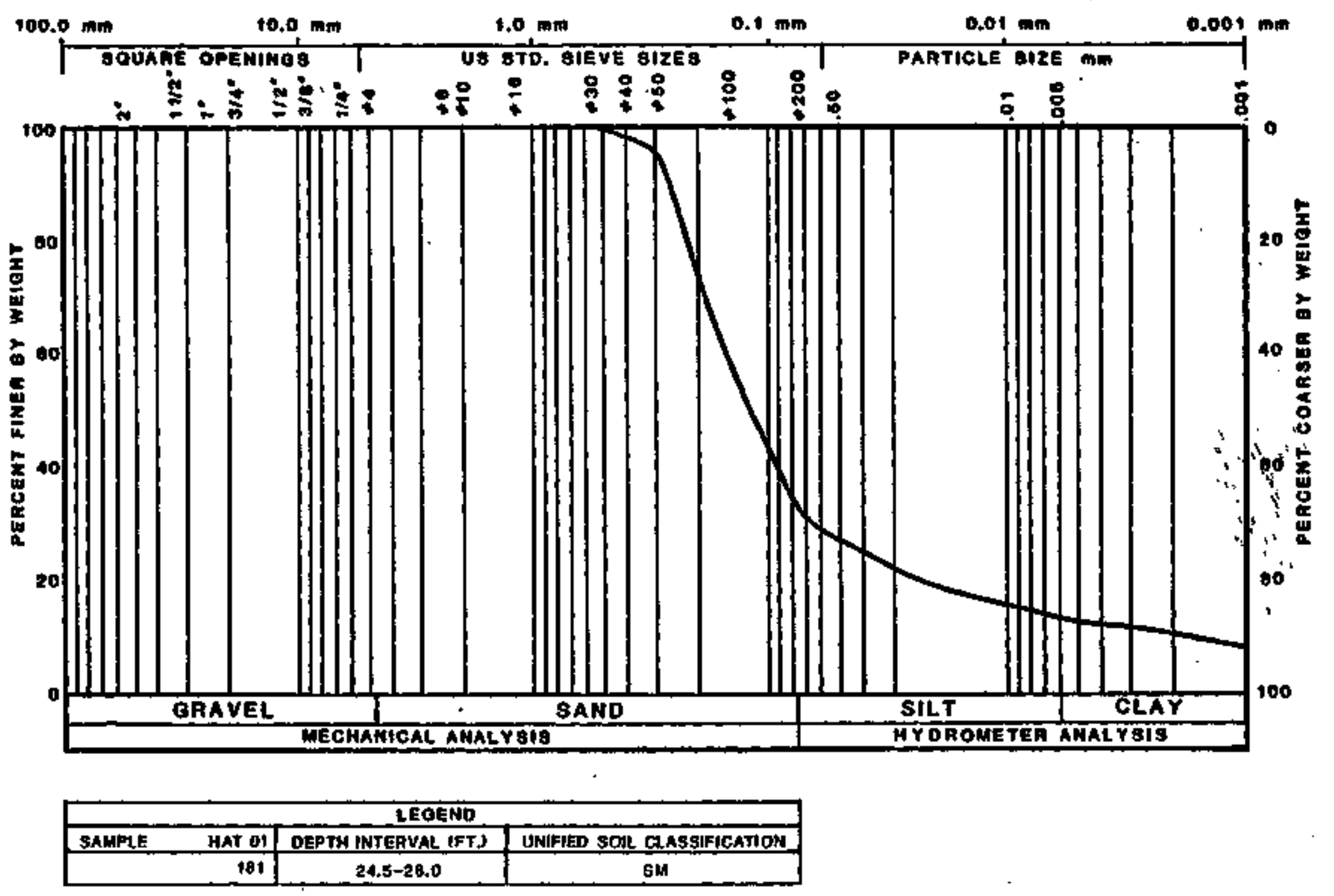

FIGUAE D5.54

PARTICLE SIZE DISTRIBUTION CURVE 
This addendum contains piezocone sounding logs. When referring to the DOE boring and test pit ID numbers. please note that the numbers used in the text refer to the numbering systemas as in the Dof computerized Database Management System (DBMS). The nưmbering systen used on the piezocone logs does not match that used in the text. Therefore, when referring to the following plezocone logs in conjunction with the text of this document it may be necessary to convert the field and laboratory numbers via Table 04.1 in Addendum 04.

\section{Results of piezocone testing}

The following pages contain the results obtained by processing the raw field data from the piezocone soundings with a plezocone data analysis and plotting computer program developed by the TAC.

The program allows the engineer to pick the various soll layer boundaries on a high resolution graphics screen plot of the data. The engineer is aided by a bar-coded, computer-calculated soll classification plotted aiongside the data plots. The tailings classification scheme, as developed through lab test correlations is presented as Figure 06.1.

The resulting graphics containing the data plots, bar-code classification, and designated layer boundarles are presented on the following pages. Following the graphic output are printouts containing various averaged plezocone readings and empirfically correlated soli parameters for each layer and for the entire profile.

\section{Bar code}

White = sand

Gray = sand-slime

Charcoal gray = slimes

Layer numbers in graphics output correspond to those 1isted in printed output that fallow graphics. 


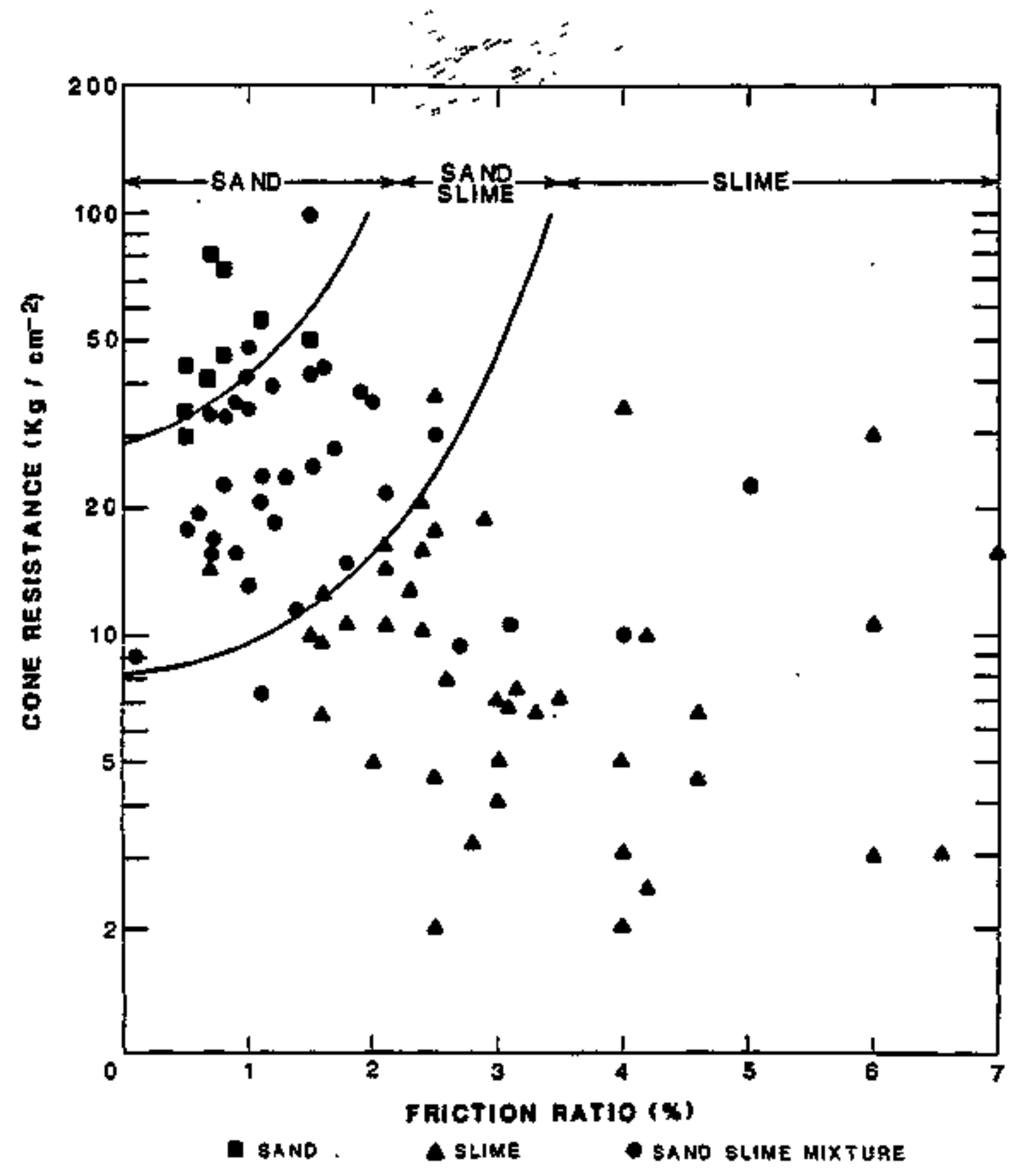

FIGUAE D6.1

PIEZOCONE CLASSIFICATION CHART USED FOR TALLINGS 
Table D6.I Geocheaical analyses of Colorado State Uaiversity borings

\begin{tabular}{|c|c|c|c|c|c|c|c|c|c|c|c|c|c|c|c|}
\hline \multirow[b]{2}{*}{$\begin{array}{l}\text { Dorling } \\
\text { Nurperara }\end{array}$} & \multirow{2}{*}{$\begin{array}{l}\text { Sanple } \\
\text { deptin } \\
\text { (feet) }\end{array}$} & & \multirow[b]{2}{*}{ DA } & \multirow{2}{*}{$\begin{array}{c}\text { Electrical } \\
\text { conductivity } \\
\text { (minos/cent imeter) }\end{array}$} & \multirow{2}{*}{\multicolumn{2}{|c|}{ 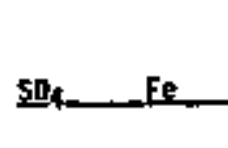 }} & \multicolumn{3}{|c|}{ Canst ituent $b$} & \multirow[b]{2}{*}{$\mathrm{cl}$} & \multirow[b]{2}{*}{ hl } & \multirow{2}{*}{\multicolumn{2}{|c|}{ 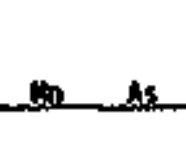 }} & \multirow[b]{2}{*}{ Se } & \multirow{2}{*}{$\begin{array}{c}\text { Cacoz } \\
\text { culvaleat. } \\
\text { (percent) }\end{array}$} \\
\hline & & & & & & & Ca. & 2010 & N. 스. & & & & & & \\
\hline 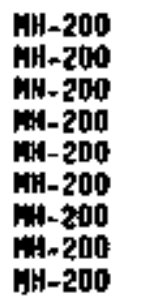 & $\begin{array}{r}2.5 \\
3.0 \\
3.5 \\
4.0 \\
4.0 \\
6.0 \\
10.0 \\
15.0 \\
19.0\end{array}$ & & $\begin{array}{l}0.1 \\
6.0 \\
0.7 \\
0.0 \\
0.2 \\
8.6 \\
0.6 \\
8.6 \\
0.5\end{array}$ & $\begin{array}{l}1.0 \\
1.0 \\
2.0 \\
1.9 \\
0.2 \\
0.1 \\
0.2 \\
0.1 \\
0.1\end{array}$ & $\begin{array}{r}3650 \\
3140 \\
4240 \\
3970 \\
200 \\
111 \\
270 \\
67 \\
130\end{array}$ & $\begin{array}{r}158 \\
130 \\
128 \\
110 \\
116 \\
64 \\
115 \\
106 \\
62 .\end{array}$ & $\begin{array}{l}2660 \\
2500 \\
4260 \\
33190 \\
222 \\
131 \\
211 \\
114 \\
155\end{array}$ & $\begin{array}{l}762 \\
507 \\
473 \\
373 \\
29 \\
21 \\
53 \\
30 \\
35\end{array}$ & $\begin{array}{r}323 \\
118 \\
161 \\
69 \\
30 \\
16 \\
41 \\
49 \\
29\end{array}$ & $\begin{array}{r}130 \\
60 \\
60 \\
40 \\
272 \\
20 \\
41 \\
80 \\
40\end{array}$ & $\begin{array}{l}612 \\
776 \\
615 \\
791 \\
288 \\
363 \\
336 \\
348 \\
248\end{array}$ & $\begin{array}{l}252 \\
224 \\
183 \\
159 \\
3 \% 0 \\
296 \\
191 \\
139 \\
224\end{array}$ & $\begin{array}{l}1 \\
4 \\
4 \\
4 \\
3 \\
2 \\
3 \\
3 \\
2\end{array}$ & $\begin{array}{l}3 \\
2 \\
1 \\
1 \\
5 \\
1 \\
4 \\
3\end{array}$ & $\begin{array}{l}10.4 \\
21.0 \\
19.3 \\
21.8 \\
35.0 \\
17.5 \\
36.0 \\
49.8 \\
10.7\end{array}$ \\
\hline $\begin{array}{l}m-205 \\
m H-205 \\
m-205 \\
m-205 \\
m-205 \\
m-205 \\
m-205 \\
m-205 \\
m-205\end{array}$ & $\begin{array}{r}5.5 \\
6.0 \\
7.0 \\
10.0 \\
12.5 \\
17.0 \\
22.0 \\
25.5\end{array}$ & $\cdot$ & $\begin{array}{l}0.5 \\
0.3 \\
9.5 \\
7.9 \\
6.7 \\
6.4 \\
6.3 \\
0.5\end{array}$ & $\begin{array}{l}0.2 \\
0.8 \\
0.2 \\
1.7 \\
0.2 \\
0.1 \\
0.2 \\
0.1\end{array}$ & $\begin{array}{r}173 \\
160 \\
190 \\
3050 \\
300 \\
150 \\
170 \\
155\end{array}$ & $\begin{array}{l}112 \\
215 \\
149 \\
96 \\
78 \\
67 \\
38 \\
61\end{array}$ & $\begin{array}{r}161 \\
1460 \\
149 \\
2110 \\
105 \\
131 \\
53 \\
130\end{array}$ & $\begin{array}{r}65 \\
165 \\
55 \\
541 \\
60 \\
44 \\
23 \\
37\end{array}$ & $\begin{array}{r}35 \\
50 \\
81 \\
268 \\
89 \\
35 \\
300 \\
30\end{array}$ & $\begin{array}{l}20 \\
<20 \\
20 \\
20 \\
30 \\
20 \\
30 \\
20\end{array}$ & $\begin{array}{l}278 \\
325 \\
306 \\
931 \\
609 \\
276 \\
671 \\
277\end{array}$ & $\begin{array}{r}222 \\
610 \\
228 \\
194 \\
69 \\
237 \\
45 \\
193\end{array}$ & $\begin{array}{r}3 \\
2 \\
3 \\
2 \\
1 \\
1 \\
1 \\
13\end{array}$ & $\begin{array}{r}5 \\
3 \\
2 \\
41 \\
1 \\
4 \\
3 \\
<1\end{array}$ & 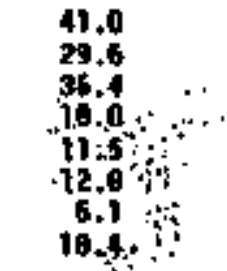 \\
\hline 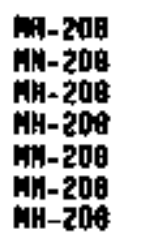 & $\begin{array}{l}27.0 \\
27.5 \\
30.2 \\
36.0 \\
30.0 \\
43.0 \\
49.0\end{array}$ & & $\begin{array}{l}6.4 \\
6.0 \\
6.5 \\
.4 .4 \\
9.1 \\
8.6 \\
6.1\end{array}$ & $\begin{array}{l}2.0 \\
0.2 \\
0.7 \\
0.7 \\
0.2 \\
0.1 \\
0.2\end{array}$ & $\begin{array}{r}5070 \\
348 \\
90 \\
99 \\
370 \\
61 \\
120\end{array}$ & $\begin{array}{r}430 \\
304 \\
130 \\
93 \\
74 \\
119 \\
52\end{array}$ & $\begin{array}{r}5470 \\
340 \\
131 \\
116 \\
699 \\
111 \\
124\end{array}$ & $\begin{array}{l}177 \\
47 \\
26 \\
92 \\
91 \\
32 \\
47\end{array}$ & $\begin{array}{l}96 \\
22 \\
24 \\
31 \\
72 \\
35 \\
30\end{array}$ & $\begin{array}{l}<20 \\
20 \\
<0 \\
<20 \\
20 \\
<20\end{array}$ & $\begin{array}{l}549 \\
253 \\
263 \\
260 \\
945 \\
342 \\
671\end{array}$ & $\begin{array}{l}12 \\
211 \\
231 \\
200 \\
120 \\
202 \\
103\end{array}$ & $\begin{array}{r}13 \\
4 \\
3 \\
2 \\
2 \\
3\end{array}$ & $\begin{array}{r}3 \\
6 \\
6 \\
4 \\
2 \\
41\end{array}$ & $\begin{array}{c}0.64: . \\
34.4 \\
32.8 \\
31.4 \\
9.8 \\
29.8 \\
10.0\end{array}$ \\
\hline
\end{tabular}

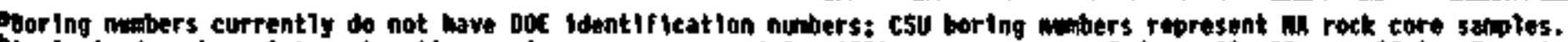

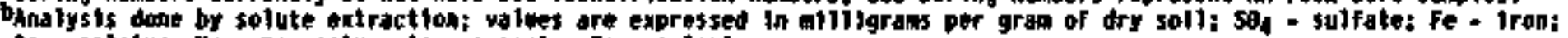

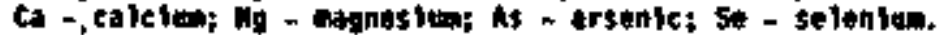


SITE ID: HATE 1

GRDUND WATER DEPTH: 50.0 (FT.)
LOCATION ID: 006

AUERAGE SOIL WEIGHT: 100.0 (PSF)

AUG. AUG.

- DEPTH - AUG. AUG. PORE FRICT. SOIL SHEAR

LY FROM TO THK. POINT SLEEUE PRESS. RATIO CLASS BC DEN FA ELAS. STREN DCR

$\begin{array}{rrrrrrrrrrrrrr}1 & 0.1 & 3.0 & 2.9 & 16.8 & 0.1 & -0.0 & 0.5 & \text { S-SL* } & 5 & 40 & 30 & 59 & 2014 \\ 2 & 3.0 & 5.1 & 2.1 & 6.5 & 0.2 & -0.1 & 3.0 \text { SLIM } & 4 & & & 23 & 778 & 6.5 \\ 3 & 5.1 & 9.1 & 4.0 & 21.0 & 0.2 & -0.0 & 1.2 & \text { S-SL* } & 7 & 45 & 31 & 74 & 2520 \\ 4 & 9.2 & 11.8 & 2.6 & 12.6 & 0.1 & -0.0 & 1.15-5 L * & 4 & 27 & 29 & 44 & 1518 & \\ 5 & 11.8 & 19.2 & 7.3 & 7.1 & 0.2 & 0.5 & 3.1 \text { SLIM } & 4 & & & 25 & 857 & 1.8\end{array}$

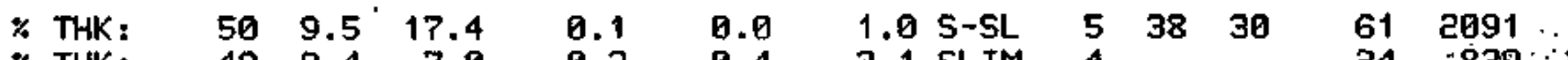

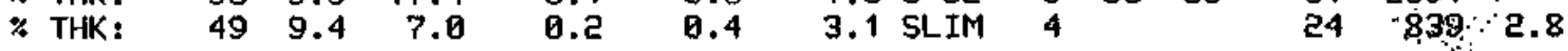


SITE ID: HATO1

GROUND UATER DEPTH: 50.0 (FT.)
LOCATION ID: 00 ?

AUERAGE SOIL WEIGHT: 100.0 (PSF)

AUG. AUG. UND.

- DEPTH - AUG. AUG. PORE FRICT. SOIL SHEAR

LY FROM TO THK. POINT SLEEUE PRESS. RATIO CLASS BC DEN FA ELAS. STREN OCR

$\begin{array}{lllllllllllll}1 & 0.1 & 1.0 & 1.0 & 9.9 & 0.2 & 0.0 & 2.0 & \text { SLIM } & 6 & 34 & 1182 & 39.5\end{array}$

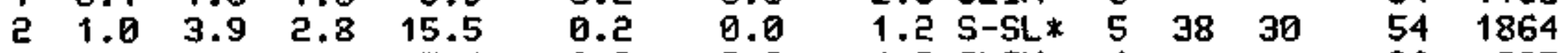

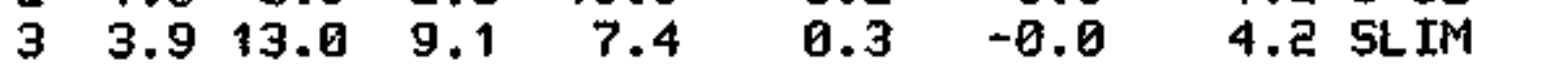

$\begin{array}{lll}26 & 887 & 3.8\end{array}$

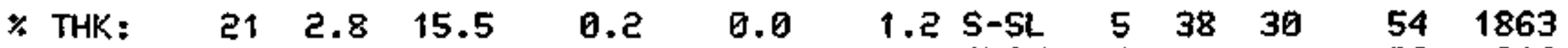

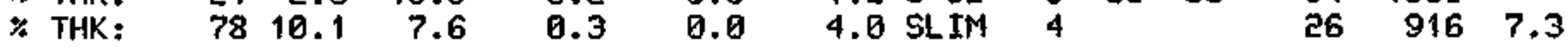


SITE ID : HATO1

GRDUND WATER DEPTH: 50.0 (FT.)
LDCATION ID: 008

AUERAGE SOIL WEIGHT: 100.0 (PSF)

AUG. AUG. UND.

- DEPTH - AUG. AUG. PORE FRICT. SOIL SHEAR LY FROM TO THK. POINT SLEEUE PRESS. RATIO CLASS BC DEN FA ELAS. STREN DCR

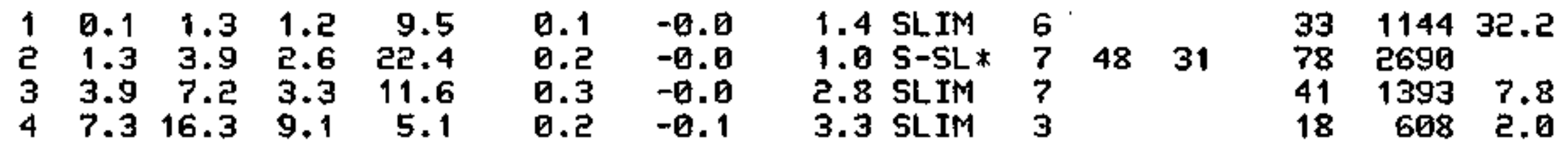

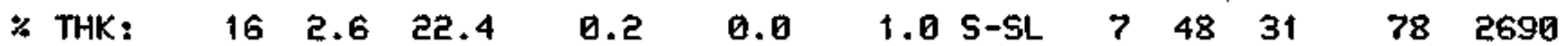

\% THK: $8313.6 \quad 7.1 \quad 0.2 \quad-0.1 \quad 3.0$ SLIM $4 \quad 6.1$ 
SITE ID: HATO1

GROUND WATER OEPTH: 50.0 (FT.)
LOCATION ID: 099

AUERAGE SOIL WEIGHT: 100.0 (PSF)

AUG. AUG. UND.

- DEPTH - AUG. AUG. PORE FRICT. SOIL SHEAR

LY FROM TO THK. POINT SLEEVE PRESS. RATIO CLASS BC DEN FA ELAS. STREN OCR

\begin{tabular}{|c|c|c|c|c|c|c|c|c|c|c|c|c|c|c|}
\hline $\begin{array}{l}1 \\
2 \\
3 \\
4\end{array}$ & $\begin{array}{l}0.1 \\
3.4 \\
4.9 \\
6.1\end{array}$ & $\begin{array}{l}3.4 \\
4.8 \\
6.0 \\
6.5\end{array}$ & $\begin{array}{l}3.3 \\
1.4 \\
1.2 \\
0.4\end{array}$ & $\begin{array}{r}18.2 \\
9.9 \\
33.2 \\
12.9\end{array}$ & $\begin{array}{l}0.1 \\
0.3 \\
0.2 \\
0.3\end{array}$ & $\begin{array}{r}-0.0 \\
0.0 \\
0.1 \\
0.1\end{array}$ & $\begin{array}{l}0.7 \\
2.8 \\
0.7 \\
2.1\end{array}$ & $\begin{array}{l}\text { S-SL* } \\
\text { SLIM } \\
\text { S-SL* } \\
\text { SLIM }\end{array}$ & $\begin{array}{r}6 \\
6 \\
11 \\
8\end{array}$ & $\begin{array}{l}42 \\
60\end{array}$ & 33 & $\begin{array}{r}64 \\
35 \\
116 \\
45\end{array}$ & $\begin{array}{l}2183 \\
1194 \\
3980 \\
1553\end{array}$ & \\
\hline
\end{tabular}

$\begin{array}{lllllllllllll}* \text { THK: } & 71 & 4.5 & 22.2 & 0.1 & 0.0 & 0.7 & 5-5 L & 7 & 46 & 31 & 77 & 2662\end{array}$

* THK: $\quad \begin{array}{lllllllll}28 & 1.8 & 10.6 & 0.3 & 0.0 & 2.6 & \text { SLIM } & 6 & \end{array}$

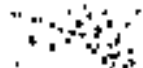


SITE ID: HATO1

GROUND WATER DEPTH: 50.0 (FT.)
LOCATION ID: 010

AUERAGE SOIL WEIGHT: 100.0 (PSF)

- DEPTH - AUG. AUG. PORE FRICT. SOIL

UND.

SHEAR

LY FROM TO THK. POINT SLEEUE PRESS. RATIO CLASS BC DEN FA ELAS, STREN OCR

\begin{tabular}{|c|c|c|c|c|c|c|c|c|c|c|c|c|}
\hline $\begin{array}{ll}1 & 0.1 \\
2 & 2.0\end{array}$ & $\begin{array}{l}2.0 \\
3.6\end{array}$ & $\begin{array}{l}1.9 \\
1.6\end{array}$ & $\begin{array}{r}14.2 \\
7.0\end{array}$ & $\begin{array}{l}0.1 \\
0.1\end{array}$ & $\begin{array}{l}-0.1 \\
-0.0\end{array}$ & $\begin{array}{ll}0.5 & 5-S L * \\
1.6 & 5 L I M\end{array}$ & $\begin{array}{l}4 \\
4\end{array}$ & 35 & 29 & $\begin{array}{l}50 \\
25\end{array}$ & $\begin{array}{r}1701 \\
842\end{array}$ & 9.0 \\
\hline $\begin{array}{l}\text { * THK: } \\
\text { \% THK: }\end{array}$ & $\begin{array}{l}54 \\
45\end{array}$ & $\begin{array}{l}1.9 \\
1.6\end{array}$ & $\begin{array}{r}14.2 \\
7.0\end{array}$ & $\begin{array}{l}0.1 \\
0.1\end{array}$ & $\begin{array}{r}-0.1 \\
0.0\end{array}$ & $\begin{array}{ll}0.5 & 5-S L \\
1.6 & 5 L I M\end{array}$ & $\begin{array}{l}4 \\
4\end{array}$ & 35 & 29 & $\begin{array}{l}50 \\
25\end{array}$ & $\begin{array}{r}1701 \\
842 .\end{array}$ & 9.0 \\
\hline
\end{tabular}


SITE ID: HATE1

GROUND WATER DEPTH: 50.0 (FT.)
LOCATION ID: 011

AUERAGE SOIL WEIGHT: 100.0 (PSF)

- DEPTH - AUG. AUG. PURE FRICT. SOIL $\quad . \quad$ UND. LY FROM TO THK. POINT SLEEUE PRESS. RATIO CLASS BC DEN FA ELAS. STREN OCR

\begin{tabular}{|c|c|c|c|c|c|c|c|c|c|c|c|}
\hline $\begin{array}{rr}1 & 0.1 \\
2 & 0.8 \\
3 & 2.5 \\
4 & 9.9 \\
5 & 10.8 \\
6 & 12.3\end{array}$ & $\begin{array}{r}0.8 \\
2.5 \\
9.8 \\
10.8 \\
12.2 \\
16.3\end{array}$ & $\begin{array}{l}0.7 \\
1.6 \\
7.3 \\
0.9 \\
1.4 \\
4.0\end{array}$ & $\begin{array}{r}36.1 \\
56.8 \\
26.2 \\
15.8 \\
21.5 \\
7.0\end{array}$ & $\begin{array}{l}0.6 \\
0.5 \\
0.2 \\
0.2 \\
0.3 \\
0.0\end{array}$ & $\begin{array}{l}0.0 \\
0.0 \\
0.2 \\
3.0 \\
1.5 \\
1.4\end{array}$ & $\begin{array}{ll}1.7 & 5-S L * \\
0.8 & 5 A N D * \\
0.7 & 5-S L * \\
1.5 & 5-S L \\
1.5 & 5-S L * \\
0.7 & 5 L I M *\end{array}$ & $\begin{array}{r}12 \\
11 \\
8 \\
5 \\
7 \\
4\end{array}$ & $\begin{array}{l}63 \\
78 \\
52 \\
32 \\
39\end{array}$ & $\begin{array}{l}33 \\
36 \\
32 \\
29 \\
30\end{array}$ & $\begin{array}{r}126 \\
199 \\
92 \\
55 \\
75 \\
24\end{array}$ & $\begin{array}{r}4326 \\
3147 \\
1891 \\
2583 \\
839\end{array}$ \\
\hline $\begin{array}{l}\text { THK: } \\
\text { THK: } \\
\text { THK: }\end{array}$ & $\begin{array}{l}10 \\
64 \\
25\end{array}$ & $\begin{array}{r}1.6 \\
10.3 \\
4.0\end{array}$ & $\begin{array}{r}56.8 \\
25.3 \\
7.0\end{array}$ & $\begin{array}{l}0.5 \\
0.2 \\
0.0\end{array}$ & $\begin{array}{l}0.0 \\
0.6 \\
1.4\end{array}$ & $\begin{array}{l}0.8 \text { SAND } \\
0.9 \text { S-SL } \\
0.7 \text { SLIM }\end{array}$ & $\begin{array}{r}11 \\
? \\
4\end{array}$ & $\begin{array}{l}78 \\
49\end{array}$ & $\begin{array}{l}36 \\
32\end{array}$ & $\begin{array}{r}199 \\
88 \\
24\end{array}$ & $\begin{array}{r}3 \\
3646 \\
839\end{array}$ \\
\hline
\end{tabular}


SITE ID: HATQ1

GROUND WATER DEPTH: 50.0 (FT.)
LOCATION ID: 012

AUERAGE SOIL WEIGHT: 100.0 (PSF)

AUG. AUG. UND.

- DEPTH - AUG. AUG. PORE FRICT. SOIL SHEAR LY FROM TO THK. POINT SLEEUE PRESS. RATIO CLASS BC DEN FA ELAS. STREN OCR

\begin{tabular}{|c|c|c|c|c|c|c|c|c|c|c|c|c|}
\hline $\begin{array}{r}0.1 \\
0.8 \\
1.5 \\
2.7 \\
3.7 \\
6.8 \\
7.5 \\
8.2 \\
8.9 \\
11.1\end{array}$ & $\begin{array}{r}0.8 \\
1.5 \\
2.7 \\
3.6 \\
6.8 \\
7.4 \\
8.2 \\
8.9 \\
11.1 \\
12.0\end{array}$ & $\begin{array}{l}0.7 \\
0.7 \\
1.2 \\
0.9 \\
3.1 \\
0.7 \\
0.7 \\
0.7 \\
2.1 \\
0.9\end{array}$ & $\begin{array}{r}17.4 \\
53.2 \\
28.7 \\
37.3 \\
21.9 \\
9.5 \\
18.4 \\
32.1 \\
19.7 \\
7.1\end{array}$ & $\begin{array}{l}0.2 \\
0.5 \\
0.3 \\
0.3 \\
0.2 \\
0.2 \\
0.2 \\
0.3 \\
0.2 \\
0.1\end{array}$ & $\begin{array}{r}-0.0 \\
0.1 \\
0.0 \\
0.1 \\
0.1 \\
0.2 \\
0.2 \\
0.2 \\
0.3 \\
0.6\end{array}$ & $\begin{array}{l}1.4 \\
0.9 \\
1.1 \\
0.7 \\
1.9 \\
1.9 \\
0.9 \\
0.8 \\
1.2 \\
1.9\end{array}$ & $\begin{array}{l}\text { S-SL** } \\
\text { SAMD* } \\
\text { S-SL* } \\
\text { SAMO* } \\
\text { S-SL* } \\
\text { SLIM } \\
\text { S-SL* } \\
\text { S-SL* } \\
\text { S-SL* } \\
\text { SLIM }\end{array}$ & $\begin{array}{r}5 \\
10 \\
9 \\
7 \\
7 \\
6 \\
6 \\
10 \\
6 \\
4\end{array}$ & $\begin{array}{l}41 \\
76 \\
56 \\
64 \\
47 \\
42 \\
57 \\
39\end{array}$ & $\begin{array}{l}30 \\
35 \\
32 \\
34 \\
31 \\
30 \\
33 \\
30\end{array}$ & $\begin{array}{r}61 \\
186 \\
101 \\
131 \\
77 \\
33 \\
64 \\
112 \\
69 \\
25\end{array}$ & $\begin{array}{c}2086 \\
3448 \\
2631 \\
1138 \\
2297 \\
385 \\
2360 \\
845\end{array}$ \\
\hline $\begin{array}{l}\text { THK: } \\
\text { THK: } \\
\text { THK: }\end{array}$ & $\begin{array}{l}13 \\
72 \\
13\end{array}$ & $\begin{array}{l}1.6 \\
8.5 \\
1.6\end{array}$ & $\begin{array}{r}44.3 \\
22.5 \\
8.2\end{array}$ & $\begin{array}{l}0.4 \\
0.2 \\
0.1\end{array}$ & $\begin{array}{l}0.1 \\
0.1 \\
0.4\end{array}$ & $\begin{array}{l}1.1 \\
1.9\end{array}$ & $\begin{array}{l}\text { SAND } \\
\text { S-SL } \\
\text { SLIM }\end{array}$ & $\begin{array}{l}8 \\
7 \\
4\end{array}$ & $\begin{array}{l}69 \\
46\end{array}$ & $\begin{array}{l}34 \\
31\end{array}$ & $\begin{array}{r}155 \\
78 \\
28\end{array}$ & $\begin{array}{r}2700 \\
978\end{array}$ \\
\hline
\end{tabular}


SITE ID: HATU1

GRQUND WATER DEPTH: 50.0 (FT.)
LOCATION TD: 013

AUERAGE SOIL WEIGHT: 100.0 (PSF) ?

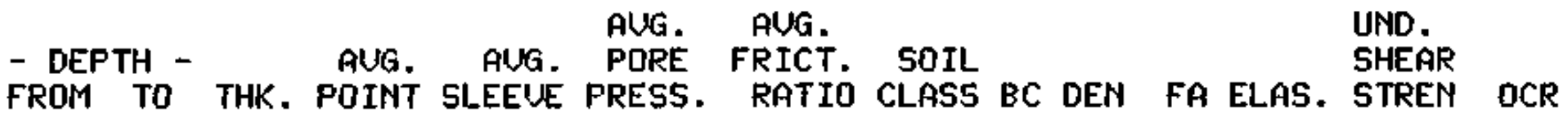

$\begin{array}{rrrrrrrrrrrrrr}1 & 0.1 & 1.0 & 1.0 & 13.2 & 0.1 & -0.1 & 0.9 & 5-S L * & 4 & 33 & 29 & 46 & 1579 \\ 2 & 1.0 & 1.7 & 0.7 & 9.7 & 0.2 & -0.1 & 1.8 & \text { SLIM } & 6 & & & 34 & 1167 \\ 3 & 1.8 & 5.5 & 3.8 & 16.6 & 0.2 & -0.0 & 1.3 & 5-S L * & 5 & 39 & 30 & 58 & 1989 \\ 4 & 5.6 & 10.1 & 4.5 & 31.2 & 0.2 & -0.1 & 0.8 & 5-5 L * 10 & 56 & 32 & 109 & 3740\end{array}$

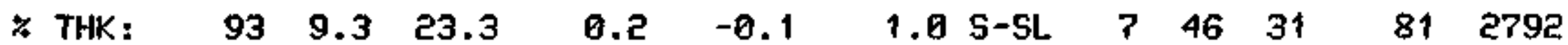

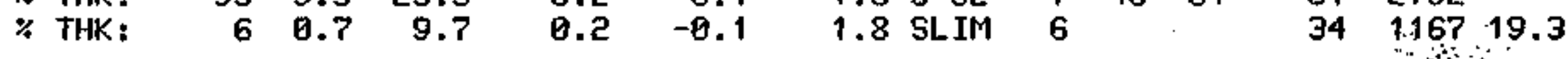


SITE ID: HATO1

GROUND WATER DEPTH: 50.0 (FT.)
LOCATION ID: 014

AUERAGE SOIL WEIGHT: 100.0 (PSF)

$\begin{array}{llll} & \\ \text { - DEPTH - } & \text { AUG. AUG. PURE FUG. } & \text { UND. } \\ \end{array}$ LY FROM TO THK. POINT SLEEUE PRESS. RATIO CLASS BC DEN FA ELAS. STREM DCR

\begin{tabular}{|c|c|c|c|c|c|c|c|c|c|c|c|c|}
\hline $\begin{array}{l}0.2 \\
1.1 \\
2.1 \\
5.1 \\
8.0\end{array}$ & $\begin{array}{l}1.1 \\
2.0 \\
5.1 \\
8.0 \\
9.7\end{array}$ & $\begin{array}{l}0.9 \\
0.9 \\
3.1 \\
2.9 \\
1.6\end{array}$ & $\begin{array}{r}17.6 \\
7.1 \\
14.0 \\
17.3 \\
45.5\end{array}$ & $\begin{array}{l}0.3 \\
0.2 \\
0.3 \\
0.3 \\
0.3\end{array}$ & $\begin{array}{l}-0.2 \\
-0.2 \\
-0.2 \\
-0.2 \\
-0.2\end{array}$ & $\begin{array}{l}1.7 \\
2.4 \\
1.9 \\
1.6 \\
0.7\end{array}$ & $\begin{array}{l}\text { S-SL } \\
\text { SLIM } \\
\text { SLIM } \\
\text { S-SL } \\
\text { SAND* }\end{array}$ & $\begin{array}{l}5 \\
4 \\
9 \\
5 \\
9\end{array}$ & $\begin{array}{l}40 \\
67\end{array}$ & $\begin{array}{l}30 \\
34\end{array}$ & $\begin{array}{r}61 \\
25 \\
49 \\
61 \\
159\end{array}$ & $\begin{array}{r}2107 \\
858 \\
1682 \\
2078\end{array}$ \\
\hline THK & $\begin{array}{l}17 \\
40 \\
42\end{array}$ & $\begin{array}{l}1.6 \\
3.8 \\
4.8\end{array}$ & $\begin{array}{l}45.5 \\
17.4 \\
12.4\end{array}$ & 0.3 & $\begin{array}{l}-0.5 \\
-0.5 \\
-0.5\end{array}$ & $\begin{array}{l}0.7 \\
1.6 \\
2.0\end{array}$ & $\begin{array}{l}\text { SAND } \\
\text { 5-SL } \\
\text { SLIM }\end{array}$ & $\begin{array}{l}9 \\
4 \\
7\end{array}$ & $\begin{array}{l}6 ? \\
40\end{array}$ & $\begin{array}{l}34 \\
30\end{array}$ & $\begin{array}{r}159 \\
60 \\
43\end{array}$ & \\
\hline
\end{tabular}


SITE IO: HATE

GROUND WATER DEPTH: 50.0 (FT.)
LOCATION ID : 015

AUERAGE SOIL WEIGHT: 100.0 (PSF)

AUG, AUG. UND.

LY FROM TO THK. POINT SLEEUE PRESS. RATIO CLASS BC DEN FA ELAS. STREN OCR

\begin{tabular}{|c|c|c|c|c|c|c|c|c|c|c|c|c|}
\hline $\begin{array}{r}0.2 \\
2.4 \\
3.1 \\
5.8 \\
7.2 \\
9.1 \\
10.6 \\
11.8 \\
16.6 \\
18.5 \\
19.9 \\
22.0 \\
22.8\end{array}$ & $\begin{array}{r}2.4 \\
3.1 \\
5.7 \\
7.2 \\
9.1 \\
10.6 \\
11.7 \\
16.5 \\
18.4 \\
19.9 \\
22.0 \\
22.7 \\
25.1\end{array}$ & $\begin{array}{l}2.2 \\
0.7 \\
2.6 \\
1.4 \\
1.9 \\
1.4 \\
1.1 \\
4.8 \\
1.9 \\
1.4 \\
2.1 \\
0.7 \\
2.4\end{array}$ & $\begin{array}{r}7.1 \\
25.2 \\
8.9 \\
27.5 \\
14.1 \\
28.8 \\
16.8 \\
27.3 \\
49.0 \\
28.0 \\
42.6 \\
17.5 \\
16.9\end{array}$ & $\begin{array}{l}0.2 \\
0.4 \\
0.2 \\
0.3 \\
0.3 \\
0.2 \\
0.3 \\
0.4 \\
0.5 \\
0.4 \\
0.5 \\
0.3 \\
0.2\end{array}$ & $\begin{array}{l}0.0 \\
0.1 \\
0.1 \\
0.2 \\
0.2 \\
0.3 \\
0.3 \\
0.3 \\
0.3 \\
0.3 \\
0.4 \\
0.3 \\
0.4\end{array}$ & $\begin{array}{l}2.9 \\
1.6 \\
2.3 \\
1.3 \\
2.1 \\
0.8 \\
1.9 \\
1.3 \\
1.0 \\
1.5 \\
1.1 \\
1.9 \\
0.9\end{array}$ & $\begin{array}{l}\text { SLIM } \\
\text { S-SL* } \\
\text { SLIM } \\
\text { S-SL* } \\
\text { SLIM } \\
\text { S-SL* } \\
\text { SLIM } \\
\text { S-SL* } \\
\text { SAND* } \\
\text { S-SL* } \\
\text { S-SL* } \\
\text { S-SL } \\
\text { S-SL* }\end{array}$ & $\begin{array}{r}4 \\
8 \\
5 \\
9 \\
9 \\
9 \\
11 \\
9 \\
9 \\
9 \\
14 \\
5 \\
5\end{array}$ & $\begin{array}{l}41 \\
54 \\
36 \\
46 \\
23 \\
21\end{array}$ & $\begin{array}{l}32 \\
32 \\
30 \\
32 \\
29 \\
31 \\
28 \\
28\end{array}$ & $\begin{array}{r}25 \\
88 \\
31 \\
96 \\
49 \\
101 \\
59 \\
95 \\
172 \\
98 \\
149 \\
61 \\
59\end{array}$ & $\begin{array}{r}846 \\
3021 \\
1071 \\
3294 \\
1697 \\
3456 \\
2015 \\
3270 \\
3355 \\
5109 . \\
2101 \\
2024\end{array}$ \\
\hline $\begin{array}{l}\text { THK: } \\
\text { THKK }\end{array}$ & $\begin{array}{r}7 \\
60 \\
31\end{array}$ & $\begin{array}{r}1.9 \\
14.9 \\
7.8\end{array}$ & $\begin{array}{l}49.0 \\
27.4 \\
10.8\end{array}$ & $\begin{array}{l}0.5 \\
0.3 \\
0.2\end{array}$ & $\begin{array}{l}0.3 \\
0.3 \\
0.1\end{array}$ & $\begin{array}{l}1.0 \\
1.2 \\
2.4\end{array}$ & $\begin{array}{l}\text { SAND } \\
\text { S-SL } \\
\text { SLIM }\end{array}$ & $\begin{array}{l}9 \\
8 \\
6\end{array}$ & $\begin{array}{l}54 \\
39\end{array}$ & $\begin{array}{l}32 \\
30\end{array}$ & $\begin{array}{r}172 \\
95 \\
37\end{array}$ & $\begin{array}{l}3289 \\
1293\end{array}$ \\
\hline
\end{tabular}


SITE ID: HATO

GROUND LATER DEPTH: 50.0 (FT.)
LOCATION ID: 016

AUERAGE SOIL WEIGHT: 100.0 (PSF)

- DEPTH - AUG. AUG. PORE FRICT. SOIL SUG. SMD. LY FROM TO THK. POINT SLEEUE PRESS. RATIO CLASS BC DEM FA ELAS. STREN OCR

\begin{tabular}{|c|c|c|c|c|c|c|c|c|c|c|c|}
\hline $\begin{array}{rr}1 & 0.0 \\
2 & 4.4 \\
3 & 5.1 \\
4 & 7.3 \\
5 & 9.2 \\
6 & 10.6 \\
7 & 15.6\end{array}$ & $\begin{array}{r}4.4 \\
5.1 \\
7.3 \\
9.2 \\
10.6 \\
15.6 \\
16.1\end{array}$ & $\begin{array}{l}4.4 \\
0.7 \\
2.1 \\
1.9 \\
1.4 \\
5.0 \\
0.5\end{array}$ & $\begin{array}{r}8.2 \\
22.4 \\
10.8 \\
28.0 \\
17.2 \\
29.2 \\
14.6\end{array}$ & $\begin{array}{l}0.2 \\
0.3 \\
0.3 \\
0.4 \\
0.4 \\
0.4 \\
0.5\end{array}$ & $\begin{array}{l}0.1 \\
0.3 \\
0.2 \\
0.8 \\
0.8 \\
0.7 \\
0.6\end{array}$ & $\begin{array}{l}2.3 \text { SLIM } \\
1.4 \text { S-SL* } \\
2.4 \text { SLIM } \\
1.5 \text { S-SL* } \\
2.2 \text { SLIM } \\
1.3 \text { S-SL* } \\
3.2 \text { SLIM }\end{array}$ & $\begin{array}{r}5 \\
7 \\
7 \\
9 \\
11 \\
9 \\
9\end{array}$ & 53 & 31 & $\begin{array}{r}29 \\
79 \\
38 \\
98 \\
60 \\
192 \\
51\end{array}$ & $\begin{array}{r}988 \\
2694 \\
1292 \\
3365 \\
2066 \\
3506 \\
1756\end{array}$ \\
\hline $\begin{array}{l}\text { THK: } \\
\text { THK: }\end{array}$ & $\begin{array}{l}47 \\
52\end{array}$ & $\begin{array}{l}7.6 \\
8.4\end{array}$ & $\begin{array}{l}28.3 \\
10.7\end{array}$ & $\begin{array}{l}0.4 \\
0.3\end{array}$ & $\begin{array}{l}0.7 \\
0.3\end{array}$ & $\begin{array}{ll}1.4 & 5-\mathrm{SL} \\
2.4 & \mathrm{SLIM}\end{array}$ & $\begin{array}{l}8 \\
6\end{array}$ & 47 & 31 & $\begin{array}{l}98 \\
37\end{array}$ & \\
\hline
\end{tabular}


SITE ID: HATO1

GROUND WATER DEPTH: 50.0 (FT.)
- LOCATION ID: 017 AUERAGE SOIL WEIGHT: 100.0 (PSF)

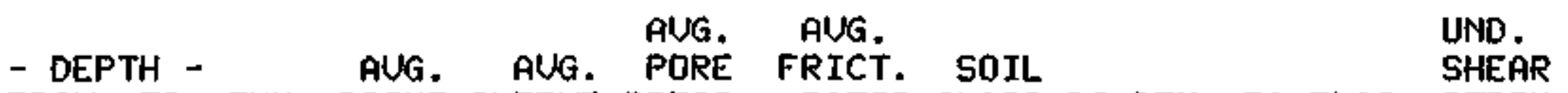
LY FROM TO THK. POINT SLEEVE PRES5. RATIO CLASS BC DEN FA ELAS. STREN OCR

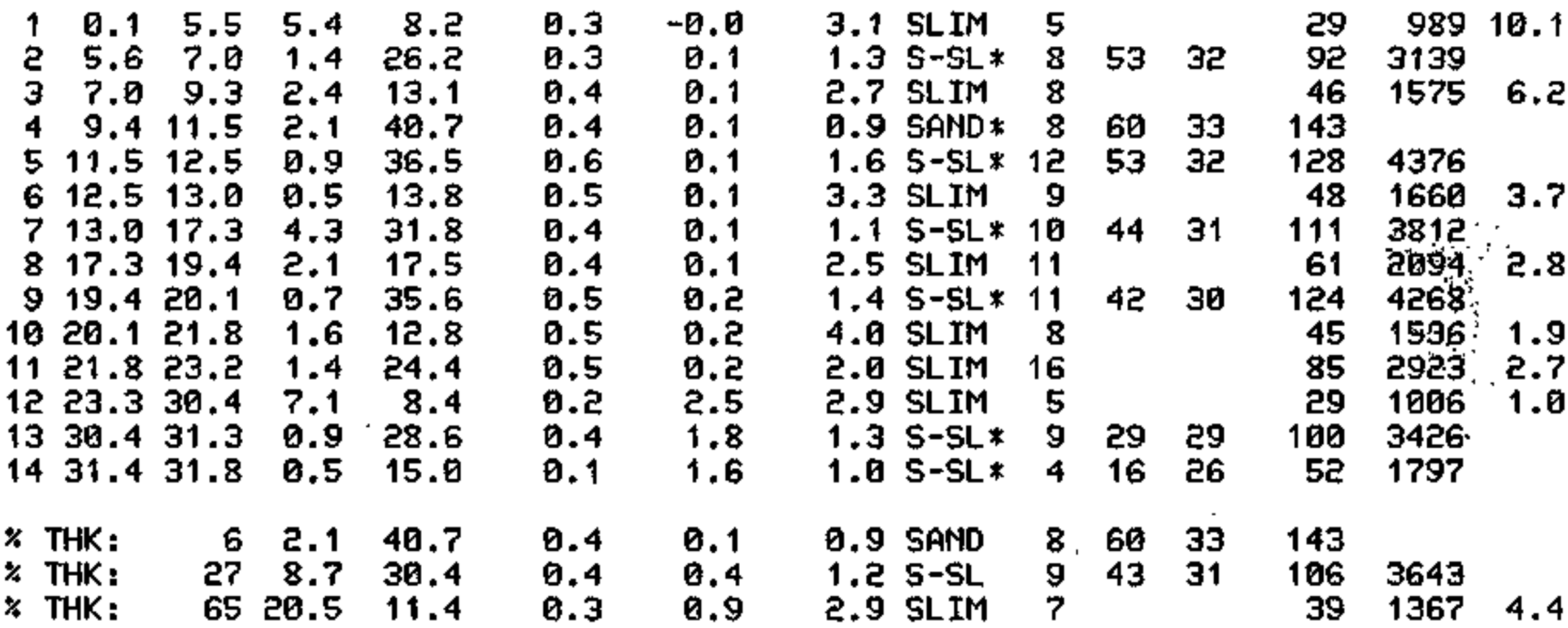


SITE ID: HATO1

GROUND WATER DEPTH: 50.0 (FT.)
LOCATION ID: 018

AUERAGE SOIL WEIGHT: 100.0 (PSF)

AUG. AUG. UND.

- DEPTH - AUG. AUG. PORE FRICT. SOIL SHEAR LY FROM TO THK. POINT SLEEUE, PRESS. RATIO CLASS BC DEN FA ELAS. STREN OCR

\begin{tabular}{|c|c|c|c|c|c|c|c|c|c|c|c|c|}
\hline $\begin{array}{rr}1 & 0.0 \\
2 & 3.7 \\
3 & 4.9 \\
4 & 8.4 \\
5 & 11.8 \\
6 & 13.4 \\
7 & 15.6 \\
8 & 16.8\end{array}$ & $\begin{array}{r}3.6 \\
4.8 \\
8.4 \\
11.7 \\
13.4 \\
15.6 \\
16.8 \\
18.4\end{array}$ & $\begin{array}{l}3.6 \\
1.1 \\
3.5 \\
3.3 \\
1.6 \\
2.1 \\
1.1 \\
1.6\end{array}$ & $\begin{array}{l}13.4 \\
13.1 \\
21.5 \\
32.4 \\
13.7 \\
25.5 \\
40.8 \\
40.6\end{array}$ & $\begin{array}{l}0.1 \\
0.3 \\
0.2 \\
0.3 \\
0.3 \\
0.3 \\
0.3 \\
0.5\end{array}$ & $\begin{array}{r}-0.0 \\
0.0 \\
0.1 \\
0.1 \\
0.1 \\
0.2 \\
0.2 \\
0.2\end{array}$ & $\begin{array}{l}1.0 \\
2.0 \\
1.1 \\
0.9 \\
2.2 \\
1.2 \\
0.6 \\
1.1\end{array}$ & $\begin{array}{l}\text { S-SL* } \\
\text { SLIM } \\
\text { S-SL* } \\
\text { S-SL* } \\
\text { SLIM } \\
\text { S-SL* } \\
\text { SAND* } \\
\text { S-SL* }\end{array}$ & $\begin{array}{r}4 \\
8 \\
7 \\
10 \\
9 \\
8 \\
8 \\
13\end{array}$ & $\begin{array}{l}47 \\
53 \\
39 \\
50 \\
48\end{array}$ & $\begin{array}{l}31 \\
32 \\
30 \\
32 \\
31\end{array}$ & $\begin{array}{r}47 \\
46 \\
75 \\
113 \\
48 \\
89 \\
143 \\
142\end{array}$ & $\begin{array}{l}1609 \\
1572 \\
2584 \\
3890 \\
1644 \\
3056 \\
4867\end{array}$ \\
\hline $\begin{array}{l}\text { 1HK: } \\
\text { THK: } \\
\text { THK : }\end{array}$ & $\begin{array}{r}6 \\
78 \\
15\end{array}$ & $\begin{array}{r}14.1 \\
2.7\end{array}$ & $\begin{array}{l}24.7 \\
13.5\end{array}$ & 0.2 & $\begin{array}{l}0.1 \\
0.1\end{array}$ & & $\begin{array}{l}\text { SAND } \\
\text { S-SL } \\
\text { SLIM }\end{array}$ & $\begin{array}{l}8 \\
7 \\
8\end{array}$ & $\begin{array}{l}50 \\
44\end{array}$ & $\begin{array}{l}32 \\
31\end{array}$ & $\begin{array}{r}143 \\
86 \\
47\end{array}$ & $\begin{array}{l}2979 \\
1614\end{array}$ \\
\hline
\end{tabular}


SITE ID: HATO1

GROUND LATER DEPTH: 50.0 (FT.)
LOCATION ID : 019

AUERAGE SOIL WEIGHT: 100.0 (PSF)

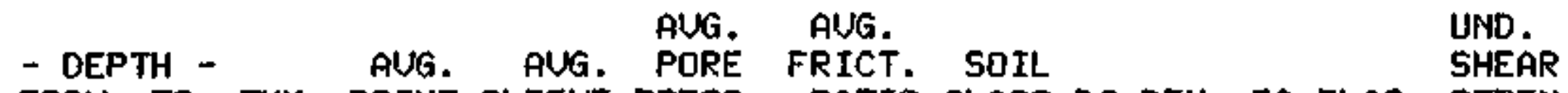
LY FROM TO THK. POINT SLEEUE PRESS. RATIO CLASS BC DEN FA ELAS. STREN DCR

$\begin{array}{llllllllllllll}1 & 0.0 & 0.8 & 0.7 & 13.4 & 0.1 & -0.1 & 0.8 & 5-S L * & 4 & 34 & 29 & 47 & 1613\end{array}$

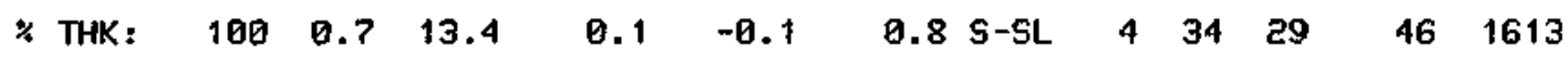


SITE ID: HATO1

GROUND WHTER DEPTH: 50.0 (FT.)
LOCATIỌN ID: DED

AUERAGE SOIL WEIGHT: 100.0 (PSF)

- DEPTH - AUG. AUG. PURE FUICT. SOIL SND. LY FROM TO THK. POIMT SLEEVE PRESS. RATIO CLASS BC DEN FA ELAS. STREM OCR

\begin{tabular}{|c|c|c|c|c|c|c|c|c|c|c|c|}
\hline $\begin{array}{rr}1 & 0.0 \\
2 & 1.0 \\
3 & 2.0 \\
4 & 3.2 \\
5 & 6.5 \\
6 & 9.4 \\
7 & 10.3\end{array}$ & $\begin{array}{r}1.0 \\
2.0 \\
3.1 \\
6.5 \\
9.3 \\
10.3 \\
11.3\end{array}$ & $\begin{array}{l}1.0 \\
1.0 \\
1.1 \\
3.3 \\
2.8 \\
0.9 \\
1.0\end{array}$ & $\begin{array}{r}15.2 \\
6.7 \\
15.9 \\
19.1 \\
27.2 \\
13.3 \\
31.7\end{array}$ & $\begin{array}{l}0.1 \\
0.1 \\
0.2 \\
0.2 \\
0.2 \\
0.2 \\
0.2\end{array}$ & $\begin{array}{l}-0.2 \\
-0.1 \\
-0.1 \\
-0.1 \\
0.0 \\
0.0 \\
0.0\end{array}$ & $\begin{array}{ll}0.8 & \text { S-SL* } \\
1.8 & \text { SLIM } \\
1.4 & \text { S-SL* } \\
2.4 & \text { SLIM } \\
0.7 & \text { S-SL* } \\
1.4 & 5-S L \\
0.6 & \text { S-SL* }\end{array}$ & $\begin{array}{r}5 \\
4 \\
5 \\
6 \\
9 \\
4 \\
10\end{array}$ & $\begin{array}{l}\mathbf{5 3} \\
29 \\
\mathbf{5 1}\end{array}$ & $\begin{array}{l}32 \\
29 \\
32\end{array}$ & $\begin{array}{r}53 \\
23 \\
56 \\
35 \\
95 \\
47 \\
111\end{array}$ & $\begin{array}{r}1827 \\
798 \\
1905 \\
1208 \\
3265 \\
1599 \\
3800 \\
3\end{array}$ \\
\hline $\begin{array}{l}\text { THK: } \\
\text { THK: }\end{array}$ & $\begin{array}{l}61 \\
38\end{array}$ & $\begin{array}{l}6.8 \\
4.3\end{array}$ & $\begin{array}{r}22.4 \\
9.3\end{array}$ & $\begin{array}{l}0.2 \\
0.2\end{array}$ & $\begin{array}{l}-0.0 \\
-0.1\end{array}$ & $\begin{array}{l}0.95-5 L \\
2.35 L I M\end{array}$ & $\begin{array}{l}7 \\
5\end{array}$ & 44 & 31 & $\begin{array}{l}78 \\
32\end{array}$ & $\begin{array}{l}2691 \\
1 \text { 1:1 }\end{array}$ \\
\hline
\end{tabular}


SITE ID: HATO1

GROUMO WATER DEPTH: 50.0 (FT.)
LOCATION ID: D21

AUERAGE SOIL WEIGHT: 100.0 (PSF)

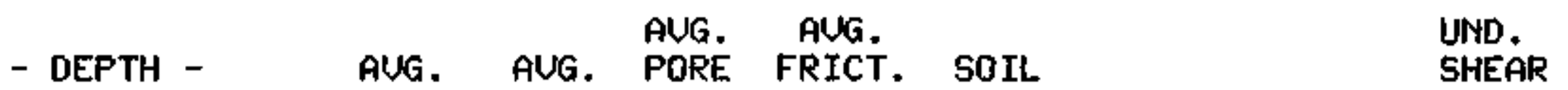
LY FROM TO THK. POINT SLEEVE PRESS. RATIO CLASS BC DEN FA ELAS. STREN OCR

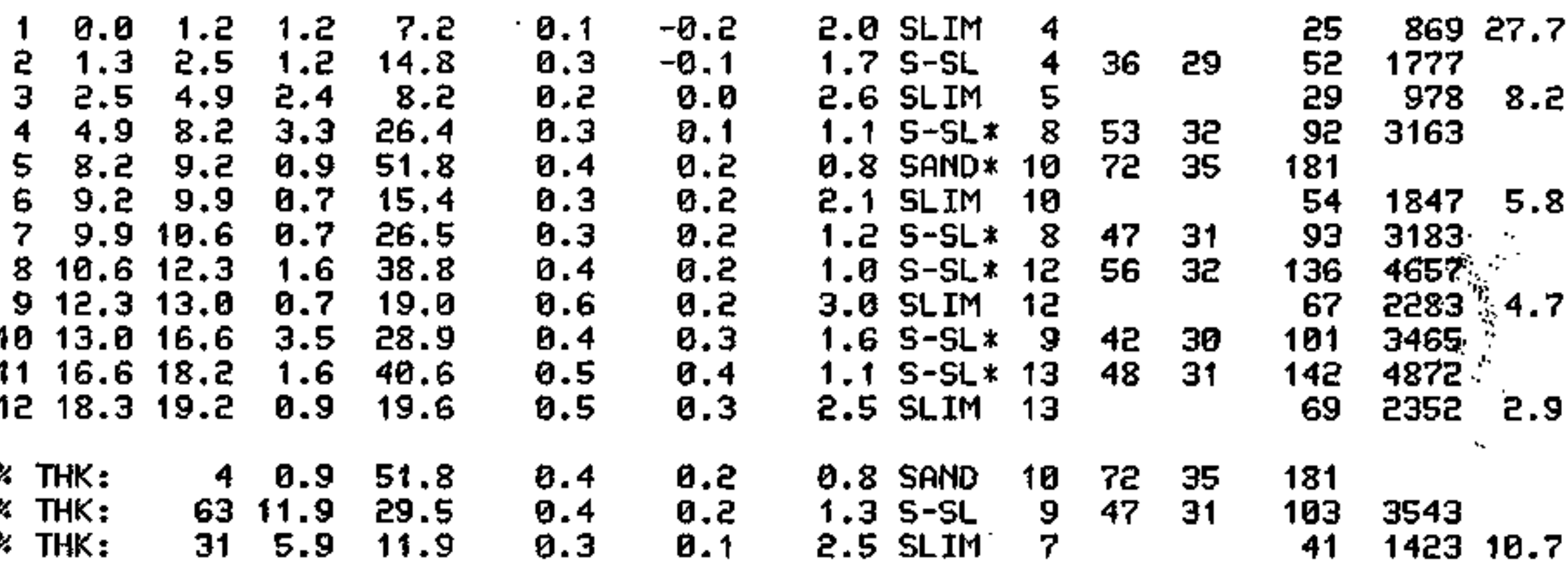


SITE ID: HATE1

GRDUND WATER DEPTH: 50.0 (FT.)
LOCATION ID: D22

AUERAGE SOIL WEIGHT: 100.0 (PSF)

$\begin{array}{llll} & \\ \text { - DEPTH - AUG. AUG. PUG. AUG. } & & \text { UND. } \\ \end{array}$ LY FROM TO THK. POINT SLEEUE PRESS. RATIO CLASS BC DEN FA ELAS. STREN OCR

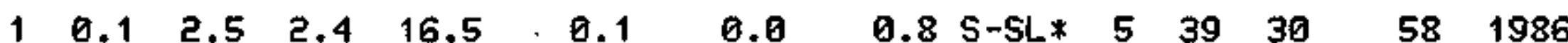

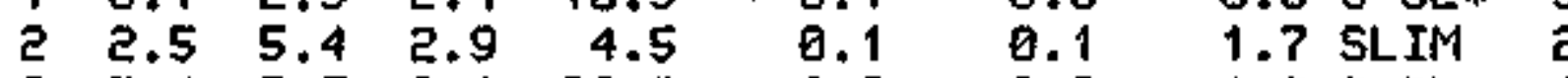

$\begin{array}{llllll}3 & 5.4 & 7.5 & 2.1 & 20.0\end{array}$

(2.

0.3

1.0 S-SL* 6

$\begin{array}{lllll}4 & 7.5 & 8.4 & 0.9 & 8.9\end{array}$

0.2

0.5

0.2

0.5

1.8 SLIM 5

0.6 S-SL* 8

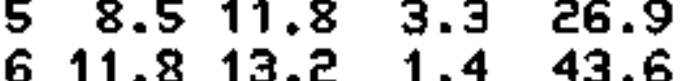

0.6

0.6 SAND* 8

0.5

$\begin{array}{lllll}7 & 13.3 & 15.1 & 1.9 & 27.9\end{array}$

0.7 S-SL* 9

0.2

0.5

0.5 SAND* 9

0.6

1.0 S-SL* 10

0.3

$\begin{array}{lllll}9 & 16.1 & 17.2 & 1.1 & 30.2\end{array}$

0.4

0.7

0.8 SAND*

8

0.7 SAND

D.6

0.3

0.4

0.2

0.2

$0.8 \mathrm{~S}-\mathrm{SL}$

1.7 SLIM

$\begin{array}{lll}8 & 54 & 32 \\ 7 & 43 & 31\end{array}$

$16 \quad 538$

$70 \quad 2405$

$31 \quad 1064$

943233

$\begin{array}{ll}47 & 31\end{array}$

153

98

3352

158

1063626

$\%$ THK:

$\begin{array}{lll}20 & 3.8 & 5.5\end{array}$

0.1

2

155

$83 \quad 2855$

$19 \quad 662 \quad 5.0$ 
SITE TD: HATQ1

GROUND WATER DEPTH: 50.0 (FT.)
LOCATION ID: 023

AUERAGE SOIL WEIGHT: 100.0 (PSF)

- DEPTH - AUG. AUG. PURE FUG. FICT. SOIL UNO. LY FROM TO THK. POINT SLEEVE PRESS. RATIO CLASS BC DEN FA ELAS. STREN OCR

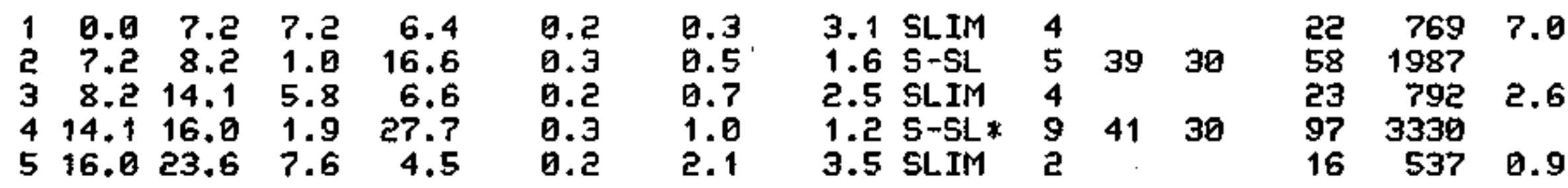

* THK: $12 \quad 2.9 \quad 23.9$

\% THK: $8720.6 \quad 5.8$

0.30 .8

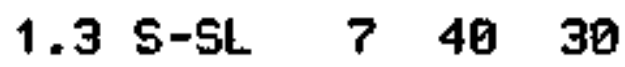

$83 \quad 2866$

0.21 .1

3.1 SLIM 3

$20 \quad 689=3.5$ 
SITE ID: HATO1

GROUND LATER DEPTH: 50.0 (FT.)
LOCATION ID: 024

AUERAGE SOIL WEIGHT: 100.0 (PSF)

AUG AUG. AUG.

LY FROM TO THK. POINT SLEEUE PRESS. RATIO CLASS BC DEN FA ELAS. STREN OCR

$\begin{array}{lllllllll}1 & 0.1 & 2.2 & 2.1 & 11.3 & 0.2 & 0.0 & 1.6 & \text { SLIM }\end{array}$

$\begin{array}{lllllllll}2 & 2.3 & 24.7 & 22.4 & 4.5 & 0.2 & 0.5 & 4.3 & \text { SLIM }\end{array}$

* THK: $10024.5 \quad 5.1 \quad 0.2 \quad 0.5 \quad 4.1$ SLIM 2
$40 \quad 1359 \quad 24.8$

$\begin{array}{lll}16 & 536 & 1.5\end{array}$

$18 \quad 606 \quad 3.5$ 
AUG. AUG. PUG. PURE
- DEPTH
FROM TO

\begin{tabular}{|c|c|c|c|c|c|c|c|c|c|c|c|c|c|}
\hline $\begin{array}{rr}1 & 0.1 \\
2 & 1.3 \\
3 & 3.0 \\
4 & 4.2 \\
5 & 5.4 \\
6 & 6.8 \\
7 & 10.6 \\
8 & 12.1\end{array}$ & $\begin{array}{r}1.3 \\
3.0 \\
4.1 \\
5.3 \\
6.8 \\
10.6 \\
12.0 \\
15.1\end{array}$ & $\begin{array}{l}1.2 \\
1.6 \\
1.1 \\
1.2 \\
1.4 \\
3.8 \\
1.4 \\
3.1\end{array}$ & $\begin{array}{r}18.7 \\
7.7 \\
20.4 \\
13.9 \\
9.5 \\
8.9 \\
13.8 \\
10.2\end{array}$ & $\begin{array}{l}0.2 \\
0.2 \\
0.3 \\
0.4 \\
0.1 \\
0.3 \\
0.2 \\
0.2\end{array}$ & $\begin{array}{l}-0.2 \\
-0.1 \\
-0.1 \\
-0.1 \\
-0.1 \\
-0.0 \\
0.0 \\
0.1\end{array}$ & $\begin{array}{l}1.1 \\
2.1 \\
1.6 \\
2.8 \\
1.4 \\
3.0 \\
1.6 \\
2.3\end{array}$ & $\begin{array}{l}\text { S-SL** } \\
\text { SLIM } \\
\text { S-SL* } \\
\text { SLIM } \\
\text { SLIM } \\
\text { SLIM } \\
\text { SLIM } \\
\text { SLIM }\end{array}$ & $\begin{array}{l}6 \\
5 \\
6 \\
9 \\
6 \\
5\end{array}$ & 43 & 30 & $\begin{array}{l}65 \\
27 \\
72 \\
49 \\
33 \\
31 \\
48 \\
36\end{array}$ & $\begin{array}{r}2245 \\
922 \\
2454 \\
1666 \\
1137 \\
1069 \\
1652 \\
1223\end{array}$ & $\begin{array}{r}11.8 \\
10.1 \\
6.3 \\
4.3 \\
4.3 \\
2.7\end{array}$ \\
\hline $\begin{array}{l}\text { HK: } \\
\text { HK: }\end{array}$ & $\begin{array}{l}15 \\
84\end{array}$ & $\begin{array}{r}2.3 \\
12.5\end{array}$ & $\begin{array}{l}19.5 \\
10.2\end{array}$ & $\begin{array}{l}0.2 \\
0.2\end{array}$ & $\begin{array}{l}-0.2 \\
-0.0\end{array}$ & $\begin{array}{l}1.39 \\
2.4\end{array}$ & $\begin{array}{l}\text { S-SL } \\
\text { SLIM }\end{array}$ & & 43 & 31 & $\begin{array}{l}68 \\
35\end{array}$ & $\begin{array}{l}2344 \\
1218\end{array}$ & 5.6 \\
\hline
\end{tabular}


SITE ID: HATO1

GROUND WATER DEPTH: 50.0 (FT.)
LOCATION ID: 026

AUERAGE SOIL WEIGHT: 100.0 (PSF)

AUG. AUG. UND.

- DEPTH - AUG. AUG. PORE FRICT. SOIL SHEAR LY FROM TO THK. POINT SLEEUE PRESS. RATIO CLASS BC DEN FA ELAS. STREN OCR

\begin{tabular}{|c|c|c|c|c|c|c|c|c|c|c|c|c|}
\hline $\begin{array}{ll}1 & 0.1 \\
2 & 5.1 \\
3 & 6.1 \\
4 & 9.0\end{array}$ & $\begin{array}{l}5.1 \\
6.1 \\
9.0 \\
9.7\end{array}$ & $\begin{array}{l}5.0 \\
0.9 \\
2.9 \\
0.7\end{array}$ & $\begin{array}{r}14.2 \\
8.2 \\
22.9 \\
13.2\end{array}$ & $\begin{array}{l}0.1 \\
0.2 \\
0.3 \\
0.2\end{array}$ & $\begin{array}{r}-0.0 \\
0.0 \\
0.2 \\
0.2\end{array}$ & $\begin{array}{l}0.9 \\
2.3 \\
1.1 \\
1.6\end{array}$ & $\begin{array}{l}\text { S-SL* } \\
\text { SLIM } \\
\text { S-SL* } \\
\text { SLIM }\end{array}$ & $\begin{array}{l}4 \\
5 \\
7 \\
8\end{array}$ & $\begin{array}{l}35 \\
48\end{array}$ & $\begin{array}{l}29 \\
31\end{array}$ & $\begin{array}{l}50 \\
29 \\
80 \\
46\end{array}$ & $\begin{array}{r}1793 \\
989 \\
2753 \\
1588\end{array}$ \\
\hline
\end{tabular}

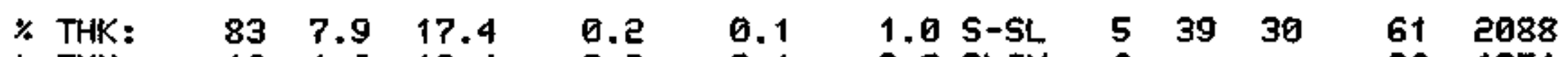

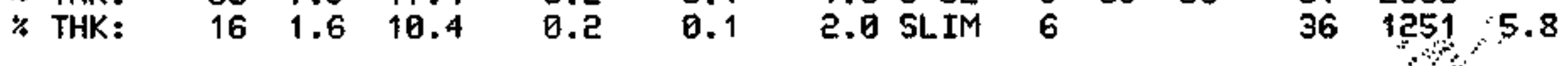


SITE IO: HATO1

GROUND WNTER DEPTH: 50.0 (FT.)
LOCATION ID: DE?

AUERAGE SOIL WEIGHT: 100.0 (PSF)

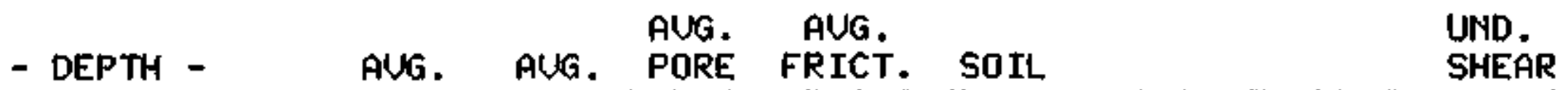
LY FROM TO THK. POINT SLEEUE PRESS. RATIO CLASS BC DEN FA ELAS. STREN DCR

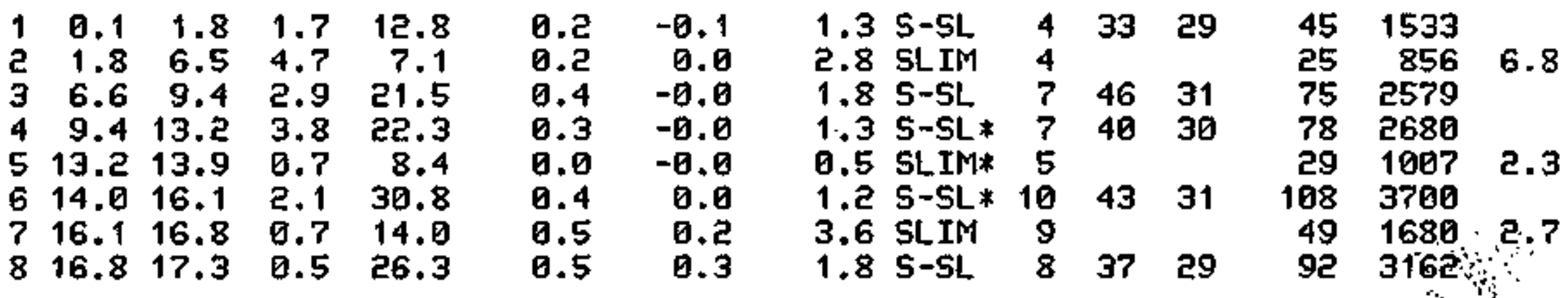

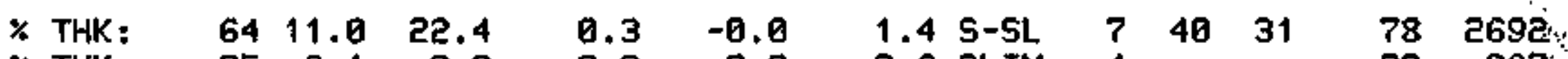

* THK: $\begin{array}{lllllllll}35 & 6.1 & 8.0 & 0.2 & 0.0 & 2.6 & \text { SLIM } 4 & 78 & 967.8\end{array}$ 
SITE ID: HATOI

GROUND WATER DEPTH: 50.0 (FT.)
LOCATION ID: D28

AUERAGE SOIL WEIGHT: 100.0 (PSF)

AUG. AUG. UND.

- DEPTH - AUG. AUG. PORE FRICT. SOIL SHEAR

LY FROM TO THK. POINT SLEEUE PRESS. RATIO CLASS BC DEN FA ELAS. STREN OCR

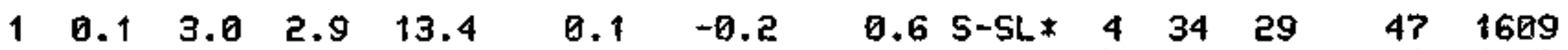

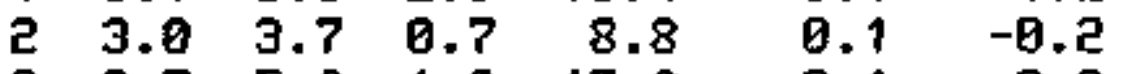

$\begin{array}{lllllll}3 & 3.7 & 5.6 & 1.9 & 17.6 & 0.1 & -0.2\end{array}$

$\begin{array}{lllllll}4 & 5.6 & 10.6 & 5.0 & 4.9 & 0.0 & -0.1\end{array}$

1.0 SLIM* 5

$\begin{array}{lll}31 & 1052 & 9.4\end{array}$

0.5 S-SL* 54130

$62 \quad 2115$

0.2 SLIM* 3

$17593 \quad 3.0$

* THK:

$\begin{array}{lll}45 & 4.8 & 15.1\end{array}$

$0.1-0.2$

$0.6 \quad 5-S L \quad 4 \quad 36 \quad 30$

$52 \quad 1899$

* THK:

$545.7 \quad 5.4$

$0.0-0.1$

0.3 SLIM 3

$18 \quad 649 \div 3.8$ 
SITE ID: HATO1

GROUND WATER DEPTH: 50.0 (FT.)
LOCATION ID: 029

AUERAGE SOIL WEIGHT: 100.0 (PSF)

- DEPTH -

AUG. AUG. PORE FRICT. SOIL

LY FROM TO THK. POINT SLEEUE PRESS. RATIO CLASS BC DEN FA ELAS. STREN OCR

$\begin{array}{lllllllll}1 & 0.1 & 7.0 & 6.9 & 11.2 & 0.3 & -0.1 & 2.6 & \text { SLIM ? }\end{array}$

$\begin{array}{llllllll}2 & 7.1 & 10.1 & 3.1 & 3.2 & 0.3 & 0.3 & 8.2 \text { SLIM } 2\end{array}$

* THK: $10010.0 \quad 8.7 \quad 0.3 \quad 0.0 \quad 4.3$ SLIM 5
$39 \quad 134210.7$

$\begin{array}{lll}11 & 383 & 2.0\end{array}$

$30 \quad 1044 \quad 8.0$ 
SITE ID: HATQ1

GROUND WATER DEPTH: 50.0 (FT.)
LOCATION ID: 930

AUERAGE SOIL WEIGHT: 100.0 (PSF)

- DEPTH - RUG. AUG. PURE FRICT. SOIL UND.

LY FROM TO THK. POINT SLEEUE PRESS. RATIO CLASS BC DEN FA ELAS. STREN OCR

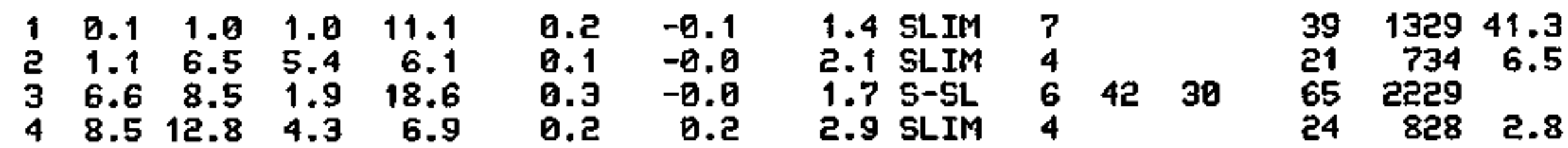

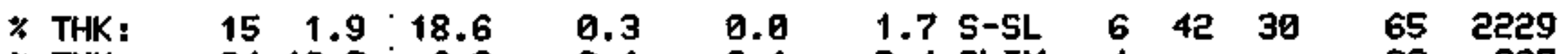

* THK: $8410.7 \quad 6.9 \quad 0.1 \quad 0.1 \quad 2.4$ 5LIM $4 \quad 83 \quad 827.8 .3$ 
SITE ID: HATO1

GROUMD WATER DEPTH: 50.0 (FT.)
LOCATION ID: 031

AUERAGE SOIL WEIGHT: 100.0 (PSF)

- DEPTH - AUG AUG AUG. AUG.

LY FROM TO THK. POINT SLEEUE PRESS. RATIO CLASS BC DEN FA ELAS. STREN OCR

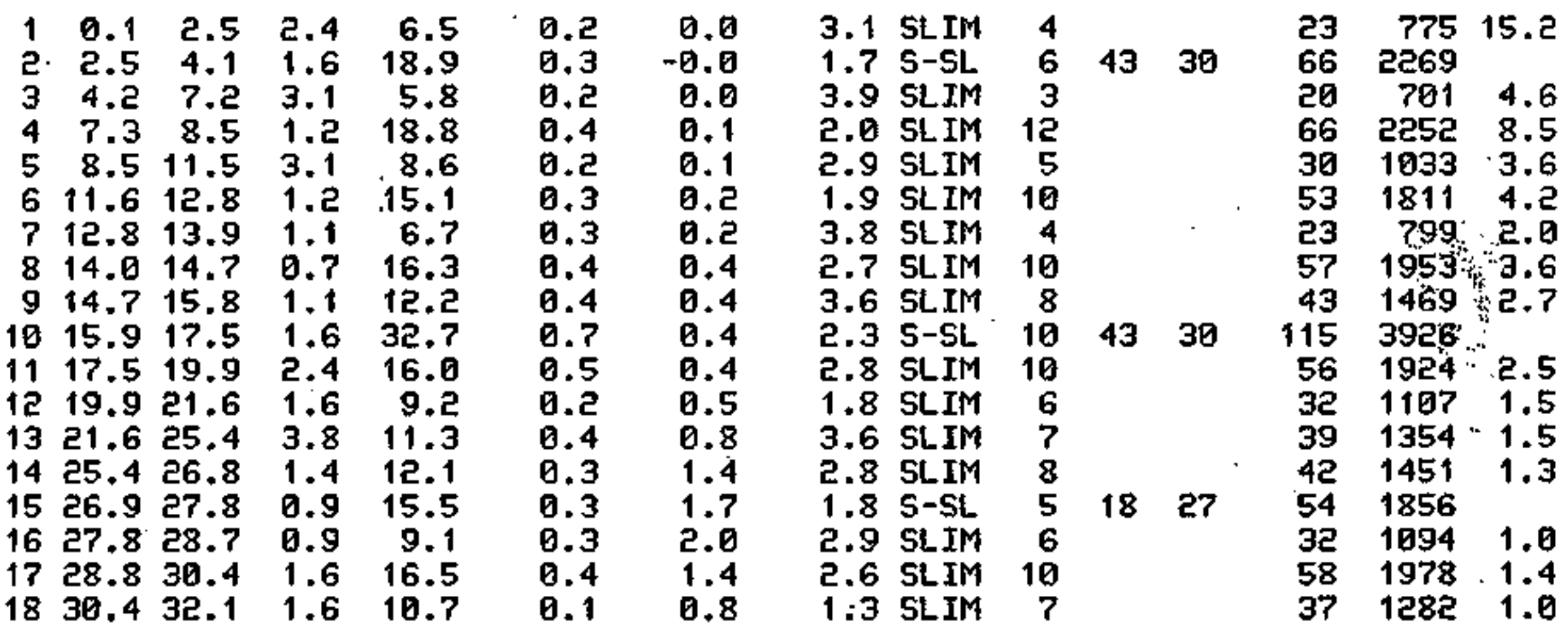

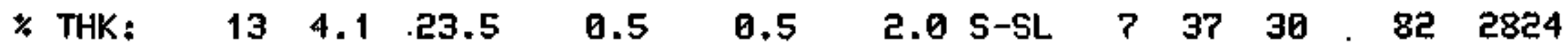

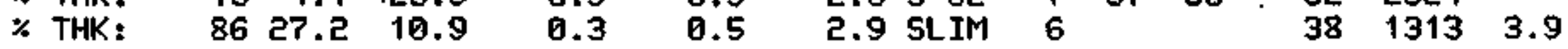


SITE ID: HATQ1

GRDUND WATER DEPTH: 50.0 (FT.)
LOCATION ID: 032

AVERAGE SOIL WEIGHT: 100.0 (PSF)

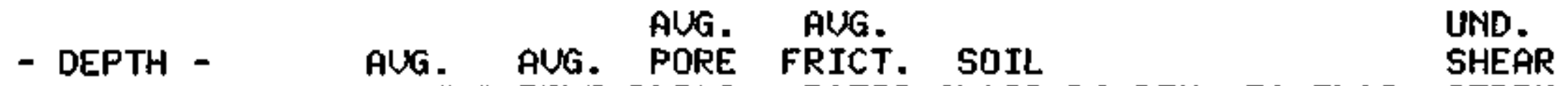
LY FROM TO THK. POINT SLEEUE PRESS. RATIO CLASS BC DEN FA ELAS. STREN DCR

\begin{tabular}{|c|c|c|c|c|c|c|c|c|c|c|c|c|}
\hline $\begin{array}{rr}1 & 0.2 \\
2 & 2.1 \\
3 & 3.6 \\
4 & 6.9 \\
5 & 12.2 \\
6 & 14.6 \\
7 & 16.5 \\
8 & 23.2 \\
9 & 25.1 \\
10 & 30.3\end{array}$ & $\begin{array}{r}2.1 \\
3.5 \\
6.9 \\
12.1 \\
14.5 \\
16.4 \\
23.1 \\
25.0 \\
30.3 \\
40.3\end{array}$ & $\begin{array}{r}1.9 \\
1.4 \\
3.3 \\
5.2 \\
2.4 \\
1.9 \\
6.7 \\
1.9 \\
5.2 \\
10.8\end{array}$ & $\begin{array}{r}8.6 \\
16.0 \\
10.2 \\
23.1 \\
22.1 \\
14.4 \\
36.6 \\
69.3 \\
22.1 \\
11.9\end{array}$ & $\begin{array}{l}0.3 \\
0.3 \\
0.4 \\
0.4 \\
0.6 \\
0.3 \\
0.7 \\
0.9 \\
0.6 \\
0.3\end{array}$ & $\begin{array}{r}0.0 \\
0.0 \\
-0.0 \\
0.0 \\
0.0 \\
0.2 \\
0.2 \\
0.2 \\
0.2 \\
0.8\end{array}$ & $\begin{array}{l}3.4 \\
2.0 \\
3.5 \\
1.8 \\
2.7 \\
2.2 \\
2.0 \\
1.5 \\
2.6 \\
2.1\end{array}$ & $\begin{array}{l}\text { SLIM } \\
\text { SLIM } \\
\text { SLIM } \\
\text { S-SL } \\
\text { SLIM } \\
\text { SLIM } \\
\text { S-SL* } \\
\text { SAMD* } \\
\text { SLIM } \\
\text { SLIM }\end{array}$ & $\begin{array}{r}5 \\
10 \\
6 \\
7 \\
14 \\
9 \\
12 \\
12 \\
14 \\
7\end{array}$ & $\begin{array}{l}43 \\
54\end{array}$ & $\begin{array}{l}30 \\
32\end{array}$ & $\begin{array}{r}30 \\
56 \\
36 \\
81 \\
77 \\
50 \\
128 \\
211 \\
77 \\
42\end{array}$ & $\begin{array}{l}1031 \\
1918 \\
1224 \\
2771 \\
2647 \\
1725 \\
4394 \\
2654 \\
1429\end{array}$ \\
\hline $\begin{array}{l}\text { THK: } \\
\text { THK: } \\
\text { THK: }\end{array}$ & $\begin{array}{r}4 \\
29 \\
65\end{array}$ & $\begin{array}{r}1.9 \\
11.9 \\
26.1\end{array}$ & $\begin{array}{l}60.3 \\
30.7 \\
14.8\end{array}$ & $\begin{array}{l}0.9 \\
0.6 \\
0.4\end{array}$ & $\begin{array}{l}0.2 \\
0.1 \\
0.4\end{array}$ & $\begin{array}{l}1.5 \\
1.9 \\
2.5\end{array}$ & $\begin{array}{l}\text { SAND } \\
\text { S-SL } \\
\text { SLIM }\end{array}$ & $\begin{array}{r}12 \\
9 \\
9\end{array}$ & $\begin{array}{l}54 \\
43\end{array}$ & $\begin{array}{l}32 \\
31\end{array}$ & $\begin{array}{r}211 \\
107 \\
51\end{array}$ & $\begin{array}{l}3684 \\
1777\end{array}$ \\
\hline
\end{tabular}


SITE ID: HATQ1

GROUND WATER DEPTH: 50.0 (FT.)
LOCATION ID: 933

AUERAGE SOIL WEIGHT: 100.0 (PSF)

$\begin{array}{llll} & \\ \text { - DEPTH - } & \text { AUG. AUG. PORE FRICT. SOIL } & \text { UND. } \\ \end{array}$

LY FROM TO THK. POINT SLEEUE PRESS. RATIO CLASS BC DEN FA ELAS. STREN OCR

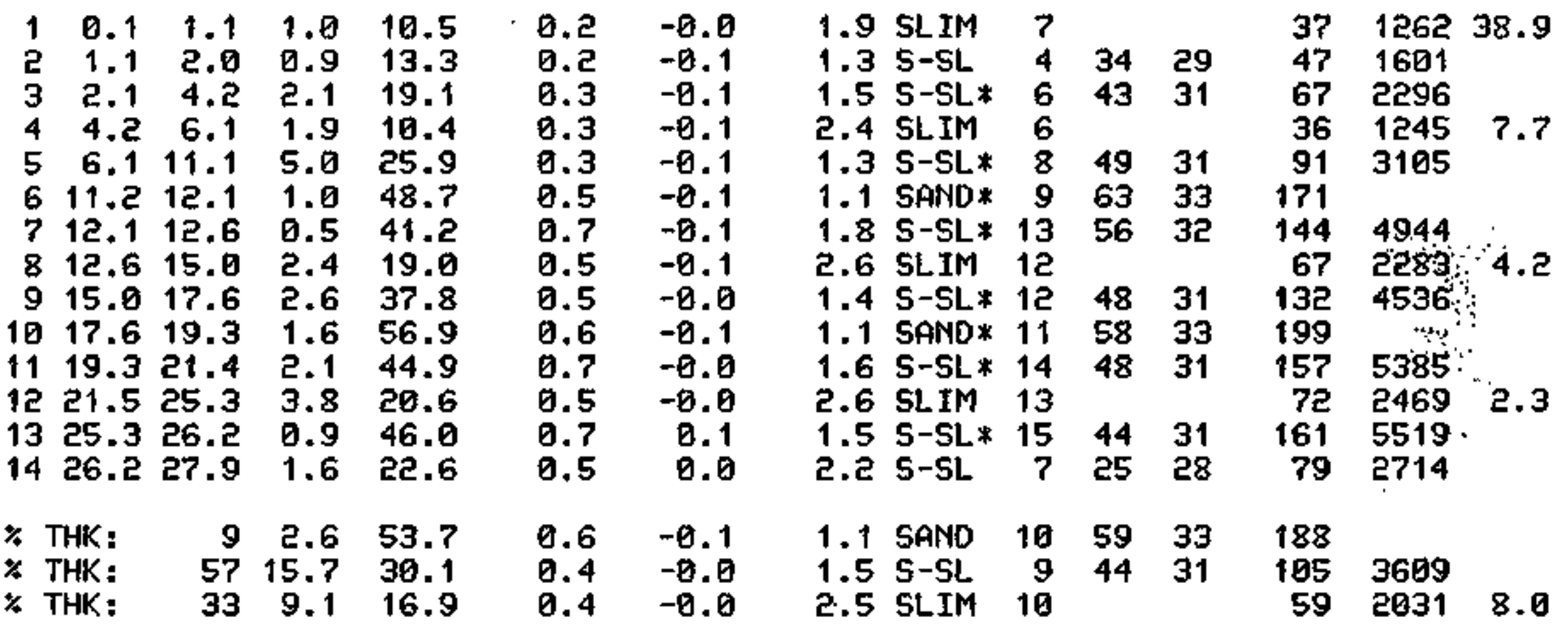


SITE ID: HATO1

GROUND WATER DEPTH: 50.0 (FT.)
LOCATION ID: 034

AUERAGE SOIL WEIGHT: 100.0 (PSF)

$\begin{array}{llll}\text { - DEPTH - } & \text { AUG. AUG. } & \text { UND. } \\ \end{array}$ LY FROM TO THK. POINT SLEEUE PRESS. RATIO CLASS BC DEN FA ELAS. STREN DCR

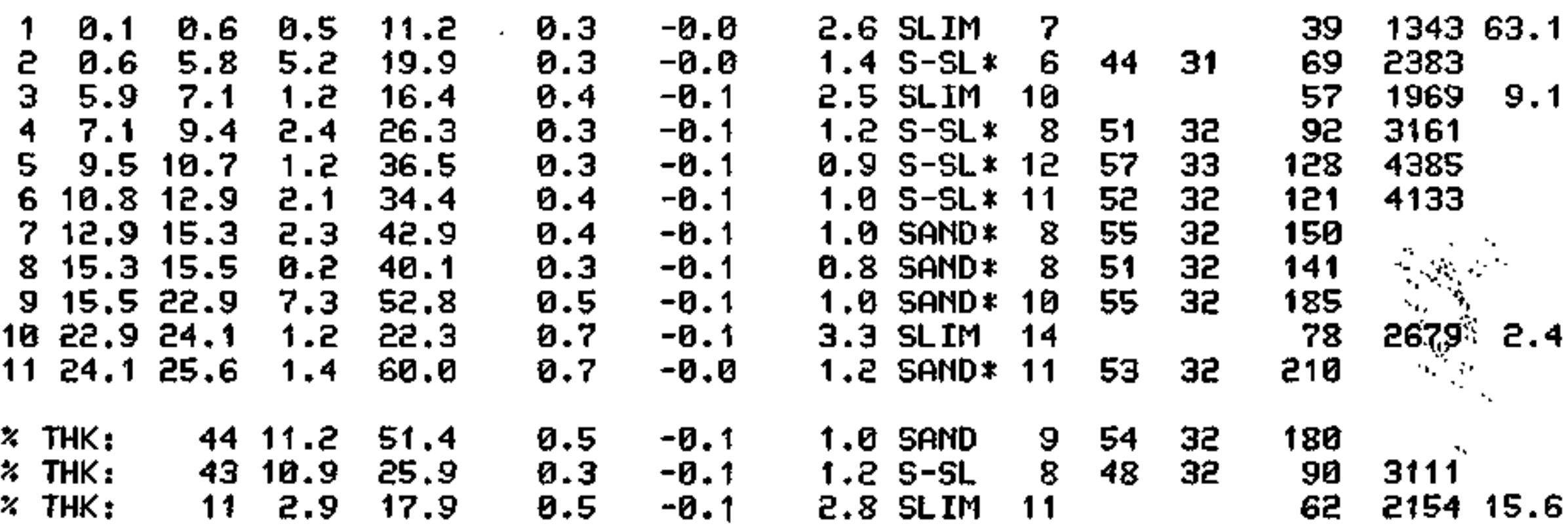


SITE ID: HATO1

GROUNO WATER DEPTH: 50.0 (FT.)
LOCATION ID: 035

AUERAGE SOIL WEIGHT: 100.0 (PSF)

AUG. AUG. UND.

- DEPTH - AUG. AUG. PORE FRICT. SOIL SHEAR

LY FROM TO THK. POINT SLEEUE PRESS, RATIO CLASS BC DEN FA ELAS. STREN OCR

\begin{tabular}{|c|c|c|c|c|c|c|c|c|c|c|c|c|}
\hline $\begin{array}{rr}1 & 0.1 \\
2 & 5.6 \\
3 & 9.9 \\
4 & 17.4 \\
5 & 23.8 \\
6 & 25.6 \\
7 & 26.7 \\
8 & 27.4\end{array}$ & $\begin{array}{r}5.6 \\
9.9 \\
17.3 \\
23.7 \\
25.6 \\
26.6 \\
27.4 \\
28.5\end{array}$ & $\begin{array}{l}5.5 \\
4.3 \\
7.4 \\
6.4 \\
1.8 \\
1.0 \\
0.7 \\
1.1\end{array}$ & $\begin{array}{l}16.5 \\
27.0 \\
38.6 \\
55.4 \\
23.2 \\
39.1 \\
18.9 \\
16.4\end{array}$ & $\begin{array}{l}0.3 \\
0.4 \\
0.4 \\
0.6 \\
0.6 \\
0.4 \\
0.5 \\
0.3\end{array}$ & $\begin{array}{l}-0.1 \\
-0.1 \\
-0.1 \\
-0.1 \\
-0.1 \\
-0.0 \\
-0.1 \\
-0.0\end{array}$ & $\begin{array}{l}1.7 \\
1.5 \\
1.1 \\
1.1 \\
2.4 \\
1.1 \\
2.6 \\
2.1\end{array}$ & $\begin{array}{l}\text { S-SL } \\
\text { S-SL* } \\
\text { S-SL* } \\
\text { SAND* } \\
\text { S-SL } \\
\text { S-SL* } \\
\text { SLIM } \\
\text { SLIM }\end{array}$ & $\begin{array}{r}5 \\
8 \\
12 \\
11 \\
7 \\
13 \\
12 \\
10\end{array}$ & $\begin{array}{l}39 \\
52 \\
52 \\
55 \\
27 \\
39\end{array}$ & $\begin{array}{l}30 \\
32 \\
32 \\
32 \\
29 \\
30\end{array}$ & $\begin{array}{r}58 \\
94 \\
135 \\
194 \\
81 \\
137 \\
66 \\
57\end{array}$ & $\begin{array}{l}1985 \\
3237 \\
4628 \\
\\
2778 \\
4693 \\
2263.1 .8 \\
1971.1 .6\end{array}$ \\
\hline THK & $\begin{array}{r}22 \\
70 \\
6\end{array}$ & 6.4 & $\begin{array}{l}55.4 \\
28.7 \\
17.4\end{array}$ & $\begin{array}{l}0.6 \\
0.4 \\
0.4\end{array}$ & $\begin{array}{l}-0.1 \\
-0.1 \\
-0.0\end{array}$ & $\begin{array}{l}1.1 \\
1.5 \\
2.3\end{array}$ & $\begin{array}{l}\text { SAND } \\
\text { S-SL } \\
\text { SLIM }\end{array}$ & $\begin{array}{r}11 \\
8 \\
10\end{array}$ & $\begin{array}{l}55 \\
45\end{array}$ & & $\begin{array}{r}194 \\
100 \\
60\end{array}$ & $\begin{array}{r}3438 \\
2084\end{array}$ \\
\hline
\end{tabular}


SITE ID: HATV1

GROUND WATER DEPTH: 50.0 (FT.)
LOCATION ID: 036

AUERAGE SOIL WEIGHT: 100.0 (PSF)

- DEPTH - AUG. AUG. PUG. AUG. LY FROM TO THK. POINT SLEELE PRESS. RATIO CLASS BC DEN FA ELAS. STREN OCR

\begin{tabular}{|c|c|c|c|c|c|c|c|c|c|c|c|c|c|}
\hline $\begin{array}{r}0.1 \\
2.3 \\
3.0 \\
3.7 \\
4.7 \\
5.4 \\
6.9 \\
7.4 \\
8.7 \\
12.2 \\
13.2 \\
14.6\end{array}$ & $\begin{array}{r}2.3 \\
3.0 \\
3.7 \\
4.7 \\
5.4 \\
6.8 \\
7.4 \\
8.6 \\
12.2 \\
13.1 \\
14.6 \\
18.1\end{array}$ & $\begin{array}{l}2.1 \\
0.7 \\
0.7 \\
0.9 \\
0.7 \\
1.4 \\
0.6 \\
1.2 \\
3.5 \\
0.9 \\
1.4 \\
3.5\end{array}$ & $\begin{array}{r}12.3 \\
22.6 \\
10.1 \\
17.2 \\
7.4 \\
30.7 \\
14.8 \\
21.7 \\
35.3 \\
41.7 \\
37.2 \\
14.2\end{array}$ & $\begin{array}{l}0.1 \\
0.2 \\
0.2 \\
0.1 \\
0.1 \\
0.3 \\
0.3 \\
0.2 \\
0.4 \\
0.3 \\
0.4 \\
0.5\end{array}$ & $\begin{array}{l}-0.1 \\
-0.1 \\
-0.1 \\
-0.1 \\
-0.1 \\
-0.1 \\
-0.2 \\
-0.1 \\
-0.1 \\
-0.1 \\
-0.2 \\
0.1\end{array}$ & $\begin{array}{l}0.8 \\
0.7 \\
2.1 \\
0.8 \\
1.9 \\
1.0 \\
2.3 \\
0.8 \\
1.1 \\
0.7 \\
1.0 \\
3.5\end{array}$ & $\begin{array}{l}\text { S-SL* } \\
\text { S-SL* } \\
\text { SLIM } \\
\text { S-SL* } \\
\text { SLIM } \\
\text { S-SL* } \\
\text { SLIM } \\
\text { S-SL* } \\
\text { S-SL* } \\
\text { SAND* } \\
\text { S-SL* } \\
\text { SLIM }\end{array}$ & $\begin{array}{r}4 \\
7 \\
6 \\
5 \\
4 \\
10 \\
9 \\
7 \\
11 \\
8 \\
12 \\
9\end{array}$ & $\begin{array}{l}46 \\
55 \\
56 \\
51\end{array}$ & $\begin{array}{l}31 \\
32 \\
32 \\
32\end{array}$ & $\begin{array}{r}43 \\
79 \\
35 \\
60 \\
26 \\
107 \\
52 \\
76 \\
124 \\
146 \\
130 \\
50\end{array}$ & $\begin{array}{r}1477 \\
2799 \\
1211 \\
2064 \\
887 \\
3685 \\
1773 \\
2598 \\
4244 \\
4459 \\
1705\end{array}$ & $\begin{array}{r}10.3 \\
6.1 \\
7.8 \\
2.7\end{array}$ \\
\hline HK: & $\begin{array}{r}5 \\
63 \\
31\end{array}$ & $\begin{array}{r}0.9 \\
11.2 \\
5.5\end{array}$ & $\begin{array}{l}41.7 \\
26.9 \\
12.9\end{array}$ & $\begin{array}{l}0.3 \\
0.3 \\
0.4\end{array}$ & $\begin{array}{r}-0.1 \\
-0.1 \\
0.0\end{array}$ & 0. & $\begin{array}{l}\text { SAND } \\
\text { S-SL } \\
\text { SLIM }\end{array}$ & $\begin{array}{l}8 \\
8 \\
7\end{array}$ & & & $\begin{array}{r}146 \\
94 \\
45\end{array}$ & $\begin{array}{l}3233 \\
1545\end{array}$ & \\
\hline
\end{tabular}


SITE ID: HATO

GROUND WATER DEPTH: 50.0 (FT.)
LOCATION ID: 037

AUERAGE SOIL WEIGHT: 100.0 (PSF)

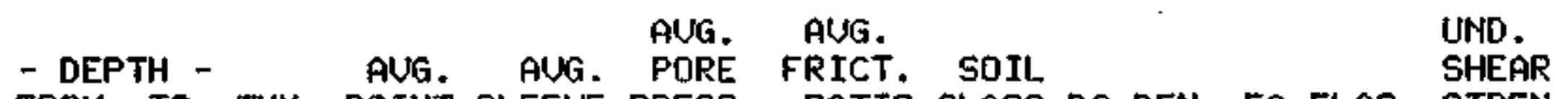
LY FROM TO THK. POINT SLEEUE PRESS. RATIO CLASS BC DEN FA ELAS. STREN OCR

\begin{tabular}{|c|c|c|c|c|c|c|c|c|c|c|c|c|c|}
\hline $\begin{array}{rr}1 & 0.1 \\
2 & 2.0 \\
3 & 5.1 \\
4 & 9.2 \\
5 & 10.4 \\
6 & 11.6 \\
7 & 13.0\end{array}$ & $\begin{array}{r}2.0 \\
5.1 \\
9.2 \\
10.4 \\
11.6 \\
13.0 \\
19.6\end{array}$ & $\begin{array}{l}1.9 \\
3.1 \\
4.0 \\
1.1 \\
1.2 \\
1.4 \\
6.6\end{array}$ & $\begin{array}{r}9.9 \\
24.5 \\
17.3 \\
24.5 \\
12.7 \\
18.1 \\
13.0\end{array}$ & $\begin{array}{l}0.2 \\
0.2 \\
0.4 \\
0.3 \\
0.3 \\
0.4 \\
0.4\end{array}$ & $\begin{array}{l}-0.0 \\
-0.1 \\
-0.1 \\
-0.1 \\
-0.0 \\
-0.0 \\
0.1\end{array}$ & $\begin{array}{l}1.9 \\
1.0 \\
2.4 \\
1.2 \\
2.6 \\
2.0 \\
2.7\end{array}$ & $\begin{array}{l}\text { SLIM } \\
\text { S-SL* } \\
\text { SLIM } \\
\text { S-SL* } \\
\text { SLIM } \\
\text { S-SL } \\
\text { SLIM }\end{array}$ & $\begin{array}{r}6 \\
8 \\
11 \\
8 \\
8 \\
6 \\
8\end{array}$ & $\begin{array}{l}45 \\
33\end{array}$ & $\begin{array}{l}31 \\
29\end{array}$ & $\begin{array}{l}35 \\
86 \\
60 \\
86 \\
44 \\
63 \\
46\end{array}$ & $\begin{array}{l}1183 \\
2940 \\
2673 \\
2946 \\
1519 \\
2177 \\
1565\end{array}$ & $\begin{array}{r}24.1 \\
8.6 \\
4.2 \\
\therefore 2.6\end{array}$ \\
\hline $\begin{array}{l}\text { \% THK: } \\
\text { * THK: }\end{array}$ & $\begin{array}{l}29 \\
70\end{array}$ & $\begin{array}{r}5.6 \\
13.7\end{array}$ & $\begin{array}{l}22.9 \\
13.8\end{array}$ & $\begin{array}{l}0.3 \\
0.4\end{array}$ & $\begin{array}{r}-0.1 \\
0.0\end{array}$ & $\begin{array}{l}1.3 \\
2.5\end{array}$ & $\begin{array}{l}\text { S-SL } \\
\text { SLIM }\end{array}$ & $\begin{array}{l}7 \\
8\end{array}$ & 45 & 31 & $\begin{array}{l}80 \\
48\end{array}$ & $\begin{array}{l}2758 \\
1656\end{array}$ & 7.5 \\
\hline
\end{tabular}


SITE ID: HATV1

GROUND LAATER DEPTH: 50.0 (FT.)
LOCATION ID: 938

AUERAGE SOIL WEIGHT: 100.0 (PSF)

- DEPTH - AUG. AUG. PURE FRICT. SOIL

UND.

SHEAR

LY FROM TO THK. POINT SLEEUE PRESS. RATIO CLASS BC DEN FA ELAS. STREN DCR

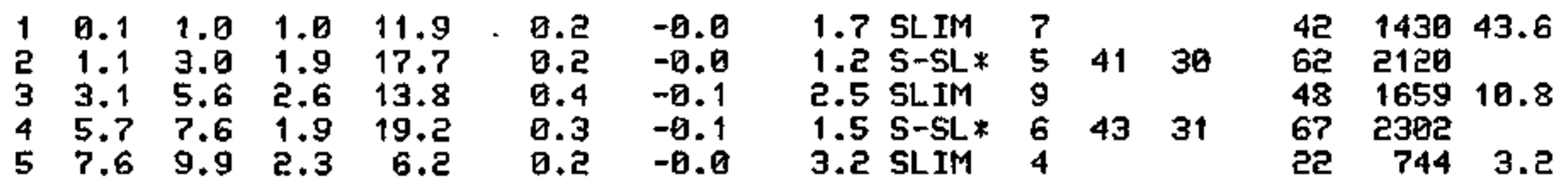

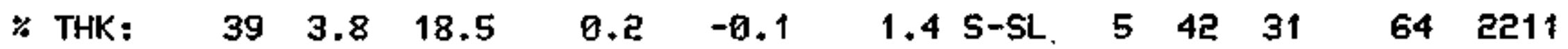

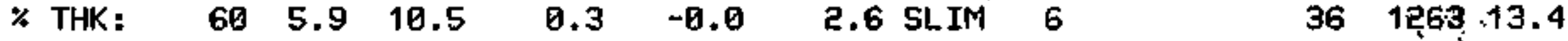


SITE ID: HATO1

GROUND WATER DEPTH: 50.0 (FT.)
LOCATION ID: 039

AUERAGE SOIL WEIGHT: 100.0 (PSF)

$\begin{array}{llll} & & & \\ \text { DEPTH - } & \text { AUG. AUG. AUG. PORE FRICT. SOIL } & \text { UND. } \\ \end{array}$

LY FROM TO THK. POINT SLEEUE PRESS. RATIO CLASS BC OEN FA ELAS. STREM OCR

$\begin{array}{rrrrrrrrrrrrrrr}1 & 0.2 & 2.8 & 2.6 & 16.4 & 0.2 & -0.0 & 1.3 & \text { S-SL* } & 5 & 39 & 30 & 57 & 1967 & \\ 2 & 2.8 & 4.2 & 1.4 & 9.2 & 0.3 & -0.1 & 3.1 & \text { SLIM } & 6 & & & 32 & 1193 & 9.3 \\ 3 & 4.3 & 5.0 & 0.7 & 22.7 & 0.4 & -0.1 & 1.6 & \text { S-SL* } & 7 & 48 & 31 & 79 & 2725 & \\ 4 & 5.0 & 5.9 & 0.9 & 8.5 & 0.2 & -0.1 & 2.9 & \text { SLIM } & 5 & & & 30 & 1015 & 6.3 \\ 5 & 5.9 & 6.4 & 0.4 & 26.1 & 0.4 & -0.0 & 1.4 & 5-S L * & 8 & 53 & 32 & 92 & 3138 & \\ 6 & 6.4 & 8.0 & 1.6 & 9.9 & 0.3 & -0.1 & 2.6 & \text { SLIM } & 6 & & & 35 & 1183 & 5.7 \\ 7 & 8.1 & 16.4 & \mathbf{8 . 3} & \mathbf{3 . 7} & 0.1 & 0.0 & 3.5 & \text { SLIM } & 2 & & & 13 & 442 & 1.5\end{array}$

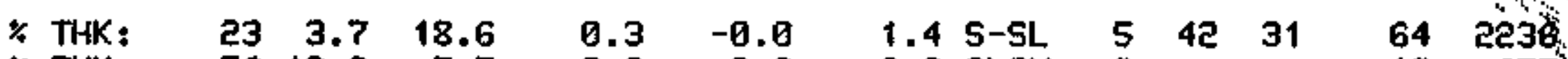

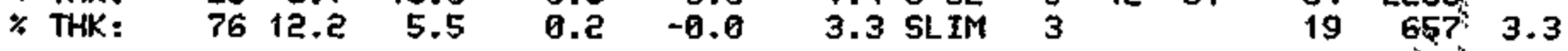


$\begin{array}{llll}\text { - DEPTH - } & \text { AUG. AUG. PORE FRICT. SOIL } & \text { UND. } \\ \text { SHEAR }\end{array}$ LY FROM TO THK. POINT SLEEVE PRESS. RATIO CLASS BC DEN FA ELAS. STREN DCR

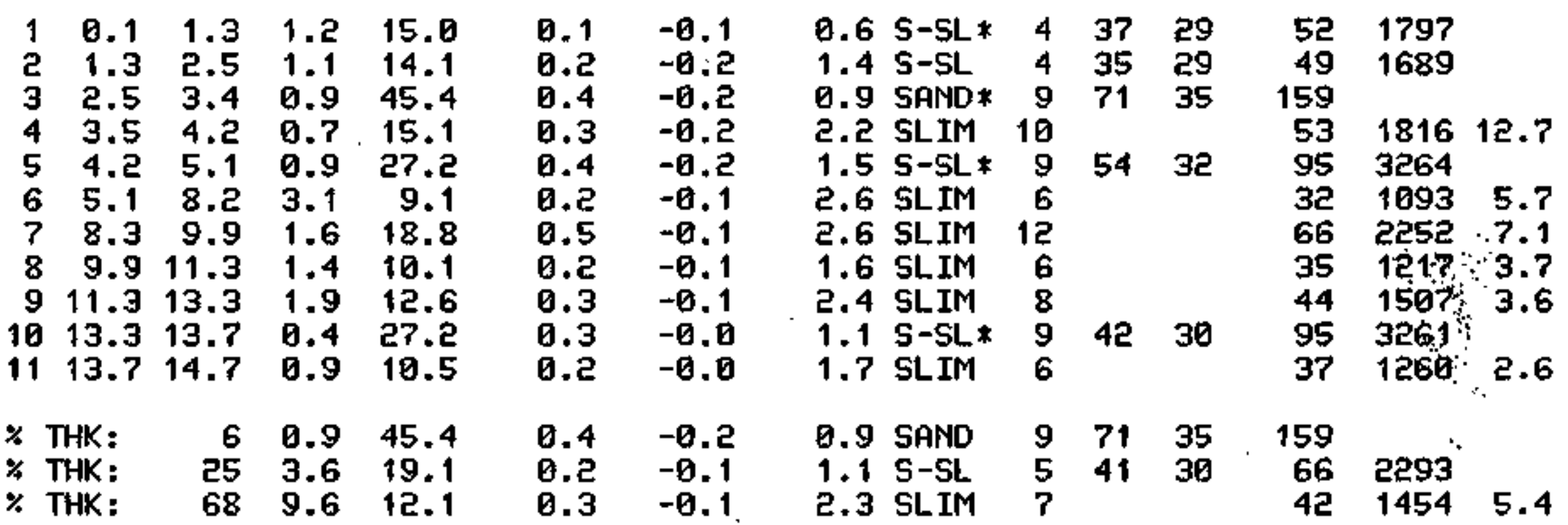




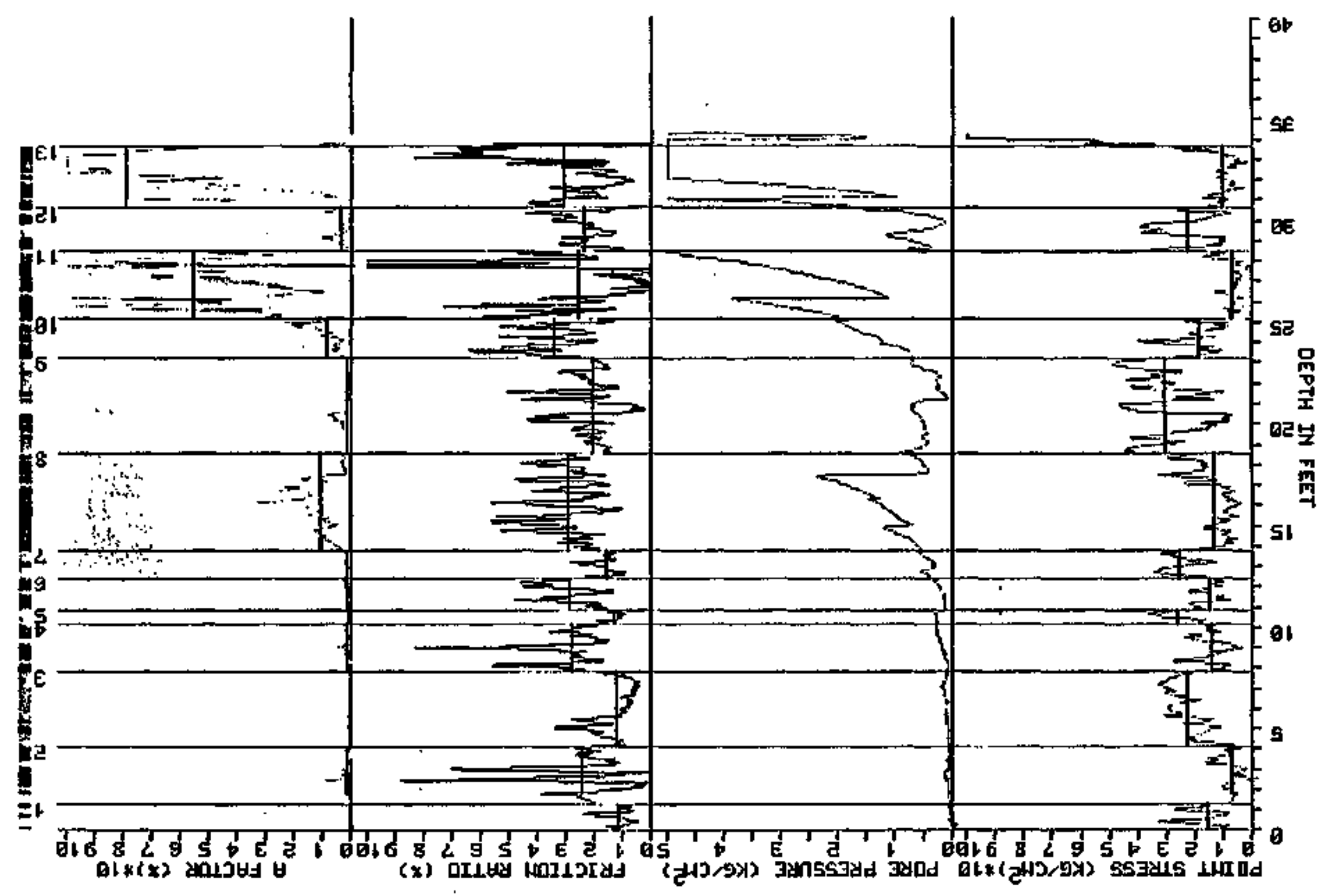

LOQ :QI NOILO307

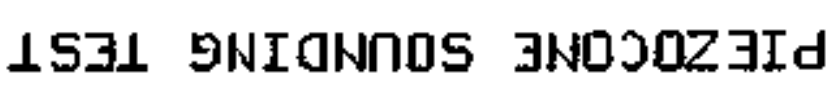




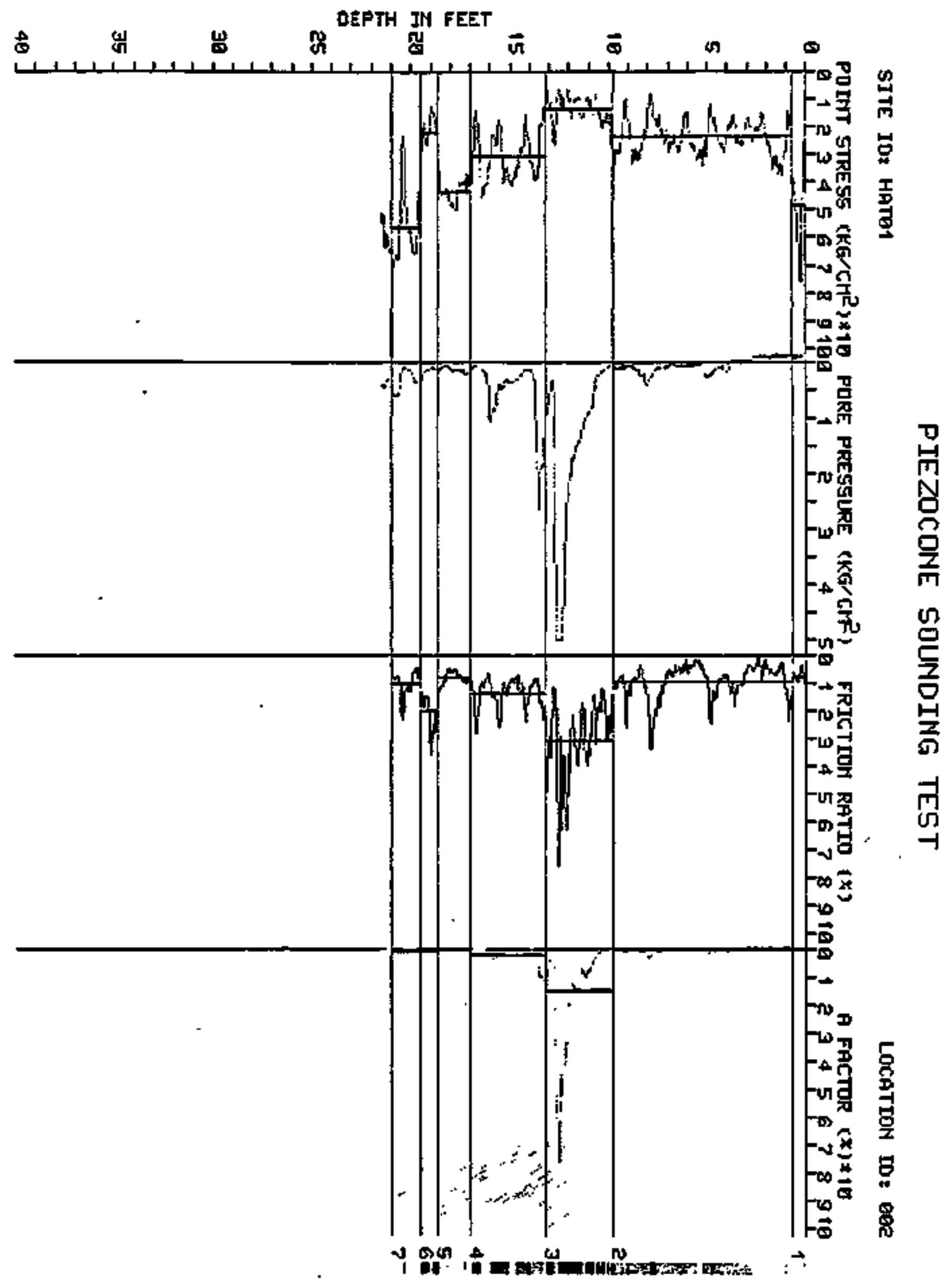




\section{PIEZOCONE SOUNDING TEST}

SITE ID: HATO1

LOCATION TO: 093

POINT STRESS (KG/CH')

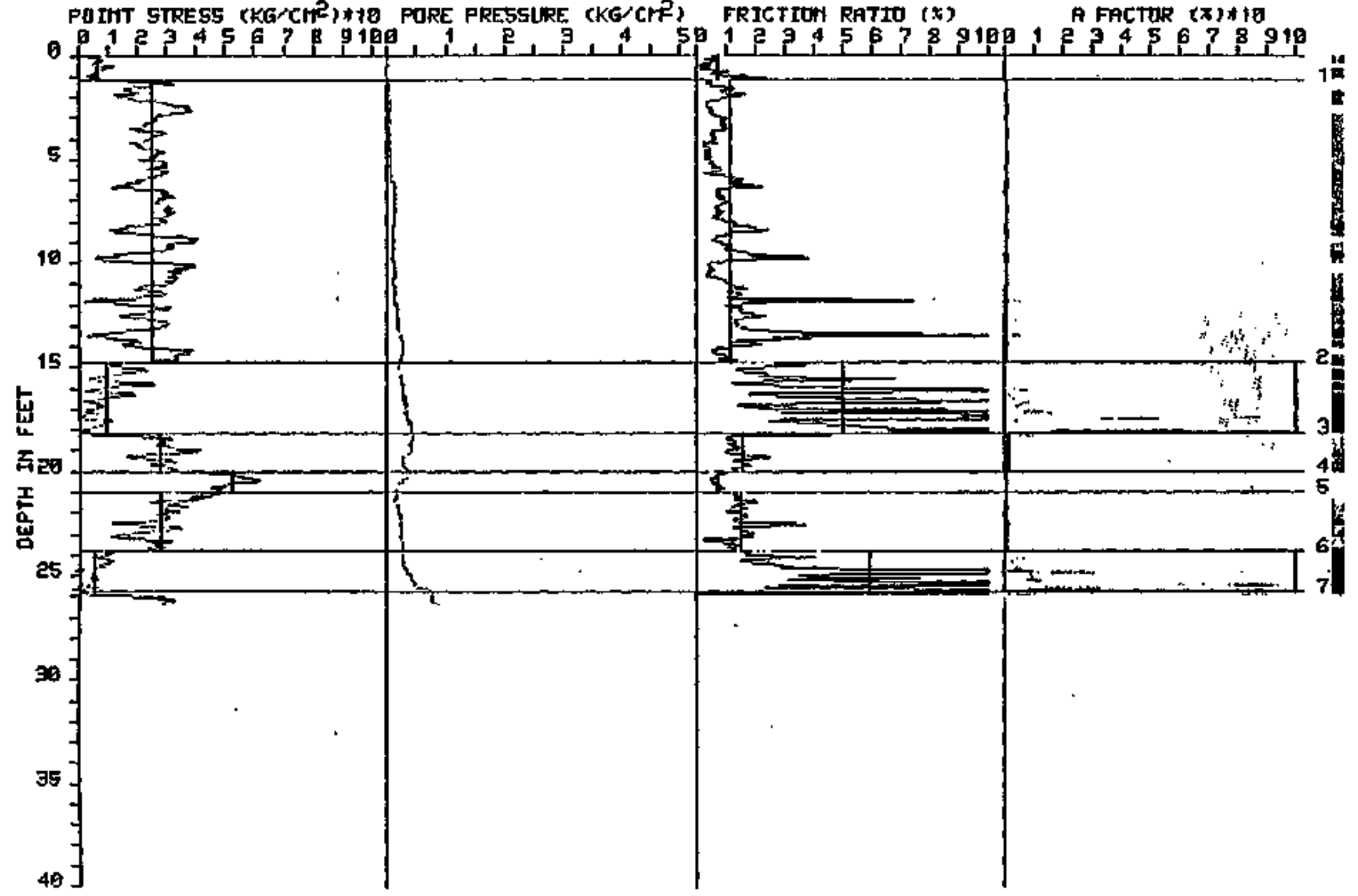




\section{PIEZOCONE SOUNDING TEST}

SITE ID: HATOS

LOCATION IO: 004

PUIMT STRESS (KG $/ \mathrm{CM}^{2}$ ) *10 PORE PRESSURE (KG COF')

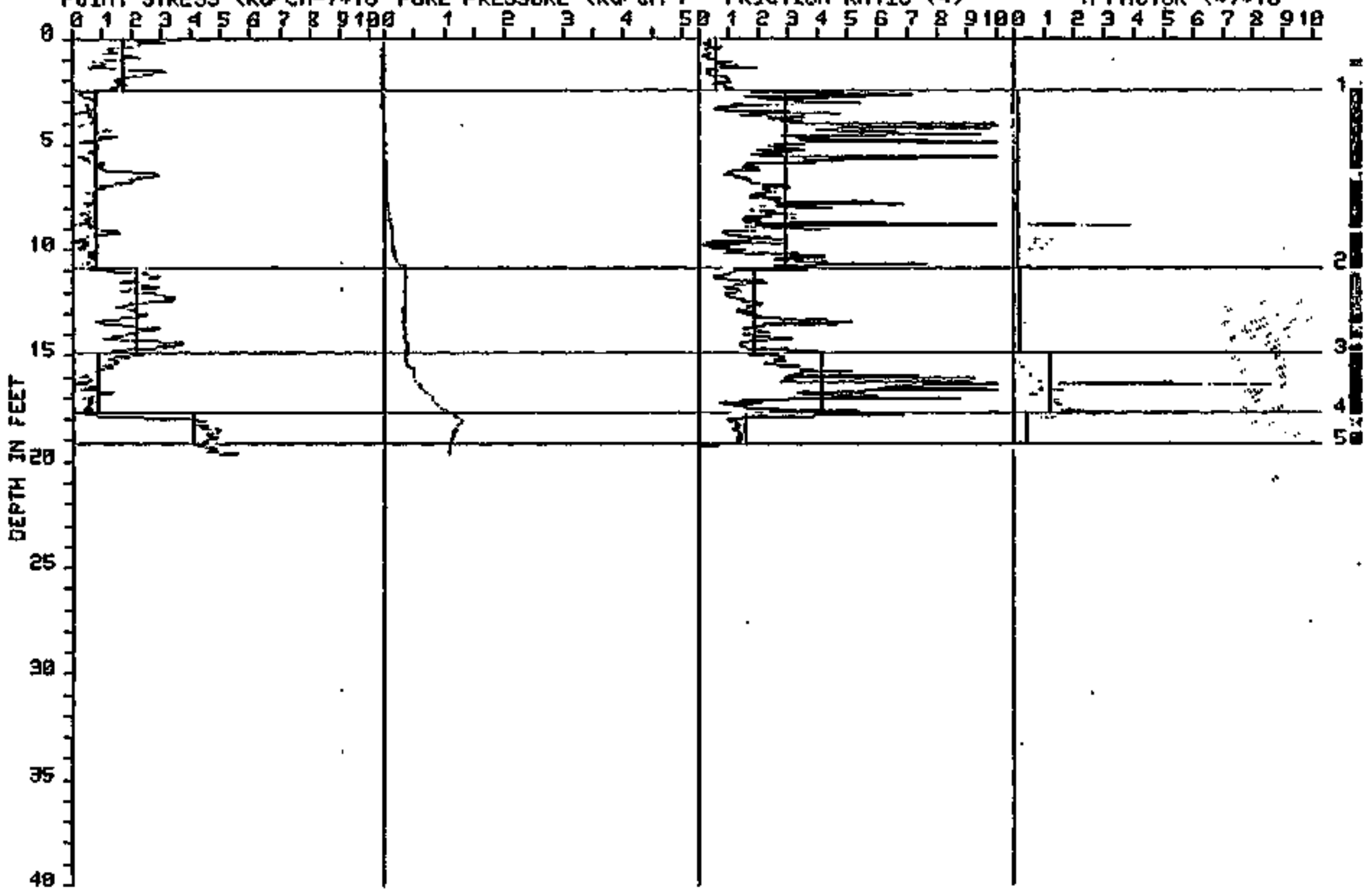




\section{PIEZOCONE SOUNDING TEST}

SITE ID: HATEI

LOCATION LD: 905

POIMT STRE55 (KG/CMP)IIV PORE PRESSURE (KG/CHF) FRICTION RATIO (*)

A FACTOR (x) *10

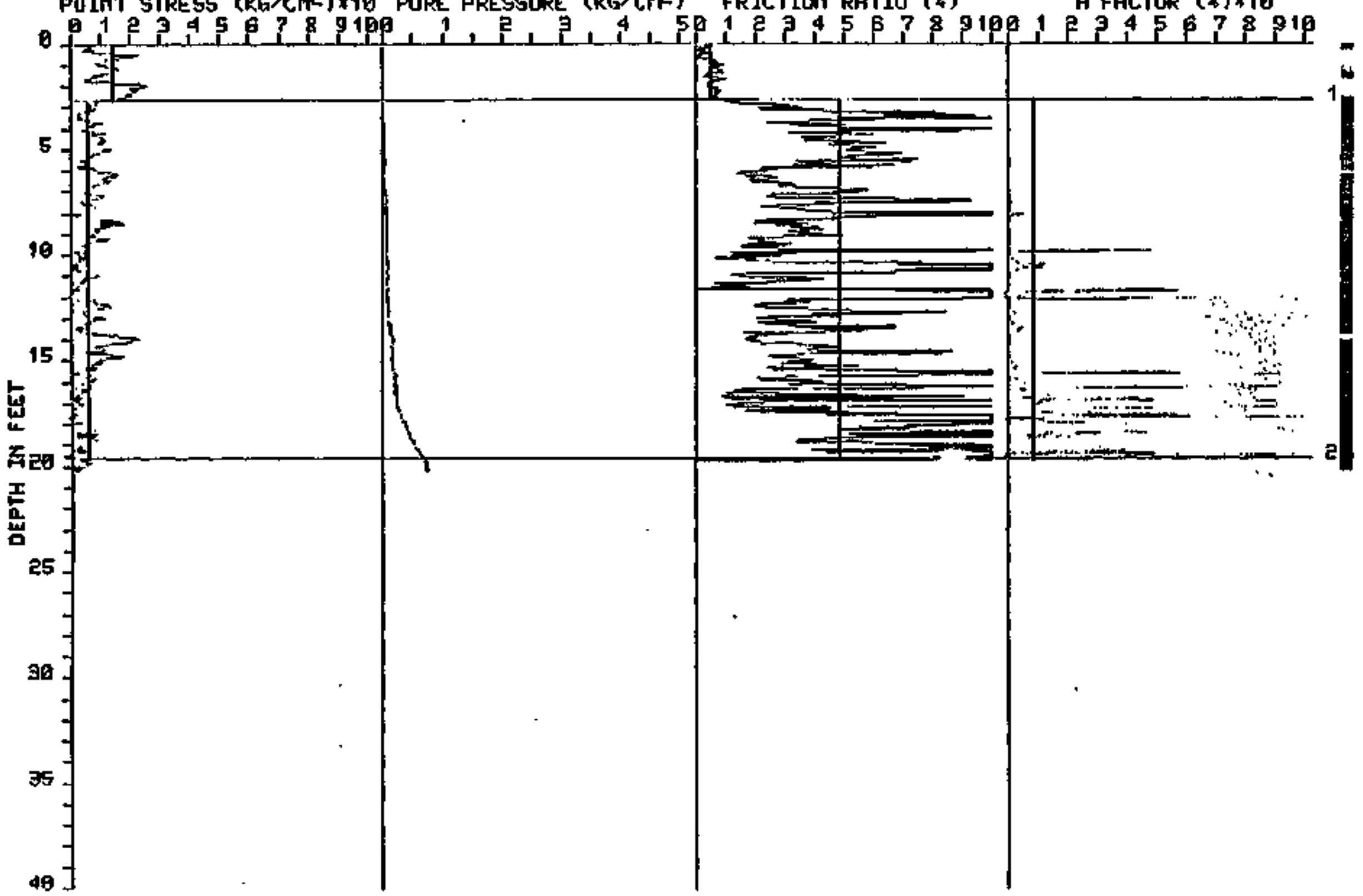




\section{PIEZOCONE SOUNDING TEST.}

SITE ID: HATO1

LOCATION ID: 006

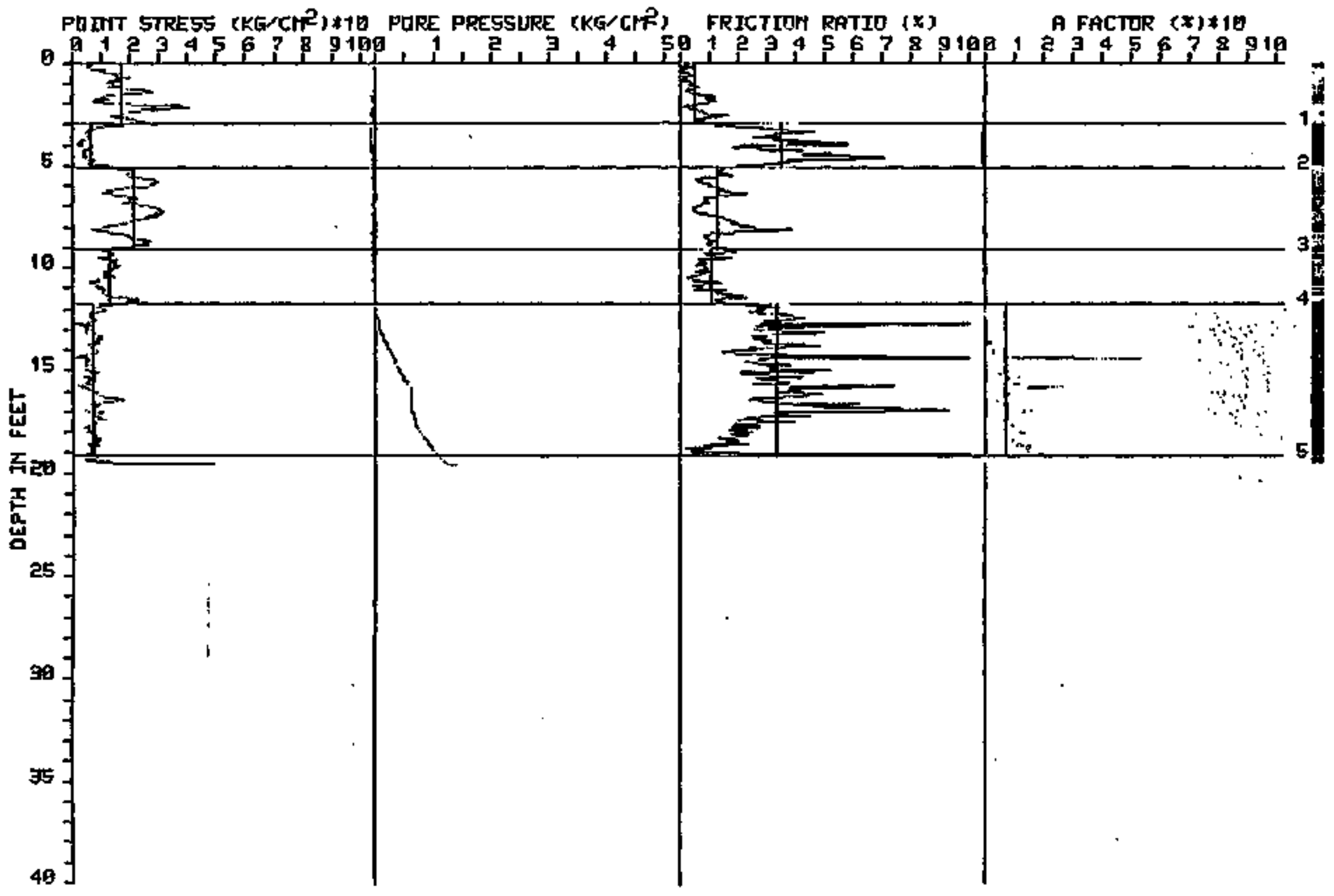




\section{PIEZOCONE SOUNDING TEST.}

SITE ID: HATOI

- LOCATION ID: 007

PUIIT STRESS (KG/CME) N10 PORE PRESSURE (KG/CMF) FRICTION RATIO (X)

A FACTOR $(x) \neq 10$

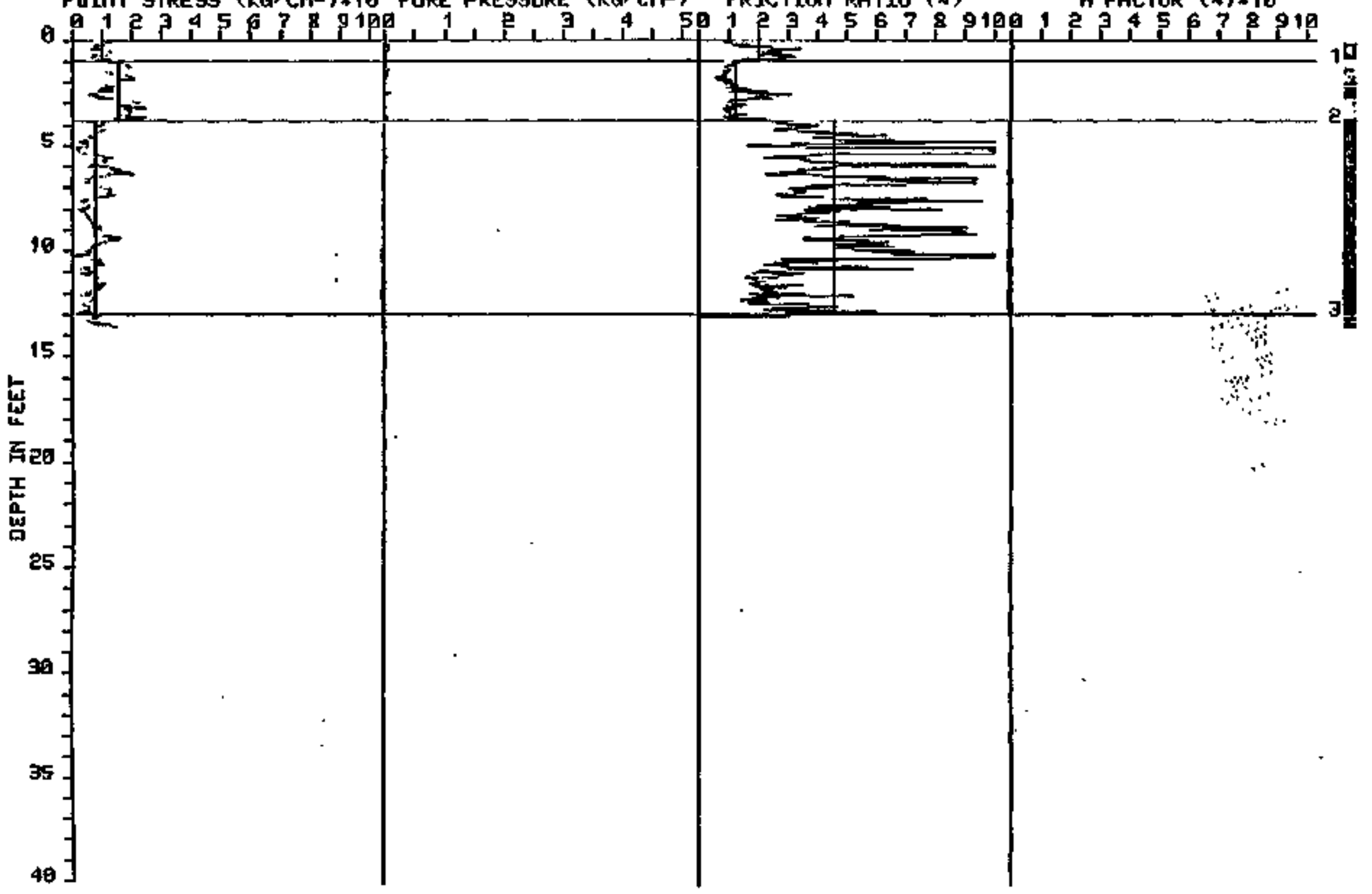




\section{PIEZOCONE SOUNDING TEST.}

SITE ID: HATO1

PU INT STRESS TKE $/ \mathrm{CM}^{2}$ ) \#10 PORE PRESSURE (KG/CMR)

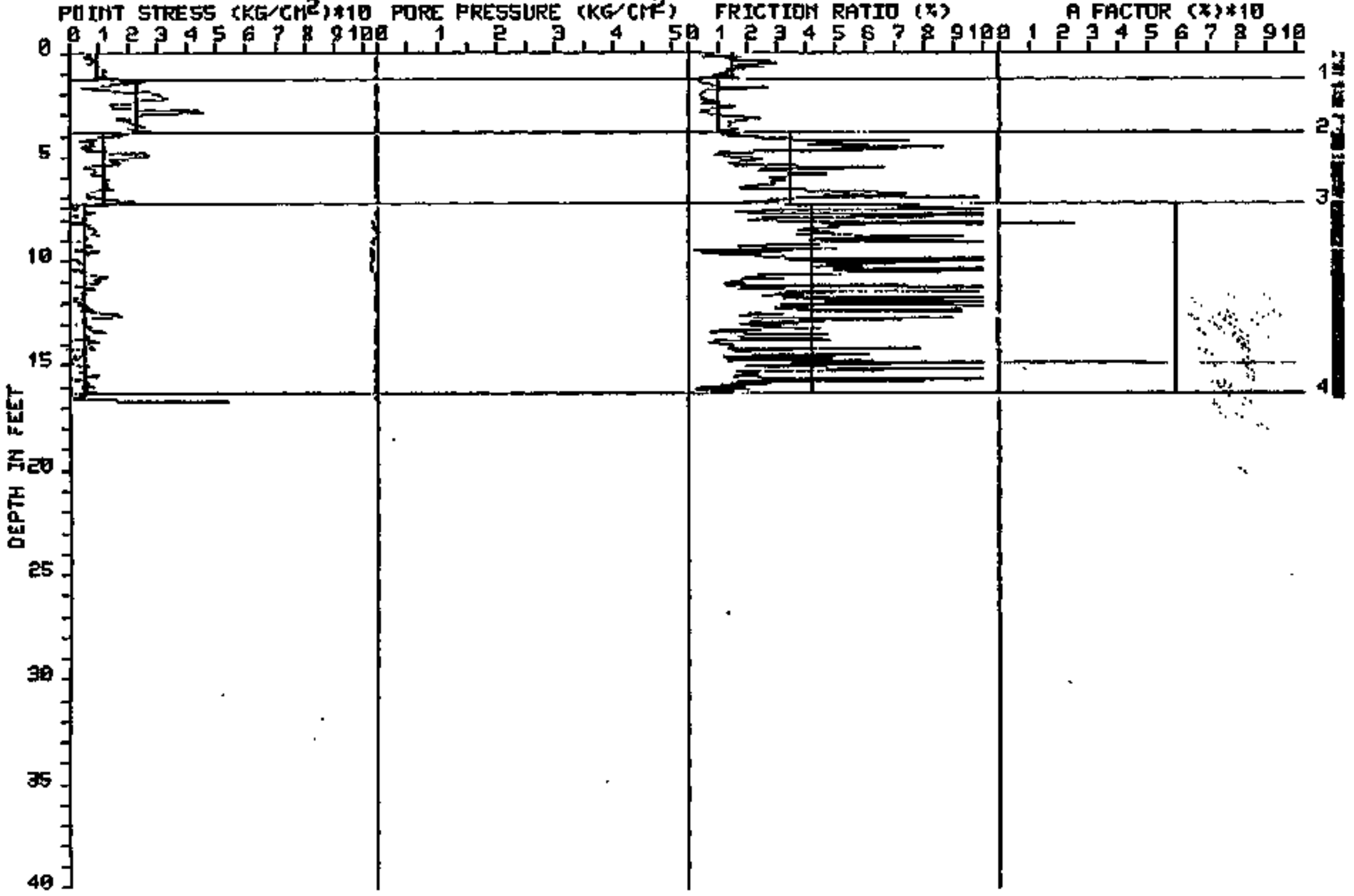




\section{PIEZOCONE SOUNDING TEST}

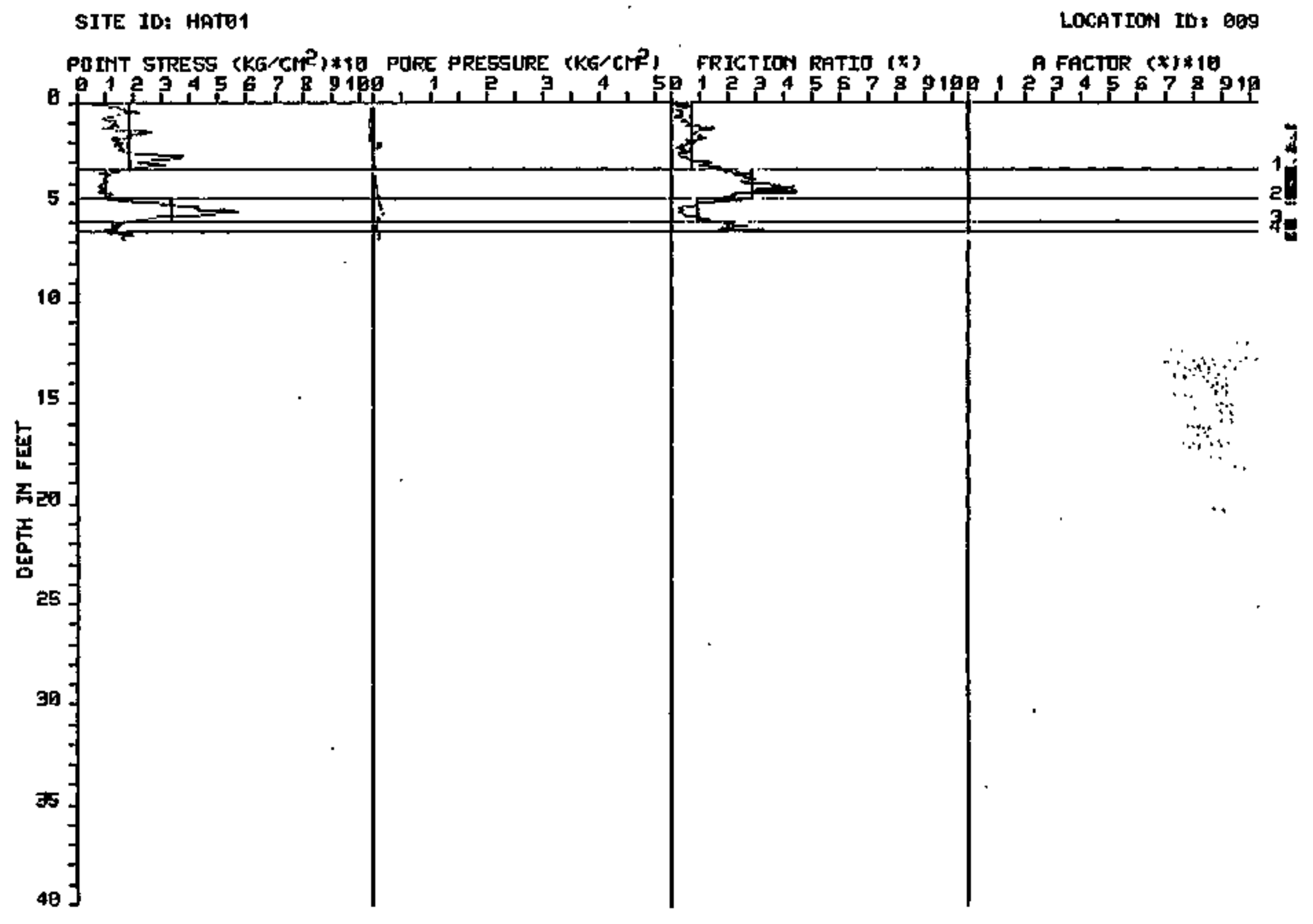




\section{PIEZOCONE SOUNOING TEST.}

SITE ID: HATOI

LOCATION TO: eto

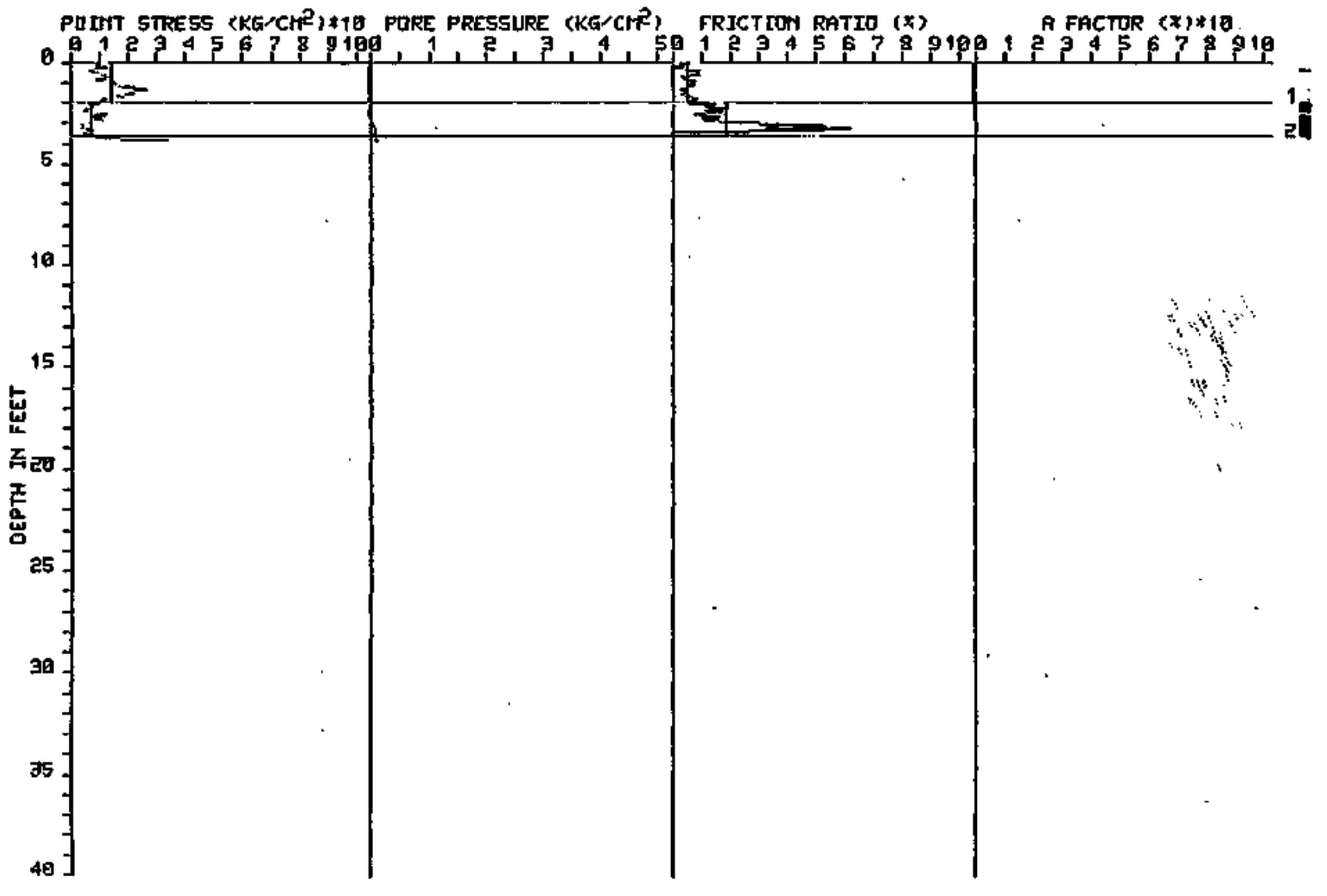




\section{PIEZOCONE SOUNDING TEST}

SITE ID: HATE1

LOCATION TO: O19

PIINT STRESS (KG/CMP)*10 PORE PRESSURE (KG/CME) FRICTIOH RATID ( $x$ )

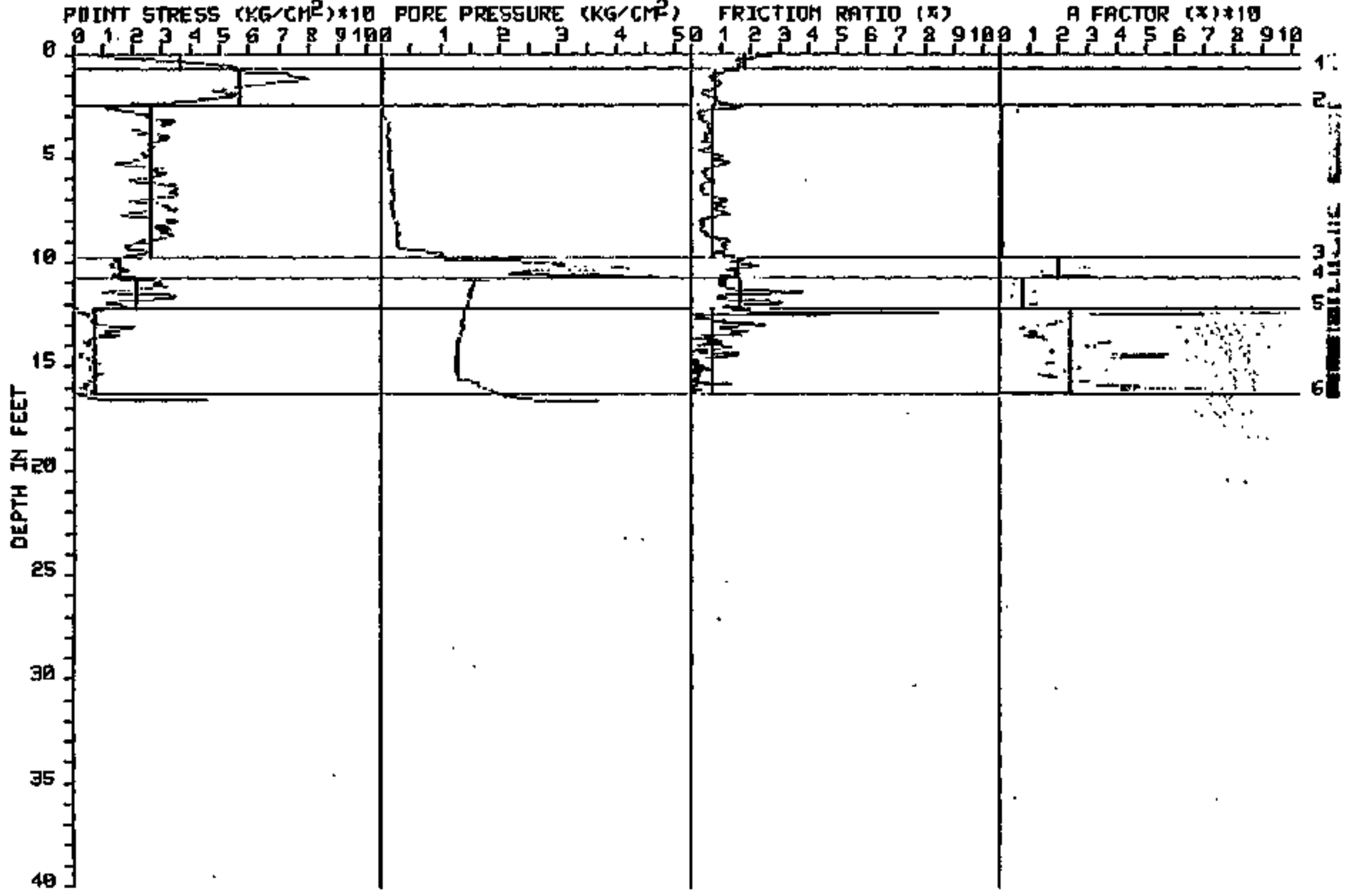




\section{PIEZOCONE SOUNDIMG TEST}

SITE ID2 HATO1

PAIMT STRES5 (KG,CM2) *10 PORE PRESSURE (KG/CHF)

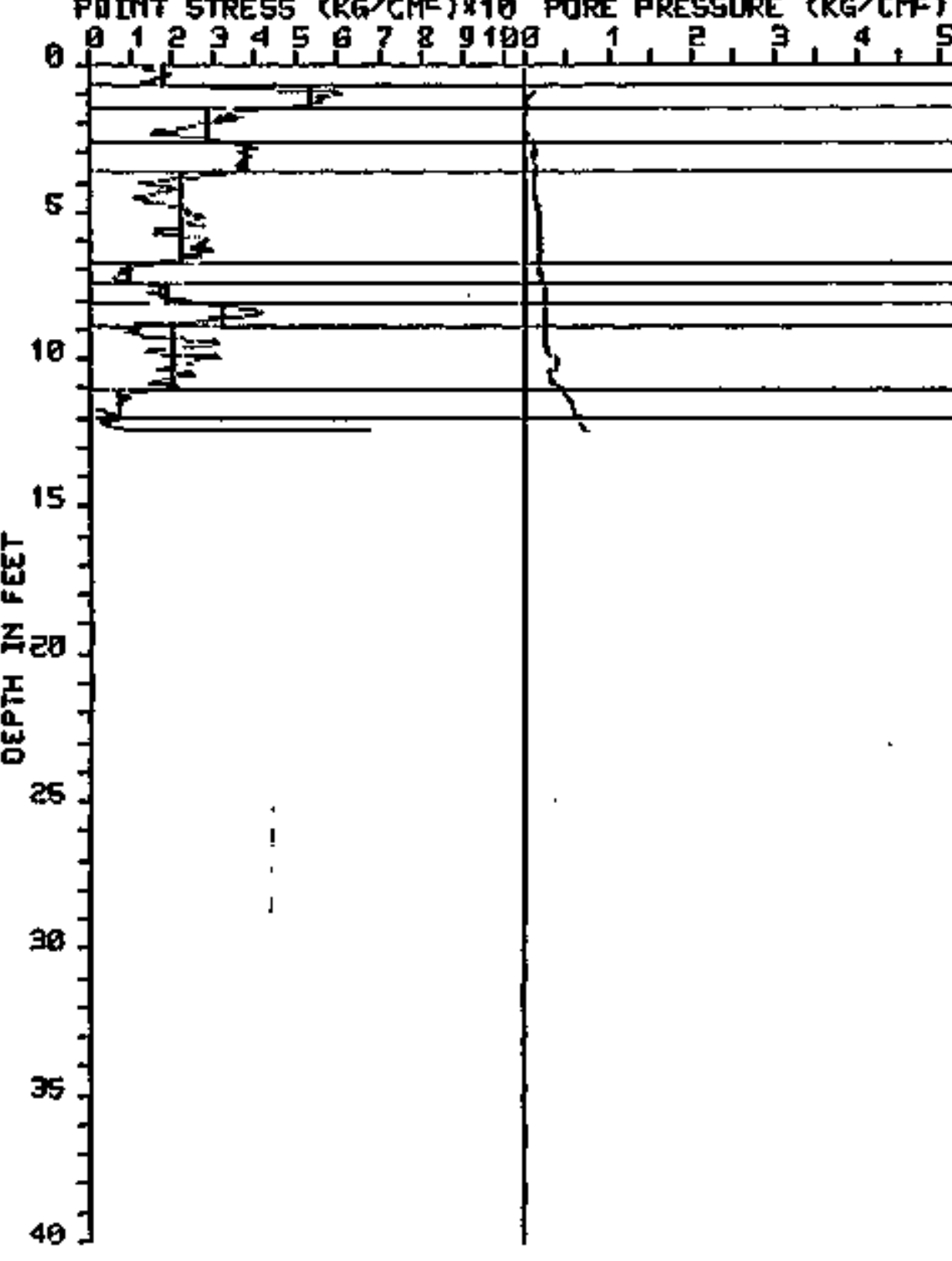

LOCATION ID, OHe \begin{tabular}{l} 
FRICTEOH RATIO (x) \\
R FACTOR (x)*1E \\
\hline
\end{tabular}

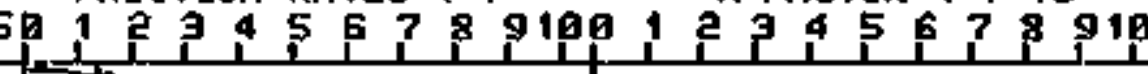

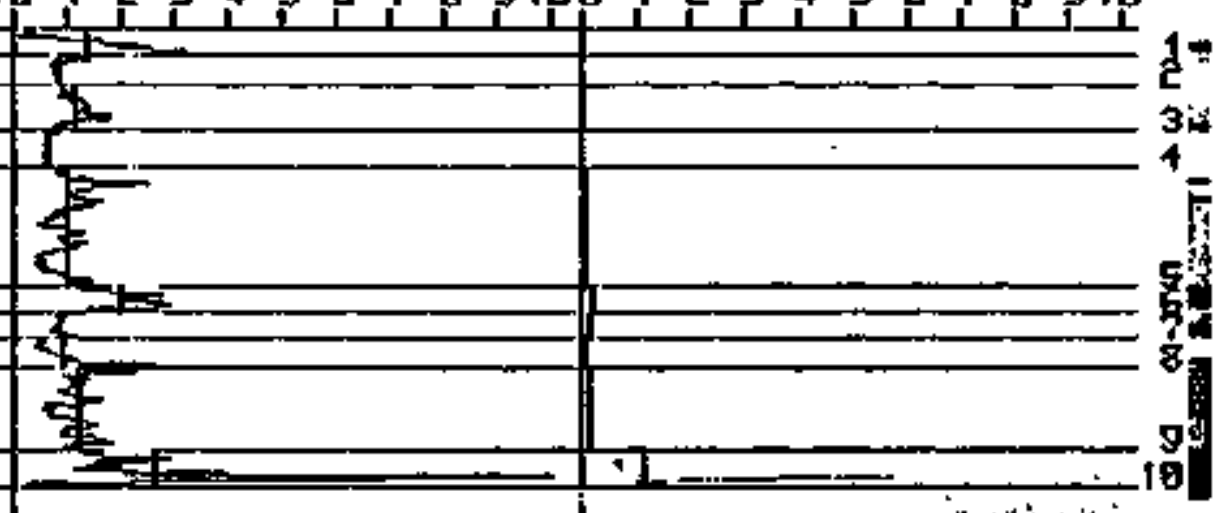


PIEZOCONE SOLNDIMG TEST

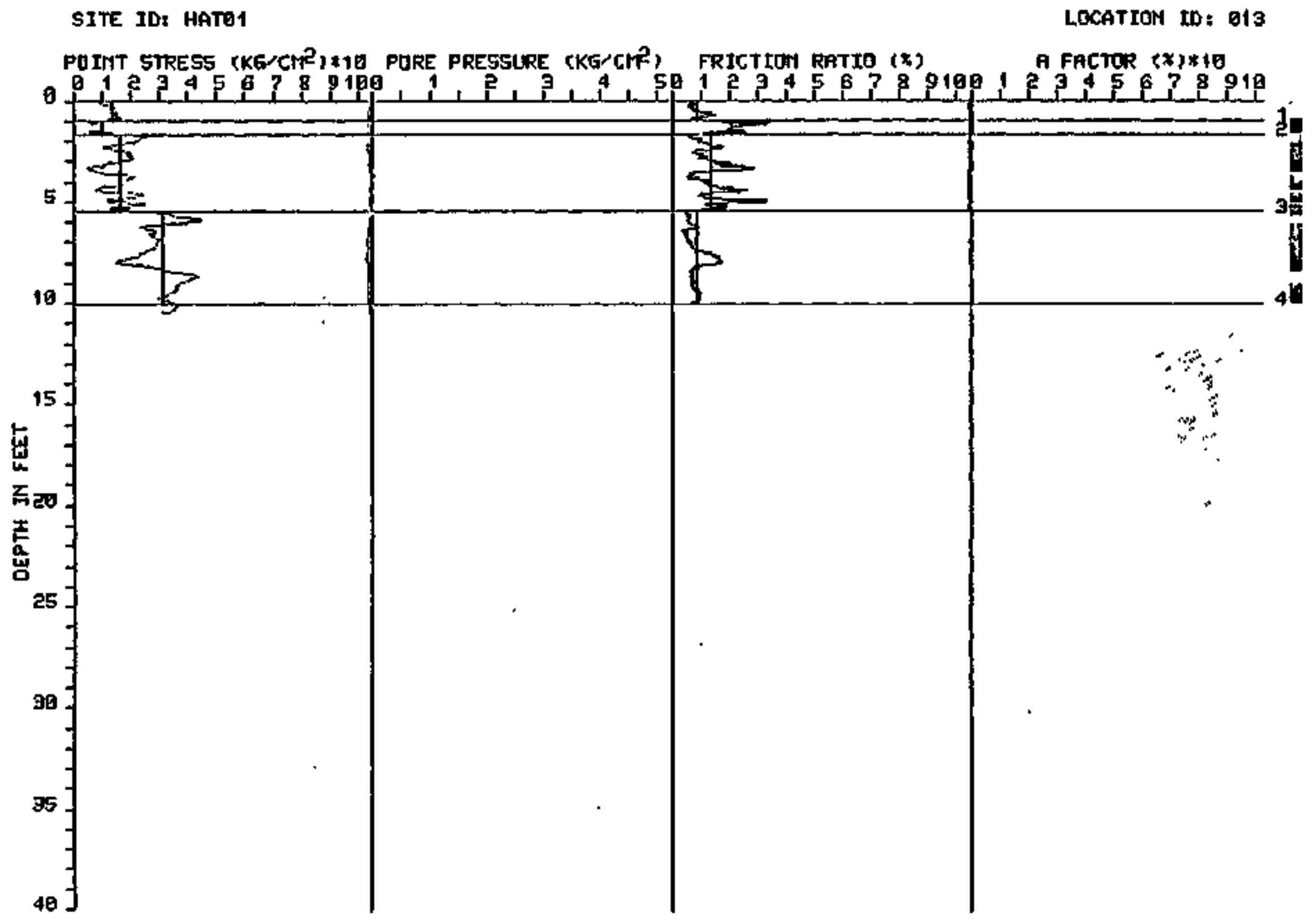




\section{PIEZOCONE SOUNDING TEST}

SITE ID: HATOI

LOCATIOH ID: 014

PQIIT SIRESS (KG/CM ${ }^{2}$ ) *10 PORE PRESSLRE (KE/CH?) FRICTIOH RATIO (\%)

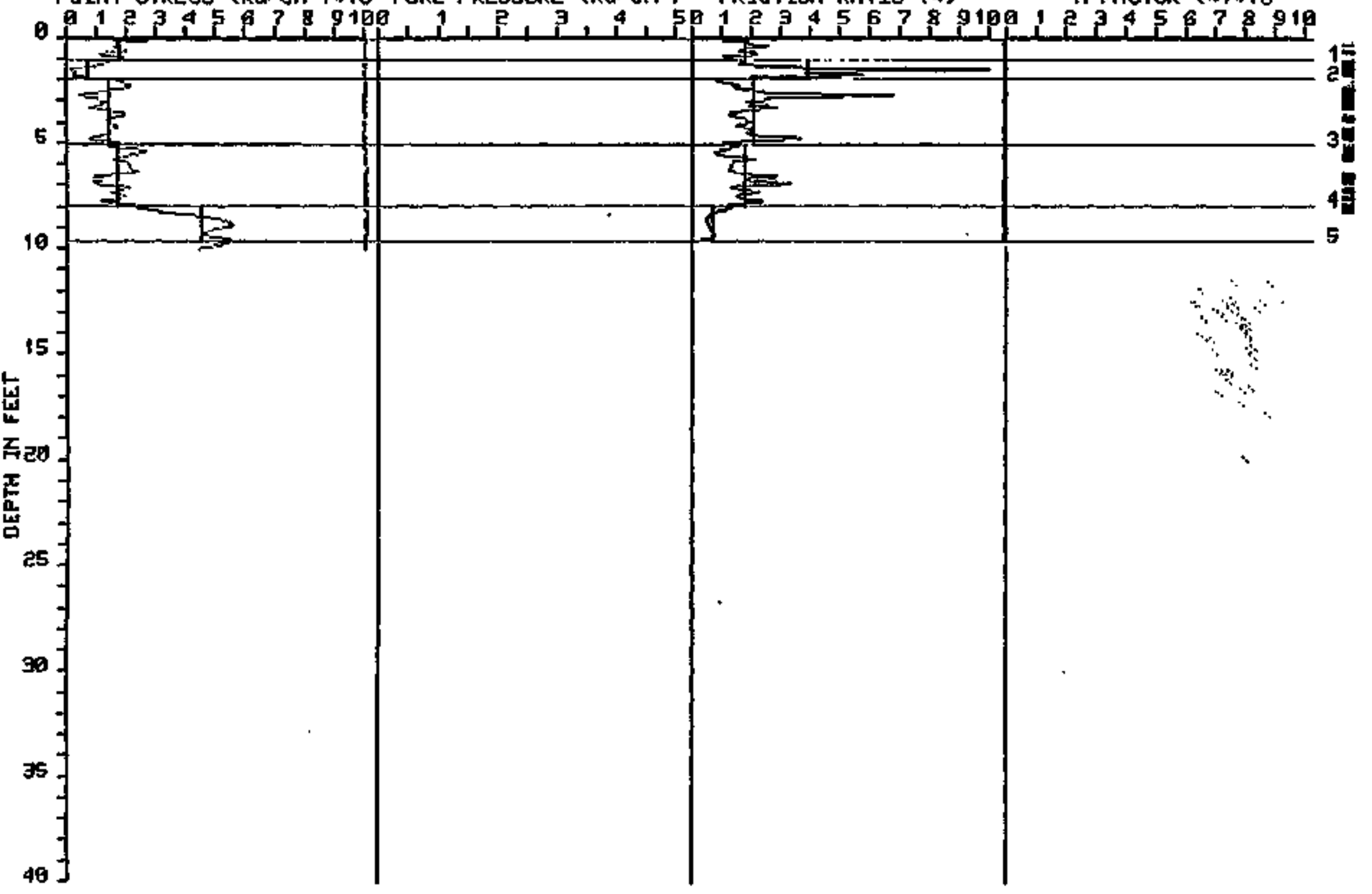




\section{PIEZOCDNE SOUNDING TEST}

SITE IDE HATOI

LOCATION TO: 015

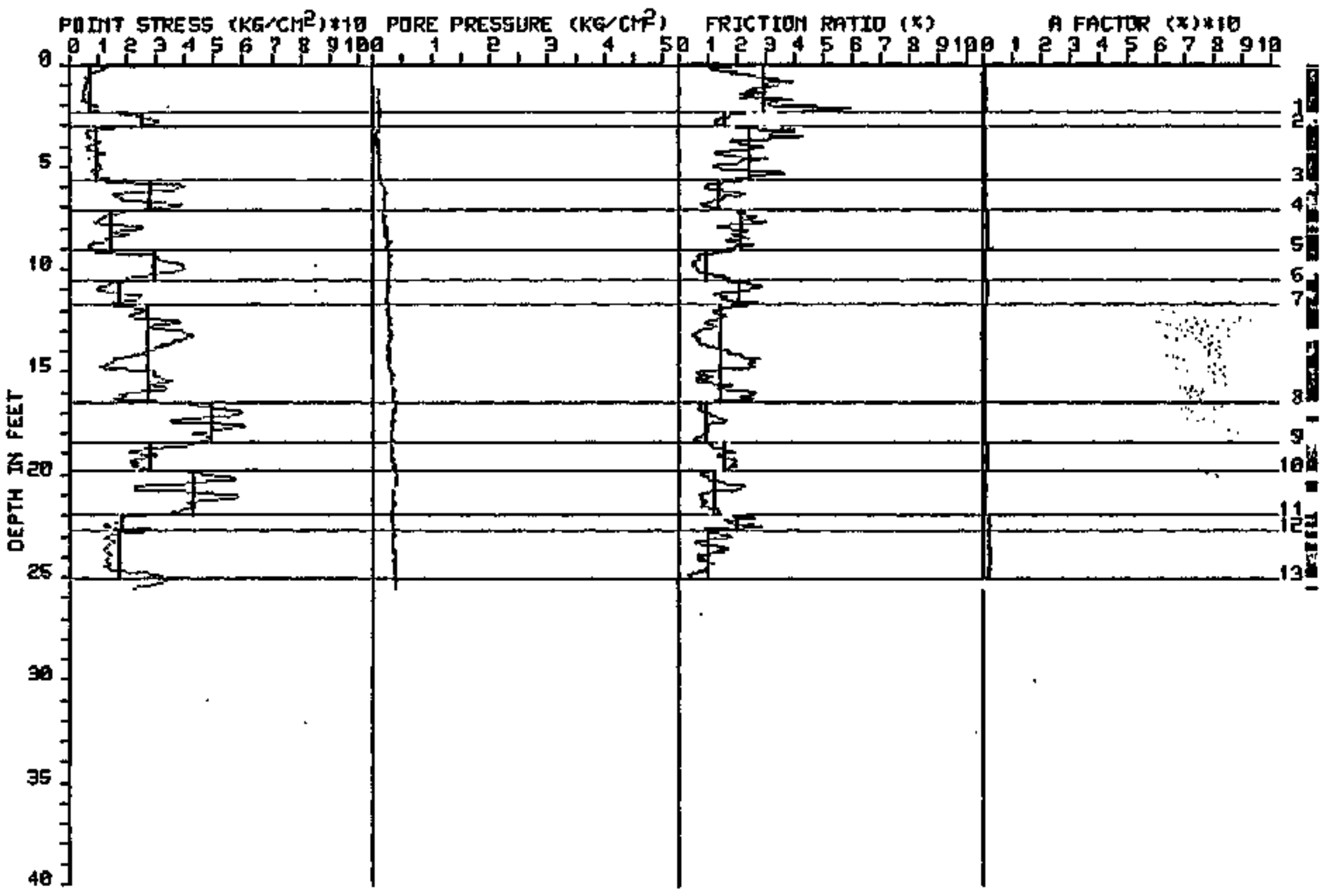




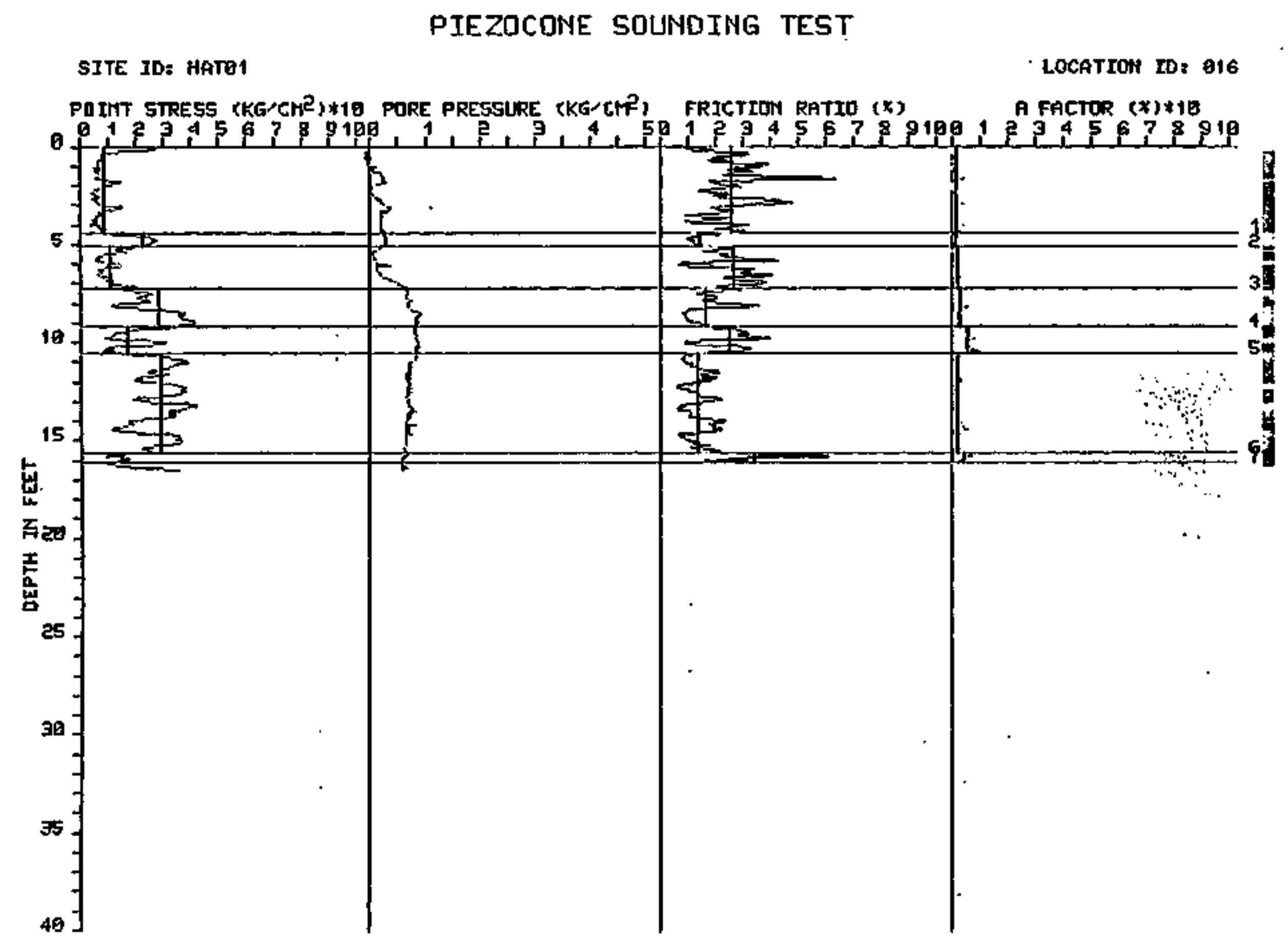


PIEZOCONE SOUNDING TEST

SITE ID: HATEI

LOCATION ID: 017

POIMT STRESS (KG/CTT2)*10 PORE PRESSURE (KG/CMR) FRICTION RATIO (x)

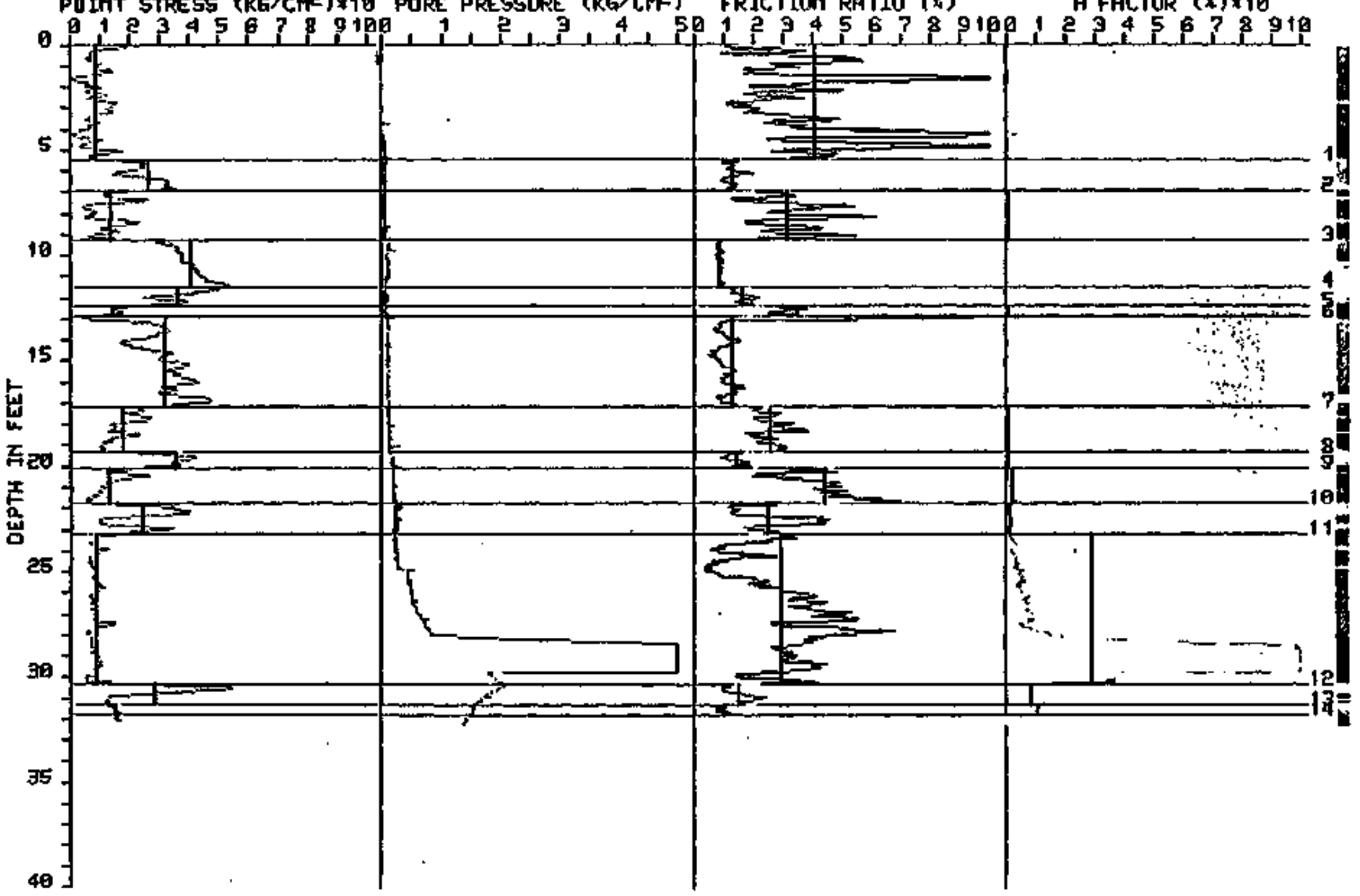




\section{PIEZOCONE SOLNDING TEST}

SIFE IDS HATO1

LOCATION ID: 018

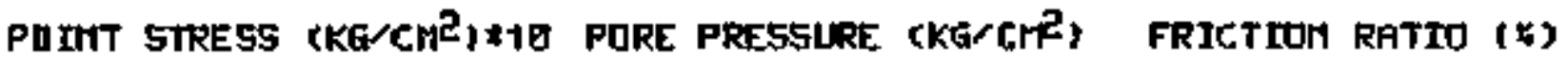

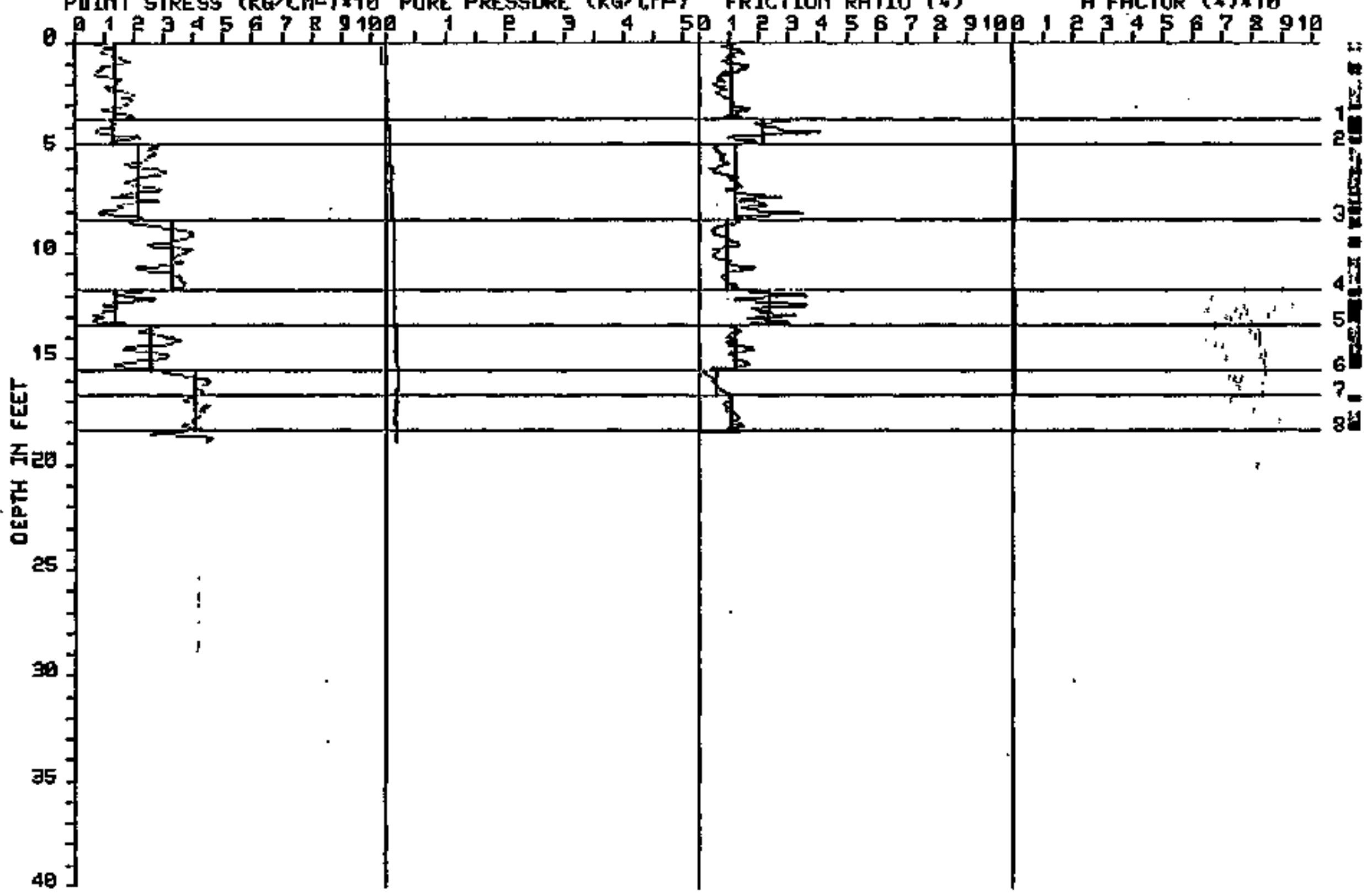




\section{PIEZOCONE SOLNDIMG TEST}

SITE ID: HATVI

LOCATION ID: OI9

PUNIT STRESS (KG/CM)

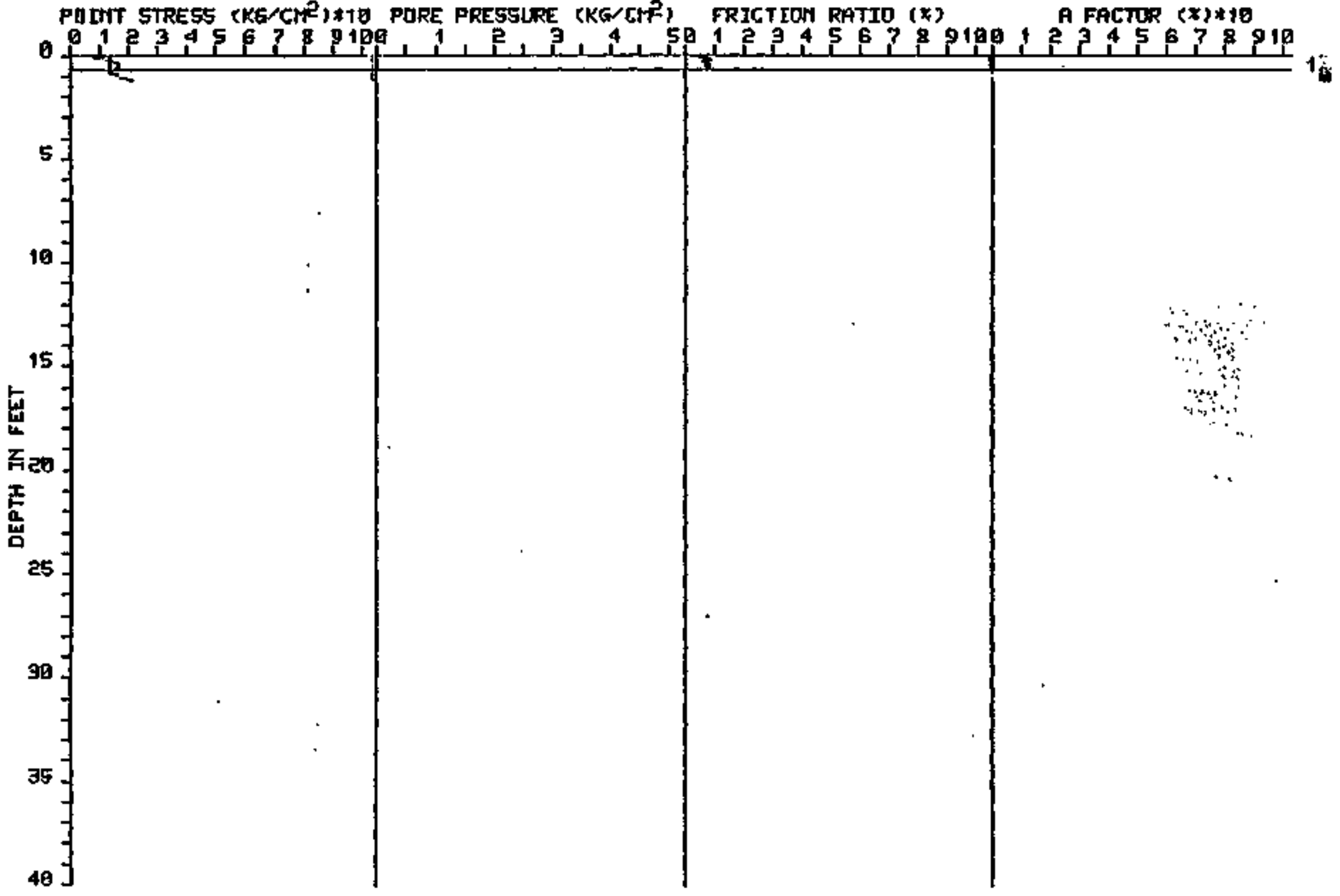


PIEZOCONE SOUNDING TEST

SITE IDI HATO1

LOCATION ID: O20

POIMT STRESS (KE八CME)

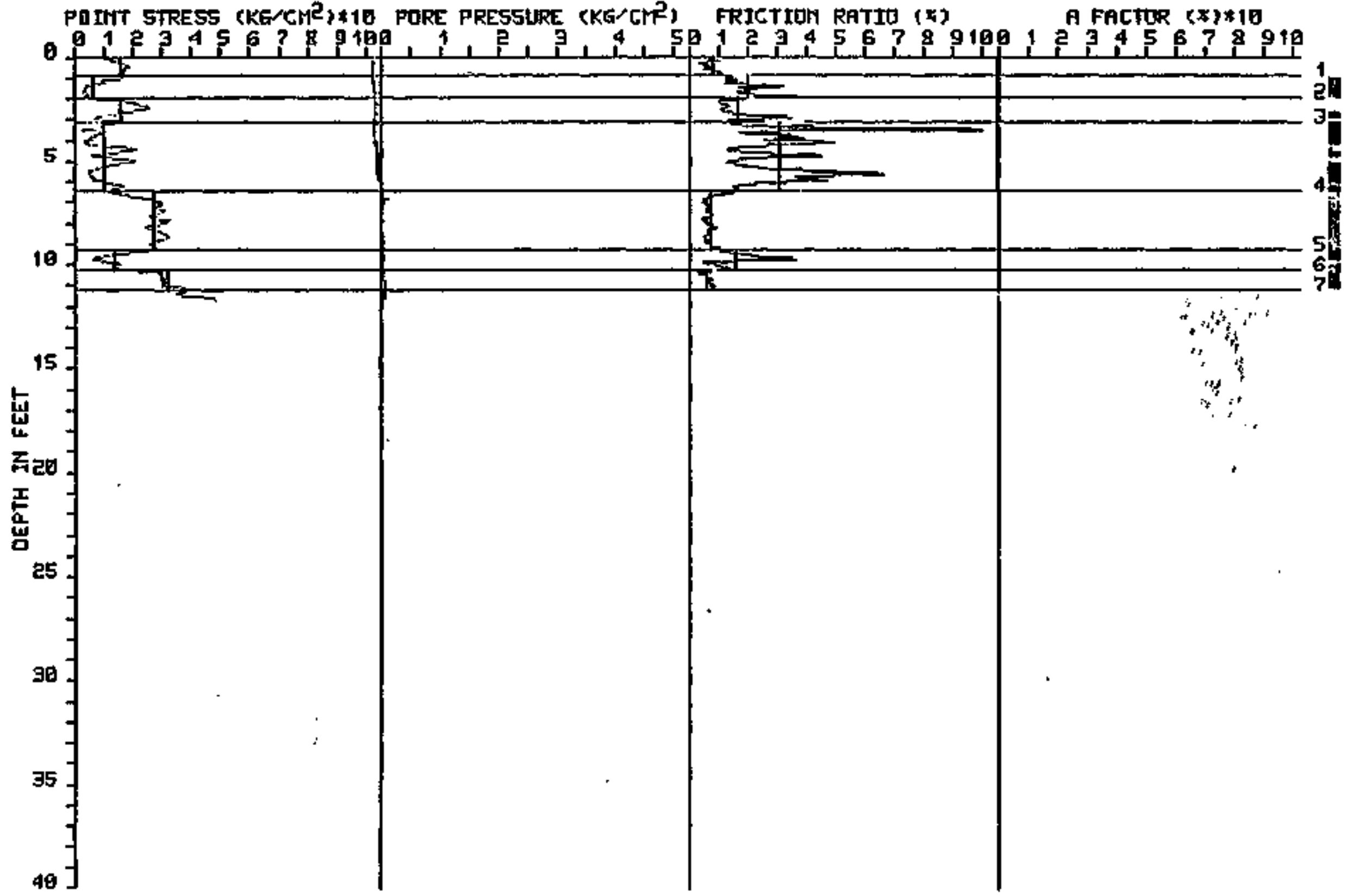

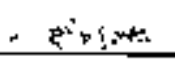




\section{PIEZOCONE SOUNDING TEST}

SITE IOZ HATE1

LOCATION DD: 021

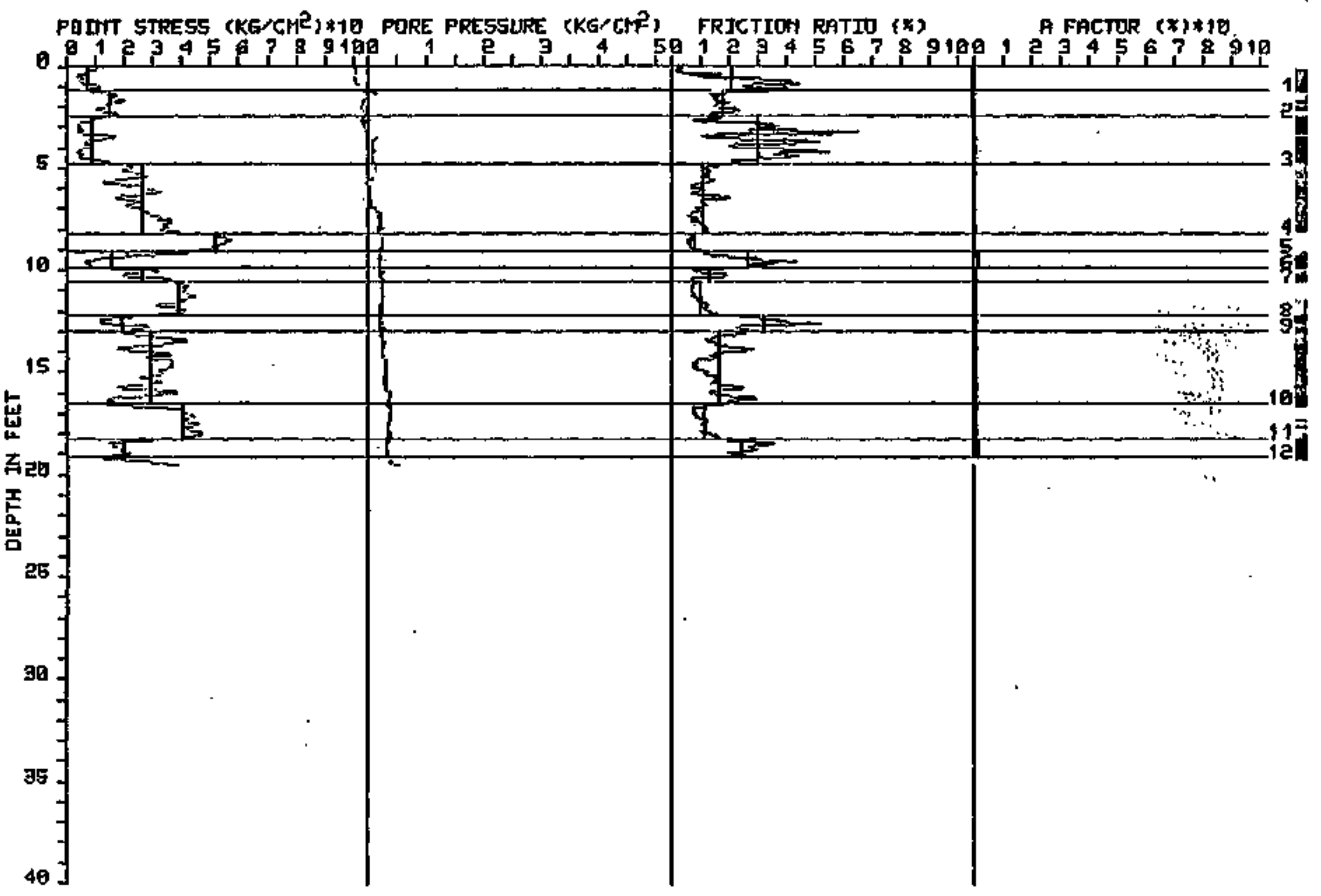




\section{PIEZOCONE SOLNDING TEST}

SITE IDI HATOI

LDCATLON ID: DER

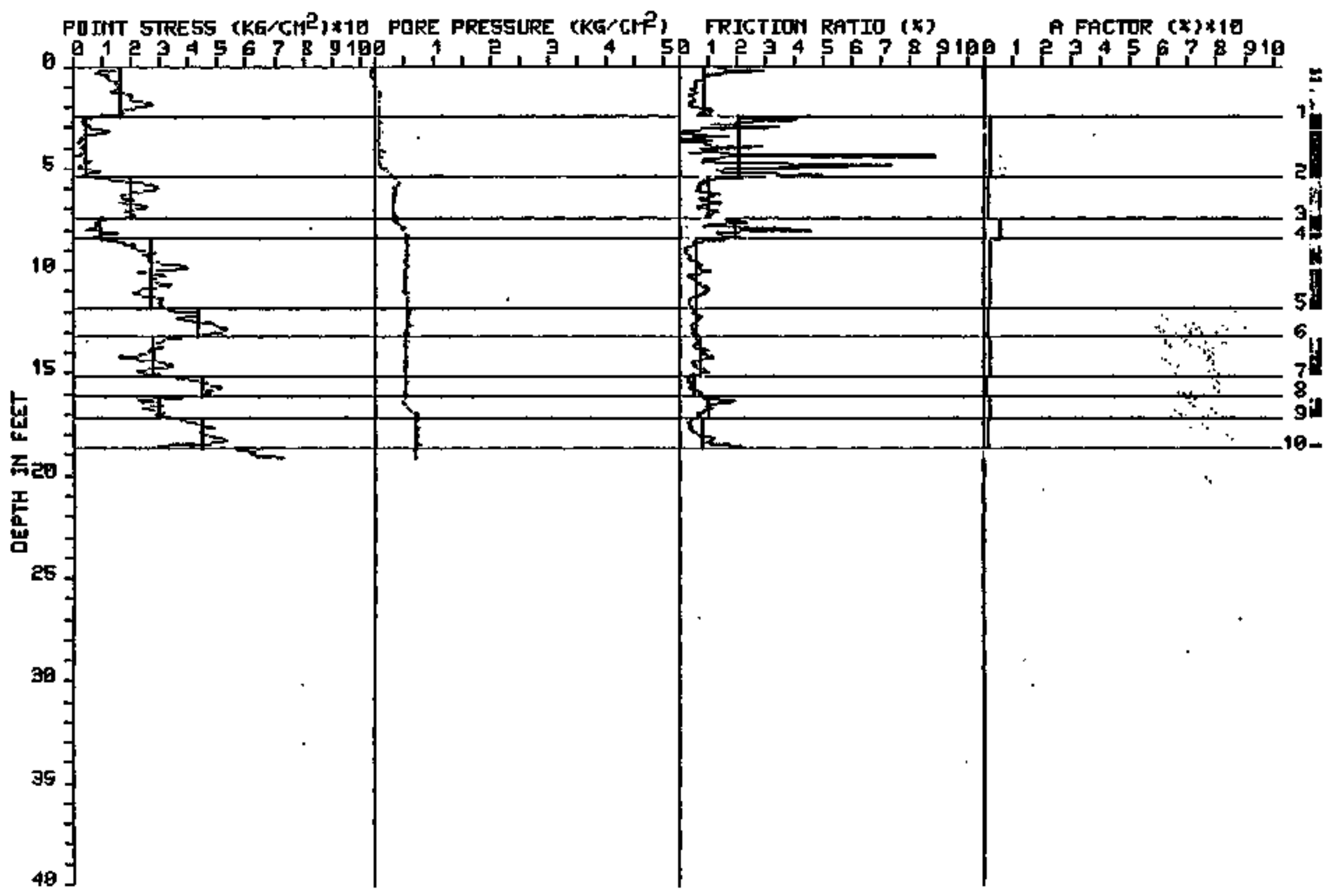


PIEZOCONE SOUNDING TEST

SITE IDI HATOI

LOCATION TD: 023

POINT STRESS (KG/CM) N10 PORE PRESSURE (KG/CMF), FRICTION RATIO (X)

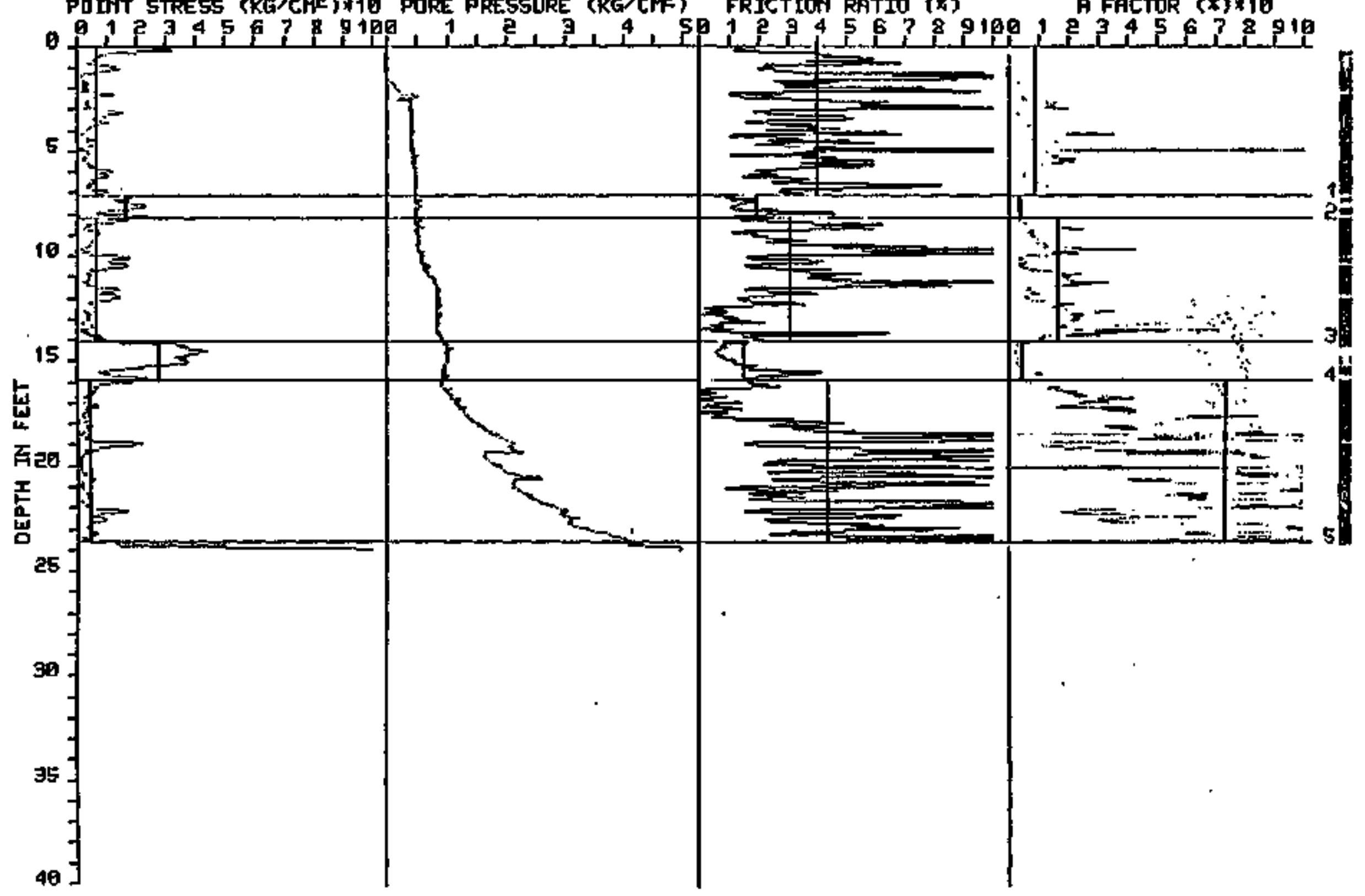




\section{PIEZOCONE SOUNDING TEST}

SJTE ID: HATO1

LOCATION IDI 024

PIINT 5TRESS (KG/CM 2 ) $\times 10$ PORE PRESSURE (KG/CTF)

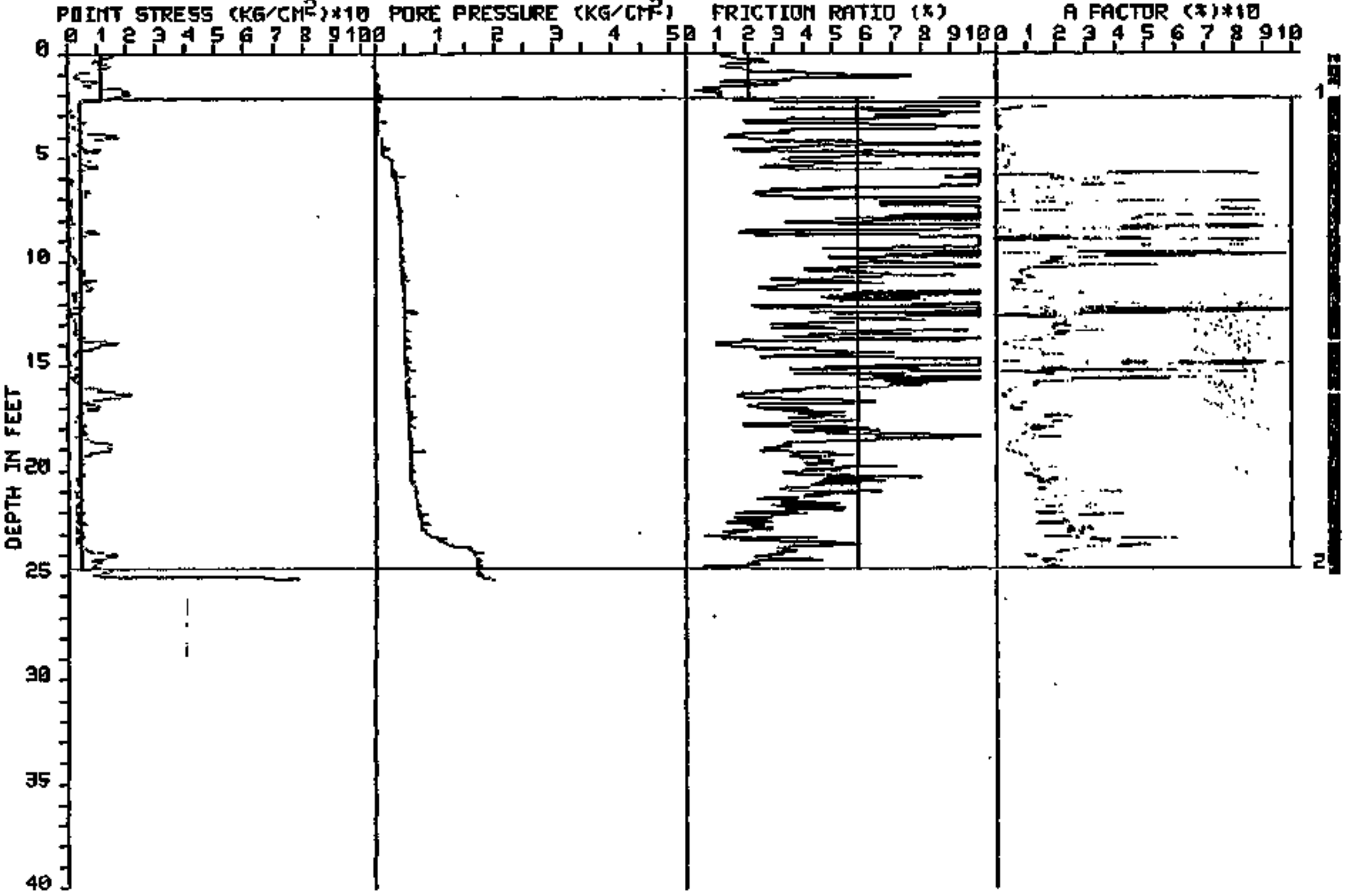

A FACTOR $(x) * 10$ 


\section{PIEZOCONE SOUNDING TEST}

SITE ID: HATQI

POIMT STIRESS (KG/CMP)*10 PORE PRESGURE (KG/CMF) FRICTIOH RATID (X)

P 12355

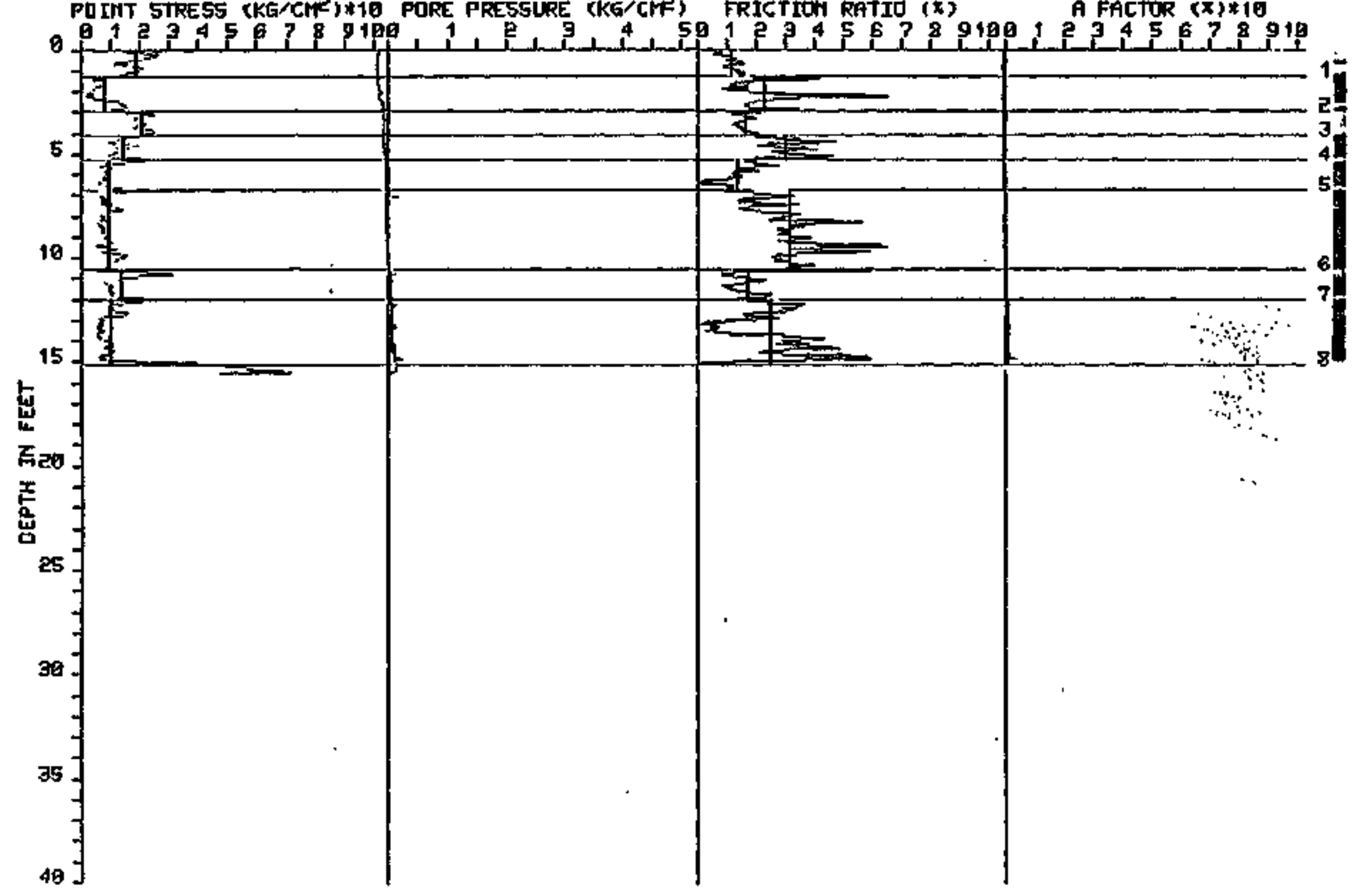




\section{PIEZOCONE SOLNDING TEST}

SITE ID2 HATO1

LOCATION ID: OEG

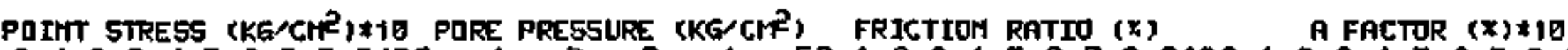

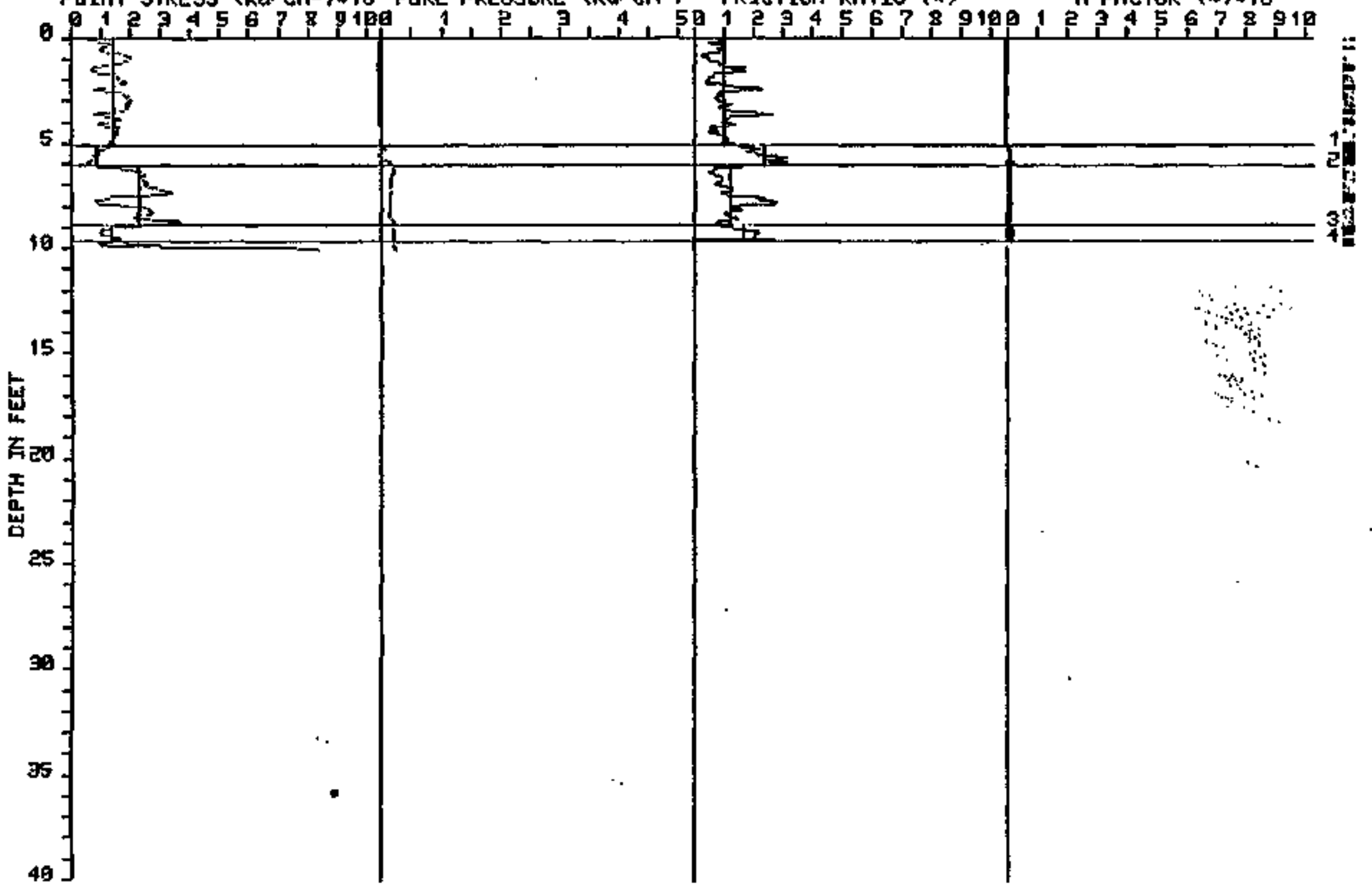




\section{PIEZDCONE SOUNDIMG TEST}

SITE ID: HATEI

LOCATIOM IO: OEP

PUINT 5TRESS (KG/CME)*10 PORE PRESSURE (KG/CMR) FRICTIEH RATTO (x)

A FACTER (*)*ia

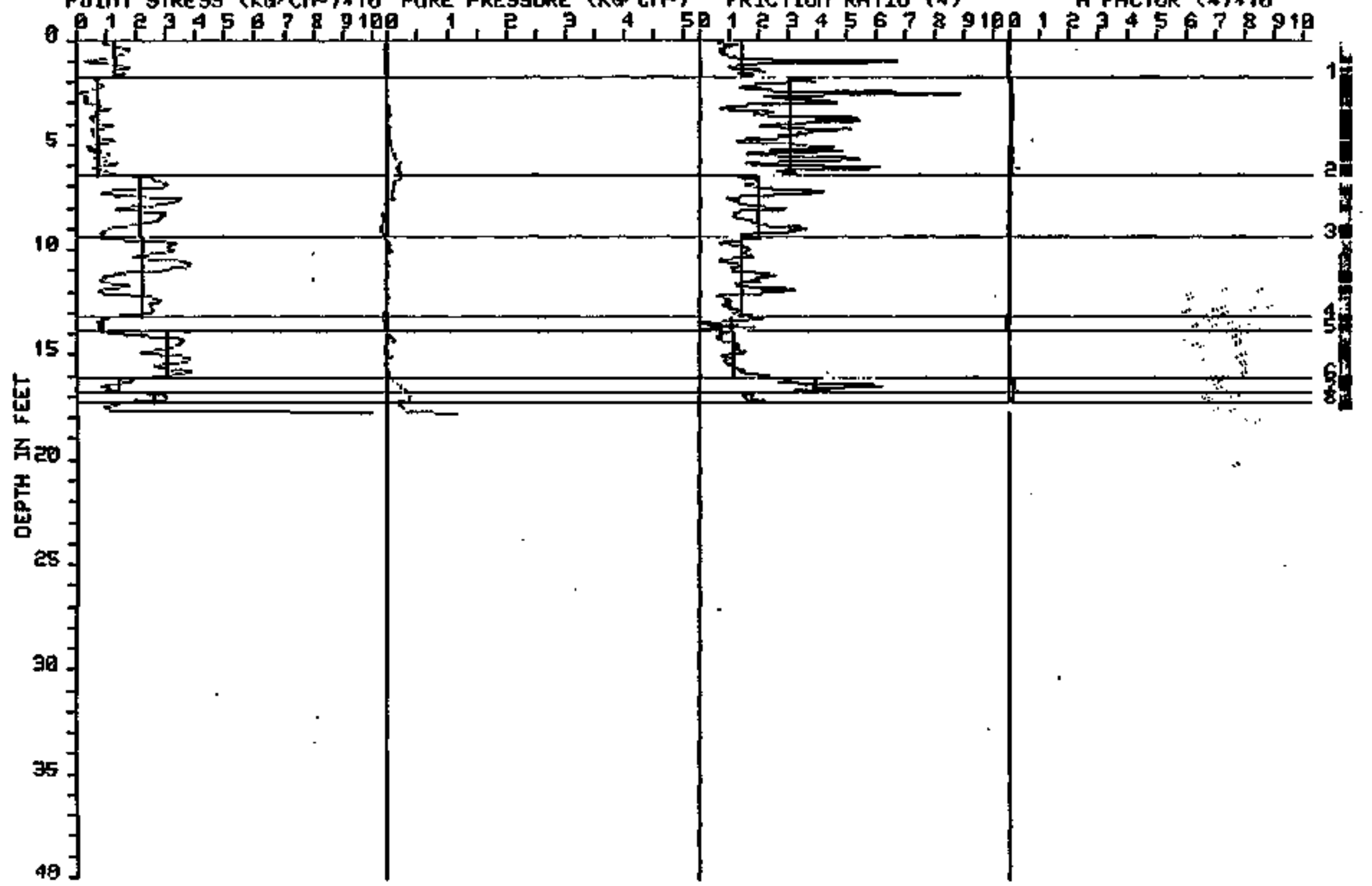




\section{PIEZOCONE SOUNDING TEST}

SITE ID: HATO1 LOCATION ID: OZE

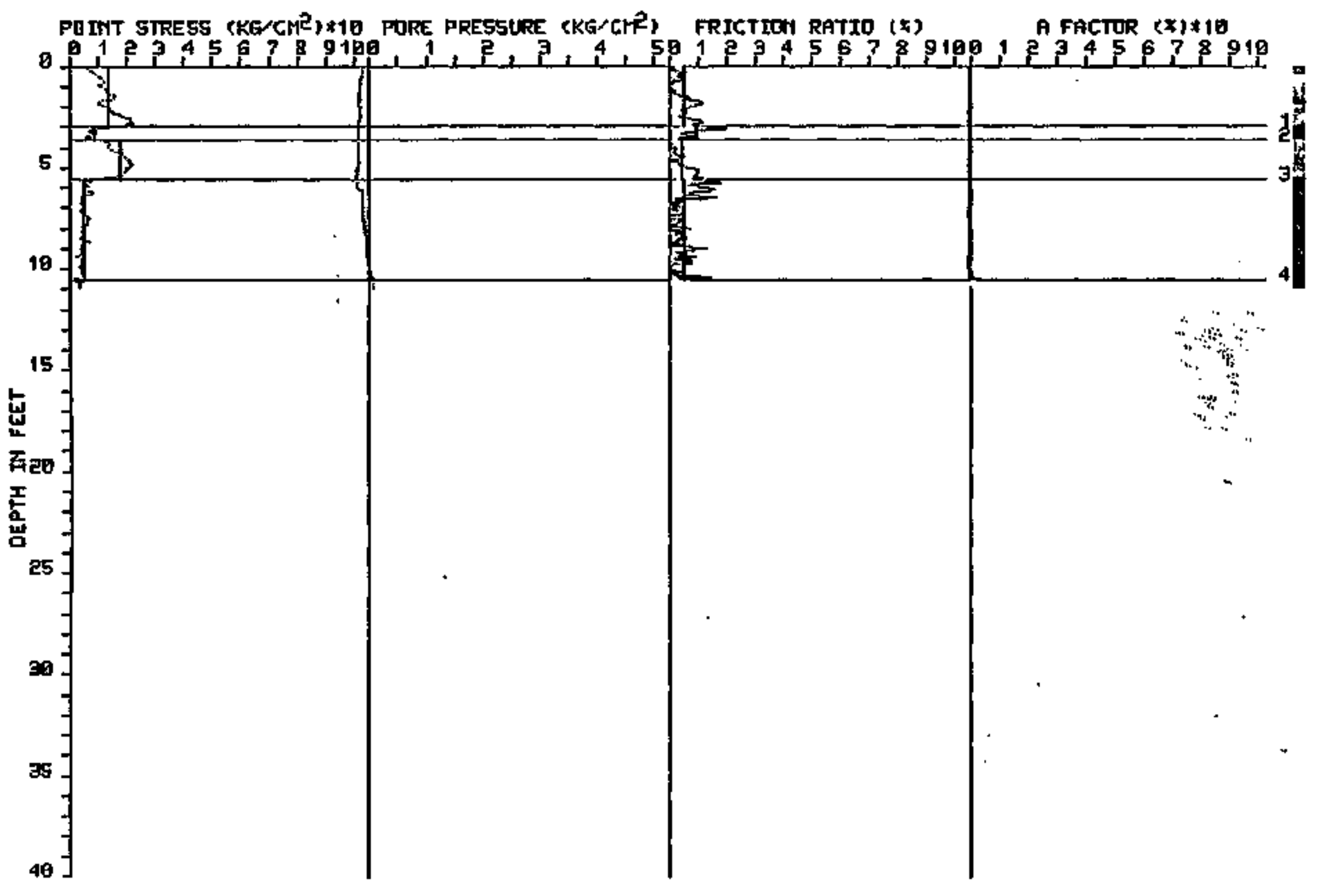


PIEZOCONE SOUNDING TEST

SITE ID? HATE I

LOCATION ID: DEP

PUIMT STRESS (KG/CMP) *10 PORE PRESSURE (KG/CMF) FRICTION RATIO (*) A FACTOR (X)*1E

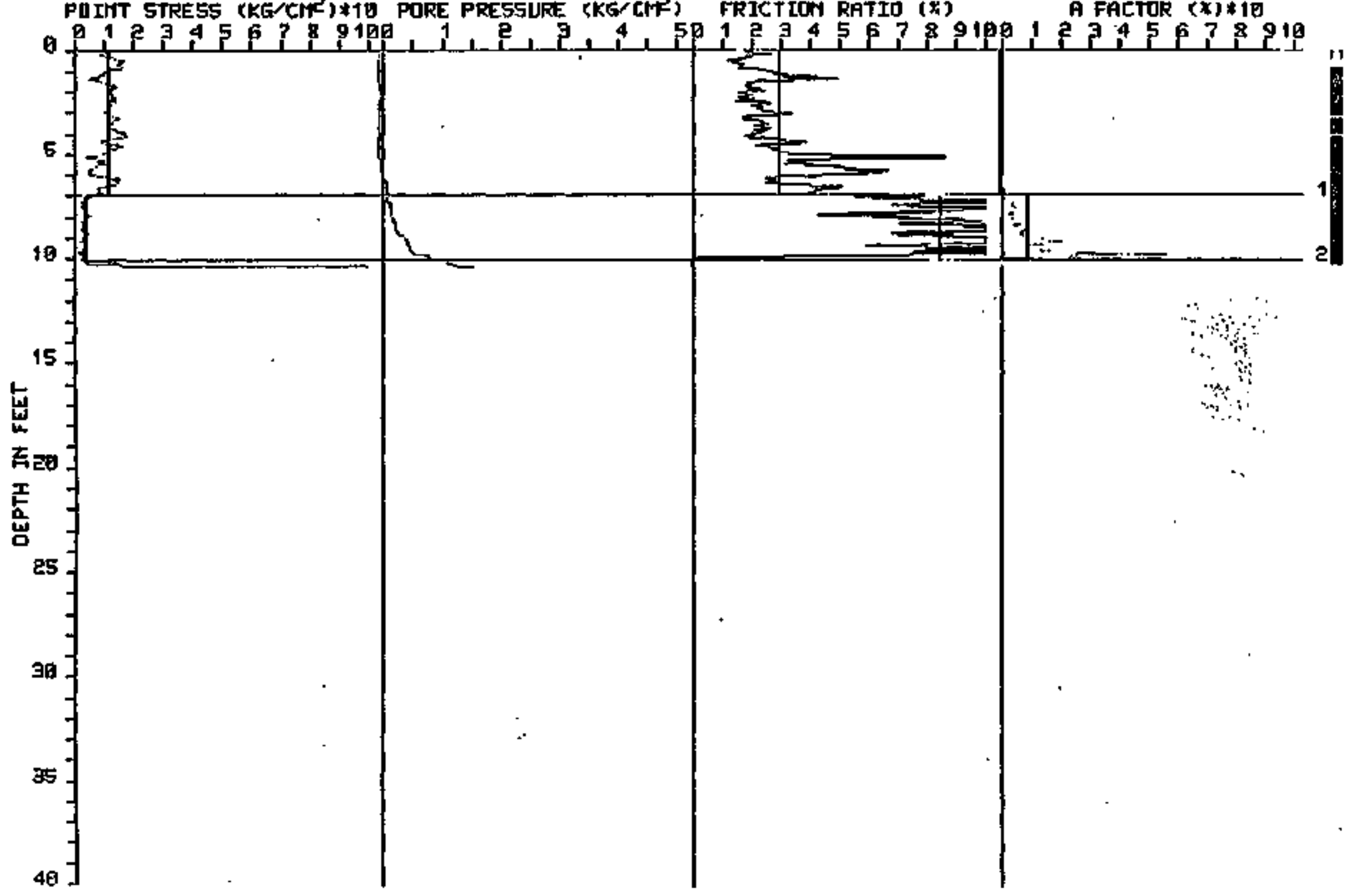




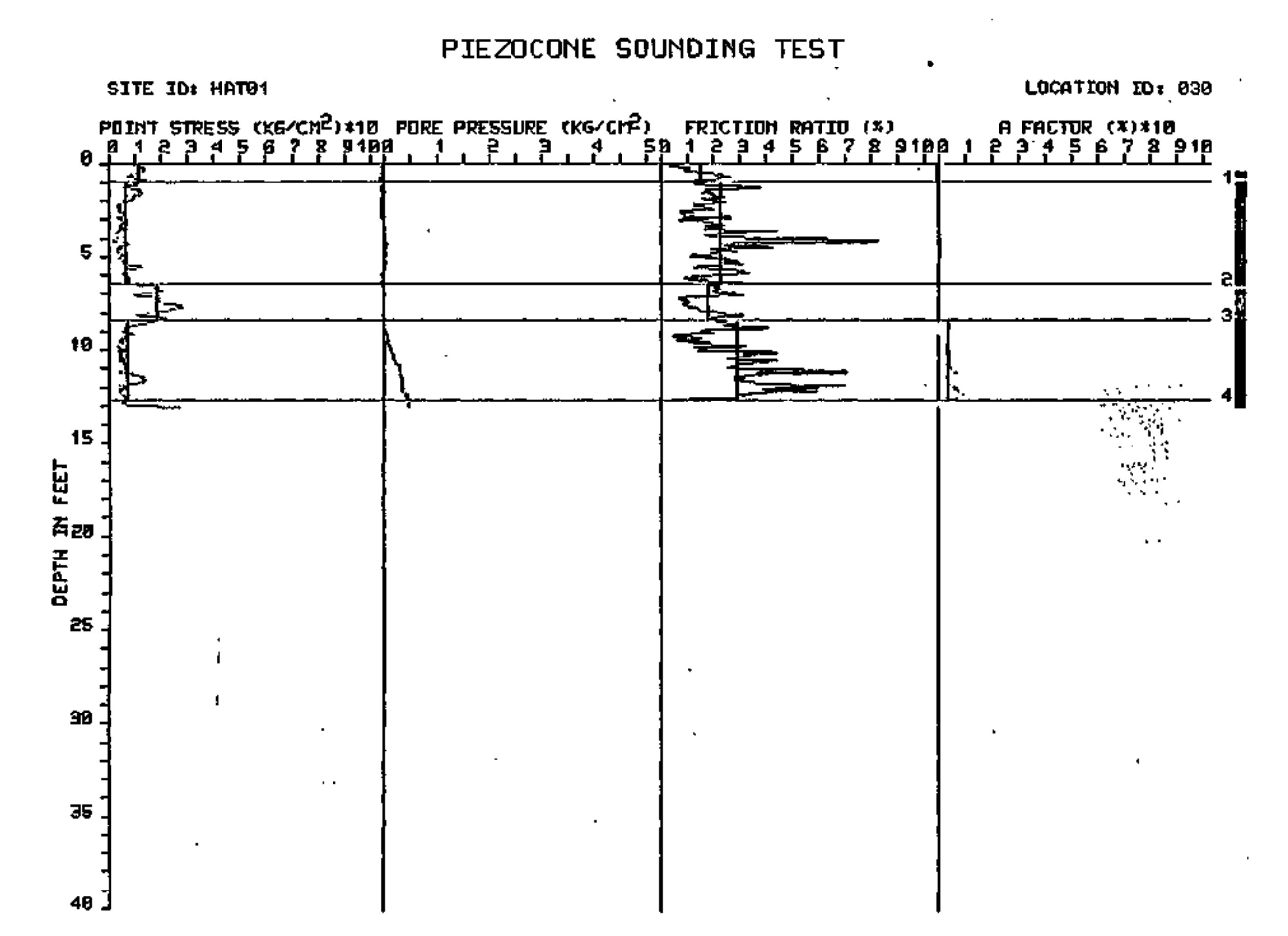


SITE IO: HATO1

GROUND WATER DEPTH: 50.0 (FT.)
LOCATION ID: 001

AUERAGE SOIL WEIGHT: 100.9 (PSF)

- DEPTH - $\begin{aligned} & \text { AUG. AUG. } \\ & \text { - AUG. PORE FRICT. SOIL }\end{aligned}$ LY FROM TO THK. POINT SLEEVE PRESS. RATIO CLASS BC DEN FA ELAS. STREN OCR

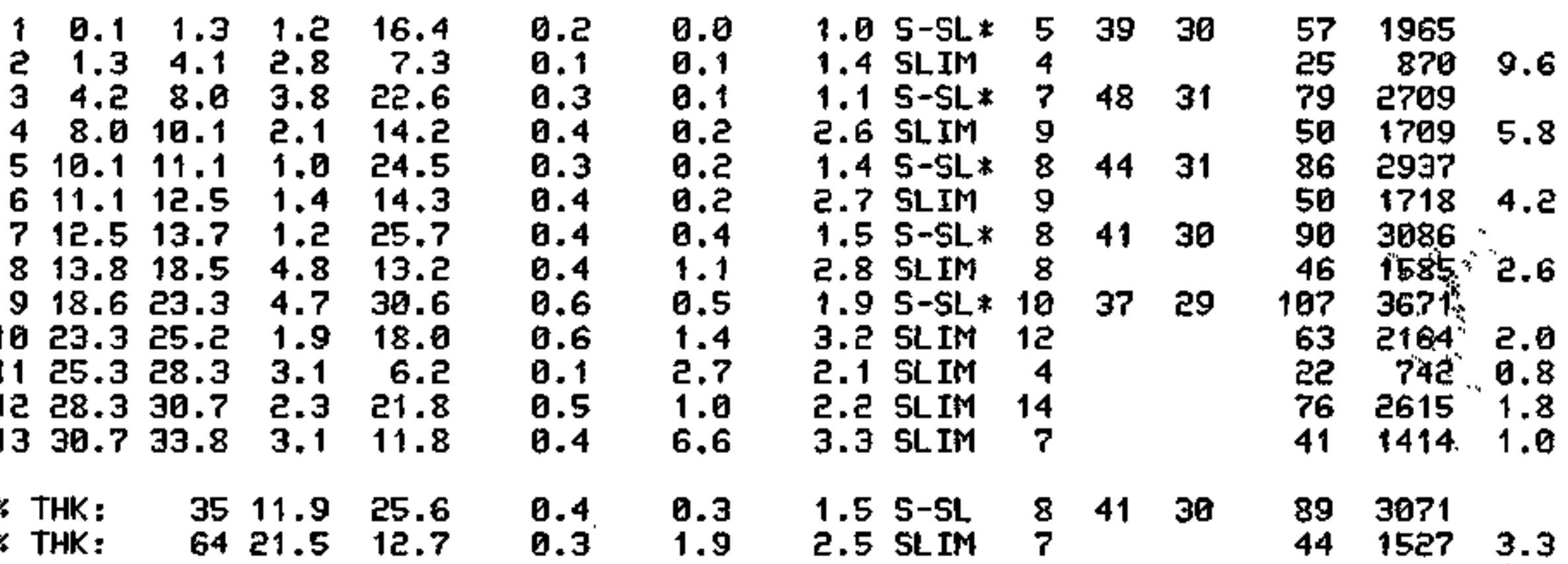


SITE ID: HATO1

GROUNO WATTER DEPTH: 50.0 (FT.)
LOCATION ID: 002

AUERAGE SLIL WEIGHT: 100.0 (PSF)

AUG AUG AUG.

LY FROM TO THK. POINT SLEEVE PRESS. RATIO CLASS BC DEN FA ELAS. STREN GCR

\begin{tabular}{|c|c|c|c|c|c|c|c|c|c|c|c|c|}
\hline $\begin{array}{rr}1 & 0.1 \\
2 & 0.8 \\
3 & 9.9 \\
4 & 13.2 \\
5 & 17.1 \\
6 & 18.7 \\
7 & 19.6\end{array}$ & $\begin{array}{r}0.8 \\
9.8 \\
13.2 \\
17.0 \\
18.6 \\
19.6 \\
21.0\end{array}$ & $\begin{array}{l}0.7 \\
9.1 \\
3.3 \\
3.8 \\
1.6 \\
0.9 \\
1.4\end{array}$ & $\begin{array}{l}48.0 \\
23.7 \\
13.4 \\
30.4 \\
43.1 \\
22.2 \\
56.3\end{array}$ & $\begin{array}{l}0.3 \\
0.2 \\
0.4 \\
0.4 \\
0.3 \\
0.4 \\
0.5\end{array}$ & $\begin{array}{r}-0.1 \\
0.0 \\
1.6 \\
0.5 \\
0.1 \\
0.2 \\
0.3\end{array}$ & $\begin{array}{l}0.7 \\
0.8 \\
2.7 \\
1.2 \\
0.8 \\
1.9 \\
1.0\end{array}$ & $\begin{array}{l}\text { SAND* } \\
\text { S-SL* } \\
\text { SLIM } \\
\text { S-SL* } \\
\text { SAND* } \\
\text { S-SL } \\
\text { SAND* }\end{array}$ & $\begin{array}{r}9 \\
7 \\
8 \\
10 \\
8 \\
7 \\
11\end{array}$ & $\begin{array}{l}43 \\
50 \\
30 \\
55\end{array}$ & $\begin{array}{l}35 \\
31 \\
30 \\
31 \\
29 \\
32\end{array}$ & $\begin{array}{r}168 \\
83 \\
47 \\
106 \\
151 \\
78 \\
197\end{array}$ & $\begin{array}{l}2845 \\
1611 \\
3647 \\
\\
2662\end{array}$ \\
\hline $\begin{array}{l}\text { THK: } \\
\text { THK: } \\
\text { THK: }\end{array}$ & $\begin{array}{l}17 \\
66 \\
15\end{array}$ & $\begin{array}{r}3.7 \\
13.8 \\
3.3\end{array}$ & $\begin{array}{l}49.0 \\
25.4 \\
13.4\end{array}$ & $\begin{array}{l}0.4 \\
0.3 \\
0.4\end{array}$ & $\begin{array}{l}0.1 \\
0.2 \\
1.6\end{array}$ & $\begin{array}{l}0.9 \\
1.0 \\
2.7\end{array}$ & $\begin{array}{l}\text { SAND } \\
\text { S-SL } \\
\text { SLIM }\end{array}$ & $\begin{array}{l}9 \\
7 \\
8\end{array}$ & $\begin{array}{l}56 \\
46\end{array}$ & $\begin{array}{l}32 \\
31\end{array}$ & $\begin{array}{r}171 \\
89 \\
46\end{array}$ & $\begin{array}{l}\because 4 \\
3053 \\
161.1\end{array}$ \\
\hline
\end{tabular}


SITE ID: HATQ1

GROUND WATER DEPTH: 50.0 (FT.)
LOCATIOM ID: 003

AUERAGE SOIL WEIGHT: 100.0 (PSF)

- DEPTH -

AUG. AUG.

LY FROM TO THK. POINT SLEEUE PRESS. RATIO CLASS BC DEN FA ELAS. STREN OCR

\begin{tabular}{|c|c|c|c|c|c|c|c|c|c|c|c|c|}
\hline $\begin{array}{rr}1 & 0.1 \\
2 & 1.2 \\
3 & 14.8 \\
4 & 18.2 \\
5 & 20.1 \\
6 & 21.1 \\
7 & 23.9\end{array}$ & $\begin{array}{r}1.2 \\
14.8 \\
18.1 \\
20.1 \\
21.0 \\
23.9 \\
25.8\end{array}$ & $\begin{array}{r}1.1 \\
13.5 \\
3.3 \\
1.9 \\
0.9 \\
2.8 \\
1.9\end{array}$ & $\begin{array}{r}6.7 \\
24.9 \\
8.8 \\
27.5 \\
52.8 \\
27.4 \\
4.8\end{array}$ & $\begin{array}{l}0.0 \\
0.2 \\
0.3 \\
0.4 \\
0.4 \\
0.4 \\
0.2\end{array}$ & $\begin{array}{r}-0.0 \\
0.1 \\
0.3 \\
0.4 \\
0.2 \\
0.2 \\
0.4\end{array}$ & $\begin{array}{l}0.6 \\
1.0 \\
3.4 \\
1.4 \\
0.7 \\
1.5 \\
4.0\end{array}$ & $\begin{array}{l}\text { SLIM* } \\
\text { S-SL* } \\
\text { SLIM } \\
\text { S-SL* } \\
\text { SAND* } \\
\text { S-SL* } \\
\text { SLIM }\end{array}$ & $\begin{array}{r}4 \\
8 \\
5 \\
9 \\
10 \\
9 \\
3\end{array}$ & $\begin{array}{l}48 \\
36 \\
53 \\
33\end{array}$ & $\begin{array}{l}31 \\
29 \\
32 \\
29\end{array}$ & $\begin{array}{r}23 \\
87 \\
31 \\
96 \\
185 \\
96 \\
17\end{array}$ & $\begin{array}{r}800 \\
2985 \\
1057 \\
3302 \\
3287 \\
574\end{array}$ \\
\hline $\begin{array}{l}\text { THK: } \\
\text { THK: } \\
\text { THK: }\end{array}$ & $\begin{array}{r}3 \\
71 \\
24\end{array}$ & $\begin{array}{r}0.9 \\
18.2 \\
6.3\end{array}$ & $\begin{array}{r}52.8 \\
25.6 \\
7.2\end{array}$ & $\begin{array}{l}0.4 \\
0.3 \\
0.2\end{array}$ & $\begin{array}{l}0.2 \\
0.1 \\
0.3\end{array}$ & $\begin{array}{l}0.7 \\
1.1 \\
3.1\end{array}$ & $\begin{array}{l}\text { SAND } \\
\text { S-SL } \\
\text { SLIM }\end{array}$ & $\begin{array}{r}10 \\
8 \\
4\end{array}$ & $\begin{array}{l}52 \\
44\end{array}$ & $\begin{array}{l}32 \\
\mathbf{3 1}\end{array}$ & $\begin{array}{r}185 \\
89 \\
25\end{array}$ & $\begin{array}{r}3064 \\
866\end{array}$ \\
\hline
\end{tabular}


PIEZOCONE SOUNOING TEST

SITE ID: HATE1

LOCATION LO: 031

POIMT STRESS (KG/CM2) *10 PQRE PRESSURE (KG/CIF) FRICTIOH RATIO (x) A FACTOR $(x) * 10$

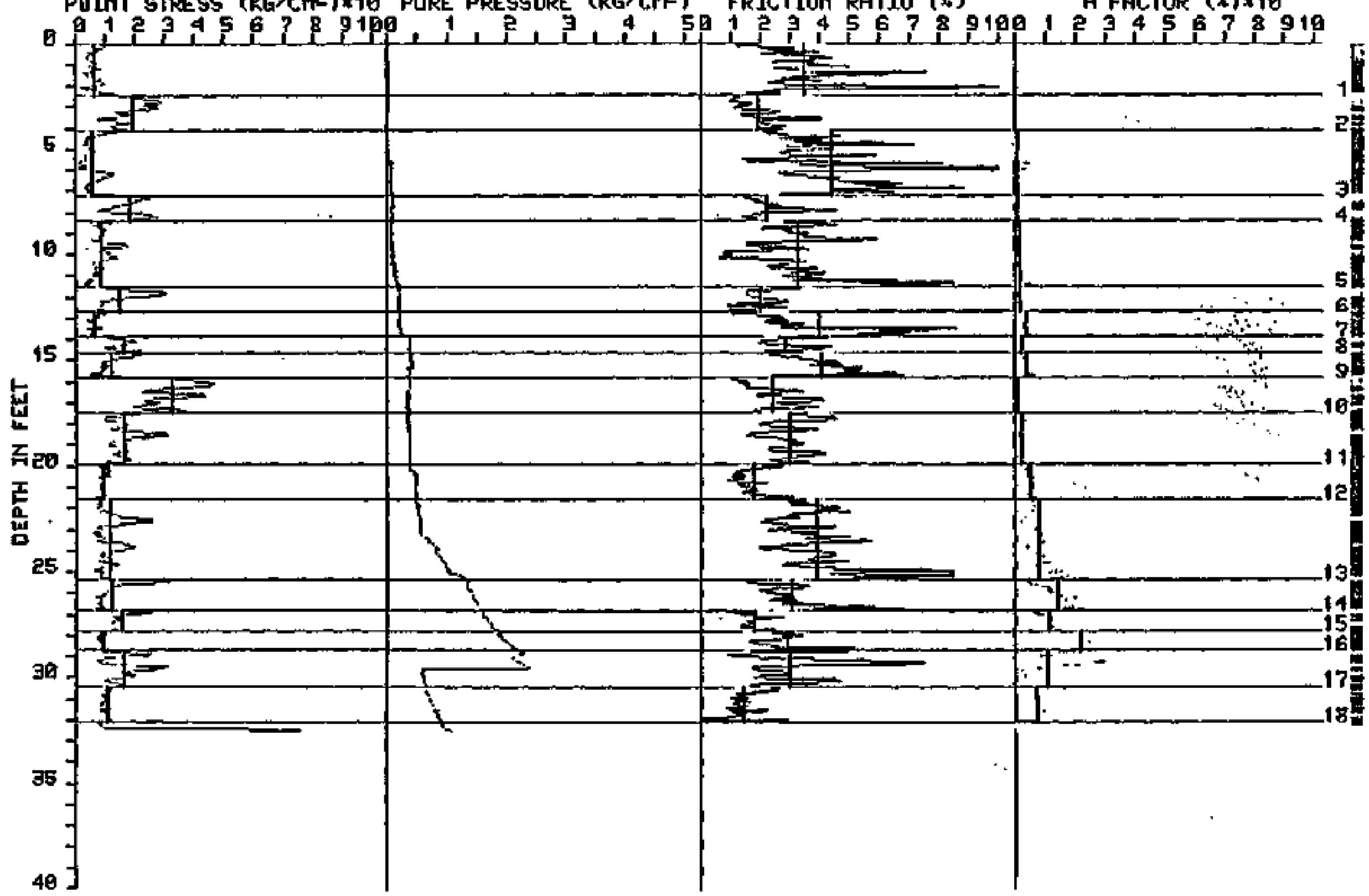


PIEZOCONE SOUNDING TEST

SITE IO2 HATO1

LOCATION ID: 032

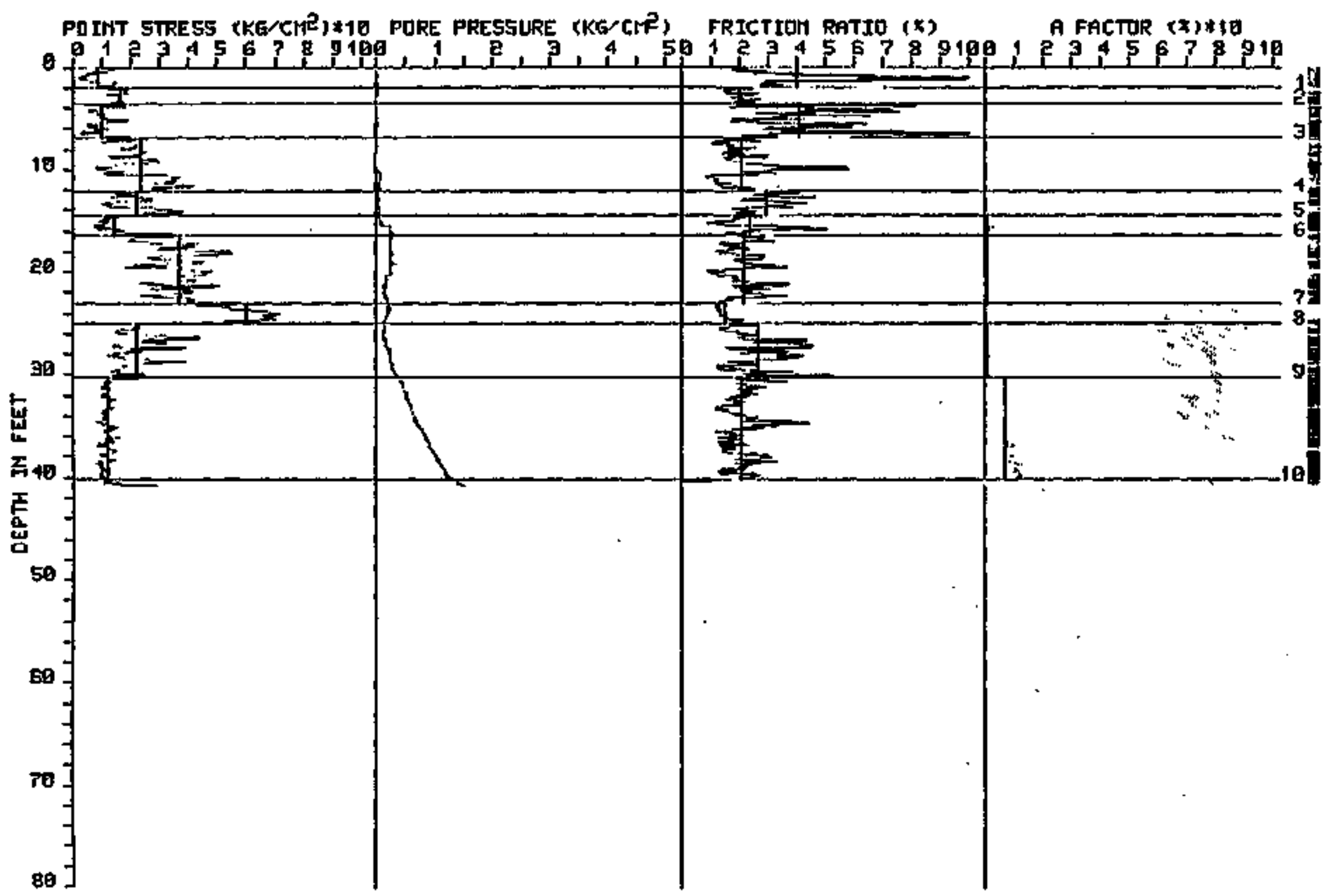




\section{PIEZOCONE SOLNOING TEST}

SITE IDI HATO1

LOCATION ID: 833

POIM STRESS (KG/CME)*1D PORE PRESSURE (KE/CMF) FRICTION RATID ( $x$ )

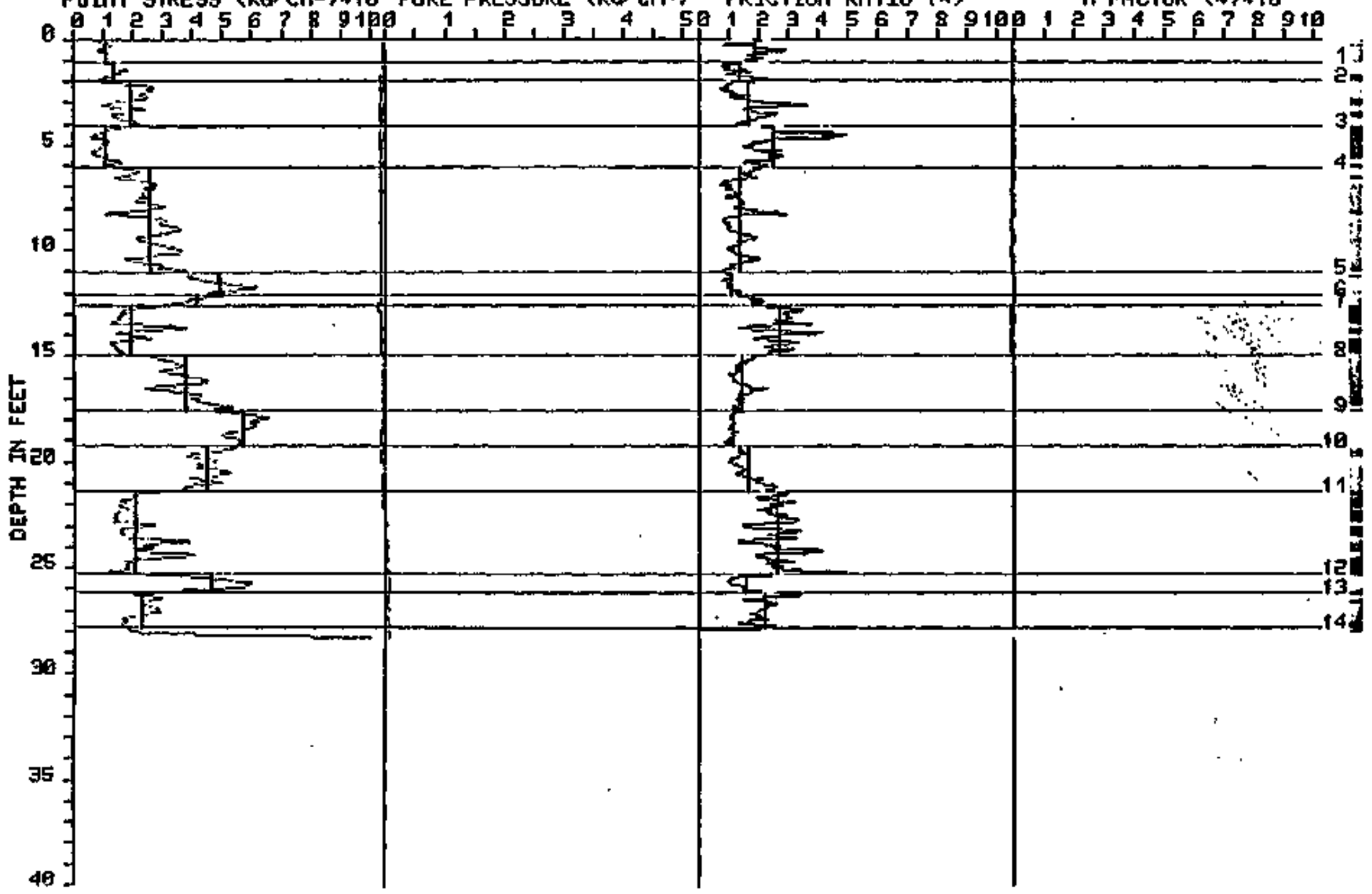




\section{PIEZOCONE SOUNDING TEST}

SITE ID; HATO1

LOCATION ID: 034

FOINT 5TRE5S (KG/CMP)*10 PORE PRESSURE (KG/CMF) FRICTLON RATID (*)

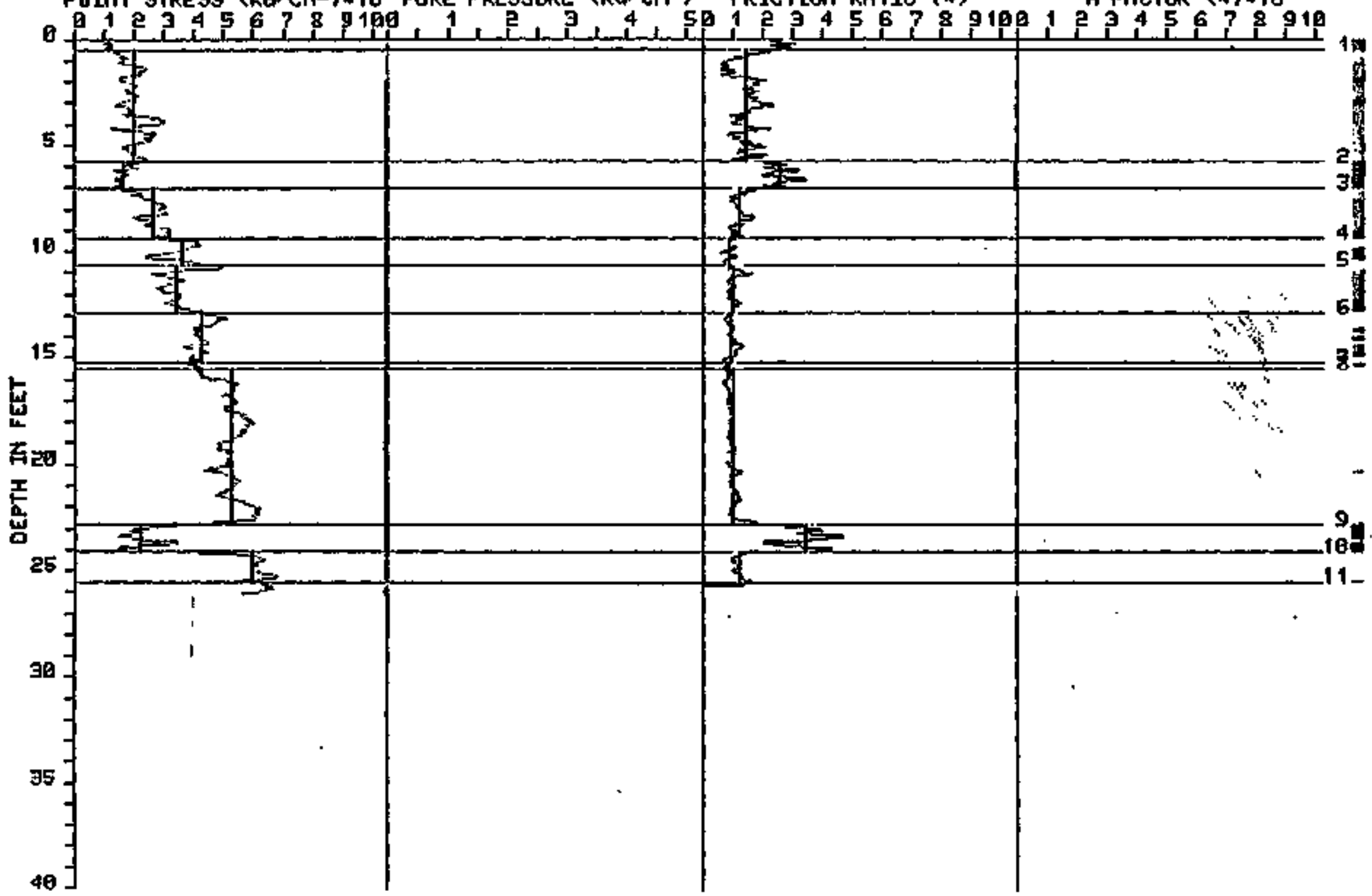




\section{PIEZOCONE SOUNDING TEST}

SITE ID: HATO1

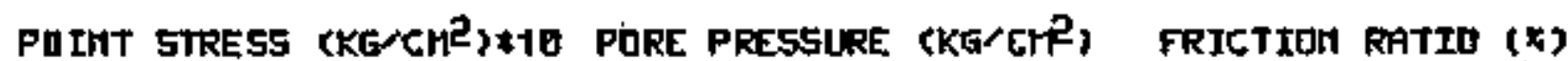

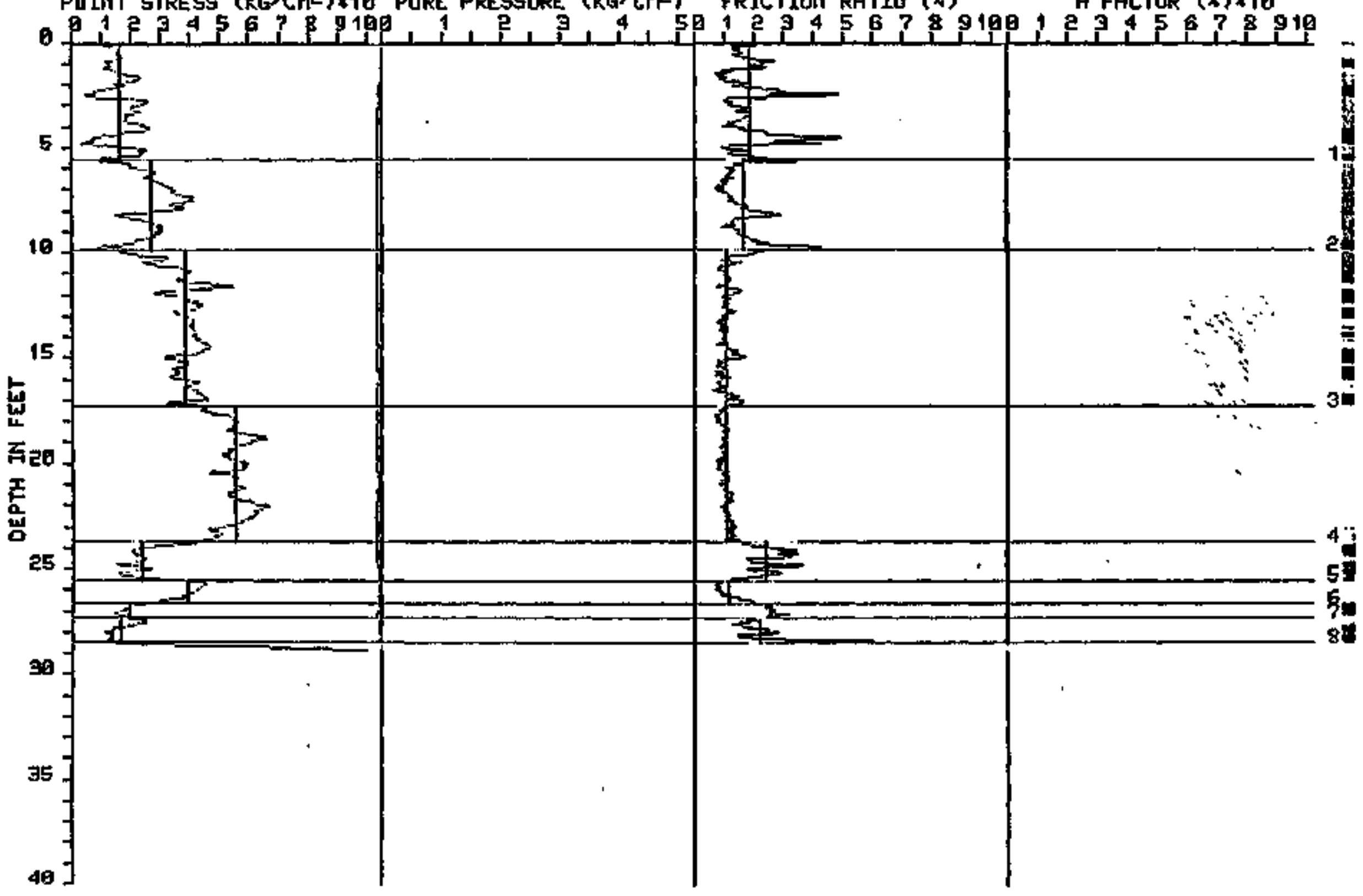




\section{PIEZOCONE SOUNDING TEST}

SITE ID: HATO1

LOCATIOY ID: 836

PUIMT STREGS (KG/CMP)*1V PORE PRESSURE (KG/CM

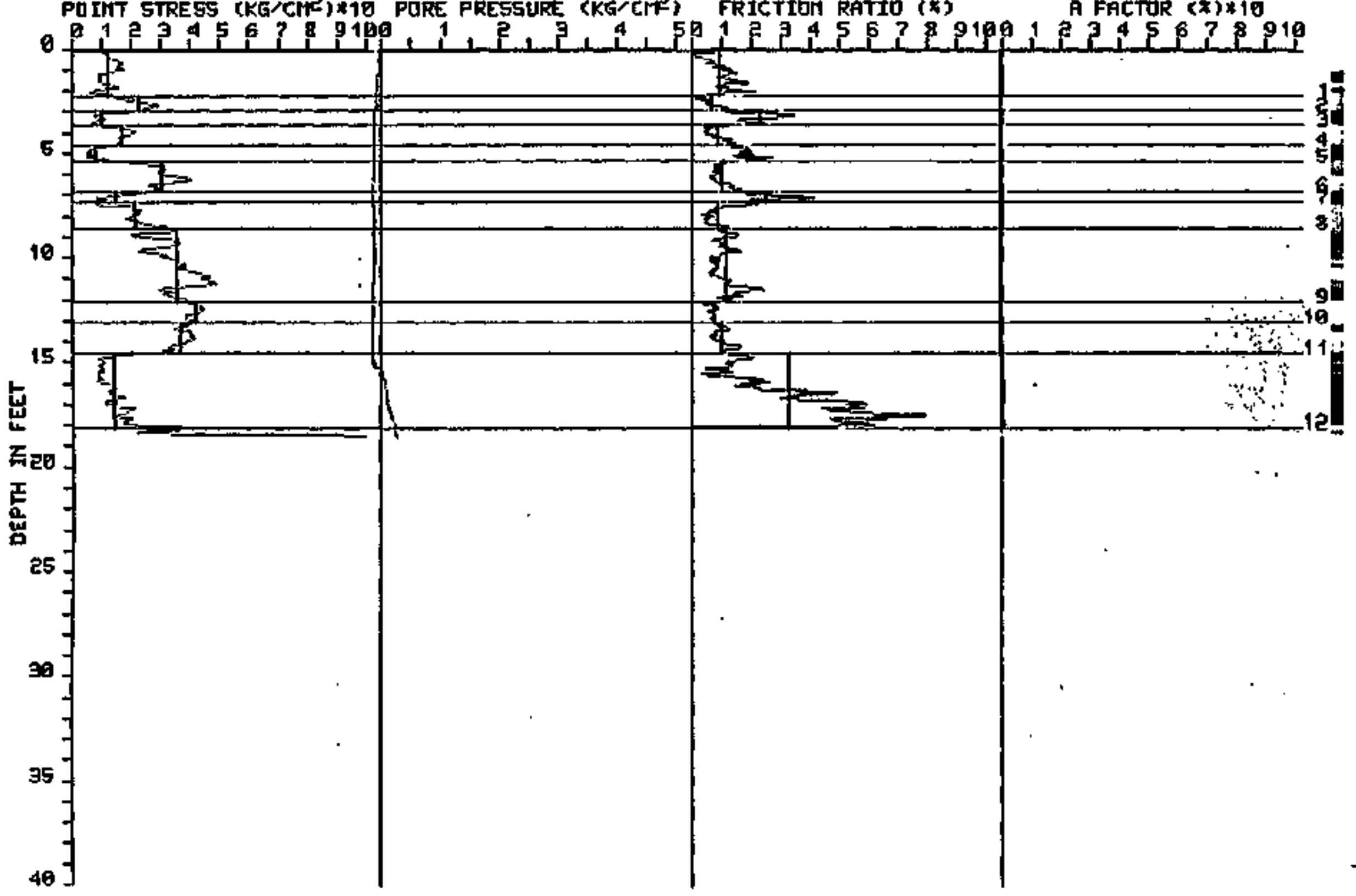




\section{PIEZOCONE SOUNDING TEST}

SITE IOZ HATO1

LOCATION ID: 037

POINT STRESS (KG/CHE)*10 PURE PRESSURE (KG/CMP) FRICTION RATIO (*)

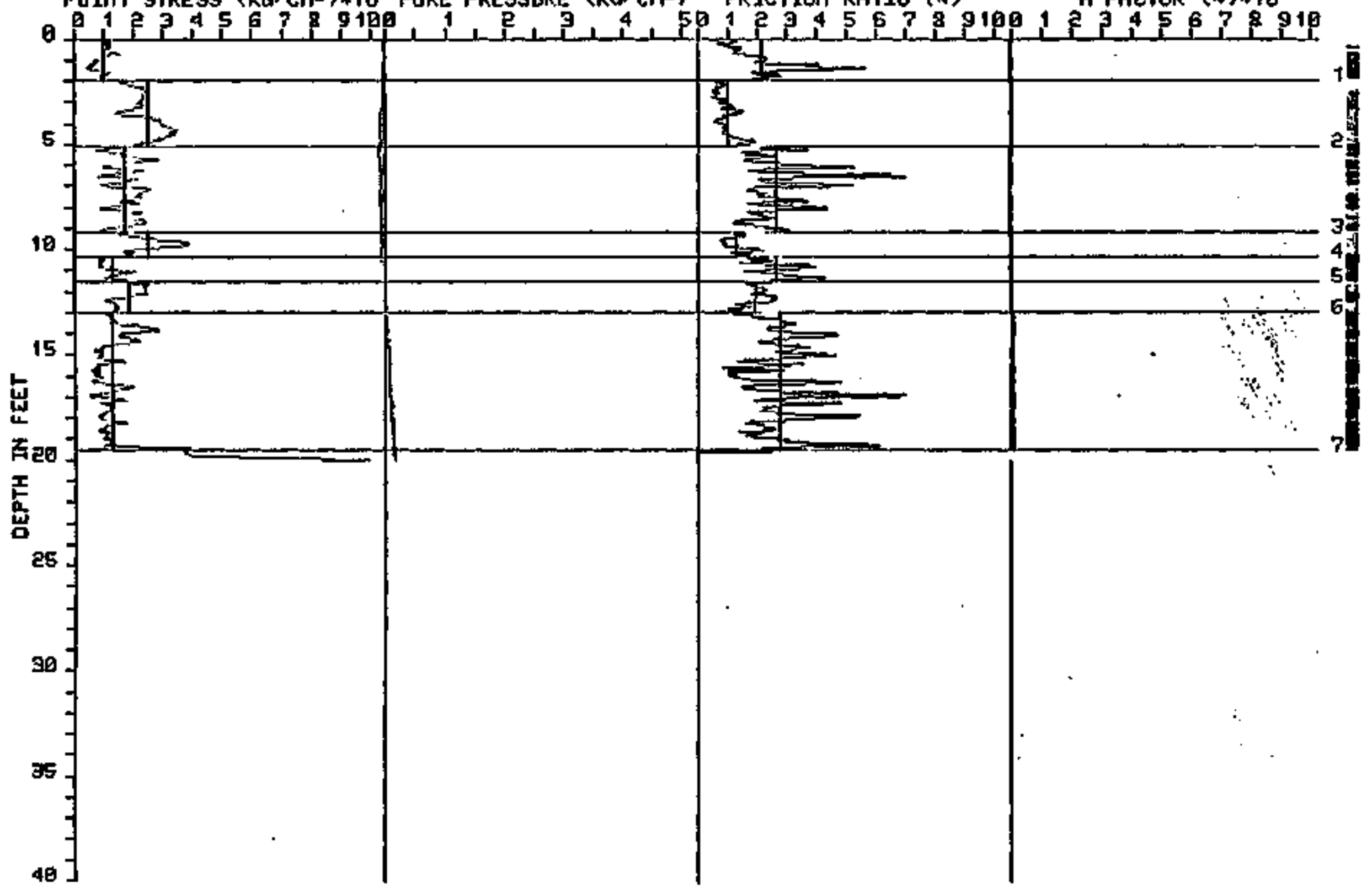




\section{PIEZOCONE SOUNDING TEST}

SITE 3D: HATO1

LOCATION TOE 038

POIMT STRESS (KG $/ \mathrm{CM}^{2}$ ) *1 PORE PRESSURE (KG/CM²) FRJCTIOAH RATID (x)

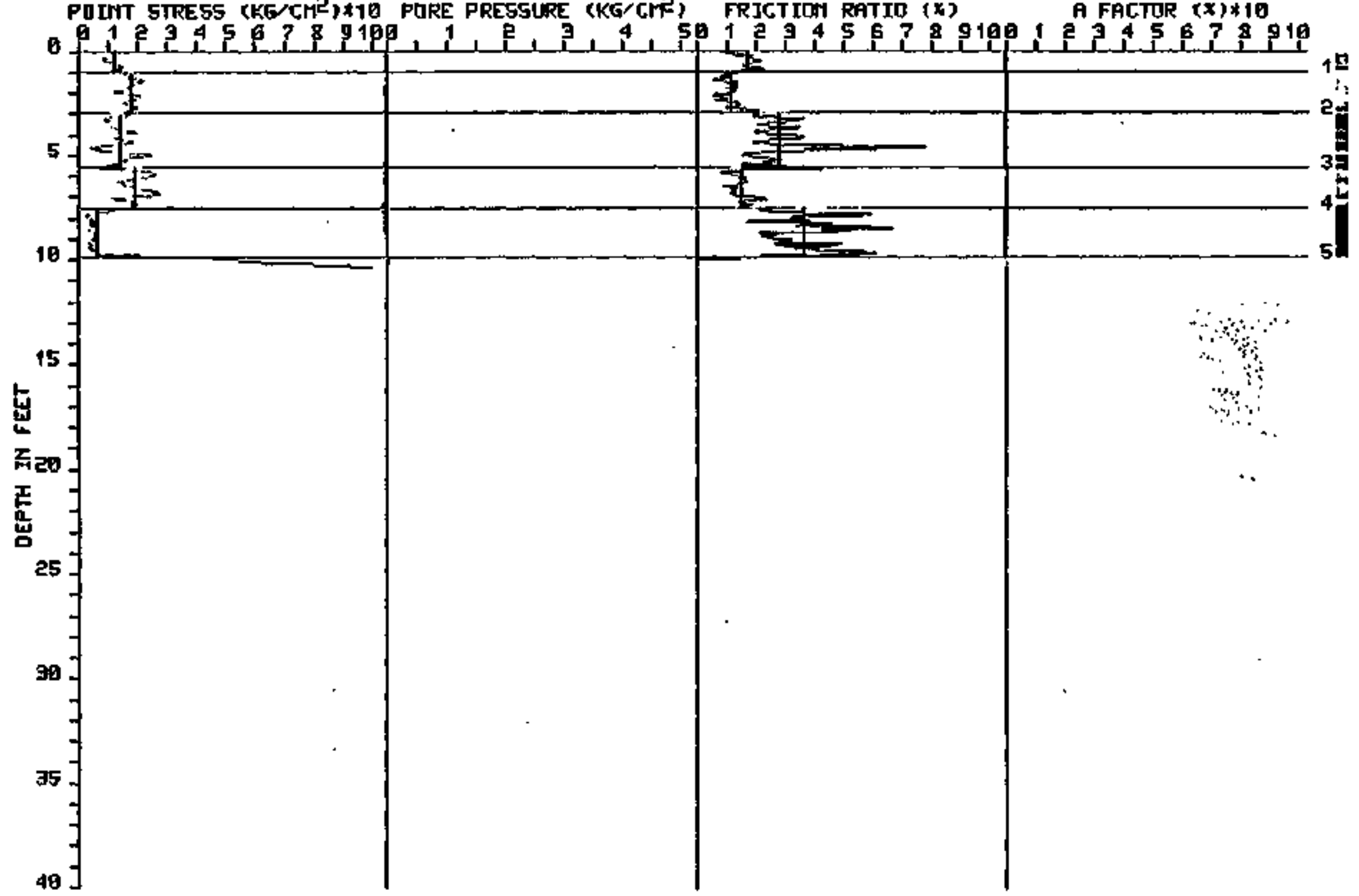




\section{PIEZOCONE SOUNDING TEST}

SITE IDS HATE1

LOCATION ID: 039

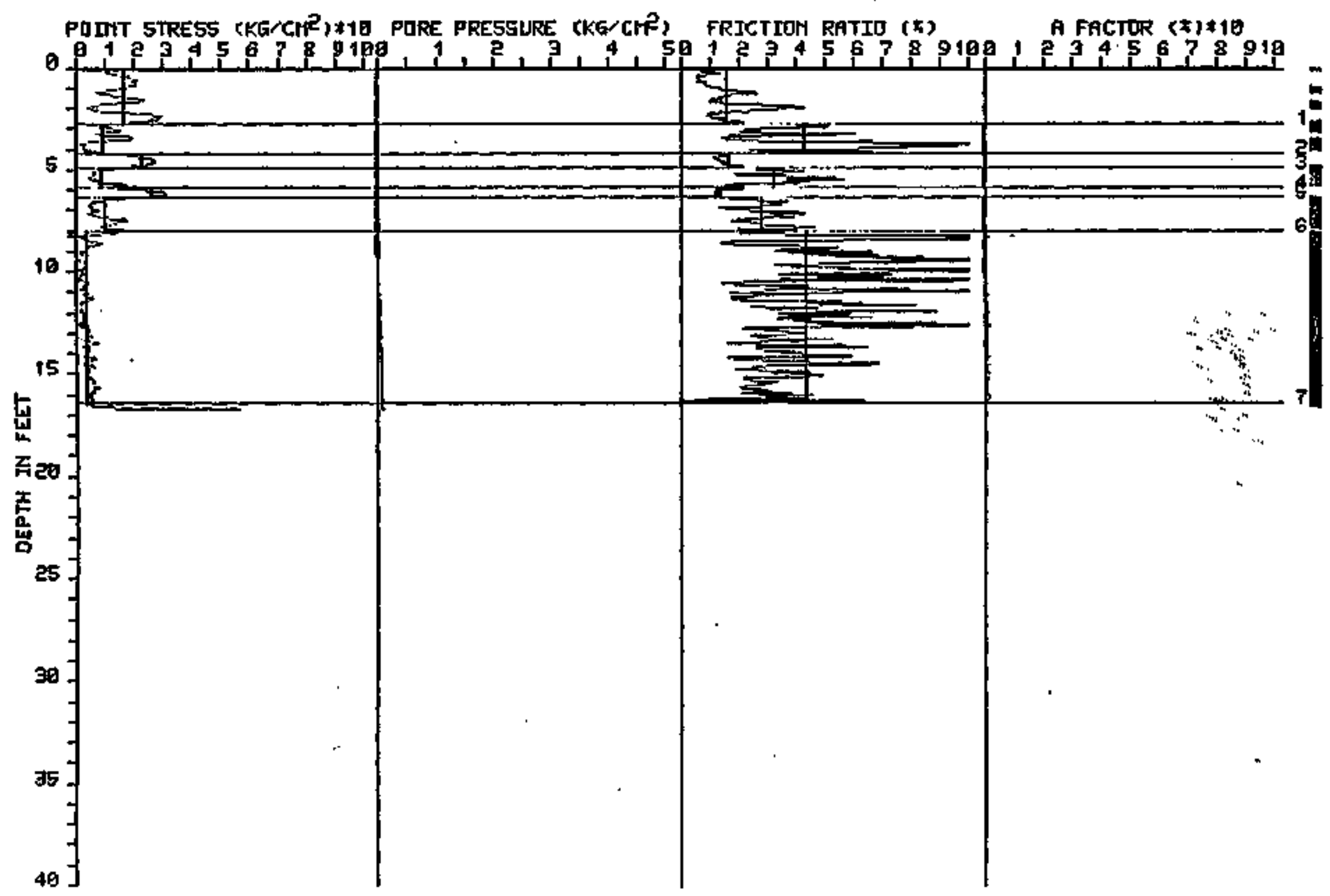




\section{PIEZOCONE SOUNDING TEST}

SITE ID: HATOI

LOCATION ID: 040

POIMT STRESS (KG/CMP)

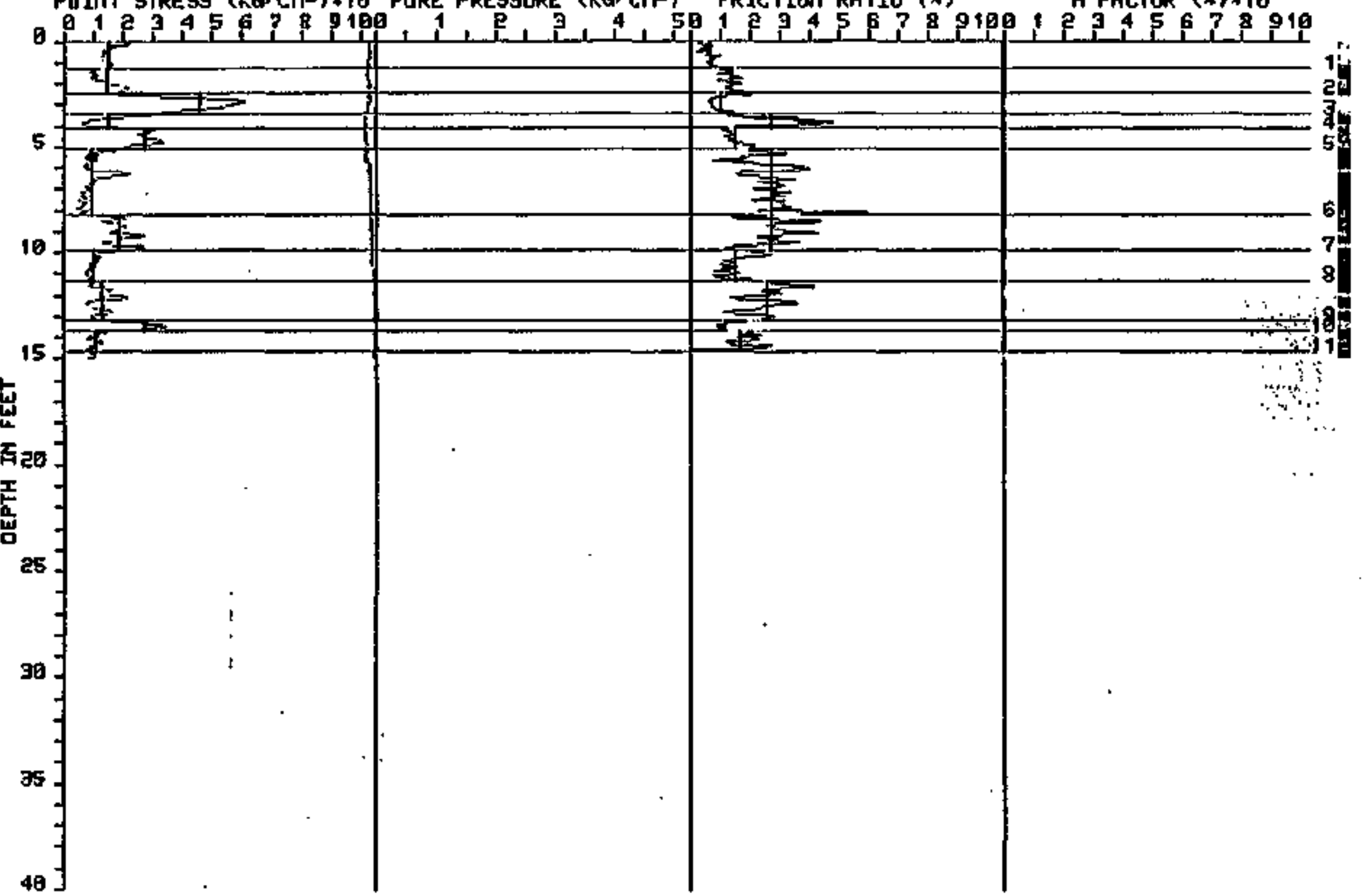


SITE ID: HATO1

GROUND WATER DEPTH: 50.0 (FT.)
LOCATION ID: 006

AUERAGE SOIL WEIGHT: 100.0 (PSF)

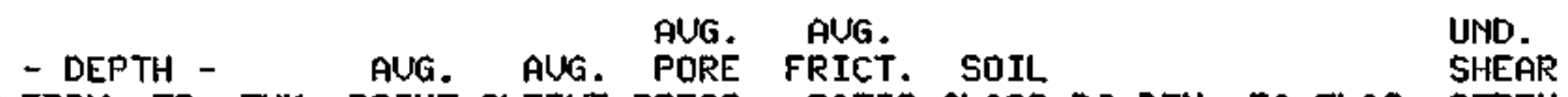
LY FROM TO THK. POINT SLEEUE PRESS. RATIO CLASS BC DEN FA ELAS. STREN OCR

$\begin{array}{rrrrrrrrrrrrrrr}1 & 0.1 & 3.0 & 2.9 & 16.8 & 0.1 & -0.0 & 0.5 & 5-5 L * & 5 & 40 & 30 & 59 & 2014 \\ 2 & 3.0 & 5.1 & 2.1 & 6.5 & 0.2 & -0.1 & 3.0 & \text { SLIM } & 4 & & & 23 & 778 & 6.5 \\ 3 & 5.1 & 9.1 & 4.0 & 21.0 & 0.2 & -0.0 & 1.2 & 5-S L * & 7 & 45 & 31 & 74 & 2520 & \\ 4 & 9.2 & 11.8 & 2.6 & 12.6 & 0.1 & -0.0 & 1.1 & 5-S L * & 4 & 27 & 29 & 44 & 1518 & \\ 5 & 11.8 & 19.2 & 7.3 & 7.1 & 0.2 & 0.5 & 3.1 & \text { SLIM } & 4 & & & 25 & 857 & 1.8\end{array}$

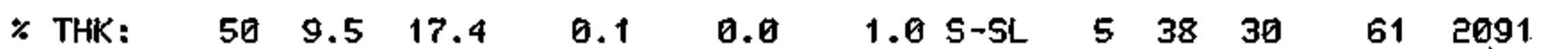

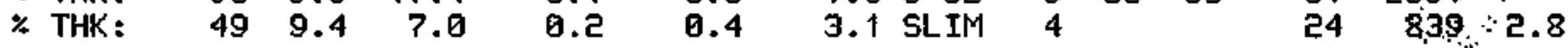


SITE ID: HATO1

GROUND WATER DEPTH: 50.0 (FT.)
LOCATION ID: OQ?

AUERAGE SOIL WEIGHT: 100.0 (PSF)

$\begin{array}{llll}\text { - OEPTH - } & \text { AUG. AUG. PUG. PUE. FRICT. SOIL } & \text { UND. } \\ \end{array}$ LY FROM TO THK. POIHT SLEEUE PRESS. RATIO CLASS BC DEN FA ELAS. STREN OCR

\begin{tabular}{|c|c|c|c|c|c|c|c|c|c|c|c|c|c|}
\hline & $\begin{array}{r}1.0 \\
3.9 \\
13.0\end{array}$ & $\begin{array}{l}1.0 \\
2.8 \\
9.1\end{array}$ & $\begin{array}{r}9.9 \\
15.5 \\
7.4\end{array}$ & \multicolumn{2}{|c|}{$\begin{array}{rr}0.2 & 0.0 \\
0.2 & 0.0 \\
0.3 & -0.0\end{array}$} & \multicolumn{2}{|c|}{$\begin{array}{l}\text { SLIM } \\
\text { S-SL* } \\
\text { SLIM }\end{array}$} & \multicolumn{2}{|c|}{$\begin{array}{l}6 \\
5 \\
4\end{array}$} & \multicolumn{2}{|c|}{38} & 30 & $\begin{array}{l}0.1 \\
1.0 \\
3 \\
3.9\end{array}$ \\
\hline
\end{tabular}

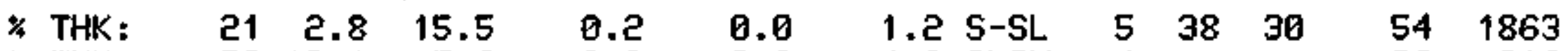

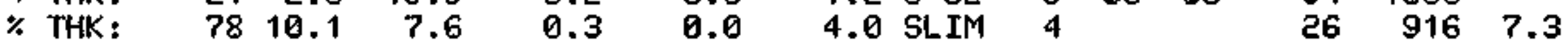


SITE TD: HATO1

GROUND WATER DEPTH: 50.0 (FT.)
LDCATIDN TD:

AUERAGE SOIL LEIGHT: 100,0 (PSF)

$\begin{array}{lll} & \text { AUG. AUG. UND. }\end{array}$

LY FROM TO THK. POIMT SLEEUE PRESS. RATIO CLASS BC DEN FA ELAS. STREN DCR

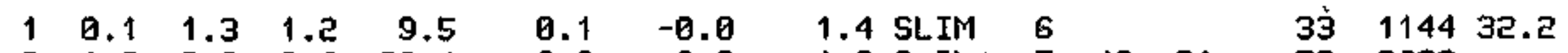

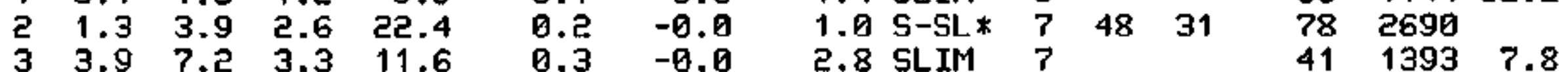

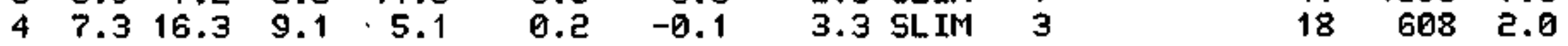

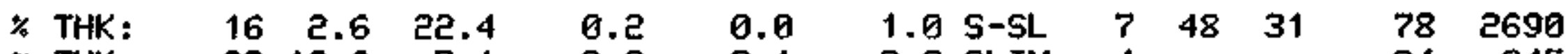

* THK: $8313.6 \quad 7.1 \quad 0.2 \quad-0.1 \quad 3.0$ SLIM $4 \quad 84$ 
SITE ID: HATQ

GROUND WATER OEPTH: 50.0 (FT.)

\section{LOCATION ID: 009}

AUERAGE SOIL WEIGHT: 100.0 (PSF)

- DEPTH -

GUG. AUG. UND.

LY FROM TO THK. POINT SLEEUE PRESS. RATID CLASS BC DEM FA ELAS. STREN OCR

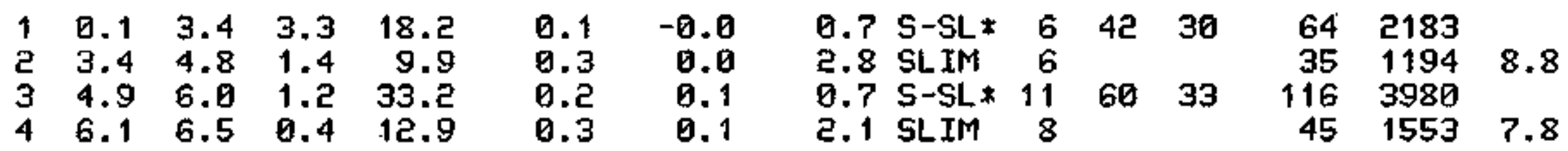

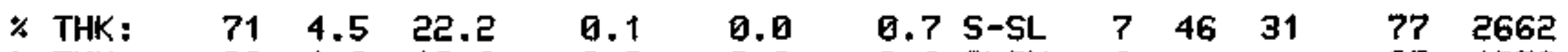

* THK: $\begin{array}{llllllll}28 & 1.8 & 10.6 & 0.3 & 0.0 & 2.6 & \text { SLIM } 6 & \end{array}$ 
SITE ID: HATV1

GROUND WATER DEPTH: 50.0 (FT.)
LOCATION ID: 010

AUERAGE SOIL WEIGHT: 100.0 (PSF)

LY FROM TO THK, POINT SLEEVE PRESS, RATIO CLASS BC DEN FA ELAS. 5TREN OCR

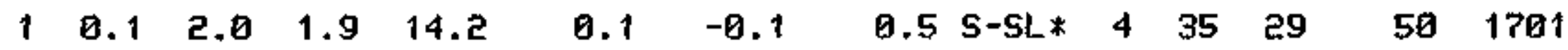

$\begin{array}{llllllllllll}2 & 2.0 & 3.6 & 1.6 & 7.0 & 0.1 & -0.0 & 1.6 \text { SLIM } & 4 & 25 & 842 & 9.0\end{array}$

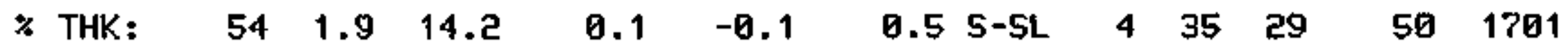

* THK: $45 \quad 1.6 \quad 7.0 \quad 0.1 \quad 0.0 \quad 1.6$ SLIM $4 \quad 258429.0$ 
SITE ID: HATQ1

GROUND WATER DEPTH: 50.8 (FT.)
LOCATION ID : 011

AUERAGE SOIL WJEIGHT: 100.0 (PSF)

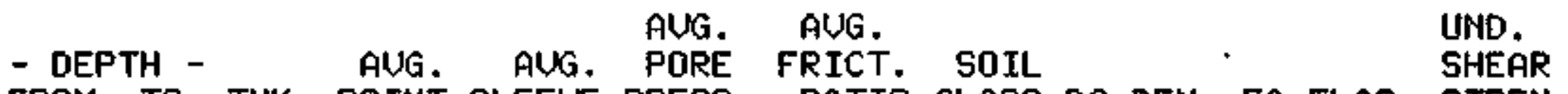
LY FROM TO THK. POINT SLEEVE PRESS. RATIO CLASS BC DEN FA ELAS. STREN DCR

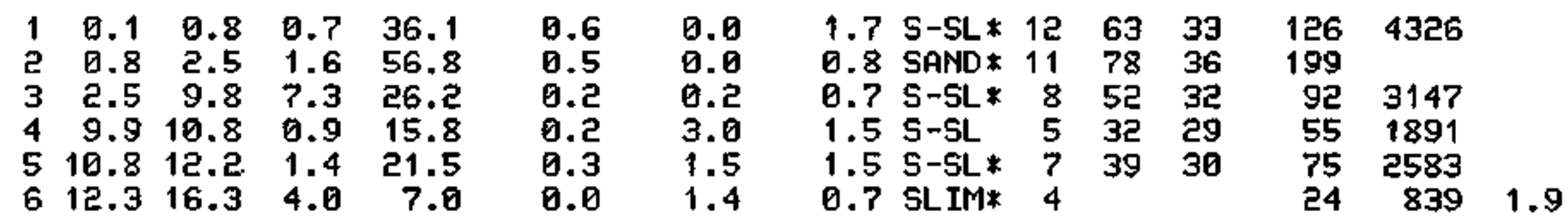

\begin{tabular}{|c|c|c|c|c|c|c|c|c|c|c|c|}
\hline $\begin{array}{l}\text { \% THK: } \\
\text { * THK: } \\
\text { * THK: }\end{array}$ & $\begin{array}{l}10 \\
64 \\
25\end{array}$ & $\begin{array}{r}1.6 \\
10.3 \\
4.8\end{array}$ & $\begin{array}{r}56.8 \\
25.3 \\
7.0\end{array}$ & $\begin{array}{l}0.5 \\
0.2 \\
0.0\end{array}$ & $\begin{array}{l}0.0 \\
0.6 \\
1.4\end{array}$ & $\begin{array}{l}0.8 \text { SAND } \\
0.9 \text { S-SL } \\
0.7 \text { SLIM }\end{array}$ & $\begin{array}{r}11 \\
7 \\
4\end{array}$ & $\begin{array}{l}78 \\
49\end{array}$ & $\begin{array}{l}36 \\
32\end{array}$ & $\begin{array}{r}199 \\
88 \\
24\end{array}$ & $\begin{array}{c}3040 \\
839\end{array}$ \\
\hline
\end{tabular}


SITE ID : HATO1

GROUND WATER DEPTH: 50.0 (FT.)
LOCATION ID: 812

AUERAGE SOIL WEIGHT: 100.0 (PSF)

AUG. AUG. UND.

LY FROM TO THK. POINT SLEEUE PRESS. RATIÓ CLASS BC DEN FA ELAS. STREN DCR

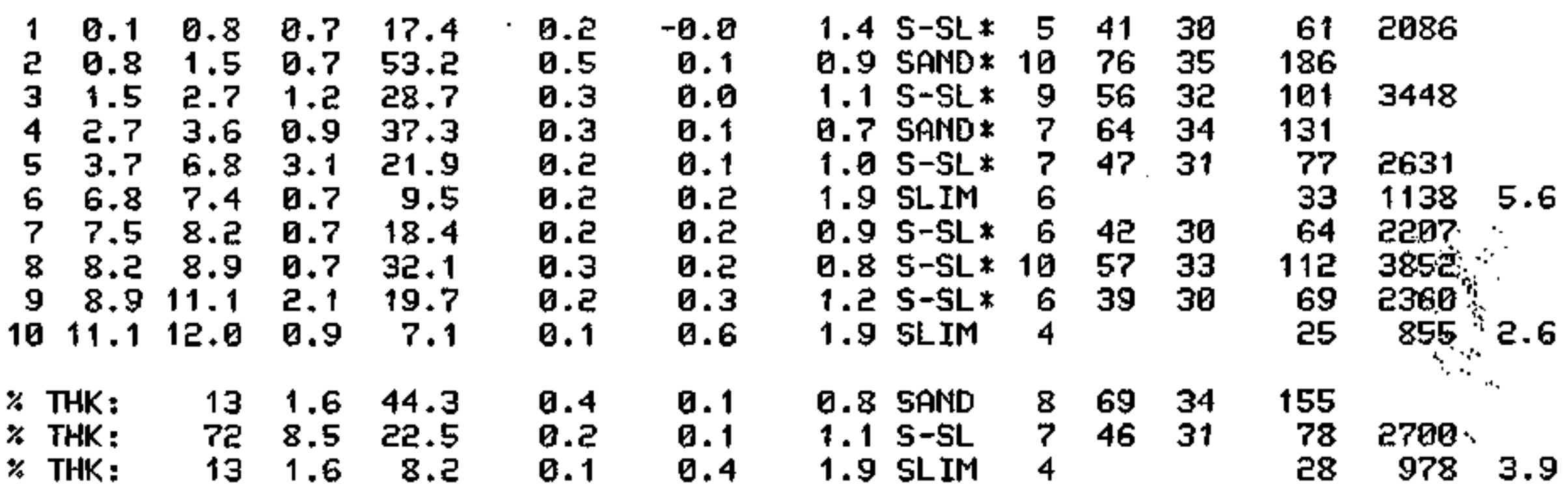


SITE ID: HATO1

GROUND WATER DEPTH: 50.0 (FT.)
LOCATION ID: 013

AUERAGE SOIL WEIGHT: 100.0 (PSF)

DEPTH - AUG. AUG. AUG. AUG.

LY FROM TO THK. POINT SLEEUE PRESS. RATIO CLASS BC DEN FA ELAS. STREM OCR

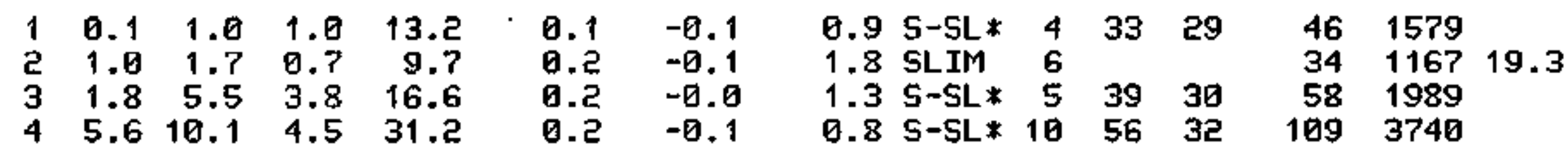

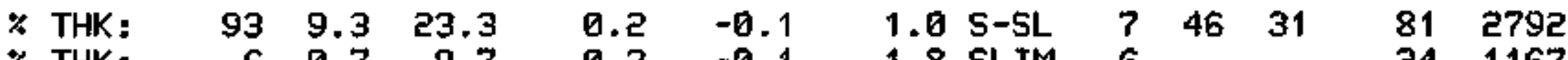

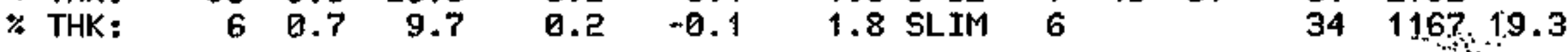

i. 
SITE ID: HATO1

GROUND IJATER DEPTH: 50.0 (FT.)
LOCATION ID : 014

AUERAGE SOIL WEIGHT: 100.0 (PSF)

AUG. AUG. UND.

- DEPTH - AUG. AUG. PORE FRICT. SOIL SHEAR

LY FROM TO THK. POINT SLEEVE PRESS. RATIO CLASS BC DEN FA ELAS. STREN DCR

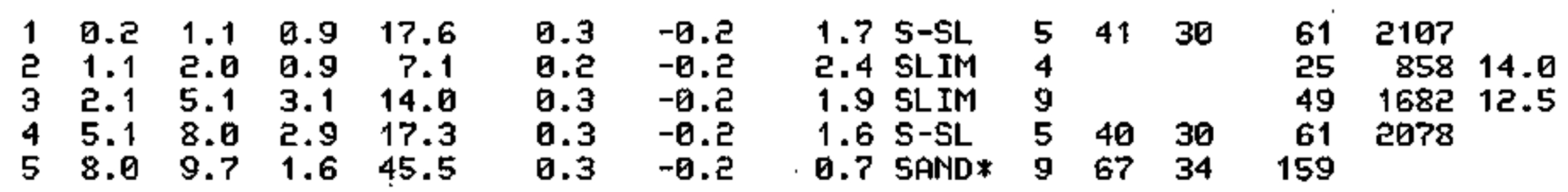

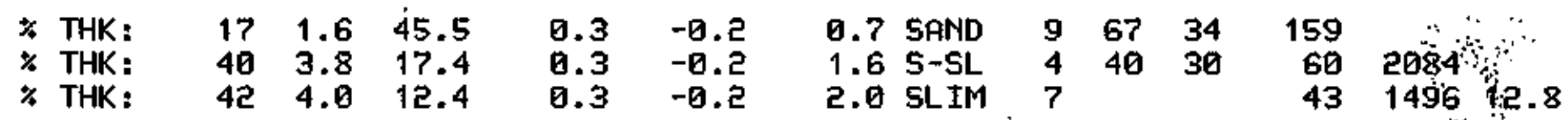


SITE ID: HATO 1

GRQUND WATER DEPTH: 50.0 (FT.)
LOCATION ID: 015

AUERAGE SOIL WEIGHT: 100.D (PSF)

- DEPTH - AUG. AUG. PURE FUG. FICT. SOIL LY FROM TO THK, POINT SLEEUE PRESS. RATIO CLASS BC DEN FA ELAS. STREN DCR

\begin{tabular}{|c|c|c|c|c|c|c|c|c|c|c|c|}
\hline $\begin{array}{r}0.2 \\
2.4 \\
3.1 \\
5.8 \\
7.2 \\
9.1 \\
10.6 \\
11.8 \\
16.6 \\
18.5 \\
19.9 \\
22.0 \\
22.8\end{array}$ & $\begin{array}{r}2.4 \\
3.1 \\
5.7 \\
7.2 \\
9.1 \\
10.6 \\
11.7 \\
16.5 \\
18.4 \\
19.9 \\
22.0 \\
22.7 \\
25.1\end{array}$ & $\begin{array}{l}2.2 \\
0.7 \\
2.6 \\
1.4 \\
1.9 \\
1.4 \\
1.1 \\
4.8 \\
1.9 \\
1.4 \\
2.1 \\
0.7 \\
2.4\end{array}$ & $\begin{array}{r}7.1 \\
25.2 \\
8.9 \\
27.5 \\
14.1 \\
28.8 \\
16.8 \\
27.3 \\
49.0 \\
28.0 \\
42.6 \\
17.5 \\
16.9\end{array}$ & $\begin{array}{l}0.2 \\
0.4 \\
0.2 \\
0.3 \\
0.3 \\
0.2 \\
0.3 \\
0.4 \\
0.5 \\
0.4 \\
0.5 \\
0.3 \\
0.2\end{array}$ & $\begin{array}{l}0.0 \\
0.1 \\
0.1 \\
0.2 \\
0.2 \\
0.3 \\
0.3 \\
0.3 \\
0.3 \\
0.3 \\
0.4 \\
0.3 \\
0.4\end{array}$ & $\begin{array}{ll}2.9 & \text { SLIM } \\
1.6 & 5-S L * \\
2.3 & \text { SLIM } \\
1.3 & \text { S-SL* } \\
2.1 & \text { SLIM } \\
9.8 & \text { S-SL* } \\
1.9 & \text { SLIM } \\
1.3 & \text { S-SL* } \\
1.9 & \text { SAND* } \\
1.5 & 5-S L * \\
1.1 & 5-S L * \\
1.9 & 5-S L \\
0.9 & 5-S L *\end{array}$ & $\begin{array}{r}4 \\
8 \\
5 \\
9 \\
9 \\
9 \\
11 \\
9 \\
9 \\
9 \\
14 \\
5 \\
5\end{array}$ & $\begin{array}{l}41 \\
54 \\
36 \\
46 \\
23 \\
21\end{array}$ & $\begin{array}{l}32 \\
32 \\
30 \\
32 \\
29 \\
31 \\
28 \\
28\end{array}$ & $\begin{array}{r}25 \\
88 \\
31 \\
96 \\
49 \\
101 \\
59 \\
95 \\
172 \\
98 \\
149 \\
61 \\
59\end{array}$ & $\begin{array}{r}846 \\
3021 \\
1071 \\
3294 \\
1697 \\
3456 \\
2015 \\
3270 \\
3355 \\
5109 \\
2101 \\
2024\end{array}$ \\
\hline $\begin{array}{l}\text { THK: } \\
\text { THK: }\end{array}$ & $\begin{array}{r}7 \\
60 \\
31\end{array}$ & $\begin{array}{r}1.9 \\
14.9 \\
7.8\end{array}$ & $\begin{array}{l}49.0 \\
27.4 \\
10.8\end{array}$ & $\begin{array}{l}0.5 \\
0.3 \\
0.2\end{array}$ & $\begin{array}{l}0.3 \\
0.1\end{array}$ & $\begin{array}{l}1.0 \text { SAMD } \\
1.2 \text { S-SL } \\
2.4 \text { SLIM }\end{array}$ & $\begin{array}{l}9 \\
8 \\
6\end{array}$ & $\begin{array}{l}54 \\
39\end{array}$ & $\begin{array}{l}32 \\
30\end{array}$ & $\begin{array}{r}172 \\
95 \\
37\end{array}$ & $\begin{array}{l}3289 \\
1293\end{array}$ \\
\hline
\end{tabular}


SITE ID: HATD1

GROUND WATER DEPTH: 50.0 (FT.)
LOCATION ID: 016

AUERAGE SOIL WEIGHT: 100.0 (PSF)

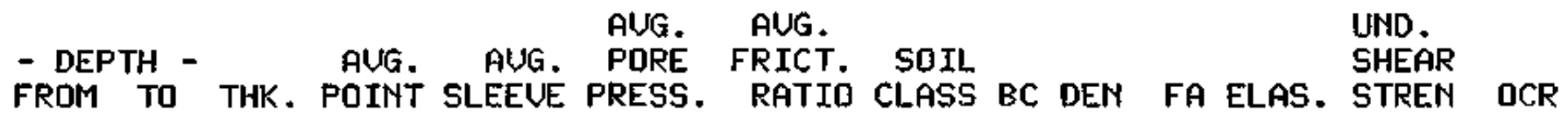

$\begin{array}{lllllllll}1 & 0.0 & 4.4 & 4.4 & 8.2 & 0.2 & 0.1 & 0.3 & \text { SLIM } 5\end{array}$

$\begin{array}{lllllll}2 & 4.4 & 5.1 & 0.7 & 22.4 & 0.3 & 0.3\end{array}$

$\begin{array}{llllll}3 & 5.1 & 7.3 & 2.1 & 10.8 & 0.3\end{array}$

$\begin{array}{llllll}4 & 7.3 & 9.2 & 1.9 & 28.0 & 0.4\end{array}$

$\begin{array}{llllll}5 & 9.2 & 10.6 & 1.4 & 17.2 & 0.4\end{array}$

$\begin{array}{llllll}6 & 10.6 & 15.6 & 5.0 & 29.2 & 0.4\end{array}$

$\begin{array}{llllll}7 & 15.6 & 16.1 & 0.5 & 14.6 & 0.5\end{array}$

0.2

0.8

0.8

0.7

1.4 S-SL*

2. 4 SI. IM 7

1.5 S-SL* 9

2.2 SLIM 11

1.3 S-SL* $945 \quad 31$

$\begin{array}{rrr}29 & 988 & 12.1\end{array}$

0.6

3.2 SLIM 9

$48 \quad 31$

$79 \quad 2694$

$38 \quad 1292$

$98 \quad 3365$

$60 \quad 2066$

$102 \quad 3506$

$51 \quad 1756 \cdot 2.9$

\% THK:

$\begin{array}{lll}47 & 7.6 & 28.3\end{array}$

0.4

4 THK:

$\begin{array}{lll}52 & 8.4 & 10.7\end{array}$

0.3

0.7

1.4 S-SL

2.4 SLIM

$\begin{array}{lll}8 & 47 & 31 \\ 6 & \end{array}$

$98 \quad 3395$

$37 \quad 1289: 9.2$ 
SITE ID: HATQ1

GROUND WATER DEPTH: 50.0 (FT.)
L.OCATION ID: 017

AUERAGE SOIL WEIGHT: 100.0 (PSF)

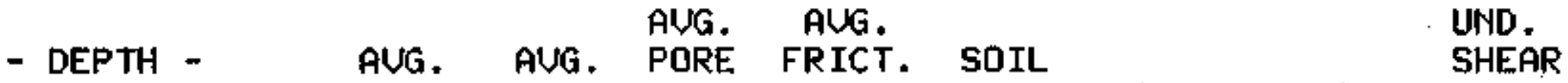
LY FROM TO THK. POINT SLEEUE PRESS. RATIO CLASS BC DEN FA ELAS. STREN OCR

$\begin{array}{llllllll}1 & 0.1 & 5.5 & 5.4 & 8.2 & 0.3 & -0.0 & 3.1 \\ \text { SLIM }\end{array}$

$\begin{array}{llllll}2 & 5.6 & 7.0 & 1.4 & 26.2\end{array}$

0.30 .1

$\begin{array}{llll}7.0 & 9.3 & 2.4 & 13.1\end{array}$

$0.4 \quad 0.1$

$\begin{array}{lllll}4 & 9.4 & 11.5 & 2.1 & 40.7\end{array}$

0.4

0.1

.1 SLIM 5

1.3 S-SL* 8

2.7 SLIM 8

0.9 SAND* 8

1.6 S-SL* 12

0.1

0.6

0.1

3.3 SLIM 9

1.1 5-SL* 10

0.4

0.1

$\begin{array}{lllll}8 & 17.3 & 19.4 & 2.1 & 17.5\end{array}$

0.4

0.1

0.5

0.2

$\begin{array}{lllll}9 & 19.4 & 20.1 & 0.7 & 35.6\end{array}$

0.5

2.5 SLIM 11

1.4 S-SL* 11

ด.

0.5

0.2

$\begin{array}{lllll}11 & 21.8 & 23.2 & 1.4 & 24.4\end{array}$

0.2

2.5

1330.431 .3

7.18 .4

0.4

1.8

1431.431 .8

0.515 .0

$0.1 \quad 1.6$

4.0 SLIM 8

2.0 SLIM 16

2.9 SLIM 5

$1.3 \mathrm{~S}-\mathrm{SL} * 9$

$53 \quad 32 \quad 29 \quad 989.10 .1$

$\begin{array}{llll}53 & 32 & 92 & 3139 \\ & 46 & 1575 & 6.2\end{array}$

$\begin{array}{lll}60 & 33 & 143\end{array}$

$53 \cdot 32 \quad 128 \quad 4376$

$\begin{array}{llll}48 & 1660 & 3.7\end{array}$

$\begin{array}{llll}44 & 31 & 111 & 3812\end{array}$

$61 \quad 2094.2 .8$

$\begin{array}{llll}42 & 30 & 124 & 4268\end{array}$

$45 \quad 1536,1.9$

$852923 \cdot 2.7$

$\begin{array}{llll}29 & 29 \quad 100 \quad 3426\end{array}$

$1.0 \mathrm{~S}-\mathrm{SL}$ * $4 \quad 1626$ 52 1797

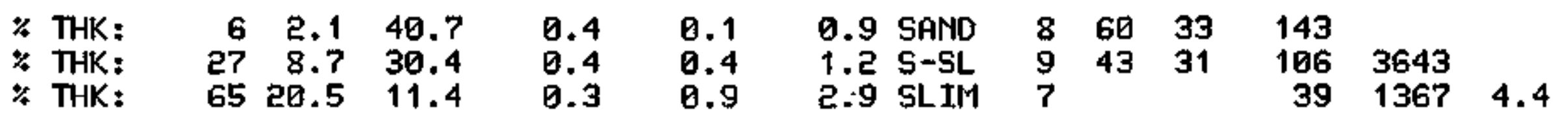


SITE ID: HATO1

GROUND WATER DEPTH: 50.0 (FT.)
LOCATION ID: 018

AUERAGE SOIL WEIGHT: 100.0 (PSF)

AUG. AUG. LIND.

- DEPTH - AUG. AUG. PORE FRICT. SOIL SHEAR LY FROM TO THK. POINT SLEEUE PRESS. RATIO CLASS BC DEN FA ELAS. STREN DCR

\begin{tabular}{|c|c|c|c|c|c|c|c|c|c|c|c|c|}
\hline $\begin{array}{rr}1 & 0.0 \\
2 & 3.7 \\
3 & 4.9 \\
4 & 8.4 \\
5 & 11.8 \\
6 & 13.4 \\
7 & 15.6 \\
8 & 16.8\end{array}$ & $\begin{array}{r}3.6 \\
4.8 \\
8.4 \\
11.7 \\
13.4 \\
15.6 \\
16.8 \\
18.4\end{array}$ & $\begin{array}{l}3.6 \\
1.1 \\
3.5 \\
3.3 \\
1.6 \\
2.1 \\
1.1 \\
1.6\end{array}$ & $\begin{array}{l}13.4 \\
13.1 \\
21.5 \\
32.4 \\
13.7 \\
25.5 \\
40.8 \\
40.6\end{array}$ & $\begin{array}{l}0.1 \\
0.3 \\
0.2 \\
0.3 \\
0.3 \\
0.3 \\
0.3 \\
0.5\end{array}$ & $\begin{array}{r}-0.0 \\
0.0 \\
0.1 \\
0.1 \\
0.1 \\
0.2 \\
0.2 \\
0.2\end{array}$ & $\begin{array}{l}1.0 \\
2.0 \\
1.1 \\
0.9 \\
2.2 \\
1.2 \\
0.6 \\
1.1\end{array}$ & $\begin{array}{l}\text { S-SL* } \\
\text { SLIM } \\
\text { S-SL* } \\
\text { S-SL* } \\
\text { SLIM } \\
\text { S-SL* } \\
\text { SAMD* } \\
\text { S-SL* }\end{array}$ & $\begin{array}{r}4 \\
8 \\
7 \\
10 \\
9 \\
8 \\
8 \\
13\end{array}$ & $\begin{array}{l}47 \\
53 \\
39 \\
50 \\
48\end{array}$ & $\begin{array}{l}30 \\
32 \\
31\end{array}$ & $\begin{array}{r}47 \\
46 \\
75 \\
113 \\
48 \\
89 \\
143 \\
142\end{array}$ & $\begin{array}{r}1609 \\
1572 \\
2584 \\
3890 \\
1644 \\
3056 \\
\therefore \\
486 ?\end{array}$ \\
\hline$T$ & $\begin{array}{r}6 \\
78 \\
15\end{array}$ & $\begin{array}{r}1.1 \\
14.1 \\
2.7\end{array}$ & $\begin{array}{l}40.8 \\
24.7 \\
13.5\end{array}$ & $\begin{array}{l}0.3 \\
0.2 \\
0.3\end{array}$ & $\begin{array}{l}0.2 \\
0.1\end{array}$ & 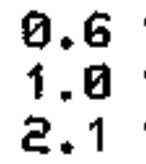 & & $\begin{array}{l}8 \\
7 \\
8\end{array}$ & $\begin{array}{l}50 \\
44\end{array}$ & $\begin{array}{l}32 \\
31\end{array}$ & $\begin{array}{r}143 \\
86 \\
47\end{array}$ & $\begin{array}{l}2970 \\
1614\end{array}$ \\
\hline
\end{tabular}


SITE ID: HATO1

GROUND WIATER DEPTH: 50.0 (FT.)
LOCATION ID: V19

AUERAGE SOIL WEIGHT: 100.0 (PSF)
- DEPTH -
LY FROM TO THK. POINT SLEEVE PRESS. RATIO SLIL $\begin{array}{llll}1 & 0.0 & 0.8 & 0.7\end{array}$

$\begin{array}{llll}* \text { THK: } 100 & 0.7 \quad 13.4\end{array}$
0.1

0.1

0.8 S-SL*

$4 \quad 34 \quad 29$

$0.8 \mathrm{~S}-\mathrm{SL}$

$\begin{array}{lll}4 & 34 & 29\end{array}$

UND.

SHEAR RATIO CLASS BC DEN FA ELAS. STREN OCR

$47 \quad 1613$

$46 \quad 1613$ 
SITE ID: HATO1

GROUND WATER DEPTH: 50.0 (FT.)
LOCATION ID: 020

AUERAGE SOIL WEIGHT: 100.0 (PSF)

$\begin{array}{llll} & & & \\ \text { - DEPTH - } & \text { AUG. AUG. PUG. PORE FRICT, SOIL } & \text { SHEAR }\end{array}$ LY FROM TO THK. POINT SLEEUE PRESS. RATIO CLASS BC DEN FA ELAS. STREN DCR

\begin{tabular}{|c|c|c|c|c|c|c|c|c|c|c|c|c|}
\hline $\begin{array}{lr}1 & 0.0 \\
2 & 1.0 \\
3 & 2.0 \\
4 & 3.2 \\
5 & 6.5 \\
6 & 9.4 \\
7 & 10.3\end{array}$ & $\begin{array}{r}1.0 \\
2.0 \\
3.1 \\
6.5 \\
9.3 \\
10.3 \\
11.3\end{array}$ & $\begin{array}{l}1.0 \\
1.0 \\
1.1 \\
3.3 \\
2.8 \\
0.9 \\
1.0\end{array}$ & $\begin{array}{r}15.2 \\
6.7 \\
15.9 \\
10.1 \\
27.2 \\
13.3 \\
31.7\end{array}$ & $\begin{array}{l}0.1 \\
0.1 \\
0.2 \\
0.2 \\
0.2 \\
0.2 \\
0.2\end{array}$ & $\begin{array}{r}-0.2 \\
-0.1 \\
-0.1 \\
-0.1 \\
0.0 \\
0.0 \\
0.0\end{array}$ & $\begin{array}{l}0.8 \\
1.8 \\
1.4 \\
2.4 \\
0.7 \\
1.4 \\
0.6\end{array}$ & $\begin{array}{l}\text { S-SL* } \\
\text { SLIM } \\
\text { S-SL* } \\
\text { SLIM } \\
\text { S-SL* } \\
\text { S-SL } \\
\text { S-SL* }\end{array}$ & $\begin{array}{r}5 \\
4 \\
5 \\
6 \\
9 \\
4 \\
10\end{array}$ & $\begin{array}{l}38 \\
53 \\
29 \\
51\end{array}$ & $\begin{array}{l}30 \\
32 \\
29 \\
32\end{array}$ & $\begin{array}{r}53 \\
23 \\
56 \\
35 \\
95 \\
47 \\
111\end{array}$ & $\begin{array}{r}1827 \\
798 \\
1905 \\
1208 \\
3265 \\
1599 \\
3800\end{array}$ \\
\hline $\begin{array}{l}\text { IHK: } \\
\text { THK: }\end{array}$ & $\begin{array}{l}61 \\
38\end{array}$ & $\begin{array}{l}6.8 \\
4.3\end{array}$ & $\begin{array}{r}22.4 \\
9.3\end{array}$ & $\begin{array}{l}0.2 \\
0.2\end{array}$ & $\begin{array}{l}-0.0 \\
-0.1\end{array}$ & 2.3 & $\begin{array}{l}\text { S-SL } \\
\text { SLIM }\end{array}$ & $\begin{array}{l}7 \\
5\end{array}$ & & 31 & $\begin{array}{l}78 \\
32\end{array}$ & \\
\hline
\end{tabular}


SITE ID: HATO1

GROUMD WATER DEPTH: 50.0 (FT.)
LOCATION ID: 021

ALERAGE SOIL WEIGHT: 100.0 (PSF)

$\begin{array}{llll} & & & \\ \text { - DEPTH - AUG. AUG. UND. } & \end{array}$ LY FROM TO THK. POINT SLEEUE PRESS. RATIO CLASS BC DEN FA ELAS. STREN DCR

\begin{tabular}{|c|c|c|c|c|c|c|c|c|c|c|c|c|}
\hline $\begin{array}{r}0.0 \\
1.3 \\
2.5 \\
4.9 \\
8.2 \\
9.2 \\
9.9 \\
10.6 \\
12.3 \\
13.0 \\
16.6 \\
18.3\end{array}$ & $\begin{array}{r}1.2 \\
2.5 \\
4.9 \\
8.2 \\
9.2 \\
9.9 \\
10.6 \\
12.3 \\
13.0 \\
16.6 \\
18.2 \\
19.2\end{array}$ & $\begin{array}{l}1.2 \\
1.2 \\
2.4 \\
3.3 \\
0.9 \\
0.7 \\
0.7 \\
1.6 \\
0.7 \\
3.5 \\
1.6 \\
0.9\end{array}$ & $\begin{array}{r}7.2 \\
14.8 \\
8.2 \\
26.4 \\
51.8 \\
15.4 \\
26.5 \\
38.8 \\
19.0 \\
28.9 \\
40.6 \\
19.6\end{array}$ & $\begin{array}{l}0.1 \\
0.3 \\
0.2 \\
0.3 \\
0.4 \\
0.3 \\
0.3 \\
0.4 \\
0.6 \\
0.4 \\
0.5 \\
0.5\end{array}$ & $\begin{array}{r}-0.2 \\
-0.1 \\
0.0 \\
0.1 \\
0.2 \\
0.2 \\
0.2 \\
0.2 \\
0.2 \\
0.3 \\
0.4 \\
0.3\end{array}$ & $\begin{array}{l}2.0 \\
1.7 \\
2.6 \\
1.1 \\
0.8 \\
2.1 \\
1.2 \\
1.0 \\
3.0 \\
1.6 \\
1.1 \\
2.5\end{array}$ & $\begin{array}{l}\text { SLIM } \\
\text { S-SL } \\
\text { SLIM } \\
\text { S-SL* } \\
\text { SAND* } \\
\text { SLIM } \\
\text { S-SL* } \\
\text { S-SL* } \\
\text { SLIM } \\
\text { S-SL* } \\
\text { S-SL* } \\
\text { SLIM }\end{array}$ & $\begin{array}{r}4 \\
4 \\
5 \\
8 \\
10 \\
10 \\
8 \\
12 \\
12 \\
9 \\
13 \\
13\end{array}$ & $\begin{array}{l}53 \\
72 \\
47 \\
56 \\
42 \\
48\end{array}$ & $\begin{array}{l}32 \\
35 \\
31 \\
32 \\
30 \\
31\end{array}$ & $\begin{array}{r}25 \\
52 \\
29 \\
92 \\
181 \\
54 \\
93 \\
136 \\
67 \\
191 \\
142 \\
69\end{array}$ & $\begin{array}{r}869 \\
1777 \\
978 \\
3163 \\
1847 \\
3183 \\
465 \\
2283 \\
3465 \\
4872 \\
2352\end{array}$ \\
\hline & $\begin{array}{r}4 \\
63 \\
31\end{array}$ & $\begin{array}{r}0.9 \\
11.9 \\
5.9\end{array}$ & & 0. & $\begin{array}{l}0.2 \\
0.2 \\
0.1\end{array}$ & $\begin{array}{l}.8 \\
.3 \\
.5\end{array}$ & $\begin{array}{l}\text { ND } \\
\text { SL } \\
\text { IM }\end{array}$ & $\begin{array}{r}10 \\
9 \\
7\end{array}$ & $\begin{array}{l}72 \\
47\end{array}$ & 31 & $\begin{array}{r}181 \\
103 \\
41\end{array}$ & $\begin{array}{l}3543 \\
1423\end{array}$ \\
\hline
\end{tabular}


SITE ID: HATO 1

GROUND WATER DEPTH: 50.0 (FT.)
LOCATION ID: ODE2

AUERAGE SOIL WEIGHT: 100.0 (PSF)

AUG. AUG. UND.

- DEPTH -
LY FROM TO THK. POINT SLEEUE PRESS. RATIO CLASS BC DEM FA ELAS. STREM DCR

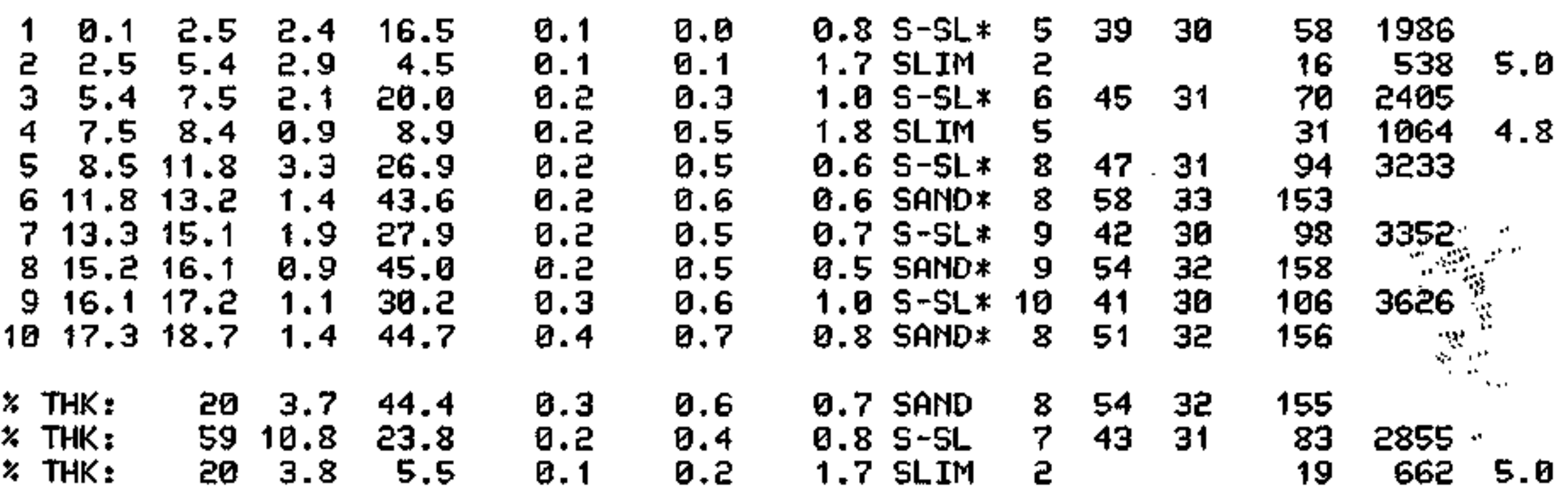


SITE ID: HATDI

GROUND WATER DEPTH: 50.0 (FT.)
LOCATION ID: 023

AUERAGE SDIL WEIGHT: 100.0 (PGF)

- DEFTH - AUG. AUG. PUGE PUG. SOIL FRICT. SOIL UND.

LY FROM TO THK. POINT SLEEUE PRESS. RATIO CLASS BC DEN FA ELAS. STREN DCR

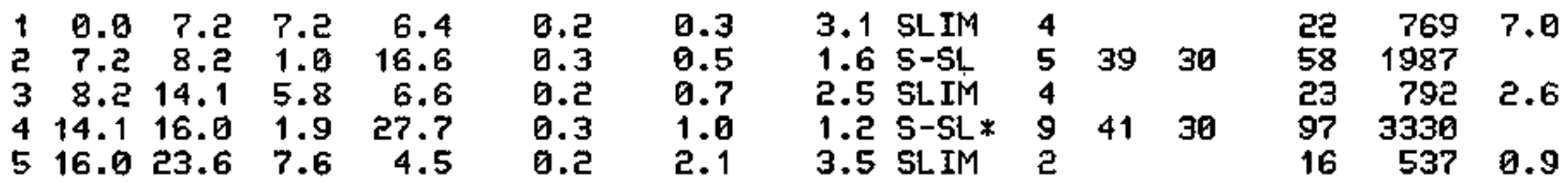

* THK

$\begin{array}{lll}12 & 2.9 & 23.9\end{array}$

0.3

$\begin{array}{llllll}0.8 & 1.3 & 5-S L & 7 & 40 & 30\end{array}$

$83 \quad 2866$

\% THK:

$8720.6 \quad 5.8$

घ.2

1.1

3.1 SLIM

3

20689 
SITE ID: HATO1

GROUND WATER DEPTH: 50.0 (FT.)
LOCATION ID: 024

AUERAGE SOIL WEIGHT: 100.0 (PSF)

AUG, AUG. UND.

- DEPTH - AUG. AUG. PORE FRICT. SOIL

SHEAR

LY FROM TO THK. POINT SLEEUE PRESS. RATIO CLASS BC DEN FA ELAS. STREN QCR

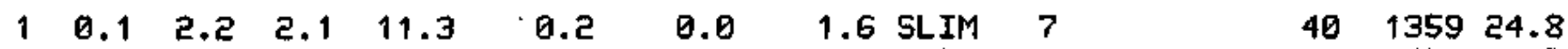

$\begin{array}{llllllll}2 & 2.3 & 24.7 & 22.4 & 4.5 & 0.2 & 0.5 & 4.3\end{array}$ SLIM 2

$16 \quad 536 \quad 1.5$

* THK: $10024.5 \quad 5.1 \quad 0.2 \quad 0.5 \quad 4.1$ SLIM 2

$18 \quad 606 \quad 3.5$ 
STITĚr IO: HATO1

GROUND WATER DEPTH: 50.0 (FT.)
LOCATION ID: DES

AUERAGE SOIL WEIGHT: 100.0 (PSF)

L DEPTH -
LYROM TO THK. POINT SLEEVE PRESS. RATIO CLASS BC DEN FA ELAS. STREN OCR

$\begin{array}{rrrrr}1 & 0.1 & 1.3 & 1.2 & 18.7 \\ 2 & 1.3 & 3.0 & 1.6 & 7.7 \\ 3 & 3.0 & 4.1 & 1.1 & 20.4 \\ 4 & 4.2 & 5.3 & 1.2 & 13.9 \\ 5 & 5.4 & 6.8 & 1.4 & 9.5 \\ 6 & 6.8 & 10.6 & 3.8 & 8.9 \\ 7 & 10.6 & 12.0 & 1.4 & 13.8 \\ 8 & 12.1 & 15.1 & 3.1 & 10.2\end{array}$

0.2

ด. 2

0.3

0.4

0.1

0.3

a.e

0.2

$-0.2$

$-0.1$

$-0.1$

$-0.1$

$-0.1$

$-0.0$

0.0

0.1

$0.2-0.2$

$0.2-0.0$

$\begin{array}{lll}1.1 & \text { S-SL* } & 6 \\ 2.1 & \text { SLIM } & 5 \\ 1.6 & \text { S-SL* } & 6 \\ 2.8 & \text { SLIM } & 9 \\ 1.4 & \text { SLIM } & 6 \\ 3.0 & \text { SLIM } & 5 \\ 1.6 & \text { SLIM } & 9 \\ 2.3 & \text { SLIM } & 6\end{array}$

$43 \quad 30$

$45 \quad 31$

$$
\begin{array}{rrr}
65 & 2245 & \\
27 & 922 & 11.8 \\
72 & 2454 & \\
49 & 1666 & 10.1 \\
33 & 1137 & 6.3 \\
31 & 1069 & 4.3 \\
48 & 1652 & 4.3 \\
36 & 1223 . & 2.7
\end{array}
$$$$
\begin{array}{lllllll}
1.3 & \text { S-SL } & 5 & 43 & 31 & 68 & 2344
\end{array}
$$$$
2.4 \text { SLIM } 6
$$ 
SITE ID: HATQT

GROUND WATER DEPTH: 50.0 (FT.)
LOCATION ID: 026

AUERAGE SOIL WEIGHT: 100.0 (PSF)

- DEPTH - AUG. AUG. PUG. AUG. FRICT. SOIL UND. LY FROM TO THK. POINT SLEEVE PRESS. RATIO CLASS BC DEN FA ELAS. STREN OCR

$\begin{array}{lllllllllllllll}1 & 0.1 & 5.1 & 5.0 & 14.2 & 0.1 & -0.0 & 0.9 & \text { S-SL* } & 4 & 35 & 29 & 50 & 1703 & \\ 2 & 5.1 & 6.1 & 0.9 & 8.2 & 0.2 & 0.0 & 2.3 & \text { SLIM } & 5 & & & 29 & 989 & 6.1 \\ 3 & 6.1 & 9.0 & 2.9 & 22.9 & 0.3 & 0.2 & 1.1 \text { S-SL* } & 7 & 48 & 31 & 80 & 2753 & \\ 4 & 9.0 & 9.7 & 0.7 & 13.2 & 0.2 & 0.2 & 1.6 \text { SLIM } & 8 & & & 46 & 1588 & 5.3 \\ * \text { THK: } & 83 & 7.9 & 17.4 & 0.2 & 0.1 & 1.0 & \text { S-SL } & 5 & 39 & 30 & 61 & 2088 & \\ * \text { THK: } & 16 & 1.6 & 10.4 & 0.2 & 0.1 & 2.0 \text { SLIM } & 6 & & & 36 & 1251 . & 5.8\end{array}$


SITE ID: HATO1

GROUND WATER DEPTH: 50.9 (FT.)
LOCATION ID: Q2?

AUERAGE SOIL WEIGHT: 100.0 (PSF)

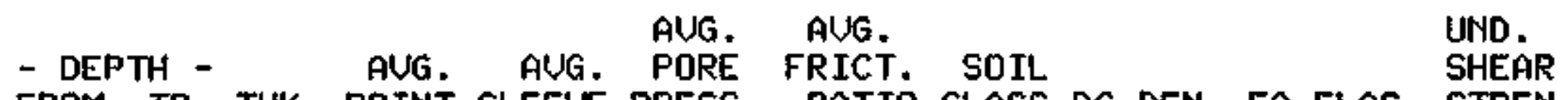
L.Y FROM TO THK. POINT SLEEVE PRESS. RATIO CLASS BC DEN FA ELAS. STREN OCR

\begin{tabular}{|c|c|c|c|c|c|c|c|c|c|c|c|c|}
\hline $\begin{array}{rr}1 & 0.1 \\
2 & 1.8 \\
3 & 6.6 \\
4 & 9.4 \\
5 & 13.2 \\
6 & 14.0 \\
7 & 16.1 \\
8 & 16.8\end{array}$ & $\begin{array}{r}1.8 \\
6.5 \\
9.4 \\
13.2 \\
13.9 \\
16.1 \\
16.8 \\
17.3\end{array}$ & $\begin{array}{l}1.7 \\
4.7 \\
2.9 \\
3.8 \\
0.7 \\
2.1 \\
0.7 \\
0.5\end{array}$ & $\begin{array}{r}12.8 \\
7.1 \\
21.5 \\
22.3 \\
8.4 \\
30.8 \\
14.0 \\
26.3\end{array}$ & $\begin{array}{l}0.2 \\
0.2 \\
0.4 \\
0.3 \\
0.0 \\
0.4 \\
0.5 \\
0.5\end{array}$ & $\begin{array}{r}-0.1 \\
0.0 \\
-0.0 \\
-0.0 \\
-0.0 \\
0.0 \\
0.2 \\
0.3\end{array}$ & $\begin{array}{l}1.3 \\
2.8 \\
1.8 \\
1.3 \\
0.5 \\
1.2 \\
3.6 \\
1.8\end{array}$ & $\begin{array}{l}\text { S-SL. } \\
\text { SLIM } \\
\text { S-SL } \\
\text { S-SL* } \\
\text { SLIM* } \\
\text { S-SL* } \\
\text { SLIM } \\
\text { S-SL }\end{array}$ & $\begin{array}{r}4 \\
4 \\
7 \\
7 \\
5 \\
10 \\
9 \\
8\end{array}$ & $\begin{array}{l}46 \\
40\end{array}$ & $\begin{array}{l}31 \\
30\end{array}$ & $\begin{array}{r}45 \\
25 \\
75 \\
78 \\
29 \\
168 \\
49 \\
92\end{array}$ & $\begin{array}{r}1533 \\
856 \\
2579 \\
2680 \\
1007 \\
3700 \\
1680 \\
3162\end{array}$ \\
\hline & $\begin{array}{l}64 \\
35\end{array}$ & $\begin{array}{l}1.0 \\
6.1\end{array}$ & $\begin{array}{r}22.4 \\
8.0\end{array}$ & 0.2 & $\begin{array}{r}-0.0 \\
0.0\end{array}$ & 6 & LIM & $\begin{array}{l}7 \\
4\end{array}$ & & 31 & $\begin{array}{l}78 \\
28\end{array}$ & 067 \\
\hline
\end{tabular}


SITE TD: HATV1

GROUMO WATER DEPTH: 50.0 (FT.)
LOCATION ID:

AUERAGE SOIL WEIGHT: 100.0 (PSF)

- DEPTH - AUG. AUG. PORE FRICT. SOIL UMG. LY FROM TO THK. POINT SLEEUE PRESS. RATIO CLASS BC DEN FA ELAS. STREN OCR

\begin{tabular}{|c|c|c|c|c|c|c|c|c|c|c|c|}
\hline $\begin{array}{ll}1 & 0.1 \\
2 & 3.0 \\
3 & 3.7 \\
4 & 5.6\end{array}$ & $\begin{array}{r}3.0 \\
3.7 \\
5.6 \\
10.6\end{array}$ & $\begin{array}{l}2.9 \\
0.7 \\
1.9 \\
5.0\end{array}$ & $\begin{array}{r}13.4 \\
8.8 \\
17.6 \\
4.9\end{array}$ & $\begin{array}{l}0.1 \\
0.1 \\
0.1 \\
0.0\end{array}$ & $\begin{array}{l}-0.2 \\
-0.2 \\
-0.2 \\
-0.1\end{array}$ & $\begin{array}{l}0.6 \text { S-SL* } \\
1.0 \text { SLIM* } \\
\text { 0.5 S-SL* } \\
\text { घ.2 SLIM* }\end{array}$ & $\begin{array}{l}4 \\
5 \\
5 \\
3\end{array}$ & 41 & 30 & $\begin{array}{l}47 \\
31 \\
62 \\
17\end{array}$ & $\begin{array}{r}1609 \\
1052 \\
2115 \\
593\end{array}$ \\
\hline $\begin{array}{l}\text { THK: } \\
\text { THK: }\end{array}$ & $\begin{array}{l}45 \\
54\end{array}$ & $\begin{array}{l}4.8 \\
5.7\end{array}$ & $\begin{array}{r}15.1 \\
5.4\end{array}$ & $\begin{array}{l}0.1 \\
0.0\end{array}$ & $\begin{array}{l}-0.2 \\
-0.1\end{array}$ & $\begin{array}{l}0.6 \text { S-SL } \\
0.3 \text { SLIM }\end{array}$ & $\begin{array}{l}4 \\
3\end{array}$ & 36 & 30 & $\begin{array}{l}52 \\
18\end{array}$ & $\begin{array}{r}1809 \\
649\end{array}$ \\
\hline
\end{tabular}


SITE ID: HATQ1

GROUND WATER DEPTH: 50.0 (FT.)
LOCATION ID: 029

AUERAGE SOIL WEIGHT: 100.0 (PSF)

- DEPTH -

AUG. AUG.

UND.

AUG. AUG. PORE FRICT. SOIL

SHEAR

LY FROM TO THK. POINT SLEEVE PRESS. RATIO CLASS BC DEN FA ELAS. STREN OCR
$\begin{array}{rrrrrrrrr}1 & 0.1 & 7.0 & 6.9 & 11.2 & 0.3 & -0.1 & 2.6 \text { SLIM } & ? \\ 2 & 7.1 & 10.1 & 3.1 & 3.2 & 0.3 & 0.3 & 8.2 \text { SLIM } & 2\end{array}$
$39 \quad 134210.7$
* THK: $10010.0 \quad 8.7 \quad 0.3 \quad 0.0 \quad 4.3$ SLIM 5
* THK: $10010.0 \quad 8.7 \quad 0.3 \quad 0.0 \quad 4.3$ SLIM 5
* THK: $10010.0 \quad 8.7 \quad 0.3 \quad 0.0 \quad 4.3$ SLIM 5
113832.0
$30 \quad 1044 \quad 8.0$ 
SITE ID: HATO1

GROUND WATER DEPTH: 50.0 (FT.)
LOCATION ID: 030

AUERAGE SOIL WEIGHT: 100.0 (PSF)

AUG. AUG. UND. LY FROM TO THK. POINT SLEEVE PRESS. RATID CLASS BC DEN FA ELAS. STREN DCR

\begin{tabular}{|c|c|c|c|c|c|c|c|c|c|c|c|c|c|}
\hline $\begin{array}{ll}1 & 0.1 \\
2 & 1.1 \\
3 & 6.6 \\
4 & 8.5\end{array}$ & $\begin{array}{r}1.0 \\
6.5 \\
8.5 \\
12.8\end{array}$ & $\begin{array}{l}1.0 \\
5.4 \\
1.9 \\
4.3\end{array}$ & $\begin{array}{r}11.1 \\
6.1 \\
18.6 \\
6.9\end{array}$ & $\begin{array}{l}0.2 \\
0.1 \\
0.3 \\
0.2\end{array}$ & $\begin{array}{r}-0.1 \\
-0.0 \\
-0.0 \\
0.2\end{array}$ & $\begin{array}{l}1.4 \\
2.1 \\
1.7 \\
2.9\end{array}$ & $\begin{array}{l}\text { SLIM } \\
\text { SLIM } \\
\text { S-SL } \\
\text { SLIM }\end{array}$ & $\begin{array}{l}7 \\
4 \\
6 \\
4\end{array}$ & 42 & 38 & $\begin{array}{l}39 \\
21 \\
65 \\
24\end{array}$ & $\begin{array}{r}1329 \\
734 \\
2229 \\
828\end{array}$ & $\begin{array}{r}41.3 \\
6.5 \\
2.8\end{array}$ \\
\hline $\begin{array}{l}\text { THK: } \\
\text { THK: }\end{array}$ & $\begin{array}{l}15 \\
84\end{array}$ & $\begin{array}{r}1.9 \\
10.7\end{array}$ & $\begin{array}{r}18.6 \\
6.9\end{array}$ & $\begin{array}{l}0.3 \\
0.1\end{array}$ & $\begin{array}{l}0.0 \\
0.1\end{array}$ & $\begin{array}{l}1.7 \\
2.4\end{array}$ & $\begin{array}{l}\text { S-SL } \\
\text { SLIM }\end{array}$ & $\begin{array}{l}6 \\
4\end{array}$ & 42 & 30 & $\begin{array}{l}65 \\
23\end{array}$ & $\begin{array}{r}2229 \\
827\end{array}$ & 8.3 \\
\hline
\end{tabular}


SITE ID: HATO1

GROUND WATER DEPTH: 50.0 (FT.)
LOCATION. ID: 931

AUERAGE SOIL WEIGHT: 100.0 (PSF)

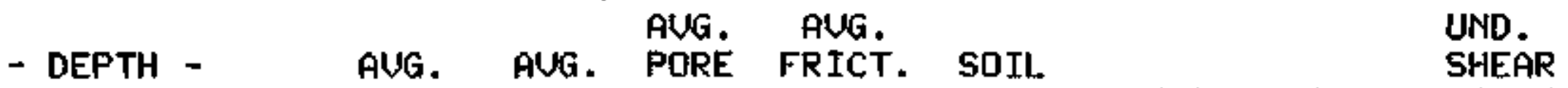
LY FROM TO THK. POINT SLEEUE PRESS. RATIO CLASS BC DEN FA ELAS. STREN OCR

\begin{tabular}{|c|c|c|c|c|c|c|c|c|c|c|c|c|c|}
\hline $\begin{array}{r}0.1 \\
2.5 \\
4.2 \\
7.3 \\
8.5 \\
11.6 \\
12.8 \\
14.0 \\
14.7 \\
15.9 \\
17.5 \\
19.9 \\
21.6 \\
25.4 \\
26.9 \\
27.8 \\
28.8 \\
30.4\end{array}$ & $\begin{array}{r}2.5 \\
4.1 \\
7.2 \\
8.5 \\
11.5 \\
12.8 \\
13.9 \\
14.7 \\
15.8 \\
17.5 \\
19.9 \\
21.6 \\
25.4 \\
26.8 \\
27.8 \\
28.7 \\
30.4 \\
32.1\end{array}$ & $\begin{array}{l}2.4 \\
1.6 \\
3.1 \\
1.2 \\
3.1 \\
1.2 \\
1.1 \\
0.7 \\
1.1 \\
1.6 \\
2.4 \\
1.6 \\
3.8 \\
1.4 \\
0.9 \\
0.9 \\
1.6 \\
1.6\end{array}$ & $\begin{array}{r}6.5 \\
18.9 \\
5.8 \\
18.8 \\
8.6 \\
15.1 \\
6.7 \\
16.3 \\
12.2 \\
32.7 \\
16.0 \\
9.2 \\
11.3 \\
12.1 \\
15.5 \\
9.1 \\
16.5 \\
10.7\end{array}$ & $\begin{array}{l}0.2 \\
0.3 \\
0.2 \\
0.4 \\
0.2 \\
0.3 \\
0.3 \\
0.4 \\
0.4 \\
0.7 \\
0.5 \\
0.2 \\
0.4 \\
0.3 \\
0.3 \\
0.3 \\
0.4 \\
0.1\end{array}$ & $\begin{array}{r}0.0 \\
-0.0 \\
0.0 \\
0.1 \\
0.1 \\
0.2 \\
0.2 \\
0.4 \\
0.4 \\
0.4 \\
0.4 \\
0.5 \\
0.8 \\
1.4 \\
1.7 \\
2.0 \\
1.4 \\
0.8\end{array}$ & $\begin{array}{l}3.1 \\
1.7 \\
3.9 \\
2.9 \\
2.9 \\
1.9 \\
3.8 \\
2.7 \\
3.6 \\
2.3 \\
2.8 \\
1.8 \\
3.6 \\
2.8 \\
1.8 \\
2.9 \\
2.6 \\
1.3\end{array}$ & $\begin{array}{l}\text { SLIM } \\
\text { S-SL } \\
\text { SLIM } \\
\text { SLIM } \\
\text { SLIM } \\
\text { SLIM } \\
\text { SLIM } \\
\text { SLIM } \\
\text { SLIM } \\
\text { S-SL } \\
\text { SLIM } \\
\text { SLIM } \\
\text { SLIM } \\
\text { SLIM } \\
\text { S-SL } \\
\text { SLIM } \\
\text { SLIM } \\
\text { SLIM }\end{array}$ & $\begin{array}{r}4 \\
6 \\
3 \\
12 \\
5 \\
10 \\
4 \\
10 \\
8 \\
10 \\
10 \\
6 \\
7 \\
8 \\
5 \\
6 \\
10 \\
7\end{array}$ & 18 & 27 & $\begin{array}{r}23 \\
66 \\
20 \\
66 \\
30 \\
53 \\
23 \\
57 \\
43 \\
115 \\
56 \\
32 \\
39 \\
42 \\
54 \\
32 \\
58 \\
37\end{array}$ & $\begin{array}{r}775 \\
2269 \\
791 \\
2252 \\
1033 \\
1811 \\
799 \\
1953 \\
1469 \\
3926 \\
1924 \\
1107 \\
1354 \\
1451 \\
1856 \\
1094 \\
1978 \\
1282\end{array}$ & 3. \\
\hline HK & $\begin{array}{r}13 \\
86\end{array}$ & 4.1 & $\begin{array}{c}23.5 \\
10.9\end{array}$ & $\begin{array}{l}0.5 \\
0.3\end{array}$ & $\begin{array}{l}0.5 \\
0.5\end{array}$ & 0.0 & $\begin{array}{l}\text { S-SL } \\
\text { SLIM }\end{array}$ & $\begin{array}{l}7 \\
6\end{array}$ & 37 & 30 & $\begin{array}{l}8 \\
3\end{array}$ & $\begin{array}{l}2824 \\
1313\end{array}$ & \\
\hline
\end{tabular}


SITE ID: HATO1

GROUND WATER DEPTH: 50.0 (FT.)
LOCATION ID: 032

AUERAGE SOIL WEIGHT: 100.0 (PSF)

AUG. AUG. UND.

LY FROM TO THK. POINT SLEEUE PRESS. RATIO CLASS BC DEM FA ELAS. STREN DCR

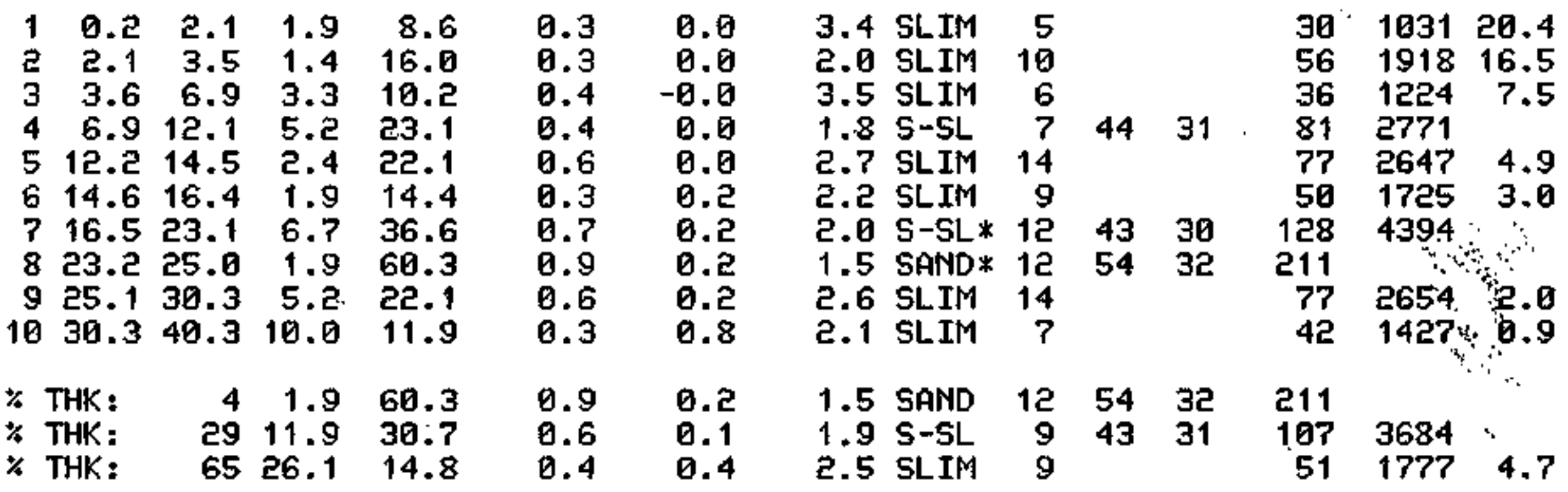


SITE ID: HATO1

GRDUND WATER DEPTH: 50.0 (FT.)
LOCATION ID: 033

AUERAGE SOIL WEIGHT: 100.0 (PSF)

$\begin{array}{llll}\text { - DEPTH - } & \text { AUG. AUG. PUG. ALG. FRE FRICT. SOIL } & \text { UND. } \\ \end{array}$

LY FROM TO THK. POINT SLEELE PRESS. RATIO CLASS BC DEN FA ELAS. STREN DCR

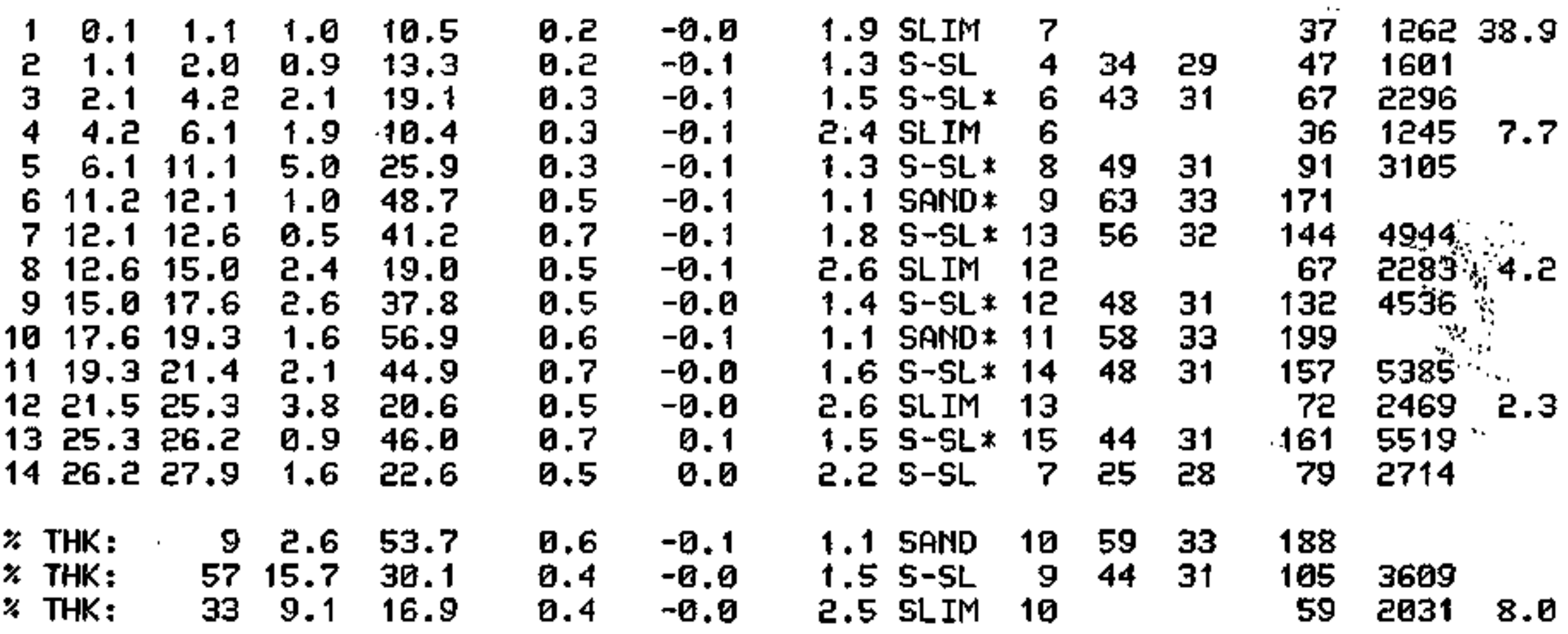


- DEPTH - AUG. AUG. PUG. AUG. PORE FRICT. SOIL UND. LY FROM TO THK. POINT SLEEUE PRESS. RATIO CLASS BC OEN FA ELAS. STREN OCR

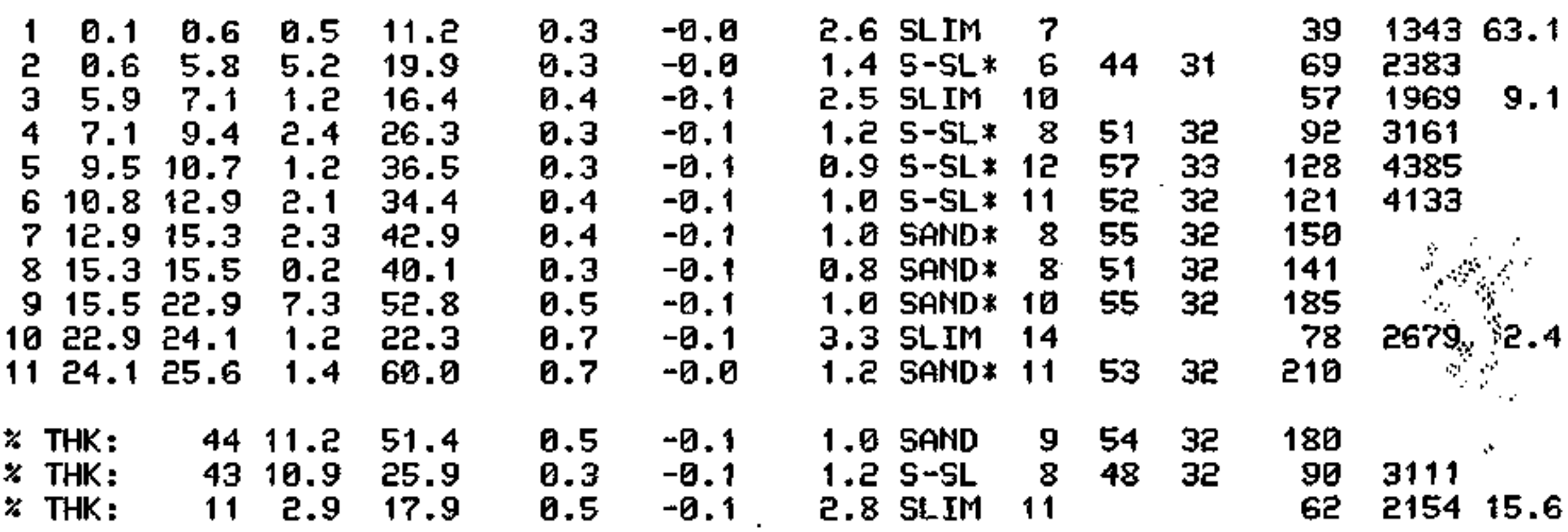


SITE ID: HATO1

GROUND WATER DEPTH: 50.0 (FT.)
LOCATION ID: 035

AUERAGE SOIL WEIGHT: 100.0 (PSF)

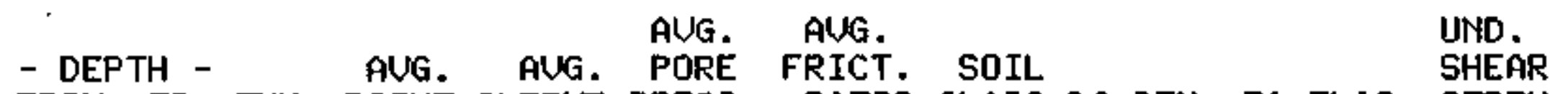
LY FROM TO THK. POINT SLEEYE PRESS. RATIO CLASS BC DEN FA ELAS. STREN DCR

\begin{tabular}{|c|c|c|c|c|c|c|c|c|c|c|c|c|}
\hline $\begin{array}{rr}1 & 0.1 \\
2 & 5.6 \\
3 & 9.9 \\
4 & 17.4 \\
5 & 23.8 \\
6 & 25.6 \\
7 & 26.7 \\
8 & 27.4\end{array}$ & $\begin{array}{r}5.6 \\
9.9 \\
17.3 \\
23.7 \\
25.6 \\
26.6 \\
27.4 \\
28.5\end{array}$ & $\begin{array}{l}5.5 \\
4.3 \\
7.4 \\
6.4 \\
1.8 \\
1.0 \\
0.7 \\
1.1\end{array}$ & $\begin{array}{l}16.5 \\
27.0 \\
38.6 \\
55.4 \\
23.2 \\
39.1 \\
18.9 \\
16.4\end{array}$ & $\begin{array}{l}0.3 \\
0.4 \\
0.4 \\
0.6 \\
0.6 \\
0.4 \\
0.5 \\
0.3\end{array}$ & $\begin{array}{l}-0.1 \\
-0.1 \\
-0.1 \\
-0.1 \\
-0.1 \\
-0.0 \\
-0.1 \\
-0.0\end{array}$ & $\begin{array}{l}1.7 \\
1.5 \\
1.1 \\
1.1 \\
2.4 \\
1.1 \\
2.6 \\
2.1\end{array}$ & $\begin{array}{l}\text { S-SL } \\
\text { S-SL* } \\
\text { S-SL* } \\
\text { SAND* } \\
\text { S-SL } \\
\text { S-SL* } \\
\text { SL.IM } \\
\text { SLIM }\end{array}$ & $\begin{array}{r}5 \\
8 \\
12 \\
11 \\
7 \\
13 \\
12 \\
10\end{array}$ & $\begin{array}{l}39 \\
52 \\
52 \\
55 \\
27 \\
39\end{array}$ & $\begin{array}{l}30 \\
32 \\
32 \\
32 \\
29 \\
30\end{array}$ & $\begin{array}{r}58 \\
94 \\
135 \\
194 \\
81 \\
137 \\
66 \\
57\end{array}$ & $\begin{array}{l}1985 \\
3237 \\
4628 \\
\\
2778 \\
4693 \\
2263 \cdot 1.8 \\
197119.6\end{array}$ \\
\hline THK: & $\begin{array}{r}22 \\
78 \\
6\end{array}$ & $\begin{array}{r}6.4 \\
20.0 \\
1.8\end{array}$ & $\begin{array}{l}55.4 \\
28.7 \\
17.4\end{array}$ & $\begin{array}{l}0.6 \\
0.4 \\
0.4\end{array}$ & $\begin{array}{l}-0.1 \\
-0.1 \\
-0.0\end{array}$ & $\begin{array}{l}1.1 \\
1.5 \\
2.3\end{array}$ & $\begin{array}{l}\text { SAND } \\
\text { S-SL } \\
\text { SLIM }\end{array}$ & $\begin{array}{r}11 \\
8 \\
10\end{array}$ & $\begin{array}{l}55 \\
45\end{array}$ & $\begin{array}{l}32 \\
31\end{array}$ & $\begin{array}{r}194 \\
160 \\
60\end{array}$ & $\begin{array}{c}3438 \\
2084 \\
1.7\end{array}$ \\
\hline
\end{tabular}


SITE ID: HATV 1

GROUND LATER DEPTH: 50.0 (FT.)
LOCATION ID: 036

AUERAGE SOIL WEIGHT: 100.0 (PSF)

$\begin{array}{llll}\text { - DEPTH - AUG. AUG. PORE FRICT. SOIL } & \text { UND. } \\ \end{array}$ LY FROM TO THK. POINT SLEEUE PRESS. RATIO CLASS BC DEN FA ELAS. STREN OCR

\begin{tabular}{|c|c|c|c|c|c|c|c|c|c|c|c|c|}
\hline $\begin{array}{r}0.1 \\
2.3 \\
3.0 \\
3.7 \\
4.7 \\
5.4 \\
6.9 \\
7.4 \\
8.7 \\
12.2 \\
13.2 \\
14.6\end{array}$ & $\begin{array}{r}2.3 \\
3.0 \\
3.7 \\
4.7 \\
5.4 \\
6.8 \\
7.4 \\
8.6 \\
12.2 \\
13.1 \\
14.6 \\
18.1\end{array}$ & $\begin{array}{l}2.1 \\
0.7 \\
0.7 \\
0.9 \\
0.7 \\
1.4 \\
0.6 \\
1.2 \\
3.5 \\
0.9 \\
1.4 \\
3.5\end{array}$ & $\begin{array}{r}12.3 \\
22.6 \\
10.1 \\
17.2 \\
7.4 \\
30.7 \\
14.8 \\
21.7 \\
35.3 \\
41.7 \\
37.2 \\
14.2\end{array}$ & $\begin{array}{l}0.1 \\
0.2 \\
0.2 \\
0.1 \\
0.1 \\
0.3 \\
0.3 \\
0.2 \\
0.4 \\
0.3 \\
0.4 \\
0.5\end{array}$ & $\begin{array}{l}-0.1 \\
-0.1 \\
-0.1 \\
-0.1 \\
-0.1 \\
-0.1 \\
-0.2 \\
-0.1 \\
-0.1 \\
-0.1 \\
-0.2 \\
0.1\end{array}$ & $\begin{array}{l}0.8 \\
0.7 \\
2.1 \\
0.8 \\
1.9 \\
1.0 \\
2.3 \\
0.8 \\
1.1 \\
0.7 \\
1.0 \\
3.5\end{array}$ & $\begin{array}{l}\text { S-SL* } \\
\text { S-SL* } \\
\text { SLIM } \\
\text { S-SL* } \\
\text { SLIM } \\
\text { S-SL* } \\
\text { SLIM } \\
\text { S-SL* } \\
\text { S-SL* } \\
\text { SAND* } \\
\text { S-SL* } \\
\text { SLIM }\end{array}$ & $\begin{array}{r}4 \\
7 \\
6 \\
5 \\
4 \\
10 \\
9 \\
7 \\
11 \\
8 \\
12 \\
9\end{array}$ & $\begin{array}{l}40 \\
58 \\
46 \\
55 \\
56 \\
51\end{array}$ & $\begin{array}{l}30 \\
33 \\
31 \\
32 \\
32 \\
32\end{array}$ & $\begin{array}{r}43 \\
79 \\
35 \\
60 \\
26 \\
107 \\
52 \\
76 \\
124 \\
146 \\
130 \\
50\end{array}$ & $\begin{array}{r}1477 \\
2709 \\
1211 \\
2064 \\
887 \\
3685 \\
1773 \\
2598 \\
4241 \\
4459 \\
1705\end{array}$ \\
\hline K K & $\begin{array}{r}5 \\
63 \\
31\end{array}$ & $\begin{array}{r}0.9 \\
11.2 \\
5.5\end{array}$ & $\begin{array}{l}41.7 \\
26.9 \\
12.9\end{array}$ & $\begin{array}{l}0.3 \\
0.3 \\
0.4\end{array}$ & $\begin{array}{r}-0.1 \\
-0.1 \\
0.0\end{array}$ & $\begin{array}{l}0.7 \\
0.9 \\
3.0\end{array}$ & $\begin{array}{l}\text { ID } \\
\text { LM }\end{array}$ & $\begin{array}{l}8 \\
8 \\
7\end{array}$ & $\begin{array}{l}56 \\
47\end{array}$ & & $\begin{array}{r}146 \\
94 \\
45\end{array}$ & \\
\hline
\end{tabular}


SITE ID: HATO1

GROUND WATER DEPTH: 50.0 (FT.)
LOCATION ID: 037

AUERAGE SOIL WEIGHT: 100.0 (FSF)

- DEPTH - AUG. AUG. UND. LY FROM TO THK. POINT SLEEUE PRESS. RATIÓ CLASS BC DEN FA ELAS. STREN OCR

\begin{tabular}{|c|c|c|c|c|c|c|c|c|c|c|c|c|}
\hline $\begin{array}{rr}1 & 6.1 \\
2 & 2.0 \\
3 & 5.1 \\
4 & 9.2 \\
5 & 10.4 \\
6 & 11.6 \\
7 & 13.0\end{array}$ & $\begin{array}{r}2.0 \\
5.1 \\
9.2 \\
10.4 \\
11.6 \\
13.0 \\
19.6\end{array}$ & $\begin{array}{l}1.9 \\
3.1 \\
4.0 \\
1.1 \\
1.2 \\
1.4 \\
6.6\end{array}$ & $\begin{array}{r}9.9 \\
24.5 \\
17.3 \\
24.5 \\
12.7 \\
18.1 \\
13.0\end{array}$ & $\begin{array}{l}0.2 \\
0.2 \\
0.4 \\
0.3 \\
0.3 \\
0.4 \\
0.4\end{array}$ & $\begin{array}{l}-0.0 \\
-0.1 \\
-0.1 \\
-0.1 \\
-0.0 \\
-0.0 \\
0.1\end{array}$ & $\begin{array}{l}1.9 \\
1.0 \\
2.4 \\
1.2 \\
2.6 \\
2.0 \\
2.7\end{array}$ & $\begin{array}{l}\text { SL IM } \\
\text { S-SL* } \\
\text { SLIM } \\
\text { S-SL* } \\
\text { SLIM } \\
\text { S-SL. } \\
\text { SLIM }\end{array}$ & $\begin{array}{r}6 \\
8 \\
11 \\
8 \\
8 \\
6 \\
8\end{array}$ & $\begin{array}{l}45 \\
33\end{array}$ & $\begin{array}{l}32 \\
31 \\
29\end{array}$ & $\begin{array}{l}35 \\
86 \\
60 \\
86 \\
44 \\
63 \\
46\end{array}$ & $\begin{array}{l}1183 \\
2940 \\
2973 \\
2946 \\
1519 \\
2177 \\
1565\end{array}$ \\
\hline $\begin{array}{l}\text { THK: } \\
\text { THK: }\end{array}$ & $\begin{array}{l}29 \\
70\end{array}$ & $\begin{array}{r}5.6 \\
13.7\end{array}$ & $\begin{array}{l}22.9 \\
13.8\end{array}$ & $\begin{array}{l}0.3 \\
0.4\end{array}$ & $\begin{array}{r}-0.1 \\
0.0\end{array}$ & $\begin{array}{l}1.3 \\
2.5\end{array}$ & $\begin{array}{l}\text { S-SL } \\
\text { SLIM }\end{array}$ & $\begin{array}{l}7 \\
8\end{array}$ & 45 & 31 & $\begin{array}{l}80 \\
48\end{array}$ & $\begin{array}{l}2750 \\
1656\end{array}$ \\
\hline
\end{tabular}


SITE ID: HATO1

GROUND WATER DEPTH: 50.0 (FT.)
LOCATION ID: 038

AUERAGE SOIL WEIGHT: 100.0 (PSF)

- DEPTH - aUg. aUg. PURE FRICT. SOIL

UND.

SHEAR

LY FROM TO THK. POINT SLEEUE PRESS. RATIO CLASS BC DEN FA ELAS. STREN OCR

$\begin{array}{ll}1 & 0.1 \\ 2 & 1.1 \\ 3 & 3.1 \\ 4 & 5.7 \\ 5 & 7.6\end{array}$

$1.0 \quad 1.0 \quad 11.9$

$3.0 \quad 1.9 \quad 17.7$

0.2

$-0.0$

1.7 SLIM

7

$\begin{array}{lll}5.6 & 2.6 & 13.8\end{array}$

0.2

$-0.0$

$0.4-0.1$

1.2 S-SL*

2.5 SLIM 9

$0.3-0.1$

1.5 S-SL*

3.2 SLIM

$-0.0$

1.4 S-SL

$0.2 \quad-0.1$

2.6 SLIM

542

$-0.0$

$\%$ THK:

$\begin{array}{lll}60 & 5.9 & 10.5\end{array}$

0.3

6

41

$\begin{array}{rrrr} & 42 & 1430 & 43.6 \\ 30 & 62 & 2120 & \\ & 48 & 1659 & 10.8 \\ 31 & 67 & 2302 & \\ & 22 & 744 & 3.2\end{array}$

31

64 221.1

$36 \quad 126313.4$ 
SITE ID: HATO1

GROUMD LIATER DEPTH: 50.0 (FT.)
LOCATION ID: 039

AUERAGE SOIL WEIGHT: 100.0 (PSF)

- DEPTH - AUG AUG AUG. AUG. UND.

LY FROM TO THK. POINT SLEEVE PRESS. RATIO CLASS BC DEN FA ELAS. STREN OCR

\begin{tabular}{|c|c|c|c|c|c|c|c|c|c|c|c|c|}
\hline $\begin{array}{ll}1 & 0.2 \\
2 & 2.8 \\
3 & 4.3 \\
4 & 5.0 \\
5 & 5.9 \\
6 & 6.4 \\
7 & 8.1\end{array}$ & $\begin{array}{r}2.8 \\
4.2 \\
5.0 \\
5.9 \\
6.4 \\
8.0 \\
16.4\end{array}$ & $\begin{array}{l}2.6 \\
1.4 \\
0.7 \\
0.9 \\
0.4 \\
1.6 \\
8.3\end{array}$ & $\begin{array}{r}16.4 \\
9.2 \\
22.7 \\
8.5 \\
26.1 \\
9.9 \\
3.7\end{array}$ & $\begin{array}{l}0.2 \\
0.3 \\
0.4 \\
0.2 \\
0.4 \\
0.3 \\
0.1\end{array}$ & $\begin{array}{l}-0.0 \\
-0.1 \\
-0.1 \\
-0.1 \\
-0.0 \\
-0.1 \\
0.0\end{array}$ & $\begin{array}{ll}1.3 & \text { S-SL** } \\
3.1 & \text { SLIM } \\
1.6 & \text { S-SL* } \\
2.9 & \text { SLIM } \\
1.4 & 5-S L * \\
2.6 & \text { SLIM } \\
3.5 & \text { SLIM }\end{array}$ & $\begin{array}{l}5 \\
6 \\
7 \\
5 \\
8 \\
6 \\
2\end{array}$ & $\begin{array}{l}48 \\
53\end{array}$ & $\begin{array}{l}31 \\
32\end{array}$ & $\begin{array}{l}57 \\
32 \\
79 \\
30 \\
92 \\
35 \\
13\end{array}$ & $\begin{array}{r}1967 \\
1103 \\
2725 \\
1015 \\
3138 \\
1183 \\
442 .\end{array}$ & $\begin{array}{r}5.7 \\
1.5\end{array}$ \\
\hline $\begin{array}{l}\text { THK: } \\
\text { THK : }\end{array}$ & $\begin{array}{l}23 \\
76\end{array}$ & $\begin{array}{r}3.7 \\
12.2\end{array}$ & $\begin{array}{r}18.6 \\
5.5\end{array}$ & $\begin{array}{l}0.3 \\
0.2\end{array}$ & $\begin{array}{l}-0.0 \\
-0.0\end{array}$ & $\begin{array}{l}1.45-S L \\
3.3 \text { SLIM }\end{array}$ & $\begin{array}{l}5 \\
3\end{array}$ & 42 & 31 & $\begin{array}{l}64 \\
19\end{array}$ & & 3.3 \\
\hline
\end{tabular}


SITE ID: HATV1

GROUND WATER DEPTH: 50.0 (FT.)
LOCATION ID: 940

AUERAGE SOIL WEIGHT: 100.0 (PSF)

$\begin{array}{llll} & & & \text { AUG. AUG. } \\ \end{array}$

LY FROM TO THK. POINT SLEEUE PRESS. RATIO CLASS BC DEN FA ELAS. STREN OCR

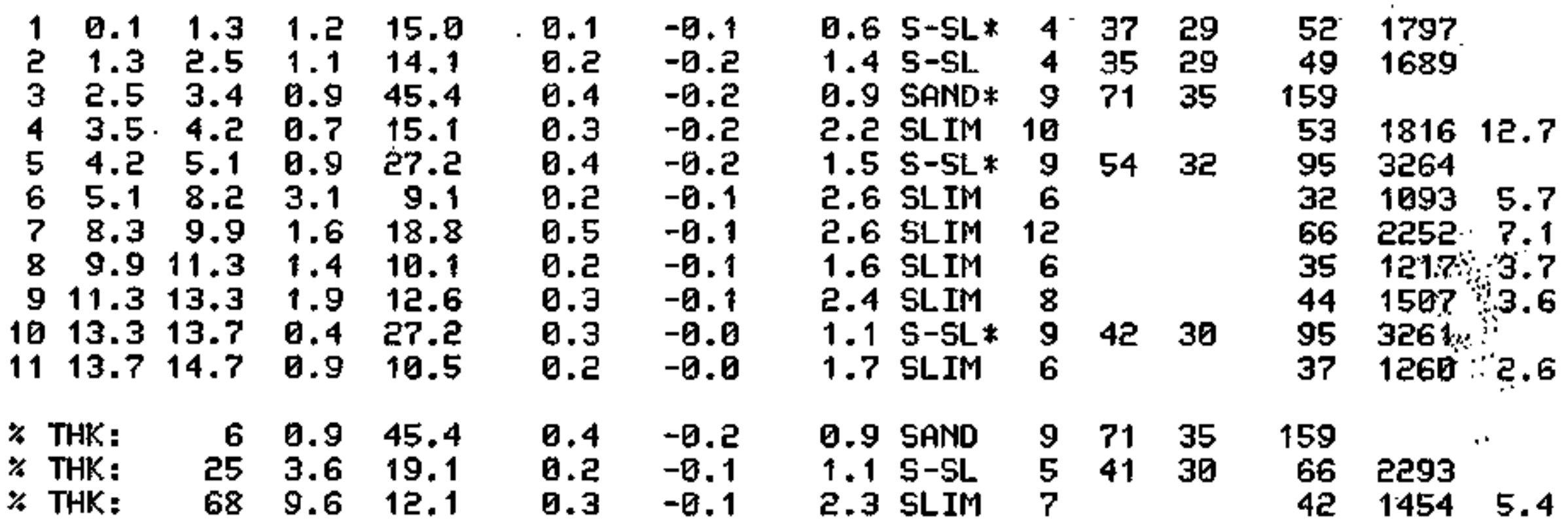




\section{PIEZOCONE SOUNDING TEST}

SITE JD: HATQ1

LDCATION ID: 001

PUINT STRESS (KG/CME) *1D PORE PRESSURE $\left(K G / C H^{2}\right.$ ) FRICTION RATID $(x)$ A FACTUR $(x) * 10$

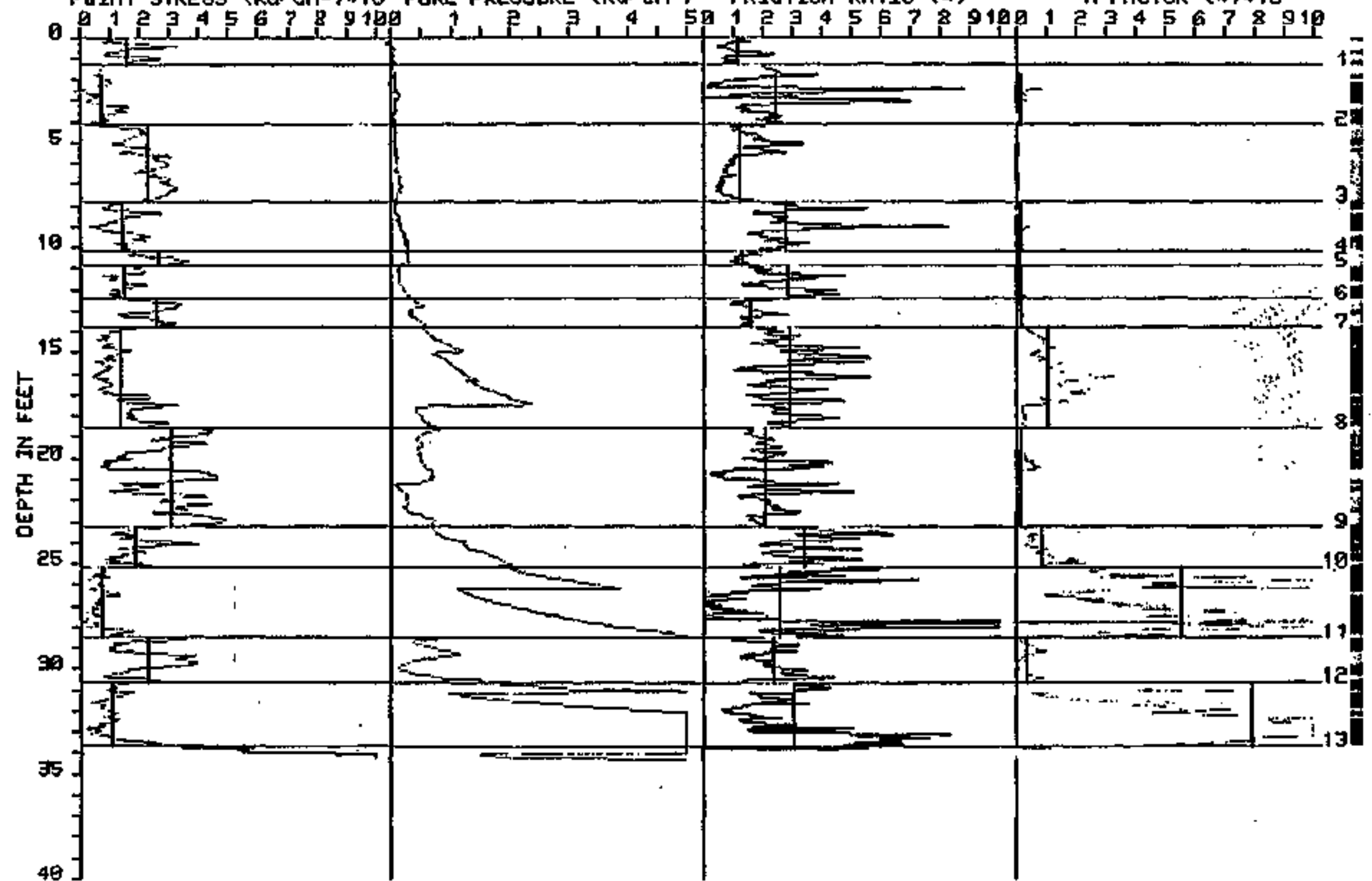




\section{PIEZOCONE SOUNOING TEST}

SITE IDE HATOI

LOCATION ID: GOE

POINT STRESS (KG/CMP)*10 PURE PRESSURE (KE CKR) FRICTION RATIO (*)

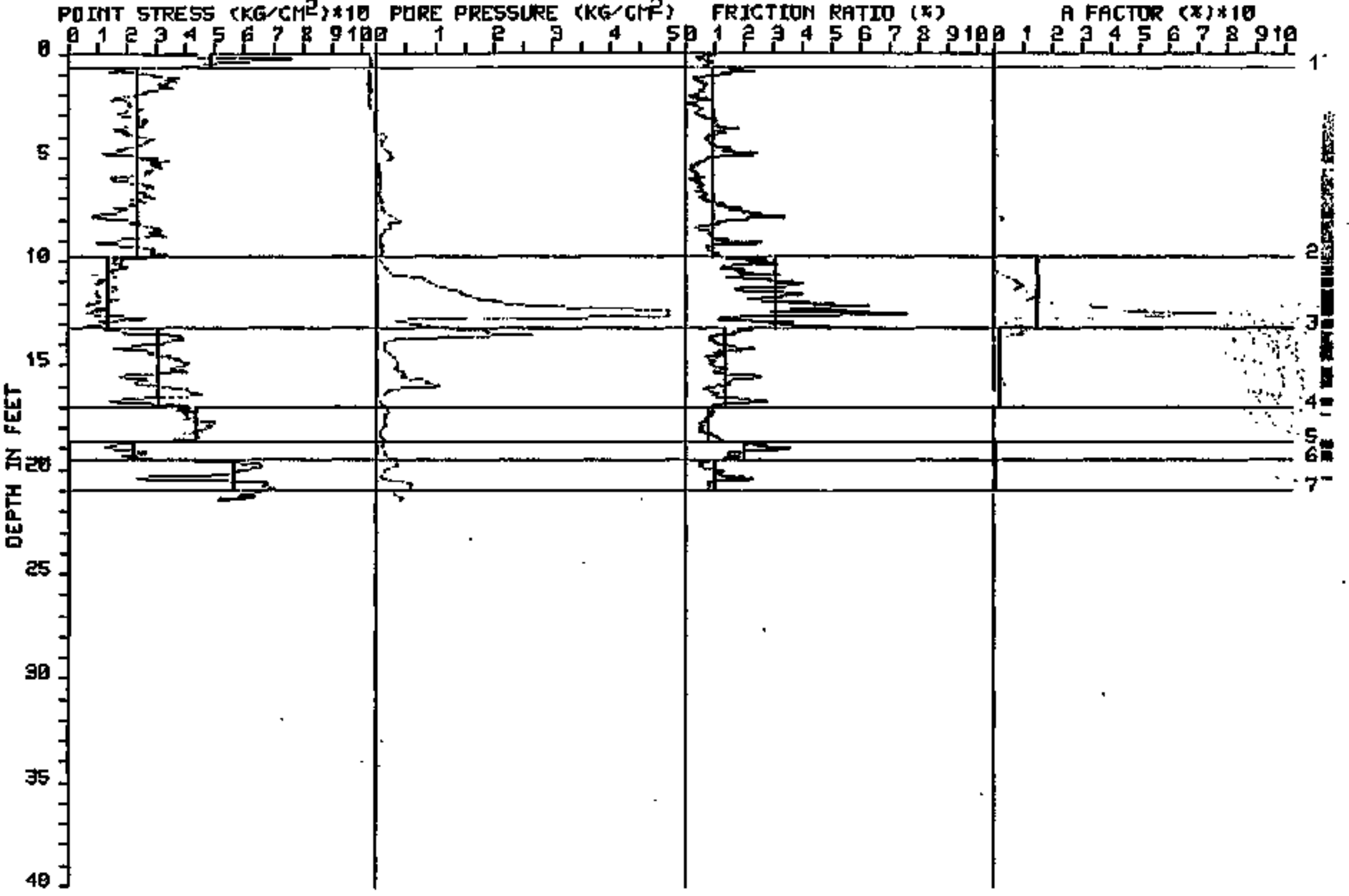




\section{PIEZOCONE SOUNDING TEST}

SITE ID: HAFEI

LOCATION IO: 603

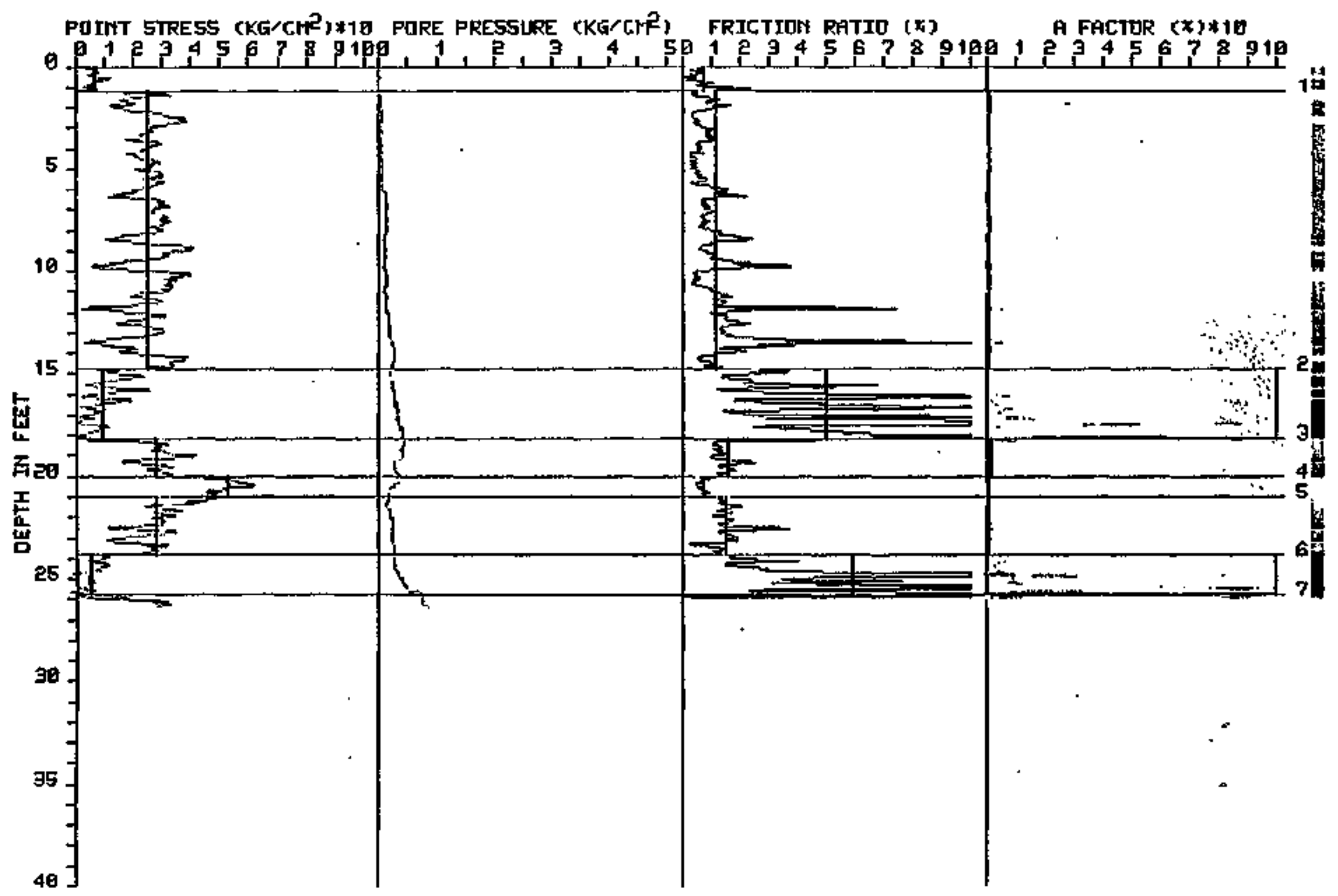




\section{PIEZOCONE SOUNDING TEST}

SITE IDI HATD

LOCATION ID: 004

POINT 5TRESS (KG/CME)*10 PORE PRESSURE (KG/CH')

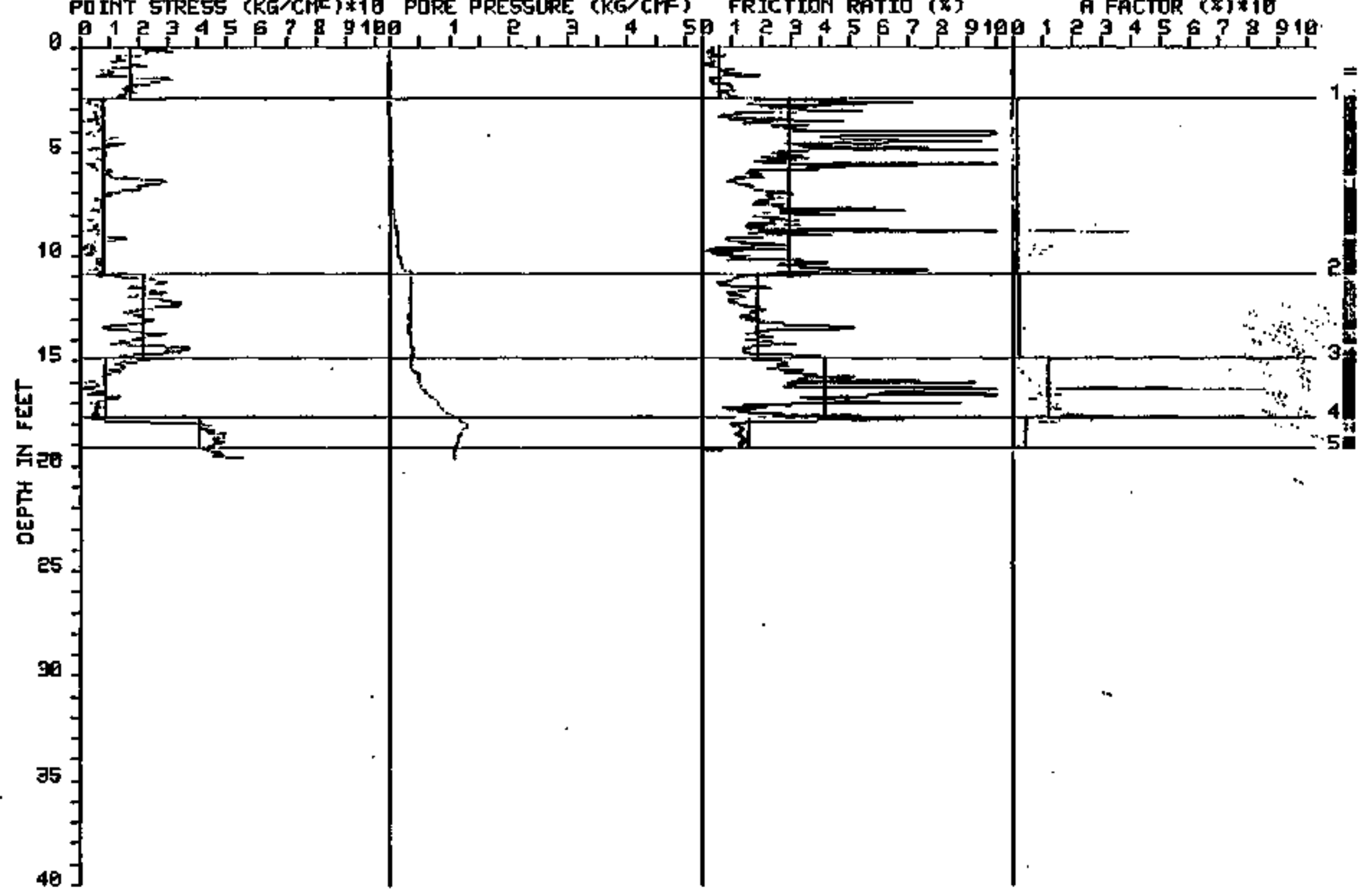




\section{PIEZOCONE SOUNDING TEST}

SITE ID: HATO1

LOCATION ID: 005

PIIIT STRESS (KE/CM 2 )*10 PORE PRES5URE (KG/CrF) FRICTIOM RATIE (x)

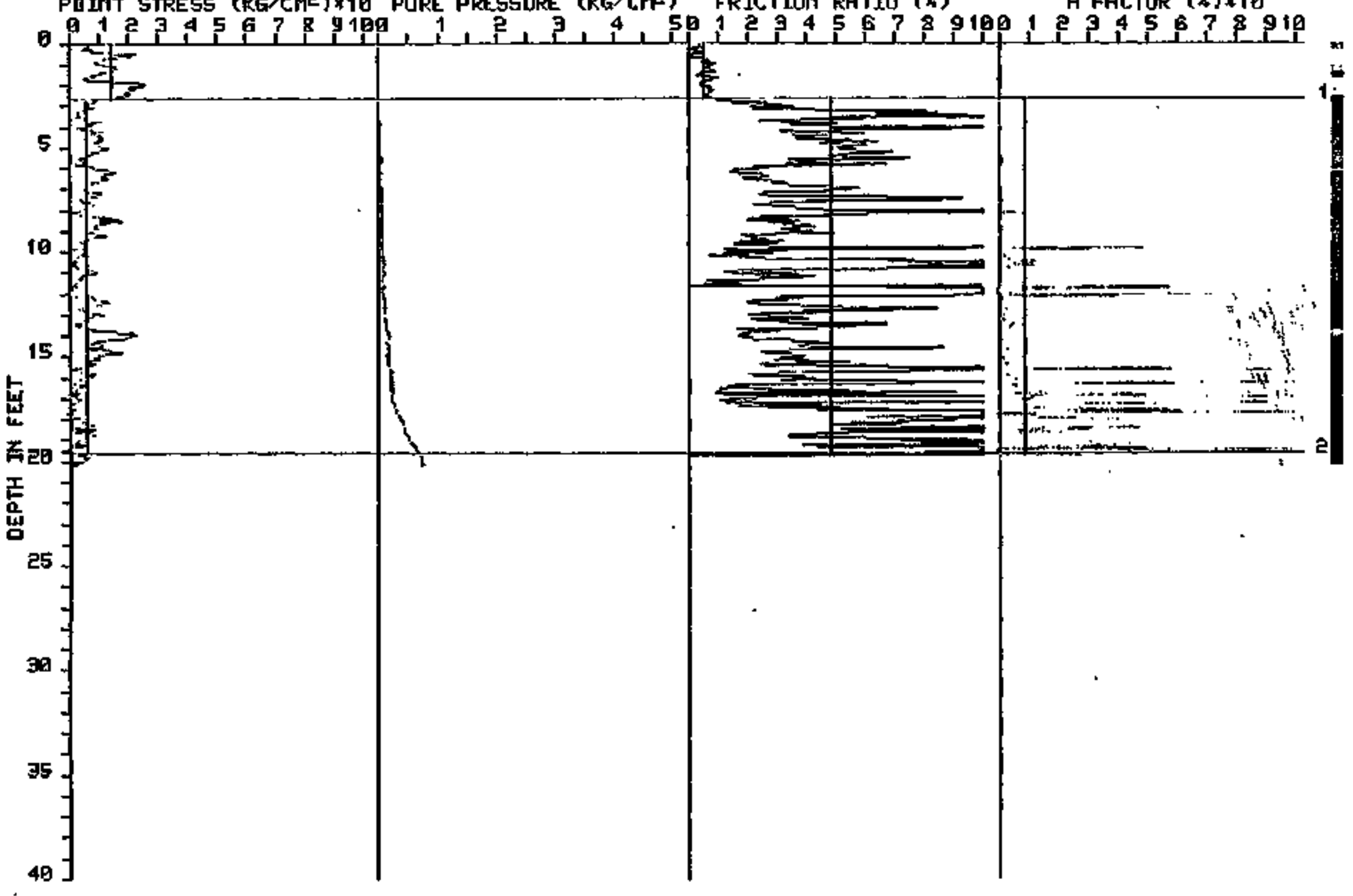




\section{PIEZOCONE SOUNDING TEST}

SITE ID: HATO

LOCATION ID: 006

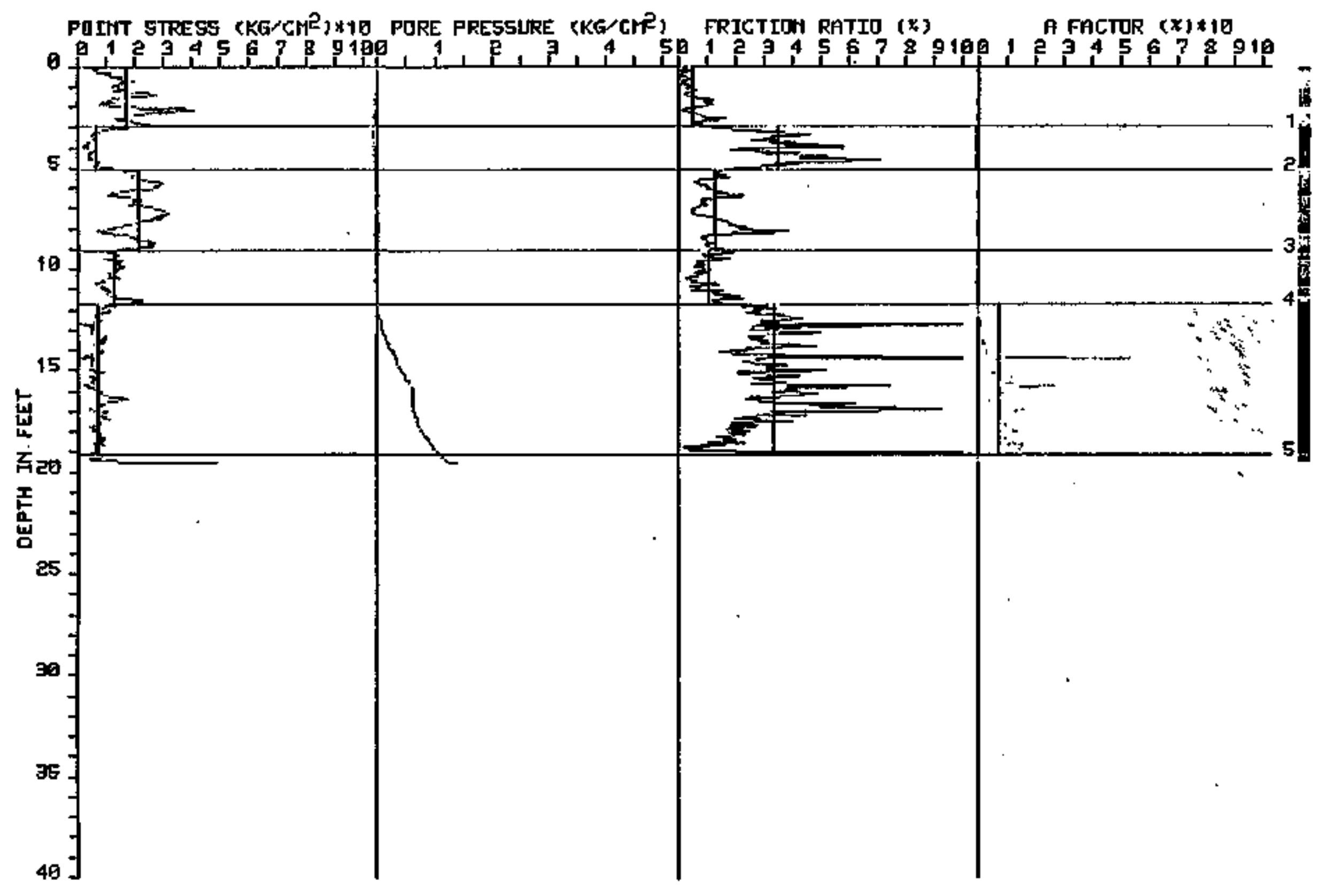




\section{PIEZOCONE SOUNDIMG TEST}

SITE ID: HATOT

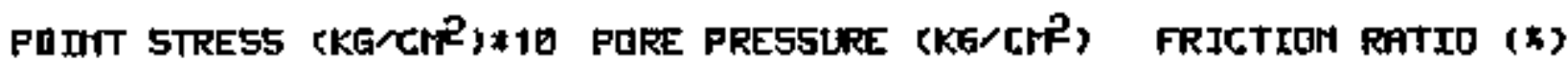

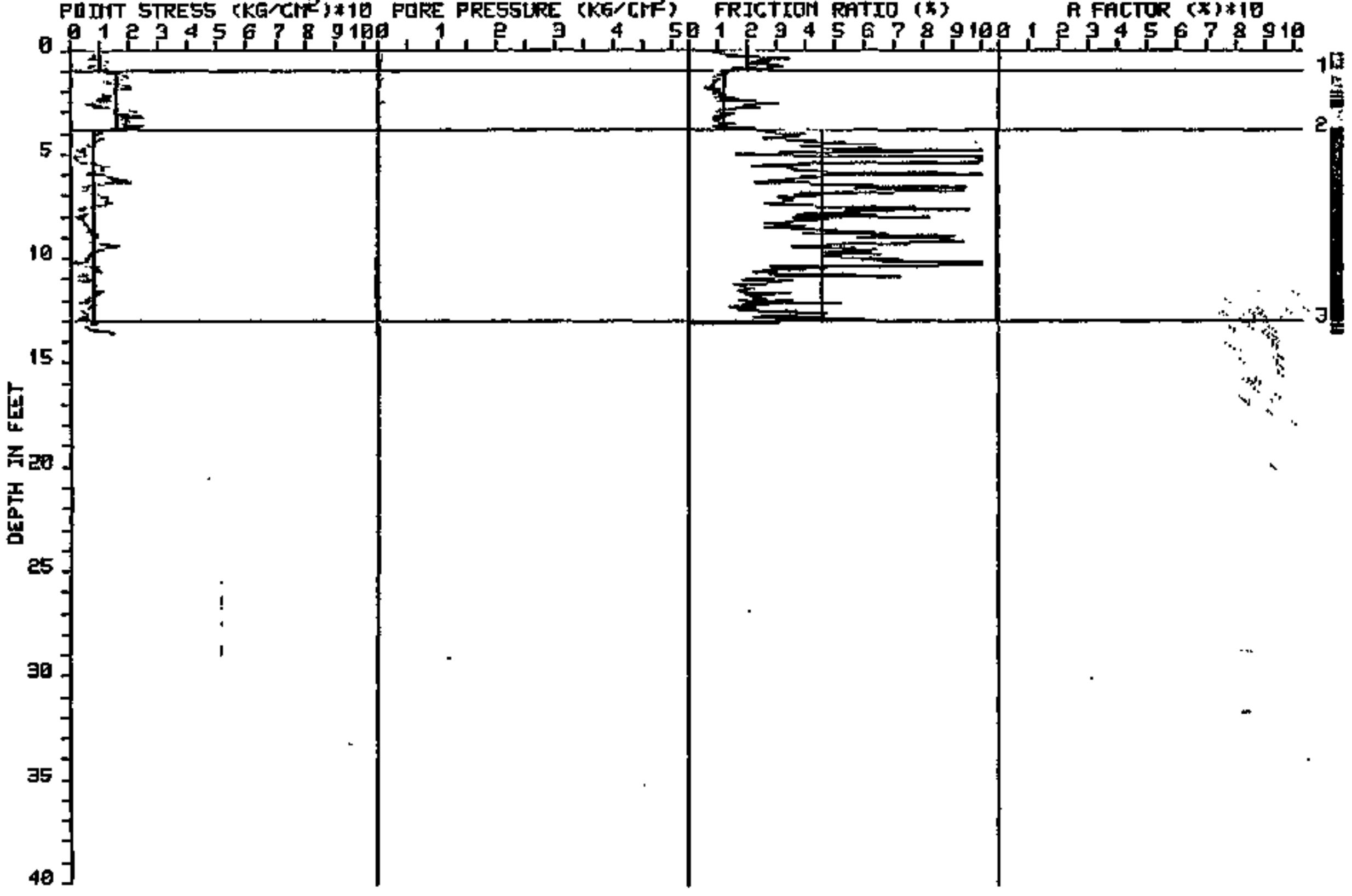




\section{PIEZOCONE SOUMDING TEST.}

SITE IOI HATOI

LOCATION ID: 6OS

POINT STRESS (KG/CH2)

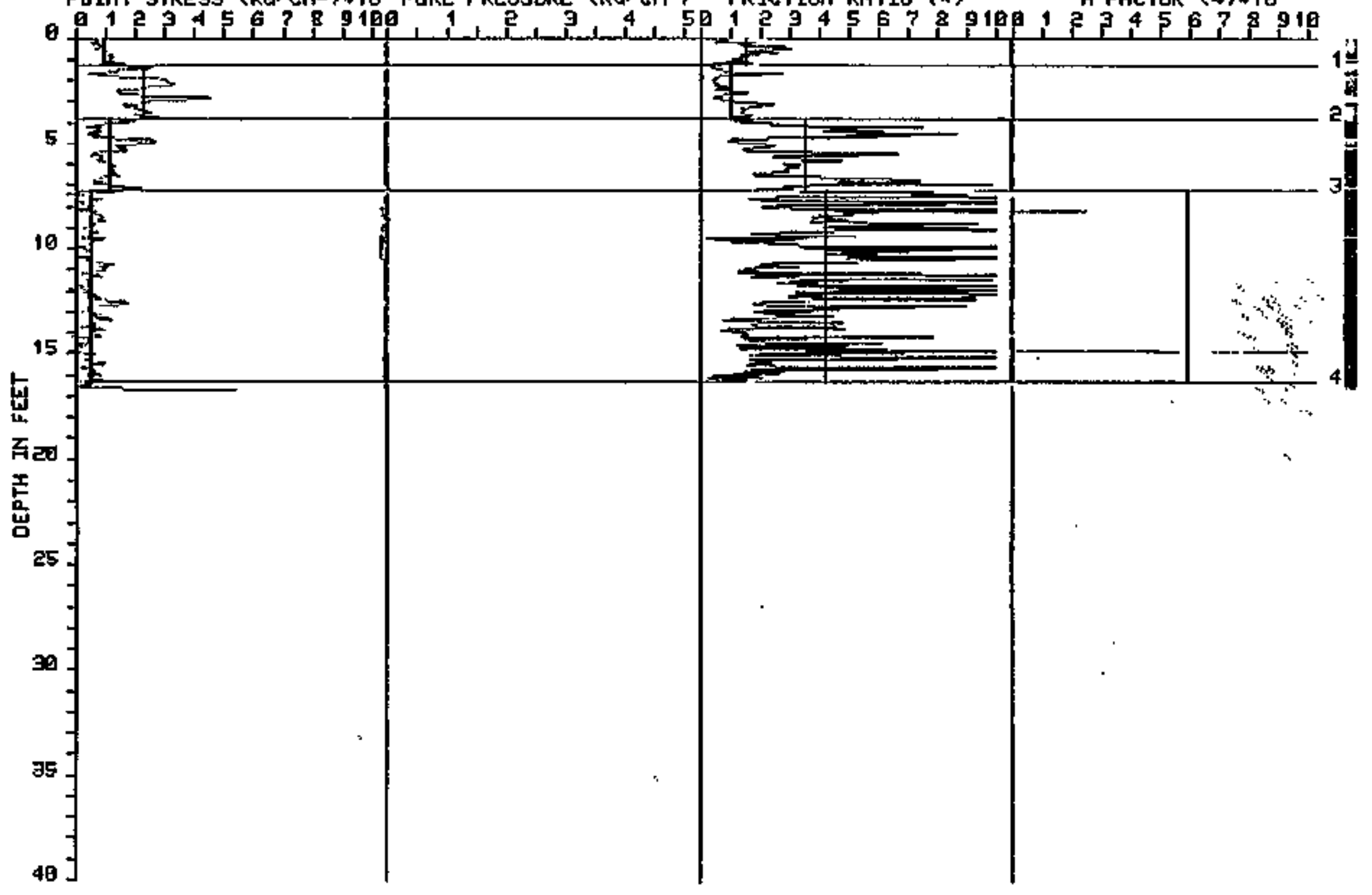




\section{PIEZOCONE SOUNDING TEST}

SITE ID: HATOI

LOCATION ID: 098

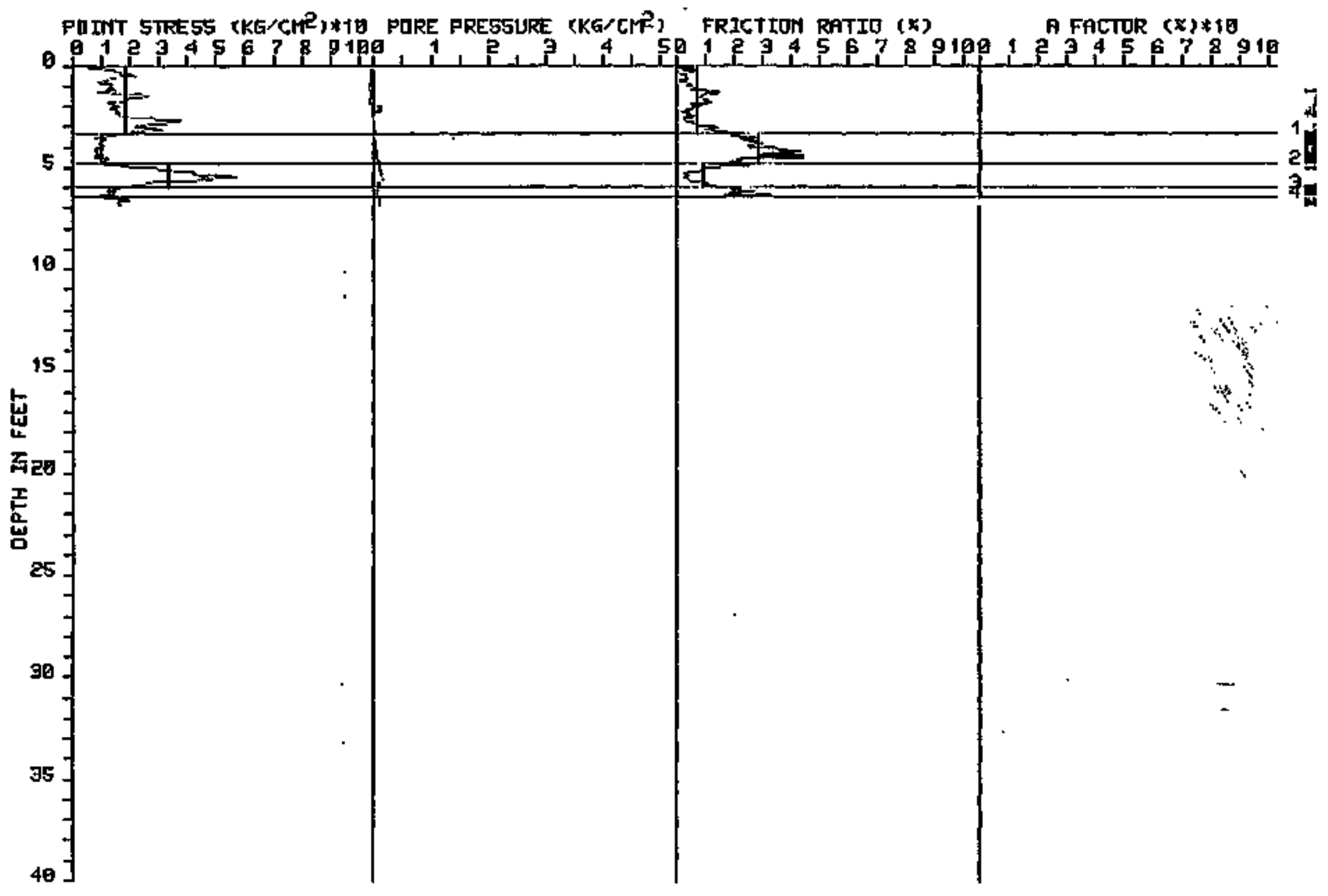




\section{PIEZOCONE SOUNDING TEST.}

SITE ID: HATOI LOCATION IDI 010

POINT STRESS (KG/CME) *1D PORE PRESSURE (KE/CIR)

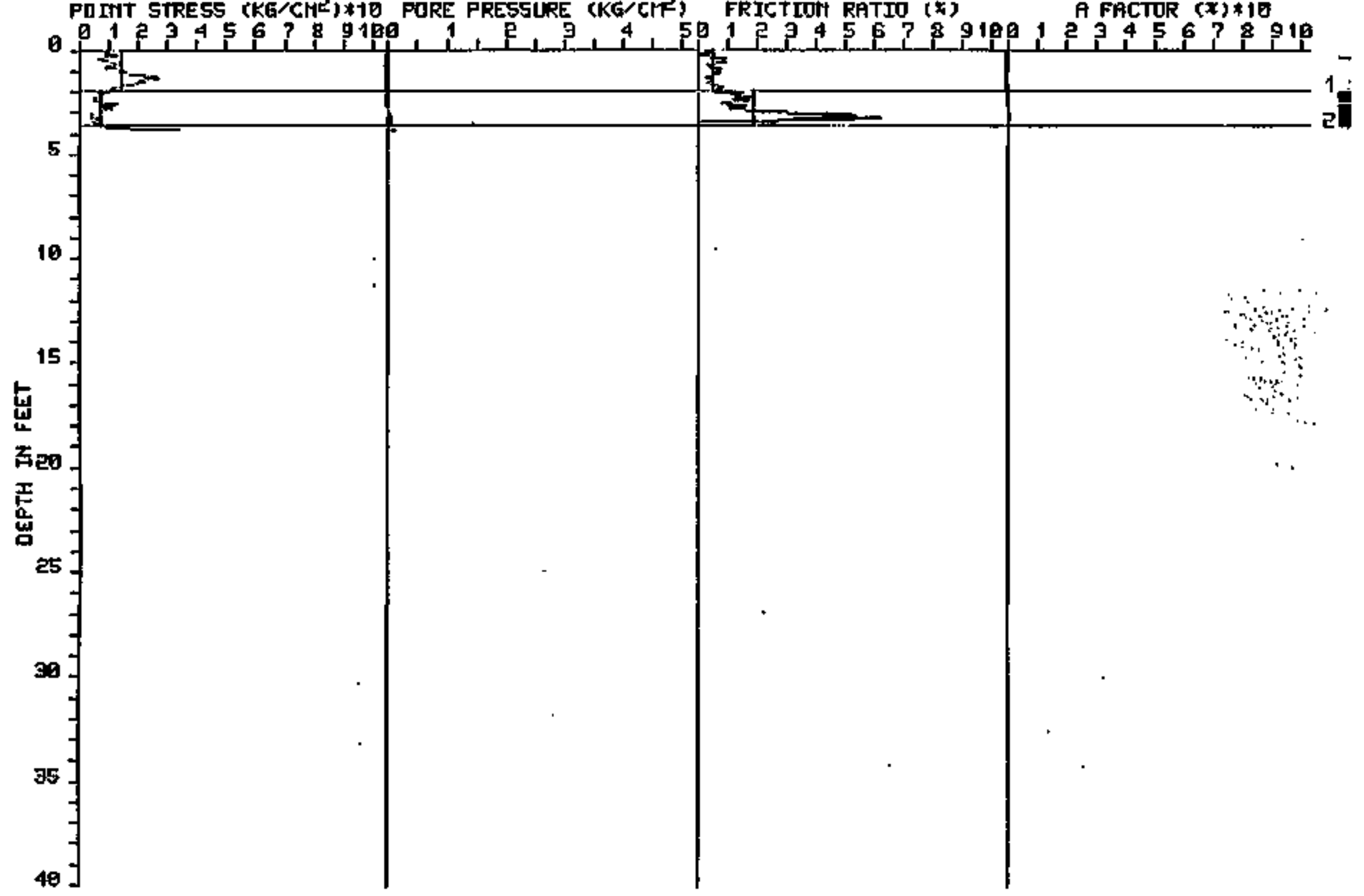




\section{PIEZOCONE SOUNDING TEST}

SITE ID: HATE1

LOCATION ID: 011

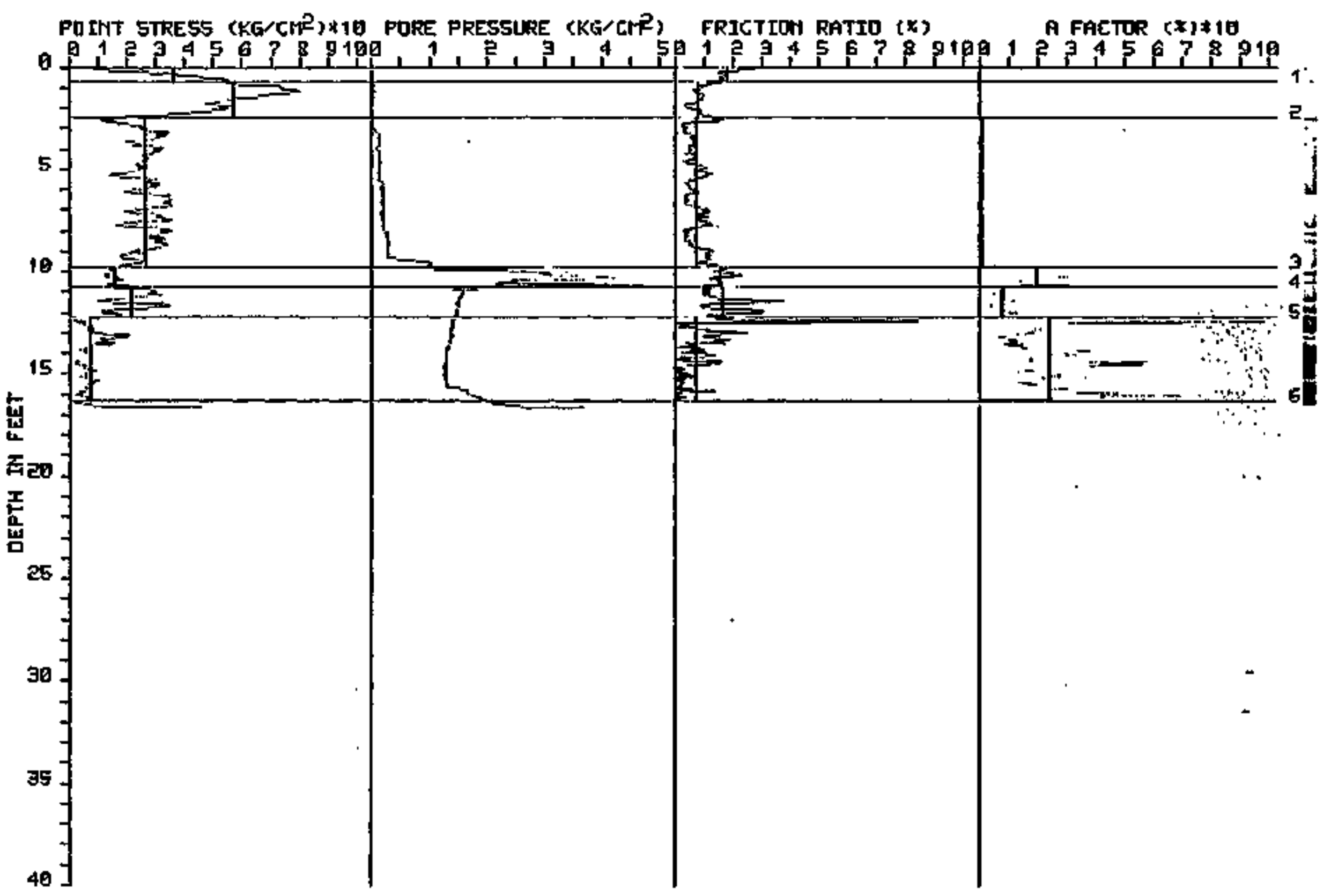




\section{PIEZOCONE SOUNDING TEST}

SITE IO: HATOI

LOCATION ID: DIE

PO EMT STRESS (KG/CHE) N10 PORE PRESSURE (KG/CHF) FRICTION RATIO ( $x$ )

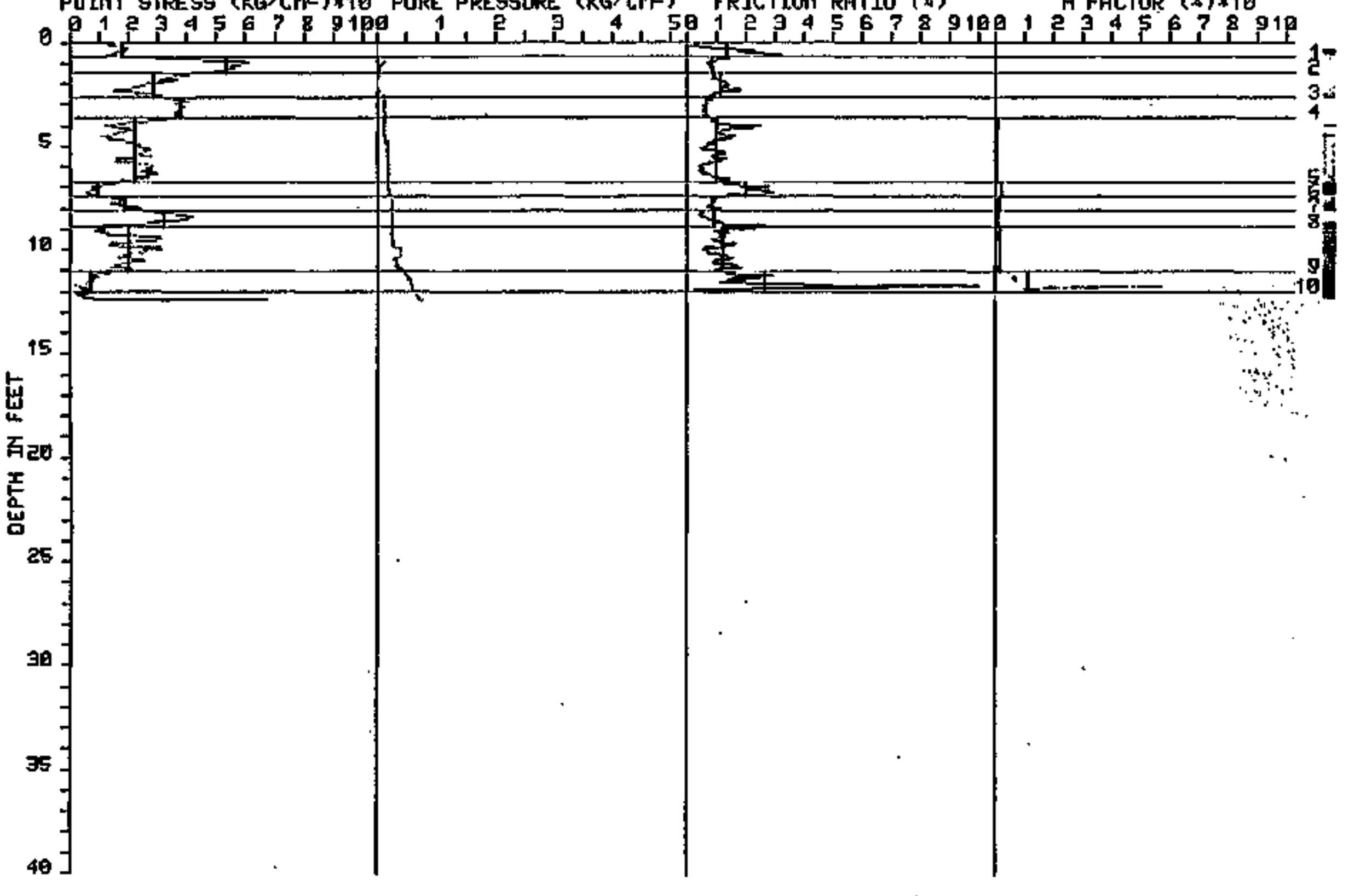




\section{PIEZOCONE SOUNDIMG TEST}

SITE ID: HATEI

LOCATION ID: 013

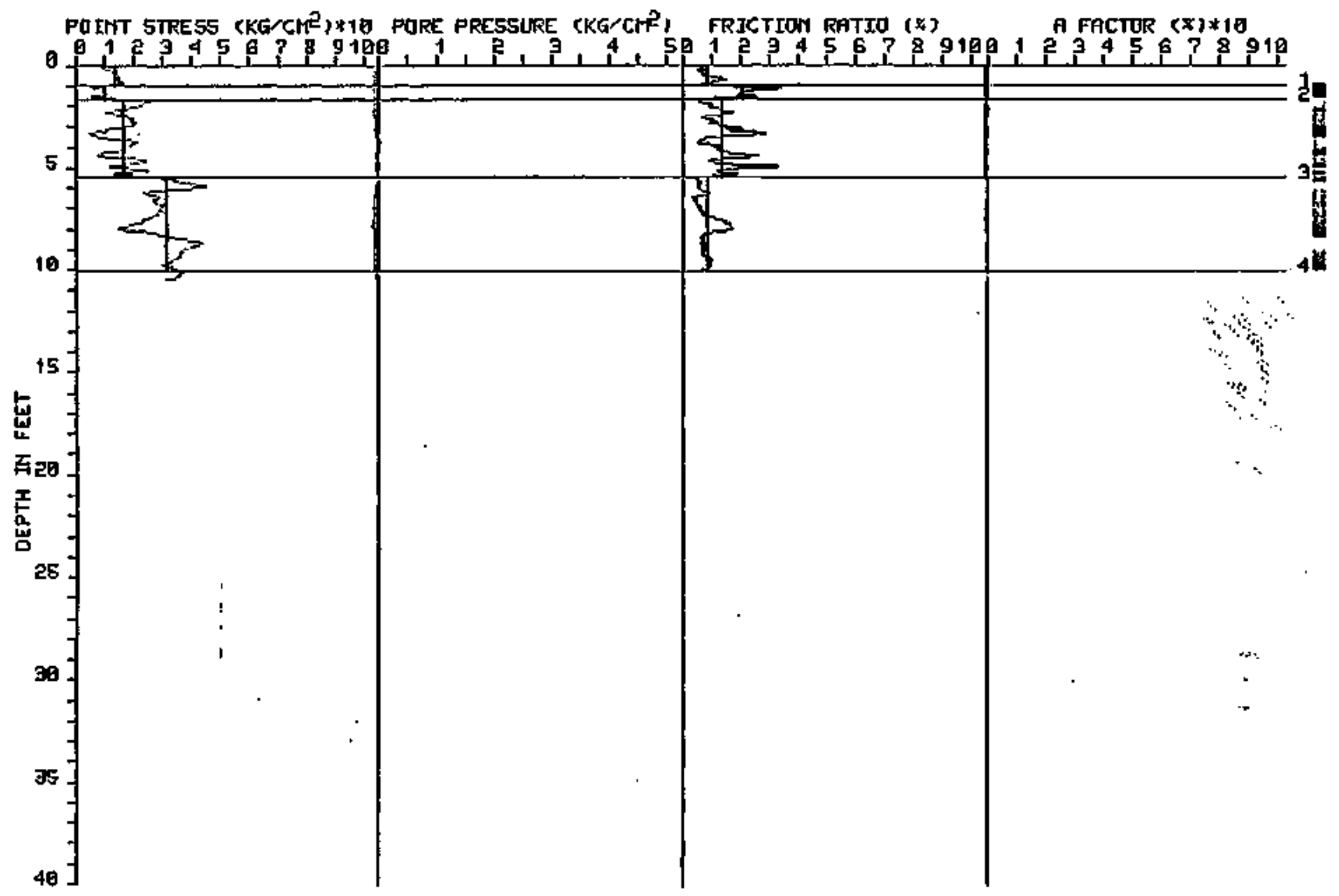




\section{PIEZOCONE SOLNOING TEST.}

STTE IDS HATE1

PUINT 5TRESS (KG/CIP) $* 10$ PURE PRES5URE (KG/CHF), FRICTIOH RATTO ( $*$ )

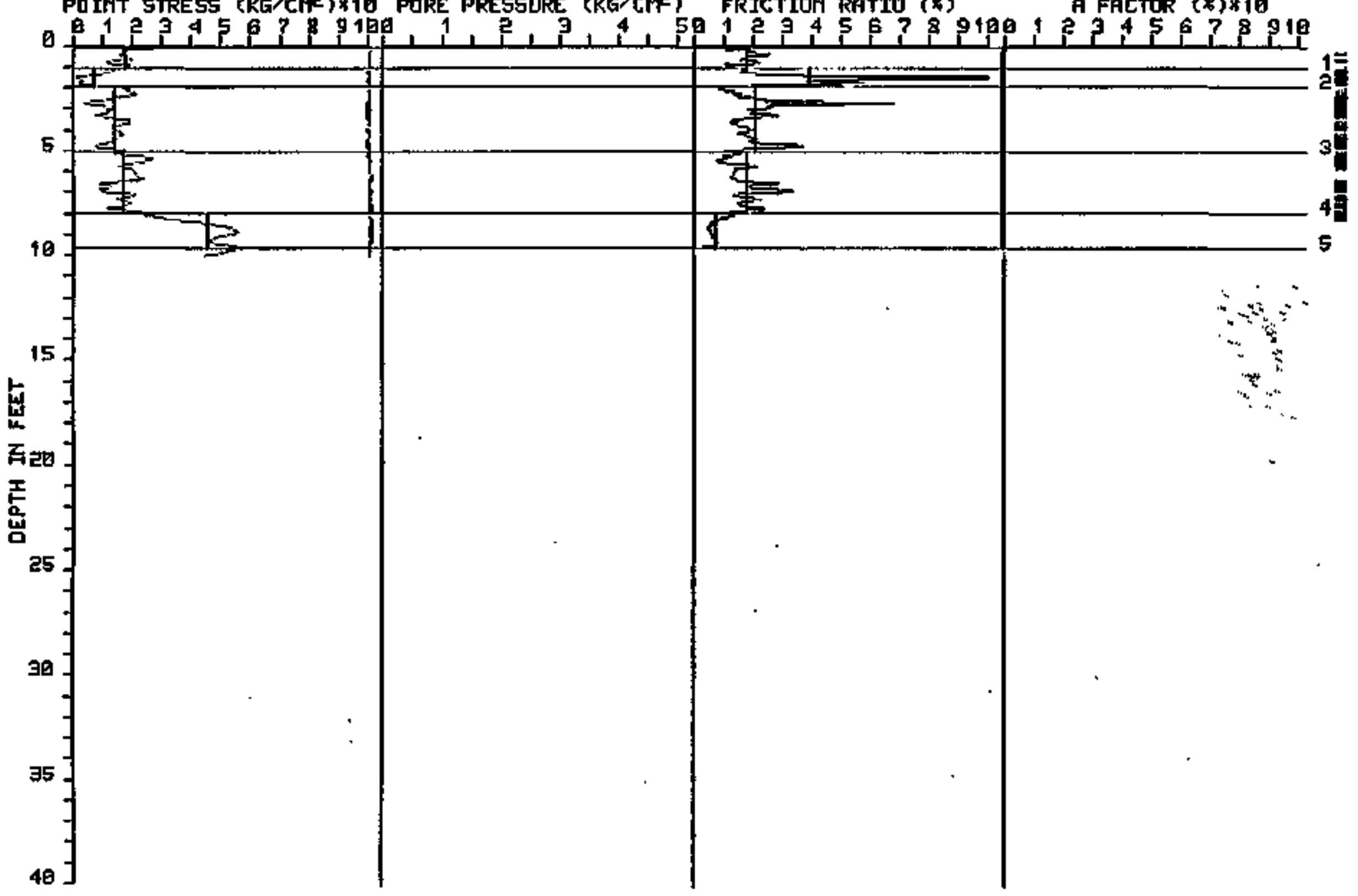


SITE ID: HATQ1

LOCATIOH IO: 015

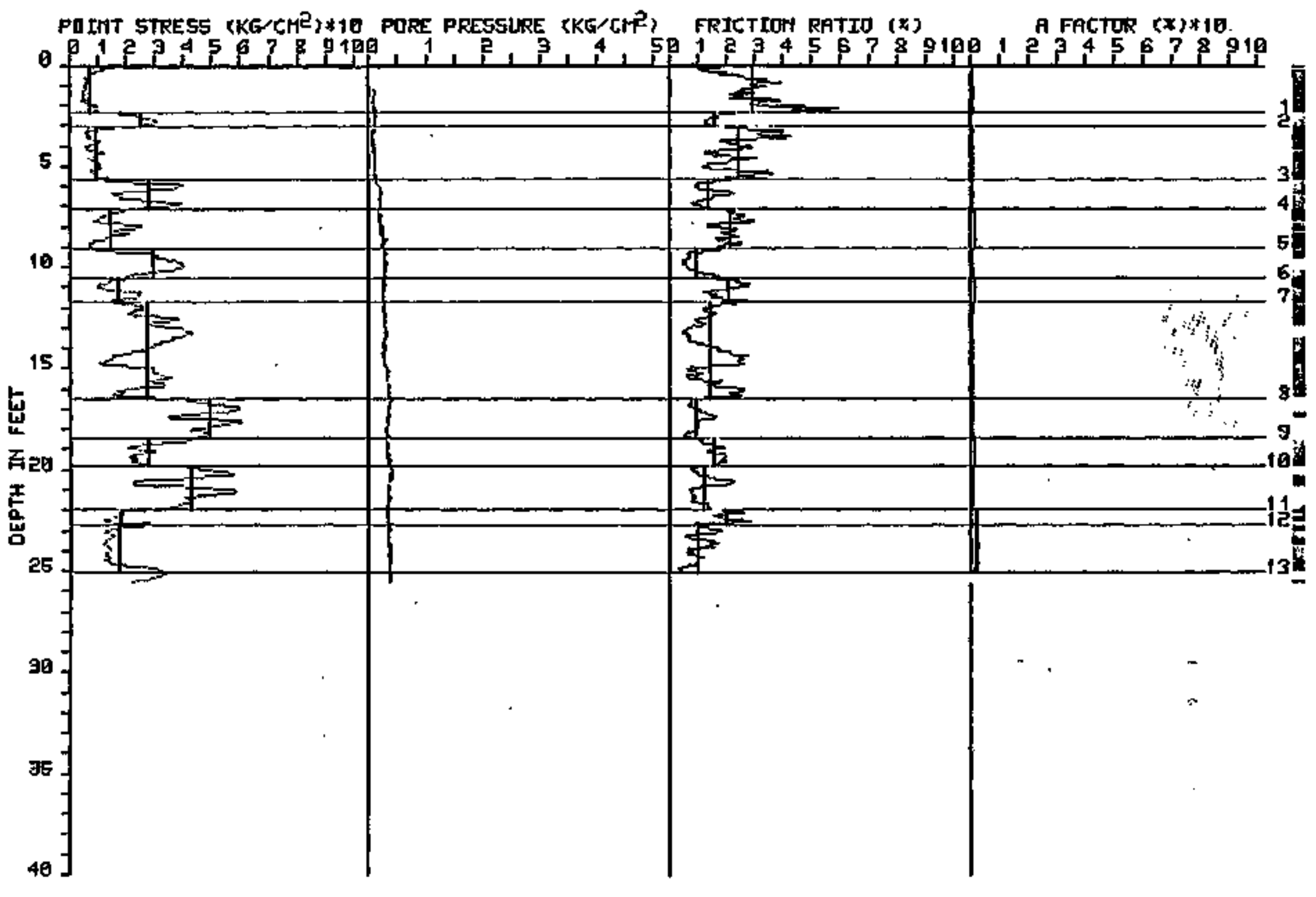




\section{PIEZOCONE SOUNDING TEST.}

SITE ID: HATO1

POINT STRESS (KG/CM2)*10 PORE PRESSURE (KG/CHF) FRICTIOH RATIO $(x)$

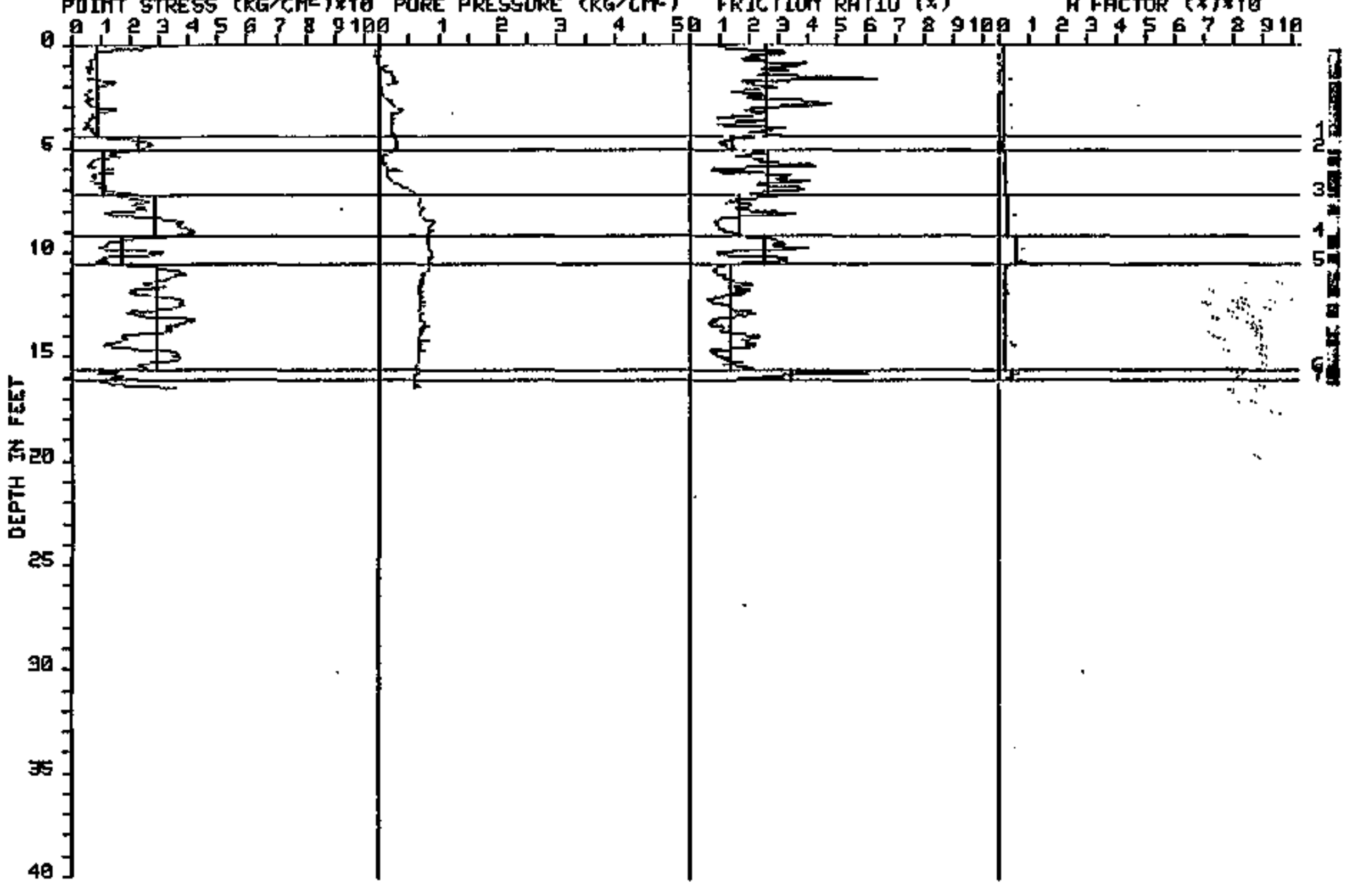


PIEZOCONE SOUNDING TEST.

SITE ID: HATET

LOCATION ID: 017

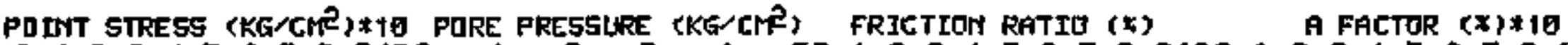

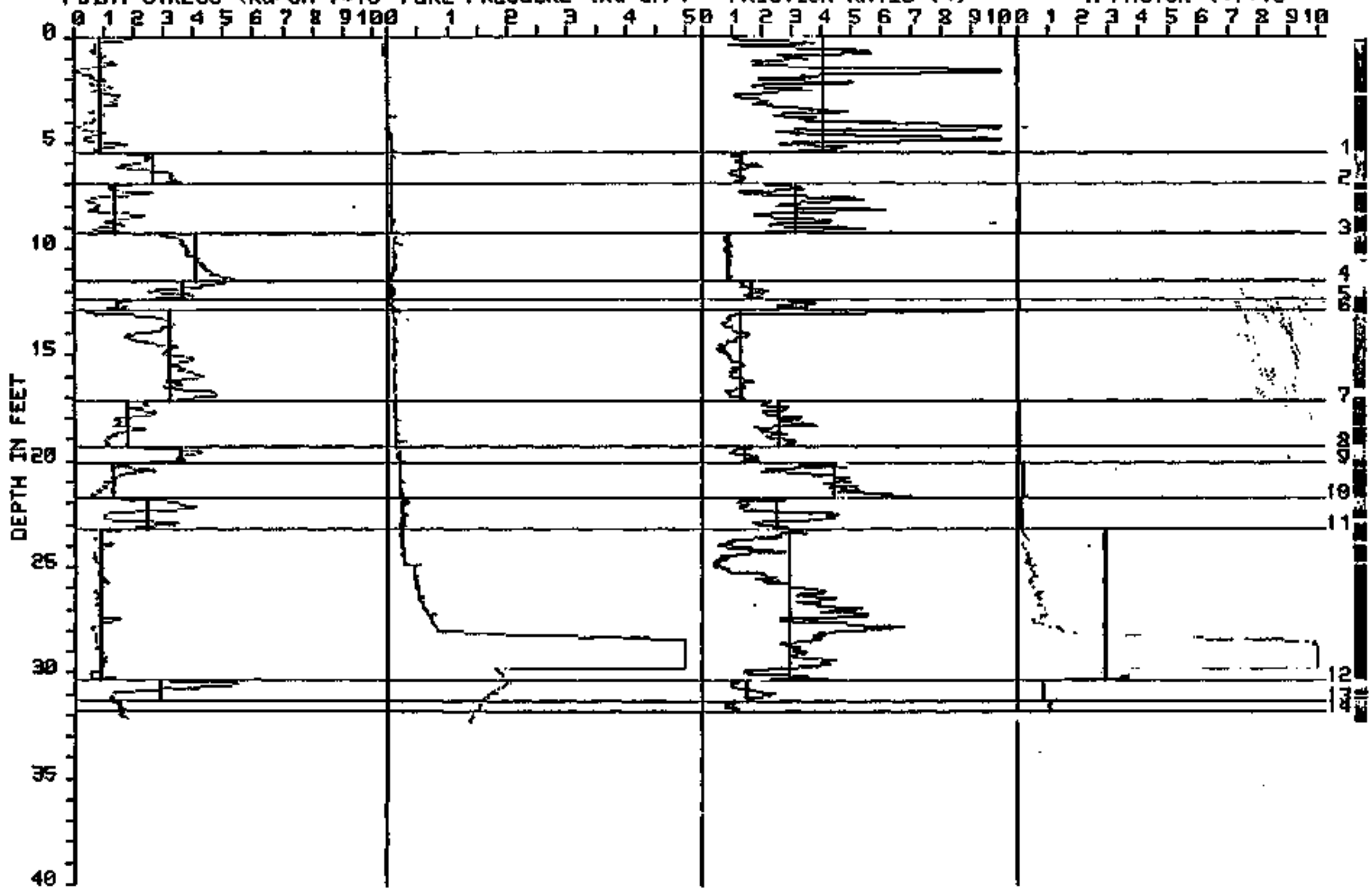




\section{PIEZOCONE SOUNDING TEST}

SITE ID: HATO1

LOCATION ID: 018

PQINT STRESS (KG/CMR)*1V PORE PRESSURE (KG/CHF) FRICTIOM RATIO (*)

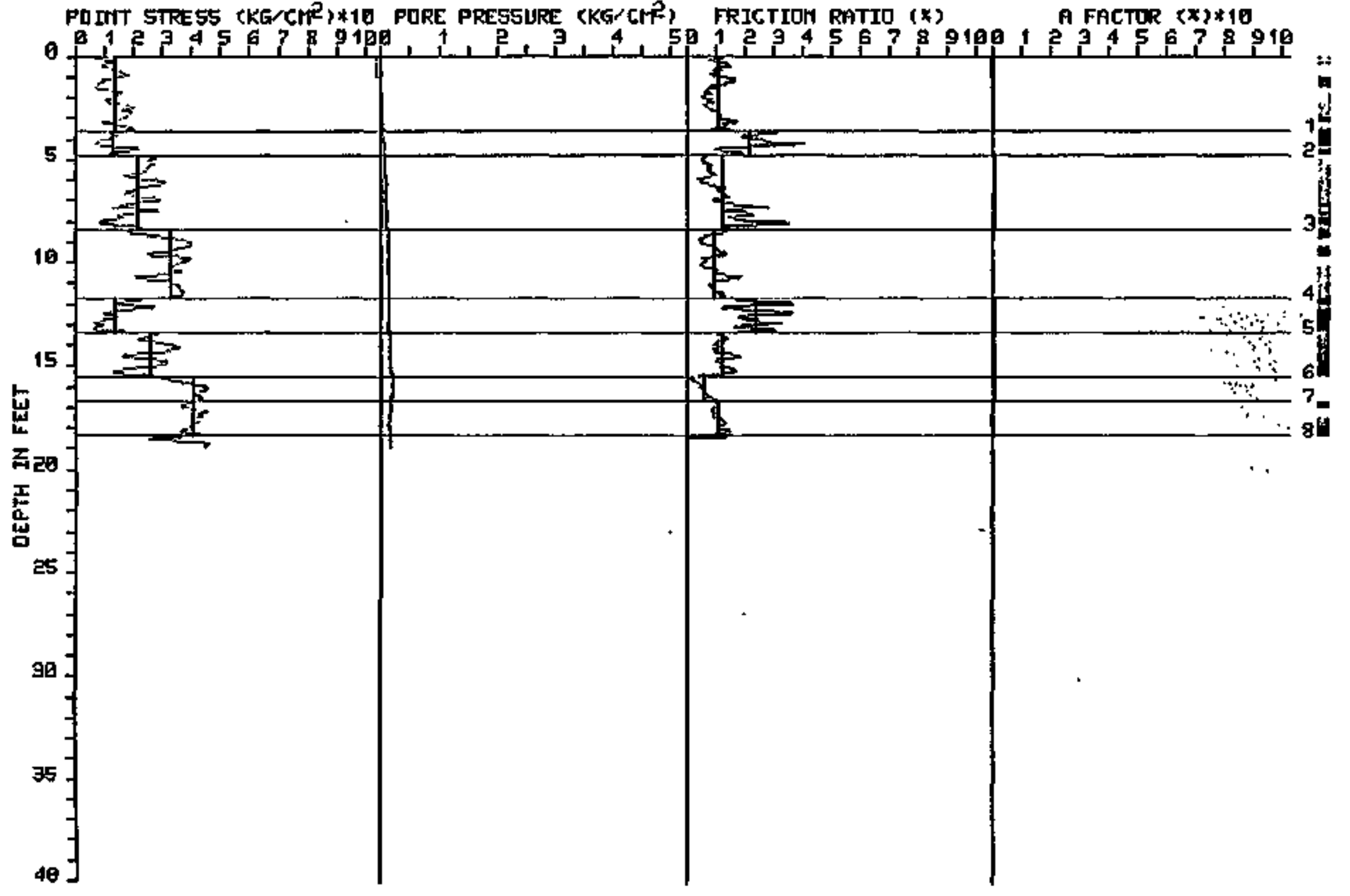




\section{PIEZOCONE SOUNDING TEST}

SITE ID: HATO1

LOCATION ID: 019

POIMT STRESS (KG/CM²)*10 PORE PRESSURE (KG/CHF) FRICTION RATID $(x)$

A FACTOR $(x) * 10$

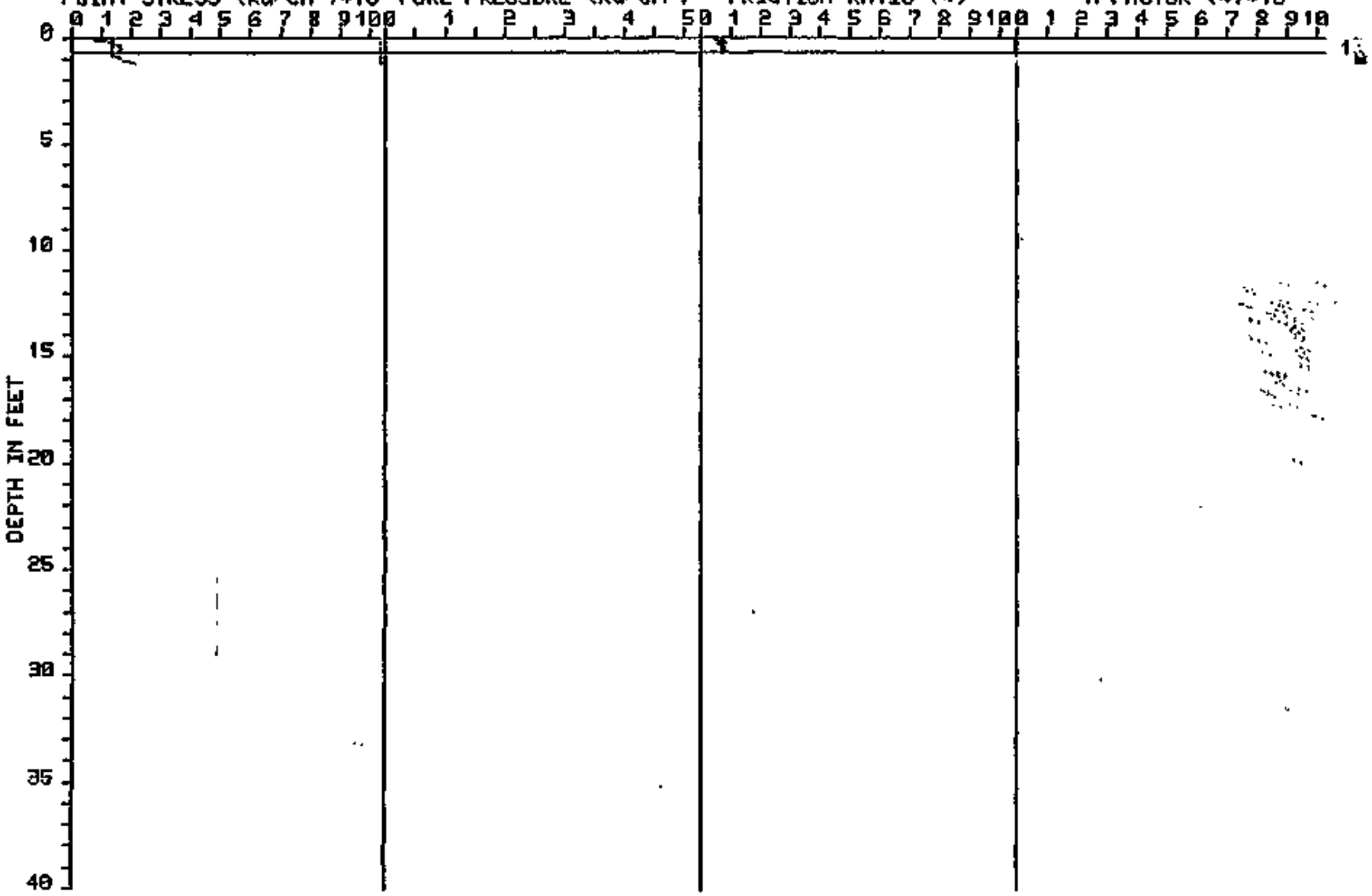




\section{PIEZOCONE SOUNDING TEST}

SITE ID: HATO1

LOCATION ID: OEO

POINT STRESS (KG/CME)*1E PORE PRESSLRE (KG/CTF)

FRICTIOH RATIO (\%)

A FACTOR $(x) * 10$

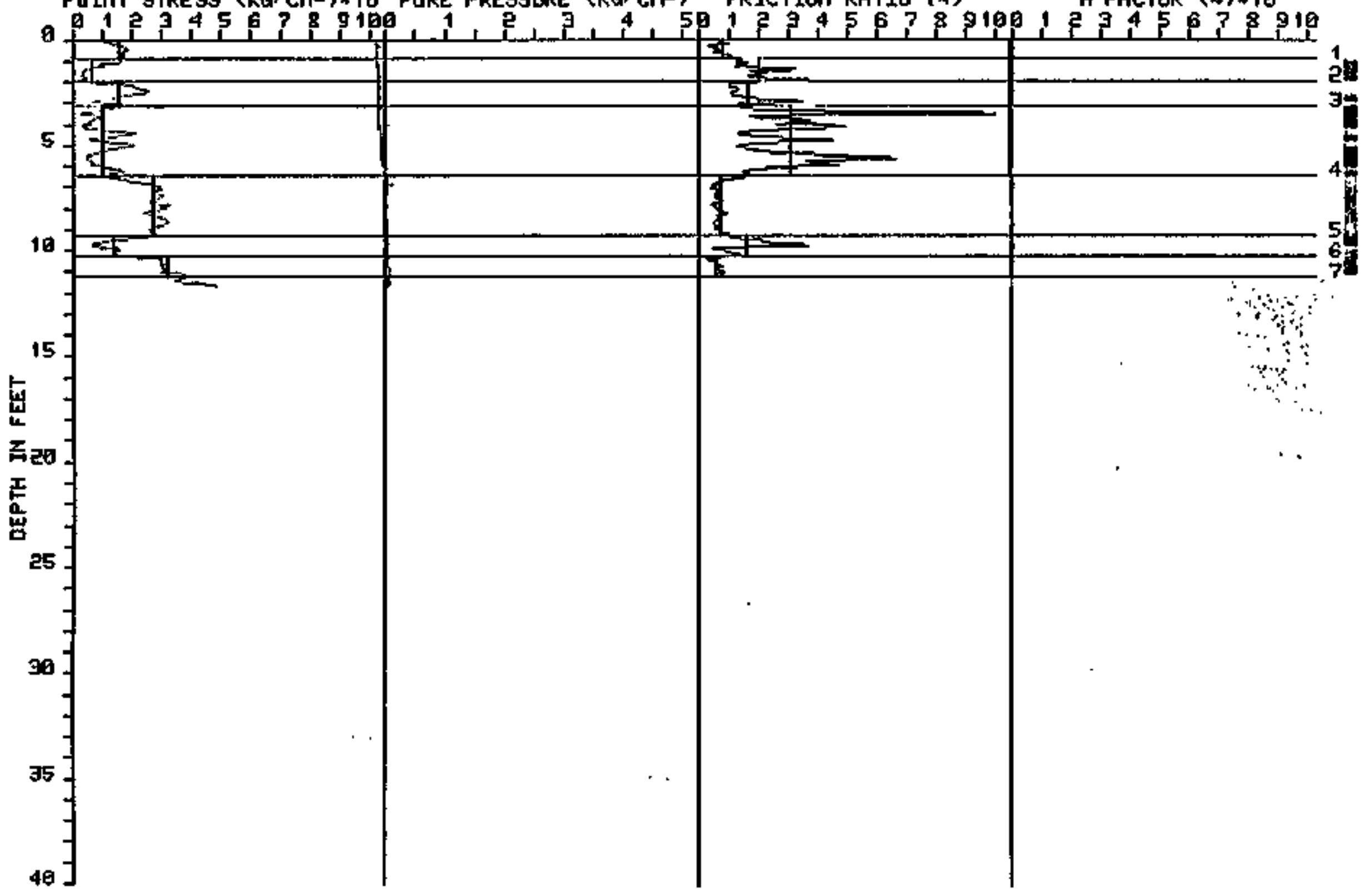




\section{PIEZOCONE SOUNDING TEST}

SITE ID: HATOS

LOCATION HO: ORT

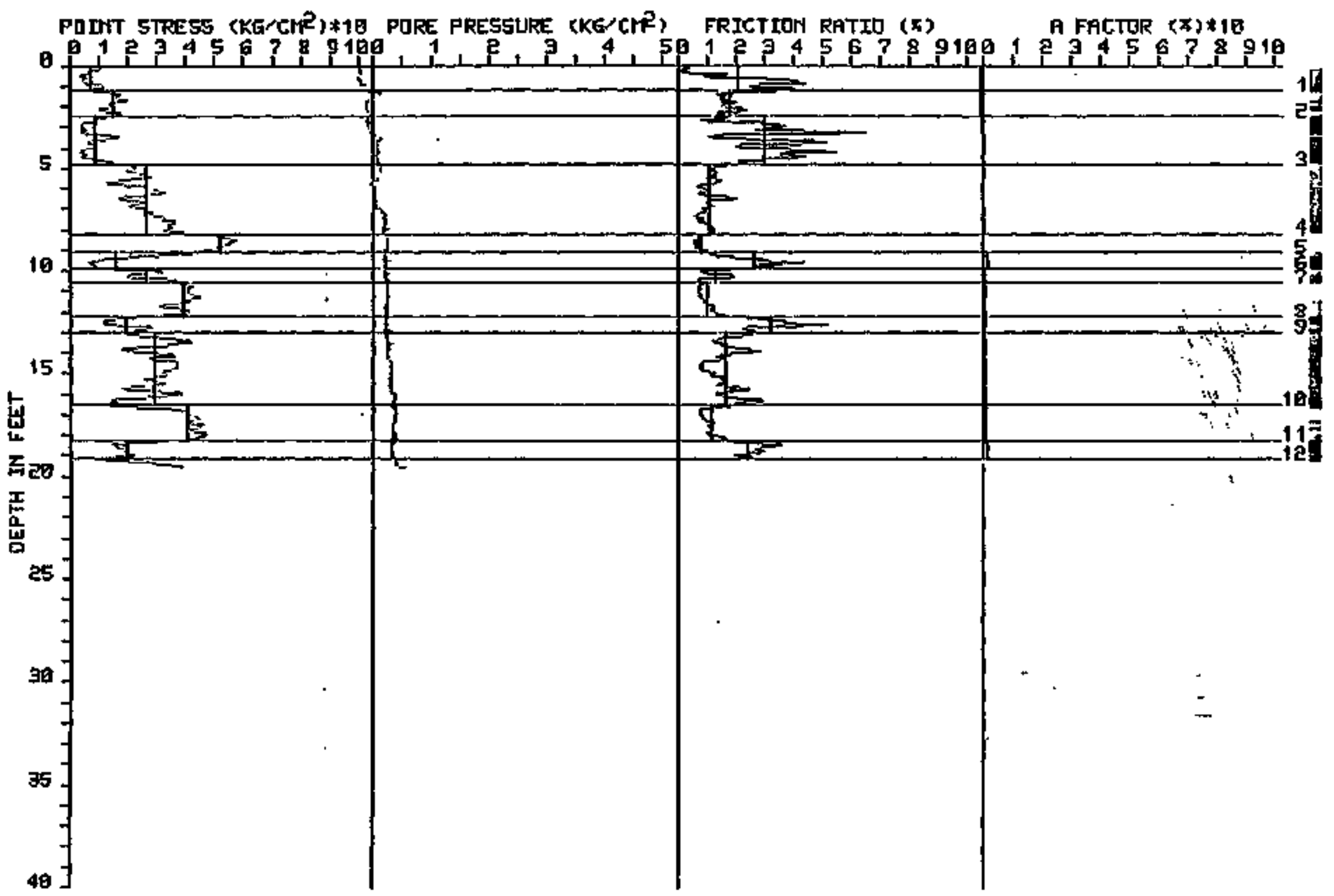




\section{PIEZOCONE SOUNDING TEST}

SITE ID: HATEI

LOCATION IO: OE2

POIMT STRESS (KG/CM2) *10 PURE PRESSURE ( $K G / \mathrm{CH}^{2}$ )

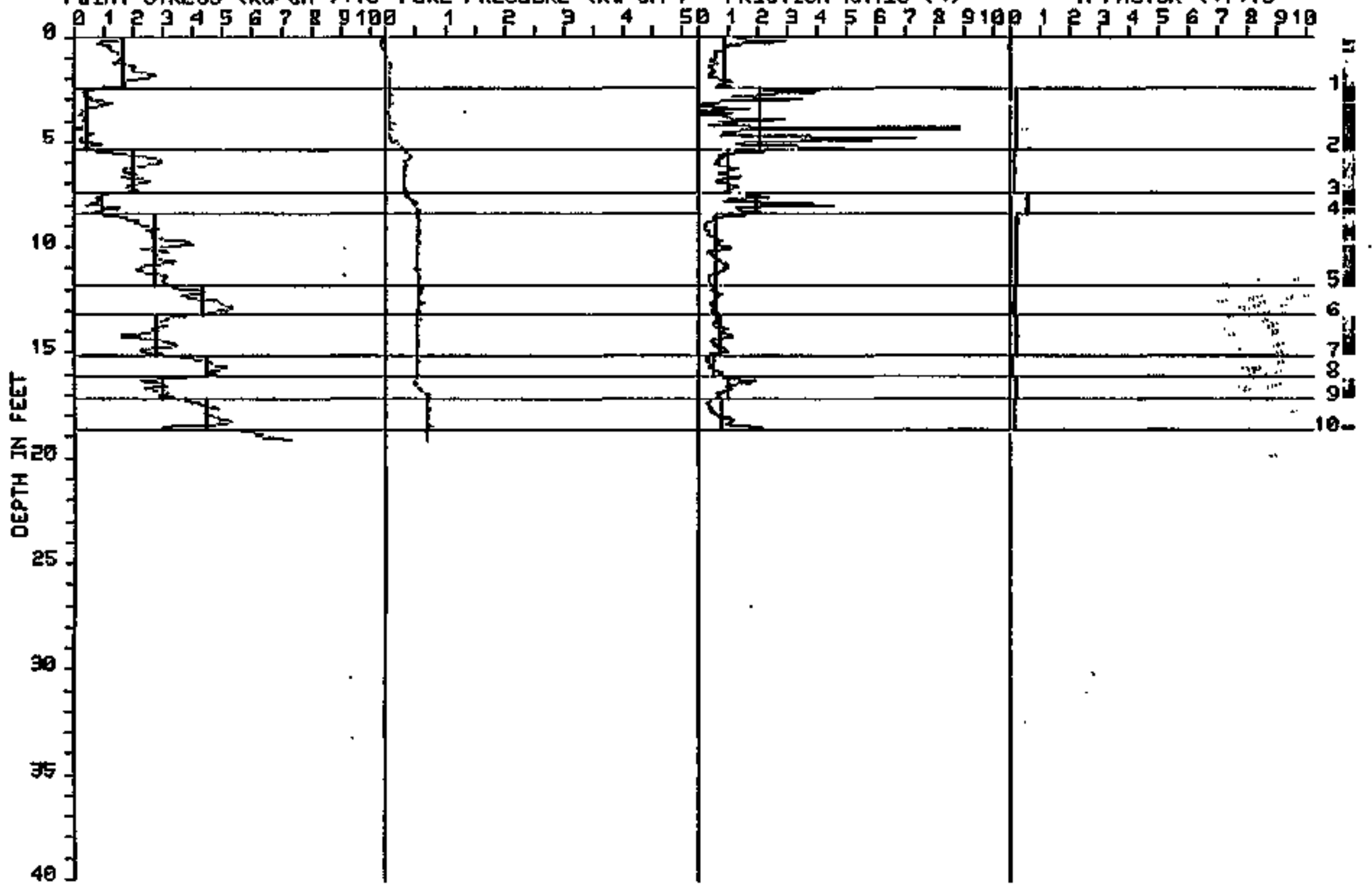


PIEZQCONE SOLINDING TEST

SITE IDE HATDI

LOCATION ID: QD3

PIIHT STRESS (KG/CME)*10 PORE PRESSIRE (KG/CIR) FRICTION RATIO (x) A FACTOR (x)*1E

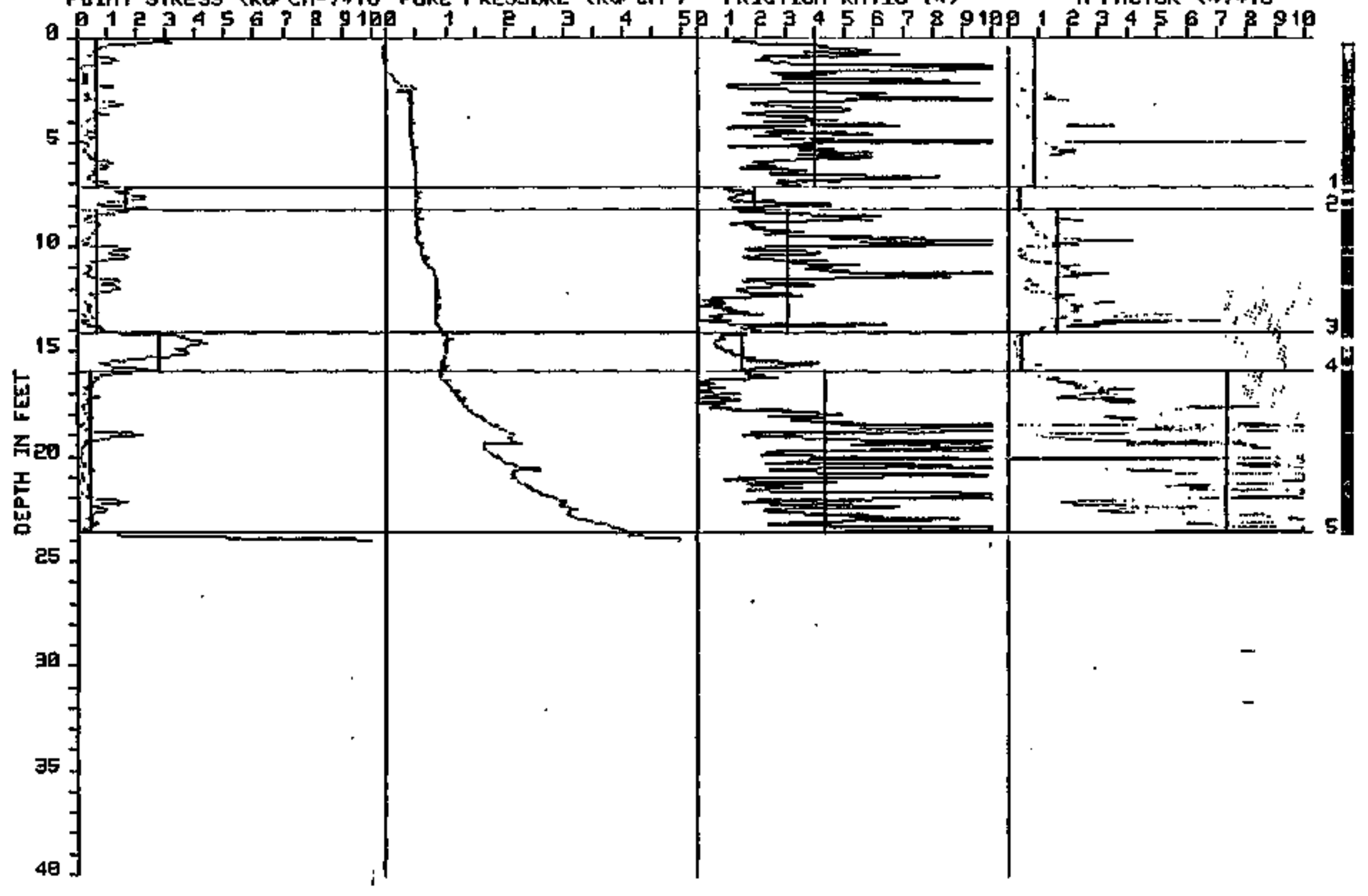




\section{PIEZOCONE SOUNDING TEST}

SITE ID: HATO1

LOCATION TO: OE4

POCWT STRESS (KG CME)

FRICTIDUN RATIO (x)

A FACTOR (*)*10

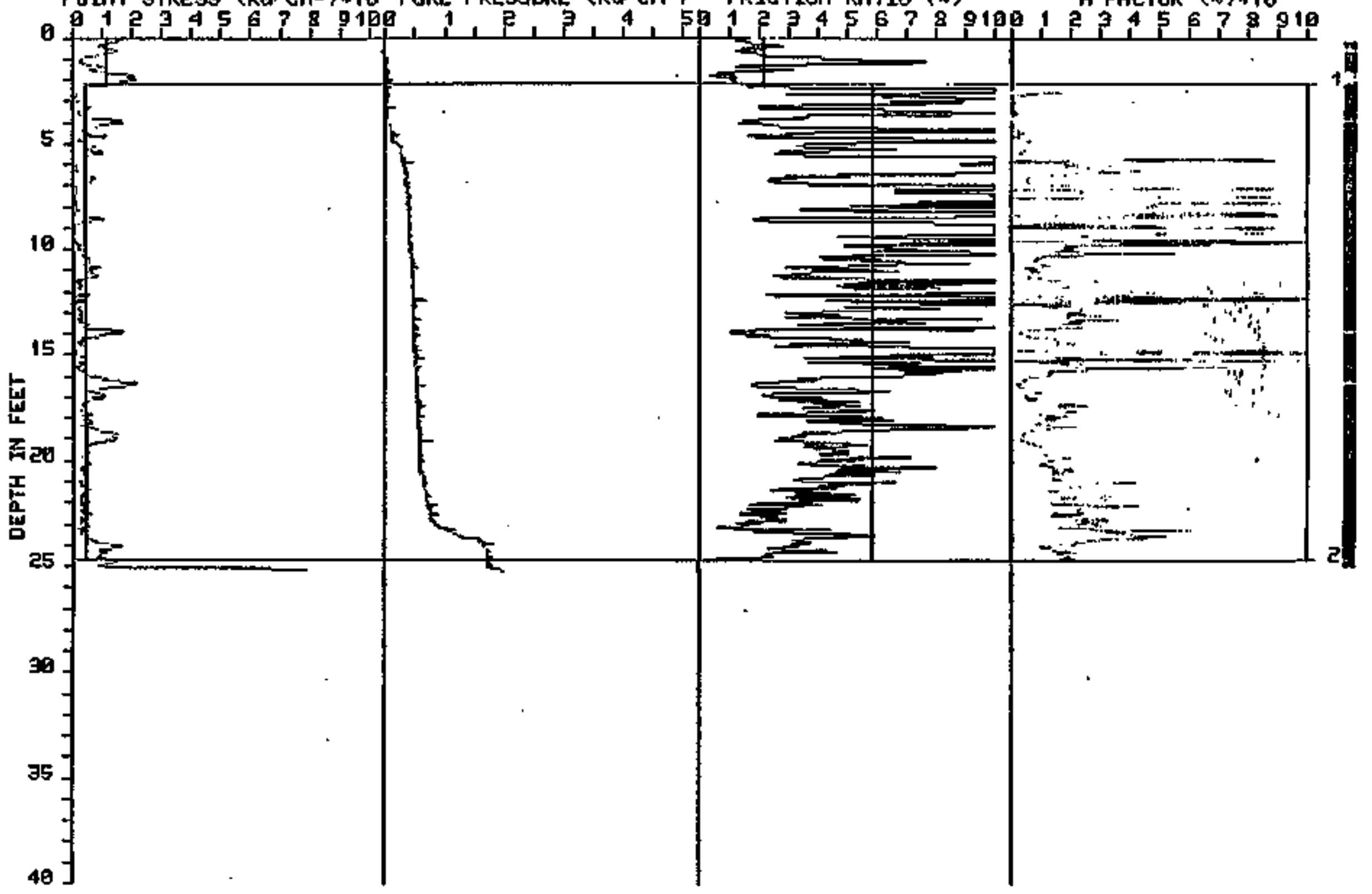




\section{PIEZOCONE SOLNDING TEST}

SITE ID: HATO1

LOCATION ID: 025

POINT STRESS (KG CME) *1D PORE PRESSURE KKG/CHF) FRICTION RATIO (*) P FACTOR (x)*1D

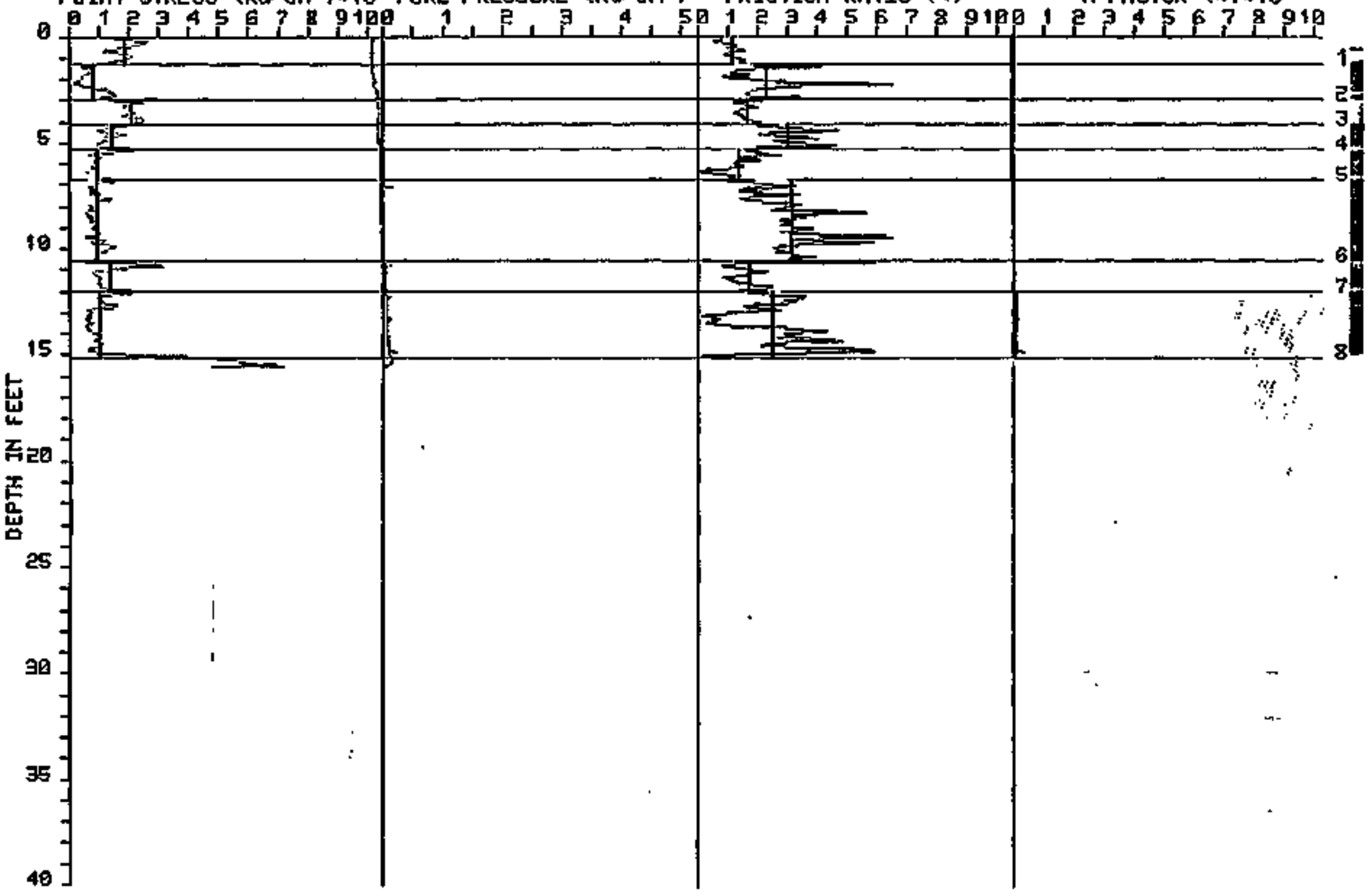




\section{PIEZOCONE SOUNDING TEST}

SITE ID: HATO1

LOCATION ID: 026

PDINT STRESS (KG/CIR) $* 10$ PORE PRESSURE (KG/CH?) FRICTIOH RATIO $(x)$

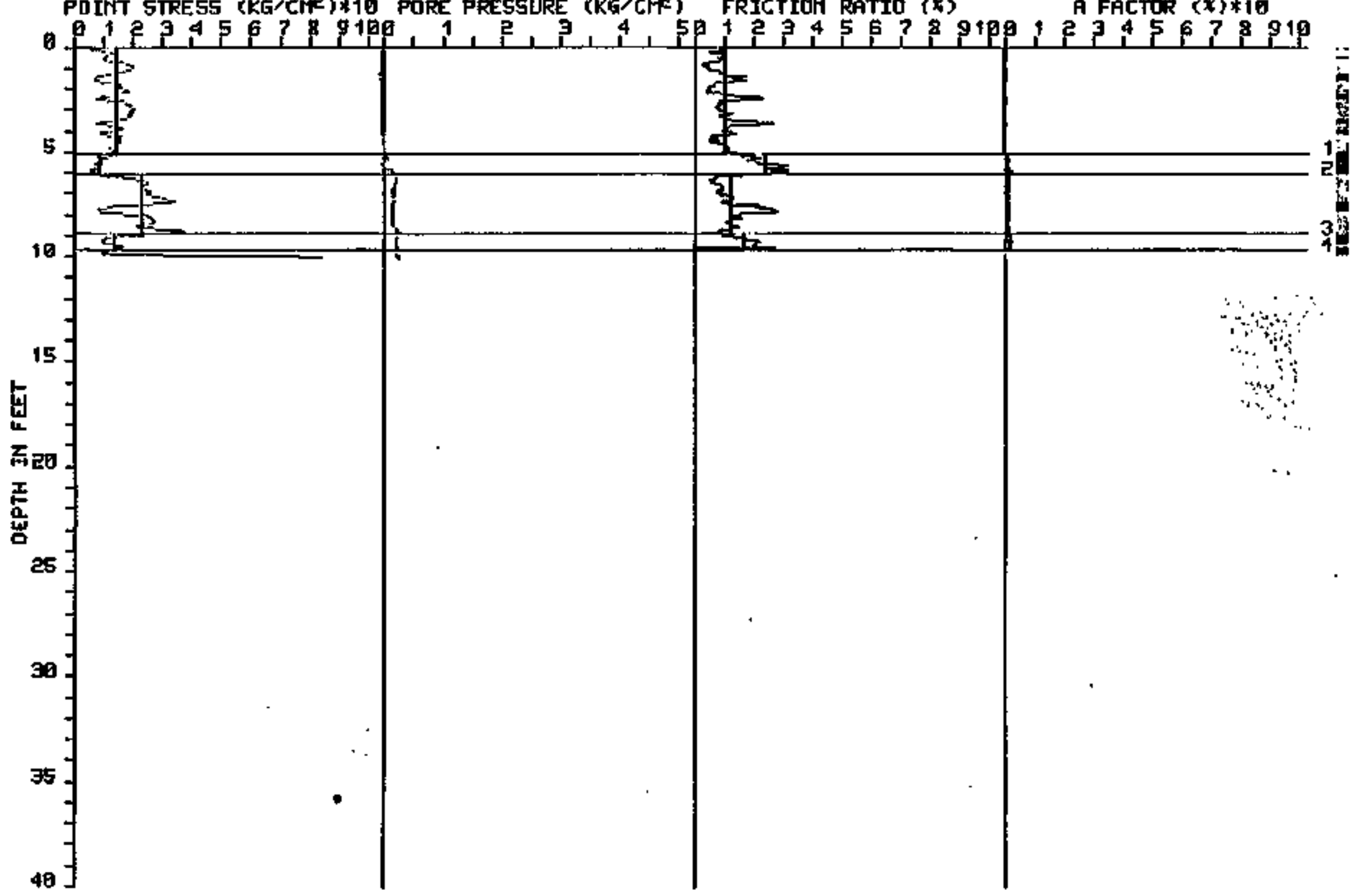




\section{PIEZOCONE SOUNDING TEST}

SITE ID: HATOI

LOCATICAY ID: QR?

PQIYT STRESS (KG/CME) $* 10$ PORE PRESSURE (KG/CM?) FRICTIUM RATID (*)

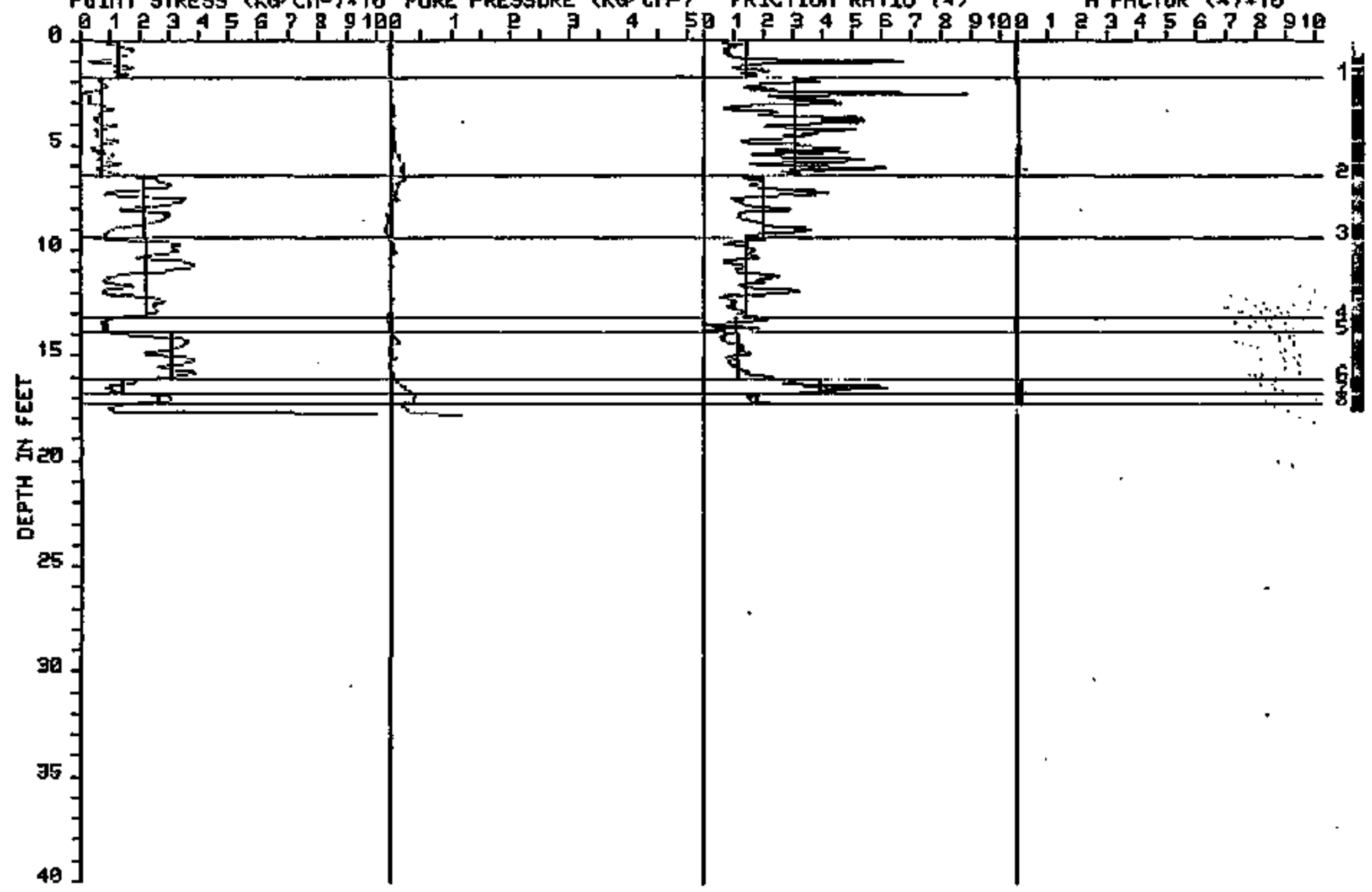




\section{PIEZOCONE SOUNDING TEST}

SITE ID: HATE1

LOCATION IOI OEZ

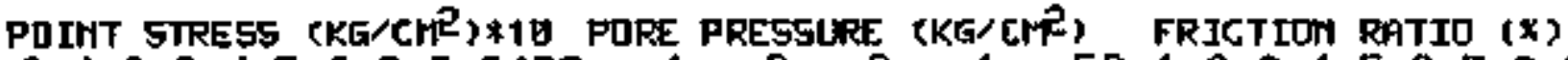

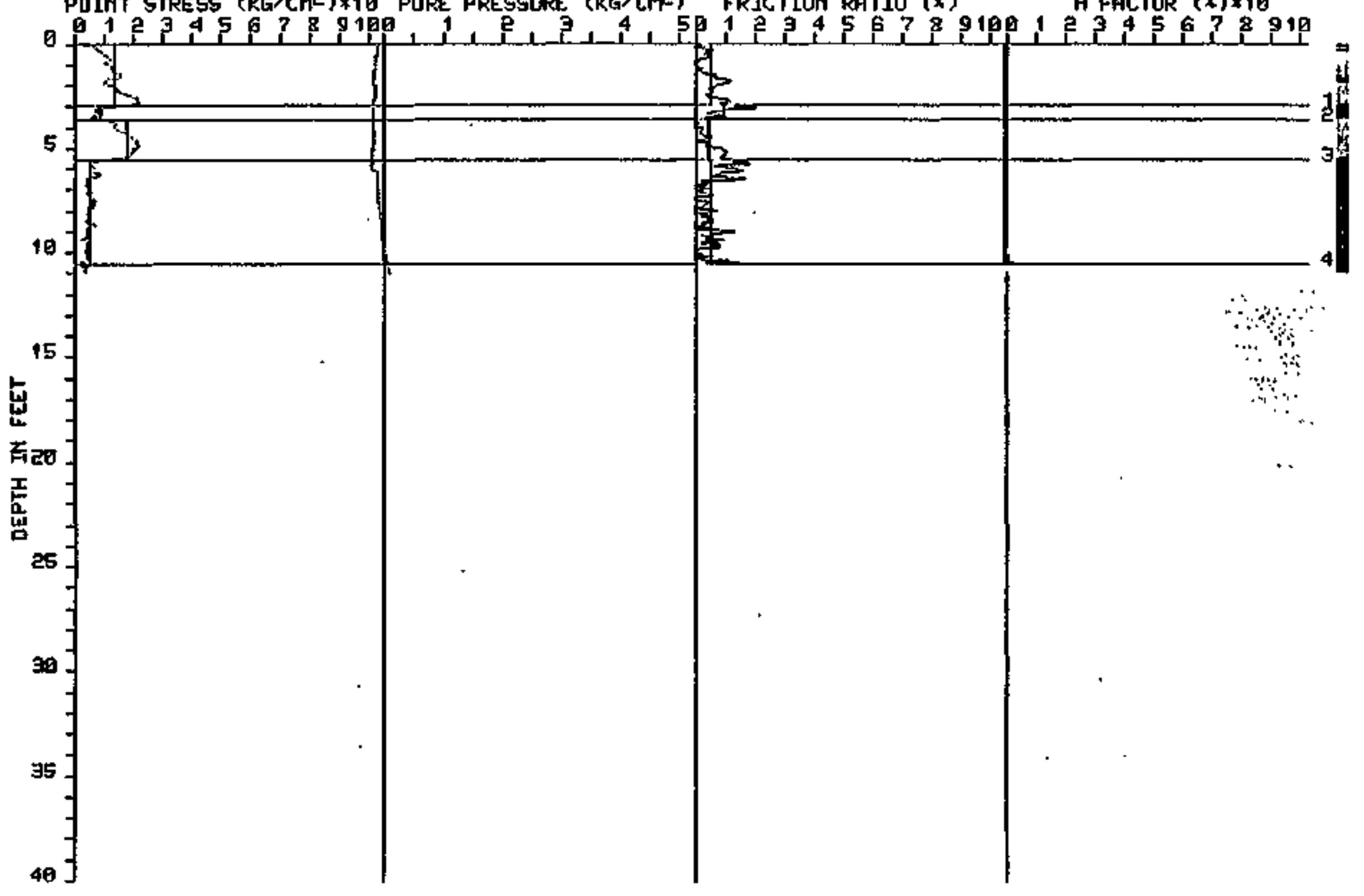


PIEZOCONE SOUNDING TEST.

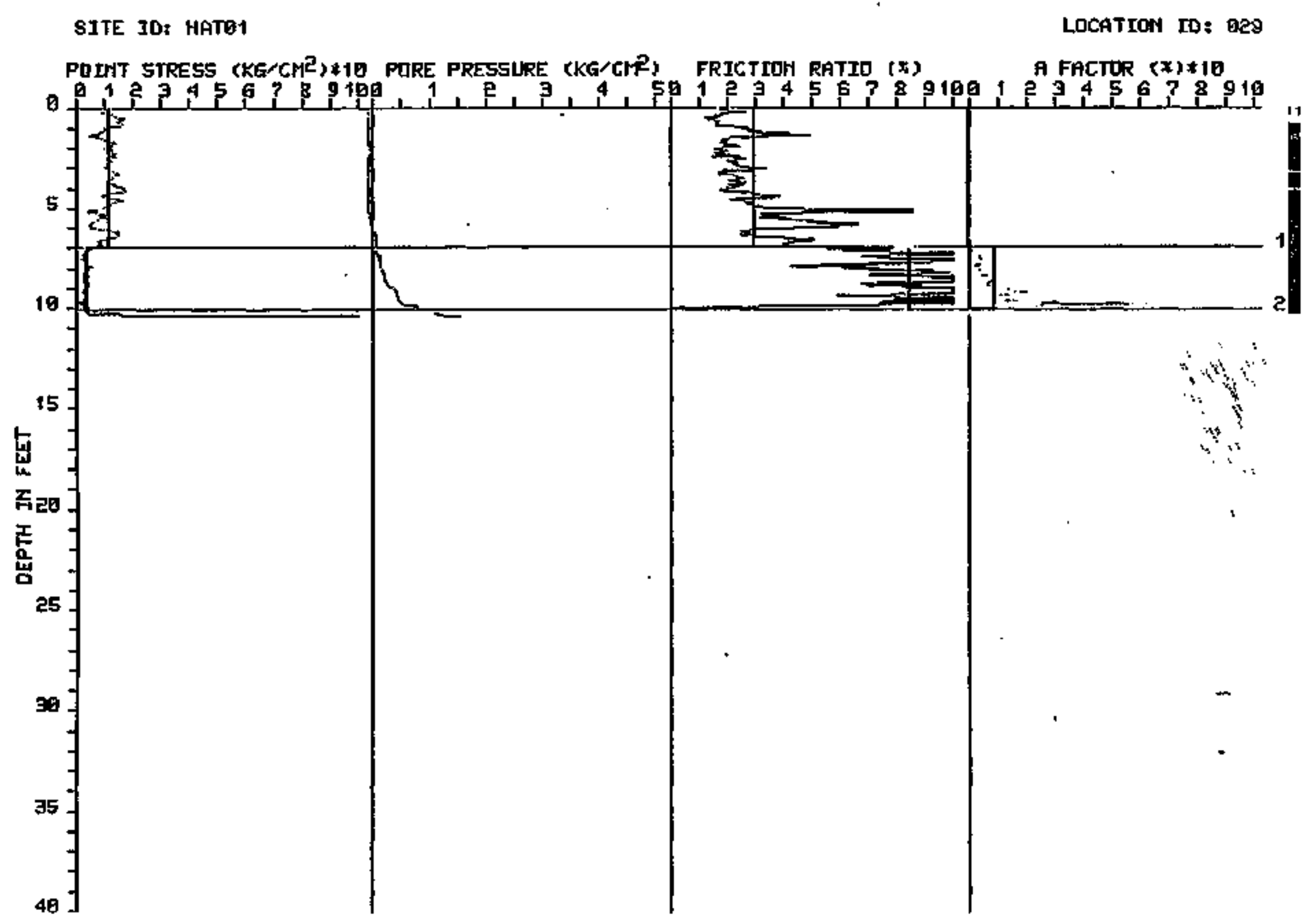

P्Pros 


\section{PIEZOCONE SOUNDING TEST}

SITE ID: HATDI

LOCATION ID: 030

POIMT STRESS (KG/CMP) 210 PORE PRESSLURE (KG/CHF) FRICTION RATIO ( $x$ )

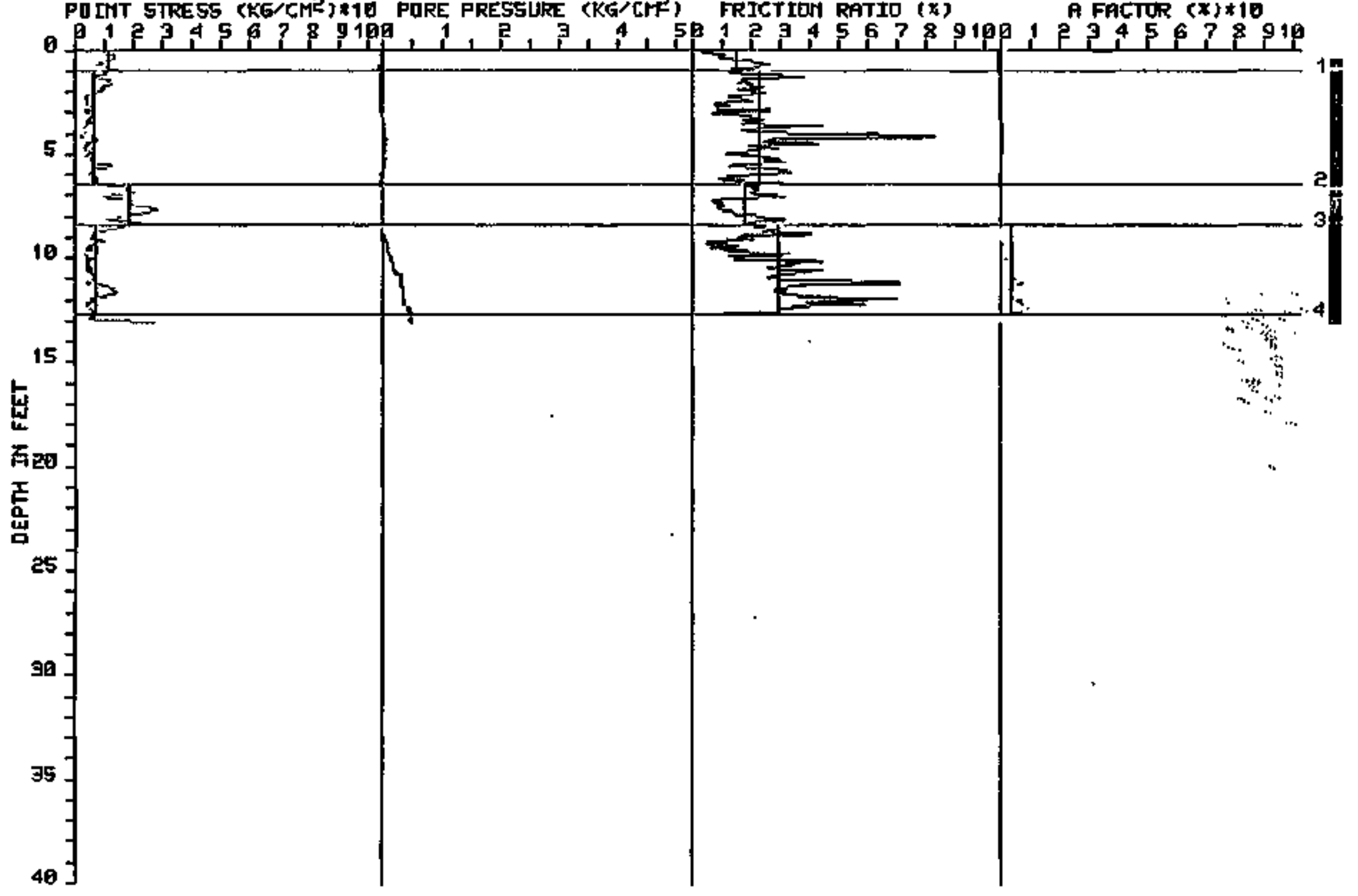


SITE ID: HATO1

GROUND WATER DEPTH: 50.0 (FT.)
LOCATION ID: 001

AUERAGE SOIL WJIGHT: 100.0 (PSF)

- DEPTH - AUG. AUG. PUG. AUG. FRICT. SOIL LY FROM TO THK. POINT SLEEUE PRESS. RATIO CLASS BC DEM FA ELAS. STREN OCR

\begin{tabular}{|c|c|c|c|c|c|c|c|c|c|c|c|c|}
\hline $\begin{array}{r}0.1 \\
1.3 \\
4.2 \\
8.0 \\
10.1 \\
11.1 \\
12.5 \\
13.8 \\
18.6 \\
23.3 \\
25.3 \\
28.3 \\
30.7\end{array}$ & $\begin{array}{r}1.3 \\
4.1 \\
8.0 \\
10.1 \\
11.1 \\
12.5 \\
13.7 \\
18.5 \\
23.3 \\
25.2 \\
28.3 \\
30.7 \\
33.8\end{array}$ & $\begin{array}{l}1.2 \\
2.8 \\
3.8 \\
2.1 \\
1.0 \\
1.4 \\
1.2 \\
4.8 \\
4.7 \\
1.9 \\
3.1 \\
2.3 \\
3.1\end{array}$ & $\begin{array}{r}16.4 \\
7.3 \\
22.6 \\
14.2 \\
24.5 \\
14.3 \\
25.7 \\
13.2 \\
30.6 \\
18.0 \\
6.2 \\
21.8 \\
11.8\end{array}$ & $\begin{array}{l}0.2 \\
0.1 \\
0.3 \\
0.4 \\
0.3 \\
0.4 \\
0.4 \\
0.4 \\
0.6 \\
0.6 \\
0.1 \\
0.5 \\
0.4\end{array}$ & $\begin{array}{l}0.0 \\
0.1 \\
0.1 \\
0.2 \\
0.2 \\
0.2 \\
0.4 \\
1.1 \\
0.5 \\
1.4 \\
2.7 \\
1.0 \\
6.6\end{array}$ & $\begin{array}{l}1.0 \\
1.4 \\
1.1 \\
2.6 \\
1.4 \\
2.7 \\
1.5 \\
2.8 \\
1.9 \\
3.2 \\
2.1 \\
2.2 \\
3.3\end{array}$ & $\begin{array}{l}\text { S-SL** } \\
\text { SLIM } \\
\text { S-SL* } \\
\text { SLIM } \\
\text { S-SL* } \\
\text { SLIM } \\
\text { S-SL** } \\
\text { SLIM } \\
\text { S-SL** } \\
\text { SLIM } \\
\text { SLIM } \\
\text { SLIM } \\
\text { SLIM }\end{array}$ & $\begin{array}{r}5 \\
4 \\
7 \\
9 \\
8 \\
9 \\
8 \\
8 \\
19 \\
12 \\
4 \\
14 \\
7\end{array}$ & $\begin{array}{l}44 \\
41 \\
37\end{array}$ & $\begin{array}{l}31 \\
30 \\
29\end{array}$ & $\begin{array}{r}57 \\
25 \\
79 \\
50 \\
86 \\
50 \\
90 \\
46 \\
107 \\
63 \\
22 \\
76 \\
41\end{array}$ & $\begin{array}{r}1965 \\
870 \\
2709 \\
1709 \\
2937 \\
1718 \\
3086 \\
1585 \\
3671 \\
2164 \\
742 \\
2615 \\
1414\end{array}$ \\
\hline $\begin{array}{l}\text { THK } \\
\text { THK }\end{array}$ & $\begin{array}{l}35 \\
64\end{array}$ & 1.9 & $\begin{array}{l}25.6 \\
12.7\end{array}$ & $\begin{array}{l}0.4 \\
0.3\end{array}$ & 1.9 & $\begin{array}{l}1.5 \\
2.5\end{array}$ & $\begin{array}{l}S-S L \\
\text { SL IM }\end{array}$ & $\begin{array}{l}8 \\
7\end{array}$ & 41 & 00 & $\begin{array}{l}89 \\
44\end{array}$ & $\begin{array}{l}3071 \\
1527\end{array}$ \\
\hline
\end{tabular}


SITE ID: HATV1

GROUND WATER DEPTH: 50.0 (FT.)
LOCATION ID: 002

AUERAGE SOIL WEIGHT: 100.0 (PSF)

- DEPTH -
FROM TO THK. POINT SLEEUE PRESS. PATIO CLASS BC DEN FA ELAS. SHTREN OCR

\begin{tabular}{|c|c|c|c|c|c|c|c|c|c|c|c|c|}
\hline $\begin{array}{rr}1 & 0.1 \\
2 & 0.8 \\
3 & 9.9 \\
4 & 13.2 \\
5 & 17.1 \\
6 & 18.7 \\
7 & 19.6\end{array}$ & $\begin{array}{r}9.8 \\
9.8 \\
13.2 \\
17.0 \\
18.6 \\
19.6 \\
21.0\end{array}$ & $\begin{array}{l}0.7 \\
9.1 \\
3.3 \\
3.8 \\
1.6 \\
0.9 \\
1.4\end{array}$ & $\begin{array}{l}48.0 \\
23.7 \\
13.4 \\
30.4 \\
43.1 \\
22.2 \\
56.3\end{array}$ & $\begin{array}{l}0.3 \\
0.2 \\
0.4 \\
0.4 \\
0.3 \\
0.4 \\
0.5\end{array}$ & $\begin{array}{r}-0.1 \\
0.0 \\
1.6 \\
0.5 \\
0.1 \\
0.2 \\
0.3\end{array}$ & $\begin{array}{l}0.7 \\
0.8 \\
2.7 \\
1.2 \\
0.8 \\
1.9 \\
1.0\end{array}$ & $\begin{array}{l}\text { SAND* } \\
\text { S-SL* } \\
\text { SLIM } \\
\text { S-SL* } \\
\text { SAND* } \\
\text { S-SL } \\
\text { SAND* }\end{array}$ & $\begin{array}{r}9 \\
7 \\
8 \\
10 \\
8 \\
7 \\
11\end{array}$ & $\begin{array}{l}43 \\
50 \\
30 \\
55\end{array}$ & $\begin{array}{l}30 \\
\mathbf{3 1} \\
29 \\
32\end{array}$ & $\begin{array}{r}168 \\
83 \\
47 \\
106 \\
151 \\
78 \\
197\end{array}$ & $\begin{array}{l}2845 \\
1611 \\
3647 \\
2662\end{array}$ \\
\hline $\begin{array}{l}\text { THK: } \\
\text { THK: } \\
\text { THK: }\end{array}$ & $\begin{array}{l}17 \\
66 \\
15\end{array}$ & $\begin{array}{r}3.7 \\
13.8 \\
3.3\end{array}$ & $\begin{array}{l}49.0 \\
25.4 \\
13.4\end{array}$ & $\begin{array}{l}0.4 \\
0.3 \\
0.4\end{array}$ & $\begin{array}{l}0.1 \\
0.2 \\
1.6\end{array}$ & $\begin{array}{l}0.9 \\
1.0 \\
2.7\end{array}$ & $\begin{array}{l}\text { SAND } \\
\text { S-SL } \\
\text { SLIM }\end{array}$ & $\begin{array}{l}9 \\
7 \\
8\end{array}$ & $\begin{array}{l}56 \\
46\end{array}$ & $\begin{array}{l}32 \\
31\end{array}$ & $\begin{array}{r}171 \\
89 \\
46\end{array}$ & $\begin{array}{l}3053 \\
1611\end{array}$ \\
\hline
\end{tabular}


SITE ID: HATQ1

GROUND WATER DEPTH: 50.0 (FT.)
LOCATION ID: 003

AUERAGE' SOIL WEIGHT: 100.0 (PSF)

AUG. AUG. UND.

- DEPTH - AUG. AUG. PORE FRICT. SOIL SHEAR

LY FROM TO THK. POINT SLEEUE PRESS. RATIO CLASS BC DEN FA ELAS. STREN DCR

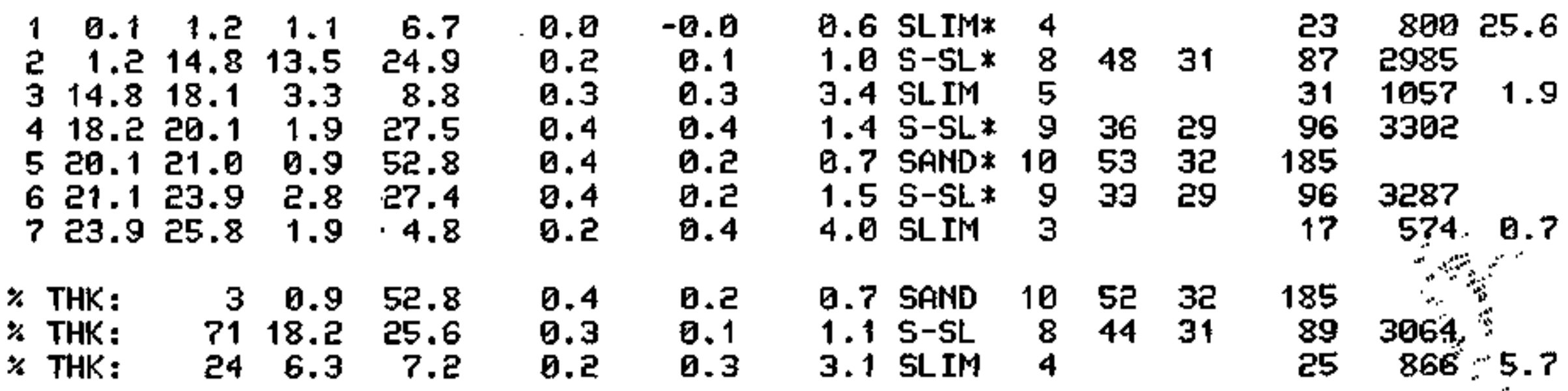


SITE ID: HATQ1

GROUND LATER DEPTH: 50.0 (FT.)
LOCATION ID: 004

AUERAGE' SOIL WEIGHT: 100.0 (PSF)

FEPTH -
FROM TO

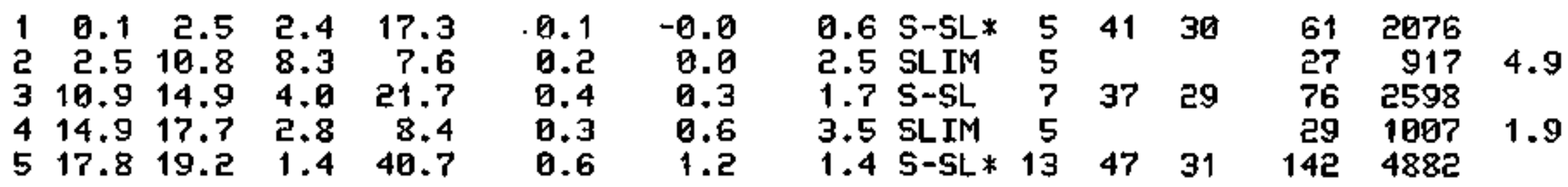

\% THK:

$\begin{array}{lll}41 & 7.8 & 23.8\end{array}$

0.3

0.4 1.3 S-SL

$7 \quad 40 \quad 30$

832847.

$\%$ THK:

$58 \quad 11.1 \quad 7.8$

0.2

0.2

2.8 SLIM

27

$939: 4 \div 1$ 
SITE ID : HATQ1

GROUND LATER DEPTH: 50.0 (FT.)
LOCATION ID: 005

AUERAGE SOIL WEIGHT: 100.0 (PSF)

AUG. AUG.

- DEPTH -

AUG. AUG. PORE FRICT. SOIL

UND.

LY FROM TO THK. POINT SLEEVE PRESS. RATIO CLASS BC DEN FA ELAS. STREN DCR

$\begin{array}{lllllllllllllll}1 & 0.1 & 2.7 & 2.6 & 13.8 & 0.1 & -0.0 & 0.6 & 5-5 L * & 4 & 35 & 29 & 48 & 1661\end{array}$

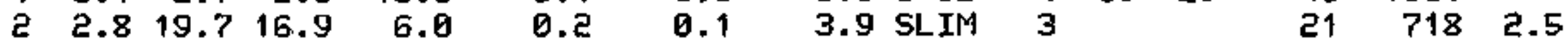

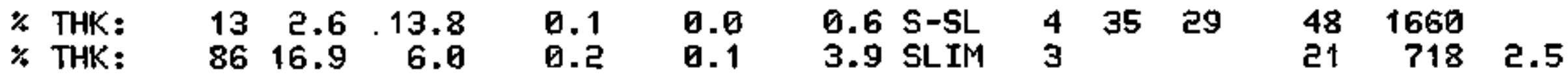




\section{PIEZOCONE SOUNDING TEST}

SITE ID: HATEI

LOCATICN ID: 031

POIHT STRESS (KG/CH')

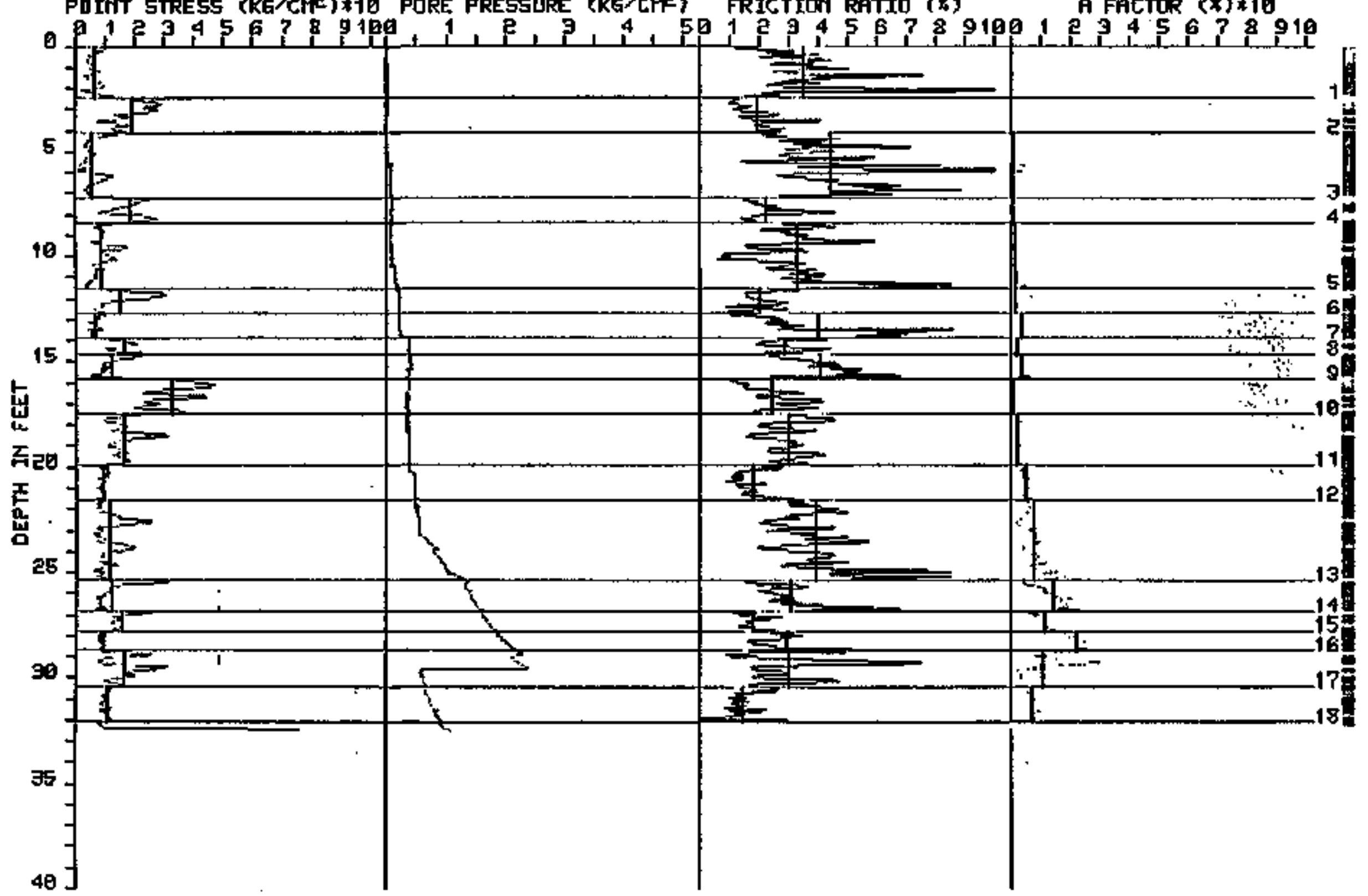




\section{PIEZOCONE SOUNDING TEST}

SITE ID: HATOI

LOCATION ID: 932

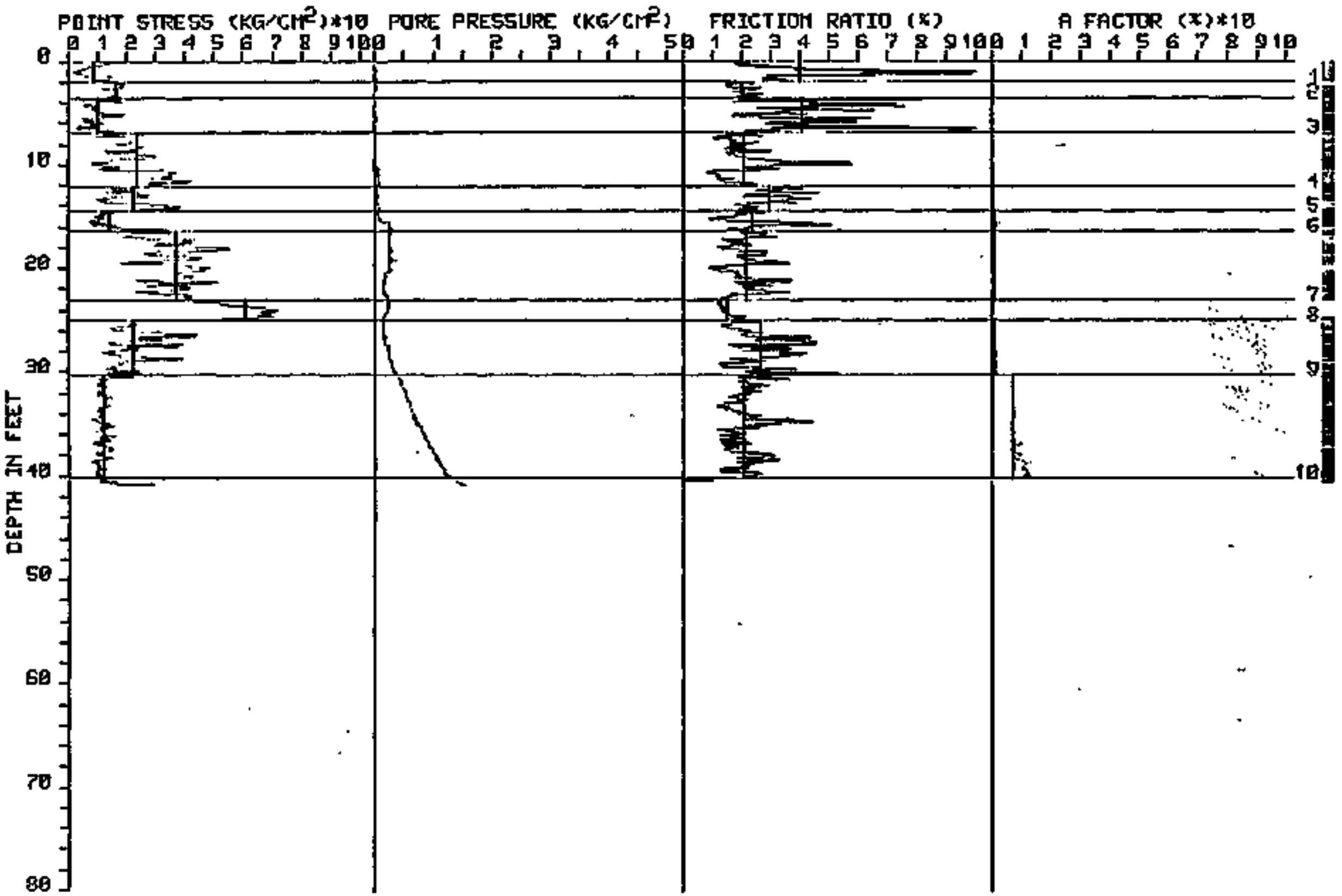




\section{PIEZOCONE SOUNDING TEST}

SITE ID: HATBI

LOCATION ID: 033

POINT STRESS (KG/CME)*10 PORE PRESSURE (KG/CMR) FRICTION RATID (x) A FACTOR $(x) * 10$

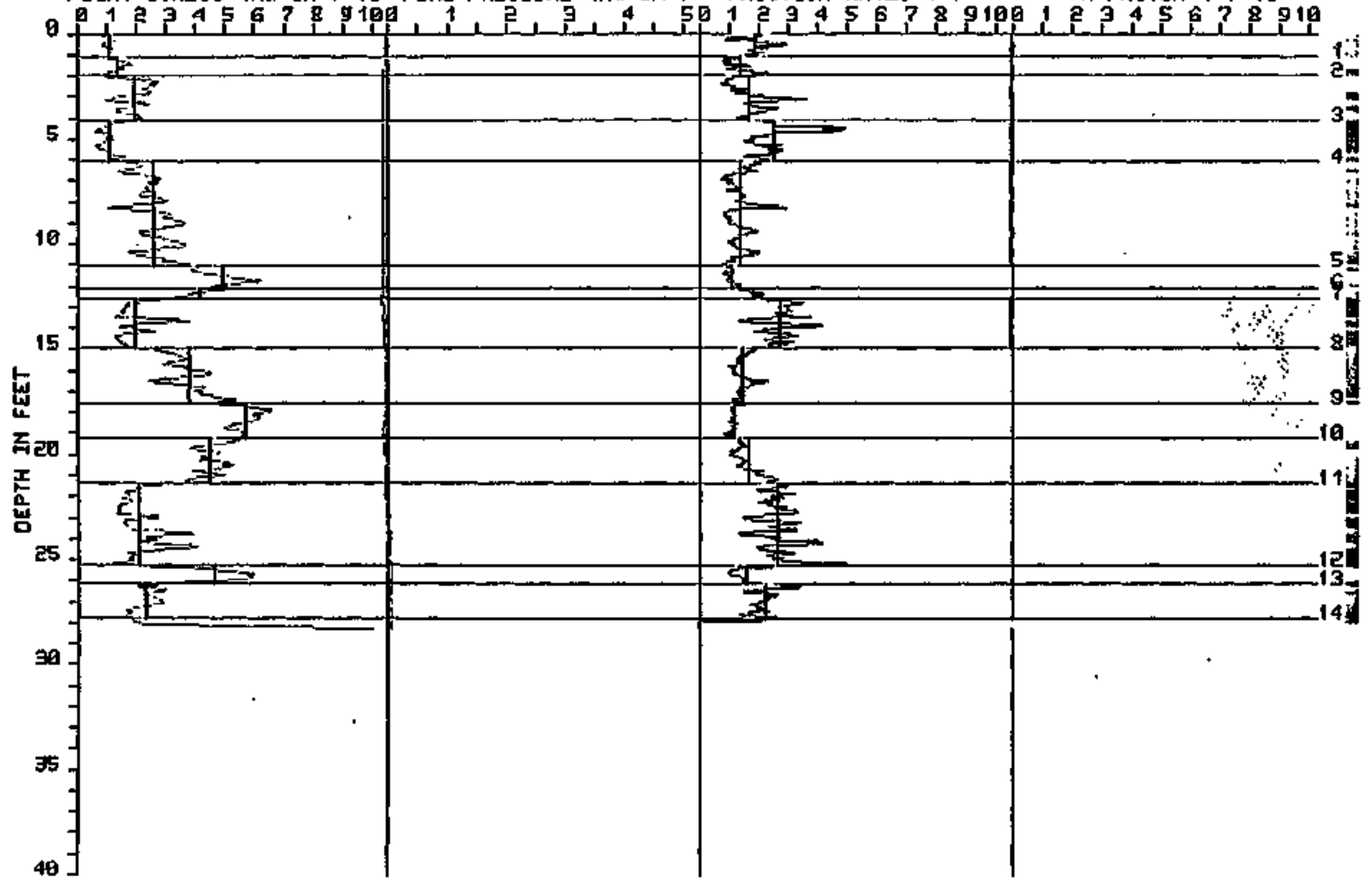




\section{PIEZOCONE SOUNDIMG TEST}

SITE ID: HATQ1

LOCATION ID: 634

PIINT STRESS (KG/CT2) *10 PORE PRESSURE (KG/CHF) FRICTIOH RATTO (*)

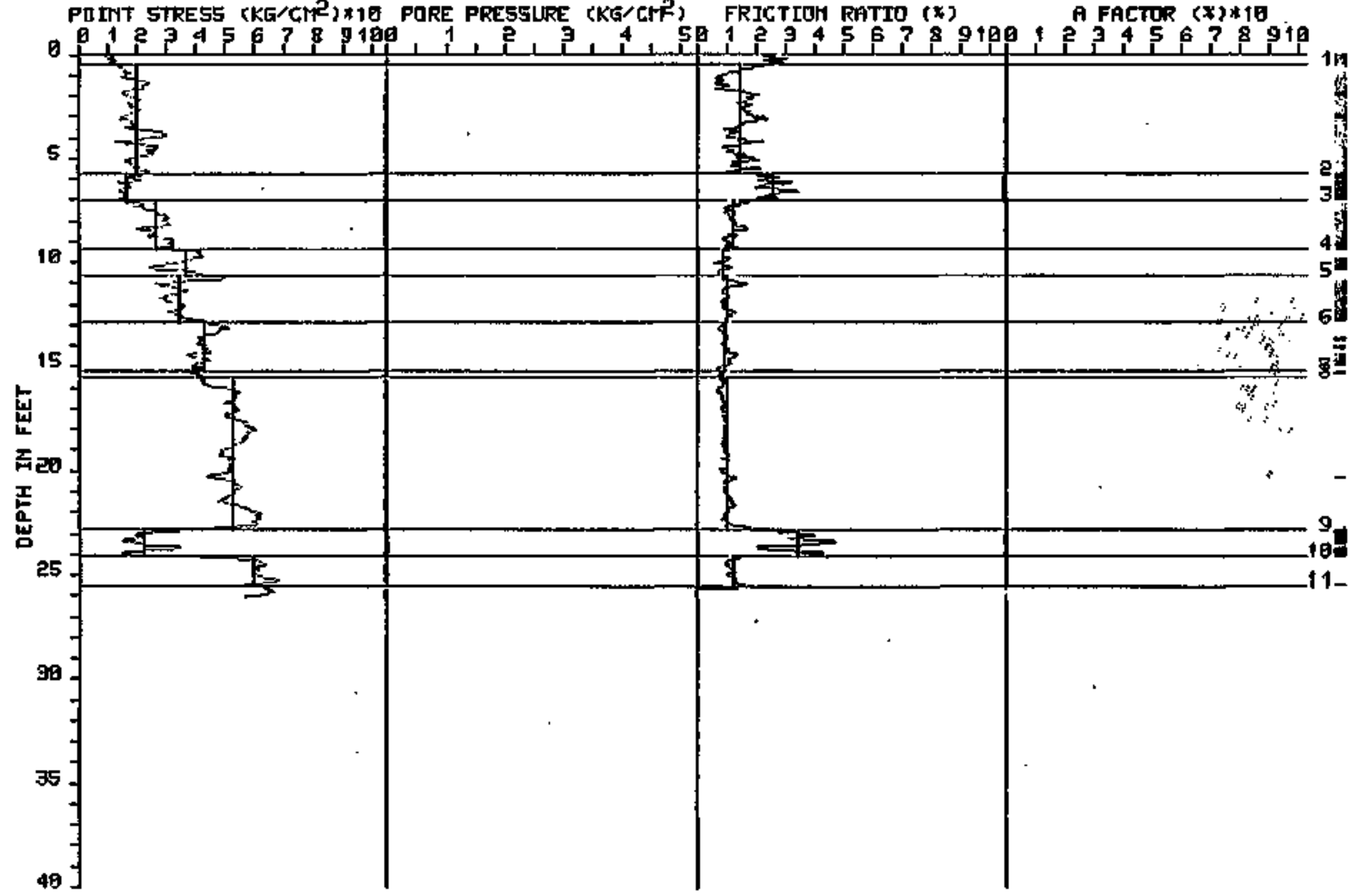




\section{PIEZDCONE SOUNDIMG TEST}

SITE ID: HATO1

PODTT STRESS (KG/CME) *10 PORE PRESSLRE (KG/CRF) FRICTIOM RATIO (x) A FACTOR (x)*10

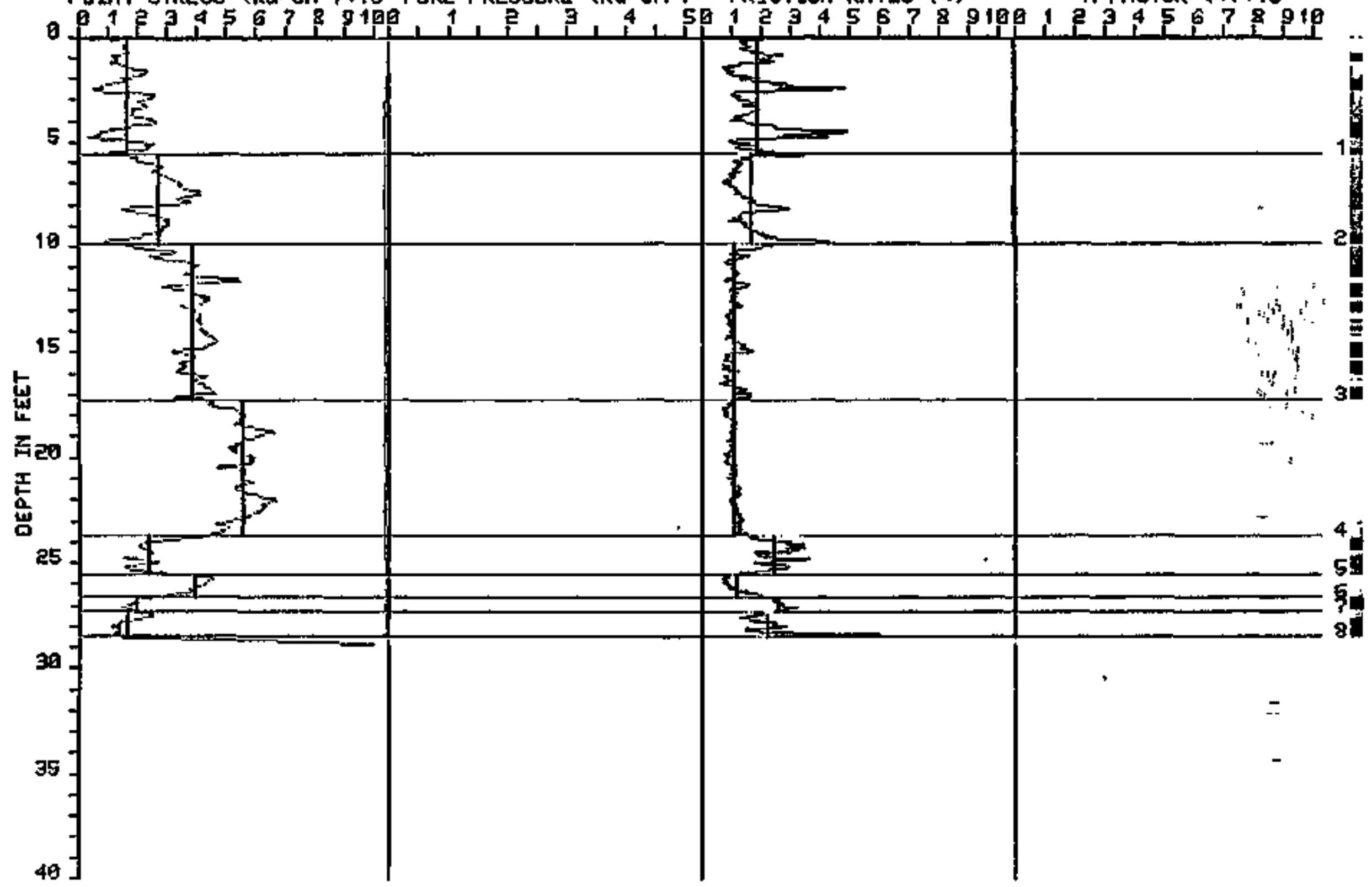




\section{PIEZOCONE SOUNDIMG TEST}

SITE ID: HATO1

LOCATION ID: 036

PDINT STRESS (KG/CMP)*10 PORE PRESSURE (KG/CHF) FRICTION RATID (*)

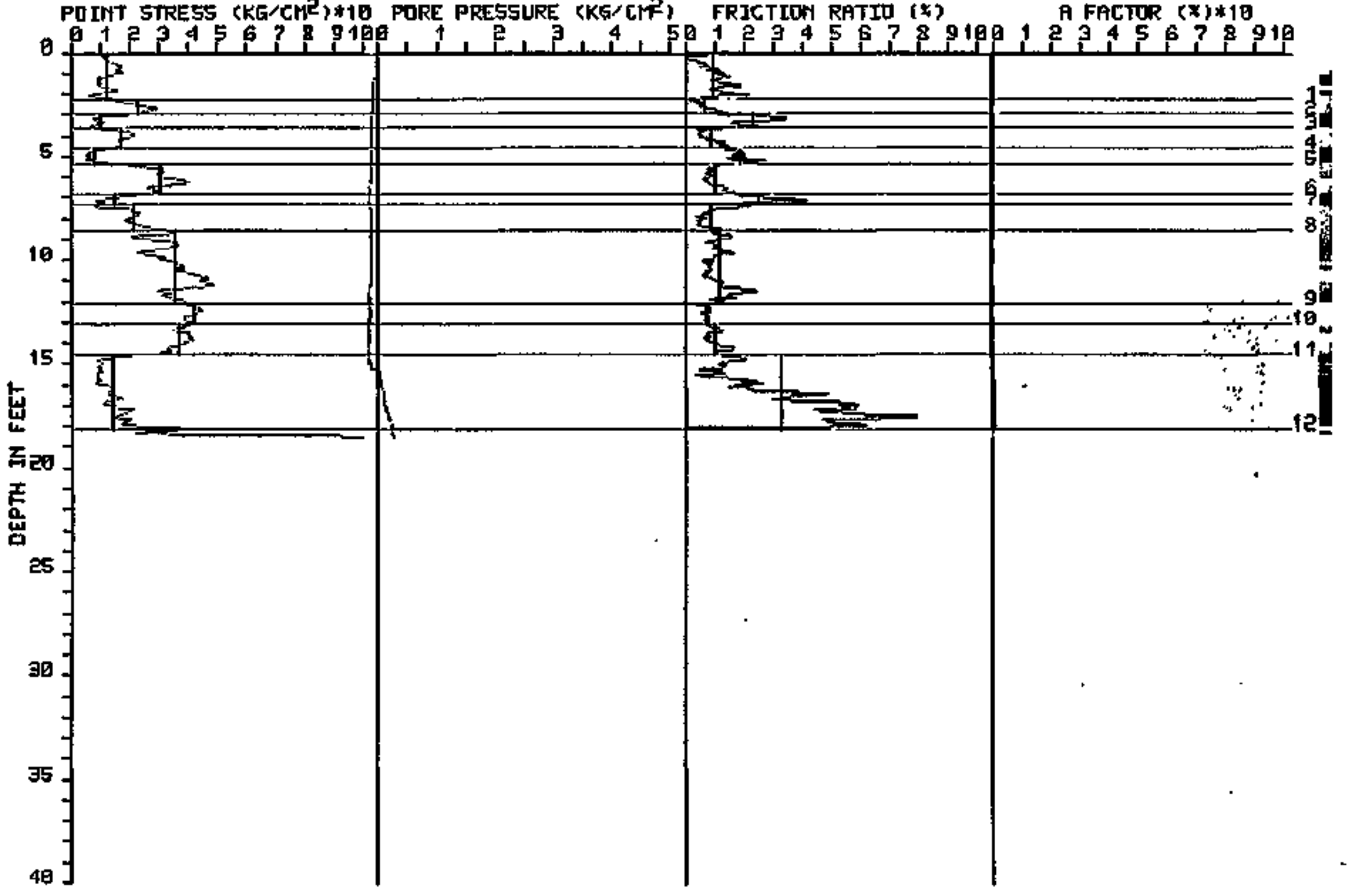


PIEZOCONE SOUNDING TEST

SITE ID; HATES

- LOCATION IDE 037

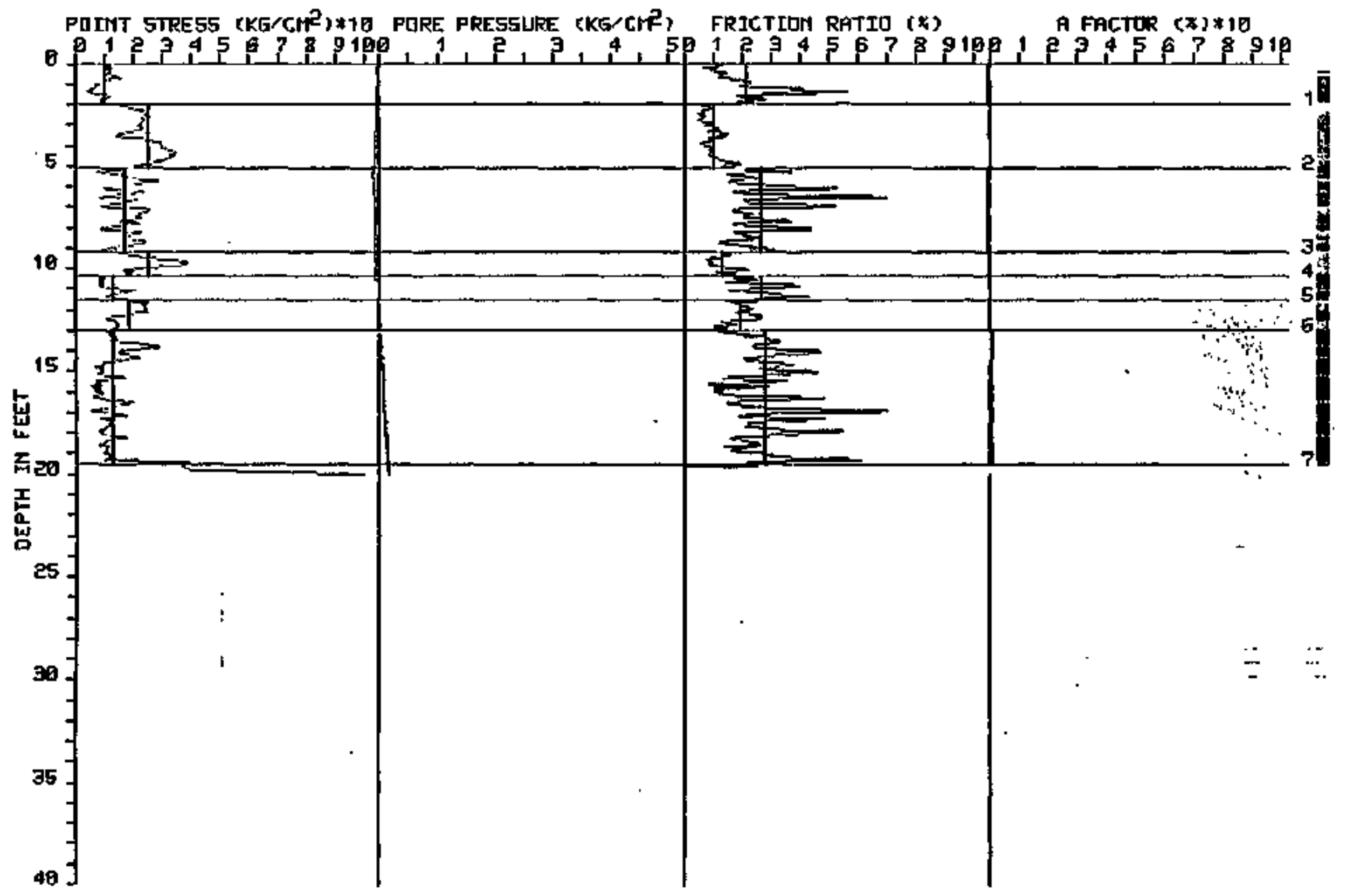




\section{PIEZOCONE SOUNDING TEST}

SITE TD: HATOI

LOCATION ID: 038

PDINT STRESS (KG/CHP) *IV PORE PRESSURE (KG/CME) FRICTIOM RATIO (*)

A FACTOR $(z) * 10$

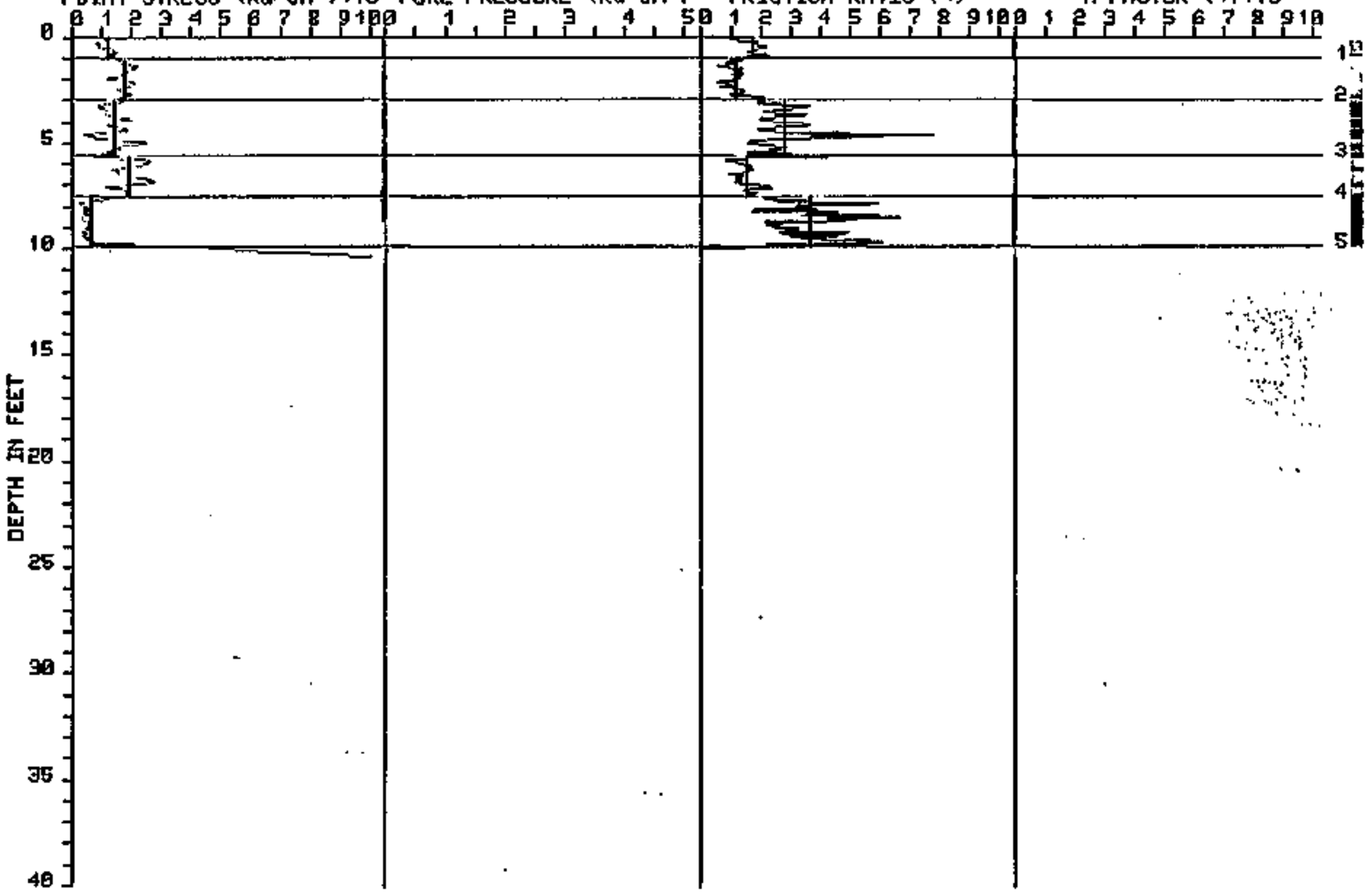




\section{PIEZOCONE SOUNDING TEST}

SITE ID: HATOI

LOCATION IO: OBS

POINT STRESS (KE/CM2) *10 PORE PRESSURE KKG CIF)

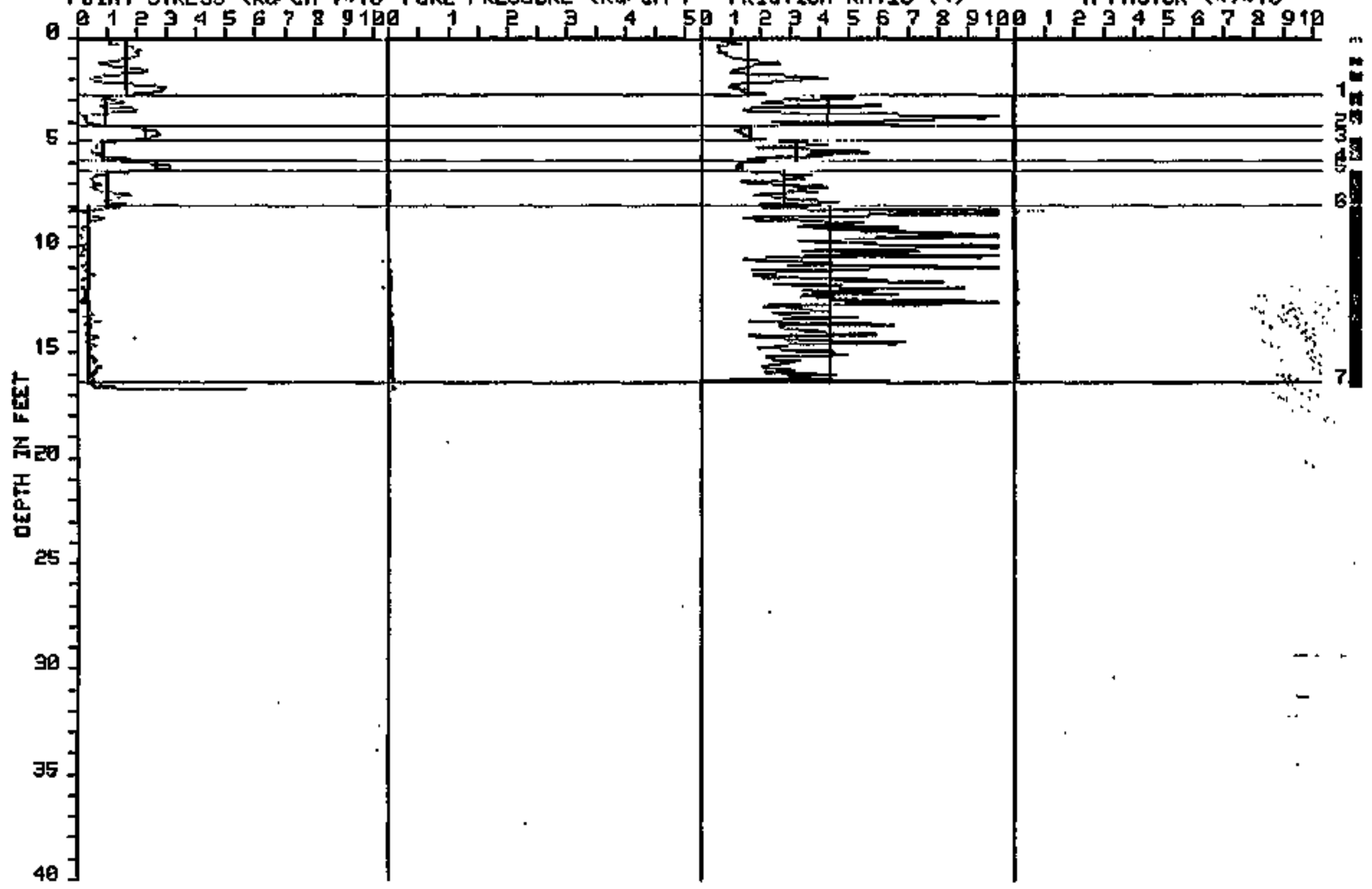




\section{PIEZOCONE SOUNDING TEST}

SITE IDE HATET

LOCATION ID: 040

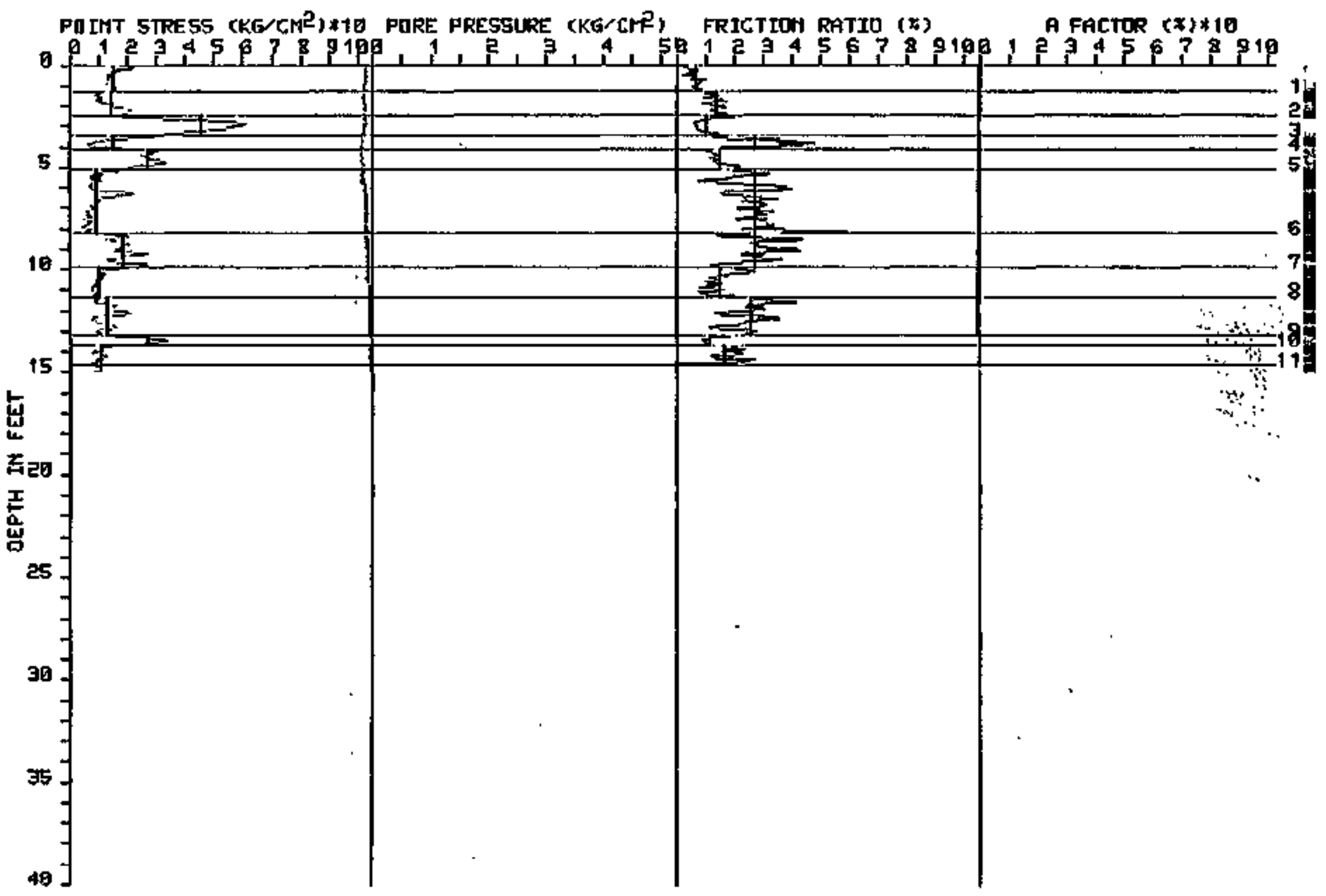


SITE ID: HATU1

GROUND WATER OEPTH: 50.0 (FT.)
LOCATION ID: 004

AUERAGE SOIL WEIGHT: 100.0 (PSF)

DEPTH AUG. AUG. UND.

- DEPTH - AUG. AUG. PORE FRICT. SOIL SHEAR LY FROM TO THK. POINT SLEEUE PRESS. RATIO CLASS BC DEN FA ELAS. STREN OCR

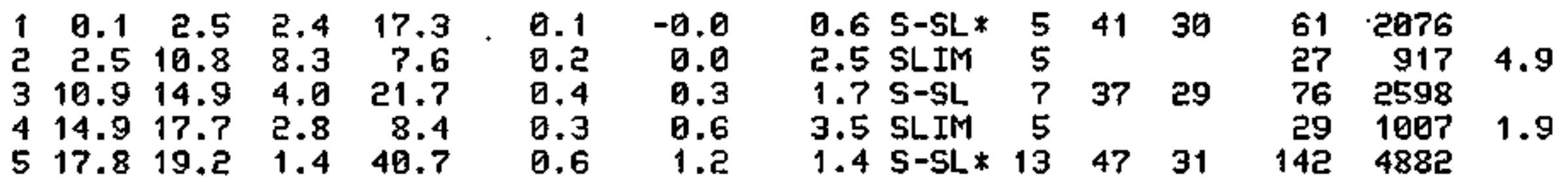

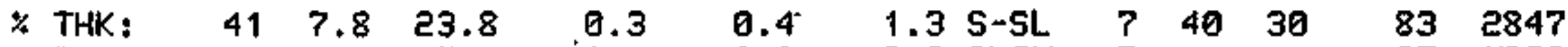

\% THK: $\begin{array}{lllllllll}58 & 11.1 & 7.8 & 0.2 & 0.2 & 2.8 \text { SLIM } 5 & 27\end{array}$ 
SITE ID: HATO1

GROUND WATER DEPTH: 50.0 (FT.)
LOCATION ID: 005

AUERAGE SOIL WEIGHT: 100.0 (PSF)

- DEPTH - AUG. AUG. PQRE FRICT, SOIL

AUG. AUG.

UND.

SHEAR

LY FROM TO THK. POINT SLEEUE PRESS. RATIO CLASS BC DEN FA ELAS. STREN OCR

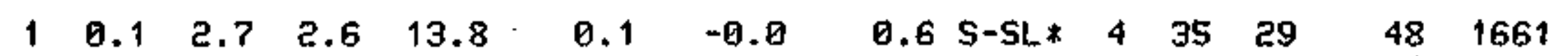

$\begin{array}{lllllllll}2 & 2.8 & 19.7 & 16.9 & 6.0 & 0.2 & 0.1 & 3.9 & \text { SLIM } 3\end{array}$

$21 \quad 718$

2.5

\% THK: 13 2.6. 13.8

0.1

0.0-0.6 S-SL $435 \quad 29$

$48 \quad 1660$

$\%$ THK:

$86 \quad 16.9$

6.0

0.2

0.1

3.9 SLIM 3

21

718

2.5 
ADOENDUH G7

ADOITIOHAL RADIOLOGICAL DATA 
Radon monitoring was performed at the Hexican Hat site from $08 / 27 / 95$ until $09 / 22 / 86$ in four separate quarterly periods: $08 / 27 / 95$ to $11 / 21 / 85$; $11 / 21 / 8$ s to $02 / 28 / 86$; 02/28/86 tó 065/06/g6; and 06/06/86 to 09/22/86. Nineteen monitoring locations were estabjished from the edge of the Hexican Hat stte to 2500 meters away. Each mopitoring location housed two to five fild racion detectors. Multiple detectors were placed at each location to determine an average Rn-220 concentration and to avoid Joss of data from a single camaged detector. The monitoring locations are illustrated in Figure p7.1. The mean radon concentration at each location for each quarterly period is given in Table or. 3 .

Table 07.1 Radon (Rn-222) concentrations, Mexican Hat site, Utah

\begin{tabular}{|c|c|c|c|c|}
\hline \multirow[b]{2}{*}{$\begin{array}{l}\text { Location } \\
\text { number }\end{array}$} & \multicolumn{4}{|c|}{ Concentration for the period (in pCi/1) } \\
\hline & $\begin{array}{c}08 / 27 / 85 \text { to } \\
11 / 21 / 85\end{array}$ & $\begin{array}{c}11 / 21 / 85 \\
02 / 28 / 86\end{array}$ & $\begin{array}{c}02 / 28 / 86 \\
06 / 06 / 86\end{array}$ & $\begin{array}{c}06 / 06 / 86 \text { to } \\
09 / 22 / 86\end{array}$ \\
\hline $\begin{array}{r}1 \\
2 \\
3 \\
4 \\
5 \\
6 \\
7 \\
8 \\
9 \\
10 \\
11 \\
12 \\
13 \\
14 \\
75 \\
16 \\
17 \\
19 \\
20\end{array}$ & $\begin{array}{l}5.9 \\
6.3 \\
12 \\
3.7 \\
0.57 \\
4.0 \\
0.57 \\
0.97 \\
0.44 \\
0.67 \\
0.60 \\
0.39 \\
-.9 \\
- \\
0.84 \\
0.45 \\
7.7 \\
0.20 \\
0.62\end{array}$ & $\begin{array}{l}12 \\
9.4 \\
15 \\
4.7 \\
0.73 \\
6.7 \\
0.91 \\
2.1 \\
0.74 \\
1.2 \\
0.77 \\
0.65 \\
0.47 \\
0.64 \\
1.3 \\
0.98 \\
2.0 \\
0.37 \\
0.94\end{array}$ & $\begin{array}{l}6.6 \\
- \\
9.5 \\
2.9 \\
0.56 \\
4.7 \\
0.51 \\
- \\
0.36 \\
0.64 \\
0.57 \\
0.31 \\
0.27 \\
0.40 \\
0.76 \\
- \\
0.97 \\
0.30 \\
0.48\end{array}$ & $\begin{array}{l}- \\
- \\
- \\
- \\
- \\
- \\
1.3 \\
1.5 \\
0.61 \\
0.86 \\
1.3 \\
- \\
0.48 \\
- \\
1.2 \\
- \\
- \\
0.36 \\
0.51\end{array}$ \\
\hline $\begin{array}{l}\text { Hean of nine } \\
\text { background } \\
\text { locations } \\
\text { Mean of four on } \\
\text { sjte locations }\end{array}$ & 0.50 & 0.76 & 0.42 & 0.74 \\
\hline
\end{tabular}

a- Indicates e1ther damaged or stolen detector. 


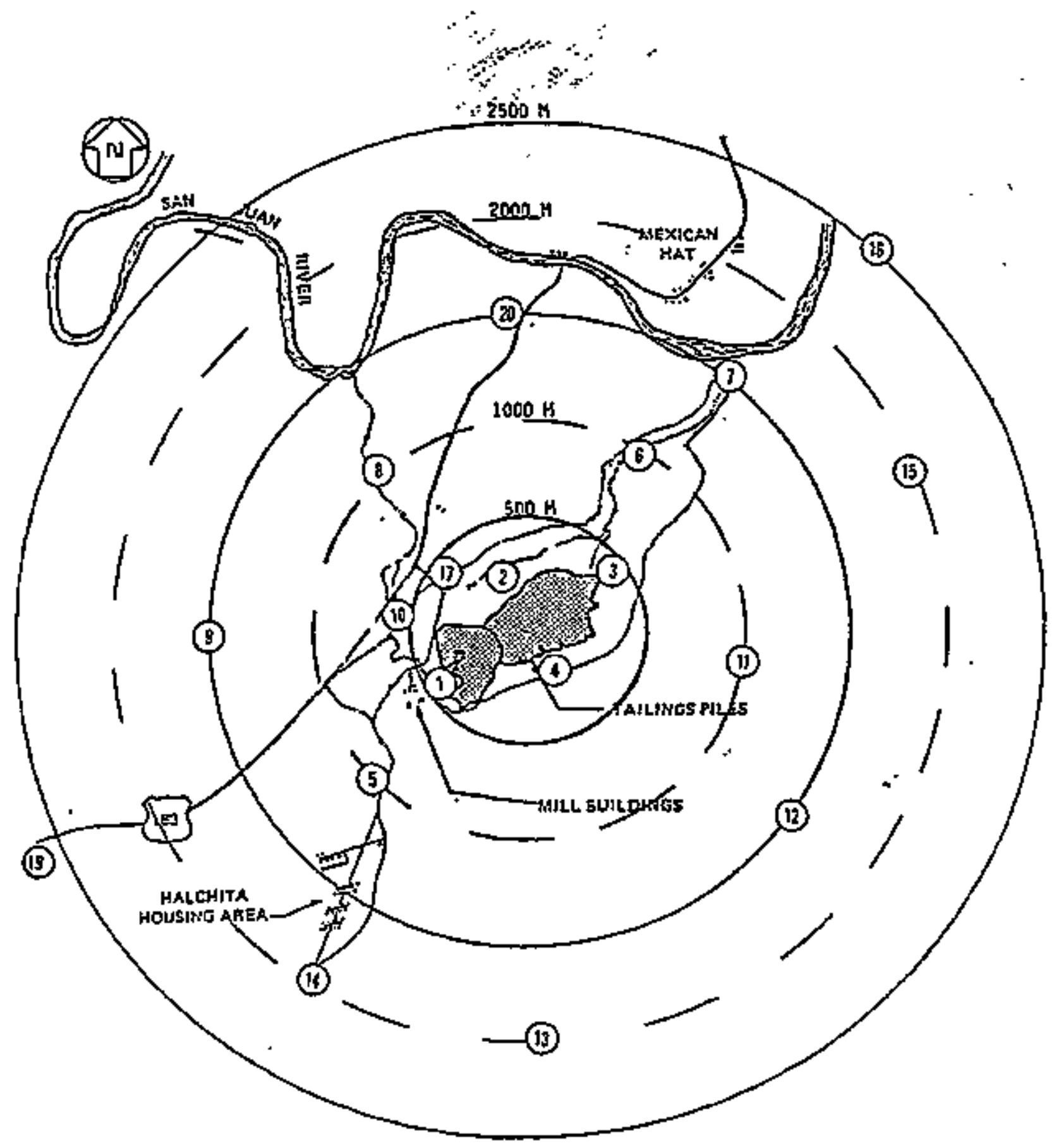

FIGUAE 07.1

RADON MONITORING LOCATIONS

MEXICAN HAT, UTAH 
Locations 1, 2, 3, and 4 are on the Mexican hat uranium m1ll tallings site and nay be used to deterinine the on-site radon concentration. The average radon concentration for the four sites ranged from 6.3 to $10.3 \mathrm{pCt} / 1$ for the four quarterly periods. jucations $5,6,8,10,11$, and 17 are not on the site and are too close to the site to be considered background locations. Monitoring locations $7,9,12,13,14,15,16,19$, and 20 are 1500 meters or more from the site and mis be considered background locations. The average background radon concentration over the nine background locations was 0.50 $\mathrm{pC}\} / 1$ for the pertod $08 / 27 / \mathrm{BS}$ to $11 / 21 / 85$; 0.78 pCj/1 for $11 / 21 / 85$ to $02 / 28 / 86 ; 0.42 \mathrm{pCi} / 1$ for $02 / 28 / 86$ to $06 / 06 / 86$; and $0.74 \mathrm{pCi} / 1$ for $06 / 06 / 86$ to $09 / 22 / 86$. The background radon concentration in afr shows a small seasonal variation wth a range of 0.42 to $0.78 \mathrm{pC} / 1$.

Background gamia measurements were performed at foisr locations in the vicinity of the Hexican Hat site using a pressurized fonization chamber. Two measurements were performed at each location, giving an average ganna exposure rate of $13.6 \mu R / h r$ for the elght measurements. The locations of the background measurements were from 0.5 to five miles from the tailings site and are beyond the influence of the stte. Gamm exposure rate is independent of the frequency and time of measurement for background measurements.

The arroyos extending beyond the $5 \mathrm{pCi} / \mathrm{g}$ windblown contanination linit are illustrated in Plate 1, Radiological Contamination Distribution Map. The contamination material was characterized using delta measurements and the data are suminarized in Table 01.2 of Addendum 01 . These data indicate that contamination may exist up to 2500 feet along the length of the four arroyos outside of the $5 \mathrm{pci} / \mathrm{g}$ windbiown boundary. Assuming a 100-foot width and a six-1nch depth of contarinated material in the arroyos gives a volume estinate of $5500 \mathrm{cy}$. The average Ra-226 concentration is $10.4 \mathrm{pCi} / \mathrm{g}$. This small volume of contaminated material will have a negligible impact on the final pile design.

Further characterization by the RAC will be necessary in the arroyos to deteraine the exact volume of contaminated materials since the contanination is largely associated with sandbars and probably does not cover the entire length and width of the arroyos, zero- to six-inch soil sanples may adequately characterize the materials tn the arroyos north and east of the site. The arroyo at the northeast corner of the site will be characterized further downstrean than is indicated on Plate 1 to a length of 1600 feet from the windblown border. This characterization will be performed by the RAC during renedial action. 